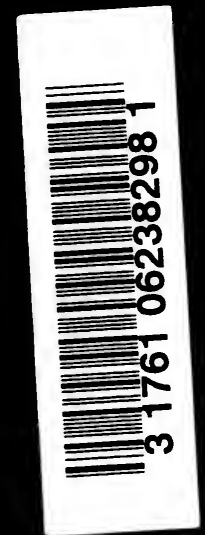

INIVGA:

lorowity

LBRART 

Digitized by the Internet Archive in 2007 with funding from Microsoft Corporation 


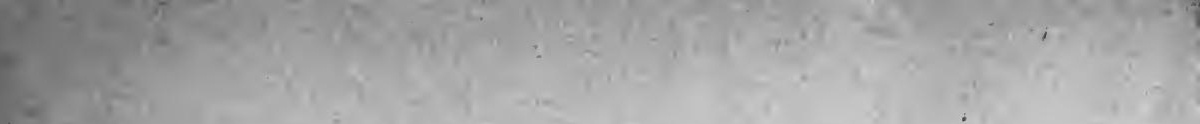

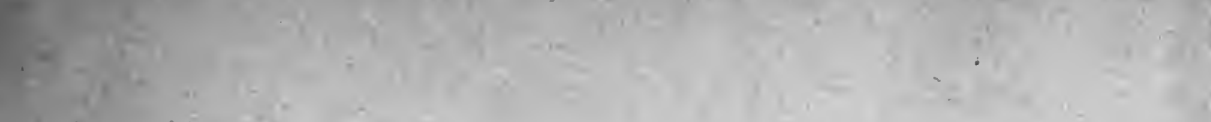

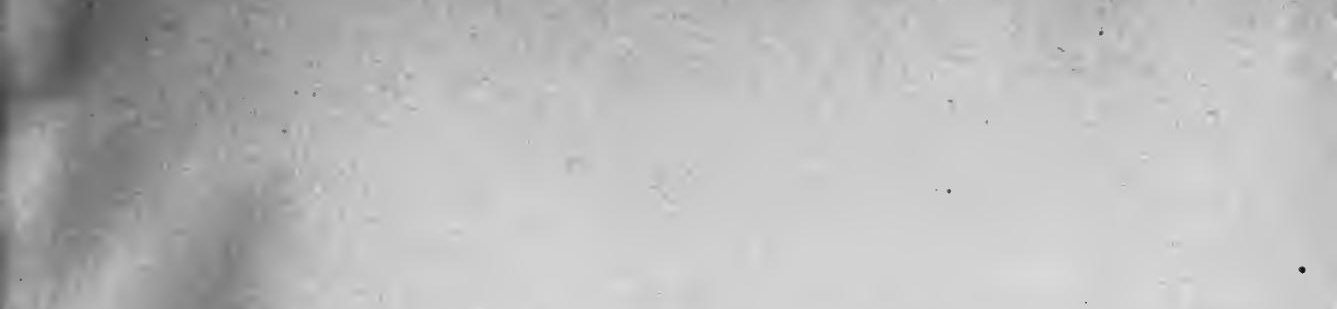

$\cdot$ 



\title{
Differential Diagnosis
}

VOLUME I

PRESENTED THROUGH AN ANALYSIS OF 385 CASES

\author{
RICHARD C. ${ }^{3}$ CABOT, M. D.
}

PROFESSOR OF CLINICAL MEDICINE, HARVARD UNIVERSITY MEDICAL SCHOOL, BOSTON CHIF'F OF THE WEST MEDICAL SERVICE

AT THE

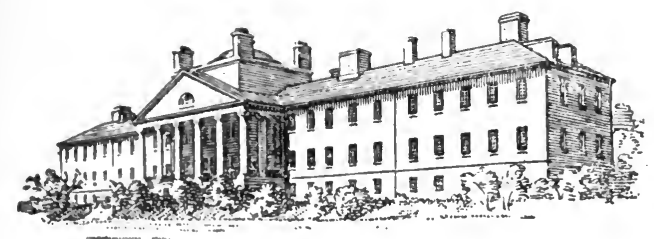

MASSACHUSETTS GENERAL HOSPITAL

FOURTII EDITION, REVISED PROFUSELY ILLUSTRATED 
Copyright, Igı, by W. B. Saunders Company. Reprinted March, rgrr, June, 19rx, and October, rgrr. Revised, reprinted, and recopyrighted February, 1912. Reprinted October, 1912, January, 1913, July, rgr3, and February, r9r4. Revised, reprinted, and recopyrighted January, rgr5. Reprinted January, r916, and July, 1917. Revised, reprinted, and recopyrighted September, rgrg.

Copyright, 1919, by W. B. Saunders Company

Reprinted May, 1920

PRINTED IN AMERICA

PRESS OF

W. B. SAUNDERS COMPANY

PHILADELPHIA 


\section{PREFACE TO THE FOURTH EDITION}

New matter has been inserted relating to pyloric spasm, the streptococcus infections of the tonsils and teeth, the "dome pain" arising from irritations in the dome of the diaphragm, the splanchnic circulatory changes and their effect upon cerebral circulation, migraine, chronic prostatitis as a cause of backache, prostatic cancer and its diagnosis, peptic ulcer and its frequency, cholecystitis and cholangitis as causes of epigastric pain and chronic appendicitis. A good deal of new matter has also been introduced relating to pain in the arms, also to spurious fevers (malingering), bed fevers, relapsing fevers, and terminal fevers.

I have availed myself of the admirable papers by Drs. Bissell and LeCount, on coma, published in the Journal of the American Medical Association, February I7, I9I7, since the last edition of this book. Their studies in the diagnosis of coma, supposed to be due to uremia, are especially valuable.

Other new matter relates to the upsetting of renal, hepatic, cardiac, and metabolic inequalities in chronic disease by acute infection.

R. C. C.

1 Marlborough Street Boston, Mass.. 



\section{PREFACE}

THE attempt to make and defend a differential diagnosis brings all one's failings into sharp relief. Though I have done my best to avoid obvious errors in this book, I am confident that it contains much that deserves-and I hope will receive-challenge from other physicians.

My best thanks are due to Dr. James H. Young for help in the diagrams, and to my secretary, Miss Edith K. Richie, who has made the index and rendered many invaluable services throughout the preparation of the book. 



\section{TABLE OF CONTENTS}

\section{INTRODUCTION}

The Presenting Symptom................................... I 7

The Grouping of Reasonable Possibilities......................... I7

Advantages of the Plan Here Adopted........................... I8

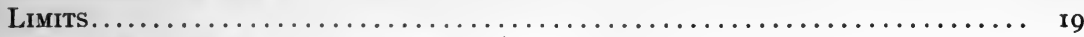

Vulnerability of All Differential Diagnosis................... I9

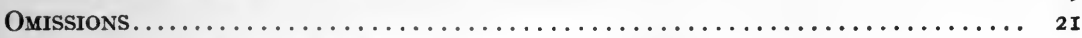

Explanation of Diagrams and Charts......................... 22

\section{CHAPTER I}

\section{PAIN}

General Considerations.................................. 24

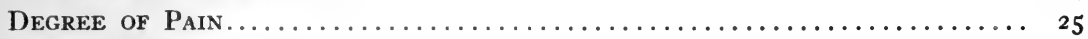

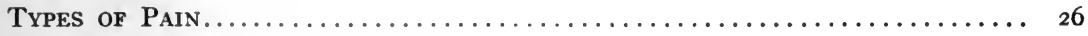

Relation of Pain to Other Facts............................ ${ }_{2} 6$

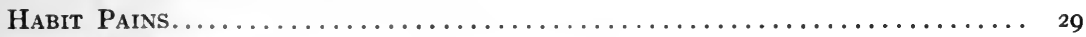

Theories Regarding the Production of Pain..................... 29

\section{CHAPTER II}

\section{HEADACHE}

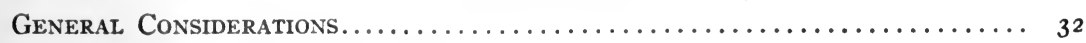

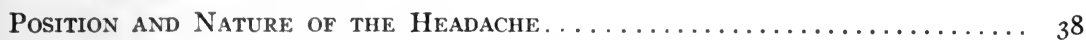

Two Traditional Fallacies About Headache.................... 39

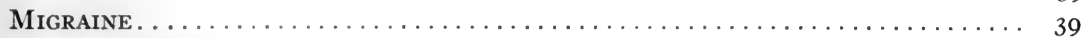

IMPORTANT TESTS. .................................. 4 Q

CASE No.

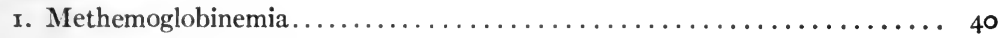

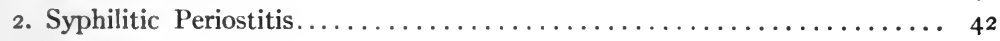

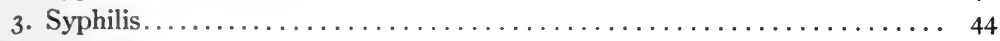

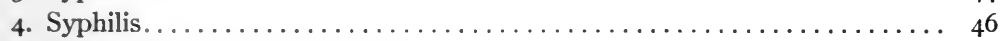

5. Headache of Psychic Origin........................... 48

6. Uremic Headache; Chronic Glomerulonephritis; Graves' Disease...... 50

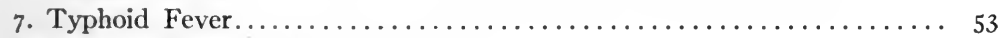

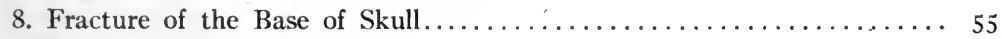

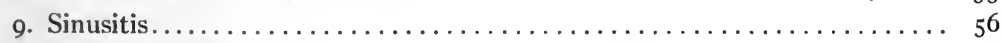

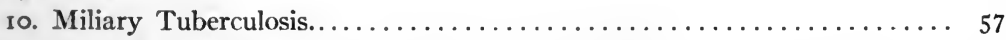

Ir. Stone in the Kidney with Abscess and Nephritis .............. 59

12. Malaria.......................................

13. Paroxysmal Tachycardia Complicating a Chronic Myocardial Insufficiency. 62

14. Postpneumonic Empyema............................ 64 
CASE No.

15. Staphylococcus Infection (Osteomyelitis)

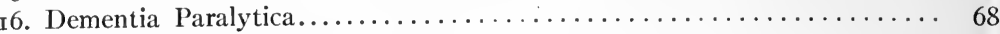

I7. Headache of Unknown Origin $\ldots \ldots \ldots \ldots \ldots \ldots \ldots \ldots \ldots \ldots \ldots \ldots \ldots \ldots$

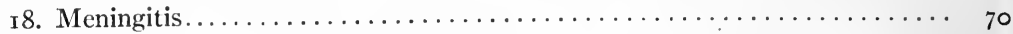

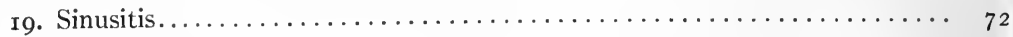

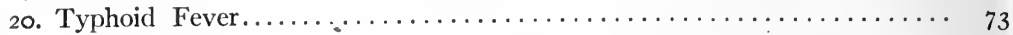

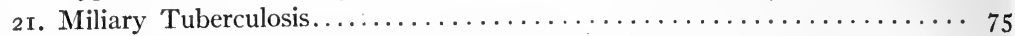

22. Cerebral Hemorrhage..................... 77

\section{CHAPTER III}

LUMBAR PAIN

Examination of Patients with Lumbar Pain..................... 88

CASE No.

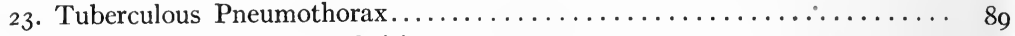

24. Hypertrophic Spinal Arthritis.......................

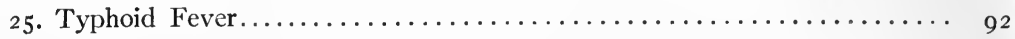

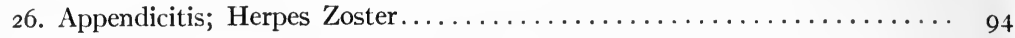

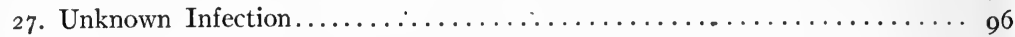

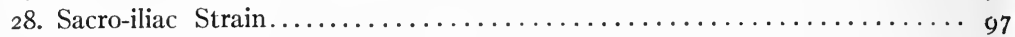

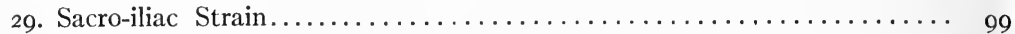

30. Renal Infection, Hematogenous or Ascending.............. roo

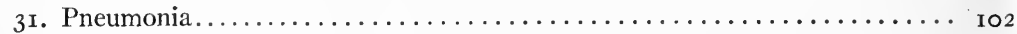

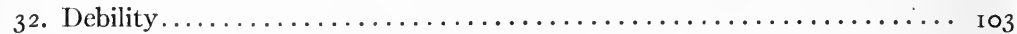

33. Typhoid and Colon Bacillus Infection $\ldots \ldots \ldots \ldots \ldots \ldots \ldots \ldots \ldots \ldots \ldots$

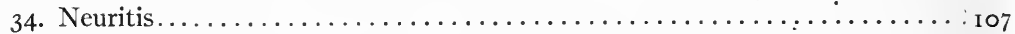

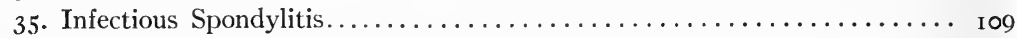

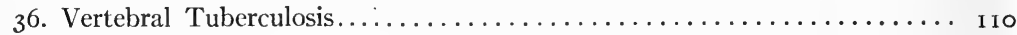

37. Aortic Aneurysm.............................. I I I

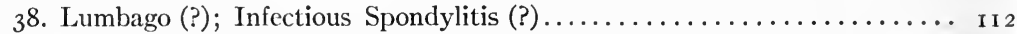

39. Renal Stone; Multiple Renal Abscess ................... II3

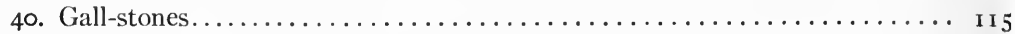

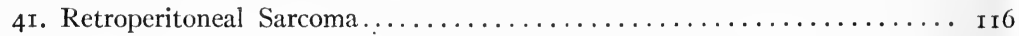

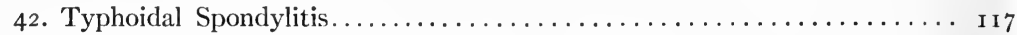

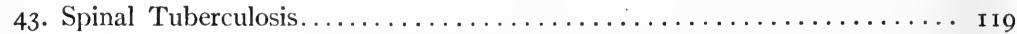

44. Old Syphilis; Acute Spondylitis..................... I 20

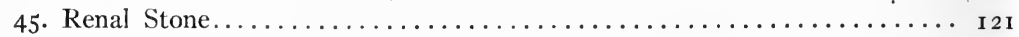

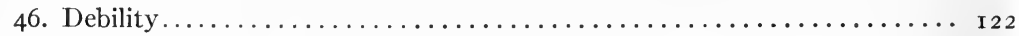

47. Streptococcus Meningitis ....................... I 23

48. Prolapsed, Retroverted, Incarcerated, Pregnant Uterus............ I 24

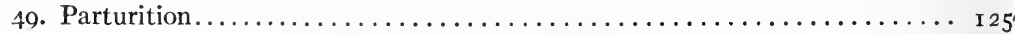

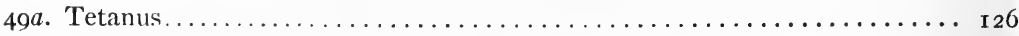

\section{CHAPTER IV}

CASE No.

\section{GENERAL ABDOMINAL PAIN}

50. Neurosis; Mucous Colitis............................. I30

51. Bad Hygiene.................................. I34

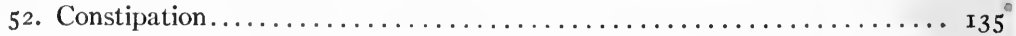

53. Peritoneal Tuberculosis............................ I36

54. Postoperative Neurosis........................... I38 
CAse No.

55. Typhoid Fever............................. I39

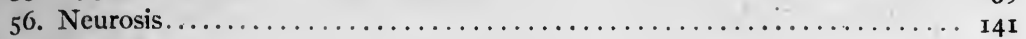

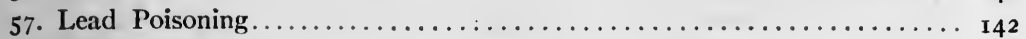

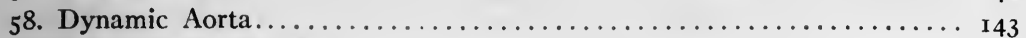

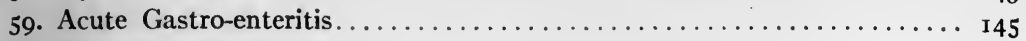

60. Cancer of the Stomach.............................. I46

6r. Recurrent Intestinal Cancer......................... r48

62. Tertian Malaria................................. 150

63. Perforative Colitis and General Peritonitis.................. I5 I

64. Cancer of the Rectum.............................. 152

65. Obstruction of the Intestine; Volvulus.................. I 53

\section{CHAPTER V}

CASE No.

\section{EPIGASTRIC PAIN}

66. Hepatic Gumma; Syphilis............................ 159

67. Plumbism ................................. I6

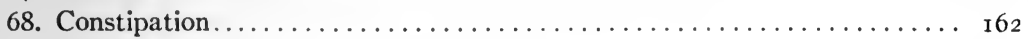

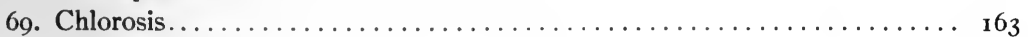

70. Tabes Dorsalis.................................. I64

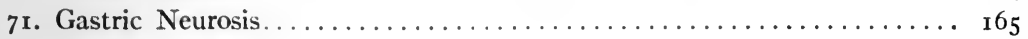

72. Duodenal Ulcer................................. I66

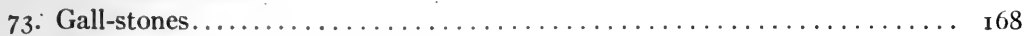

74. Hyperchlorhydria (Alcoholism?) ....................... I70

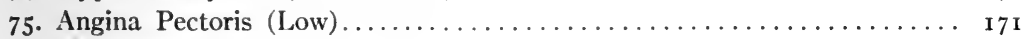

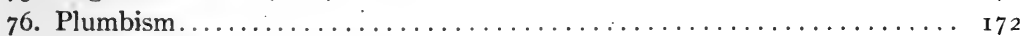

77. Sarcoma Testis with Metastases...................... I 74

78. Tuberculous Peritonitis............................. I75

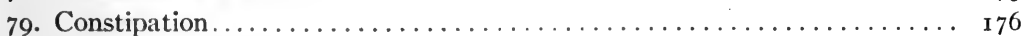

8o. Pyloric Adhesions............................... 177

8I. Cholelithiasis and Gangrenous Gall-bladder.................. I 79

82. Cholelithiasis with Perforations...................... I $8_{1}$

83. Acute Pericarditis............................. $18_{2}$

84. Arteriosclerosis; Vascular Crises......................... I 84

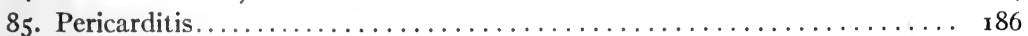

86. Gastric Cancer.................................... 187

87. Pancreatic Cancer (Chronic Pancreatitis ?) .................. I 89

88. Gastric Ulcer.................................... 190

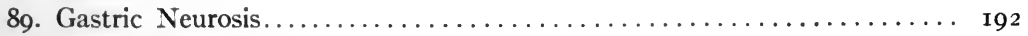

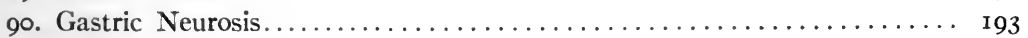

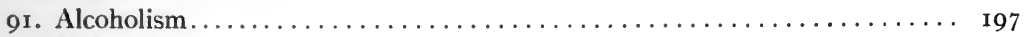

92. Melancholia....................................... 198

93. Hepatic Congestion (Uncompensated Valvular Heart Disease)........ 200

94. Gall-stones.................................... $20 \mathrm{I}$

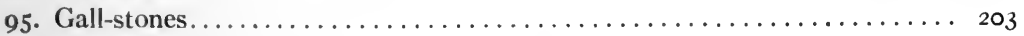

CHAPTER VI.

RIGHT HYPOCHONDRIAC PAIN

Case No.

96. Pericardial Effusion.

97. Renal Stone. 
Case No.

98. Catarrhal Jaundice..............................

99. Hepatic Cancer................................... 215

100. Alcoholic Gastritis................................ 2 I 8

ror. Fibrous Endocarditis of the Mitral and Aortic Valves, with Insufficiency of Both.................................. 2 I9

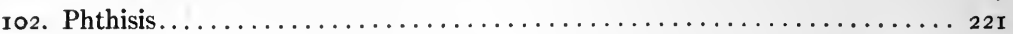

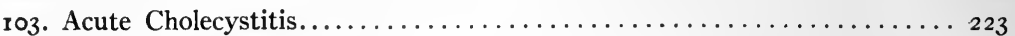

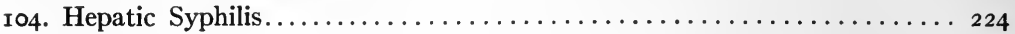

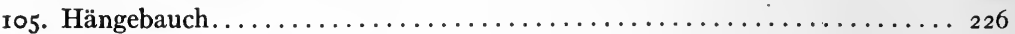

106. Stone in Ductus Choledochus.......................... 228

107. Debility; Floating Kidney............................. 229

108. Debility.......................................

Iog. Subdiaphragmatic Abscess........................... 233

I 10. Pancreatic Cancer..................................... 234

II I. Pericecal Tuberculosis................................ 237

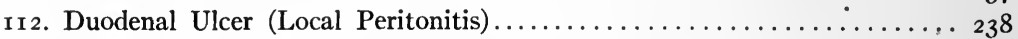

I 3. Hysteria Minor.................................... 240

I14. Cholecystitis Complicating Typhoid Fever.................. 242

\section{CHAPTER VII}

\section{PAIN IN THE LEFT HYPOCHONDRIUM}

CASE No.

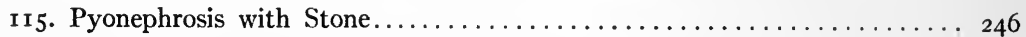

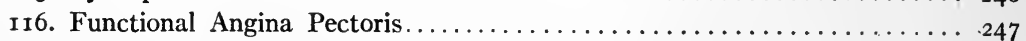

I17. Congenital Cystic Kidneys................................ 250

I18. Myeloid Leukemia....................................... 25 I

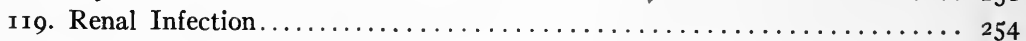

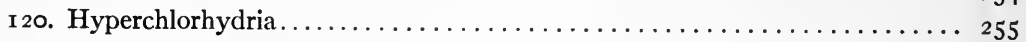

121. Renal Stone..................................... 257

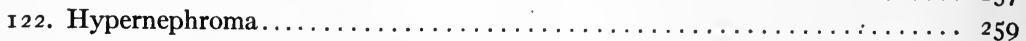

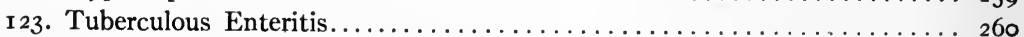

\section{CHAPTER VIII}

CASE No.

\section{RIGHT ILIAC PAIN}

124. Pericecal Tuberculosis............................. 262

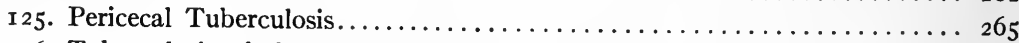

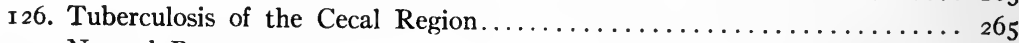

1 27. Normal Pregnancy...................................... 266

128. Ovarian Cyst with Twisted Pedicle........................ 267

129. Tabes Mesenterica.................................. 268

130. Ovarian Cyst with Twisted Pedicle....................... 269

I3I. Ruptured Ovarian Cyst............................. 270

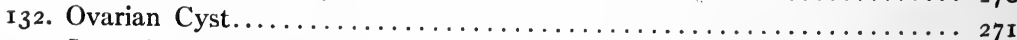

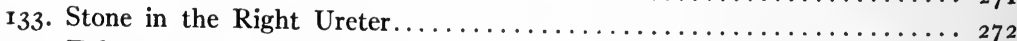

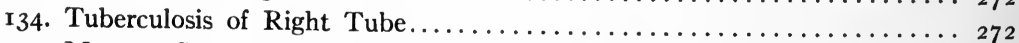

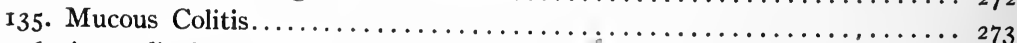

136. Appendicular Colic (Chronic Appendicitis) ..................... 274

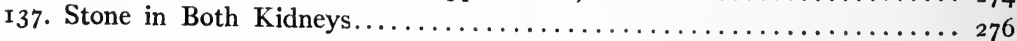




\section{CHAPTER IX}

\section{LEFT ILIAC PAIN}

CASE No.

PAGE

138. Perforated Gastric Ulcer......................

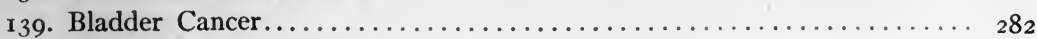

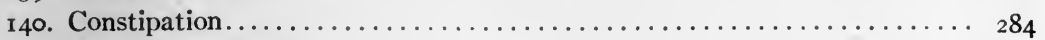

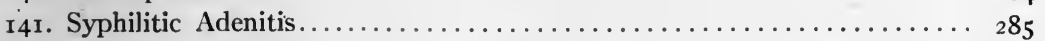

142. Multilocular Ovarian Cyst (Twisted Pedicle) ............... 286

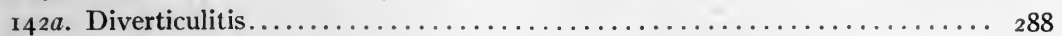

General Consideration of the Diagnosis of Abdominal Pain.......... 290

\section{CHAPTER $\mathrm{X}$}

CASE No.

\section{AXILLARY PAIN}

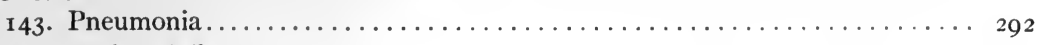

144. Broken Rib................................... 296

145. Unknown Infection $\ldots \ldots \ldots \ldots \ldots \ldots \ldots \ldots \ldots \ldots \ldots \ldots \ldots \ldots \ldots$

146. Angina Pectoris............................... 299

147. Syphilitic Heart and Aorta...................... 300

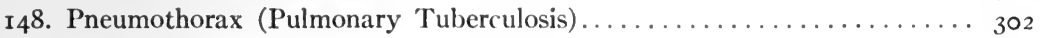

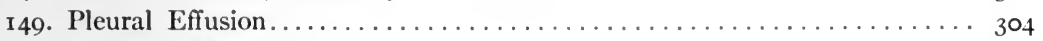

150. Artificial Menopause........................... 306

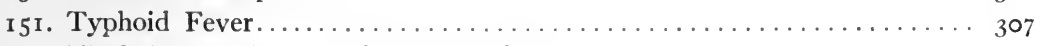

152. Weak Heart; Acute Pulmonary Edema.................... 309

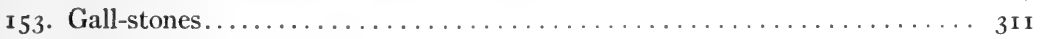

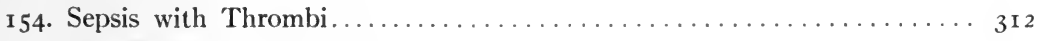

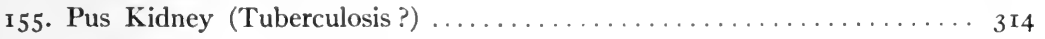

156. Neurosis . . . . . . . . . . . . . . . . . . . . . . . 316

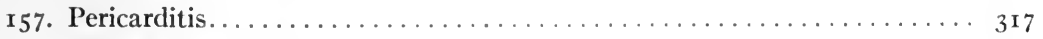

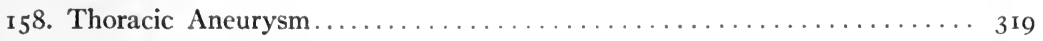

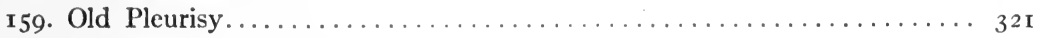

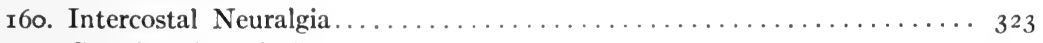

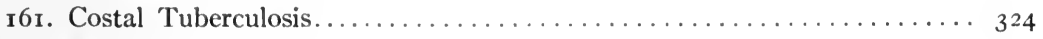

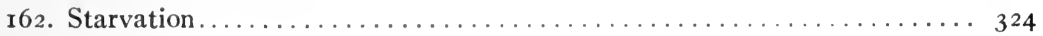

\section{CHAPTER XI}

CASE No.

\section{PAIN IN THE ARMS}

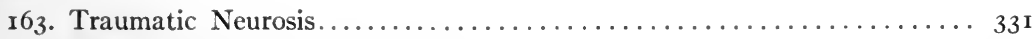

164. Aneurysm (Called Rheumatism) .................... 333

165. Mediastinal Neoplasm (Metastatic) .................. 335

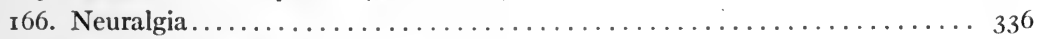

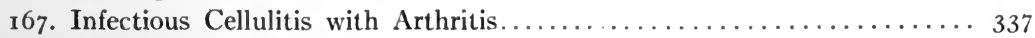

168. Tuberculosis of the Humerus....................... 339

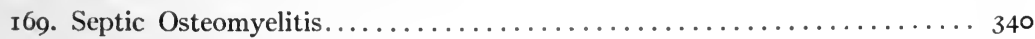

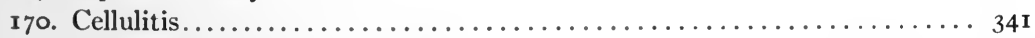

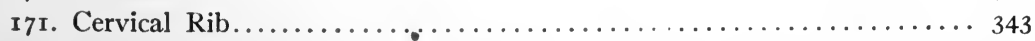

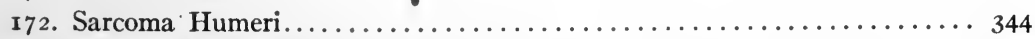

173. Sarcoma Humeri................................ 345 
CASE No.

I74. Septic Osteomyelitis

I75. Thoracic Aneurysm

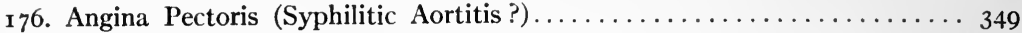

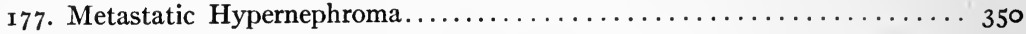

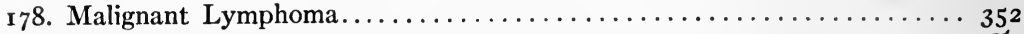

\section{CHAPTER XII}

\section{PAIN IN THE LEGS AND FEET}

CASE No.

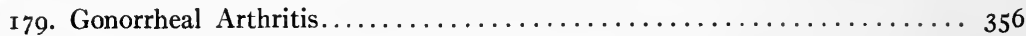

180. Arteriosclerosis; Chronic Nephritis; Pleural Effusion; Terminal Infection . . 359

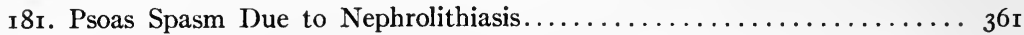

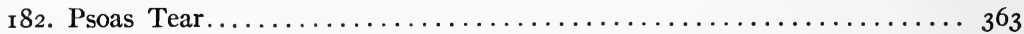

183. Pott's Disease with Psoas Abscess; General Tuberculosis . . . . . . . . . 364

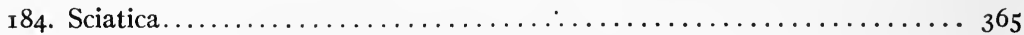

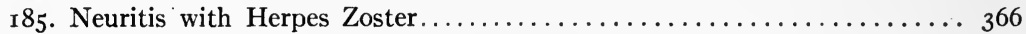

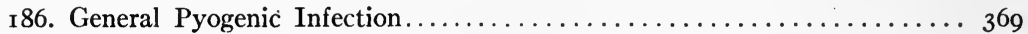

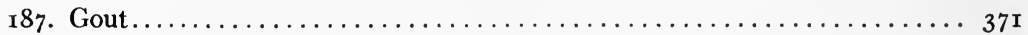

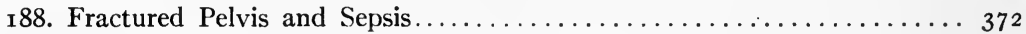

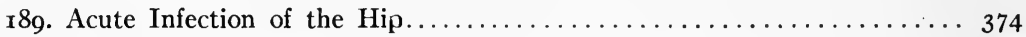

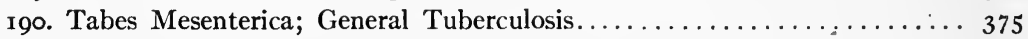

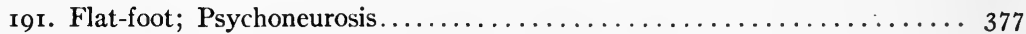

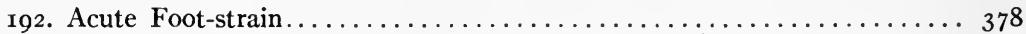

193. Alcoholic Neuritis. . . . . . . . . . . . . . . . . . . . . . 379

194. Pneumococcus Arthritis, Endocarditis (?), and Pneumonia . . . . . . 38 I

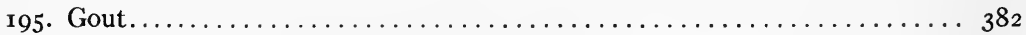

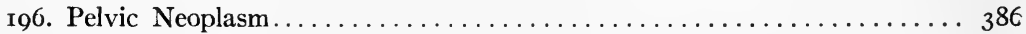

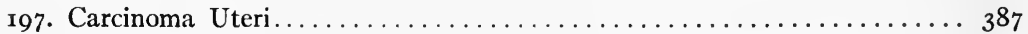

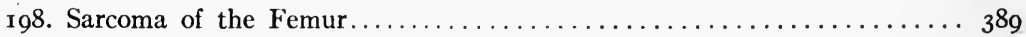

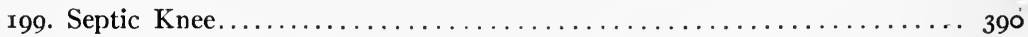

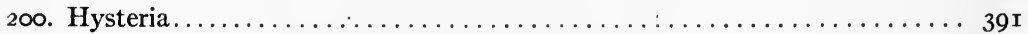

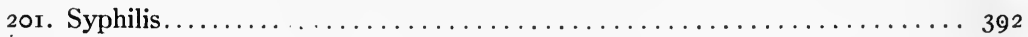

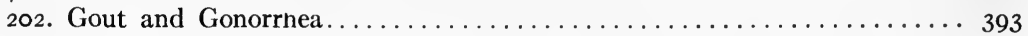

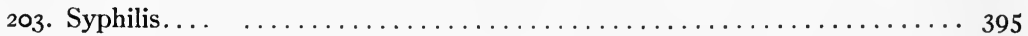

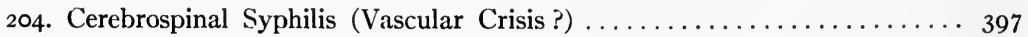

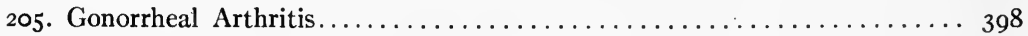

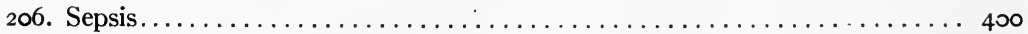

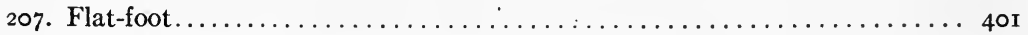

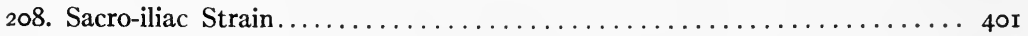

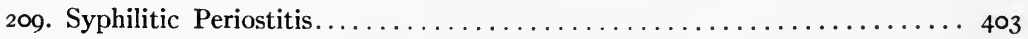

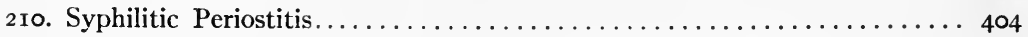

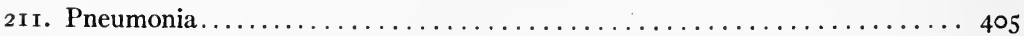

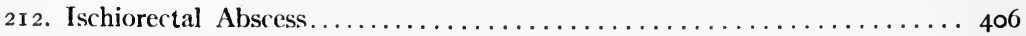

\section{CHAPTER XIII}

\section{FEVERS}

Short Fevers................................... 4 II

Spurious Fevers (Malingering) $\ldots \ldots \ldots \ldots \ldots \ldots \ldots \ldots \ldots \ldots \ldots \ldots \ldots \ldots \ldots \ldots \ldots$ II 
CASE No.

Bed Fevers

Relapsing Fevers.

Terminal Fevers.

Non-Infectious Fevers.

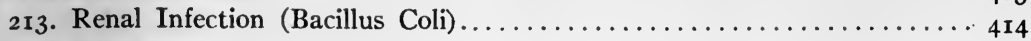

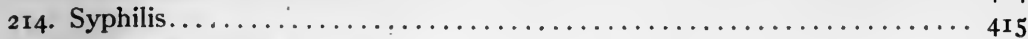

215. Pulmonary Tuberculosis........................ 4 17

2I6. Septic Thrombosis of the Lateral Sinus and Jugular Vein......... 4I8

217. Perirectal Abscess; Perinephric Abscess................. 4 I9

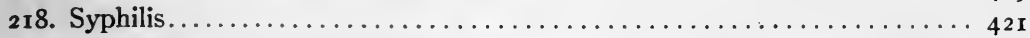

219. Interlobar Postpneumonic Empyema................... 423

220. Poliomyelitis; Renal Infection..................... 424

221. Typhoid Fever with Relapse....................... 426

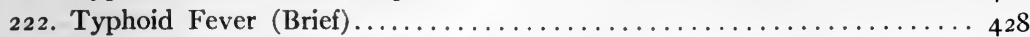

223. Typhoid Fever (Afebrile when First Seen) ................ 430

224. Typhoid Fever; Impaction; Dysuria................. 43 I

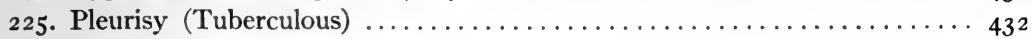

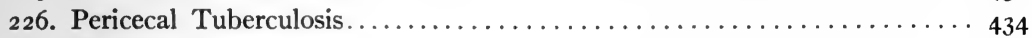

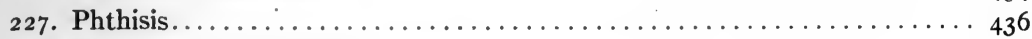

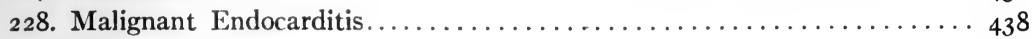

229. Vascular Crisis.................................. 440

230. Pneumonia and General Pneumococcus Infection . . . . . . . . . . 443

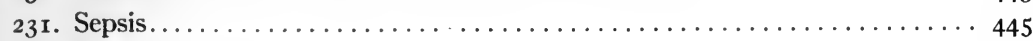

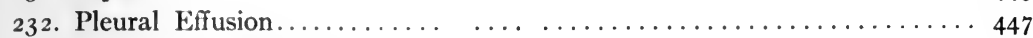

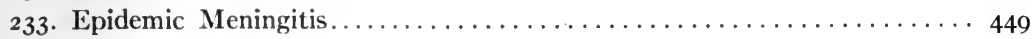

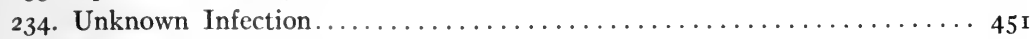

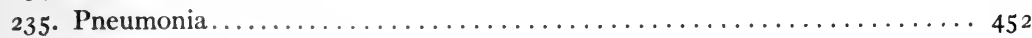

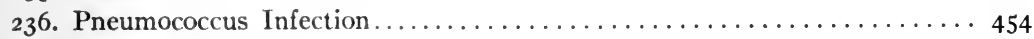

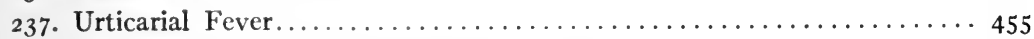

238. Pharyngeal (and Transpharyngeal) Infection ............... 457

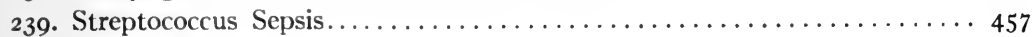

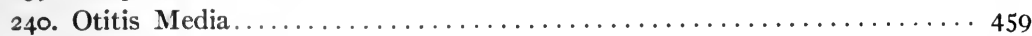

241. Glandular Fever.............................. 460

242. Catarrhal Jaundice........................... 462

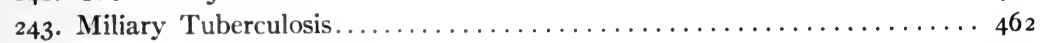

244. Estivo-autumnal Malaria........................ 464

\section{CHAPTER XIV}

\section{CHILLS}

CASE No.

245. Hepatic and Pulmonary Abscess..................... 470

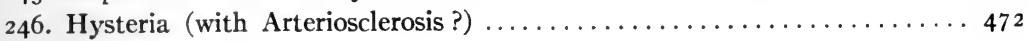

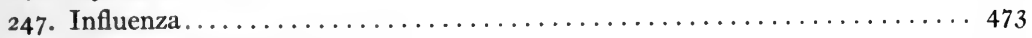

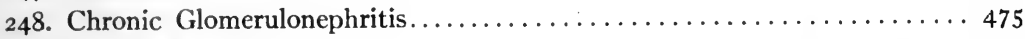

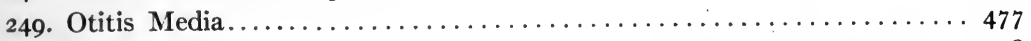

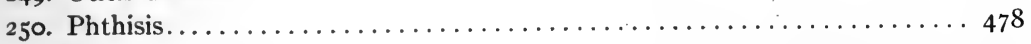

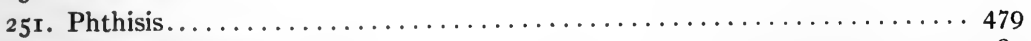

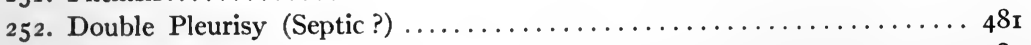

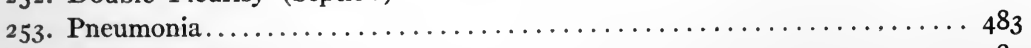

254. Visceral Syphilis........................... 485 
CASE No.

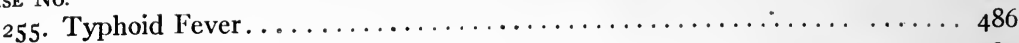

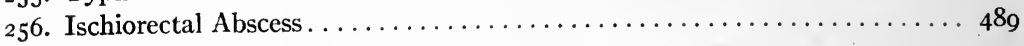

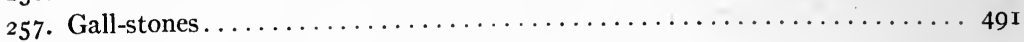

258. Deep Axillary Abscess. . . . . . . . . . . . . . . . . . . 492

\section{CHAPTER XV}

\section{COMA}

Examination of Comatose or Convulsive Patients................... 494

Certain Hoary Errors to be Avolded............................. 494

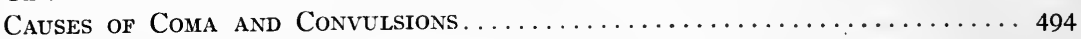

Valuable Clues..................................... 500

Infection in Uremia and Other Chronic Diseases................. 5 OI

CASE No.

259. Stokes-Adams' Disease .............................. 503

260. Mitral Disease (and Hysteria ?) ........................ 505

26r. Chronic Valvular Disease; Sudden Heart Failure from Unknown Cause.. 506

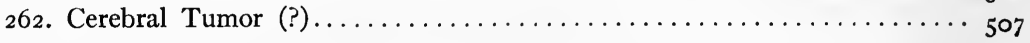

\section{CHAPTER XVI}

CASE No.

\section{CONVULSIONS}

263. Alcoholism........................................ 5 10

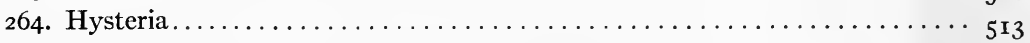

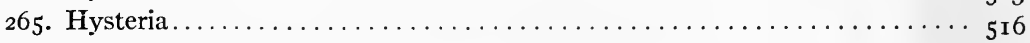

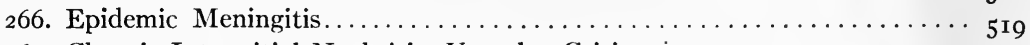

267. Chronic Interstitial Nephritis; Vascular Crisis ................ $55^{22}$

268. Chronic Interstitial Nephritis; Uremia..................... $55^{24}$

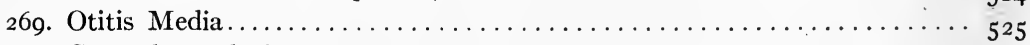

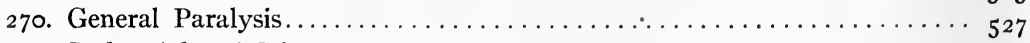

271. Stokes-Adams' Diseasc................................ 529

272. Stokes-Adams' Disease ............................... 53 I

273. Tonsillitis and Congenital Heart Disease.................. 533

274. Cerebral Tumor................................... 534

275. Dementia Paralytica................................... 536

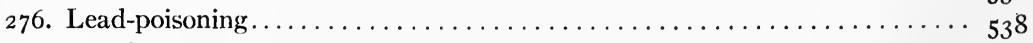

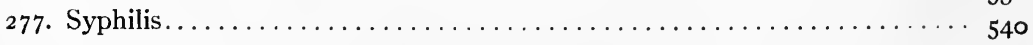

\section{CHAPTER XVII}

CASE No.

WEAKNESS

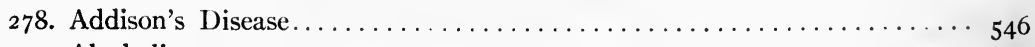

279. Alcoholism ................................... 549

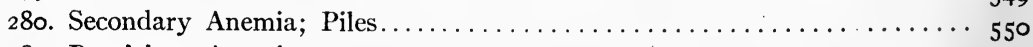

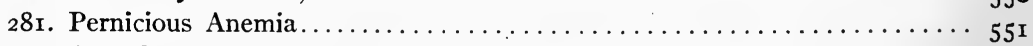

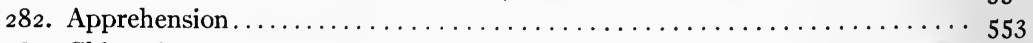

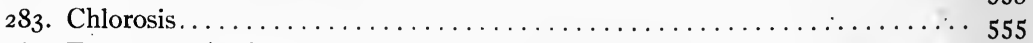

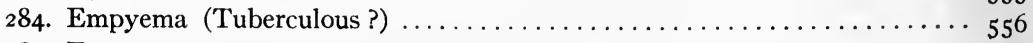

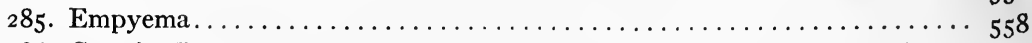

286. Gastric Cancer.................................. 559 


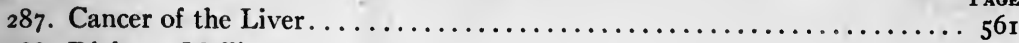

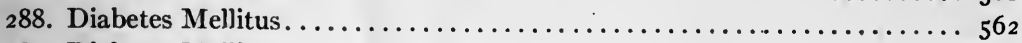

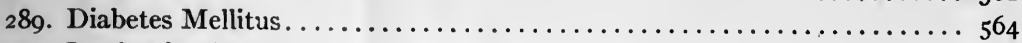

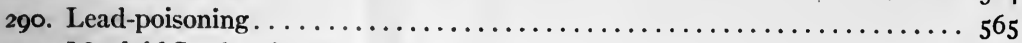

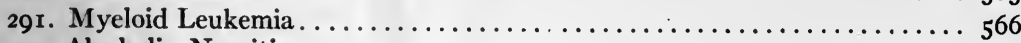

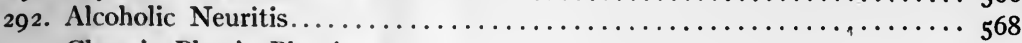

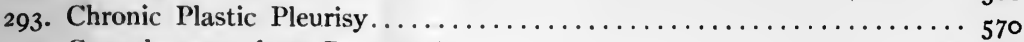

294. Convalescence from Pneumonia..................... 57 1

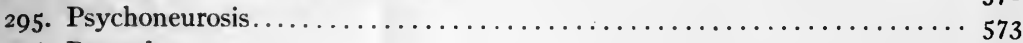

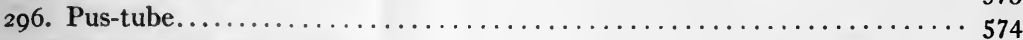

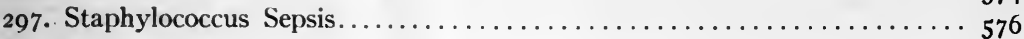

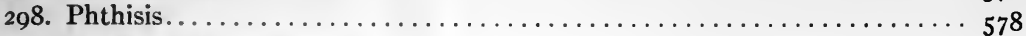

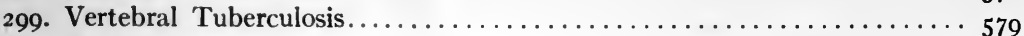

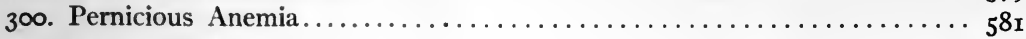

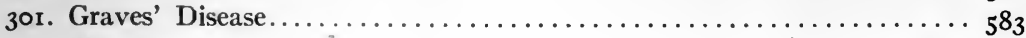

302. Myxedema................................... 584

\section{CHAPTER XVIII}

\section{COUGH}

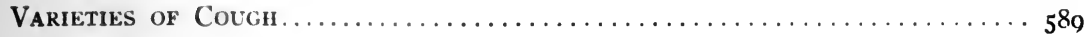

CASE No.

303. Pulmonary Abscess.................................... 590

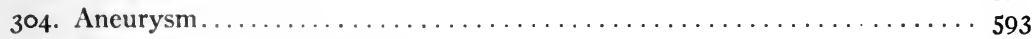

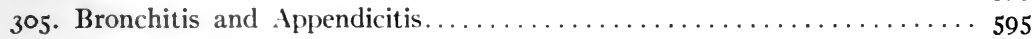

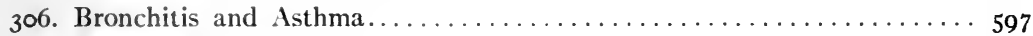

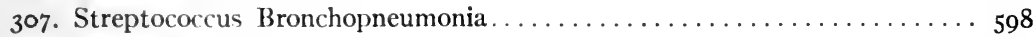

308. Bronchiectasis . . . . . . . . . . . . . . . . . . . . . 600

309. Bronchitis; Bronchopneumonia; Bronchiectasis; Emphysema..........60I

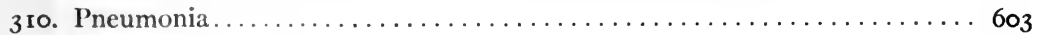

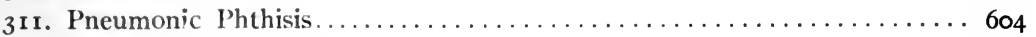

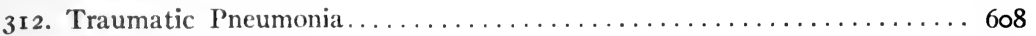

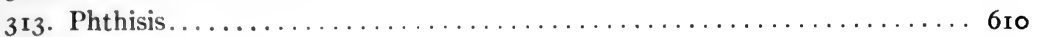

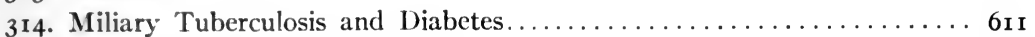

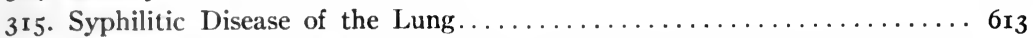

316. Tuberculosis of the Lungs, Chronic Interstitial Nephritis, Hypertrophy and Dilatation of the Heart, Tubercular Ulcers of the Intestine,

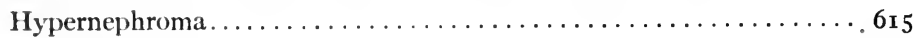

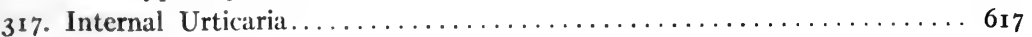

\section{CHAPTER XIX}

\section{VOMITING}

Important factors in the Production of Vomiting.................. 624

CASE No.

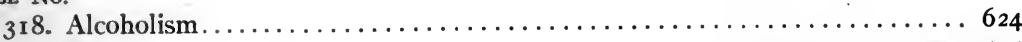

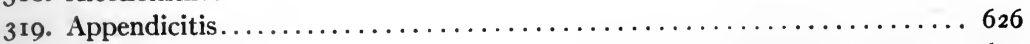

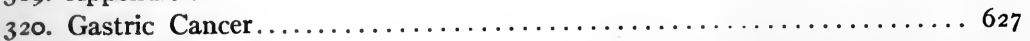

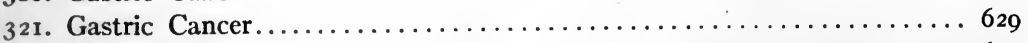

322. Cancer of the Sigmoid................................... 63 I 
CASE No.

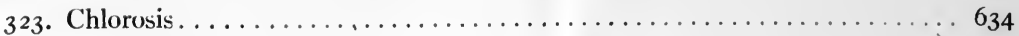

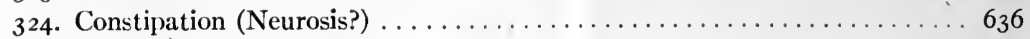

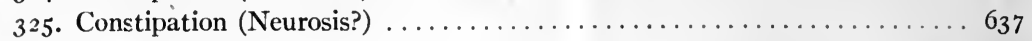

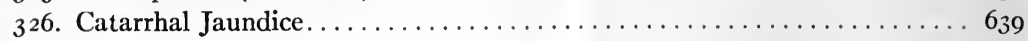

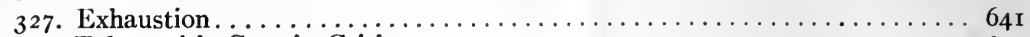

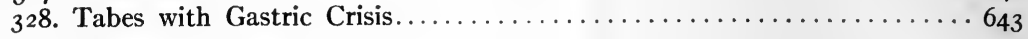

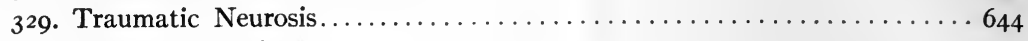

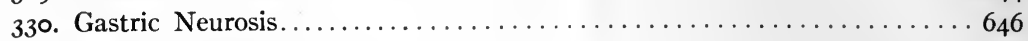

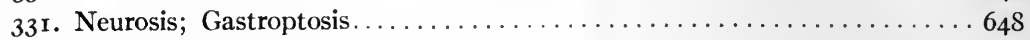

332. Gastric Ulcer; Pyloric Stenosis............................. 650

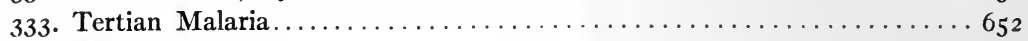

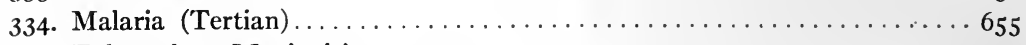

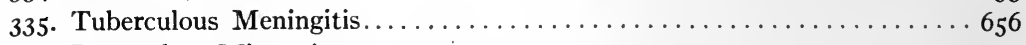

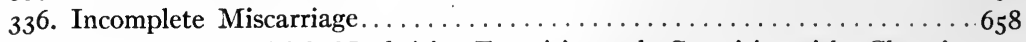

337. Chronic Interstitial Nephritis, Enteritis and Gastritis with Chronic

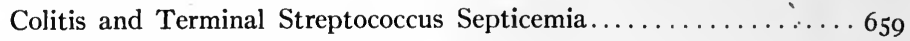

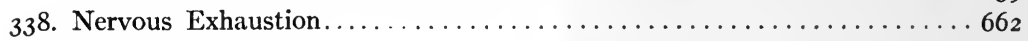

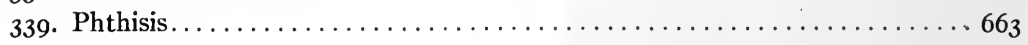

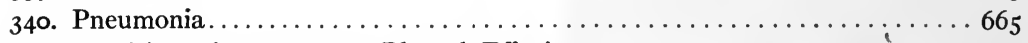

34r. Vomiting of Pregnancy; Pleural Effusion.....................667 667

342. Mitral Stenosis. ....................................... 669

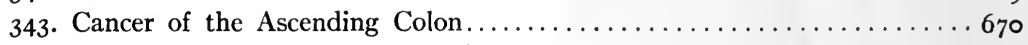

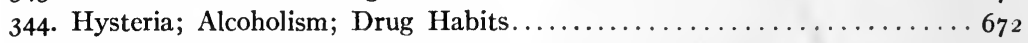

345. Chronic Intestinal Obstruction, Probably Due to Malignant Disease. . . . 674

346. Neurosis; Morphin........................................... 675

\section{CHAP'TER XX}

\section{HEMATURIA}

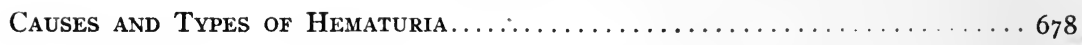

CASE No.

347. Tuberculosis of the Kidney and Bladder..................... $68_{2}$

348. Tuberculosis of the Bladder; Renal Tuberculosis (?) $\ldots \ldots \ldots \ldots \ldots \ldots 64$

349. Renal Neoplasm.................................. 685

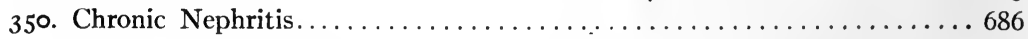

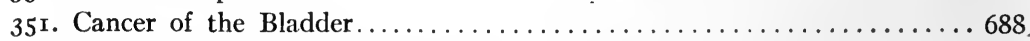

352. Cystitis Due to the Bacillus Coli.....................689

353. Papillary Cystadenoma of the Kidney ...................690

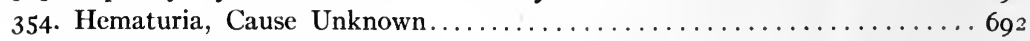

355. Renal Irritation from Oxaluria ........................ 693

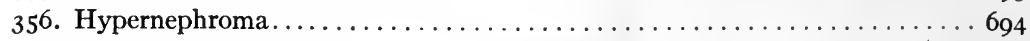

357. Gastric Ulcer; Hematuria, Cause Unknown.................. 695

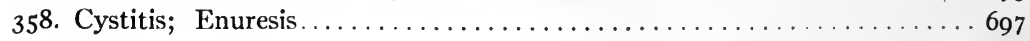

\section{CHAPTER XXI}

\section{DYSPNEA}

Causes of Dyspnea.................................... 703

The Effect of Position and of the Time of DAY ................ 704

Cheyne-Stokes' Breathing................................ 704 
359. Hysteria; Polypnea. . . . . . . . . . . . . . . . . . . . . . 705

360. Aortic Stenosis and Regurgitation.................... 708

36r. Infectious Endocarditis, Myocarditis, and Pericarditis; Mitral Stenosis

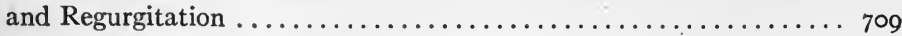

362. Hyperplastic Endometritis; Debility ................... 7 I $2^{2}$

363. Bronchitis and Emphysema; Epilepsy.................. 7 14

364. Tuberculous Empyema and (Presumably) Phthisis............. 715

365. Acute Cardiac Dilatation, Cause Unknown................. 7 I8

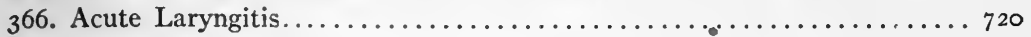

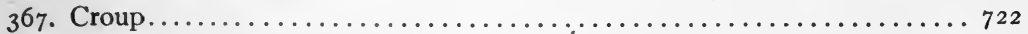

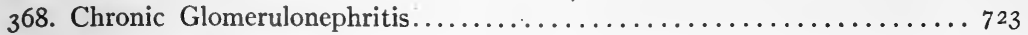

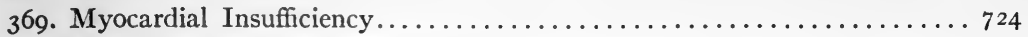

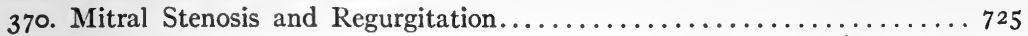

\section{CHAPTER XXII}

\section{JAUNDICE}

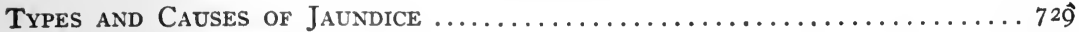

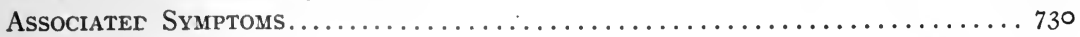

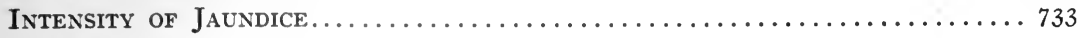

CASE No.

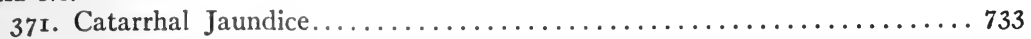

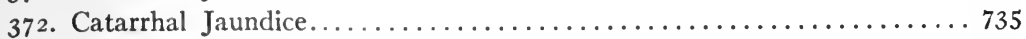

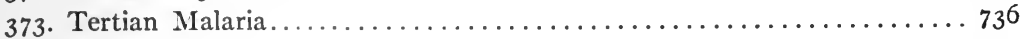

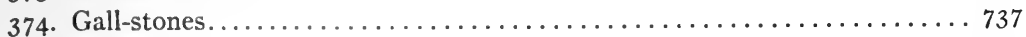

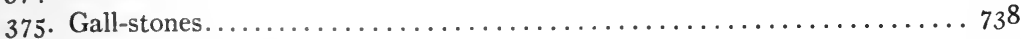

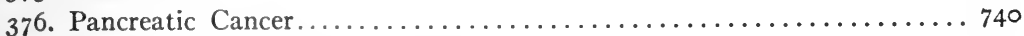

377. Acute Yellow Atrophy of the Liver..................... 74 I

\section{CHAPTER XXIII}

CASE No.

\section{NERVOUSNESS}

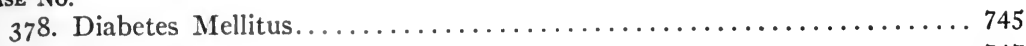

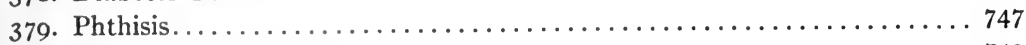

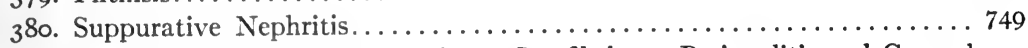

38r. Endothelioma of the Pleura; Acute Serofibrinous Pericarditis and General

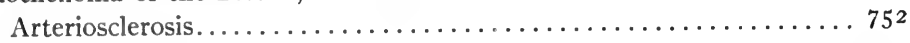

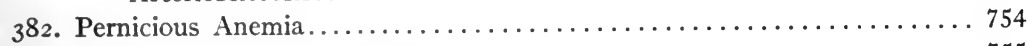

383. Chronic Interstitial Nephritis.......................... 755

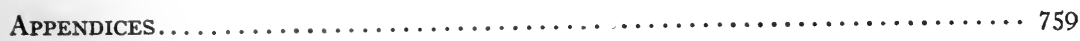

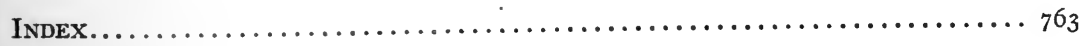





\section{DIFFERENTIAL DIAGNOSIS}

\section{INTRODUCTION}

\section{THE PRESENTING SYMPTOM}

CASES of disease present, as we say, certain leading symptoms. They thrust forward, like a soldier who presents arms, a complaint such as pain, cough, or "nervousness," so that it occupies the foreground of the clinical picture. Such a "presenting symptom," comparable to the "presenting part" in obstetrics, may turn out to be of minor importance when we have studied the whole case. But at the outset it has the power to lead us toward right or wrong conclusions in diagnosis, prognosis, and treatment, according as we have or have not learned the art of following it up.

This book is an attempt to study medicine from the point of view of the presenting symptom. I hope to show how the complaints of the patient-fragmentary expressions of the underlying disease-should be used as leads, and how their lead can be followed to the actual seat of the disease.

The plan thus outlined has three parts:

(a) To present a list of the common causes of the symptoms most often complained of by patients, $e$. g., the causes of pain in the back, of vomiting, or of hematuria.

(b) To classify these causes in the order of their frequency, so far as this is possible.

(c) To illustrate them by case-histories in which the presenting symptom is followed home until a diagnostic problem and its solution are presented.

\section{THE GROUPING OF REASONABLE POSSIBILITIES}

Diagnoses are missed-(a) Usually because physical signs are not recognized; (b) occasionally because we do not think correctly.

This book will not help any one to recognize the signs of disease, but it ought to aid physicians to solve those clinical puzzles wherein the diagnosis is missed because the patient's disease is not among those 
considered and looked for. In other words, correct diagnosis depends upon what enters the doctor's head as possible, and on what his head does to sift the possibilities after they have entered it, as well as on the direct recognition of signs by physical examination.

To throw open the mind's door and allow all disease to enter into consideration each time that we are called to a bedside is foolish in the attempt, and impossible in the performance. Each case should lead us to arrange before the mind's eye a selected group of reasonably probable causes for the symptoms complained of and for the signs discovered: What we select should depend upon the clues furnished us by the patient himself, or by the results of our own examination.

When, for example, a patient pronounces the word "headache," a group of causes should shoot into the field of attention like the figures on a cash register. Blue lips and finger-nails call up quite another group of ideas. Each clue or combination of clues should come to possess its own set of radiations or "leadings," determined partly by what we know of anatomy and physiology, partly by the hard knocks of clinical experience.

\section{ADVANTAGES OF THE PLAN HERE ADOPTED}

This way of working into a knowledge of medicine has the advantage of following the course of procedure by which we often question and examine patients in the office or in the clinic. We begin with the chief complaint and work inward and backward to the causes, the organic lesions, the evolution, probable outcome, and rational treatment of the case. Cases do not often come to us systematically arranged like the account of typhoid in a text-book of practice of medicine. They are generally presented to us from an angle, and with one symptom, often a misleading one, in the foreground. From this point of view we must reason and inquire our way back into the deeper processes and more obscure causes which guide our therapeutic endeavors.

Why do so many practitioners treat symptoms only? Why are their diagnoses and the resulting treatment so full of vagueness, groping, hedging, and "shot-gun" prescriptions?

Because they do not know how to get beyond symptoms. They have not been taught from the point of view of practice-i.e., of the presenting symptom. What are the possible causes and linkages of any symptom? Which of them are most probable? By what methods of questioning or of examination can the actual cause be founc? This book aims to put into the physician's hand the means of answering these questions. 
I quite realize that the art of forming reasonable hypotheses about a case of disease and then of testing these hypotheses by such experiments as shall establish the correct and nullify the incorrect, is useless unless the methods of physical and chemical diagnosis have been mastered and unless the natural history of all common diseases has been learned by observation and reading. But experience shows that a man may possess a considerable acquaintance with physical diagnosis and with the course of disease, and yet be quite helpless in the presence of a suffering person, simply because he cannot apply his knowledge to this case. He can observe, he can remember, but he cannot constructively think and experiment. Every item of physical or chemical examination is an experiment made to test the soundness of an idea about the case in hand. Skill in thinking and in putting our thoughts to such a test of experiment are not learned either by drill in physical diagnosis or by reading upon the history of disease.

To give such practice in thinking and working one's way into the mastery of a case of disease, through the intelligent verification of our thoughts by physical examination, is my object in the following chapters. They follow the method of case-teaching which I have used for eight years at the Harvard Medical School, applying there a method long employed at the Harvard Law School, and first described by Dr. W. B. Cannon.

\section{LIMITS}

To keep the book within reasonable limits I have selected I 2 symptoms (see Table of Contents) which are most often complained of by patients. I am well aware that others, such as diarrhea, constipation, loss of weight, paralysis, pallor, edema, purpura, or palpable tumors, might well have been discussed did space permit.

\section{VULNERABILITY OF ALL DIFFERENTIAL DIAGNOSIS}

The discussions which here follow each printed case are concerned with differential diagnosis, a very dangerous topic-dangerous to the reputation of physicians for wisdom. It is, I suppose, owing to this danger that so little has been written on differential diagnosis and so much on diagnosis (non-differential). To state the symptoms of typhoid perforation is not difficult. To give a set of rules whereby the conditions which simulate typhoid perforation may be excluded is exceedingly difficult. Physicians are very naturally reticent on such matters, slow to commit their thoughts to paper, and very suspicious of any attempt to tabulate their methods of reasoning. 
Yet all diagnosis must become differential before it can be of any use. All recognition of a lesion or a disease involves distinguishing possible sources of error and excluding them by a reasoning process-more or less definite and conscious. To be of any value, then, diagnosis must descend into the arena where it is questioned and assailed, where all sorts of errors and uncertainties arise to unsettle our wisdom. Those differential tables which we all distrust so much are really no more untrustworthy than the diagnoses we make in practice-for every diagnosis expresses the results obtained by using such a table more or less unconsciously, as we exclude possible errors and alternative diagnoses.

I am very well aware, therefore, that the differential diagnostic statements which fill this book are one and all subject to such limiting phrases as "in most cases," "as a rule," etc. This must always be so as long as the list of possible causes or diagnoses which we call to mind when we attack any diagnostic problem is an incomplete list (or possibly an overinclusive one). To decide which of the known causes of jaundice is the cause of the yellowness of Miss Smith we investigate, by the experiments known as "history," "physical examination," and "therapeutic test," a list of these known causes. But some day we may meet a case in which none of these well-known causes is present. Some new cause, so far unlisted, may, in fact, be at work. There are probably as many fish in the sea as ever came out of it; the unrecognized infections, poisons, and maladjustments are probably as many as those already described in text-books.

All this unconquered territory lies about us, full of hidden dangers to our differential diagnosis-i.e., to all practical diagnosis.

One other limitation must be mentioned. Whenever one says: "The symptoms produced by typhoid (or by peritonitis or by renal stone) are such and such," one should tacitly add- "provided that it produces any characteristic symptoms at all." It is certain that the three diseases just mentioned may exist without producing any symptoms of which the patient is aware. It is probable that this is true of all other diseases. But as we can have no direct dealing with these silent types of disease, we can give them place in the theater of our reasonings only in that outer circle reserved for "possible sources of error," a great and distinguished company whose presence serves to keep us within the bounds of humility and of scientific caution.

Meantime we must go on with our work of finding the most probable among the known causes and discoverable types of disease. 


\section{OMISSIONS}

Some diseases are omitted by choice, others by necessity. The 385 cases which I have selected for study were all seen in private or hospital practice. To prevent the possibility of their recognition by the individuals concerned I have changed or omitted certain personal details. In essentials the cases are reproduced as they were observed.

I have chosen no cases in which diagnosis was obvious and none in which it was impossible or dependent chiefly on good luck. To avoid the obvious, I have omitted discussion of such clinical pictures as the following:

Patient of twenty-five, who has had two attacks of rheumatic fever, complains of dyspnea, dropsy, and cough. Examination shows a rapid, irregular, transversely enlarged heart, with a presystolic murmur and thrill at the apex and an accentuated pulmonic second sound. There is evidence of passive congestion of the lungs, liver, legs, and gastro-intestinal tract, with dropsy of the serous cavities.

There may be many difficulties in physical examination here, but none in the reasoning processes which lead us to the examination and thence to our conclusions. Obvious maladies, such as pharyngitis, peripheral gangrene, or talipes, have been omitted for the same reason; likewise all those in which diagnosis is made only by incision; $e$. g., acute pancreatitis, certain breast tumors.

While selecting cases in which diagnosis was difficult, but not impossible, I have tried to choose those in which in the end we could attain a reasonable certainty. Absolute certainty is attainable only as the result of operation or autopsy, and not always then. Hence it is possible that certain of my readers may disagree with the diagnosis finally reached in some cases. This is inevitable in a book of this kind, as it is in actual practice. Book and practice alike can only reflect the existing state of medical knowledge, medical uncertainty, and ignorance. But I sincerely hope that my errors may be pointed out by correspondents.

After restricting the field in the way just mentioned, I have tried to exemplify in each chapter all the diseases which often lead a patient to consult his physician, complaining of the symptom which forms the subject of that chapter. Now and then, however, I have altogether omitted some important disease because I could not find any suitable example of it within my own cases or among those which I had myself studied.

In a few cases certain items have been omitted here because they were likewise omitted in the version of the case given me by the attending physician. My task was to notice their conspicuous or inconspicuous 
absence, and to act accordingly. It seems justifiable, therefore, to impose a similar task upon my readers.

\section{EXPLANATION OF DIAGRAMS AND CHARTS}

The book contains figures, tables, diagrams, and charts. The two last need some explanation.

The diagrams, which are introduced in each chapter just before the illustrative cases, represent an attempt (the first that I know of) to estimate the relative frequency of the commoner causes for each symptom discussed. This estimate, which can be but approximate, rests upon the following data:

(a) An enumeration of the total number of cases of every disease treated at the Massachusetts General Hospital during the last six years. About 180,000 cases are thus classified according to diagnosis, and the relative frequency of each disease in this material is thus roughly computed. But these figures do not give us the relative frequency of any of the symptoms (such as jaundice or headache) studied in this book. Many cases of gall-stones are not jaundiced; hence we cannot directly compare the number of gall-stone cases with the number of cirrhoses (for example), but must estimate the percentage of jaundiced cirrhoses and jaundiced gall-stone disease in each group. This is done by consulting-

(b) Statistical articles from the literature in which the percentage occurrence of each symptom in a large series of cases is worked out. Such statistical articles, however, are not common. In Rolleston's magnificent monograph on the liver almost every statement has a statistical basis, and the wearisome recurrence of phrases like "as a rule," "not infrequently," "sometimes," etc., is replaced by concrete quantitative estimates. But there are not many such books. Hence I have been forced in some instances to compute the percentage occurrence of a symptom by-

(c) The study of the symptom and of the frequency of its occurrence in $25^{\circ}$ cases of the disease in question; these cases were taken trom the more recent records of the Massachusetts General Hospital.

By the methods described under $(a),(b)$, and $(c)$ the length of every line in every diagram has been calculated. I am well aware that there are numerous sources of error in these calculations. The diagnoses in the Massachusetts General Hospital records may be faulty in some instances, though the large number of cases used tends to minimize such errors. The statistical articles referred to under (b) may be incorrect. and do not often include a very large bulk of cases. Finally, the number of cases 
referred to in the calculations under $(c)$ is smaller than I should wish. More important than any of these errors are the absolute omissions which are sure to be discovered among my tables of causes. I hope for much aid from my critics in supplying such missing links. Indeed, I am confident that some one will be so indignant at my mistakes that he will at once begin to write a better book on similar lines-a result which I most earnestly desire.

The sources of my information regarding the figures used in the diagrams are given in Appendix A, p.759.

The list of causes represented in these gridiron-shaped diagrams is not wholly the same as that exemplified in the illustrative cases. Only the commonest, clearest, and most important causes are drawn in upon the "gridirons." Still a third group of causes, which do not lend themselves either to diagrammatic or to detailed illustrative treatment, are mentioned briefly in the introductory section of each chapter. Hence the complete list of causes discussed is to be found-(a) In part in the gridirons; $(b)$ in part in the illustrative cases; $(c)$ in part in the introductory section of each chapter.

The Charts.-Beside the three lines, which represent in the ordinary way the course of temperature, pulse, and respiration, there is a fourth line interwoven with the respiratory curve, and distinguished by the presence of cross striæ, like the railroads on a map. This line stands for the twenty-four-hour amount of urine measured in ounces.

In the charts the line of this type: indicates the amount of urine in ounces, while the line cut by stars, as follows, *—— $*$ represents the blood-pressure. 


\section{CHAPTER I}

\section{PAIN \\ GENERAL CONSIDERATIONS}

BEFORE we begin to study the cause or the cure of any pain, we need to convince ourselves that it really exists. Not only in the cases of deliberate deception or malingering, but in dealing with perfectly honest people, we are liable to error. Many persons, especially of the less educated classes, do not distinguish between pain and the other varieties of discomfort, such as itching or a sense of pressure. Many patients whe say at first that they have a headache or a stomachache may be brought; by a little questioning, to recognize that they are referring to a sense of weight, constriction, or vague discomfort, rather than to pain in the narrower sense.

As evidences of pain we are accustomed to scrutinize:

(a) The facial expression and bodily movements.

(b) The account of some onlooker, such as a nurse or relative.

(c) The results, such as emaciation or muscular weakness, often produced by long-continued suffering.

(d) The blood-pressure.

When a patient's face is contorted and his body writhes, stiffens, or doubles up, we can have no doubt that he is suffering, unless we believe him an impostor, but obviously these evidences of pain may be easily simulated or exaggerated.

It is in such cases that we need the testimony of some third person who can watch the patient at a time when he supposes himself to be alone. Many patients who do not intend to deceive us show far greater evidences of suffering when a doctor, a nurse, or a friend is near at hand than when they believe they are unobserved. This is partly due to the fact that a perfectly genuine though distinctly mild lesion is very much more painful to the patient when his self-pity is aroused by the presence of a sympathetic onlooker.

When a patient who bears the ordinary marks of blooming health states that he has been suffering excruciating pain for many months, the lack of any of the ordinary evidences of suffering naturally and properly make us take his statement with a grain of salt. Chronic suffering is pretty sure to leave its mark on the face and body.

In cases of suspected malingering, when an individual states that a certain motion or a certain pressure upon a supposedly tender point 
causes great suffering, we may control his statement to a certain extent by measuring the peripheral blood-pressure at the time. Severe pain almost always causes a notable rise in blood-pressure, and if we find nothing of the kind, we may rightly conclude that if pain is present, it is probably not intense.

\section{DEGREE OF PAIN}

I have long been accustomed to compare, as a matter of routine and in every case, the extent and quickness of the knee-jerks with the patient's statement regarding his own suffering. I have found that those who describe all their troubles as "terrible," "awful," "fearful," and the like, are very apt to have lively knee-jerks, and that those who are more moderate in their expressions have usually less active reflexes. It seems quite probable that there is a parallelism here between reflex sensibility and sensitiveness to pain. Those who respond to a given stimulus by an exaggerated knee-jerk might well be expected to respond to a given cause of pain by an exaggerated complaint. So it has seemed to me as a result of many observations, and I have come to believe that people are more likely to be oversensitive and to exaggerate their sufferings when the knee-jerks are unusually lively. This belief is strengthened when the gag reflex in the pharynx is diminished or wanting, as is often the case.

This is, of course, a very rough and uncertain method of measuring pain, and would perhaps be more truly described as an attempt to measure the severity of the cause of pain, rather than of the pain itself. We are greatly in need of some more accurate method of estimating how much people suffer. For the present, we have to judge largely by such uncertain evidences as were mentioned in the last section-facial expression, bodily movement, the accounts of onlookers, and the evidences of such physical changes as pain might produce. In addition to these we get a certain amount of information by asking:

"Does the pain prevent you from working?"

"Does it prevent sleep?"

"Does it take away appetite, the capacity for movement and enjoyment in the ordinary functions of life?"

We know that certain races-for example, the Chinese-are much less sensitive than others to pain in that they exhibit far less evidence of "shock" after a bullet wound or a disembowelment. We can only guess at the sensory side of this phenomenon, but the absence of the ordinary organic effects produced by the same injury in a Caucasian gives us some ground for believing that the suffering is proportionately small. In all probability there are similar differences between individuals of the same race. 
Though women are generally believed to be more highly organized and more sensitive than men, it is a well-known fact that they bear pain, especially prolonged pain, better than men. I have never heard any plausible explanation of this fact.

\section{TYPES OF PAIN}

Most of the adjectives which are attached to the complaints of patients, either by themselves or in the text-book description, give us no information of value because they are not regularly associated with any one disease. Boring pains, tearing pains, and knife-like pains do not characterize any particular disease. Nevertheless, there are a few distinctions of importance.

Pains that recur rhythmically, or at regular intervals, working up gradually to a climax each time, and then disappearing suddenly or gradually, are often associated with hyperperistalsis within some hollow tube, such as the intestine, the ureter, the bile-ducts, or the uterus. To such pains the name of "colic" is traditionally attached, though it is often used much more vaguely to denote any type of severe and sudden pain in the abdomen.

Throbbing pains, increased momentarily with each beat of the heart, are characteristic of vascular hyperemia, such as occurs about the roots of an inflamed tooth. In connection with vasomotor headaches and in dysmenorrhea we occasionally see the same phenomenon.

Pain with a sense of constriction is of great diagnostic value when it occurs in the precordial region, pointing, as it does, in the great majority of cases, to angina pectoris as its cause. Other diseases producing pain in this region are rarely, if ever, accompanied by this sense of constriction, which the patients often express in very vivid phrases, e. g., "as if I were squeezed in a vise," or "as if some one gripped my heart in his hand."

Thoracic or abdominal pain increased or produced by exertion and promptly relieved by rest is almost always due to the cause just mentioned-angina. Many pains supposed by the patient to be due to indigestion, to rheumatism, or to neuralgia may thus be recognized as anginoid.

Pain that shoots and darts, especially if it follows the course of some nerve-trunk, usually turns out to be neuralgic. In many cases such a pain is associated with prickling, burning, numbness, or other paresthesias.

\section{RELATION OF PAIN TO OTHER FACTS}

A careful history of the bearing of various factors in the patient's habits and environments upon the occurrence or the severity of pain is of 
prime importance in diagnosis. Among the elements to be taken account of are the relation of pain to:

(a) The time of day.

(b) The position of the body.

(c) The taking of food.

(d) The effect of motion involving the painful part, or of jolting of the entire body.

(e) The effect of emotional excitement.

(f) The effect of occupation.

(g) The effect of season and the weather.

(h) The mode of relief-e. g., by heat, cold, food, vomiting, medicine, rest, occupation.

Neurasthenic headaches and the pains of chronic joint troubles are apt to be worse in the morning and to improve as the day goes on. Any pain associated with fever and infection is likely to be worse in the evening, when the temperature is at its highest.

Pains affected by position are especially those due to diseases of the joints and muscles, such as lumbago, sacro-iliac strain, all the types of arthritis, stiff neck, and the like. Almost all varieties of pelvic disease are worse when the patient is on her feet, as the position is likely to involve some pressure or dragging upon painful points. For the same reason the surgical affections of the kidney and all diseases which involve splenic enlargement are usually more painful when the upright position is assumed. Occasionally a headache is distinctly improved or aggravated when the patient lies down. The distress accompanying uncompensated cardiac disease is always aggravated by recumbency.

Most muscular pains are aggravated by the use of the muscle; hence the presence of such an aggravation may help us to distinguish muscular pains from those of different origin. It must be remembered, however, that in some cases the pains of neuritis are increased by use of the part, even when no muscular lesion is discoverable. The motion of coughing brings great distress in pleurisy, pneumonia, and all diseases involving the intercostal muscles. Anginoid pains are increased not only by motion, but by any other cause which raises blood-pressure (gastric digestion, mental exertion, or excitement).

On the other hand, some pains are made worse by rest; for example, all types of habit pains, to which I shall refer more in detail in the next section. The pains of chronic joint troubles are worse immediately after rest, when the patient attempts to move his stiffened joints.

The effect of jolting, as in riding on a rough road or a rough-gaited horse, is traditionally associated with an increase of the distress produced by stone in any part of the urinary tract. Doubtless this is a true observation, but there are many exceptions to the rule. 
Aggravation of any pain by the taking of food properly inclines us to believe that the pain is produced in the stomach (gastric cancer, gastric neurosis). Many intestinal pains, however, are likewise produced or increased when food enters the stomach. Thus the sufferings due to enteritis and to chronic intestinal obstruction are often much worse immediately after a meal. It appears to be true, moreover, that pain due to gall-stones may be set agoing by the presence of food in the stomach presumably through the production of pyloric spasm. (See also page I59.) I have already referred to the excitement of anginoid pain through the rise of blood-pressure produced by the act of digestion. Possibly an accompanying gaseous distention may help to call out the attack. Relief of pain by food or soda is characteristic of peptic ulcer, gastric or duodenal, and of hyperchlorhydria, as well as of the vaguer gnawings due to hunger.

Many types of muscular, articular, and neural pains are subject to aggravation as the result of various meteoric conditions, of which we understand all too little. It cannot be questioned, I think, that the muscular pains involved in lumbago and stiff neck are more apt to be present in darip, rainy weather, such as occurs in the spring and fall, than in dry heat or dry cold. Probably infections arising in the tonsils or the teeth are responsible for many of these spring attacks. The persons who can foretell a storm by the disagreeable sensations in the neighborhood of diseased joints are very numerous, but I have never been able to associate this form of prophecy with any one type of disease. I am also convinced that the approach of a thunderstorm may precipitate a headache not only in those predisposed to migraine, but in other sensitive persons. Whether this is due to barometric, to electric, or to quite unknown conditions I am unable to say. Many of my patients have noticed that their headaches are more apt to occur on especially bright, bracing days, when the air is unusually clear. Relief by vomiting does not prove that the disease is of gastric origin. Intestinal pain, biliary colic, renal colic, and the sufferings of duodenal ulcer may also be relieved by emesis.

Relief by heat or by cold cannot be predicted for any variety of pains. The same disease in different individuals may be assuaged now by the one now by the other agencies. It is wholly a matter of experimentation. But in my experience most of the pains which cold relieves are more completely and more permanently abated by heat.

Relief by alkali, sodium bicarbonate, or one of the magnesium compounds is especially characteristic of hyperchlorhydria and of peptic ulcer. But lesser degrees of the same relief may occur in almost any stomach trouble. 


\section{HABIT PAINS}

The term is a misleading one, and needs more explanation than the fact. The genesis of the latter may be described as follows:

(a) Some exciting, terrifying, or mortifying event draws the patient's attention to a certain part of his body-the cardiac region or the pharynx, for example. Then-

(b) As the microscope discovers bodies invisible to the unaided eye, so the patient's focused and concentrated attention discovers sensations due probably to some of the physiologic changes occurring normally in the part to which attention has, unfortunately, been directed. These changes go on normally without producing any sensation noted by the brain. But when the brain is sensitized, especially in relation to the part attended to, even the heart-beat may be felt as painful, or the normal blood, lymph, and nerve-currents of the pharynx may be magnified into painful events.

(c) The "set" of attention produced by habit keeps the brain "on edge," keyed up to perceive the slightest glimmer of sensation, such as we ordinarily disregard.

(d) Finally, some actual disturbance of the function of the part may follow this abnormal interference of consciousness in activities which should be subconscious. The heart-beat becomes irregular; the pharynx secretes abnormally. This redoubles, of course, the patient's alarmed concentration upon the part, and so a vicious circle is established.

Such a circle is broken, and the diagnosis of habit pain confirmed when we succeed in switching off the patient's attention upon other subjects-and thus making him forget, at any rate for a time, his habitual sufferings.

\section{THEORIES REGARDING THE PRODUCTION OF PAIN}

I wish to refer briefly to the beliefs of McKenzie and Head, also to those of J. Pal, regarding the means whereby pain is produced under certain conditions.

To James McKenzie ${ }^{1}$ and to Henry $\mathrm{Head}^{2}$ we owe the elaboration of a theory whereby pain and cutaneous hyperesthesia are viewed as associated manifestations of morbid irritability in one or another group of spinal ganglion-cells. According to their theory, this irritability is due to impulses transmitted from a diseased organ, which, though not itself the seat of pain, yet causes in the corresponding spinal segment a disturbance which is transferred thence to the periphery of the body.

1 James McKenzie, Symptoms and their Interpretation, Shaw and Sons, London, Igog.

2 Henry Head, On Disturbances of Sensation, Brain, I893, vol. xvi, p. I ; also in subsequent numbers, 1794, 1896, 1900, etc. 
and there recognized by the individual as pain in a place often far distant from the organ diseased.

Thus these writers account for the umbilical pain experienced in intestinal obstruction, no matter where the stoppage occurs, by supposing that all parts of the intestine are represented in the cord by the same spinal segment, and that the umbilical region is the seat of centrifugal impulses from that center, resulting in cutaneous hyperesthesia, as well as pain.

The best confirmation and exemplification of this theory are seen in the so-called radiations of the pain known as angina pectoris, and in the similar radiations from the region of the dome of the diaphragm on either side. Infarcts of the spleen, pus working up to the superior surface of the liver, and anything else which irritates the dome of the diaphragm on either side, gives shoulder pain. It is difficult to account for the arm pains of angina and the shoulder pains of gall-stone disease or any other hypothesis, and if all other types of pain could be traced with similar accuracy to a spinal segment, rather than to an organ directly underlying the painful spot, the theory of McKenzie and Head would serve our unqualified assent. In point of fact, however, the examples given above are almost the only ones in which the theory is clearly verifiable. The pain of appendicitis, of pleurisy, most kidney pains and splenic pains do not well accord with the tneory, and the zones of cutaneous hyperesthesia which are essential to the confirmation of their theory have seldom been found by other observers. In spite of my profound respect for the originators of this theory, I have been unable to apply it successfully in clinical work, except in the two diseases just referred to, and in the localization of spinal lesions.

More useful, on the whole, is the book on Gefasskrisen, ${ }^{1}$ in which Pal elaborates, upon the basis of careful observation, both at the bedside and at the dead-house, a theory of the origin, not of all pains, but of certain paroxysmal types of suffering associated especially with the vessels of the brain, the heart, and the kidney, but to a lesser extent with those of the intestine and of the extremities. He supposes that arterial spasm (favored and prepared for by arteriosclerosis, by uremia, by lead-poisoning, and by the nerve lesions of tabes) is the cause of a large group of pains, paralyses, and other functional disturbances which had never before been brought together under any single explanation. Taking lead-poisoning as an impressive example of the theory, he points out that we have here a notable rise of blood-pressure, associated sometimes with cerebral crises (headache, convulsions, coma), often with abdominal crises (lead colic), and occasionally with anginoid seizures. In arteriosclerosis

${ }^{1} \mathrm{~J}$. Pal, Gefasskrisen, Leipzig, rgo5. 
we have likewise cerebral, abdominal, and cardiac crises, and, in addition to these, well-marked peripheral crises (intermittent claudication). In uremic and eclamptic poisoning we have likewise cerebral and abdominal crises. In tabes dorsalis the abdominal crises are the most familiar.

In all these affections postmortem examination may demonstrate that there is no gross lesion, such as cerebral hemorrhage or thrombosis, coronary occlusion, or blocking of a peripheral artery. Indeed, the arteries and the surrounding tissues may appear almost or quite normal postmortem. It is natural, therefore, to assume some functional change, such as spasm, to account for the pain, paralysis, and other functional changes recognized at the bedside. In favor of the hypothesis of vascular spasm, or Gefasskrise, are two considerations:

(a) A rise of blood-pressure has many times been demonstrated by Pal before, as well as during, the crisis. This hypertension cannot be accounted for as a result of pain, since in many of Pal's cases it preceded the pain. He has found it in the gastric crises of tabes, as well as in the uremic, saturnine, and arteriosclerotic cases.

(b) During an attack of transient blindness occurring in a patient who had been subject to various other "crises," ophthalmoscopic examinations showed a high-grade spasm or contraction of the retinal arteries.

So much for the theory and the evidence on which it is based. It seems to me a good working hypothesis as an explanation of many of the transient amauroses, aphasias, monoplegias, hemiplegias, and headaches associated with chronic nephritis. Like other theories, it is to be tested partly by what it enables us to discover. Like the atomic theory, it may lead us to perceive and so to fill in certain gaps, such as appear in the following table:

CRISES

\begin{tabular}{l|c|c|c|c|c|c}
\hline & Cerebral. & Cardiac. & Abdominal. & Peripheral. & Pulmonary. & Laryngeal. \\
\hline I. Arteriosclerosis...... & + & + & + & + & + ? & - \\
2. Nephritic hyperten. & & + & + & & & - \\
sion ("uremia").... & + & + & $+?$ & - & + ? & - \\
3. Tabes dorsalis...... & - & - & + & + & - & + \\
4. Plumbism.......... & + & + ? & + & - & - & - \\
\hline
\end{tabular}

I may here acknowledge my deep indebtedness to Rudolf Schmidt's book on Pain,1 which has guided and confirmed my own observations on many points.

1 Pain-its Causation and Diagnostic Significance, by Rudolf Schmidt; translated by Karl M. Vogel and Hans Zinsser, J. B. Lippincott Company, I908. 


\section{CHAPTER II}

\section{HEADACHE}

\section{GENERAL CONSIDERATIONS}

In discussing this, probably the commonest of all symptoms, I shall exemplify by cases only such causes as are likely- $(a)$ to be complained of by the patient as his leading symptom, and $(b)$ to occasion diagnostic difficulties. Others will be briefly mentioned here.

I. Anemia of any type-pernicious, chlorotic, posthemorrhagicis now and then accompanied by headache, usually as a minor symptom. It is noteworthy, however, that intense anemia often persists for months without producing any headache whatever. It may well be doubted whether anemia is ever in itself the cause of headache. ${ }^{1}$

2. Fatigue, hunger, and bad air often produce a headache (perhaps due to the circulation of "fatigue poisons") whose cause is made obvious by its disappearance after rest, food, and fresh air.

3. Poisons, such as alcohol, morphin, and lead. Except after a drinking bout, I have never known a patient whose chief complaint, as a result of any of these poisons, was headache. Other symptoms usually occupy the foreground.

4. Arteriosclerosis.-It has long been stated in medical lectures and text-books that the headaches of elderly persons are frequently caused by arteriosclerosis. My own experience, however, coincides entirely with that of Thomas, of Walton, and of Paul, who deny any such association. In my experience, it is only when the kidney is extensively involved and blood-pressure thereby raised that headache results from arteriosclerosis.

5. Indigestion and Constipation.-Gastric stasis, arrested digestion, and the resulting abnormal fermentation of food often lead to a headache which needs no further mention here. The patient can usually make the diagnosis for himself. The same is often true of the headaches

1 Of 697 cases of pernicious anemia studied by me, 300 had no headache at any time. See Osler's Modern Medicine, vol. iv, p. 622.

2 Walton and Paul, Jour. Amer. Med. Assoc., I908; Thomas, Osler's Modern Medicine, vol. vii, p. 336 . 


\section{Causes of Headache}

1. FATIGUE, BAD AIR, AND HUNGER
2. CONSTIPATION AND INDIGESTION ("BILIOUS-
NESS")
3. ALCOHOL (THE "DAY AFTER" HEADACHE)
4. EYE-STRAIN AND INTRINSIC DISEASES OF
THE EYE
5. INFECTIOUS DISEASES (ONSET)
VAGUELY ENUMERABLE FOR
G. MENSTRUATIC REPESENTATION.

7. PSYCHONEUROSES 1039

8. NEPHRITIS 602

9. MENINGITIS 172

10. SINUSITIS 157

11. TRIGEMINAL \} NEURALGIA

12. "INDURATIVE"

13. MIGRAINE

14. BRAIN TUMOR

$\left.\begin{array}{l}\text { 15. SYPHILITIC } \\ \text { PERIOSTITIS }\end{array}\right\}$

16. UNKNOWN CAUSE

1 The diagnosis of intracranial syphilis seems to me still so uncertain that I have not included it here. 

resulting from constipation, miscalled "lithemia," "biliousness," or "torpid liver."

A remarkable feature of this type of headache is its swift disappearance, in certain cases, after defecation. From several very intelligent patients I have heard repeatedly the story of a headache that disappeared, wholly or mostly, within a few minutes of the time of defecation. This is hard to reconcile with any chemical theory regarding the origin of such a pain, and is probably due to changes in the splanchnic circulation conditioned by variations in intra-abdominal pressure. These changes are doubtless compensated by corresponding changes in cerebral circulation such as might account for the disappearance of headache. Somewhat parallel phenomena are exemplified in the frequency of faintness after loose movements of the bowels and the cerebral symptoms produced by straining at stool.

6. Many common infections-rhinitis, tonsillitis, the exanthemata, etc.-are often accompanied by headache, which, however, is rarely the patient's chief complaint. There are other infections-examples of which will be given below-which cause so severe and persistent a headache that it becomes the "presenting symptom."

7. The headache sometimes accompanying otitis media and other forms of aural disease gets its recognition, in the vast majority of cases, from the concurrent aural symptoms.

8. Menstruation is often preceded or followed, less often accompanied, by headache the exact origin of which is very obscure.

9. Trigeminal neuralgia, with or without the paroxysms and spasms of tic douloureux, presents, as a rule, no serious difficulties in diagnosis, and will, therefore, not be further mentioned here. Mild types may originate in dental caries or other peripheral irritations. The severer forms appear to be due to changes in the Gasserian ganglion.

Io. Insolation, with or without actual sunstroke, has often been listed among the causes of headache. In my experience, however, there is usually a large neurasthenic element in these cases, and the history of insolation is often vague and forced.

II. Adolescence is frequently associated with a headache for which no local cause can be found. We connect such headaches vaguely with adolescence, because they pass off with the end of that period.

12. Cerebral concussion - as in a foot-ball game-is a common cause of headache, which usually presents no diagnostic difficulties.

I3. Indurative Headache.- "This-probably the most frequent form of headache-seems to be unknown to the majority of physicians, 
although it has been described in text-books for decades" (Edinger, in Die Deutsche Klinik ${ }^{1}$.

The term "indurative" expresses an attempt to characterize the malady without committing ourselves to any theory regarding its cause or morbid anatomy. In some of the older books it is referred to as "rheumatic headache." Its distinguishing feature is the presence of painful "indurations" near the insertions of the muscles at the occiput. Bits of the trapezii, sternocleidomastoids, scaleni, or splenii become sensitive, uneven, and nodular, "as if something were deposited in the substance of the muscle." (See Fig. I.)

Pain which is chiefly, but not exclusively, occipital is associated with these "indurations," and disappears when they are removed by massage. It is on this account that the disease is so much better known to

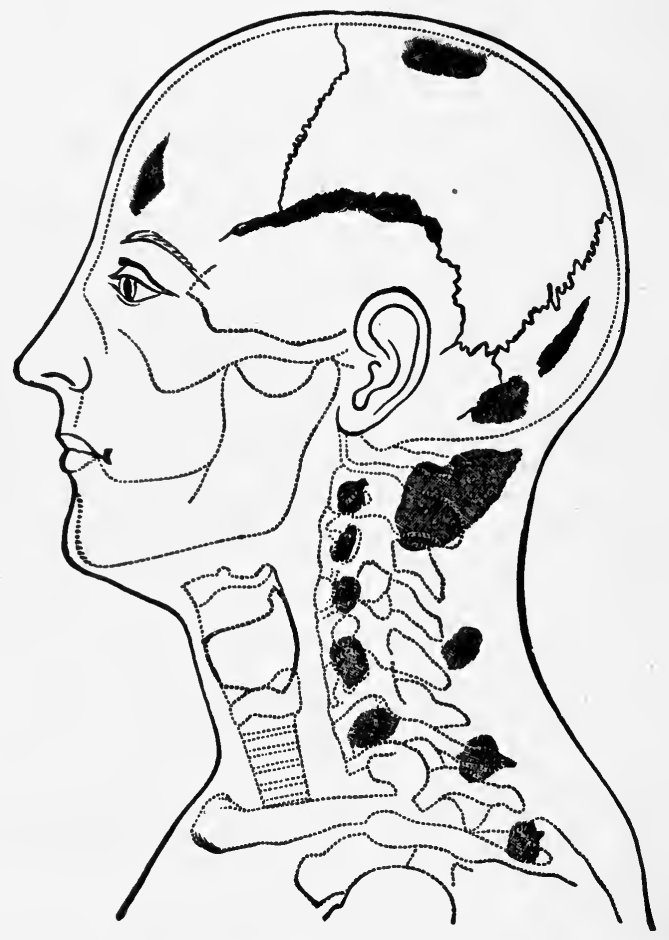

Fig. I. - The points upon which indurations are most frequently found (Edinger).

the masseurs and to the physicians who have studied and practised massage than to the medical profession at large. Writers on massage

1 Translated under title of Modern Clinical Medicine, in the volume on Diseases of the Nervous System, p. 863, Appleton, 1908. 
do not hesitate to speak of the "indurations" as foci of "chronic myositis," but there are, so far as I know, no histologic examinations on which we can base such a term. Edinger ${ }^{2}$ apparently considers the condition a neuralgia. Swelling of the neighboring lymph-glands and of the cervical sympathetic ganglia is mentioned by some writers.

The sensitiveness to touch extends to the aponeuroses over the skull, to the vertex and even to the frontal region; also down along the outline of the trapezius on the shoulder. In this as in many other respects it resembles "lumbago" and "stiff neck."

The disease is often referred to as "rheumatic," because it seems in some cases to follow exposure to cold and wet, e. g.: "A few days before the appearance of the symptoms he had been overtaken by a hailstorm while riding a bicycle." To some these statements still carry conviction, e. g., to Edinger, who says: "It is certain that refrigeration may produce the disease." In view of the modern conviction that much hitherto called "rheumatism" is really due to streptococcus infection, it may be proper to call these symptoms "rheumatic." Without implying any belief in the causal efficacy of cold, I am inclined to believe that the indurations described by Edinger and the masseurs are, in fact, areas of streptococcic myositis and represent a selective localization of organisms which find in this particular situation conditions that make their life and growth possible. Very possibly the streptococci present at first in the general circulation seek the tendinous ends of muscles for reasons like those which drive small fish into shallow water at the edge of a pond when a pike is chasing them.

I have, I regret to say, no cases in my own experience which exemplify this disease. I have referred to it here because it seems to me to deserve more careful study by clinicians and because of Edinger's statement, based on his extensive experience at the Neurological Institute in Frankfurt-am-Main, that it is probably the most frequent form of headache, and that: "The examination of the insertions of the muscles should never be neglected in any case of headache."

I4. Vasomotor Headaches.-Though vasomotor disturbances ma" occur in various types of headache, especially in migraine, there remains a group of cases in which only the vasomotor trouble (vasoparalysis and vasodilatation) is discoverable as cause. These patients have very red faces in the attack, and usually show reddish blotches or striæ over the rest of the body. The diagnosis is made by the presence of the above signs and by exclusion of all other known causes.

${ }^{1}$ Page 865 in the volume above cited. 


\section{POSITION AND NATURE OF THE HEADACHE}

(I) Many text-books map out the surface of the skull with special "headache areas," reminding one of a phrenologic map, but in my experience there is not often much to be learned from the position of a headache. Ocular headaches often begin or center near the eyes; pains due to otitis media often spread from an initial focus near the ear. Inflammations of the antrum or frontal sinus cause pain over the affected cavity. The pain of syphilitic periostitis corresponds with the position of the lesion. Migraine, with its unilateral distribution, and trigeminal neuralgia have also a typical distribution.

On the other hand, ocular and aural headache is often not thus localized, and the pain due to any of the other familiar causes (uremia, infection, brain tumor, constipation, menstruation, neurasthenia) may be in any part of the head, and is often unilateral, so as to be mistaken for migraine.

(2) The kind of pain is likewise of very little significance: throbbing, dull, burning, boring headaches are encountered in all sorts of diseases. A sense of constriction and pressure is mentioned by many patients of the psychoneurotic group, especially if they have been to France and have been told that they have a "tête en casque."

(3) The severity of headache is probably greatest in organic diseases of the brain or periosteum (cerebral tumor, meningitis, syphilitic periostitis), in the paroxysms of tic douloureux, and in those of migraine.

(4) Chronic headaches, sometimes lifelong, are associated with all the psychoneuroses (neurasthenia, hysteria, psychasthenia), and are sometimes present without any discoverable cause. They are often referred to the "base of the brain" (meaning the nape of the neck). Blows on the head, sunstroke, arsenical poisoning, and all sorts of "reflex" disturbances (pelvic, ocular, gastro-intestinal) are often vainly invoked as causes, and the term "constitutional" is often attached to such pains, but a frank confession of our ignorance seems to me better.

(5) The time of day markedly influences some headaches; those associated with frontal sinus disease often begin at the same hour each morning, last a certain time, and pass off. This is also true of the psychoneurotic group, but the time of seizure and of relief is much less accurately recurrent.

Headaches due to syphilis, to brain tumor, and to uremia are often worse at night, but syphilis has no monopoly of this characteristic.

Headache felt immediately on waking in the morning is often due to chronic nephritis; such a headache should always make us suspect 
that disease. Nevertheless there are other types of headache which show themselves at the same hour.

\section{TWO TRADITIONAL FALLACIES ABOUT HEADACHE}

(a) The belief that physiologic and pathologic states of the female generative organs often produce headache is widespread. Textbooks, such as Butler's, list dysmenorrhea, "uterine disease," disease of the ovaries, and even of the bladder (!) as causes of headache. No proper justification for these ideas has yet been attempted, so far as I am aware. Headache is, of course, exceedingly common in menstruation, but so it is in eclampsia; yet no one to-day connects the eclamptic headache in any direct way with the condition of the uterus. Toxemia of the puerperium, toxemia of the menstrual period, is a much more plausible, though not a demonstrable, hypothesis. (For further evidence on this point see p. 84.)

(b) "Lithenia" and "rheumatism" are also frequently invoked to explain headache. Neither word is defined by those who use them in this connection. "Lithemia" means constipation and the indigestion of lazy, gluttonous people. conditions which certainly do produce headache. (See p. 35.)

"Rheumatic headaches" refer usually to the type associated with "stiff neck" and indurations in the bellies of muscles attached to the occiput or the temporal region. Such headaches are presumably the result of streptococcus infection. (See above, p. 36.)

There seems, therefore, a good reason for reviving the tradition which applies the word "rheumatism" to such lesions, provided we recognize that rheumatic means streptococcic.

\section{MIGRAINE}

It may be doubted whether migraine represents a definite etiologic or pathologic entity. The word is generally applied to headaches explained by none of the causes thus far referred to in this chapter and remaining as an unexplained residuum. These headaches have, however, certain positive characteristics, namely, (a) their irregularly persistent recurrence, especially at the time of the menstrual period, but also between periods when the patient is especially tired; (b) their hereditary character, as they show a strong tendency to run in families; $(c)$ their tendency to diminish or disappear at the timeof the menopause. 
Although they also occur in men, they are much less common than in women. Such headaches are apt to begin in the early morning and last ten to twenty-four hours, occasionally longer. They are often associated with nausea and great prostration. I have never known a case cured.

\section{IMPORTANT TESTS}

The following tests should be made in all puzzling cases:

I. Thorough examination of the eyes (including retinoscopy), the pupil, and the testing of intra-ocular tension (glaucoma).

2. Temperature record (infections).

3. Blood-pressure measurement (nephritis, tumor).

4. Urinalysis (albumin, sugar, acetone).

5. Palpation of the insertion of the nape muscles at the occiput.

6. Examination of the nose and its accessory sinuses.

In the history, the following clues should be attended to:

(a) Is the headache hereditary, of paroxysmal occurrence and fixed duration (usually, twelve to twenty-four hours), accompanied by disturbances of vision and great prostration (migraine)?

(b) Is the history that of a psychoneurosis?

(c) Does the pain recur at precisely the same hour each day?

\section{Case 1}

A married woman of forty-two consulted me March I7, I904, for longstanding headaches which had been present, off and on, during the last five years, since an attack of what was called "grip," followed by deafness and ringing in the left ear. The patient lives in a very malarious part of a specially malarious suburb of Boston, but has never had the disease, so far as she knows.

For the past year the headaches have been much more severe, and have come with especial frequency at night, together with a burning sensation over the left side of the head, and to some extent over the entire body, and accompanying this burning sensation she feels chilly, but the temperature has never been taken. The menopause occurred a year ago, and since that time she has noticed that she is getting stouter, that her skin is very dry, harsh, and sallow, with scarcely any perspiration, and that her lips look bluish. Pain and the sense of coldness are often felt in the lower left axilla. Each winter she feels the cold more and more.

Some months ago she noticed edema of the feet and face; at the 
present time there is none, but she gets out of breath upon the slightest exertion, and her heart then beats violently, rapidly, and irregularly. Her urine is thick, dark, offensive, and at times its passage is followed by vesical tenesmus. The headache often wakes her in the night, and as soon as she wakes she has to pass water, which gives relief to the headache. She thinks she passes more urine at night than in the daytime. She is very irritable, and has much twitching and quivering of the lips. Her only child was born ten years ago, and died within the first year.

On examination the hands and lips were of a dark, slaty-blue color, yet quite warm. The face showed a yellow pallor, the total effect being that often seen under the Cooper Hewitt mercury light, such as is used in automobile garages. The heart was negative, save for a slight systolic murmur at the base. The lungs showed nothing abnormal. The edge of the spleen was easily felt on full inspiration. Its consistency seemed increased. The abdomen was otherwise negative. The temperature was $99.2^{\circ} \mathrm{F}$. at $5 \mathrm{P}$. M. The urine, save for high color and other evidences of concentration, showed no abnormality.

Discussion.-The possibilities which were first considered in this case included cardiac disease, myxedema, malaria, and another presently to be mentioned. The diagnosis of the attending physician was "some queer kind of heart disease," but on examination I could find no heart disease, queer or otherwise, although the breathlessness and cyanosis made it natural to search for a cardiac lesion.

Myxedema was suggested by the cutaneous changes and the sensitiveness to cold, but on cross-questioning neither of these two characteristics was at all well marked, and there were no mental changes, no subnormal temperature and no special alteration in the physiognomy except as regards the extraordinary coloration before mentioned. It was easily made clear that this cyanosis did not depend upon any disease of the heart or lungs. The enumeration of the red cells showed but $4,180,000$, proving that the color of the lips was not due to polycythemia. There was nothing in the symptomatology nor in the gross characteristics of the feces to suggest a cyanosis of intestinal origin, nor did the coloration appear to be of the vasomotor type, so often seen in neurotic and hysteric patients. There was no ebb and flow about it, no variation in the tint from hour to hour, nor from day to day. By rough tests there was no notable deafness and no mastoid tenderness.

After excluding the causes above referred to, it was natural to think of methemoglobinemia, such as is often produced by overdose of headache powders containing acetanilid. Her attending physician had given her no such powders nor any diug belonging to the group prone to pro- 
duce methemoglobinemia, but on questioning the patient I learned the following facts: For the last five years she had been taking headache powders in increasing numbers. Her husband obtained a box of them from the local druggist once or twice a week, and by calculation it appeared that she had averaged roo grains a week for some months, great relief being thus obtained for the headache.

A drop of her blood soaked into the bibulous paper of the Talqvist hemoglobin scale produced a chocolate-brown stain, quite incomparable with any of the hemoglobin tints of the scale. Spectroscopic examination showed the familiar spectrum of methemoglobin.

Outcome.-The patient was ordered at once to stop the headache powders and to take no medicine containing acetanilid or any member of that group. May $3 \mathrm{~d}$ she reported that her headaches were much less, her sleep and breathing much better, and her sensitiveness to cold much less troublesome. She was still weak and pale, but her appetite was much improved, and she had gained eight pounds since March 17 th.

January 26, 1907, the attending physician writes me: "A year after you saw her the general condition was much better, although she occasionally had severe headaches. The color of the blood was improving, but at the time of the last examination which I made, a year after you saw her, blood still showed a tinge of brown."

Diagnosis.-Methemoglobinemia.

\section{Case 2}

A longshoreman of thirty-six was first seen March 8, I904. The patient has been in the habit of taking three glasses of whisky a day. He had gonorrhea at twenty-six, and chancre twelve years ago, followed by sore throat, a mucous patch, and an eruption.

He had typhoid and pneumonia at thirty. Family history good.

For a good part of the past five years he has had frontal headache. Last October he began to take potassium iodid, but in November the headache became worse, and a swelling appeared on the forehead over the left eye. The pain lasted a week and then disappeared. A week ago, after being exposed to a violent draft on a sleeping-car, he had a similar attack. This time his eyes were closed by a swelling of the lids. His forehead was tender and swollen, especially on the left. Now he complains of severe pain in the forehead, with swelling and tenderness.

Two years ago he had what seemed like a similar process in the metacarpal bones of the right hand, the bone becoming enlarged and very tender. His general condition is now better than six months ago. $\mathrm{He}$ has taken potassium iodid, but finds that it makes the pain worse. 
He has taken as much as 225 grains a day, but not regularly. His appetite is good, his bowels regular. He has had no symptoms of iodism, and feels perfectly well but for his headache.

On physical examination the points mentioned in the history were verified, and nothing else was discovered. The second left metacarpal bone was much enlarged and irregular in outline. There were also enlargements at the base of the first phalanx of the left index-finger, and a slight rounded prominence over an area the size of an egg above the left eyebrow. The temperature ranged between $98^{\circ}$ and $99.5^{\circ} \mathrm{F}$. The leukocytes were $\mathrm{I} 7,200$ at entrance, 78 per cent. of them being polynuclear. The hemoglobin was 70 per cent., and the red cells showed a slight achromia.

I. What further inquiries and examinations are likely to throw light on this case?

2. What inferences can be drawn-(a) From the effect of a draft on the pain; $(b)$ from the effect of potassium iodid?

3. Why is eye-strain an unlikely cause for this headache?

4. What points against frontal sinus disease?

5. What three common chronic ulcerative processes involve the skin and deeper tissues most frequently.

Further inquiry into the past history revealed the fact that he had had a chancre at twenty-eight, followed by a rash and a sore throat, with white patches in his mouth. A Wassermann test (not known in 1904) would have helped toward diagnosis.

Probably the "effect" of the draft was coincidence, at most, an exciting or favoring cause. Many headaches miscalled "rheumatic" are really syphilitic. There is no reason to believe that "rheumatism" ever causes headache except in acute infectious cases. The failure of potassium iodid is discussed below.

Eye-strain does not often begin at thirty-six in a man who uses his eyes for such work as a longshoreman's.

The long duration of the headache is against disease of the frontal sinus. Physical examination, however, must determine the point.

Three common causes for chronic ulcerative processes (excluding varicose ulcer) are: Syphilis, tuberculosis, malignant disease.

[The man was seen in consultation by Drs. Bowen, Shattuck, Fitz, Gannett, A. T. Cabot, and J. P. Clark. All concurred in the diagnosis of syphilitic periostitis.]

Outcome.-The day after entrance two distinct craters about the size of a half-dollar were felt on the forehead. The headache was given some immediate relief by Io grains of phenacetin with 2 of caffein, but 
sulphonal and trional, Io grains each, were also needed for sleep. He was given inunctions of mercury and potassium iodid grains Io, increasing to Ioo. Black-wash was also applied to the forehead, and on the twelfth the iodid was omitted on account of marked swelling of the left eyelid. By that time the swelling of the forehead was much less, and after omitting the iodid, the swelling of the eyelid also became normal. By the fifteenth of March his symptoms had almost disappeared. Obviously, the mercury, rather than the iodid, was what helped him. $\mathrm{He}$ showed at no time any signs of salivation.

In view of the above facts the diagnosis of syphilis is not in doubt, and needs no further discussion in this case. The relation of syphilis to headache seems to warrant us in dividing syphilitic headaches into three groups:

(a) An acute infectious headache, occurring at or near the time of the roseola, adenitis, and other "secondary" lesions.

(b) A chronic periosteal headache, with or without obvious external lesions on the forehead.

(c) A headache with symptoms of brain tumor, due to localized syphilitic processes within the skull, forming syphilomatous tumors or causing internal hydrocephalus.

The latter group is of especial importance, since they are often mistaken for genuine brain tumor, whence follow a hopeless prognosis, a neglect of vigorous antisyphilitic treatment, and much unnecessary suffering. I have three times seen recovery after antisyphilitic treatment in cases given up to die of brain tumor. The only safe rule is: Give mercury (in moderate doses) and potassium iodid (in doses gradually becoming enormous) in every case presenting the signs and symptoms of cerebral tumor.

The prognosis is for immediate relief and subsequent recurrence in one or another form. The expectation of life is much less than for non-syphilitics.

Treatment-mercury especially - should be given at intervals for life. Potassium iodid is needed only when definite lesions are recognized.

Diagnosis.-Syphilitic periostitis.

\section{Case 3}

A married Russian housewife of thirty-seven entered the hospital May I7, I904. In I90I she had been in the surgical wards for a stricture of the rectum of inflammatory origin, for which an inguinal colostomy was done. After this operation she had no trouble with her bowels 
(which had been seriously constipated), the inguinal wound was closed, and she remained well until May I, I904, when she began to have pain in the back of her head, at first mild, and relieved by "bromo-seltzer,' but for the past week very severe and extending over the whole head. It now lasts through the entire twenty-four hours, and has prevented sleep for the past two nights. Day before yesterday she had an attack of nausea and vomiting. The headache is so severe that she wants to jump through the window and kill herself. She feels first hot, then cold, sweats a great deal, especially at night, and easily becomes tired.

Physical examination, including the fundus of the eye, shows nothing abnormal except an inequality of the pupils. Their reactions, however, are normal, likewise the blood and urine.

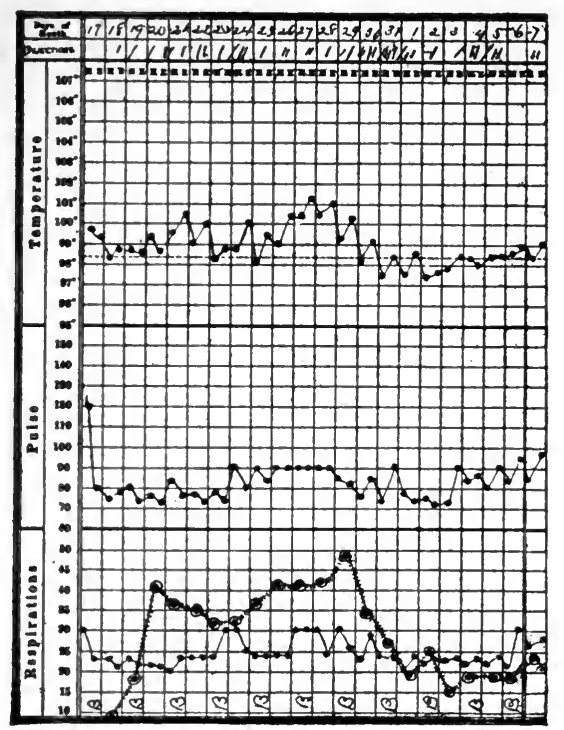

Fig. 2.-Chart of case 3 .

For the first two days her headache was continuous and severe, despite lactophenin, ro grains, caffein, 5 grains, sodium bromid, 30 grains, potassium iodid, Io grains, three times a day. Compound jalap powder, I dram, and high enemas of oil and suds were given in the hope that the headache might be relieved by purgation. In spite of all these remedies the headache was undiminished at the end of the first week in the hospital.

Discussion.-No certain diagnosis can be made in this case. The relief following potassium iodid may well be a coincidence, for many headaches of unknown origin subside without any treatment after, a period similar to the course of this case. 
The rectal stricture was of the type ordinarily regarded as always or usually syphilitic, but on insufficient evidence. There is no good histologic evidence for syphilis in such strictures, whereas it is well known that gonorrhea is prone to produce stricture in any tube. The presence of syphilitic lesions elsewhere in the body often gives color to the diagnosis of syphilis in a rectal stricture, but in this case there were no such lesions.

The study of the previous history is of prime importance in the diagnosis of such cases. It revealed in this case that the woman had been sterile, but had had no miscarriages and no lesions suggesting syphilis, so far as she knows.

If the case is syphilitic, it is probably due to an intracranial lesion of the type simulating brain tumor. (See Case I, p. 40.)

The prognosis is for immediate recovery, but probable recurrence, if the diagnosis of syphilis is correct, though the recurrence may involve any other organ (liver, aorta, bones, subcutaneous tissues).

Antisyphilitic treatment should be continued at intervals for life. The interval depends on the character, circumstances, and physical condition of the patient.

Outcome.--The potassium iodid was increased after the first week to 15 grains and then to 30 three times a day. The headaches had rapidly diminished in severity and frequency. Slight signs of iodism had in the mean time appeared. By the eighth of June she was feeling well and ready to go home. Three months later there had been no recurrence.

Diagnosis.-Syphilis ?

\section{Case 4}

A married cloakmaker, forty years old, was seen June 2I, I894. Eight years previously she had begun to have womb trouble, characterized by bearing-down pain in the upper abdomen and back. Five years ago she had an accident to her head, and I 7 stitches had to be taken. Since then she has had unilateral "sick headaches" about four times a year, lasting usually one day. She has been pregnant six times, and has three times produced an abortion.

Four weeks ago she began to have pain in the back of her neck, sometimes darting, sometimes constant, worse in the day-time, not preventing sleep. With the pain there seemed to be a swelling, which impressed her as being both inside the throat and in the nape. She had no difficulty in swallowing, though her throat was somewhat sore at the same time. Three weeks ago this pain extended to the whole head, affecting 
especially the temporal regions, which feel swollen and tender. She has had no other symptoms.

Physical examination showed the patient sallow and covered with a reddish, papular eruption, with a shot-like feel under the skin. It is most marked upon the face and trunk. Visceral examination is otherwise negative, as is the blood. The urine is alkaline, high in color, Io23 in gravity, with the slightest possible trace of albumin The sediment shows large squamous epithelial cells in clumps, also polynuclear cells, triple phosphate crystals, and some octahedral crystals which resist the action of acetic acid. The fundus oculi is normal.

Discussion.-Three types of headache are immediately suggested as we read the story of this patient:

I. Due to trauma.

2. Due to migraine.

3. Due to syphilis.

Traumatic headaches, following violent cerebral concussion, as in foot-ball or coasting, are apt to follow an initial period of coma, and usually persist steadily for weeks or months. Periodic pain, such as is here described, is not often associated with trauma.

Migraine is apt to appear before the thirty-fifth year, and to occur more frequently than in this case.

It is important to realize that unilateral periodic headache accompanied by nausea and vomiting deserves the term "migraine" only when all known causes of headache can be excluded. The headaches associated with nephritis or cerebral tumor are often migrainoid in type, especially in the earlier stages of the malady. The study of the urine and of the fundus oculi is thus often omitted because the attacks are so described by the patient that "typical migraine" is assumed and treated from the start.

A migrainoid headache which later became constant, aroused, therefore, the suspicion of nephritis and of cerebral tumor. Nephritis, however, could be excluded in this case by the absence of urinary changes and of vascular hypertension. The fundus oculi was negative; there were no focal symptoms (such as aphasia, paralysis, Jacksonian or general epilepsy, paresthesia, or astereognosis), and the absence of vertigo, vomiting, and vascular hypertension also militated against the diagnosis of cerebral tumor, which, however, could not be absolutely excluded.

Syphilis is suggested by the rash. Further examination showed a postcervical adenitis. The absence of any knowledge of infection is of no importance. Only positive evidence is of value in relation to syphilis, 
and it cannot be too positively stated that in any person, young or old, rich or poor, whatever his character or circumstances, syphilis is always a possible diagnosis. The opportunities for the non-venereal acquisition of syphilis are very many.

In this case the rash was not typical, but might have been an ordinary skin infection. Its generalized distribution, the associated adenitis, and the persistent headache made it, however, more suspicious.

The absence of miscarriages is less significant; since the abortions may have anticipated the course of nature. On the whole, syphilis seems the most probable diagnosis.

The prognosis and treatment of syphilis have been sketched on $\mathrm{pp}$. 44 and 46.

Outcome.-The headache was relieved temporarily by 5 grains of phenacetin with $\frac{1}{2}$ grain of codein. Later, some morphin was required on one or two occasions. Mercury and iodid of potash were given by mouth, in small doses, and in a week she was very much better. In two weeks the headache was very slight, the rash nearly gone, the glands barely palpable. July I 2 th she was discharged well, with the advice to continue the iodid of potash in 5-grain doses three times a day for a number of months.

Diagnosis.-Syphilis.

\section{Case 5}

A Jewish shoemaker of thirty-seven was seen July 8, I908. He has had some trouble with his stomach since he first came to this country, five years ago. Five days ago he began to have "pain over his heart," followed by shortness of breath and fainting. This attack lasted only a few hours, but since that time he has had severe headache, loss of appetite, and gastric distress without vomiting. His bowels have become constipated, and his sleep is disturbed by bad dreams.

On examination a few fine transient râles were found at the base of each lung. Respiration at the left base and axilla was somewhat louder than on the right. There was slight epigastric tenderness, and the edge of the spleen was easily felt $\mathrm{I}_{2} \frac{1}{2}$ inches below the costal margin. There was anterior and internal bowing of the right tibia, with prominence, but no roughening. The temperature at entrance was $100^{\circ} \mathrm{F}$; pulse, 75; leukocytes were 4800 ; the Widal reaction negative. Bloodculture was negative; urine normal.

Discussion.-What are the causes of prominence or enlargement of the tibiæ?

Significance of the râles in this case? 
The pulmonary signs are not characteristic of tuberculosis nor of any other disease of the lung.

The prominence and bowing of one tibia might be due to old rickets, to osteitis deformans (Paget's disease), or to syphilitic changes, but the latter are usually accompanied by roughness, unevenness, and cutaneous changes, while Paget's disease should affect the femora and the clavicles more extensively than the tibix. Rickets seems the more likely explanation. .

An acute headache (five days' duration) with fever suggested, naturally enough, an infectious disease. The acute infectious diseases most often causing headache in a temperate climate are the milder respiratory infections ("common colds"), tonsillitis, sepsis, and typhoid -malaria less often.

The negative blood examination, the normal temperature, and the paucity of visceral lesions rule out these infections.

There are many items pointing to a psychic origin for this headache. It began immediately after an attack of thoracic pain, which was evidently believed by the patient to be due to heart disease- that terrifying affliction. The subsequent bad dreams and gastro-intestinal disturbances are very common results of a scare about one's heart, especially in high-strung people like the Jews.

The therapeutic test also bears on the diagnosis here. As soon as the patient was assured (after a searching and thorough examination) that his vital organs were sound, his headache and other trouble began to improve. The application of suggestion (in the form of menthol) completed the cure. It is, of course, impossible to exclude some obscure infectious or toxic disease, but the weight of evidence is against it.

Outcome. - A diagnosis of typhoid had previously been made, but the next morning the temperature was normal and the man complained of nothing but headache. This continued for several days, but was relieved by a 25 per cent. alcoholic solution of menthol applied to the painful part. Reassurance played a considerable part in his recovery.

Diagnosis.-Headache of psychic origin.

This is a fit place to consider the so-called "neurasthenic," "essential," or "constitutional" headaches. There are some persons so abnormally sensitive to sensory stimuli that the weight of the body gives pain, even when they are seated on soft chairs; the pressure of the clothing, the ordinary changes in atmospheric temperature, hurt them as a decaying tooth is hurt by simple pressure, heat or cold. In such persons the circulatory or neural processes in the head may be sufficient to cross the pain threshold and to present themselves as pain. Not all such persons 
have any of the mental or physical characteristics of the neurasthenic, and it seems to me to darken counsel if we class such headaches as "neurasthenic" merely because we discover no organic basis for them.

In another group of persons the headache is clearly dependent upon psychic states, which can be used both to produce and to allay the pain. The suffering is forgotten when the person is active and interested, returning when the sufferer's attention relapses upon himself.

In a third group there are no general hyperesthesia and no variation of the pain with psychic states. I have followed several such cases through the period of adolescence and up to their disappearance with the end of this state.

Others occur in later life, and may be steady or vaguely periodic. Of this large group. we know practically nothing, and this should, I think, be plainly indicated in our terms. I believe then that we should distinguish within the so-called "neurasthenic" group:

(a) Headaches due to constitutional or chronic hyperesthesia.

(b) Headaches due to psychic causes.

(c) Headaches whose cause is absolutely unknown.

As an example of the type last mentioned, I recently studied the case of a hearty, vigorous Italian laborer who began to suffer from constant headache in July, I908. In August I saw him in consultation, but could discover no cause for his steady suffering, which now disabled him from work. I sent him to the Massachusetts General Hospital, where the most careful study of his internal viscera, body fluids, eyes, ears, nose, throat, and bony sinuses revealed absolutely nothing. Just as we reached the end of this fruitless search the headache-after nine weeks' duration-suddenly ceased altogether without treatment, though quinin, mercury, and potassium iodid had been proved ineffectual by thorough trial previous to his entering the hospital. Up to the present time (September, I9ro) there has been no recurrence of pain.

\section{Case 6}

A housewife of forty-seven entered the hospital December 23, 1907. Since the beginning of her menstruation at the eleventh year she had noticed a fullness in the front of her throat, which became more prominent at the time of her first pregnancy in her twenty-sixth year. It became smaller after delivery, but increased with the next and with each of the succeeding eight pregnancies. Each time the swelling increased more during the pregnancy than it diminished after delivery, so that the total effect has been an increase of the tumor. It has never caused any discomfort or inconvenience. 
For the past two years she has had "sick headaches," beginning early in the morning or long after eating, lasting twenty-four hours, coming about once in two weeks, until lately, when they have begun to come twice a week and have been accompanied by nervousness. She feels hot most of the time, and prefers cold weather. For six months she has noticed a weakness of her hands. Two weeks ago she had the "grip," and has since then noticed considerable shortness of breath, amounting of late to orthopnea. She has lost 30 pounds in the last two years. The bowels have been slightly loose; the appetite excellent.

Physical examination showed emaciation, cyanosis, a slight exophthalmos (not previously noticed by the patient), a fine tremor of the hands, and marked asymmetric enlargement of the thyroid, its greatest circumference being $16 \frac{1}{2}$ inches. The heart's impulse was in the sixth interspace, $\frac{3}{4}$ inch outside the nipple-line, 4 inches to the left of midsternum. The action was rapid, ranging between roo and I20, accompanied by some irregularity. The first sound was very sharp at the apex, and was preceded by a presystolic roll. The pulmonic second sound was much greater than the aortic second sound. Systolic bloodpressure, $175 \mathrm{~mm}$. Hg. Shadows and movements of the intestinal coils were visible over the abdomen. There was considerable tenderness in the epigastrium and about the navel. The edge of the liver was felt 3 inches below the costal margin. There was no ascites, but both legs showed soft edema throughout. The leukocytes were 15,400 . The blood was otherwise normal. The urine was pale, Ioro to Ior 2 in specific gravity, without a trace of albumin. There were many leukocytes; no casts. The twenty-four-hour amount ranged between 5 and 30 ounces.

Discussion.-I. What was the nature of the thyroid tumor?

2. What caused the headache?

3. Can any reason be given for her preferring cold weather?

4. How are the characteristics of the urine explained?

5. Is there more than one possible explanation of the presystolic roll heard in this case?

6. Under what conditions are intestinal movements visible through the abdominal wall?

7. What diseases produce loss of weight despite good appetite and digestion?

The discussion of these questions will involve a statement of the diagnosis, prognosis, and treatment.

Regarding the thyroid tumor, it is clear that its duration (thirty-six vears) excludes malignant disease. We have left the so-called "simple 
goiter" and "exophthalmic goiter." The case illustrates well the transition from the first to the second condition; also the relation of the thyroid to pregnancy. From her eleventh to her forty-fifth year the patient had no symptoms from her goiter. It was increasingly unsightly, nothing more. After the forty-fifth year came the familiar symptoms of hyperthyroidism-loss of weight despite good appetite, a sense of increased bodily heat corresponding with the abnormally rapid metabolism; finally tachycardia, tremor, and exophthalmos.

The only important diseases causing loss of weight despite good appetite are diabetes (either form), Graves's disease, and some cases of arteriosclerosis. In one of my cases of Graves's disease loss of weight was the symptom which brought the patient to me. He wanted to know why he was losing weight despite an excellent appetite. $\mathrm{He}$ mentioned no other complaints.

In the present case the emaciation explains the visibility of intestinal peristalsis, for emaciation is all that is necessary to produce this symptom. In patients not emaciated such a sign usually means intestinal obstruction.

The condition of the urine in this case can hardly be explained (as one might at first think) as a result of renal stasis due to a dilated heart, for the low specific gravity and pale color are the opposite of what we expect in renal stasis. When taken in connection with the abnormally high blood-pressure, these features of the urine suggest nephritis. Such vascular hypertension is unusual in Graves's disease. WT The headaches are also much more comprehensible if we suppose that the patient had both nephritis and Graves's. I have already referred to the frequency of so-called "sick headache" in nephritis, as well as in brain tumor and syphilis. In simple hyperthyroidism headache is not common.

The cardiac signs suggest, first of all, a mitral stenosis with dilatation of the heart, but another possibility is to be remembered, namely, that the dilatation itself may be the cause of the murmur. It has been repeatedly noted of late that not only in connection with aortic regurgitation (the so-called Flint's murmur), but in any form of cardiac hypertrophy and dilatation affecting the left ventricle, a presystolic roll may be heard at the apex. Thus in adhesive pericarditis and in simple nephritic hypertrophy we often hear such murmurs. There is no way of deciding in this case whether or not mitral stenosis is present, but it is a good rule, often borne out by postmortem experience, to assume as few lesions as can be made to explain the facts. On this principle, the diagnosis of this case should be Graves's disease; chronic nephritis with resulting cardiac hypertrophy and dilatation. 
The prognosis is for a few months of life at best. In treatment rest is the essential. Morphin, followed by bleeding, purgation, and diuretics, may give some relief. Digitalis is not likely to be effectual.

Outcome. - The pulse-rate steadily declined during her first week in the hospital, and the swelling became less; but on January Ist the patient became noisy and mentally confused, the respiration slow and deep, the breath having an ammoniacal odor. There were frequent attacks of severe dyspnea. In the next thirty-six hours she was, for the most part, semiconscious, but never unconscious, and was comfortable except during the attacks of dyspnea. Pilocarpin produced no sweating, and attempts to give a hot-air bath were unsuccessful. It was impossible to purge, as she refused to swallow anything. Her heart continued strong and not rapid.

She died on the third of January.

Autopsy showed chronic glomerulonephritis with hypertrophy and dilatation of the heart and general dropsy; simple adenoma of the thyroid; obsolete tuberculosis of the spleen.

One of the interesting points in this case is the existence of nephritis without albuminuria during the period under observation. The bloodpressure gave more correct indication for diagnosis, as is often the case.

Diagnosis.-Uremic headache; chronic glomerulonephritis; hyperthyroidism.

\section{Case 7}

A young woman of twenty-five, a student, entered the hospital November 7, 1907. One of her aunts died of consumption. She was treated by Dr. R. W. Lovett for three years for some spinal trouble, beginning with her fifteenth year. When she was eighteen her menstruation stopped and her spleen became enlarged. She was then treated for some time by Dr. Franz Pfaff. Two years ago menstruation again ceased during the winter. The intervals between her periods are still five or six weeks long, and she always has headache during the catamenia. She has been overworked for the past three years, and has been nervous, but has had no actual breakdown and no hysteric symptoms; she has been especially tired most of the time since the first of October. She takes two cups of tea and one of coffee a day.

Eight days ago she found it very hard to concentrate her mind upon her work. The next day she had severe headache, and that night was sleepless. Six days ago the headache became still worse, and she coughed up a little blood and phlegm. Ever since then she has coughed a little, but without sputa. Five days ago she had photophobia and felt 
tender lumps upon the back of her head. That evening she had chill, followed by sweating. Three days ago she had another chill, and her teeth and her left ear ached. This time she began taking aspirin in 5-grain doses for the relief of her headache, and found it very effectual. The last two days her headache has been less severe, but it is still present in the back of her head. She has been slightly constipated and has felt somewhat weak.

Temperature, IоI. $3^{\circ} \mathrm{F}$; pulse, 83 ; respiration, I8. White cells normal.

Physical examination was negative, save for a short, sharp, whistling systolic murmur in the pulmonary area, transmitted only along the left

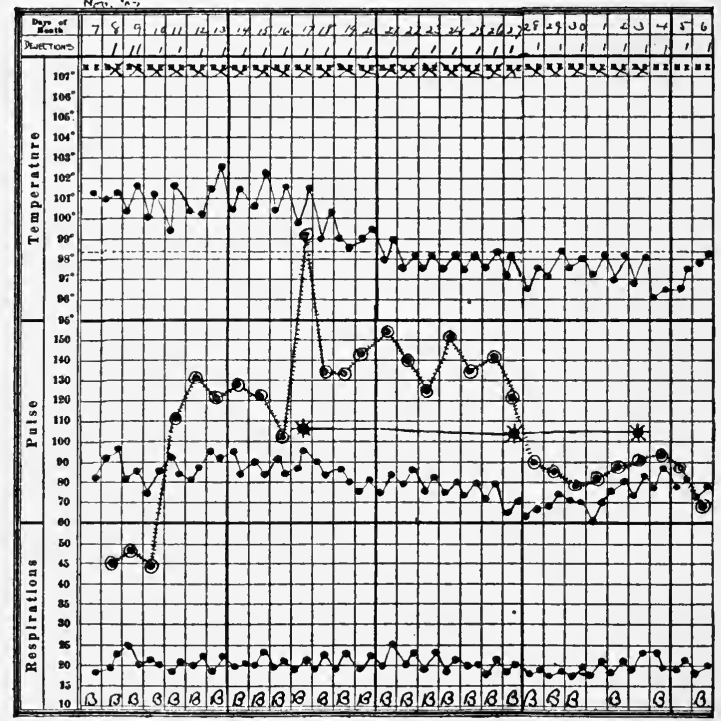

Fig. 3.-Chart of case 7 .

side of the sternum. The abdomen was held slightly rigid throughout, but was tympanitic and free from tenderness. The spleen was not felt.

Discussion.-Certain features in this case suggest that the headache may be due to tuberculous meningitis. The history of consumption in the family and of a prolonged treatment addressed to the spine, together with the cessation of menstruation at her eighteenth year, are all factors which make us think of tuberculosis. The photophobia, too, is a common meningeal symptom.

Against meningitis, however, is the absence of any tuberculous focus now discoverable on physical examination, the absence of any lesions referable to the cranial nerves, and the recent subsidence of the headache without any oncoming coma. 
Could this be a neurasthenic headache, so called? She is at the age when such things are commonest, and there is a history of nervousness and overwork. But the continued fever seems to me to make this impossible. I do not think there is any good evidence that a fever such as is shown in the accompanying chart ever results from neurasthenia, hysteria, or any psychoneurosis.

Malaria is suggested by the chills and the headache, but is excluded by the absence of parasites in the blood. The lumps complained of in the occipital region were not discoverable on physical examination. Had they turned out to be glandular, syphilis might have been suspected.

With the exclusion of the above possibilities we have to consider what diseases are the most frequent in patients who have fever with a negative physical examination and a low leukocyte count. The answer must, I think, be as follows: If the fever is a short one, it is generally labeled "grip" under these conditions, though I prefer to call it an unknown infection. If the fever persists for two weeks or more without the development of physical signs, typhoid usually turns out to be present, as was the case here.

Outcome.-On the right side of the abdomen there developed later two red macules which decolorized on pressure. The course of the temperature is seen in the accompanying chart. The Widal reaction was positive at entrance. Blood otherwise negative. The course of her illness was uneventful, and she was discharged well on the seventeenth of December.

It is worth emphasizing the fact that constipation, cough, and chills are common symptoms at the onset of typhoid, also that the headache is usually earlier and more prominent than in other infections. (For the treatment of this case see Appendix, p. 759.)

Diagnosis.-Typhoid.

\section{Case 8}

A Russian clerk, eighteen years of age, entered the hospital February 27 , I908. The only history which could be obtained from him was that two days ago he fell downstairs and since then he has had a good deal of headache.

Physical examination showed that he was drowsy, his right pupil slightly larger than his left, both reacting normally. His throat was red and slightly swollen. There was considerable rigidity of his neck, but no actual retraction. Rotation and backward flexion were normal, but the head could not be bent forward. Visceral examination was entirely negative, with the exception of Kernig's sign, which was present 
on hoth sides. The temperature was $100.3^{\circ} \mathrm{F}$; the pulse, 60 ; respiration, 25. Fundus oculi normal. Blood and urine normal. Bloodpressure, I45. During the night he became unconscious, and the next morning had marked retraction of the head, unequal and unresponsive pupils, a strabismus, absence of superficial reflexes, Babinski's reaction on the right, and a rectal temperature of $102.8^{\circ} \mathrm{F}$.

Discussion.-Concussion of the brain and meningitis were the diagnoses at first suggested in this case. Any headache following a fall on the head is rightly suspected as being due to concussion, but there are certain symptoms in this case not thus to be explained, $i$. e., the inequality of the pupils, the rigidity of the neck, and the presence of Kernig's sign.

These three signs, together with the presence of fever and slow pulse, the rapidly developing coma, strabismus, and Babinski's reaction, all point to meningitis, which was the diagnosis made at the outset. Acting upon this a lumbar puncture was done, and 35 c.c. of bloody, turbid fluid were removed. The examination of this fluid, however, showed nothing but macerated red corpuscles, no micro-organisms either in cover-glass or culture. This speaks strongly against epidemic meningitis, while the great rapidity of onset and the absence of any lymphocytosis in the spinal fluid make tuberculous meningitis unlikely. The presence of blood in the spinal fluid suggests cerebral hemorrhage or fracture of the base of the skull.

Normal urine and normal blood-pressure rule out uremia, and normal blood excludes malaria. Brain tumor may manifest itself suddenly after a long latent period by symptoms like those in this case, but the absence of paralysis, of changes in the fundus oculi, and the presence of the rigid neck and the bloody spinal fluid militate against this diagnosis. No absolute decision was arrived at before death, which occurred on the first of March.

Outcome.-Autopsy, March Ist, showed fracture of the base of the skull, multiple contusions of the cerebellum and frontal lobes, with hemorrhage.

Diagnosis.-Fracture of the base of the skull.

\section{Case 9}

A domestic of twenty-three was seen March I4, I908. She was perfectly well until noon of the day before, when she was seized with sharp, cutting pain in the forehead and a slight sore throat, with fever. She went to bed and slept well, but awoke with the same headache, and vomited when she got out of bed. The headache has continued since. 
When seen at the hospital the patient's temperature was ro2. $5^{\circ} \mathrm{F}$. her pulse, I25, the skin hot and dry. The pupils were equal, regular, and reacted normally; the fundus negative; the throat slightly reddened and swollen; face flushed. The viscera, negative. Leukocytes, 9000. Blood and urine were otherwise normal. Blood-pressure, 125. During the first thirty-six hours of her stay in the hospital she suffered a good deal with headache, relieved more or less by phenacetin and an ice-bag in the frontal region.

Discussion.-I have known tuberculous meningitis to manifest itself first by intense pain at the root of the nose, as in this case. All the ordinary symptoms of that disease, however, except headache and fever, are absent in this case. Typhoid, malaria, and most other infections are ruled out by the negative physical examination and the short course of the disease, which was practically gone in four days. On the third day careful questioning showed that the pain was limited to the region of the frontal sinuses. On the eighteenth she was able to go back to work.

In view of these facts an infection of the frontal sinus seems the most likely cause of her headache. In some cases of this disease the headache appears in a characteristic way at the same hour each morning, perhaps owing to the accumulation of secretions during the night. Sometimes the diagnosis is assisted by the sudden appearance of a nasal discharge coincident with the cessation of pain; in other cases the close limitation of the pain to the region of the frontal sinuses is our best clew to diagnosis.

Outcome.-On the eighteenth of March she went back to work.

Diagnosis.-Sinusitis.

\section{Case 10}

A cook of twenty-three entered the hospital April 9, I908. Her family history and past history were excellent. Two months ago she stopped work for a fortnight because of fatigue and persistent headache. Ten days ago the headache returned and has persisted since. It is severe in the frontal and occipital regions. Four days ago she began to vomit, and since then has vomited about six times every twenty-four hours. Even water is rejected. There has been no abdominal pain, but persistent nausea. She has had no cough and no other symptoms. The course of the temperature is seen in the chart on page 58 . The white cells were 4400 at entrance, 4900 on April 2oth; the Widal reaction always negative; the blood otherwise negative. The urine ranged between 20 and 30 ounces in twenty-four hours, with a specific 
gravity between 1026 and 1036; a trace of albumin; a few hyaline and fine granular casts. The pupils were equal and reacted to light and distance; the fundus oculi, normal; the chest and abdomen negative, save for slight tenderness and rigidity in the epigastrium. During the first week she seemed rather hysteric at times, complained continually of headache, and was hungry, but no diagnosis could be made.

Discussion.-Typhoid is suggested by the course of the temperature, the subnormal leukocyte count, and the headache. Indeed, there is nothing in the case, as here stated, positively to exclude typhoid. Against it, however, are the long duration of the headache, which is usually gone after the first ten days in typhoid. The persistent nausea

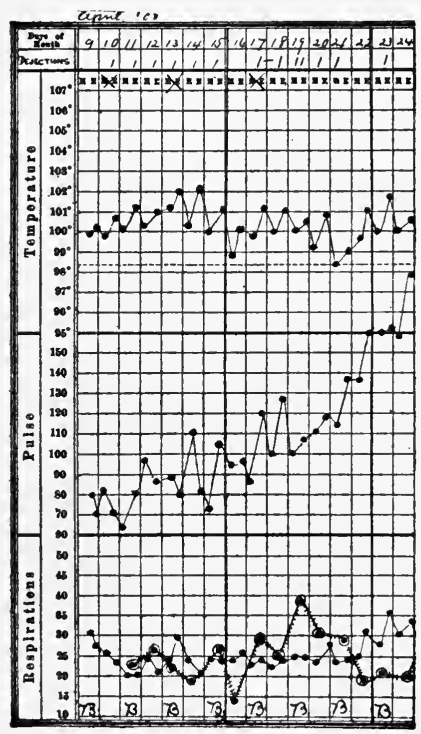

Fig. 4.-Chart of case Io. is also very unusual in typhoid. Perhaps the strangest symptom, however, is the excellent appetite, which is almost unknown in a patient seriously sick with typhoid.

The question of hysteria must be considered. All the symptoms in the case are consistent with this diagnosis, with one exception-the continued fever. There is, in my opinion, no such thing as a hysteric fever of this type. An elevation of less than one degree over a considerable period or a sharp sudden, short-lived rise occurs in hysteria, but not a persistent fever of this type.

The two diseases seriously to be considered are cerebral syphilis and tuberculous meningitis. As a matter

- of fact, the diagnosis of syphilis was made in this case by a skilled neurologist. The entire absence of any history and of any visible lesions of this disease is not in itself at all conclusive against it, neither is the age of the patient, although the great majority of cases of cerebral syphilis occur in older persons. More important evidence against syphilis is the subnormal leukocyte count, which is distinctly rare in syphilitic cases.

Epidemic meningitis comes on more suddenly, almost always produces a leukocytosis, and usually runs a shorter course. Nevertheless, it cannot be excluded without an examination of the spinal fluid.

Outcome.-April 22d lumbar puncture was done, and ro c.c. of 
clear pale fluid was obtained, the sediment showing 72 per cent. of lymphocytes, 28 per cent. of epithelial cells. In the Thoma-Zeiss counter, this fluid showed 42 lymphocytes per c.mm. On the twenty-third the patient seemed to be restless, the left pupil slightly larger than the right. During the forenoon the left hand became flexed. At noon, the left leg and the left side of the face became paralyzed, and the reflexes absent. Syphilis, producing softening from thrombosis in the region of the right internal capsule, was suspected. The patient soon after became semicomatose. The head was drawn sharply to the right. At times the patient would recognize and talk with her relatives, and is even able to move the left arm and leg.

On the twenty-fourth knee-jerk of the right leg disappeared, and a pin could be passed through the skin of either leg without pain.

On the twenty-fifth there was left lateral conjugate deviation with lateral nystagmus, more constant in the right eye. Respiration became labored. Edema appeared in the hands, and the patient died at noon on the twenty-fifth.

Autopsy showed miliary tuberculosis of the lungs and spleen; tuberculous meningitis; tubercular ulcers of the ileum; tuberculosis of the retroperitoneal glands.

It should be distinctly stated that cases of proved tuberculous meningitis have recovered. Probably this outcome takes place in less than I per cent. of the cases, but it is important to know that it is possible.

Diagnosis.-Miliary tuberculosis.

\section{Case 11}

A house-painter of forty-two entered the hospital December 4, 1907. $\mathrm{He}$ is in the habit of taking three drinks of whisky a day, but has had no previous illness. A year and a half ago he began to have headaches, vertigo, cramps, and vomiting; was sick for three or four days. He was treated in the Somerville Hospital for five weeks, but did not improve much, and has been unable to work since. He is now troubled much with occipital headache, worse in the morning and after he has been drinking. He now rarely vomits. Last night he had a nosebleed. $\mathrm{He}$ has had no abdominal pain of late. He has had occasional nightsweats, but none for two weeks. Headache is his chief complaint.

Physical examination of the chest is negative except for a short systolic murmur at the apex of the heart and accentuation of the aortic second sound. The pulse tension seemed to be high. The blood-pressure was $160 \mathrm{~mm}$. Hg. On the right side of the abdomen, at the level of the navel, a smocth, rounded, slightly tender mass is felt. The 
patient subsequently said that he had had blood in his stools for fifteen or sixteen weeks, averaging a gill a day. An expert proctoscopic examination showed no sufficient cause for this blood. On examination in a warm bath the tumor previously described was much easier to feel. It appeared to be about the size of a grape-fruit, and connected with the kidney. The urine was about 30 ounces in twenty-four hours, milky, Ior 3 in specific gravity, the sediment containing a large amount of pus, and an occasional granular cast. The hemoglobin was 70 per cent.

Profuse bleeding from the rectum continued, and vomiting of large amounts of green material with a little fresh blood happened several times. The right lung was full of coarse moist and dry râles. $X$-ray showed a shadow, probably of stone in the right kidney. The profuse intestinal hemorrhage suggested malignant disease, but no other evidence of it could be found. The amount of urine passed became smaller and smaller. On the seventeenth of December the sputum became bloody and the patient continued to vomit blood and to pass it by rectum. Numerous purpuric spots appeared on the skin. $\mathrm{He}$ sweated profusely in the hot-air baths and seemed better after them.

Discussion.-Although the patient is somewhat alcoholic, there certainly is not enough evidence to make us believe that whisky is the cause of his sufferings.

Lead-poisoning is naturally suggested by the patient's occupation, by the history of abdominal cramps, and headaches. If lead-poisoning were present, it might also account for the renal symptoms and for the rather high blood-pressure. As a matter of fact, the patient was treated for five weeks for lead-poisoning, and the fact that he did not improve during that time is, in itself, against the diagnosis. More important, however, is the tumor above described, which cannot possibly be explained by lead. The presence of this tumor, together with the pus in the urine and the results of $x$-ray examination, point strongly to stone or tuberculosis of the kidney. The headaches, pains, and sweats are quite explicable if there is suppuration of tuberculous or calculous origin in the renal pelvis.

We have still to explain in some way the mysterious hemorrhages from the rectum, stomach, and respiratory tract. The accentuation of the aortic second sound, the blood-pressure, the low specific gravity of the urine, and its constantly diminishing amount suggest a concomitant nephritis.

Outcome.-Operation was considered, but postponed, owing to the man's poor condition. During the last two days of life he passed practi- 
cally no urine. He was irritable and unreasonable, his mind wandering. He died on the twenty-second of December.

At autopsy the right kidney was found to contain a very large stone and several smaller ones. Three-quarters of the organ was converted into a bag of pus, and the remaining portion showed as cystic degeneration. The left kidney showed the lesions of chronic glomerulonephritis.

Diagnosis.-Stone in the kidney with abscess and nephritis.

\section{Case 12}

An electrician of thirty-three entered the hospital September 10, 1907. He was perfectly well until two weeks ago, when he began to have

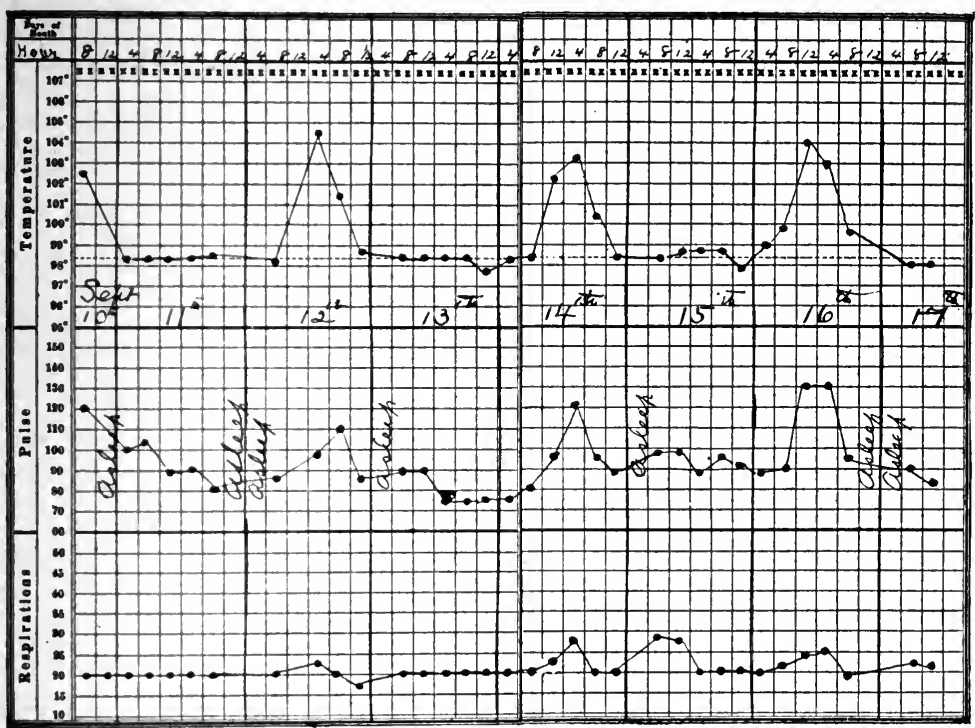

Fig. 5.-Chart of case 12.

severe, shooting pain in the forehead, spreading to the rest of the head. His face was puffy and red every forenoon and his hands became swollen. Yesterday he became very dizzy and could hardly see to walk, but did not fall. He lost three pounds in two weeks and is thirsty and nervous.

The patient was semicomatose and answered no questions. $\mathrm{He}$ moved restlessly upon the bed with his eyes shut and his hand to his head. He was not asleep or drunk, and there was no evidence that he had been drugged.

On examination, the face was distinctly puffy. The muscles about the eyes twitched involuntarily from time to time. Fundus oculi negative. The incisor teeth were worn down-the patient says because he grinds 
them at night. The spleen was not palpable. Physical examination was otherwise negative. Blood-pressure, $100 \mathrm{~mm}$. Hg. Temperature, 102.6 $6^{\circ} \mathrm{F}$. White cells, 3400 . Urine negative. The blood showed no malarial organisms. The symptoms seemed to point strongly toward uremia at the time of entrance, but the urine was absolutely negative.

At entrance the patient was put into a hot bath, but collapsed twenty minutes later, his blood-pressure being very low.

Discussion.-Nephritis is suggested by the drowsy condition, the edema of the face and hands, and the headache. The negative urine does not necessarily exclude chronic nephritis, but the low blood-pressure and the normal size of the heart are strongly against this diagnosis.

Brain tumor is suggested by the headache, the vertigo, and the drowsiness. Against it are the negative fundus examination, the low blood-pressure, the absence of focal symptoms.

Migraine may produce symptoms similar to those in this case, but one almost never sees a patient of thirty-three in his first attack of migraine, and this patient had had no previous attacks like this.

There is no evidence of reflex causes. In fact, the diagnosis was not suspected until the fall of temperature to normal next morning, and its sutsequent rise on the succeeding day suggested malaria.

Outcome.-On the fourteenth he had a chill. The blood showed a number of fully grown malarial parasites. Under quinin the patient was well within a few days.

Diagnosis.-Malaria.

\section{Case 13}

A Russian housewife of fifty-eight entered the hospital November 30 , I906. She entered the hospital first in April, I906, suffering from "interstitial myocarditis" with paroxysmal tachycardia. She was next seen on the thirtieth of November; her physician states that since leaving the hospital she has had attacks of tachycardia every few weeks, the attack usually lasting two days and often accompanied by headache. Between attacks she felt well; her appetite was good, her bowels regular, there was no loss of strength.

Nine days ago she began to have constant headache, precordial distress, insomnia, and anorexia. There is now no cough and no dyspnea, but she feels weak and tired.

Physical examination showed slight pallor and marked pulsation in the neck. The left border of cardiac dulness was six inches to the left of the midsternum in the fifth space, the right border one inch to the 
right of midsternum; sounds rapid, but regular; the first apex sound sharp; the second, barely audible; a rhythm like that of the fetal heart, the rate something over I9o. All the heart-beats were transmitted to the wrist, though the tension was low.

Physical examination was otherwise entirely negative. During the first part of her stay in the hospital the tachycardia showed only slight remissions, sometimes for a minute, sometimes for several hours. The rate did not seem to be affected by sleep, talking, or food. Digitalis had no effect. Tincture of aconite had no effect. Except for weakness and some mental anxiety, the patient seemed well.

Discussion.-Although the headache was much complained of in this case, it was at once thrown into the background by the general

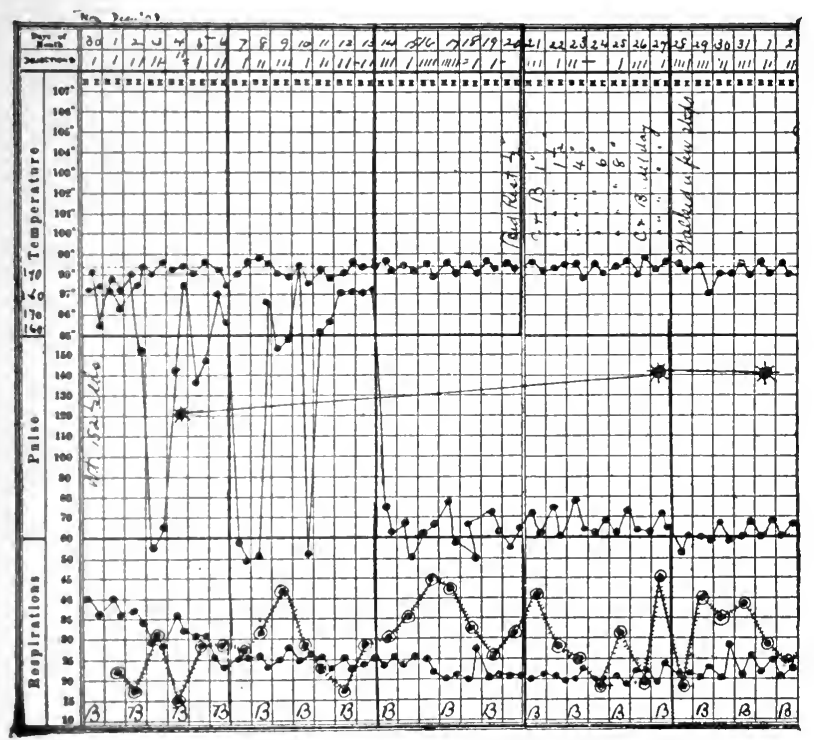

Fig. 6.-Chart of case 13 .

physical examination, which made tachycardia prominent. Our chief problem is to interpret the tachycardia existing in this case, more especially as regards prognosis, which is always the essential point in tachycardia.

Cases of paroxysmal tachycardia may be divided into three groups:

(a) Those having an obvious exciting cause.

(b) Those occurring in the course of a chronic cardiac disease.

(c) Those of whose origin we have no idea.

The first and the last of these groups carry a good prognosis. For practical purposes this is the most important point. I was once sum- 
moned in hot haste to the bedside of a woman of forty, where I found the family assembled awaiting her death. The attending physician thought she had but a few hours to live. Her pulse was 2 IO, her heart action absolutely regular and of the fetal type, her heart not enlarged, her breathing slow and easy in a recumbent position. The tachycardia had come on six hours previously, during a family quarrel, the patient being partly drunk.

Vigorous reassurances were given to the family in the patient's hearing, but without addressing her. Within an hour the tachycardia ceased.

I have seen a similar attack in a high-strung young girl who was in the dentist's chair during menstruation. The dentist was excessively alarmed, as the pulse was over 200 and barely perceptible, but the patient was as well as usual next day.

Attacks may follow a gastric upset or come after a surgical operation.

Tachycardia of this type occurring in patients who have definite signs or history of cardiac insufficiency, whether from valvular or myocardial lesions, are more serious, but I have never known a patient to die during or soon after such an attack. The prognosis is that of the underlying lesion, and is not appreciably modified by the occurrence of tachycardia.

Treatment.-Some cases are immediately relieved if the patient is placed head downward for a few seconds; others have been known to recover immediately after by drinking ice-water after emptying the stomach or after moderate exercise. Drugs have no obvious effect.

Outcome.-On the twelfth of December the tachycardia ceased during the night, and on the sixteenth she had two days without any. From this point on the attacks grew shorter and occurred at longer intervals. There was no evidence that they were influenced in any way by any drug or other treatment given her, and she left the hospital much relieved, on the third of January, though the myocardium still showed evidence of weakness.

Diagnosis.-Paroxysmal tachycardia complicating a chronic myocardial insufficiency.

\section{Case 14}

A school-boy eight years old entered the hospital May I6, I907. Since early childhood he and his brother and his sister have had vomiting spells about once a month. In such a spell he goes to bed feverish, vomits in the night, is feverish and sleepy the next day; after that he is perfectly well. It is surmised that these effects are due to eating too much candy. 
Five days ago he had headache and fever and vomited once. The headache and fever have continued since, and he has been unable to go to school. He has had a slight loose cough, but no expectoration. Last night he slept poorly and complained of epigastric pain. The course of the temperature is seen in the accompanying chart (Fig. 7).

Physical examination of the head, neck, and heart was negative. The abdomen was slightly distended, tympanitic, firmly held, and very tender throughout. The child breathed rapidly, with short, groaning. expiration. He was admitted to the hospital with a diagnosis of acute appendicitis. The right lung showed dulness from the apex to the fourth rib in front and over the entire back, associated with bronchial breathing, increased voice, and fremitus.

Discussion.-I have known several cases like this operated upon for appendicitis owing to the lack of a thorough physical examination. Especially in children it is essential to make a thorough examination of the chest whenever the presenting symptom is abdominal pain. The backs of the lungs are often not thoroughly examined, because we shrink, very naturally, from having a patient sit up or even turn upon his side; but in a case of this kind this is a shortsighted kindness.

Outcome.-On the twenty-second the temperature reached normal and the patient felt finely. On the twenty-fifth the temperature

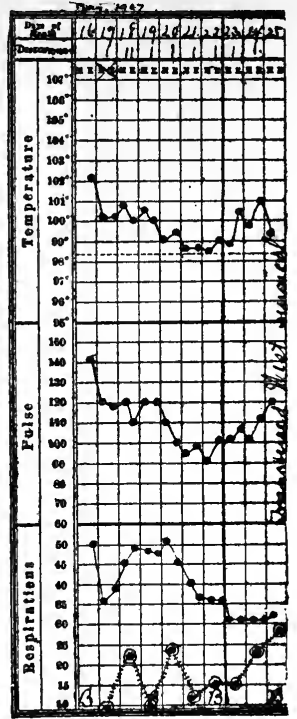

Fig. 7.-Chart of case I4. again rose, and the white cells, which had been 35,000 at entrance, were found to be still at approximately the same figure, with $9^{2}$ per cent. of polynuclear neutrophiles.

When fever persists in a case of this kind and the percussion dulness does not clear up, one of three possibilities is generally entertained: One thinks of an unresolved pneumonia, of a pleural thickening, or of postpneumonic empyema. In nine cases out of ten the latter turns out to be the true diagnosis. Unresolved pneumonia is mostly a myth. In the vast majority of cases it spells empyema. Pleural thickening causes no such elevation of the leukocyte count.

A needle introduced at the right base drew pus containing polynuclear leukocytes and pneumococci.

Diagnosis.-Infection (post-pneumonic empyema). 


\section{Case 15}

A little girl of fifteen, a chocolate dipper by trade, entered the hospital December 27, 1900, complaining chiefly of headache, which she has had for three days. It has been accompanied by some aching of the feet, general fatigue, and weakness. Her appetite has been good, but her food has been frequently vomited. She has had to stay in bed for the past three days. The course of her temperature is seen in the accompanying chart (Fig. 8).

Physical examination showed good nutrition, flushed face, heavy eyes, pupils equal and reacting, tonsils enlarged and red, a soft, sharply localized systolic murmur at the apex of the heart; the spleen palpable on inspiration. The internal viscera were otherwise negative; the first

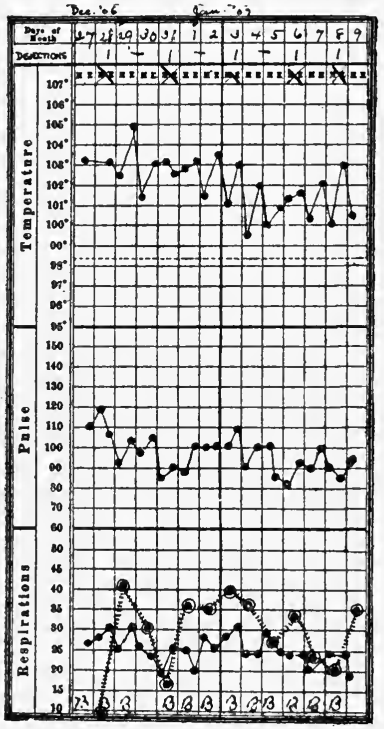

Fig. 8.-Chart of case I5 . phalanx of the right ring-finger was a little red and swollen. On the ulnar side there were a patch of granulation tissue and a large bleb, from which pus could be expressed. From the history and spleen typhoid seemed to be the most probable diagnosis.

The Widal reaction was absolutely negative. White cells, II,ooo. There was a diazo-reaction in the otherwise negative urine. The headache continued very troublesome.

On the night following entrance the patient complained of a little pain in her right knee, the inner side of which was found to be very slightly swollen and tender, not red or hot. The next three or four days there was the same complaint at the same time every night. The knee gradually became more swollen, and there was a suggestion of floating of the patella.

Discussion.- In the early days of this case, with headache, high fever, and nothing to show for it, it was probably impossible to make a definite diagnosis. The presence of the heart murmur suggests an endocarditis, with or without general sepsis. Such infections are very common in girls of this age. The white count of $I r, \infty 00$ is somewhat against this; the condition of the ring-finger favors it.

Typhoid seems more probable in many respects. The history and the enlargement of the spleen especially favor that diagnosis, and the diazoreaction would be generally considered confirmatory evidence, while the 
absence of the Widal reaction by no means excludes typhoid. In fact, the only decided evidence against typhoid during the early days of her illness was the leukocyte count.

The headache and splenic enlargement are quite consistent with malaria, but the time of year makes this unlikely and the blood examination excludes it.

With the appearance of pain in and about the right knee thirty-six hours after entrance a new crop of possibilities springs up. Rheumatic arthritis or gonorrheal arthritis comes first to mind. The fact that only one joint is involved is against ordinary "rheumatism," and in any type of arthritis we should expect more pain when the fever and constitutional manifestations are as marked as in this case.

I have recently seen a case of trichiniasis with symptoms a good deal like those in this case, and absolutely without eosinophilia during the first week under observation. In this case the diagnosis was made by finding the trichinella embryo in the peripheral blood.

Osteomyelitis should always be considered in a case presenting the symptoms here described. It is not at all unusual to have the fever and constitutional manifestations precede, by a considerable interval, any localization of the process. We get strongly the impression that the infection is first general and later local.

Occasionally we see a case beginning exactly like this one, but going on to rapid recovery without ever presenting symptoms any more definite than those here described. We have then to be content with surmising that some low-grade infection has been overcome.

Outcome.-On January $2 \mathrm{~d}$ the temperature was still high, and the knee intermittently painful. At times the patient awoke from sound sleep complaining bitterly of knife-like pain in her knee. The swelling increased over the inner condyle of the femur, where there was also the greatest tenderness. There was no cording of the veins, no glandular enlargement or tenderness, no edema of the leg.

January 6th: "The swelling of the knee has been increasing. The whole leg is now somewhat swollen. At the knee it measures $I_{2}^{\frac{1}{2}}$ inches more in circumference than the left. The patella now floats. Leukocyte count is now 16,000 . At entrance it was only II,000. The temperature is also lower, and in the past two days there has been some decrease in the swelling. There was a diazo-reaction in the urine at the time of entrance, and this has persisted since.

"January 9th: Pain in and around the knee has been very severe in the last three days. The leukocyte count is now I9,300."

January Ioth: Incision over the outer condyle of the femur liberated 
two ounces of greenish staphylococcus pus. Three perforations were found in the periosteum at the lower end of the femur, with pus all around the bone. The bone was opened and pus found in the lower epiphysis and the lower end of the shaft. Convalescence normal.

Diagnosis.-Staphylococcus infection (osteomyelitis).

\section{Case 16}

A laborer of thirty-six entered the hospital September 25, I906. For three years he has complained of indefinite stomach symptoms. For sixteen months these symptoms have been more marked, but have not amounted to actual pain, though they have been severe enough to prevent his working; there has been no vomiting. During these sixteen months he has had fairly constant headache, not localized, not very severe, but often accompanied by vertigo. A year ago he was so sick that he was in bed four months, after which he was much improved, and has not been in bed since.

His bowels move from one to three times a week, and only with purgatives or enemata. He has no appetite and has lost about thirty pounds. He has had many doctors, many diagnoses, and much treatment. He denies alcoholic excess and venereal disease.

Physical examination shows slight irregularity and sluggish reactions in the pupils; the left is larger than the right, and there is right external strabismus. There is a well-marked tremor of the tongue when protruded, and at times his lips are tremulous, as are his hands. The edge of the liver is palpable on deep inspiration. The knee-jerks are lively, Achilles jerk normal. White cells, I2,000; urine normal. Gastric examination with the stomach-tube showed the lower border of the organ reached two inches below the umbilicus; its functions and secretions appeared to be normal. Visceral examination otherwise negative.

Under daily lavage and Zander treatment with vibrations he showed some improvement. He took a good deal of exercise and gradually acquired a good appetite.

Discussion.-The questions which we naturally ask ourselves in this case are as follows:

I. Can this be a "neurasthenic" headache?

2. Can it be due to eye-strain?

3. Has it any connection- $(a)$ With the gastrectasis or $(b)$ with the pupillary changes?

A "neurasthenic" headache-i.e., one of unknown cause and benign outcome-is suggested by the long course of the symptoms, by the absence of fever and visceral lesions, and by the apparent nervousness 
manifest in trembling of the lips and hands. But against this hypothesis is, in the first place, the fact that he is a day-laborer and has, therefore, no right to such troubles unless under the influence of alcoholism or some severe and obvious mental strain. Further, this hypothesis does not explain the irregularity and sluggishness of the pupils nor the tremor of the tongue.

Eye-strain causes chronic headache, and the strabismus here present might well be a favoring cause. How long that strabismus has existed the patient has no idea, but it is certainly a very old affair as compared with the headache. Again, it is inherently unlikely that a day-laborer should begin to suffer from eye-strain at thirty-three. The point could only be definitely settled by a more accurate examination of his eyes.

Dementia paralytica is distinctly suggested by the association of pupillary defects with the tremor of the tongue and lips and the chronic headache. The absence of a syphilitic history does not exclude the existence of that disease. We might expect more change in the reflexes and more obvious mental symptoms, but these are by no means necessary. The diagnosis could be made much more certain in case the spinal fluid obtained by lumbar puncture contained an excess of cells with a lymphocytosis.

Outcome.-By the eleventh of October his stomach ceased to trquble him, but he showed a marked lack of initiative; he was perfectly content to sit and gaze absent-mindedly at nothing in particular. He expressed himself as greatly improved, and had gained a couple of pounds. It was subsequently ascertained that he had been in an insane asylum in November and December, I905. There they obtained a history of convulsive attacks, said to be brought on by eating, and characterized by twitching of both arms, with numbness of hands, occurring daily for about a week and lasting something less than an hour. During these attacks he was sometimes unconscious, and after coming out of them, failed to recognize people for a considerable time.

While at the asylum his eyes showed typical Argyll-Robertson pupils. The knee-jerks were exaggerated, and there was a Babinski reaction on the left, with marked incoördination of the upper extremities and in the gait. Examination of the eyes was entirely negative. Mentally, he seemed more cheerful than the situation justified.

Diagnosis.-Dementia paralytica.

\section{Case 17}

A widow of seventy-three was seen March 8, 1907. She had a fall at twenty-one, was hurt inwardly and doctored for ten years. She had 
"brain fever" at twenty-four, and was four months in bed. Ten years ago she had an attack similar to the present one, but less severe. She has become very nervous in the last few years. Six weeks ago she was taken with sharp pain in the eyes, spreading later to the top of the head and the left side of the face, sometimes shooting along the jaws or behind the ears. The pain has been steady during these weeks - at times sharp enough to make her cry out. Light hurts her eyes. Cold increases the pain, and her jaw is so painful that she cannot chew.

Physical examination showed obesity, but was otherwise negative. When the patient's attention was turned from herself, she seemed to be perfectly happy. One night she kept the whole ward awake because of an indefinite fear that something was going to happen to her.

Discussion.-In this case, as in the last one, dementia paralytica is suggested, but there is really very little to support that supposition. The tremors and pupillary signs present in the last case are quite absent here.

Although the pain here started in the eyes, there is nothing else in the case to suggest eye-strain, and as the suffering has not been closely limited to the region of the frontal sinuses, we have no good reason to suppose any inflammation there.

In genuine neuralgia we cannot ease the pain by diverting the patient's attention.

On the whole, the headache seems to be one of that large class of mysteries from which we divert our attention because we are unable to give them a name and because they pass off fairly quickly. No doubt in this case the psychic condition was in some way an important cause.

Outcome.-On examination by an eminent alienist she showed no proof of insanity, but was believed to be a nervous, hypochondriac, weak-minded old lady. Magnesium sulphate, $\mathrm{I}_{2}^{\frac{1}{2}}$ ounces daily, seemed to do her good. She was easily controlled by reason and by appealing to her better nature. Since the first night when she raised the roof for a time she had no bursts of temper or loss of self-control. The pain did not seem to mean much, and she was discharged on the nineteenth.

Diagnosis.-Headache of unknown origin.

\section{Case 18}

An Irish housewife of twenty-three entered the hospital April 30, 1907. She was confined eighteen days ago, the labor being accompanied by a large loss of blood. At the end of a week she complained of a severe pain in the side of her face; later in the other side as well, but was 
able to get up and take care of the baby. Last night the doctor found her in a slight stupor, which has increased during to-day. The course of the temperature is seen in the accompanying chart (Fig. 9).

The patient was semicomatose, had considerable pigmentation of the face and neck, normal pupils, twitching right eye-brow, pulse of high tension, viscera otherwise negative; reflexes normal; urine normal; red cells, 3,832,000; white cells, Io,000, with 76 per cent. polynuclears. By May $2 d$ Kernig's sign, photophobia, and marked stiffness of the neck had developed. The patient moaned continuously, and had headache unless she was kept under morphin.

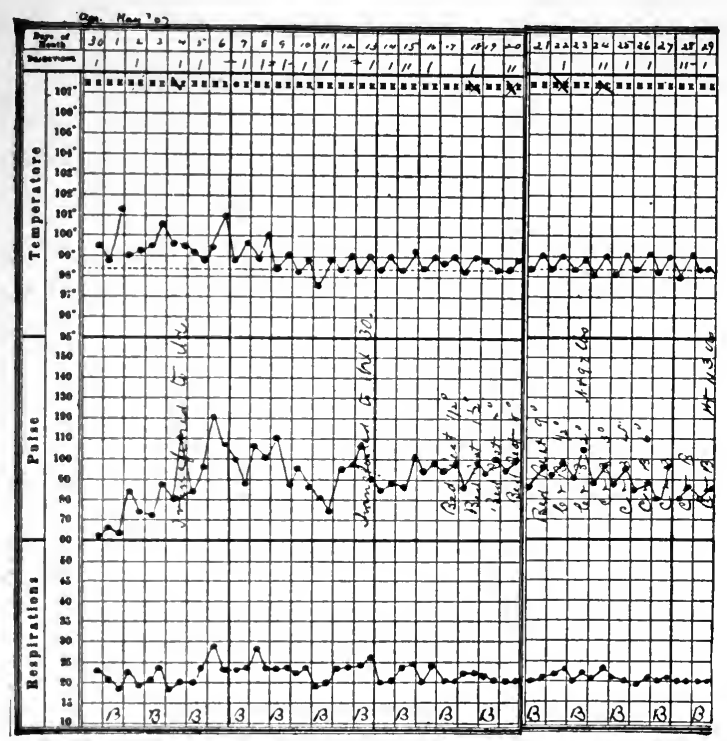

Fig. 9.-Chart of case $\mathbf{I} 8$.

Discussion.-Any headache near the time of parturition naturally suggests uremia or some related autointoxication, but in this case nothing was found in the examination of the urine or of the heart to support these ideas.

Cerebral hemorrhage or embolism is not uncommon near parturition, but would probably have a more sudden onset and produce paralysis or aphasia.

Cerebral tumor should be considered and cannot be excluded without an examination of the fundus. The absence of focal symptoms and the presence of Kernig's sign, photophobia, and retracted head militate against it.

Meningitis is left as the most plausible diagnosis, though the temperature-chart and the leukocyte count are against it. 
Outcome.-On lumbar puncture a clear fluid spurted eight inches through the cannula; immediate and great relief followed. The patient ceased moaning and went to sleep. A sediment of the fluid thus obtained showed very rare leukocytes or degenerate mononuclear cells and a few Gram-decolorizing bacteria not characteristic. Cultures remained sterile; urine, normal. After the lumbar puncture the pupils, which were previously inactive, became normal, the Kernig sign less marked, and the head, though still stiff, was not retracted. By the thirteenth of May there was marked improvement. The temperature, as seen by the chart on P. 7I, was entirely normal. Less morphin was required to control the headache. Consciousness returned on the ninth of May. May I $3^{\text {th }}$ she fed herself.

May Igth: Marked improvement. Sits up daily. No stiffness of neck.

May 28th: Red cells, 4,380,000; leukocytes, 4000; hemoglobin, 65. per cent.

May 29th: The patient anxious to go home and is discharged.

Just what type of meningitis was present could not be determined. At the present day an injection of Flexner's antimeningeal serum would probably be indicated, despite the dubious results of this lumbar puncture.

Diagnosis.-Meningitis.

\section{Case 19}

A married woman of thirty-five entered the hospital December 9, I897. She had septicemia after the birth of her baby, six years ago. She has never been quite as well since. For three weeks she has had a. little cold in her head and a little headache, gradually getting worse, until four days ago, when she went to bed. Three days ago she began to have severe "neuralgic" headache, localized just above the left eye. She has had a hard, dry cough, which is now somewhat better; and for three days there has been some pain in the left chest on full inspiration.

Physical examination showed the evidences of intense suffering from headache, marked tenderness at the exit of the left supra-orbital nerve, and less marked tenderness over its distribution. There is considerable voluntary spasm of the right rectus abdominis. The temperature is $100.5^{\circ} \mathrm{F}$; p pulse, 90; respiration, 25; white cells, 14,000; urine, normal. Freezing the supra-orbital nerve with ethyl chlorid gave no relief. Morphin in $\frac{1}{3}$-grain dose eased the pain, but soon after she became hysteric, noisy, apprehensive, and almost delirious. She sat up in bed, trembling, breathing rapidly, with widely dilated pupils, said she could not get her breath, and wanted something to counteract the effect of the morphin. 
Discussion.-The problems presented by this case are:

I. Is the headache due to neuralgia, to frontal sinusitis, or to some other cause?

2. What is the significance of the thoracic pain and of the abdominal spasm?

3. What was the nature of the acute attack following the administration of morphin?

The fact that no relief was afforded by freezing the supra-orbital nerve argues against neuralgia. Sinusitis is made more likely by the direct sequence of the symptoms upon a cold in the head. There is nothing in the history to suggest any other diagnosis.

Regarding the cause of the thoracic pain and the abdominal spasm, we must say, in the light of the outcome, "ignoramus." It should be said with emphasis that in almost every carefully studied case there are one or two facts like these which stray across the clinical field quite wild and untamed, and never submit to any rational explanation. If a case does not manifest some such symptom, but reels itself off like a textbook account, I always suspect that it is carelessly reported.

At the time of the acute attack above described meningitis was suspected on account of the association of the mental symptoms and headache, but there was at no time any fever, and the results of treatment (see below) made it obvious that it was one of those semihysteric attacks of excitement which not infrequently follow the administration of morphin in idiosyncratic individuals.

Outcome.-She was reassured in regard to her breathing, and given common salt in water to counteract the morphin, after which she was quiet for the rest of the night. The next morning the pain had almost disappeared. The temperature was normal, and on the third day she was allowed to go home.

Diagnosis.-Sinusitis.

\section{Case 20}

A farmer of thirty-five was seen October 8, I906. About August ist he began to have eruptions described as resembling giant urticaria in various parts of his body. He had previously been treated for an attack of angioneurotic edema. In the middle of August he had smothering sensations in his chest, which lasted from one to three hours. The coagulation time of his blood was then two minutes.

Four and a half days ago he began to have headache, which has grown rapidly worse. Two days ago he had a chill at 3 P. M., and yesterday one at 7 P. M. Fever has been continuous since the onset. 
The bowels have moved but once in three days. He has lost much money of late, but says he does not worry about it.

Physical examination showed palpable glands in the neck, axillæ, and groins. Examination of the chest and abdomen was negative. The blood showed no Widal reaction.

Discussion.-The questions which naturally present themselves in this case are:

I. Can the headache and fever be due to some of the urticarial group of lesions, which, as we know, are sometimes associated with fever and sometimes manifest themselves in the internal organs (respiratory and gastro-intestinal tracts)? The smothering sensations complained

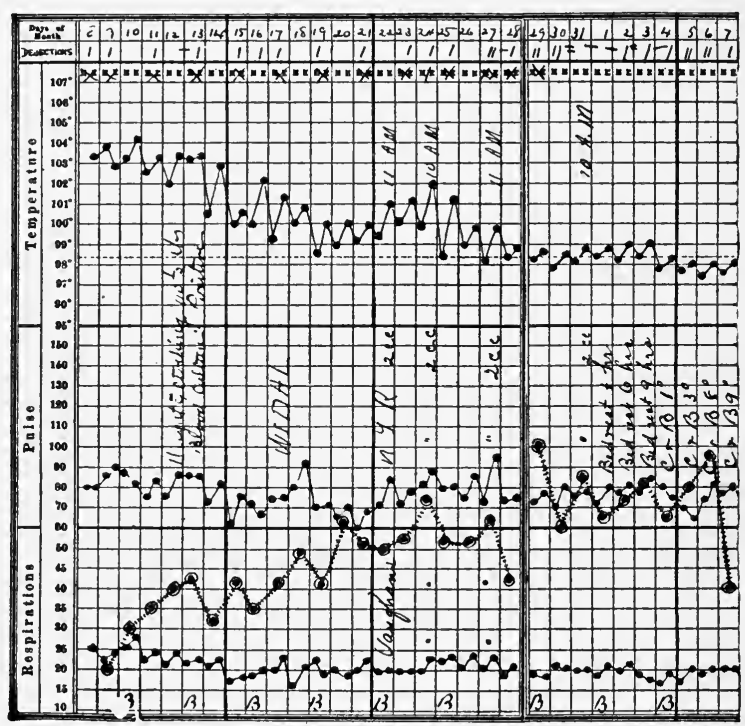

Fig. 10.-Chart of case 20 .

of in August may have indicated the involvement of the respiratory tract by lesions of the urticarial group.

2. Can financial worry, owing to his money losses, account for his symptoms?

3. What is the significance of the general glandular enlargement? leukemia? syphilis?

In relation to the first question it must be said, first of all, that urticarial or erythematous lesions almost never occur on mucous surfaces and serous membranes alone. If the fever and headache were of this type, there ought to be some external lesion.

Neither worry nor any other psychic event produces a fever like that here shown. 
The text states that glands are palpable in the neck, axillæ, and groins, but this is far from indicating that the glands are now in a diseased condition. Glands are palpable in health in a large majority of adults in one or more of the above-mentioned situations. Nevertheless, the possibility of leukemia cannot thus be dismissed. I recently saw a leukemic case with signs much like those here described, and with a total leukocyte count nearly the same, the differential count, however, showing 95 per cent. of lymphocytes. As a matter of fact, this examination was made in the case here under discussion, but the blood was wholly normal.

General glandular enlargement certainly suggests syphilis, but such enlargement was not present in this case, the glands being no bigger than normal. There is nothing else in the case to suggest syphilis, though a fever of this type is quite compatible with syphilis.

The suggestion of malaria (chills) was promptly negatived by the blood examination.

The clinical picture then is that of a fever with nothing to show for $i t$. This makes us suspect typhoid, especially in October. The absence of Widal reaction at this stage of the fever is, of course, not evidence against typhoid. Still the diagncsis is not certain. Is there any way of making it more certain? Blood culture should certainly be undertaken.

Outcome.-A blood culture showed a bacillus giving all the reactions of the typhoid organism. White cells, 6000. The Widal reaction did not appear until the seventeenth. The course of the fever was uneventful. He was discharged well on the eighth of November.

This case weil illustrates the value of blood-cultures, which are most likely to be positive at the very time when the Widal reaction oftenest fails us, viz., at the beginning of the disease.

For the treatment of this case see Appendix B.

Diagnosis.-Typhoid.

\section{Case 21}

A sailor of twenty-seven entered the hospital November 26, 1906. He has lost one sister of "meningitis." Six months ago he had malaria, with chills every second day for three weeks. He has not felt perfectly well since. He denies venereal disease. Two weeks ago he began to have slight, throbbing headache, with blurring of eyes and general fatigue. Three days later he felt feverish. Eight days ago the headache became severe enough to confine him to bed, where he has been since. His appetite has been poor. Vomiting has been frequent. He 
has lost much weight and strength. The course of the fever is seen in the accompanying chart (Fig. II).

On physical examination the right pupil was found to be slightly larger than the left; both reacted normally; heart and lungs normal, except that respiration at the left apex was rather harsh, with slight dulness. A rare sibilant râle was heard over this area. White cells, 8300; polynuclear cells, 80 per cent.; there were no malarial parasites. Widal reaction negative, November 26th, 29th, and December Ist.

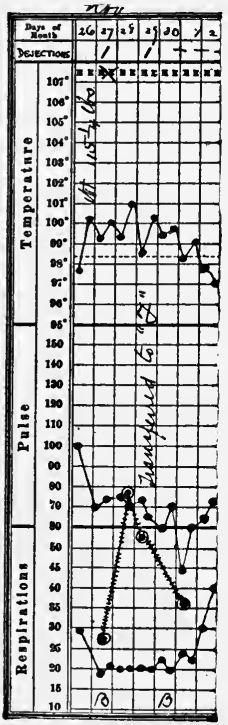

Fig. II.-Chart of case $2 \mathrm{I}$.

The urine was normal; fundus oculi perfectly normal; sputa negative; stools normal.

Discussion.-Naturally, our first thought is of typhoid, but after ten days of fever the temperature should be higher in typhoid, unless, indeed, we are dealing with one of the rare abortive cases which finish themselves up within ten days, so that we are here seeing only the tail end of the disease. Against this, however, militates very strongly the total leukocyte count (almost always subnormal at this stage of typhoid), and especially the high percentage of polynuclear cells, which is practically unknown under these conditions.

The history of a previous malaria makes that disease worth a moment's consideration, but as this individual has not been out of a temperate climate for many months, it is practically impossible that he should have acquired an estivo-autumnal malaria, which is the only type compatible with a fever-curve like that shown below. The patient's occupation brings syphilis to our minds as a passibility, but there is nothing else about the case to support this supposition.

Brain tumor often produces a remarkably slow pulse, such as is seen in this case, but there is nothing else about the patient to verify this hypothesis. The fact that the patient is obviously sick and yet has a very slow pulse directs our attention still further to the possibility of a brain lesion. Can he be suffering from tuberculous meningitis? There are no disturbances of the cranial nerves nor retraction of the head, and no leukocytosis, but the lung signs suggest a possible tuberculosis there. Lumbar puncture should certainly be done unless further evidence soon appears to clear up the diagnosis.

Outcome.-On the twenty-eighth slight stiffness of the neck on forward bending was noticed; otherwise there was no change. 
On the twenty-ninth he became slightly delirious, and in the evening required restraint and refused to swallow.

On the first of December he became comatose, and the stiffness of his neck disappeared. On the third of December he died. Autopsy showed general miliary tuberculosis and tuberculosis of the mesenteric and retroperitoneal glands.

Diagnosis.-Miliary tuberculosis.

\section{Case 22}

A bricklayer of sixty-four entered the hospital May I5, I908. Three uncles upon his father's side died of consumption; his family history is otherwise good. He takes from a pint to a quart of whisky a day; has had gonorrhea many times; had chancre fourteen years ago, for which he was treated three years. He was down South at the time the present illness began, two weeks ago; he does not seem to know exactly how he got there. He has been in bed for a week and a half, complaining of nothing but headache and poor appetite.

On examination, his pupils are equal, regular, and react normally. His temperature is as seen in the accompanying chart. His tongue is covered with a thick, dry coat. The heart-sounds are faint. A faint, systolic murmur is heard all over the precordia, transmitted into the axilla. The aortic second sound is slightly accentuated; heart not enlarged; the arteries palpable. In the lower half of the right lung, behind, slight dulness, diminished breathing, many medium and coarse crackling râles; abdomen and reflexes normal; white cells, I3,600; urine normal; Widal reaction negative.

The patient was sent in with a diagnosis of typhoid fever, but showed at entrance only headache and bronchitis in an alcoholic subject.

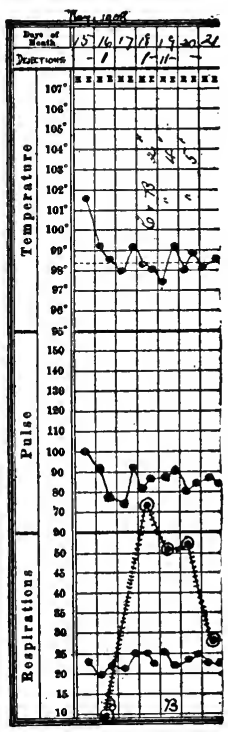

Fig. I2.-Chart of case 22 .

May Igth: The hospitai record states that he does not need hospital treatment, and will be sent home in a day or two.

May 2ist: On the morning visit he seemed "dopey"; for the past two nights he has complained of severe headache. At I I P. M. May 2Ist, he was found unconscious.

Discussion.-The family history, the presence of lesions suggestive of a pleurisy at the base of the right lung, suggest the possibility of a tuberculosis with involvement of the meninges. This could only be 
partially excluded by lumbar puncture, and must remain a possibility in the diagnosis of this case.

Headaches with nocturnal exacerbations suggest syphilis, especially in a patient who has certainly had that infection in previous years. It is impossible, however, to go beyond suspicion unless we can get further evidence, such as disturbances of the cranial nerves, of the reflexes, a positive Wassermann reaction, or other syphilitic lesions.

The history naturally suggests alcoholism ("wet brain"), but in the absence of any sign of delirium tremens this seems unlikely, since the amount of alcohol consumed in the last ten days has been almost nil.

Typhoid and other infections disappeared from consideration when the temperature fell to normal and stayed there.

Can the diagnosis be malaria? The patient has recently come from a malarial country, where he may have acquired a type of the infection not characterized by the familiar tertian or quotidian chills seen in temperate climates. In a case very similar to this, occurring in a drummer who had recently returned from a southern trip complaining of fever, headache, and prostration without chills, I found large numbers of estivo-autumnal "rings" in the red cells. The present case, however, showed no such evidences in the blood.

It is much to be regretted that we made no measurement of bloodpressure in this case. An elevated pressure would support the supposition that some brain lesion (tumor, hemorrhage, softening, or meningitis) existed. As it was, no diagnosis was made during life.

Outcome.-In the evening the pupils ceased to react; the left arm and leg were cooler than the right; Babinski on both sides; abdominal reflexes absent; no paralysis made out. He died on the twenty-second.

Autopsy showed subdural cerebral hemorrhage; hemorrhage into tegmentum of epencephalon; arteriosclerosis; atheromatous endocarditis of the aortic valve; fibrous endocarditis of the mitral valve; hypertrophy of the heart; syphilitic cirrhosis of liver; bronchopneumonia; acute fibrinous pleuritis; congenital cyst of kidney; round ulcer of stomach; fibrocalcareous tuberculosis of the lungs; chronic pleuritis; subcapsular hemorrhage of kidney.

Diagnosis.-Cerebral hemorrhage. 


\begin{tabular}{|c|c|c|c|c|c|c|c|c|c|c|c|c|}
\hline 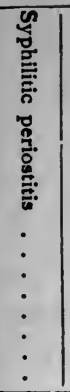 & 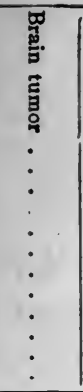 & 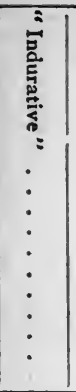 & \begin{tabular}{l}
\multirow{3}{*}{} \\
$\frac{0}{0}$ \\
$\vdots$ \\
$\vdots$ \\
$\vdots$ \\
$\vdots$ \\
$\vdots$ \\
$\vdots$ \\
$\vdots$ \\
$\vdots$
\end{tabular} & 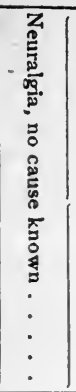 & 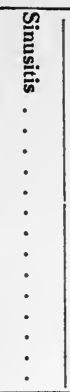 & 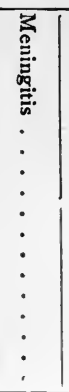 & $\begin{array}{l}\frac{z}{4} \\
\frac{8}{3} \\
\frac{a}{5} \\
\vdots \\
\vdots \\
\vdots \\
\vdots \\
\vdots \\
\vdots \\
\vdots\end{array}$ & \begin{tabular}{c|} 
\\
0 \\
0 \\
0 \\
0 \\
0 \\
0 \\
0 \\
0 \\
0 \\
0.0 \\
$\vdots$ \\
$\vdots$ \\
$\vdots$ \\
$\vdots$ \\
$\vdots$ \\
$\vdots$
\end{tabular} & 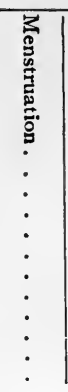 & 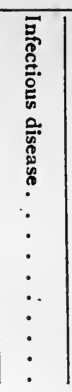 & 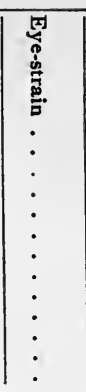 & 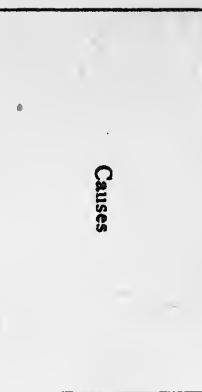 \\
\hline : & $\because$ & $\vdots$ & $\mid$ & مै & $\vdots$ & $\vdots$ & $\vdots$ & 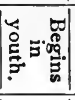 & : & $\vdots$ & 岕 & Age. \\
\hline 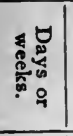 & 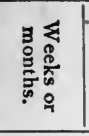 & 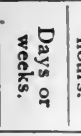 & 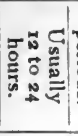 & 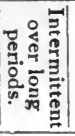 & $\mid$ & 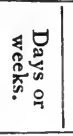 & 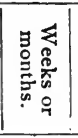 & 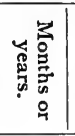 & 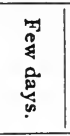 & 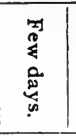 & 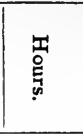 & Duration. \\
\hline$\circ \circ+$ & $\circ \stackrel{+}{+}$ & $\circ \varrho+$ & 。 & 。 & 。 & + & $\circ \circ+$ & + & $\circ \circ+$ & 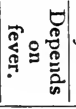 & 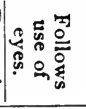 & $\begin{array}{l}\text { Constant sense of } \\
\text { pain or of pres- } \\
\text { sure. }\end{array}$ \\
\hline 。 & 。 & ○ & + & 。 & 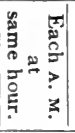 & $\circ$ & 。 & 。 & 。 & ○ & 。 & $\begin{array}{l}\text { Regular recur- } \\
\text { rence. }\end{array}$ \\
\hline$\circ$ & $\circ$ & 。 & 。 & ○ & 。 & 。 & 。 & 。 & + & $\circ$ & $\circ$ & $\begin{array}{l}\text { Definite relation } \\
\text { to catamenia. }\end{array}$ \\
\hline$\circ$ 아 & 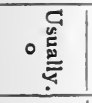 & 。 & 。 & $\circ$ & $\circ \circ+$ & + & 。 & 。 & $\circ$ & + & 。 & $\begin{array}{l}\text { Fever and other } \\
\text { signs of infec- } \\
\text { tion. }\end{array}$ \\
\hline$\circ$ & $\circ \supseteqq+$ & 。 & 。 & 。 & 。 & + & $\stackrel{\circ}{\stackrel{\vec{m}}{!}}+$ & 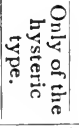 & $\circ$ & 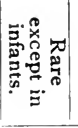 & $\circ$ & $\begin{array}{l}\text { Convulsions, } \\
\text { coma, delirium. }\end{array}$ \\
\hline$\circ$ & + & $:$ & $\circ$ & $\circ$ & $\circ$ & $\circ$ & $\circ$ & $\circ$ & $\circ$ & $\circ$ & $\circ$ & F'ocal symptoms. \\
\hline ○ & $\mid$ & ० & $\circ \stackrel{+}{+}$ & $\circ$ & 。 & 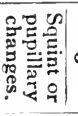 & $\circ \circ+$ & $\circ \%+$ & $\circ \circ+$ & $\circ$ & + & Ocular anomalies. \\
\hline$\circ$ & ○ & 。 & ○ & $\circ$ & 。 & 。 & $\circ$ & 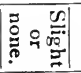 & 。 & $\circ$ & + & $\begin{array}{l}\text { Relief from treat- } \\
\text { ing the eyes. }\end{array}$ \\
\hline ○ & 。 & $0 \%+$ & + & $\circ$ & $\circ$ & 。 & $\circ$ & $\circ \circ+$ & $\circ$ & $\circ$ & $\circ$ & $\begin{array}{c}\text { Vasomotor phe- } \\
\text { nomena about } \\
\text { the head "con- } \\
\text { gestion." }\end{array}$ \\
\hline 。 & 唃。 & ○ & $\circ:+$ & $\circ \circ+$ & $\circ$ & 。 & 。 & + & $\circ$ & : & $\circ \%+$ & $\begin{array}{c}\text { Other signs of } \\
\text { neurosis. }\end{array}$ \\
\hline+ & ○ & 。 & 。 & 。 & 。 & . & 。 & $\circ$ & 。 & $\circ$ & $\circ$ & $\begin{array}{l}\text { H is t ory and } \\
\text { other evidence } \\
\text { of syphilis. }\end{array}$ \\
\hline 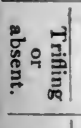 & 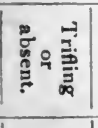 & 。 & 。 & $\circ$ & 。 & 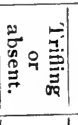 & + & $\circ$ & 0 & 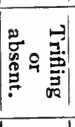 & 0 & $\begin{array}{l}\text { Albuminuria, } \\
\text { cylindruria, car- } \\
\text { diacc b y er- } \\
\text { trophy, edema, } \\
\text { etc. }\end{array}$ \\
\hline 。 & 。 & + & 。 & 。 & 。 & 。 & $\circ$ & 。 & 。 & 。 & 。 & $\begin{array}{l}\text { Indurated nodes } \\
\text { in muscles of } \\
\text { neck or scalp. }\end{array}$ \\
\hline$\circ \%+$ & $\circ$ & $\circ$ & ○ & $\circ$ & $\circ$ & + & $\circ$ & $\circ$ & $\circ$ & $\circ$ & $\circ$ & $\begin{array}{l}\text { Result of lumbar } \\
\text { puncture. }\end{array}$ \\
\hline
\end{tabular}




\section{CHAPTER III}

\section{LUMBAR PAIN}

Some years ago, when I was doing a good deal of work on the blood, I was asked to substitute as visiting physician to a convalescent home intended primarily for tired domestics and shop-girls. The matron met me with that patient and respectful expression which long service under many enthusiastic young physicians produces in some nurses. "I hear," she said, "that you are specially interested in the blood. Dr. R., the gynecologist, who was visiting last autumn, found that all the patients were gynecologic. When Dr. C. visits us in summer, he finds them all nose and throat cases-that's his specialty. Now that you are to visit us, I suppose they will all turn out to be blood cases."

It must be explained that there was no election on the patients' part. They did not seek the institution because they heard that Dr. X. (a specialist in their particular trouble) was on duty. They were sent there by a variety of other physicians who had no knowledge of the interests of the different attending specialists.

Now, in a similar way, we may explain, I think, the various interpretations of backache given by different physicians, each according to his point of view.' To the gynecologist backaches are usually gynecologic symptoms; to the orthopedist, they result from sacro-iliac disease or postural strain; to the neurologist, they represent one phase of habitpain due to a psychoneurotic make-up. There are stomach specialists who explain backache as a result of malnutrition, gastroptosis, or constipation (loaded colon).

So it goes! The one thing which remains unchanged is the backache. When we find $I_{5}$ or 20 drugs recommended for one disease, we are inclined to believe that none of them has much value. Similarly, when we find many and various explanations for one condition, it is natural to doubt whether any of them are true.

The one thing clear about the obscure backaches called "functional," "postural," "uterine," "sacro-iliac," etc., is relief by mechanical compression exerted about the pelvis and lower lumbar region by means of corsets, plaster strapping, belts, or plaster-of-Paris.

In many cases a strong neurotic element can be traced-the mental or nervous weakness acting on the back through a reduction of muscular tone. Flabby mind, flabby muscles, unsupported joints, pain. Doubt- 


\section{Causes of Lumbar Pain}

1. FATIGUE AND. DEFECTIVE BALANCE ("FUNCTIONAL
BACK")
$\begin{array}{ll}\text { 2. CHILDBIRTH } \\ \text { 3. INFECTIOUS DISEASES } \\ \text { 4. POSTOPERATIVE }\end{array} \mid \begin{aligned} & \text { CASES TOO MANY } \\ & \text { AND TOO VAGUELY } \\ & \text { ENUMERABLE FOR } \\ & \text { GRAPHIC REPRE- } \\ & \text { SENTATION. }\end{aligned}$

\section{SACRO-ILIAC DISEASE (NON-INFECTIOUS)}

6. LUMBAGO 549

$\left.\begin{array}{l}\text { 7. HYPERTROPHIC } \\ \text { ARTHRITIS }\end{array}\right\}$ 351

8. HERPES ZOSTER ) ("SHINGLES")

9. INFECTIOUS ARTHRITIS OF SPINE

10. ACUTE SPRAIN OF THE
BACK

11. RENAL STONE 109

12. SPINAL TUBERCULOSIS 72

13. RENAL SUPPURATION 65

14. PERINEPHRIC ABSCESS 26

15. RENAL TUMOR - 16

16. CANCER OF THE SPINE

$\left.\begin{array}{l}\text { 17. RETROPERITONEAL } \\ \text { TUMOR }\end{array}\right\}$ 

less any of these factors (and probably various others) may so "activate" the rest that in various ways the back may be made to ache. I do not think that any one knows much about it.

On the gynecologic side the most careful study of backache (and other pains) in relation to pelvic disease is that reported by Dr. C. T. Dercum, ${ }^{1}$ of Philadelphia, in which she shows statistically what I have long believed from unrecorded but fairly extensive observations in the Women's Medical Clinic of the Massachusetts General Hospital, viz., that there is no type of backache or other "reflex" pain which can reasonably be referred to pelvic disease. All types of pain in the back, head, and extremities occur with equal frequency with and without pelvic disease. All types of pelvic disease exist with and without backache. Even deep-seated cancerous growths may be latent and symptomless for many months.

The tables on page 8I from Dr. Dercum's article show to my satisfaction the mutual independence of backache and pelvic disease.

Aside from this huge group of backaches cured by mechanical support and lateral compression of the pelvis, but explained in many ways, as gynecologic, neurasthenic, or functional, as sacro-iliac strain or as loss of balance, ${ }^{2}$ etc., we have two other affections which I have found it convenient to bracket with it under the clumsy title of the orthopedic group of backaches. These diseases are:

\section{Lumbago.}

2. Hypertrophic spondylitis.

These may be for a time indistinguishable from each other and from the larger and vaguer group above referred to.

The "kidney group" of causes for backache is a term which I shall use throughout this chapter to denote the "surgical" diseases in or near the kidney: tuberculosis, stone, neoplasm, abscess, cystic degeneration.

The "pressure group" refers to diseases which involve a progressive compression of the lumbar cord or its nerves: aneurysm, neoplasm, vertebral tuberculosis.

Some of the commonest causes of lumbar pain will not be discussed in any detail here. Probably more persons experience such discomfort as a result of -

(a) Fatigue and simple weariness than from any other single cause. The patient usually finds this out when the pain goes off after a rest.

1 The Nervous Disorders in Women Simulating Pelvic Disease; An Analysis of $59^{\mathbf{r}}$ Cases, Jour. Amer. Med. Assoc., March 13, 1909, p. 848.

${ }^{2}$ Reynolds and Lovett, An Experimental Study of Certain Phases of Chronic Backache, Jour. Amer. Med. Assoc., March 26, r910, p. ro33. 


\section{TABLE I.}

Location of Pain or Tenderness. Pelvis

Both groins (so-called ovarian tenderness) $\ldots \ldots \ldots \ldots \ldots \ldots$ 70

Left groin (so-called ovarian tenderness) .............. 40

Right groin (so-called ovarian tenderness).............. 20

Under both breasts (inframammary tenderness) ........... 2

Under left breast (inframammary tenderness)............ 26

Under right breast (inframammary tenderness) .......... I

On either side of the spine in the cervical region.......... 3

On either side of the spine in the dorsal region............. I4

On either side of the stine in the lumbar region............. 9

Over the sacrum.......................... I4

At the end of the coccyx..................... ro

Above the spines of the scapulæ................. 4

Clavus hystericus........................ 5

Deep intrapelvic pain (hysteric) ................. ro

Painful areas on mucous surfaces, vagina, vulva, rectum, and

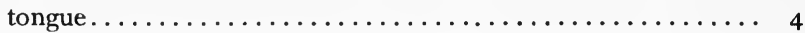

Limbache (legs, thighs, arms, and shoulders).......... 40

Sacral backache.......................... 6o

Lumbar backache......................... 2

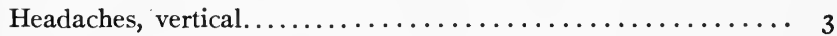

Headaches, diffuse...................... 6

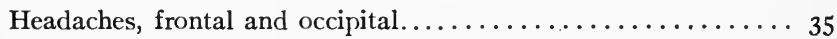

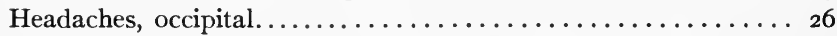

Headaches, frontal...................... 6

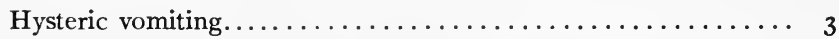

Hysteric pain in one eye during menstruation. .......... I

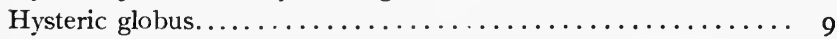

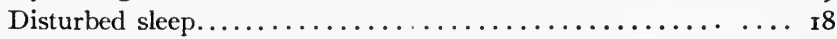

Insomnia. ........................... 3

Gastro-intestinal disturbances of nervous origin, such as constipation, flatus, gastric distress, diminished secretions, and ano-

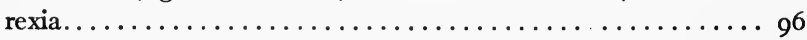

TABLE II.

\section{Pathologic Condition.}

Cystic degeneration of the cervix.

Tubo-ovarian inflammations and exudates. ........... 53

Fibroid growths, including one weighing $\mathrm{I} 7$ pounds......... I I

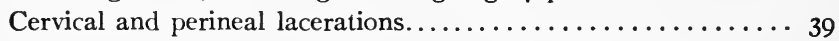

Dysmenorrhea......................... 7

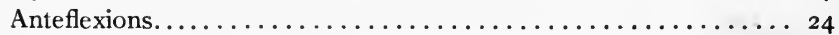

Retropositions............................. 44

Splanchnoptosis (relaxation of uterine supports).......... I9

Lacerations that were repaired .................. 2

Where both ovaries had been removed .............. 9

Where one ovary had been removed................. 2

Where the appendix had been removed............... 4

Carcinoma (no nervous symptoms found in any case of car-

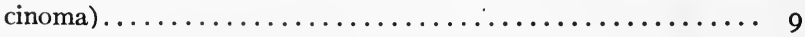

Pelvic organs normal........................ o
Backache, headache, and other hysteric or neurasthenic symptoms.

\section{sent. \\ Present.}

3


Sometimes, however, the fatigue has become cumulative, and is so chronic that it has developed, as it were, into a member of the household. Its source and origin may have been forgotten, and come to light only after close questioning or as a result of a therapeutic test, viz., a thorough rest. In persons of high-strung, hypersensitive, and neurotic temperament, these simple fatigue pains merge into what may be called-

(b) The psychoneurotic backaches, which have certain characteristics worth noting here. Pains of this type are often confined to the region of the coccyx, and, unfortunately, they are apt to lead the patient into the hands of some fervid and eager surgeon, who speedily does an operation on the coccyx. If the operation is followed by prolonged rest with hypernutrition and a considerable amount of reëducation given consciously or unconsciously by the surgeon or his assistant, the patient may recover, but the credit is falsely given to the operation, which would have been quite useless-as, indeed, it often proves-without the nutritive and educational influences linked to it.

Another type of psychoneurotic backache makes the patient abnormally conscious of the whole length of his vertebral column, which is affected, not only by pain, but by a variety of paresthesix, tingling, sensations of heat or cold, sensations of pressure or crawling. This type of trouble may arise without any obvious reason, but it is also often met with following some accident, whence the term, "railway spine." In the vast majority of these cases, however, the accident has served merely to direct the patient's attention to a certain part of the body, in this case the spine, and also to perturb his moral consciousness through the expectation of damages and court-room scenes.

A third type of psychoneurotic backache, to which further reference will be made below, is recognizable by its obvious connection with psychic and especially emotional states. A depressing emotion will produce it, a joyful event will cure it; but one must beware of doing the patient injustice by dubbing the pain imaginary or unreal, either in this or any other type of psychoneurotic trouble. What the facts show is that a certain direction and morbid concentration of attention is followed by pain, and that a new habit of life, physical and mental, leading to a more profitable direction of attention, is followed by relief. The most plausible hypothesis, and also the most useful one, because the most helpfully comprehensible to the patient, is that which assumes the following: Numberless physiologic changes are occurring every. moment in every part of our anatomy-the circulation of blood, the distention and contraction of blood-vessels, the movements of lymphcurrents, the varying tension and pressure of muscular masses, ligament. 
ous strands and fascix-all these and presumably many other phenomena go on very busily but quite unconsciously when our minds are normal; but when attention gets caught and concentrated upon the spine or the coccyx or the back of the neck, and when the patient has made a mental picture of the organ which he supposes to be diseased " the base of the brain," "the whole spinal cord," "the outlet of the stomach," "the left ovary"), then this unfortunate begins to be aware of physiologic processes normally unfelt. This very awareness, through the formation of brain habits and possibly also through vasomotor influences acting upon the points supposed to be diseased, reinforces and increases the sensations referred to this point until they finally attain the dignity of pain, which ultimately becomes a habit ("habit pain").

I shall not try to exemplify in any detail this type of pain, though it is one of the most common in the practice of all busy physicians.

(c) Lumbar pain due to parturition is only rarely mistaken for any other variety, and offers, as a rule, very little diagnostic difficulty. Obviously, it is one of the commonest of all such causes.

(d) Backache from infectious disease of any type, from a simple cold to the severest septicemias and pneumonias, is, I suppose, the next commonest variety. Occasionally this type offers some difficulties in diagnosis, examples of which will be considered later. In the great majority of cases, however, the presence of fever, headache, and widely distributed pain in other parts of the body enables us to identify infectious backache without much difficulty.

(e) Postoperative backache appears usually about twenty-four hours after the operation, and is troublesome for the next two or three days. Though often associated with gaseous distention of the lower bowel, there seems to me to be no good reason to believe that the distention causes the pain, since similar distention is so commonly present in typhoid, pneumonia, and other infectious diseases without any backache. The postoperative lumbar pain seems to be more common after prolonged operations in which the patient's back rests upon a flat table, so that the normal spinal curvature is no longer maintained by muscular tone, which the anesthetic relaxes. Pressure by the surgeon or his assistant upon the patient during operation may contribute to the result. If this explanation be correct, the backache should be prevented by padding or curving the surface of the table to correspond with the normal lumbar curve of the spine.

The types of lumbar pain next to be discussed all differ from those above mentioned in two important respects: those listed so far have been far commoner than those still to be mentioned, and far less depend- 
ent upon direct physical examination for their recognition. It is for this latter reason that diagnostic difficulties are far commoner in the still remaining groups already mentioned on p. $8 \mathrm{r}$.

$(f)$ The Orthopedic Group.-What was almost universally called lumbago ten years ago has now been split up into three main subtypes of disease: spinal osteo-arthritis, sacro-iliac disease (non-tuberculous), and a residue still known under the name of lumbago. Despite the important differences which have now been demonstrated and have given rise to this separation, these three diseases are still loosely bound together by the fact that their treatment is very similar. It is, however, altogether for reasons of convenience in the discussion of differential diagnosis that I have linked them together under the title of the orthopedic group. They differ sharply, both in prognosis and treatment, from all the types of disease above referred to, as well as from those next to be described.

(g) The pressure group of diseases causing lumbar pain includes vertebral tuberculosis (Pott's disease), aortic aneurysm, and neoplasm in or near the spinal column. I am quite aware that this term has no other merit than that of convenience for discussion, since in two members of the lumbago group pressure is also the cause of the pain.

(h) The kidney group of causes for lumbar pain includes renal stone, tuberculosis, neoplasms, hematogenous infection of the kidney, and paranephritic abscess as its chief members. Among the rarer causes for lumbar pain may also be mentioned renal infarct, hydronephrosis, pyonephrosis, and cystic kidney.

(i) Lumbar neuralgia or neuritis, clearly recognizable only in the presence of the vesicular eruption (herpes zoster or shingles), is a comparatively rare cause for lumbar pain. Of about equal rarity as a cause of such pain is-

(j) Cholelithiasis.-Perhaps one case of gall-stones in a hundred shows itself by pain starting in the back and working toward the gall-bladder instead of in the opposite direction, as is usual.

(k) Chronic Prostatitis.-Young, Geraghty, and Stevens (Johns Hopkins Hospital Reports, Vol. 13, 1906) emphasize the importance of chronic prostatitis as a cause of pain in the back. Sometimes the pain in the back due to this cause is the presenting symptom of the case, though more often the backache is associated with sexual and urinary symptoms. Out of $35^{8}$ cases of chronic prostatitis, they found 87 , or 24 per cent., complaining of severe or mild backache. In 64 of these cases the pain was in the lower lumbar region; in 8, over the kidney; in 5 , over the sacrum. In ro additional cases the 
pain simulated renal colic so closely that operation for that disease had been performed in 3 cases; while in 6 others that diagnosis had been definitely made. The attacks in some of these cases were so sharp that morphin was needed for their control. In several cases blood was also present in the urine. Prostatic massage gave prompt and permanent relief.

With lumbar pain or tenderness due to ulcer or cancer of the stomach or bowel I have had no experience, though I have asked and examined for such pain many times. Schmidt ${ }^{1}$ mentions very specifically that in lead-poisoning sharp lumbar pain is occasionally associated with the ordinary abdominal colic.

To investigate the cause of lumbar pain it is well to ask the following questions:

(I) Is it unilateral (diseases of the renal group especially) or bilateral?

(2) Is it of long duration? Chronic lumbar pain points especially to the psychoneuroses and to the pressure group of causes.

(3) Is it made much worse by stooping or sidewise bending? This is the characteristic of the lumbago group and of many psychoneurotic cases, while diseases of the pressure group and the kidney group are not thus characterized.

(4) Is the lumbar region sensitive to pressure or percussion? Such sensitiveness is especially common in diseases of the renal group, but if localized over the sacro-iliac joint, it often points to disease there.

(5) Does pain radiate along the course of the intercostal nerve? This occurs especially in the lumbago group and the pressure group.

(6) Does the urine contain blood or pus?

A valued correspondent sends me the following note on backache: "Young and middle aged patients are especially apt to have backache from the following three causes: (I) Early generalized tuberculosis. (2) Late syphilis. (3) Any change of occupation, as, for example, from fireman to engineer, from driver to loader, from cloth salesman to shoe department salesman, from clerk to collector."

\section{EXAMINATION OF PATIENTS WITH LUMBAR PAIN}

Incredible though it seems, there are physicians in practice to-day who do not hesitate to treat lumbar pain without stripping the patient so that the naked back can be examined. I have known a case of herpes zoster to be treated for "rheumatism" (salicylates, alkalis,

\footnotetext{
${ }^{1}$ Pain, Its Causation and Diagnostic Significance, by Rudolph Schmidt, translation published by Lippincott.
} 
vegetable diet, etc.) simply because the vesicular eruption was unknown to the patient and had never been looked for by the physician.

Osler mentions a case of aneurysm of the descending thoracic aorta, which presented as a pulsating tumor near the angle of the left scapula, quite undiagnosed through many weeks of treatment for lumbago and neuralgia. The attending physician had never examined the exposed back, presumably because the patient, being a male, wore clothes which opened in front and did not offer to remove them.

Once we have formed the habit of examining the naked back, we should note especially:

(a) Is the spine rigid locally or throughout? (Allowance must be made for the moderate rigidity of normal old age.)

(b) Is there any tenderness over the spinous processes?

(c) Is there any dulness on percussion of the bases of the lungs? (Renal abscess or neoplasm may push up the diaphragm and encroach upon the thoracic space.)

(d) Does the patient stand or walk with a list to one side?

(e) Has he any fever?

\section{Case 23}

A Swedish tinsmith, twenty years of age, of excellent family history, past history, and habits, entered the hospital on the twenty-fifth of June, 1908. On June 7 th, while sitting in a chair upon his piazza, he had a sudden attack of sharp pain in the right lower back. This pain continued severe for the next six days, and on the day after its onset he began to be short of breath on slight exertion. A dry cough began at the same time, and has persisted since. His appetite has been poor, but he has not been in bed. He has had no constipation or other symptoms.

When first seen, his temperature, pulse, and respiration were normal. His heart's apex was $I_{2}^{\frac{1}{2}}$ inches to the left of the nipple-line in the fifth space, the right border of cardiac dulness two inches to the left of the midsternum line in the fourth space. The heart-sounds were of good quality, and there were no murmurs. The upper part of the right chest was slightly dull as low as the third rib. Below this there was tympany extending two inches to the left of the midsternal line, below the costal margin, and to the middle of the right axilla. Tactile fremitus was diminished over this area, and breath-sounds distant or altogether absent, except at the right apex, where the voice sounds were increased and the breathing was bronchovesicular.

In the back, with the patient sitting up, there was relative dulness down to a point $\mathrm{I}_{2} \frac{1}{2}$ inches below the angle of the scapula, the line of 
resonance rising from that point obliquely across the axilla to the level of the third rib in front. Below this there was tympany.

Over the dull area in the back fremitus is diminished, and at the extreme base absent. Otherwise the signs are the same as in the corresponding area in the front. There are no rales, no friction or other abnormal sounds.

Physical examination is in other respects negative. The blood and urine are normal.

Discussion.-As we read the signs set down in this case, pneumothorax is naturally our first thought. But can pneumothorax occur so suddenly in a person of excellent health and without any of the known causes of pneumothorax (phthisis, trauma)? Let us consider the other possibilities before answering this question.

Pain, dyspnea, and cough suggest pneumonia, but the absence of fever and of any evidence that the patient has had and passed a crisis exclude this.

A sharp thoracic pain, followed by dyspnea and cough, constitutes the ordinary onset of pleurisy, but the physical signs of this case, especially the tympany at the base of the chest, together with the absence of the friction sounds, exclude this.

Passing to other possible explanations of the tympanitic resonance just referred to, we think of emphysema; but this cannot be so localized, and is never of sudden onset. The presence of gas below the diaphragm, either in the bowel or in an abscess cavity, would explain many of the signs in this case; but there is no history of any previous abdominal symptoms, such as usually lead to the so-called subphrenic pyopneumothorax. There has been nothing to suggest appendicitis, perforating gastric ulcer, or hepatic abscess. There are not enough fever and constitutional disturbance.

We, therefore, return to the first supposition, viz., pneumothorax. Investigation of any large number of cases of this disease shows that its symptoms may be either stormy and virulent, or so mild as to be practically negligible. Twice I have seen pneumothorax (proved to be such by the liberation of air through puncture) in patients who felt practically well and were examined almost by chance. This means that the cause present and leading to the vast majority of all cases of pneumothoraxnamely, tuberculosis-may be absolutely latent and symptomless. This is, of course, a well-known fact, but the sudden appearance of a tuberculous pneumothorax brings the truth home to us in a startling way.

Outcome.-The patient was given 3 milligrams of tuberculin after 


\section{LUMBAR PAIN}

five days of normal temperature, and the temperature thereafter rose to ror $^{\circ} \mathrm{F}$. and was accompanied by headache and malaise.

The patient was accordingly transferred to a sanatorium for tuberculosis.

The prognosis in a case of this kind and the treatment are those of the underlying process-phthisis. The advent of pneumothorax does not render the outlook much graver. In the great majority of cases the air is readily absorbed, and no special treatment need be directed to it. If the air persists in the chest unchanged for a number of weeks, or if its amount is so large as seriously to embarrass the action of the heart and lungs, it may be removed by puncture, after which it may, or may not, reaccumulate.

Diagnosis.-Tubercular pneumothorax.

\section{Case 24}

A stationary fireman of fifty entered the hospital November 9, rgor. Seven years ago, following an injury to his left elbow, the joint gradually grew stiffer, and he was told that there was a growth of bone there. He came to the out-patient department for treatment, and the elbow was baked daily for five weeks, with considerable benefit, but he has never been able fully to extend the arm since that time.

Three weeks ago he began to have shooting pains across the small of his back, brought on by any motion. Three days ago these pains became so severe that he could scarcely move. The pain now starts in the small of the back and extends down the left leg as far as the ankle. Three days in bed has given him no relief.

Physical examination showed well-marked Heberden's nodes on the fingers. The physical examination was otherwise negative, except that the left elbow could not be flexed beyond 80 degrees or extended beyond 45 degrees. There was tenderness along the back of the left thigh from the popliteal space to the sacrum, also over the Achilles tendon, pressure on which causes pain to shoot up the thigh.

So long as the patient remained absolutely quiet he was comfortable, but coughing, sneezing, any movement of the leg or body caused pain to shoot from the sacrum to the foot. Fixation with a ham splint afforded no relief, nor did the application of cold along the nerve. Drugs were without effect. Heat, on the other hand, relieved him somewhat. Tight criss-cross strapping of the lower back and, later, a supporting belt, gave still more relief, although numbness of the thigh and calf developed as the pain diminished. 
Discussion.-The great majority of cases of pain in the back fall into three groups:

I. The infectious group.

2. The orthopedic group.

3. The renal group.

The first and the last of these may be excluded by the absence of fever and of urinary signs. Within the group which I have called orthopedic fall chiefly lumbago, sacro-iliac strains and displacements, spinal osteo-arthritis.

Lumbago is pretty definitely excluded by the long duration of the disease. After three weeks of pain we must find some other cause, especially as the pain is no longer confined to the lumbar muscles, but extends down the left leg.

Sacro-iliac disease (strain, sprain, displacement, or pinching of joint fringes) should cause the patient to stand with a list to one side, and should produce tenderness over the sacro-iliac joint, together with pain increased when the leg is raised without bending the knee. Direct physical examination of the sacro-iliac joint usually reveals nothing in these cases except localized tenderness. In this case the above tests were all negative.

Spinal osteo-arthritis is favored by the age of the patient, and by the presence of similar joint outgrowths elsewhere (elbow and fingers). Pain on coughing and sneezing is also rather characteristic of osteoarthritic processes, because they so often involve the costovertebral joints, which have to move sharply and suddenly when we cough or sneeze. This symptom, however, also occurs in all the orthopedic group of diseases above referred to.

Malignant growths in or near the spinal column might account for all the symptoms here present, and can only be excluded by $x$-ray examination or by the outcome of the case.

Outcome. $-X$-ray showed osteo-arthritic outgrowths in the lower lumbar region. By December 5 th he was able to walk about with crutches, and by the eleventh he was able to go home very much relieved.

Diagnosis.-Hypertrophic spinal arthritis.

\section{Case 25}

A motorman of twenty-four entered the hospital August 19, I907. His habits and previous history were good, but for the past two weeks he has had pain across the small of his back. For the past four days the pain across the small of his back has become more severe and he has been nauseated when he tried to eat, although he has felt hungry. 
Six days ago he felt chilly in the evening and shivered a little; but he did not give up his work until two days before his entrance to the hospital. This morning he had a brief spell of tingling in the left arm. He continues to feel hungry, but cannot eat. He does not feel at all weak. His bowels move once daily.

At entrance the patient's temperature was I03.8 $8^{\circ} \mathrm{F}$.; pulse, 88; respiration, 24. He was mentally alert, and did not look very sick. There was a harsh, systolic murmur heard all over the precordia, loudest in the pulmonary area, where there is a suggestion of a systolic thrill. The pulmonary second sound was slightly greater than in the aortic. The heart shows no evidence of enlargement. A slightly tender mass was felt to descend below the left costal margin on full inspiration.

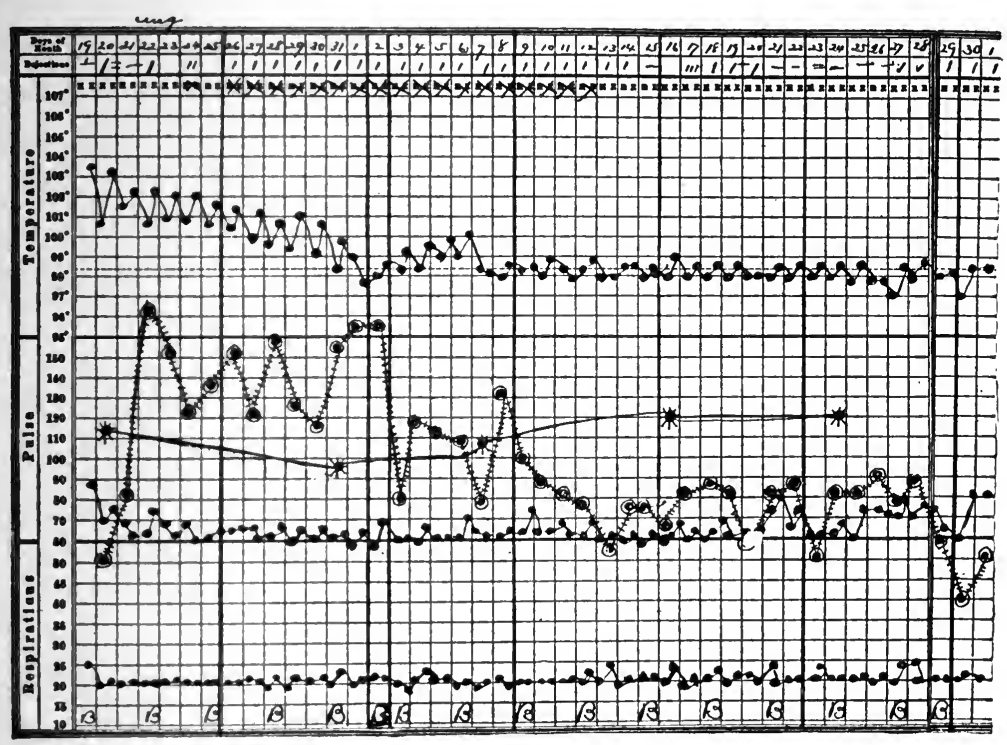

Fig. 13.-Chart of case 25 .

Physical examination was otherwise negative. The urine was normal. The Widal reaction was negative; the white cells were 5400 .

Discussion.-The presence of continued fever excludes most of the so-called orthopedic group discussed in the last case. We have left the infections, local and general. Local infections producing pain in the back are chiefly spinal tuberculosis, hematogenous renal infection, and perinephric abscess. These are excluded in the present case, because the spine, the region of the kidney, anteriorly and posteriorly, and the urine, are all negative. We are left with the question, What general infections are most apt to cause backache? The answer is: "grip," tonsillitis, typhoid, and sepsis. Of tonsillitis and sepsis we have no 
positive evidence, though the harsh systolic murmur mentioned in the text might suggest a sepsis of the type known as ulcerative endocarditis. There is, however, nothing conclusive about this murmur as described, and nothing else in the case to support the diagnosis of sepsis. The murmurs most suggestive of a septic endocarditis are those that rapidly change their characteristics under observation, especially diastolic murmurs.

The good appetite and the mental alertness are not characteristic of typhoid, but there is nothing in the case absolutely inconsistent with that diagnosis. The tender mass felt below the left ribs might be the spleen. The absence of a Widal reaction is not unusual at the onset of a typhoid.

In the absence of a well-marked infection of the upper air-passages with the influenza bacillus predominating in the discharges there is never any good reason for the diagnosis "grip." Such a diagnosis is usually a rather equivocal way of saying "I don't know." The word is used largely to satisfy the patient.

On the evidence thus far presented, then, one can only guess at the diagnosis of this case. Only as the chart develops do we begin to feel any more confidence that our original guess, typhoid, is correct.

For treatment see Appendix.

Outcome.-The subsequent course of the temperature is shown in the accompanying chart. The Widal did not appear until August 25th. The mind was clear and alert throughout, and after the twentyeighth the mass was no longer felt in the left hypochondrium. The patient sat up on the twenty-second of September, and went home well on the first of October.

Diagnosis.-Typhoid.

\section{Case 26}

A widow of fifty entered the hospital March I7, I908. Her history was always negative up to a month previously, when she began to have pain in her lower back and in her right hip, making it difficult to lie down. The pain was sharp, constant, and increased by motion. It was usually relieved by heat, but last night she had to take morphin to get to sleep. She has worked until four days ago, though her appetite has been poor, and she has some nausea and loss of weight.

On the right back, on a line with the spine of the ilium, is a group of broken vesicles covering an area of $2 \frac{1}{2}$ inches by one inch, and extending at right angles to the spinal column. Temperature, pulse, and respiration normal. 
Dr. R. B. Osgood found nothing wrong in the bones and joints. Vaginal examination was negative, as were the blood and the urine. Dr. James J. Putnam considered the pain due to herpes. She slept well on the night of the twenty-first after Io grains of veronal, and aspirin in doses of 10 grains also relieved her. Acetanilid 5 grains with 2 grains of caffein was later given to insure sleep. Aconitin in $\frac{1}{2} \frac{1}{50}$ to $\frac{1}{100}$ grain doses had no effect, although it was pushed up to the point of toxic symptoms, when she remarked that it made her feel cold and clammy, like a chicken just taken off the ice. Later her pain became more severe, and was not relieved either by aspirin, by quinin, or by strychnin. Phenacetin and salol relieved her more, and by the sixteenth of April she was able to sleep without any drugs at night. Veronal and codein, the former ro grains, the latter half a grain, were repeatedly needed before April roth for sleep.

After she had been in the ward five weeks with normal temperature and pulse throughout, her temperature suddenly rose to I03.5 $5^{\circ} \mathrm{F}$, and her leukocytes, which had previously been normal, rose to 19,200 .

The lungs were negative, but there was marked tenderness in the right lower abdomen, without spasm. The patient was so hypersensitive when she was touched at any point that it was difficult to know how much weight to lay upon her abdominal pain.

By the twenty-fifth the temperature had again reached normal; the white cells were still above $15, \infty 00$, and there was indefinite sensitiveness in the right lower quadrant.

On the twenty-ninth, after she had been sitting up, she appeared to be very sensitive in the right iliac fossa.

Discussion.-Lumbar pain without fever and without evidence of any disease of the orthopedic group or of the kidney group should always suggest the possibility of a neuritis. The group of vesicles, though covering so limited an area, gives strong support to the hypothesis. Neuritis of the thoracic region, involving, presumably, in every case a lesion of the spinal ganglion corresponding, is especially apt to be accompanied by that vesicular eruption which we call herpes zoster or "shingles." In the majority of cases the painful area is much larger that the vesiculated area. It need not surprise us, then, that in this case the vesicles cover such a small spot, and we have no good reason to hesitate regarding the diagnosis-herpes zoster. Presumably this is due to a local infection of the spinal ganglion similar to that which has been demonstrated in the ganglia corresponding to the facial herpes in pneumonia.

Regarding the treatment of this painful affection, it is worth noting 
that the application of an ethyl chlorid spray over the corresponding spinal ganglion sometimes gives very striking relief to the pain.

Can the abdominal pain, occurring in the sixth week of this case, be attributed to a second attack of the same trouble? Experience has taught us never to multiply causes or diagnoses if the facts can be explained otherwise. But in this case the occurrence of fever and leukocytosis, with the new pain, should make us look for some local inflammatory cause. We should search for evidence of a local abscess, of tonsillitis, of phlebitis, arthritis, or pneumonia. By the twenty-ninth, when tenderness in the right iliac fossa was marked, there seemed to be every reason to suspect the appendix.

Outcome.-On the second of May the white cells had risen to 31,000 , and a distinct mass could be felt in the right iliac region.

On May $3 \mathrm{~d}$ the abdomen was opened and an ounce of pus evacuated from the region of the appendix.

The patient's recovery was complete.

This case constitutes one of those exceptions which prove the rulethe rule, namely, that we do not often deal with two diseases as the explanation for a group of symptoms. In the light of the findings at operations we naturally ask ourselves whether the whole thing, from start to finish, might not have been due to appendicitis. I should answer decidedly, "No." The location of the original pain, the absence of fever, and the presence of the vesicular eruption seem to me to make this supposition impossible, though it is conceivable that there may have been a common cause both for the zoster and the subsequent appendicitis.

Diagnosis.-Appendicitis; herpes zoster.

\section{Case 27}

A married woman of twenty-one had "grip" three times last winter, but has otherwise been well until two weeks ago, when, after her last attack of "grip," she began to have pain in her back, and to a less extent in her arms, chest, and knees, without any limitation to the movement of the joints. For the past week she has been in bed, but for the past two nights she has slept little on account of pain in the back.

When the patient was first seen, March 26, I908, her temperature was IOI ${ }^{\circ} \mathrm{F}$., pulse, IIO, and respiration, 25 .

The temperature remained elevated for four days; after that it was, for the most part, normal. The action of the heart was regular and rapid; with a gallop rhythm. The pulmonic second sound was accentuated, and the first sound at the apex was accompanied by a rough systolic 
murmur heard all over the precordia and in the axilla. There was no obvious enlargement of the organ. Physical examination was otherwise negative, except that the white cells numbered r6,300.

Rest in bed, ro grains of salicylate of strontium every four hours, with an ice-bag over the precordia, an occasional A. S. and B. pill, and an occasional $\frac{1}{s}$ grain of morphin, gave her relief within a few days. Later, she complained of piercing pains in the precordia, which made her very nervous. Nothing was found there on physical examination.

Discussion.- I have included this case because it seems best that my book should mirror some of the most annoying defects of our present knowledge, as well as its strong points. This is the sort of case which is ordinarily called "grip" at the start, while we watch for developments. If none come, the diagnosis is formally confirmed.

For what other possibilities should we be on the watch in a case of this kind? Endocarditis, first of all, on account of the cardiac murmur, the leukocytosis, and the early joint pains. Only the disappearance of these symptoms with the lapse of a few days excludes endocarditis.

Typhoid is made practically impossible by the presence of wellmarked leukocytosis.

As I have already said in the discussion of previous cases, I think "unknown infection" should be our verdict. It is time to drop the equivocal use of the word "grip" as a cloak for our ignorance.

It is worth noting that the use of an ice-bag over the precordia very probably accounted for a good deal of the patient's later suffering. It drew her attention to the possibility of heart trouble. In a nervous person this is enough to produce heart pains.

Outcome.-Nervousness was throughout a prominent feature, but by the sixteenth of April she was nearly well, and was discharged to finish her convalescence at home.

Diagnosis.-Unknown infection.

\section{Case 28}

A night watchman of sixty-nine entered the hospital January $3 \mathrm{I}$, ${ }_{190 \%}$, complaining that when he got up two days before he "felt his hip catch." Within three hours he was unable to bear any weight on the left foot and went back to bed. The pain has continued since, and he has been helpless.

On physical examination it was found that any motion of the left hip or back caused exquisite pain. There was some tenderness at the upper point of exit of the nerve. Physical examination otherwise nega- 
tive. Temperature oscillated between $98^{\circ}$ and ror. $4^{\circ} \mathrm{F}$. for four days, then normal. Whites, 8000 .

Flexion of the thigh, with the knee kept straight, caused pain referred to the left sacro-iliac joint.

Discussion.-Can the symptoms be due to strain of the back? What tests should be employed to confirm or exclude the diagnosis of lumbago, of sacro-iliac disease, of hip disease, of spinal osteo-arthritis? What further data are necessary?

In answer to these questions I should say that it is wholly unlikely that strain entered into the causation of these symptoms, since the pain was first felt after the blameless action of getting out of bed.

For lumbago the main tests are for the production of pain by any use of the lumbar muscles, together with the absence of any disease of the bone or kidney.

In relation to sacro-iliac disease we should endeavor to ascertain whether the patient stands with a list to the other side, whether the pain and tenderness are referred especially to the sacro-iliac joint when the thigh is flexed with the knee straight, whether there is any sacro-iliac pain on compressing the wings of the ilium.

The therapeutic test, the effect of attempting to immobilize the joint by strapping or otherwise, is also of importance. Hip-joint disease is to be excluded in case the motions at that joint are really free.

Osteo-arthritis is difficult to exclude or to identify positively. We suspect it in the presence of long-standing lumbar pain associated with radiations along the thoracic, lumbar, and sciatic nerves, aggravated if when the muscular protection is relaxed in sleep, the patient attempts to turn over. It is aggravated also by coughing and sneezing. An $x$-ray picture and the exclusion of sacro-iliac disease complete our task.

The present case offers a fairly characteristic picture of what is ordinarily known as sacro-iliac sprain or strain. The pathology of this affection is still very obscure. It may be that one of the joint fringes gets pinched owing to slight relaxation or subluxation of the joint when the muscular or ligamentous protection is imperfect. A person becomes debilitated or tired, muscularly or nervously. His muscles are no longer as alert and well toned for protection as they should be. A slight slip occurs, and a joint fringe or some other sensitive joint structure is impinged upon. If this were true, it would explain the frequent association of the trouble with neurasthenic and debilitated states. 
Outcome.-The patient was considerably relieved by ro grains of aspirin every four hours and tight cross-strapping of the back and hip. He was able to leave the hospital by the twenty-fifth of February.

Diagnosis.-Sacro-iliac strain.

\section{Case 29}

A nurse of thirty-six who had previously suffered from dysentery when nursing in the Philippines, entered the hospital March 2I, I908, complaining that for the past four months she had had pain in the lower part of her back, extending down the right leg. She has also had swelling of the right foot and stiffness of the neck off and on during these four months. The pain is somewhat relieved by heat, but she has had to have morphin pretty continuously in order to keep her comfortable.

She has been unable to work since the previous December, and has lost 20 pounds in the past five weeks.

On physical examination the thyroid gland was found to be slightly enlarged. Temperature, pulse, and respiration were normal, the chest and abdomen negative. Urine normal.

The pulsations of the aorta were violent in the epigastrium. The knee-jerks were extremely lively, but there was no clonus and no Babin.ski. Cross-strapping gave her a great deal of relief.

Discussion.-Here is a long-standing pain which, in a woman of thirty-six, should make us consider Pott's disease and cancer; but examination shows no evidence of either of these troubles, and a closer study of the case shows two causes whereby the duration of the pain may well have been inordinately prolonged. I refer to the use of morphin and to the evidence of a hypersensitive temperament, shown in the exaggerated knee-jerks and violent pulsation of the abdominal aorta. Coming then to the milder possibilities, we should naturally think of lumbago, because the patient has also suffered from stiff neck (so often associated with lumbago). The duration, however, is somewhat too great. She should have been relieved by rest within a week or two.

The pain extends down the right leg, and is accompanied by swelling of the right foot. Can it be due to neuritis? There were no nerve tenderness and no disturbance of sensation. The ordinary tests for sacro-iliac disease (see above) were positive.

Outcome.-Dr. Goldthwait saw the case in consultation and made a diagnosis of chronic strain in the right sacro-iliac joint.

Diagnosis.-Sacro-iliac strain. 


\section{Case 30}

A school-girl, eight years of age, entered the hospital May 26, I908, complaining of dull, constant pain in the right side of the lower back,

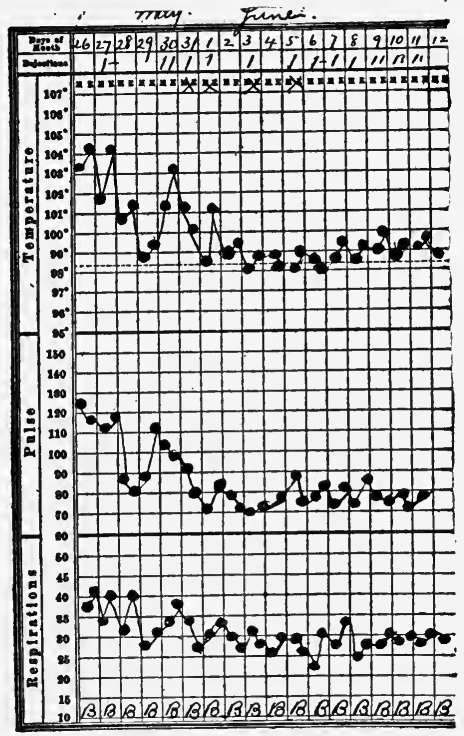

Fig. I4.-Chart of case 30 . worse at night, accompanied by fever, vomiting, and constipation. Her bowels have not moved for four days. There has been no injury to the back, no cough, and no chill. Family history and previous history are negative.

Physical examination showed a herpes on the lips. Nothing abnormal was found in the chest or abdomen except for a general tenderness, especially marked in the costovertebral angles and in the flanks.

The urine showed a large amount of pus, and the culture revealed a characteristic growth of colon bacilli.

The temperature remained above $\mathrm{roI}^{\circ} \mathrm{F}$. for a week. (See accom-

panying chart.) The patient was at first very sick, with a white count of $24,000,82$ per cent. of the cells being polynuclear.

Any lumbar pain with fever in a small girl suggests Pott's disease. This being excluded by the absence of any kyphos or muscular spasm about the spine, we have next to note that the patient is rather young for any of the orthopedic group of diseases.

If it is an infection, as the fever suggests, is it local-that is, renal or perirenal-or is it general? The condition of the urine and the leukocytosis point strongly to a local urinary infection.

Outcome.-The leukocyte count fell to normal along with the temperature. The treatment consisted of alcohol sponges at $80^{\circ} \mathrm{F}$. every four hours; urotropin, 4 grains, three times a day, an abundance of water to drink, and a liquid diet.

By the eleventh of June the urine was nearly normal and the child practically well.

In view of the rapidly favorable outcome in this case there was no need for any attempt further to verify the diagnosis by cystoscopy or ureteral catheterization. 
The renal infections, among which the hematogenous are not always to be distinguished from the ascending affections, may be subdivided into the following four groups:

I. Those presenting in girl babies or young girls an apparently unaccountable fever, without anything to suggest its source. It is not always easy in these cases to collect and examine the urine, hence this most important clue is often neglected. The presence of a moderate or considerable number of leukocytes in the sediment of such a urine, when vaginal contamination is excluded, strongly suggests a urinary infection. A pure culture of colon bacilli can usually be obtained from the urine, as it was in this case, and the therapeutic test (rapid improvement under forced water-drinking and urotropin) puts the diagnosis upon a fairly firm foundation.

2. In other persons the disease often sets in in an acute and threatening way, like appendicitis or acute cholecystitis. Fever, leukocytosis, pus in the urine, and tenderness in the costovertebral angle are a very suggestive group of symptoms and demand cystoscopy as confirmation. Nephrotomy or nephrectomy may be necessary to save life if the symptoms do not rapidly abate after the ingestion of urotropin and large amounts of water.

3. Relatively mild and chronic cases, characterized by pyuria, with waves of irregular fever and possibly some bladder symptoms, often occur in women before or after parturition. In some of these chronic cases the urotropin and water treatment may be assisted by the use of a vaccine prepared from the organism isolated from the urine-almost always the colon bacillus.

4. There seems to me to be good reason to believe that most, if not all, cases of perinephric abscess represent neglected forms of the hematogenous infections just classified. It is a notable fact that in the past two years, since our attention was called to the frequency of hematogenous renal infections by the papers of Brewer and Cobb, the number of cases of perinephric abscess has greatly diminished.

In my opinion there is no longer any ground for supposing that a primary pyelitis, distinct from ascending infections, exists at all. It has neither a pathologic nor a clinical basis.

Diagnosis.-Renal infection, hematogenous or ascending. 


\section{Case 31}

A waitress of twenty-six, of good family history and previous history, entered the hospital January 30 , I908. Up to yesterday morning she had been well. She then was seized with pain in the right lumbar

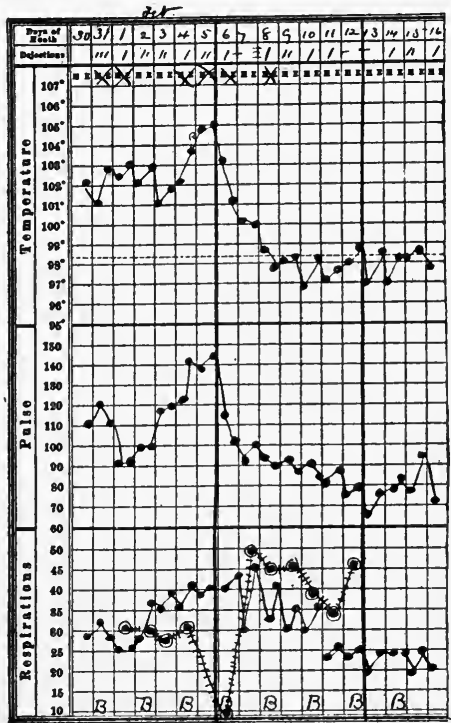

Fig. 15.-Chart of case 3I. region and lower back. This pain has persisted and become worse ever since. She has vomited a clear liquid several times, and has had some cough, with thick white sputum. She has no abdominal pain, but considerable headache.

Physical examination showed many papules scattered over the entire body. The conjunctivæ were injected and watery; the breath offensive. At the angle of the right scapula the respiration was slightly diminished, and the whisper slightly increased. The right kidney was doubtfully felt, and there was some tenderness there, but more marked tenderness under the right costal border and in the right iliac fossa.

The general abdominal tenderness was so marked that the patient was seen by a surgical consultant who, however, found no evidence of peritonitis. The urine was negative.

The temperature ranged for seven days above IоI $^{\circ} \mathrm{F}$. (see accompanying chart), and the white count between $1_{3}, 000$ and r $_{5}, 000$. The chest was strapped, with very slight relief.

Discussion.-An acute lumbar pain, accompanied by fever, headache, leukocytosis, cough and some tenderness about the right kidney, suggests several possibilities.

I. Since the pain began upon the right and is accompanied by some tenderness in the region of the right kidney, a renal infection must be considered, especially as the right kidney is far more often affected than the left by such infections. But in the presence of a negative urine all the other possibilities must first be carefully canvassed before proceeding to cystoscopy or any such bothersome tests. 
2. The orthopedic group of troubles seems unlikely in view of the acute febrile onset and the absence of confirmatory tests.

3. Against the diagnosis of local peritonitis (gall-bladder, appendix, perforating gastric ulcer) is the entire absence of muscular spasm and the very wide area of tenderness.

4. Despite the exclusion of all these possibilities, the diagnosis remained uncertain. The rather doubtful signs at the base of the right lung were sufficient, however, to make us examine this part very frequently in anticipation of the possible development of pneumonia. So many cases beginning with abdominal symptoms have ultimately turned out to be pneumonia, escaping laparotomy narrowly, if at all, that we are always on the watch for such an event.

Outcome.-On February $2 \mathrm{~d}$ the signs of solidification finally appeared at the right base. Abdominal distention and tenderness were marked. The patient had a crisis on the evening of the sixth of February, and by the fourteenth was out of bed and convalescent, though loud pleural friction, entirely unaccompanied by pain, persisted from the aleventh of February until her discharge from the hospital on the sixteenth.

It is a familiar and a puzzling experience that many infections, especially pneumonia, cholecystitis, and appendicitis, begin with vague general symptoms (fever, wide-spread pains, chills, vomiting) before settling down to business in any discoverable locality. Looking back over the course of such a chain of events, after the pneumonia or the appendicitis has been found, we are apt to suppose that the local trouble was really there all the time. The weight of evidence, however, seems to me to point the other way. The local manifestation of an infection is often, I believe, a late event in fact, as well as in our diagnoses.

Diagnosis.-Pneumonia.

\section{Case 32}

A married woman of thirty entered the hospital October $27,1899$.

Four and a half years ago she had had a miscarriage, induced by the introduction of a sound into the uterus, and a second miscarriage, without known cause, four years ago. Otherwise than this she had been always well until seven weeks ago, when she was taken with severe pain in the small of the back, which has lasted ever since, and which extends at times to the front of the abdomen. Her bowels are very costive, moving about once in five days. The pain in her back is not affected by motion, but has been severe enough to confine her to bed for the first 
two weeks of her sickness. Since that time she has been up part of each day, but has gained very little in strength, and has lost 20 pounds in weight. The range of temperature and pulse are seen in the accom-

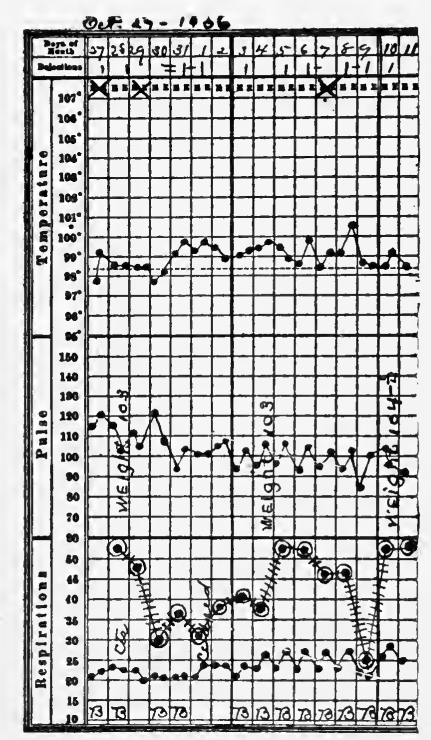

Fig. I6.-Chart of case $3^{2}$. panying chart. The right lobe of the thyroid gland is palpable, and seems about the size of a plum. The patient has noticed this lump for several months, and says that it varies greatly in size, at times being scarcely palpable.

The chest shows nothing abnormal.

The abdomen shows slight general resistance and considerable general tenderness, the latter most marked in the left iliac fossa. Motions of the back are limited in all directions by muscular spasm, and seem to cause pain, especially when she bends to the right. Pelvic organs normal.

Examination of the stomach through a tube shows a capacity of only I6. ounces. The position of the organ after distention with air was apparently normal. After a test-meal the stomach-contents showed o.I per cent. of free $\mathrm{HCl}$, no lactic acid, and no blood.

In the course of two weeks all the pains disappeared. Dr. Goldthwait found no lesion of the spine, hip, or pelvic joints. A firm binder about the hips gave no relief. Tonics, sodium bromid, enemata, and hypnotics were given for the control of symptoms as they appeared from time to time.

By the eleventh of November the patient seemed nearly well.

Discussion.-The case is afebrile, and apparently not of the renal or orthopedic groups. The pain is not affected by motion and, therefore, is not due to lumbago. There is no evidence of sacro-iliac or spinal disease. The most definite and important feature in the case is the fact that the woman is debilitated, has lost 20 pounds in weight, is badly constipated, and probably has a wide-spread depression of other functions. There is no reason to suppose that the thyroid enlargement has any bearing on the symptoms.

Nothing certain can be said regarding the diagnosis of such a case until the lapse of some time has made it clear that nothing else is going 
to develop. After this we may settle down more or less discontentedly, with the diagnosis of what some orthopedic specialists call a "functional back." This is a very familiar clinical entity, whatever its real cause and best nomenclature may be.

Two things seem to me clear about this trouble: first, that psychic causes enter into it, and, second, that they are not the whole of it. For example, I have seen a young woman drag herself painfully down the street to the post-office with lumbar pain at every step, while each foot seems to weigh a ton and every muscular contraction is an effort. She calls at the post-office, gets a certain kind of letter, and walks home erect and free from pain. We are apt to say that such symptoms are imaginary, but this seems to me wholly unscientific. Certainly psychic causes enter powerfully into their production and destruction. May we not plausibly suppose that discouragement has slackened the muscles as it does those of a tired army on the march? A psychic cause renders them taut-a band of music, a long-expected letter; they there-upon begin to support the sagging joints, and the pain disappears as sensitive parts are relieved of pressure. It is in cases of this type that practitioners are apt to seek a cause for the symptoms in the pelvis, with what scanty justification I have endeavored to show in the introduction to this chapter.

Diagnosis.-Debility.

\section{Case 33}

A metal polisher of thirty-six entered the hospital June 24, I908, because of pain in the back, beneath the twelfth rib, on both sides. This pain had been present for one week before entrance, accompanied by fever for the past four days, and vomiting for the past three days. Ten days ago micturition was frequent and painful for one day and the urine bloody.

The urine at entrance showed much pus, a little blood, a slight trace of albumin. The specific gravity varied between 1003 and Ioro. The twenty-four-hour amount was from 80 to Ioo ounces a day. An occasional granular cast was found in the sediment. The leukocytes ranged from 16,000 to $19, \infty \infty 0$ per cubic millimeter. Widal's reaction was negative; the range of the temperature and pulse was as seen in the accompanying chart.

On physical examination the man was emaciated, pale, with sunken eyes. The edge of the spleen was easily felt. Physical examination was otherwise negative, except for considerable tenderness in both 
costovertebral angles. On the second of July a macular erythema appeared upon the back of the trunk and hands, and was seen by Dr. Charles J. White, who stated that he could not definitely recognize the nature of these macules. His bowels were moved by calomel and enemata, and he was given liquid diet. A culture specimen of urine showed a pure growth of colon bacilli. By the thirteenth of July pus had disappeared from the urine. The

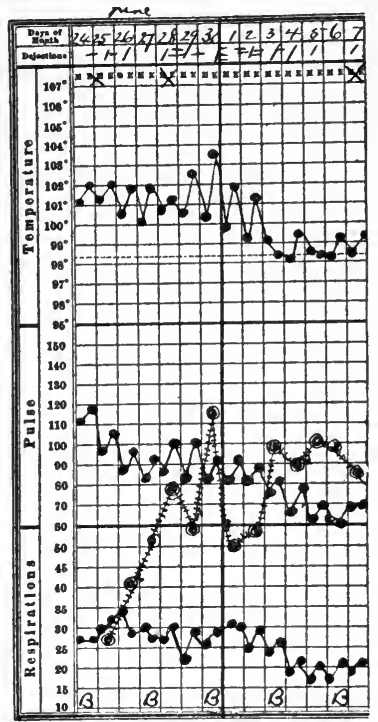

Fig. 17.-Chart of case 33 . white cells were 8700 . The Widal reaction was negative, as it was throughout the illness.

Discussion. - The symptoms point obviously to the kidney, but the enlargement of the spleen suggests the possibility of some other cause for the fever. With such a urine, with costovertebral tenderness and leukocytosis, a urinary infection must form at least a partial explanation of the symptoms. Owing to the persistence of fever and the splenic enlargement, a routine blood-culture was taken, which, to everyone's surprise, showed typical typhoid bacilli. In view of this fact it may well be questioned whether the macular erythema was not, in fact, due to some form of typhoid rose spot-in other words,

whether it was not due, like the ordinary crop of rose spots, to the lodgement of typhoid bacilli beneath the skin.

Evidently we were dealing, in this case, with a double infection, both typhoid bacilli and colon bacilli being active pathogenic agents. The colon bacilli, in process of elimination from the body, presumably caused the renal infection. The lumbar pain was probably of the general infectious type, and not due to kidney lesion.

Outcome.-The patient was given urotropin, $7 \frac{1}{2}$ grains three times a day, and left the hospital well on the twenty-sixth of July.

Diagnosis.-Typhoid and colon bacillus infection. 


\section{Case 34}

A laborer of thirty-nine entered the hospital June II, 1907.

In 1899 he wrenched his back in lifting a heavy jack, and was lame for three or four weeks afterward. In February, I906, he had sciatica. For the past two months he has noticed an ache in his back when he gets up in the morning. Ten days ago he noticed tingling and numbness in his toes and the pain in his back increased. Since then he has slept very little, and six days ago he had to have morphin, which has been frequently used since then, but lately with only slight relief.

Both legs, from the knees to the heels, are now sensitive and prickling. His feet feel freezing cold. He denies alcohol and venereal disease.

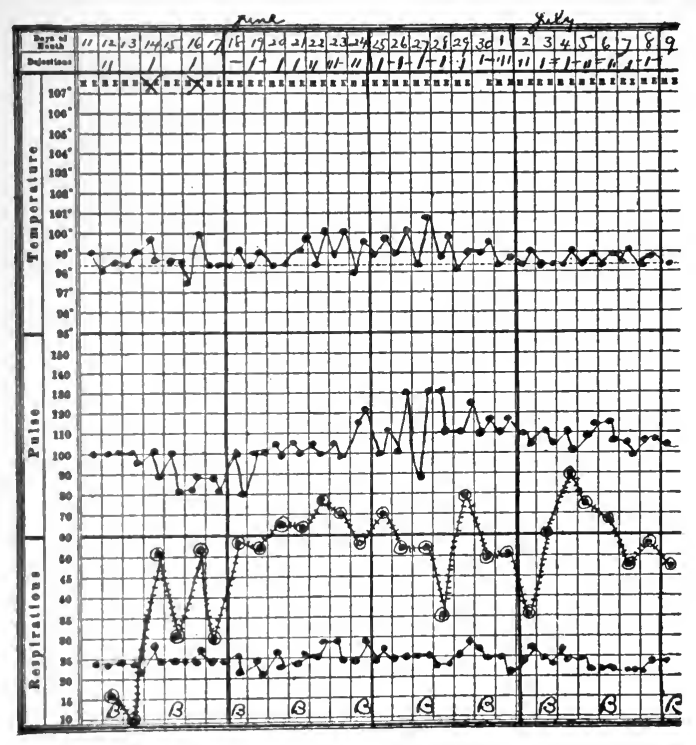

Fig. 18.-Chart of case 34 .

Present Condition.- The range of temperature and pulse are as seen in the accompanying chart. The patient is well developed and nourished, but looks worn out and in much pain. Indeed, he can scarcely lie still a moment. The chest and abdomen show nothing abnormal. From the third to the eighth dorsal vertebra the backbone is bowed posteriorly. There is much tenderness on pressure over the seventh cervical spine. There is no disturbance of sensation in the feet or legs, and motion is everywhere normal.

The urine is normal in amount, I028 specific gravity, with the slightest possible trace of albumin and a few finely granular casts. No blood or pus. The white cells number 14,800 . The red blood-cells show 
no stippling; $x$-ray is negative. The lines of expression in both sides of the face are flattened out. The right side moves less than the left. The patient cannot whistle, and protrudes his tongue slightly to the right.

The grasp in both hands is equal, but markedly diminished.

The knee-jerks are absent, the superficial reflexes normal, the mind clear.

Discussion.-Clearly, we cannot blame the old wrench for the present trouble. The sciatica also seems to be ancient history, though both of these events may be of some importance as indicating a locus minoris resistenđia.

In contrast with all the types of lumbar pain previously discussed this case stands out, marked by the presence of sensory symptoms (numbness and prickling) in the legs. It is also notable that the face and arms are affected, though not at the beginning of the case.

Though the backward bowing of the spine brings the possibility of Pott's disease to mind, there is nothing else in this region to support any such hypothesis, and neither here nor in the cervical region, where some tenderness was present, did $x$-ray show any lesions. The entire absence of muscular spasm helps to exclude spinal tuberculosis. No other disease of bone or joint is definitely suggested, and there is nothing to point to the urinary system or to any general infection as the source of these troubles. The urine cannot be called normal, but its abnormalities are of a very vague and general nature, consistent with the presence of almost any disease and with the absence of all known disease, so that in this differential diagnosis they may be disregarded.

In view of the general sensory symptoms, the loss of muscular power and tone and the diminished reflexes, multiple neuritis is the natural diagnosis. Were the spinal cord involved, one would expect pupillary changes, increased reflexes, relaxed sphincters, and the absence of such wide-spread sensory symptoms.

As to the cause of the neuritis, we are here, as in so many other cases, quite in the dark. Alcohol and lead can be definitely excluded. There is no reason to suspect arsenic. The presence of moderate waves of fever and a continued leukocytosis makes it reasonable to suppose that an infectious process is at the bottom of the symptoms.

Outcome.-The leukocytes continued to range high, and the pain and the tenderness continued to be very troublesome. Sterile water was given at times instead of morphin, and the patient was gradually weaned from his fondness for the drug.

By the thirtieth of June the pains were less severe and could be con- 
trolled by aspirin, Io grains, once or twice a day, or by placebos. There was moderate muscular wasting, and tenderness along the nerve-trunks was present.

By July 8th the grip was much improved. The patient was up and about the ward, and soon went home to finish his convalescence.

Diagnosis. - Neuritis.

\section{Case 35}

A negro of fifty, with a negative previous history, entered the hospital August 2, 1906, complaining that for three weeks he had been suffering from loss of appetite, nausea, fever, and weakness, and had been in bed a good deal of the time.

Ten days ago he was beginning to recover his strength, but four days ago he suddenly began to have shooting pain in the lower back and buttocks, the pains running down the backs of both legs, especially the left, and intensified by motion. He is fairly comfortable when quiet in bed. At the onset of his disease he took large doses of quinin with relief. His bowels now move every two or three days.

On physical examination, temperature, pulse, and respiration were found to be normal, the chest and abdomen likewise normal, the blood and urine negative. Extension of the left leg with the knee straight caused marked pain in the left sacro-iliac joint. Movements of the leg with knee flexed were not painful. There was tenderness over the left sacro-iliac joint and over the lower dorsal spines. $X$-ray was negative. The motions of the spine were markedly limited in all directions.

Discussion.-Apparently the symptoms in this case followed a three weeks' febrile illness, the nature of which we do not know. The possibility of typhoid and a post-typhoidal spondylitis is naturally suggested, but if typhoid is often complicated by a late spondylitis, presumably other infections may have a similar sequel.

The marked limitation of spinal motion and the tenderness in the lower dorsal region make it necessary to consider spinal tuberculosis. The negative $x$-ray, however, goes far to exclude this possibility. Only the course of time and the effects of treatment can make us more certain on this point. The same remarks apply to the possibility of malignant disease.

Outcome.-Dr. Robert B. Osgood, who saw the case in consultation, considered it one of infectious arthritis of the spine and the left sacro-iliac joint; strapping of the back, with enemata and tonics, was the treatment. The patient was able to leave the hospital almost well in twelve days.

Diagnosis.-Infectious spondylitis. 


\section{Case 36}

A Swedish housemaid of twenty-five, with an excellent family history, entered the hospital March 27, 1907. She states that eight or nine years ago she was in bed for six weeks with "catarrh of the lungs," and that since she was ten years of age she has had frequent attacks of tonsillitis. Otherwise she has been well. At Christmas, I906, she caught cold and was weak and feverish. At this time her back became very sore and painful on motion, and she had to give up work the first of January. Since then she has not improved at all, and has been in bed a considerable part of the time. At entrance, the temperature, pulse, and respiration were normal, the chest and abdomen negative, the spine held rigidly and all motion painful. There was no kyphos and no sacro-iliac tenderness.

Discussion.-The long duration of the symptoms holds our attention at once. Chronic backaches may be due to functional causes, to osteoarthritis and the pressure group (Pott's disease, new-growths, and aneurysm). It is notable that in this case a rest in bed has not produced any marked improvement, neither has there been any alarming advance in the severity of the symptoms, such as would probably occur with malignant disease. The physical signs are confined to the evidence of a rigid and painful spine. Renal lesions and general infections are easily ruled out. Any ordinary lumbago would have been cured long before this. The spinal rigidity and tenderness make it very improbable that sacroiliac disease is the only lesion present.

The so-called functional, neurasthenic, or hysteric affections of the spine are naturally suggested by the long duration of the symptoms, by the age and sex, and by the absence of fever, kyphos, and other obvious lesions. The outcome of the case shows the great importance of not jumping at such conclusions until every method of physical examination, including the $x$-ray, has been used. This is especially true of all dubious and chronic cases.

Outcome.-At entrance, the diagnosis was "acute osteo-arthritis with a neurasthenic background." An $x$-ray taken the first of April showed that the body of the second lumbar vertebra was extensively diseased, and a knuckle was later developed in the lumbar region. The patient was put at once into a plaster jacket, and by April 6th was able to sit up with comfort. On April gth she left the hospital.

Dr. Osgood's diagnosis was early Pott's disease.

Diagnosis.-Vertebral tuberculosis. 


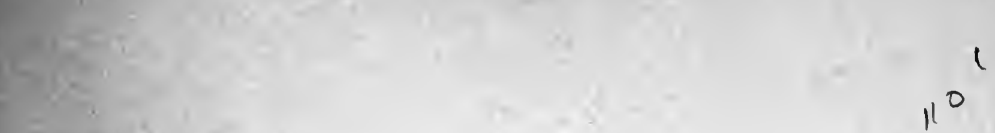

$11^{\circ}$ 


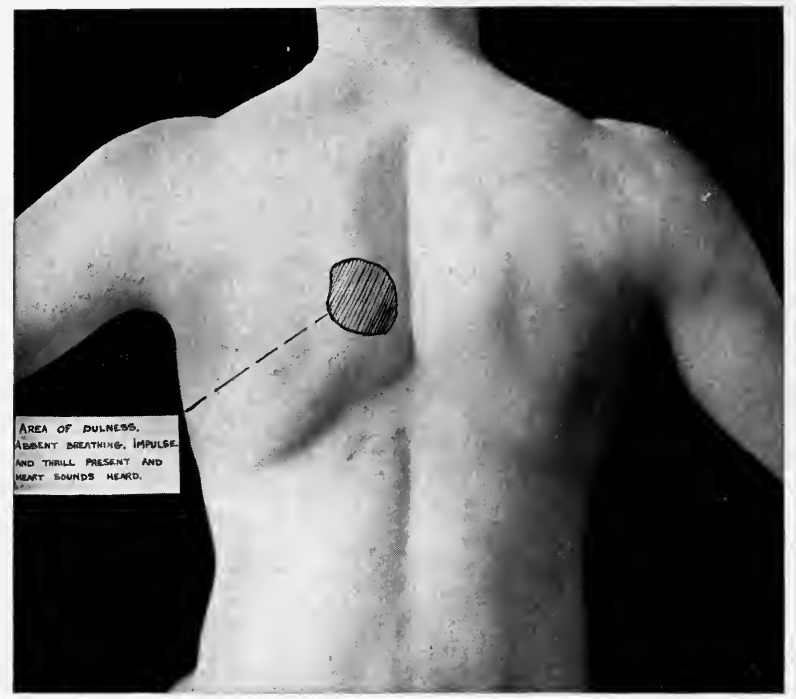

Fig. 19.-Area of pulsation at a point often overlooked in physical examination. Complaint, pain in the back. 


\section{Case 37}

A carriage painter of thirty-four entered the hospital March 20, 1907. His father died of a paralytic shock; his family history was otherwise excellent. He remembers no sickness in his life. Had a soft sore eleven years ago, and a bubo about the same time; had no rash, sore throat, falling of hair, or pains following it, but was treated for a year after it, with what medicine he does not know.

In his work he lifts from 1oo to 200 pounds every day. He was perfectly well until five months ago, when he began to feel weak. Since that time he has been losing weight and has done no work. Three months ago he strained his back, and since then he has had a burning pain in the small of his back and below the region of the heart on the left. This pain has increased considerably in the last three weeks, and is now so severe that he has to bend forward and to the left to ease it. It is made worse by walking, and interferes with sleep. He has no dyspnea and no other symptoms.

Physical examination showed normal temperature and respiration; pulse somewhat accelerated, keeping most of the time between roo and 120. His pupils are equal and react normally; his heart and lungs negative, except as shown in the diagram (Fig. Ig), his right radial pulse slightly larger than the left.

There is resistance and dulness in the epigastrium, but no definite mass made out. The glands are considerably enlarged in the groins and axillæ.

Discussion.-As in the previous case, the element of duration is a most important one in the diagnosis. A steady pain lasting three months is not likely to be due to functional causes when it occurs in a carriage painter of thirty-four. Lead-poisoning, suggested by the occupation, never produces such a pain as this without other symptoms. The general infections and the renal group of lesions are easily excluded by the physical examination. This leaves us with the diseases which I have called the pressure group (Pott's disease, aneurysm, and neoplasm) especially deserving of consideration. Only one diagnosis is possible in this case, provided it occurs to our minds at all. The danger is that it will not be thought of, and, therefore, will not be found in physical examination. Nothing but aneurysm produces an impulse and thrill with dulness and absent breathing between the spinal column and the left scapula. Pulsating pleurisy and pulsating sarcoma do not present themselves at this point.

Outcome. $-X$-ray showed a distinct shadow in the area of pulsation, 
as figured in the diagram. The pain felt over the lower ribs in front seemed to be explained by pressure of aneurysm on the intercostal nerve.

There is now no pain in the region of the tumor. The patient was given iodid of potash, 15 to 30 grains, four times a day; nitroglycerin, $\frac{1}{100}$ grain, every three hours; when needed for pain an occasional dose of morphin, $\frac{1}{6}$ grain.

The patient left the hospital slightly relieved on June $4^{\text {th. }}$

Diagnosis.-Aortic aneurysm.

\section{Case 38}

A laborer of twenty-two entered the hospital July 4, 1906, with a negative family history. All last winter, he says, he suffered from "rheumatism around the heart"; otherwise his past history and habits are good.

For the past two weeks he has been ailing, especially on account of pain in the abdomen, the back, the neck, or the head, every day. The pain in the back has prevented any continuous sleep for the last five nights, but he also aches all over, although he was able to work until two days ago. For the past week he has had a bad taste in his mouth in the morning. He says a number of his friends have the same trouble, and call it the "grip." His appetite is poor, and he has nausea after eating. The bowels are regular; there are no other symptoms. A soft, systolic murmur is heard all over the precordia, loudest in the pulmonary area. The pulses are of low tension and dicrotic. The chest and abdomen are negative. On the forearms are a number of sharply defined macules and papules, which decolorize on pressure (mosquito bites?). In the left hypochondrium is a group of rose-colored macules, five in number.

During the first three days of his stay in the hospital he had fever, ranging from $100^{\circ}$ to $103^{\circ} \mathrm{F}$., accompanied by considerable pain in his back.

Leukocytes were 5900; Widal reaction-persistently negative. No malarial organisms were found in the blood. The urine was negative. His abdomen was always rigid, and his bowels difficult to move. On the twenty-first of July, after four days of normal temperature, his back still showed limitation of motions in all directions, with considerable tenderness on his shins. A diagnosis of lumbago was made this day by Dr. Joel E. Goldthwait. Under criss-cross strapping his pain was almost gone by the twenty-fifth. His lips were cyanotic throughout his stay in the hospital; his appetite, enormous.

His treatment consisted of salicylates and aspirin for the pain; also 
the acetate and the iodid of potash, an occasional dose of morphin, and laxatives. At entrance he was treated as for typhoid.

Discussion.-The diagnosis of lumbago is very plausible in this case, owing to the fact that the patient has general limitation of the lumbar motions, and has previously suffered from stiff neck and other apparently muscular pains. But there are other features about the case which make it seem more like a post-febrile spondylitis of the type most often seen after typhoid. Lumbago does not produce a fever like that here described, and there are many other facts pointing to the existence of a general infection. The rapid recovery under a simple strapping treatment does not necessarily prove that the diagnosis is lumbago, but does tend to exclude all other possibilities, except the two above mentioned. The cyanosis and the enormous appetite are not explained.

Diagnosis.-Lumbago(?) Infectious spondylitis(?)

\section{Case 39}

An ice-man of twenty-five entered the hospital April 10, I906. His family history was negative, his past history good. He had urethritis six months before. He has taken five or six glasses of beer a day, and one or two glasses of whisky a week, as a rule, but has seldom been drunk.

Except for the urethritis, he was well until two weeks ago, when he began to have a dull, aching pain in the right side of his back and flank, not severe enough to make him give up work nor to keep him awake. After a couple of days this pain disappeared, but returned five days ago. This time it extended into the right leg, but not into the groin or testes. The painful area is tender, and the pain is constant. He has noticed no change in his urine; he thinks, however, that he passes more urine in the night than in the day. He has some shortness of breath and palpitation on exertion.

He had no temperature above $99.5^{\circ} \mathrm{F}$. during his stay of ten days in the hospital. The abdomen was held firmly above the navel, was everywhere tympanitic, and in the right upper quadrant was tender. At this point a mass the size of the fist was felt, moving with respiration, apparently lobulated, and coming down a hand's-breadth below the ribs on full inspiration. It was easily felt bimanually, and could be partially replaced behind the ribs.

The urine was between 60 and 80 ounces in twenty-four hours, and contained a very slight trace of albumin. In the sediment were, many intracellular diplococci, decolorizing by Gram's stain. Twenty minims of the sediment of urine was inoculated into a guinea-pig. The animal was killed two months later, and showed no evidence of tuberculosis. 
On the twentieth of April $x$-ray showed a definite shadow in the region of the right kidney. Dr. Davis catheterized the right ureter and obtained pus containing gonococci.

Discussion.-Everything points to the kidney as the source of this patient's troubles. Our suspicions in that direction are promptly confirmed as the result of cystoscopy, $x$-ray examination, and animal inoculation, a group of procedures demanded in almost every case of chronic renal pyuria.

Since "surgical kidney" is excluded by the cystoscopic examination, and tuberculosis by the results of animal inoculation, the only important possibility left is renal stone, a supposition strongly supported by the $x$-ray evidence.

Outcome.-The patient was transferred to the surgical ward and operated upon on May 2 d. A stone was removed. The patient's convalescence took place without any incident and he was discharged May 26th.

He was readmitted December 5, I907. After leaving the hospital he was well and strong, and worked hard until three weeks ago, when he began to pass blood and pus in his urine and suffered pain in the right lumbar region, similar to that which he had previously had. He now suffers from two sorts of pain: (a) A dull ache in the right side, present most of the time; and $(b)$ a stinging pain occurring only after micturition, starting from the urinary meatus and running up into the right side. The urine continued bloody for the first week of this attack, the last two or three spoonfuls of each discharge being bright blood with threads of yellow pus. Of late, no blood has been visible. He has lost appetite and has been very thirsty, although he has not been conscious of any fever. He has lost about ro pounds in weight. The patient entered the hospital with a temperature of $\mathrm{IO}_{2}{ }^{\circ} \mathrm{F}$., pulse I20. After two days the temperature subsided to normal. His leukocytes were 10,000 at entrance. The abdomen was altogether normal, but in the right flank there was a visible prominence and a palpable, tender, dull, rounded, lobulated mass, apparently retreating under the ribs on pressure.

The urine, as at the previous entry, was persistently of low gravity, ranging from IorI to IOI 4 , and rather large in amount-from 50 to 70 ounces a day. The sediment was composed almost entirely of pus in moderate amounts. The pus persisted in his urine, and the patient continued to have considerable pain in the right flank. $X$-ray showed only doubtful shadows of a possible stone.

Operation, December 24th, showed no stone, but many pockets of pus scattered throughout the kidney, with smaller foci of round-cell 
infiltration between them. The kidney was enlarged, and at one end was fibrous. Its pelvis was normal. The patient did well after nephrectomy.

\section{Diagnosis.-Renal stone; multiple abscesses.}

\section{Case 40}

A housewife of fifty-one entered the hospital August II, I906, for the third time. At her first entry, in June, I899, a diagnosis of gallstones had been made; at the next entry, June, rgor, neurasthenia was the diagnosis. Her attacks of illness between February, I899, and December, I90I, were very frequent and of a similar character. There was a sudden occurrence of pain, severe and cramp-like, doubling her up. It always started in the right side of the back, thence radiating to the right hypochondrium, but never to the right shoulder. It would last from two hours to two days, and was relieved occasionally by household remedies, but always by morphin. After relief there would be no recurrence for weeks or months. The pain was associated with romiting, but showed no special tendency to occur at night. The urine and feces were normal, and there was no fever with the attacks. Twice she entered the hospital for these attacks, but has always been free from pain while here. For the past two and a half weeks she has had an attack every day, sometimes in the afternoon, sometimes at night. Morphin has been injected several times, and she has had morphin pills on hand. Her bowels are moved daily, but she has had no appetite.

Her physical examination, including blood and urine, temperature, pulse, and respiration, was wholly negative. On the thirteenth, at 3 A. M., she began to have severe pain. A rounded tumor was easily felt below the ribs, in the region of the gall-bladder, moving with respiration, and easily mapped out by percussion.

Discussion.-Colicky pain in the right lumbar region naturally suggests renal stone. In the absence of any urinary changes, however, an $x$-ray would be necessary to confirm the diagnosis. The account of the pain does not sound like that of lumbago, which is not promptly driven away by morphin, and is rarely so severe as to call for its use.

Another cause for the pain is suggested by the rounded tumor in the right hypochondrium. This tumor might be connected with the stomach or intestine, but the absence of gastric or intestinal symptoms between the attacks of colic makes this unlikely. It seems more probable that the tumor is due to distention of the gall-bladder, the absence of jaundice being due to the fact that the common duct is patent.

It is well to say a word in condemnation of the previous diagnosis 
of neurasthenia, based, apparently, on the fact that the patient happened to be in the hospital during an interval between her severe attacks. Such a diagnosis, based wholly on negative findings, is always unjustified; for the patient it is often adding insult to injury. It is far better to make no diagnosis at all and watch for a recurrence of the previous symptoms.

Outcome. $-X$-ray of the renal region was negative. On the fifteenth the abdomen was opened, and a number of gall-stones were found in the gall-bladder.

Diagnosis.-Gall-stones.

\section{Case 41}

A longshoreman of forty-four with a good family history entered the hospital March 7, r907.

$\mathrm{He}$ had used a quart of ale daily until five weeks ago. In I883 he had malaria in India. In 1890 he had blood-poisoning of the arm, and was in the Royal Infirmary, Liverpool, twenty-five days.

Two years ago he had "pleurisy," and half a gallon of fluid was taken from his left chest.

March 28, 1906, he had some operation done on his right testis, just for what cause he does not know. Since then he has been well until five weeks ago. He entered the hospital March 7, I907, complaining of constant pain across the small of his back. It has been severe for the past two weeks, so as to prevent work or sleep. For a week he has had frequent cramps in his calves, and lately has been short of breath. $\mathrm{He}$ has lost I4 pounds in the five weeks.

Physical examination of the abdomen showed in the right hypochondrium and epigastrium two smooth, rounded masses, palpable bimanually, descending with respiration. (See Figs. 20 and 2r.) On inflation of the stomach the masses appeared to be behind it. Physical examination was otherwise negative, except that the urine was of low gravity-roo7ranged in amount from 70 to I 20 ounces during the week of his stay in the hospital, and contained in its sediment a few hyaline and fine granular casts. $X$-ray of the spine was negative.

The gastric contents contained no hydrochloric acid after a testmeal. The size of the stomach was normal, and there were no organic acids or fasting contents.

Discussion.-The occupation is one of those often associated with lumbago or spinal osteo-arthritis, but for simple lumbago the pain has been rather too steady and prolonged. The question of osteoarthritis will be referred to later.

The history of pleurisy, together with a severe and long-standing 


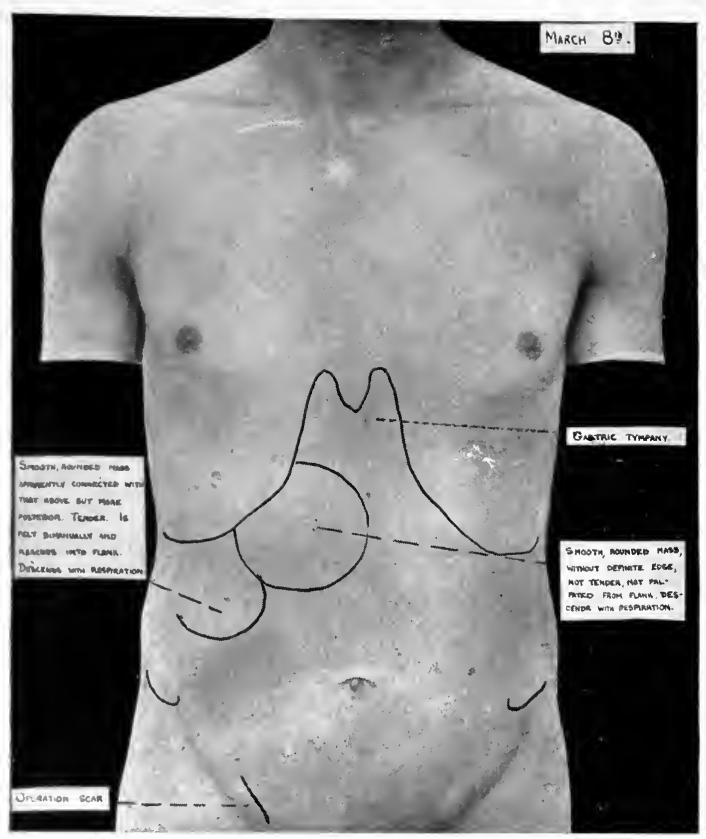

Fig. 20.-Outline of masses felt March 8th, Case 4T. (See also Fig. 2I.)

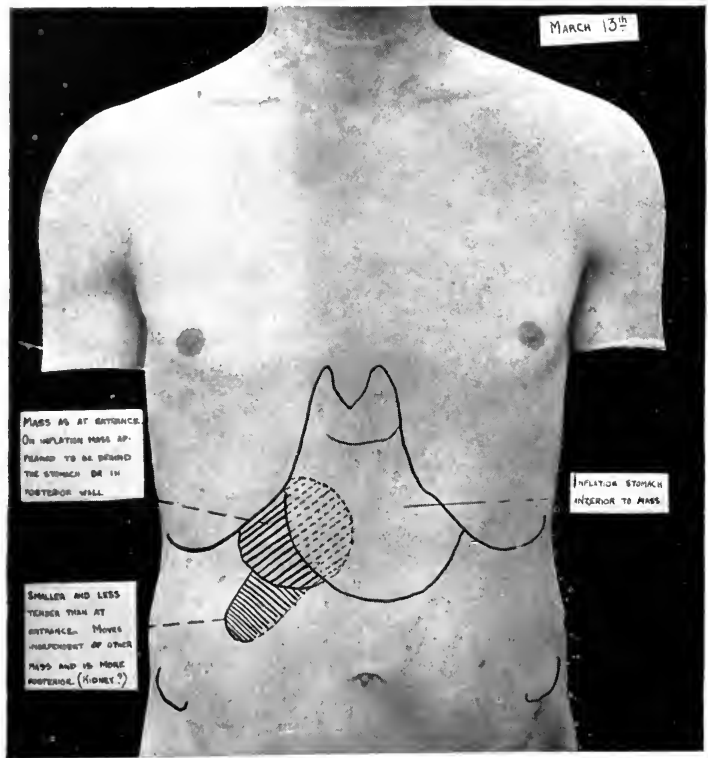

Fig. 21.-Outlines recorded March ${ }^{3} 3^{\text {th. }}$. Chief complaint, lumbar pain. (See also Fig. 20.) 

spinal pain, often points to a spinal tuberculosis. It is quite possible, also, that the previous operation may have been for tuberculous epididymitis. Against this diagnosis, however, is the absence of fever and muscular spasm, as well as the negative $x$-ray examination.

The two latter facts are also against the diagnosis of osteo-arthritis, though this cannot possibly be excluded.

We naturally desire to connect all the symptoms and signs in the case into a mutually explaining group, and this brings us to the consideration of the abdominal tumors. Cystic kidney (congenital) would produce such a tumor and such a urine, but as it is invariably bilateral, we should expect to get some evidence of a tumor in the left hypochondrium. Further, cystic kidneys never cause pain in the back of anything like the severity here complained of.

Hydronephrosis would explain the tumor, and possibly the urine, but would not account for the pain.

Can the tumor be in the stomach, possibly with spinal or glandular metastases to account for the pain? This is suggested by the absence of hydrochloric acid in the gastric contents, but it must be remembered that a similar lack of hydrochloric acid has been frequently demonstrated in association with malignant tumors of any organ, $e . g$., cancer of the breast, as well as in a variety of debilitated conditions. Since no gastric symptoms are complained of, and there are no changes in the size or motility of the stomach, a gastric tumor seems unlikely.

Retroperitoneal growths certainly deserve consideration. The previous tumor of the testis may well have been sarcoma, and if so, a metastasis in the retroperitoneal lymph-glands would be very likely. Further than this one cannot go without exploratory operation.

Outcome.-The abdomen was opened on March i $5^{\text {th }}$, and a retroperitoneal mass the size of a grape-fruit was found behind the pylorus. It was afterward learned that the tumor of the testis was sarcoma.

Diagnosis.-Retroperitoneal sarcoma.

\section{Case 42}

A medical student of twenty-three entered the hospital July I8, I907. He had typhoid fever in the Massachusetts Hospital in August and September, I906. After that he went back to college for the second half-year in February, 1907, taking his work easily, but finding it hard to concentrate his attention, having a good deal of pain in the forehead after studying, and needing to lie down every afternoon. On March rst he had an attack of severe pain in the small of his back; this lasted five days, with much stiffness. Four weeks ago he had another attack, 
following exposure to cold and wet, lasting four days. For the past three weeks he has been in bed with the same trouble. Ten days ago he woke up in the night, doubled up with pain, and had to have morphin to relieve it.

On physical examination the knee-jerks were found to be exaggerated. Kernig's sign was marked, aind ankle clonus present.

Temperature at entrance was $102^{\circ} \mathrm{F}$., pulse, I20, but after fortyeight hours both pulse and temperature were normal.

The white count was 3200 ; urine was negative.

The spine was held rigidly in extreme lordosis, with well-marked spasm of the erector spinæ group. The patient was unable to stand or

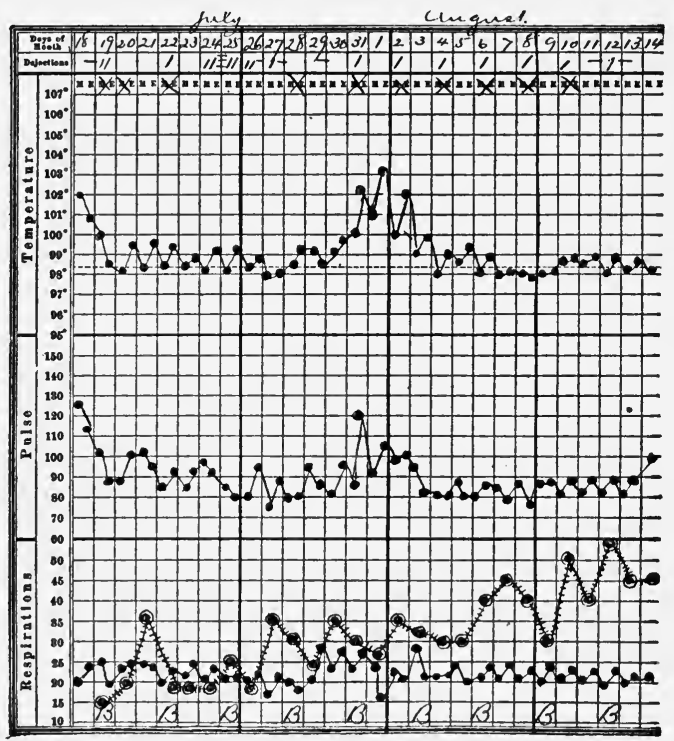

Fig. 22.-Chart of case 42.

to sit erect. The hip motions were free and normal, except that hyperextension of the right hip is painful. There is slight tenderness on pressure over the right sacro-iliac joint.

Discussion.-A rigid, tender spine following typhoid fever and associated with some neurotic symptoms is almost the typical picture of post-typhoidal spinal arthritis. The chief objection to such a diagnosis in this case is the long duration of the interval between the typhoid fever and the present symptoms. Almost all cases of "typhoid spine" come on within three months, while in this case the interval is almost six months. This, however, is by no means convincing evidence against the diagnosis. 
In the earlier editions of Osler's text-book this disease was described as a neurosis without anatomic basis. The reason for this belief is suggested in the present case, as in the majority of all cases. Mental symptoms, of the type usually referred to as neurotic or neurasthenic, constitute one of the varieties of post-typhoidal psychosis, and may be obstinate and long continued. Various types of insanity are also met with as sequelæ of typhoid, though nearly all of them recover. It is easy to see how mental depression, associated with muscular relaxation, might accent and aggravate the symptoms of an otherwise latent spondylitis. That spondylitis may be latent is proved by the occasional finding of rigid spines in patients who have never experienced any previous pain, and in whom $x$-ray demonstrates osteo-arthritic lesions.

Outcome.-Dr. R. B. Osgood saw the case in consultation, and considered it a typhoidal arthritis of the lower spine and sacro-iliac joints.

On the twenty-third a plaster jacket was applied, with complete relief to the pain in the back. The patient had a good deal of vomiting, and for some days took nothing but cracked ice by mouth. At this time the urine showed a trace of albumin, with hyaline, granular, and epithelial casts in small numbers.

The Widal reaction was positive.

The second week of his stay in the hospital he had a rise in temperature (see accompanying chart) lasting five days. The patient was very hysterical, and a false chart hung at the head of his bed had a salutary effect. After the application of his plaster jacket he had no pain. On the eighth of August he was able to sit up in a chair. On the fourteenth he was discharged, much relieved. On the twenty-sixth of August he reported that he had been walking as much as twice a day without pain. He was still hypochondriacal and introspective, but was otherwise well.

Diagnosis.-Typhoidal spondylitis.

\section{Case 43}

A Jewish housemaid of twenty, with a good family history and past history, entered the hospital September 2, 1907. She said that for two years she had had a steady and rather severe pain in the small of the back. At the beginning of this period she was in bed for three months, after which she was able to work, although her back was stiff and her trunk bent toward the right. Last winter she had for several weeks a painful cough, without expectoration; she had no fever at any time. Although in constant pain, she has worked until three days ago.

There was no fever. Examination of the chest and abdomen, 
blood, and urine was negative. The left knee-jerk was considerably livelier than the right. The spinal muscles were rigid.

Discussion.-The most important fact about this particular case of lumbar pain is that it has lasted far longer than any other hitherto described. Such prolonged suffering suggests either some member of the "pressure group" (aneurysm, tuberculosis, or neoplasm), or a functional neurosis; no general infection, no form of renal disease, and none of the orthopedic group of diseases would last so steadily and so long.

A functional neurosis is not likely in a girl who keeps steadily at work, although in constant pain. The difference in the knee-jerks is also decidedly against this diagnosis. The patient is rather young either for neoplasm or for aneurysm. The muscular rigidity, the long duration of the pain, and the history of a previous cough support the suspicion of tuberculosis.

Outcome.-Just below the level of the twelfth rib a knuckle the size of a small apple was later made out; it was very tender and hard, not red or hot. The patient was then in exquisite pain, but on the application of a plaster jacket was greatly relieved.

Diagnosis.-Spinal tuberculosis.

\section{Case 44}

A married woman of fifty entered the hospital October IO, I907. Her family history was good. She passed the menopause one year ago. Her menstruation has always been irregular, profuse, and painful. She has had no children and no miscarriages. In childhood she had rheumatism, typhoid fever, and abscesses on the forearm. For the past fifteen years she has had stomach trouble, symptoms consisting of lack of appetite, distress after eating, and constipation. For the past two months she has had frequent severe pains in the back, chest, neck, and legs; also occipital headache, "pins and needles" in the legs, noises in the head, buzzing in the ears, palpitation of the heart, insomnia, and great nervousness. Examination of the throat showed a linear aperture threequarter inch long in the soft palate in the median line.

There was anterior bowing of both shin bones, with roughening of their front surfaces, and three large white scars; also two or three deep scars on the extensor surface of the left forearm. Spinal motions were limited in all directions, but the pain was greatiy relieved by strapping and rest. Dr. E. G. Brackett examined the spine and considered the trouble an acute infectious osteo-arthritis.

Discussion.- In any patient who has such a multitude and variety of symptoms as this we naturally suspect a psychoneurosis, especially 
as the menopause has recently occurred. There are a number of data, however, brought out by the physical examination, which point in another direction. The hole in the soft palate is almost.pathognomonic of old syphilis, especially when taken in connection with the scars on the extremities and the roughening and the prominence of the shin bone.

There is no reasonable doubt, then, that this patient has suffered from syphilitic infection. The question remains whether this can explain her present complaints. That syphilis may attack the spinal column has been satisfactorily demonstrated by $x$-ray evidence. At the same time, it is quite possible that her present troubles may be due to an acute infectious process of some other origin, or to purely functional derangements. Only by further observation and by noting the effects of treatment can the diagnosis be definitely established.

Outcome.-The patient was also given sodium salicylate, ro grains every hour, until toxic. Citrate of potash, 45 grains four times a day, until the urine became alkaline. Later, iodid of potash, I5 grains three times a day, increasing Io grains daily, when the other drugs were omitted.

Diagnosis.-Old syphilis; acute spondylitis.

\section{Case 45}

An Italian fruit-dealer of twenty-three is in the habit of carrying heavy loads, and thinks he has strained his back. He has never been sick otherwise, and has good habits and a good family history. He was first seen August I6, I907. For five years he has had attacks of pain in the right side of his back almost every day. The pain is sharp, and he says it feels as if something was "rolling over" in his back. Six days ago the pain lasted all day. It never radiates to any other point, and has not often kept him awake. It does not hurt him to stoop.

Physical examination was entirely negative, except for the presence of numerous musical râles, with slightly prolonged expiration throughout both chests.

Discussion.-Muscular strain or lumbago is our first thought in this case; it was the patient's own explanation of his troubles. The long duration and paroxysmal occurrence of the symptom, however, and its independence of stooping, make this idea impossible. Any lumbar pain that lasts so long suggests one of the pressure group of causes, but physical examination does, not bear this out. The pain should be steadier and less intermittent were it due to pressure. The same considerations, together with the absence of radiation or night attacks, tend to exclude 
osteo-arthritis and sacro-iliac disease. The absence of local tenderness and urinary changes militates against the idea of renal disease.

Vertebral tuberculosis was suggested by the prominence of certain vertebral spines, and by the doubtful phenomena in the lungs. The absence of any muscular spasm or tenderness makes this more unlikely, but $x$-ray should be taken in confirmation. On the whole, from the paroxysmal nature of the attack, some renal lesion seems the most likely.

Outcome.-Aug. Igth there was no muscular spasm or tenderness about the spine or sacro-iliac joints, but he could not bend to the left as well as to the right. The vertebral spines from the eighth to the twelfth dorsal were slightly more prominent than their neighbors. There were slight prolongation of expiration and a shade of dulness at the right apex. Numerous musical râles were scattered through both chests. There was no fever. Blood and urine were still normal.

Physical examination was otherwise negative. The patient was free from pain and said he felt perfectly well. $X$-ray showed a stone in the right kidney. Operation on the twenty-fourth verified this diagnosis.

Diagnosis.-Renal stone.

\section{Case 46}

A housewife of twenty-three was first seen December 29, I907. For three months she has been having pain in the left side of her back, worse at the menstrual period, and accompanied by constipation and general weakness. She has kept at work until two days ago. Family history, past history, and habits are good. The physical examination is negative in all respects.

Discussion.-The chronicity and steadiness of the pain are like those often seen in spinal tuberculosis, and this disease can oniy be positively excluded by $x$-ray examination and by the course of the case, though it is made unlikely by the absence of muscular spasm $_{9}$ of fever and of local tenderness or prominence.

Kidney lesions cause unilateral pain like that here described, but there is no further evidence to support any such hypothesis.

The orthopedic group of lesions is excluded by the mobility of the spine and the absence of local tenderness.

Since there is no fever, we have no good reason to suspect any infectious disease.

If the $x$-ray proves negative, the case must be treated as one of functional pain, while we await further developments. 
Outcome.-After a week's rest in bed with German powder as a laxative the patient's symptoms were entirely relieved, and as the $x$-ray was wholly negative, she was allowed to resume work.

Diagnosis.-Debility.

\section{Case 47}

A blacksmith of thirty-one was seen July 21, I906. Seven days ago he began suddenly to have sharp stabbing pains in the lower part of both chests and on both sides of his back, and was unable to take a deep breath on account of the pain. Three days ago he gave up his work. Two days ago he went to bed. He has felt feverish, especially at night; for the last two days has had general headache and has slept poorly. Just before the onset of the present illness a horse had thrown him heavily against a building. He had a negative past history and family history and good habits.

On physical examination the pupils were found to be equal and to react normally. The chest showed nothing abnormal. The abdomen was full and rather rigid, but showed nothing else of interest. The spleen was not palpable. Flexing the neck caused pain in the back, but there was no rigidity of the neck muscles and no Kernig sign.

The white cells were 5200. Stained specimen negative. Widal reaction and blood culture negative. The urine was normal.

The temperature ranged between $102.5^{\circ}$ and IC5. $5^{\circ} \mathrm{F}$. for ten days, the pulse gradually rising from IoO to $\mathrm{I} 2 \mathrm{O}$, the respiration most of the time ranging between 40 and 50 to the minute. The abdomen became more distended, and on the twenty-fourth the patient developed delirium and tremor. On the twenty-sixth his neck was found to be entirely rigid, though rotation was possible without pain.

Discussion.-The onset of the present symptoms immediately after an accident makes it natural that we should attempt to connect them with some injury then sustained, but the negative visceral examination and the presence of continued fever make it probable that the accident had nothing to do with the case.

I have known tertian malaria to begin exactly in this way, with sharp stabbing pain in the lower part of both chests, but in that case the characteristic course of the fever, with remissions on alternate days, quickly led me to examine the blood and to demonstrate malarial parasites. In the present case the temperature curves and the results of blood examination enable us to exclude malaria.

With the rapid onset of thoracic pain, fever, headache, and accelerated respiration we should consider pneumonia, which may be present 
even without denıonstrable signs in the chest and without leukocytosis. Within a few days, however, repeated and painstaking examinations of the lungs usually demonstrate some evidence of solidification, even when cough and sputum are absent. No such signs developed in this case.

Typhoid fever was the diagnosis made during the first five days of the patient's illness, and in the absence of all physical signs, with continued fever and low white count, this was probably as good a guess as we could expect to make. With the appearance of stiffening of the neck on the twenty-sixth of July the diagnosis was promptly changed to meningitis, though the condition'known as meningismus complicating typhoid was also a possibility; indeed, between meningitis and meningismus-i. $e$., between cerebral congestion and actual exudation of the pus-formation-we have no certain way of distinguishing.

Outcome.-Kernig's sign and leukocytosis appeared next day, and the delirium ceased, though a low muttering and twitching of the arms continued. Lumbar puncture was tried on the twenty-seventh, but no fluid was obtained.

Throughout, the patient's behavior was strikingly like that seen in typhoid. Death occurred on the second of August.

Autopsy showed acute purulent leptomeningitis; septicemia (streptococcus pyogenes); hypertrophy and dilatation of heart; septic hyperplasia of the spleen; obliterated extra ureter on the left side; fatty metamorphosis of the liver; fibrous cord from umbilicus to the mesentery.

\section{Case 48}

An unmarried seamstress of nineteen entered the hospital January 25, I908. The girl had never been sick until a few days ago, when she began to have pain in the small of the back, relieved by lying down, a good many headaches, and an occasional vomiting spell. There was no costovertebral tenderness; the urine was negative. The spine was normally flexible without pain, and no tenderness in the sacro-iliac joints could be elicitated by any maneuver. Fever was absent. The catamenia had been absent for three months.

Vaginal examination showed a mass the size of a horse chestnut, reddened and eroded, protruding slightly from the vulva, but reducible. In the posterior culdesac was a mass the size of a large apple, not at all movable, apparently in the back of the uterus. There was milk in the breasts, and the areolæ were darkly pigmented. Under light ether anesthesia it was easily possible to free the fundus from the sacrum and to put the whole organ into normal position. Examination then 


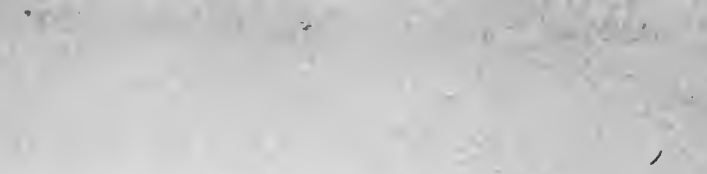

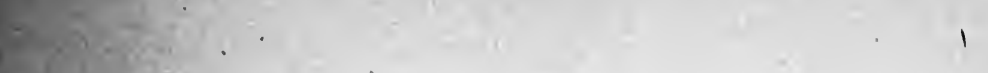

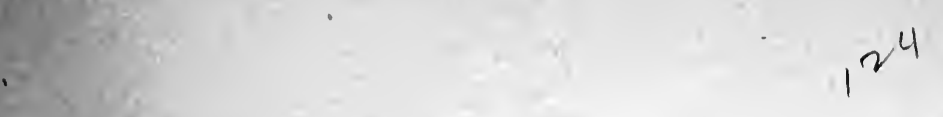




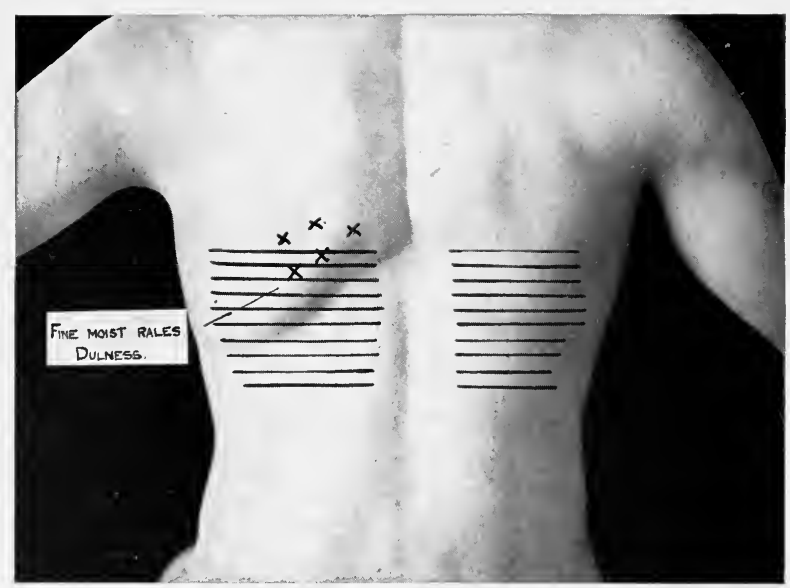

Fig. 23.- Signs as recorded in Case 49. Lumbar pain is the chief symptom. (See also Fig. 24.)

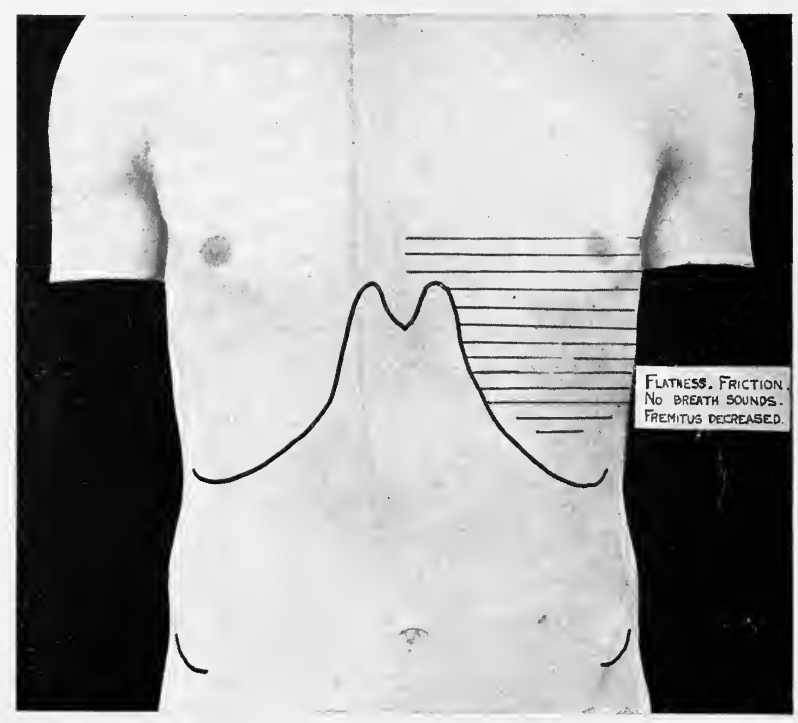

Fig. 24.-Results of physical examination of the chest in a case of lumbar pain. (See also Fig. 23.) 
showed a normal uterus enlarged about the size of a three and a half mont'ns' pregnancy with a very soft, patulous cervix.

Discussion.- In the absence of all the causes of lumbar pain heretofore discussed, and in view of the amenorrhea, a pelvic examination was obviously indicated. The only remaining question is whether the symptoms are likely to have been due to the condition of the uterus. The anatomic position of the displaced and enlarged organ as here described seems to me to put it in a different category from any of the minor pelvic disorders to which I have previously referred as unlikely of themselves to cause lumbar pain. The question seems to me solved in all reasonable probability by the-

Outcome.-The patient was entirely relieved by these procedures. Diagnosis.-Prolapsed, retroverted, incarcerated, pregnant uterus.

\section{Case 49}

A Russian housewife, twenty-eight years old, entered the hospital December 10, I908, complaining of sharp pain in the back and on both sides of the chest below the ribs, which has lasted a week. She has also had a cough for the past three weeks. She is eight months' pregnant. At entrance her temperature is $\mathrm{IOI}^{\circ} \mathrm{F}$.; pulse, I25; respiration, 32. She is slightly cyanotic. The heart's apex is in the fifth interspace, anterior axillary line, $\mathrm{I} 4 \mathrm{~cm}$. to the left of midsternum. A harsh systolic murmur is heard at the apex and in the axilla. The pulmonic second sound is accentuated. The superficial veins over the chest are very prominent. Near the junction of the second rib with the sternum on each side are seen tortuous arteries which pulsate visibly. In the lower left axilla there is flatness, absence of breath-sounds, and fine crackling sounds. (See Fig. 24.) The abdomen is distended as by a pregnant uterus. A fetal heart is heard in the left lower quadrant; rate, I48. The pain in the back is intermittent.

Discussion.-Only one question need be seriously considered in this case. Is the pain due to an infectious disease or to the contractions of a pregnant uterus?

Infection is suggested by the fever, the three weeks' cough, and the signs in the left lower axilla, which are quite consistent with a pleurisy.

On the other hand, the intermittence of the pain is what we should expect if it coincided with uterine contractions. The next thing to do, then, is to watch the patient continuously with the hand over the uterus, and see whether the pains coincide with the uterine movements. In a somewhat similar case, occurring in a young, neurotic Jewess six and a half months pregnant, and suffering also from a moderately advanced 
tuberculous process in the lung, I stood by the patient, with my hand upon the abdomen, until I convinced myself that the lumbar pain was dependent upon her restless movements and not upon uterine contractions. In this latter case the patient went on to full term, though the tuberculous process developed ominously. The signs at the bases of the lungs were presumably due to a high position of the diaphragm and some relaxation of the lung margins.

Outcome.-On observation the pain was soon determined to coincide with uterine contractions. On December I2th she gave birth to a seven-and-a-half-pound boy.

Diagnosis.-Parturition.

\section{Case 49a}

Called May 9th, r9r r, to a girl eight years of age, who complained of severe pain in back and thighs, with difficulty in walking. The father is addicted to the too liberal use of intoxicants, but is otherwise in good health. Mother in good health. The patient is the third child in a family of seven children, all living and well. On questioning, it appeared that two days before, while playing at school, she was thrown down a bank; she thinks that the vertebrae in the dorsal region struck a stone. No history of any previous illness or injury. could be obtained. She had pain in the back during the forenoon of the injury, and while walking home at noon she lay down beside the road for some time because of the pain in back and legs, and the consequent difficulty in walking. She felt unable to return to school in the afternoon, but went as usual the next day.

On the third day she was seen by a physician. The brows were then contracted, the eyebrows raised at their inner ends, and the muscles of the face rigid. There was stiffness of the back and legs. When she was turned on her side the legs would remain separated, with no support for the upper one except the tonic spasm of the muscles. The hands were rigidly flexed at the metacarpophalangeal joint. There was no anesthesia. No signs of injury along the spine or elsewhere on the body were found on casual examination. At this time a consultant saw the case and was unable to decide between myelitis and meningeal hemorrhage.

Next morning there was a general muscular tonic spasm, lasting one or two minutes, with involuntary micturition and defecation. The mind was perfectly clear. This condition continued for about twelve days, the temperature varying from $100^{\circ}$ to $102^{\circ} \mathrm{F}$., with several tonic convulsions daily. The jaws were not tightly closed, but would not open over a third of an inch. The respirations were "grunting" in character, and during the spasms there was marked cyanosis. No 
cough. The patellar reflexes were present at the time of the first examination, but were not tried for after that. Physical examination (including the urine) was otherwise negative.

Discussion.-In view of the history of trauma to the spine, one thinks first of some abnormal pressure upon the cord, perhaps a hemorrhage. But with hemorrhage into the cord one would expect a more definite localization of the symptoms below the level of traumatism. The muscles of the face would not be affected as they are here. Paroxysmal and general tonic spasm is also uncharacteristic of hemorrhage into the cord. Fracture of the spine seems to be excluded by the physical examination and by the free power of locomotion.

In view of the presence of fever, pain, and muscular weakness, with relaxation of the sphincters, acute myelitis or poliomyelitis might be considered. The latter is easily excluded by the absence of definite paralysis and the very widespread tonic spasm. In transverse myelitis or diffuse inflammation of the cord, anesthesia or other sensory symptoms are almost always present, and convulsions with involvement of the face are, so far as I know, unknown.

The muscular spasms present in this case have something in common with those seen in poisoning by strychnin, which may have been taken accidentally or with suicidal intent. Continued fever, however, is not usually present in strychnin-poisoning. The face is not often involved and the sphincters are rarely relaxed. No strychnin was found in the house, and none had been given therapeutically.

Uremia may be ruled out by the absence of changes in the heart and blood-pressure, and the negative urinary examination.

Epileptic convulsions may occur, as in this case, without loss of consciousness, but so far as I know they almost always include clonic as well as tonic spasms, whereas in this case clonic motions were altogether absent. Continuous fever without loss of consciousness is also rare in epilepsy.

Hysteria may produce tonic spasm not unlike that here described, but is practically never associated with continued fever nor with involuntary micturition and defecation.

A rigidly resistant condition of all the muscles is sometimes seen as a feature of the negativism in dementia præcox, but this disease can here be ruled out by the great suddenness of this patient's attack without any accompanying or preceding mental abnormalities, and by the presence of continuous fever and relaxed sphincters.

With the exclusion of all these possibilities one naturally comes 
to ask one's self what infectious disease can produce fever like that here present, associated with widespread muscular tonic spasm. Obviously tetanus is such a disease, but we have no history of any wound or injury whereby the bacillus of tetanus could have been introduced. There has been no subcutaneous injection of any substance which could contain the tetanus bacillus as an impurity (e.g., diphtheria antitoxin, gelatin). Nevertheless cases are on record in which it was not possible to discover the portal of entry for the bacillus, though such a portal had to be assumed, since the bacillus was later isolated from the tissues. It is not generally believed that infection can enter through the gastro-intestinal tract. On the whole, tetanus is the best choice among available alternatives.

Outcome.-After the diagnosis of tetanus had been decided upon and tetanus antitoxin administered, repeated and prolonged inquiries were again instituted regarding any previous injury, and it was learned that two weeks before the onset of symptoms there had been an abrasion of the knee from the edge of a rough board; a sliver had been removed and the wound had healed. A closer examination of the knee was accordingly made; it revealed a small bluish area on the inner side of the right knee, posterior to the hamstring and superficially healed except for one small spot from which a drop of pus could be expressed. This area was incised and curetted and a further sliver of wood about one-third of an inch in length was thus found and removed. The wound was swabbed out with iodin and a second injection of antitoxin administered. No cultures were made. On the fifteenth day of the illness the patient was convalescent, and twenty-four days from the onset was well. There had been marked loss of flesh and a decidedly round-shouldered condition of the upper spine persisted; also occasional muscular pains.

Diagnosis.-Tetanus. 


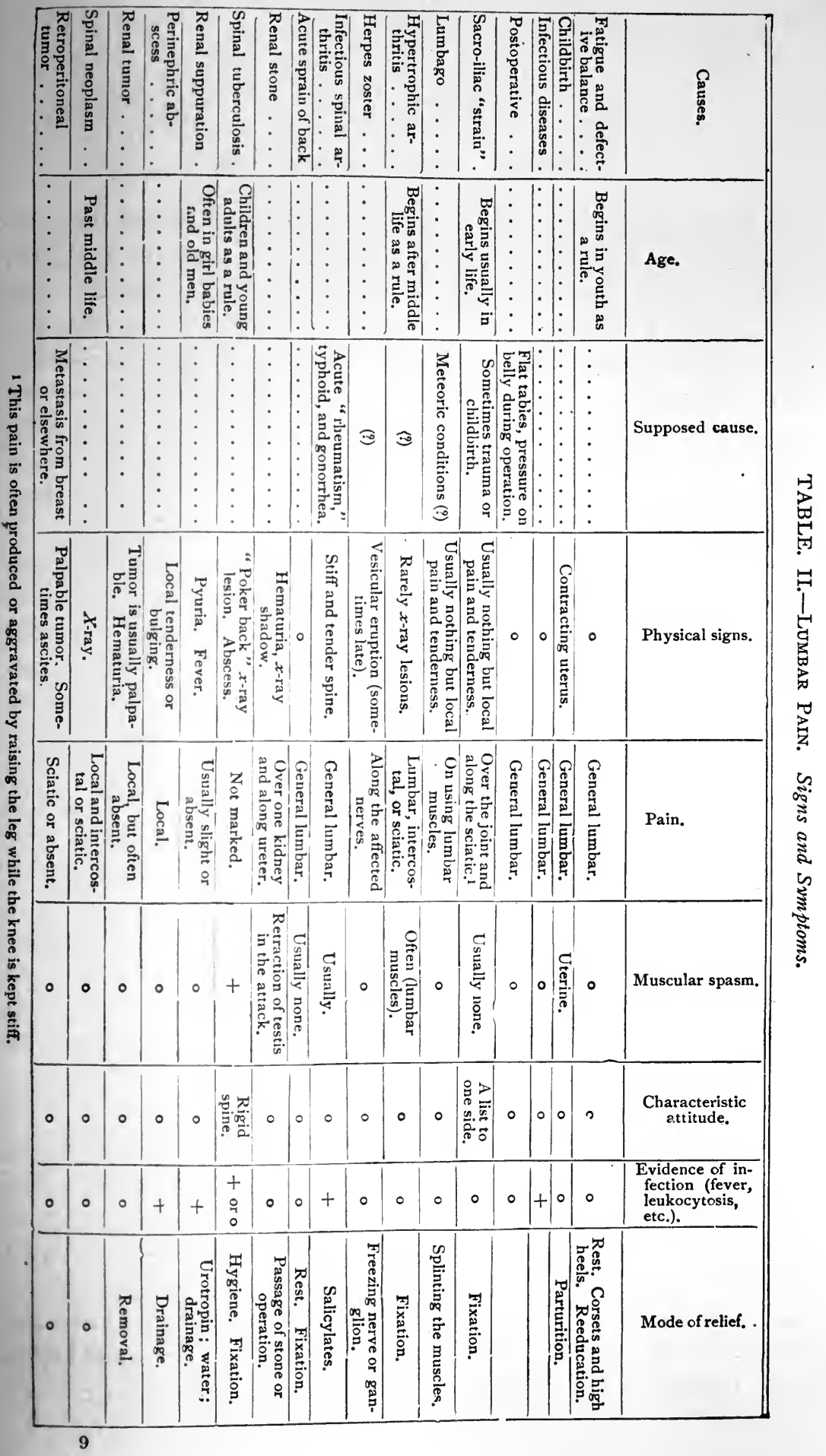




\section{CHAPTER IV}

\section{GENERAL ABDOMINAL PAIN}

THE diagnosis of the causes of abdominal pain is one of the most unsatisfactory, as well as one of the most important, in medicine; unsatisfactory, because our methods of examination are so inadequate. The chest, the cranium, and the extremities present far less difficulty, partly because their diseases are more accessible to direct inspection, partly because (in relation to thoracic disease) we have developed the technic of auscultation, percussion, and $x$-ray examination to a point quite out of the question in dealing with the belly. Our methods of investigating the abdomen are rough and primitive compared to those for the study of the chest.

Aside from the information obtained by study of the urine, blood, gastric and intestinal contents, practically all our knowledge depends upon palpation and a good history of the case. The latter is of crucial importance in this diagnosis of gall-stones, peptic ulcer, gastric cancer, colica mucosa, and many other common diseases. Palpation is materially assisted by immersing the patient in a bath as hot as he can bear. In some cases the procedure gives us almost as complete a relaxation of the belly walls as can be obtained under anesthesia. It should be employed in all dubious abdominal cases (questionable tumors, unexplained pains, etc.), especially if spasm of the muscles makes ordinary palpation difficult. ${ }^{1}$

Further reference will be made at the end of this chapter to another obstacle to correct abdominal diagnosis; namely, the tendency of local lesions to produce generalized pain, and of generalized lesions to produce localized pain. These obscure radiations often deceive even the expert.

\section{Case 50}

A clerk of thirty-nine, of good family history and habits, entered the hospital January 3I, I907. He had rheumatic fever eight years ago. Three and one-half years ago he was doubled up by an attack of pain and aching all over his bowels. He was seen in the Brockton Hospital by Dr. Daniel F. Jones, who said it was not appendicitis. Since this attack he has been well up to three weeks ago, when, after lifting, he had a stitch in his back, could not straighten up, and had to

${ }^{1}$ Women can be protected by making the water opaque with soapsuds. 


\section{Causes of General Abdominal Pain}

\section{CONSTIPATION \\ 2. DIARRHEA AND ENTERITIS}

3. APPENDICITIS

4. TYPHOID

5. GENERAL PERITONITIS

ING

$C L \cup D \mid N G$ STRANGULATED HERNIA

8. TUBERCULOUS PERITONITIS AND TABES $M$ E S N NTERICA

9. TABES (GASTRIC CRISIS)

10. Eヘ́TRA-
$\left.\begin{array}{l}\text { UTERINE } \\ \text { PREGNANCY }\end{array}\right\}$

$\left.\begin{array}{l}\text { 11. NEUROSES } \\ \text { (GASTRIC) }\end{array}\right\}$ ।

Among the rarer causes are many varieties of abdominal tumor (which usually produce local rather than generalized pain), malaria (especially in children), and spinal tuberculosis. 

stop work. The pain in his back was eased by lying down, and has not recurred. His bowels have since been very costive; hence after taking laxatives without result he took two injections, which caused cramps in the abdomen so severe that he "almost went crazy." The doctor came, gave him some "dope," and explained that he had appendicitis or gallstones. He is very nervous and sleeps poorly. He has sometimes a voracious appetite.

Physical examination showed good nutrition and slight pallor. Temperature, pulse, urine, and blood normal. The patient was a cribber. Physical examination was entirely negative, except for slight tenderness in both iliac fossæ. There was considerable mucus in the feces, binding the whole stool together into a single tenacious mass, like sputum. The patient was very much afraid of appendicitis, and complained frequently of terrible pain relieved by cooking soda. Guaiac test persistently negative.

Discussion.-Appendicitis is and should be our first thought in any case beginning with such symptoms, but the suspicion is shown to be groundless by the absence of elevation of the pulse, temperature, or leukocyte count, and by the fact that there is no tenderness or spasm in the appendix region.

Inflammation of the gall-bladder is ruled out for similar reasons.

Lead colic is consistent with all the symptoms here mentioned, but no such diagnosis can be made in the absence of all other evidence that lead is in the system (lead-line, stippled red corpuscles, occupation involving lead).

Pain relieved by cooking soda is often the result of duodenal ulcer, a disease always to be thought of in patients with acute abdominal symptoms. The history and the physical examination, however, offer no confirmatory evidence. No blood has apparently been discharged, either by the mouth or by the bowels, and we have not the usual history of long-standing digestive disturbance. Mucous colitis or colica mucosa is a diagnosis consistent with all the symptoms here described. The chronic constipation, the suggestion of a neurotic constitution, the occasional attacks of severe abdominal pain, and the presence of a large amount of mucus in the stools passed soon after such pain complete a typical picture of this disease.

Three groups of cases are often met in practice:

(I) Those with much nervousness, some pain, and some mucus. (2) Those of much pain, some nervousness, and some mucus. (3) Those of much mucus, some nervousness, and some pain.

In all three groups constipation is the underlying factor. Treat- 
ment must be directed to the relief of this and of the accompanying neurosis.

Outcome.-His points of tenderness varied from day to day, but at no time did he have tenderness in the right iliac fossa. After his bowels got to moving regularly, the pains disappeared and he gained 4 pounds inside of a week. Simultaneously his urinary excretion increased from 30 to 60 ounces. He left the hospital well on the eleventh of February. Diagnosis.-Neurosis; mucous colitis.

\section{Case 51}

A stenographer of twenty-four entered the hospital March 26, Igo8. Six years ago she had six attacks of cramp-like pain in the abdomen, each lasting six or eight hours, and relieved by morphin. The pain was not localized in any one place, but after an attack she had soreness in the left lower quadrant. Since that time she has had a more or less continuous "hard ache" in the left lower quadrant, never moving to any other place. She also has stiffness in both legs down as far as the knees. Her pain is not aggravated by motion. She has had no vomiting at any time. Working at the typewriter seems to cause cramp-like pains in the stomach. On account of these she was operated on in August, 1907, for appendicitis, and was told that "chronic appendicitis" was found and cured. The pains have continued as before. Her appetite and sleep are good, but she is markedly constipated. Last August she weighed 126 pounds; now she weighs II8. She often has pain on micturition, and occasionally difficulty in passing her urine.

On physical examination her pupils are widely dilated, equal, and react normally. The gums are normal. There is a short, rough, systolic murmur heard all over the precordia and in the left axilla. There is no enlargement of the heart nor accentuation of the pulmonic second sound. The abdomen is negative; likewise the blood, urine, temperature, pulse, and respiration.

Discussion.-The gist of this case seems to be: non-localized abdominal pain, with a negative physical examination in all essentials. Lead-poisoning is easily ruled out by the absence of changes in the blood or in the gums. Since the pupils react normally, tabes dorsalis seems very unlikely, though there is nothing said about the reflexes in the description as given above.

Dilatation of the pupils is common in a great variety of psychoneurotic states; nevertheless, it should always suggest the possibility of a cocain habit, especially if any heart trouble is complained of or comes 
to light on physical examination. In the present case there was no such evidence, and the habit was firmly denied.

In a considerable number of cases of pulmonary tuberculosis there is dilatation of one or both pupils, and the presence of this sign always leads me to examine the pulmonary apices with particular care. In this case such an examination was negative.

The controverted question of chronic appendicitis is raised afresh in - this case, but I suppose no one will maintain that an appendix can produce symptoms seven months after it has been removed. When the patient's symptoms persist unchanged after the removal of a socalled chronic appendix, it is generally agreed upon that in this case the appendix was not the cause of the symptoms. Indeed, this is one of the few points regarding chronic appendicitis on which physicians do very generally agree. Personally, I believe that in a considerable proportion of the cases operated upon as chronic appendicitis the appendix has nothing to do with the symptoms. The disappearance of symptoms following operation is not always a proof that the appendix was the offending member. The operation itself, with the postoperative rest, diet, physical and mental training, may well have been the cause of the relief.

In the present case, if we take account of the age and sex, the marked constipation, and the variety of "wild symptoms," such as painful micturition and stiffness of the legs, it seems more than likely that a general neurosis based on faulty habits and unfortunate environment is at the root of all the troubles. The domestic and industrial background should be looked into.

Outcome-On further investigation it appeared that insufficient food, hurry, worry, and sedentary occupation in a close office had much to do with her condition. All the reflexes were lively.

Diagnosis.-Bad hygiene.

\section{Case 52}

A Russian Jew, apparently without occupation, forty-eight years old, entered the hospital December 26, 1907. For seven weeks he has been having pain and "burning" in the center of the abdomen, not very severe, but constant and worse at night, though he sleeps well. It is worse, also, immediately after eating. His appetite is poor; he has taken nothing but a little milk of late. His bowels are very irregular, usually constipated. He does not vomit or cough.

On physical examination a regularly distributed, rose-colored macular exuption is found in various parts of his body, and there are marks of 
scratching on the upper arms. The chest shows nothing abnormal. Beneath the umbilicus, and extending out toward the right flank, is a smooth, rounded, cylindric mass, about three inches long, one and a half inches wide, freely movable, not hard or tender, feeling not unlike a kidney. Physical examination, including the blood, urine, temperature, pulse, and respiration, is otherwise entirely negative.

Discussion.-The important objective findings are the macular eruption and the cylindric mass in the abdomen; the former suggests syphilis, the latter, an abdominal tumor. Against syphilis, however, is the itching of the eruption, as evidenced by scratch-marks. There is also no evidence of a primary lesion, and the patient denies all knowledge of the disease.

Russian Jews in general, and unoccupied Russian Jews in particular, are very prone to neuroses and vague unexplained pains. It is striking how often they refer to these pains as "burning." "Es brennt mir das Herz," or "Es brennt mir überall," are very common complaints among them.

It is noteworthy also that this pain, though worse at night, does not prevent him from sleeping well.

Turning now to the abdominal mass, we note that it occupies the position in which a displaced kidney is often to be felt, especially in women. It seems, however, rather too short and too little sensitive. In view of his chronic constipation a mass of retained feces may well be the explanation. It seems reasonable, then, to explain his indigestion, eruption, and anorexia as the result of constipation, the latter in turn being the commonest of all manifestations of a general neurosis.

Outcome.-The patient was given an A. S. and B. pill, and the next morning the tumor had entirely disappeared. The following day it was again felt just at the level of the umbilicus, and considerably smaller than at entrance. Similar masses were then felt in the left iliac fossa. These also disappeared with free movements of the bowels. On December 3 Ist his abdomen was wholly negative, his eruption gone, and he had a wonderful appetite.

Diagnosis.-Constipation.

\section{Case 53}

A storekeeper of twenty-six, of good family history and habits, entered the hospital October 17, I907, stating that he had always had a weak stomach and had been troubled by pains in the chest and limbs off and on for the past ten years. Nevertheless he kept about and did his work in this condition until January, $190_{7}$, when he was confined to 
bed for fifteen days by an attack of pain "in the lungs and back." In March he was again confined to bed for two days with pain across the upper abdomen. In April and May, he felt poorly, but kept at work. In June he first noticed general abdominal tenderness and considerable enlargement, with painful micturition. He was then in bed for three weeks. After that he worked until August, when he was suddenly taken with violent headache, chills, sharp pain under the left breast, in the back and in the loins, with enlargement of the abdomen. He remained in bed thirty-five days, his temperature rising every afternoon to $102^{\circ} \mathrm{F}$. or $102.5^{\circ} \mathrm{F}$. He sweated profusely every night. Since then he has been poorly and his night-sweats have continued, but the size of his belly has diminished. During the past nine months he has lost II pounds in weight. He had at times a slight cough, with sputa rarely blood-specked. During the past few days there has been slight swelling of his legs.

Examination of the lungs and heart showed nothing abnormal. The abdomen was symmetrically distended; there was slight tympany in the flanks; the belly elsewhere was dull, tense, firm, slightly tender throughout. There was vague resistance at and about the umbilical region.

The blood and urine showed nothing abnormal, and the temperature, pulse, and respiration were not elevated during the seven weeks of his stay in the hospital. After the injection of 5 milligrams of tuberculin there was no rise of temperature, but he felt sick and weak, and his belly became much more tender.

Discussion.-Chronic abdominal pain and tenderness, with fever and sweating, form a clinical picture characteristic of very few diseases occurring in the male sex. Subphrenic abscess may produce such symptoms, but not without further physical signs, either in the abdomen, near the costal margin, or in the chest through displacement of the diaphragm. Perforative peritonitis could not be so chronic without either healing or killing.

Typhoid fever might produce such a pyrexia, and would account for most, if not all, of the abdominal symptoms, but during his stay in the hospital his abdominal symptoms continued despite the absence of all fever. Typhoid would not explain this.

Can he be suffering from chronic intestinal obstruction? The abdominal pain and distention suggest it, but his bowels have moved regularly throughout. There has been no vomiting, visible peristalsis, or other evidence of local lesion.

In my own experience there are only two diseases which present a 
clinical picture at all like this: (a) The psycho-neurotic state, and (b) abdominal tuberculosis. Since the former can be ruled out by the five weeks of daily fever, only one diagnosis seems reasonable.

Outcome.-On the second of November the spine of the fifth dorsal vertebræ was found to be very tender on pressure. This, in connection with the fact that sitting erect caused sharp pains in the chest and abdomen, suggested spinal tuberculosis, but an orthopedic consultant thought it more likely to be glandular tuberculosis in the abdomen.

Two other consultants thought the symptoms probably due to chronic appendicitis.

On the sixth of December the abdomen was opened, and the intestines found to be everywhere adherent to each other, to the omentum and to the abdominal wall. A large chain of glands was matted together in the appendix region, and many others were scattered about. There was no fluid. Microscopic examination of a piece excised showed tuberculosis.

Diagnosis.-Peritoneal tuberculosis.

\section{Case 54}

A housewife of forty-four who had been in the hospital in May, I905, and been operated on for inflamed tubes and ovaries (which were removed), chronic appendicitis, and sigmoid adhesions, entered the hospital February 20, 1908. Ever since May, 1905, the symptoms which then led to operation have persisted. She has been treated in the medical, surgical and orthopedic departments for out-patients, and has.worn flat-foot plates and abdominal supporters without relief. She has been unable to do any work on account of soreness in the lower abdomen, together with sharp attacks of pain starting in the back and passing around the sides to the center of the abdomen. These attacks come on when she steps or moves quickly, even when she turns over in bed at night. The pain is somewhat less sharp when her bowels are open, but she is exceedingly constipated. She complains of a "drawing, scratching" feeling in her bowels, as if they were trying to move, but could not. She has gained 20 pounds since the operation at which the tubes and ovaries were removed.

Physical examination shows extreme obesity, slight tenderness in the left lower quadrant of the abdomen, and nothing else, except slight soft edema over the shins.

Discussion.-In an analysis of "One Hundred Christian Science Cures," printed in McClure's Magazine for August, I908, I pointed out that patients who have had many doctors and many diagnoses are very 
apt to be successfully rounded up and cured by Christian Science, owing to the fact that in such cases no organic disease is present.

The history of the present patient and of the vicissitudes through which she passed suggest that she belongs in this group. Doubtless many of her symptoms represent only the discomforts inseparable from extreme obesity, especially when it is associated with constipation.

If this be true, the question may be asked how the edema of the leg is to be accounted for, but I think it is generally recognized that obesity is in itself sufficient to account for such a swelling, without supposing any insufficiency of the heart or kidneys.

Doubtless this patient's symptoms are due in part to the nervous instability often following the removal of the ovaries, but the constipation, the obesity, and the firmly acquired "doctor habit" are also important factors.

Such a diagnosis, though satisfactory enough from our point of view, may be of very little use to the patient, whose sufferings often go on unabated unless we can succeed in the almost superhuman task of changing most of her habits, mental and physical.

Outcome.-When the patient is alone in the ward, she does not seem to suffer, but her complaints are very numerous whenever a doctor or a nurse approaches. She complains that she is restless at night, but snores loudly. A tight abdominal binder and vibratory massage had relieved her considerably by the eleventh of March.

Diagnosis.-Postoperative neurosis.

\section{Case 55}

A school-boy of nine was first seen September 23, I907, with the statement that he had never been sick before, except that six months ago he had an attack similar to the present. Seven days ago he began to have general abdominal pain. Five days ago the pain was much aggravated, and seemed to be more troublesome on the right side of the abdomen. Four days ago he had a sore throat. His appetite has been good; his bowels regular. He has had no headache or nausea.

Examination September $23 \mathrm{~d}$ was negative, except for a temperature of $103.6^{\circ} \mathrm{F}$., and the leukocyte count of 22,000 , with a negative Widal reaction. There was at that time slight' tenderness at and above McBurney's point.

September 26th the fever still continued; physical examination was negative in all respects. The Widal reaction was negative; white cells, 8400 ; the course of the temperature was as shown in the accompanying chart. 
Discussion.-During the early days of my attendance on this case I could make no diagnosis. The fever, the leukocytosis, and the abdominal signs favored appendicitis, though the absence of all spasm and of all but very slight tenderness in the appendix region made this doubtful. The sore throat was practically gone before I saw him, and could not be hold responsible for the symptoms then present.

On the twenty-sixth, however, the clinical picture had quite changed. Continued fever with a low white count and a negative tuberculin reaction were now the essential features. This means, in all probability, either typhoid fever or some of the unknown infections unwisely called "febricula" or "grip." The latter possibilities were soon ruled out

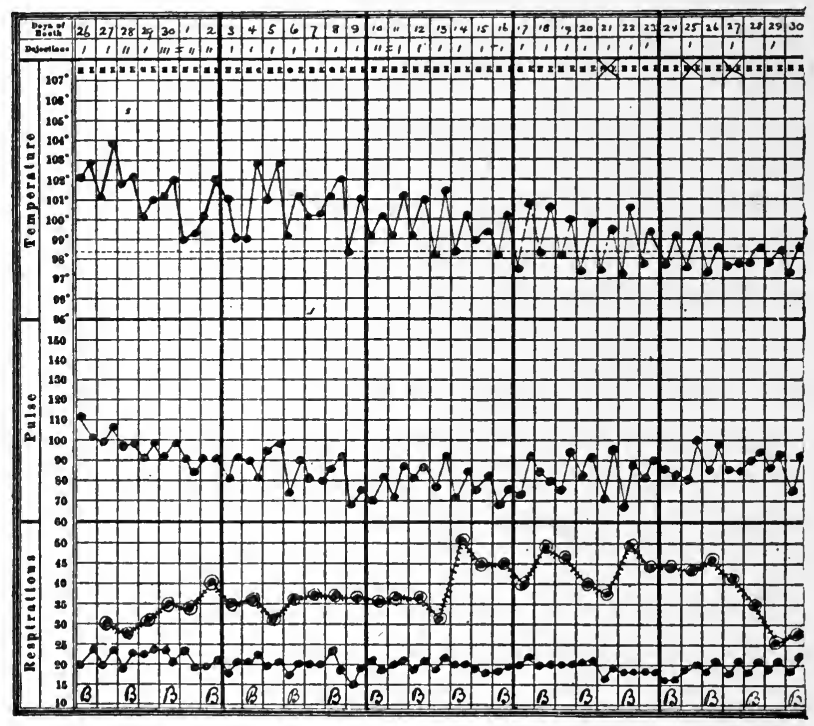

Fig. 25.-Chart of case 55 .

by the long duration of the fever. Under the hypothesis of typhoid fever it was left for us to explain the initial leukocytosis and the absence of the Widal reaction. No such explanation, however, was then forthcoming. The phenomena just referred to remained as examples of the wild, untamed, residual items so characteristic of any accurately described case of illness.

Outcome.-On the third of October the Widal reaction appeared. On the eighth he was given a drop of tuberculin in the left eye, without any subsequent reaction.

On November 9 th he was discharged well.

Diagnosis.-Typhoid. 


\section{Case 56}

A Portuguese housewife of thirty-two entered the hospital October 25, I907, with a negative family history and good habits. She had a miscarriage two years ago, and two other miscarriages since her marriage three years ago. She has one healthy child. For seven years she has been subject to general abdominal pain, not severe.

Three weeks ago she began to have dull, steady pain, starting in the left lower quadrant, whence paroxysms of more severe pain extended across the abdomen and up both sides of the chest to the neck. The appetite is poor; there is occasional nausea, but no vomiting. The bowels are constipated. For the past three weeks micturition has been somewhat painful.

Physical examination shows obesity. The chest is normal, the abdomen tympanitic in the upper part, dull in the lower part, where tenderness is so great that palpation is impossible. The blood-pressure is roo millimeters of mercury; the white count, I4,900. Urine, temperature, pulse, and respiration are normal. During the week of her stay in the hospital she complained of pain in every part of her body.

Discussion.- Syphilis is the first possibility that occurs to us in this case, in view of the frequent miscarriages. It is impossible, however, to incriminate any particular organ or to obtain any more definite history of the disease, which must remain in the background as a possibility incapable, at present, of further verification.

We naturally ask ourselves next whether the abdominal tenderness and painful micturition are not due to gonorrheal infection of the tubes and bladder. This possibility cannot be absolutely excluded, but in the absence of fever, leukocytosis, and urinary changes, it seems decidedly unlikely.

The very wide distribution and radiation of the pain, and its association with nausea, constipation, and anorexia, lead us to conclude that if any inflammatory lesion has existed in the pelvis it is now burnt out and exerting its effect chiefly through the nervous system.

Outcome.-A few nights before her discharge she was rolling and groaning with pain, but a subcutaneous injection of sterile water gave immediate relief. Vaginal tampons also improved her mental condition.

Obviously, the therapeutic test was here of considerable diagnostic value. I believe, however, that the same important information can be obtained through the investigation of the psychic state, and without any of the charlatanry which seems to me inherent in the methods here employed.

Diagnosis.-Neurosis. 


\section{Case 57}

A factory girl of twenty-six, a Canadian by birth, was first seen May 28, 1907. In April, 1906, she had a sickness similar to the present one. At that time medication gave no relief, but a six weeks' vacation in Roxbury entirely relieved her. Her home is in Blackstone, Mass.

In March, 1907, she began to have dull, colicky pain and tenderness in the lower part of her abdomen, constant, showing no relation to meals nor to the kind of food eaten, often keeping her awake at night, usually relieved by pressure. Frequently she has to sleep upon her belly all

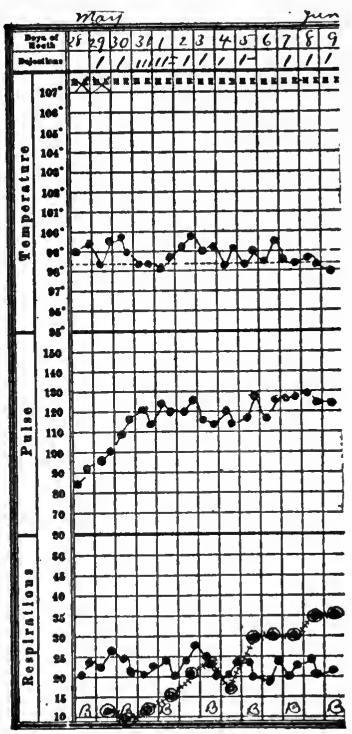

Fig. 26.-Chart of case 57 . night. With the pain she has constipation, and has noticed that she is getting pale.

On physical examination the abdomen was full, soft, tympanitic throughout, and showed no tenderness at any point. The chest was likewise normal. A blood-smear showed 60 per cent. hemoglobin, some achromia and many stippled cells.

The urine averaged about 25 ounces in twenty-four hours, and contained a trace of albumin, many hyaline and granular casts with an occasional cell adherent.

Discussion.-Although this case puzzled a number of physicians, there would have been no puzzle about it but for the neglect of a routine blood examination, for there is only one disease which often produces basophilic stippling of the red cells in the absence of marked anemia.

That disease is chronic lead-poisoning. Other diseases (e.g., diabetes) have been known to produce a similar blood-picture, but this is rare.

Lead-poisoning is a very common disease, but the failure to recognize it is, in my experience, still commoner. This is not because it is difficult of diagnosis, for the very reverse is the case, but because physicians so often fail to suspect its possibility and to examine patients for definite evidence of its presence. When once our attention is turned toward this diagnosis, we shall note, as in the present case, a very striking group of confirmatory signs. A chronic abdominal pain relieved by pressure would be likely to have more relation to meals if it were due to duodenal ulcer or to any cause other than lead. Association with constipation, pallor, and albuminuria should certainly make us search for a lead line 
on the gums-one of the signs which is most often forgotten in routine physical examination.

Outcome.-The gums showed a typical lead line. Her mother and sister have a similar but less severe trouble. The patient was given magnesium sulphate, an ounce every morning; iodid of potash, 5 grains three times a day, and an occasional dose of morphin and atropin was also needed. Turpentine stupes relieved her pain more or less.

By June 6th her appetite had improved, her cramps were gone, and her color had begun to return.

By the ninth of June she was ready to go home. It appeared that the whole family got their water from a well through a lead pipe 75 feet long.

The reader will note the striking rise in pulse-rate and its continued rapidity after the first week of treatment. The bradycardia of plumbism has often been recorded, but never, I think, satisfactorily explained.

Diagnosis.-Lead-poisoning.

\section{Case 58}

An Italian meat-cutter of thirty-five was seen June 20, I907. He stated that he had never been sick before until a year and a half ago (six months after his arrival in this country), when he began to notice that his abdomen was slightly larger than normal. He also noticed a beating in the pit of the stomach with vague abdominal pains, much loss of strength, occasional chills, and slight fever. At times his abdomen has seemed to be swollen, but of late it has been smaller. The pain is steady, dull, worse on dark, cold days. He is easily fatigued and has done no work for six months, but his weight has remained steady. He has had dizziness and buzzing in his head for three months, and for one month night-sweats. He eats and sleeps well, but his bowels move only with laxatives.

Physical examination of the chest was negative. The abdomen showed dulness in the left flank, which, however, did not shift with change of position. There was slight tenderness in the region of the umbilicus. Near this tender point a violent pulsation was felt, synchronous with the heart-beat. It was expansile in character, and during palpation a systolic thrill could be appreciated over it. A systolic murmur was audible at the same site. The tuberculin reaction (subcutaneous) was entirely negative. The urine averaged about 22 ounces in twentyfour hours, was free from albumin, but contained rare hyaline and granular casts. Blood examination was negative; white cells, 7000. Temperature, pulse, and respiration were normal. 
Discussion.-In the presence of chronic abdominal pain with swelling of the abdomen, weakness, night-sweats, and constipation, the possibility of tuberculous peritonitis should always be entertained, especially when the patient is an Italian recently settled in America. In the present case, however, the absence of fever at the present time, the negative tuberculin reaction, and the fact that no characteristic lesionseither of "dry" or of "wet" tuberculosis - can be detected in the abdomen make this diagnosis unlikely.

More plausible is the idea of aortic aneurysm, and this was, in fact, the diagnosis of the attending physician. Against it, however, were two very important facts: the pain was in the wrong place and there was no tumor. The pain of abdominal aneurysm is almost entirely in the back and legs. Further, the diagnosis of aneurysm is never well grounded unless we can feel a definite tumor with a beginning, middle, and end. However violent the pulsation we may find in the abdomen, - and I have seen it sufficient to shake the bed in which the patient lay,we have no right to make the diagnosis of aneurysm unless we have, in addition to the pulsation, a definite tumor or severe pain in the back. Expansile pulsation, thrill, and systolic murmur can be appreciated over any abdominal aorta which is superficial enough to be reached with the fingers and with the stethoscope.

It seems almost incredible that an illness so prostrating as this could be produced by the mere accident of having one's attention directed to the normal, though lively, pulsation of one of one's own bloodvessels; but such was really the case here. Dynamic aorta-that is, a somewhat unusual liveliness in the pulsation of a perfectly normal blood-vessel in a person of neurotic constitution-is very frequently mistaken for abdominal aneurysm. Indeed, I should say that five out of every six cases in which I have known the diagnosis of abdominal aneurysm to be made have turned out to be nothing but dynamic aorta. Nothing but the experience of following such a case to complete and lasting recovery, as the result of the policy of disregarding all the symptoms and turning the attention in other directions, can convince the patient and his physician of the facts just quoted.

In true abdominal aneurysm the tumor is seldom in the median line. It is much larger and more globular, and pulsates less violently than the dynamic aorta. One of the most astonishing things about the latter is that it often appears just beneath the skin of the abdominal wall, seemingly separated from our finger-tips only by the thickness of a piece of blotting-paper. As we recall our dissecting-room experiences, it does not seem possible that the aorta can lie so close to the abdominal 
wall. Doubtless this is due to a somewhat atypical curve of the spinal column.

There can be no doubt, I think, that three factors enter into the production of the neurosis known as dynamic aorta:

(I) An unusually superficial position of the abdominal aorta.

(2) A sensitive and impressionable temperament, such as shows itself in rapid bodily motion, quick excitable speech, lively knee-jerks and easily excited heart action.

(3) The abnormal concentration of attention upon the pulsation. This latter condition is favored by the physician's obvious interest and concern, as expressed in his careful and repeated examinations of the part, his overclouded countenance, and sometimes his unguarded utterances. If by any mischance the patient begins to suspect that he has an aneurysm, he is pretty sure to learn from a dictionary or otherwise what the disease really means. Thereafter he passes his days and nights feeling very much as though he had inside of him a dynamite bomb which might explode at any minute. This, of course, reacts upon his mental condition, and makes him watch himself all the more carefully, thereby increasing the pulsation and soon leading to the development of pain; but it should be reiterated that the pain is in the spot to which his attention has been directed, and not in the place where it would be were aneurysm really present.

I have dwelt at considerable length upon the nature of this trouble and the means of its recognition, because it is by no means uncommon, is prone to lead to a great deal of unnecessary misery when mistaken for aneurysm, and because it is not treated at any length in most text-books.

Outcome.-Gas in the abdomen and the perception of the pulsating artery were apparently the cause of his symptoms. This was explained to him, and by June $27^{\text {th }}$ he was free from complaint. He returned to work after ten days more and has since (IgIO) remained well.

Diagnosis.-Dynamic aorta.

\section{Case 59}

A printer of twenty-seven entered the hospital August 19, I907. His family history and habits are good. He states that he had "renal colic" last May for two days, and has since then been well. Two weeks ago his bowels began to be rather loose. His appetite has remained good and he has slept well. Beginning this morning he has had severe abdominal cramps, his bowels have moved six times, and he has vomited six times. The pain is felt throughout the abdomen.

Physical examination shows two glands the size of marbles in the 10 
right axilla. No other glands seem to be enlarged. There is a soft systolic murmur at the heart's apex. The chest is otherwise negative. The abdomen is slightly retracted. There is general muscular rigidity, especially in the epigastrium, and in the right side near the navel. On percussion the belly is tympanitic, except in the left flank-no definite mass or tenderness found. Temperature at entrance $99.8^{\circ} \mathrm{F}$; white count, I6,600, with 96 per cent. of polynuclear cells. The next day the temperature and the white count were normal. The diarrhea had ceased.

Discussion.-What further evidence should be searched for in this case? In any printer who complains of abdominal pain we should at once look for a lead line on the gums and search for basophilic granulations in the stained blood-smear. Both these lesions were absent in this case. The presence of diarrhea is also very uncommon in lead-poisoning.

An $x$-ray examination is indicated in view of the patient's statement that he had renal colic a few months before. There is nothing, however, pointing to any such disease in a physical examination.

Perforative peritonitis would account for the pain, vomiting, fever, leukocytosis, spasm, and tenderness, but the presence of a diarrhea with good appetite and sleep makes this very unlikely, especially as there is no local point of maximum pain and tenderness.

But for the definite evidence afforded by the blood examination, it would be necessary to consider an acute lymphoid leukemia. I have seen leukemia presenting the symptoms here described with no more striking glandular enlargement. The blood examination, however, was distinctive.

Why should it not be a simple gastro-enteritis, especially in view of the time of year at which the symptoms occurred? Severe abdominal cramps, a general muscular rigidity in the abdomen, transitory fever and leukocytosis are all quite consistent with that diagnosis; there seems to be nothing of importance against it.

Outcome. $-X$-ray showed no evidence of renal calculus, after rest in bed and regulated diet, ten half-grain doses of calomel, and an ounce of magnesium sulphate, the patient was discharged well on the twentysecond.

Diagnosis.-Acute gastro-enteritis.

\section{Case 60}

A teamster of forty-four, with a negative family history, was first seen August 24, 1907 . 
For many years. he has been in the habit of taking from twelve to twenty glasses of beer and three to five glasses of whisky daily. $\mathrm{He}$ chews a five-cent plug of tobacco a day, and smokes three or four pipefuls besides. He has always been very well and strong until five months ago, when he began to have dull pain in the abdomen, not definitely localized, but more marked in the lower half. This was accompanied by distress and flatulence after meals, and frequent vomiting immediately after the taking of food. The vomitus is bitter, yellow-green, never bloody. His appetite is poor, his bowels constipated, and he has been short of breath for the past four weeks. For the past two weeks he has had to pass his urine twice each night. Two years ago he weighed I55 pounds; to-day he weighs I2I.

On physical examination the skin is dry and satiny. There is a marked alcoholic odor on the breath. The arteries are all palpable, and there is a lateral pulsation in the brachials. The chest and abdomen showed nothing abnormal.

Examination of the blood showed red cells, 2,030,000; white cells, 7200; hemoglobin, 25 per cent. The stained specimen showed achromia, slight poikilocytosis, many off-colored cells, no nucleated red cells.

The urine was negative. After a test-meal the stomach-contents showed no free hydrochloric acid. The gastric capacity was 23 ounces. His stools were brownish-black, with a well-marked reaction to guaiac. Rectal examination was negative. The prostate was not enlarged.

Discussion.-The excesses in alcohol and tobacco above described would naturally lead one to suspect cirrhosis of the liver. The longcontinued gastric symptoms, as well as all the minor complaints, could be thus explained. The guaiac reaction in the feces might be the result of blood poured out from dilated veins in the esophagus or stomach. Against this supposition, however, is the extreme degree of anemia, without any history of severe hemorrhage. Even if the blood were discharged by rectum, the patient would probably be made aware by faintness, weakness, and thirst, of the loss of an amount of blood sufficient to explain the present anemia. It is unusual, furthermore, that a cirrhosis disables the patient and produces such marked symptoms as are here present, without manifesting itself by any change in the size of the liver or by the accumulation of ascites.

Whenever a patient past forty years of age, and previously free from stomach trouble, begins to have any sort of gastric discomfort, severe or mild, gastric carcinoma should be considered. This diagnosis would explain all the symptoms in this case, including the anemia. It is remarkable, however, that there should be no more definite evidence 
of gastric stasis, no food in the vomitus or in the stomach-washings. If cancer is present, it is probably not at the pylorus-its usual seat.

So extreme a degree of anemia, associated with gastric symptoms and achylia gastrica, brings the thought of pernicious anemia to mind. The blood, however, is very uncharacteristic, and is, indeed, typical of secondary anemia.

On the whole, gastric cancer is the most probable diagnosis.

Outcome.-On the morning of the twenty-seventh of August the right middle finger was blanched and cold up to the knuckle-joint. Examination of the patient in the warm bath showed a sharp edge in the region of the liver, descending with respiration. (See Fig. 27.)

On the third of September the abdomen was opened, and an inoperable cancer of the anterior stomach-wall found. The mass thought to be liver before operation proved to be part of the gastric tumor.

Diagnosis.-Cancer of the stomach.

\section{Case 61}

An Italian shoemaker of thirty-two has complained for a year of general bellyache with diarrhea, at times bloody. Much intestinal noise. Has lost 28 pounds in two months. For the past week he has been costive.

Examination was negative, excepting for a palpable spleen and a hemoglobin of 65 per cent. During his fortnight under observation (September I-I4, I904) he had no fever, no diarrhea, and gained eight pounds. He had slight abdominal pain, especially at night. There was slight tenderness in both iliac fossæ. Colitis, possibly tuberculous, was the diagnosis in the out-patient department and in the wards.

Next spring (May 22, I905) he was again at the hospital. His pain, he said, had never ceased. Constipation has been obstinate and is getting worse. The rumbling noises are still loud. $\mathrm{He}$ has lost I4 pounds since his previous entry.

Slightly above the region of the cecum is a firm, regular mass, about the size and shape of the kidney, freely movable in all directions, distinctly tender on pressure. No reaction to tuberculin (two large doses). Stools foul, watery, no blood, no tubercle bacilli, some mucus.

Discussion.- In view of the information which came to light when this patient entered the hospital for the second time, there are oniy two diseases to be considered as at all likely to produce these symptoms, viz., cancer of the cecum and pericecal tuberculosis. The latter is made uniikely by the negative reaction to tuberculin.

The interesting question remains: could the cancer which now 


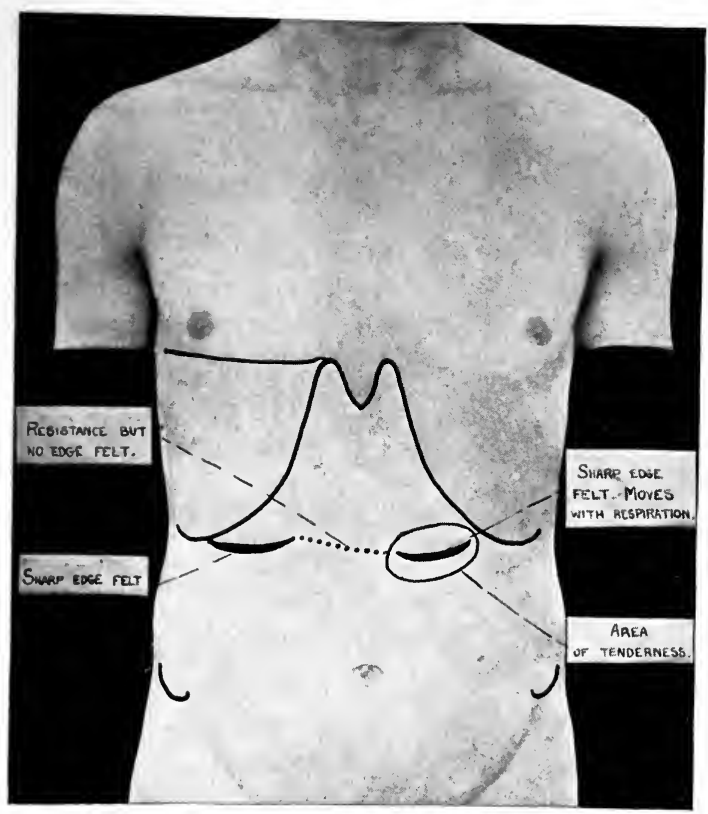

Fig 27.--Diagram of the findings in Case 6o. Chief complaints, dull abdominal pain, vomiting, and flatulence. 

shows itself at the cecum have been suspected in 1904? Certainly no positive diagnosis of this disease could have been made, but it seems to me that whenever we have the history of very loud and marked intestinal noise, accompanied by pain experienced at short intervals throughout a. year's time, we ought to suspect that some sort of disease has caused intestinal stricture with muscular hypertrophy of the gut behind it. It is true that in many cases of diarrhea from colitis intestinal noise is heard, but it is especially in the acute varieties that we meet with this symptom. In cases lasting a year it is much more uncommon. Again, a good many women are troubled by intestinal noise at the time of the menstrual period, or whenever they are especially nervous, but the process is never so continuous as in the present case.

Except for this symptom, the diagnosis of chronic colitis was certainly justifiable in I904. The case, however, reënforces in a striking way the well-known rule that in all long-standing diarrheas intestinal obstruction should be suspected, especially, but not exclusively, in elderly people. It is, of course, a very familiar fact that many cases of cancer of the sigmoid begin with diarrhea.

Despite such warnings as are given us by a case like this, the diagnosis of intestinal cancer is often entirely impossible with our present methods of investigation. There is good reason to believe that it is often present and quite latent for years. The symptoms we see are merely terminal. For example, a patient whom I saw in I9o6 for pain high up in the rectum, accompanied by discharges of blood and mucus, had been troubled by severe periodic pains with considerable constipation, referred to appendicitis, for at least fifteen years. At the autopsy in June, I907, cancer of the sigmoid was found, but no appendicitis. In another group of cases the patient is aware of the presence of tumor in the abdomen for three or four years, without any pain or disturbance of the bowels, yet the tumor turns out on exploration to be cancerous. Not infrequently pain may be referred to the pit of the stomach, and so closely associated with ordinary gastric symptoms that all our attention is drawn in that direction.

Outcome.-Dr. Conant diagnosed tuberculous colitis and advised operation.

A growth the size of an orange was found in the cecum (adenocarcinoma by microscopic examination) and excised. Discharged well June 23 d.

A year later (June 5, I906) he returned. The operation had given relief for months, and he had gained 20 pounds, but of late pain and bloody stools have returned, this time in the left lower quadrant, where 
there is a mass I by $2 \frac{1}{2}$ inches, and tenderness. Operation showed inoperable cancer of the sigmoid. Cecal region normal. Inguinal colostomy. Discharged July 7 , r9o6, to out-patient department.

Diagnosis.-Recurrent intestinal cancer.

\section{Case 62}

A boy of eleven was seen September 28, 1903. Since his third year, when he had malaria, he has had fleeting pains in his arms and legs, especially at night. The feet often show toe-drop.

For three months he has been troubled with attacks of bellyache, accompanied often by chill and vomiting and by an increase in the troubles in his arms and legs.

Twice he has had tonic-clonic convulsions.

Discussion.-When a child has a stomachache in summer, it would be folly to conclude that malaria is the cause; but it is equal folly not to suspect that malaria may be the cause. For some unknown reason the malarial attacks of children and of young adults are much more likely to be atypical than those of older persons.

(a) Malaria often exists in children without producing any symptoms at all, and is demonstrated only by blood examination.

(b) In many cases it produces only a recurrent headache and listlessness, due, in fact, to a rise in temperature every twenty-four or fortyeight hours, without any chill ("dumb ague").

(c) Vomiting recurring at regular intervals, daily or every other day, has been the only suggestion of malaria in some of my cases until the blood was examined.

(d) An intractable diarrhea is sometimes associated with a malarial infection of the blood, and promptly cured by the administration of quinin.

(e) Abdominal pain of the type exemplified in this case is perhaps the most common of the atypical manifestations of malaria. In some cases it is localized in the right iliac fossa. In one week's service at the Massachusetts General Hospital three patients were sent in to be operated upon for supposed appendicitis. All of them had malaria, and were promptly cured by quinin. These have been referred to by Dr. James M. Jackson, in his article published in the Boston Medical and Surgical Journal, June 26, I902. I have already referred, in the discussion of a previous case (see p. I23), to a case of malaria beginning like pneumonia with violent thoracic pain.

( $f$ ) In adults we not infrequently see cases of malaria with predominating cerebral symptoms, such as acute mania or coma. 
Now if malaria can assume such a bewildering variety of clinical aspects, what is to guide us toward correct diagnosis. I should answer that in practically all these atypical forms a thorough blood examination should be suggested by the presence of an irregular fever and the low: leukocyte count. Enlargement of the spleen and the firm, painless edge which the organ presents to the palpating finger are generally to be recognized in these cases, and should also put us upon our guard against malaria. The therapeutic test is valuable, but should not be abused by continuing to pour quinin into the patient at the rate of 20 to 40 grains a day for a week or more. This is not a therapeutic test: it is a stupid blunder. Two or three days is enough to settle the matter in 999 cases out of rooo, and in the remaining case no further information is obtained by prolonging the administration of quinin.

Outcome.-The blood was found to be swarming with tertian parasites. Wrist-drop and toe-drop. Knee-jerks absent.

Diagnosis.-Tertian malaria.

\section{Case 63}

A woman of fifty, a lawyer's clerk, entered the hospital January 2, I906, stating that she had had many attacks similar to the present one, but had always been able to work. Two days ago she felt some abdominal discomfort in the afternoon. Early yesterday morning she awoke with a sharp, steady pain, especially in the right side of the abdomen, but not definitely localized. This was accompanied by distention and obstinate constipation. Last night the pain was felt in the left side. She has vomited several times, and has slept poorly on account of pain. (For temperature, see chart.)

The abdomen is distended, tympanitic, and generally tender; white cells, 4600; urine, Io29; a very slight trace of albumin; many fine, granular casts. Physical examination was otherwise negative. A glycerin enema and hot-water bottle to the abdomen gave her some relief, but on the morning of the fourth, the temperature continuing to rise, though the white cells were still only 4000 , she was operated upon.

Discussion.-A definite diagnosis was impossible here, but the general appearance of the patient made it

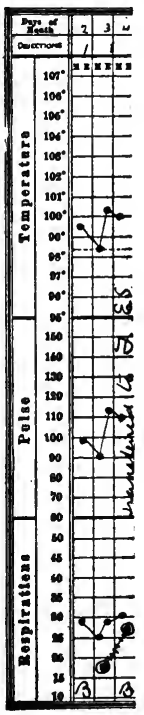

Fig. 28.-Chart of case 63 . clear that she was very ill, while the course of the symptoms went on progressively from bad to worse. It was for these reasons that the 
abdomen was opened, although without any clear notion of what would be found.

Outcome.-Chronic ulcerative enteritis and colitis, with numerous strictures and diverticula, were found. One of these diverticula, which contained an orange-seed, had perforated and gave rise to general peritonitis, from which she died. At autopsy the enteritis seemed to be due to tuberculosis or syphilis.

This case is introduced chiefly to show how short and how slight may be the symptoms associated with a fatal general peritonitis. The pain was never sharp during the time when she was under observation, and there was no muscular spasm. The subnormal leukocyte count was doubtless due to the virulence of the process, but previous to the operation it was impossible to be sure of this.

It is probable that this patient had previously had many slow and partial perforations of the gut, which were protected by adhesions so that no general peritonitis resulted. In a normal intestine an orange-seed does no harm; only in the presence of severe ulceration and thinning of the intestinal wall, such as was present here, could such a foreign body be dangerous.

Diagnosis.-Perforative colitis ar.d general peritonitis.

\section{Case 64}

A woman of fifty-four has had for a year cramps after meals in various parts of the abdomen. The pain has not been severe, but has led her to cut out from her diet one food after another in search of relief, until now she eats very little, and has lost 45 pounds during the year.

Eight months ago the bowels began to move more and more often, and now act eight to ten times a day, with blood and pain on defecation.

On examination the internal viscera, the urine, the temperature, pulse, and respiration are normal. Digital examination of the rectum shows a relaxed external sphincter, with ballooning above it. The red cells are I,792,000; hemoglobin, 25 per cent.; leukocytes, I2,400, 86 per cent. of which are polynuclear.

Discussion.-Chronic colitis is so common in elderly persons that it is naturally our first thought in this case. It is especially frequent when there is a slight degree of interstitial change in the kidneys, as evidenced by high blood-pressure, with or without characteristic urinary changes. This possibility certainly cannot be excluded by any of the facts so far given.

Pernicious anemia produces a reduction in red corpuscles such as is here present, and is often associated with a chronic diarrhea, but in 
the finer points revealed by blood examination the picture is one of secondary anemia.

Any case presenting these symptoms demands a very careful examination of the rectum and lower sigmoid by means of a speculum, since cancer of this part of the gut is a frequent cause of all the symptoms here presented.

Outcome.-Through a rectal speculum with an adequate light a large cauliflower mass could be seen nearly occluding the upper part of the rectum. From it there was a foul serosanguineous discharge. An excised bit proved to be cancer.

Diagnosis.-Cancer of the rectum.

\section{Case 65}

An active, muscular young man of twenty-six, a machinist by trade, had always been perfectly well until three years ago, when he had an attack of acute appendicitis for which an operation was performed. A five-inch incision was necessary; the wound was drained for a long time, and later a large ventral hernia developed. Theręafter he seemed perfectly well until five days ago, when he had an attack of acute generalized abdominal pain lasting for about eighteen hours, and accompanied by constipation. He was then perfectly well for the two succeeding days, when a second attack of pain came on, accompanied by nausea and vomiting. This had persisted nearly twenty-four hours when he was seen in consultation.

When examined, the head, chest, and extremities showed nothing remarkable. The abdomen was slightly tender throughout, and there was a moderate amount of spasm not localized. Attacks of colicky pain, now here, now there, but for the most part in the umbilical region, recurred every few minutes. There was no bulging at the seat of the scar, and no palpable mass anywhere. There was slight dulness in the flanks, which shifted with change of position. The temperature was normal; the pulse IIo and of low tension. The face was drawn and showed evidences of severe pain; indeed, the patient looked exceedingly ill. The blood and urine showed nothing abnormal. There was no lead-line on the gums. An enema brought away a small movement, normal in character.

Discussion.-Intestinal obstruction and general peritonitis are the most likely hypotheses. There is nothing in his occupation nor in the examination of the blood and the gums to make lead-poisoning at all probable. If perforative peritonitis were present, there would be apt to be more tenderness and some fever. Yet I have several times seen 
acute virulent peritonitis demonstrated without any fever or tenderness. We have no evidence pointing to any source for peritonitis, and nothing to connect the symptoms with the stomach or the gall-bladder, while the appendix has already been excluded surgically. What can we argue from the presence of shifting dulness in the flanks? In the absence of diarrhea there is every reason to believe that this sign indicates fluid free in the peritoneal cavity, but this is fully as common in cases of intestinal obstruction as in general perforative peritonitis.

Many of the symptoms here present could be explained by simple constipation. Indeed, on paper this seems quite a reasonable diagnosis. In the living patient, however, this could be quite readily excluded by the obvious severity of the patient's sufferings and of the prostration accompanying them. By the same tokens it was easily possible to rule out those multiform neuroses which are, on the whole, the commonest cause of general abdominal pain.

By, exclusion, intestinal obstruction seems the most probable diagnosis.

Outcome. - The abdomen was opened at once, and the mesentery of the lower ilium was found to be tightly twisted on itself, the twist leading to a group of intestinal coils which were distended and dark purple in color. There were many adhesions near the site of the appendix, but apparently these were not responsible in any direct way for the strangulation. There was about a quart of bloody serum free in the abdomen.

The intestines were untwisted and returned to their proper position, the wound sewed up, and the patient made an uneventful recovery,

This case illustrates the truth of the rule that in young people most cases of intestinal obstruction are connected in some way with the results of a previous peritonitis or operation, while in old people the great majority of cases are due to cancer. For some unknown reason twists occur much more frequently in those whose peritoneums have been damaged by a previous operation or inflammation, even when no constricting band of adhesions can be found.

Diagnosis.-Obstruction of the intestine; volvulus. 


\begin{tabular}{|c|c|c|c|c|c|c|c|c|}
\hline 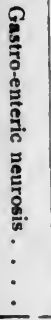 & 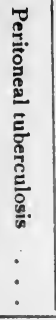 & $\begin{array}{l}\dot{0} \\
\bar{\omega} \\
\phi \\
\vdots \\
\vdots \\
\vdots \\
\vdots \\
\vdots\end{array}$ & 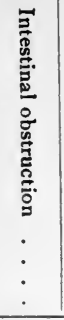 & 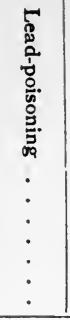 & 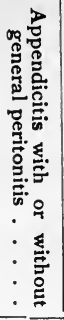 & 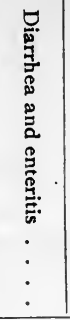 & 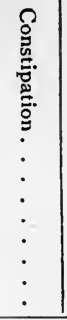 & 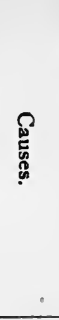 \\
\hline$\vdots:$ & $\begin{array}{l}: \\
\vdots \\
\vdots\end{array}$ & 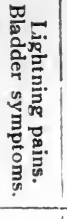 & 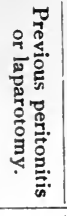 & 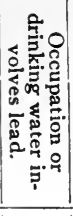 & 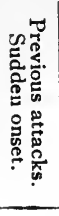 & 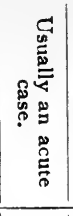 & 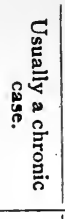 & 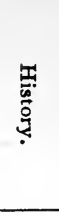 \\
\hline$\vdots$ & $\begin{array}{l}\vdots \\
\vdots\end{array}$ & . & 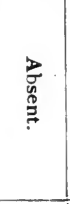 & 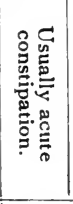 & 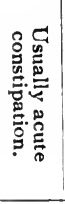 & 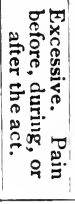 & 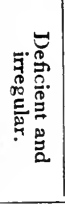 & 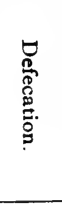 \\
\hline 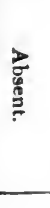 & 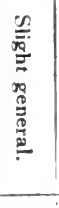 & 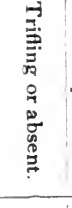 & 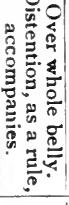 & 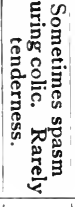 & 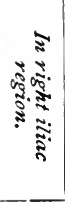 & 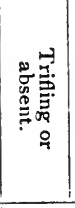 & 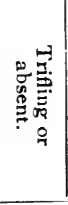 & 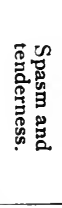 \\
\hline$\circ$ & 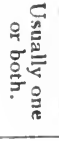 & $\circ$ & $\stackrel{0}{\stackrel{0}{?}}$ & 0 & 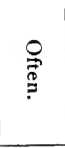 & 0 & 0 & 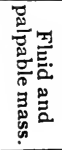 \\
\hline 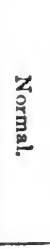 & 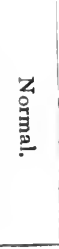 & 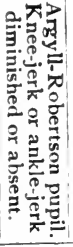 & 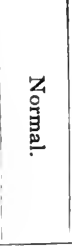 & 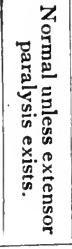 & 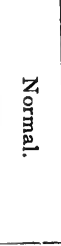 & 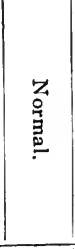 & 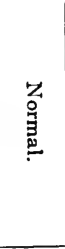 & 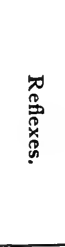 \\
\hline$\vdots$ & 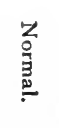 & 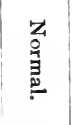 & 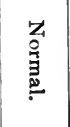 & 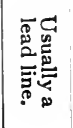 & 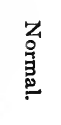 & 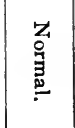 & 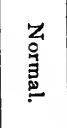 & 尽 \\
\hline 0 & 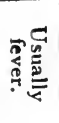 & 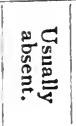 & + & 0 & + & 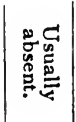 & $\circ$ & 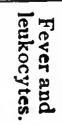 \\
\hline 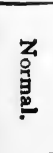 & 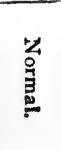 & 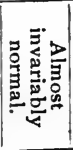 & 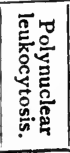 & 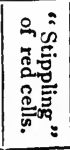 & 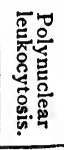 & 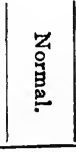 & 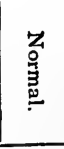 & \\
\hline
\end{tabular}




\section{CHAPTER V \\ EPIGASTRIC PAIN}

Next to headache and backache, epigastric pain, or "stomach ache," is probably the commonest of all complaints. When we have assured ourselves by careful questioning that we are dealing with genuine pain and not merely with a sense of pressure, burning, or vague discomfort, we may be guided in the further investigation of the case by the following considerations:

(a) Pyloric spasm is probably the commonest of all causes for epigastric pain. This spasm may be brought about not only by disease within the stomach-such as ulcer or hyperchlorhydriabut also and more often by duodenal ulcer, acute appendicitis and gall-bladder disease. Most characteristic of pyloric spasm is its relief by the passage of gas upward through the esophagus or downward through the intestine. Just what the connection is between relief of spasm and the passage of gas I do not know.

(b) Intestinal hyperperistalsis, or intestinal colic, associated with diarrhea or constipation, is probably the next commonest cause for epigastric pain. Other causes are listed in the table on page $\mathrm{r}_{57}$.

As evidence bearing on the frequency of peptic ulcer as a cause of epigastric pain, the following figures are of some interest. In 3000 consecutive autopsies performed at the Massachusetts General Hospital, peptic ulcer of the stomach was the cause of death in 36 cases, peptic ulcer of the duodenum in 29 cases, making a total of 65 . Peptic ulcer of the stomach was also found as a minor item, not the cause of death, in 29 other autopsies, and duodenal ulcer in $\mathrm{r}_{7}$ other autopsies, making a total of 46 cases in which peptic ulcer was a minor feature. The total incidence of peptic ulcer of the stomach or duodenum in 3000 autopsies was 111 cases of 3000 , or 3.7 per cent.

(c) Cholecystitis and cholangitis have recently attracted much attention as possible causes of epigastric pain and chronic dyspepsia. These lesions are now displacing the popular favorite of the last decade, namely, "chronic appendicitis," as an operable cause of dyspepsia. As to the groundlessness of the belief that chronic appendicitis can be diagnosed as a cause of dyspepsia, I shall have 


\title{
Causes of Epigastric Pain
}

\author{
CONSTIPATION \\ DIARRHEA AND ENTERITIS
}

ACUTE INDIGESTION

APPENDICITIS

NEUROSES

LEAD-POISONING

INTESTINAL OBSTRUCTION

\section{TABES}

TABES PERITONITIS AND TABES MESENTERICA;

NO ACCURATE STATISTICS AVAILABLE.

ONLY THE FIRST THREE ARE COMMON AS CAUSES OF EPIGASTRIC PAIN.

1. GASTROHEPATIC CONGESTION DUE TO CIRRHOSIS OR CARDIAC DISEASE

2. APPENDICITIS 350

3. PEPTIC ULCER 347

4. GALL-STONES 329

5. HYPERCHLOR326 HYDRIA $\left.{ }^{1}\right\}$ 898

6. GASTRIC CANCER 133

7. PERICARDITIS

8. GASTRIC NEUROSIS 72

9. PANCREATITIS

10. PYLORIC ADHESIONS

11. ANGINA ABDOMI- $\}$ I

1 Many of these cases may actually be cases of peptic ulcer. Only operation or autopsy can decide. 

something to say later on. (See page I95.) Here I may merely indicate my skepticism as to the supposed frequency of gall-bladder disease as a cause of dyspepsia. In the last 60 cases of gall-bladder disease operated on at the Massachusetts General Hospital during the year I9I6, only II had any distress or dyspepsia between the sharp attacks of colic on which the diagnosis rested.

\section{Case 66}

An Italian laborer of forty entered the hospital November 21, I906. For sixteen days he had been having pain at the "pit of the stomach." The pain came on rather suddenly, and had since been dull and steady, at times interfering with sleep. He has not been able to work since the onset of the pain. It has no relation to food or posture. There are no gastric or urinary symptoms, no jaundice, no constipation, and no loss of weight. The patient denies venereal disease, and has never, to his knowledge, been sick before.

Physical examination, except in so far as relates to the abdomen, was negative: There a hard, apparently nodular mass was felt just below the ensiform cartilage and a little to the left of the median line. It was not tender, and descended readily with inspiration. The liver dulness extended as high as the fourth interspace, but the edge was not felt. The blood and urine showed nothing abnormal. The capacity of the stomach was enlarged to 72 ounces, and the lower border reached $4 \frac{1}{2}$ inches below the navel; the upper border, I inch above it. Free $\mathrm{HCl}$ was absent. The total acidity was 0.12 per cent. There were no organic acids, no blood, no fasting contents. There was no reaction to guaiac in the stools. The patient's temperature ranged, for the most part, about $99^{\circ} \mathrm{F}$., often reaching $100^{\circ} \mathrm{F}$. and occasionally ror ${ }^{\circ} \mathrm{F}$. His pulse and respiration were within normal limits. At times there was considerable tenderness orer the epigastric mass.

Discussion.--Tuberculous peritonitis is remarkably common in the newly arrived Italian immigrant. The presence of fever and of abdominal pain without fulminating or alarming symptoms is quite suggestive of tuberculous peritonitis, but we have no evidence either of free fluid in the abdomen or of the tenderness, spasticity, and localized masses which are necessary for the diagnosis of this disease when fluid is absent.

Some of the gastric signs in this case are quite consistent with gastric cancer, but against this are the sudden onset, the absence of emaciation, stasis, or blood. 
The induration about a partially perforated gastric ulcer sometimes produces a mass in the left hypochondrium similar to that here described. But as the symptoms seem to have no relation to food, and as there is no tenderness or spasm about the indurated point, there seems to be no good reason to consider this possibility seriously.

The tumor is on the wrong side for gall-bladder disease, and the absence of colic and jaundice makes it unnecessary to look further in this direction.

Malignant disease of the liver might cause such a fever as is here described, and occasionally arises without any previous or coincident growth in the stomach. This possibility cannot be excluded, especially as the liver seems to be enlarged upward; but the left hypochondrium is a very unusual place for a hepatic neoplasm.

On the other hand, the position of the mass here described corresponds with the point at which hepatic syphilis most often shows itself. This diagnosis would explain the fever, and is more consistent with the history and with the good nutrition of the patient. In the absence of any further evidence, however, one could not do more than suspect syphilis. Treatment by mercury and potassium iodid and the use of the Wassermann test are indicated as a means to a more certain diagnosis.

Outcome.-On December 6th the abdomen was opened, and the liver was found to be adherent to the abdominal wall by fine, soft adhesions. The mass felt through the abdominal wall was found to consist of an irregular, boggy, yellowish-white elevation, from which a considerable amount of pus-like material was removed by cautery.

Microscopic examination showed it to be a gumma. The patient left the hospital on December 29th feeling perfectly well.

Diagnosis.-Hepatic gumma; syphilis.

\section{Case 67}

A Lithuanian of twenty-nine, working in a rubber factory, and never consciously sick in his life before, entered the hospital April ro, 1907. Sometimes he takes as much as eight beers and four whiskies daily. At other times he goes without alcohol for at least a week. For two weeks he had been having severe epigastric pain, with loss of appetite and obstinate constipation.

The patient was very nervous, trembling, and pale. The red cells were 2,750,000; hemoglobin, 65 per cent.; white cells, 7200. The stained smear showed 78 per cent. of polynuclear cells and very marked stippling of the reds. The abdomen was flat, moderately stiff, and slightly tender. The reflexes were very lively, and there was hyper- 
esthesia of the feet. The aortic second sound was accentuated. The urine showed a very slight trace of albumin; otherwise it was negative, as was the rest of the physical examination. There was no lead line.

Three days after entrance the patient became maniacal in the night and had to be restrained. This continued for six days, after which he became sane. His temperature was frequently above $99^{\circ} \mathrm{F}$., and once reached Ior. $4^{\circ} \mathrm{F}$. This was at the height of his maniacal attack.

Discussion.-The history, the maniacal attack, and the gastric symptoms point toward alcoholism. The anemia, however, is not thus to be explained.

Tuberculous peritonitis and meningitis are suggested by the combination of a spastic, tender abdominal wall, and the maniacal attack accompanied by fever. This form of tuberculosis, however, rarely produces anemia, and mania is very unlikely in it, unless other cerebral symptoms (lethargy, coma, squints, headache, or vomiting) are also present.

Work in a rubber factory often produces a stubborn type of general debility, but it does not lead, so far as I know, to fever, to mania, or to anything like this grave anemia.

Nephritis must be considered. It would explain the albuminuria, the accentuated aortic second sound, and the mania; but a nephritis which had lasted long enough to produce such an anemia would almost certainly produce a demonstrable enlargement of the heart and some other uremic manifestations, such as headache, vomiting, or hemorrhages.

Lead-poisoning should always be considered in a case presenting the combination of anemia and cerebral symptoms, especially if the red cells contain a basophilic granulation, as in this case. Looked at from this point of view, all the symptoms seem to fall very naturally into linelead colic, lead anemia, lead nephritis, lead encephalopathy.

Outcome.-The treatment consisted at the beginning of glycerin enemata and magnesium sulphate, with morphin for the pain. Iodid of potash, Io grains, was given three times a day, while hot applications and turpentine stupes were also used for the pain. Chloroform anesthesia was once needed during his attack of mania. Fifteen grains of trional were given several times for sleep.

April igth the red cells were $3,600,000$, and no stippling was found.

$\mathrm{By}$ the twenty-third of April he had nearly recovered and was ready to go home. It was subsequently found that he drank water which came through a lead pipe, and that he seldom let the water run before drinking in the morning. Another possible source for the Jead was the patient's occupation. Many rubber workers acquire lead-poisoning. 
in connection with this case I will mention briefly a patient to whom I was called because of anemia and convulsive attacks. She was a young married woman with a baby three months old. She lived in a rural district, and did no work outside her own house. Epilepsy and uremia were the diagnoses previously considered, but examination showed that she and every other member of the household except the baby had a well-marked lead-line on the gum and all the other evidences of leadpoisoning. After giving up a water-supply heavily impregnated with lead, this patient rapidly recovered.

Diagnosis.-Plumbism.

\section{Case 68}

A blacksmith of twenty-three entered the hospital November 24, I906, with a negative family history and past history and good habits. He had been complaining for three weeks of epigastric pain, usually coming on about eleven o'clock in the morning, seeming to bear no relation to food-described as "pulling" in character, and relieved by lying down. There had been slight tenderness in the epigastrium, especially under the right ribs. The bowels had been very constipated, moving only once in three days. Three days ago he began to vomit, and had done so once or twice a day since. The vomitus contained no blood or food, but was yellow in color. His pain was never present when he waked in the morning; it was sometimes brought on by drinking water. He appeared to be 15 pounds lighter than in the previous June.

Physical examination was entirely negative, except that lumps were felt in the sigmoid region.

Discussion.-It does not seem likely that a blacksmith of twentythree is suffering from a pure neurosis, and he is at an age when cancer of the stomach is very rare. The pain comes at a time when the stomach is likely to be empty, and, therefore, suggests hyperchlorhydria or duodenal ulcer. The fact that his vomitus contains no food goes to strengthen this hypothesis, and the negative physical examination is entirely consistent with it.

Is it possible that the lumps felt in the sigmoid region may be due to a fecal accumulation behind a stricture, cancerous or of othe origin? I have known cancer of the intestine in a boy of twenty-one, so that the youth of this patient does not exclude that possibility, and the vomiting and constipation are quite consistent with it. In the absence of more definite symptoms, however (such as visible peristalsis, blood in the stools, and abdominal distention), there seems to be nothing further $t c$ verify this idea. 
May not the symptoms be due to simple constipation, so called? Why then should he have symptoms just now and not previously?

On the whole, the youth of the patient and the short duration of the symptoms make cancer and constipation less likely than the other alternative above mentioned, but no certainty can be attained on the basis of the facts here presented. Only by the therapeutic test-the results of treating the patient for duodenal ulcer (a treatment identical, in its early stages, with that of hyperchlorhydria) — can greater certainty be obtained.

Outcome.-After castor oil by mouth and enemata of oil, large movements followed. Guaiac test negative. Olive oil by mouth also relieved him very much, likewise a gastric ulcer diet. In five days he seemed to be entirely well.

Diagnosis.-Constipation.

\section{Case 69}

A chambermaid of twenty-two, with a negative previous history and family history, entered the hospital March 2, 1907. In February, I906, she had what was called "grip," followed by abdominal pain, weakness, and the loss of Io pounds in weight. The pain was sudden and nagging, coming sometimes immediately after meals, sometimes later, never lasting long, and never severe. She has had recurrences of this pain at intervals ever since. Four weeks ago the pain became more troublesome, and was accompanied by belching and constipation. It did not always remain in the epigastrium, but might shift to the lower abdomen, to the left chest, and to the back. ' It seemed to be produced especially by toast, potatoes, and meat; it was sometimes relieved by drinking hot water, but not by cooking-soda. It had kept her awake during the past two nights. She also complained of "palpitation in her stomach." She had very rarely vomited. At the present time her bowels are regular, and she feels fairly well except for weakness.

On physical examination it was noted that her cheeks were red, but her lips pale. The chest, abdomen, and urine showed nothing remarkable. Blood examination showed: Red cells, 4,976,000; white cells, 5600; hemoglobin, 60 per cent. The stained specimen was normal except for moderate achromia. The patient was treated by a careful diet.

Discussion.-Lead-poisoning is always one of the possibilities when a patient demonstrably anemic complains of abdominal pain. Lead may be excluded, however, in my opinion, by the absence of basophilic stippling in the red cells. I have never known a clear case of 
plumbism without stippling. There was nothing else, moreover, to favor the suspicion of lead-poisoning in this patient.

If the patient were somewhat older, the history would be quite consistent with gastric cancer, which would also explain the anemia; but as these symptoms have lasted a year, we should almost certainly find more evidence of cancer if that were the cause of the patient's sufferings.

Chlorosis is generally accompanied by constipation and hyperchlorhydria, which appear to be present in this case. The age and the occupation favor this diagnosis, which may be provisionally accepted, subject to confirmation by the results of treatment. The pain in this case is very typical of that most often associated with constipation, whether or not the latter is its cause.

Outcome.-The bowels were regulated by cascara and enemata. She was given Io grains of Blaud's pill after each meal. Recovery was uneventful.

Diagnosis.-Chlorosis.

\section{Case 70}

A married woman of thirty-five entered the hospital December 5, I906. She has always been well, but subject to what she calls bilious attacks. She was operated on four years ago for strangulated hernia. Since then she has had a great deal of severe, cramp-like epigastric pain, sometimes relieved by a movement of the bowels. On December Io, I905, the catamenia failed to appear, and she had vomiting and headache. In January, I906, she was operated on for extra-uterine pregnancy. In convalescence she was troubled by diarrhea and gaseous distention of the bowels. Later on she was obstinately constipated. She felt as if her intestines would fall out, but found relief by holding them up with her hands. Two months ago she woke up with a violent pain in her right hand. The next morning the hand was swollen up so that she could not close it. This trouble soon passed away, but ever since that time, she says, she never knew when a sharp, shooting pain would strike her. The pains were felt in all parts of the body, and lasted from a minute to four hours. She has had to take morphin for them at times. She says that she had never been nervous or hysteric before. She now enters the hospital expecting an operation for intestinal obstruction, having been sent in by one of the visiting surgeons with a diagnosis of intestinal obstruction of mechanical origin.

Physical examination showed that the pupils were irregular and did not react to light. Knee-jerks were present, but diminished. The ankle-jerk was absent; otherwise examination of the reflexes was negative. 
Sensation and coördination appeared to be perfect. In the right loin a mass was felt descending below the ribs on deep inspiration, slightly tender.

Physical examination, including the blood and urine, was otherwise negative.

Discussion.-Certainly a very complicated case. No doubt constipation accounts for a part of the symptoms, but the pains are very wide-spread and unusually intense for constipation. Moreover, there are certain facts in the physical examination which cannot possibly be thus explained.

Intestinal obstruction by bands or adhesions is always a menace in those who have had a strangulated hernia and an operation for extrauterine pregnancy; but for the same reason as mentioned in the last paragraph, intestinal obstruction cannot account for all the facts in this case.

Much in the patient's behavior and appearance, and something in her symptoms, point toward a neurosis, but this would not account for the absence of ankle-jerks and pupillary reactions.

The signs just mentioned practically assure us that this patient has tabes dorsalis. The only important question remaining is whether the tabes explains all the symptoms. Certainly the pains are very characteristic of tabes, and the gastro-intestinal symptoms may well be interpreted as "crises." The mass in the loin is certainly not due to tabes, but in all probability does not represent evidence of any disease whatever, but is merely a sagging kidney.

On the whole, it seems reasonable to believe that all the symptoms are now due to tabes. At any rate, the patient should be treated on that basis for the present. The chief lesson of the case is the necessity for self-restraint on the part of earnest surgeons when the patient's pupils and Achilles tendons fail to react.

Outcome.-The patient remained only two days in the hospital, whither she had come reluctantly and under the impression that a second operation would be necessary. When it was decided that no operation was advisable, she declared that she felt well and went home at once.

Diagnosis.-Tabes dorsalis.

\section{Case 71}

A married woman of forty-two, of negative family history and past history, entered the hospital December I, I906. On January 28, I906, she broke her leg and was confined to bed for eight weeks, during which time she lost her appetite, had palpitation of the heart, a grinding pain 
in the epigastrium, and a feeling as if there were strings inside her hitched to the navel and to the backbone. She had occasional vomiting of whitish material. She was given various medicaments without relief. In July she began to walk on crutches, but her symptoms were unrelieved. Her appetite was poor, and she lost 30 pounds in weight between January and December.

Her physical examination was entirely negative, except for a leukocytosis of 20,000 . The gastric capacity was 27 ounces; the stomach considerably prolapsed. There were no fasting contents, and after a test-meal free hydrochloric acid was found to the amount of 0.23 per cent. There was no blood. Three days later the white cells had fallen to 10,000 , and ranged between that and I6,000 during the three weeks of her stay in the hospital. At no time was there anything abnormal about her temperature, pulse, or respiration.

Discussion.-It is natural to fear cancer in this case, for gastric symptoms of recent origin always threaten cancer when the patient is over forty. The presence of abundant free hydrochloric acid in the stomach-contents by no means excludes cancer. The most hopeful feature in this regard is the absence of tumor or stasis, one of which would, in all probability, be manifest after a year of suffering.

To those who are always on the look-out for psychic causes in gastrointestinal disease, the fact that this patient had no stomach trouble until she broke her leg and was confined to bed, offers an important clue. It should lead us to investigate very carefully the patient's mental condition.

Outcome.-It turned out on careful questioning that she feared she was suffering from cancer. She was greatly encouraged by the negative results of the gastric tests, and in eighteen days gained $7 \frac{1}{4}$ pounds, mostly as a result of forced feeding, with laxatives and myrrh pill, one or two at night, aromatic chalk mixture, sodium bicarbonate when in distress, and a quassia cup before meals. She was also relieved by ro grains of sodium bromid after meals, and on two or three occasions had trional at night. The main point, however, in her recovery, was forced feeding.

The leukocytosis is not explained, but must be listed as one of those wild and untamed facts which $\mathrm{I}$ have grown to expect as a normal element in every well-studied case.

Diagnosis.-Gastric neurosis.

\section{Case 72}

A factory hand of thirty-eight entered the hospital December 29, 1907. Seven years ago he began to suffer from tape-worm, of which large segments were passed until three years ago, when the whole worm 
was removed. Since this time he has had attacks of epigastric pain and vomiting, often associated with jaundice. His family history and habits are good. Eight days ago he was again seized with pain in the epigastrium, relieved by vomiting. An hour later the pain returned and he vomited again. This happened five times that day. The next day he kept quiet and had no pain or vomiting. On the third day he went to work, and the pain and vomiting recurred. On the fourth day he was quiet and felt well. On the fifth day he again worked, and again had pain and vomiting. For the past three days he has not worked and has felt well. This association of pain with work has been present in all his past attacks. He has never had pain at night, on Sundays, or on holidays; and during the time that he has had these attacks he has changed his work three times. His pain bears no special relation to the time or kind of food. The vomitus consists of small amounts of greenish material and saliva. He has never seen food or blood either in the vomitus or in his stools. During the attacks his appetite is poor and his bowels constipated. He states that he has been considerably jaundiced during this last attack. He has lost five pounds in the course of the last year.

On physical examination no jaundice is found. Many teeth are missing; the rest are in fair condition. There is a systolic murmur at the apex, not transmitted. The heart-apex is in the fifth interspace, inside the nipple-line. The aortic second sound is louder than the pulmonic second sound. The tension of the pulse is apparently high, the lungs normal. The abdomen is level, slightly rigid, tympanitic throughout, and very slightly tender on pressure in the epigastrium. There are slight dulness and resistance in the region of the gall-bladder, but no jaundice. The liver is not felt. Physical examination, including the blood and urine, is otherwise normal.

Discussion.-The tape-worm is obviously "a blind." It is very unlikely that the epigastric pain and vomiting from which the patient suffered from 1900 to 1904 had any real connection with the tape-worm. It is perhaps worth mentioning here that practically all the symptoms traditionally associated with tape-worm are mythical. In the vast majority of cases tape-worm produces no symptoms whatever.

Since the death and burial of "gastralgia," that ancient foe of clear diagnosis and helpful treatment, such pain as this patient suffered has been shown to be generally due to one of two causes-duodenal ulcer or gall-stones. Since the attacks have apparently been associated with jaundice, our first thought is gall-stones, but on a closer study of the case we find that he has now no jaundice, although he now considers himself as yellow as in the previous attacks. This makes us doubt 
whether he really was ever jaundiced. I have many times found reason to discount patient's own statements in this matter. Patients and their friends often use the word "jaundice" to denote nothing more definite than a sallow complexion. To the consideration of duodenal ulcer I shall return later.

Aneurysm or angina abdominalis is suggested by the fact that the pain is increased by exertion and the pulse tension high. On the other hand, a pain which produces and is relieved by vomiting is rarely due to either of the causes just mentioned. The physical examination shows no evidence of aneurysm.

Is it likely that the lack of a good set of teeth explains some or all of this patient's symptoms? It does not seem to me so. Despite the many positive statements regarding the close association of digestive troubles and poor or deficient teeth, I have never seen any clinical evidence which would enable us to say more than "perhaps," so extraordinarily common is it to examine people who have lived their lives quite free from digestive troubles, though only one or two blackened fangs remain in each jaw. I by no means deny the possibility that malnutrition or poor digestion may in certain cases be due to defective teeth, but I think we need a great deal more definite study and evidence before we shall have justification for the positive statements and the expensive municipal crusades that are now so rife.

A definite diagnosis in this case would be easier if we knew-(a) Whether there is blood in the stools and $(b)$ whether hyperchlorhydria is present. Even in the absence, however, of these data $I$ think the diagnosis of duodenal ulcer is justifiable. Between this disease and the hyperchlorhydria which leads to it diagnosis is not always possible, as will be exemplified in a subsequent case. The absence of any temperamental or occupational cause for the worry and irritability so often associated with hyperchlorhydria makes me incline, on the whole, toward ulcer.

Outcome.-On January I, I908, the abdomen was opened. The gall-bladder and ducts were found to be normal, but a small duodenal ulcer was present. No aneurysm. The patient made a good recovery.

Diagnosis.-Duodenal ulcer.

\section{Case 73}

A married woman of forty-seven, with negative family history and good habits, entered the hospital December 2I, I907. She stated that for eighteen years she has had abdominal cramps every three or four months, but that for the last two weeks these have come much more 
often-seven times in two weeks. The pain starts in the epigastrium very suddenly and without known cause, without relation to food, to menstruation, or to the time of day. It radiates to the right flank, lasts about three hours, and often wakes her from sleep. It is usually accompanied by vomiting of food or brownish liquid. There is no history of jaundice, and between attacks she feels perfectly well, although the pain is so severe as to require morphin. Her bowels are regular, her urination normal, although for the last three days she has passed less urine than usual. She thinks she has lost a great deal of weight.

Physical examination is negative, except for considerable epigastric tenderness. The white cells number ${ }_{5} 5,800$; the stained smear negative. The urine contains a slight trace of albumin; gravity, Io3०; a few hyaline and granular casts.

Discussion.-Such symptoms might be due to constipation, but her negative statement upon this point was confirmed by our observation in the hospital. The history is also suggestive of lead-poisoning, except for its extreme duration, but the condition of the blood and of the gums enables us to rule this out.

The negative physical examination, which included tests of the pupillary and other important reflexes, makes tabes with gastric crisis out of the question. The regularity of the bowels and the long duration of symptoms render chronic intestinal obstruction (cancer) very unlikely.

Gastric cancer is always to be feared at the age of forty-seven when the patient has vomited a brownish liquid at frequent intervals, has had a great deal of epigastric pain, and is believed to have lost a great deal of weight. By the use of the stomach-tube we were able to establish the fact that there were no gastric stasis and no blood in the stomach-contents or in the vomitus. The size of the stomach was normal, and no tumor palpable.

Duodenal ulcer often gives a history of very prolonged suffering, similar to that in this case, and there is nothing in the history to exclude it. Even the fact that blood was absent from the vomitus, the artificially abstracted gastric contents, and the feces by no means excludes ulcer. The radiation of the pain, however, its sudden onset and its sudden relief by morphin, are less characteristic of duodenal ulcer than of the disease next to be considered. We note also the absence of any relation between the pain and the digestive activities.

Gall-stones might explain all the symptoms in the case, although the diagnosis is not forced upon our notice, as it would be were jaundice present. We are no longer surprised, however, to find gall-stones in the 
absence of jaundice, and, on the whole, no other diagnosis seems as likely. The negative physical examination does not militate at all against this idea, nor does the condition of the urine incline us to change our minds, though it is not at all obvious why the albumin and casts are present.

Outcome.-On December 26, 1907, the abdomen was opened and I5 large stones were found in the gall-bladder. The patient made a good recovery.

Diagnosis.-Gall-stones.

\section{Case 74}

A tailor of forty-nine with a good family history and good habits entered the hospital on June 15, I907. For the last eighteen years he has had occasional spells of dull epigastric pain coming on in the afternoon for a month or so. These attacks had never troubled him much, and were often absent for a month at a time; but for the last ten years they have become more frequent, and the pain has appeared in the morning, as well as in the afternoon, accompanied by a feeling of weight in the abdomen, but rarely by vomiting. About a year ago the pain began to come regularly between Io and I 2 in the morning, and between 4 and 6 in the afternoon, except during the periods when he was under treatment. The pain is now sharp, and radiates sometimes from the epigastrium to the back, rarely to the left hypochondrium. It is partly relieved by eating, and wholly by cooking-soda, but never by pressure. He frequently belches gas.

Two months ago, after a day during which he had been constantly regurgitating sour fluid, he vomited at one time almost three quarts of sour, foaming yellow fluid, with great relief. Two weeks ago he vomited a similar quantity, and at the end of it was a little chocolate-colored stuff. He thinks he has lost 20 pounds in the last six months, yet he worked until May 29th and until very recently felt as strong as ever, and has eaten and slept well.

Physical examination was negative, except that the stomach capacity was 74 ounces, the organ extending three inches below the navel and showing visible peristalsis.

Discussion.-Here is a history nearly typical of duodenal ulcer. I have given it here to prove that in some such cases no ulcer is demonstrable at operation. One of the wisest clinicians of my acquaintance recently said in a personal letter: "In my experience 'hyperchlorhydria" generally spells duodenal ulcer." I agree with this statement if it is taken literally - that is, if we distinguish "generally" from "always." 
My object at the present time is to exemplify one of the weak points in clinical diagnosis - our inability, namely, clearly to distinguish the two diseases above referred to. Had we known at the outset that this patient was an alcoholic, the balance might have inclined a little more toward hyperchlorhydria, as this trouble is not infrequently associated with alcoholism. But still we should have been wandering in the region of probabilities.

Outcome.-Operation on the ninth of July showed no dilatation, ulceration, or scar formation anywhere in the stomach or duodenum. The pyloric ring was of good size. The patient made a good recovery, and on July 28, 1908, reports that he had had similar attacks of pain, but less severe. He now admits that at times he drinks liquor freely, but thinks that these sprees have no relation to his gastric attacks.

Diagnosis.-Hyperchlorhydria (alcoholism?).

\section{Case 75}

A farmer of forty-six, with a negative family history and good habits, entered the hospital February 19, 1907. For the past two years he has had many severe attacks of epigastric pain, coming without apparent cause, and relieved about once a month by vomiting. For the past two weeks the pain has increased in severity. He localizes it accurately just below the ensiform cartilage, and describes it as sharp, increased by coughing, by exertion, or by a meal containing pork, eggs, or veal. It is usually worse at night, especially just after he goes to bed. It is somewhat relieved by hot-water bottles, but it generally keeps him awake most of the night.

Physical examination shows the heart's impulse two inches outside the nipple-line in the fifth space. There is a presystolic thrill and murmur at the apex, ending in a sharp first sound. A short systolic murmur is also heard at the apex. Both murmurs are transmitted to the axilla. The pulmonic second sound is very difficult to hear. At the base of the heart a soft systolic thrill can be felt in the aortic area, and a high-pitched diastolic murmur heard under the sternum at the level of the third rib and above this point, together with a soft systolic murmur, which is audible throughout the precordia. No second sound can be heard in the aortic region. The pulse is of the plateau type; the arteries are tortuous and thickened. There is a lateral excursion of the brachials. Blood-pressure, I95. The edge of the liver is felt on inspiration, and there is moderate tenderness, sharply localized below the ensiform cartilage, and accompanied by muscular spasm.

Discussion.-In this case, as in most of those preceding and follow- 
ing it, the pain is worse at night. This symptom has often been referred to as characteristic of gall-stone pain or duodenal ulcer, and there are other features in the case consistent with one of those two diagnoses, but it is of crucial importance in the study of this case to note that the pain is increased by exertion and by coughing. This is not usually the case with duodenal ulcer or gall-stones, although inflammatory adhesions may be so situated that muscular action stretches them painfully.

The presence of the well-marked heart lesions (aortic stenosis and regurgitation), and especially of the high blood-pressure, makes us suspect any pain of being connected with the circulatory system. The relation to exertion is very characteristic of angina pectoris. Does pain of this type ever occur as low as the epigastrium? It certainly does, although the term "angina abdominalis" is perhaps more appropriate. I have seen a great many cases of this type treated quite unsuccessfully by stomach specialists without regard to the circulatory condition. To get further clearness on the diagnosis, one would need to observe carefully the effect of rest and of nitroglycerin. Certainly no type of stomach or gall-bladder trouble is relieved by nitroglycerin.

Outcome.-A few days' observation in the hospital ward demonstrated the truth of our suspicions: rest rendered the attacks less frequent, and those which occurred were promptly relieved by nitroglycerin.

Diagnosis.-Angina pectoris (low). What about cardiovaseu

\section{Case 76}

A salesman of forty-nine came to the hospital on December I0, 190.7, complaining of pain, constipation, and vomiting. He is in the habit of taking several drinks of whisky a day, but has never been sick until the present illness, and his family history is good. For five weeks he has suffered from abdominal pain. The pain began at a time when he was "not feeling well," and had stopped work for a few days. It is in the epigastrium, worse at night, relieved by eating, and accompanied by much wind and belching. It usually begins about 4 P. M., and reaches its maximum severity between II P. M. and 4 A. M., after which it subsides. Of late it has come every night. He often vomits with the pain, and last night did so three times. He has small movements of the bowels every second or third day. Two months ago he weighed I60 pounds. Now he weighs $I_{3} 6$ pounds.

Physical examination, including the urine, is negative. No leadline is to be seen. The leukocytes number ro,400; hemoglobin, 90 per cent. In the differential count the polynuclear cells are 80 per cent.; 
lymphocyies, I 8 per cent.; eosinophiles, 2 per cent. There is very marked stippling and abnormal staining of many red cells.

Three days later the urine was found to contain a trace of albumin, with numerous hyaline, finely and coarsely granular casts, many with cells adherent.

Discussion.-Our first impression is naturally that "rum done it," but on second thought there seems no special reason why he should suddenly begin to suffer at this time as the result of so long continued a habit.

The fact that his bowels are so constipated raises the question whether this trouble may not account for all his symptoms, whether it be of the ordinary functional type or dependent upon a stricture (malignant?). But, as before, the question arises, why should he suddenly begin to suffer from constipation at the age of forty-nine? The functional types of the affection usually make their appearance long before that age. Only some special aberration in diet or some great nervous strain would account for the sudden appearance of functional constipation in a man of this age.

It is possible, of course, as I have previously stated, that cancer of the bowel may exist for months or even years without manifesting its presence by any symptoms, but when we look over the history and examine the patient with this possibility in mind, there seems to be nothing to support it, although the loss of weight is suggestive.

A pain relieved by eating often occurs in connection with hyperchlorhydria or peptic ulcer, and there is nothing in the case absolutely to exclude these affections, which, like cholelithiasis, must always remain in the background of our minds when paroxysmal epigastric pain is the presenting symptom.

Before making any further investigation or following up any other clue, we should test the possibilities suggested by the presence of marked stippling in the stained red corpuscles despite the absence of anemia. Although no lead line is seen and nothing in the patient's occupation suggests plumbism, this blood lesion is so characteristic that every effort should be made to follow it as a clue.

Outcome.-During the first three days the diagnosis was not made; and later it was discovered that he has for three years used drinkingwater coming through 30 feet of lead pipe. His blood-pressure was found to be $\mathrm{r} 85 \mathrm{~mm}$.

On December 17 th his attacks of colic were less marked, but sudden muscular weakness in both arms appeared for the first time. On December $24^{\text {th }}$ he was free from colic and the urine had cleared up, but the arms 
and back showed very marked muscular weakness. On this day (the 24 th) a well-marked lead line was found on the gums, visible only on the inner side of the teeth of the lower jaw.

Diagnosis.-Plumbism.

\section{Case 77}

A negro of sixty-four entered the hospital August 7, 1907. He stated that his mother died at eighty-five "of worry." His family history is otherwise not remarkable. He now complains of severe epigastric pain which had been present for three months. During the Civil War he drank a quart of whisky daily. Fifteen years ago he had a venereal sore which was treated at the Boston Dispensary with calomel locally and iodid of potassium internally. He was treated for six months and noticed no secondary symptoms. He says it was his habit to take three or four glasses of whisky a day and three or four beers, but for the past four months he has abstained. He smokes and chews five cents' worth of tobacco a day.

At the onset of the pain, three months before, he fell in the street, although he was not unconscious. Since that time the pain is apt to radiate from the epigastrium across his chest or up his left side and through his back. Occasionally it shoots from the lower part of his back up to his left shoulder, or from his right hip down his leg, but it is worst in the epigastrium.

Four weeks ago he was examined at the Boston Dispensary and thinks that he was ruptured at that time. He has had no vomiting, headache, or palpitation. In January, I907, he weighed I 80 pounds; in June, I45 pounds; now, I40. His digestion is good.

Physical examination shows a pallor of the mucous membranes. The heart is negative except for accentuation of the aortic second sound. The carotid arteries are prominent and easily palpable. The bloodpressure is $I 30 \mathrm{~mm}$. of mercury. The right lung shows a considerable number of coarse râles below the scapula, with modera dulness extending to the base of the lung. One and a half inches below the right costal margin is a rounded nodule an inch and a half in diameter, considerably elevated, apparently not connected with the skin. It is somewhat movable, not tender, and does not descend with respiration. There is dulness in both flanks, shifting with change of position. The penis is six inches in circumference, markedly edematous, as is the perineum. The motions of the back are limited and painful. A rectal examination shows that the prostate is the size of a small grape-fruit, very firm, im- 
movable in the pelvis, and encroaching markedly upon the rectum. The right testis is enlarged and tender.

Red cells, 2,696,000; differential count normal; white cells, I4,200; hemoglobin, 45 per cent.

Discussion.-Abdominal aneurysm must certainly be considered as a cause of pain like that described in this case, especially when there is so well authenticated a history of syphilitic infection. The enlarged testicle would then naturally be explained as syphilitic orchitis. The sudden onset of the pain and its prostrating effects might be accounted for by a partial rupture of the aneurysmal sac.

Against this diagnosis, however, is the evidence furnished by rectal examination. I know of no syphilitic lesion which will produce such changes in the prostate. Another fact of importance, which came to light later, was the inefficiency of a prolonged course of antisyphilitic treatment which he had recently undergone. Malignant disease is certainly the commonest cause for an extensive, hard, immovable tumor connected with the prostate gland. This would easily account for the anemia and for the nodule in the abdominal wall, though both of these might possibly be accounted for also by syphilis.

If malignant disease is the correct diagnosis, why was the patient so suddenly stricken that he fell in the street three months before? I can give no confident answer to this question. Possibly his habits have something to do with explaining it.

Outcome.-The patient died on the tenth of August. Autopsy showed sarcoma of the right testis, with metastasis in the prostate, adrenal glands, small intestine, bronchial lymphatic glands, pleura, pericardium, and abdominal wall.

Diagnosis.-Sarcoma testis with metastases.

\section{Case 78}

A colored woman of twenty-four entered the hospital August r, I907. Seven months ago she began to complain of a severe steady pain about the center of the abdomen, a little more on the left than on the right. At this time a large, hard tumor was discovered near the navel. For three months following this she had many attacks of pain in the same region, and her temperature ranged from $100^{\circ}$ to $105^{\circ} \mathrm{F}$. The lump in the mean time decreased in size. For the last four months she has had. occasional spells of pain lasting two or three days. She does not feel feverish. For the past four months she has had severe epigastric pains, coming on every fifteen minutes, lasting two or three minutes, and often leading to vomiting, but for the past twenty-four hours she has been free 
from pain. She has lost twelve pounds in the past seven months, but until the last four days has not felt very much weakness. Nose-bleed has been frequent all her life, and has been more apt to come at the menstrual period. Her bowels have been constipated for years, but with medicine have usually moved once a day. Temperature, never above $99^{\circ} \mathrm{F}$. Hemoglobin, 80 per cent.; leukocytes, 8800; urine, normal.

Physical examination shows nothing abnormal in the chest. The abdomen is held very stiffly, especially in the lower portion, where there is slight dulness. Much tenderness is complained of throughout. Nothing else could be made out on account of this tenderness. By vagina a band could be felt to the right of the uterus, but the fundus could not be palpated on account of abdominal spasm. Immersion in a warm bath failed to relax the abdominal muscles, and even under ether the spasm did not entirely relax.

Discussion.-Clinical experience teaches that whenever a negress is sick and the symptoms are below the waist, fibroid tumor of the uterus usually turns out to be the diagnosis. The abdominal examination was so unsatisfactory in this case that nothing definite could be said regarding the uterus. The lump which was so readily felt some months before would play in very well with the idea of a fibroid tumor, but its apparent decrease in size, the prolonged fever (three months' duration), and the generalized abdominal spasm do not fit well with this diagnosis.

Pelvic peritonitis originating in a pus-tube would explain the band felt by the vagina and the tenderness of the lower abdomen, but would not account for the long fever, the wide extent of the abdominal spasm, and the tumor near the umbilicus. Tuberculous peritonitis, however, will explain all these facts, and is, moreover, exceedingly common in young colored folks.

Outcome.-On August 7 th the abdomen was opened and showed tuberculous peritonitis, the viscera irregularly matted together; no fluid.

Diagnosis.-Tuberculous peritonitis.

\section{Case 79}

A married woman of thirty-eight, a French Canadian, entered the hospital December I0, I907, for chronic abdominal pain which has lasted for several weeks and apparently has incapacitated her for any work. This pain has troubled her on and off for three years and a half. At times it is very severe and interferes much with her sleep. Now it is present every day; formerly she would have respite from it for many weeks at a time. It is not affected by eating nor by the time of day. Her appetite is fair, and she has never been jaundiced. She 
vonits occasionally, the vomitus not being in any way characteristic. Her bowels move about once in three days. She has no cough and no headache, but thinks she has lost 20 pounds in the past eight months, and has been unable to work during that time on account of pain.

Physical examination showed considerable loss of weight and pallor of the mucous membranes. Temperature, pulse, and respiration normal. The chest was normal, the abdomen somewhat retracted, rigid, tympanitic throughout, and tender in the epigastrium; no masses felt. The blood and urine showed nothing abnormal.

Di cussion.-The symptoms are strikingly like those of the last case (tuber ulous peritonitis), but in the present case there are weeks of freedom from symptoms and no fever has been recorded. All the ordinary clues suggested by the cases last studied were followed up quite fruitlessly. We could obtain no positive evidence of an intestinal stricture, of lead-poisoning, of peptic ulcer, cholelithiasis, or of any form of peritonitis. There seemed no reason to suspect the kidney or any part of the urinary tract.

Under these conditions it is proper to ask ourselves whether the symptoms may not be due to pure constipation? It seems extraordinary that a loss of 20 pounds in weight should be brought about by this cause. Only the therapeutic test, however, can decide the question. If the symptoms all disappear when the bowels are properly regulated, and if so long as they continue regular there is no recurrence of pain, the diagnosis will be justified.

Outcome.-Under careful diet, with sodium bicarbonate $\frac{1}{2}$ dram after meals and mild laxatives, the patient ceased to have pain and left the hospital in six days. Her subsequent history has been uneventful (I9I0).

Diagnosis.-Constipation.

\section{Case 80}

A Russian Jew of thirty-two entered the hospital February II, I908. $\mathrm{He}$ has complained for five months of epigastric cramps beginning about 4 P. M., lasting all night and until noon the next day. In previous years he has had similar attacks occasionally. The pain has no relation to eating, but on the days in which his stomach has been washed out in the out-patient department he has been relieved. He has a good appetite and eats well, but vomits daily, sometimes spontaneously, sometimes purposely for relief of distress. The amount of vomitus is largeoften as much or more than he has eaten since he last vomited. His bowels often go five and six days without moving. About a week ago 
he woke at 2 o'clock in the morning feeling very faint. He soon began to be "choked up," and for twenty-four hours had great difficulty in breathing. About a year ago he weighed I45 pounds. His present weight is II4 pounds. He was formerly a painter, but has had nothing to do with lead for thirteen years.

Physical examination is negative, except that there are tenderness and some spasm under the right costal border. The blood and urine are normal. His stomach holds I08 ounces of fluid. The contents, obtained by washing, smell strongly of organic acids, and it is difficult to get the wash-water clear. On inflation, the lower border of the stomach reaches to a point midway between the navel and the pubic bone. Sahli's test was administered, with the following result: 300 c.c. of the test fluid were given. After one hour the total residue was $3 \mathrm{I} 5$ c.c., of which rog c.c. are test fluid and 206 c.c. secretion; therefore the percentage of test fluid passed from the intestine in one hour is 63 per cent. as compared with the normal of 75 to 90 per cent.; the hydrochloric acid of the pure gastric juice, 3.4 per cent.; average normal, 3.5 per cent. Diagnosis: deficient motility with hypersecretion. His chief complaints during his stay in the hospital were a burning epigastric pain, flatulence, and constipation. He received no relief from diet, medication, or gastric lavage.

Discussion.-We repeated in this case the therapeutic test used so successfully in the last, but even when the bowels were in a perfectly satisfactory condition, the suffering continued without respite. Constipation, therefore, was not the trouble; it was the result, not the cause.

Lead-poisoning was excluded by the study of the blood and the gums.

Tenderness and spasm under the right costal border occurring in a patient who suffers from paroxysmal epigastric pain compel us to consider gall-stones. This possibility cannot be ruled out, and was one of the alternatives in the mind of the surgeon who later opened the abdomen.

Obviously, however, there must be something wrong outside the gall-bladder, for the patient's stomach is markedly dilated and does not empty itself properly. Gastric stasis, however, may be one of the disasters following in the train of repeated gall-stone attack and as a result of the adhesions thus produced.

For gastric cancer-that commonest of all causes of pyloric stenosisthe history seems to be too long in this case. Yet can we explain the loss of weight on any other hypothesis? In answering this last question it is worth while to state emphatically that patients may lose a fifth or a quarter of their weight within a few months as a result either of gallstones or of peptic ulcer. 
In the present case all that was certain before operation was the existence of an obstruction to the outflow of gastric contents. As a cause for this, the scar of a duodenal ulcer and the adhesions resulting from repeated gall-stone attacks were the alternatives most seriously considered.

Outcome.-Accordingly, on February Igth the abdomen was opened. No disease was found in the stomach, duodenum, or gall-bladder, but the pylorus was considerably obstructed by adhesions. Gastro-enterotomy was done. After the operation the patient improved, and by

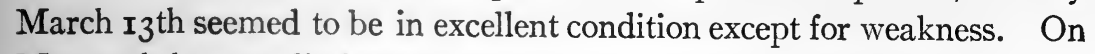
May 20 th he was discharged, wholly free from gastric symptoms.

Diagnosis.-Pyloric adhesions.

\section{Case 81}

A married woman of thirty-two has been complaining for some months of acute epigastric pain coming immediately after meals, lasting about fifteen minutes, and relieved by the belching of gas. She entered the hospital on July 29, I907. She had suffered from typhoid fever at the age of fifteen, from diphtheria at twenty, scarlet fever at twentytwo, "peritonitis" five years ago. She has been married fifteen years, but has had no children and no miscarriages. Five years ago she weighed 250 pounds, and she thinks she has gained in weight lately. She is in the habit of taking two or three drinks of whisky a week for the "blues." Four days ago she ate very heartily at supper-time. At I o'clock the following morning she was taken with severe epigastric pain, which has persisted ever since.

After palpation of the epigastrium the pain becomes spasmodic and seems to go straight through to the back. It is worse with every deep breath, and is increased by emotion.

The bowels were moved last night for the first time during this illness, as a result of laxative pills. The pain has prevented sleep, and last night she thinks she was delirious. The patient's temperature is I0 $2^{\circ} \mathrm{F}$.; pulse, I00; respiration, 30 . There is tenderness on percussion over the lower part of both lungs behind, but nothing else abnormal is made out. The abdomen is somewhat hollow above the umbilicus, rather full below; the abdominal wall very thick and flabby. There is slight rigidity in the lower part, less in the epigastrium, where the pain is worst. Deep pressure elicits expressions of pain in both the lower quadrants and in the right hypochondrium. The edge of the liver cannot be made out. 
Next morning the pain was more definitely localized in the epigastrium, and the temperature and pulse remained elevated, while the white corpuscles had risen from $\mathrm{I} 3,400$ to $\mathrm{I} 7,000$.

Discussion.-Out of this very checkered past history, with its suggestions of dyspepsia, peritonitis, and alcoholism, no clear indications for diagnosis emerge. The constipation and the very wide-spread character of the pains, both in the back and the front of the body, are common features of some types of neurosis, but the presence of fever and leukocytosis make neurosis very unlikely. In the foreground of the clinical picture are the epigastric pain and tenderness of acute onset. Many possibilities may emerge, but at present no clear diagnosis is possible.

The problem here presented is a very familiar one. We have good reason to believe that in the course of twenty-four or forty-eight hours the diagnosis will be much clearer, but is it not dangerous to wait so long? Should not an operation be done at once before more dangerous symptoms appear? No definite rules can be given by following which we can solve this difficulty in every case. The decision rests mainly upon two points of observation:

I. How sick is the patient?

2. Is she getting worse from hour to hour?

An answer to the first question depends on long and mature clinical experience. A general impression is gained, of which no very clear account can be given. The look of the patient's face and the quality of the pulse are perhaps the most important items in the judgment.

More important is the demonstrable change under observation of some of the measurable data, such as temperature, pulse, respiration, leukocytosis, the degree and area of spasm, tenderness, and pain. While we are watching the course of these variables, it is quite likely that the pain and tenderness will have time to "settle." Careful observation of most cases of this kind brings out three stages:

I. The initial pain, its location being of great diagnostic value if the history is clear and definite.

2. The subsequent radiations of this pain; often most confusing.

3. Its final "settling" in a single spot, most important in diagnosis, but often dangerous to wait for.

The symptoms do not.seem to be violent enough for perforated peptic ulcer or for acute pancreatitis, though neither of these can be ruled out. Gall-stones is the next most frequent cause for pain of this type, provided lead, tabes, constipation, pericarditis, and angina pectoris are excluded, as is easily possible in the present case. Since 
there are fever and leukocytosis, it is reasonable to believe that some cholecystitis has also occurred here.

Outcome.-On the afternoon of the thirtieth of July operation showed an enlarged, edematous, partially gangrenous gall-bladder, with one faceted stone within.

The patient made a good recovery.

Diagnosis.-Cholelithiasis and gangrenous gall-bladder.

\section{Case 82}

A woman of forty-eight entered the hospital February I4, I908. She has had four children, all of whom are now dead. The first was a congenital idiot; the second had water on the brain; the third was still-born, and the fourth died at three years of pneumonia. She had repeated convulsions during the latter months of her third pregnancy. During the others she had no such trouble. She has had no miscarriage. Her habits are good, but she has usually passed her water eight or ten times each night during the past ten years.

For the past seventeen years she has had many attacks of epigastric pain, with distention and belching. The pain has never been colicky or accompanied by jaundice, but has radiated to the back, and has sometimes been severe enough to require morphin. The attacks of pain have no relation to mental conditions nor to the character or time of meals. Her weight is unchanged.

Two and a half weeks ago she had a sudden attack of pain, worse than at any previous time, and vomited several times in the first twentyfour hours. She had fever for five days, and has been in bed ever since. She has had daily chills, lasting from fifteen to twenty minutes each, and recurring about the same hour. Her bowels have been constipated, and she has taken only liquid food for two weeks.

Temperature, pulse, and respiration are normal. The patient is very obese. The sclera shows a very slight yellowish tinge. The chest is negative, and the abdomen shows nothing but general tenderness. At a point $2 \frac{1}{2}$ inches below the costal margin a rounded edge (presumably the liver) is felt to descend on inspiration, and there is considerable tenderness at this point and just above it. The surface of the liver seems irregular. The right sacro-iliac joint is tender to pressure, and she feels better with a pillow under the lumbar spine. Her pain and vomiting continued after the patient entered the hospital, and despite laxatives, counterirritants, and starvation. The leukocyte count at entrance was 8000 , but rose on the third day to 17,000 , with 90 per 
cent. of polynuclear cells. The temperature at the same time rose to IO2 ${ }^{\circ} \mathrm{F}$.

Discussion.-When a woman's pregnancies have resulted as in this case, syphilis must always be thought of as a possible cause for any subsequent symptoms. The presence of chills and the suggestion of an irregular liver point toward that organ as possibly the seat of a syphilitic process. On account of such chills I have twice known patients to be drenched with quinin for weeks at a time, when syphilis of the liver was the true diagnosis.

In this case, however, the normal temperature makes us wonder whether the chills may not be of nervous origin. Trembling and shivering are very common nervous symptoms, with or without the sensation of cold, and under these conditions often get mistaken for a chill, which usually carries with it the presence of fever. We are by no means certain, however, that the temperature has always been normal previous to February I4th; indeed, the patient's statement directly contradicts such an idea. At any rate, we cannot be content with the diagnosis of psychoneurosis in view of the quite definite physical signs described above.

Can her troubles all be due to sacro-iliac arthritis? Attention is drawn to this point by the tenderness over the sacro-iliac joint and the relief following support of the lumbar spine, but the jaundice, enlarged liver, and the persistent vomiting cannot be thus explained. Pain and tenderness in various parts of the abdomen may be produced through the nerve radiations originating in sacro-iliac disease. Both gall-stones and appendicitis may thus be simulated. But in this case we have other objective signs.

By far the commonest lesion associated with a picture like that here given is cholelithiasis, and although the case is atypical in various respects, this seems to be the most reasonable diagnosis.

Outcome.-Operation showed an enlarged, thickened, and perforated gall-bladder, surrounded by a considerable amount of pus, and containing numerous gall-stones.

Diagnosis.-Cholelithiasis with perforations.

\section{Case 83}

A school-boy of thirteen entered the hospital February I4, I908. In November and December, I906, he had an acute urethritis, and gonococci were demonstrated in the discharge. He has had "rheumatism" for about one year in the past three years, in periods lasting from six weeks to three months. His family history is not remarkable, and he has been well for the past two years. 


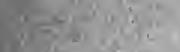

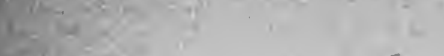

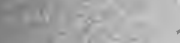

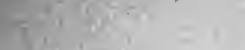

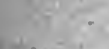




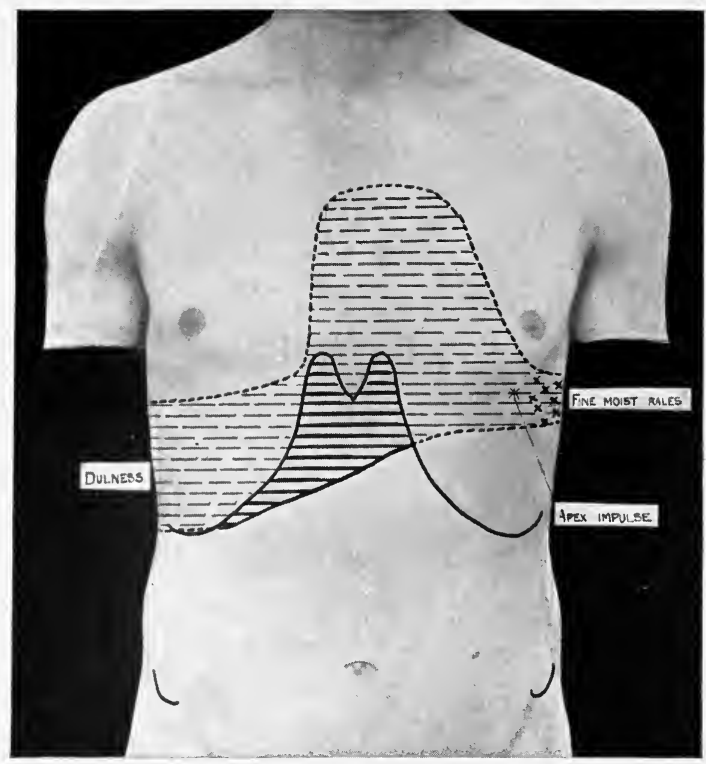

Fig. 29.-Percussion outlines in a patient complaining chiefly of epigastric pain. (See also Fig. 30.)

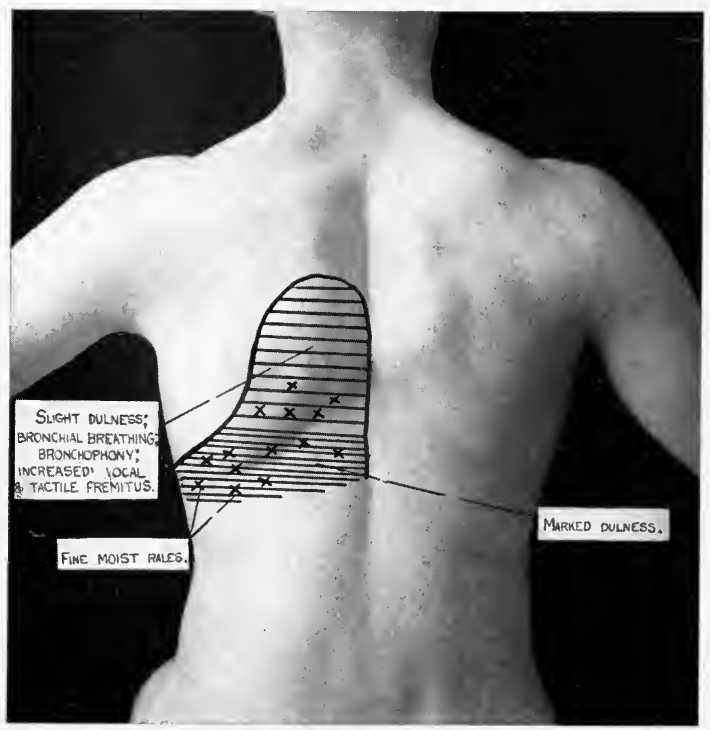

Fig. 30.-Signs demonstrable in a case of endopericarditis. (See also Fig. 29.) 
Seven days ago he began to have epigastric pain. Five days ago his knees became swollen and painful on motion, and he took to his bed, where he has since remained. In the past two days his knees have improved and no other joints have been involved. Yesterday morning he began to breathe very rapidly, but has had no cough and no vomiting.

Physical examination shows slightly labored breathing, with pallor of the mucous membranes. Temperature, I00. $2^{\circ} \mathrm{F}$; pulse, II 2 ; respiration, 28. The heart's dulness extends into the sixth-interspace, two inches to the left of the nipple-line. The right border extends $\mathrm{I} \frac{3}{8}$ inches to the right of midsternum. The cardiohepatic angle is obtuse. All over the precordia, but loudest at the apex, a systolic murmur and a rough diastolic murmur are heard. The latter is also heard over the lower end of the sternum. In the left back there is dulness extending up to a point one inch above the lower angle of the scapula, thence sloping down through the axilla to meet the cardiac dulness. Over most of the dull area bronchial breathing, increased voice-sounds, with increased tactile fremitus, and fine moist râles, are heard. At the extreme base, where dulness is most marked, the intensity of voicesounds and breath-sounds is very slight. Later a capillary pulse was demonstrated, and the diastolic murmur was shown to be loudest along the left edge of the sternum, but also fairly loud in the second right interspace.

At no time was there any cough. The leukocytes ranged between I2,000 and I3,000; the urine was between 30 and 40 ounces in twentyfour hours, and free from albumin.

Discussion.-Obviously, this boy has an arthritis, and gonorrhea is its probable cause. The problem of present importance is to determine what complications have occurred. Evidently some infectious disease is still going on, and the physical signs call our attention especially to the heart and the lungs.

Pneumonia, with or without empyema, would explain the signs in the right back, and it is a very familiar fact that pneumonia and pleurisy often begin in children with abdominal pain. The absence of cough by no means excludes pneumonia.

But the cardiac signs have also to be explained. The increased area of dulness and the double apical murmur are the ordinary evidences of endocarditis with disease of the aortic and mitral valve. But the percussion lines on the right side of the heart (see diagram) are more indicative of pericarditis, though no typical friction is described. If a pericardial effusion were present, it might account not only for the percussion outlines and the auscultatory abnormalities, but also for the 
signs in the back of the left lung, since this is just the area of lung on which a pericardial effusion exerts pressure in bed-ridden patients. By such pressure sufficient condensation of the lung is produced to simulate the signs of pneumonia. It is impossible to exclude a patch of pneumonia complicating the other troubles present, but experience shows that we are more apt to be right when we explain a clinical picture by one diagnosis rather than by two. Pericarditis, therefore, seems the most reasonable working hypothesis.

Outcome.-The temperature gradually subsided in ten days. The murmurs disappeared, and the boy seemed entirely well by February 28th.

Diagnosis.-Acute pericarditis.

\section{Case 84}

A sexton of sixty-five was first seen December 16, 1907, complaining of paroxysmal abdominal pain relieved only by morphin. About three years ago he began to suffer from dyspnea and swelling of the legs: This trouble has been present, off and on, erer since, but he notices that it is better if he is working hard than if he sits around the house.

In July, 1907, he had an attack of sudden, cramp-like pain in the upper abdomen, accompanied by dyspnea and persistent vomiting of foul green fluid. After twenty-four hours the pain was relieved by a subcutaneous injection of morphin. Since that time he has had similar attacks, gradually increasing in frequency and diminishing in severity. He now has them every second or third day, but does not vomit with them. In the last three months he has noted that during the day and night before an attack he passes large amounts of pale urine, and on the day following an attack small amounts of dark urine. His abdomen is often bloated, but this subsides without treatment.

Physical examination shows that the pupils are equal and react well. The tongue is large and smooth, especially in the posterior portion. The apex of the heart extends one inch outside the nipple-line in the fifth space. The first sound at the apex is weak, the second sound everywhere accentuated; no murmurs are heard. Blood-pressure ranges between $\mathrm{I} 40$ and $\mathrm{I} 60 \mathrm{~mm}$. The artery walls are stiff and tortuous. The abdomen shows general voluntary spasm, and the edge of the liver is felt one inch below the costal margin. The knee-jerks cannot be obtained even on reënforcement. The urine averages about 40 ounces in twenty-four hours, with a gravity of about Io20. There is no albumin, but a few hyaline granular casts are seen in the sediment. The white corpuscles are 6roo. 
During his month in the hospital the patient had many attacks of abdominal pain, always coming on at night, relieved by morphin so completely that next morning he felt well and wanted to get up. Nitroglycerin and amyl nitrite were repeatedly tried without any relief. Most of the attacks of pain were preceded by slight shortness of breath. The patient sometimes vomited during an attack.

Dr. James J. Putnam examined the patient and said that the loss of knee-jerk might be due either to spinal arteriosclerosis or, more probably, to the diphtheria of his youth.

Discussion.-In a patient who has no knee-jerks and complains of paroxysmal abdominal pain, the thought of tabes should automatically rise in our minds. In this case tabes must remain a possibility unexcluded to the last, though it is very unusual to find the pupils normal and the other signs of tabes (lightning pains, sphincteric disturbances, sensory abnormalities, ataxia, syphilitic history) all absent.

Angina pectoris (or angina abdominalis) is the natural inference when we come to take account of the evidences of failing heart power and of arterial degeneration. But angina is almost never accompanied by vomiting, and it is rare to find a case absolutely unrelieved by the nitrites. Moneren he fies

Though the pain is not in the typical place and has not the typical radiations of cholelithiasis, there are a number of points suggesting that diagnosis. It would be very unusual, however, to find no fever or chill in the history of a patient who has had gall-stone pains for six months. Further, the association of the pain with dyspnea and with changes in the amount of urine would be very unexpected in cholelithiasis.

Peptic ulcer might produce such a pain, but the brief paroxysms completely relieved by morphin are not at all characteristic of that disease. Further, it is very rare to find an active peptic ulcer coincident with evidences of failing heart.

Plumbism, simple constipation, and intestinal obstruction by cancer can easily be ruled out.

It seems to me of importance to notice the background of this case. For nearly three years previous to the onset of the symptoms now complained of the patient had suffered from dyspnea and edema of the legs. Physical examination at the present time seems to indicate that this is not due to primary valvular trouble, but rather to vascular degeneration. It is possible that all the symptoms may be due to this same cause acting upon different organs.

It is a well-known fact that in arteriosclerotic subjects there appear from time to time a great variety of paroxysmal attacks which in former 
years were attributed solely to the obliteration, embolic closure, or rupture of one or another blood-vessel. In the light of more careful postmortem study we have come to speak of these paroxysmal attacks as vascular crises. ${ }^{1}$ The idea of vascular spasm takes the place of the older idea of gross vascular lesion, in view of the fact that postmortem there is often no gross vascular lesion to be found. Under this general heading of vascular crises belong in all probability many of the transient hemiplegias, monoplegias, aphasias, comas, local or general spasms formerly explained as due to permanent anatomic lesions. Cardiac vascular crises may be supposed to account for the cases of fatal angina pectoris without marked narrowing of the coronary arteries. The gastric and other crises occurring in tabes are very possibly to be accounted for in the same way.

In the present case there are three sets of data supporting the hypothesis of vascular crisis: (a) The curious urinary changes which strongly suggest the "urina spastica" seen in vasomotor affections and hysteric states; (b) the swelling of the abdomen during attacks; and $(c)$ the association with dyspnea.

I have already stated that it is impossible to exclude tabes in this case. Were that the correct diagnosis, the mechanism by which the attack was produced would be the same as under the hypothesis of vascular crisis without the other lesions of tabes.

Outcome.-He died of pneumonia at the end of a month; the autopsy showed arteriosclerosis with hypertrophy and dilatation of the heart. The celiac axis and the coronary arteries were only slightly involved in the arteriosclerotic process. No tabes.

Diagnosis.-Arteriosclerosis; vascular crises.

\section{Case 85}

A school-boy of ten entered the hospital January 28 , I908, on account of epigastric pain which came on night before last after a supper of pork and beans with cheese. It has prevented him from sleeping since then.

He says that it feels as if some one had punched him in the stomach. Breathing or gaping gave pain at this point and in the left axilla. $\mathrm{He}$ has almost constant nausea, and has been feverish since yesterday morning. He has a brother who has been treated at the Children's Hospital for tuberculosis of the knee.

At entrance his temperature was $102.4^{\circ} \mathrm{F}$; pulse, 98; respiration, 30, and accompanied by a grunt. He now complains of pain both in the epigastrium and at the top of the sternum. The car-

\footnotetext{
1 For Pal's account of these crises see p. 30.
} 



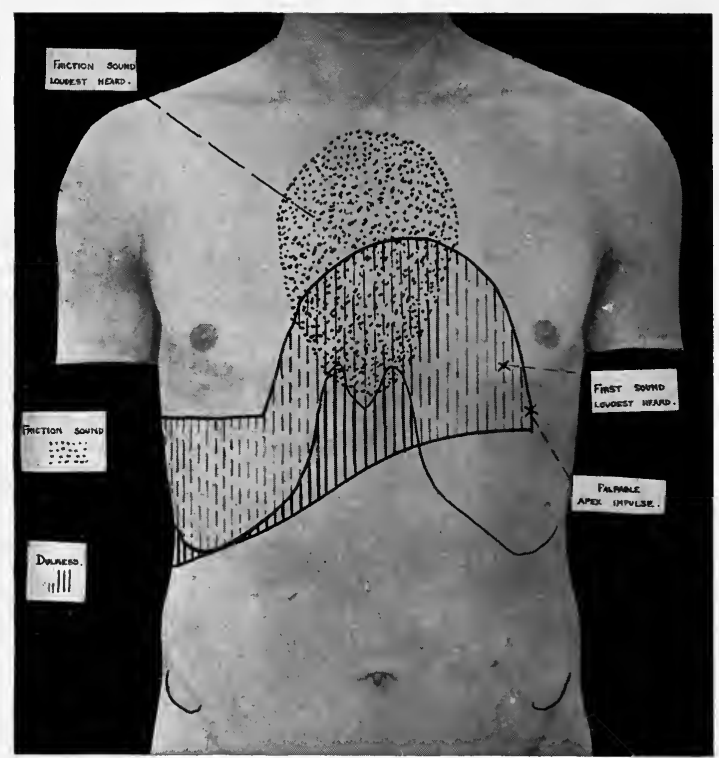

Fig. 3r.-Friction-area and percussion outlines in Case 85. Chief complaint is epigastric pain. 
diohepatic angle is obtuse, and over the area shown in the diagram (Fig. 3I) there is a to-and-fro friction sound, loudest in the second right interspace. Physical examination is otherwise normal. The white cells number 9600 ; the urine is negative. The day after entry the friction-rub disappeared and the temperature fell to normal on the second day. On February 5 th he was playing about the ward, and a fairly loud systolic murmur was heard at the apex and in the axillæ. The cardiohepatic angle was now acute.

Discussion.-Some digestive disturbance is naturally the first explanation which occurs to us, since the symptoms followed so immediately upon the taking of a heavy meal; but a simple digestive upset of this kind would not account for a temperature of $102.4^{\circ} \mathrm{F}$. forty-eight hours after. In all probability the digestive upset was a result, not a cause, of the present trouble.

Tuberculosis of the spine is said to be associated with epigastric pain, such as is here present, and the presence of tuberculosis in the patient's brother makes it proper for us to consider this disease seriously. There is nothing, however, in the physical examination to support any such idea-no spasm of the erector spinæ group and no prominence or tenderness of any vertebra; nor are there any indications of tuberculosis elsewhere.

With these two alternatives excluded and with due regard for the results of the physical examination the only reasonable diagnosis is pericarditis. Indeed, the diagnosis could hardly have been missed except by reason of the all too common error-the failure to look for it.

Outcome.-It was learned subsequently that when the patient was three years old he had considerable pain and weakness in his legs, accompanied by fever. Recovery was uneventful.

Diagnosis.-Pericarditis.

\section{Case 86}

A brass-finisher of fifty-six entered the hospital on January 30, I908, with a negative history up to eight weeks ago, although he had been in the habit of taking about five drinks of whisky a day for a good many years. Eight weeks ago he began to have abdominal pain, worst in the pit of the stomach. This pain is sharp and piercing, almost constant of late, keeping him awake at night. For the past week or two it has run up under the left costal margin at times. There have been no vomiting and no belching, but he has gradually lost his appetite entirely. Food does not affect the pain in any way. His weight has fallen $4^{2}$ pounds in three months. His bowels are regular. 
On physical examination temperature, pulse, and respiration are normal, likewise the lungs. The heart shows no evidence of enlargement, and its sounds are regular and of good quality. At the apex there is a faint systolic murmur transmitted to the axilla, heard also in the pulmonary area and more faintly in the aortic area. All over the precordia and over the left pectoral is heard, during inspiration alone, a faint, grating, systolic sound, loudest in the third space and anterior axillary line. In the fourth space, near the left edge of the sternum, is heard a crackling systolic sound not affected by respiration. The aortic second sound is considerably accentuated; the artery walls are somewhat thickened. Examination of stools shows nothing remarkable, the guaiac test being negative. The stomach was found to hold 76 ounces of water. The lower border descended $\mathrm{I}_{4}^{\frac{3}{4}}$ inches below the navel. After a test-meal the gastric contents showed no free hydrochloric acid and no lactic acid; the guaiac test was negative.

Discussion.-Whisky is so old a friend of this patient that it is not likely to begin to disagree with him in his fifty-sixth year. Probably it has nothing to do with the symptoms in this case.

Peptic ulcer might produce such pain, and is perfectly consistent with the loss of 42 pounds' weight in two months. But the lack of appetite, the entire absence of vomiting and belching, and the short duration of the symptoms make this unlikely.

What are we to make of the curious signs in the chest? Have they anything to do with the symptoms complained of? Inspiratory systolic sounds, absent during expiration, and best heard along the margins of cardiac dulness, constitute the commonest type of so-called cardiorespiratory murmur. The phenomenon has no clinical significance except that in a considerable proportion of cases it is found to be associated with pleural or pleuropericardial adhesions, which may be due to tuberculosis. The same may be said of systolic crackling sounds, which occasionally mystify the practitioner.

It is well to make it a rule always to hunt for evidence of gastric sancer when a patient past forty comes to us with a recent and unexplained history of gastric symptoms, mild or severe. Errors in diet, worries, and such causes are not apt to take effect for the first time after a person has lived fifty-six years. If gastric symptoms are due to any cause other than cancer, careful questioning of the patient will usually prove that they have existed at intervals for years. In the nresent case the evidence of enlargement of the stomach and the absence of hydrochloric acid from the gastric contents are chiefly of confirmatory value as evidence, the history being the important thing. 
Very characteristic of gastric cancer is the gradual but complete loss of appetite in this case. On the other hand, the absence of vomiting and of any relation between the pain and the taking of food is rather unusual.

Outcome.-His symptoms were somewhat relieved by Io grains of orthoform, given four times a day, and 15 minims of dilute hydrochloric acid, given twenty minutes after each meal.

The patient died on March I5th. Autopsy showed cancer of the stomach.

Diagnosis.-Gastric cancer.

\section{Case 87}

A bricklayer of fifty-two entered the hospital April 7, 1908, with a diagnosis of gall-stones. His family history and past history were negative; his habits good. For three months he has complained of pain in the epigastrium, not severe, but worse after eating, and usually radiating to the right back. For six weeks he has noticed white stools, dark urine, and jaundice. Throughout this time, however, his appetite has been good, and he has had no vomiting.

On physical examination he was found to be deeply jaundiced, his lungs hyperresonant in front, with slightly prolonged expiration. Over the sacrum was a soft, flattened, subcutaneous prominence the size of a dollar. Nothing else was detected on physical examination, abdominal palpation being unsatisfactory, owing to constant rigidity. On April IIth the abdomen became less resistant, and an indefinite mass was felt in the region of the gall-bladder. A' stomach-tube was passed, and the capacity of the organ was found to be 42 ounces of water, the lower border extending to a point one inch below the navel. No contents were found in the fasting stomach. After a test-meal, however, hydrochloric acid was found to be 0.09. Lactic acid test and guaiac test were negative.

Discussion.-Excluding congenital cases, an afebrile, persistent jaundice usually presents to us the problem of deciding between three causes:

I. Gall-stones and their effects.

2. Cancer, either of the pancreas or bile-ducts, occasionally of the liver itself.

3. Cirrhosis.

Hepatic syphilis is considerably less frequent as the cause of longstanding jaundice, and the duration is here assumed to be sufficient to exclude the acute infectious and the catarrhal type of jaundice. 
Against gall-stones in the present case is the intensity of the jaundice without variation in six weeks, the absence of colic, and the presence of a mass in the region of the gall-bladder. Long-standing jaundice due to gall-stones is usually associated with a normal sized or contracted gall-bladder (Courvoisier's law). It is quite possible, however, that the mass in the region of the gall-bladder is not due to distention of that viscus.

Cirrhosis almost never produces an intense degrce of jaundice. The coloration is slight or moderate. It is rarely associated with pain, and usually produces either enlargement of the liver or some evidence of portal stasis.

Cancer then seems the more likely diagnosis; whether it is of the pancreas or the bile-ducts we have no means of determining. That it is probably not in the liver itself is to be argued from the absence of gastric symptoms and of objective manifestations of gastric disease.

Outcome.-Operation, April 23d, showed moderate enlargement of the liver, distention of the gall-bladder, and a mass of hard, apparently cancerous, tissue in the region of the pancreas. The patient made a good recovery from the operation.

Diagnosis.-Pancreatic cancer; [chronic pancreatitis](?).

\section{Case 88}

A chef of thirty-two entered the hospital on April 8, I908, with the statement that his mother had died of a "complication of diseases"; his father had had a persistent cough for four years; one brother hari died of consumption at the age of twenty-four, and a sister died of "rectal abscess" at the same age. The patient had been exposed to tuberculosis.

Ever since he was nineteen years of age he has had attacks called epilepsy. These have always come during sleep, and do not awaken him. In the morning he wakes with a headache and general pains, usually finding that he has bitten his tongue. At first these attacks came about once a month; now they come only about once in six months. Nevertheless, he was well and strong until four years ago, when he vomited about four quarts of fluid. Following this he was sent to a hospital for tuberculosis and remained there six months, although, so far as he knows, he has never had a cough and nothing abnormal has been found in his lungs. Two and a half years ago he weighed I60 pounds; now he weighs I 37 pounds. His habits are good.

For the past six weeks he has been treated for abdominal pain not ciosely localized. Four days ago he vomited a few times, and this vomit- 
ing has persisted and rather increased since then. In the last two days he has vomited up about two quarts of dark-brown material, together with some food which he thinks was eaten at least twenty-four hours before. His pain is now most severe in the epigastrium and under both costal margins. It is sometimes relieved by vomiting, and is never worse after eating. Yesterday he noticed palpitation for the first time. $\mathrm{He}$ has a good appetite, but has had some constipation for three weeks.

Physical examination was negative except for slight tenderness in the left epigastrium and under both costal margins. His vomitus was found to contain free hydrochloric acid, and the guaiac test was positive, both in the stomach-contents and in the stool. Despite careful diet, he continued to vomit and have pain.

Discussion.-Abdominal symptoms of any kind, when occurring in a patient with so strong a tuberculous history, compel us to make a most searching examination for evidences of tuberculous peritonitis. This is true even when the onset is much more acute than in this case. ${ }^{1}$ But in the absence of fever and of all the local manifestations of tuberculous peritonitis (free fluid, generalized tenderness, spasm, and tumorlike masses) this disease may be excluded.

Is it possible to connect in any way the history of epileptiform attacks with the present symptoms? Such attacks might be due to cerebral syphilis, and the same disease attacking the liver and spleen might now produce acute abdominal pain. But in the absence of any enlargement of the liver or spleen, and without fever, anemia, or other lesions pointing to syphilis, we have no good reason for considering this disease seriously.

In the treatment of cases characterized by pain and vomiting I have often been misled so as to forget the possibility of chronic intestinal obstruction-misled, I mean, by the prominence of symptoms apparently referable to the stomach. Especially when there is constipation, as in the present case, this possibility should never be lost sight of; but it must remain a mere possibility unless there is other evidence to support it. In the present case the positive guaiac test in the stool is all that we have in the way of physical signs favoring obstruction. In the absence of tumor, visible peristalsis or intestinal noise, chronic obstruction deserves no further consideration.

If, then, the symptoms are of gastric origin, as seems, on the whole, most probable, there are but two diseases deserving serious consideration-cancer and ulcer. In the absence of alcoholism and of any other cause for chronic congestion of the stomach (heart disease, cirrhosis),

${ }^{1}$ As an illustration of the acute onset of symptoms in tuberculous peritonitis see p. 435 . 
cancer and ulcer are the only diseases likely to produce hemorrhage both from the stomach and the bowel, associated with persistent vomiting and epigastric pain. This likelihood is increased when the patient fails to improve after careful dieting.

Against cancer is the fact that the patient is relatively young, has no steady gastric stasis, and especially the persistence of a good appetite. The presence of free hydrochloric acid is also somewhat against the diagnosis of cancer. On the whole, peptic ulcer, gastric or duodenal, is the best working diagnosis.

Outcome.-On the fifteenth of May his stomach was opened and a puckered scar found on the posterior wall of the stomach. Posterior gastro-enterotomy was done. The patient did well.

Diagnosis.-Gastric ulcer.

\section{Case 89}

A waitress of twenty-eight entered the hospital on May 5,1898 . She said that she had "malaria of the stomach" seven years ago, and was sick for three days with fever and chills. At that time she had no vomiting and no pain, and has otherwise been well except for occasional "chills," until three years ago, when she began to have a gnawing in the stomach, coming immediately after eating and followed by gastric distention and belching, which continues until about two hours after eating. This belching has been worse for the past year. At times enormous quantities of gas are expelled with much noise. For relief from the gnawing sensation she sometimes makes herself vomit, the vomitus usually consisting of about half a pint of white phlegm in which she has several times seen specks of blood. Her appetite has been good and her bowels regular.

Physical examination shows a very marked pulsation near the navel; over it a thrill is felt and a systolic murmur heard. There is slight tenderness in the center of the epigastrium. The examination revealed nothing abnormal.

The guaiac test in the stool was negative. She was put on a diet of carbohydrate and fats, with a diagnosis of gastric neurosis, and was at once relieved of her symptoms.

Discussion.--Any one who had the opportunity to hear the thunderous noise with which this patient expelled gas from the stomach would be strongly biased, I think, toward a diagnosis of gastric neurosis, for these explosions are almost always preceded and brought about by the habit of "cribbing," or swallowing air, which in turn is usually the result of gastric neurosis. The most important question is, can we exclude pep- 
tic ulcer? Many of the symptoms suggest this disease, and the patient's neurotic constitution by no means excludes it. On the other hand, it is unusual for the patient to be relieved of pain and other gastric symptoms at a time when the stomach is empty. Though many gastric ulcers exist without producing hemorrhages, it would be impossible, I think, to make a diagnosis of ulcer in this case unless hemorrhage occurred. 'The specks of blood in the vomitus are, of course, of no special importance, and the epigastric tenderness has no diagnostic value.

The thought of aneurysm is apt to disturb both doctor and patient, when, as in the present case, abdominal pain is associated with a marked pulsation, palpable thrill, and systolic murmur near the navel. The evidences by means of which aneurysm may be excluded in this and similar cases have already been fully discussed on p. I44. Malaria was considered in the diagnosis of this case, but a careful temperature record enabled us to exclude it. The diagnosis remained in doubt, gastric ulcer and gastric neurosis being the main alternatives.

Outcome.-On June 6th she was once more on house diet, up and about the ward, and seemingly quite well. With care about diet and an improved environment, the patient has continued well up to the present time (I9I0).

The continued good health, after so short a period of treatment, seems to me to argue strongly against ulcer.

Diagnosis.--Gastric neurosis.

\section{Case 90}

A Jewess of thirty had been operated on, July 30, 1900, for cholecystitis. The gall-bladder was drained. After this operation she remained well, and has had three children. She entered the hospital March I3, I907, complaining of epigastric pain of two years' duration, coming at irregular intervals, and worse after eating. For the past month the pain has increased in severity and has radiated to the back, but not to either side; it often awakens her at night. Her bowels are constipated, and she has eaten little for the past four weeks, though her appetite was previously good. She has lost much strength, and for the past four days has remained in bed.

At entrance and thereafter her pulse ranged most of the time above 90 , and not infrequently reached $\mathrm{I} 20$. Her evening temperature was usually above $99^{\circ} \mathrm{F}$., but below $100^{\circ} \mathrm{F}$.

Physical examination showed nothing abnormal in the chest. There was general abdominal rigidity, especially above the navel, where there 
was marked diffuse tenderness. The white cells were 27,000 at entrance, and 88 per cent. of these cells were polynuclear. Three days later the tenderness was gone, and the leukocytes were found to be normal; they remained so thereafter. The urine was at all times negative.

Examination of vomitus showed free hydrochloric acid in abundance, and a positive guaiac test for blood was obtained. Tube examination was negative. In the stool the guaiac test was twice negative. The patient complained of marked abdominal pain, but obtained great relief from the subcutaneous injection of sterile water.

The patient was treated by careful feeding, the administration of $\frac{1}{4}$-grain doses of cocain, and dram-doses of Hoffmann's anodyne for gastric distress. On one or two occasions $\frac{1}{6}$ grain of morphin was administered. Nutrient enemata were tried, but were always expelled within a short time. The patient took liquids well after the first few days and was, for the most part, free from pain and vomiting.

Discussion.-The symptoms seem to be very much the same as those previously proved to be due to cholecystitis. Since the gallbladder was drained, and probably, for the most part, obliterated, it is unlikely that there is any return of inflammation at that point, especially as she seems to have had five years of freedom from symptoms. The same considerations, however, lead us to wonder whether adhesions may not have formed in the vicinity of the gall-bladder, resulting in gastric stasis and precipitating the attacks of pain. The absence of any gastric stasis, however, as evidenced by the stomach-tube examination, makes this supposition less likely.

The local signs at the time of entrance and the leukocytosis point rather toward a local peritonitis, possibly from a gastric ulcer. Were this the case, however, we should not expect the disappearance of all these signs within three days. One cannot help being influenced by the fact that this patient's pain was greatly improved by the "lie cure" (injections of sterile water, mistaken by the patient for morphin).

Chronic appendicitis has not been yet extensively discussed in this book, for the reason that I find it hard to arrive at any very definite conclusion upon the subject, but certainly this case is very similar to those which surgeons are accustomed to operate on with that diagnosis. The childhood attacks often seen in chronic appendicitis are not here mentioned. There was at no time any local tenderness or spasm in the right iliac region, nor any radiation of pain to that region. Nevertheless, it is certainly true that cases no more typical than this have been relieved of all symptoms after the removal of an adherent, kinked appendix. In this connection I wish to call attention to the article by Dr. E. A. 
Codman in the Boston Medical and Surgical Journal of October 2, I913, an article the great value of which seems to me to have been insufficiently appreciated. Dr. Codman there demonstrates to my satisfaction that the diagnosis of chronic appendicitis is impossible. He points out in the first place that competent pathologists generally deny the existence of any. such entity, while admitting that many cases of healed acute appendicitis are recognizable postmortem. He points out further that in roo consecutive abdominal sections performed for various diseases at the Massachusetts General Hospital, " $7 \mathrm{r}$ showed definite lesions of the appendix, although no such preoperative diagnosis was made from the history" or from the physical examination. He shows also that in 98 cases diagnosed by him as chronic appendicitis, only 6r showed any evidence of disease in the appendix at the time of operation. In other words, the lesion existed in $7 \mathrm{I}$ per cent. of cases in which it was not diagnosed and only in 6I per cent. of the cases in which it was diagnosed. Moreover, in 50 out of these 6r cases there was a history of definite acute attacks on which to base the diagnosis, not of chronic appendicitis but of a healed or healing appendicitis. Omitting these, we have left but I I per cent. correct diagnosis.

In 17 cases operated with a diagnosis of chronic appendicitis the real cause of symptoms as determined after operation was as follows:

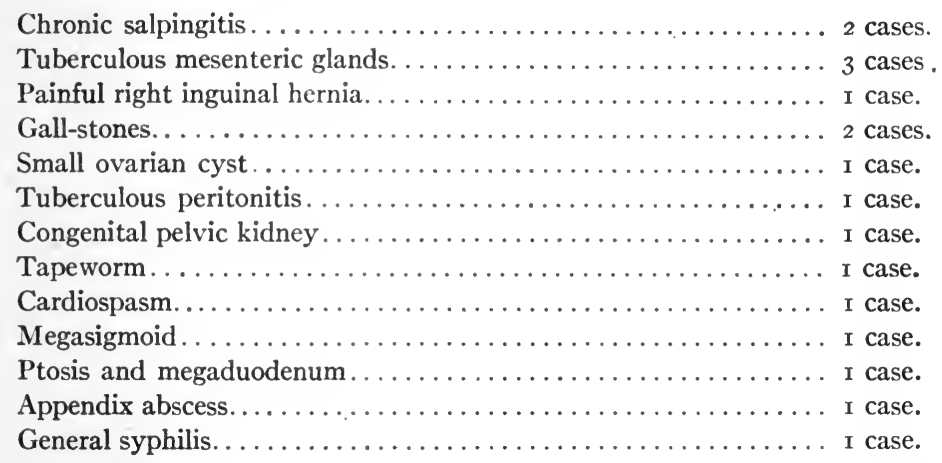

Six of these I 7 cases also showed evidence of a healed appendicitis. In 26 of the 98 cases diagnosed as chronic appendicitis, the appendix was normal at operation.

During the period covered by these studies Dr. Codman and his colleagues had also operated on an equal number of cases of abdominal disease under diagnoses other than chronic appendicitis, and yet at operation found nothing wrong in the abdomen except a so-called "chronic appendicitis," i. e., healed appendicitis. 
Dr. Codman further shows that no symptom or group of symptoms such as have been supposed to be characteristic of chronic appendicitis was present in these cases which were proved at operation to have a "chronic" or healed appendicitis. Hyperacidity symptoms were present in only $\mathbf{I} 2$ of the whole number.

Against Dr. Codman's well-marshalled disproof of the diagnosability of chronic appendicitis, the chief argument raised is that there are many patients who have had a variety of abdominal symptoms cured by the removal of a shrivelled, stenosed, or kinked appendix. This line of argument I believe to be fallacious. Similar arguments were used in support of the now generally disused operation for the fixation of a movable right kidney, also for the obsolete practice of removing adenoids as a cure for enuresis.

After very careful study of Case 90 we were unable to arrive at any definite diagnosis. We could not definitely incriminate the stomach, the gall-bladder, or any other viscus, yet we were by no means certain of the absence of severe disease calling for surgical interference. Accordingly, on March 24th the abdomen was opened, but careful search revealed no disease of any kind. The patient made an uneventful recovery.

Diagnosis.-Gastric neurosis.

Apropos of Case 90, I have received the following very interesting letter from Dr. Walter U. Kennedy, Professor of Surgery at the National University, St. Louis, Mo.:

"Why is cholangitis here excluded in favor of gastric neurosis? May a coincident pain, rigidity, rise of temperature, and leukocytosis be disregarded? Some years ago I pointed out as an objection to the prevailing type of cholecystotomy that if the bladder be sutured to the wall, there is a possibility of such later traction on the common duct (since it is the flexible attachment of a structure fixed at one end) as regularly or intermittently to lessen the lumen of the common duct; result, stasis and its sequences.

"Might you not have had stasis with infection from the above or other cause, and yet at operation no macroscopic evidence differing from the conditions apparently referable to the first operation? And if such could occur, would not a happy drainage clear up the matter? I have seen a distended gall-bladder giving typical symptoms empty itself after the abdomen was open and the patient had an unusually severe attack of vomiting. Nothing was done to the bladder and the patient recovered speedily from his pain and jaundice. Repeatedly I have done secondary operations wherein the gall-bladder had 
been attached directly to the wall, and histories quite similar to your Case 90 be found. In those, removing the bladder mass, or at least the attachment, has given relief."

In comment I would merely say that Prof. Kennedy's opinion is better than mine, and that I think he is very probably right.

\section{Case 91}

A dressmaker of twenty-three, whose mother died of cancer of the stomach, was seen January 28, 1907. She admitted that for a year she had taken a great deal of beer, wine, and whisky, and for the past two weeks she had taken from a pint to five pints of whisky a day. During this last period she had eaten practically nothing, and has been in bed most of the time. A few days ago, whenever she closed her eyes, she saw big animals and other apparitions. For the past three days she has vomited almost constantly, and had some epigastric pain, which has become more severe during the past two days, especially when she breathes deeply. Last night her respiration was very difficult and shallow in consequence. There has never been any blood in the vomitus.

Temperature, pulse, and respiration were normal, the left pupil considerably larger than the right, but both reacted normally; a heavy brownish coat was found on the tongue, and a marked tremor in the fingers. 'The chest showed nothing abnormal. The abdomen was rigid and tender throughout; exquisitely so in the epigastrium. Liver dulness was not increased, and there was no shifting dulness in the flanks.

Discussion.-The chief problem in this case is to decide whether the alcoholism from which she is suffering will account for all the symptoms. We are not accustomed to associate extreme abdominal tenderness and rigidity with delirium tremens or with simple alcoholism. On the other hand, if perforative peritonitis (stomach, gall-bladder, appendix) were present, there should be some rise of temperature, pulse, respiration, or leukocyte count, none of which occurred. There is nothing in the case to justify the suspicion of lead-poisoning, tabes, chronic intestinal obstruction, passive congestion of the liver, pericarditis, pneumonia, or any of the other causes of epigastric pain which have been discussed on previous pages.

Is it possible that the symptoms may be due merely to the excessive vomiting, with the wrenching strain thereby brought upon the abdominal muscles? We decided to take our chance of this diagnosis, and planned our treatment accordingly. 
Outcome.-The next day the pain was much less, likewise the tenderness and tremor, and there has been no vomiting. By February $3 \mathrm{~d}$ she was entirely free from complaints, and on the sixteenth she left the hospital well.

Her treatment consisted of milk diluted one-third with lime-water, 4 ounces every two hours when awake; orthoform, to grains, every four hours; hot stupes to the abdomen every hour when awake; whisky, $\frac{1}{2}$ ounce every four hours; triple bromids, 30 grains, and tincture of capsicum, I5 minims before meals. After the first two days the whisky was omitted. The other medicines were not needed after the thirteenth.

Diagnosis.-Alcoholism.

\section{Case 92}

A teamster of forty-eight entered the hospital August 12, I907. Gastric ulcer and abdominal aneurysm were the diagnoses suggested by the out-patient physician. The family history was not remarkable, except that one sister has been in the Worcester Insane Asylum. The patient's habits and past history are good. Ten weeks ago he began to have steady epigastric pain, usually dull, sometimes sharp. After two or three days he had to give up work on account of pain and weakness, but he has not been in bed for the whole ot any day. Previous to this illness he has never had pain of this sort. It is worst about one hour after eating, but it is not relieved by food, and does not radiate to any other point. During the same period he has also had aches and darting pains in his neck, legs, and the right side of his chest. For the past two or three weeks he has felt sleepy and nervous in the daytime, while at night pain and nervousness have often kept him awake. He has headache during most of every morning. The last four or five weeks he has been short of breath, but has noticed no swelling of his feet. His bowels move only once in four days. His appetite is poor, but he has not vomited.

On examination he seems to be emaciated. The chest shows nothing abnormal. The abdomen is decidedly concave and somewhat tender in the epigastrium, where there is marked pulsation visible and palpable from a point two inches below the sternum to a point one inch below the navel. Otherwise physical examination is negative, and the blood, urine, and temperature-chart indicate nothing abnormal. The patient was depressed, seemed very apathetic, and at times refused nourishment. The stomach-tube proved that the stomach held 30 ounces of water and showed no evidence of enlargement. After a test-meal the extracted contents showed free $\mathrm{HCl}$, o.I2 per cent., no lactic acid, no blood. 
Discussion.-Although abdominal aneurysm was considered in this case, the physical signs are clearly those of dynamic aorta, the differential diagnosis of which has been already discussed. (See p. 144.)

Gastric cancer is always a threatening possibility when a man of forty-eight begins to have digestive symptoms for the first time in his life. The emaciation present in this case lends support to this hypothesis, and the negative results of examination by the stomach-tube do not enable us positively to exclude cancer. We will return to the discussion of it below.

Peptic ulcer does not cause pains so wide-spread as those here described. If this were the diagnosis, we should expect also some relief after food, and very possibly some blood in the stomach-contents. Yet while ulcer would not account for all the facts here present, we must hold judgment in reserve regarding it, as we have already done regarding cancer.

Could the symptoms be explained as the result of simple constipation combined with starvation which his emaciation suggests? Very possibly they may, but we still require some reason for the sudden appearance of constipation in a healthy teamster of forty-eight.

We cannot afford to leave out of consideration the psychic symptoms in this case. A middle-aged laboring-man does not begin to be sleepless and nervous without obvious cause. The ordinary cause for such symptoms is alcoholism, which could be definitcly excluded here. In view of the patient's depression, his persistent headaches, his nervousness, insomnia, and apathy; a mild type of insanity (depressive maniac psychosis) seems probable, especially since no cause for his depression can be found in any of the recent events of his life. Assuming this to be true, the question remains: Can the abdominal symptoms, the anorexia, and emaciation be thus explained? To this it is to be answered that in sanatoria and asylums for the insane it is a very common experience to find the foreground of the clinical picture occupied mainly by gastro-intestinal symptoms almost as severe as those seen in organic disease. The further course of these cases, however, demonstrates the absence of any such disease, and leads us to the conclusion that the gastro-intestinal symptoms are simply one item in the symptom-complex called insanity.

Assuming then that this patient is mildly insane, we are justified in supposing that his stomach symptoms are dependent upon this psychosis, even though, were he normal mentally, we should be strongly inclined to believe that he had gastric ulcer or cancer.

Outcome.-The patient became more and more depressed. Two 
special consultants pronounced the case simple melancholia, and he was removed to an asylum.

Diagnosis.-Melancholia.

\section{Case 93}

An Italian laborer forty years old had "rheumatism" five years ago and one year ago. Many joints were swollen, painful, and tender for a few weeks in each attack, but he has regained perfect function in all the joints.

He takes two whiskies before breakfast and four beers during the day. Denies venereal disease.

For six weeks he has had gnawing pain in the epigastrium and right hypochondrium, gradually getting worse, sometimes disturbing sleep, but never influenced by food. Nocturia, I to 3 times.

Physical Examination.-The cardiac impulse extends I cm. outside the nipple in the fifth space. No enlargement to the right is detected. Cardiac action regular-8o per minute; the apex first sound is replaced by a long, blowing murmur, which is also audible in the left axilla. At the third left costal cartilage is the maximum intensity of a diastolic murmur, which is also faintly heard in the second right interspace. The pulmonic second sound is accentuated.

All the superficial arteries pulsate strongly, and there is a "Corrigan" and capillary pulse.

Nails slightly incurved. Lungs negative.

In the upper right abdominal quadrant is a mass easily felt bimanually, descending over an inch on full inspiration, with a rounded edge and a semifluctuant consistence. The liver dulness extends $8.5 \mathrm{~cm}$. below the ribs (nipple-line) and $12.5 \mathrm{~cm}$. below the ensiform. Whether or not the liver is continuous with the mass described above cannot be certainly determined. The liver edge is sharp on the left of the median line, but cannot be felt distinctly on the right.

The spleen is palpable $2 \mathrm{~cm}$. below the ribs. Abdomen otherwise negative; likewise the rest of the body. Urine, 40 ounces; specific gravity, то2 I. No albumin, pus, blood, or casts. Blood normal.

Cystoscopy showed evidence of normal functioning in each kidney.

Discussion.-Clearly enough this patient has incompetence of the aortic and mitral valves, presumably of rheumatic origin. The interesting problem remaining concerns the mass in the right hypochondrium. Is it liver, kidney, or retroperitoneal tumor?

The alcoholic history may have produced a cirrhosis, but cirrhosis rarely causes pain, and the cirrhotic liver is hard, not semifluctuant. 
Moreover, we do not expect to feel the liver bimanually, though that is by no means impossible. There seems reason to believe that the liver is enlarged in this case, but apparently there is something else wrong.

A mass palpable bimanually in the right flank usually turns out to be connected with the kidney, and it was with this in mind that cystoscopy was done. The results of this examination go far toward excluding renal disease, and were interpreted in this sense.

Tumors of the retroperitoneal glands produce not infrequently a mass like that here described. Diagnosis of such tumors, however, is impossible unless there are more definite pressure symptoms (pain in the back and legs), or unless there has been malignant disease elsewhere in the body, with possible metastasis in the region now under consideration.

Syphilis of the liver and cancer of the liver or colon would not account for so soft a mass as is here described. Is it possible that simple passive congestion due to the cardiac lesion might produce so soft an enlargement of the liver? Against this is the absence of much stasis in the lungs, legs, or abdominal cavities, and the fact that the questionable mass cannot with certainty be connected with the liver edge palpable to the left of the median line. A surgical consultant considered the symptoms due to a tumor of the gall-bladder or of the kidney. On the whole, there seems to be enough doubt upon this point to justify exploratory laparotomy.

Outcome.-Laparotomy showed the kidneys and gall-bladder to be normal. A large, dark, congested liver was the only finding.

This case seems to me to be of unusual interest, since it shows that passive congestion of the liver is one of the items which must be seriously considered in a diagnosis of diseases involving the right upper quadrant. So far as I am aware, this is one of the few cases on record in which laparotomy has been done for passive congestion of the liver.

Diagnosis.-Hepatic congestion.

\section{Case 94}

A private secretary, sixty years old, entered the hospital March 2, 1907. Her father died of consumption. She had diphtheria at twelve. Twenty-five years ago she had inflammatory rheumatism and ophthalmia, was in bed a week, and has had a slight similar attack since. In the past thirty years she has had about twelve attacks of colic, characterized by sudden painful cramps in the abdomen. The last attack was in July, I906. Ten years ago an appendix abscess was opened and drained. She has never been jaundiced, but always has had a strong tendency to con- 
stipation. Her best weight was 182 pounds six months ago. Six weeks ago she had several attacks of indigestion within a week; after this she was well until four weeks ago, when she had a sudden severe attack of epigastric pain lasting an hour. She has had five or six similar attacks since, most of them coming after breakfast and lasting several hours until relieved by morphin.

The pain does not seem to radiate in any direction. For three days she has been jaundiced.

Physical examination showed an obesity and a marked jaundice, but was otherwise negative. By the sixth of March the jaundice had cleared up and the patient was comfortable except for slight sore throat.

Discussion.-Since tuberculous peritonitis may manifest itself for the first time with symptoms as acute as those here present, it deserves a moment's consideration, especially in view of the tuberculous family history. But there are no physical signs corresponding to this disease, and in the absence of fever it need not be further discussed.

Attacks of abdominal pain in a patient who has no knee-jerks should always remind us of tabes, yet there are no other confirmatory facts, and it is quite possible that the diphtheria which the patient passed through at the age of twelve may have produced a neuritis which accounts for the loss of knee-jerks.

In elderly persons with a strong tendency to constipation we need no further explanation for many uncomfortable abdominal symptoms; but constipation practically never produces pain so sharp as to require morphin unless, indeed, it be due to organic obstruction. Her age and the character of the pain are quite consistent with this diagnosis, and experience has shown that intestinal obstruction is always a serious danger for those who have been operated upon for appendicitis, especially if the formation of adhesions has been favored by drainage of the wound. But if the intestine were obstructed, we should expect distention and vomiting, while the attacks of pain would probably not occur so frequently and at such short intervals.

Peptic ulcer is, as in so many cases, a possibility impossible to exclude, but the presence of jaundice, the sudden relief by morphin, and the absence of any definite relation between the pain and the taking of food turn our attention rather to gall-stones. Since the appearance of the jaundice this diagnosis has been tolerably obvious. It is favored by the age and sex, the obesity, and the character of the pain.

Outcome.-The abdomen was opened on the ninth of March, and showed a small gall-bladder completely filled with stones.

Diagnosis.-Gall-stones. 


\section{Case 95}

Mrs. H., a widow of seventy, was seen in consultation November 8, I90I. Her mother died of old age at eighty-one; her father of diabetes at sixty. Three sisters died of pulmonary tuberculosis; one from an accident; one of unknown cause; one is still living.

Mrs. $\mathrm{H}$. has had ten children: By first husband, eight; two of these died of pulmonary tuberculosis, one of "dropsy"; one daughter died from "effects of a surgical operation"; three died in infancy, cause unknown; one living. The two children by her second husband are living and well.

She had the usual children's diseases, but otherwise was always well until 1890 , when she had strangulated hernia and was operated upon. During the following year she did not feel well, had fever, chills, vomiting, and pain, and in I89I was operated for right empyema. This discharged for six months, but finally healed. Since that time she has complained of dyspepsia, sour, bitter eructations, dull pain in epigastrium, headache, malaise, and gradual loss of flesh-about 20 pounds in all.

In May, I90I, she had an attack of severe pain in the epigastrium, midway between umbilicus and ensiform; the pain was relieved by hot drinks. A month later had a similar attack; a physician was called, who said it was acute neuralgia of the stomach. He gave her something to make her vomit, and she vomited for twenty-four hours almost continuously, the vomitus consisting mostly of "green, bitter stuff." She had a similar attack September I, I90I, relieved by hot drinks. There was some vomiting in this attack. Next attack, September 8th; then, September I4th; the last two relieved by morphin, $\frac{1}{4}$ grain. The final attack about October Igth. This last attack was the most severe. Between attacks patient was fed on liquids and semisolids, and complained of no pain or indigestion. The pain seemed to start at a spot in the right back on the level of the sixth or seventh rib, radiating straight forward to "pit of stomach," thence down the left side of the belly. There was nothing to be seen at this dorsal spot, but it was painful to touch. After receiving a subcutaneous injection of morphin, she began to vomit and continued to vomit about every half-hour for thirty-six hours. She became very weak, but had a normal temperature and a pulse of 60 . She passed but little urine during this thirty-six hours, but at the end of it she voided nearly two quarts. Examination of this urine showed specific gravity I022, color high, about O.I per cent. of albumin. Sediment contained few hyaline and fine granular casts, with fat- 
drops adherent. Aspecimen of urine sixteen hours later was smoky, contained o.I albumin, and in addition to sediment in previous urine was full of blood and calcium oxalate crystals. The patient now complained of pain in both flanks and soreness all over abdomen, especially on right side. The temperature now is $100^{\circ} \mathrm{F}$. and pulse 88. There is headache. Blood-pressure, I45. No jaundice now or in any of these attacks, but the patient says she always looked a little yellow.

She is a well-preserved lady, rather fat. Liver normal in size. A point of extreme tenderness is situated half-way between ensiform and umbilicus. Heart and lungs negative. Colon distended with gas. The sclera near the iris is clear blue. On drawing back the eyelid a slight tinge of yellow is visible at the periphery.

Discussion.-Intestinal obstruction is naturally our first thought when a patient complains of acute abdominal symptoms with persistent vomiting, and has previously had an operation for strangulated hernia. But in this case there is no abdominal distention, no constipation or diarrhea, no visible peristalsis, and an unusual degree of comfort between attacks.

When a patient is relieved as markedly as in this case by the taking of hot drinks, gastric flatulence with pyloric spasm seems a natural explanation. But this symptom in practically all cases is dependent upon some deeper cause, such as peptic ulcer or gall-stones. The long history of dyspepsia leading up to sharp attacks of pain is consistent with either of the above diagnoses, which will be further discussed below. One of the confusing elements here is the condition of the urine. Can the symptoms be due to uremia, which is traditionally supposed to lead to attacks of abdominal pain in certain cases? The urine does not suggest acute nephritis, and if any type of chronic nephritis were present, there should be hypertrophy of the heart and a higher blood-pressure. In all probability, therefore, the urinary findings are to be explained as the result of some toxic irritation of the kidney, and are not of any serious significance. In one of the later examinations the presence of macroscopic blood in the urine is noteworthy as suggesting a possible stone or tumor of the kidney, but one remarks that this specimen of urine was passed not long after the bladder had been emptied of two quarts of urine following an acute retention. This chain of events is notoriously prone to produce hematuria. On the whole, then, in the absence of any palpable mass in the kidney region, there seems no good reason to suspect that organ.

We are left with the two diseases so often suspected and discussed heretofore-gall-stones and peptic ulcer. The tender spot in the 
back corresponds rather to the pain of gall-stones than to that of ulcer, and it is especially significant that in one of the attacks the pain started at this point and radiated thence forward. The immediate relief of pain by morphin and the absence of indigestion between attacks incline us to the diagnosis of gall-stones, especially since the less accessible portions of the sclera have begun to show a yellowish tinge. ${ }^{1}$

The prolonged vomiting after the administration of morphin is presumably to be ascribed to one of the not uncommon idiosyncrasies in relation to this drug.

Outcome.-Next day slight jaundice was evident in the sclera. This gradually deepened until her skin was almost a coffee color. The stools were carefully sifted, but no stone found. Liver tender. In two days the gall-bladder could be felt. Urine heavy with bile; stool clay colored. Temperature, $100^{\circ}$ to $\mathrm{IOI}^{\circ} \mathrm{F}$; ; pulse, 80 to 100. Pain in both flanks. The spot on her back has developed into a mark that looks as if some local application had been made. It is shaped like this: $\mathbf{O}$, has sharply defined edges, is not tender, not swollen, and not hot.

Operation showed stones in the common duct. Diagnosis.-Gall-stones.

1 It is perhaps worth noting here that when we are expecting or suspecting a slight degree of jaundice, we should examine especially the peripheral portions of the sclera, which show a yellowish tinge long before there is any coloration around the iris. It is only in the more pronounced grades of jaundice that the yellow color actually meets the iris. Attention to this point sometimes renders the more delicate tests of the serum unnecessary. 


\begin{tabular}{|c|c|c|c|c|c|c|c|c|c|c|}
\hline 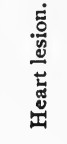 & 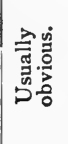 & ० & 。 & ○ & 。 & 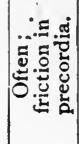 & 0 & $\begin{array}{l}: \\
0\end{array}$ & ' & ' \\
\hline 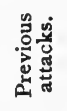 & . & 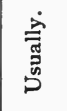 & 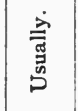 & 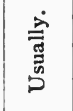 & : & 0 & $:$ & 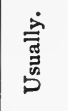 & ○ & 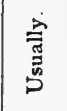 \\
\hline 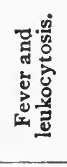 & 0 & + & 0 & ० & 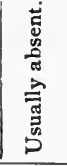 & + & ० & 离 & + & ○ \\
\hline 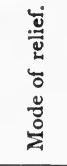 & 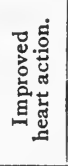 & $:$ & 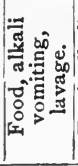 & 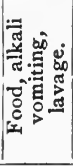 & 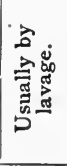 & 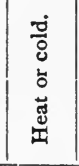 & . & 竧 & $:$ & 若 \\
\hline 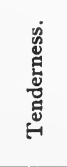 & 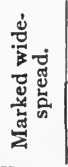 & 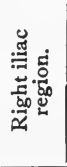 & 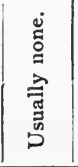 & 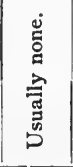 & 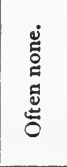 & 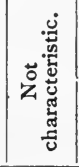 & 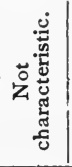 & 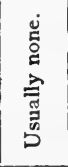 & 苞 & 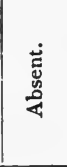 \\
\hline 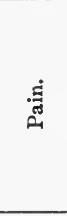 & 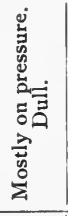 & 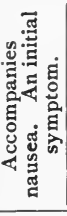 & 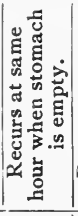 & 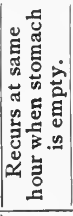 & 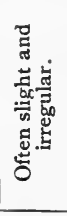 & 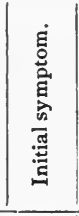 & 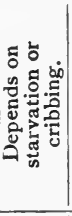 & 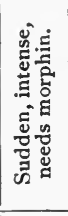 & 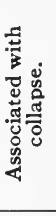 & 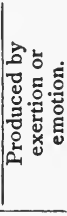 \\
\hline 盛 & $\dot{\vec{\theta}}$ & $\dot{\overrightarrow{\mathrm{C}}}$ & 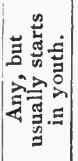 & 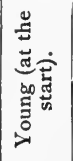 & 安 & d. & $\dot{\overrightarrow{\hat{\theta}}}$ & 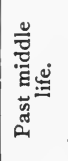 & 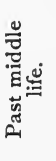 & 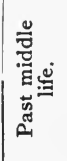 \\
\hline 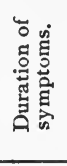 & 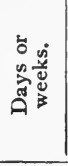 & 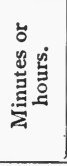 & 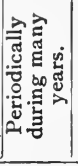 & 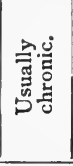 & 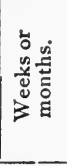 & 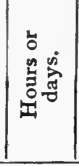 & 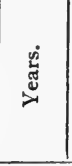 & 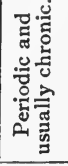 & 站 & 苞 \\
\hline 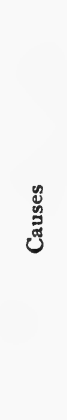 & 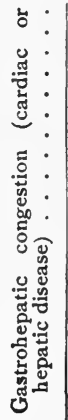 & 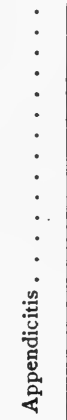 & 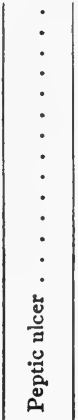 & 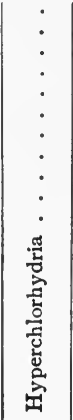 & 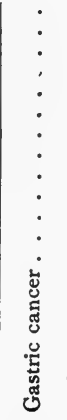 & 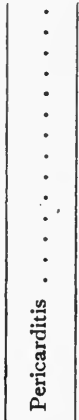 & 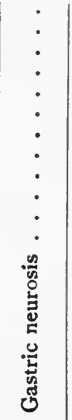 & 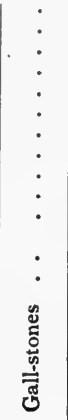 & $\begin{array}{c}\vdots \\
\vdots \\
\vdots \\
\vdots \\
:\end{array}$ & 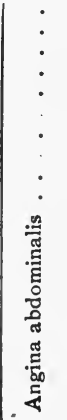 \\
\hline
\end{tabular}




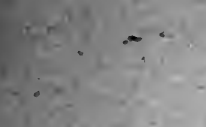

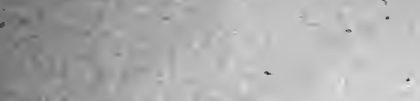

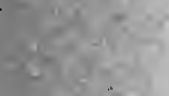

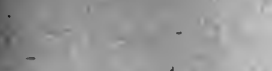

, 1, i Ixper $1 .$.

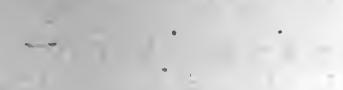




\section{Causes of Pain in the Right Hypochondrium}

1. PASSIVE CONGESTION OF THE LIVER $\left\{\begin{array}{c}\text { CASES TOO MANY AND TOO VAGUELY } \\ \text { ENUMERABLE FOR GRAPHIC REPRE- } \\ \text { SENTATION. }\end{array}\right.$

2. GALL-STONES

AND ACUTE

CHOLECYS -
TITIS

3. HIGH APPENDIX

(INFLAMED) $\}$

4. PLEURISY

5. CANCER OF THE

6. URETERAL STONE

7. RENAL STONE

8. PEPTIC STONE

$\left.\begin{array}{l}\text { 9. SUBDIAPHRAG- } \\ \text { MATICABSCESS }\end{array}\right\}$ ।

Rarer causes are: Hydro- and pyonephrosis, renal and perirenal infections, sacro-iliac lesions, and retroperitoneal neoplasms. 


\section{CHAPTER VI \\ RIGHT HYPOCHONDRIAC PAIN}

Case 96

A boy of twelve entered the hospital April 6, I908, complaining of tenderness and pain in the right hypochondrium. He had a temperature of $100^{\circ} \mathrm{F}$. Gall-bladder inflammation was the diagnosis suggested by his physician. His previous and family history suggested nothing, but he had been suffering almost constantly for two months with the pain above described. This pain has been gradually growing worse, and is now aggravated by deep inspiration. Occasionally he has a sharp pain in the right shoulder; otherwise than this he has had no symptoms, and has been able to go to school until five days before his entrance to the hospital. $\mathrm{He}$ has been decidedly constipated.

Physical examination showed that the heart's impulse was best seen and felt in the fourth interspace, just outside the nipple-line. The sounds were regular and of good quality. A soft systolic murmur was heard at the apex, not transmitted widely; the pulmonic second sound was slightly accentuated; the pulse not remarkable. The lungs were normal, likewise the abdomen, with the exception of tenderness and considerable voluntary spasm in the right hypochondrium and right iliac fossa. The temperature record is shown in the accompanying chart. The leukocytes numbered 9200 April 7 th.

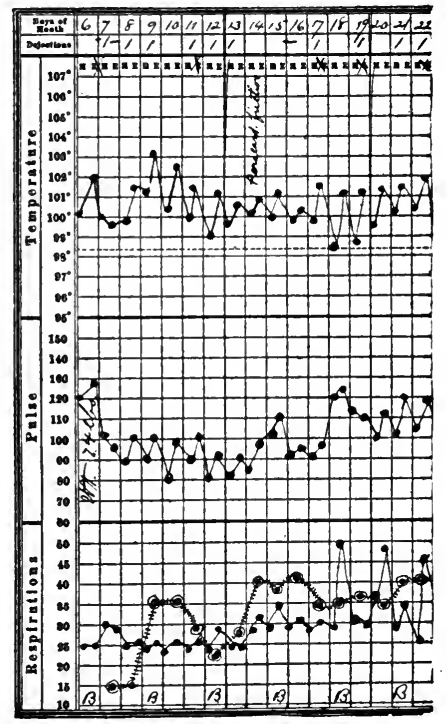

Fig. 32.-Chart of case 96.

April 8th, two days after entrance, the temperature rose to 102.4 F. and the pain increased. A surgical consultant saw the boy, and said that the case was one for exploration of the bile-ducts, but it was 
decided to wait until the boy's parents could be communicated with. In the meantime, dulness and diminished breathing were found in the lower right back, and on April Ioth the abdominal rigidity had almost disappeared. On this day a hypodermic needle was introduced in the back over the dull area, but no fluid obtained. An $x$-ray taken April 13 th showed no lesion of the lung or pleura and no enlargement of the cardiac area to the right. So far the diagnosis was wholly in doubt.

April $5_{5}$ th, nine days after entrance, a double pericardial friction sound was heard for the first time, and the right border of cardiac dulness on the level of the fifth rib was found to be two inches from the midsternal line.

Discussion.-Gall-stones are so rare in boys of twelve that one should be very slow to make the diagnosis, no matter how much the symptoms resemble that disease. Pain and spasm constitute the whole of our positive evidence pointing toward gall-stones, though the fever shown on the accompanying chart would be quite consistent with gall-bladder inflammation. Without more characteristic colic, without jaundice or palpable gall-bladder, we should not make the diagnosis of gall-stones until every other possibility has been disproved.

Abdominal pain in children always points toward disease of the chest (pneumonia or pleurisy) as well as of the abdomen. In the wellmeant desire to solve the problem through some diagnosis of this kind an area of dulness and diminished breathing was worked out in the lower right back, a most dubious region, owing to the varying height of the liver dulness. Such signs as were found were not substantiated in any way by the results of exploratory puncture and $x$-ray examination. In view of this they may be set down as hallucinations of hearing, due to what the psychologists call "expectant attention."

From the lips of the majority of physicians we should surely hear of "rheumatism" or "neuralgia" as explanations of an obscure pain like this, but in the present case these antiquated blanket-diagnoses may be excluded without qualification. Boys of twelve do not have neuralgia or rheumatism at the sites where pain is complained of here. We must demand that the pain shall be localized at or near a joint before the word "rheumatism" can find any place, while all pain called "neuralgic" should follow the known anatomic course of some nerve.

Inflammation of an undescended (subhepatic) appendix is suggested by the position of the pain and spasm. The onset has not been as sudden nor the leukocyte count as high as in most cases of appendicitis associated with so much fever and pain. . Nevertheless, until the spasm 
disappeared and the pericardial friction made itself apparent, a "high appendix" could not be ruled out.

We may ask ourselves whether the position of the cardiac impulse (fourth interspace, just outside the nipple) indicates any pathologic condition or has any bearing upon the diagnosis. The answer should be in both cases, no. At this boy's age the heart's apex is not infrequently thus situated.

Until the appearance of the pericardial friction-rub I do not believe that a diagnosis could have been made in this case, nor do I believe that the pericarditis, which ran its course in so typical a way after that date, was itself the cause of all the previous symptoms. There seems to me good reason to believe that many infections, especially in young people, are in their early stages as wide-spread and unlocalized as their symptoms. It is probably by a further step in the progress of the infectious process that inflammation appears in a well-marked circumscribed area with an exudate and the resulting pathologic changes. It was with the idea of producing such a localization of a previously general process that French physicians have employed subcutaneous injections of turpentine to bring about what they call a "fixation abscess."

Possibly blood cultures would have helped us in this case. They must be, for the present, our only means of recognizing many infections in their early, unlocalized stage.

Outcome.-On the nineteenth the area of cardiac dulness had considerably increased in size, and now extended well out into the left axilla. The leukocyte count had meantime risen from 9200 at entrance to I9,900 on the eighteenth. The friction sound had meantime disappeared, while dulness and diminished breath-sounds were detected in the left lower back.

On the twentieth dulness in the left axilla was found to extend nearly to the posterior axillary line. The leukocytes numbered 22,000, with 80 per cent. of polynuclear cells. The diagnosis of pericardial effusion was then made, and a trocar was inserted in the fifth space, one inch outside the left nipple, and just beyond the palpable cardiac impulse. Seven ounces of turbid, blood-tinged fluid were obtained, with a specific gravity of IO22; 2.I per cent. albumin. The sediment of this fluid showed 87.5 per cent. of polynuclear cells. No tubercle bacilli were found. Immediately after the tapping a double friction-sound could again be heard all over the precordia, and great pain was complained of in this region. Pain and audible friction continued, with some intervals of relief, during the next three days. 
April 23 d the case was again seen by a surgical consultant, and on the twenty-fourth the pericardium was opened and drained by resecting a costal cartilage. The boy afterward developed a left pleural effusion, which finally became purulent, but after rather a tedious illness he completely recovered.

Notes of Treatment.-The bowels were moved by calomel, $\frac{1}{4}$ grain every fifteen minutes until ten doses were given; afterward by cascara and by an enema. For the pain, hot fomentations and turpentine stupes were given. A mustard poultice to the abdomen also gave some relief, and later an ice-bag was placed over the heart and about $\frac{1}{8}$ grain of morphin was given daily by subcutaneous injection.

Diagnosis.-Pericardial effusion.

\section{Case 97}

A highly neurotic Jewish boy of eighteen was seen June 19, 1907. His illness began in November, I906, when for two weeks he wás troubled by pain in the right loin and right back, together with "dizzy headaches" and weakness in his legs. He believes that he strained himself in lifting a heavy packing-case in October, 1906. In the latter part of December he had a similar but milder attack. He states that since January 2oth he has suffered from constant pain in the right loin, frequently catching him with a severe stitch on inspiration. Occasionally the pain has shot down from his side toward the groin or up toward the epigastrium. His urine is usually clear, but sometimes stained red, and full of floating particles. He has gained in weight, but lost in strength since February.

In January he was carefully examined, but no disease found. On June I2th his urine showed a slight trace of albumin, with many leukocytes and blood-cells in the sediment. On June I9th a physical examination was negative except that the right rectus abdominalis was spastic, and there was tenderness over the right side, most marked at the edge of the ribs, in the right nipple-line, and in the right iliac fossa.

At the time of this examination the lower edge of the right kidney was palpable on deep inspiration, and there was a slight tenderness along the lower dorsal and lumbar spine. The movements of the spine were free. He had no fever and no increase in the leukocytes. The urine varied greatly in gravity, being twice below roo 8 and three times above Io20 within twenty-four hours. It always contained a very slight trace of albumin, and in the sediment a very small number of bloodcells and leukocytes. One specimen showed a blood-clot the size of a bean. 
Cystoscopy was done on the twenty-sixth, and showed on the floor of the bladder "a brownish, cylindric, putty-like plug." The orifice of the right ureter was greatly dilated, and a little pus was seen to issue from it. A strong, clear stream of urine issued from the left ureter.

Discussion. - In the actual presence of this patient it was far more difficult than in reading the printed case to avoid being unduly impressed by his neurotic temperament. Any one so manifestly and annoyingly self-centered, especially if he be of the Jewish race, runs a considerable risk of being falsely accused or falsely suspected of being "merely a neurotic." Our better judgment, however, should make it clear that there is something else in the background.

The patient himself was inclined to attribute all his symptoms to the strain suffered in the previous October, but on careful questioning it was clear that the symptoms did not make their appearance until some weeks after the date of the supposed strain.

We may note that in the physical examination there are no data regarding the condition of the sacro-iliac joints. Many of the symptoms here described could be accounted for by some of the acute lesions of those joints. In fact, however, the joints were normal, although this is not stated in the text.

The chief moral of this case is the impossibility of a satisfactory diagnosis through the ordinary methods of physical examination in many cases involving the right upper abdominal quadrant. Without cystoscopy a "high appendix" (see case 96) could not have been excluded, and the diagnosis must have remained long in doubt; indeed, the case is introduced largely to illustrate the importance of cystoscopy in cases involving neither bladder symptoms nor ordinary "renal colic."

It remains merely to discuss what lesion we should expect to find in the kidney on the basis of the facts here given. Malignant disease of the kidney is rare at eighteen, and cannot be recognized in the absence of tumor and hematuria. Tuberculosis of the kidney should produce fever, pyuria, and vesical discomfort. In the majority of cases also a tumor would be palpable after eight months of suffering. Renal stone seems the most reasonable diagnosis.

Outcome.-An $x$-ray plate taken on the twenty-eighth showed a shadow apparently in the pelvis of the right kidney. On the same day operation confirmed the findings of the $x$-ray, though the stone crumbled up into fine sand when touched. The patient made a good recovery.

Diagnosis.-Renal stone. 


\section{Case 98}

A factory-hand of twenty-six, whose family history was unimportant, had typhoid fever when he was eight years of age, and has suffered from constipation for the past ten years. With the exception of 20 cigarettes a day, his habits are good.

For the past four months he has been more constipated than usual, his bowels moving only once in four or five days. For the past two weeks he has been troubled by headache, which, however, has disappeared to-day. During this time his appetite has been poor.

Eight days ago he began to have a steady, moderately severe pain at the right costal margin. Five days ago he noticed that his eyes were yellow, and that his urine was of a deep-red color.

On physical examination his sclera was found to be moderately yellow, and his skin considerably discolored. Both tonsils were slightly enlarged, and there were a few white spots upon the right tonsil. The heart's impulse was not seen or felt. The sounds were best heard in the fourth interspace, three inches from the median line. There were no murmurs nor other modifications of the sounds. There was rigidity in the right upper quadrant, with tenderness and dulness extending an inch and a half below the ribs. A sharp edge could be felt to descend on full inspiration at this point. The upper border of liver dulness was at the sixth rib. The abdomen was otherwise negative, as were the other organs. The urine contained bile and a very slight trace of albumin, but was otherwise normal. There was no anemia and no leukocytosis.

The patient was first seen on the twenty-second of February. Under sodium phosphate, 20 grains after meals, and a hot-water bag to the hypochondrium, he became comfortable, and by March $4^{\text {th his }}$ yellow color had considerably faded. His constipation was later treated by cascara and by enemata.

Discussion.-Any case involving jaundice and a past history of typhoid fever suggests a typhoid cholecystitis with the resulting gallstones, and this possibility cannot be excluded here. Without colic, fever, chills, or vomiting, and without a palpable gall-bladder, we cannot get beyond suspicions in this direction.

Cases of relatively short jaundice, with or without slight enlargement of the liver, such as is here present, are traditionally labeled as "catarrhal jaundice" if nothing more definite appears in sight; but it is always quite possible that we may be dealing in these cases either with a transient obstruction due to stone or to an infectious cholangitis traveling down 
the ducts rather than up. There is little if any proof that so-called catarrhal jaundice spreads upward from an inflamed duodenum. For the present, however, and until our knowledge of the subject is considerably increased, we must be content with the old term.

Outcome.-On the fifteenth of March his color was practically normal and the bile was gone from his urine. He felt perfectly well and was discharged. There has been no recurrence in three years.

Diagnosis.-Catarrhal jaundice.

\section{Case 99}

A widower of seventy-seven entered the hospital February 25, I908. He has always followed the trade of carpenter and has been strong and well except for two attacks of malaria, one during the Civil War (when he served for three years), and the other eight years ago.

Seventeen years ago he was kept out of work for fourteen months on account of symptoms supposed by one doctor to be due to cancer of the stomach, by other doctors to be caused by liver trouble. At that time he suffered pain under the right costal margin; this pain shot through into his back and was associated with vomiting and frequent black stools. He never vomited blood, was never jaundiced, and had no chills, fever, or colic. The pain was always worse at night, but had no relation to the character of food nor to the time of taking it.

$\mathrm{He}$ completely recovered from this attack, and has been at work ever since except for a period of two months, seven years ago, when he was in the Massachusetts General Hospital for an attack diagnosed as duodenal ulcer. At that time he frequently passed blood in his stools and his weight fell to 200 pounds, where it has since remained.

One year later he had an attack of vomiting with tarry stools, similar to those passed the year before, but was well again in a few days. Three years ago he had an attack of vomiting lasting nine hours; there was no blood in his stools at that time, but he had to remain in the house for two weeks. Between the attacks, i. e., for most of the last fifteen years, he has called himself well. Twenty months ago he had a severe attack of pain under the right costal margin, accompanied this time by the appearance of a red spot on the skin just below the ribs. He was told by his doctor that he probably had an abscess of the liver. After a day or two of this pain his urine suddenly became pink and remained so for ten days; the pain and the red spot then gradually subsided, and the urine became normal in appearance.

$A$ year ago he had an attack of indigestion, with pain under the right 
costal margin and fainted, so that he fell out of his chair while the doctor was talking to him. His habits have always been excellent.

For the past six months he has had a continuous, dull pain under the right rib-margin. This pain gets worse on moving about, is not affected by food, and occasionally becomes severe, radiating to other points of the abdomen and to the back. He had such an attack three nights ago, but was relieved by drinking three glasses of cold water.

Six weeks ago he noticed under the right costal margin a swelling, which has steadily increased in size and become exceedingly tender to the touch. He has had no fever, no jaundice, no vomiting, and no change in the amount or color of his urine. He has noticed nothing remarkable about his stools.

Physical examination reveais no emaciation and nothing abnormal in the chest. The right costal margin is markedly prominent, and in the center of this prominence is a rounded protrusion which is very tender. (See Fig. 33.) The tender mass is firm and somewhat movable, sometimes reaching the median line in the epigastrium. The edge of the liver is felt just below the mass, and is apparently somewhat irregular.

Physical examination is otherwise negative, likewise the blood and urine. His stools contain no occult blood. After further observation it was found that the tumor would move with a change in the patient's position until it reached the left costal margin; with this motion the upper border of liver dulness also moved downward.' Examined by means of a stomach-tube, the stomach was found to reach one inch below the navel when inflated. The upper border was at the tip of the ensiform cartilage. The stomach-contents after a test-meal showed hydrochloric acid, O.II per cent., and total acidity, 0.I7; no occult blood.

Discussion. - The early history of this case points straight to the diagnosis of duodenal ulcer. Between these initial symptoms, however, and the sufferings of the last six months, there are two curious episodes which may be first briefly discussed.

How are we to explain the appearance of the red spot in the right hypochondrium and the close sequence of pink-colored urine? Since these symptoms began together and ceased together, it is reasonable to look for a common cause. We may conjecture that the spot on the hypochondrium was due to a "purpuric" extravasation of blood, and that the urinary coloration was due to a similar ecchymosis in the kidney. Such occurrences would be easily explicable were jaundice present, for we are well accustomed to see all sorts of oozing and hemorrhages in jaundiced patients. It has been pointed out, however, by Dr. 


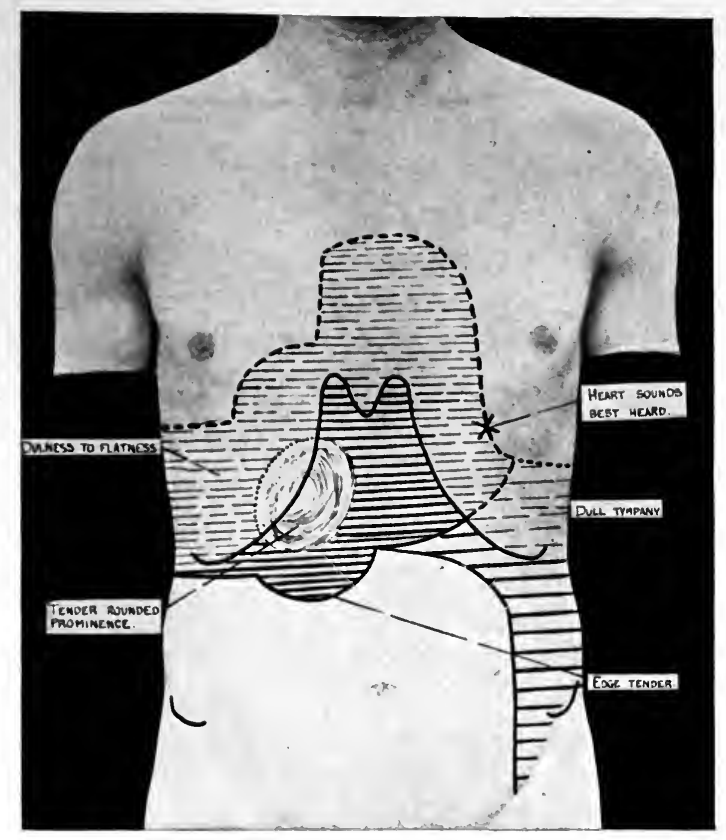

Fig. 33.-Diagram of signs recorded in a patient who complains of pain and swelling under the right ribs. 

Maurice H. Richardson and others, that the hemorrhagic tendency in diseases of the liver is not confined to those which produce jaundice. If, therefore, we assume, as seems warranted by the outcome of the case, that this patient may have had liver disease at the time of the phenomena we are now attempting to explain, the idea of multiple hemorrhage would be plausible.

What shall we say of the fainting attack which occurred a year ago? Since this patient has had repeated and profuse intestinal hemorrhages, presumably from duodenal ulcer, it seems not unlikely that the faintness was due to the repetition of such a hemorrhage.

Coming now to the events of the last six months, we find them characterized by continuous pain in the region of the liver, apparently unconnected with the taking of food, but complicated later by enlargement and irregularity of the liver.

In patients who have never lived under conditions favorable to hydatid infection (association with sheep and sheep-dogs, especially in Greece, Australia, and Iceland), we need consider only two diseases to explain a nodular enlargement of the liver, viz., cancer and syphilis. The nodules due to cirrhosis are rarely if ever palpable through the abdominal walls. The hepatic enlargements due to passive congestion, fatty infiltration, leukemia, pseudoleukemia, amyloid disease, obstructive jaundice, and abscess do not produce a nodular surface. Our problem, then, is reduced to narrow limits-cancer or syphilis. I have never known syphilis to produce so much pain as was suffered in this case. The absence of fever is also against this diagnosis. The same is true in a lesser degree of the absence of syphilitic history and syphilitic lesions in other parts of the body.

Cancer of the liver-which seems the most probable explanation of this man's present sufferings-is rarely primary. We may suppose it to be secondary to a growth implanted in the site of the peptic ulcer which we have good reason to believe existed some years ago. Yet we have no definite evidence of any such growth in the stomach or duodenum, and the starting-point of the disease must be left in uncertainty.

Outcome.-On the seventh of March the abdomen was opened and showed a firm, nodular mass of malignant disease in the liver about the size of a cocoanut. The abdomen was closed and the patient left the hospital on the nineteenth of March. He died three months later.

Diagnosis.-Hepatic cancer. 


\section{Case 100}

The patient is a stable-man of thirty-six who was first seen March 7 , 1908. He had a good deal of trouble with his stomach three years ago, but since then has been well until four weeks ago, when he began to vomit and to have severe pain in the right upper quadrant. His vomitus sometimes contains large quantities of food. The pain is very severe, and for the last two weeks has forced him to walk the floor every night and to take morphin tablets.

At present his pain is at its worst about two hours after meals; it is also very troublesome at night; sometimes it shoots across to the left costal margin and up to the right nipple. For three weeks he has eaten onlv bread, milk, and tea.

On physical examination his right pupil was found to be slightly larger than his left. Both react normally. The skin was everywhere notably smooth and satin-like to the touch. His radial arteries were considerably thickened, and his aortic second sound was greater than his pulmonic; otherwise nothing wrong was found in the chest. There was moderate tenderness in the right upper quadrant. Physical examination, including the blood and urine, was otherwise normal. A stomach-tube passed before breakfast showed no fasting contents. The capacity of the stomach was 24 ounces, and the percussion outlines after distention with air indicated no dilatation of the organ. Microscopic and chemical tests of the gastric contents after a test-meal revealed nothing abnormal.

It was later ascertained that this attack followed a debauch in which he took whisky, beer, and ale to excess for a week, "which," he says, "scalded his insides." Before that he had taken no liquor for years.

Discussion.-By force of ancient tradition we are accustomed to think of syphilis as a cause for all pains which are worse at night. We have seen, however, from the cases already studied in this book, that pain due to hyperchlorhydria, to peptic ulcer, gall-stones, and lead-poisoning, is also aggravated at night in many cases. In the present case the suspicion of syphilis is somewhat increased by the finding of thickened radial arteries, accentuated aortic second sound, and unequal pupils; yet there is nothing sufficiently definite in the physical examination to justify a diagnosis of visceral syphilis(Yur said it!)

Of the other causes of pain above mentioned there is not sufficient evidence, though only lead can be positively excluded. The most signiticant point of the physical examination is the satin-like surface of the skin. This quality, when well marked in workingmen, is strong evidence of recent alcoholism, and when, as in this case, the history 
does not at once suggest any such habit, the evidence obtained through a routine physical examination, which includes a note on the condition of the skin, may be most important. This is especially true when no other cause can be found for the sudden appearance of marked gastric disturbances in an adult.

Outcome.-The patient was put on Lenhartz's diet, and in a couple of weeks seemed perfectly well.

Diagnosis.-Alcoholic gastritis.

\section{Case 101}

A young Jewish house painter eighteen years old, who was first seen March I6, I908, has had several attacks of rheumatism, but nevertheless has considered himself well until five weeks ago, when he began to suffer from pain in the right upper quadrant, together with dyspnea on exertion, weakness, and cough, with frothy white sputa. For the past ten days he has been in bed, and found it impossible to lie down at night on account of cardiac distress.

On physical examination he was found to be pale and slightly cyanotic. The veins of his neck were markedly distended and showed a systolic pulsation. The carotids also pulsated vigorously. The heart sh',wed a diffuse pulsation in the second, third, fourth, and fifth left interspaces, but the maximum impulse was seen and felt in the sixth space $1 \frac{1}{8}$ inches outside of the nipple-line. The area of cardiac dulness extended $2 \frac{1}{4}$ inches to the right of midsternum, and the cardiac impulse could be felt for nearly an inch beyond the right of midsternum. The heart was regular; rate, I Io. At the apex a systolic and a presystolic murmur were heard. In the left axilla and along the left sternal border the systolic murmur was much more intense, and a musical diastolic murmur was heard. The pulmonic second sound was much accentuated; the aortic second sound was absent. The pulse was of low tension and of the Corrigan type, but no capillary pulse was demonstrated. The lungs were normal except for the presence of a few moist râles at the base of the left axilla. There was dulness in the flanks, shifting with change of position. The edge of the liver was felt three inches below the ribs. The organ was tender, and moved with each systole. The urine averaged 30 ounces in twenty-four hours, with a specific gravity of 1025 . There was a slight trace of albumin and a few granular casts. The blood showed nothing abnormal. There was no fever.

Discussion.- In relation to the prognosis and treatment of this case it is important to form some estimate of its duration. Even a cursory study of the cardiac lesion must convince us that the heart 
was diseased for some time previous to the last five weeks, during which he has called himself sick. In view of the size of the heart and the character of the murmurs (which suggest stenoses and therefore chronicity) we may assume that the disease has existed for months, if not for years.

Cardiac lesions involving marked hypertrophy are most often due to:

(a) Valvular disease.

(b) Chronic nephritis. Essential hypertension

(c) Adherent pericardium, with or without interstitial myocarditis.

Renal disease may be ruled out by the low tension of the pulse and by the characteristics of the urine. Adherent pericardium by itself cannot produce so marked a diastolic murmur and would not account for the arterial changes (Corrigan pulse). We cannot exclude the possibility of adherent pericardium complicating other lesions, but alone it would not account for the facts.

The signs certainly point to the existence of disease at the aortic valve, and probably to a similar condition at the mitral. Is the aortic lesion single or double? The physical signs give us assurance only of aortic incompetence, but postmortem experience has led me to believe that whenever aortic regurgitation is recognized in a cardiac case of considerable duration occurring in a young person, aortic stenosis is almost always present as well, whether the physical signs indicate it or not. In other words, aortic disease due to endocarditis almost always produces stenosis as well as regurgitation if it has lasted longer than a few weeks.

As this case occurred previous to the discovery of the Wassermann reaction, we had no means of ascertaining whether the aortic lesions were possible or probably due to syphilis.

As regards the mitral valve, we have no way of being sure whether or not an endocarditis has been at work there. The systolic murmur might be due to relative insufficiency without lesion of the valve itself, while the presystolic murmur might be of the type described by Austin Flint. But the strong accentuation of the pulmonic second sound gives us reason slightly to favor a definite mitral lesion.

Obviously, there is passive congestion of the liver, explaining the pain and tenderness in the right hypochondrium, and these facts, together with the gathering ascites (shifting dulness in the flank), make it clear that the tricuspid valve is leaking badly. This still further inclines us to assume an organic mitral disease. The urine is typical of passive renal congestion.

Why should the heart have begun to fail just at this time? We are 
apt to explain such events through a so-called break of compensation supposedly of mechanical origin. The individual is supposed to have reached and overpassed the limits of his cardiac reserve power. It has been pointed out, however, especially by Dr. Charles Hunter Dunn, ${ }^{1}$ that many of the so-called breaks of compensation, occurring as they usually do without any known strain or overexertion, are, in fact, due to a fresh outbreak of the endocarditis which has previously been smouldering upon the diseased valve. This possibility is especially to be thought of when the supposed break of compensation comes, as it were, out of a clear sky, and is accompanied by a polynuclear leukocytosis, with or without a slight elevation of temperature.

Outcome.-The boy lived in the hospital from March 6th to April 8th. At no time did he show any improvement; and despite digitalis, strychnin, morphin, magnesium sulphate, diuretin, calomel, squills, and other drugs, he died on April 8th.

Autopsy showed fibrous endocarditis of the mitral and aortic valves, with stenosis and insufficiency of both. There was also a fresher verrucose process on both valves, and some acute degeneration of the myocardium.

Diagnosis.-See last paragraph.

\section{Case 102}

An Irish laborer fifty-eight years old entered the hospital February I8, I908. His family history was unimportant, but he stated that for the past two years he had had "bronchitis," and that he had used each week 70 cents' worth of tobacco all his life, until eighteen months ago; very little since. Since the middle of October his "bronchitis" has been very severe, and he has felt weak and tired, but as the rest of the family were out on a strike, he had to keep at work. Three days ago he got wet through, and since then he has been in bed. He complains of pain in the right hypochondrium, with dyspnea and cough, especially when he is working. He raises yellow sputum in considerable amounts, but has never raised blood. Last November he was troubled for some weeks with pain in the left side of his chest. Two years ago he weighed I85 pounds; now he weighs 135 . Whenever he coughs he has an aggravation of the pain in the right side of the abdomen beneath the ribs.

Physical examination showed small, irregular pupils, which reacted normally. The throat was reddened and slightly swollen; the heart's action irregular in force and rhythm, but showing no other abnormality.

${ }^{1}$ Dunn, Jour. Amer. Med. Assoc., February 9, 1907. 
His pulses were apparently of increased tension, and his arteries easily palpable, but on measurement his blood-pressure showed only I20 $\mathrm{mm}$. Hg. There was no dyspnea in the recumbent position and no edema anywhere. The lower two-thirds of the right lung behind showed dulness. At the base there was intense bronchial breathing, with marked increase of voice-sounds and fremitus and many fine, moist râles. These sounds became less marked in the upper portion of the dull area.

During a ten days' stay in the hospital there was no notable change in the physical signs. The patient had an irregular pyrexia, reaching as high as $102^{\circ} \mathrm{F}$. at frequent intervals, but always falling below normal at some time in the twenty-four hours.

Discussion.-Chronic bronchitis (usually with disseminated bronchiectatic cavities) is the commonest cause of a long-standing winter cough in elderly people. The fact that this patient's cough appears to last all the year round does not exclude this type of bronchiectasis, but the fact that it is accompanied by loss of weight, by pain in the right hypochondrium, and by intense bronchial respiration at the base does not fit in well with bronchitis and bronchiectasis. The sputum examination is very important in solving this problem.

The irregularity of the heart and the evidence of degeneration in the peripheral arteries make us wonder whether the signs at the base of the right lung may not represent a hydrothorax due to cardiac weakness. The signs, to be sure, are by no means typical of hydrothorax, but might possibly be consistent with that condition, were it not that the absence of dyspnea and edema points strongly against the existence of any cardiac weakness sufficient to account for hydrothorax.

If we fix our attention upon the physical signs alone, there is much to suggest a pulmonary abscess, dependent either upon a postpneumonic empyema rupturing into a bronchus or upon some unknown cause ("primary pulmonary abscess"). But the long duration of the symptoms and the lack of any suggestion of acute onset make this rather unlikely.

But for the unusual position of the signs, it would be natural to consider pulmonary tuberculosis first of all in this case. Even as it is this disease is by no means to be excluded. Repeated and thorough examinations of the sputa are called for.

Outcome.-The sputum showed many tubercle bacilli and also many pneumococci, both within and outside of the leukocytes. The patient remained in the hospital until the second of March without showing any considerable change in any respect, except that he gained 4 pounds in weight. He is troubled greatly with insomnia, for which 
he was given chloral hydrate, I 5 grains, on two occasions, and veronal, Io grains, once. His coughing was relieved by $\frac{1}{4}$ grain of codein, and his bowels were kept regular by A. S. and B. pills. After the first few days he was able to be up and out-of-doors, and gained considerably in strength.

Diagnosis.-Phthisis.

\section{Case 103}

An English tailor thirty-eight years of age, who entered the hospital March II, I908, had been complaining of pains throughout his body, especially in his lower legs, for the past seven weeks. The pains were so severe as to compel him to give up work, but were relieved by treatment. Three days ago he began to have pain in the right upper quadrant, radiating to other parts of the abdomen and downward. His appetite has been poor, but he has not vomited. His bowels have been moved by cathartics. Three days ago he had two severe chills, and since then he has sweat a good deal at times. Nothing abnormal was noticed about his water.

Physical examination revealed nothing wrong in the chest. The abdomen was full, tympanitic throughout, and held rather rigidly. The patient appeared to suffer a good deal of pain, but when his attention was distracted, one could palpate deeply without discovering any tenderness. Attempts to move the bowels were not satisfactory. The leukocyte count at entrance was 14,000 ; next day it had risen to 21,000 , and on the third day to 25,200 . His temperature ranged between IoI $^{\circ}$ and $102{ }^{\circ} \mathrm{F}$; his pulse, between 90 and 100. His urine showed nothing abnormal.

Discussion.-The patient's account of himself leaves us still in the dark as to the nature of his trouble. Discovering that his abdominal tenderness apparently disappears when his. attention is distracted, we are in danger of discounting his other and more serious symptoms. But with pain, chills, and an increasing leukocytosis there is almost certainly a focus of infection somewhere. Our best guide in all probability is the initial pain, since he has not yet arrived at that third stage in the development of an infection at which, after scattering itself in confusing radiations, the pain and tenderness finally "settle" over the site of the disease. (See further discussion of this point on p. 2II.)

There is nothing in the data here presented to incriminate the kidney or the stomach. On the whole, therefore, the most likely place for investigation is the gall-bladder. Nevertheless, there are many other possibilities. I have seen a case much like this in which throm- 
bosis of a mesenteric artery was found at operation, but I have never known that diagnosis correctly made before operation. Appendicitis and portal phlebitis are also possible.

Outcome.-Laparotomy on the thirty-first revealed an acute cholecystitis.

Diagnosis.-Acute cholecystitis.

\section{Case 104}

A Hungarian woman of sixty entered the hospital August 22, I907, complaining of two months' pain in the right upper quadrant of the abdomen, but asserting that her sickness was wholly due to the behavior of her step-daughter. As a result of this the patient has lost her appetite, become constipated and rather sleepless, but has not given up work. She has had five children and no miscarriages, and has always considered herself well. She passed the menopause twenty years ago; her past history and family history have been wholly good.

On examination she was found to be decidedly pale. There was no glandular enlargement. There was ptosis of the left upper eyelid, but the eyes were otherwise normal except for marked irregularity of the pupils and a failure to react to light. The chest revealed nothing abnormal. The abdomen was large and flabby. The whole of the right half of it was occupied by a hard, smooth, irregular mass, immovable, not tender, and very sharp at the edge. The dulness over this area was continuous with the liver dulness, which began at the sixth rib. The lower border of the tumor was six inches below the costal margin (Fig. 34). There were slight edema along the shins and marked varicosity of the veins in both legs.

The hemoglobin was 20 per cent.; leukocytes, 2000; the urine normal. Vaginal and rectal examinations were negative. The gastric contents extracted after a test-meal showed no free hydrochloric acid and no occult blood. The capacity of the stomach was 50 ounces. There was no residue before breakfast.

Discussion.-The problem here is of a tumor in the right hypochondrium with anemia - a tumor which gives every evidence of being coarsely irregular in shape. Under these conditions the possibilities for diagnosis are as follows:

(a) Liver-cancer, syphilis; much less probably hydatid, enlarged gall-bladder, downward displacement of the normal organ.

(b) Kidney-tuberculosis, hydronephrosis or pyonephrosis, cystic degeneration, neoplasm.

(c) Retroperitoneal tumors displacing or pushing forward the liver. 


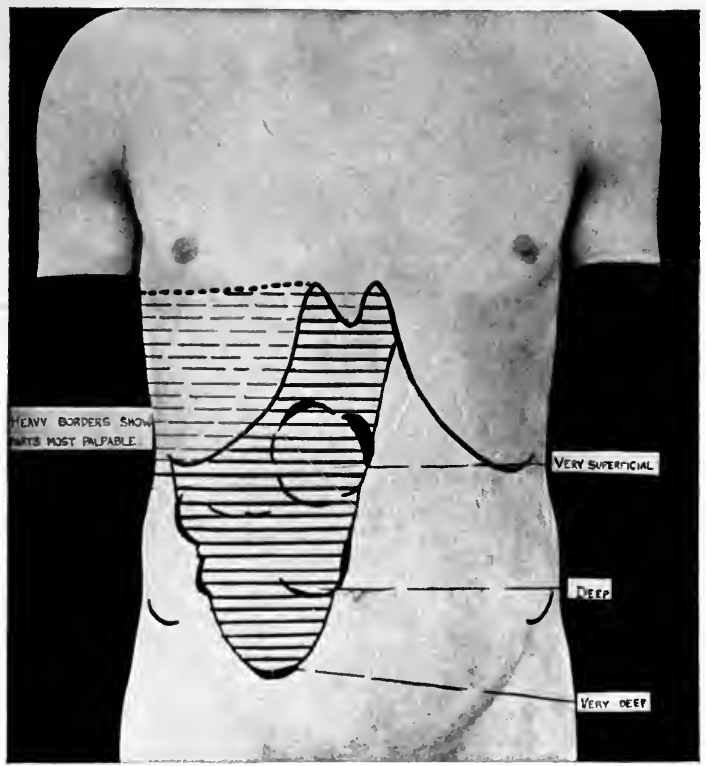

Fig. 34.-Outlines of a mass felt in Case ro4. Chief complaint is pain in the right hypo chondrium. 

Tumors of the stomach or intestine are practically out of the question. Masses of exudate and matted intestines, such as occur with tuberculous peritonitis, are smaller and have no sharp edge.

Returning, then, to the three main groups listed above, we may exclude displacements of the liver and enlargements of the gall-bladder, since the shape of the tumor here present does not correspond at all with any of these.

Hydatid cysts of the liver do not produce so grave an anemia and are usually large enough to be perceptible by the individual and complained of by him before the physician discovers them. This patient was wholly unaware of her tumor.

Cancer and syphilis of the liver remain as possibilities, to the discussion of which we shall return presently.

Of the tumors connected with the kidney, those due to tuberculosis are perhaps the commonest. They almost invariably produce pyuria and bladder symptoms, which are not present here. Further, the shape of this mass and its position in the abdomen are not at all characteristic of tumors originating in the kidney. Fever and pain would also be expected in a patient suffering from renal tuberculosis, though these symptoms are less constant than those above mentioned.

Hydronephrosis and pyonephrosis produce smooth, rounded tumors, usually elastic in feel, and more deeply situated in the loin than the mass here in question. They often appear intermittently, their disappearance being accompanied by an increased flow of urine.

Cystic kidneys are practically always congenital and bilateral. They are not associated with anemia; indeed, none of the renal lesions hitherto mentioned produces any considerable anemia in the great majority of cases.

New-growths of the kidney may produce grave anemia, but when this is the case, they are practically always associated with hematuria, which has been absent here.

Retroperitoneal tumors originating in the prevertebral glands occasionally present a picture much like that here seen. The fact that the tumor is immovable tends to identify it with a retroperitoneal structure, rather than with the liver. Not infrequently these retroperitoneal tumors displace the liver downward and forward, so that what our hands feel is, in fact, not the new-growth itself, but the normal liver. I have taken part in long and fruitless discussions as to what disease of the liver is present in a case of this kind, only to discover at operation or autopsy that we have been suspecting the wrong organ. The nodular 
surface of the growth from which this woman is suffering excludes the latter possibility.

The tumor is certainly not the normal liver: it is either a diseased liver or a new-growth arising elsewhere.

With these possibilities in mind we return to the general study of the case, and are struck by the fact that the patient has a ptosis and pupils unresponsive to light, both of which symptoms are characteristic results of old syphilis. This naturally makes us inclined to follow up the clue and try the therapeutic test. A course of iodid and mercury will do no harm to any malignant new-growth, and will probably produce marked improvement, local and general, if the liver be syphilitic.

Outcome.-Under mercurial inunctions and potassium iodid, 5 to 50 grains, the patient improved very markedly in ten days, and the size of the tumor rapidly decreased. Except for occasional doses of veronal, 5 grains, and the painting of a 25 per cent. alcoholic solution of menthol over the epigastrium for the relief of pain, no other medication was given.

Diagnosis.-Hepatic syphilis.

\section{Case 105}

A Russian Jewess of forty-two has been complaining for eighteen months of a burning pain in the right upper quadrant, almost constant, often keeping her awake, sometimes shifting into the back, but never colicky or paroxysmal. She has vomited occasionally, but has never been jaundiced. For the same period she has had distress across the upper half of the abdomen after meals, with belching and constipation, her bowels moving only every four or five days. For three months all these symptoms have been aggravated, and she has vomited green material nearly every day. She has never vomited any blood or any food. She thinks she has lost much weight. She has no appetite and has been in bed much of the time of late.

The patient was obese, the chest negative, the abdominal wall loose, flabby, and soft. The right kidney could be felt at three fingers' breadth below the ribs, and the edge of the liver was also palpable. Physical examination, including the blood, pulse, temperature, respiration, and blood-pressure, was normal. The urine ranged between 25 and 35 ounces in twenty-four hours, with a specific gravity from ror 2 to ror9; there were very slight traces of albumin and a few hyaline, granular, and brown granular casts. Examination of the stomach-contents and of the stools revealed nothing abnormal.

Discussion.-When a Russian Jew complains of a "burning pain," it usually turns out, on closer questioning, that he has a burning and 
not a pain. The word "burning" (brennend) is used by the Jews far more often in describing their symptoms than by any other race, and, as a rule, patients who use this term turn out to be free from organic disease. Whether it is a cutaneous paresthesia connected with nervous debility, or whether it is connected with gastric stasis and fermentation, is often very difficult to determine.

Gastric symptoms appearing for the first time in a person over forty always make us think of cancer of the stomach, but if that disease had existed for eighteen months, we should be almost certain to find stasis, emaciation, or anemia. Peptic ulcer cannot be positively excluded, but the symptoms are not definite enough to warrant our beginning treatment for that affection until more probable alternatives have been tried out.

The palpable kidney and the presence of albumin and casts in the urine make it our duty to consider whether the symptoms may be due to some form of renal disease. These symptoms could be produced by the kidney if the latter exerted direct pressure upon the pylorus or intestine, so as to retard their movements in the course of digestion. But this seems very unlikely in view of the moderate size and free mobility of the organ. The kidney might also be responsible for suffering like that here described if it were the seat of a chronic nephritis with uremia, but the normal condition of the heart and blood-pressure makes this unlikely, and the urine is not at all typical of acute nephritis.

Evidently the patient has a general visceroptosis, and this, with her obesity, her incompetent abdominal muscles, and her constipation, might well be sufficient to account for her complaints.

It may be well to say a word here about the psychic significance of green vomiting. Of course, every physician is aware that, from the physical point of view, any long-continued or violent vomiting produces green-colored vomitus through the compression exerted upon the gall-bladder by the abdominal walls. But in the patient's mind green vomiting has often a dark and terrifying significance, so that it is well explicitly to reassure any patient who complains of this symptom, remembering that he does not share our understanding of its harmlessness.

Outcome.-A snugly fitting abdominal bandage gave the patient very marked relief, and when her bowels had been regulated by the use of calomel, $\frac{1}{2}$ grain every fifteen minutes until ten doses, followed in half an hour by a seidlitz powder and thereafter by cascara, she was able to leave the hospital, much relieved, at the end of two weeks.

Diagnosis.-Hängebauch. 


\section{Case 106}

A carpenter of fifty-four entered the hospital June I9, I908, with the statement that six weeks ago, while at work, he had a sudden attack of pain in the right upper quadrant, radiating to the right shoulder. This pain was relieved by a hot drink, and disappeared in about three hours. He vomited once that night. He went to work the next morning. A week later the pain returned, and it has since been nearly continuous, though for the past two days it has been less severe. At the onset it was accompanied by a swelling of the abdomen and by jaundice. $\mathrm{He}$ has had dark urine, light stools, and much itching for the past five weeks. Fever and vomiting have been absent. His appetite has been poor, and he has had moderate constipation.

On examination, moderate jaundice and marks of scratching were everywhere evident. The chest was normal. The abdomen showed tenderness in the epigastrium and for several inches to the right of this point. The upper right rectus was more resistant than the left. The edge of the liver could be felt an inch and a half below the rib margin. The patient has lost 42 pounds in the past eight weeks.

Discussion.-The diagnostic problem confronting us concerns the cause of emaciation, jaundice, steady pain, and enlargement of the liver in a man of fifty-four. Cancer of the pancreas or of some portion of the bile-ducts would produce all these symptoms, and is their commonest cause in men of this age, but it is hard to understand why any of these lesions should produce so sudden an attack of pain and of jaundice. The supposed cause-cancer-being an affair of gradual growth, one would expect the symptoms to develop gradually, not suddenly. Nevertheless, clinical experience has shown that cancer may manifest itself suddenly, and with the symptoms here described. We must face the fact, whether we understand it or not. Against cancer is the absence of an enlarged gall-bladder, which is the rule when cancer obstructs the bile-ducts. But this objection is not sufficient to make us certain that cancer is not present. The possibility must still be entertained.

Stone in the common duct might produce all the symptoms under discussion, and would account, better than cancer does, for the sudden onset and the biliary colic. The loss of 42 pounds in eight weeks as a result of cholelithiasis alone is at first sight astounding, but experience shows that it is not at all unusual. More unexpected is the absence of fever, chills, vomiting, and of variations in the intensity of the jaundice, all of which are the rule when a stone blocks the common duct. The 
moderate degree of jaundice, on the other hand, favors stone rather than cancer.

Obliteration of the bile-ducts by the scar of an inflammatory process, due to syphilis or some other cause, is a very rare lesion. It is usually gradual in onset and does not produce sharp pain.

Nothing is said in the history about the patient's habits. If we choose to assume that he was a confirmed alcoholic, his jaundice might be due to cirrhosis of the liver, especially as the edge of that organ is easily palpable. The sudden pain, however, could hardly be due to cirrhosis, and, as the case stands, we have nothing to support such a hypothesis. The habits should be further investigated.

On the whole, the diagnosis must remain in doubt as between stone and cancer, the odds slightly favoring stone.

Outcome.-On the twenty-second of June the abdomen was opened and two large stones were removed from the common bile-duct. The patient made an uneventful recovery.

His itching was relieved by a powder consisting of sodium salicylate, talc, and starch in equal parts, dusted on the skin, and also by an alkaline bath.

Diagnosis.-Stone in ductus choledochus.

\section{Case 107}

An unmarried woman of twenty-nine entered the hospital March II, I908. Since the age of sixteen she has had at times "stoppage of the bowels," worse when she is on her feet. When questioned as to the nature of this stoppage, she stated that it consisted of pain in the right upper quadrant, so severe that she cannot stand the pressure of her clothes, accompanied by the presence of a lump which is more prominent when she exercises. This trouble has been especially bad for the past five months. Her bowels rarely move without medicine, and her stools are small, hard, and often black. She has a poor appetite, but never vomits. She had considerable cough and sputa off and on for years, but has never raised blood. For four months she has had much dyspnea and palpitation. Her urine is at times scanty, never bloody, and never passed in large amounts. She has lost five pounds in the last two years.

The patient is pale (hemoglobin, 75 per cent.). Scattered on the right half of the trunk and the inner aspect of the right upper arm are numerous light-brown, irregularly shaped spots. The glands are palpable in the axillæ and groins. The tongue is bat shaped-widest at the tip. It is protruded very far, and during this act the anterior 
pillars of the fauces are drawn forward. A low-pitched systolic murmur is heard over all the precordia, but not transmitted beyond that area. The heart shows no enlargement. The pulmonic second sound is greater than the aortic second. In the epigastrium a violent pulsation, vertical and lateral, is felt, raising the hand three-quarters of an inch at each beat of the heart. Beneath the margin of the right ribs a smooth, rounded mass, about four inches long and two inches wide, can be grasped. between the hands and moved about in all directions. It is very tender.

Discussion.-The points deserving discussion in this case are the nature of the "stoppage of the bowels," the interpretation of the heart murmur in connection with the patient's dyspnea and palpitation, the significance of the way in which the tongue is protruded, the nature of the rash upon the chest, and the importance of the mass in the right hypochondrium.

It is clear that she has no stoppage of the bowels. We have to explain, however, why the lump complained of in the upper right quadrant and the pain which accompanies it are more prominent on exertion. This is the case not infrequently with a tender, passively congested liver, the result of cardiac insufficiency. But have we any such insufficiency in this case?

Since the heart is not enlarged and the pulmonic second sound no louder than we should expect it to be in a woman of twenty-nine, we have only the murmur to suggest heart disease. But from a systolic murmur alone it is never wise to infer the presence of any disease of the heart, especially when the patient is anemic. It seems reasonable to consider this murmur as hemic or functional. We have no reason, then, to believe that the heart is failing or that the lump in the right upper quadrant has any relation to it.

A patient who protrudes his tongue in the way described above, so that the whole of it can be seen, has usually been in the habit of looking at his tongue in a mirror. The pillars of the fauces are then drawn forward by the effort to get the tongue completely into the outer world. These facts give us a certain inkling of the patient's mental condition and of its possible bearing on the interpretation of his symptoms.

The eruption here described seems to correspond with that produced by tinea versicolor. Though other possibilities are open, this seems the most reasonable one, provided the lesions are of long standing. This is the most common position for an eruption of that origin.

The mass in the right hypochondrium corresponds accurately to 
the description of a floating kidney, though not all such kidneys are tender. It is probably the lump which the patient felt at the times when she supposed herself to have stoppage of the bowels. This would account for its greater prominence when she is on her feet.

The association of floating kidney with a great variety of so-called neurasthenic symptoms is a very familiar fact clinically. That this patient is of a neurotic temperament is suggested by the violent beating of the abdominal aorta (dynamic aorta), by the way she puts out her tongue, and by her chronic constipation. In the absence of any other lesions discoverable on physical examination the diagnosis of floating kidney associated with slight anemia in a neurotic person seems the best explanation of the symptoms. The dyspnea may well be due to the anemia.

Outcome.-The patient was given a close-fitting abdominal binder, which apparently gave much relief. Reassurance and general tonic treatment (Blaud's pill, Io grains thrice daily, tincture of nux vomica, Io to 50 drops before each meal) played a large part in her recovery.

Diagnosis.-Debility; floating kidney.

\section{Case 108}

An American woman of forty-six has been doctoring for stomach trouble for five months. A month ago she was taken suddenly with a profuse black, watery diarrhea. This was followed by vomiting, chills, and pain in the epigastrium and back. For a week she was kept more or less under opium, after which the gastric and intestinal symptoms abated, but she has remained in bed most of the time since then, in a very exhausted condition, and suffering most of the time from pain in the right upper quadrant and in the small of the back. This pain is constant, with occasional exacerbations. Opium has frequently been given. For several weeks she has taken only liquids. Although the pain appears only in relation to eating, she vomits nearly every day at irrregular intervals. She has never vomited blood, and has never been jaundiced. She has had a slight cough for five years, and dyspnea on exertion for one year. She has lost $\mathrm{I} 3$ pounds in the past two years. The pain is often severe enough to keep her awake at night.

On physical examination the mucous membranes are found pale. The chest is negative, except for slightly diminished respiration in the right back, below midscapula. The abdomen is entirely negative, except that the edge of the liver is palpable on deep inspiration.

The blood shows red cells, 4,032,000; white cells, 6800; hemoglobin; 55 per cent. 
The stained smear shows some achromia and poikilocytosis. The differential count and the other features of the blood are normal. Careful examination in a warm bath, with complete and satisfactory relaxation of the abdominal muscles, shows absolutely nothing abnormal. Examination of the stomach shows no fasting contents. The gastric capacity is 36 ounces - the lower border of the organ one inch below the navel after inflation. After a test-meal no free hydrochloric acid and no organic acids are found. The guaiac test is negative in the gastric and intestinal contents.

After three weeks' stay in the hospital the patient gained three pounds, but continued to complain of pain and seemed very miserable.

Discussion.-The black color of the stools, associated with a longcontinued gastric disturbance, forms an important portion of the picture of peptic ulcer. We must remember, however, that as she has been doctoring for stomach trouble for five months and has taken a great deal of opium, it is quite possible that her symptoms may be due wholly or in part to the treatment. Black stools may well be due in this case to that commonest of gastric medicaments, subnitrate of bismuth. In patients who have been through five months of this kind of treatment it is not at all surprising to find hydrochloric acid absent from the gastric contents. Her stomach empties itself normally, shows no enlargement and no blood. One more point serves to increase our confidence that no visceral disease is present, namely, the complaint of a year's dyspnea by a patient whose heart and lungs are normal. This dyspnea antedates the occurrence of stomach symptoms by at least seren months. This would be quite natural if we supposed that the dyspnea and the stomach symptoms were alike due to the anemia shown by the present blood examination. If, on the other hand, the anemia resulted through hemorrhage from an ulcer, the dyspnea should not have antedated the stomach symptoms.

Yet, after the use of treatment based upon the idea that anemia was the cause of her symptoms, there was no clear proof that we were right and it seemed best to explore the abdomen.

Outcome.-Operation by Dr.F.B. Harrington revealed absolutely nothing, but the patient seemed greatly improved after it, and when last heard from had continued in good health. Cases of this type should be borne in mind when discussion arises regarding those operations for "chronic appendicitis" in which the appendix shows signs of appendicitis only to the eye of the surgeon, while the pathologist remains unconvinced.

"But the patient's symptoms abated after the operation," says the 


$$
2^{3^{2}}
$$




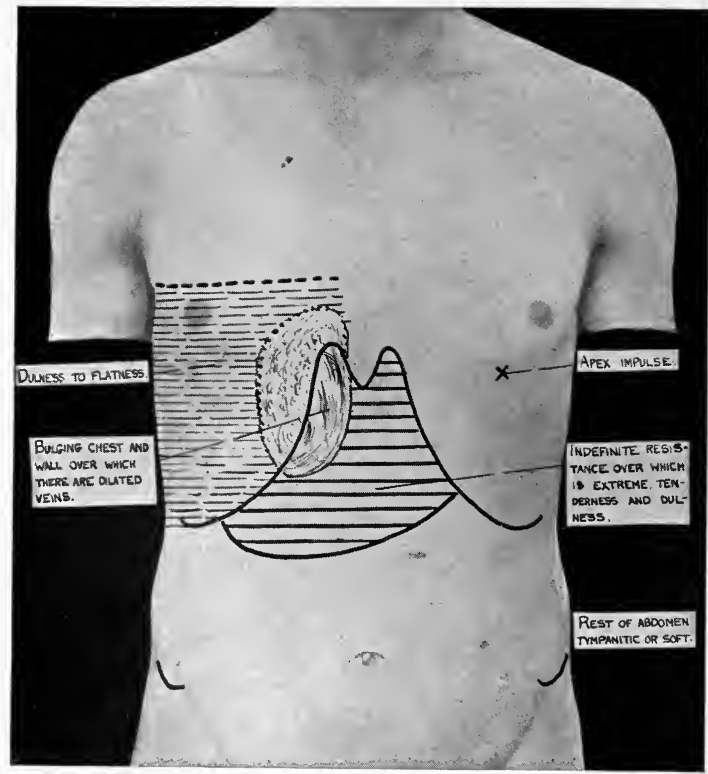

Fig. 35.-Diagram of bulging and resistance as recorded in Case rog. Symptoms, pain over the above area, weakness, dyspnea, and cough. (See also Fig. 36.)

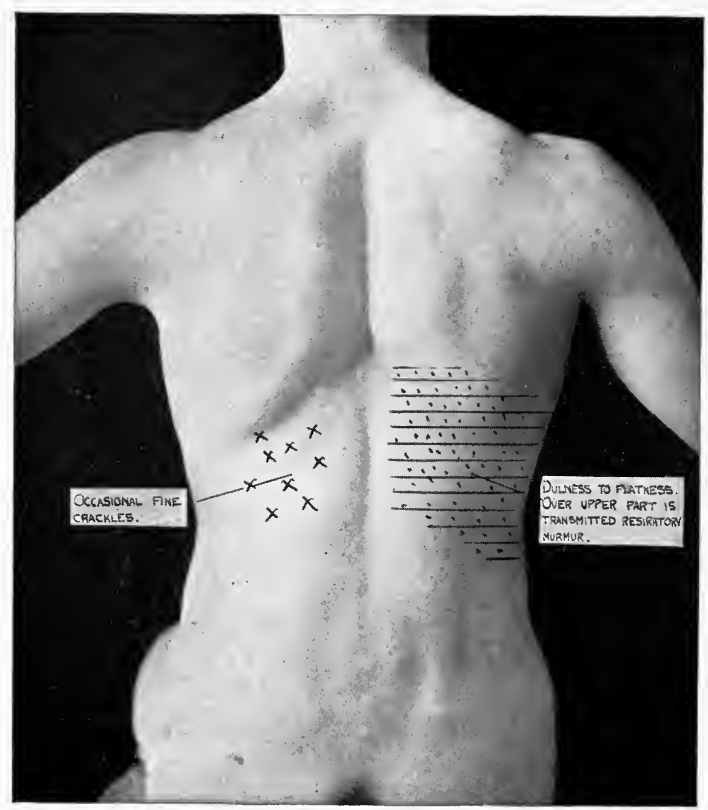

Fig. 36.-Signs discovered in the back of patient described on page 233. "(See also Fig. 35.) 
surgeon. True, but so they did in the case just described, though nothing was removed. There is abundant experience to prove that operations and postoperative hygiene (mental and physical) are in themselves enough to produce a marked improvement in the symptoms of many a patient.

Diagnosis.-Debility.

\section{Case 109}

March 18, 1907, I examined a Russian tailor thirty-nine years old, with the history of pain in the right upper quadrant lasting fifteen weeks, accompanied by frequent dry cough, shortness of breath, and increasing weakness, but no fever, so far as he knows. For the past two weeks he has had night-sweats, tenderness in the left shoulder, and inability to sleep on the left side. He has lost weight and strength, but has worked irregularly until two weeks before entrance. His previous history, family history, and habits are otherwise excellent.

Examination showed a sallow, emaciated, worn-looking man. Nothing abnormal was detected in the examination of the heart. In the right back there was dulness below the angle of the scapula, with diminished breath and voice-sounds. The condition of the abdomen is best explained by Fig. 35 .

The patient's temperature ranged for eight days between $99^{\circ}$ and IOI ${ }^{\circ} \mathrm{F}$. His red cells were 4,000,000; white cells, I I,000; hemoglobin, 60 per cent. The stained smear showed nothing abnormal. The urine was equally blameless. In the stools numerous eggs of the Trichuris trichiuria were found. Rather large, palpable glands were found above both clavicles; the chest, head, and extremities negative, except as above noted. Free purgation produced no change in the physical signs.

Discussion.-There is a good deal to suggest phthisis in the first impression of this case-cough, dyspnea, weakness, night-sweats. But though there are some abnormal signs in the right back, they are not sufficient to account for the symptoms. Empyema is, perhaps, more likely, but I have never heard of an empyema which worked to the surface so near the ensiform cartilage. It is unfortunate that an $x$-ray was not taken, owing to the patient's great prostration. By this means one might have obtained some evidence as to whether the trouble was above or below the diaphragm. Our attention naturally centers on the region of the prominence shown in Fig. 35; at first sight the mass certainly appears to be below the diaphragm.

Tumors of the liver should first engage attention. Cancer of the liver almost never presents itself in this situation onlv. We find almost 
invariably a general enlargement of the liver downward, and multiple nodular masses below the ribs. I have never known a hepatic cancer to produce a localized bulging of the chest-wall such as was present in this case. This latter observation applies also to tumors of the colon, gall-bladder, retroperitoneal glands, and kidney. The hypothesis of cancer somewhere receives some support from the presence of enlarged glands over the clavicle, which might represent metastases; but it is very hard to see where the cancer could be situated.

Hydatid cyst of the liver was much discussed in the numerous bedside consultations over this case, but it was pointed out that hydatid does not produce so much prostration and pain, not, at any rate, until it has produced a tumor much larger than that in this present case. The striking thing about most hydatid cysts of the liver is the slight impression that they seem to make either upon the patient's consciousness or upon his general health and nutrition. The feel of the tumor in this case is not at all characteristic of hydatids.

Can local disease of the chest-wall explain these symptoms? Tuberculosis, syphilis, actinomycosis, or neoplasm might appear at this point, but they should involve the ribs or intercostal tissues themselves, whereas in this case the ribs seem to be quite unaffected-merely pushed forward by something behind them.

Hepatic abscess or subdiaphragmatic abscess often causes a prominence at exactly this point, and some of the symptoms of the.case-the pain, cough, dyspnea, weakness, and night-sweats-could be thus explained. On the other hand, we have no history of the ordinary causes for either of these varieties of abscess-no dysentery, no appendicitis, no peptic ulcer or gall-stones. It seems remarkable, moreover, that the leukocytes should not be more increased if there is an abscess large enough to produce such a tumor. Despite these objections, however, the picture corresponds more nearly with that of subdiaphragmatic abscess than with any other disease.

Outcome.-A week later the patient's abdomen was opened and a subdiaphragmatic abscess found. Its source remained doubtful.

Diagnosis.-Subdiaphragmatic abscess.

\section{Case 110}

A fireman of fifty-eight worked on the Panama canal in r9o4 and 1905, but had to return in December, 1905, on account of a long attack of dysentery. Though always a heavy drinker, he was otherwise well until May, I906, and then weighed 212 pounds. In May irregular colicky pains began in the right upper quadrant, which were 
much worse at night and which did not, as a rule, bother him in the day-time. At times he suffered enough to require morphin. There was no radiation and no vomiting, but there was nausea, and considerable relief was obtained by belching gas. The bowels were rather loose, and a movement caused relief of pain. At times the movements were clay colored; at other times they were brown. About June Ist the stools became persistently clay colored, the skin was noticed to be yellow and the urine dark colored.

From June Ist until the present time (August 8th) he has had no pain, but to-day about noon he suddenly began to have a series of very severe cramps, vomited for the first time, and had a chill. His present weight is $\mathrm{I} 6 \mathrm{I}$, a loss of 50 pounds, but he had been able to work until six weeks previously.

Inspection of the patient's body revealed nothing abnormal except intense jaundice, with brownish mucous membranes and an increase of liver dulness, such that the organ extended from the fifth rib in the nipple-line to a point one inch below the costal margin. Below this edge a soft, rounded mass could be made out, about the size of a lemon. The spleen was not palpable. The white cells were 16,600 ; hemoglobin, 90 per cent. The Cammidge test was positive. In the afternoon after entrance the patient suddenly sat up in bed and yelled with pain; it was referred to the epigastrium, and was, he said, unlike any that he had had before. The abdomen was now rigid throughout, with marked tenderness, especially in the epigastrium. The patient vomited several times this afternoon.

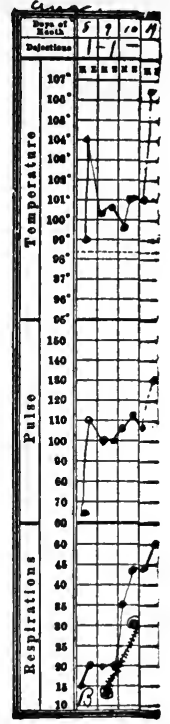

Fig. 37.-Chart of case iro.

Discussion.-A history of dysentery and a residence in the tropics, when followed by symptoms which appear to involve the liver, should always remind us that hepatic abscess is a common complication of tropical dysentery. This idea seems all the more plausible in the present case, because there have been chills, fever, leukocytosis, and an increase of liver dulness. Yet the pain of hepatic abscess could rarely be described as "cramps." The disease is often painless; if there is any pain at all it is usually a steady, dull, but increasing type. The soft tumor below the ribs, moreover, cannot be due to hepatic abscess, and we do not expect such intense and persistent jaundice as the result of that disease.

The colicky pains and the jaundice might well have been due to a 
stone in the common duct. Had this stone been near the entrance of the cystic duct, infection might well have extended to the gall-bladder. Suppurative cholecystitis and final perforation of the gall-bladder would then account for the chill and intense pain on the day of entrance.

Against this we have the fact that a gall-bladder which has previously nourished stones is not often so distensible as to form a tumor, such as projected below this patient's liver. The intensity and steady persistence of the jaundice are also somewhat unusual for choledochus stone.

Cirrhosis does not seem probable, though the usual cause of that disease appears to have been operative. A pain like that here described is rarely if ever due to cirrhosis, and the jaundice which occurs in a certain proportion of cirrhotic cases is almost never intense. Cancer of the pancreas or of the bile-ducts is the commonest cause of intense and persistent jaundice in a man of this age. The tumor below the ribs might be the enlarged gall-bladder which generally results from this disease. On the other hand, the pain is severer and more sudden in its onset than is to be expected in cancerous obstruction of the bileducts. The colic and the variation in the color of the stools seem more like cholelithiasis. No one of these facts, however, excludes cancer, which seems, on the whole, the most reasonable diagnosis.

How are we to explain the chill and attack of pain described in the last few lines, which are intended to convey an idea of the greatest possible severity of suffering? In my experience an abdominal pain of this description, such that the patient suddenly yells out in agony, is almost always due to one cause-perforative peritonitis. This might be accounted for under either of the diagnoses last discussed; either stone or cancer may have caused ulceration of the ducts and set up at first a localized peritonitis, which later perforated and set free a virulent fluid into an unguarded peritoneum.

Outcome.-He died three days later. The variations of his temperature are shown in the accompanying chart. He was able to take very little food, and during the last twenty-four hours was delirious. Autopsy showed cancer of the head of the pancreas, almost occluding the common bile-duct. The pancreas was practically destroyed. The gall-bladder was enlarged, much distended, not inflamed, and contained a single gall-stone. There was an extensive cancerous infiltration of the posterior wall of the stomach. There was also an acute general peritonitis, for which no cause could be found!

Diagnosis.-Pancreatic cancer. 


\section{Case 111}

A negro of thirty-five has complained of steady pain in the right hypochondrium for two months. This pain came immediately after eating and lasted about two hours. His appetite is good. His bowels are constipated, moving only once in from two to seven days with medicine. He has also suffered from numbness and tingling in his legs, with weakness, and has had a cough for the past two weeks, with slight white sputum.

Physical examination shows an irregular fever (see chart), the skin very dry and scaling, the heart not remarkable, the lungs negative. There is a nodular mass indistinctly felt in the right iliac fossa, tender, but at times difficult to outline. The abdomen is retracted, and peristalsis can be seen near the navel. On the left hand and the dorsum of the right foot are elevated areas of reddened skin, with a pink, smooth center, about one inch in diameter. The chest is negative; likewise the blood and urine.

Examination of the stomach by means of a stomach-tube showed that the organ would hold about only 23 ounces of water without distress. Its lower border after inflation reached just below the navel. The contents extracted after a test-meal showed free $\mathrm{HCl}$, O.I per cent.; no occult blood; no lactic acid. No contents could be obtained from the stomach before breakfast.

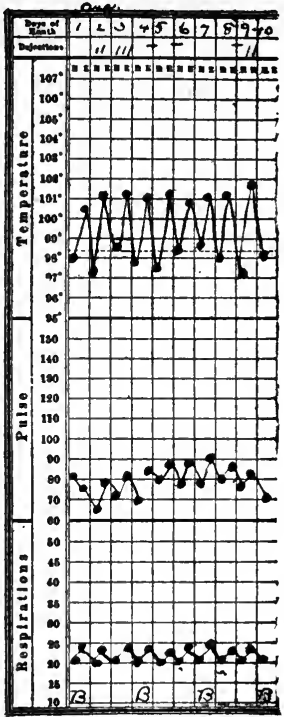

Fig. 38.-Chart of case III.

Discussion.-There is no need of extended discussion here. A nodular mass in the right iliac fossa, accompanied by visible peristalsis, marked constipation, and fever, means chronic intestinal obstruction in the region of the cecum. Obstruction at this point is practically never due to fecal impaction alone; there is almost always some stricture of the gut behind which feces accumulate. Such a stricture might be syphilitic, cancerous, or tubercular. Occasionally a chronic appendicitis with adhesions produces obstruction.

Imagine that an abscess has occurred earlier in the history of the case; this may later have become walled off and massed into a tumor something like that here felt. The adhesions associated with it might then lead to the symptoms of obstruction here described. 
A retracted abdomen and an irregular fever without leukocytosis is distinctly more suggestive, however, of tuberculosis.

If the stricture is tuberculous, the tumor mass is probably made up of caseating glands adherent to the cecum, itself infiltrated by tuberculosis. The fact that the patient is a negro and the presence of fever-especially fever of the type shown in this chart-make tuberculosis more probable than cancer. Syphilis rarely produces so large a mass in this region. Extensive syphilitic infiltrations are generally found near the rectum. The diagnosis of pericecal tuberculosis is further supported by the character of the cutaneous lesions, which are distinctly suggestive of tuberculosis.

No good reason can be given why the pain is referred in this case to the right hypochondrium, rather than to the region of the tumor, as is the rule.in such cases.

Outcome.-The patient remained ten days in the ward, complaining continually that he did not receive enough medicine, but showing no improvement in any respect. At the end of that time the abdomen was opened and showed a nodular mass of tuberculosis in the cecal region, with general adhesions but without fluid. At death, a month later, tuberculosis was found also in the lungs, adrenal glands, lymphglands, skin, and in almost every other organ.

Diagnosis.-Pericecal tuberculosis.

\section{Case 112}

A young farmer of twenty-five, whose father had died of tuberculosis but whose family history was otherwise good, came to the hospital January 25, 1906, with the following story: For the past fifteen years he has had from time to time pain in the right upper quadrant, in attacks lasting from three to five days, then gradually subsiding but leaving him much used up. For the past three years the pain has been so sharply localized that it could be covered with one finger.

Up to one year ago he averaged about two attacks a year, but within the past year the attacks have been from one to four weeks apart, apparently depending upon the performance of heavy work or the eating of hearty food. During these attacks the pain is not constant, but comes in spasms and is relieved by taking a "pain-killer" and using a hotwater bag, or by leaning over the back of a chair so as to bring strong pressure to bear upon the painful spot. In the last attack the pain radiated to the back, but never to any other point. Three years ago he thought he noticed in the region of the pain a bunch, which was tender, but gradually disappeared. The painful spasms last from 
fifteen to twenty minutes each. He was very sallow three years ago, and thinks he has been so since then. His severest attacks are accompanied by chills and fever. During the past year he has been able to do only very light work. He occasionally vomits during an attack, the material being usually greenish. During an attack he has nightly emissions. Walking on hard pavements or hard floors, especially during the time of an attack, causes pain in the right upper quadrant, and sometimes shortness of breath. His appetite between attacks is always good and his bowels are usually constipated. In the fall of 1905 he weighed 185 pounds. Now he weighs 165 pounds. Work that requires stooping or heavy lifting will often bring on an attack within two or three hours.

Physical examination showed no jaundice. There was nothing abnormal in the chest or abdomen, and nothing wrong with the blood or urine. After staying four days in the hospital entirely free from symptoms he was discharged.

May 24, 1907, he entered the hospital for the second time, and stated that, since leaving the wards fifteen months previously, he had had many attacks of pain similar to those previously described. His worst attack was ten months ago, when the pain failed to yield to morphin or chloroform, and lasted four hours. For a week after this he was unable to leave his bed. This spring he had nearly steady pain for five or six weeks following the ingestion of a large quantity of maple syrup. After an attack his urine is always high colored, almost black; the color of his stools is not abnormal.

As on a previous occasion, physical examination was entirely negative, but this time the use of a stomach-tube revealed that the percentage. of free hydrochloric acid after a test-meal was 0.29 , and that in the fasting contents the percentage of hydrochloric acid was 0.23 . There was no reaction to guaiac in stools or gastric contents.

Discussion.-There is much to make us think of gall-stones in this case, though the age and sex are against this diagnosis. The association of such a pain as is here described with chills and fever, with deep discoloration of the urine, which may well have been due to bile, and with a bunch which may have been the gall-bladder, goes far to complete the clinical picture of cholelithiasis. This picture becomes still clearer as we note the freedom from digestive symptoms between the attacks of pain. Moreover, it may be that on stooping he shifts the position of a stone in the gall-bladder in such a way that it becomes impacted and produces colic.

But this trouble has been going on for fifteen years, and gall-stones 
are practically unknown in a boy of ten, which was the age of our patient at the beginning of his attacks. Again, it is difficult to see why a gallstone colic should not be relieved by morphin or by chloroform, and why it should not produce tenderness in the region of the gall-bladder. Patients who have had many attacks of gall-stones almost always experience some of the typical radiations of the pain, which, with one exception, have been wholly lacking here. The absence of jaundice and enlarged gall-bladder adds a certain weight to the arguments already adduced against gall-stones.

Next to gall-stones, by far the commonest cause of symptoms like these is peptic ulcer, gastric or duodenal. The long history of his attacks and the gradually shortening intervals between them, the excess of hydrochloric acid in the gastric contents, and the relief of pain by pressure are facts tending to convince us that peptic ulcer is present. On the other hand, it is curious that we were unable to obtain any reaction to guaiac in the gastric contents or in the stools. Why the pain should be increased by walking on hard pavements or hard floors, and why the attacks should be associated with nocturnal emissions, are problems not explained by any knowledge that I possess.

A "high" (undescended) appendix comes to our minds as a possibility, but who ever saw a case of appendicitis-high or low-in which the pain was relieved by strong pressure, as in this case?

Outcome.-On May 29th the abdomen was opened; a duodenal ulcer was found. It had perforated and become adherent to the gallbladder. In connection with the relief of pain by pressure in this case I recall a case of duodenal ulcer which I saw with Professor Osler at Oxford in the summer of Igo8. The man told us, without a ghost of a smile, that the pain was so bad that his wife often had to kneel on his stomach for half the night.

Diagnosis.-Duodenal ulcer (local peritonitis).

\section{Case 113}

A single woman of thirty-seven entered the hospital on July $20,1906$. $\mathrm{Up}$ to the age of five years she was subject to convulsions with loss of consciousness, but these have not recurred since. She had diphtheria with paralysis of the palate at the age of twelve. In I892 she fell in a gymnasium and hurt her back, since which time she has done no work, and has suffered from severe pain in the middle of the back and on the top of her head. At times she has a sense of constriction in her throat. She consulted an orthopedic specialist in I902, and has since then worn a brace for her back off and on, with very little relief. A 
spur was removed from her nose one year ago. Three weeks ago she began to have very severe pain over the right side of the face, and was operated on for disease of the antrum, but none was found. Her pain was immediately relieved, and sleep induced by the subcutaneous injection of sterile water.

Since that time she has suffered especially from pain in the right hypochondrium-worse in the early morning, somewhat relieved after the morning urination or by vomiting.

Physical examination shows rigidity of the abdomen with marked sensitiveness of the right half of the head and of the back, especially in the dorsal region. The internal viscera, the blood and urine are normal, likewise the temperature and pulse. Respiration ranges between 30 and 45 . She is often awakened by spasmodic pain in the neck, much increased by attempts to walk. She looks well, but still complains "of soreness in the bowels, which prevents her from eating and causes her to vomit and her head to ache."

Discussion.-We get a strong impression, on reading this case, that we are dealing with nervous invalidism reinforced and made more obstinate by a variety of meddlesome treatments. But in any case which gives us this first impression we should do our best to combat it by endeavoring to establish the existence of some form of organic disease. Only in this way can we avoid doing serious injustice to many patients who have both organic disease and a nervous make-up, with the latter in the foreground. One of the problems which first engaged our attention was this: Why should her pain be relieved after the morning urination? This combination of symptoms is not at all unusual, and in my experience it signifies that the pain has resulted from gaseous distention of the colon, which is relieved when the emptying of the bladder shifts the pelvic tensions enough to allow the escape of intestinal gas.

The unilateral distribution of sensitiveness over the head and trunk, the relief of pain by the subcutaneous injection of sterile water, the rapid respiration, and the history of her medical fortunes justify us, I think, in believing that our negative physical examination represents the truth, and that we are justified in making that dangerous diagnosis: hysteria. But it is only by experimental therapeutics, that is, by trying out the results of treatment based on the hypothesis that we are dealing with habit-pain and nervous invalidism, that we can get any further certainty upon the diagnosis. To such experiments, accordingly, we addressed ourselves.

Outcome.- Under a combination of scolding, encouragement, and reèducation she was able, at the end of a month, to walk fifteen yards 
without support. Two weeks later she could walk an eighth of a mile, and the pain in her head was much relieved. She still complained, however, of soreness in the bowels, and this she has had at intervals ever since that time, especially when she gets run down.

This case seems to me to illustrate well that fallacy about the importance of "reflex causes" for general nervous disturbances which had so strong a hold on the last generation of medical men. The wonder is that this patient escaped without appendectomy and hysterectomy. In many clinics she would also have undergone a gastro-enterostomy. I think the opinion is coming to prevail that when the history and the physical signs point strongly toward a general neurosis, attention to socalled reflex or local sources of irritation not only does no good, but makes the patient distinctly worse by concentrating his attention upon the part, by increasing the period of invalidism, and by withdrawing him from the normal supports and stimuli of the working life.

Diagnosis.-Hysteria minor.

\section{Case 114}

A Portuguese tailoress, forty years old, entered the hospital December 21, 1908. When a little girl in Portugal she had typhoid fever. In the preceding June she was in bed for a week with "malaria," and has not been well since. She has now had fever for five weeks. She has been at work for the first two weeks of this time, but has had headache, anorexia, vomiting, diarrhea, and cough for most of the time in the last five weeks. She has been in bed for ten days.

Physical examination shows no emaciation. Many fine râles are scattered over both chests, and a few squeaks distributed among them. Whichever side she lies on appears to contain the greatest number of râles. 'There is a slight cyanosis, with movements of the nostrils as she breathes. Voice-sounds are slightly increased at the right base. The white cells are 6800,56 per cent. of them being polynuclear. Widal reaction positive. The spleen was palpable on full inspiration.

On the fourth of January she was suddenly seized in the night with sharp pain in the right upper abdominal quadrant, accompanied by vomiting, sweating, and a weak, rapid pulse. Despite $\frac{1}{3}$ grain of morphin and $\frac{1}{30}$ grain of strychnin, the pain and vomiting persisted. Next morning there was distinct tenderness over the seat of pain, and a tender, rounded mass was vaguely felt in the region of the gall-bladder.

The white cells, January 5th, were I2,800 at Io A. M., 23,600 at 9 P. M.; 22,800, January 6th at 9 A. M. There was still no abdominal spasm. 
Discussion.-This patient has a fever of long duration. In temperate climates, as I have elsewhere shown, ${ }^{1}$ there are but three common fevers which last more than two weeks, viz., typhoid, tuberculosis, and sepsis. This woman has cough, cyanosis, râles in both lungs, and, since the nostrils move visibly, she probably has dyspnea. May it not be that she has'miliary tuberculosis with tuberculous peritonitis, the latter showing itself in one of those acute paroxysms which are so often mistaken for appendicitis, cholecystitis, intestinal obstruction, and other abdominal emergencies? But if this is so, why is the patient not more emaciated after five weeks of illness? Unless we can get evidence of tuberculosis either in the family history, in the sputa, or in some other part of the body, there is no way of verifying this hypothesis any further.

As we read that the Widal reaction was positive, it seems at first unnecessary to discuss the diagnosis further. There is nothing in the case to exclude typhoid, since lung signs like those here described may represent simply the ordinary bronchitis of typhoid. But as she has previously passed through an attack of typhoid fever, it may be that her Widal reaction is one of the residual results of that illness. We know that the Widal reaction may persist for thirty years or more after an attack of typhoid. The splenic enlargement is quite consistent either with typhoid or with tuberculosis. As there seems no good evidence of a generalized septic infection, and as the leukocyte count is at the outset so low, there seems no good reason to consider any disease other than typhoid and tuberculosis. The rarer causes of prolonged pyrexia (meningitis, rheumatism, syphilis, leukemia, malignant disease) do not deserve serious consideration.

But there seem to be two acts to this drama, and the secondwhich began January $4^{\text {th }}$ - throws considerable light upon the first, for the new pain gives every evidence of being due to cholecystitis, and cholecystitis is a common complication of typhoid, not of tuberculosis.

Outcome.-Operation was done on the sixth and showed an injected, distended gall-bladder filled with pus, with a spot of gangrene on the wall and several stones within.

The disease showed thereafter the ordinary course of typhoid. Eberth's bacilli were recovered from the gall-bladder.

Diagnosis.-Cholecystitis complicating typhoid

${ }^{1}$ R. C. Cabot, The Three Long-continued Fevers of New England, Boston Med. and Surg. Jour., August 29, 1907 . 


\begin{tabular}{|c|c|c|c|c|c|c|c|c|c|c|c|c|c|}
\hline 芶高 & 0 & 0 & 0 & 0 & + & 0 & 0 & + & 0 & 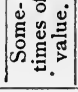 & $\circ$ & $\circ$ & 。 \\
\hline 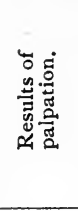 & 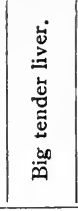 & 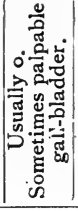 & 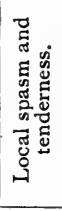 & $\circ$ & $\circ$ & 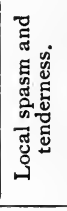 & 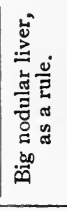 & ० & 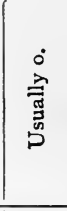 & 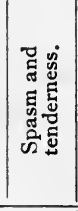 & $\vdots$ & $:$ & 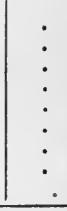 \\
\hline 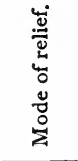 & 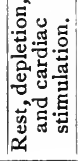 & 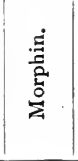 & 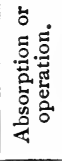 & 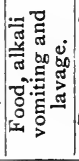 & 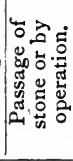 & 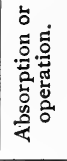 & $\vdots:$ & 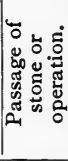 & 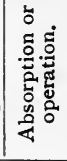 & 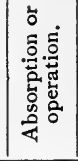 & 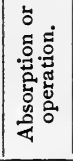 & 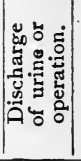 & 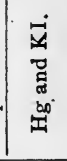 \\
\hline 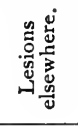 & 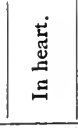 & $\circ$ & 0 & $\circ$ & $\circ$ & $\circ$ & 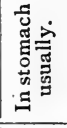 & ० & 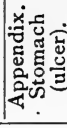 & 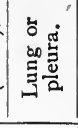 & ० & $\circ$ & 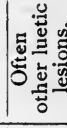 \\
\hline 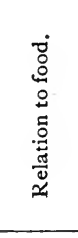 & $\circ$ & 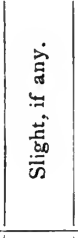 & ० & 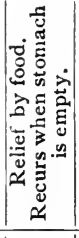 & 0 & 0 & 0 & 0 & 0 & $\circ$ & $\circ$ & ○ & ० \\
\hline 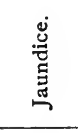 & 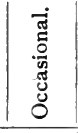 & $\begin{array}{l}\text { 节 } \\
\text { 离 } \\
\text { 点 }\end{array}$ & 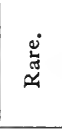 & ○ & $\circ$ & ○ & 巳ँّ & $\circ$ & $\circ$ & ○ & 。 & ○ & 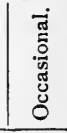 \\
\hline 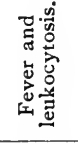 & 0 & 竞 & + & 0 & $\circ$ & + & 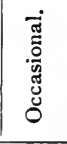 & 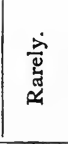 & + & + & + & 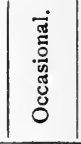 & 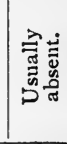 \\
\hline 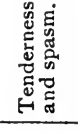 & 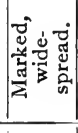 & 总过 & $\begin{array}{l}\text { 馬 } \\
\stackrel{\Xi}{0}\end{array}$ & $\circ$ & 总 & త్త & 。 & $\circ$ & 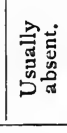 & 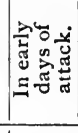 & 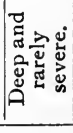 & 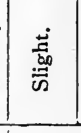 & 莺 \\
\hline 离 & $\overrightarrow{\bar{\Xi}}$ & 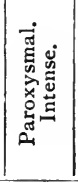 & 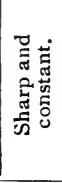 & 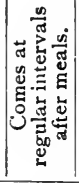 & 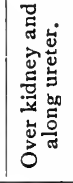 & 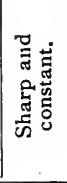 & 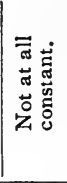 & 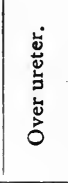 & 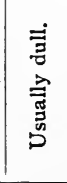 & 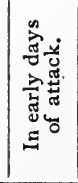 & 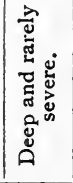 & 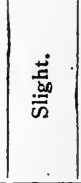 & $\begin{array}{l}\frac{\dot{\vec{n}}}{\mathrm{~m}} \\
\frac{\bar{v}}{\bar{\Omega}}\end{array}$ \\
\hline 宬 & : & 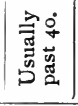 & : & 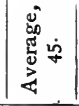 & : & : & 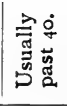 & $:$ & $\vdots$ & 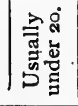 & $:$ & 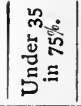 & $\vdots$ \\
\hline 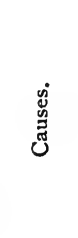 & 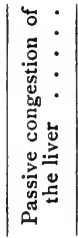 & 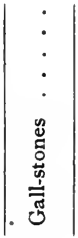 & 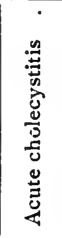 & 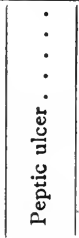 & 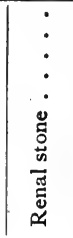 & 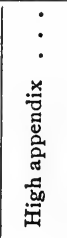 & 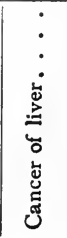 & 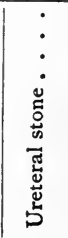 & 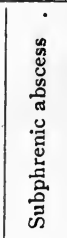 & 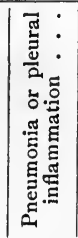 & 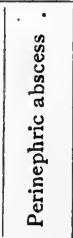 & 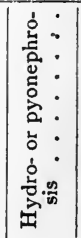 & 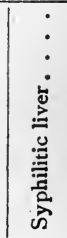 \\
\hline
\end{tabular}




\section{CHAPTER VII}

\section{PAIN IN THE LEFT HYPOCHONDRIUM}

THE left hypochondrium is not a common place for puzzling pains. I have known very few diagnostic problems which centered there. Many discomforts arising from the stomach are felt in the left hypochondrium, but, as a rule, their origin and nature are tolerably clear.

I. Flatulence, the commonest of all causes of pain in the lower left axilla, is also responsible for many complaints below the left ribs. The relief by escape of gas distinguishes many such pains, but we must remember that in many cases the flatulence itself requires explanation. Gas-formation may be the result, and its discharge the relief, of pain due to:

(a) Angina pectoris.

(b) Peptic ulcer and hyperchlorhydria.

(c) Chronic appendicitis.

(d) Gall-stones (rarely).

Even toothache may cause recurrent flatulence and be temporarily relieved each time the gas (air?) is discharged.

2. Surgical disease of the kidney (stone, tuberculosis, neoplasm, local infection, hydronephrosis) occasionally causes pains in the left hypochondrium. More often, however, the pain is in the loin, in the lumbar region, or along the course of the ureter. The presence of a tumor and of urinary disturbances usually makes it clear that the kidney is the source of the pain.

3. Adhesions about a spleen enlarged by leukemia, splenic anemia, malaria, syphilis, or polycythemia often produce pain in the left hypochondrium and above that point, but the obvious enlargement of the organ puts us on the right track unless we neglect physical examination altogether.

4. Cancer of the splenic flexure of the colon rarely gives pain over its own site. Usually such pains are in the umbilical region or diffused over the whole belly.

Some of the other diseases mentioned in Table $\mathrm{V}$ may cause pain in the left hypochondrium as well as in the right (e. g., pneumonia and pleurisy, especially in children), but no separate discussion of them is needed here. 
On the whole, then, it appears to me that most pains in the left hypochondrium have either an obvious origin from one of the easily recognized sources mentioned above under I, 2, 3, and 4, or are to be explained by reasoning identical with that already applied to the right hypochondrium. Some of the possible occasions for doubt are exemplified in the following cases:

\section{Case 115}

A white-lead worker of twenty-one entered the hospital July I6, I906, with negative family history, past history, and habits, except that he had syphilis four years ago.' Five years ago he passed some bloody urine, with clots, and at times nearly pure blood; this lasted for about ten days. He was well after that until two years ago, when he began to have dull, dragging pain under the left ribs, fairly constant day and night for two weeks, preventing work, but not preventing sleep. At this time he passed some "white stuff" looking like pus in his urine, mostly at the end of micturition. After two weeks he was well enough to be about and work, but he still notices the white stuff and occasional little strings in his urine. At times the urine is entirely clear, but for the past five months he says it has been clear for only five consecutive days. There has been no blood since four or five years ago. At times the urine smells very badly. Eight months ago and a year ago he had similar attacks of pain, relieved, as formerly, by the passage of pus.

The present attack came on five months ago; he began to have dragging pain under the left ribs, severe enough to prevent work, but not sleep. At times it doubles him up. At the beginning of this period he thinks he had high fever. He now passes urine every hour. He has lost nearly 20 pounds.

Physical examination of the chest is not remarkable. In the left hypochondrium is an irregular mass, palpable bimanually, hard, and slightly tender. Its position is fixed.

White cells, II,700; the temperature ranges most of the time about $99.5^{\circ} \mathrm{F}$. There is no elevation of pulse or respiration.

The urine shows pus, at times in large amounts, at times in very small amounts. It is not otherwise remarkable. Five milligrams of tuberculin were injected subcutaneously and caused fever, constitutional symptoms, and increased pain in the tumor.

Discussion.--If we fixed our attention chiefly upon the history of this case, our first impression as to diagnosis would naturally be lead-poisoning. Any abdominal pain in a lead-worker may be lead colic. We know also that lead affects the kidney. On the other hand, the physical ex- 
amination includes data not thus to be explained, and assures us that lead cannot play more than a subordinate part in the case. The mass, palpable bimanually, and the pus in the urine have nothing to do with lead.

Abdominal pain in patients who give a history of syphilis should lead us to consider tabes with gastric crises. As we look over the case with this idea in mind, we note that there is no record concerning the pupillary reactions, the knee-jerks, or the ankle-jerks. We know that tabes often leads to bladder troubles, and sometimes to a retention of urine. In this way a cystitis and pyuria might have been produced, and thence, by ascending infection, a pyelonephritis. In this way all the facts might be accounted for. Actually, however, the pupillary and other reactions were normal, and there was nothing to support the hypothesis of tabes.

Local renal disease giving rise to pyuria and tumor, with slight leukocytosis and fever, turns out most often to be due to renal tuberculosis. The positive reaction to tuberculin is not especially significant in an adult, since many adults react to tuberculin whether they are sick or well. More significant, however, is the increase of pain and sensitiveness over the tumor immediately following the injection. There seems to be no way of obtaining further insight into the nature of the trouble here present until we have further information in regard to the following points: (a) Can tubercle bacilli be demonstrated in the sediment of the centrifugalized urine? (b) If not, what is the result of injecting this sediment into a rabbit or a guinea-pig? (c) What does $x$-ray show in the region of the kidney? Even without these data, however, renal tuberculosis seems the most probable diagnosis.

Outcome.-On July 2 Ist the kidney was opened and a considerable amount of pus evacuated from a trabeculated cavity in which were fragments of stone. There was no positive evidence of tuberculosis.

Diagnosis.-Pyonephrosis with stone.

\section{Case 116}

A carpenter of thirty-seven, whose mother died of consumption, had an attack of "brain fever" eighteen years ago, and was in bed ten days. Ten years ago he fell while carrying some heavy lumber and broke four ribs. He was laid up for twelve weeks, and his left side "has never been strong since." He has had bleeding piles for seven years. His habits are good.

Four years ago he began to have needle-like pains under the left costal margin, coming on about every fifteen minutes, usually not severe. Oc- 
casionally the pains have been decidedly severe, radiating to the reginn of the heart and into the back. During these attacks he usually sweats, and at times, but not during the attacks of pain, his heart seems to pound. He has worked irregularly, and although at times he felt faint, he has never actually fainted.

Twelve days ago he awoke in the night with great difficulty in breathing, severe knife-like pain about the heart, radiating to the left arm, cold sweat upon the forehead, and great weakness. The attack lasted five minutes. After that he staid in bed for a week with slight, needle-like pains as before, and an annoying general soreness about the heart, in the left arm and in the back. Four days ago he awoke with a severe grasping pain in the region of the left nipple, extending through to the back, but not increased by deep breathing. He staid in bed for the next three days. To-day he got up and felt much better, but still feels heaviness and soreness in the left side.

On physical examination the heart's apex is seen and felt in the fourth interspace, four inches to the left of midsternum. There is no enlargement at the right. The sounds are regular and of good quality. A soft systolic murmur is heard at the apex, transmitted a short distance into the axilla. The artery wall is somewhat thickened above the elbow, but not beaded.

Blood-pressure, I $50 \mathrm{~mm}$. of mercury at entrance; a week later, I30. Blood and urine normal.

In the left lower back, below and around the lower angle of the scapula, over an area the size of the palm, breath- and voice-sounds are diminished and fremitus is lessened. An area about two inches in diameter in the left midaxillary line, over the sixth and seventh ribs, is tender on pressure. There are scattered areas of tenderness over the ribs below this point.

Discussion.-Flatulence is the commonest cause of pain like that here described, but the pain of flatulence is rarely so severe, and since there are no gastric symptoms to speak of, we cannot account for the patient's complaints in this way.

The signs in the back of the left chest are consistent with a chronic pleural thickening, such as might result from tuberculosis, and the family history of that disease strengthens this possibility. But although it is quite possible that the patient has had tuberculous pleurisy, we cannot account for the paroxysmal painful attacks in this way, especially as they seem to be independent of respiration.

The callouses due to broken ribs sometimes include nerve terminations and produce pain similar to that in the stump of an amputated 
limb. Presumably, we should interpret in some such way the patient's statement that his left side "has never been strong" since he broke his ribs twelve years ago. But it seems very unlikely that the recent paroxysmal attacks are due to his broken ribs. How large a part his old pleurisy may have played in his consciousness of weakness in the left side and in the heaviness and soreness which he still complains of it is difficult to say. The tenderness still complained of certainly cannot be due to pleurisy.

Functional angina pectoris is the natural explanation for severe precordial pain extending to the left arm in a patient whose age and relatively low blood-pressure do not suggest organic disease of the cardiovascular apparatus. This idea is favored by the long duration of his sufferings and by the fact that there is no demonstrable relation to exertion.

In connection with paroxysmal attacks of this character, and more especially with precordial and left axillary pain of moderate severity and long duration, the physician must never forget the mental aspect of the case. Pain supposed by the patient to be in the region of the heart is always made up of two elements- $(a)$ The pain itself; and $(b)$ what he thinks of it. The latter element is all the more important when it is largely unconscious. Dr. H. F. Vickery taught me years ago that, in dealing with patients who complain of pain in the precordial or left axillary region it is always well, after excluding organic disease by physical examination, to ask the following question:

"Suppose you had that same pain in your shin, would you have come here to see me to-day?"

This clever little psychologic device of Dr. Vickery's enables the patient to separate the pain itself from what he thinks of $i$, and to decide. whether or not his fear of heart disease and its consequences has added to his sufferings. To think of the pain in his shin is to think of it freed from the additions and vague dreads sure to be associated with pain "around the heart." The very vagueness of these fears magnifies their organic effects, their tendency to aggravate pain. It is really astounding how rapidly such a pain will abate when the patient understands that his heart is entirely sound.

Outcome.-On further questioning it appeared that the patient smokes and chews tobacco constantly while at work. After ten days in the hospital, during most of which time the patient felt perfectly well, he said that he wanted to go gunning; accordingly he was advised to stop the use of tobacco and discharged.

Diagnosis.-Functional angina pectoris. 


\section{Case 117}

A laundress of forty-five, with negative family history and past history, entered the hospital March 2, I904. She passed the menopause six years ago. She has been markedly alcoholic for years. One month ago she began to have pain in the left hypochondrium, relieved by painting with tincture of iodin. Three weeks ago she had a similar attack, relieved in the same way. Nine days ago she had some pain in the lower abdomen, relieved by a vaginal suppository. Since then she has been in bed for about half the time, owing to nausea and pain in the left hypochondrium. She says she has vomited blood, but her daughter has seen only greenish and dark-brown material. For a week the urine has been reddish. The patient has been pale for about five months.

At entrance the patient was apparently in a uremic condition. The chest showed nothing abnormal. All the superficial lymph-glands were considerably enlarged. Only a few ounces of urine could be drawn from the bladder, and this nearly clear blood, some pus, no casts. Blood-pressure, 215. The patient was semicomatose, with coarse tremor of the hands. She died on the fourth of March.

Discussion.-Peptic ulcer is naturally our first thought, but on further study of the case there seems to be little to support it. The condition of the abdomen and the high blood-pressure cannot possibly be thus explained.

Cirrhosis of the liver, with associated splenic enlargement, might explain the abdominal symptoms. The vomiting of blood would then be the result of passive congestion of the stomach. The alcoholic history makes this explanation plausible, but on careful palpation we do not get the impression that the abdominal masses shown in the diagram represent enlargement of the liver and spleen. 'There is no sharp edge on either side, and the respiratory mobility is slight.

The general enlargement of the superficial lymph-glands might be due to syphilis. Enlargement of the spleen and liver is also frequently the result of this disease, and the pain of which the patient complains might be due to local peritonitis (perihepatitis and perisplenitis). The gastric hemorrhage might be explained under this hypothesis as a result of splenic fibrosis, the circulatory mechanism being the same as in splenic anemia. Against this, however, may be urged the same considerations which incline us to rule out cirrhosis: the abdominal masses do not suggest spleen and liver.

By the same reasoning and by the negative results of blood examina- 


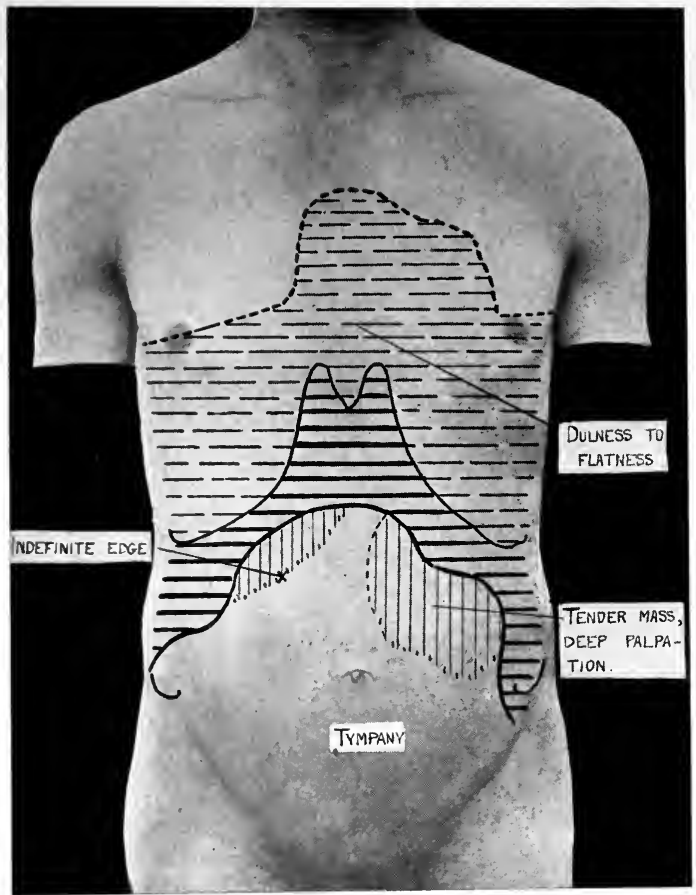

Fig. 38a.-Abdominal findings in Case $\simeq 7$. 

tion we may exclude leukemia, although this disease would account for the glandular enlargement, and (through a cerebral hemorrhage) might explain the high blood-pressure and the semicomatose condition.

Tuberculous peritonitis as part of a general tuberculosis might produce nearly all the symptoms of the case. This disease produces masses more or less vaguely felt in the abdomen, is often associated with abdominal pain, and, if we suppose an accompanying tuberculous meningitis with internal hydrocephalus, would explain the high blood-pressure and the psychic state. We should expect, however, some cranial nerve paralysis, some fever, and some signs in the lungs, even if only those of diffuse bronchitis; also some indication of a focus whence the disease, previously local, may have spread. Free fluid would probably be demonstrable in the abdomen.

It is not definitely stated in the text that the abdominal masses were palpable bimanually, or that a connection with the kidney was thus suggested. Whenever we have reason to believe that some renal lesion exists, and whenever this lesion-although apparently of a gross, "surgical" nature-is associated with high blood-pressure, we should remember the possibility of cystic kidney. It is rare to find any other nonnephritic lesion of the kidney associated with hypertension. Cystic kidney is generally a bilateral, congenital condition. Why, then, should these symptoms have appeared only within a month? Why should the disease have remained so strikingly latent? In answer, I can only say that this is the usual course of the disease, which encroaches upon the renal substance so slowly and so gradually that the system becomes accustomed to it, as to any other form of chronic interstitial nephritis, which is practically equivalent to the condition here described. Just what determines the final breakdown we usually cannot discover.

Outcome.-Autopsy showed congenital cystic kidneys; there was almost no kidney substance remaining. There was hemorrhage into several of the cysts and pus in the pelvis of the left kidney.

Diagnosis.-Congenital cystic kidneys.

\section{Case 118}

A housewife of thirty-nine lost one sister of phthisis thirteen years ago. Family history otherwise good. In her seventeenth and in her twentyfifth year she was in poor condition and was told that she had anemia. Five years ago she had her first attack of fever, with pain in the left lower abdomen. Since then she has had more or less pelvic trouble, especially after standing or after working hard. About Christmas-time, I9o6, she had frequent attacks of pain in the left upper abdomen; the pain doubled 
her up, and was ascribed to gas in the stomach. During the winter the pain grew less, but the abdomen seemed to be enlarged. In March. I907, she noticed in the left upper abdomen a visible prominence, which has steadily increased up to the present time. By May she had to let out her clothes three inches, and thought she could feel a lump in the left side.

Now (June, I907) there is a dragging pain after standing, and a feeling of pressure when she lies on her left side. Since early spring she has had frequent attacks of palpitation, associated with pulsation in the neck, roaring in the ears, and slight dyspnea. Once during the summer she saw red spots in front of her eyes, but she has noticed no bleeding from any point. Her gums have several times been swollen. Three weeks ago, while urinating, she heard a sound in the chamber-pot, and looking in saw that the urine was very red and contained several hard, darkbrown masses about the size of a large pin's head. She felt no pain and noticed no stoppage of water.

When examined at the hospital, her urine showed nothing worthy of note.

Physical examination was negative except as regards the left hypochondriac region, where she felt an enlargement (as figured in the diagram, Fig. 39). The mass is only slightly tender, and moves freely with respiration; it is very firm.

Discussion.-When a patient tells us that his stomach is so. sore that he can't bear the weight of his clothes on it and that it is "all puffed up," examination generally shows nothing in particular, no actual distention or prominence. Such symptoms usually occur in the neurotic, and represent the referred pain described so admirably by Henry Head. In the present case, however, physical examination shows that the patient is perfectly correct in supposing that the abdomen has enlarged. Indeed, the results of abdominal palpation make it unnecessary to consider any organs except the spleen and the kidney.

The present tumor seems to be spleen rather than kidney, for the following reasons:

(a) It has a sharp, hard edge, superficial and easily felt. Tumors of the kidney usually have no distinct edge, but shelve off into the depths of the abdomen. They are rarely as hard and superficial as those connected with the spleen.

(b) In the present case the tumor descends at least an inch with full inspiration. Kidney tumors sometimes move half an inch, often not at all. 


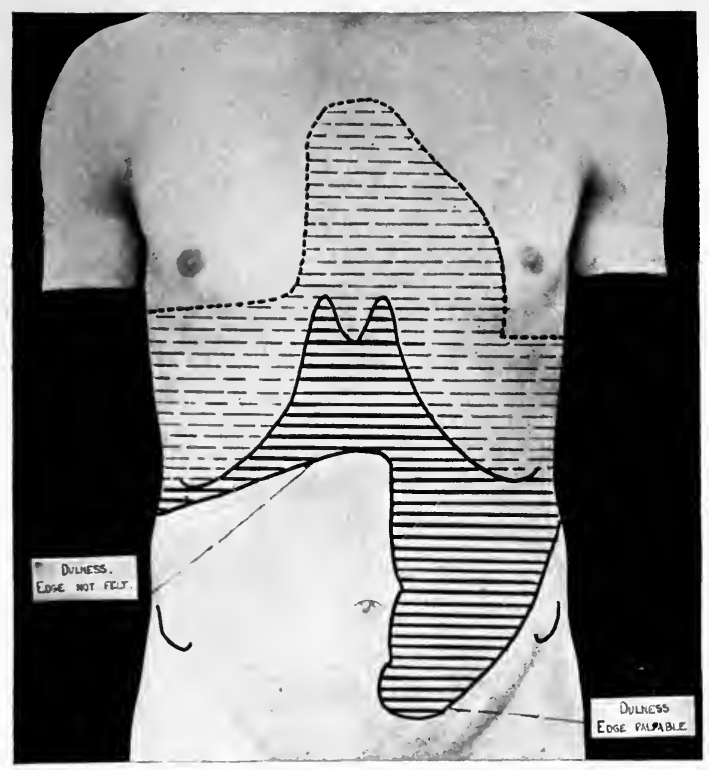

Fig. 39.-Percussion outlines in a case of left-sided abdominal pain. 

(c) One cannot grasp this tumor bimanually, while bimanual palpability is especially common in renal tumors.

(d) We are not told whether or not the air-distended colon overlies the tumor, but in view of its superficiality this seems very unlikely. Tumors overlain by the air-distended colon usually originate in the kidney or retroperitoneal glands.

All the signs, therefore, in this case lead us to believe that the tumor is due to the spleen. Assuming, then, that this is the case, we have to consider the following possibilities:

(a) Leukemia (proved or disproved by blood examination).

(b) Malaria (proved or disproved by blood examination).

The spleen may remain enlarged long after the malaria has died out, a fact very frequently illustrated in Armenian patients. In such cases, however, the patient presents no symptoms.

(c) Syphilis (anemia, hepatic enlargement, and ascites often accompany the splenic enlargement); the history, the evidence of syphilis elsewhere, the result of treatment and of Wassermann's test, must decide.

(d) Splenic anemia (diagnosis based upon the presence of a chronic anemia, secondary in type, often associated with gastric hemorrhages. All other causes for splenic enlargement must be excluded).

(e) Cirrhosis of the liver and Banti's disease. In cirrhosis we have a hepatogenous splenic enlargement; in Banti's disease, a splenogenous hepatic cirrhosis. The end-result is the same. Without evidence of cirrhosis, which is absent here, neither diagnosis can be made.

(f) Splenic enlargement of unknown cause is a rare but well-recognized clinical entity. It produces no symptoms other than those dependent upon the weight and dragging of the enlarged organ. The diagnosis rests, of course, upon the exclusion of all known causes, such as have been listed above. Abscess, neoplasm, and echinococcus of the spleen are so rare that, for practical purposes, they may be disregarded. The splenic enlargements accompanying acute infectious disease never reach any degree comparable to that shown in the accompanying diagram (Fig. 39).

The next step in differential diagnosis evidently depends upon blood examination.

Blood examination showed 277,000 white cells; 4,800,000 red cells; 75 per cent. hemoglobin. Among the white cells were 35 per cent. of myelocytes; 4 per cent. of eosinophiles; 2 per cent. of mast cells; $5^{2}$ per cent. of polynuclear cells.

Diagnosis.-Myeloid leukemia. 


\section{Case 119}

A girl of six years, whose mother died of quick consumption, entered the hospital September 2, I907. She drinks three cups of tea a day and eats considerable candy. She was recently operated on for congenital cataract at the Eye and Ear Infirmary. For nine days she has been suffering from weakness, with tenderness and pain in the left upper quadrant of the abdomen. September ist the white cells were I9,000. The temperature was $103^{\circ} \mathrm{F}$. The Widal reaction was negative. There were no parasites in the blood. The urine showed a moderate amount of pus, but nothing else remarkable.

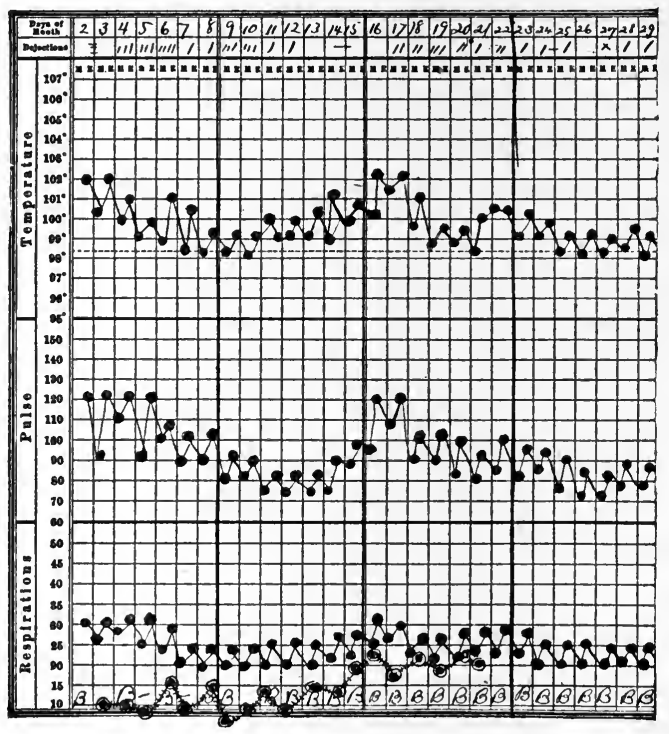

Fig. 40.-Chart of case IIg.

Examined on September 2d, the child is found to have moderate photophobia and seems apathetic.

Examination of the abdomen is negative except that in the left upper quadrant there are considerable tenderness and slight spasm extending through the left flank into the back. There is also dulness from the seventh rib (anterior axillary line) to the costal margin. The patient is tender in the costovertebral angle. Culture from the urine shows a strain of colon bacillus, and a heavy pus sediment which lasted throughout her stay in the hospital. $X$-ray of both kidneys was normal. The temperature was as shown in the accompanying chart (Fig. 40). 
Discussion.-Surgical disease of the kidney is rare at this age. Renal tuberculosis and renal stone, which might account for such pain, are especially rare in small children.

The dulness in the axilla, the pain, fever, and tenderness, might be accounted for by pleurisy. The text does not state whether or not these signs were supported by auscultatory evidences of disease. We should seek for diminution in the respiratory murmur, with decreased transmission of voice-sounds and of tactile fremitus. Friction-sounds might also be heard. As a matter of fact, however, neither of these confirmatory signs was present, nor was there any evidence of pneumonia. I mention pneumonia and pleurisy especially because in children they are frequently ushered in by abdominal pain without any reference to the chest.

The most notable feature in the physical examination is the presence of pus in the urine. Not many years ago this might have been passed over with very little attention, but since so much has been said and written of acute infection of the kidney, either hematogenous or ascending, the urinary sediments are more carefully scrutinized. We are especially on the alert in young girls who, from babyhood up, are particularly apt to acquire renal infection, presumably of the ascending type. The presence of the colon bacillus in pure culture in urine obtained under aseptic precautions, such as were observed in this case, lends support to the hypothesis of renal infection. In view of the negative results of $x$-ray, renal infection may be accepted as a working diagnosis. (For a discussion of clinical types of renal infection see Lumbar Pain, p. Iог.)

Outcome.-By the thirteenth of October the patient was well. The treatment consisted of counterirritants, laxatives, urotropin, 3 grains, three times a day, and abundant water.

Diagnosis.-Renal infection.

\section{Case 120}

A wool-spinner of forty entered the hospital March 3, I908. He had been in the hospital four years before for "gastric indigestion." One sister died of cancer of the stomach at thirty-five. He takes four to six cups of tea a day. His habits are otherwise good.

Since the fall of 1900 he has had intermittent pain in the left hypochondrium, worse on deep breathing, associated with belching, considerable nausea and vomiting, loss of appetite, and constipation. The vomitus consisted at first of sour liquid, later of yellow or greenish, bitter liquid containing no pus, blood, or mucus. Taking food sometimes 
makes the pain better, sometimes worse. He has often taken morphin to relieve the pain. For the past two weeks he has had the pain almost constantly. He has done no work. His best weight is 125 pounds. Now he weighs II 7 .

The patient is pale and sallow, with pigmentation about the eyes. His pupils are small, equal, and react very slowly, either to light or distance. The tonsils are somewhat enlarged. The chest shows nothing abnormal, nor does the abdomen. The knee-jerks are very lively. Water-distention of the stomach with a tube showed that the organ held 28 ounces. On inflation, the lower border reached about one inch below the navel. After a test-meal free hydrochloric acid was $0.3^{2}$ per cent. Lactic-acid and the guaiac tests were negative. No fasting contents were obtained.

Discussion.-We have here a long period of suffering from chronic dyspepsia, which has led, as it so frequently does, to the taking of morphin. It is well to remember this fact, since abdominal pain that leads to a call for morphin is often associated in our minds with the diagnosis of gall-stones.

Further analysis shows us that the motor power of the stomach is good and its outlet free. There has been no vomiting of food, but only of liquid which may be interpreted as gastric secretion. Tube examination shows no stasis. Since most gastric cancer produces pyloric stenosis and stasis, the absence of stasis in this case, especially in view of the long duration of the symptoms (1900-r908), makes cancer unlikely.

Of leukemia, pleurisy, and the other extragastric causes for left hypochondriac pain, physical examination shows no evidence. Cancer of the splenic flexure might produce most of the symptoms here present, but there is no palpable tumor nor visible peristalsis, no diarrhea, and no blood in the feces. The constipation here described might be due to many causes.

The high percentage of hydrochloric acid in the gastric contents is in itself a partial diagnosis, and might account for many of the symptoms. Our chief remaining problem is to determine whether anything more serious than hyperchlorhydria is present. Many-probably most-cases in which hyperchlorhydria is associated with symptoms so long continued and so severe turn out sooner or later to be peptic ulcer. No further exactness of diagnosis is possible without operation. 'The absence of the reaction to guaiac, both in the stomach-contents and in the feces, by no means excludes ulcer.

What treatment should be advised here? The proper rule in such cases seems to me to be this: give a fair trial to treatment by hygiene, 
diet, and drugs; if these fail to make the patient reasonably comfortable, advise operation. It does not seem evident, from the data here presented, that any persistent attempt has been made to control the symptoms by non-operative measures. Such measures should, therefore, be tried first. I have had excellent success with a modification of Lenhartz's diet, suggested by Dr. H. F. Hewes, which consists essentially of the following regimen:

For the first two or three days: For thenext two or threeweeks:

2 ounces of milk. 6-8 ounces of milk.

I powdered soda-cracker.

I ounce of sugar.

To be given every two hours while the patient is awake.
4 powdered crackers. I-2 ounces of sugar. Eight such feedings in twenty-four hours.

For the final two months:

Milk and crackers.

Indian-meal mush with creant or salt.

Potato purée; jelly.

Milk and whites of two eggs.

Soft custard.

Chocolate.

Pea purée.

Eight feedings in twenty-four hours.

If the patient is uncomfortable despite this diet, he should take cooking-soda in doses sufficient to relieve him. What this dose is can be ascertained only by experiment. It may be anywhere between Io grains and 2 drams.

Outcome.-On a subsequent examination free hydrochloric acid after a test-meal was o.II per cent. The patient complained of "cold sweats" at night, but under careful diet, small doses of calomel and seidlitz, olive oil, two teaspoonfuls after meals, and an occasional lavage, he seemed practically well by the eleventh of March. Rest and freedom from worry seemed to have much to do with his recovery, which by the nineteenth was complete.

Diagnosis.-Hyperchlorhydria.

\section{Case 121}

A teamster of forty-four entered the hospital April 4, I908. He has always been well until four years ago, when he was working on the great Clinton dam; a blow in the left side by a heavy pile laid him up for six weeks, during which he suffered from pain in the left side and had bloody urine. Since that time he has never been entirely free from pain in this region, and after any unusual exertion he has passed bloody urine. Last fall he had to give up work because of the severity of the pain. Three weeks ago he had a specially sharp pain in the left hypochondrium just below the ribs, the pain traveling down the left leg, occasionally to the left testis, and up toward the heart. Since then he has had three 
or four attacks, lasting from five to twenty-four hours, all of the same character. When the pain is severe he vomits, and is relieved to a certain extent thereby. There is tenderness under the ribs in the left hypochondrium during and after his attacks. He has been in bed most of the last three weeks, but has passed no bloody urine. His weight, eighteen months ago, was 197 pounds; now it is 167.

Physical examination shows nothing wrong in the chest. The arteries are easily palpable. The aortic second sound is louder than the pulmonic. Blood-pressure, I40. In the left lumbar region there is slight voluntary spasm and tenderness, increased by inspiration.

The temperature, pulse, respiration, and blood are normal.

The urine amounts to 40 ounces in twenty-four hours; 1022 in specific gravity; it shows a trace of albumin and many fresh red bloodcells. No casts.

Discussion.-Can we connect the symptoms with the injury sustained four years previously? The patient had hematuria immediately after this, and he has had it more or less ever since. Can we conceive any type of trauma which would produce an effect so lasting? I do not see that we can. The trauma, I think, must be regarded as having no important connection with his present disease.

In the absence of all bladder symptoms, causes of hematuria arising there deserve no further consideration. The clinical picture is one of renal colic associated with hematuria and a loss of 30 pounds in weight. Malignant disease of the kidney would produce these three symptoms, but would hardly have lasted so long. Either it would have killed the patient or it would have produced a palpable tumor. The hemorrhages from renal tumor are apt to be longer continued and of larger amount, leading to decided anemia.

Tuberculosis of the kidney of anything like this duration would have produced tumor and pyuria. It may be easily excluded.

Chronic nephritis, either of the glomerular or the interstitial type, may be complicated by sudden attacks of hemorrhage unassociated with any special increase in the other urinary manifestations of disease (casts, cells, deficient solids). Such hemorrhages may be painless, or may lead to colic, owing to the formation of clots and the difficulty of their expulsion into the bladder. The present case, however, shows no signs of nephritis.

We have left the two commonest and most puzzling occasions for hematuria: $(a)$ stone and $(b)$ unknown cause. The latter is, I believe, one of the most frequent of all the types of hematuria. Between this and stone our chief means of distinction is the $x$-ray examination. 


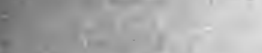

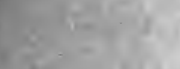

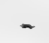




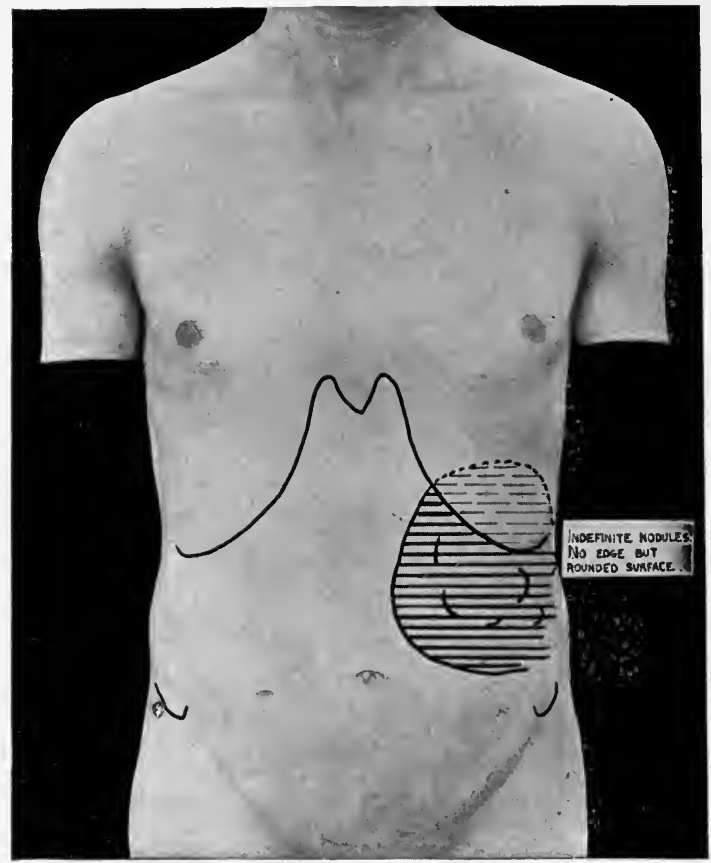

Fig. 4I.-Outlines of mass referred to on p. 259. Palpable bimanually. 
Outcome. $-X$-ray taken April 8th showed a small round shadow in the region of the left kidney. Cystoscopy helped to confirm the diagnosis of stone. The stone was subsequently found at operation.

Diagnosis.--Renal stone.

\section{Case 122}

A clerk of thirty-five entered the hospital October 21, 1907. He was operated on for appendicitis four years ago. He had left-sided pleurisy at the same time. He says he has always been pale. Eight weeks ago he began to have a sore, uneasy feeling, first in the left lower quadrant, later in the left hypochondrium, left hip, and over the left kidney in the back. He has also had numbness in the leg, extending from groin to knee. Three and a half weeks ago he first noticed a lump in the left upper quadrant, and began at the same time to have a very obstinate constipation-the bowels moving scantily by enema only. No blood seen in the stools.

Examination showed pallor of the mucous membranes and negative chest, while in the left upper quadrant there were marked resistance and tenderness. There is also considerable tenderness over the anterior muscles of the left thigh. Four days later palpation of the left flank had become easier, and a mass filling the whole flank from back to front, immobile and slightly tender, was easily felt. Blood-pressure normal. The inflated colon lay in front of the mass. Urine: 40 ounces in twenty-four hours; normal color; I020; no albumin; sediment negative. (See Fig. 4I.) Physical examination otherwise negative.

Discussion.- - Is it possible that this patient's pleurisy of four years ago is in any way connected with his present symptoms? It is a familiar fact that after any pleurisy most patients have a certain amount of pain in one or another part of the affected side of the chest, a pain that lasts on, oftentimes, for months and even years. But in such cases we expect to find some residual signs of the old pleurisy, and there seems to be nothing of the kind here. It is obvious, moreover, that pleurisy could not explain more than a small fraction of the facts in this case.

Leukemia would explain the lump and the pallor. Even in advance of blood examination, however, leukemia is practically excluded by the fact that the colon passes in front of the tumor. The blood examination was also negative.

Cancer of the splenic flexure of the colon would produce a mass in just this situation, and might account for all the pains here described. We should expect, however, if such a cancer existed, to get some of the ordinary evidences of intestinal obstruction, such as visible peri- 
stalsis, intestinal noise, gross or occult blood in the stools, diarrhea, or constipation. None of these symptoms was present except the constipation, which may well have been due to other causes.

The tumor is in the position usually occupied by growths arising from the kidney. Tuberculosis, cyst, and neoplasm may be considered. Against tuberculosis is the fact that we have no fever and no pyuria. The amount of pain and the extent of its radiations exceed what we usually find in renal tuberculosis. The latter remark applies also to renal cysts, which often attain a much larger size than the mass here present without producing any pain at all. Most chronic renal cysts also produce an elevation of blood-pressure, which did not exist here. New-growths of the kidney might explain all the symptoms that are here present, but in most cases would also cause hematuria. The nodular surface of the growth, if the observation be correct, would identify it almost certainly with a neoplasm. In some cases, however, the irregularities of a cystic kidney or of a tuberculous kidney feel very much like the nodules of malignant disease.

Outcome.-The patient was operated on March 25th, and hypernephroma found.

Diagnosis.-Hypernephroma.

\section{Case 123}

A single woman, thirty-three years old, was first seen June 28, I90I. Family history, personal history, and habits excellent. Eight years ago she weighed I22 pounds; now, IO4.

For two years she has had almost daily attacks of severe general bellyache with rumblings; the pain is worse in the left hypochondrium, lasting one to twelve hours, doubling her up, making her cry aloud, and radiating to the left shoulder. The pain has no clear relation to food. When the pain occurs, she usually vomits, and is promptly relieved thereby, but in the last seven months she has vomited only twice. Vomitus consists, as a rule, of food eaten recently, but on several occasions it has contained food eaten two days before and exceeding the amount of the last meal.

She has distress and acid eructations one-half to one hour after meals. Diarrhea often comes with the attacks of pain (3 or 4 movements). Mucus, but no blood, has been seen in the feces.

She has worked except during paroxysms of pain.

Examination.-Well nourished. Visible peristalsis below the navel, with slight general fulness of the abdomen. Much gurgling. No 
tenderness. Leukocytes, 8800; hemoglobin, 95 per cent. Borborygmi can be heard all over the house.

Stomach and its contents negative. Temperature (three weeks) normal.

Discussion.-The complaint of long-standing gastric pain and the evidences of gastric stasis make it reasonable to consider briefly the possibility of hyperchlorhydria or of a constricting ulcer near the pylorus. Most of the patient's complaints might be thus accounted for. Two facts, however, militate against this diagnosis: (a) Peristalsis is visible below the navel. In a well-nourished patient this has considerable diagnostic value, and points to the intestines rather than to the stomach as the source of trouble. (b) Very loud intestinal noise is a feature of the case. This, like the peristalsis, directs our attention away from the stomach.

The record of the physical examination is printed here as it was given me by the attending physician. In it we lack the data necessary to exclude lead-poisoning and tabes, either of which might account for part, if not for the whole, of the symptoms. My own examination disclosed no lead dotting of the gums, no basophilic stippling of the red cells, no abnormalities of the ocular or tendon reflexes. The age and symptoms are consistent with gastric neurosis were it not that visible peristalsis is revealed by examination.

With the exclusion of the possibilities mentioned above, chronic intestinal obstruction is left as the most plausible diagnosis. But what is.its cause? In any patient who has had no known cause for the formation of adhesions within the peritoneal cavity (appendicitis, pyosalpinx, or gall-bladder disease, with or without operation), cancer is the commonest cause for chronic intestinal obstruction. The age of this patient does not enable us to exclude this disease. More important evidence against cancer is the duration of the symptoms. Cancer of the gut often lasts two years or more, but in such cases it usually produces a palpable tumor. In the absence of any such tumor our best diagnosis is: chronic intestinal obstruction of unknown origin; the most significant symptoms being the visible peristalsis and the loud intestinal noise.

Outcome.-Operation, July $I 7$ th, showed strictures $I$ to 6 inches long in the small gut. The gut was thickened and, in the contracted portions of it, tubercles could be seen.

Diagnosis.-Tuberculous enteritis. 


\section{CHAPTER VIII \\ RIGHT ILIAC PAIN}

\section{Case 124}

A GIRL, fifteen years old, was first seen July 2I, I898; six months ago she began to get run down. Pallor, dyspnea, anemia, and weakness brought her to the out-patient department, where, March 26th, the hemoglobin was found to be 55 per cent. Patient had moderate general abdominal pain throughout her illness, but did not complain loudly of it until June 2Ist, when it began to be localized chiefly in the right iliac region. It is more soreness than pain, she says. Jolting in a wagon or rising from a chair aggravates it. She limps in walking lately. Otherwise feels well. No fever (two weeks' observation). Bowels regular. Last menstrual period ten days ago.

Examination.-Negative save for a large hard "cake" filling most of the right iliac region nearly to Poupart's ligament. On the lower side of the mass is a tender prominence diagonally placed. Leukocytes, 7400; hemoglobin, 95 per cent. Urine negative. Vaginal examination negative.

Discussion.-The essential point in this case is the presence in the right iliac region of a large mass, associated with anemia and preceded in its development by a considerable period of general constitutional symptoms, such as weakness and dyspnea. All this in a girl of fifteen can hardly be due to the cause which ordinarily produces such symptoms in the latter half of life-namely, malignant disease.

For appendicitis or pyosalpinx the onset seems rather too gradual, the preceding constitutional symptoms too marked, the fever and leukocyte count too low. What was known as to the girl's circumstances seemed to render gonorrheal infection very unlikely.

Ovarian tumors, especially those of the dermoid type, may occur in girls of this age, but rarely produce so much constitutional disturbance, and are not apt to be described as a "cake," being, as a rule, elastic and globular. The catamenia have been regular, the last period occurring so recently that extrauterine pregnancy seems impossible.

Many points in the case suggest pericecal tuberculosis. These points are especially the early general weakness and anemia, the slow 


\section{Causes of Right Illiac Pain}

1. APPENDICITIS

2. $P \cup S-T \cup B E$ (AND PELVIC

ADHESIONS)

3. DYSMENORRHEA

4. EXTRA-UTER-

INE PRE G-
NANCY

5. O VAR I A N CYST WITH TWISTED PEDICLE

6. PSYCHONEUROSIS AND THE FEAR OF APPENDICITIS

7. COLICA MUCOSA I

8. URETERAL

STONE

Obstruction in the ileocecal region (neoplasm, tuberculosis, adhesions) occasionally produces right iliac pain. As a rule, however, the pain is not thus localized.

Inguinal hernia produces usually an inguinal pain with radiations which may involve the iliac and other neighboring regions.

Any of the causes of generalized abdominal pain $(e . g$., tuberculous peritonitis) may produce right iliac pain. Conversely, the local causes above mentioned may in exceptional cases lead to generalized pains.

Many of the dragging "bearing down "inguinal pains of debilitated women (see page 8I) extend now and then to one or the other iliac region. 

onset and moderate degree of abdominal soreness, the large size of the mass. Against this diagnosis is the absence of fever and of any considerable disturbance of the bowels. One expects constipation, with or without intervals of diarrhea. The diagnosis then lies between ovarian cyst and pericecal tuberculosis, inclining rather toward the latter.

Outcome.-Operation, August 4th, showed a tumor the size of a lemon, studded with tubercles-pericecal abscess with the tube also involved.

Diagnosis.-Pericecal tuberculosis.

The two following cases do not seem to me to admit of any accurate differential diagnosis previous to operation. They are introduced here to suggest the variety of clinical pictures which pericecal tuberculosis may present.

\section{Case 125}

A little girl of six was first seen September 19, r905. She had whooping-cough January, 1905. Since then she has made frequent complaints of pain in the right iliac fossa, worse after meals, and has vomited almost every day. For five months she has had tenderness in the painful region. No other complaint.

Appetite good; bowels regular; urine normal.

Examination.-Poorly nourished. Chest negative. Belly negative, save for slight tenderness in the region of the appendix. Leukocytes, 8000 . No fever.

Operation as for appendicitis. Tuberculosis was found in a loop of small gut about four inches long. This was excised and the diagnosis confirmed by microscope.

A year later (November 28, I906) was in "splendid general condition. Appetite, bowels, and sleep satisfactory. Some thickening in cecal region." "Several abscesses have broken through," and in October she entered the Children's Hospital and was very sick for twelve days.

Pain occasionally wakes her at night (spasmodic pain with rumbling), but she soon drops asleep. She sometimes vomits with painonce daily on an average. Wets bed once or twice a week. Is listless and disinclined to exertion. No dyspnea. Weight, $3^{\mathrm{I}}$ pounds. Slight resistance in appendix region. No spasm; no tenderness.

Diagnosis.-Pericecal tuberculosis.

\section{Case 126}

A young Assyrian was admitted March 19, 1906, for chronic appendicitis. Several attacks of right iliac pain in the past year. Diarrhea 
with one attack. No vomiting. Most of the attacks last a few hours only. Bowels regular.

Examination.-Chest negative. Belly negative except for a large mass indefinitely outlined in the right iliac region, with slight tenderness and spasm.

Mass was not affected by free catharsis. Comfortable. No fever. Pulse, 9o.

Operation March 2Ist: Some free fluid. A nodular mass in the ileocecal region. Similar smaller masses could be felt in the mesentery and along the cecum. Cecum adherent.

April I4th discharged well.

Microscopic examination of excised piece showed tuberculosis.

Diagnosis.-Tuberculosis of the cecal region.

\section{Case 127}

Consulted October 23, 1902, by a married woman of thirty-one who has had left tube and ovary removed at Boston City Hospital in 1897. For eighteen months she has been more or less constantly in pain, referred to the right lower quadrant. For the past six weeks it has been severe. No fever or chills. Last menses in July, and again three weeks before entrance, when she flowed for five days, using five napkins a day. Many clots came away, one the size of a hen's egg.

The diagnosis of the attending physician is extrauterine pregnancy.

Examination.-Tenderness over the uterus and in appendix region. Movable pelvic mass on the right, thought to be closely attached to the uterus, which does not seem enlarged.

The patient ran a slightly elevated temperature with a normal pulse. Her general condition was excellent. Twelve days' observation.

Discussion.-The essentials in this case are right iliac pain of eighteen months' duration and amenorrhea of three months. The latter fact strongly inclines us to believe that the genital tract is involved, and tends to exclude a simple appendicitis. Amenorrhea is consistent with any of the following possibilities: Normal pregnancy, extrauterine pregnancy, pyosalpinx, ovarian cyst, ${ }^{1}$ fibroid tumor, peritubal tuberculosis. It is, however, less frequent in ovarian cysts and in pyosalpinx, and very much less frequent when fibroid tumors are present than in either form of pregnancy. The flow which is said

1 I shall make no attempt in this or in subsequent cases to distinguish between ovarian and parovarian cysts, nor between either of these and a cyst of the broad ligament or a hydrosalpinx. I do not believe that these can often be distinguished by physical examination alone. 
to have occurred three weeks before entrance, came at a time not corresponding to the menstrual period. In many ways it sounds like a miscarriage, but one must be on one's guard when patients give a history such as this, for not infrequently stories of pure fabrication are designed to induce the physician to curet the uterus and thereby to bring about a miscarriage.

Very possibly the diagnosis might have been made clearer had a uterine sound been introduced, but in view of the possibility of pregnancy this was obviously improper. On the whole, the diagnosis seems to me to be impossible, and the case is introduced merely as an example of the present limitations of our diagnostic skill.

Outcome.-Operation for ovarian cyst revealed normal pregnant uterus (three months) strongly right latero-verted. Subsequently tried to miscarry at six months but failed, and child was born at term (Boston Lying-in Hospital).

Diagnosis.-Normal pregnancy.

\section{Case 128}

A married woman of forty-two; has one child two years old, and suffered a miscarriage three years ago.

For three months has had periodic attacks of pain in the right lower abdomen which make her feel "sick all over." These came at first every four weeks, now every two weeks. Vomiting, constipation, distention, relieved by enemata. Catamenia normal.

Last attack began ten days ago, and pain has persisted since. It shoots into right hip and flank. When she reaches out for anything she has a sense of tension in the right lower belly.

Examination.-Hard, smooth tumor in right iliac region, fairly tender, about size of a large orange. No fluctuation. Vaginal examination cannot determine whether or not tumor is connected with uterus. No fever. Leukocytes normal.

Discussion.-We rightly consider appendicitis in every patient who complains of right iliac pain, but in the present case this possibility may be promptly dismissed. An appendix abscess rarely if ever lasts so long or attains such a size as this without producing more constitutional and local disturbance.

Tubal abscess would probably produce more tenderness, and rarely attains this size. The woman's age is not typical for tubal disease, though this, in itself, is not a point of great importance.

The tumor suggests especially uterine fibroid and ovarian cyst. Fibroids are more apt to be situated in the median line and to be obvi- 
ously connected with the uterus. They are rarely smooth. Unless they lead to profuse flowing, they usually cause no symptoms of any importance until a considerably greater size has been reached.

Except for its extreme hardness and the absence of mobility, the tumor is fairly typical of ovarian cyst. Cysts of this size rarely produce marked symptoms unless the pedicle becomes twisted, with resulting necrosis, hemorrhage, or local peritonitis. Any of these conditions may be here present.

Outcome.-Operation showed a cyst the size of a child's head. Its pedicle was twisted. The patient was discharged in three weeks. A year later she was heard from and had remained entirely well since her operation.

Diagnosis.-Ovarian cyst with twisted pedicle.

\section{Case 129}

An Italian laborer of twenty-four entered the hospital August 22, I908, complaining of right iliac pain which has been severe only for ten days, but had troubled him off and on since March. He has had no constipation, vomiting, jaundice, or headache.

The pain is worse at night, is somewhat relieved by applications of iodin, and somewhat increased by the taking of food.

Worked until four days ago. Family and previous history good.

Examination.-Scars in the neck near the angle of the jaw. Tenderness throughout the belly on deep pressure, most marked in the right iliac region. Physical examination, including the blood and urine, temperature, pulse, and respiration, showed nothing else that was abnormal.

Discussion.-This case was operated upon as one of acute appendicitis. Against this diagnosis, however, were urged the following considerations, to which, as I think, insufficient attention was paid. The patient's pain was never sharp and never well localized. The same was true of his tenderness. He never suffered from constipation, vomiting, or fever; his blood showed no leukocytosis. In view of these facts it seems to me that all the other possibilities should have been considered.

His symptoms have been of long standing and have increased little in severity. The long history of the case, the scars in the neck, and the fact that the patient is a recently arrived Italian immigrant, make abdominal tuberculosis a genuine possibility. Many cases of abdominal tuberculosis produce no more symptoms than are here described, although the absence of fever is somewhat surprising.

The pain has none of the radiations characteristic of stone in the ureter, and there has been nothing in the urine to suggest this disease. 
Gall-stone pain is sometimes referred to the right iliac region, but no diagnosis of gall-stone disease is possible upon the evidence here presented. There seems no good reason to suspect any part of the gastrointestinal tract.

Young Italian laborers rarely suffer from functional neuroses. I have once known a case somewhat similar to this in which the patient turned out to be a malingerer, but he had obvious reasons for his lies, while this patient has none. On the whole, I think that, had tuberculosis been seriously considered by the surgeon who performed the operation, the diagnosis of appendicitis would never have been made.

Outcome.-Operation revealed a normal appendix. Many glands of the size of marbles were felt in the mesentery and along the spinal column. Two of them seemed a little soft on one side. The patient made a rapid and permanent recovery.

The outcome of this case seems to me to prove that the glands were tuberculous. The patient's recovery proves that they were not malignant, and there is nothing to make us suspect typhoid. What should have been the treatment had the diagnosis been known before operation? Clearly, I think, it should have been purely a hygienic and dietetic one, similar to that applied in pulmonary tuberculosis.

Diagnosis.-Tabes mesenterica.

\section{Case 130}

A young married woman complains that since her second child was born, four months ago, she has had intermittent right iliac pain in spells of one to two weeks. It is worse on standing or exertion.

Examination.-Slight enlargement of the thyroid. Flat, globular, smooth mass, the size of a grape-fruit, is felt in right iliac region. It can be moved to the other side of pelvis. Distinct fluctuation wave over it. No connection with uterus can be made out.

Next day (September I 5 th), at 4 P. M., sudden right iliac agony with vomiting. It lasted until I2 P. M. Then she slept (no drug). Free fluid was demonstrated in the peritoneal cavity.

September I6th, comfortable in day-similar attack in evening.

September 17 th: Operation: Ovarian cyst with twisted pedicle (free bloody fluid-as usual).

Discussion.-This is a typical case, quite easy of diagnosis. The smooth, globular, painful mass in the right iliac region, the free mobility of the tumor, the sudden advent of agonizing pain, and the evidences of free fluid in the peritoneal cavity make up the typical picture of ovarian cyst with twisted pedicle. In many, perhaps most, cases, however, we 
cannot be so sure either of the cyst or of the twist because we have had no opportunity to question and examine the patient previous to the advent of any acute symptoms. Very large, centrally placed cysts are recognizable in case they project sharply forward, leaving the flanks comparatively free from bulging and still resonant on percussion. The diagnosis is much aided if the patient has been able to notice that the tumor originated at one side of the abdomen and only assumed its central position at a later date. But the majority of patients remember nothing of the kind and pay no special attention to their condition until it gradually dawns upon them that the enlargement cannot be due either to fat or to the so-called "high stomach." Under these conditions it may be difficult or impossible to distinguish the disease from tuberculous peritonitis. The other and commoner causes of ascites (cirrhosis, cardiac or renal disease, cancerous peritonitis) are more easily recognized.

In another group of cases the cyst is smaller and bears no great resemblance to an ascitic accumulation, but is of such a board-like hardness that we can scarcely imagine its contents to be fluid. A careful examination under ether and the introduction of a uterine sound will usually determine the point.

As a rule, it is useless to attempt any distinction of the different varieties of ovarian tumor. Occasionally the smaller and more solid tumors (ovarian fibroid, cancer, or sarcoma) may be recognized by their consistency, and especially by their association with ascites, which is much commoner with solid than with cystic tumors.

The occurrence of a twist in the pedicle of an ovarian tumor is often recognized without difficulty, provided we have seen and studied the case before the twist occurred. If we know that an ovarian tumor is present, the occurrence of any kind of acute abdominal symptom is strongly suggestive of a twist. But if we see the patient for the first time after the acute symptoms have appeared, it may be quite impossible to make out anything which enables us to distinguish the condition from perforative peritonitis or intestinal obstruction. The abdomen may be so tender and its muscles so spastic that nothing definite is distinguished on physical examination, while the pain, vomiting, constipation, and general prostration are quite equivocal.

Diagnosis.-Ovarian cyst with twisted pedicle.

\section{Case 131}

A seventeen-year-old school-girl has had three attacks like the present: one, the last eight months ago. Catamenia regular and normal. 
Yesterday general abdominal pain, with vomiting and diarrhea, brought her to her physician.

Examination.-Temperature, IO2 $^{\circ} \mathrm{F}$.; pulse, I05; respiration, 25. Slight rigidity and considerable tenderness in right iliac region. Leukocytes, 14,000. Operation: Normal appendix. Considerable bloody fluid in pelvis. Ruptured ovarian cyst one inch in diameter, whence oozed gelatinous material.

Discussion.-Much that was said in the discussion of the last case applies equally to this one. With no accurate knowledge of her condition previous to the present attack, appendicitis was the most natural and reasonable diagnosis. Such mistakes cannot be avoided. It is on this account that $I$ have not discussed ruptured ovarian cyst in detail among the possibilities to be considered in differential diagnosis, as I have intended to deal chiefly with the recognizable and verifiable possibilities.

Diagnosis.-Ruptured ovarian cyst.

\section{Case 132}

A married woman of forty-seven was seen January 25, I908. Eight months ago, on getting out of bed, she felt sudden sharp right iliac pain, which ceased in one hour on lying down. Many attacks sincelately, three or more every week.

Two months ago noticed a lump in right side of belly. Thought she was getting fatter there; lump seemed larger during the attacks of pain. Thinks she has lost weight in the rest of her body.

Examination.-Thin, worn face. Belly prominent, especially to the right of the median line below the navel. Dull here, tympany elsewhere. A large, slightly compressible mass, extending from the pelvis to a hand's breadth above the navel. Not tender; freely movable. Vaginal examination adds nothing.

Operation revealed a multilocular ovarian cyst about $24 \mathrm{~cm}$. in diameter. There were no adhesions except a few about the appendix. Well in two weeks.

Discussion.-This case is introduced to exemplify the occurrence of attacks of pain in connection with an ovarian cyst easily recognizable as such. These attacks, however, were demonstrably not due to a twisting of the pedicle. Their cause is not explained.

Diagnosis.-Ovarian cyst. 


\section{Case 133}

A man of forty-eight has had for two days pain in right side of belly, extending to the back, tending to shoot upward, and increased by motion. Dull ache with exacerbations. No other symptoms.

Temperature, $100^{\circ} \mathrm{F}$; pulse, 62 . Leukocytes, 12,000 .

Tenderness in right loin and along the ureter down to McBurney's point. No muscular spasm. Urine normal.

The tenderest areas are: $(a)$ Midway between the ribs and the anterior superior iliac spine; $(b)$ over the right kidney.

Discussion.-Although this case was diagnosed and operated upon as appendicitis, there are several points distinctly against that diagnosis. In the first place, it is important that the pain-and especially the tenderness - centered rather in the loin and over the ureter than at McBurney's point. The absence of muscular spasm is also distinctly against appendicitis. Dull aching pain with exacerbations occurs in appendicular colic, but also in colic of other origin (intestinal, biliary, renal, uterine).

So much in this case suggests kidney that, even though the urine is normal, cystoscopy and the introduction of a catheter into the ureters seems indicated.

Outcome.-Operation showed in the ureter a stone the size of a large bean. Recovery was uneventful.

Diagnosis.-Stone in the right ureter.

\section{Case 134}

A factory girl of twenty-four entered the hospital June 2I, I906. She had pleurisy eighteen months ago. One month ago, without known cause, her abdomen began to be sore and tender on pressure, especially in the lower portion and on the right side. There has been no actual pain, but she has been too weak to work, and has been part of the time in bed. The menses have been regular and normal.

Physical examination showed normal temperature, pulse, and respiration, nothing abnormal in the chest, general rigidity of the abdomen, especially in the right lower quadrant, where there are marked tenderness and an oval mass, the size of half a lemon, raised above the surface.

Discussion.-The presence of a raised mass in the region of the appendix narrows the field of possibilities considerably. The most important differential point in the case seems to me to be the gradual onset of the symptoms and signs, without anything that the girl will call pain. Appendicitis and pyosalpinx may have a gradual onset, but almost never does this occur without marked pain. If these two possibilities 
are for the moment put on one side, we have left ovarian cyst, cancer, and tuberculosis of the cecal region and extrauterine pregnancy. If we believe the girl's story, the latter is excluded by the regularity of menstruation and the absence of pain. Cancer is very rare at her age, and should produce symptoms more distinctly referable to the intestine. Ovarian cyst cannot be excluded, but there are two points which incline us to the only remaining alternative, tuberculosis. These points are: (a) the occurrence of a pleurisy (i.e., of a tuberculosis) eighteen months previously; and $(b)$ the wide distribution of tenderness and rigidity over the abdomen. Ovarian cysts generally cause very little either of tenderness or of muscular spasm, except in the presence of other acute symptoms, such as are absent here.

Outcome.-The patient was operated on June $23 \mathrm{~d}$. A large tubercular abscess originating in the right tube was drained.

Diagnosis.-Tuberculosis of right tube.

\section{Case 135}

A married woman of thirty-nine entered the hospital July 29, I908. Her father died of consumption; one of her sisters is partially paralyzed. The patient was a seven-months' baby, and was said to have weighed only a pound at birth(?). She has had measles four times, and many attacks of grip. A year ago she had an attack similar to the present one. She formerly took alcohol in considerable quantities "to give her strength," and for six months she has not felt well and has had darting pains in various parts of the abdomen, especially in the right iliac region, also in the back, knees, and other joints. Three days ago she began to have frequent loose, slimy movements, with much pain in the right iliac region. The pains in the joints and back have also been increased.

Physical examination shows a slight systolic thrill at the apex of the heart, with a systolic murmur, which, however, is louder in the pulmonary area, and not heard in the axilla. There is no enlargement; slight general abdominal tenderness, more marked in the right iliac region; blood, urine, pulse, temperature, and respiration are normal. The stools show a few food elements and large amounts of mucus. The patient lies in bed with her eves closed most of the time, paying no attention to what is going on about her, but complaining of pains in different parts of her body.

Discussion.-The suspicion of tuberculosis which is naturally excited when we learn that the patient's father died of consumption receives very slight support from any of the other facts in the case. It is true that the patient has slight general abdominal tenderness, but at 
no time has there been any fever or any evidence of free fluid or tuberculous masses in the abdomen. Nor do we get any very distinct help in diagnosis from the knowledge that she has been alcoholic at times, and that she apparently had a very poor start in the world. Possibly her alcoholism may have something to do with her mental state or with the various pains of which she complains.

The darting character of these pains and their distribution correspond quite accurately with the "lightning pains" of tabes. The physical examination as it is here reproduced gives us no evidence wherewith to support or to attack this idea, but from my own examination of the case I know that all the reflexes were normal.

The essential symptoms in the case seem to me at the present time as follows: Right iliac pain, accompanied by frequent bowel movements containing much mucus. Occurring in a patient of the temperament and physique which may be inferred from the above description, these symptoms suggest especially the condition known as "colica mucosa" or mucous colitis. Three types of this disease are familiar to most practitioners: (a) Those characterized mostly by pain, with a moderate amount of constipation and neurasthenia; $(b)$ those characterized mostly by constipation, with a moderate amount of pain and neurasthenia; and $(c)$ those characterized mostly by neurasthenia, with a moderate amount of constipation and pain.

In all these cases the stools contain varying amounts of mucus, sometimes mixed with fecal matter, sometimes making up practically the whole of the dejection. In my opinion, however, the fundamental and underlying factor in all cases is the neurasthenic state which is the cause of the constipation, and thereby of the pain and mucus. The most successful treatment must address itself to the cure of the constipation, but this cannot be permanently relieved unless the patient's mental habits and point of view can be reconstructed.

Outcome.-Under treatment for constipation, with 5 grains of Blaud's pills three times a day, the patient was discharged relieved on the nineteenth of August.

Diagnosis.-Mucous colitis.

\section{Case 136}

A school-girl seventeen years old was first seen December 4, I908. Six days before she had a stomachache, which lasted about twenty-four hours and then got better. Three weeks before she had had a similar, but less severe, pain. Since then she has had similar attacks three or four times a day. 
On examination the temperature, pulse, and respiration are normal; the chest negative, the abdomen level, generally tender, with slight muscular spasm over the whole right side.

The last menses came two weeks ago. The diet has been blameless. The present attack followed immediately after some high jumping in the gymnasium. The pain was almost as great in the back as in front. The bowels moved normally during three days of observation. The temperature was steadily normal, likewise the pulse. Pain, however, persisted and kept her awake most of three nights. At times it was rhythmic, coming every fifteen minutes and lasting about two minutes. The girl and her family were all well acquainted with the symptoms of appendicitis, and much afraid of it. The leukocytes ranged close to Io,coo. Pressure on the left side of the belly caused pain in the appendix region. Physical examination was otherwise wholly negative.

Discussion.-The extension of pain and tenderness to the back, the absence of temperature, elevated pulse, and increased leukocyte count, and the apparent relation to a strain at the time of the onset, inclined me at first to believe that this case was due to a wrench either of the back muscles or of the sacro-iliac joint. I could not rule out the possibility of a pure neurosis, since the patient was an exceptionally high-strung and nervous girl, who had known and feared appendicitis all her life. Indeed, this diagnosis was furnished to me, ready made, as I entered the sick-room.

But against both these possibilities there was the fact that the pain was not relieved either by a complete rest in bed with cross-strapping of the back and elevation of the lumbar region on a pillow, nor by repeated assurances that she was not suffering from appendicitis. On the contrary, the pain continued with very little abatement. Heat gave it only very slight relief; aspirin was equally inefficacious. Judgment was still more affected, however, by the pain's rhythmic character, which usually indicates spasm produced in some hollow, tubular structure. This could not fit in with either of my previous diagnoses, and the verdict had to be shifted to appendicular colic. At no time was there any indication of an involvement of any part of the urinary tract. The pain never followed the course of the ureter, nor showed any of the typical radiations of nephrolithiasis. The urine remained wholly negative.

Outcome.-Operation, December 8th, showed an appendix bent upon itself, and covered with old adhesions, but not inflamed.

Diagnosis.-Appendicular colic (chronic appendicitis). 


\section{Case 137}

A Scottish housewife of thirty-five was first seen February 7, 1908. Her family history is good, though her mother died of cancer. She had polyarthritis, with fever and prostration, seven years ago (soon after marriage).

For one year she has had nearly constant pain in the right lower quadrant of the belly. There is no colic, but the steady pain often needs morphin. Pain is relieved by lying down and always disappears at night. She sleeps well and has worked until three weeks ago. She is not in bed.

Sometimes the pain extends down the right leg, but it has no other radiations. No jaundice. No urinary, circulatory, respiratory, or infectious symptoms.

Examination.-No emaciation or anemia. Visceral examination was negative except that in the right upper quadrant there was a mass palpable bimanually, irregular of surface, descending to the navel with inspiration. Tenderness of right lower lumbar muscles. (See Fig. 42.) Cutaneous tuberculin reaction negative.

A catheter specimen of urine showed microscopic blood and pus. Cystoscopy showed a normal bladder. Turbid urine was obtained from the right ureter; injected into a guinea-pig; five weeks later negative autopsy.

Discussion.-It is noticeable in this case that, although the pain is in the right iliac fossa, the tenderness is in the lumbar region, where a mass is felt bimanually. The fact that the pain disappears when the patient lies down tends still further to connect it with the kidney, rather than with any structure in the neighborhood of the cecum.

Tumors of the kidney produce pain, enlargement of the organ, and often a urine such as that here described, but it would be unlikely that the amount of pus would be so large in proportion to the amount of blood. There has been, indeed, no true hematuria, and after a year's duration kidney tumors usually produce a hematuria so profuse as to result in anemia. Emaciation would probably be present also by this time.

Renal tuberculosis would explain all the symptoms, though it usually does not give rise to such severe and long-standing pain, and almost always produces bladder symptoms, which are not complained of here. Nevertheless, it is only the results of animal inoculation that enable us to exclude tuberculosis in this case.

Is it possible that a simple looseness and displacement of the kidney, 


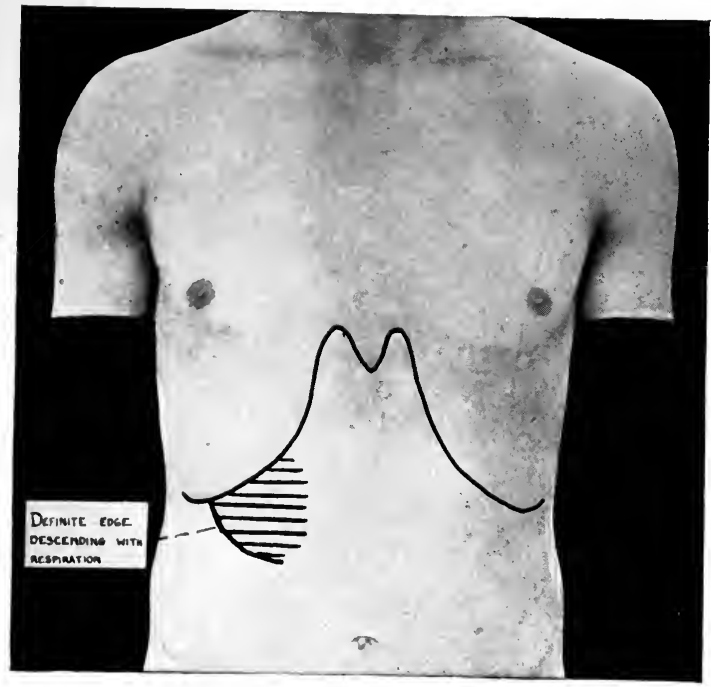

Fig. 42.-Position of the mass described in Case 137 . 

could produce such symptoms? This idea is favored by the disappearance of pain in the recumbent position, but we do not expect a kidney, not in itself diseased, to secrete a urine turbid with blood and pus, although when the kidney gets in such a position as to twist its blood-vessels, we may have hematuria from congestion. The enlargement here present seems sufficient to exclude a simple floating kidney.

The important evidence which we still lack is that obtainable through the $x$-ray examination of the kidneys with special reference to stone. The only point distinctly against stone here is the absence of any colic. The good preservation of nutrition is more in harmony with the diagnosis of nephrolithiasis than with any other condition producing enlargement of the kidney. It is not easy to see just why the kidney should be enlarged as the result of stones in the pelvis, unless there were obstruction to the flow of urine, a complication of which we have no evidence here. Yet it is a very familiar fact that kidneys which turn out to be the seat of no disease other than nephrolithiasis uncomplicated, seem considerably enlarged when palpated before operation.

Outcome. $-X$-ray shows stones in both kidneys.

Operation: in right kidney a stone with a body the size of a plum and three branches one inch long was found; in left kidney three stones, the largest as large as a marble, the smallest the size of a marrow-fat pea. November I2th: Discharged well.

Diagnosis.-Stone in both kidneys. 


\begin{tabular}{|c|c|c|c|c|c|c|c|c|}
\hline 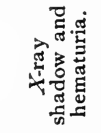 & 0 & $\circ$ & 0 & $\circ$ & 0 & $\circ$ & $\circ$ & + \\
\hline 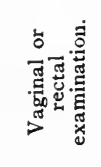 & 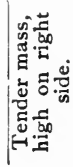 & 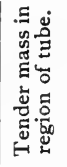 & $\circ$ & $\circ$ & 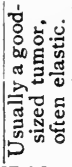 & 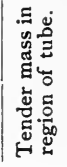 & 。 & $\circ$ \\
\hline 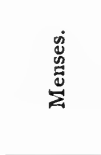 & $\vdots$ & 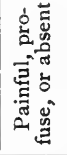 & $\vdots$ & $\vdots:$ & $:$ & 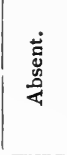 & $:$ & $\vdots:$ \\
\hline 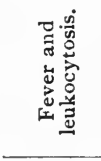 & + & + & 0 & 0 & 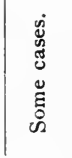 & 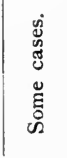 & 。 & 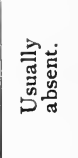 \\
\hline 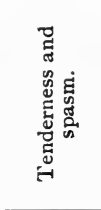 & 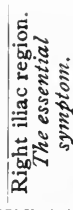 & 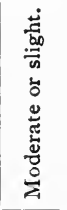 & ○ & ○ & 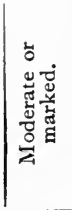 & 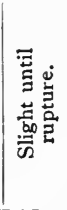 & 。 & 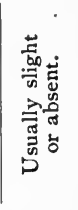 \\
\hline 范 & 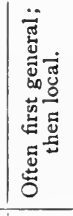 & 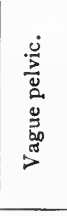 & 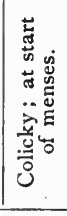 & 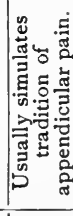 & 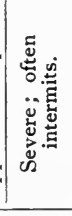 & 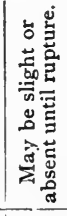 & 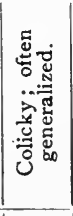 & 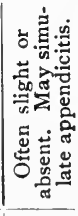 \\
\hline 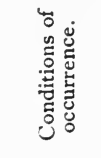 & 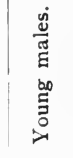 & 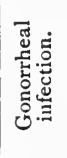 & 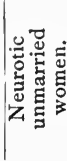 & 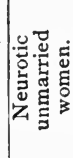 & 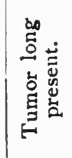 & 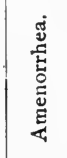 & 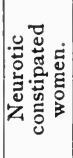 & : \\
\hline 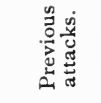 & + & a. & + & a. & 离 & a. & + & 。 \\
\hline 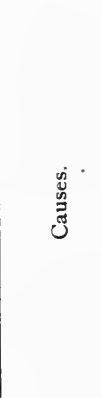 & 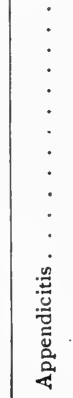 & 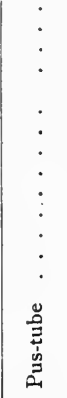 & 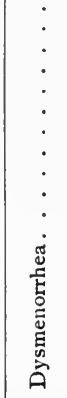 & 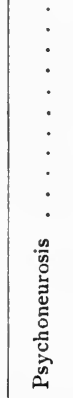 & 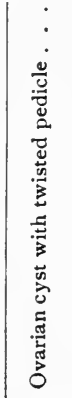 & 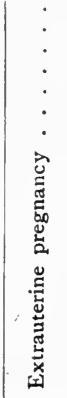 & 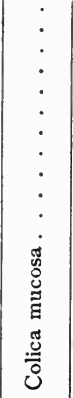 & 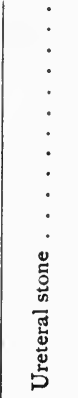 \\
\hline
\end{tabular}





\section{Causes of Left lliac Pain}

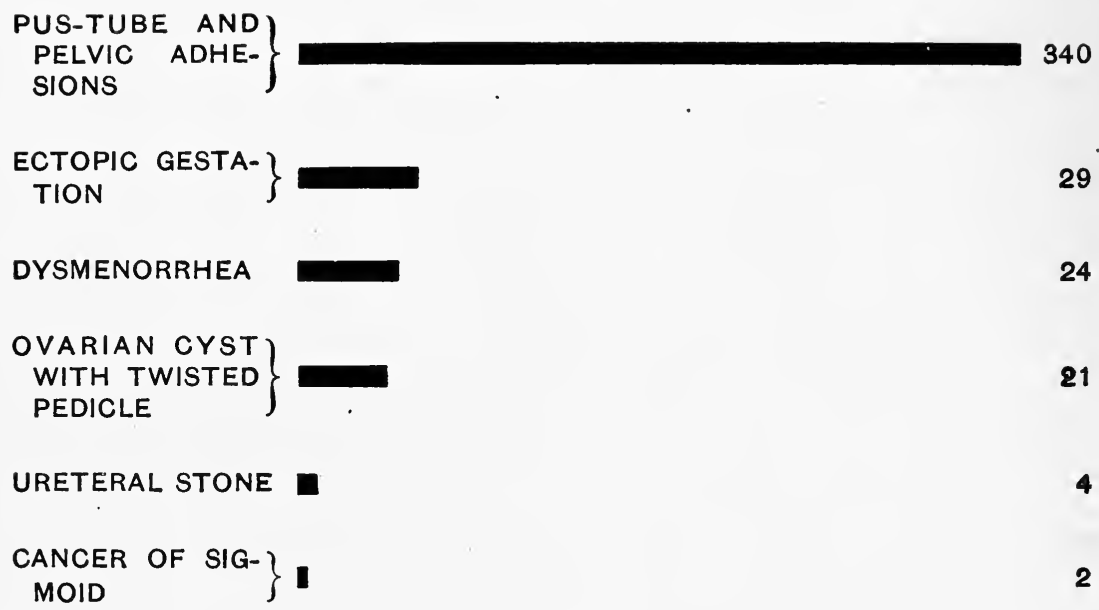

Inguinal hernia, the debility of neurotic women, and the temporary and atypical localization of some of the causes of diffuse abdominal pain are also to be mentioned. 


\section{CHAPTER IX}

\section{IEFT ILIAC PAIN}

\section{Case 138}

A HousewiFe of forty-six was seen in consultation May 10, 1907. The attending physician's diagnosis was cancer of the intestine, probably in the sigmoid. The patient has had for three or four years a "stomach trouble" characterized by pain near the left costal margin, with vomiting of greenish fluid and "coffee-grounds," the vomitus being sometimes sour, sometimes bitter. Vomiting relieved the pain. For the past year she has had no vomiting and only moderate soreness in the left side. Six weeks ago she felt a sudden knife-like pain in the left lower quadrant, which lasted twenty-four hours, following which she was in bed for five weeks. The bowels moved every second day. She has lost five or six pounds.

Physical examination showed fair nutrition; marked pallor. Red cells, 3,332,000; hemoglobin, 50 per cent.; polynuclear cells, 52 per cent.; considerable achromia; urine negative; chest negative. Above and to the left of the umbilicus a hard, movable, sausageshaped mass, extending from the median line obliquely outward and downward for three inches.

A stomach-tube showed no fasting contents and no enlargement of the organ. After a test-meal, free $\mathrm{HCl}$ was 0.28 per cent.; total

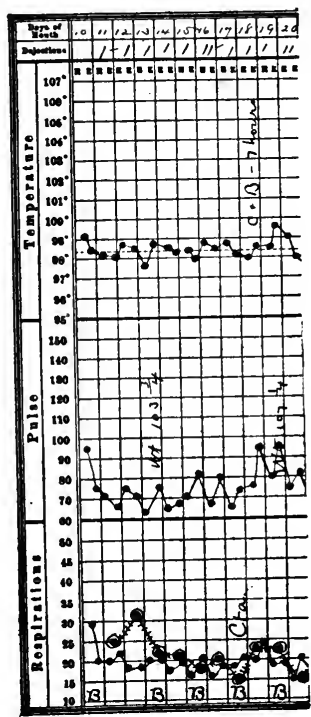

Fig. 43.-Chart of case 138 . acidity, 0.35 per cent. The guaiac test was negative, both in the gastric and intestinal contents.

The diagnosis of cancer of the sigmoid was generally agreed to.

Discussion.-At least three years of a stomach trouble which has produced anemia and hyperchlorhydria, but which has not led to any gastric stasis-such is the background against which the recent symptoms 
of this case stand out. A sudden acute attack of left iliac pain and in the same region a tumor, regarding the age of which we have no knowledge, are the facts which must in some way be woven into a satisfactory diagnosis.

With such a tumor and such a pain, a diagnosis of sigmoid cancer seems at first inevitable. But a cancer which has existed long enough to be palpable as a tumor of this size should also manifest itself by visible peristalsis, intestinal noise, gross or occult blood in the stools, diarrhea, or marked constipation. That none of these symptoms is present should certainly give us pause.

Were the tumor situated higher up in the abdomen, we should certainly be inclined to consider a perigastric exudate resulting from the attempt of a gastric ulcer to perforate. The long previous history, the present hyperchlorhydria, the anemia, and the recent acute attack of pain are all quite consistent with this diagnosis. It seems somewhat remarkable, however, that the symptoms should have come to so complete a standstill as has apparently occurred since the attack six weeks ago.

Though nothing is said in the text regarding the results of pelvic examination, I may add here that nothing could be found in the pelvis to connect any of its organs with the disease under consideration.

Outcome.-On the twenty-first of May the abdomen was opened. The mass proved to be composed of a perigastric exudate adherent to the abdominal wall. Behind this was the narrow neck of an hour-glass stomach, which barely admitted the little finger and was evidently due to the scar of an old gastric ulcer. Gastro-enterostomy was done. Six days after operation the patient was doing well.

Diagnosis.-Perforated gastric ulcer.

\section{Case 139}

A housewife of twenty-six entered the hospital December 27, I906. For two months she has been having pain in the left iliac region, at first darting in character and extending through to the back; later, dull and constant, sometimes more severe at night. Pain has been accompanied by weakness and frequent micturition. Her appetite has been good, her bowels regular, her urine dark and cloudy for a month.

The course of the temperature is seen in the accompanying chart. The urine was alkaline and contained always a large amount of pus, and sometimes a great deal of blood in clots. The specific gravity was always low, averaging about ror 2, and the amount of albumin large; no casts were ever found. Examination of the chest and abdomen was negative; likewise $x$-ray examination of the renal regions. After entrance to the 
hospital the urine was sometimes quite normal, at other times composed almost wholly of blood. Several small concretions were passed in the early days of January, 1907. On the sixth, one obstructed the urethra and had to be removed. It was shown to be composed of calcium phosphate upon a nucleus of mucin. Its passage was not attended with pain. Repeated examinations of the urinary sediment showed no tubercle bacilli.

Discussion.-The essential features of this case are left iliac pain of two months' duration, associated, during the past month, with the frequent passage of an alkaline, cloudy urine containing large amounts of pus and blood. The continued fever is also of importance.

All these symptoms may be produced by renal tuberculosis, and this diagnosis cannot be possibly excluded upon the evidence here presented. Animal inoculation is necessary. Nevertheless, the absence of any evident enlargement of the kidney, demonstrable by palpation or $x$-ray examination, the presence of an alkaline

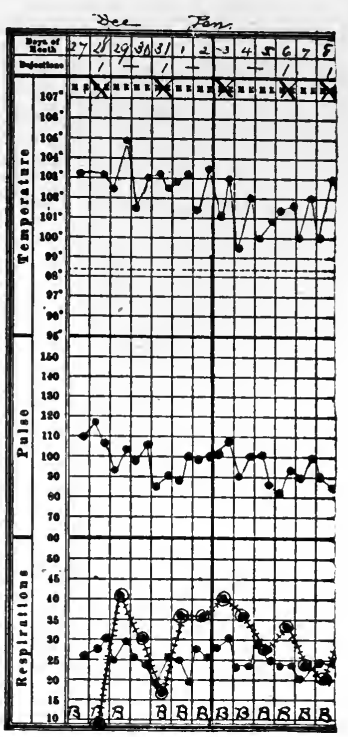

Fig. 44.- Chart of case I39. urine, and the constant abundance of blood, are facts which tend to support the negative results of the search for tubercle bacilli.

In the hands of a competent operator we may say that $x$-ray examination, declared by him to be negative, is very strong evidence against the existence of renal stone. The predominance of bladder symptoms here, the absence of anything suggesting colic, and the apparently steady discharge of blood and pus tend to rule out nephrolithiasis.

Malignant disease of the kidney rarely produces such a predominance of bladder symptoms or so large an amount of pus in the urine. Unless we suppose the neoplasm to be complicated by bladder disease, we could not account for the alkalinity of the urine.

Stone in the bladder is rare in women if we leave out of account the secondary calculi incrusted about a hair-pin or some other foreign body. There is no history of the introduction of any such body in this case, and if we take the history on its face value, this is evidence against bladder stone. In one sense, of course, we are quite sure that stones have been in the bladder, since several small ones have been passed; but from the rarity of primary bladder calculi in women and the absence of any of the 
exacerbations due to jolting or moving, we may suppose that the small stones which have emerged were formed as a secondary result of some other disease. The question remains as to what that disease is likely to be.

Chronic cystitis is now universally recognized to be, in practically all cases, a symptom of some deeper cause. The days of primary or idiopathic cystitis are passed. Gonorrheal cystitis is distinctly rare unless as a part of a much more obvious acute and general infection of the genito-urinary tract.

Tuberculosis of the bladder is a frequent cause of cystitis, and is practically always secondary to renal tuberculosis, reasons for excluding which have been already given.

Tumor of the bladder is the only remaining cause of cystitis frequently occurring in women, and against that diagnosis there seem to be no important data. The occurrence of small concretions in and about tumor of the bladder is a familiar fact.

Outcome.-On the twenty-third cystoscopy showed an exceedingly foul bladder and a ragged tumor mass on the left side. Operation on the twenty-sixth confirmed this diagnosis. A cutting from the mass was examined histologically and pronounced undoubtedly malignant. The walls of the bladder were much thickened and contracted.

Diagnosis.-Bladder cancer.

\section{Case 140}

A laundress of forty-four entered the hospital December 24, I907. She had lost one sister of consumption; her family history was otherwise good. She has been subject all her life to occasional sick headaches. At half-past nine this morning, while washing, she suddenly began to have steady, severe pain half-way between the navel and the left flank. Soon after she vomited her breakfast. The pain was so severe that she could not lie down until night. Her suffering has been constant, though varying in intensity, and she has continued to vomit a thin, yellowish fluid. There is some soreness in the region of the pain, but no headache at the present time. The bowels moved two days ago with medicine, not since. She has been very constipated for years, sometimes. going a week without a movement.

Physical examination of the chest was negative save for accentuation of the aortic second sound. The urine and blood were normal. The right kidney descended two fingers' breadth below the costal margin on full inspiration. During the first two days in the hospital the patient vomited everything that was taken by mouth. Finally, the bowels were 
started by calomel and enemata, and by the twenty-seventh the patient was taking milk and feeling happy. The first urinary examination showed sugar, acetone, and diacetic acid. After that there was no sugar, but acetone and diacetic acid persisted until the twenty-eighth.

Discussion.-The chronic constipation leading to acute pain and obstinate vomiting cannot but incline us very strongly toward the diagnosis of sigmoid cancer, especially since the woman is forty-four years old. But what are we to say when, after we have made such a diagnosis, we succeed in getting the patient's bowels to move naturally and all the symptoms disappear? I have introduced this case in order that I might emphasize the point that such a recovery by no means excludes cancer. In the early stages of that disease, when the growth is little bigger than a signet ring, temporary obstruction with fecal impaction behind the stricture often leads to symptoms quite like those here described, which, nevertheless, disappear under treatment and may not recur for weeks or months. It is only by a careful following of the case that we can be justified in excluding cancer.

Outcome.-On the first of January sugar was again present in the urine. On the second it was gone and did not return, although the patient was allowed a full mixed diet. Thereafter the patient's bowels were kept regular by the use of an A. S. and B. pill four times a day. There has been, so far as known, no return of symptoms.

Diagnosis.-Constipation.

\section{Case 141}

A hostler of thirty-two entered the hospital June 3, I902. His family history and past history were negative. Until the previous fall he had always taken five or six beers and three or four whiskies a day. He denied venereal disease. Yesterday morning he awoke with a chill, chattering teeth, fever, vomiting, headache, and pain in the left groin. He slept poorly last night. The course of the temperature was as seen in the accompanying chart. On the sixth the glands were discovered to be tender and considerably enlarged in the left groin. There was an operation scar over the upper part of the left tibia; the bone underneath it very rough. Below this the skin was bluish red, and several ulcerated areas from the size of a silver dollar to that of the palm were present. An $x$-ray showed that the tibia was considerably thickened in its upper third, and the fibula throughout its entire length. Physical examination, including the blood and urine, was otherwise negative.

Discussion.-This story seems to narrow itself down to a case of fever with painful glands in the groin. Our chief task is to consider the 
probable cause of the glandular enlargement. The ulceration on the lower leg may well produce sufficient irritation to stimulate the glands into a work-hypertrophy, ordinarily known as a bubo. Leukemia being ruled out by the negative result of the blood examination, and pseudoleukemia by the absence of glandular enlargement elsewhere, it remains only to consider the probable nature of the ulcerations which

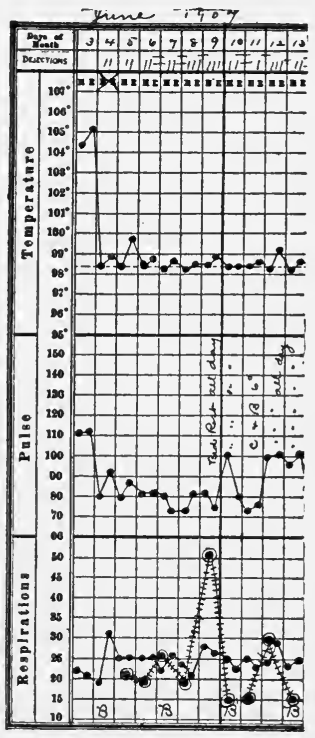

Fig. 45. - Chart of case $\mathrm{I} 4 \mathrm{I}$. have led to the adenitis and so to the pain and fever.

Ulcerations in this situation are most frequently due to the malnutrition following varicose veins, hence the term varicose ulcer. Next to this, syphilis is the most common cause, though it is more apt to produce ulcerations in the calf or above the knee than upon the shin. In view of the $x$-ray evidence, which shows a bony change very commonly associated with syphilis, this seems the most reasonable diagnosis.

Regarding the cause of the acute infection, with its attendant pyrexia and chill, nothing very definite can be said. Possibly there was some secondary invasion of the tissues induced by a sudden lowering of their vitality, for which there are many occasions in the life of such an individual.

Outcome.-Under iodid of potash the glands became smaller, the fever went down, the leg ulcers began to heal; on the fourteenth the patient was discharged.

Diagnosis.-Syphilitic adenitis.

\section{Case 142}

A housewife of twenty-seven entered the hospital June 2I, I908, for pain in the left iliac fossa, her second severe attack within three weeks. The first attack (twenty days ago) was very severe, but lasted only about one minute. Yesterday at 2 A. M. sudden severe pain began again at the same point, lasted until ro A. M., then suddenly ceased until this morning about five, when it returned as she was getting up. At times she has seen and felt a swelling in the region of the pain.

sine has had three children, the youngest three months old. Menses normal. No other illnesses.

Examination.-Abdomen promịnent in lower left quadrant, where 
there are circumscribed dulness and a large, hard, irregular mass, movable and very tender. It was apparently not connected with the uterus, but could be felt per vaginam. Physical examination, pulse, temperature, blood, and urine negative.

Discussion.-The association of left iliac pain with a hard, irregular mass in the same region naturally suggests malignant disease. The sigmoid flexure of the intestine is the commonest site for such a growth in this part of the body, and the age of the patient by no means excludes this possibility. I have seen a cancer of the sigmoid demonstrated at autopsy in the body of a boy who died before his twenty-first year. In the present case, however, we have no intestinal symptoms sufficient to incriminate the sigmoid, and a growth of the size above described would certainly have produced such symptoms if the gut were involved.

Ovarian tumor seems more probable. We do not expect the commoner varieties of ovarian tumor to be as firm of surface as the description of this tumor suggests, but I have often been deceived in this respect and seen at operations a cystic tumor which felt as hard as a piece of wood when examined through the abdominal wall, so that I am no longer willing to trust my tactile sensations. Solid tumors of the ovary are considerably less common, especially in women of this age, and rarely reach so large a size without previously attracting any attention. Fibromyoma of the uterus would probably show an obvious connection with that organ and would be less likely to be situated so much at one side.

Uncomplicated ovarian tumors do not produce acute symptoms like those above described, but there are many accidents to which such tumors are exposed and by which severe pain may be produced. As we have no way, in the great majority of cases, of distinguishing these accidents clinically, it is safest to assume that the commonest of them -twisting of the pedicle-has occurred.

Outcome.--Operation showed a gangrenous, strangulated, multilocular ovarian cyst with a double twist in its pedicle and a quart of bloodserum in the peritoneal cavity.

It may be well to mention here some of the varieties in the symptomatology of strangulated ovarian cyst, so as to bring out features not exemplified in the case just discussed.

(a) In many cases there are repeated attacks which are clinically similar in type, but lesser in intensity than that above described. Many of these attacks are due, doubtless, to patches of local peritonitis such as result in the adhesions which often confront the operator years later.

(b) General abdominal tenderness and spasm, associated with vomiting and great prostration, often make the clinical picture much like that 
of acute peritonitis, which can be excluded only when the patient or her physician has previously known of the tumor's existence.

(c) Tumors occupying the right side of the abdomen are fully as common as left-sided growths. In a considerable proportion of cases the cyst is to be found in the median line, and the diagnosis is thereby considerably obscured.

(d) Moderate fever and leukocytosis are the rule, the former ranging between $100^{\circ}$ and $102^{\circ}$ in most cases, while the leukocytes are usually between 14,000 and 20,000 .

(e) If menstruation occurs during such an attack of pain, the latter is often relieved.

Diagnosis.-Multilocular ovarian cyst (twisted pedicle).

\section{Case $142 a$}

A widow of sixty-seven called her physician in September, I908, on account of pain in the left iliac fossa. For five or six years she has noted a bloody discharge with some odor. This discharge has been supposedly due to hemorrhoids and has been treated as such, but examination now shows it to come from the vagina. For the past week this discharge has been active and the blood has been bright. Four weeks previously to this time she had a week's flowing, and similar periods have occurred from time to time during the last five years.

The present illness began three weeks ago with pain, tenderness, and enlargement of the left lower quadrant of the abdomen, accompanied by fever which averaged $\operatorname{IOI}^{\circ} \mathrm{F}$. for the first week of her illness. This gradually fell to normal, so that ten days ago the local physicain was able to discontinue his visits for three days. With the subsidence of temperature the weakness, tenderness, and pain of which she had previously complained gradually disappeared, but a week ago all the symptoms returned, and during the last six days fever has averaged $100^{\circ} \mathrm{F}$. The pain is now referred not only to the left iliac fossa, but to the left thigh and hamstring muscles. The bowels are moved by enema.

The appetite has been very poor and there has been marked prostration, so that she has been in bed most of the time during the last four weeks. Her weight has fallen considerabley. There has been no vomiting, no cough, and no pain other than that described above. The menopause occurred thirteen years ago.

When seen in consultation October 19, I910, the patient's tem- 
perature was IOI.2; there was moderate emaciation; at the apex of the right lung the physiologic peculiarities of that space on auscultation and percussion seemed somewhat exaggerated. Otherwise the chest showed nothing abnormal. The left lower quadrant of the abdomen was filled by a smooth, resistant, apparently elastic mass, protected by a considerable amount of muscular spasm and rather tender. The same mass was felt by vagina, but seemed to be unconnected with the uterus, which was normal.

The leukocytes numbered 25,600, 90 per cent. of which were polynuclears. There was no anemia. The urine was normal.

Three weeks later the attending physician reported that the patient was about the same, the temperature still reaching about ror ${ }^{\circ} \mathrm{F}$. each night, being normal or subnormal in the morning. There was then very little pain and the vaginal discharge had ceased.

Discussion.-Cancer of the uterus was first suspected on account of the ill-smelling vaginal discharge. That this was not of the ordinary type, involving the cervix uteri, was readily shown by the vaginal examination. Cancer of the body of the uterus was not excluded, as no intra-uterine examination was made. It is very unlikely, however, that so much fever and left iliac tenderness would be produced by a neoplasm of the body of the uterus.

Cancer of the sigmoid was next considered. The position of the tumor mass, the age of the patient, and the presence of a bloody discharge, which the patient believed to have come from the rectum, favored this diagnosis. On the other hand, nothing definite could be felt by rectum. There was no evidence of intestinal obstruction and no diarrhea, while the presence of continued fever for more than a month made uncomplicated neoplasm very unlikely. The same holds true of ovarian neoplasm. Pyosalpinx was considered, but seemed exceedingly unlikely in view of the patient's age and character.

Diverticulitis is strongly suggested by all the facts of the case. The age of the patient, the position and consistency of the tumor, and the continued fever with leukocytosis are typical.

Outcome.-On operation, November ${ }^{3} 3^{\text {th }}$, a large inflammatory mass was found tying together the bladder, the lower sigmoid, and the adjoining parts. In the center of the mass, close to the sigmoid, a pus-cavity containing about a tablespoonful of pus was found. Leading out of this cavity was a sinus connecting with the interior of the lower sigmoid, which was greatly thickened and infiltrated for a considerable distance above and below the sinus. Microscopic 
examination later showed that the sinus originated in a diverticulum. The pus was evacuated and drained, a portion of the sigmoid resected, and an end-to-end suture done. The patient made a somewhat slow, but uninterrupted recovery.

Diagnosis.-Diverticulitis of the sigmoid.

\section{GENERAL CONSIDERATIONS ON THE DIAGNOSIS OF ABDOMINAL PAIN}

Though I have followed current practice in separating the causes of localized from those of generalized abdominal pain, it must be admitted that the separation is not always true to fact. Diseases like appendicitis, whose pain belongs in the right iliac fossa, are very apt to set their pain loose all over the belly. On the other hand, lead-poisoning, which usually causes wide-spread "dry" bellyaches, may anchor its colic to a single spot in a most misleading way.

Hence one who looks under one chapter for some familiar type of pain may wonder at its absence and be surprised to find it in another. Some causes of suffering, on the other hand, are listed under two different headings (e. g., ectopic gestation, strangulated ovarian cyst), because they are about equally common on the right and on the left.

When searching out and thinking out the probable cause of an abdominal pain we are all guided, I take it, by the following obvious rules:

I. Suspect, first of all, the gastro-intestinal tract, and if its simpler troubles (such as constipation and colitis) can be excluded, consider especially appendicitis, peptic ulcer, neoplasms of the stomach or large gut, and the remoter consequences of these lesions (peritonitis, intestinal obstruction).

2. Suspect next (in women) the genital tract (pus-tube, ovarian cyst, uterine fibroid, ectopic gestation).

3. The gall-bladder and its ducts are especially to be considered if the patient is past middle life.

4. The urinary tract, especially in elderly men or young girls, comes next in the order of causes for abdominal pain.

In investigating any of these causes the history, palpation, the blood, the urine, $x$-ray, and cystoscopy are the most valuable aids. 


\begin{tabular}{|c|c|c|c|c|c|c|}
\hline $\begin{array}{l}C \\
C \\
\frac{1}{0} \\
\frac{0}{0} \\
\frac{0}{0} \\
0 \\
\vdots \\
\vdots \\
\vdots \\
\vdots \\
\vdots \\
\vdots \\
\vdots \\
\vdots\end{array}$ & 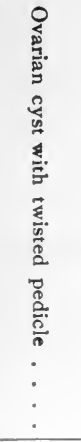 & 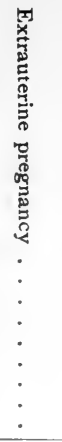 & $\begin{array}{l}尺 \\
0 \\
0 \\
0 \\
0 \\
0 \\
0 \\
00 \\
0 \\
0 \\
0 \\
\vdots \\
\vdots \\
\vdots \\
\vdots \\
\vdots \\
\vdots\end{array}$ & 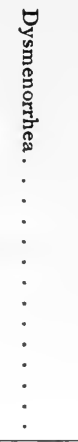 & 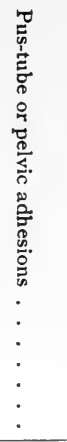 & 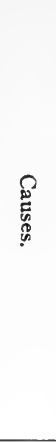 \\
\hline $\begin{array}{l}\vdots \\
\vdots \\
\vdots \\
\vdots\end{array}$ & 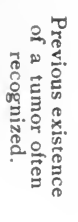 & 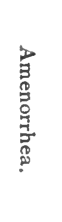 & 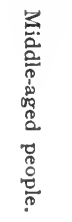 & 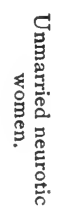 & 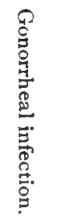 & 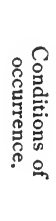 \\
\hline 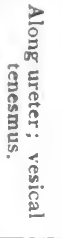 & 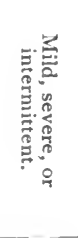 & 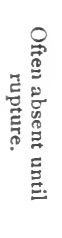 & 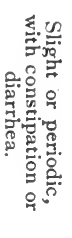 & 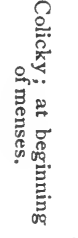 & 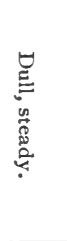 & . \\
\hline 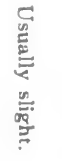 & $\begin{array}{l}0 \\
\stackrel{0}{0} \\
9 \\
\square \\
0 \\
0 \\
0 \\
0 \\
0\end{array}$ & 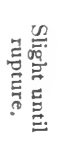 & 0 & $\circ$ & 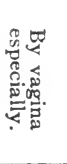 & 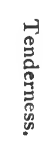 \\
\hline 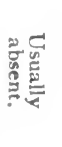 & 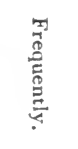 & 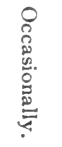 & $\circ$ & $\circ$ & $\begin{array}{l}3 \\
0 \\
\frac{2}{0} \\
\frac{0}{20} \\
\frac{0}{8}\end{array}$ & 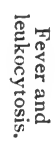 \\
\hline 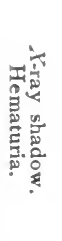 & 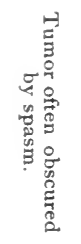 & 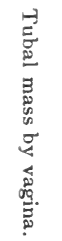 & 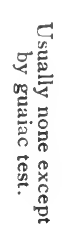 & ० & 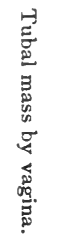 & 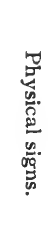 \\
\hline 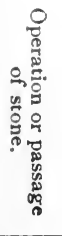 & 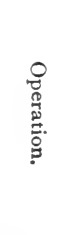 & 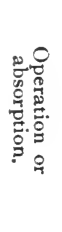 & 0 & 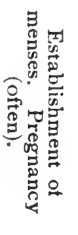 & 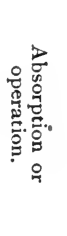 & $\begin{array}{l}\frac{\pi}{0} \\
\stackrel{0}{0} \\
\stackrel{n}{n}\end{array}$ \\
\hline
\end{tabular}




\section{CHAPTER X}

\section{AXILLARY PAIN}

\section{Case 143}

A Stableman of thirty-nine entered the hospital January 24, I908, with negative family history and good habits. Past history uneventful except for an attack of malaria in September, I907. Three days ago, while at work, he had a severe chill. He went home, but did not go to bed. The next morning he went to work as usual, but had to give up

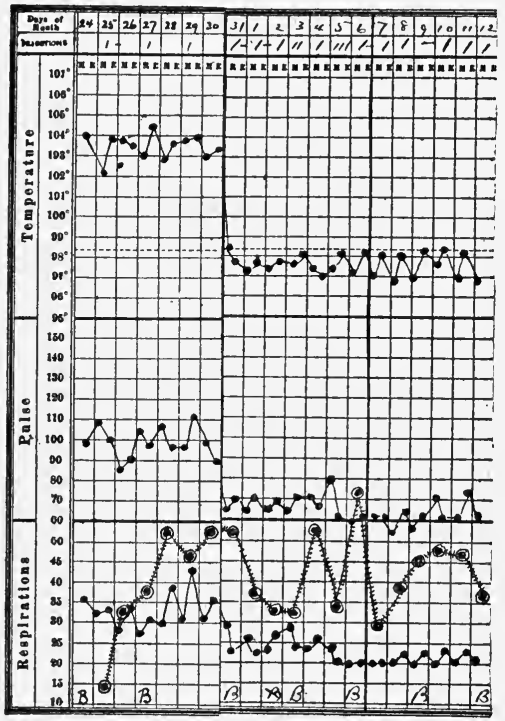

Fig. 46. - Chart of case 143 . about noon and take to bed, where he has remained since, with headache, high fever, pain in the left chest, sore throat, nausea, thirst, and frequent vomiting. His bowels were moved by laxatives this morning. When he swallows, he sometimes feels a sharp pain which shoots from his throat toward his left ear.

The patient's temperature is seen in the accompanying chart. At entrance he was breathing easily, and there was no motion of the nostrils. He complained of deafness and buzzing in his ears, especially the left. There was internal strabismus on the right, which he says is of long standing. There was a mild spasmodic cough, but no sputa. Visceral examination was negative, save that in the lower left back there was a little dulness, and the voice-sounds were a trifle nasal in character. Just below the scapula the breath-sounds were somewhat diminished, whispered voice slightly increased, and an occasional clicking râle was audible. No bronchial breathing.

The white cells were 20,000; urine, 32 ounces; specific gravity, ro23. There were a few hyaline, many fine granular casts, and a slight trace of albumin. 


\section{Causes of Axillary Pain}

3. PNEUMONIA

4. FRACTURED RIB

5. INTERCOSTAL NEURALGIA

6. RADIATIONSI FROM HYPERTROPHIC SPINAL ARTHRITIS

Among other lesions not graphically represented here, because of their rarity as causes of axillary pain, are :

Herpes zoster.

Costal tuberculosis.

Costal neoplasm.

Costal actinomycosis.

Angina pectoris.

Deep axillary abscess.

Fractures and dislocations of the shoulder and humerus often produce axillary pain, but usually present no diagnostic difficulties so far as the source of the pain is concerned.

Finally, there is a large group of axillary pains, apparently of muscular origin, akin to lumbago and "stiff neck." The bombastic term "pleurodynia" is often attached to these pains, but since their actual nature is unknown and their diagnosis never certain, I have attempted no estimate of their relative frequency. 

Discussion.-Obviously, we are dealing with an infectious disease, though gastric symptoms occupy the foreground of the clinical picture. For simple tonsillitis the patient is apparently too sick, and there was nothing in the tonsillar region sufficient to justify the diagnosis.

The deafness, the buzzing sounds, and the shooting of pain toward the ear might indicate otitis media, but unless pain is more definitely localized and continuous in or about the ear, one could not make such a diagnosis in the absence of any discharge or any knowledge of the condition of the drum membrane.

Acute meningitis may begin in this way, and there is nothing said in the text regarding the condition of the neck muscles or of the hamstrings (Kernig's sign). The strabismus would be of great diagnostic importance if we disregarded the patient's statement that it has existed for many years. As a matter of fact, however, investigation showed that there was no stiffness of the neck or of the ham-string muscles. Without lumbar puncture no further certainty can be obtained on this point, and meningitis must remain a possibility unless we can find some more plausible explanation for the symptoms.

It was subsequently learned that the patient had been given large doses of quinin before he entered the hospital, the chill and the previous attack of malaria having led to the exhibition of this drug.

Although the pulmonary signs are very slight and not distinctive, they seem to me sufficient to warrant a diagnosis of pneumonia when we link them with the continued fever, the leukocytosis, the chest pain, the chill, and the gastro-intestinal symptoms. Cases of pneumonia which do not show early and well-marked signs of pulmonary solidification are very apt to begin with several days of gastro-intestinal symptoms, the significance of which would be very obscure but for their association with fever and leukocytosis.

Outcome.-Rusty, tenacious sputum was later raised, and in it the pneumococcus was the predominating organism.

At no time were the signs in the chest any more definite than at entrance. On the twenty-sixth there were moist râles in various parts of the lungs, and the patient was somewhat delirious. On the thirtyfirst, the day after the crisis, there was a friction-rub in the sixth left space, anterior axillary line.

On the twelfth of February the patient left the hospital perfectly well.

The treatment consisted of laxatives, a tight swathe, ice-bag, and hot-water bottle for pain, and an occasional dose of morphin.

Diagnosis.-Pneumonia. 


\section{Case 144}

An Italian farm-laborer, sixty-six years old, was first seen January 30, 1908. His family history and past history were negative, his habits good. Seventy-two hours ago. while standing on a chair to put a cloth over his canary's cage, he lost his balance and fell to the floor, striking his left side on the back of the chair. He was unconscious for some minutes, and later experienced a sharp pain in the left side of the chest, worse on coughing or deep breathing. This pain has troubled him ever since, and has been accompanied by a slight dry cough. For two days he has been feverish.

At entrance, the patient's temperature was $99.4^{\circ} \mathrm{F}$; pulse, 79 ; respiration, 20. There was a marked posterior convexity of the lower dorsal and upper lumbar spine. The breath was foul. There was a slight, diffuse, systolic pulsation under each clavicle, especially on the left. The heart was negative. Scattered throughout both lungs were squeaks and crackles. There was marked tenderness over the eighth and ninth left rib in the midaxillary line. Pressure over the vertebral end of the ninth rib caused pain over the same rib in the midaxilla. No definite crepitus was obtained. A rough grating was heard with inspiration in the painful area. At the top of the left axilla was a suggestion of bronchial breathing. On the left forefinger and the back of the last phalanx was a raised, reddened, tender area, half an inch in diameter, crusted in the center. From this a little seropurulent fluid could be expressed. He has had this trouble for a month. The next day the fluid in this lesion was distinctly purulent.

Discussion.-Fever, cough, râles, and axillary pain in a man of sixty-six lead straight to the diagnosis of pneumonia if we are in the habit of judging by symptoms alone, and so far as the pulmonary signs are concerned, they are perfectly consistent with the existence of a central pneumonia or of pneumococcus infection which has not yet become localized anywhere. More important evidence against pneumonia is furnished by the temperature chart, the low respiration rate, and the absence of gastro-intestinal symptoms. Up to the time when I saw the patient no leukocyte count had been made, and as this seemed to me one of the most important diagnostic data, I made the count at once. There were 6500 leukocytes per c.mm. So low a count rarely occurs in pneumonia unless the patient is more ill than this man seemed to be.

The pulsations beneath the clavicles had given rise to considerable anxiety in the mind of the attending physician, who thought they might be connected with an aneurysm, which he suspected of producing pain 
in the side. But there was really no evidence of aneurysm, and the pulsation was not greater than is often seen in thin persons whose subclavian arteries happen to lie near the surface.

Of pleurisy there were no certain physical signs, and although this diagnosis is often made on the basis of the patient's account of his pain, and often definitely at his suggestion, experience does not justify any such diagnosis. Many patients and not a few physicians allow themselves to speak of "pleurisy pains" when they would not seriously maintain that they had evidence of any form of pleurisy. The rough grating sound referred to was probably due to another cause, soon to be mentioned.

Pain of muscular origin - the so-called pleurodynia-akin to stiff neck and lumbago-must be shown to vary directly with the amount of muscular motion; apparently there was no such variation. Pleurodynia produces general widespread tenderness, much less local than was present in this case.

The protuberance of dorsal and lumbar vertebræ makes us ask whether any form of spondylitis may be responsible, through radiations along nerve-roots, for this patient's pain. Pain of this type is often made worse by coughing or deep breathing. It does not, however, lead to tenderness in midaxilla, and would be very unlikely to appear suddenly after a fall.

The fact that pressure on the ninth rib near the spine produces pain localized in the axillary portion of that rib is strong evidence that that rib is cracked, and the local tenderness and the rough grating sound following such a fall point strongly in the same direction. In the absence of crepitus no further evidence can be obtained, unless a callus forms. This diagnosis would doubtless have been made at the start had not the patient chanced to be feverish. Presumably the fever was due to the slight infection on the forefinger.

Outcome.-The chest was strapped with plaster and in two days the patient was well enough to go back to work of a light character.

Diagnosis.-Broken rib.

\section{Case 145}

A housemaid of twenty-five entered the hospital July 20, I906. Nine days ago she suddenly experienced sharp, shooting pain in the lower ribs and in the right axilla, not worse on cough nor on deep breathing. ,This pain lasted one day; she then began to have pains in her head and knees, with fever, chill, and general weakness. Four days ago she had to give up and go to bed. Her bowels have moved regularly, but she has vomited once. 
Physical examination was entirely negative.

The white cells were 2500; no Widal reaction; urine normal, except for the presence of a diazo-reaction.

The course of the fever is shown in the accompanying chart.

Discussion.-At the outset it was impossible to exclude pneumonia, although the association of so low a white count with a good general condition seemed very much unlike pneumonia. Had the leukocyte

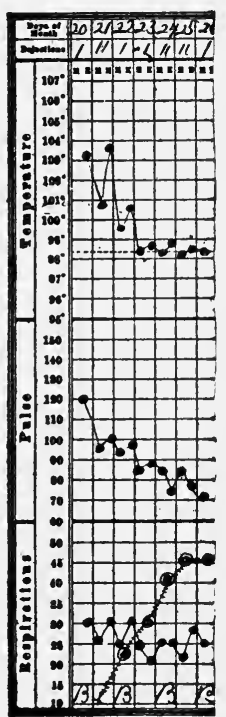

Fig. 47.-Chart of case $\mathrm{I} 45$. count been high, I should have suspected pneumonia, present or to come, even in the absence of definite signs in the chest.

I have known a case altogether similar to this to be counted among the successes of a physician who thought he could abort typhoid fever. It is true that typhoid fever not infrequently shows under observation no longer a period of pyrexia than was here recorded, but the presence of a diazo-reaction is by no means sufficient evidence on which to base a diagnosis of typhoid under these conditions. Only by the demonstration of typhoid bacillus or at least of a well-marked Widal reaction can the diagnosis be justified when the fever is so brief.

Pleurisy is excluded by the short duration of the pain and by the absence of physical signs.

I have known tertian malaria to produce symptoms strikingly like those with which this case began, but the pain and fever were then much more definitely intermittent and did not cease permanently until quinin was given. In the case here under consideration no quinin was exhibited.

It is the fashion to call such cases as this "grip" or "influenza," but although these words are not taken very seriously by the physician who makes the diagnosis, they seem to me sufficient to mislead the patient, and incidentally the physician himself. They hide from us the fact that we are facing something which we do not understand. A well-known name easily transforms itself into the impression that we know something of the disease to which we are applying it. This tends to make progress impossible. It seems more sensible to recognize that the unnamed infections are probably as numerous as those already listed and named in our text-books, and that in a case like this we are confronted with one of this unnamed and unknown multitude.

Outcome.-In six days the patient seemed perfectly well; the treatment consisted mainly of an occasional laxative and hypnotic.

Diagnosis.-Unknown infection. 


\section{Case 146}

A master painter of sixty entered the hospital November I8, 1907. His family history was good. From the, age of sixteen up to the age of thirty-two he suffered from neuralgia in the right side of his forehead, but was finally cured in 1879 . He had typhoid at eighteen, just after the Civil War. Twenty years ago he was laid up for six weeks with lumbago, and has had several less severe attacks of this pain since. He has never had lead colic, nor any pain in his joints. His habits are good.

For six years he has been troubled with pain in the left upper chest, the attacks gradually growing worse and more frequent. Now the pain is nearly constant unless he takes medicine. The pain is of three sorts: (I) A dull, burning pain, present in the chest most of the time; (2) a terribly severe pain, with a feeling as if he were gripped in a vise. This comes from once a week to once a month, and has several times waked him in the night. (3) A sharp, shooting, knife-like pain, beginning in his left chest, running up to his shoulder and neck, and sometimes felt also in his arms. This comes at irregular intervals-more often within the last two or three years. There are no gastric symptoms. The pain does not seem to have any relation to food. There is no dyspnea, cough, palpitation, or edema. Years ago exertion seemed to make him worse, but now, he says, it seems to make him better, and lately he has dreaded bed-time. He has been treated in the out-patient department since May, 1902. He still directs his business and works irregularly.

Physical examination shows an obese man, with normal temperature, pulse, and respiration; the blood-pressure, I50 $\mathrm{mm}$. The urine averages 40 ounces in twenty-four hours; specific gravity, I027; no albumin and no casts. The white corpuscles range between I2,000 and I4,000 per c.mm. No stippling of red cells. No lead-line.

The first sound at the heart's apex is followed by a soft murmur, best heard in the aortic area, not transmitted to the axilla. The aortic second sound is greatly accentuated. There is no demonstrable cardiac enlargement. The pulses are equal and regular, the artery wall not remarkable.

Physical examination is otherwise negative, except that there is some dulness in the flanks, which, however, shows no shift attendant upon change of position.

Discussion.-When a house-painter complains of a pain of any kind, our knowledge of the pathology of lead-poisoning naturally leads us to do what we can to connect the pain with the patient's occupation. In this case, however, there is no definite evidence of lead-poisoning 
(stippling is often present in lead-workers who show no evidence of illness), and the pain is not such as we are accustomed to see produced by that disease.

The previous history of lumbago makes us seek to find evidence of that disease in the patient's present symptoms, but there seems to be no such close relationship between the pain and movement of the affected muscles as would be expected in lumbago.

The situation and continuity of the pain are such as we are accustomed to associate with aortic aneurysm, and only by $x$-ray examination (which was not made, owing to the patient's poor condition when he first entered the hospital) can aneurysm be positively excluded.

Angina pectoris produces pains the character and location of which correspond accurately with those here described. The patient's statement that exertion now seems to make him better is practically the only consideration that seems to contradict this diagnosis, and this is not sufficient to exclude it. As to the nature and prognosis of the affection, our judgment would be much assisted if we knew whether the patient was an excessive consumer of tobacco. No further certainty can be obtained without the therapeutic test (nitroglycerin or amyl nitrite) and an $x$-ray examination.

Outcome.-He has used nitroglycerin in doses of $\frac{1}{10} \overline{0}$ grain from the first, and for years a single tablet gave prompt relief. Gradually the necessary dose has increased, until of late he takes as much as $\frac{35}{100}$ in twenty-four hours.

$X$-ray showed no evidence of aneurysm. During the patient's stay in the hospital he usually had an attack each night, best relieved by amyl nitrite. Sitting up or walking about the ward seemed to. bring on attacks, relieved in the same way.

On the second of December he was discharged not relieved.

Diagnosis.-Angina pectoris.

\section{Case 147}

A French-Canadian cabinet-maker of thirty-six entered the hospital November 3, 1906. His family history and past history were not remarkable, but he has used a great deal of tobacco and taken three or four drinks of hard liquor every day for fifteen years.

Five years ago he began to have pain in the left side of the chest and in the pit of the stomach, brought on by exertion or excitement, gradually increasing in frequency and in severity. The pain stabs like a knife, lasts about half a minute, makes him stop whatever he is doing and stand 
bracing himself back. Occasionally it comes on at night, and then he has to sit up in bed "holding onto himself."

Last winter he began to have palpitation and dyspnea on exertion. Four months ago he stopped work by his physician's advice and went into the country, following which he promptly became worse and for a time could not sleep on less than four pillows. His abdomen also swelled, and the upper part of it was tender. These symptoms have now so far subsided that he can sleep on one pillow. Two or three years ago his wife noticed that one pupil was larger than the other. He has lost twelve pounds in the last three years.

On physical examination the above observation regarding the pupils was confirmed. Both were slightly irregular in outline, but reacted

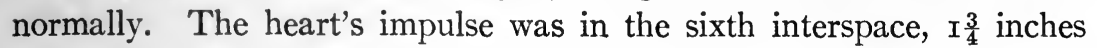
outside the nipple. A systolic murmur was heard, loudest at the apex, transmitted also over the whole precordia and into the axilla. In the axilla and back, a harsh diastolic murmur was also heard replacing the second sound. No second sound at all was heard in the aortic area. The pulse was of the Corrigan type. The systolic blood-pressure was $165 \mathrm{~mm}$. The daily amount of urine averaged 30 ounces, with a trace of albumin and no casts. At times a presystolic rumble was heard at the apex. During the first ten days' stay in the hospital he was given magnesium sulphate, an ounce every morning, tincture digitalis, Io minims every six hours, iodid of potash Io grains four times a day, $\frac{1}{100}$ grain of nitroglycerin when needed. His progress during this period was uneventful. On the night of the fourteenth he was rather uncomfortable. On the fifteenth he vomited several times. His pulse was more rapid and weaker.

Discussion.-The pain is strongly suggestive of angina pectoris, but the patient seems rather young for the organic type, dependent on arteriosclerosis, and too ill for the functional type. As in the previous case, we are unable to exclude aneurysm, as the patient is too ill to be moved to the $x$-ray room. The pain and the inequality of the pupils remind us distinctly of that disease.

As regards the type of cardiac lesion, there seems to be distinct evidence of aortic insufficiency with hypertrophy and dilatation of the heart. In a patient of this age the occurrence of aortic disease with no preceding rheumatic attacks justifies us in treating the case as one of syphilis, especially when the cardiac lesions are associated with irregular and unequal pupils. This assumption rests upon the fact that syphilis of the cardiovascular system usually begins in the arch of the aorta and extends thence to the aortic valves. 
Outcome.-About 7 P. M. he remarked that he had had rather an uncomfortable day, and felt that it was his duty to stay in bed, but that he hoped to be allowed to get up the next day. About 8 o'clock be became unconscious and died within a few minutes.

At the autopsy (No. I8I6) no cause for the suddenness of death was discovered. The heart was greatly dilated and hypertrophied. There was a chronic fibrous myocarditis, and the heart-wall was much thinned near the apex of the left ventricle. There was stenosis of the coronary orifices and a fibrous deformity of the aortic valve. Just above the aortic valve, and in the arch of the aorta, were very many fibrous plaques. A chronic pleuritis and chronic perihepatitis with adhesions was also found.

Microscopic examination of the aortic wall showed a number of the organisms of syphilis (treponema pallidum).

Diagnosis.-Syphilitic heart and aorta.

\section{Case 148}

A Jewish laborer of nineteen entered the hospital November 4, I907. His family history and past history were good, also his habits. In February, 1907 , he began to have a loud, ringing, brassy cough, and to raise considerable sputa. At the same time he had hoarseness and pain in the left upper chest, both front and back. He improved at first, later losing all he gained. Yet he has felt less thoracic pain of late, although he has coughed considerably. Three days ago, following a severe paroxysm of coughing, he was seized with intense pain in the left lower chest, both front and back. The pain has gradually improved since, but is still severe on coughing. During the same period he has been somewhat short of breath - a new symptom for him-and has felt feverish.

The movement of his temperature, pulse, and respiration is seen in the accompanying chart (Fig. 48). The iris of his left eye is bluish; of the right, brownish. The right pupil is smaller, markedly irregular, and situated more toward the inner side of the eye. The vision of this eye is much diminished. The right border of the cardiac dulness extends $3^{\frac{1}{4}}$ inches beyond the midsternal line, and reaches a point just inside of the right nipple. The left border of dulness extends about an inch beyond the midsternum. The cardiac sounds are best heard in the second and third right interspaces. Here the rhythm is fetal; the sounds sharp and clear. To the left of the sternum they are difficult to hear. The left chest is hyperresonant throughout, while the right is somewhat dull. Breath-sounds are markedly diminished on the left, increased on the 


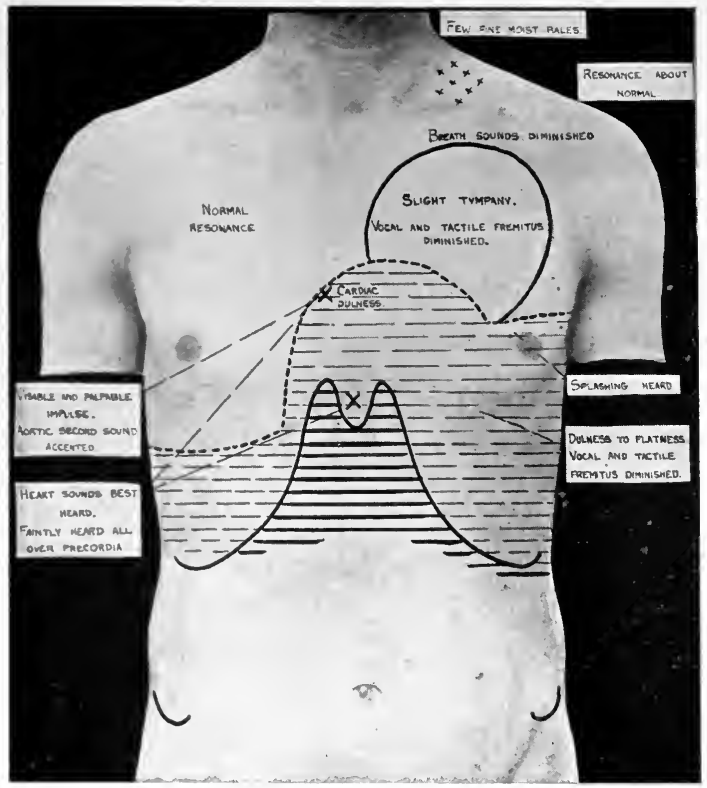

Fig. 49.- Physical signs in Case I 48. Cough, fever, and a sudden attack of pain in the lower left axilla are the chief complaints.

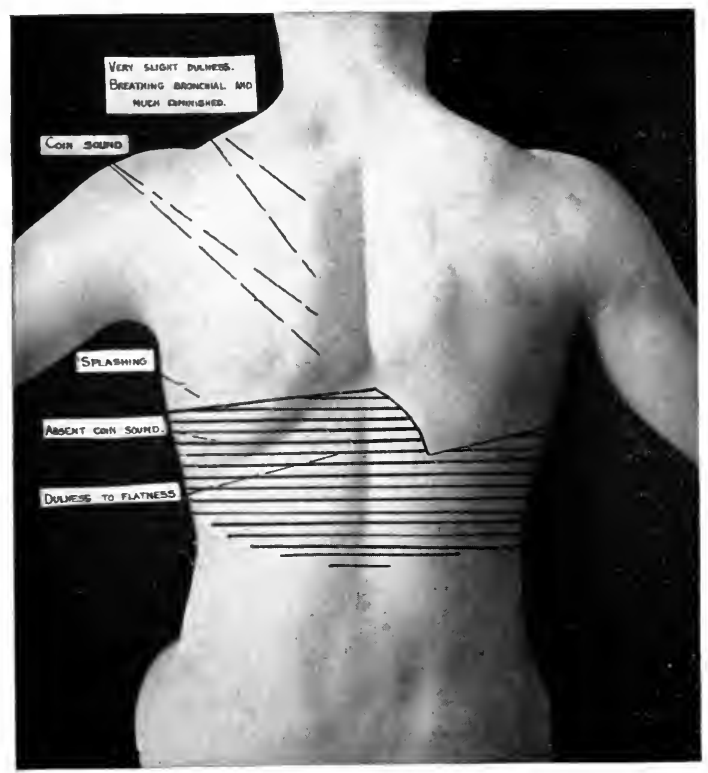

Fig. 50.-Physical signs found posteriorly in Case 148. (See also Fig. 48.) 

right. Tactile and vocal fremitus are almost absent on the left. Physical examination of the abdomen and the rest of the body is normal.

By the seventeenth of November the patient was much more comfortable, though the physical signs had not changed. At the apex of the left lung a few fine moist râles were heard, with distant bronchial breathing and slight dulness (Fig. 49).

$X$-ray revealed a shadow in the left chest about the level of the angle of the scapula. There were evidences of fluid below this point.

Discussion.-Although fever, chest pain, and cough are so often the precursors of pneumonia, these symptoms have lasted far too long, in the present case, to be at all typical, and as soon as we scrutinize the de-

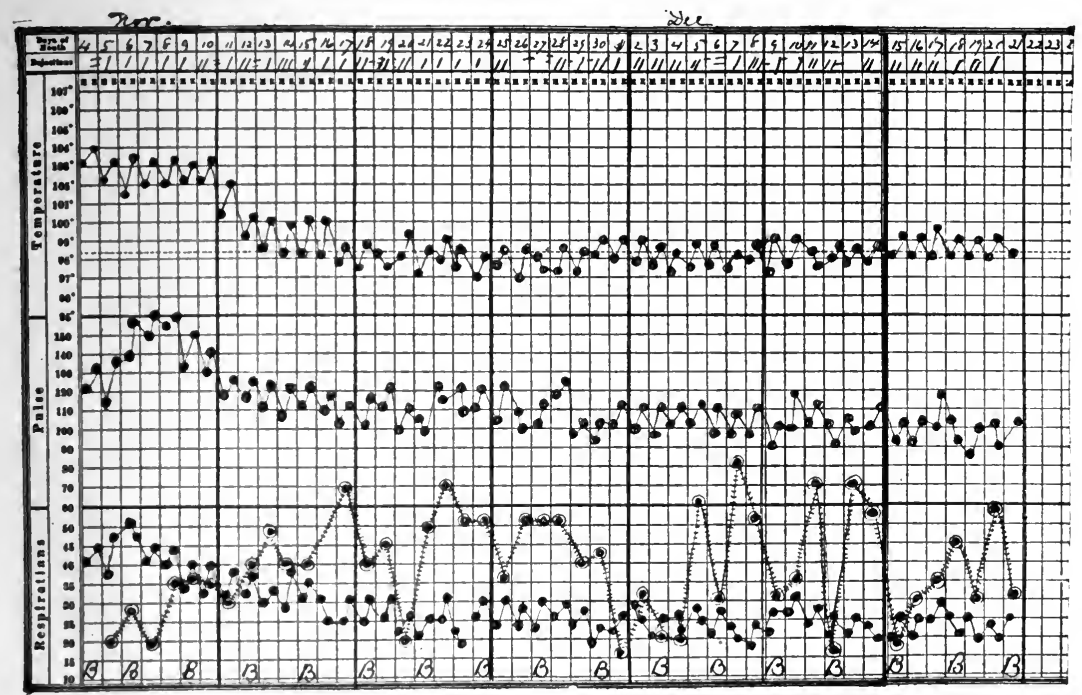

Fig. 48. - Chart of case I48.

tails revealed by physical examination, it is obvious that the picture is quite unlike that of pneumonia.

The extension of dulness, continuous with that of the heart's area to the right of the sternum, the hoarseness and brassy cough, and the pain in the chest suggest aneurysm. But the pain is on the left, and the extension of cardiac dulness on the right. There are no pressure signs, pulsations, or $x$-ray shadows to support the suspicion of aneurysm; only the patient's complaints are favorable to that diagnosis.

When the heart is displaced to the right, as seems to be the case here, we naturally investigate the causes of this displacement, beginning with the commonest-left pleural effusion. In favor of this condition we have the diminution of respiratory murmur and the absence of vocal and tactile 
fremitus in the left chest. But in spite of these signs, pleural effusion. serous or purulent, may be unconditionally excluded on the evidence of a single sign, viz., the hyperresonance of the whole left chest. Hyperresonance of a portion of one chest-for example, the lower axillary region or the upper quarter-is quite consistent with pleural effusion, but total hyperresonance has never been recorded, so far as I know, with pleural effusion. Over a pneumonic consolidation situated deeply in the lung substance the percussion-note is not infrequently hyperresonant or tympanitic, but this never occurs, I believe, throughout a chest containing a pleural effusion. Hyperresonance of one chest then, with displacement of the heart toward the opposite side, is practically distinctive of pneumothorax, which seems the reasonable diagnosis of this case.

Emphysema produces general hyperresonance, but it is never unilateral, never dislocates the heart, and never causes pain.

The prolonged cough, with the râles and dulness at the apex of the left lung, are presumably due to that disease which almost invariably underlies pneumothorax-phthisis. The $x$-ray shadow and the evidences of fluid which gradually developed at the base of the left chest are doubtless due to the accumulation of an exudate, converting the pneumothorax into hydropneumothorax according to the ordinary rule.

Some account of the two main clinical types of pneumothorax has already been given. Hence nothing further is added here.

Outcome.-The sputum contained many tubercle bacilli. A succussion splash was once made out.

On the nineteenth of December there was still no change in the patient's condition so far as the signs in the chest were concerned; the patient was feeling much better, had gained considerably in weight, and had almost no cough. On the twenty-first of December he was allowed to go home.

Diagnosis.-Pneumothorax (pulmonary tuberculosis).

\section{Case 149}

A teamster of fifty-two entered the hospital April 3, I908. His family history and habits were good. He had right-sided pleurisy in I872, and was in bed ten weeks with fever and pain in the chest. $\mathrm{He}$ was not tapped. Since then he has been well. In October, I907, he was struck on the right chest by a roll of cotton duck weighing 400 pounds. He had some pain there, which went off after a few days. He thinks no ribs were broken. Three weeks ago he began to have dull, constant pain in the right chest, worse on deep breathing. This pain lasted a week. 
March 3 oth he went to work, but the pain soon returned and compelled him to stop work. Now that he is in bed he has practically no pain, no cough, no fever, an excellent appetite, and feels in most respects very well.

His temperature, pulse, and respiration are normal, likewise his blood and urine. He lies comfortably in bed without dyspnea. His heart is negative. The artery walls are tortuous, with visible pulsation in the radials, brachials, and axillaries. The right chest is flat below the fourth rib in front and midscapula behind. Over this area respiration is absent, likewise voice and fremitus.

Discussion.-As this patient has previously had pleurisy on the right side, we need to consider whether the organized results of that attackpleural adhesions-might account for the symptoms which are now present. I should say decidedly not. An inflammation which has entirely died out thirty-six years earlier does not lead to acute pain. The pain of a pleural effusion may linger on for months, or even for a year or two, but never for thirty-six years. Pleural adhesions may cause dulness and diminished breathing, but not flatness and absent breathing.

Can the trauma of October, $\mathrm{I}_{90} \mathrm{7}$, be the cause of the present trouble? The interval of five months between the time of the blow and the onset of the present pain makes this rather unlikely. Hemothorax never results, so far as I know, from an injury of this kind without fracture of a rib or puncture of the pleura. Serous pleurisy has also, in my opinion, no connection with such an accident.

Dropsical effusions due to disease of the heart or kidney have a predilection for the right chest, but we have no evidence of any such disease in the present case, although there appears to be some arteriosclerosis in the peripheral vessels. Further, dropsical effusions do not produce pain.

These alternatives can be easily excluded, and the diagnosis of pleural effusion is then so automatic that it may be questioned whether I am justified in introducing this case in a book supposed to deal with diagnostic difficulties. On this point I can only say that I have repeatedly seen in consultation cases of serous pleurisy which had not previously been recognized because the patient had complained so little of the chest that no thorough physical examination had been made. Under these conditions the diagnosis is usually "typhoid," "slow fever," "autointoxication," or "ptomain-poisoning."

It is worth while to note in passing that this patient was in bed ten weeks with his untapped pleurisy of 1872 , whereas in 1908 his illness lasted less than two weeks. 
Outcome.-A paravertebral triangle was demonstrated, its dulness 3 inches wide at the base. The right chest was tapped, and 32 ounces of fluid obtained. Specific gravity, IoI7; albumin, 2.7 per cent.; lymphocytes, 87 per cent.

The fluid did not reaccumulate. On April gth he was discharged well, with the caution that he must always be rather more careful than other men as regards fresh air (day and night), regular meals, and the avoidance of all excesses.

Diagnosis.-Pleural effusion.

\section{Case 150}

An Italian housewife of thirty-five entered the hospital April 25, 1907. Three years ago she had an operation, following which she has had no menstruation, but frequent "hot flushes" rising from the at. domen to the head, accompanied by sweating and headaches which sometimes "made her crazy." During the past five months she has had frequent attacks of pain in the epigastrium and left chest. The pain is never severe enough to make her lie down. It lasts sometimes most of the day. It sometimes runs down the inner side of the left arm to the fingertips. The pain comes on suddenly, feels like needles pricking the skin, and is often accompanied by a sensation of heat all over her abdomen. Her urine scalds her during micturition.

She has worked up to the time of entrance, although she eats and sleeps poorly and her bowels are costive.

Temperature, pulse, and respiration are normal. Physical examination of the chest and abdomen is wholly negative. There is a thin, yellowish, vaginal discharge. The urine shows considerable pus.

Discussion.-Everything inclines us to explain many of the symptoms in this case as the result of an artificial menopause. We must make sure, however, that the familiar and typical phraseology used by such a patient does not sometimes mislead us into overlooking some deeper organic disease, such as pulmonary tuberculosis. If this occurs to us as a possibility, the use of a thermometer will soon make clear in the vast majority of cases that there is no fever, the sweating and sense of heat being due to vasomotor changes.

The presence of pus in the urine makes it reasonable to inquire whether some local infection of the genito-urinary tract may not be connected with the cardiac symptoms, since gonorrheal endocarditis is not nearly so rare as is often supposed. The first point, however, is to make sure that we are dealing with a genuine pyuria, not with an admixture of urine and vaginal discharge. In the present case a specimen of urine 
drawn by catheter showed no pus. A smear from the vaginal discharge showed a variety of saprophytic organisms, but no gonococci.

Returning now to the main complaint-the thoracic and epigastric pain -we notice first that it is accompanied by paresthesiæ, that it has no special relation to exertion, and is often prolonged over many hours. These facts, together with the negative results of physical examination, tend to show that it is not due to the organic type of angina pectoris, but belongs in the loose group of pains to which the name of "functional" or "false" angina has been given. As in so many other cases of this group, the patient's own interpretation of the pain have led to forebodings' and apprehensions, and so to a concentration of attention which greatly increases the suffering. The clinical importance of this fact is that it should lead us to a much greater vehemence, directness, and circumstantiality in our reassurances than would seem to be warranted by the patient's own statement. The organic effects of a fear are often in proportion to the patient's reticence upon the subject.

Outcome.-After eloquent reassurance and a few days' rest with full diet the patient seemed so much better that she was allowed to go home.

Diagnosis.-Artificial menopause.

\section{Case 151}

A Portuguese lumberman of forty entered the hospital May 30, I908. His family history, past history, and habits have been good. Three years ago he began to have pain in the left side of the chest, with cough and thick yellow sputa; also a headache, backache, lack of appetite, occasional vomiting. For the past week he has been worse. Three days ago he took to bed. His throat is now rather sore. The course of the temperature, pulse, and respiration is seen in the accompanying chart (Fig. 5I).

The patient was found to be slightly delirious, with rapid respiration and slight dry cough. The leukocytes were 5000 per c.mm. the urine, negative. Widal reaction negative. The heart was negative. There were coarse râles scattered throughout both chests. In the left back, just outside the lower end of the scapula, the voice-sounds were slightly nasal. The right clavicle was somewhat more prominent than the left, and expiration just below it was somewhat prolonged. The abdomen was held rather rigidly, and there was slight general tenderness there. The spleen was not felt, and there were no rose spots.

Discussion.-A low leukocyte count in a patient who is not apparently very ill may be taken as important evidence against the diag- 
nosis of preumonia, especially if the patient's lungs give little evidence of disease.

Pulmonary tuberculosis seems more probable in view of the long duration of cough with sputa, but unless we suppose that we are dealing with a miliary tuberculosis, there is not enough in the lungs to account for so sudden and severe an illness. Miliary tuberculosis cannot be excluded. The evidence is suggestive, but not compelling. This possibility should, therefore, be held in reserve until other alternatives are exhausted.

Acute influenzal bronchitis, or bronchitis of some other type, might account for most of the facts in this case. This diagnosis also it is im-

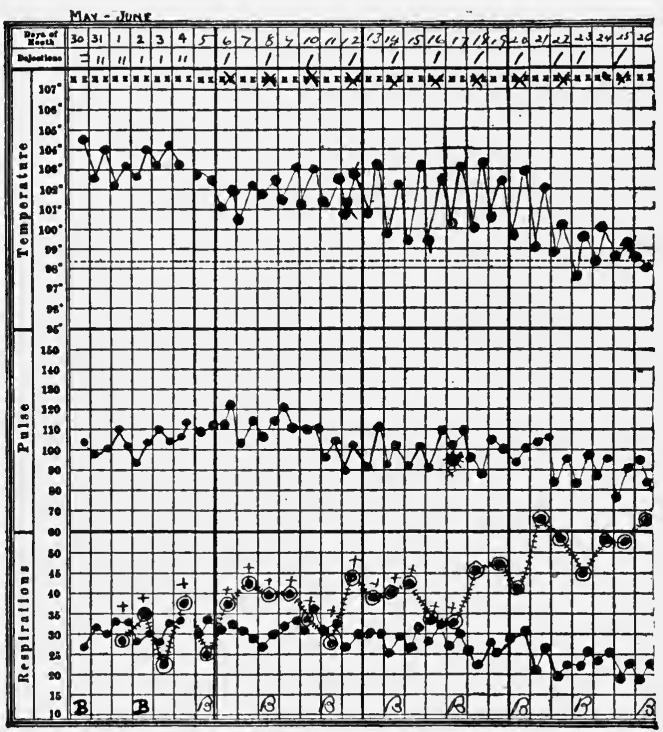

Fig. 5I.-Chart of case $15 \mathrm{I}$.

possible to exclude, although my impression as I saw the patient was that he was ton sick for simple bronchitis. The grounds of this impression, however, are hard to convey. Bronchitis and miliary tuberculosis, therefore, remained as possibilities to be accepted or rejected as the further course of the case might determine.

Influenzal infection of numerous small bronchiectases (such as occur very frequently with the clinical picture of chronic winter cough) is strongly suggested by the history and is compatible with the physical sign here described. It rarely causes so high a temperature, however, usually produces leukocytosis with profuse nummular sputa, and often has an emphysema associated with it. 
Meantime it is important not to forget the possibility of typhoid fever, although the time of the year is not the usual one, and although no definite evidence of typhoid has yet been presented. It seems to me essential, however, that we should consider typhoid in every febrile patient with vague and colorless symptoms which do not compel us to incriminate any one organ or group of organs. Typhoid is, beyond all other infections, the disease which produces fever with nothing particular to show for it in the way of local lesions. Hence in all such cases we should remember it and test for it by all the available methods.

Outcome.-Blood-culture taken into bile was positive for typhoid bacilli.

The course of the disease was uneventful. The patient went home well on the thirteenth of July. There was but little cough or sputa. The treatment consisted of $\frac{1}{4}$ grain of calomel given every fifteen minutes for ten doses, at the time of entrance, followed by a suds enema; thereafter he had alcohol and water sponges at $80^{\circ} \mathrm{F}$. every four hours when the temperature was above $102.5^{\circ} \mathrm{F}$; urotropin, 7 grains, three times a day twice a week, and turpentine stupes from time to time. In convalescence he had a good many boils, from one of which the staphylococcus was isolated. For this, staphylococcus vaccine was given.

Diagnosis.-Typhoid.

\section{Case 152}

A Turkish rug-repairer of forty-seven entered the hospital May 2, I908, stating that when he was twenty-six he was sick for three weeks, and had shortness of breath on exertion. He has since been well until three weeks ago, when he began to have pain in the back of his neck and the left side of his chest, with dyspnea, orthopnea, and nocturia. For ten days he has had cough and yellowish sputa.

The patient's temperature during the nine weeks of his stay in the hospital was generally subnormal; his pulse averaged about roo, his respiration 27. The daily amount of urine was generally diminished, averaging 25 ounces; specific gravity, 1023; no albumin or casts were found.

The heart's impulse was best seen and felt in the third space, four inches to the left of the midsternal line; the right border one inch to the right of the median line. In the fourth space the impulse was barely felt. The sounds were loudest and the palpable impulse strongest just below the ensiform. The sounds were regular and of good quality, the pulmonic second accentuated. The pulse was of good volume and tension. In front the percussion-note was dull below the left fourth rib, 
below the fifth rib on the right, below the angle of the left scapula, while in the right back the dulness extended one inch higher. Over these duli areas breathing, vocal and tactile fremitus were diminished. There were many fine, crackling râles at the left base, and a few coarse crackles after cough at the left top, behind. The systolic blood-pressure was I45.

The spleen was easily palpable. The abdomen and extremities otherwise negative.

On the night of the fourth of May the patient's respiration became rapid and difficult-respiration, 42, with pulse, I30; tracheal râles could be heard half-way across the ward. The first heart-sound was almost inaudible, the second loudly accentuated. The pulse was very weak. The outline of the heart was normal on percussion. The patient was livid, cyanotic, and covered with perspiration.

Discussion.-But for the persistently subnormal temperature coming on, as it has, with acute axillary pain and dyspnea, one might think of pneumonia in this case, although the duration is somewhat too great. The signs in the lungs point to fluid accumulation in both chests. Is this an exudate or a transudate, due to inflammation or to dropsy? Double pleural effusion is very rare. The absence of fever and of pain connected with respiration makes pleural effusion still more unlikely. Indeed, this possibility would scarcely have been considered but for the fact that there seems hardly enough in the condition of the heart or kidney adequately to account for so much effusion as a dropsy.

In the urine there is really no evidence of renal disease, the slight variations from normal being more characteristic of passive congestion. In the heart, accentuation of the pulmonic second sound is the chief abnormality, and this is indicative less of any cardiac lesion than of a blocked condition of the lungs, however produced. The displacement of the apex impulse is also to be regarded rather as the result of the pleural effusion than of any disease of the heart itself. On the whole, therefore, there is no direct evidence of heart disease obtainable by examination of the organ itself, and if we are to predicate any weakness of the heart's action, we must do so upon the evidence of passive congestion in the pulmonary circuit. This is not satisfactory, but it is a very familiar dilemma, and one in which experience has shown that it is usually safe to assume a myocardial lesion provided that there is no evidence of nephritis, goiter, or adherent pericardium. Such diagnoses as "myocarditis" used to be much more frequent than they are at the present day, since the habit of routine blood-pressure measurements has led us to recognize so many latent cases of chronic nephritis not evident by urinarv examination. In the present case it seems inevitable that we 
should blame the heart-wall for the circulatory disturbance, though it may be wiser to speak of "myocardial weakness" (adopting the vaguer functional term), rather than of "myocarditis."

The acute attack of May 4th tends to confirm our opinion that the heart is organically weak. This attack will be easily recognized as one of acute pulmonary edema-one of the most interesting and mysterious of clinical pictures. The vast majority of such attacks occur in persons whose cardiovascular system has shown a distinct but not extreme grade of degeneration and weakness. In many cases the kidney has also shown evidence of chronic disease, but this is about the sum of our knowledge on the subject. As to the nature and determining cause of the attacks, we know almost nothing, and in a few cases we are not even warned or guided by any definite evidence of cardiac or renal disease; the edema appears, as it were, out of a clear sky. It will be understood, of course, that the types of edema here briefly referred to are distinguished from the ordinary, long-standing, gradually increasing edema of uncompensated heart disease.

Outcome.-He was bled a pint from a vein of the left arm and given strychnin, $\frac{1}{30}$ grain, and digitalone, 20 minims, subcutaneously. Following this the pulse-rate fell at once to Ico, and the perspiration and dyspnea diminished. The left chest was then tapped, and three pints of fluid removed. After this the pulse feli to 9o. After $\frac{1}{6}$ grain morphin subcutaneously the patient went at once to sleep and slept five hours, waking vastly improved, with good color, strong and regular heart action.

The fluid removed from the chest had a gravity of IoI I, with 2.7 per cent. albumin. In the sediment lymphocytes made up 76 per cent., polynuclears, I4 per cent., endothelial cells, to per cent.

Two nights after this he again became uncomfortable; the other chest was aspirated and four pints of fluid withdrawn. 'The specific gravity was again IOII; the albumin only I.2 per cent.; lymphocytes, 77 per cent. The patient was then given magnesium sulphate $\frac{1}{2}$ ounce every morning, a dram of French Vermouth in a small amount of water just before dinner and supper, diuretin, I5 grains four times a day. Following the tapping of the chest the amount of urine increased markedly. On the sixth of July he left the hospital much relieved.

Diagnosis.-Weak heart; acute pulmonary edema.

\section{Case 153}

A single woman of twenty-five, a nurse, entered the ward on January 2, 1906. The night before she had had some pain in the left side. She 
was a wakened by it several times in the night. She finds it uncomfortable to lie on the right side or on the back, but pressure on the left side, or lying on that side, relieves the pain. She has an extremely tender spot under the right border of the ribs in front.

Examination showed spasm in the right hypochondrium, with tenderness. The pain, however, was consistently referred across the abdomen to a point in the left axilla on a level with the left nipple. Physical examination, including blood and urine, was otherwise negative. The temperature ranged between $99^{\circ}$ and $10 \mathrm{I}^{\circ} \mathrm{F}$. The pain did not

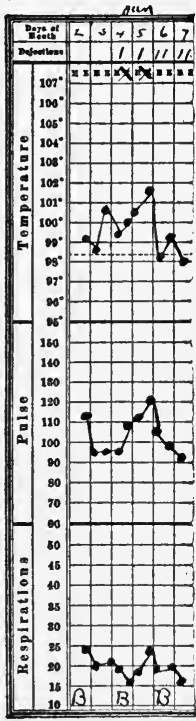

Fig. 52.-Chart of case 153 . seem to be affected by morphin, and came on two or three times a day in spasms lasting one-quarter to one-half hour. The tender point in the right upper quadrant grew steadily more rigid and more sensitive. Pressure there caused pain to shoot to the left axilla. She was seen daily by a surgical consultant, who did not advise operation. On the fourth day the white cells rose to $\mathrm{I} 4,000$.

Discussion.-This case is introduced to exemplify an unusual reference of pain to a point far removed from the lesion producing it. The tenderness and spasm turn out here, as in so many other cases, to guide us better than the pain, when the two diverge. A rhythmic or spasmodic character in any painful seizure usually turns out to mean frustrated peristalsis within a hollow muscular organ. But there is no such organ in the left axilla; the nearest hollow muscular organ is the heart, and there is nothing else in the clinical picture to connect the pain with that organ. The rising leukocyte count and the fever are data not ordinarily associated with angina of any type.

Outcome.-Three days later the abdomen was opened and a much distended and twisted gall-bladder found; the cystic duct was dilated, twisted, and occluded by a large stone. Three other stones were also found in the gall-bladder, which was acutely inflamed.

Diagnosis.-Gall-stones.

\section{Case 154}

A Swedish machinist aged twenty-five entered the ward February 8, 1907. Five weeks previously he had suffered from tonsillitis. In two weeks he was back at work, but began to have pains in his legs and feet; at one time both knees were red and swollen. Ten days ago he gave 
up work and went to bed, with fever, headache, loss of appetite, and weakness. His chief complaint for the past week has been pain in both chests, worse in the right front. His legs have shown only indefinite stiffness and soreness in the past few days.

Physical examination of the chest showed in the right axilla very slight dulness, with diminished breath-sounds and a suggestion of friction. The heart was negative. There was no redness or swelling of any joint, but some pain on motion of the right knee, and a slight rigidity of the neck. Belly negative. Temperature, $101.8^{\circ} \mathrm{F}$.; pulse, I20; respiration, 3०; hemoglobin, 70 per cent.; white cells, 24,000; urine normal.

On the twelfth of February there was still no evidence of any localization of the infection except that the signs in the lower right axilla had slightly increased. The patient looked decidedly sick, and the white count had risen to 25,400 . On the fourteenth, pain and edema of the whole right leg appeared without tenderness; the next day swelling appeared in the left foot and the veins below the left knee were distended; there were still no tenderness and no change in the signs in the right chest. By the sixteenth the swelling of the left leg had considerably increased, and there was tenderness over the red, cord-like veins of the left calf. The white count remained the same, 88 per cent. of the cells being polynuclear.

The patient remained in the hospital until August I2th-six months. There was some sloughing of the superficial tissues of the right foot. A well-marked nephritis appeared on the twenty-fourth of February, and lasted until July, but finally disappeared altogether. Pleurisy appeared in the left side on the tenth of May, but disappeared in the course of a week. Thrombosis appeared in both arms in the early part of March, and in the middle of the month there was bloody expectoration for a couple of days, without any special pulmonary signs to account for it. By April ist the arms were normal and the left leg nearly so.

A marked anemia gradually developed, so that on the thirteenth of April the red cells were $2,725,000$, with 65 per cent. of hemoglobin. Late in June there were purpuric spots on the dorsum of the left foot, but they disappeared within a few days.

Discussion.-As the history of this case opens with a tonsillitis, it may be well to consider some of the lesions which the clinical experience of the last fifteen years tends to associate with tonsillar inflammation. Although the majority of cases of tonsillitis progress beyond their origin no further than the lymphatic glands at the angle of the jaw, the very striking prostration which accompanies and follows the acute infection probably indicates that the disease rarely remains local. It seems to be 
shown beyond reasonable doubt that in many cases an infection first demonstrable in the tonsil appears soon after in one or another synovial membrane or joint surface, in the endocardium, in the kidney, or on some serous surfaces. This may be taken to indicate that bacteria are circulating in the blood-stream in a considerable proportion of cases, though they have not often been isolated by blood culture.

The case above described is remarkable chiefly because it narrates the fortunes of a patient who suffered, one after another, most of the common complications of tonsillitis above referred to. Beginning with multiple arthritis and right-sided pleurisy, he next suffered a series of infections of the peripheral veins, leading to multiple thrombi. Then came the nephritis, which I have often seen occurring in tonsillitis as the only manifestation of the body's effort to expel invaders. The pulmonary bleeding is probably to be explained as analogous to the purpuric spots which appeared for a few days in the latter part of his illness. Only histologic examination could decide whether these pulmonary and cutaneous hemorrhages were due to embolism or to some other cause. The development of a marked anemia in a six months' illness of this severity is not to be wondered at, since chronic sepsis always tends to produce anemia. But it is quite remarkable that the heart escaped, apparently without injury. Possibly the transient rigidity of the neck might be interpreted as a larval infection of the meninges ("meningismus"), since we know that all the serous membranes-pleura, pericardium, peritoneum, meninges---may be attacked in cases of generalized sepsis.

Another very remarkable feature about this case was that the patient's final recovery was complete. The treatment consisted essentially of good nursing.

Diagnosis.-Sepsis with thrombi.

\section{Case 155}

An Italian laborer of twenty-nine entered the hospital on March I8, 1908. The family history was negative; his past history likewise so; his habits good.

Four weeks ago he began to have pain in his left chest, chiefly low down in the axilla, accompanied and aggravated by cough or deep breathing. 'There was slight dyspnea on exertion. For three days he has felt chilly and feverish, especially at night. He has noticed nothing remarkable about his urine, and no pain except as above described.

On physical examination the heart showed nothing abnormal. The right side of the chest moved better than the left, and there was slight dulness at the right pulmonary apex as low as the second rib, with broncho- 
vesicular respiration and increased voice. At the base of the left axilla the percussion-note was flat below the sixth rib. Tactile fremitus was absent, voice- and breath-sounds diminished. Over the area of flatness were scattered a few fine râles, and some were audible as high as the second rib. In the back the area of flatness reached up to the lower angle of the scapula. Tactile fremitus was diminished over the whole left back, and absent, together with voice- and breath-sounds, below the angle of the scapula. A paravertebral triangle was percussed out on the opposite side. The abdomen showed considerable general rigidity and in the extreme flanks some dulness, which did not, however, exhibit any change with change of position. During the next ten days his temperature ranged between $99^{\circ}$ and ror ${ }^{\circ} \mathrm{F}$., his pulse between 70 and 80 , his respiration between 20 and 25 , his urine between 40 and $5^{\circ}$ ounces in twenty-four hours, with a slight trace of albumin, a moderate amount of pus, many hyaline and granular casts, some of which have blood or fat adherent. The leukocytes were 6700; hemoglobin, 75 per cent.

By March 22d the dulness in the chest had somewhat diminished, and the paravertebral triangle was not evident. The dulness on the left side seemed to rise higher in the axilla than near the spinal column. A large mass was now felt in the left flank, but could not be definitely outlined on account of the rigidity of the whole abdomen, which did not relax even in a warm bath.

On the twenty-fourth of March the $x$-ray showed apparently a large stone in the left kidney.

On March $27^{\text {th }}$ the area of dulness in the left chest had not changed. but there were coarse, moist râles in the right lower back and axilla. The urine still showed a slight amount of pus. Cystoscopy showed this pus to issue from the left ureter, while normal urine came from the right.

Discussion.-The signs at the base of the left axilla and in the back seem to indicate a localized pleurisy, with or without a small effusion. In view of the later developments of the case, however, I believe that the paravertebral triangle was percussed out largely as the result of "expectant attention"-i.e., of the interne's conscientious determination to find it. Even at the beginning of the case every one who saw the patient felt that the pleural effusion was not sufficient to account for the marked fever and constitutional symptoms. We all thought there must be "something back of it."

Our first clue to that "something" in the background was the finding of pus in the urine. This led us to search more carefully the region of the kidneys, whence the mass in the left flank came to light. As I read the record now it is amusing to note how promptly the chest signs 
retire into the background of the clinical picture as the kidney begins to loom up in the foreground. How far this represents the actual course of events in the patient and how far it is a matter of the historian's psychology it is now difficult to say.

Outcome.-On March 28th operation showed a large kidney filled with thick pus and adherent to the diaphragm and other structures. No stone was found, but there was a calcareous plate near the surface of the kidney; no histologic report is preserved.

The patient made a good recovery.

Diagnosis.-Pus kidney (tuberculous ?).

\section{Case 156}

A widow of forty entered the hospital February I3, I908. She had typhoid fever three years before, and was operated on for extra-uterine pregnancy seven years before. Otherwise she has never been sick, but has had many colds this winter. Six weeks ago she began to suffer from pain in the left side of the chest. Four weeks ago she had to give up her work on account of vomiting immediately after eating. The vomitus rarely contained food. It usually was greenish. There was constant soreness in the epigastrium, and a good deal of pain in the left arm and left side of the chest. She has taken almost no solid food for several weeks. She has considerable dyspnea and palpitation, and has lost twenty-six pounds. Twice she has had shivering spells lasting several hours at night. She admitted the occasional use of alcohol, and it was apparent on her breath at the time of entrance. Some nights she passes urine at frequent intervals.

Physical examination was negative except for considerable tenderness in the epigastrium and moderate enlargement of the axillary glands on both sides. Blood-pressure, I35.

Discussion.-On p. $75^{2}$ of this book I have referred to a case diagnosed and treated as neurasthenia, but dying shortly afterward of cancer of the pleura. The symptoms in that case were not unlike those described above, and my remembrance of the former mistake leads me to be especially cautious in the diagnosis of supposedly neurasthenic pains in the side of the chest. The presence of enlarged glands would be quite consistent with malignant disease of the chest, and is often one of the most important clues to the discovery of that trouble. The import. ance of this enlargement, however, is weakened by the fact that it is bilateral. Adenitis secondary to malignant disease is usually, unilateral. In the present case I did my best to find signs of malignant disease by physical examination of the chest, but could find nothing. 
By the negative results of physical examination, which included a temperature record, we were able also to exclude pleurisy.

The extension of the pain to the left arm, the presence of dyspnea and palpitation, and the age of the patient are data quite consistent with the diagnosis of angina pectoris. Against this, however, is the absence of any relation to exertion -as a cause of pain, the long-standing and moderate character of the suffering, and the low blood-pressure.

After the exclusion of these and all the other possibilities which we could call to mind, it seemed best to make a diagnosis of neurosis and use that as a working basis for a therapeutic test.

I interpret the left axillary pain as due to that commonest of all causes for such a complaint, viz., fatulence. When the stomach is overdistended, whether by atmospheric air which has been swallowed and "cribbed" or by the products of gastric fermentation, the "Magenblase," or bubble, which is usually to be seen near the cardia by fluoroscopy, swells to huge dimensions, invades the axillary region, and often causes much discomfort. The patient usually thinks she has heart disease; the thought increases her nervousness and thus her flatulence. The vicious circle is then in complete working order.

Outcome.-The patient was put to bed and given a diet of liquids and soft solids, with paraldehyd, half to one teaspoonful, on two successive nights. Within two days the vomiting had ceased and she felt much better. She had apparently been working hard, and was of a neurotic type, easily frightened by the slightest unexpected noise or occurrence. She was well enough to go to work again on the twentieth of February.

Diagnosis.-Neurosis.

\section{Case 157}

A Greek waiter of twenty-one, with a negative family history, entered the hospital November I3, I907, with the statement that he has been feeling rather poorly for the past four months, but had no definite symptoms until three weeks ago, when he began to have frequent severe pains in the front and left side of his chest and a distressing cough without expectoration. The pain and cough were both worse at night, but he has been very comfortable lying flat, and has complained of no shortness of breath. He has been chilly and feverish and had much vertigo and frontal headache. During the past three weeks he has lost 20 pounds.

At entrance the patient was unable to lie down on account of dyspnea. The cardiac apex was neither visible nor palpable. The area of cardiac dulness, as shown in the diagram, had a total width of $\mathrm{II}_{2} \frac{1}{2}$ inches, the left border of dulness being $7 \frac{3}{4}$ inches to the left of the midsternum in the 
fifth interspace. In the upper front of the right chest there was tubular breathing over the dull area, and in the left back, near the angle of the scapula, all the signs of solidification were present. The abdomen was everywhere dull except in the umbilical region and in the left flank. The patient's temperature was irregularly elevated for the whole of his three months' stay in the hospital. (See Fig. 53.)

The blood showed 9900 white cells and 90 per cent. hemoglobin. The urine was not remarkable. A paradoxic pulse was demonstrated on the fifteenth.

Discussion.- - If we accept as accurate the record of a to-and-fro friction-sound at the point shown in the diagram, there seems no reason for doubt that pericarditis is present. It remains to discover, if we can, whether a pericardial effusion is present and by what other lesions the pericarditis is complicated.

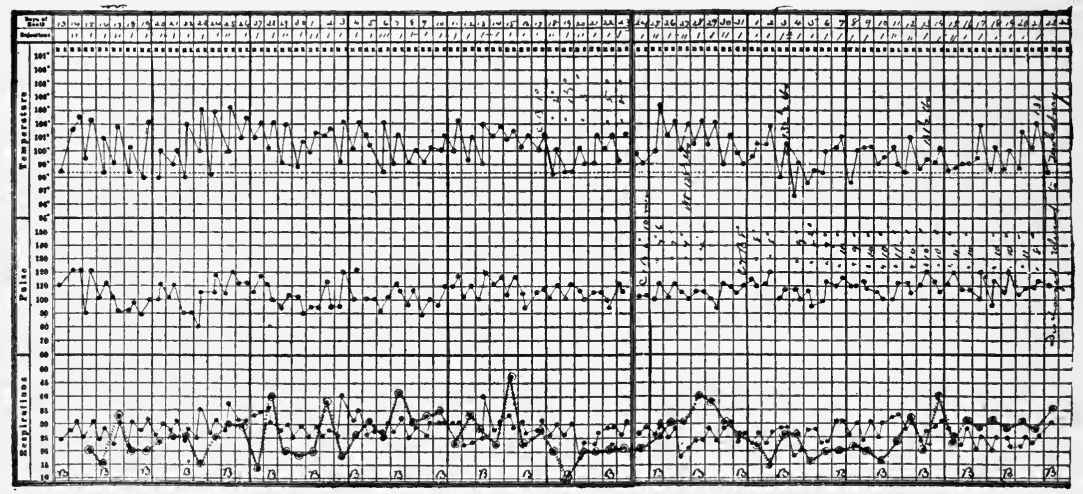

Fig. 53.-Chart of case I57.

The diagnosis between a pericardial effusion and a dilated heart is notoriously difficult, often impossible. In the present case we have no good cause for such a dilatation of the heart-no valvular or arterial lesion, no goiter or chronic nephritis, no history of beer-drinking. The area of solidification in the left back is, in all probability, due to pressure exerted upon the lung either by a pericardial effusion, a pleural dropsy, or a greatly dilated heart. The latter possibility is very rarely mentioned in text-books, but I have been convinced by postmortem evidence that a heavy, distended heart in a patient who lies persistently on the back may compress the left lung so as to produce an atelectasis or pseudopneumonic condensation of the lung similar to that often caused by pericardial effusion. A common mistake in these cases is to suppose that a lobar pneumonia is present. Experience has shown that when we hear the signs 


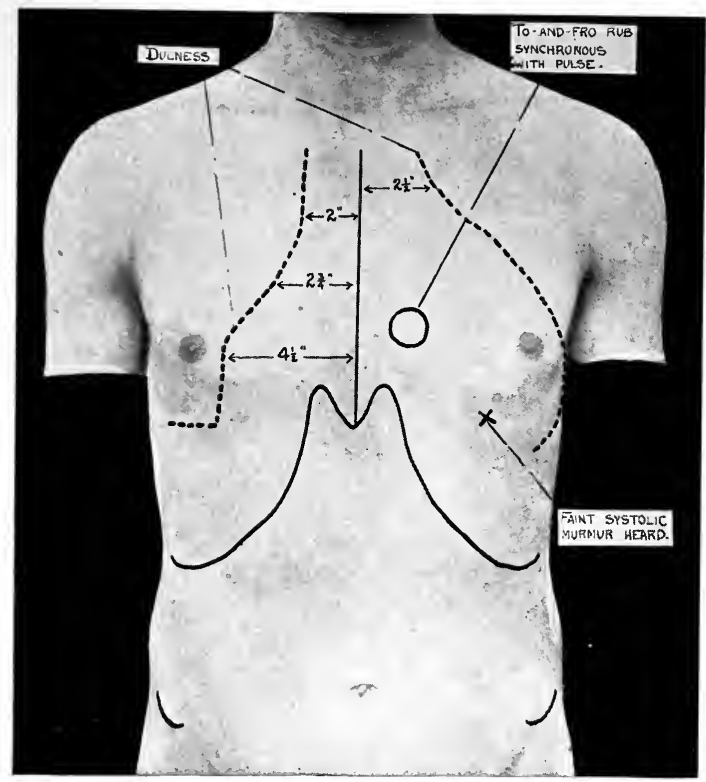

Fig. 54.-Lateral limits of an area of percussion dulness found in Case $\mathbf{1 5 7}$ 

of solidification near the angle of the left scapula in the course of a case showing a greatly dilated heart, with or without pericardial effusion, these signs turn out in the great majority of cases to be due to pressure exerted on the lung by the heart, or by a pericardial or pleural effusion, and not to an exudate in the lung.

The presence of a high continued fever and the absence of any cause for cardiac dilatation in the present case incline me to believe that there is a pericardial effusion. The long duration of the case without any notable improvement suggests that the pericarditis may be tuberculous.

The extensive dulness in the abdomen is in all probability due to fluid which may be the result either of tuberculous peritonitis or of stasis. Ascites is especially apt to accumulate as the result of a chronic pericarditis which has gone on to complete obliteration of the pericardial sac, but it does not seem probable that the inflammation has lasted long enough in this case to bring about that result. Further evidence as to the nature of the fluid in the peritoneum might be obtained by tapping, for a dropsical fluid would probably be of lower gravity than one due to tuberculous peritonitis.

Outcome.-He was admitted to Tewksbury Almshouse January 22, I908.

The left lung continued to show the flatness and loss of voice-sounds below the fourth rib in the axillary line. Sputum was examined twelve times and found negative for the tubercle bacillus. Slight dulness and bronchial breathing spread to both lungs.

In February his temperature rose daily, going as high as $103^{\circ}$ and IO4 ${ }^{\circ} \mathrm{F}$., but usually reaching IO $^{\circ} \mathrm{F}$.

He died June 4, I9n8. No autopsy.

Diagnosis.-Pericarditis.

\section{Case 158}

A night watchman of forty-six entered the hospital August 24, I906. $\mathrm{He}$ has been a hard drinker up to eleven months ago. He had syphilis twenty years ago. For over two years he has been troubled by a hacking cough without sputa, accompanied by night-sweats and a slight pain in the left side of the chest. He has gradually increasing dyspnea on exertion, but can still lie flat and with the greatest comfort on the right side. For the past ten months he has been having pain in the left upper chest and paroxysms of distressing cough. At times he loses his voice for a few hours, but is never constantly hoarse. Nitroglycerin has given him considerable relief, but he has rattled and wheezed all summer, 
especially during the last four days. 'He sleeps poorly, has lost much weight, and has no appetite.

The heart's apex is in the fifth space, one-half inch outside the nipple, the right border of dulness two inches to the right sternal margin in the fourth space. There is marked bulging of the left chest over the area shown in the accompanying diagram, and considerable pulsation in the third and fourth left spaces. The veins of the neck and arms are distended. Loud groaning, whistling sounds are audible throughout both lungs. Physical examination of the heart, blood, and urine is otherwise negative (Fig. 55).

Discussion.-If a careful physical examination were made and duly meditated on in this case, the only hesitation in diagnosis would be on the question whether aneurysm or malignant disease of the chest is the cause of the patient's sufferings. In the absence of such an examination, however, I have known a case very similar to this to be treated as consumption for a number of months, the cough, night-sweats, emaciation, and pain in the chest being accepted as sufficient evidence of phthisis.

In another case the wheezing and rattling led straight to a diagnosis of bronchial asthma and to all sorts of therapeutic attempts based on that diagnosis.

Returning to the only diagnostic problem which ought to exist in this case (aneurysm or malignant disease of the chest), I may say in the first place that in a considerable number of cases in which $I$ have known this discussion to arise, the outcome has always shown aneurysm. In this man the history of syphilis, the absence of any glandular enlargement, and the slow march of the symptoms, which apparently have lasted two years, all favor aneurysm. The loss of weight is perfectly characteristic of aneurysm, and occurs, as I have previously shown, ${ }^{1}$ in the vast majority of all cases. I emphasize this point because in the discussion of this differential diagnosis I have several times heard emaciation adduced as evidence against aneurysm and in favor of malignant disease.

Outcome. $-X$-ray showed a large shadow corresponding to the area of dulness. The mass seemed to grow and then to decrease in size in the next few days, the pulsation varying much from time to time in amount and in extent. There were two main projecting points-one over the precordia, and one above it, under the clavicle. Sarcoma of the chest-wall was considered seriously. The patient died on the sixteenth. Autopsy showed aneurysm of the first portion of the aorta; rupture into the

${ }^{1}$ Two Possible Causes of Emaciation Not Generally Recognized, R. C. Cabot. M. D., Jour. Amer. Med. Assoc., March I7, I906. 


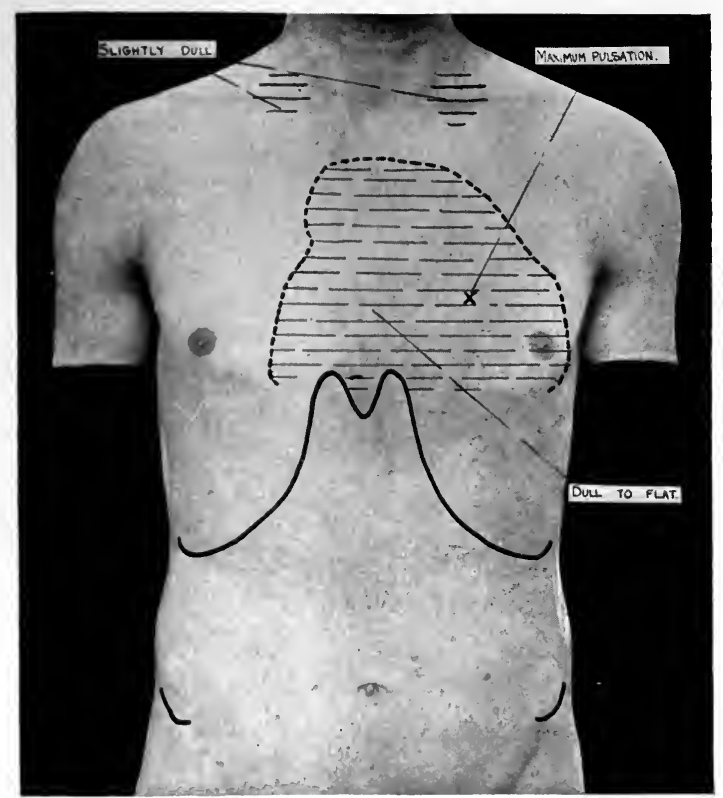

Fig. 55.-Physical signs found in a patient who complained of dyspnea, cough, emacia tion, night-sweats, and pain in the chest. 

pericardium; compression atrophy and bronchopneumonia of the left lung. The aneurysm was filled by a very thick clot lying in front of and above the heart.

Diagnosis.-Thoracic aneurysm.

\section{Case 159}

In March, r898, a housewife of thirty-three came to the hospital for hemoptysis, supposedly due to phthisis. No sign was found in the lungs. In October, 1898 , she was again treated for pleurisy with effusion and fistula in ano. She had had a nervous breakdown in 1896 , and had been very irritable and self-centered since that time.

In April, r899, she began to suffer from pain in the right side of the chest, much aggravated by coughing and laughing.

The urine contained a trace of bile and a good many leukocytes; otherwise it was negative, as were the blood, temperature, pulse, and respiration.

Physical examination, April 23d, was negative save for a patch at the right base near the scapular angle, where there were slight dulness, diminished voice, respiration, and fremitus.

Discussion.-Pulmonary hemorrhage of any amount-an ounce or more-means pulmonary tuberculosis in 999 cases out of Icco, if disease of the heart and aorta be excluded, as they easily can be in most cases. The other traditional causes of hemoptysis-disease of the throat, vicarious menstruation, hemorrhagic conditions-amount practically to nothing; that is, they are usually quite obvious, like purpura hæmorrhagica, or quite mythical, like vicarious menstruation. Pulmonary hemorrhage due to distomiasis never occurs in North America except among Japanese immigrants.

The fact that signs are absent on examination of the lungs after a hemoptysis due to tuberculosis is entirely according to rule when hemoptysis is the first evidence of disease. We almost never find any signs of disease until some months later; in many cases we never find them at all, and only the postmortem examination proves tuberculosis.

All this, however, refers to an event over a year old. Is it not possible that her suffering, at the present time, is connected with her nervous condition and due to habit pain? Against this hypothesis we have the fact that she has previously had pleurisy with effusion and fistula in ano, both of them tuberculous affections in practically every case. Bearing these troubles in mind, we naturally assume that her present pain is in some way produced by her old pleurisy, of which there seems to be still some evidence at the right base. There are, however, two other possibilities which must first be considered briefly: 
The urine contains bile. This directs our attention to the liver; but enlargement of the liver upward may produce in the right back all the signs here described-signs which, if interpreted as pleurisy, might be due either to a small effusion or to marked pleural thickening. I have known abscess of the liver to produce exactly these signs, so that it was mistaken for empyema. Against the possibility of liver disease there is not a great deal to be said, as our methods for detecting liver disease are so few and unsatisfactory. We may note, however, that there seems to be no enlargement of the liver downward, no bile staining of the skin or conjunctiva, none of the ordinary causes for cirrhosis, hepatic abscess, passive congestion, amyloid or fatty metamorphosis, no change in the spleen, glands, or blood to suggest leukemia or Hodgkin's disease. This is the best that we can do to exclude liver disease. Had these same signs appeared in the back following an appendicitis, amebic dysentery, or cholelithiasis, the situation would suggest hepatic abscess.

I once made a diagnosis of purulent pleural effusion in a case bearing a good deal of resemblance to this one. I put in a needle an inch and a half below the angle of the scapula, drew pus, and promptly handed over the case to a surgeon for drainage. He opened the pleura, found it smooth and clean, and indulged in disparaging remarks on medical diagnosis. Further exploration, however, showed that the diaphragm was pushed up nearly to the angle of the scapula, and that through its domed surface fluctuation could be detected. A second puncture, ten days later, after the pleura had healed without infection, liberated a quart of pus from the region of the kidney. Since that time I have always remembered the possibility of perinephritic or subdiaphragmatic abscess when dealing with what appears at first sight to be an effusion (serous or purulent) at the right base. The presence of leukocytes in the urine makes it all the more necessary to consider the kidney in this case, but we must first make sure that those leukocytes come from the urinary tract by obtaining a catheter specimen of urine. When this was done, the urinary sediment no longer showed leukocytes, and as there were no other facts pointing distinctly to the kidney, I returned to my original ideapleurisy at the right base.

This case is one of many which exemplify the long duration of pain and of physical signs after the healing of a pleural effusion. Perhaps in the majority of cases there is more or less suffering for a year.

Outcome.-The pain remained mostly in the back, and not in the side, during the five days of her stay in the hospital, but soon disappeared with rest, full diet, and counterirritation.

Diagnosis.-Old pleurisy. 


\section{Case 160}

A typewriter of twenty-three lost her mother and one brother of phthisis. Two and a half years ago she was in bed several weeks on account of pain in the left axilla. The whole attack lasted three months.

Lately she has noticed pain in the left side when she is nervoussharp for a few minutes, and leaving an ache for two or three days afterward. Sometimes exertion relieves it. Coughing or sneezing does not increase it.

Five months ago the pain increased. For six weeks she slept almost none and walked the floor much. Her weight fell from $I_{32}$ to Io8. The pain is chiefly in the left side, but there is also a constant sense of pressure in the right breast and back, with occasional sharp pains. Suffering is worse at night. She has a good deal of indigestion and constipation.

Examination.-Cardiac apex in fifth space, nipple-line. Accompanying the first sound is a systolic murmur, loudest at the apex, but audible also over the whole precordia and in the left axilla. The pulmonic second sound is slightly louder than the aortic.

Tender spots near the left lower scapular edge, in post-axillary line, in the axillary line in fifth, sixth, seventh spaces, and along sternal border [fourth to eighth ribs] were found. Sensation normal.

Discussion. - In view of the site of the pain and the family history of tuberculosis, it would be wrong not to consider pleurisy in this case; but there was no definite evidence of it on physical examination, and without such evidence the diagnosis can never be made.

Pain due to dyspepsia and flatulence would hardly be so constant, and this same characteristic excludes both types of angina pectoris. Muscular pains (pleurodynia) would show exacerbation rather than relief by exertion. Of local diseases of the chest-wall we have also no evidence.

Intercostal neuralgia is characterized by pain like that here described, and especially by tender points corresponding approximately with those which physical examination has revealed. I believe intercostal neuralgia to be a rare disease, although the diagnosis of it is so common. By intercostal neuralgia one means ordinarily the so-called "primary" type, unrelated to any cause of pressure, such as aneurysm or spondylitis. Pressure pains of this type are, of course, by no means uncommon, but primary intercostal neuralgia unaccompanied by herpes and without any known cause is, I believe, distinctly rare. The diagnosis, like all diagnoses of a "primary" or obscure lesion, is one with which we are 
never quite content, and which we can tolerate only when we have done our best, by rigid scrutiny and thorough sifting of all other recognized possibilities, to find a cause. In the present case, for example, I should not be satisfied unless disease of the spinal column had been, so far as possible, excluded.

Outcome.-The pain gradually disappeared in six weeks. Many forms of treatment were tried, but none of them had any effect that I could discover.

Diagnosis.-Intercostál neuralgia.

\section{Case 161}

An alcoholic Irish teamster of twenty-eight has noticed for a week a pain in his right lower axilla. The pain has several times been associated with vomiting and a slight cough. No injury is remembered.

Family and past history good.

Physical examination is negative, save for a rounded swelling about I $5 \mathrm{~cm}$. in diameter near the right costal margin in the axilla. The swelling is brawny, with a slightly fluctuant crater in the center.

Discussion.-There seems every reason to believe that the pain and the tumor are connected in this case. It remains to ask, What is the nature of the tumor?

The commonest causes are: septic osteomyelitis or tuberculous osteomyelitis of a rib. The patient might have broken one or more ribs without knowing it during one of his drinking bouts, but the resulting calluses would not produce a mass like that here described.

A fatty tumor or an empyema necessitatis would not have a brawny surface. Either of these lesions, if fluctuant, would be fluctuant throughout. Malignant disease of the chest-wall does not often show itself at this point: Actinomycosis cannot be excluded; it is, however, a rare lesion, and the commoner causes of a swelling at this point should be considered first.

Further diagnosis is impossible without incision.

Outcome.-Two ounces of pus were removed by incision and a sinus found leading to a rib. Rough bare bone was found at the bottom of the sinus. There was no evidence of actinomyces. The patient seemed greatly debilitated.

Diagnosis.-Costal tuberculosis.

\section{Case 162}

An unoccupied girl of twenty-eight entered the hospital January I5, I908. Nine months before she caught cold at a dance, and a week after 
began to have pain in the left chest. Ever since it has been a constant ache, at times becoming severe, worse after eating; it is not affected by respiration or motion. Her appetite is good, but she has complained of a great deal of "gas upon her stomach," and for some months has lived upon a diet excluding meat and eggs, sweets, salt, and fried stuff. She never vomits. Her bowels are constipated, and she has considerable dyspnea on exertion. One year ago she weighed I 50 pounds; she thinks she has lost weight since.

Physical examination shows a rather obese girl weighing $149 \frac{1}{2}$ pounds. There is a harsh systolic murmur audible all over the precordia, but not transmitted elsewhere. The apex is neither visible nor palpable. The left border of dulness is in the nipple-line and fifth space. The aortic second sound is louder than the pulmonic second sound. . Physical examination, including blood and urine, is otherwise normal.

Discussion.- The only objective abnormality in the physical examination is the cardiac murmur and the accentuation of the aortic second sound. These items are not sufficient, separately or in combination, to warrant any inference of disease. The history shows that she has been starving herself, yet on physical examination she is obese. Possibly she is trying to reduce her weight, which may have been greater six months ago.

In the absence of any local cause for the pain one naturally thinks of neuralgia, especially since the diet is so insufficient. But there are no tender points corresponding to the nerve exits, while the fact that pain is worse after eating is very uncharacteristic of neuralgia. While this diagnosis cannot be positively excluded, it seems rather unlikely.

Muscular pain (pleurodynia) should be more distinctly related to exertion and less to food.

In view of these facts and of the absence of any apparent connection between the cardiac murmur and the pain, it seems reasonable to believe that it is due to a digestive disturbance favored by insufficient food and associated with gaseous distention. On p. 292 I have already mentioned the great frequency of axillary pain due to this cause. Such pain is very common as an element in the clinical picture of the gastric neuroses, with or without starvation.

No cause of stomach trouble in women is commoner than starvation. The vicious circle is established in the following manner: Some temporary fatigue or depression of vitality results in digestive disturbance. The food eaten last or most abundantly is blamed by the patient and excluded from the subsequent meals. Digestive disturbance continues. Other foods are excluded. The nutrition of the whole body, including 
that of the stomach itself, begins to suffer, and digestion is still further delayed by gastric stasis or insufficient secretion. The suffering thus produced makes the patient aspirate air into the stomach ["cribbing"], which in time increases the discomfort and renders her still more timid about eating. The circle is then complete. To break it one must force the patient to eat, despite considerable pain, until some gain can be made in the general and so in the local nutrition. A weakened stomach, like a weakened muscle, cannot be strengthened without exercise; and this entails, for a time, increase of suffering.

Outcome.-The patient was given a full diet, a tight swathe, $\frac{1}{2}$ dram bicarbonate of soda after meals, and half a dram of the elixir of the valerianate of ammonia before meals.

By January 2ist she seemed perfectly well and able to go home. Diagnosis.-Starvation. 


\begin{tabular}{|c|c|c|c|c|c|c|c|}
\hline 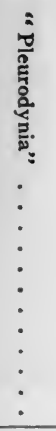 & 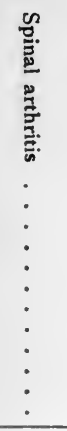 & 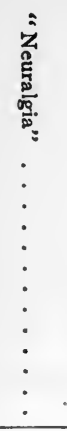 & 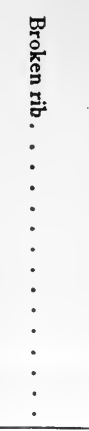 & 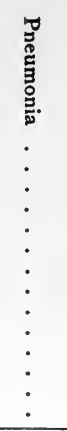 & 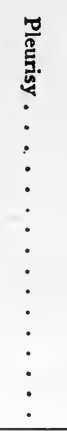 & 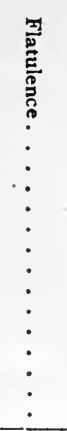 & 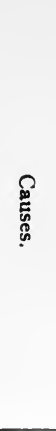 \\
\hline 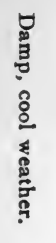 & 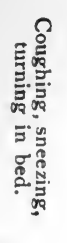 & $\vdots$ & 불 & 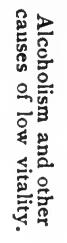 & 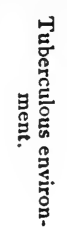 & 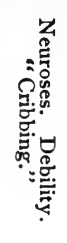 & 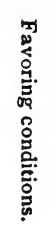 \\
\hline 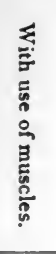 & 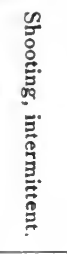 & 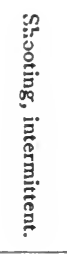 & 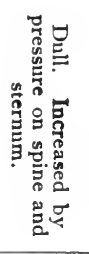 & 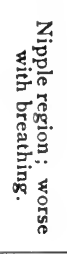 & 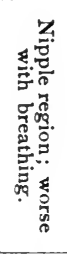 & 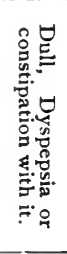 & 苛. \\
\hline ० & ○ & ○ & $\circ$ & + & 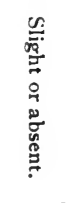 & 0 & 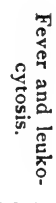 \\
\hline 。 & 0 & ० & 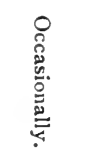 & + & + & ० & 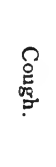 \\
\hline ० & 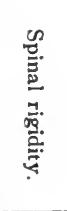 & 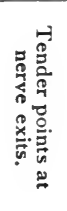 & 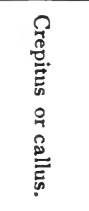 & 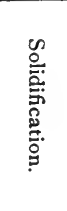 & 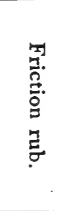 & 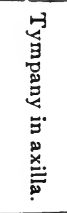 & 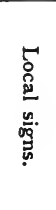 \\
\hline 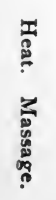 & 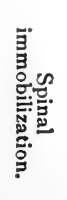 & $\bullet$ & 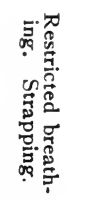 & 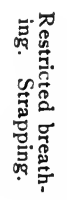 & 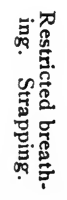 & 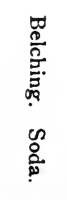 & $\begin{array}{l}3 \\
0 \\
0 \\
0 \\
0 \\
0 \\
0 \\
0 \\
0 \\
0 \\
0\end{array}$ \\
\hline
\end{tabular}




\section{CHAPTER XI}

\section{PAIN IN THE ARMS}

ASIDE from neurotic cases and angina pectoris, pain in the arms is not a common symptom. The majority of cases, as one meets them in practice, are due to $(a)$ occupational or other neuroses. They come in people who are tired and who have experienced some sort of "psychic trauma," such as the expectation of money compensation for supposed occupational disease and the emotional strains connected with love affairs. In such cases physical examination is, of course, wholly negative. The pain is rarely confined to the arms. Headache and backache are usually more or vless troublesome as well.

I think it is rarely the case that the occupation itself or the degree of labor put upon the arm muscles is the chief cause of the trouble. The vast majority of persons who do exactly the same labor, for example, cigar making or typewriting, do not suffer from such pains. Some other etiologic factor must co-operate in order to produce the trouble.

(b) Elderly people with arteriosclerosis often suffer prolonged severe pain about one or both shoulders or in the upper arms. The cause of such pain is not clear. It is often attributed to arteriosclerosis, but without any first rate reason. The mere presence of arteriosclerosis in such a patient is by no means proof that the armpain results from it. Another explanation which is now beginning to be popular is hypertrophic arthritis of the upper spinal joints. Occasionally such bony changes can be demonstrated by $x$-ray; but it is undeniable that they are also demonstrable in people who suffer no such pain. Moreover, they are not demonstrable in all the cases in which precisely similar arm-pains occur in elderly people.

(c) Subacromial bursitis is often adduced as a cause for these pains, and occasionally this is a correct explanation. But in most cases such a bursitis, if it occurs, can be shown to have developed subsequently to the beginning of the arm-pains and is probably the 


\section{Causes of Brachial Pain}

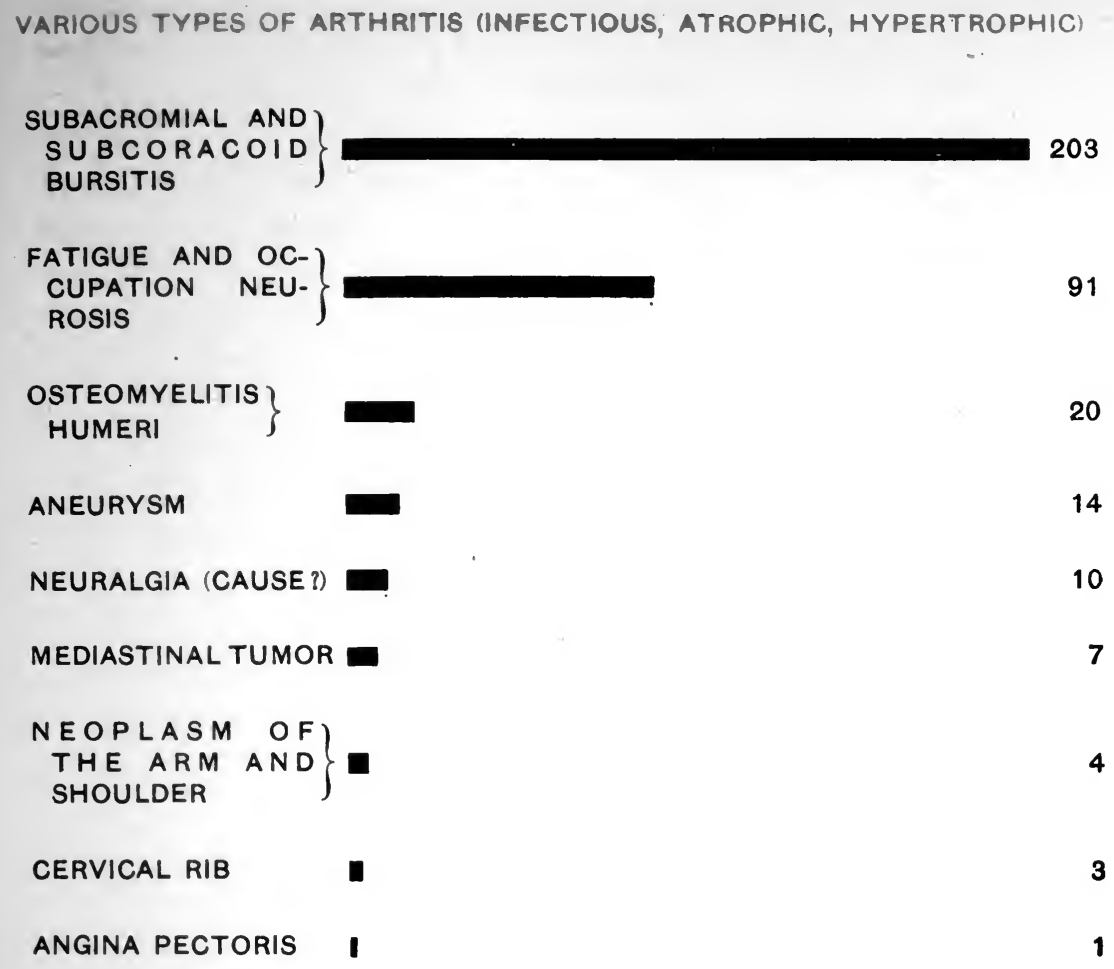

Among the other causes not here represented are:

(a) Wounds, with or without lymphangitis or thrombosis :

(b) Bruises, fractures, sprains, and strains;

(c) Poliomyelitis and cortical irritation (tumor, gumma). 

result of the unnatural immobility of the arm and shoulder which the pains bring about. Bursitis, then, is result, not cause. It is also to be remembered that in the great majority of all cases of demonstrable subacromial bursitis there is little or no pain in the arms. Stiffness, not pain, is the characteristic complaint.

(d) Angina pectoris must be remembered as a possible cause of pain beginning not in the chest, but in the left wrist or near the elbowjoint. Occasionally such a pain is felt in both arms and it may be confined to the arms, not appearing in the chest at all. In some cases, however, a breast pang is associated with the arm-pain.

The cause of these pains should be easily recognized if one traces out the dependence of the pain upon exertion or emotion and its relief by rest and peace. Probably of this type was the pain which I vividly remember witnessing in a patient with pernicious anemia who as soon as he rose from his chair and began to walk would experience excruciating pain in both forearms. Rest gave prompt relief. This patient had no evidence of arteriosclerosis, but probably experienced a pain due to a blood-supply insufficient for his needs during exertion, and therefore similar to the pain occasioned by narrowed coronary arteries. The impoverished blood of pernicious anemia was insufficient for the needs of the body during exertion, even though the arteries were not narrowed or stiffened.

(e) Cervical rib is, I believe, a decidedly rare cause of pain or other abnormality in the arms, but it deserves mention because it is curable by operation.

\section{Case 163}

A theatrical advance agent of thirty-five entered the hospital Jan. 10, 1907. Ten days ago he strained his arm while swinging on a trapeze. A week ago he was suddenly taken with aching and soreness in the muscles of the right arm, with a slighter amount of pain in the other arn and in the legs. The joints were not affected, and there was no fever or chill, but the right arm was somewhat swollen above the elbow, where it was more tender than in any other part. He had severe, constant, frontal headache and a harassing, dry cough. He stayed in bed for the first day, but, feeling no better, got up again and has been up most of the time ever since. Four days ago he began to be short of breath, especially on exertion, and for three days he has had chilly sensations. To-day he complains chiefly of dyspnea, cough, soreness all over his body, head- 
ache, weakness, and a sharp pain in his right wrist on motion. He gets up three or four times at night to pass his water.

On physical examination, temperature, pulse, respiration, blood, and urine were found to be normal. The patient looked sick and breathed with some difficulty. The lungs were slightly dull in both backs, and showed many fine and coarse râles with a few squeaks on both sides. The heart was negative, likewise the abdomen. The râles in the chest disappeared the next day. The principal complaint thereafter was of pain in the whole right arm, and in it there were slight general swelling and apparently great tenderness. The arm was held rigid most of the time. Dr. Goldthwait found nothing abnormal about the bursæ or joints.

The pain did not prevent sleep at all, and the temperature remained normal.

On the eighteenth of January the patient dropped and broke a cup. Immediately after this he had a convulsion, in which his body became rigid and his eyes rolled up, while the lids flickered.

Discussion.-All that physical examination reveals in this case is the evidence of a slight bronchitis and a tender arm, very possibly due to a strain. There is no evidence of inflammation or of any lesion of bone or joint. The pain does not follow the course of any nerve, is independent of exertion, and associated with no evidence of cardiac or vascular disease. Cervical rib, aneurysm, and tumor were excluded by careful examination.

In view of all this negative evidence, and in consideration of certain neurotic mannerisms which were obvious, but not easily described, we were strongly inclined from the outset of the case toward the diagnosis of traumatic neurosis. After the fit, which was clearly hysteric in nature, we felt much surer of our previous diagnosis, and instituted treatment based upon it.

Outcome.-From the fit above described he could not at once be aroused. He was, therefore, ignored, and after about twenty minutes he sat up and acted as if nothing had happened. Up to this time the arm had been held rigidly, and all attempts to move it had been resistedas he said, because of severe pain. After the convulsion he was given a severe scolding, and the arm was raised and the fingers were bent and straightened again by force for about five minutes, in spite of his shrieks and protestations. Motions not anticipated by the patient were found to be free. The next day the patient was up and about the ward, the use of his arm as good as ever, and there was no sign of his previous incapacity. He is now anxious to go out and get to work. The chest is clear, and he was discharged well. 


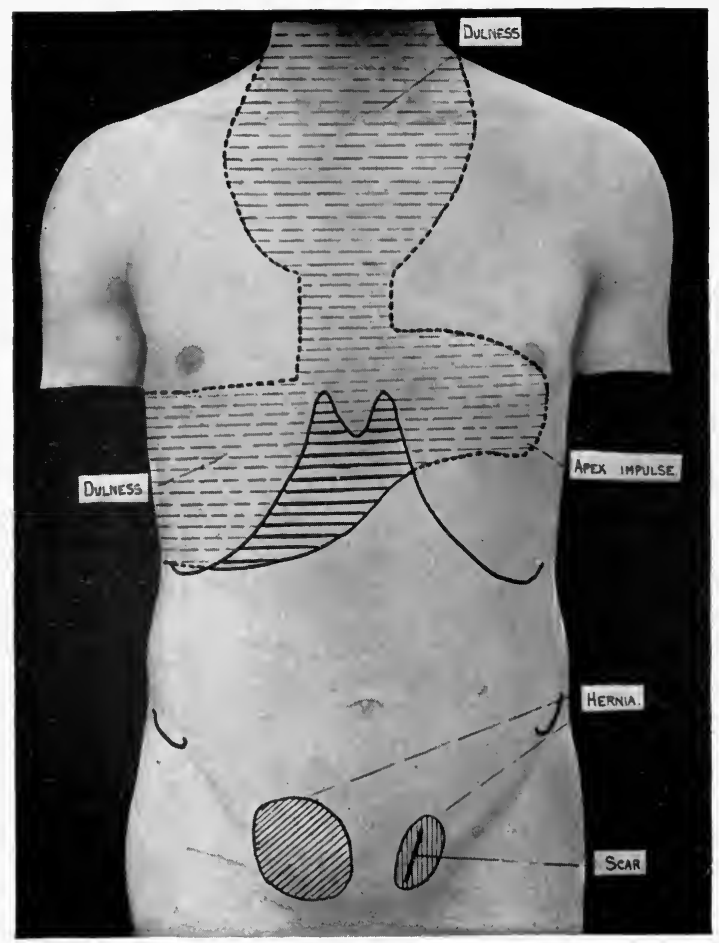

Fig. 56.-Physical signs in a case characterized for nearly three years by pain in the arms. 
It is worth noting in this case that there was no suit for damages in contemplation. Some writers on traumatic neurosis and many lawyers engaged in defending suits for damages try to persuade us that the expectation of a money payment as the result of litigation produces most of the symptoms of the traumatic neuroses. Cases like that here described upset such assertions.

Diagnosis.-Traumatic neurosis.

\section{Case 164}

A Turkish jeweler forty-five years old entered the hospital December 26, 1907. His family history and past history were not remarkable. He denied yenereal disease. Three years ago he had his first attack of "rheumatism" in the right hand and forearm, later in the other hand and other arm. There was no involvement of the joints and no increase of pain by motion. Six months later the pain extended up to the shoulders and to the neck. For this rheumatism he has been treated by many doctors, but without relief. Three years ago he gave up work and has never resumed it. Fifteen months ago he began to have a distressing cough with foamy sputum and this has continued ever since. For about the same period he has noticed hoarseness and dyspnea on exertion. For the past five months he has been unable to lie down at night. His appetite remains excellent, but he sleeps poorly.

A loud ringing cough is the patient's most striking symptom, and no cause for this could be found on examination of the lungs. Over the base of the heart a loud, harsh systolic murmur is heard. There is an area of percussion dulness as shown in the diagram (Fig. 56). Physical examination is otherwise negative.

Discussion.--In view of the symptoms which have recently developed in this case it no longer presents any diagnostic problems of special difficulty. Any patient who has a long-standing violent cough, with dyspnea, hoarseness, pain in the arm, and a dull area over the manubrium, with negative heart and lungs, has either aortic aneurysm or mediastinal tumor in all human probability. To this residual problem I will return later.

The great interest of the case centers in the three years which have led up to the appearance of the present distinctive symptoms of mediastinal pressure. Until very recently this case was regarded, as most such cases are, as one of "rheumatism." I have taken occasion in various parts of this book to illustrate the dangers and fallacies inherent in most diagnoses of rheumatism. No other word in the doctor's vocabu- 
lary stands so frequently for a dangerous mistake, one for which the physician bitterly reproaches himself when he discovers it. How are these dangers to be avoided?

I. Let us never use the word rheumatism unless there is evidence of acute infection, with distinct and predominant involvement of joints. Muscular pains will then be ruled out, their distinguishing characteristic being an increase of pain, especially when the muscle is used. The recognition of nerve pains, distinguished by the close relation of the suffering to the anatomic distribution of one or more nerves, will still further to restrict the unchartered freedom with which we pronounce the word "rheumatism." Pain due to inflammation involving the subcutaneous tissues or deeper parts may ordinarily be recognized by the other familiar evidences of exudation (tenderness, redness, swelling, heat).

2. When muscular pains, neuralgias, and subcutaneous exudations are excluded, we have left a very large group of lesions in or near the joints-bony outgrowths, periosteal inflammations, septic and tuberculous osteomyelitis, malignant disease of the bone, cartilage, or periosteum, joint fringes and foreign bodies, joint atrophies, traumatic synovitis, gout, hemophilic arthritis, joint suppurations, and other less common affections. From all these true rheumatism (i.e., acute infectious polyarthritis of unknown origin) may be distinguished, in the vast majority of cases, by the fact that it produces no permanent changes in any of the joint structures and gives a negative $x$-ray picture. Joint fringes, traumatic synovitis, and suppurative arthritis may show nothing characteristic in the $x$-ray picture; but the history and the accompanying symptoms usually make the diagnosis clear. The point which must be insisted upon, however, is that if we are to be even approximately secure in a diagnosis of rheumatism we must have a satisfactory $x$-ray picture of the joint in any case persisting over two weeks.

3. It is, I trust, worth while to mention here some of the diseases which I have known frequently diagnosed as rheumatism. The list includes many cases of tabes dorsalis, aortic aneurysm, and osteomyelitis (septic or tuberculous), a smaller number of cases of malignant disease involving the mediastinal, prevertebral, or abdominal glands and the long bones; also a good many cases of pressure neuritis (due to spondylitis, subacromial bursitis, or cervical rib).

Returning now to the case under discussion, we must attempt a diagnosis between aneurysm and mediastinal tumor. The strongest evidence against tumor is the long duration of the symptoms without any involvement of the external lymphatic glands and without more ob- 
vious depression and exhaustion of the patient's physical condition. As has been already said, diagnostic problems involving the differentiation between aneurysm and mediastinal tumor are usually settled sooner or later by the discovery of aneurysm.

Outcome. - The $x$-ray confirmed the diagnosis of aneurysm. On the eighth of January a diastolic murmur was noted, best heard at the apex. The pulse showed no change. At times the murmur was loudest in the anterior axillary line in the fifth space, and could be heard indistinctly as far back as the posterior axillary line. The murmur was long and wholly replaced the second sound at the apex. Gelatin injections produced great pain, but no relief.

The patient left the hospital on February 24th.

Diagnosis.-Aneurysm (called rheumatism).

\section{Case 165}

A washwoman of fifty-nine entered the hospital February Io, I908. Three years ago she had what she was told was a benign tumor in the left breast, which was removed in September, I905. Otherwise, she has been well until three months ago, when she began to notice pain on motion of the right upper arm and shoulder. Since Christmas, I907, she has been able to do little or no work. Until very recently there has been no pain when the arm is kept still. Coughing produces pain; breathing does not. For two weeks she has had a somewhat similar soreness in the right groin and hip.

Physical examination showed no emaciation, normal temperature, pulse, respiration, blood, and urine. The chest and abdomen were also normal, but it was found that the patient could not raise the right arm without marked pain. The greatest tenderness was in the front of the upper arm. There was no atrophy.

Counterirritation and small doses of morphin did not relieve the pain at all. On the nineteenth it was found that the right arm and the right side of the chest were almost completely anesthetic. An orthopedic consultant considered the case one of subacromial or subcoracoid bursitis. A neurologic consultant agreed. The pain in the right groin disappeared after a short stay in the hospital.

Discussion.-Against the diagnosis of subacromial bursitis the most important datum is the area of anesthesia, which involves not only the right arm, but the right side of the chest, and was apparently overlooked by the other consultants. I have never heard of a bursitis producing so wide-spread an anesthesia. Less important considerations antagonistic 
to the diagnosis of bursitis are the absence of any trauma or of any evidence that abduction or rotation is especially painful, and the fact that the pain is not especially worse at night. In the great majority of cases of bursitis the opposite is true.

Three months' suffering with shoulder pain and disability, associated with so wide-spread an anesthesia, should always lead at once to the investigation of the mediastinum by radioscopy, especially since we have no positive evidence that the mammary tumor removed in 1905 was as benign as the patient had been led to suppose.

Outcome. $-X$-ray taken on the twenty-sixth showed a wide shadow in the mediastinum. On March 4 th the patient began to complain of a smothering sensation in the chest, and some edema appeared in the right hand. The veins in the neck, especially on the right, now began to be engorged, though the pain was diminished. The patient left the hospital March 2Ist, not relieved.

Diagnosis.-Mediastinal neoplasm (metastatic).

\section{Case 166}

A clerk of forty-nine entered the hospital June 25, I908. He had previously been in the hospital twenty-two years before, suffering from what was considered facial neuralgia, but since that time he had been perfectly well until five months ago, when he began to have sharp pain under the right shoulder and finally down the whole of the right arm. After the first two or three days the pain never bothered him at night, but seven weeks ago it compelled him to give up work. He has had to have morphin for it once. The pain is most severe near the elbow. The joints do not seem to be involved. There is no limitation of motion. His appetite and sleep are poor. For a month he has had five or six loose movements of the bowels a day.

Physical examination was entirely negative. There was no tenderness along the course of the nerve-trunks. $X$-ray showed nothing abnormal in the chest, the neck, or in the joints. On the second day of his stay in the hospital he had a return of the facial neuralgia, which he had not previously had for twenty-two years.

Discussion.-Neuralgia, $i$. e., nerve pain of unknown origin, is always an unsatisfactory diagnosis, and one that we should make with the greatest hesitation and as a consequence of a long process of exclusion, whereby all known causes for such a pain are sought for without result. In the present case we can make a diagnosis of neuralgia only by satisfying ourselves that there is: 1 
(a) No relation to exertion (angina pectoris).

(b) No injury of the part (unrecognized fracture, traumatic neuritis, contusion or traumatic traction of nerve-trunks, tearing of muscular, capsular, or ligamentous fibers).

(c) No evidence of bursitis (limitation of motion, tenderness at the point of the shoulder or in the region of the bicipital groove).

(d) No signs of inflammation involving the veins, lymphatics, or subcutaneous tissues.

(e) No local lesion of the bone or periosteum (septic or tuberculous osteomyelitis, periostitis, benign or malignant neoplasm).

$(f)$ No evidences of pressure, such as cervical rib, aneurysm, mediastinal, supraclavicular, or axillary glands, or pulmonary tumor.

(g) No atrophic or hypertrophic arthritis ( $x$-ray evidence).

(h) No occupation neurosis.

(i) No systemic, infectious, or vertebral disease.

In the present case it seems possible, by rigid cross-questioning and examination, to exclude all these possibilities. We had reason to believe that the patient was already subject to nerve pain of unknown source. The diagnosis of brachial neuralgia was, therefore, finally made.

Outcome.-Under aspirin, Io grains every hour for eighteen hours, hot and cold douching, rest, and generous diet, the pain was very much relieved by the second of July. On the seventh he left the hospital practically well.

Diagnosis.-Neuralgia.

\section{Case 167}

A colored housewife of twenty-eight entered the hospital July 23, 1907. She has never menstruated, but has otherwise been well until seven days ago, when she woke up with a sore throat and stiffness throughout the whole left side, such that she could not raise her arm or leg. Since then she has had much pain in both arms and has taken a great deal of morphin. At entrance the arms, knees, and lower legs were tender and swollen, the tenderness being as great in the muscles as at the joints. Physical examination was otherwise negative, though the temperature ranged between $100^{\circ}$ and $I O I^{\circ} \mathrm{F}$. for a week, gradually falling to normal in the course of another week. The blood showed a moderate polynuclear leukocytosis. The urine contained bile for the first five days, and she had severe nose-bleed several times in the first four days of her stay. The conjunctivæ were distinctly bile-stained.

Discussion.- It seems obvious that we are dealing with an infection 
of some kind. The well-marked pyrexia, the jaundice (hemolytic presumably), the polynuclear leukocytosis, and the evidences of local inflammation all point to a bacterial origin.

Swelling of the extremities is not a common symptom when the heart and kidneys are sound, as they appear to be in this case. This is especially true of the arm. Occlusion of the vein by infectious thrombosis should produce a well-localized cord-like induration along the course of one or more veins. We have nothing of the kind here.

Lymphangitis is usually the result of some infection involving a break in the skin. It generally produces a red blush, extending from the point of injury or its neighborhood up the extremity toward the nearest lymphglands. But of such an inflammation there is no evidence.

Of septic myositis we know so little that it is hard to make any definite statements about it in a diagnostic discussion. I have never heard of so diffuse a myositis except that resulting from the disease next to be mentioned.

Trichiniasis might produce almost all the symptoms in this case, though it is not often limited to the extremities and rarely associated with so much edema. ${ }^{1}$ The patient's color, the polynuclear leukocytosis, and the absence of the eosinophilia strengthens the case against trichiniasis.

With the exclusion of all these possibilities, there is nothing left but an inflammation of the subcutaneous tissue and joint structure, not involving the veins or lymphatics, not due to an infected wound or to any known parasite. In the great majority of such cases the tenderness and swelling soon "settle" in the joints, leaving the other tissues free. Because of this fact and because the joints ultimately recover entirely, such cases are usually labeled "rheumatism." For the reasons previously discussed on p. 333, I believe this term should be restricted to articular disease involving no permanent joint changes nor lesions of the subcutaneous tissues around the joints. The present case, therefore, should be labeled provisionally as a cellulitis and arthritis of unknown origin.

Outcome.-The patient was given hot fomentations surrounding the extremities, and Io grains of sodium salicylate every hour. By the third of August she was greatly improved. By the thirteenth she was up and walking about, all pain and swelling having gone except from the left hand. This also gradually got well in the course of six weeks.

Diagnosis.-Infectious cellulitis with arthritis.

${ }^{1}$ A case of trichiniasis involving still more wide-spread edema was reported by Dr. Donald Gregg in the Boston Med. and Surg. Jour., December 3, 1909. 


\section{Case 168}

An unmarried girl of eighteen has always been well save for a bunch over the left collar-bone which formed five years ago, broke and discharged for several months.

For six months she has had slight pain and considerable disability in right shoulder. Rotation is painful and creaky, but abduction is not especially limited. The deltoid is very weak and markedly atrophied.

Physical examination, including temperature, pulse, respiration, blood, and urine, is otherwise negative.

Discussion.-Weakness, soreness, and stiffness of the shoulder lasting six months make a clinical picture raising many diagnostic possibilities before our minds. Since the general physical examination reveals nothing abnormal in the internal viscera or in any other part of the body, we are justified in fixing our attention upon the local lesion.

Subacromial bursitis might produce all the symptoms here described, but the history does not suggest any of the ordinary causes of this disease, such as trauma, prolonged fixation, or sepsis. If subsequent examination $(x$-ray) reveals no other disease of the bone or joint, bursitis will present strong claims upon our notice.

Tuberculous osteomyelitis involving the head of the humerus might also account for all the symptoms of which this patient complains. The fact that she has previously had a chronic suppuration originating in a bunch on the left side of the neck (presumably a tuberculous gland) inclines us toward the belief that the bone also is tuberculous. Although there is no apparent involvement of the soft parts overlying the joint, the tuberculous process may be confined to the destruction of bone (caries sicca). Further evidence must be sought by $x$-ray examination.

Only by this means can we exclude an unrecognized fracture of the upper end of the humerus. It would be strange, however, if, in a young girl apparently free from disease of any other part of the body, we should find a fracture of the humerus without any known trauma. In the early stages of such a lesion the history should have mentioned the presence of ecchymosis and swelling, especially on the inner side of the arm. Six months after the time of fracture we should expect the symptoms either to be gone altogether or to be associated with some bony deformity.

Circumflex paralysis rarely occurs without some much more obvious 
cause than is stated here. In case of such a paralysis there would be no visible or palpable contraction of the deltoid fibers if the patient were to make an effort to raise the arm (abduction). In the present case there were distinct wrinkling and hardening of the deltoid under the palpating hand during the patient's effort, although no considerable motion resulted.

Atrophic or hypertrophic arthritis would be almost certain to involve some other joint to a greater or lesser extent. The age and sex are typical for atrophic arthritis, not at all so for hypertrophic lesions. Further evidence regarding such disease could only be obtained by $x$-ray examination.

A deep axillary abscess, small and high up under the pectoral, sometimes produces a fixation of the shoulder-joint and pain on any motion involving it; but careful examination of the upper axilla behind the pectoral should disclose a deep tenderness and induration, and there should be some fever. In the present case such an abscess is unlikely on account of the long duration of the symptoms.

Outcome. $-X$-ray showed considerable necrosis of the head of the humerus, which was therefore excised. Examination of the portion resected showed tuberculosis. The girl ultimately made a good recovery, with very fair use of the arm.

Diagnosis.-Tuberculosis of the humerus.

\section{Case 169}

An Armenian factory hand of thirty-one received a blow on the right shoulder six weeks ago. Afterward the shoulder swelled and stiffened. The patient is not able to give any more detailed history of his illness.

Examination.-There is almost complete loss of active motion in the right shoulder. Passive motions are also somewhat restricted in all directions; there is marked tenderness over the upper third of the humerus. No swelling, no hollowing of the deltoid, but marked atrophy of the whole upper arm. The axilla is full of tender glands.

Temperature, $100^{\circ}$ to $103^{\circ} \mathrm{F}$; leukocytes, 8000 .

Discussion.-The signs seem to point toward some type of osteomyelitis, but why does not the man get well? Why are there atrophy of the whole arm and such marked loss of power in the shoulder? Six weeks of disuse might alone cause atrophy and limitation of motion. Is there some malignant disease behind it all, some lesion of the central nervous svstem. or tuberculosis? The presence of temperature and 
tender axillary glands tends to show that there is still infection going on, although the leukocyte count is so low. The fact last mentioned inclines us slightly toward tuberculosis as the cause of the osteomyelitis.

Obviously, however, the chief need of the case is for an $x$-ray examination, to be followed in all probability by a more thorough investigation of the conditions below the deltoid.

Cases of this type offer an extensive field of possible alternatives for differential diagnosis. The history of trauma makes it necessary to consider fracture or dislocation of the humerus and subacromial bursitis. Contusion or hematoma would presumably have been well before the end of six weeks, but there may always be an element of traumatic neurosis in the case.

On the other hand, it is essential to remember that the history of trauma is often evolved quite out of whole cloth by the patient, whose mind imperatively demands some such explanation for a painful and tender swelling, due, in fact, to neoplasm, to tuberculosis, to septic osteomyelitis, or other disease in which trauma plays a very subordinate rôle.

Further, we must realize that a subacromial bursitis is sometimes brought about by the prolonged immobilization of the shoulder resulting from a shoulder contusion which is coddled by a neurotic patient or an overanxious mother.

Taking up now these alternatives, we may eliminate fracture and dislocation by the negative results of $x$-ray examination; bursitis, by the absence of characteristic limitations of mobility; neoplasms, by the results of $x$-ray. The tenderness is distinctly suggestive of osteomyelitis, especially if neoplasm can be ruled out. The results of exploratory incision will be important here. Tuberculosis, whether in the form of caries sicca or whether including subcutaneous tissues, should be shown up by the results of $x$-ray examination.

Outcome. $-X$-ray showed a large cavity in the head of the humerus and a smaller one in the shaft; shoulder-joint obliterated. After operation, the patient recovered. The excised bone showed no tuberculosis.

Diagnosis.-Septic osteomyelitis.

\section{Case 170}

Two months ago a teamster's left arm suddenly became stiff and pained at night near the neck of the humerus. Two days later the 
fingers and palm began to swell and to get shiny. Three days after this the whole arm swelled. The pain then extended into the upper back. He was then treated in the South Framingham

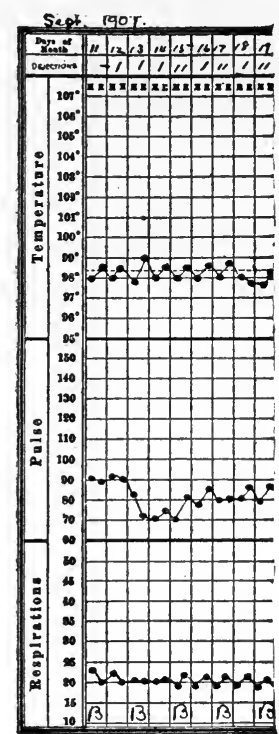

Fig. 57.-Chart of case I70. hospital for neuritis, and was two weeks in bed, but without fever. Now he is much better. Edema gone.

Examination.-All motions of the left shoulder were made voluntarily. The muscles were still very weak, and there was tenderness over the scapula, which later improved with counterirritation and sodium salicylate. $X$-ray negative.

Discussion.-The earlier symptoms remind us of tuberculosis of the humerus or of subacromial bursitis. But neither of these diseases produces so much swelling of the lower arm. Tuberculosis may be, with reasonable probability, excluded by the negative results of $x$-ray examination, bursitis by the absence of spasm or characteristic limitation of motion and the presence of diffuse extensive edema.

This edema might be explained by the pressure of an intrathoracic tumor or a cervical rib upon the veins of the arm, but the fact that the edema so promptly disappeared and that physical examination shows no evidence of these causes of pressure, suffices to exclude them.

Swelling of the arm without obvious cause is occasionally due to a thrombophlebitis, but such a diagnosis cannot be made unless we find induration and tenderness along the course of some vein or veins.

Brachial neuralgia is a possible diagnosis, although the presence of edema and the absence of tenderness following sharply the course of any known nerve make it rather unlikely.

Diffuse inflammation of the subcutaneous tissues (so-called cellulitis) is not, in my opinion, a very rare condition, even in the absence of any known cause. We hear but little of such affections, because they are apt to be called muscular rheumatism, as the present case was. In view of the outcome of the case cellulitis seems to me to be the best diagnosis.

Outcome.-The patient made an uneventful recovery in the course of three weeks.

Diagnosis.-Cellulitis. 


\section{Case 171}

A housewife of thirty-five has suffered for many years from "rheumatism " in her right shoulder.

For three years the pain has been worse and has been referred especially to the region of the right clavicle and to the whole right arm. Sometimes it is localized at the lower end of the ulna.

Within a month she seems to be losing strength in the arm, and the pain often keeps her awake at night.

Examination shows a pulsating mass above the left clavicle, with a sense of firm resistance below and around it. Backward motions of the arm cause sharp pain. The outer side of the pulsating mass is very tender. There is no considerable atrophy or limitation of abduction. Temperature range, $98^{\circ}$ to $99.5^{\circ} \mathrm{F}$. Pulse, 90 to $\mathrm{I} 20$. Urine pale, acid, IоI6; albumin, slightest possible trace. Sediment. Numerous blood-globules; small round mononuclear cells, some of which are fatty. Many calcium oxalate crystals. Internal viscera negative.

Discussion.-The diagnosis was not suspected in this case until the conditions were actually seen at operation. This seems to me wrong, for there are very few causes which produce a pulsating mass above the clavicle. Aneurysm is naturally our first thought, but this is a very unusual place for an aneurysm, although diffuse dilatations of the subclavian or carotid arteries often occur as a result of aortic regurgitation and in connection with a diffuse dilatation of the arch. This condition is not aneurysm, and should not be confounded with it, since there is no breaking of the arterial coats and no tendency to end in rupture of the artery. Further, an aneurysm of two years' standing is very rare in this situation, and the source of the marked resistance around the pulsating mass would not be explained by the diagnosis of aneurysm.

Can the pulsation be transmitted through some tumor or glandular mass by a normal artery beneath? It would seem very unlikely that a tumor which would produce pressure pains in the arm for three years should not have attained greater size and pulled the patient down more, and metastasis would probably have occurred.

The presence of a slight fever gives some color to the idea of glandular tuberculosis, but such a process rarely if ever causes much pain, and would scarcely have existed so long without abscess formation.

Brachial neuralgia is a diagnosis which one never has a right to make in the presence of anything which can possibly be interpreted as a mechanical' cause of the pain under investigation. With a mass like that here described the diagnosis of neuralgia has no justification. 
A pulsating mass above the clavicle means cervical ribs in nine cases out of ten, the pulsation being due to the subclavian artery which overlies the rib, while brachial pain results from pressure on the brachial plexus.

The firm resistance below and around the pulsating mass was the rib underlying the artery. Had an $x$-ray been taken, the diagnosis should easily have been clinched before operation, but even without an $x$-ray one might make a reasonably certain diagnosis on the history and physical signs, provided one had ever seen a similar case.

Outcome.-The brachial plexus and subclavian artery were found at operation to be elevated on the blunt head of a cervical rib which joined the first dorsal rib about two inches from the sternum.

After excision of the cervical rib the pain disappeared within ten days and did not return.

Diagnosis.-Cervical rib.

\section{Case 172}

A very alcoholic clerk of thirty-three was sent into the hospital for "osteomyelitis humeri." He has had three months' pain in right upper arm, at times sharp; occasionally it shifts to the elbow or forearm. Day and night make no difference.

One month ago it began to swell and the soreness and tenderness increased. Otherwise he feels well.

Examination.-Whole upper right arm $2 \frac{1}{2}$ inches larger in circumference than the left. Hard (bony?) enlargement is felt beneath the muscles. The whole mass is hot and tender.

A plexus of veins is prominent over upper inner side of the arm.

Discussion.--The fact of enlargement of the upper arm below the shoulder and at the point of pain excludes many of the conditions discussed in previous cases. Subacromial bursitis, arthritis of the shoulder-joint, circumflex paralysis, brachial neuritis, tuberculous disease without abscess formation (caries sicca), all produce atrophy, not enlargement.

The heat and tenderness dispose us to consider a septic osteomyelitis, a periostitis, or a tuberculosis with abscess and infiltration of the overlying tissues, but in such diseases one would expect fluctuation rather than such extreme induration. Rarely, moreover, does an osteomyelitis or periostitis result in enlargement of the superficial veins.

Syphilitic disease of the bone, or gumma involving the skin, would probably produce far less pain and little or no enlargement. After 
three months' duration there would almost certainly be some involvement of the skin, some discoloration or ulceration.

The enlargement of the veins associated with an increase in the size of the whole arm, with marked induration, is very characteristic of malignant disease involving the bone.

Outcome. $-X$-ray examination showed only a slight increase in the area of bone-shadow-apparently a periostitis. The Wassermann reaction was negative. Operation showed osteosarcoma.

Diagnosis.- Sarcoma humeri.

\section{Case 173}

A school-boy of twelve was struck on the right arm just below the shoulder eight weeks ago. The arm became at once swollen, and in the past few weeks has been so painful as to require morphin, especially at night.

Examination.-A swelling one-half the size of an orange occupies the deltoid region, and extends one-third of the way down the arm, about half encircling it. The shoulder motions are free and painless. The veins over the lower portion of it are enlarged. The mass is rather soft, very tendcr, and apparently adherent to the bone. One enlarged, non-tender gland is felt in the right axilla (normal microscopically).

Discussion.-The acute swelling and pain near the head of the humerus are rather characteristic of septic osteomyelitis, especially in a boy of this age. But in the course of eight weeks one would rather expect that the pus would have burrowed to the surface or brought about a general septicemia.

Experts in legerdemain accomplish their tricks by setting a trap for our attention and attracting our gaze to the wrong place at the wrong time. By a similar psychologic mechanism a history of injury like this becomes one of the commonest and most dangerous of traps set to catch unwary diagnosticians. Our attention gets concentrated upon a group of lesions, such as dislocation, fracture, hematoma, or bursitis, which might result directly from trauma. While we are puzzling to decide between these alternatives, or perhaps carrying out treatment designed to relieve one of them, the actual but unsuspected neoplasm or tuberculosis progresses without hindrance. We forget for the moment that osteosarcoma is common in this situation and at this age.

The plexus of swollen veins over the swelling is rather suggestive of tumor, but against it, apparently, is the normal microscopic structure of the enlarged axillary glands, which one would expect to find trans- 
formed as a result of metastasis from the bone tumor. It must always be remembered, however, that the examination of a gland under conditions like these sometimes proves very misleading. Twice I have known malignant disease of the mediastinum associated with a large axillary gland, which, when removed, showed nothing abnormal in its structure. Diagnostic conclusions from the examination of glands in the neighborhood of doublful lesions are of value only when the results of examination are positive. Negative results are valueless, as was, indeed, exemplified in this case by the outcome.

Outcome.-Incision allowed the escape of some soft material resembling grains of sago. On microscopic examination these grains showed the structure of round-cell sarcoma.

Diagnosis.-Sarcoma humeri.

\section{Case 174}

A boy of ten was sent to the hospital for a tumor of the humerus.

One month's pain in the right upper arm, with subsequent gradual swelling but no tenderness, was the gist of his history.

Two weeks ago the pain became severe. No known cause.

Examination.-Looks worn out. Right forearm and upper arm swollen (radial pulse good). Motions free. The lower half of the humerus is tender.

Discussion.-The boy is at the age when septic osteomyelitis or malignant tumors are apt to attack the end of the long bones. The wornout appearance of the boy and the absence of tenderness rather favor tumor, but it is to be noted that tenderness is absent only in the upper part of the arm, while the lower part is notably sensitive.

Why is the whole arm swollen? We have no evidence of pressure from tumor, aneurysm, or cervical rib, no sign of phlebitis or cellulitis. Such a swelling would be very unusual were we dealing with tuberculous osteomyelitis.

It does not appear that the diagnosis can be made any clearer without $x$-ray evidence or operation. To these procedures, accordingly, we must turn.

Outcome.- $X$-ray shows thickened periosteum over a swollen humerus with a dark area in the middle of the lower one-third of the bone. Three ounces of pus were evacuated from a cavity in the medullary portion overlain by thickened bone and periosteum. Staphylococci in pure culture from the pus. Temperature, $99^{\circ}$ to $100^{\circ} \mathrm{F}$.

Well in a week.

Diagnosis.-Septic osteomyelitis. 


\section{Case 175}

A hardwood finisher of forty-seven fell down stairs in I9oI, striking the right shoulder and the back of the neck. For three months after this the shoulder continued sore.

In I903 he began to have attacks of sharp pain between his shoulders, disabling him from work for several weeks at a time, not relieved by any medicine.

In September, 1904, pain in the nape troubled him and continued until January, 1905. In December, I904, the pain between the shoulders and in the right shoulder became severe again, and has lasted until the present time (January I 7, I905). This pain is not affected by motion or position, but often keeps him awake at night.

Cough with profuse white sputa, two months. The cough produces an increase of pain in the right shoulder and at the root of the neck in front.

Has lost 20 pounds in two years.

Examination.-Left pupil larger than the right. The patient stands with a well-marked stoop. An impulse lifts the manubrium with each heart-beat. A diastolic murmur, loudest in the second right space, is audible over the whole heart, which shows no obvious enlargement. The pulse collapses markedly. The larynx and trachea are normal. There are dulness, tenderness, bronchial breathing, and increased voicesounds at the right apex. The right clavicle and shoulder are tender to touch, but all motions are free. There is no muscular atrophy. Physical examination is otherwise negative.

Discussion.-The history of the case naturally suggests that the present symptoms are due to trauma, especially as the shoulder is still tender. But a more careful reading shows that the interval between I 901 and 1903 is too long for any such explanation.

Apparently there is no lesion of the joint, muscle, or nerve. All articular motions are free; muscular action does not increase the pain, and the suffering is not definitely localized along any nerve-trunk.

The long-continued cough (two months), the emaciation, the abnormal physical signs at the right apex, and the chest pain had led to a diagnosis of pulmonary tuberculosis by the attending physician. But there seems to be no fever, no evidence of breaking down within the lung (râles, purulent sputa), and a great deal more pain in the shoulder than one expects to see in phthisis. Especially notable in this respect is the long duration of pain before the cough began. There 
seem to have been nearly two years of suffering before there was any cough.

By some orthopedic specialists many pains in the back, shoulders, and arms are explained by the so-called "round-shoulder deformity" - the ordinary stooping habit. Up to date I have not been convinced of the validity of these explanations. The difficulty with all such explanations is that they fail to show why the stoop has persisted so many years longer than the pain supposed to be due to it. In any case it is not at all probable that a stoop will be advanced to explain such severe and definitely localized pain as is here complained of.

This patient's pain is in a very queer place. One very seldom hears patients complain of pain high up between the shoulders, and whenever one hears such complaints, some cause of intrathoracic pressure should be suspected. Such causes are, for practical purposes, three and only three, viz., aneurysm, vertebral tuberculosis, and malignant disease. Turning now to the circulatory system with the thought of aneurysm in mind, we note that there is evidence of aortic regurgitation, such as often accompanies aneurysm. We notice also the inequality of the pupils, and we are led thus to suspect that the pulmonary lesions may be the result of pressure upon the lung itself or upon one of the larger bronchi. Obviously, this possibility-aneurysm - has much in its favor, especially when we consider the long duration of the symptoms. Intrathoracic neoplasm would probably have produced more obvious and alarming symptoms if it had existed so long. Tuberculous or other disease of the cervical or upper dorsal vertebræ should produce some stiffness or tenderness of the spine, and after so long a course some evidences of caseation, telescoping, kyphos, or fever would be expected.

Outcome. $-X$-ray shows an extensive shadow to the left of the sternum. Had in the ward several attacks of severe precordial pain, with great anxiety, relieved by nitroglycerin. Pain then ceased for five weeks.

In March, 1905, he began to have pain in the top of the right shoulder, with a scalding feeling in the arm above the elbow.

The heart apex was then found to be in the sixth space, six inches to the left of the median line. The right pulse is smaller than the left, and of "Corrigan" type. Tracheal tug. The patient remained in the hospital until April 6th, suffering very little pain. His treatment consisted of potassium iodid, aspirin, and laxatives.

Diagnosis.-Thoracic aneurysm. 


\section{Case 176}

A cook, fifty-nine years old, colored, born in Martinique, entered the hospital March 28, I908. He has always been well except for "rheumatism" many years ago, which attacked many joints but did not keep him in bed. He denies venereal disease.

For two years he has had attacks of pain in the left shoulder, radiating thence to the breast-bone and to the pit of the stomach. These attacks of pain have come at considerable intervals until within the past two weeks, when they have come every other day, and have forced him to stop work. The pain is not severe, and is always relieved by rest or drinking hot water. He says that his left arm is weak, especially after an attack of pain. His ankles have been painful and swollen for two weeks, and he has had a hacking cough for five months. At one time he noticed that he passed more urine at night than in the day-time, but this is not now the case.

His appetite is good; he has no indigestion and no headache.

On physical examination the painful shoulder showed no objective abnormalities. The cardiac apex seemed to extend one inch outside the nipple-line in the fifth space. A systolic murmur was heard at the base and down to the fourth left space. The aortic second sound was faint, the pulmonic second sound somewhat louder, but not accentuated. The pulses seemed to be of high tension, but the blood-pressure read only $138 \mathrm{~mm}$. of mercury. The radials and brachials were markedly thickened and tortuous. The edge of the liver was felt two inches below the ensiform. In the fourth left interspace, near the sternum, a faint diastolic murmur was later made out. At no time was there any capillary pulse or Corrigan pulse. $X$-ray was negative.

Discussion.-We may exclude all varieties of arthritis (rheumatic and other), because the joints are at present normal. Muscular, periosteal, and nerve lesions can be ruled out by the absence of swelling, tenderness, and heat, the absence of any relation of the pain to muscular movements or to the anatomic position of the nerve. There is no important evidence pointing to any source of pressure within the chest.

When these possibilities are excluded, we note that the pain comes in paroxysms which are relieved by rest, and that it has very wide radiations. Any pain of this type occurring in a man of fifty-nine suggests aneurysm or angina pectoris, especially if the patient is a negro. Of aneurysm we have no definite evidence, though it cannot be ruled out without $x$-ray examination. Most cases of angina pec- 
toris are associated with a greater elevation of the blood-pressure, but the disease cannot be ruled out on that account. Angina is, therefore, the most reasonable diagnosis. Greater certainty can be attained through the therapeutic test, but only time can exclude aneurysm.

Outcome.-The patient was given 5 grains of potassium iodid three times a day, with $\frac{1}{100}$ grain nitroglycerin and cascara as needed; later, I5 minims of tincture of digitalis three times a day were added.

By April 4th he had made marked improvement, and was sleeping

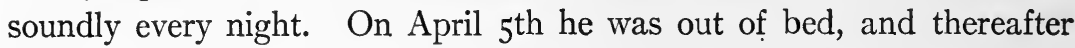
was almost free from symptoms until his discharge on the eleventh.

This case is introduced as an example of a somewhat unusual distribution of pain in angina pectoris. In other cases the pain may be wholly epigastric, wholly or largely in the arms or in the back. We are justified in grouping all these widely separated pains under the single heading of "angina," because all of them are associated with arteriosclerosis and with cardiac disease which is fairly well compensated. It is important that all of them are produced and relieved in the same way. The four specially characteristic occasions for anginal pain are all of them occasions of suddenly raised blood-pressure. These are:

(a) Muscular exertion.

(b) Strong emotion.

(c) Digestion, especially if it be impeded in any way.

(d) Getting up in the morning.

The vast majority of anginal attacks are produced by one of these four causes, which I have arranged in the order of their frequency. Much less common is angina that wakes the patient from sleep. The relief of pain when one of these causes has been removed usually enables the patient and his physician to be quite clear as to its cause. The relief by some one of the nitrite preparations, which tend to lower blood-pressure, is also of great diagnostic value.

Diagnosis.-Angina pectoris [syphilitic aortitis?].

\section{Case 177}

A tailor of sixty entered the hospital July 2I, I906. He stated that for eight or nine weeks he had had rheumatism in his right shoulder, which is now much better and troubles him very little. A little later he noticed a lump just above and to the right of his breast-bone. This has gradually increased in size until the last week, when it has grown very rapidly. It is hard, not tender, and seems to "beat." He now 
notices pain on lifting his right arm or turning on his right side. There is no history of injury. For the past two months he has been hoarse.

Physical examination shows that the pupils are equal and react normally, though they are slightly irregular. The heart shows nothing abnormal. To the right of the sternum, above the second rib, is found an expansile, pulsating tumor, the size and shape of an egg. The right clavicle is pushed forward, and the sternal end seems to be buried in the tumor. The manubrium is eroded and the first rib completely cut off from the sternum. There is no dulness beneath the manubrium, and no other abnormal pulsation. There is a faint systolic murmur over the tumor.

Physical examination of the lungs, abdomen, extremities, blood, and urine is otherwise normal.

Discussion.-Hoarseness, shoulder pain, irregular pupils, and a pulsating lump near the breast-bone seem at first almost indisputable evidence of aneurysm, and so, in fact, they did seem to most of those who saw this case in the hospital wards. Certain points, however, were, at any rate, atypical, to wit:

(a) The pain: why should it decrease? It rarely does decrease in cases of aneurysm unless the patient takes to bed and adopts other measures for slowing the circulation.

(b) The percussion area: why should there be no substernal dulness? The aneurysm must be supposed to arise from the arch of the aorta, and ought, therefore, to produce dulness under the manubrium.

(c) Aneurysms rarely begin above the level of the sternum in the neck or behind the clavicle. Unusual pulsations at this point rarely turn out to be aneurysm.

(d) The patient is rather old for aneurysm, though this by no means excludes it.

(e) An aneurysm situated in this position would probably involve the subclavian artery or the innominate sufficiently to produce inequality of the pulses.

Decisive evidence might probably have been obtained by $x$-ray examination.

If not aneurysm, what else could this lump be? Gummatous tumors are common in this situation. They are not usually painful and destroy much less bone than appears to have disappeared in this case. They pulsate only in case they have perforated the sternum, which is a rare occurrence.

Tuberculosis of the bones composing the thoracic wall usually shows 
more evidence of caseation, produces but little pain and that confined to the diseased focus itself, and never pushes the clavicle forward.

Malignant disease originating in the ribs, in the sternum, or in some of the mediastinal structures would produce most of the signs here described. The marked pulsation seems less inconsistent with a vascular neoplasm than with syphilis or tuberculosis. The patient's age is suggestive of neoplasm rather than of aneurysm.

Outcome.-Despite the considerations just adduced, a diagnosis of aneurysm was made. The patient left the hospital on the twentyfifth of July, and not long after consulted Dr. Maurice H. Richardson, who removed an incapsulated vascular tumor which suggested, on histologic examination, a metastasis from hypernephroma. There was no aneurysm. Some months later the patient entered the Cambridge Hospital for profuse renal hemorrhage, probably due to the primary tumor.

Diagnosis.-Metastatic hypernephroma.

\section{Case 178}

A milliner of twenty-seven entered the hospital March 9, I907. Her family history was negative, and she remembered no illness until within the past year, when she has had dysentery with eructations of gas after eating, especially after taking fried food. She has had to get up to pass water once or twice at night for the past year. For two months she has been conscious of her heart-beat. Eighteen months ago she weighed II 2 pounds, which was about her a verage weight. Now she weighs 97 pounds.

Three months ago she began to have cough, which sometimes is so intense as to make her vomit. She spits almost nothing. For the same period she has noticed shortness of breath on slight exertion. January 30, 1907, she was admitted to the Rutland Sanatorium for tuberculosis, and five examinations of her sputa were made, with negative results. Her temperature while there was normal the greater part of the time, but at irregular intervals it would rise to $100^{\circ}$ or $100.5^{\circ} \mathrm{F}$. She comes to the hospital directly from Rutland. On more careful questioning she admits that for a year she has been having dull pains in the left side of her neck, and pain and numbness in the left arm. This pain is apt to increase gradually for two or three minutes and then suddenly stop. Eggnog or anything containing alcohol makes the pain distinctly worse. It has quite frequently kept her awake at night. Lying on the left side makes it worse. 
Physical examination shows slight brownish pigmentation of the skin. The left chest is somewhat fuller in front than the right, and the veins over it are prominent. Over the left clavicle is a small mass the size of an English walnut, hard and movable, not tender. The heart is negative. The left lung shows dulness just above and below the clavicle. Throughout the left front, breathing is distant, and the same is true of the left back below the scapula, where there is dulness and diminished fremitus as well. The abdomen is negative. The left upper arm measures $2 \mathrm{I}$ centimeters; the right, 9 centimeters.

Discussion.-The mistaken diagnosis of tuberculosis was quite excusable in this case. Cough, dyspnea, pain, with dulness at one pulmonary apex, loss of weight, and a slight pyrexia are certainly very strong evidence in favor of tuberculous infiltration. It was only after repeated negative examinations of the sputa that it seemed necessary to reconsider the diagnosis. The fact that no râles had appeared during a considerable period of observation, and especially the early appearance and long persistence of pain, began to make it seem likely that some deeper and more serious disease was at work.

The most significant fact in this case is, I think, the long interval (nine months) between the beginning of pain sufficient to keep her awake and the onset of cough. This, I think, should have made us suspicious and doubtful of our diagnosis from the first.

High Pott's disease must be reckoned with. There need be no kyphos in such cases, and the pain is often referred to points distant from the spinal lesion. The pain, however, is the only symptom which points toward vertebral tuberculosis. We have no muscular spasm, no stiffness or torticollis, none of the evidences of caseation or abscess formation such as might well be expected after a year's duration of the disease.

When the arm began to swell and the lump appeared above the left clavicle, there was no longer any considerable doubt that a mediastinal tumor of some type was pressing upon the brachial plexus. Such tumors, whether they arise from mediastinal glands, from the root of the lung, or from the pleura, usually begin with symptoms of ordinary pleural effusion, for which they are frequently mistaken. In their early stages there are often no pain, no external tumor, and no swelling of the arm. The pleural effusion, however, reaccumulates with astonishing swiftness after aspiration. It may or may not be bloody, and its cellular constituents may or may not be identical with those of ordinary (tuberculous) pleurisy. But it is especially the rapid refilling of the chest after tapping that finally awakens our suspicions of malignant disease. 
Outcome. $-X$-ray of the chest showed a diffuse shadow, chiefly on the left side, but extending also a short distance to the right of the spinal column. The nodule at the base of the neck was removed and examined by Dr. Wright, who pronounced it malignant lymphoma. The evidences of fluid at the base of the lung steadily increased. The patient did not react to $3.5 \mathrm{mgm}$. tuberculin. On the twenty-fourth of March she was discharged not relieved.

Diagnosis.-Malignant lymphoma. 


\begin{tabular}{|c|c|c|c|c|c|c|c|c|c|c|}
\hline 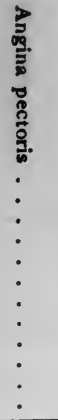 & $\begin{array}{l}8 \\
\frac{8}{0} \\
\frac{0}{2} \\
\frac{3}{5} \\
\vdots \\
\vdots \\
\vdots \\
\vdots \\
\vdots \\
\vdots\end{array}$ & 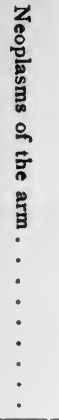 & 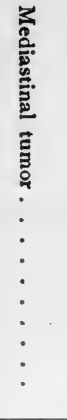 & 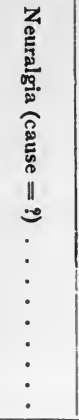 & 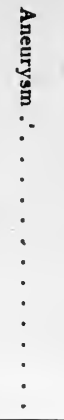 & 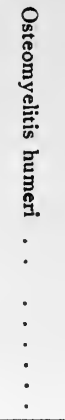 & 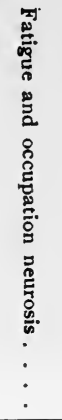 & 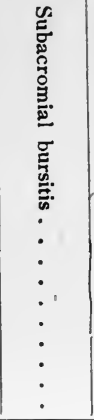 & 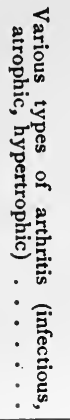 & 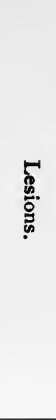 \\
\hline 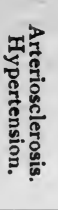 & $\infty$ & $\infty$ & $\infty$ & $\infty$ & 息 & $\begin{array}{l}\overrightarrow{D_{0}} \\
\stackrel{0}{0} \\
\stackrel{0}{0}\end{array}$ & 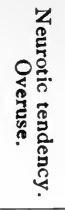 & 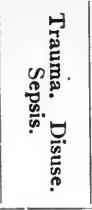 & 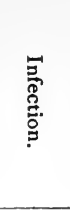 & 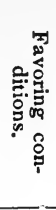 \\
\hline 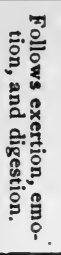 & $\begin{array}{l}\vdots \\
\vdots \\
\vdots\end{array}$ & 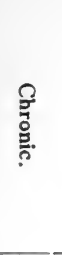 & $\begin{array}{l}\stackrel{\rho}{0} \\
\stackrel{0}{0} \\
\stackrel{0}{0}\end{array}$ & 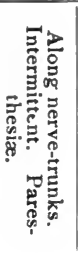 & 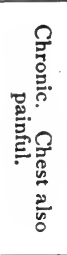 & 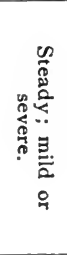 & 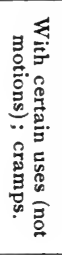 & 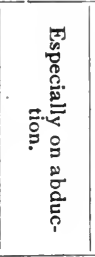 & 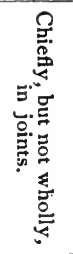 & $\begin{array}{l}\cdot \\
\overrightarrow{0} \cdot \\
\overrightarrow{0} \cdot\end{array}$ \\
\hline ○ & ० & ○ & ० & 0 & ० & + & 。 & 0 & 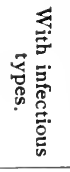 & 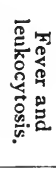 \\
\hline ० & 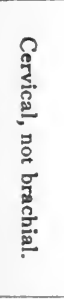 & 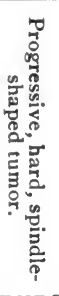 & 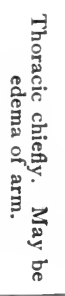 & 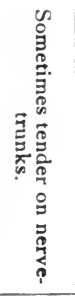 & 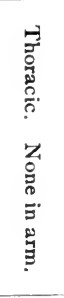 & 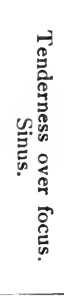 & 。 & 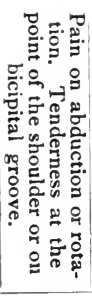 & 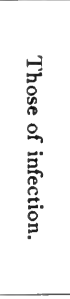 & 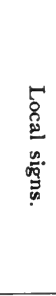 \\
\hline$\circ$ & 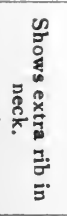 & 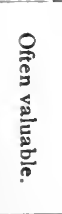 & 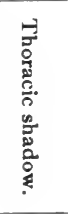 & ० & 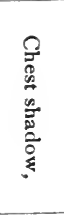 & 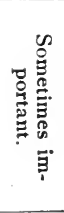 & 0 & 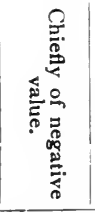 & 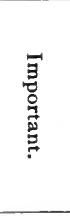 & 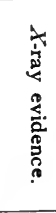 \\
\hline 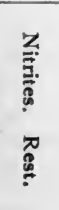 & 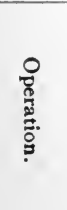 & 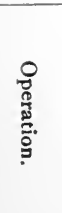 & & 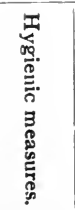 & 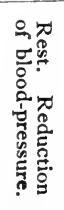 & 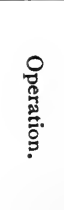 & 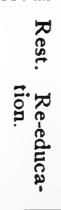 & 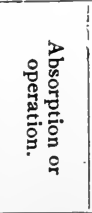 & 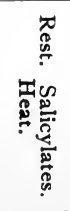 & 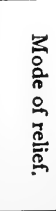 \\
\hline
\end{tabular}




\section{CHAPTER XII}

\section{PAIN IN THE LEGS AND FEET}

\section{Case 179}

A Hackman of twenty-five entered the hospital March 6, 1907. His family history was negative. A year ago he had urethritis and was sick for a month. For a week his left ankle was swollen and red and he was unable to use it for a month. Six days ago he noticed a cutting pain in his right hip, relieved by sitting down. Four days ago he was unable to get out of bed. Yesterday his left ankle was swollen and sore.

Physical examination showed normal temperature, pulse, and respiration. The chest and abdomen were normal. There were slight tenderness, redness, swelling, and pain across the instep of the left foot. Motions of the right hip caused marked pain in the sacro-iliac joint. There was also tenderness there.

Discussion.-We are dealing with lesions of the right hip and left ankle-in all probability some type of arthritis. The diagnosis of rheumatism must be avoided like a blasphemy unless we are forced to it by the exclusion of all other possibilities. To those possibilities we will . accordingly turn our attention.

Hypertrophic arthritis (osteoarthritis) does not attack these joints in a man of twenty-five. It will be remembered that in the hip-joint this lesion constitutes the malum coxe senilis and leaves youngsters unscathed.

Atrophic arthritis might involve these joints in a young man, but always involves other joints as well (particularly those of the hand), and it is very prone to a symmetric distribution, e. g., both wrists, both ring fingers, both hips, both feet.

Were the sacro-iliac joint alone affected, it might not be necessary to assume the presence of any inflammatory lesion. Some strain or displacement of the joint might suffice to produce the pain. But since the opposite ankle-joint is also involved, we have no reason to connect the two lesions mechanically. Infection is the only other familiar link, especially as we have no definite evidence of any metabolic defect, such as gout. 


\section{Causes of Pain in Legs and Feet}

\section{INFECTIOUS DISEASES (AT ONSET F.SPECIALLY)}

2. FLAT-FOOT

2204

3. INFECTIOUS ARTHRITIS $\}$

4. VARICOSE VEINS

5. TABES

6. HYPERTROPHIC 265 ARTHRITIS

7. PHLEBITIS

9. OSTEOMYELITIS

10. TUBERCULOSIS

11. SPRAINED ANKLE

12. SPRAINED KNEE

$\left.\begin{array}{l}\text { 13. SYPHILITIC } \\ \text { PERIOSTITIS }\end{array}\right\}$

$\left.\begin{array}{l}\text { 14. ATROPHIC } \\ \text { ARTHRITIS }\end{array}\right\}$

15. TENOSYNOVITIS

16. ALCOHOLIC ) NEURITIS

17. SARCOMA OF LEG
BONES

18. GOUT

19. MORTON'S META-
TARSALGIA 

If the joint troubles are of infectious origin, the first question to be answered is: Could a urethritis last so long? Can the joint trouble be due to a gonorrheal infection? To answer this question we must investigate the urethra.

Outcome.-A urethral smear showed gonococci. Vaccines were given beginning March 8th, and within two days were followed by considerable improvement.

On the seventeenth there was much pain in the sacro-iliac region, and this lasted until the twenty-second, after which he improved rapidly. The opsonic index was low most of the time until the twenty-eighth,

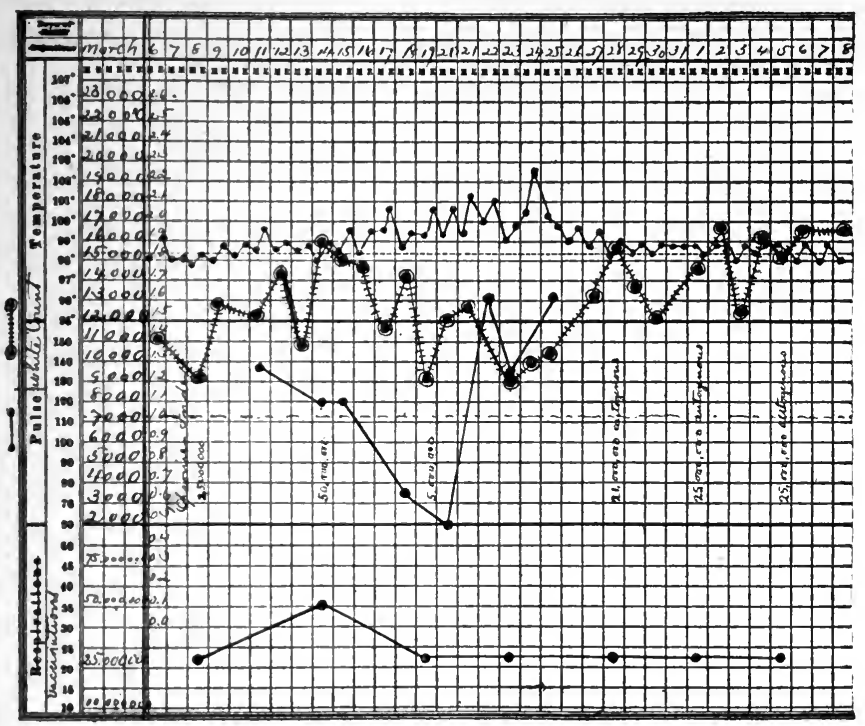

Fig. 58.-Chart of case $I 79$. The uppermost line represents the temperature; the next is the leukocyte count; the third stands for the opsonic index, and the fourth for the dose of vaccine.

after which it rose and stayed high. Its variations are shown in the accompanying chart. On April 7 th he was walking about without any difficulty, and on the ninth he was discharged much relieved.

Diagnosis.-Gonorrheal arthritis.

\section{Case 180}

A colored man of sixty-four entered the hospital July II, I907. His family history is negative. He stated that he almost died of a "bad cold" at fifteen, that he had had spinal curvature since he was thrown from a horse at fourteen. In the eighties he was at the Boston Insane Asylum for a time. 
Since spring his right hip has pained him, and for the last three weeks the pain has been so severe as to interfere with sleep, and when he wakes there is much pain and stiffness in both legs, though it wears off considerably with exercise. Three weeks ago his feet were swollen for some time. This has now gone. He drinks much water and usually passes urine three or four times at night. His bowels move every day or two, and only with medicine.

The movements of the patient's pulse, temperature, and respiration are seen in the accompanying chart. At entrance his white cells were 7700 , but a differential count showed that 90 per cent. of these were

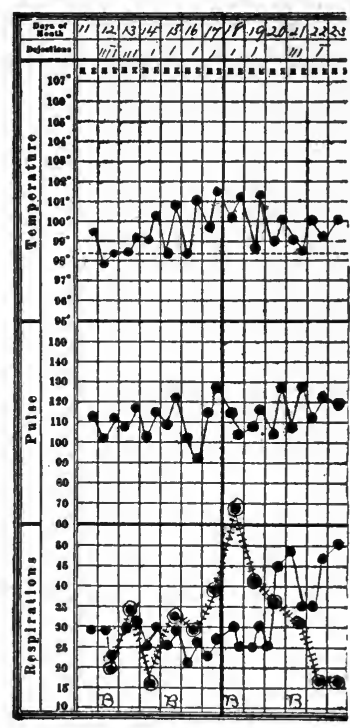

Fig. 59.-Chart of case 180 . polynuclear. There was no anemia. The spine showed scoliosis, resulting in a marked prominence of the ribs of the left back. There was an old bony deformity of the right elbow-joint, which was stiff. He was poorly nourished. There was marked arcus senilis. The heart showed nothing of interest. The radial arteries were tortuous and stiff. The front of the chest was negative except for a few fine râles over the right clavicle. Behind, the right chest was dull below the spine of the scapula, with diminished or absent breathing; the left back was full of moist râles. The abdomen showed slight tenderness in the region of the gallbladder. There were glands the size of walnuts or almonds in the groins, axillæ, and neck. There was practically no motion in the spine. The urine averaged about 35 ounces during his stay in the hospital, with a specific gravity of Ior 5 , a slight trace of albumin, and very many hyaline and fine granular casts, with cells adherent, some of which were fatty.

On the fourteenth the chest was tapped and 27 ounces of fluid removed, with a specific gravity of I0I5, albumin, 2 per cent., lymphocytes, 8I per cent. The sputa showed nothing remarkable.

From the seventeenth of July until the twentieth he was delirious.

Discussion.-There appear to be many widely diverging clues in this case. The history gives us hints of psychic stigmata, of tuberculosis, of renal or cardiorenal disease, of multiple arthritis and multiple adenitis. Certainly it is a difficult case to untangle. We seem to have reasonably good evidence of a chronic interstitial nephritis. The 
nocturia, the swollen feet, and the character of the urine point in this direction, but it is practically certain that he has something else the matter with him.

On the other hand, that "bad cold" which he had at fifteen, following immediately upon the spinal trouble, which appears to have resulted in a rigid spine, makes us very suspicious of tuberculosis, especially as the symptoms occur in a colored man. The effusion in the right chest (evidenced by dulness and absent respiration) may be due either to tuberculosis or to mechanical causes (dropsy). The low specific gravity inclines me to believe that the fluid is not a pure exudate. The multiple adenitis is not inconsistent with tuberculosis, though it might also indicate syphilis. All types of leukemia are excluded by the blood examination.

That some infection has invaded the patient's body seems indicated by the continued fever and the delirium. We might suppose that this is a terminal sepsis due to the streptococcus or some other of the common terminal invaders, the rest of the symptoms being then explained under cardiorenal disease. But this would not account for the stiff spine, the stiff elbow-joint, the general glandular enlargement, and the early history.

A positive diagnosis seems impossible, but more facts can be accounted for by assuming a tuberculous infection than by any other hypothesis. As a matter of fact, however, this diagnosis was not made.

Outcome.-He became comatose on July 2 oth and on the twentythird he died.

Clinical diagnosis: Arteriosclerosis; chronic nephritis; pleural effusion; terminal infection. Autopsy showed old tuberculosis of the spine; tuberculosis of the kidneys; tubercular ulcer of the ileum; miliary tuberculosis of the bronchial lymph-glands, with suppuration; tuberculosis of the lungs, liver, spleen, kidneys, and epicardium. The guinea-pig which was inoculated with 25 minims of the sediment of the pleural effusion was killed August $23 \mathrm{~d}$ and showed no evidence of tuberculosis.

Diagnosis.-See last paragraph.

\section{Case 181}

A housekeeper of thirty-one entered the hospital November 4, I907. Her family history was negative. She had been operated upon at the Massachusetts General Hospital for stone in the right kidney in 1903, but no stone was found. All the summer of 1907 she had been run down, 
had been easily nauseated, and had vomited frequently. The vomiting had sometimes been brought on by worry.

For five weeks she has been tired, restless, and overemotional. Appetite and sleep have been poor. Three weeks ago she first noticed that she limped, favoring the right leg. This limp has steadily increased, and for the past two weeks she has been constantly in bed. Two weeks ago she began to have sharp pain in her right groin, in the right hip and to some extent in the right lower back. The pain is worse at night and often keeps her awake; it comes in paroxysms, leaving her entirely for a few hours at a time. When tired, she passes urine every two hours or so, but she has noticed no change in it. The course of the temperature is seen in the accompanying chart.

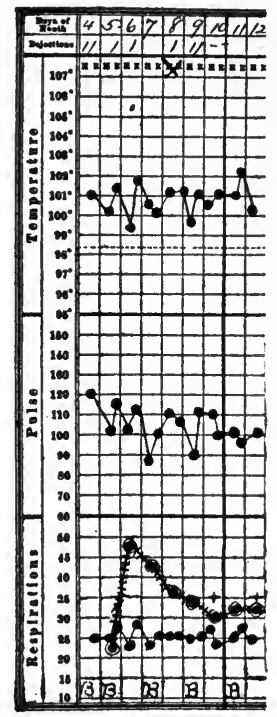

Fig. 60.-.Chart of case $18 \mathrm{r}$.

Examination of the chest was negative.

The abdomen was tympanitic throughout and held more rigidly on the left than on the right. On deep palpation there seemed to be some tenderness on the right. The right leg was kept continually flexed upon the body. Extension of the hip-joint or outward rotation was painful; other motions were good. The scar of the previous operation was seen in the right flank. On deep inspiration a rounded, tender mass could be indistinctly felt in the right flank.

Examination by an orthopedic consultant convinced me that the psoas contraction was not due to any hip lesion. The kidney and the mesenteric glands were suggested as possible causes.

On November $5^{\text {th }}$ and $7_{\text {th }}$ the urine showed a large amount of pus in the sediment; a very slight trace of albumin; specific gravity, ror3; the amount, about 40 ounces in twenty-four hours.

Discussion.-In this and the succeeding case we are dealing with a hip pain associated with a psoas spasm. There seems no evidence that the hip-joint or spine is involved. One looks accordingly for the other and less common causes which lead to contraction of the psoas. Deep tenderness on the right side of the abdomen, associated with fever and psoas spasm, is a well-known feature of appendicitis. But appendicitis rarely begins with a limp before there is any right iliac pain. It should produce some muscular spasm of the abdominal wall, but there is none of this here, nor is there any localized tenderness or "cake" over the appendix region. 
Tuberculosis of the mesenteric gland and occasionally other causes of mesenteric adenitis may lead to psoas spasm. Such a diagnosis is hard to make, harder still to deny. One inclines toward it if there is nothing to suggest any other recognized cause of psoas contraction. Probably adenitis accounts for some of the mysterious cases of "idiopathic" or "hysteric" spasm of the psoas. Psychic causes are often invoked when our diagnostic resources are exhausted.

Various kidney lesions (hematogenous infection, perinephritic abscess, tuberculosis, stone) have been known to bring about a contraction of the psoas. This patient has pus in the urine, and an investigation of the kidney is, therefore, of the first importance.

Outcome.-On November Ioth the flexion of the leg had become more marked. The patient ate and slept poorly. Three $x$-ray plates were taken. They showed apparently two renal stones on the right.

Operation November I6th showed two stones and a little pus in the kidney. Even under deep anesthesia the leg could not be extended, but later, in convalescence, this spasm entirely disappeared and she walked well.

Diagnosis.-Psoas spasm due to nephrolithiasis.

\section{Case 182}

An Italian hod-carrier of thirty-two entered the hospital June 26, 1906. Three weeks ago, while carrying bricks on a ladder, he felt a peculiar sensation in the left hip, described as "throbbing" (probably clonic spasm). Since then there has been pain in the hip, with marked stiffness, the pain being increased on motion.

Visceral examination (including blood and urine) was negative. The left thigh was partly flexed, and could not be straightened without pain. Flexion and rotation caused no pain. There was no other obvious spasm and no tenderness. The left groin was slightly fuller than the right. $X$-ray showed no sign of hip-joint disease, renal disease, or of aneurysm, which had been suggested by Dr. Goldthwait in the out-patient department; although there was greater pulsation in the vessels of the affected side, the temperature in both legs was the same. There was slight dulness in both flanks, not shifting on change of position.

Tuberculin was given, but no rise of temperature followed.

On July ist Dr. Goldthwait thought that some fibers of the iliopsoas were probably ruptured.

Discussion.-In many respects this case resembles the last. In studying it we interrogate, by means of physical examination-(a) The 
hip-joint; (b) the spinal column; $(c)$ the appendix region; $(d)$ the renal region and the urine. We consider enlargements of the mesenteric glands, always so easy to include and so hard to exclude in cases of this type. We look for evidence of abdominal tumors or aneurysm of the aorta.

In the present case we are able, apparently, to exclude all these possibilities except tabes mesenterica, and this, in view of the negative tuberculin reaction, seems very unlikely. Since there is no reason for accusing the stolid Italian laborer of the "vapors," we have to fall back upon a hypothetic strain involving the psoas. There seems no reason, a priori, why this muscle may not be subject to strain or sprain like any other, but it is obvious that, until we have followed our patient far into convalescence, we cannot place any reliance on such a diagnosis.

Outcome.-By July gth the patient was walking well, without limp or pain. Uninterrupted recovery followed, apparently as the result of the magnificent air which he breathed in the surgical wards of the Massachusetts General Hospital.

He was given no other treatment.

Diagnosis.-Psoas tear (?).

\section{Case 183}

A beef-carrier of fifty-three entered the hospital January 29, I907. His family history is negative. He has never been sick until the present illness, but has been in the habit of getting drunk once to three times a week. Two weeks ago he woke in the night with a pain in the right hip. Since that time he has been confined to bed with pain and fever, wandering in his mind, and constant twitching of the arms. His wife says he has had no alcohol for two weeks. He has been treated for lumbago and for diabetes. Later it was learned that five years ago he had had some abscesses on his neck which discharged for a year. They were finally cured by an extensive operation.

Physical examination showed good nutrition, but the patient's mind was cloudy, though he would answer simple questions. All his muscles were held rigidly, especially those in the neck and arms, but there was no paralysis. The pupils were slightly irregular, but reacted normally. The eye motions were normal, the chest and abdomen negative. The white cells were 13,000 ; the Widal reaction suggestive, but not positive; the blood otherwise normal, likewise the urine. Marked subsultus was the most prominent feature. At entrance the case was taken for an acute abdominal emergency and immediate operation was 
urged. On the second day the patient became unconscious, with profuse sweating.

Discussion.-Hip pain, fever, and delirium are the presenting symptoms. The character of the delirium suggests alcoholism, but two weeks' abstinence from alcohol should have steered him past the danger of delirium tremens. The general muscular rigidity, moreover, the hip pain, and the irregularity of the pupils could not be thus accounted for.

The mental condition, the muscular twitchings, the fever, and suggestive Widal reaction furnish us with some of the material whence a diagnosis of typhoid might be built up. But the leukocyte count is remarkably high for that disease, and we should still be left without an explanation of the hip pain, the muscular rigidity, and the condition of the pupils.

Rigidity of the neck in a febrile patient always makes us fear meningitis, and all the other facts in this case go to strengthen this hypothesis. If he had been treated for diabetes, as the history states, he has probably had sugar in his urine. Transient glycosuria is not uncommon in meningitis of any type.

But if he has meningitis, can we in any way explain the hip pain? Certainly not by the epidemic or aural type of meningitis, but meningeal tuberculosis might well originate in a tubercular hip, the probability of which is increased as we note that he has had chronic discharging

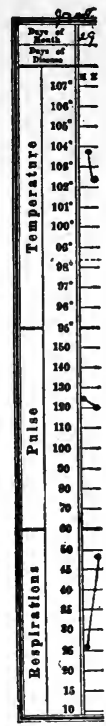

Fig. 6r. Chart of case 183 . abscesses of the neck, presumably tuberculous.

Outcome.-He died on the thirtieth of January. Autopsy showed tuberculosis of the bodies of the fourth and fifth lumbar vertebræ, with large psoas abscesses; tubercular meningitis; tuberculosis of the retroperitoneal glands; obsolete tuberculosis of the left apex.

Diagnosis.-Pott's disease with psoas abscess. General tuberculosis.

\section{Case 184}

An architect of thirty entered the hospital May 3, I907. His family history, past history, and habits are good. Five weeks ago, while jumping to catch a base-ball, he felt a sharp pain in the left hip. He got home with difficulty, and has been in bed ever since, suffering almost continual pain in the left hip and along the back of the thigh. Opiates have been necessary to produce sleep, and even then only a few hours' sleep at a time has been obtained. The pain has never been 
in the back and has gradually diminished in intensity, but the patient is still unable to walk or to put the foot to the ground. The left thigh is held slightly flexed, and there is a tender point two inches outward and upward from the tuberosity of the left ischium. There is also tenderness along the course of the sciatic nerve, but none over the sacroiliac joints. It was afterward learned that five years ago he had a similar attack, following bicycling; he was then laid up for five weeks. Later he brought on another attack by jumping while playing tennis.

Discussion.-As in the previous case, the presenting symptom is sciatic pain, but here its origin is not insidious and obscure, but abrupt and apparently traumatic. In studying it we must go through the same series of investigations intended to bring to light any cause for pressure upon the nerve (pelvic tumors, bony outgrowths from the femur, spinal osteoarthritis, sacro-iliac displacement) and any metabolic disturbance, such as diabetes, whereby a toxic neuritis or neuralgia might arise. [It should be noticed in passing that no one seems adequately to have investigated the possibility that diabetic sciatica may be due not to a chemical cause, but to muscular weakness, destroying the support of the pelvic articulations. Certainly toneless, flabby muscles play an important part in many cases of sacro-iliac trouble.]

Many cases of sciatic pain seem, like the present one, to begin after an injury which is usually of the type here described, i.e., a wrench such as might bring about violent extension of the hip-joint and possibly some strain or stretching of the sciatic nerve. It has been more frequently assumed, however, in recent discussions, that the trauma has affected the sacro-iliac joint primarily, the nerve only secondarily. This seems to me to be a matter rather of fashion than of reasonable conviction.

Outcome. $-X$-ray showed no evidence of spinal involvement or of sacro-iliac disease, and an orthopedic consultant considered the case one of "simple sciatica." From the time of entrance until the thirteenth of May he was treated, chiefly with a view to relieving the pain, by means of ice-bags, hypnotics, and an occasional dose of morphin. On the thirteenth he was given hydrotherapy and Zander treatment, which within a few days produced remarkable improvement. On the seventeenth he was discharged, much relieved.

Diagnosis.-Sciatica.

\section{Case 185}

A farm hand, thirty years old, entered the hospital February I6, I907. Three years ago he had a compound fracture of the right thigh. $\mathrm{He}$ 
was in bed seven months, and has had half an inch of shortening in that leg ever since. After being out of bed about a month, he had an attack of what was called "sciatic rheumatism," which, so far as he remembers, was exactly like his present illness. He was then confined to bed for two months and was treated by electricity and drugs. He denies venereal disease, takes about 25 cents' worth of beer and whisky a week, and chews ro cents' worth of tobacco a day.

Three days ago, without any known cause, he felt a sharp pain in the right hip-joint. This pain has continued ever since, is worse on motion or pressure, radiates down the back of the leg to the ankle, and is accompanied by a burning sensation, also described as like electricity. He has never any pain in his back. He worked until last night, but then the pain was so severe that he was unable to sleep, even with morphin. This morning for the first time he noticed blisters on the leg, due, he thinks, to a poultice.

Physical examination of the chest and abdomen was negative, except for a sausage-shaped mass in the left iliac fossa, which disappeared in the course of a couple of days.

The knee-jerk was very active on the left, less so on the right. On the left buttock was a series of vesicles filled with straw-colored fluid. On the right, opposite the upper part of the sacrum, and over the thigh, in the region of the great trochanter, was a line of ruptured vesicles. Pressure over the sciatic nerve, especially near its exit from the pelvis, in the popliteal space and in the calf, was painful. Sensibility was normal. There was no tenderness over the spine or pelvic bones. Rectal examination was negative. The pain was excruciating in all positions, and was very little affected by morphin. Ice at times gave slight transient relief. After the twenty-second the pain became more bearable, following the administration of three grains of quinin every two hours until the ears rang. Static electricity seemed to increase the pain. Aspirin did not help at all.

Discussion. - The history of pain coming on for the first time soon after a severe fracture of the femur naturally directs our minds to the possibility that by the callus formed at the site of fracture, pressure may be exerted upon the sciatic nerve or adhesions formed involving it. The difficulty with this supposition is that the patient has been free from pain for over two years, although nothing has been done which would remove adhesions or alleviate pressure. Possibly there may be some less direct connection between the fracture and the present pain, but it is difficult to get beyond the region of conjecture. Only by $x$-ray examination and rectal palpation can we get any further evidence in this direction. 
Any sciatica which involves both legs is very suggestive of pelvic new-growth. In this case we have apparently a bilateral herpetic eruption, the usual manifestation of a lesion of the ganglion with its corresponding nerve-root. The pain, however, is unilateral, and we have no definite evidence to support the idea of pelvic new-growth.

In every case characterized by sciatic pain we should remember that diabetes is one of the commonest causes for such pain. There is no statement about the urine in the above record of this case, and evidence should certainly be sought in that direction.

Largely through the influence of Dr. J. E. Goldthwait the medical profession has now learnt to search for osteoarthritis of the lumbar spine or for some lesion of the sacro-iliac joint in all cases of sciatic pain. The nature of the connection between the pain and the bone lesions has not, I think, been fully explained as yet. Most of the important evidence of such a connection consists in the results of a therapeutic test-fixation of the spinal and sacro-iliac joints by strapping, belt, or plaster-of-Parisand on the relief of symptoms following such fixation. This is of great practical importance, but does not answer all the questions regarding the mode of production of sciaticas thus relieved. In the present case we find no evidence of spinal or sacro-iliac disease.

The term "sciatic rheumatism" is now happily falling into disuse, and with it, I believe, will soon go out of existence the hoary and overworked theory that cold produces such troubles. Doubtless it was their connection with joint lesions such as those just referred to that first suggested the term "rheumatic," with the theory of cold as the cause. In view of the negative result of all the examinations directed toward finding a cause for the pain we shall be obliged to leave it as an unexplained symptom (" primary," "idiopathic," or "simple" sciatica). Since it is associated with herpetic eruption, and since we know that many cases of herpes are due to infectious disease, it is fair to surmise that the neuritis with which we are now dealing may be of the infectious type. All this, of course, presupposes that the results of urinalysis and $x$-ray examination are negative.

Outcome. $-X$-ray of the femur showed a large callus with a projecting spicule, but as there had been no pain for two years, this seemed probably not responsible for the pain. Dr. J. J. Putnam considered the case neuritis with herpes zoster. Dr. Goldthwait agreed.

On the twenty-fifth the patient was discharged much relieved.

Diagnosis.-Neuritis with herpes zoster. 


\section{Case 186}

An Italian pressman of forty-five entered the hospital March 26, I906. Three weeks ago he gave up work on account of pain in his hands and feet, which has been severe ever since, and has recently kept him awake. His appetite is poor and he has vomited several times. He attributes his pain to the fact that he gets very wet with perspiration at his work and then rides home upon a car. He got very cold in this way, just before the present illness.

The course of the temperature is shown in the accompanying chart. There was soft edema of the backs of both hands. The right wrist

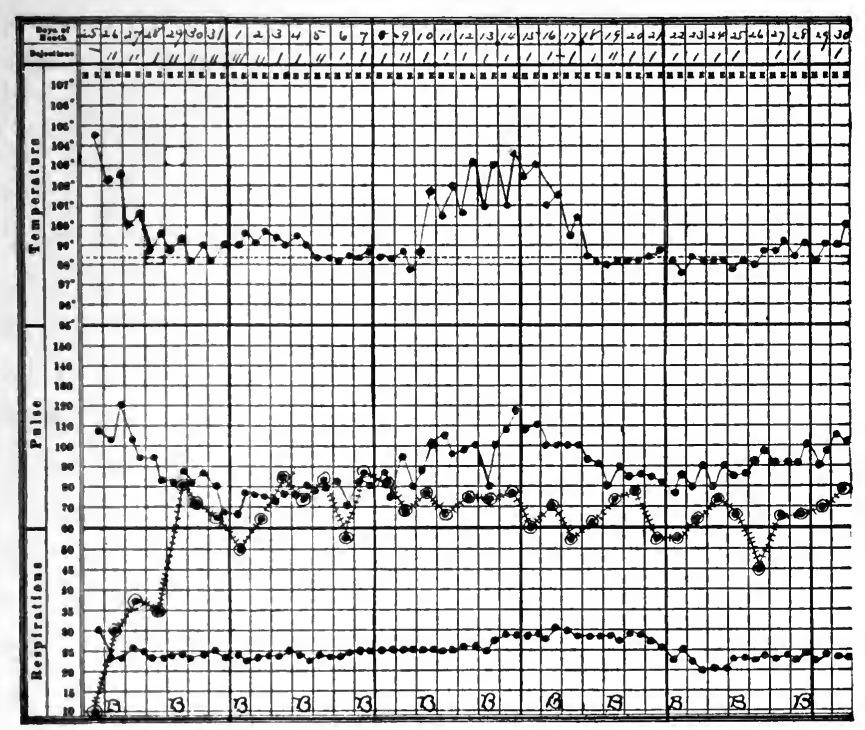

Fig. 62.-Chart of case 186.

and left elbow-joint were swollen, slightly stiff, and tender. There was tenderness on moving the fingers, and convincing evidence of fluid in the left knee-joint.

Both ankles were somewhat red, swollen, and tender. There was tenderness on pressure in the calves of the legs and over the muscles of the forearm, a bright red macular rash over his back, with small, shiny papules scattered through it, and in the arm-pits numerous small, discrete, transparent vesicles. He was seen by Dr. Goldthwait on the twelfth of April. He found at this time an infectious process, chiefly in the cellular tissue, with very little involvement of the joints.

The temperature fell to normal about the twenty-eighth of Marcn, but on the thirty-first the patient was delirious and chattered a great 
deal in the night. After omitting the salicylates, which had been given steadily up to that time, the delirium cleared up within twelve hours. The knee-jerks were present, but the Achilles reflex absent. The eyes reacted better to accommodation than to light. The urine averaged 60 ounces in twenty-four hours, the specific gravity varying very widely between 1009 and I020. Hyaline and granular casts were numerous, and there was always pus in the sediment. The blood showed I2,800 leukocytes, 8I per cent. of which were polynuclear.

On April 4th the swelling of the hands still continued. No obvious change in his condition accompanied the fever of April roth to Igth.

Purulent conjunctivitis was present throughout his stay in the hospital. The smear showed no gonococci; a variety of other organisms were present. May ist he was discharged, not relieved.

Discussion.- Judging from the condition of the pupils and of the ankle-jerks, there seems reason to believe that this patient has tabes, but evidently that is not his most important malady at the present time, so that our interest centers in the question: What else is the matter with him? We have obvious evidence that an infectious process has invaded the subcutaneous tissues, the joints, and the conjunctivæ. In all probability the pus in the kidney is to be attributed to a genito-urinary infection due to the same organism which is attacking his other tissues.

At one period in the case it seemed as if the meninges, also, were infected, but the immediate cessation of meningeal symptoms when the salicylates were stopped makes it pretty clear that we were dealing with a salicylate delirium, which should always be borne in mind when any delirium occurs during the administration of salicylate in large doses. This is a very frequent occurrence. Indeed, it is impossible to avoid it if we are in the habit of pushing this drug rapidly to its physiologic limit, as we should do in most cases of acute arthritis. No considerable harm results, as the delirium always ceases promptly when the drug is withdrawn.

We have evidence, then, of a very wide-spread infection of the body. Presumably this is due to one of the pus-forming organisms, since we have no definite evidence of tuberculosis, glanders, or syphilis. No further certainty can be arrived at without blood culture.

Milder cases of this type are often called "inflammatory muscular rheumatism" (see above, p. 338), just as the milder septic infections of the joints pass as articular "rheumatism." But in both cases there is no reasonable doubt that we are dealing primarily with an infection of the blood-stream, following which the micro-organisms take root and multiply here or there, following laws of distribution which we do not 
understand. Evidently the joints present especially favorable conditions for the growth and multiplication of micro-organisms. But we see many instances where an infection which seems to start in and to be distributed by the blood-stream gets its only recognizable localization in the heart, lung, kidney, or beneath the skin. I am inclined to think that the gall-bladder, the meninges, the peritoneal cavity, and possibly also the appendix, should be added to this list. I shall return to the further discussion of the types of pyogenic infection in the section on Fevers.

\section{Diagnosis.-General pyogenic infection.}

\section{Case 187}

A clerk of forty-nine entered the hospital January 3, I907. $\mathrm{He}$ had previously been in the hospital in I889, with a diagnosis of acute rheumatism and mitral endocarditis. Since that time he has had many similar attacks. The attacks seem to be brought on by cold, indiscretions in diet, and alcoholic drink. He had syphilis in 1884 , and later on had trouble in controlling the movements of the bowels, following an operation for piles and fever.

At times he has been a heavy drinker. Ten days ago he "got cold" and passed bloody urine. Since then he has had several acute attacks of diarrhea.

On examination his pupils are slightly irregular, but are equal and react normally. Marked pronation of both feet, with flattening of the arches, is noted. The second joint of the right big. toe is immovable, thickened, not red or tender. There is some enlargement of the joints of the fingers and toes. $X$-ray shows thin, eroded areas on the fingers and toes, also some bony outgrowth. The urine shows nothing of note.

Discussion.-What type of arthritis are we dealing with here? The association of the previous attack, in 1889 , with a mitral endocarditis gives us some ground for calling it a rheumatic arthritis, although we cannot be quite sure of the endocarditis, since there are no signs of it at present. It is impossible categorically to deny that a mitral endocarditis can heal, leaving no sign of its presence, but we have no good reason for believing so at the present time. Patients with true rheumatism often attribute their attacks to cold, but rarely to alcoholism or indiscretions of diet. This feature of the history, as well as some others presently to be mentioned, does not fit the ordinary picture of rheumatic arthritis.

Syphilitic disease of the joints is not at the present time a very sharply defined clinical entity, but the cases on record have not been 
characterized by such a tendency to recurrence and speedy recovery as have occurred in this patient.

Since the arches of the patient's feet are markedly flattened, we must consider whether this deformity is a cause or result of his symptoms. The periodic and paroxysmal character of the patient's sufferings is not at all characteristic of mechanical weakening of the arch. Ordinary flat-foot is apt to cause pain until it is relieved by treatment. It does not appear and disappear so suddenly. Against flat-foot also is the presence of eroded areas and bony outgrowths, as shown in the $x$-ray plate.

But although flat-foot is very unlikely' as a cause of this patient's troubles, it may well be viewed as a result of them, since almost any form of arthritis affecting the joints of the foot may be followed by flat-foot which remains as a cause of weakness and pain after the inflammatory trouble has passed. Thus it comes about that many cases of true arthritis of rheumatic or other origin are best treated, when they reach the doctor, by flat-foot plates and exercises designed to strengthen the adductors of the foot. The inflammation has passed, and its sequel is mechanical weakening, not an infectious process.

The $x$-ray evidence, the thickening and stiffening of the right big toejoint, and the apparent relation of the symptoms to indiscretions in diet suggest gout. Nothing is said in the history of acute night-attacks of pain in the great toe, nor of the presence or absence of tophi. But further inquiry showed that both these gouty symptoms were present. Still unexplained is the relation between the gouty diathesis and the bony outgrowths seen in this and other cases of gout, as well as in the hypertrophic form of arthritis.

Outcome.-On the fourth of February the patient was discharged quite free from symptoms. Tophi were still present in his ears, and crystals of sodium biurate were obtained both in this attack and four years previously.

Diagnosis.-Gout.

\section{Case 188}

A housewife of twenty-nine entered the hospital January I4, I908. She was delivered of her first child on December 2d, but previous to that delivery she had much pain, owing, as she supposed, to a partially retained placenta. She was douched and cureted twice a day until she decided to get a new doctor. The second physician omitted the cureting. She has since been better.

Two days after delivery both legs becáme swollen, and were still so when she was seen January 14 th. On entering the hospital she com- 
plained bitterly of pain in the left buttock. Physical examination showed nothing but moderate jaundice and a bed-sore over the left sacroiliac joint. The white count was 15,800 ; two days later, 38,200. On the second day after entrance she began to be delirious, and this continued twenty-four hours, after which she was more rational, but had occasional hallucinations at night. There was marked dulness throughout the lower abdomen. The uterus was soft, flabby, and somewhat tender, but there was no vaginal discharge.

By the sixteenth the edema had practically disappeared from the right leg, and was less in the left. A blood culture was taken, which showed no growth. Nevertheless, antistreptococcus serum was injected. The urine as drawn by catheter was bright green, but showed no other striking abnormalities. There was some tenderness in the left groin, but no other evidence of thrombosis. By the eighteenth this tenderness had increased and there was considerable fulness in the same region.

Discussion.-Fever occurring after childbirth and accompanied by jaundice, by marked leukocytosis, and by pain in the left buttock and groin, points to the existence of some deep-seated septic process originating in parturition. Though there is edema in both legs, we find no good evidence of peripheral thrombosis. Pelvic

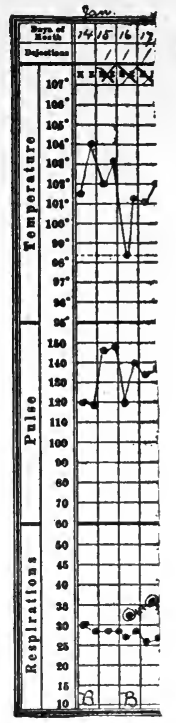

Fig. 63.-Chart of case 188 . thrombosis possibly, or some other cause for pelvic obstruction to the circulation, is our natural conjecture, since all the other symptoms appear to originate in the pelvis. ${ }^{1}$

The green color of the urine is presumably due to biliverdin, a result -like the yellowing of the conjunctiva-of hemolysis. Nothing more definite can be said as to diagnosis. Pelvic sepsis we doubtless have; its form, extent, and origin can only be revealed by surgery or by the lapse of time.

Outcome.-Incision allowed the escape of 25 ounces of pus, the source of which was extraperitoneal and apparently extended back to the region of the left sacro-iliac joint. A culture showed streptococcus. The patient died a week later.

1 Any one who has seen postmortem the condition of the uterine and the periuterine tissues in the days soon after a normal labor cannot but wonder how any woman escapes sepsis and embolic infarctions of the lung. 
Autopsy showed several fractures of the pelvic bones, deep burrowing pus without obvious point of origin, and streptococcus septicemia.

The course of the temperature is seen in the accompanying chart.

Diagnosis.-Fractured pelvis and sepsis.

\section{Case 189}

A medical student of thirty-three entered the hospital March 2, I907. On February 2ist his left great toe-joint swelled up, but the swelling was gone the next day. He then began to have pain and stiffness in the left hip. This has gradually increased ever since. Yesterday

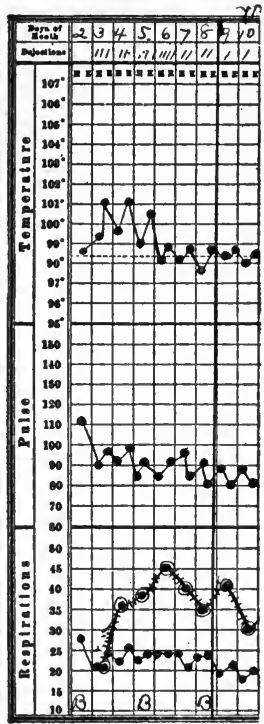

Fig. 64.-Chart of case $\mathrm{I} 78$. it took him twenty minutes to walk three blocks. No other joint has been affected. Any hip motion causes pain down the back of the leg. The greatest tenderness is over the tuberosity of the ischium.

At the onset of his symptoms, hives appeared at night all over his body, some of the lesions being as large as half a dollar. They always disappeared in the day-time. For the past two days he has not had them.

Physical examination was negative, except that all motions involving the hip-joint caused intense pain extending from the tuberosity of the ischium down the back of the leg.

Rectal examination showed marked tenderness on the right, but no mass or fluctuation. The case was considered an ischiorectal abscess by the surgeons. To an orthopedic consultant it appeared to be an infectious arthritis of the hip.

The white count at entrance was 27,400 , with 89 per cent. of polynuclear cells; on the fifth there were 15,000 ; on the twelfth, 9000 .

The course of the temperature is shown in the accompanying chart.

By the tenth of March the pain and tenderness were much less and the motions of the thigh freer. By the thirteenth he was almost free from symptoms and was able to walk about. $X$-ray was negative. Hot fomentations and sodium salicylate helped him very much in the early days of his illness.

He was discharged on April gth well.

Discussion.-Pain and tenderness in the hip following a similar pain in the toe a week earlier are the presenting symptoms here. The hip pain has sciatic radiations, and is accompanied by fever and leuko- 
cytosis. No source of infection is obvious. There has been no injury to account for the symptoms.

Our first business is to examine the hip, sacro-iliac joint and spine. As a result of this search it seems that only the hip-joint is affected, the sciatic pain being doubtless secondary to this. What, then, is the infection of the hip? Tuberculosis, the commonest of hip infections, has rarely so acute an onset, and usually occurs in younger persons. The high white count, the hives, and the acute brief pyrexia seem more like some pyogenic infection. We have no positive evidence of gonorrhea or of any other infection from without.

The marked tenderness over the tuberosity of the ischium and on rectal examination suggested a deep ischiorectal abscess, especially as the leukocyte count was so high. There is no way by which this diagnosis can be excluded, though it is rare to see such an abscess clear up without breaking or being evacuated externally. It is a well-known fact that some cases of acute arthritis at the hip produce pain in the situations complained of by this patient. In view of these facts and of the favorable course of the disease without external manifestations of abscess it seems most probable that the case was one of acute arthritis of unknown origin, such as usually receives the name of "rheumatism."

Diagnosis.-Acute infection of the hip.

\section{Case 190}

A widow of forty-five, with a negative family history, passed the menopause two years agọ. She is a heavy drinker. Has been strong and well, but in the past two years has lost 36 pounds. She now weighs 90 pounds.

She has had a cough since last fall, with a grayish sputa. Has been unable to work for a year. In bed most of the last five weeks, because of pain in both legs and hips. Bowels move five or six times a day for the past six months. She entered the hospital August I5, I904.

Examination.-Left pupil larger than the right, and reacts to light but not to accommodation. Ptosis of right eyelid. At left base behind and in the left axilla the breathing, vocal and tactile fremitus are diminished, with slight dulness and many fine, crackling râles. Heart negative. Considerable tenderness in the whole belly. Dulness in the right hypochondrium and flank, shifting slightly on change of position.

Mass felt bimanually in this region. It is movable anteroposteriorly and with respiration, and is apparently continuous with the liver. Navel flushed. Lower abdominal veins prominent. Slight edema of the belly- 
wall and of feet. Liver dulness reaches from the fifth space to four inches below the ribs. (See Fig. 66.)

Temperature, IoI $^{\circ}$ to I0 $^{\circ}$ F. (see Fig. 65). The white cells were 53c0. Urine negative.

On the third day after entrance fluid was found in the belly. The Widal reaction was negative. On the sixth day she had three hemorrhages $-\frac{1}{2}$ pint each-from the bowel.

Discussion.-The past history is of special importance in the interpretation of these symptoms. It is to be noted that a woman not previously subject to cough has now coughed steadily for nearly a year, and lost continually in weight, though she is at the menopause. As-

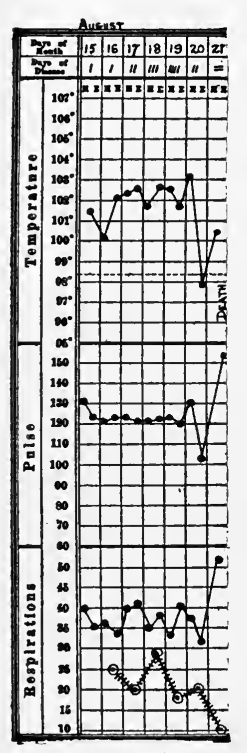

Fig. 65.-Chart of case 190.

sociated with her cough the chief symptoms have been diarrhea and leg pain.

Summing up the physical examination, we may say that there are indications of very wide-spread lesions; the ptosis and pupillary changes indicate something wrong at the base of the brain. The lung signs must be interpreted (in the absence of cardiac or renal abnormalities and in the presence of fever) as pleurisy with effusion or thickening. Finally, below the diaphragm, there are evidences of pressure exerted apparently upon the vena cava and its radicles (as well as upon the spinal nerve-roots), by the mass figured in the diagram. The liver also seems to be much enlarged.

Cancer, syphilis, or tuberculosis are the three diseases most capable of producing symptoms distributed through the body as widely as those in this case. Syphilis would account for the ptosis and pupillary changes. If we interpreted the mass below the diaphragm as a syphilitic liver, the edema, ascites and fever would be explicable under the same hypothesis. The pleurisy and leg pains would remain unaccounted for, likewise the prolonged cough. The diarrhea might be due to amyloid disease of the intestine as a result of the syphilis.

Malignant disease of the liver is sometimes associated with fever, and would explain the abdominal symptoms very well, but would not help us to account for the ocular signs, the chronic cough, the pleurisy, or the diarrhea. In the great majority of cases hepatic neoplasm is preceded by marked and long-continued gastric suffering, due to a preceding neoplasm of the stomach. We have no such suffering here.

Tuberculosis involving the base of the brain, the pleura, the intestine, and peritoneum would account for all the facts in this case. Under 


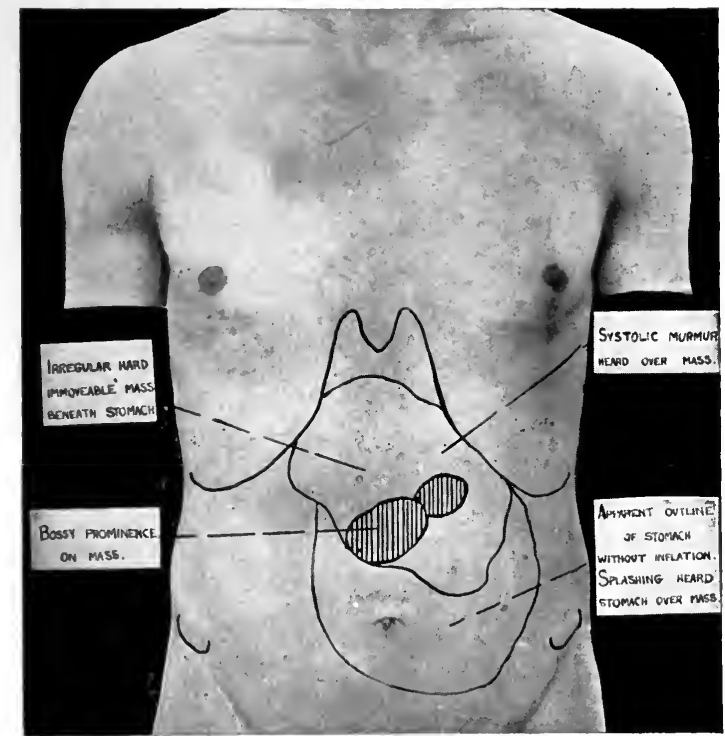

Fig. 66.-Condition of the abdomen in Case 190; bedridden by pain in both legs. 

this hypothesis the intestinal hemorrhages result from ulcerations of the gut, while the mass above the umbilicus represents a conglomeration of caseous glands and adherent intestinal coils. Enlargement of the liver might be due to fatty or amyloid metamorphosis. By strict reasoning this diagnosis seems the most probable.

Outcome.-The patient died August I8th. Autopsy showed extensive tuberculosis of the mesenteric and retroperitoneal lymphatic glands, also of the large and small intestine, with ulcerations evidently the source of hemorrhage. There were long-standing tuberculosis of both lungs and a general miliary infection.

Diagnosis.-Tabes mesenterica. General tuberculosis.

\section{Case 191}

A housewife of thirty-seven entered the hospital October I8, I907. Her family history, past history, and habits are good. For the past four and one-half years she has had frequent attacks of severe pain in the back of the left thigh, running down the leg, preceded often by a mild chill, and relieved after five or six hours of sweating. She is also troubled by nervousness and apprehension, and has worried a good deal since last winter about a prune-stone that she swallowed. She wonders where it is now. Her sleep and appetite are poor, and she has frequent attacks of headache and nausea, with some flatulence after eating and considerable constipation.

Physical examination showed great restlessness; no swelling, tenderness, or limitation of motion in any part of eitherleg. The arches of both feet were found to be much flattened. The rest of the examination, including the pelvis, the blood, and the urine, was negative.

Discussion.- In view of the negative results of a searching physical examination and of general observation under hospital conditions, we seem driven to the diagnosis of a psychoneurosis with flat-foot and sciatica. Only by the continued study and prolonged observation of such cases can we realize the harm done by semiconscious fears based on such an incident as the swallowing of a prune-stone. Especially in persons who have no knowledge of anatomy and physiology, the imagination runs riot in speculation over the possible paths which such a stone might travel. Very great benefit follows in such cases if the patient can be assured, as a result of exhaustive physical examination, that no organic lesion exists.

An element in this benefit is the result of the patient's opportunity to bring to full consciousness, as the result of the physician's questions, the vague and unformed dreads from which he has been suffering. 
As soon as they are forced to take shape, many of these apprehensions are alleviated, as the child's terror is gone when it has recounted its nightmare to its mother. To this familiar psychologic rule the name of the "cathartic method" has been given by Breuer and Freud. The essential point is that ideas or emotions which do the most harm to the body are often the most deeply hidden beneath the superficial layers of consciousness. The patient himself may be altogether unaware of their existence or may manifest his vague cognizance of them only by a systematic refusal to face them squarely, either in his own mind or in conversation with his physician. It is for this reason that the physician must sometimes employ what Freud calls "psycho-analysis" - the effort to find, by a persistent process of drawing the patient out, submerged ideas which resist more or less unconsciously the attempts to drag them to the surface. The process is risky, but occasionally valuable.

Outcome.-After a week's rest and several long talks with her physician, counterirritation to the thigh, laxative medicines and proper shoes, she was discharged much relieved.

Diagnosis.-Flat-foot; psychoneurosis.

\section{Case 192}

A cook of thirty-six entered the hospital March 14, 1907. At irregular intervals for five or six years she has had sharp pains in her arms and fingers, sometimes lasting as long as a week, usually worse in summer. During the last five years she has grown very stout, her average weight being $\mathrm{I} 75$ pounds. Otherwise her past history is good, likewise her family history and her habits. She was perfectly well until eight days ago, when she began to have pain in her heels, later passing around to the front of the foot, but never to the toes nor to the ankles. The pain kept her awake at night, and the foot has been swollen, red, and tender to touch. She has been in bed for the last three days, and seems to have been getting worse.

The patient is 5 feet 4 inches tall, very obese; chest and abdomen are negative; reflexes normal; no tenderness over the joints of the feet. After a few days in bed the patient's pain was gone. There was no fever, and physical examination, including the blood and urine, was otherwise negative.

Discussion.-This seems to be a case of obesity with pain in the feet; the nature of this pain it is our problem to discover. Is it of mechanical or infectious origin? The redness, tenderness and swelling look like infection, but there is no fever or leukocytosis, no involvement of 
any other joints, and experience has shown that even redness and swelling may result from the mechanical causes leading to the acuter forms of flat-foot. We are influenced especially toward the latter hypothesis when we find that there was no tenderness in the foot-joints, but only in the soft parts. The fact that she gets better as soon as she is off her feet is evidence pointing in the same direction.

Very similar symptoms are often seen in gout, but I see no way of coming to any closer terms with this possibility, since we have no tophi, no night attacks of pain in the great toe, and no knowledge of a heredity or habit of life predisposing to gout.

Outcome.-Padding the feet gave temporary relief. Much more permanent benefit followed the adjustment of flat-foot plates.

This case well illustrates one of the indirect evils resulting from obesity. There are many cases of obesity which do not call for treatment by reason of the inconvenience or unsightliness of the fat, but which entail, nevertheless, a genuine risk to the patient. At any time the heart may be slightly weakened or the feet slightly strained by some temporary cause. In the obese the results of these otherwise trivial injuries may be a serious and obstinate illness. During this illness it is rarely wise to attack the obesity. Later, when the acute suffering is past, the patient may be unwilling to submit to the privations entailed by the attempt to reduce his fat. Thus many patients go on from bad to worse. Their good resolutions cannot be summoned at the right time.

Diagnosis.-Acute foot-strain.

\section{Case 193}

A bartender of twenty-nine, with negative family history and past history, entered the hospital January 29, 1908; he has been in the habit of taking 25 glasses of beer a day, and one whisky every morning. For the past six months he has been growing short of breath, and lately has needed two or three pillows at night. He has no digestive symptoms, but rarely eats any breakfast. Five weeks ago he began to notice a swelling of his legs below the knee, accompanied by soreness and stiffness. The swelling disappeared after five days, but he continued to feel poorly and three weeks ago gave up work. Throughout his illness he has had slight cough and white, frothy sputum. Eight days ago he began to have considerable pain in both ankles and the left knee, without any swelling, redness, or fever.

His pains have never been of a darting character.

His eye-sight is good. He has had no headache. For the past eight days he has been in bed. 
As seen by the accompanying chart, the patient had a slight fever the first five days of his stay in the hospital. This was accompanied by a leukocytosis, which on January 29 th reached $\mathrm{I} 7,800$; January 3 oth, I8,800. The urine was sufficient in amount, averaging Ior 7 in specific gravity, with a very slight trace of albumin, but no casts. His pupils reacted well to light and distance. The aortic second sound was markedly accentuated. The heart was otherwise normal, also the lungs. The pulse tension was apparently increased.

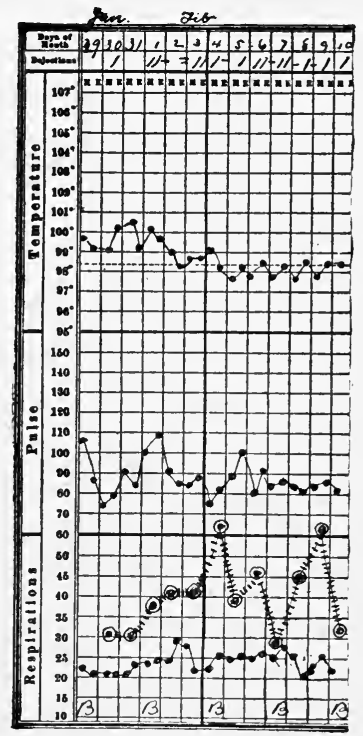

Fig. 67.-Chart of case 193 .
The edge of the liver was felt one finger's breadth below the edge of the ribs, likewise the spleen. There were considerable tremor of the fingers and obstinate insomnia.

Within a few days he began to have pain in both arms, accompanied, as in the legs, by tenderness to pressure, although the reflexes were everywhere normal.

Discussion.-Chronic alcoholism, six months' dyspnea and cough, and five weeks of leg pain are the essential data of the history. Tabes is always to be thought of in men of these habits, but there is nothing in the physical examination to verify this conjecture. Doubtless the great majority of such cases are destined to be labeled "rheumatism," chiefly because they do not present a clear picture of any more definite
no good reason to fall back upon this ancient malady. But there seems no good reason to fall back upon this ancient darkener of counsel when we have no fever and no special tenderness over the joints.

Alcoholic neuritis is the natural explanation of diffuse leg pains occurring in an alcoholic without fever or evidence of local inflammation. But in this as in most cases called alcoholic neuritis, we cannot answer the question, Why is this man stricken at this particular time; Why does the result appear so tardily when the cause has been busy throughout so many years? Doubtless there is some other determining factor of which we are, as yet, quite ignorant.

Outcome.-The patient was given sodium bromid, 20 grains, after breakfast and dinner, and 30 grains at night. Twice he needed $\frac{1}{6}$ grain morphin. For his cough he was given a prescription containing 3 grains of codein, 15 minims of spirits of chloroform, 3 ounces of syrup of wild cherry. Of this mixture a dram was given every two hours 
when the cough was troublesome. On the first of February he was given 15 grains of sodium salicylate four times a day. By the ninth of February he was free from symptoms, but had slight toe-drop and slight tenderness in the calves.

He was advised to avoid alcohol, and went home on the tenth of February.

Diagnosis.-Alcoholic neuritis.

\section{Case 194}

A plasterer of thirty-seven entered the hospital June I2, I907. $\mathrm{He}$ had his first attack of rheumatism at seventeen, when he was sick for several months. He has since then had five or six other attacks, and since his last attack, which lasted a month (five months ago), he has had a weak heart and more or less pain in various parts of his body. He has had urethral discharge off and on since he was seventeen, until five years ago; not since then. He takes from two to six glasses of beer and one or two whiskies a day. Two weeks ago he began to have pain and swelling in his feet and knees, and got transient relief from a Turkish bath. He has also had considerable severe pain in the region of his heart and right lower ribs for the past two weeks. He has had very little fever, but has sweated a great deal. For the past fortnight he has been troubled with many attacks of "hives," which, however, have not bothered him for the last two or three days. Throughout his illness he has had a cough, with whitish, frothy sputa. His appetite is poor. His bowels move twice a day. He has slept fairly well. The course of the temperature is seen in the accompanying chart.

Examination.-The heart's apex was seen and felt in the fourth space, four inches from the midsternum in the nipple-line. There was no enlargement to the right. The sounds were regular and of good quality; the pulmonic second sound accentuated. A blowing, systolic murmur was heard best at the apex, very faintly over the rest of the precordia and in the axilla. The pulses showed nothing remarkable.

The right lung was dull below the third rib in front and below the angle of the scapula behind. Over this area distant bronchial breathing with increased fremitus was detected. Just above the dull area, faint crackling râles were heard. The abdomen was negative. The right knee and shoulder, left shoulder and elbow, were slightly stiff and painful on motion.

No sputum examined. The leukocyte count was 22,000 at entrance, 16,000 on the first of July, I2,000 on the third of July, and ranged lower after that time. The urine was essentially normal. 
Discussion.-We can arrive at no clear conclusion, nor even at any helpful clue, from reading the first paragraph of this record. The patient has had many attacks of arthritis, some or all of which may have been due to gonorrhea, but it is not probable that his present joint pains are gonorrheal in origin, as he has had no local signs of that disease for five years. His other symptoms-cough, sweating, chest pain, urticaria, and anorexia-are very indefinite. Pleurisy is perhaps the possibility most indicated.

On physical examination we find the evidence of multiple arthritis, of solidified lung (right lower lobe), and possibly of mitral regurgitation. All of these might be due to a single infectious agent, such as the pneumococcus or tubercle bacillus. So far as I know there is no good evidence that the gonococcus can produce pneumonia, although it might explain the other lesions from which the patient is suffering. The temperature chart (Fig. 68) is by no means characteristic of pneumococcus infection, nor, indeed, of any other acute infection. It is more suggestive of tuberculosis.

If we are to clear up the diagnosis any further our chief need seems to be a knowledge of the sputa, which should be repeatedly and carefully examined. I have known tuberculous pneumonia to begin with just such a history and with very similar symptoms, including even the joint pains. On the other hand, many of the irregular, low-grade pneumonias, associated with a cardiac lesion and with some organism other than the pneumococcus, present a picture much like this.

Outcome.-The patient was treated by tight chest swathe; I5 grains sodium salicylate every four hours, chloroform liniment, an occasional dose of trional or morphin, and hot applications to the joints. On the seventh of July his only complaint was of weakness. At the right base there was still dulness, but the breathing and fremitus were diminished. These signs gradually disappeared, and he was discharged well on the seventh of August.

Diagnosis.-Pneumococcus arthritis, endocarditis (?), and pneumonia.

\section{Case 195}

A bartender of fifty entered the hospital March 24, I908. Four weeks ago he had an attack of rheumatism in his feet, ankles, and in his shin, just above the ankles. The ankles were swollen, red and tender. He took 5 grains of aspirin every four hours on the fourth day of his trouble, and in a day or two his pain had gone, but ever since then he has been feeling mean and cannot sleep. He still has difficulty 


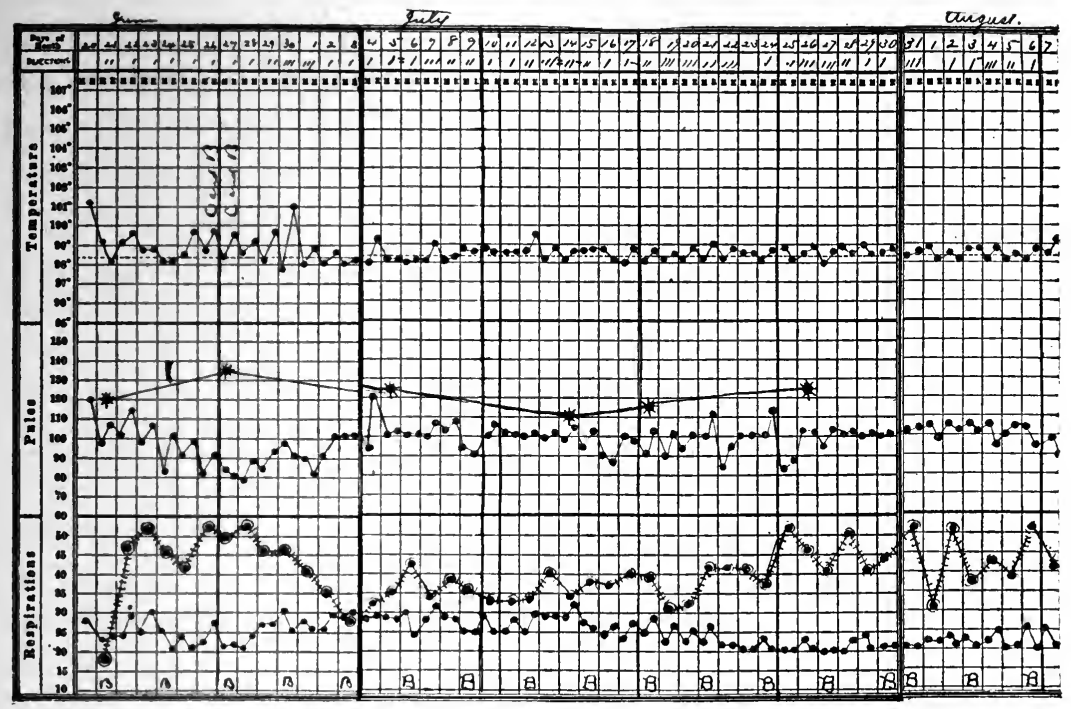

Fig. 68.-Chart of Case I94. 

in walking, but can hop round fairly well. His appetite and bowels are normal. He gets up six or eight times at night to pass water, and thinks he passes more at night than during the day. (This observation was verified during his stay in the hospital.) .

There were various rose-colored macules scattered over the trunk. The pupils were found to be irregular, but reacted normally.

Along the margin in each ear there were some white, firm nodules, the size of a pin's head, resembling sebaceous cysts, but surprisingly hard. The radials were tortuous; pulse of high tension; blood-pressure I75; aortic second sound slightly accentuated. No cardiac enlargement could be demonstrated, and the heart showed no other abnormality. The breathing was slightly harsh in the left back, below the angle of the scapula; otherwise the lungs showed nothing abnormal. The abdomen was normal. There was flattening of the arches of both feet, especially the left; blood and urine were normal, except that the urine was persistently of low gravity,-_or I, - with the slightest possible trace of albumin, but no casts.

Discussion.-Arthritis, hypertension, nocturia, irregular pupils and flattened arches are the main points on which we may be clear from the start in this case. There seems good reason to believe that the patient's kidneys are somewhat atrophic, although no cardiac enlargement can be made out as a support for this hypothesis. The remaining question is: Does flat-foot account for all the rest of his symptoms, or is the weakening of his arches secondary to some form of arthritis? This brings us to the more careful consideration of the nodules on the patient's ears, for any case of doubtful joint lesion, especially in the feet, calls for a careful scrutiny of the aural cartilages. If the nodules on the ear were sebaceous cysts, they would be soft, never hard. Such multiple, firm white nodules along the ear margin may represent the sodium biurate deposits of gout. They may also occur when the ear has been frozen. The crucial test is to ascertain whether we can dig out of one of these nodules a chalky, gritty powder, showing fine, needle-like crystals under the microscope. In the present case we obtained such crystals and our diagnosis was made.

Outcome.-He was given wine of colchicum root, 20 minims every four hours; veronal, to grains, for the first two nights; magnesium sulphate, $\frac{1}{2}$ ounce every morning. By the twenty-ninth his digestive disturbance was gone and he felt much better. The colchicum seemed to produce diarrhea, and was promptly omitted. Thereafter he was given a liberal diet, and by April 2d was discharged, relieved.

Diagnosis.-Gout. 


\section{Case 196}

A widow of fifty-five entered the hospital December ro, 1907. Her family history is good. Fifteen years ago she had cataract in both eyes, and was very successfully operated on, so that now she has very fair vision. As long as she can remember she has passed urine five or six times every night. She passed the menopause two years ago, without event.

A year ago she began to have transitory numbness in the right hip and along the back of the right thigh. Six months ago she began to have a burning pain extending from the right knee to the right hip whenever she remained seated for any length of time. She took osteopathic treatment during the summer, and was assured that her hip had been out of joint, but was now properly set. Nevertheless she did not improve. In July the pain was sharp, shooting, and often kept her awake. Since August it has been very bad until the first of December, since when it has been rather better. When the pain is severe, there is often involuntary twitching of the foot and leg. This was more frequent six weeks ago than it is now.

At present the leg feels fairly comfortable during the night and in the morning, but after she has been up for half an hour or so it begins to feel numb, and in a short time there is a burning and shooting pain which comes and goes through it. The back of the thigh and sometimes the lower leg are markedly tender to touch. There has been no pain in the back, no eruption, no fever. She has been in bed or on a sofa most of the time for the last four months, and has lost about 25 pounds in weight.

The aortic second sound is louder than the pulmonic, and is preceded by a faint murmur transmitted up to the clavicle and down to the third space. Another murmur is heard with the first sound at the apex, but is not transmitted. In the lumbar and dorsal region there is considerable curvature of the spine with convexity to the left. The ribs to the left of the spine are prominent. The abdomen and all the deep reflexes normal. In the right groin there are glands somewhat larger than the average. There is tenderness along the right sciatic nerve and in the right calf. The patient is apparently more relieved by $\frac{1}{24}$ gr. of codein taken from her own bottle, of which she is very fond, than by larger doses of morphin and codein given her in the hospital. There seems to be a large mental element in her suffering.

Discussion.- In seeking the origin of this pain it is natural to think first of the spinal curvature, which has forced the ribs apart on the 


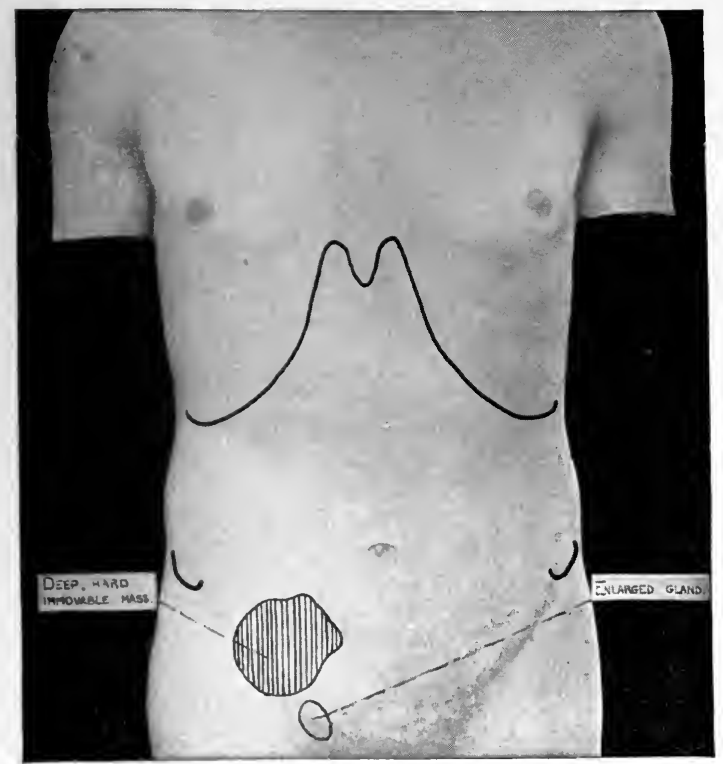

Fig. 69.-Outline of the mass referred to on p. $3^{87}$.

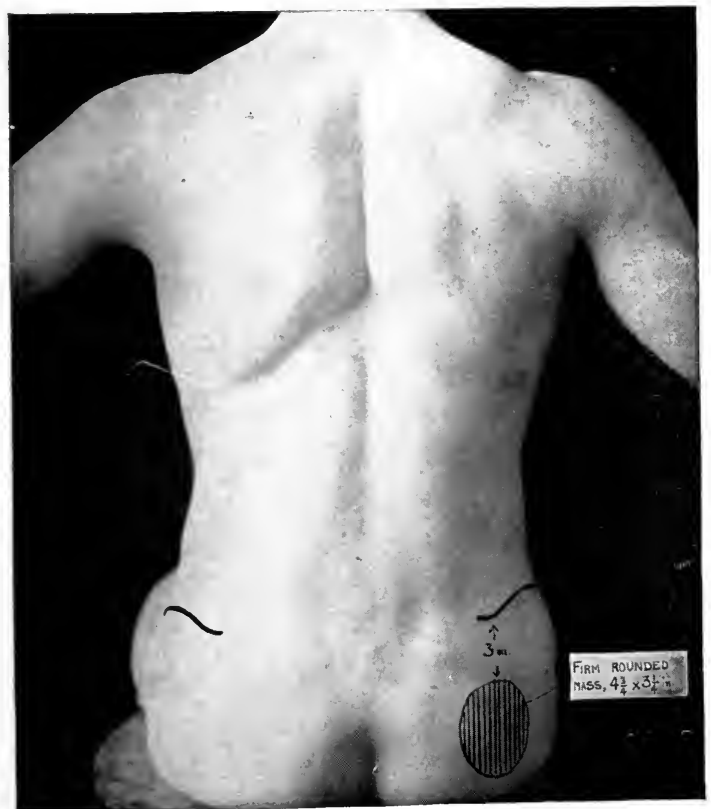

Fig. 70.-Mass apparently the cause of sciatic pain. 

left and jammed them together on the right. But it is hard to see how this could produce suffering confined to the leg. Some of the intercostal nerves would probably be involved.

We next consider the different varieties of arthritis involving the hip, spine or sacro-iliac joint. Infectious arthritis would hardly last so long. Osteo-arthritis would probably cause some pain in the back, and would be unlikely to be worse in the sitting posture. Further, the pain produced by it is hardly ever confined to the leg. $X$-ray examination might help positively to exclude this disease. Sacro-iliac disease seems more probable. Against it, however, is the gradual onset, the age and sex, and the absence of any tenderness, pain or palpable abnormality in that joint.

Some facts stated in the record incline us to believe that the pain may be of the functional or neurotic type. But before one settles down upon such a diagnosis or tries to content himself with calling the trouble a "primary sciatica" the pelvis should be thoroughly investigated for possible sources of pressure. The slight enlargement of the inguinal glands makes such an investigation all the more important.

Outcome. - Vaginal examination showed in the right side of the pelvis a firm mass, tender on pressure, seemingly attached to the pelvic wall (Fig. 69). The right thigh and calf were found to be $\frac{1}{2}$ inch smaller than the left, but there was only slight weakness of the leg; no paralysis.

Later, a large mass was found in the region of the right buttock (see Fig. 70). $X$-ray showed no definite abnormalities. On the second of January one of the glands was removed from the groin, and histological examination showed it to be malignant disease.

On the fourteenth of January the patient was discharged somewhat relieved.

Diagnosis.-Pelvic neoplasm.

\section{Case 197}

A colored scrub-woman of forty-nine, whose husband had previously been treated at the hospital for syphilis, but whose own family history, past history, and habits were not in any way remarkable, entered ine wards December 26, 1907. Since February she has been gradually running down, but worked until four days ago. During these months she has grown very weak and thin. Her meals have been scanty and irregular for some time, and once or twice a week she has vomiting spells, apparently without relation to the nature of her food. Since last winter she has been troubled by cold sensations in the left leg and more or iess constant aching there. For the last two or three months 
she has limped, and occasionally she has been short of breath in going upstairs.

The chest showed nothing abnormal. The edge of the liver was easily felt. The left knee-jerk could hardly be obtained, although the right one was easily brought out. The left Achilles jerk could not be obtained at all. The leg was quite warm to touch, although the patient complained of its being cold. Both legs could be extended more than normal upon the flexed thigh, without pain. There was no tenderness along the course of the sciatic nerve, but slight sensitiveness on firm

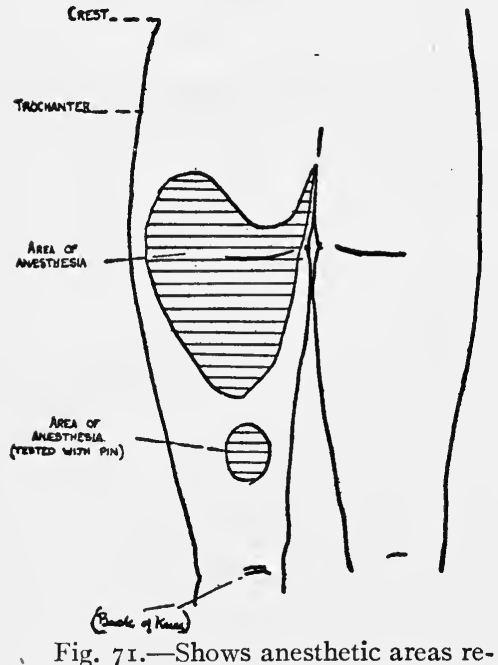

ferred to on p. 388 . Complaints: Aching and paresthesiæ (coldness) in left leg.

pressure over the left calf. An area of anesthesia was found, as shown in the accompanying diagram. Lifting the left leg with the knee stiff caused pain throughout the leg. Lifting the right leg produced no discomfort.

Discussion.-Evidently we are dealing here with a neuritis involving the sciatic and probably other nervetrunks. But as usual in such cases the diagnostician's chief task is to search for a cause for the neuritis. It seems probable that the patient has had syphilis, but syphilitic lesions so localized as to produce a neuritis confined to one extremity do not occur, so far as I am aware.

Tuberculosis is so common in the negro race that it is natural to suspect it whenever a negro is seriously sick. But there seems to be no limitation of motion in any joint and no other evidence of muscular spasm, burrowing abscess, telescoping of joints, fever, or any other result of tuberculosis. The area of anesthesia and the long, steady duration of the pain make it more than ordinarily probable that we are dealing with a pressure neuritis, the position of which must be investigated by radioscopy and by pelvic examination.

Outcome.-Inspection of the cervix uteri shows the cervical canal to be open, $\frac{3}{8}$ inch in diameter and lined with small, projecting nodules. The patient has a slight uterine flow each day, but no foulness. The uterus extends half-way up to the navel. Lifting the straightened left leg causes moderate pain; lifting the right, no pain.

January $7^{\text {th }}$ a nodule was removed from the uterus, and shown by 
microscopic examination to be cancer. Presumably there were metastases in the broad ligament, causing pressure upon the pelvic nerves.

Diagnosis.-Carcinoma uteri.

\section{Case 198}

A colored housewife of thirty-two entered the hospital June 7, I908. Her family history and past history were excellent, her habits good. Since last fall she has had some pain and stiffness, without swelling, in the left knee. On February I3th she fell and injured the knee. Her physician said that she had sprained it. Since then there has been little swelling, but considerable pain. After three days in bed she got up and hobbled around with a crutch, the knee being somewhat stiff, but not painful, until two weeks ago, when pain and swelling commenced and have confined her altogether to bed for the last six days. During the last two weeks she has had occasional night-sweats and nose-bleeds. Her appetite is poor, and her bowels constipated.

The chest and abdomen showed nothing abnormal. The reflexes were all present. The blood and urine were blameless; there was no fever. The left knee was found to be swollen and flexed to an angle of 70 degrees. Its circumference was $I_{\frac{1}{4}}^{1}$ inches greater than the right knee. Most of the swelling was on the anterior surface, and there was a suggestion of posterior subluxation of the lower leg. The skin over the knee was brownish, shiny, and slightly warmer than the right. There was some induration and some infiltration, with moderate tenderness on pressure. All attempts at motion caused extreme pain.

Discussion.-Although there is much in the history pointing to a traumatic cause for this pain, the severity and long duration of the symptoms argue something more serious.

Septic osteomyelitis has generally a more sudden onset, produces severer pain, disability and fever. This patient has had night-sweats, but, so far as we are aware, no fever.

Tuberculous osteomyelitis might produce almost exactly this picture, though it would probably be accompanied by more fever and less pain. After so long a duration one would rather expect some sinus formation, but this does not always occur. Without $x$-ray evidence we cannot either affirm or exclude tuberculosis.

Were there any evidence of spinal disease (tabes, syringomyelia), one might suspect a Charcot joint, though such joints are usually painless. But in this case there is no evidence of the primary disease whence Charcot's joint proceeds.

Malignant disease of the bone-presumably sarcoma-would account 
for all the symptoms in the case. Between this and tuberculosis the diag. nosis must remain in doubt on the basis of the data here presented.

Outcome. $-X$-ray examination showed extensive destruction of the lower end of the femur, with a fracture just above the condyles. June I $3^{\text {th }}$ the leg was amputated for sarcoma of the femur.

Diagnosis.-Sarcoma of the femur.

\section{Case 199}

A Russian tailoress of seventeen entered the hospital July I3, I907. Six days ago her right knee and lower thigh became slightly swollen and very tender. Since then she has felt a little chilly, and has had a poor

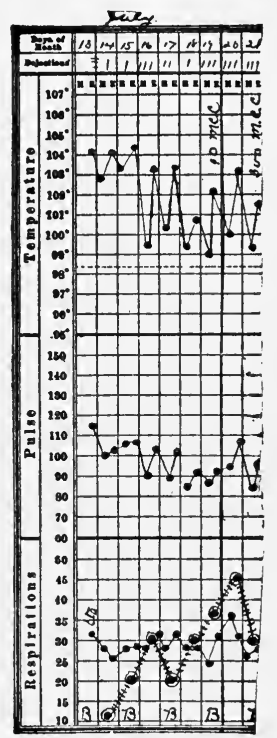

Fir. 72.-Chart of case 199 . appetite, but no other symptoms of any kind.

The course of the patient's temperature is shown in the accompanying chart.

Physical examination of the internal viscera showed nothing abnormal. The right knee was red, very tender, slightly swollen. The white cells ranged between 8000 and II,400. The urine was about normal in amount and in weight. There was no albumin, but a few hyaline and finely granular casts were found. Widal's reaction was negative. On the eighteenth the knee was less tender, but larger and the thigh was also swollen.

Bier's treatment was given, one hour off, two hours on, night and day, without much relief.

Discussion.-We are dealing with a monarticular inflammation which has involved also the soft parts in the vicinity of the joint. Such a condition is never rheumatic, and the atrophic and hypertrophic varieties may also be excluded, because they are practically never confined in febrile cases to a single joint.

Gonorrhea is perhaps the commonest cause of monarticular inflammation, but such infections are very rare in the young, unmarried Russian Jewesses of Boston. The patient had no vaginal discharge, and there was nothing else about her to make us suspect gonorrhea. Nevertheless, this infection cannot be positively excluded. The course of the disease is too acute and too painful for tuberculous esteitis.

To obtain any further light on the subject the joint should be aspirated under aseptic precautions, as may be very easily done with an ordinary hypodermic needle. In my opinion joint puncture is far too 
rarely performed. If done with rigid cleanliness, it has no dangers, produces scarcely any pain, and often gives us information of the highest value. Since I have been in the habit of using this procedure frequently I have been astonished to see how commonly one finds turbid or purulent exudates with demonstrable micrococci in joints which have been only moderately painful, and would certainly have been classed under ordinary rheumatism but for the puncture. In some cases our treatment is made far more effective when the joint puncture makes it possible to prepare a vaccine from the invading organism.

Outcome.-On the twentieth the right knee was aspirated and six ounces of fluid pus withdrawn. From this as well as from the circulating blood a pure culture of the yellow staphylococcus was obtained. On the twenty-third the knee was surgically drained. Recovery followed, though there was limitation of motion in the knee.

Diagnosis.-Septic knee.

\section{Case 200}

A waiter of twenty-four entered the hospital December 29, 1906. He had been in the hospital twice previously for exophthalmic goiter. The last time was in May, I905. Since then he has worked steadily at hard jobs and has felt well. Four nights ago he came home with a pain in his left instep. The next day the pain extended up the leg, and in the afternoon was in both knees. It confined him to bed and took away his appetite. In October he weighed I50 pounds - a week ago, I3o.

Physical examination showed both eyes slightly prominent. The pulse ranged between 90 and 1oo. Examination was otherwise negative except for spasm of the leg muscles, both legs being held flexed. The patient insisted at first that they could not be moved, but was finally induced to straighten them out. Later, the right hand was held very stiffly, with the thumb flexed into the palm. The patient persisted that it too was paralyzed, but was finally persuaded that it was normal.

Discussion.-The pain is probably due to muscular spasm, as in the familiar cramps most of us have experienced if the foot or leg is bent in an unusual position. We can hardly doubt that these cramps are of the functional or hysteric type, in view of the results of moral suasion, but it is important to remember that a latent tuberculosis, recognizable only by $x$-ray, may produce contractures of the legs fully as severe as those here described. If the contractures were not so wide-spread, one might suspect flat-foot with leg pains due to compensatory effort. The onset of the case reminds us distinctly of this lesion, but its later course makes this very unlikely. 
The case illustrates the importance of firmness and confidence in our treatment-a confidence such as can be based only on the conviction built up in us by most painstaking physical examination and interrogation of the patient. Any doubt, vacillation, or hesitation in the management of such a case may lead to disastrous results. Decisive action, on the other hand, may be of incalculable benefit to the patient by nipping hysteric tendencies in the bud. Like so many other diseases, hysteria can be checked most often and most effectually in the incipient stages.

Outcome.-A liberal diet with 30 grains of bromid every four hours for two days, preceded by an ounce of castor oil at the time of entrance, was followed by marked improvement. By the third of January the patient seemed practically well. He had still, however, a slight fine tremor of the hands, a remnant, no doubt, of his hyperthyroidism.

Diagnosis.-Hysteria.

\section{Case 201}

A housewife of forty-four, who has had two miscarriages, one child of nine years and one of five, entered the hospital December 5, I906. She herself was born with crooked legs, which were straightened by splints at her home in Sweden. She has had pneumonia four times.

Nine years ago she had bad pains in her shoulders and arms, so that she could not raise her hands to her head. At that time lumps came out upon her arms, and ever since then she has had fleeting pains, now in one place, now in another. Thirteen days ago she was wakened out of sleep by pain in her feet. Now the pain comes suddenly and lasts from two to ten or more minutes, often shooting from the hips to the knees. It is almost as sharp as labor pain at times, and is accompanied by a dragging-down sensation. Her feet have been a little swollen. There has been some dyspnea on exertion and a little cough without sputa. She has attacks of rapid heart action almost every day. Five days ago she fainted, and had to sit up in bed all that night. Her appetite is poor, her bowels regular. There has been no nocturia.

The patient was a neurotic-looking individual, and constantly demanded attention to trivial wants. The pupils were irregular, but reacted normally. The uvula was missing, and replaced by a white scar; the throat and lungs otherwise normal. The glands were palpable, but not enlarged, in the neck, axillæ, and groins. Occasional squeaks were heard scattered through both lungs. The chest was otherwise negative; likewise the abdomen, blood, and urine. The shafts of both tibiæ were enlarged and bowed forward, their surface rough and nodular. The deep reflexes were all present. 
On both forearms, especially on the extensor surfaces, there were a dozen nodules from the size of a pea to half a horse-chestnut. They were oyster-shaped, discrete, of rubbery consistency, not tender, freely movable under the skin. Vaginal examination was negative.

Discussion.-Fleeting pains in various parts of the body are often the most distressing symptom, and the earliest, in tabes dorsalis. The history of miscarriages and the tibial deformities increase the probability of syphilis, and, therefore, of tabes. But this disease may be ruled out of consideration because of the fact that the pupils and the deep reflexes are normal.

The patient's statement that her legs were crooked from birth makes us hesitate to attribute the present condition of the shins to syphilis, and as the patient has two healthy children, the miscarriages may well have had a non-syphilitic origin. But the scar in the soft palate and the absence of the uvula are decidedly more characteristic of syphilis, and in any patient who presents such lesions we must do our best to find any connection that may exist between the old infection and the present symptoms. Very possibly the vascular lesion so commonly produced by syphilis may be connected with the pains here complained of. "Vascular crises" are certainly more common in those who have suffered a luetic infection, and through such crises, with or without a syphilitic neuritis, the pains of this patient might be accounted for.

We must also consider, however, the nodules present upon the forearms and mentioned in the history as having appeared nine years earlier. The fact that these tumors have lasted so long makes it sure that they are not of a malignant type, and their limited distribution assures us that they are not connected with the much more widely distributed pain of which the patient complains. Their physical characteristics are those of lipomata, which are practically the only tumors which could last so long without more disastrous effects.

Outcome.-The patient was given mercury and potassium iodid. Her leg pains were greatly relieved by injections of sterile water, especially in the first two days after entrance. The lumps on the arms were taken to be fatty tumors.

She was discharged much relieved on the twelfth of December.

Diagnosis.-Syphilis.

\section{Case 202}

An engineer of forty-five entered the hospital July 25, I906. His family history was negative. He had urethritis twenty years ago, also five weeks ago, the latter attack followed by "rheumatism." He had 
"slow fever" twenty years ago, and was five weeks in bed. Ten years ago he had inflammatory rheumatism, lasting three weeks, in both feet. No other parts were affected. He takes an occasional glass of beer, but denies any other use of alcohol. Eleven days ago his left foot became red, swollen, and tender. This gradually improved, but yesterday the right foot became similarly affected. He has been unable to work since the onset of the symptoms. He has had a poor appetite, constipation, slight headache and fever.

The patient was obese, slightly cyanotic. The first sound at the apex of the heart was replaced by a short systolic murmur, not transmitted. The aortic second sound was accentuated, the heart not enlarged. The lungs were normal, likewise the abdomen, except for dulness in the right flank, which does not, however, shift with change of position. The second joint of the right toe was much swollen, hot, and tender. The same joint in the other foot was similarly affected, but the swelling also extended up the foot toward the ankle.

Discussion.--In any patient who complains of subacute pain in both feet, and is not flat-footed, suspect gout. Most of the ordinary joint infections do not long remain confined to the feet, while gout is very prone to do so.

Naturally, however, the first possibility to be investigated in this patient is gonorrhea, as he had so recently suffered from that infection.

Next we must search the cartilages of the ears and nose, the great tendons near the elbow and ankle, and the vicinity of the great toe-joints for signs of uratic deposit. Thirdly, we must investigate the plantar arches, since precisely these symptoms might be produced by flat-foot. Other infectious and non-infectious lesions are far less probable.

Outcome.-A smear from the urethra showed a biscuit-shaped diplococcus both within and without the leukocytes. It did not stain by Gram's method. The ears showed several small, yellowish-white, soft lumps. A scraping from one of these showed crystals corresponding to those of sodium biurate. $X$-ray showed areas of atrophy or erosion of the second phalanx of one great toe, which were believed by an $x$-ray expert to be due to gout.

By the sixth of August the patient was practically comfortable. His treatment had consisted of sodium salicylate, 20 grains every hour for the first two days, then ro grains every hour. Hot fomentations applied to the painful parts, an ounce of magnesium sulphate every morning, Io grains of urotropin four times a day. He was not in bed after the twenty-ninth, and was discharged relieved on the sixth of August.

Diagnosis.-Gout and gonorrhea. 


\section{Case 203}

A restaurant-keeper of forty-nine entered the hospital September I8, 1907. His mother died at seventy-two, after suffering from consumption for fifteen years. The patient has had "rheumatism" in his joints in two attacks of three weeks each-three years ago and eighteen months ago. He has had four attacks of urethritis, the last twenty-five years ago, but denies syphilis.

He says that he was as strong as an ox until four years ago, when he sold his business and had difficulty in getting a new start. He then began to have almost constant pain near the right costal margin. These symptoms he has had off and on ever since. He has rather frequent attacks of vertigo and weakness, and his appetite is often poor. As long as he can remember his fingers have been clubbed, as they are at present. He usually takes two glasses of beer and two or three of whisky a day, and his use of tobacco is distinctly excessive. Yesterday he noticed that the corners of his mouth cracked. Off and on for four years he has felt feverish, and sometimes chilly and shivery in the evenings. His knees and ankles have burned, especially after he gets to bed.

These joint symptoms have been getting steadily worse, and two days ago he had to give up and go to bed on account of pain in his legs and knees. Yesterday the right knee became a little

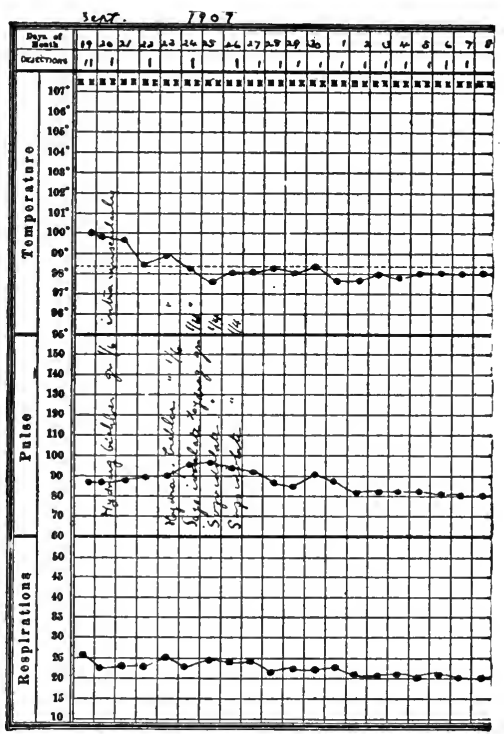

Fig. 73.-Chart of case 203. better, the left worse. At the same time his left thumb began to be red, swollen, tender and painful. This time he had true chill.

Physical examination showed that the left pupil was larger than the right, though both reacted normally. The heart was normal. There was slight dulness below the right scapula, with slight increase of vocal and tactile fremitus, and a few râles. Expiration was everywhere rough and prolonged. The abdomen was negative. The right knee was swollen, hot and shiny; the leg was kept bent at a right angle, and motion was painful. A similar condition was found in the right foot and 
ankle. Both feet were pronated. There was marked clubbing, cyanosis, and curvature in both planes in the fingers and thumbs, and to a less degree in the toes. Scattered over the chest and back was a reddishbrown, macular eruption, the spots about the size of the little finger-nail.

Discussion.-There seems to be no way by which we can connect the mother's consumption or the patient's alcoholism with the present symptoms. The joints are obviously not tuberculous, and alcohol does not attack articular structures.

Though it would be natural to connect the former attacks of urethritis with the present joint pains, the gap of twenty-five years between the two renders this impossible if the history is taken on its face value. In looking over the body for any other lesion which we can connect with the joint symptoms, we notice the irregular pupils, the clubbed-fingers, and the cutaneous eruption.

If the clubbing of the fingers be assumed to be such as is described, it is not likely to have any connection with the arthritis. Bony outgrowths near the finger-ends (Heberden's nodes) bear some resemblance to clubbed-fingers, but could hardly be mistaken for them. Such outgrowths, if present, might incline us to conjecture that the right knee and ankle were the seat of a similar process.

The irregular pupils and the cutaneous eruption look like syphilis, and since there is nothing very definite known about the differential symptomatology of syphilitic arthritis, it seems reasonable to interpret the joint manifestations in this case as syphilitic until this is disproved by therapeutic test. If no improvement follows the vigorous use of mercury and iodin, the joint should be tapped in search of some other infective agent.

Outcome.-Under daily inunctions of mercury and the administration of potassium iodid-ro grains after each meal-the joints rapidly improved, and within ten days were practically well.

The clubbing of the fingers remains in this, as in many other cases, a mystery. If clubbing were more carefully searched for as a matter of routine in cases presenting no pulmonary or cardiac lesions, it would be found, I believe, to occur in a great variety of diseased conditions and in a good many persons who have no demonstrable disease. Personally, I have obscrved it chiefly in chronic diseases of the liver (cirrhosis, abscess, gall-stone disease), in tuberculous peritonitis, and in ill-nourished children.

Its occurrence in connection with long-standing cardiac disease (congenital or acquired), with chronic pleurisy or empyema, phthisis and bronchiectasis, is, of course, familiar.

Diagnosis.-Syphilis. 


\section{Case 204}

A plumber of thirty-seven entered the hospital April II, I908. He drinks and smokes to excess. Last evening he came home complaining of severe pain in both legs, especially in the left one. About one o'clock this morning he awoke unable to speak or to move the right arm and leg. Soon after the patient became unconscious, with stertorous breathing.

The right forearm was in flexion, the fingers of the right hand flexed and spastic, the mouth drawn to the left; he made only inarticulate sounds. The right leg was spastic. By April I3th he had regained consciousness and he could move the toes slowly; otherwise he had no muscular control. His tongue came out to the right when protruded. There was no lead line. The chest and abdomen showed nothing abnormal. The blood-pressure was 155 , the blood and urine normal, the right kneejerk lively in comparison with the left knee-jerk. There were no other changes in the reflexes at this time.

By April I5th Babinski's reflex had appeared in the right foot. Lumbar puncture was done on the seventeenth, and the cells in the fluid which was withdrawn were 50 to the cubic millimeter. Practically all of them were lymphocytes.

Discussion.-The patient's occupation naturally leads us to attempt to explain the symptoms as a result of lead-poisoning, especially as paralysis and cerebral symptoms are present. But we do not expect pain or hemiplegia in plumbism, and we practically always find changes in the staining properties of the red blood-corpuscles.

Against apoplexy, which, as the commonest cause of hemiplegia, naturally occurs to us next, is the patient's age, the very moderate blood-pressure, the absence of cardiac hypertrophy, and especially the results of lumbar puncture.

The examination of the spinal fluid taken in connection with the absence of fever and the well-marked cerebral symptoms lead us straight to the diagnosis of cerebrospinal syphilis. A similar lymphocytosis occurs in the chronic forms of meningitis, especially tuberculous meningitis, but the clinical picture is quite different from that here under consideration.

The most interesting point in this case is the occurrence of a pain which, though referred to the legs, seems to be cerebral or spinal in origin. Such pains are seen not uncommonly in infantile paralysis, in some of the types of acute myelitis and meningitis, and especially in cerebrospinal syphilis. I recently studied a case in which attacks of Jack- 
sonian epilepsy, involving the right hand and forearm, were preceded, again and again, by severe pain referred to the parts about to be convulsed. Many of these central pains are preceded or accompanied by paresthesiæ.

Outcome.-Under mercury and potassium iodid the patient was able to walk by the twenty-second, though his mind was still very sluggish. The next day he was sent to a State infirmary.

Diagnosis.-Cerebrospinal syphilis (vascular crisis?).

\section{Case 205}

A machinist of thirty-nine entered the hospital May 29, 1908. His father died of apoplexy, his mother of dropsy. Seven years ago he was in bed five days, owing to swelling, redness and pain in the left knee.

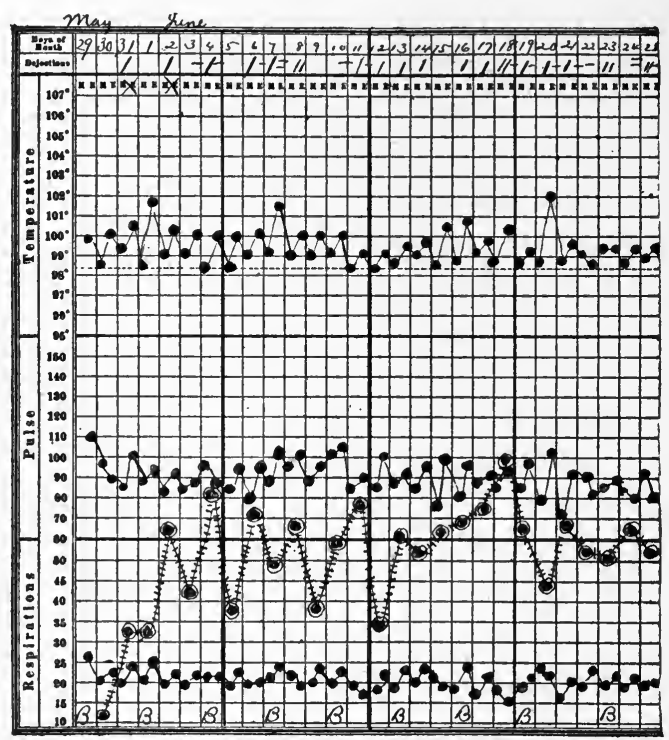

Fig. 74.-Chart of case 205 .

In the past five years he has had tonsillitis six or eight times, once severely enough to keep him in bed. He had urethritis twenty-three years ago.

He takes two or three glasses of whisky and two or three of beer each week. Two weeks ago he had a sudden chill accompanied by pain in the lower back, in the hands and the calves of his legs. He took to bed and has been there since, with persistent fever. The next morning his right knee and the joints of the left hand were painful and stiff. 
Six days ago the knee became red and swollen, while the left hand greatly improved.

He has had no urinary symptoms; his appetite has been good; his bowels regular; there has been no sore throat and no cough. The course of the temperature may be seen in the accompanying chart.

The chest and abdomen were negative, the knee-jerks normal; the plantar reflexes were not obtained. There was no glandular enlargement. The right knee was flexed at an angle of 45 degrees, any motion causing severe pain; all the evidences of fluid were found in the joint.

Discussion.-As in many of the cases discussed in this section, we have here a general infection which shows, at first, no hint of its ultimate localization.

Since the urethritis occurred too long ago for us to connect it with the present symptoms, it seems at first likely that the joint trouble may be due to the patient's repeated attacks of tonsillitis, and as gout and traumatism can be excluded by the lack of any evidence of them, tonsillitis would perhaps be the best guess we could make, were we debarred from any further and more direct investigations. But, as I have previously insisted, all monarticular infections of any seriousness or obstinacy should be tapped, since the information thus to be derived may be of the greatest therapeutic value. (See p. 39I.)

In all probability the infecting organism is one of the pyogenic cocci, but it may be of great importance to know which, as a treatment by autogenous vaccines has much to recommend it.

Outcome.-On the first of June the joint was aspirated and $35 \mathrm{~cm}$. of fluid withdrawn. Specific gravity, I008; albumin, 3.6 per cent.; in the sediment, 94 per cent. of the cells were polynuclear. Among them were numerous intracellular diplococci which did not stain by Gram's method. After this information had been obtained, the patient admitted a urethritis ten weeks ago, but insisted that there had been no discharge for the past four weeks. From the fluid withdrawn from the joint, gonococci were isolated in pure culture. From this a vaccine was prepared and injected. He improved quite rapidly after this, and by the sixteenth was able to go to the Zander room daily.

On the twenty-fourth the knee was smaller and much more comfortable. Bier's treatment was given after that date, and he was soon taught to apply it for himself. On the fourth of July he was discharged, much relieved.

Diagnosis.-Gonorrheal arthritis. 


\section{Case 206}

A metal worker of fifty entered the hospital March 27, 1908, stating that he had never been sick before, and giving a good account of his habits. Seven weeks ago, while at work, he was taken with a sudden chill and went home and to bed. In the night he awoke with a sharp pain in the right shoulder and the left knee. He managed to get to sleep, however, and was much surprised to find the next morning that the pain had left the shoulder, but that the knee was hot and swollen, painful, red and tender. The knee has increased in size since, and he has been confined to bed, but has had no more fever or chills and no pain except in his knee.

On physical examination the patient was very apprehensive and emaciated; there was a moderate, coarse tremor of the hands and feet; his face was dusky and dark under the eyes, his mucous membranes pale, though his leukocyte count was 80 per cent. His heart's apex was in the fifth space, an inch outside the nipple-line. The sounds were rapid and weak, the aortic second louder than the pulmonic second. There was no enlargement to the right and no murmur. The pulses were of very low tension, and the artery wall barely palpable.

The lungs were negative; the abdomen showed considerable voluntary spasm and slight dulness in the extreme flanks, not shifting on change of position. The left knee was markedly enlarged, tender, hot, red and very painful on motion. The swelling was most marked on the front of the knee, but extended up to the middle of the thigh and two inches below the tubercle of the tibia. The front of the thigh was fluctuant, tender, and covered by a tracery of prominent veins. A fluid wave could be transmitted from the knee to the middle of the thigh.

Discussion.-This case, though very similar to the last, is given as an awful example of what may result from the neglect of early joint puncture in monarticular arthritis. It is a sin and a shame that this patient should have gone seven weeks without any effective etiologic or radical treatment. From the facts presented, no trained observer could doubt that there is pus in and around the joint. The nature of the infection is the only remaining diagnostic problem.

Outcome.-On the twenty-seventh the knee was tapped and thick pus obtained. A culture from this pus showed streptococci. On March 28th the knee was opened and almost a quart of pus obtained, which apparently came from outside the knee-joint. The patient ran a jagged, septic temperature for a month, and developed a metastatic 
abscess in the axilla, whence a colon bacillus was obtained. Despite amputation, he finally died.

\section{Diagnosis.-Sepsis.}

\section{Case 207}

A physician forty-six years of age entered the hospital June 5, I906. He had a primary lesion on his thumb one year ago; a secondary eruption with adenitis and sore mouth followed. A thorough antisyphilitic treatment has been given since. Two weeks ago a swelling appeared in the left foot. Within a few days the soles of both feet became red, swollen and tender. Ten days ago he was laid up in bed for three days. In every other way he is perfectly well.

Physical examination showed considerable irregularity of the pupils, but was otherwise negative except as relates to the left foot, which was red, tender and slightly swollen over the dorsum and on the sole opposite the head of the second metatarsal bone.

Discussion.- It is difficult to decide whether the syphilitic infection of a year ago has any connection with this patient's present suffering. It seems rather improbable, in view of the absence of specific lesions at the present time.

As the patient has now no fever, one naturally thinks of flat-foot as a cause of such foot-pain, even though redness and tenderness would otherwise incline us to assume an inflammation. The mutual relations of arthritis and flat-foot have been previously discussed. (See p. 372.) In any such case the first and best thing to do is to try two therapeutic tests: $(a)$ The effect of taking the patient off his feet, and $(b)$ the effect of padding the arches.

Outcome.-Though no medicine was given, the pain was entirely gone after a few days' rest, and as soon as foot-plates had been fitted, the patient was able to walk without pain.

We have still on our hands, however, the question: Why did the arches break down just at this time? Possibly some latent and unrecognized phase of his old syphilis may provide the answer.

Diagnosis.-Flat-foot.

\section{Case 208}

A laborer of forty-seven entered the hospital July 6, I906, complaining of sciatica. He had a similar trouble nine years ago, which lasted three weeks. Otherwise he has been well until seven months ago, when he gradually began to notice pain in the back and left hip, running down the left thigh behind and extending into the calf. He has had tc give up work, but has walked about with a marked limp. 
For the last.six weeks the pain has been much worse and has kept him awake at night. He has had some tingling and other curious sensations in his lower left leg. He has lost I5 pounds in weight, though his appetite is good.

Physical examination shows that the patient cannot stand erect, but supports himself with the spine curved to the left and forward. The motions of the back are inhibited by a pain referred to the sacro-iliac joint. Full extension or flexion of the left leg is impossible on account of pain referred to the same point. There is tenderness over the region of the left sciatic nerve and slight atrophy of the muscles of the left leg, making about one inch difference in the circumference of the thighs and calves.

Discussion.-In the out-patient records of the Massachusetts General Hospital previous to the year I90o there are to be found notes of a large number of cases with the diagnosis "lumbago and sciatica." At the time when we were dealing with these cases it always seemed remarkable to me, and I imagine also to many of my colleagues, that a disease affecting a muscle (lumbago) should occur simultaneously with a neuritis (sciatica). The case reported above is typical of a great many of those which we used to label "lumbago and sciatica."

Looking at it from the point of view of the present day, one would say, first of all, that the lumbar pain has lasted too long for lumbago, which, like other muscular pains, is a transient though perhaps recurrent affair, producing its symptoms for not more than a week or two at a time.

The other half of the old diagnosis-sciatica-we should now be unwilling to make without a far more searching investigation of the possible causes for sciatic pain, especially diabetes, disease of the lumbar spine or sacro-iliac joint, and pelvic tumors.

The present case is fairly typical of what is now called sacro-iliac strain, a diagnosis based most firmly upon the therapeutic test-the means by which it is relieved. The etiology and pathology of the affection are still very obscure, and the theories usually advanced do not seem satisfactory to me.

Outcome.-The patient was seen by Dr. Goldthwait, who made a diagnosis of "chronic strain" of the left sacro-iliac joint.

The pain was entirely relieved by a pillow under the knee and a folded sheet under the lumbar spine, with rest in bed. A plaster jacket was then applied, and by August $2 \mathrm{~d}$ he was free from pain and could walk a little. On that day he was discharged, much relieved.

Diagnosis.-Sacro-iliac strain. 


\section{Case 209}

A housemaid of twenty-five entered the hospital August 16, 1907, stating that for three weeks she had had swelling, pain and tenderness in the lower legs, especially at night.

Physical examination shows yellowish pallor and only 25 per cent. of hemoglobin; the white cells varied between 28,000 and 43,000 in the course of the next four days, the polynuclears making up 82 per cent. of this increase. For temperature see the accompanying chart.

Physical examination was negative save for an indefinite resistance in the right upper quadrant of the abdomen. The front of both legs showed numerous sharply defined, punched-out ulcerations on a reddened base; the right lower leg showed soft nodules the size of a pea, raised one-half inch above the surrounding skin, covered with unbroken skin. They were of a doughy consistence and tender.

The subcutaneous ulcerations were drained by surgical incisions and considerable pus liberated.

Discussion.-Obviously, we are dealing with some type of acute infection, the most probable source for which seems, at first examination, to be the gall-bladder. But on iurther scrutiny it is equally obvious that we need some source for the very marked and apparently chronic anemia which has reduced the hemoglobin to 25 per cent. $^{1}$ In cases accompanied by marked secondary anemia I have found that in moving toward a diagnosis it

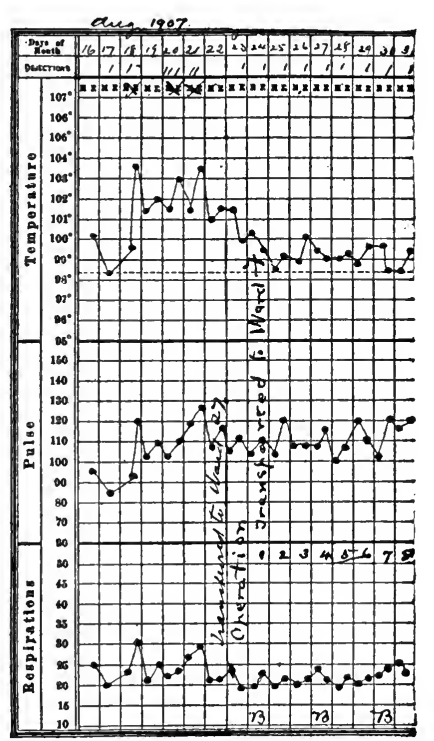

Fig. 75.-Chart of case 209. is a useful strategic maneuver to fix attention, first of all, upon this anemia, and to investigate what causes of such an anemia are possible in this patient. The patient may, of course, be chlorotic, but as she certainly has something else the matter with her, we must make two diagnoses (which we are always loth to do) in case we call it chlorosis.

${ }^{1}$ I regret that no estimation of red cells was recorded in this case. It was evident, however, from the appearance of the stained smear, that they were not greatly reduced and that the anemia was of the secondary type. 
Aside from chlorosis, what are the possible causes of a severe secondary anemia in a woman of twenty-five who has had no hemorrhage, no malaria, and no evidences of malignant disease? The lesions on the shins, and especially those covered with unbroken skin, suggest gummata, and although there is certainly a secondary infection, the hypothesis of syphilis should be put to the therapeutic test.

Outcome.-Microscopic examination of an excised nodule showed gumma with secondary infection. The lesions quickly cleared up under antisyphilitic treatment.

Diagnosis.-Syphilitic periostitis.

\section{Case 210}

A hostler of thirty-two was first seen June 3, I907. He takes five or six beers and three or four whiskies daily, but denies venereal disease. Yesterday morning he woke with a chill followed by headache, fever

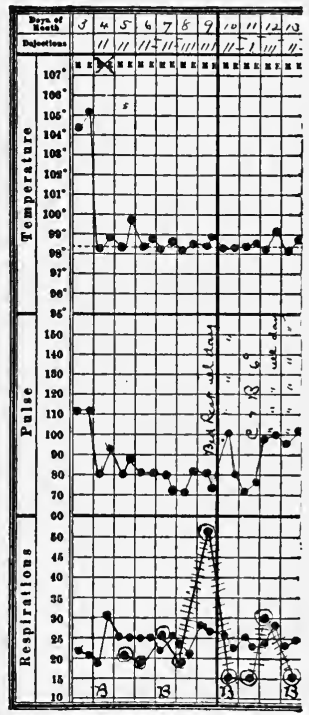

Fig. 76.-Chart of case 2 Io. and aching bones. To-day his chief complaint is of pain in his legs.

Physical examination of the chest and abdomen is negative. The right tibia is rough and nodular; the skin bluish red and containing three ulcerated areas from the size of a silver dollar to that of the palm. The course of the temperature and pulse is seen in the accompanying chart. The leukocytes are I2,500. The glands in the right groin are enlarged; urine normal. $X$-ray shows evidences of a syphilitic periostitis. Under large doses of iodid of potash, the glands of the groin became smaller and the pain disappeared within ten days.

Discussion. - This case is introduced chiefly to show the importance of $x$-ray examination of the shin bones in all cases involving an obscure pain referred to the lower

leg. Without the evidence thus obtained a diagnosis would here have been impossible.

Doubtless there was also a certain degree of secondary infection in the ulcerated area, whence the chill, high fever and other acute symptoms may be explained.

Diagnosis.-Syphilitic periostitis. 
Case 211

A shoemaker of nineteen entered the hospital May 14, I908, with a good family history, past history and habits. Three days ago he began to have pain in his legs and to a lesser extent in his left side. At night he vomited twice and has since kept his bed. The pain has been more severe in his chest, until to-day, when it has diminished. $\mathrm{He}$ has slept and eaten poorly and has been constipated. He has had no cough and no chill.

Physical examination showed dulness in the lower half of the left back, with bronchial breathing; increased voice and fine, crackling râles.

The leukocytes were 22,000. The urine and the rest of the physical examination were normal. The course of the temperature is shown in the accompanying chart. A tight swathe prevented all pain. On the nineteenth he was put in a chair, and by the twenty-fifth was able to go to his home. Throughout his illness he had practically no cough or expectoration.

Discussion.-This case is introduced in order briefly to exemplify a pain due to general infection, but confined to the legs. Some of these pains are very mysterious, and give not the slightest indication, during the first two or three days of the patient's sufferings, where the trouble is finally to settle. Obviously, in the present case the pain was ushering in a pneumonia. I recently saw a woman who suffered for two days from quite intense pain throughout all the tissues of the thighs and legs. We could find absolutely no cause for it, though the presence

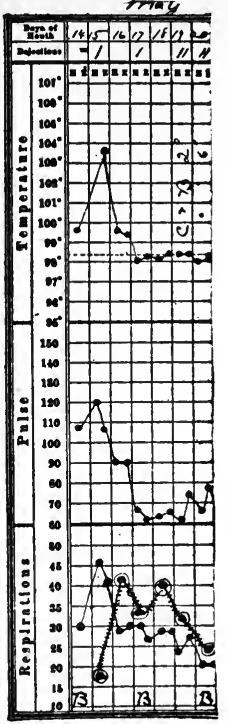

Fig. 77.-Chart of

case 2 II. of an accompanying fever and leukocytosis made us believe that some infective agent was at work. The joints, the nerves, the muscles and subcutaneous tissues, the arteries and veins were searched for evidence of a cause for the pain, but none was found. On the third day an acute dysentery made its appearance, and the pain in the legs quickly disappeared.

In view of these and similar cases we must always bear in mind, when examining the legs for a cause of pain referred to them, that a general unlocalized infection bearing no special relation to the leg may have invaded the body. Disease of the brain or spinal cord should also be remembered as among the long-range causes for leg pains.

Diagnosis.-Pneumonia. 


\section{Case 212}

A homeopathic confrère called me in consultation October 3 Ist to see a curious case of grip with pelvic neuralgia and perhaps malaria.

The patient was a young architect of twenty-seven, always previously well until he began, October Ist, to have what he called "grip" -i. e., a fever of 103. $6^{\circ} \mathrm{F}$., accompanied by aching in his head, back, and legs. After a couple of days the temperature fell to Ior $^{\circ} \mathrm{F}$., and the patient had what was called a right facial neuralgia. From October $5^{\text {th }}$ to October I2th the temperature did not rise above $100^{\circ} \mathrm{F}$., and the patient seems to be convalescent, though complaining somewhat of piles. He then went off for a week's vacation, but on his return, October Igth, said that he had been poorly while he was away, suffering a great deal from pain in the testes, which was especially severe every night about 6 P.M., and was associated either with a rectal tenesmus, a urinary frequency, or both. The pain radiated to both hips and groins.

On October $25^{\text {th }}$ the temperature was again IOI $^{\circ} \mathrm{F}$., and since that time it has risen to that point or a degree higher every day. On the twenty-seventh, quinin, the doctor said, seemed to stop the pelvic neuralgia, but for the last two days he has been eating poorly, and at II P.M. to-day his temperature was $103^{\circ} \mathrm{F}$. and the pelvic pains so great as to require morphin. The urine has been high colored, but not cloudy, and shows no gross sediment. The blood has not been examined.

Physical examination of the chest was negative; the spleen was not enlarged, and the blood showed no malarial organisms. There was no evidence of an influenzal infection of the upper air-passages or elsewhere. The urine was high colored, but showed no other important abnormality. The leukocytes numbered $28,000,83$ per cent. of which were polynuclear.

The local examination, which had been hitherto neglected, showed a reddened, tender, and resistant area close to the rectum on the left.

Discussion.-The points of interest in this case are the slow "settling" of the infection at the point where it was finally discovered, and the unwisdom of treating symptoms without careful physical examination. In view of the local conditions one could hardly doubt that the patient was suffering from an abscess near the rectum, the wide radiations of the pain being due, doubtless, to the burrowings of pus which should have been liberated long before.

Incision allowed the escape of a pint and a half of pus. The abscess cavity healed up in the course of three weeks, and by December Ist the patient was back at work.

Diagnosis.-Ischiorectal abscess. 


\section{CHAPTER XIII}

\section{FEVERS}

THE distinction between "long" and "short" fevers-i. e., those continued for two weeks or more, and those of briefer span-allows us to narrow the diagnostic possibilities of the "long" group practically to three alternatives: tuberculosis, sepsis, typhoid.

In the following table ${ }^{1} \mathrm{I}$ have classified 784 cases in which a fever lasted two weeks or more without touching normal:

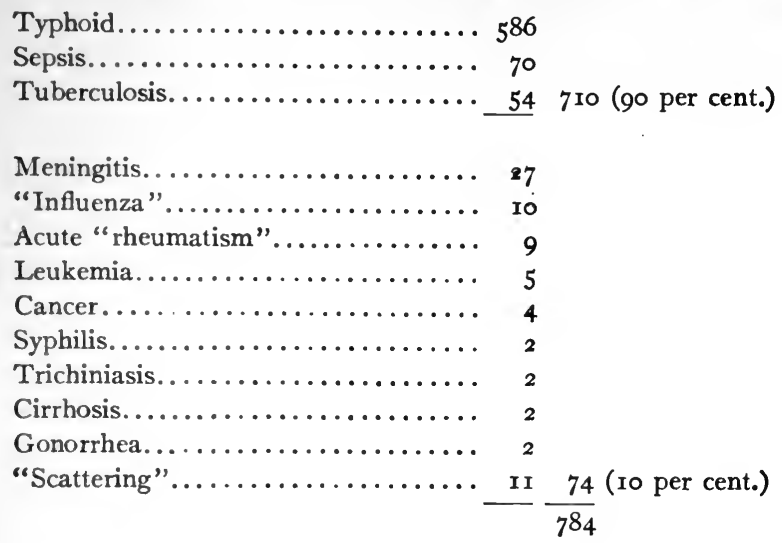

It will be noted that most of the ro per cent. of long fevers not due to typhoid, tuberculosis or sepsis are due to diseases easy of diagnosis because of their local or distinctive signs. Thus meningitis, with its evidences of cerebrospinal irritation, "rheumatism" with its joint lesions, leukemia and trichiniasis with their blood changes, cancer with the easily palpable tumors which febrile cases practically always show, gonorrhea and cirrhosis with their characteristic local manifestations-all these are, or should be, easily recognized. Obscure longcontinued fevers, then, will include only the dominant three, plus "influenza" and syphilis. In this group the dominant three make up 98 per cent.

Instead of "influenza" we should write "unknown infection" against most of the $\mathrm{I} .2$ per cent. of obscure fevers so diagnosed in my statis-

1 R. C. Cabot, The Three Long-continued Fevers of New England, Boston Medical and Surgical Journal, August 29, 1907 . 
tics, for bacteriologic proof of influenza was rarely obtained in this series. I do not doubt that long as well as short fevers may be produced by true influenzal infection, but I believe that the diagnosis is rarely well founded on cultural evidence.

The proportion of typhoid in the figures above quoted is far too high, because in the Massachusetts General Hospital, whence these figures were gathered, the typhoid cases of a large area are aggregated. In fact, the number of long typhoid fevers is generally far less than the number of long tuberculosis or septic fevers; but these are treated at home and therefore missed in hospital statistics.

The manifold manifestations of tuberculosis-in the spine, the hip, sacro-iliac, and other joints, in the lymph-nodes, peritoneum, meninges, and genito-urinary tract, as well as in the lungs and pleura-may all produce long as well as short periods of fever.

Under "sepsis" I mean to include here an extensive variety of clinical pictures, mostly due to streptococcus infection; such are(a) vegetative endocarditis (also called benign, malignant, ulcerative, or septic); (b) puerperal fevers; (c) deep-seated abscesses originating in the appendix, the gall-bladder, the genito-urinary tract, the stomach, and duodenum; $(d)$ empyema; $(e)$ wound sepsis; $(f)$ lymphangitis, erysipelas, and phlegmonous inflammation; $(g)$ sepsis - originating in the tonsils or about the roots of the teeth.

Yet only a small minority either of tuberculous or of septic fevers are obscure in origin or lead us to any diagnostic puzzles. The osseous, lymphatic, peritoneal, and meningeal forms of the disease are usually easy of recognition. It is chiefly the pulmonary and renal forms of tuberculosis that are latent and produce obscure fevers. Among the fevers due to sepsis also the great majority are plain enough. It is chiefly in the cases of vegetative endocarditis, and in some of the deepseated abscesses-especially those in or about the liver and kidneythat local symptoms are absent.

Hence we may say that, when studying obscure fevers of long duration, we should search especially for:

(a) Pulmonary and renal tuberculosis.

(b) Typhoid.

(c) Hepatic, subphrenic, renal, or perirenal suppurations.

(d) Vegetative endocarditis.

The lung, the liver, the kidney, and the blood are especially to be suspected and examined. Auscultation, $x$-ray examination, bloodcounts, cultures, biologic tests, cystoscopy and a carefully taken history will help us most in difficult cases. 


\section{Causes of Long Fevers}

1. TYPHOID

1172

2. SEPSIS 140

3. TUBERCULOSIS

$108^{1}$

4. MENINGITIS

5. INFLUENZA

6. INFECTIOUS ARTHRITIS

7. LEUKEMIA

8. CANCER

1

8

9. SYPHILIS I

10. CIRRHOSIS

11. GONORRHEA I

12. "SCATTERING"

1 In statistics of hospitals for chronic diseases this figure is often much larger proportionally. 


\section{Causes of Short Fevers}

(Omitting those listed under "Long Fevers" and excluding the exanthemata and diphtheria.)

1. "COMMON COLDS," Including:
(a) ACUTE BRONCHITIS $\}$

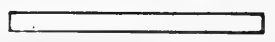

1620

(b) ACUTE TONSILLITIS

1405
(c) ACUTE PHARYNGITIS
(d) ACUTE "INFLUENZA"

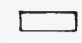
388

2. ACUTE APPENDICITIS 1504

3. ACUTE ARTHRITIS 1016

4. SALPINGITIS 871

5 PNEUMONIA 803

6. LYMPHANGITIS 365

7. SINUSITIS 259

8. ERYSIPELAS 241

9. POLIOMYELITIS 


\section{SHORT FEVERS}

Excluding the exanthemata and the milder types of the infections just mentioned, we may say, I think, that the majority of short fevers are either due to streptococcus or are of unknown origin. The habit of attributing such fevers to "common colds," to "grip," "influenza," "febricula," "auto-intoxication," "rheumatism," constipation, etc., is a pernicious way of concealing our ignorance not only from our patients, but from ourselves.

The temperature-pulse ratio has never seemed to me of much practical value in diagnosis. It may confirm a diagnosis established mainly in other ways, but in my experience it is as apt to lead us wrong as right. In typhoid the pulse may be relatively slower than in fever of similar degree due to pneumonia, sepsis or tuberculosis, but there are many exceptions to this rule.

The rapidity of respiration is also a very unreliable guide. Many non-respiratory infections (e. g., typhoid, erysipelas, liver abscess) may notably quicken the respiration, while the sudden fall of respiration at the crisis in pneumonia, when the lung signs remain quite unchanged, inclines us to believe that even in pneumonia the polypnea is due to the general rather than to the local pulmonary condition.

\section{SPURIOUS FEVERS (MALINGERING)}

Afebrile patients who wish to prolong their stay in a hospital, to continue the conditions of invalid life at home, or to excite the sympathy which alone makes life worth living to them, sometimes deceive their nurses or doctors into believing that fever is present. One device is to dip the thermometer bulb into hot water immediately before the nurse comes in to read it. Another is to put the thermometer bulb against a hot water bag at the appropriate time. Still another is to shake the thermometer with the mercury bulb upward so as to force the column up above normal. There are other patients who can somehow raise the mercury in the thermometer without using any of the devices just mentioned. We detect these cases only by having a nurse stay with the patient throughout the period during which the thermometer is registering or by taking the record with the thermometer in the rectum. Under these conditions a temperature chart which has recently been most alarming, flattens down and becomes normal. How these patients manage to work up the mercury in the thermometer I do not know. It has been surmised that they can do it by friction with the tongue, but this does not 
seem to me very probable. It is conceivable that there are local inequalities of the temperature so that the mouth temperature might be very high while that of the rest of the body was normal.

These forms of deception are to be distinguished from the cases in which a genuine though slight fever, $99.5^{\circ}$ or $100^{\circ} \mathrm{F}$., is present either for a short time or continuously, as the result of nervous influences in psychoneurotic patients.

\section{BED FEVERS}

At the end of the course of typhoid a prolonged pyrexia may sometimes be cut short by getting the patient out of bed. The same occurs in fevers from various other causes. Hence when fever persists beyond what we have reason to expect should be its natural term, we should always suspect that we may be dealing with a "bed fever." Just what this means we do not know, but its practical importance is due to the fact that it disappears when the patient gets up. Perhaps the conditions of metabolism and heat production are disturbed by the unnatural conditions of life in bed. But whatever the explanation, it is of considerable practicable importance to remember the possibility of this type of fever.

\section{RELAPSING FEVERS}

Besides the relapsing fevers due to one or another type of spirillum and confined practically to those who live in the tropics or have shortly returned from the tropics, one sees two types of disease in which periods of fever lasting days or weeks alternate for weeks or months at a time with periods of normal temperature. (a) Fever due to Hodgkin's disease (malignant lymphoma). There need be no considerable enlargement of the visible lymph-glands in these cases. The tumors may be inaccessible owing to their position deep in the abdomen or in the mediastinum. Pressure symptoms, such as ascites or dyspnea, may not appear for many weeks. Finally an external gland may make its appearance, and when removed may give us histologic evidence sufficient for diagnosis. (b) Fevers due to infection in the biliary tract, $i$. e., to a stone in the common duct or to cholangitis unassociated with stone. In these cases there is usually a history of jaundice at some time during the course of the disease. Biliary colic may or may not be present. Chills are sometimes seen, but may be absent. Such cholangitis may complicate a cirrhosis. 


\section{TERMINAL FEVERS}

(a) Streptococcus infections of the endocardium, pericardium, the pleura, together with those present in the blood stream without further localization, are probably the commonest of all terminal fevers.

(b) Toxic fevers are often seen at the end of life in uremia, poisoning by illuminating gas, cirrhosis of the liver, and other auto-intoxications.

(c) Thrombus fevers result from the presence of clots in the heart or pulmonary vessels. Careful study postmortem may reveal no other cause for fever, and it is often impossible to isolate any micro-organisms either from the clots themselves or from the blood stream.

\section{NON-INFECTIOUS FEVERS}

(a) Brain injuries and diseases of any kind may produce fever of various types. Thus cerebral hemorrhage, tumor, and acute delirium due to alcohol or other causes, often raise the temperature considerably.

Other important causes are:

(b) Malignant tumors (such as cancer of the liver, Hodgkin's disease), especially when extensive and of rapid growth.

(c) Leukemia and all types of severe anemia.

(d) Poisoning by belladonna and illuminating gas.

(e) Uremia, eclampsia, hepatic toxemia, gout, and hyperthyroidism (Graves' disease).

(f) Sunstroke.

Whether pure "nervousness" or hysteric states of one or another type can produce fever is a question which frequently arises.

Pyrexia not exceeding $100^{\circ} \mathrm{F}$. and of short duration certainly accompanies many such psychoses. Temperatures taken when a patient first enters a hospital often register $102^{\circ}$, IO $3^{\circ}$, or $104^{\circ} \mathrm{F}$., but are followed by normal records within twelve to twenty-four hours. Exhaustion and alarm doubtless contribute to produce these temporary abnormalities. Aside from the two types of fever just mentioned, I have no experience of pyrexias due to psychic causes. 


\section{Case 213 A}

A fourteen-months-old girl baby was seen December 23, 1902. She was born in Cuba, had malaria before she left the island, and since she came to live in Cambridge, Mass., had, during the summer just past, a large number of mosquito-bites. November I6th the baby began to vomit, lost appetite and soon became weak and listless. She was fed on Eskay's food and milk. From that time on she ran a continuous fever, ranging from $100.6^{\circ}$ to $104^{\circ} \mathrm{F}$., with long excursions almost every day. She was fretful and listless, dozing most of the time, rolling her head back and forth upon the pillow, running her tongue repeatedly over the region of the expected incisor teeth, but exhibiting no more definite localizing symptoms.

The symptoms were ascribed to teething, but the child grew steadily worse, and by December $2 \mathrm{~d}$ voluntary motion of the extremities had almost altogether ceased. Repeated physical examinations elicited nothing either in the legs or elsewhere. December 3 d the child seemed to be markedly "anemic," and it was difficult to obtain blood from the toe. Nevertheless, the hemoglobin was 80 per cent. The Widal reaction was negative; the white cells, 6500 . Iodophilia was very marked. The child was seen by Dr. C. P. Putnam daily for a week, but no diagnosis was made.

December 6th a squint was noticed. This disappeared within twenty-four hours and has not recurred since. December $23 \mathrm{~d}$, the fever continuing unabated, while the child grew constantly thinner, I saw her in consultation, but could make no diagnosis. The blood showed at this time:

Red cells, 4,892,000; white cells, 39,000; hemoglobin, 80 per cent.; iodophilia, very marked; among the leukocytes, 93.6 per cent. were polynuclear.

A week later Dr. T. M. Rotch saw the baby, noticed a slight "rosary," made a diagnosis of rickets, and directed the treatment accordingly. Nevertheless the child continued to go down-hill.

Discussion.-As in the case previously mentioned, there was no culture made from the urine, and the possibility of urinary infection was not, so far as I know, considered. One heard nothing of such infections in I9O2. The ears were examined, with negative result.

As the child had been healthy at birth, had been properly fed during most of its life, and showed no more signs of rickets than a large proportion of healthy children, there seemed to me no good reason to attribute its serious and progressive symptoms to that disease. 
$12-9790$ 


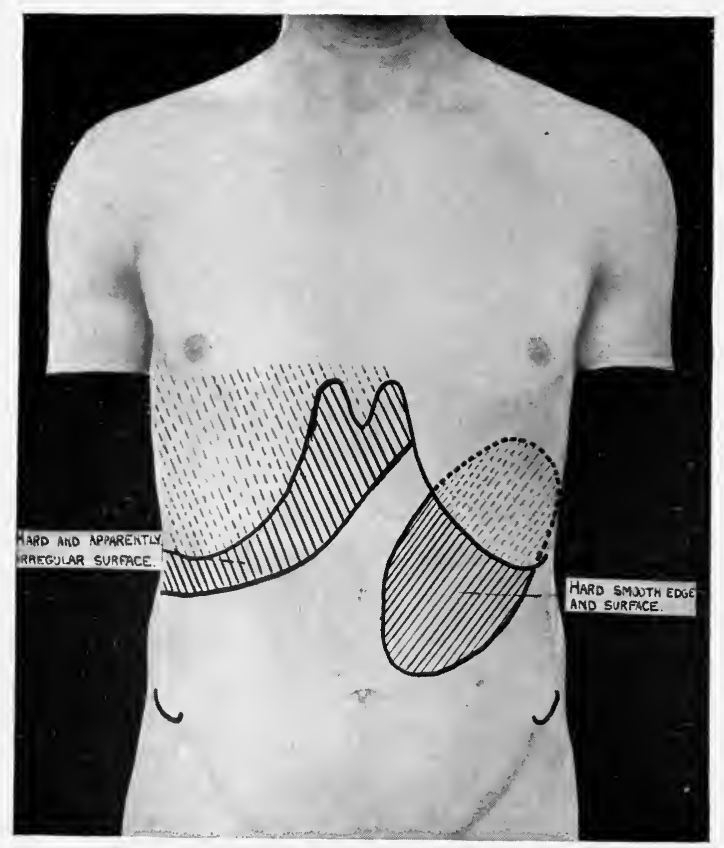

Fig. 78.-Condition of the spleen and liver in Case $2 \mathrm{I} 4$. 
Outcome.-January $23 \mathrm{~d}$ the child died. Autopsy by Dr. W. T. Councilman showed in the kidneys numerous foci of hemorrhage between the tubules; also here and there infiltrations of leukocytes; so extensive as to constitute small abscesses with destruction of the tubules and epithelium. Organisms of the colon group were found in these lesions.

At the time when this baby's illness occurred the frequency and importance of the urinary infections of girl babies was not recognized. Naturally, therefore, no one thought of this diagnosis during the life of the child, though in all probability this life might have been saved had the urinary tract been investigated earlier.

Diagnosis.-Renal infection (bacillus coli).

\section{Case $214 x$}

A real-estate broker of thirty-nine was seen June I9, I909. He had "typhoid" when he was six, and again when he was twenty-one. For the ten years succeeding this attack he had gall-stone colic in frequent paroxysms, which were finally cured by an osteopath in I900. He had no fever at that time. His wife died in 1900 . He married again in I908.

February 24, 1909, he went to Alabama feeling perfectly well. About six weeks ago he lost his appetite and began to have a headache, with much pulsation in his head. Soon after he noticed that climbing a slight hill exhausted him completely. For the past thirty-three days he had been aware that he had fever, ranging between $99^{\circ}$ and $103^{\circ} \mathrm{F}$., and usually reaching the lower figure once or more in every forty-eight hours. With this fever he had repeated chills and lost fifteen pounds.

He returned from the south a month ago, and has been in bed for ten days, troubled a good deal with gas in his bowels, with occasional belly pain and much weakness. Some weeks ago a homeopathic pathologist found a malarial parasite in his. blood, and since then he has received at least 20 grains of quinin a day. Nevertheless, he has continued to have fever and has grown steadily paler, thinner and weaker.

On physical examination he shows a yellowish pallor, hemoglobin being 55 per cent. The conjunctivæ are not discolored; the urine shows no bile-pigment. The chest and extremities are negative, the abdomen as per diagram (Fig. 78). The edge of the spleen and liver are both very sharp and hard; the surface of the liver seems to be somewhat irregular. There is no ascites.

Discussion.-As will be at once surmised from the treatment referred to above, malaria was the diagnosis up to June Igth. The chills, the anemia, the large, hard spleen and the report of malarial parasites in the 
blood had led very naturally to this diagnosis. Yet in my opinion malaria could be absolutely excluded, owing to the fact that the fever, though approaching the tertian type more nearly than any other, did not yield appreciably to large doses of quinin, which were obviously absorbed, as the patient's ears had been ringing steadily for weeks. My examination of the blood revealed no trace of malarial parasites. The red cells numbered 3,120,000; the leukocytes, 4800, the different varieties being present in approximately normal percentages.

By the blood examination just reported leukemia could be excluded. I have seen a very similar clinical picture produced by myeloid leukemia, but the blood was in that case very distinctive and the chills less numerous.

As the patient has a history of gall-stone colic and has now an irregular fever with chills and enlargement of liver and spleen, it is natural to consider for a moment the possibility that he may now be suffering from gall-stone fever. The condition of the abdomen and the course of the temperature are consistent with that diagnosis, although the spleen is unusually large; but gall-stone fever is almost always accompanied either by attacks of pain or by more or less transient yellowing of the conjunctiva during some part of the attack.

The irregular surface of the liver, if it be taken as an established fact, is of great diagnostic importance, as there are but two common diseases which produce hepatic enlargement with irregularities of surface palpable through the abdominal wall, viz., cancer of the liver and syphilis of the liver. Both of these diseases may be associated with fever, though this is more common in syphilis. The age of the patient, the freedom from marked gastric symptoms, and the size of the spleen point distinctly toward syphilis rather than cancer.

As soon as I asked the patient the direct question, he admitted that he had had syphilis seven years previously, and been treated for it by a wellknown specialist whose diagnosis I knew to be irreproachable. The patient had concealed this portion of his history even from his attending physician, who had not happened to ask him the direct question.

Outcome.-The patient was at once put on intramuscular injections of mercury with 15 grains of potassium iodid after each meal. By June 28th his fever was abating and general improvement quite noticeable. $\mathrm{He}$ afterward made a complete recovery.

Diagnosis.-Syphilis。 



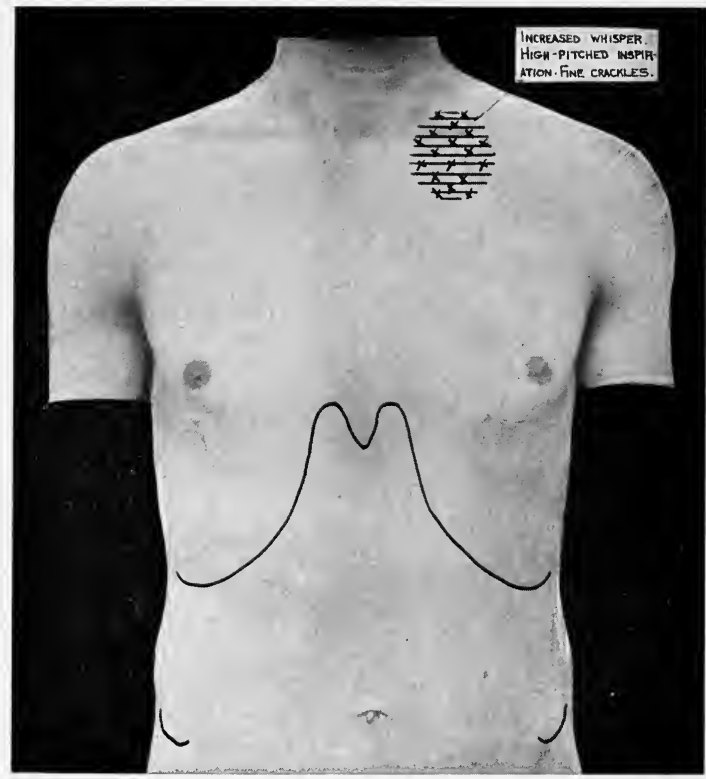

Fig. 79.-Physical signs in Case 215. No dulness; no bacilli in sputa; died of phthisis in two weeks.

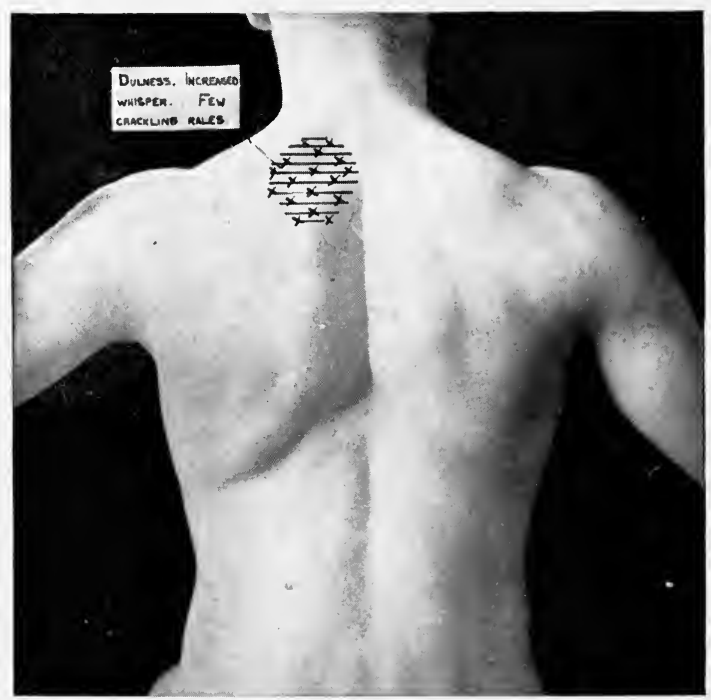

Fig. 80.-Physical signs in a case of unexplained fever. Practically no cough. 


\section{Case 215}

I was called October 24, I905, to see a young man of twenty-four-a steam-gage fitter. I reproduce the history as it was given to me.

He had complained of a week's increasing dyspnea and great lassitude. The attending physician, who saw him at the beginning of this illness, had kept a temperature chart which showed that there had been fever each day, rising to $\mathrm{IOI}^{\circ}$ or $\mathrm{IO}^{\circ} \mathrm{F}$. at night. The pulse range was from IOO to II2. The respiration rate showed a steady rise -28 for four days, 30 for the succeeding two days, and 36 for the past twenty-four hours. There was much sweating with the fever, but no pain and no other symptom except a slight, dry, hacking cough, which was not complained of and produced nothing until the day previous, when a single small mucopurulent mass was expectorated. This was examined at the Board of Health laboratory and found to be negative. The urine-ro25-contained a trace of albumin, a few fine and coarse granular casts, and a positive diazo-reaction.

The blood showed no Widal reaction. There was no wound or other known source for sepsis; no history of syphilis; no recent gonorrhea. The chest and abdomen had been examined with negative results.

What possibilities should be here investigated?

I. The past history should be scrutinized.

2. The physical examination should be repeated with special reference to the presence of-

(a) Central pneumonia; (b) endocarditis and pericarditis; typhoid; (d) miliary or generalized tuberculosis.

Further investigation of his past history showed that he had always been well, although in the previous August he had had some swollen glands in the side of his neck, which persisted for three weeks and were accompanied by night-sweats. After that he felt very well and went to work again.

Physical examination showed the signs indicated in Figs. 79 and 8o. The heart and pericardium showed nothing abnormal. The temperature chart [showing a normal or subnormal temperature each morning] was practically sufficient, considering the previous course of the illness, to exclude typhoid and central pneumonia. The leukocyte count, which was normal, added to the evidence against pneumonia.

The boy did not cough at all during my visit, but the character of the signs, when taken in connection with the fever and other symptoms, seems to me to point strongly toward pulmonary tuberculosis, of which disease he died two weeks later. 
The attending physician was much surprised and rather skeptical at my diagnosis, "for," as he said, "the boy has practically no cough, almost no sputa, and what he does raise has been examined and found negative." It cannot be too strongly insisted, in view of this and many other similar cases, that a negative sputum examination, unless it has been many times repeated, should never be considered as evidence against pulmonary tuberculosis. Even then it is by no means conclusive, as bacilli may not appear for many weeks or even months after the onset of the disease in the lung.

Diagnosis.-Pulmonary tuberculosis.

\section{Case $216 t$}

On January $18, \mathrm{I} 897$, soon after the discovery of Widal's reaction in typhoid fever, I was asked to examine the blood of a febrile case in which that diagnosis seemed fairly certain. Some confirmation, how-

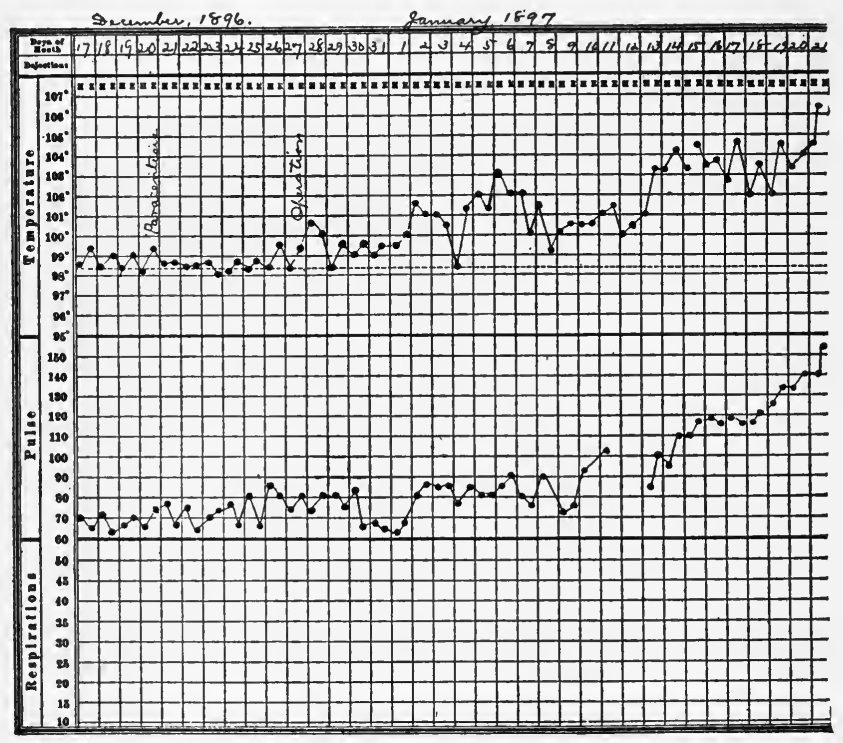

Fig. 81.-Chart of case 216 .

ever, was desired. Four weeks previously the patient had had a mastoid operation following an attack of otitis media. All had gone well, and the wound was now almost healed; only a small area of healthy granulations remaining in the mastoid region. Nevertheless, soon after the operation the patient had begun to have fever, the course of which is shown in the accompanying chart (Fig. 8). 
Throughout its course he had complained of nothing except such discomforts as could be reasonably attributed to the fever itself. $\mathrm{He}$ had had no headaches, no tenderness at or near his wound, no symptom that would serve to localize any cause for the fever.

At the time of my examination a group of typical rose spots were scattered over the abdomen. Each spot was about $2 \mathrm{~mm}$. in diameter, and disappeared wholly on pressure. The spleen was not palpable, and visceral examination was otherwise negative, with the exception of a few scattered râles at the base of each lung.

The blood examination showed leukocytes, 23,000, 88 per cent. of which were polynuclear. The Widal reaction was entirely negative, even in dilutions of $\mathrm{I}$ : 10 .

Discussion.-I reported to the surgeon in charge of the case that it was not one of typhoid fever, and that I believed some type of wound infection must be present. At that time I did not know of the commonness of infectious thrombosis of the lateral sinus and jugular vein, since so thoroughly studied by Libman ${ }^{1}$ in its relations to bacteriemia. Doubtless micro-organisms might have been cultivated from the circulating blood had I known at that time the importance of the test.

A great skepticism of my results was expressed at the time. The chart was so typically that of typhoid, the rose-spots so diagrammatic, the patient so completely free from any local symptoms or complaints, that it seemed absurd to exclude typhoid on the evidence of so academic a laboratory test as blood examination. This was before we had been shown by thousands and tens of thousands of blood-counts that uncomplicated typhoid never produces such a leukocytosis as that here recorded, and that the absence of a Widal reaction after four weeks of fever is strong evidence against the existence of typhoid.

Outcome.-The patient died January 2Ist; autopsy showed a septic thrombosis of the lateral sinus and jugular vein.

Diagnosis.-Septic thrombosis of the lateral sinus and jugular vein.

\section{Case 217}

A physician of thirty-nine was seen November 30, 1905. Six years previously he had had the grip, followed by weakness, emaciation and night-sweats. Pulmonary tuberculosis was suspected, but not proved. He went south for two months and recovered entirely, and has since then worked very hard, "mostly," he says, "on his nerve."

August 17,1905 , a hair-follicle on his finger got infected. It was

1 The Importance of Blood Culture in the Study of Infections of Otitic Origin, by E. Libman and H. L. Celler, Trans. Assoc. Amer. Physicians, rgo9, p. 36r. 
opened and cureted on the nineteenth under cocain. He felt much exhilarated thereafter, and made his medical calls as usual throughout the rest of the day. In the evening he collapsed, and had a very severe pain in the right intercostal region, accompanied by high fever not relieved by poulticing, and only modified by $\frac{3}{4}$ grain morphin. Next day the signs of pleurisy were found, and two days later an area the size of an orange appeared near the angle of the right scapula. Over this the breathing was bronchovesicular, with dulness and crackling râles. These signs lasted without much change for four weeks, and were not wholly gone for two weeks more. An irregular fever persisted throughout.

October 6th, though still weak, and despite the presence of highpitched respiration over the area described above, he felt well enough to be moved to the White Mountains, where he rapidly improved, ate well and slept well, took four-mile walks, and had no cough to speak of. He had several bad headaches, but otherwise felt well and returned to work October 26th. At this time his lungs were examined and found normal; his sputa contained no bacilli and no elastic fibers. The day after his return he got overtired and again collapsed, i.e., could not talk, eat, or sit up, had a bad headache, and was awake all night.

Next day he felt better, and the day after felt "like a fighting cock." During the next ten days he did his medical work as usual, although he felt somewhat poorly every second day. November $3 \mathrm{~d}$ he did a very hard day's work, and at the end of it felt chilly and languid. His temperature was found to be $102^{\circ} \mathrm{F}$. From November $3 \mathrm{~d}$ to November 3oth-the day on which I saw him-he had an irregular fever, accompanied by headaches. All his symptoms tended to be worse every second day.

Two of his colleagues saw him in consultation November Ioth, the diagnoses considered being grip, malaria, and simple nervousness. The spleen was felt, and accordingly quinin, 24 grains daily, and Fowler's solution, 5 minims three times a day, were administered. The quinin hammered the temperature down, but it rose again as soon as the drug was stopped. The blood was twice examined at this time, and found to be normal; no anemia, no leukocytosis, no Widal reaction. The urine was also normal (November I 3 th).

By this time the doctor-always of a very high-strung nervous temperament - had gotten so worked up about himself that he was again sent to the country, but while there still had fever, ranging from $100^{\circ} \mathrm{F}$. in the morning to Ior. $4^{\circ} \mathrm{F}$. in the evening, despite the administration of quinin, 24 grains a day. During the last two weeks he has had ten days of pain over the lower left back, in the region of the diaphragmatic 



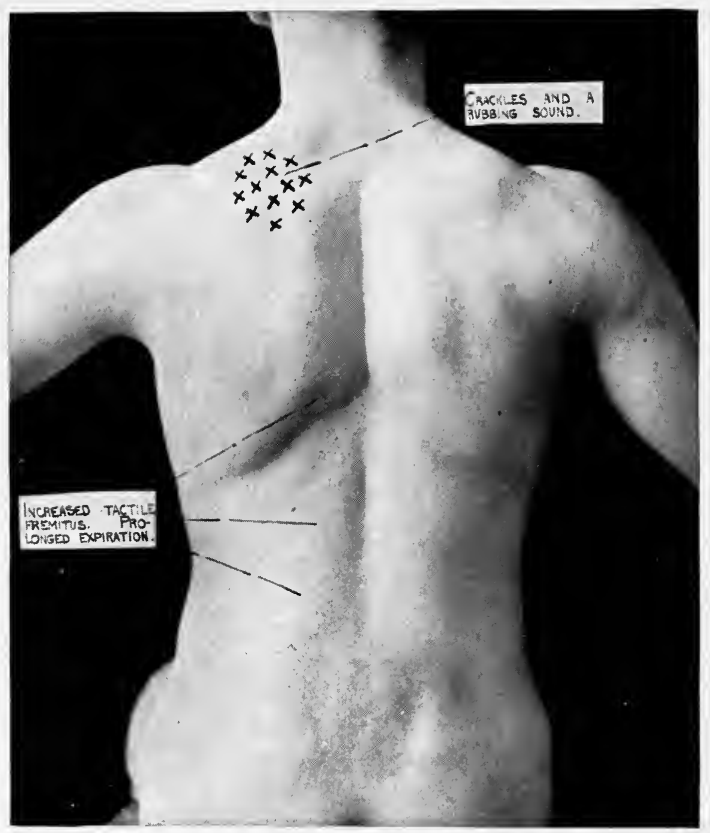

Fig. 82.- Physical signs simulating pulmonary tuberculosis in a case of sepsis with pros tatic and perinephric abscess. Complete and lasting recovery followed. 
attachment. Throughout the last ten days of his fever he has also had pain in urination, and for the past few days some distress in the rectum and perineum.

On the twenty-ninth of November he returned to his home feeling pretty poorly and eating very little.

Examination November 3 oth showed temperature $100^{\circ} \mathrm{F}$., no emaciation, abdomen negative, spleen not felt, lungs as per diagram (Fig. 82).

Discussion.-Typhoid and malaria, it seemed to me, could be easily ruled out. I could find no evidence of any form or focus of sepsis. Accordingly, I made the diagnosis of pulmonary tuberculosis. December Ist the prostatic symptoms became more marked; tenderness and fluctuation appeared in the perineum and a large prostatic abscess was evacuated.

December roth, tenderness and swelling appeared in the region of the left twelfth rib. Incision liberated a large amount of pus from the region of the kidney, which was not felt or seen. The patient made an uneventful recovery, and has been well ever since (October, r9ro).

I made two chief mistakes in this case: first, in forcing myself to make some diagnosis, even an improbable one, because everything else seemed more improbable. The proper course would have been to wait until something more distinctive appeared.

My second blunder was in paying so little attention to symptoms on the part of the bladder and rectum, which, though very trifling at the time when I saw the patient, were enough to suggest the presence of a septic focus which became evident within twenty-four hours.

Diagnosis.-Perirectal abscess; perinephric abscess.

\section{Case 218}

A married woman of thirty-two consulted me in October, r9o8, accompanied by her physician, who was an intimate friend of the family. The diagnosis was splenic anemia, and the problem presented to me for consideration was whether splenectomy should be done.

The patient's complaints were of general weakness, languor, and a dragging sensation in the left axillary region. A slight continued fever was found to be present. The spleen reached almost to the navel, and appeared to be unusually immobile, perhaps owing to the presence of adhesions. Visceral examination was otherwise negative. The blood showed 3,500,000 red cells, 8000 leukocytes, 45 per cent. of hemoglobin. The differential count showed nothing vrorthy of note. The red cells showed in the stained smear a marked achromia with slight variations in size. No nucleated red cells were seen. 
The patient was advised to enter the hospital for more careful study, and probably for an eventual splenectomy. She delayed, however, nearly three months before accepting this suggestion. Meantime there had been a considerable accumulation of fluid in the abdominal cavity, and tapping had already been required about two weeks before her entrance to the hospital.

A reëxamination of the patient at this time showed, except for the ascites, no especial change as compared with the conditions previously found, although the anemia had slightly increased. The temperature continued slightly elevated, the pulse, respiration, and urine normal. Blood-pressure, r25. Although I was somewhat apprehensive that hepatic changes might have progressed so far as to prevent the splenectomy from relieving her symptoms, it seemed as if she were going on from bad to worse in spite of all that good hygiene and the administration of iron and arsenic could do; hence it seemed best to go on with the splenectomy, perhaps preceding it by a direct transfusion of blood.

At this juncture Dr. Wilder Tileston saw the patient at my request, and, in conversation with him, the patient mentioned that she had been troubled for a long time with catarrh and cold in her head. "It had been there so long," she said, "that I am getting quite used to it; but a little while ago, as I was blowing my nose, something came away, and I noticed that there was a passage from one nostril to the other, inside."

Discussion.-Following up this hint, Dr. Tileston learned that she had had "some sort of skin disease" in her scalp, as a result of which there were still marked unevennesses over the cranial vault, though the skin was wholly normal.

No other evidences of her previous syphilis were demonstrable either in the history or in the physical examination, but the facts seemed to me to warrant an immediate abandonment of the plan for splenectomy and a thorough trial of antisyphilitic treatment, which she had never had. As a result of this she gradually returned to perfect health, the spleen diminished to one-quarter its former size, the anemia and ascites disappeared, and the patient was enabled to take up her usual mode of life.

This was a very narrow escape from a serious mistake. There was nothing in the histoiy, as given to me, to suggest syphilis. Doubtless I was misled partly by the obvious innocence of the woman, partly by the fact that her physician, who was intimate both with her and with her husband, had clearly no idea that the husband had been infected previous to marriage. Nevertheless, I ought to have considered syphilis merely from the association of the enlarged spleen and ascites with an 

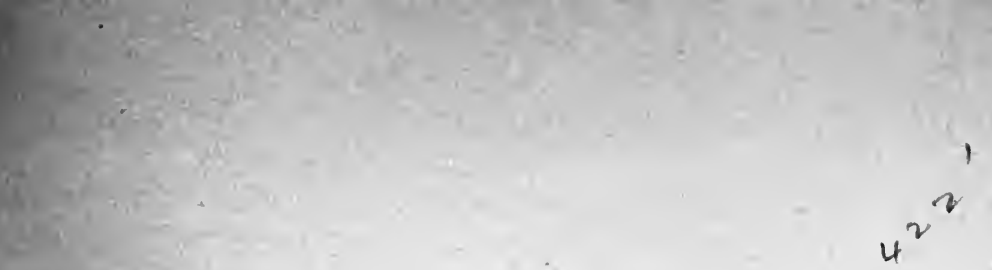


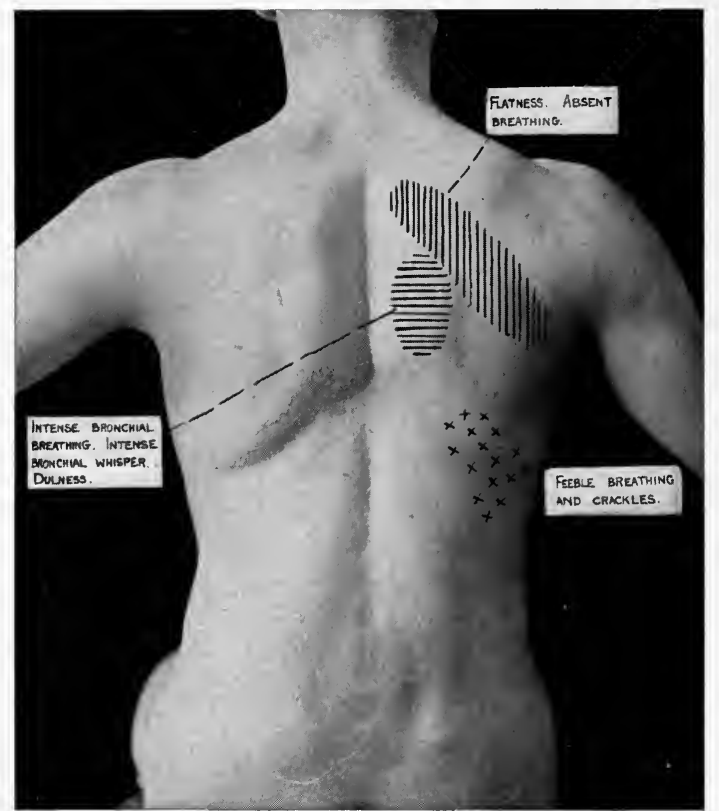

Fig. 83. - Chest signs obtained on physical examination of Case 219.

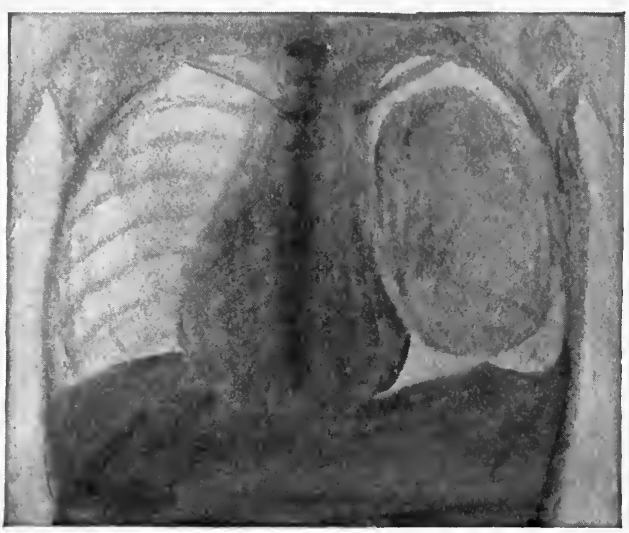

Fig. 84.-Pencil sketch from an $x$-ray plate of Case 219. 
anemia of unknown cause, for in that text-book which we should all know by heart I find the following, under Syphilis of the Liver:

"In a second group of cases the patient is anemic, the liver is enlarged, perhaps irregular, and the spleen also is enlarged. Dropsical symptoms may supervene." (Osler's Practice of Medicine, seventh edition, p. 276.)

\section{Diagnosis.-Syphilis.}

\section{Case 219 f}

A boarding-school boy of sixteen was seen December I2, I907. He had had a "regular cold" with a little fever which seemed to be ended three days ago, but next day the temperature rose again to ${ }_{102}{ }^{\circ} \mathrm{F}$. Yesterday morning crackles were heard for the first time at the right base. Last night at midnight he vomited and complained of pain in the right axilla on coughing. When examined at 7 P. M. his temperature was $102^{\circ} \mathrm{F}$, his pulse 90 and dicrotic. Except for slight distention of the belly, the abdomen and extremities showed nothing abnormal, likewise the left lung. Examination of the base of the right lung behind showed in some positions nothing but enfeebled vesicular respiration, but when lying on the right side there were crackles, increased whisper, and a small patch of feeble bronchial breathing near the angle of the scapula.

Although these signs were not very distinctive, their association with a typical rusty sputum and a high leukocyte count seemed to me to justify a diagnosis of lobar pneumonia. On the nineteenth, as the temperature suggested an empyema, a needle was put in near the angle of the scapula, but only an ounce of bloody serum was obtained. On the twenty-fourth he was tapped again, this time in the axillary line, and an $x$-ray was taken of the chest, which showed nothing abnormal.

January $3 \mathrm{~d}$ the temperature was normal, the boy hungry and sleeping well, but the chest signs were still far from normal. On January 6th the temperature rose again, and though the boy was still eating, sleeping, and feeling finely, the signs were as in the accompanying diagram (Fig. 83). The front of the chest and the axilla showed nothing of importance. The boy's temperature was ror. $6^{\circ} \mathrm{F}$. in the morning, $99.4^{\circ} \mathrm{F}$. in the afternoon. January 7 th it was $102.2^{\circ} \mathrm{F}$. in the morning, $100^{\circ} \mathrm{F}$. in the afternoon. Between this date and the twenty-second of January two other unsuccessful taps were made. The boy continued in excellent condition despite his daily fever. The sputum was repeatedly examined, with negative results.

At this time he was moved to New York city and put in charge of 
Dr. Evan Evans. A second $x$-ray made at this time showed the appearances sketched in Fig. 84. January $22 \mathrm{~d}$ pus was finally found under the scapula by a puncture made through the axilla. The boy made an' excellent recovery. 1

Diagnosis.-Interlobar postpneumonic empyema.

\section{Case 220}

A girl of six entered the hospital November 18, 1907. She has always been weak, and often complained of her ears. She has had measles, chicken-pox, and whooping-cough. Three days before entrance she fell and hurt her head. That night she was feverish and complained of headache. The next day, her mother said, she "never opened her eyes." She has vomited watery material several times, and continued to complain of pain in her head, also in the abdomen. She has been somewhat constipated. She has been in bed two days.

Physical examination showed a red throat, but two cultures, taken November I8th and November 22d, were negative for diphtheria. The ears were also negative; no stiffness of the neck; no glandular enlargement. The mucous membrane of the mouth was normal. The chest and abdomen normal. The edge of the spleen was felt. The urine was free from albumin and sugar. There was no edema. The blood was normal. The chart was as follows:

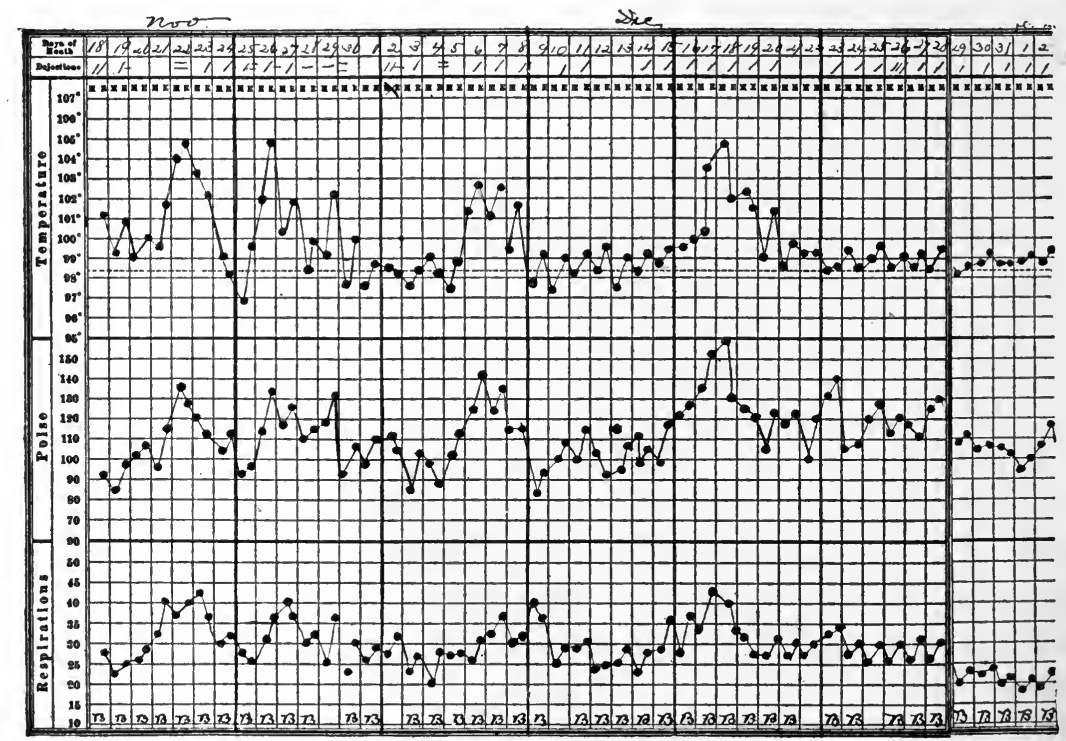

Fig. 85.-Chart of case 220.

${ }^{1}$ Dr. H. B. Whitney, of Denver, writes me of a similar case in which he drew streptoroccus pus by puncture in the interscapular space, to the right of the sternum at the level of the fourth and fifth dorsal vertebræ. The patient recovered without any further operation. 
Discussion.-The fevers of children give rise to far more diagnostic difficulties than those of adults. Children's temperatures undergo far wider and more numerous oscillations in perfect health than adults' temperatures. Besides these supposedly normal variations, there are a great many short periods of pyrexia occurring in children who are more or less out of sorts without any reason at present assignable.

In addition to the variations just alluded to, children are subject to many fevers lasting several days "with nothing to show for them"$i$. e., without any obvious local physical signs and without any complaint to direct our search to any organ or tissue. Among the commoner causes ultimately discovered for such fevers are:

(a) The onset of the exanthemata.

(b) Infections of the heart and pericardium, with or without joint pains ("rheumatic").

(c) Otitis media (without any discharge or complaint on the child's part).

(d) Urinary infections ("pyelitis," ascending or hematogenous).

(e) Empyema (without pain, cough, or dyspnea).

( $f$ ) Poliomyelitis.

(g) Tuberculous meningitis.

(h) Typhoid fever.

In all such cases the best that we can do is to make repeated and comprehensive examinations of the child, who is meantime kept in bed, given an easily digested diet and plenty of water to drink. Sooner or later, if we are vigilant, something comes to light. The points neglected in the present case will be obvious from the outcome.

Outcome.-On the twenty-fourth repeated examinations from head to foot still showed no cause for her illness. She slept and ate fairly well, and took an interest in what went on.

November 29th: "Several nights ago she complained of pain in the left leg. Next morning the left knee-jerk was absent, the right easily obtained. It was found that the child's mother had been bringing her chocolate candy and that the child had eaten about a quarter of a pound of it, hiding the box at night under her bed-clothes."

That night her urine was reported to be full of non-motile bacilli resembling colon bacilli.

December 8th: "The knee-jerk on the left is sometimes present, sometimes absent, sometimes obtained after long trials. On walking the child drags the left foot ever so little. There is no muscular atrophy. A considerable amount of pus appeared in the urine on the fifth of Decem- 
ber, and this has increased since. Urotropin, 5 grains thrice daily, and abundant water were given."

On the twenty-fourth of December a little drooping of the left shoulder was perceptible, and the left foot still dragged a little.

January $2 \mathrm{~d}$, the urine being free from abnormalities, the child was discharged well.

Diagnosis.-Poliomyelitis; renal infection.

\section{Case 221}

An Irish laborer of eighteen entered the hospital May 20, I908. On the recommendation slip from the out-patient is written: "Appendicitis? Typhoid? Tuberculosis?" His father and one brother died of phthisis. The past history is good. On the fourth of December he began to have

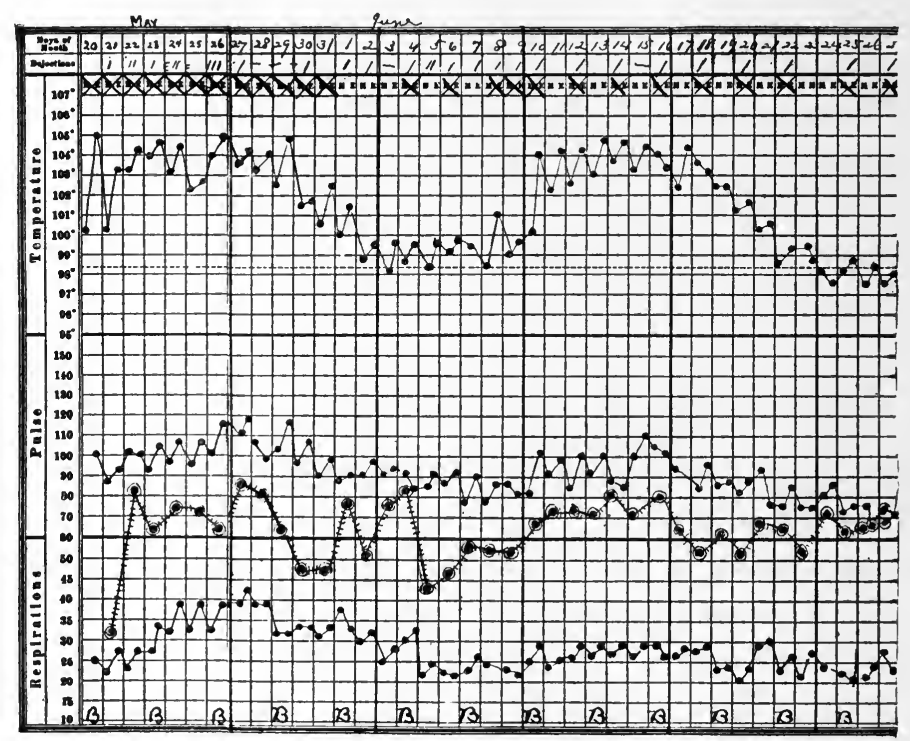

Fig. 86. - Chart of case 221 .

pain in the stomach, which has kept him awake at night off and on ever since. There is no vomiting; no appetite. He has also been coughing for the same period, with a good deal of sputa.

Physical examination shows slight emaciation, enlarged tonsils, especially the right, but no exudate. The heart is normal. The lungs show a few scattered crackles and squeaks. The right half of the abdomen is slightly more resistant than the left, and in the region of the cecum there are marked local tenderness and a mass about the size of an egg. The edge of the spleen is just felt on full inspiration, likewise the 
edge of the liver. The knee-jerks are obtained with difficulty. There are old, irregular scars on the backs of both hands and at the lower end of the right ulna. Leukocytes, 2800 .

At no time was there any considerable abdominal spasm. By May $22 \mathrm{~d}$ the tenderness in the abdomen was gone.

Discussion.-Remembering the great susceptibility of the Irish to tuberculosis, the patient's family history, and the long persistent cough of which he complains, we cannot but consider the possibility of a tuberculosis, pulmonary or generalized. The signs in the lungs are consistent with miliary tuberculosis, but not in any way characteristic of that or of any other pulmonary affection. The sputa should, of course, be repeatedly examined. (This was done, but with negative results.) A tuberculin reaction might be tried, but would be significant only in case it was negative, as the vast majority of adults react positively, owing to the latent obsolete foci of tuberculosis. Had the disease been of the ordinary pulmonary form, the signs in the lungs should have been more extensive, in riew of the long duration of the cough.

Tuberculous peritonitis with glandular masses and adherent coils of intestine near the cecum might explain many of the symptoms, though one would expect more abdominal spasm and tenderness.

Appendicitis must, of course, be considered, though the local signs are few and slight, and the cough and splenic enlargement could not be thus. explained. The leukocyte count is also surprisingly low for appendicitis.

The scars upon the back of the hands and on the right forearm resemble those sometimes produced by syphilis. The splenic and hepatic enlargement, the cough, and fever might thus be explained, and the absence of any history of this infection is of no importance. Without further evidence, however, one would not resort to the therapeutic test, at any rate until other probabilities had been excluded.

The diagnosis of typhoid fever would explain the present symptoms very well. Many cases of typhoid exhibit a certain amount of tenderness in the appendix region, and this patient's lung signs are those usually found in typhoid. We are puzzled, however, to explain the long duration of symptoms. This man can hardly have had typhoid from December 4th to May 2oth, and if we suppose the typhoid to have begun more recently, we have no means of conjecturing what other disease he may have had previously. Evidently, what we most need at the present juncture is a Widal reaction and blood culture.

Outcome. - The Widal reaction was found to be positive May 2oth, and typhoid $\mathrm{b}$, illi were isolated at the same time from the ear blood. 
The course of the disease thereafter was uneventful. The patient went home perfectly well on the fourteenth of July.

Diagnosis.-Typhoid with relapse.

\section{Case 222}

A salesman of nineteen entered the hospital June 22, I908, with a negative family history and good habits. Four months ago, in Georgia, he had a fever which kept him in bed for six weeks and a half. The blood was not examined. He had been given capsules with considerable relief. Six days ago he had a chill, followed by headache, fever, and

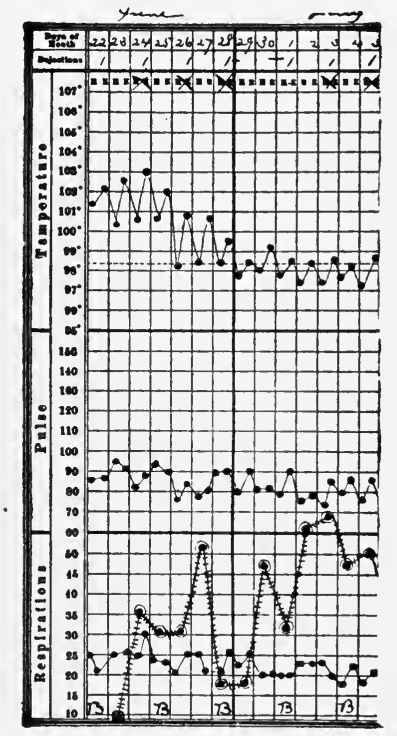

Fig. 87.-Chart of case 222 . nosebleed. Four months ago he weighed I54 pounds, now he weighs I 24 pounds.

Physical examination showed a soft systolic murmur, heard all over the precordia, while the first sound at the apex was very faint. The pulmonic second was greater than the aortic second sound. There was no enlargement or irregularity. The arteries were palpable between beats. Liver dulness extended from the sixth rib to a point two inches below the costal margin in the parasternal line. The soft edge of the spleen was felt on inspiration.

The course of the temperature is shown in the accompanying chart. The white cells were 4300 . Widal reaction negative. No malarial parasites were found in the blood.

Discussion.-Estivo-autumnal malaria is naturally our first guess in the case of a febrile patient who has recently returned from Georgia, but this is at once ruled out by the negative examination of the blood ${ }^{1}$ and the good condition of the patient. If he had had estivo-autumnal malaria in his system for four months, his spleen would have been harder and probably larger, his general condition worse.

Endocardial fever is suggested by the presence of a cardiac murmur and long duration of symptoms, but the leukocytes are rarely so few in this disease, and the murmur may well be explained as "functional."

What inference should be drawn from the extension of liver dulness

${ }^{1}$ Very rarely malarial parasites are not to be found in the peripheral circulation at a single examination during the febrile stage of estivo-autumnal malaria. I have known of but one such case. 
two inches below the costal margin? Should we consider some of the hepatic diseases which are often associated with fever (hepatic syphilis, abscess, cholangitis, leukemia)? I think not, for we have no good reason to believe that the liver is enlarged. The extension of dulness below the costal margin should never, in my opinion, be taken as evidence of hepatic enlargement unless the edge of the organ is also palpable. Dulness below the right ribs, continuous with that of the liver, is to be found in countless cases which never show any other evidence of hepatic enlargement.

The loss of thirty pounds in four months makes us suspect tuberculosis hidden somewhere in the body, but there seems to be no good evidence to support this suspicion, though tuberculosis cannot be positively excluded.

We must ask ourselves the question, Can this be the "fag-end" of a typhoid despite the absence of a Widal reaction? The time of year is not at all the usual one for such an infection, and at first sight we should suppose that after so long an illness the patient would either be well or dead if he had had typhoid all that time. Experience shows, however, that just such a history of long, indefinite illness is to be obtained in many cases which turn out eventually to be unmistakable typhoid. No one, so far as I know, has adequately accounted for this fact, but no one who has seen much typhoid will dispute it. It is commonly explained by saying that the patient has probably had most of his typhoid before he came under observation, and that what we are seeing represents the end of a relapse-perhaps the second or third relapse that he has had. This is perhaps the most plausible explanation, although we should expect the patient to be much more exhausted as we recall the appearance of patients who have had two or three relapses under treatment. We must reject the blasphemous thought that the patient may be in good condition because he has had no treatment.

The present case, however, is hard to explain, even by this rather far-fetched hypothesis, for he had his six weeks and a half of fever four months ago, and has, since that time, been up and about his business until he was suddenly seized with a chill on June I6th. It remains to me a mystery, although a very familiar one, many examples of which I see each autumn when patients in the typhoid ward relate very circumstantially the course of an illness which has lasted all summer.

Outcome.-On the twenty-fourth of June the Widal reaction was positive. The patient was out of bed July Ioth and discharged well on July i8th.

Diagnosis.-Typhoid (brief). 


\section{Case 223}

An Italian laborer of twenty-eight entered the hospital September 23, 1906. His family history, past history, and habits are good. Three weeks ago he went to bed with a headache and has been there ever since. His appetite is good, but he has not been allowed to eat much. His bowels have been constipated. There has been no cough. He has had three nosebleeds.

On physical examination the pupils were found to be slightly irregular, the right larger than the left. Both reacted normally. The glands in the neck, axillæ and groins were palpable, but not enlarged. Physical

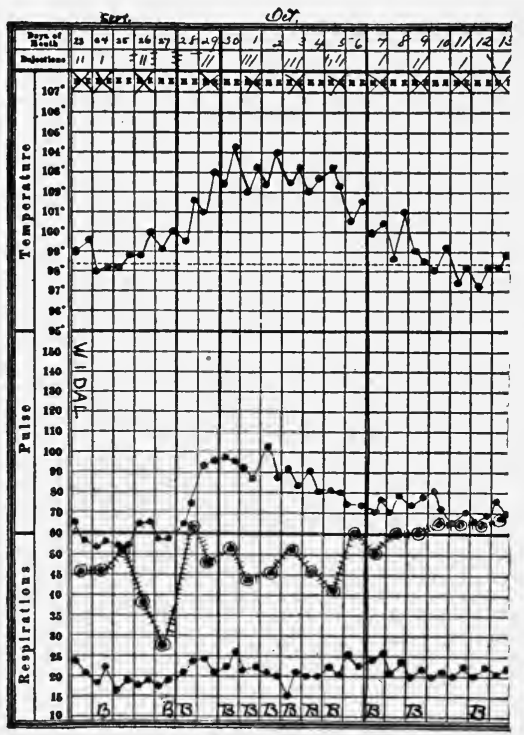

Fig. 88.-Chart of case 223 .

examination was otherwise negative, also the urine. The eye-grounds were normal. White cells, 7000 .

Discussion.-One's first impression would be that there is really not much the matter with this man. His temperature is practically normal, his organs negative to physical examination. But on second thought we must recognize that a young Italian laborer does not stay in bed three weeks for the fun of it. Something must be the matter with him, and his doctor says that he has had a fever.

A very considerable proportion of Italian laborers appear to have had syphilis. The irregularity of the pupils and the palpable glands seemed to support this idea; but it was not possible to get beyond the 
region of conjecture as regards syphilis, for the Wassermann reaction was not then in use.

The slow pulse and the rather persistent headache might be taken as evidence pointing toward brain tumor or other cerebral lesions; but this suggestion, like the others, had to be left hanging, as there were no sufficient grounds for a more positive decision.

At this time of year and in a patient with this history it is always advisable to try a Widal reaction. The result of it was, in this case, extremely interesting, as is indicated by the outcome.

Outcome.-The Widal reaction was strongly positive at entrance. The later course of the temperature is shown in Fig. 88. The patient went home, apparently well, on the twenty-second of October.

Diagnosis.-Typhoid (afebrile when first seen).

\section{Case $224 x$}

A housewife of thirty-seven was seen March 16, 1907. Her family history was good. She has never been sick before. She has been nursing her twelve-year-old girl, who has been sick for three weeks with fever,

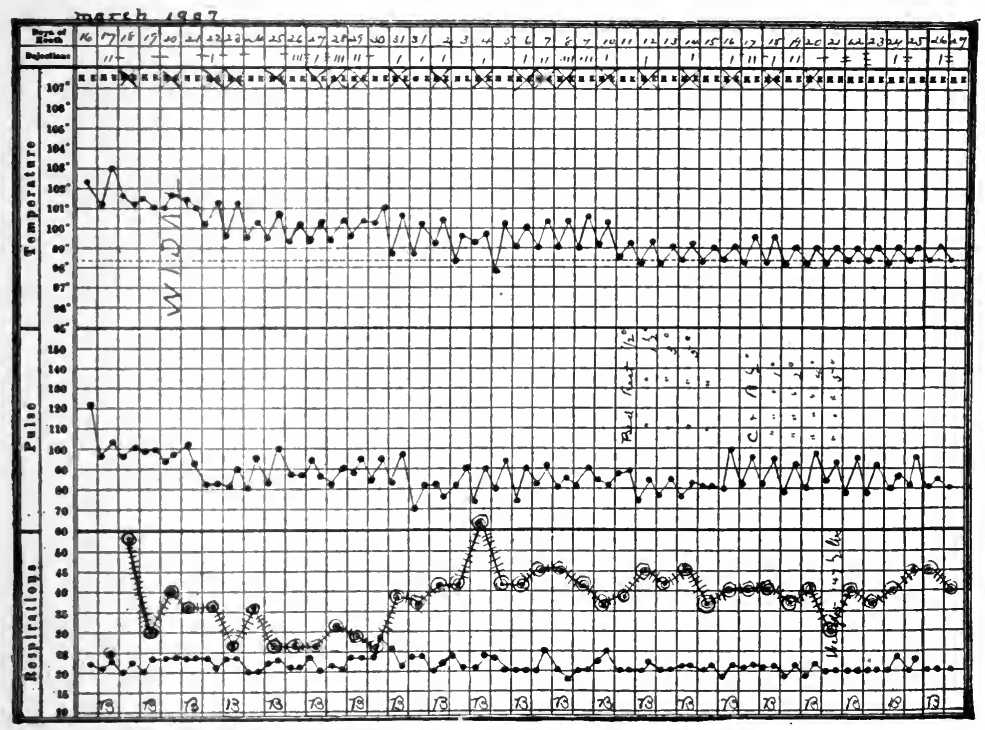

Fig. 89.-Chart of case 224.

diarrhea, thirst, and stupor. Yesterday her boy of fourteen was also taken sick. She has felt tired from nursing her children, but did not call herself sick until the doctor took her temperature at 6 P. M. to-day, and 
found it $102^{\circ} \mathrm{F}$. She sleeps well, but is constipated and has a rather poor appetite.

Examination showed an obese, apathetic woman, her scalp covered with crusts. A soft, blowing systolic murmur was heard over the precordia, loudest in the pulmonary area. The pulmonic second sound was accentuated, the heart not enlarged. The lungs and abdomen were negative. White cells, 4600; Widal reaction negative. The bowels moved daily. On the twenty-sixth she began to suffer from diarrhea with distressing tenesmus, which lasted three days, and on the same day she passed a small amount of blood, the pulse not being at all affected.

On the twenty-eighth, rectal examination revealed a large mass of feces just inside the anus. Removal of this relieved all the symptoms.

On the third of April she complained of a burning micturition. The urine showed nothing abnormal except extreme acidity. Citrate of potassium and cream of tartar water promptly relieved this symptom. She was discharged well on the twenty-seventh.

Discussion.-When a woman has a fever and nothing to show for it; when the leukocytes are subnormal and two others in the same family have febrile illnesses, the chances are strongly in favor of the assumption that she has typhoid fever, probably acquired by contact. In the present case the Widal reaction appeared March 2oth, but the diagnosis was easily made before that.

The case is introduced here to exemplify the occurrence of diarrhea and tenderness due to fecal impaction in typhoid fever, even though the bowels had been moving daily. Such cases are not at all uncommon, and if rectal examination is neglected, the trouble is rarely recognized, and may cause much suffering. It usually occurs toward the end of the case, at or near the period of defervescence, coming on quite suddenly and without warning. The accumulation is often so great that it has to be removed mechanically. The lesson forced upon me by my failure in one such case was never to neglect rectal examination in a case of diarrhea.

Of some interest also is the dysuria, which the therapeutic tests apparently prove to be due to hyperacidity of the urine.

Diagnosis.-Typhoid; impaction; dysuria.

\section{Case 225}

A rubber worker of thirty-seven, a Swede by birth, entered the hospital June 10, 1908. His family history and past history were good, except that he had "typhoid" at the age of eighteen, and "malaria" for a week a year ago.

Two weeks ago, while at work, he had a severe chill and abdominal 
cramps, which doubled him up. After three hours he went to work again and kept on for the next two days, when he had to give up on account of weakness and pain in his stomach. He has been in bed for a week. To-day he vomited twice; he has had no appetite, poor sleep, moderate constipation. He has passed urine only twice in each twenty-four hours during the last two weeks. What he passes is very red.

Physical examination showed obvious loss of weight. Cardiac dulness extended one inch beyond the right border of the sternum. No cardiac impulse was seen or felt. There was nothing abnormal about the sounds. The left lung showed bronchial respiration above the clavicle, bronchovesicular respiration and increased voice-sounds down

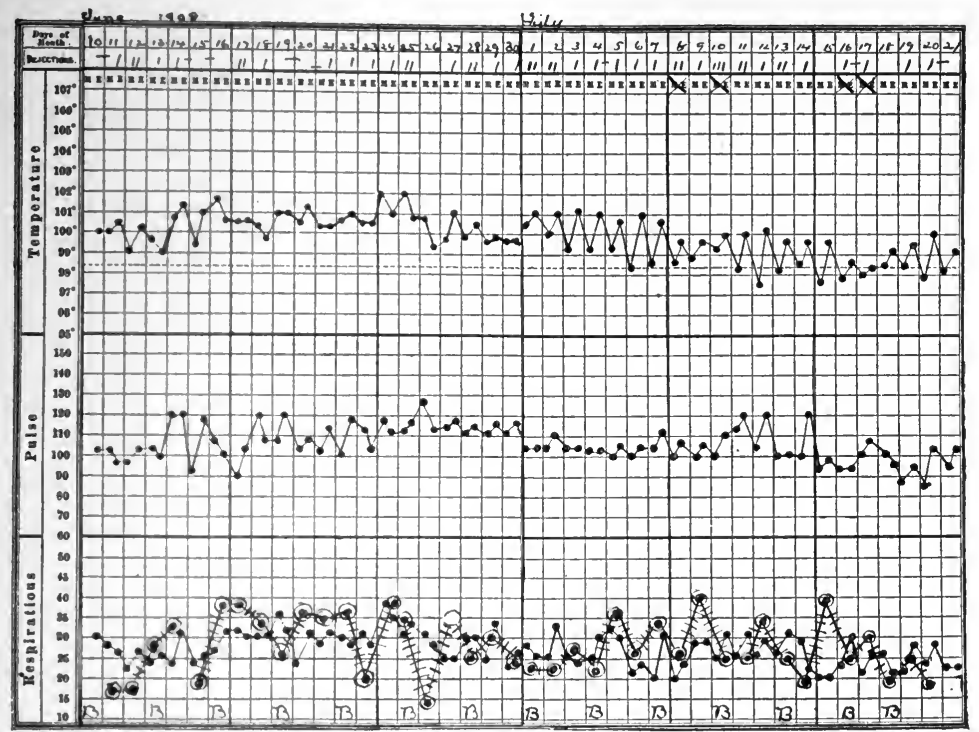

Fig. 90.-Chart of case 225 .

to the second rib. Below that level voice-sounds, breath-sounds, and tactile fremitus were diminished; percussion was dull to flat. The abdomen was quite negative. The white cells were 7400; the urine negative.

The chest was tapped on the eleventh and 40 ounces of clear, pale yellow fluid removed. Specific gravity, IoI7; albumin, 2.8 per cent.; lymphocytes, 97 per cent.

On the sixteenth 64 ounces more were removed from the chest. On the twentieth it was tapped a third time, but only Io ounces found. On the twenty-eighth it was again tapped and 70 ounces were removed. His weight a month before entering the hospital was I55 pounds. At the time of his discharge it was I24 pounds. 
Discussion.-In rubber workers we meet with all sorts of obstinate and debilitating symptoms which oftentimes refuse to be grouped into any recognizable disease, although lead colic sometimes emerges from the obscurity, in case the workers deal with that part of the process of manufacture in which lead is used. But, so far as I am aware, none of the toxic effects of work in a rubber factory produces fever.

The patient's account of himself gives us no inkling of what may be the cause of the fever. Physical examination and the results of aspiration leave no doubt that the patient has been suffering from a pleural effusion. It is unusual, however, to observe so rapid a reaccumulation of the fluid. In the vast majority of cases of ordinary tuberculous pleurisy a single tapping suffices, or if recurrence takes place, it is far less rapid than in the present case, which suggests another and more ominous possibility.

Whenever rapid and frequent reaccumulation of pleural fluid occurs in a case believed to be one of ordinary (tuberculous) pleurisy, we should always suspect malignant disease of the lung, pleura, or mediastinal glands, no matter how young the patient and despite the absence of all pain. I have twice made the mistake of diagnosing as pleurisy a case which turned out to be malignant disease with secondary effusion. Malignant disease not infrequently produces a bloody effusion, but this is by no means invariable.

The $x$-ray gives us usually but little assistance in doubtful cases of this type, as the collapsed lung may simulate the shadow produced by malignant disease. The cellular elements of the sediment may be identical in both diseases. The first clue obtained in most doubtful cases is the appearance of a metastasis in one of the external lymph-glands or elsewhere. Later the steady decline in the patient's strength makes it obvious that something more serious than pleurisy underlies the effusion.

Outcome.-After July 28th there was no further reaccumulation and the patient rapidly improved. On August 6th he went to Rutland Sanatorium.

Diagnosis.-Pleurisy (tuberculous).

\section{Case 226}

A young married woman of twenty was first seen January 27, I904. Two months ago her second child was born. Hemorrhage and cureting followed.

Fever and chills for three weeks. (See Fig. 9I.) No pain whatever. No other complaints. 
Physical examination negative. Widal, negative. Whites, 7000. The case was considered by Dr. R. H. Fitz a mild septicemia. The uterus was dextroretroverted. Cervix very soft. Uterine body very hard. Culs-de-sac free. The uterus was dilated and cureted.

February I 9 th vaginal examination showed some edema in right iliac region.

Discussion. - This woman complained of nothing in the world but fever. As she had rather recently emigrated from Italy, had had repeated chills and irregular fever, her blood was many times examined for malarial parasites, but none were found.

After this, typhoid was considered, although the chart was very unlike it, and the patient showed at no time any hebetude. The Widal

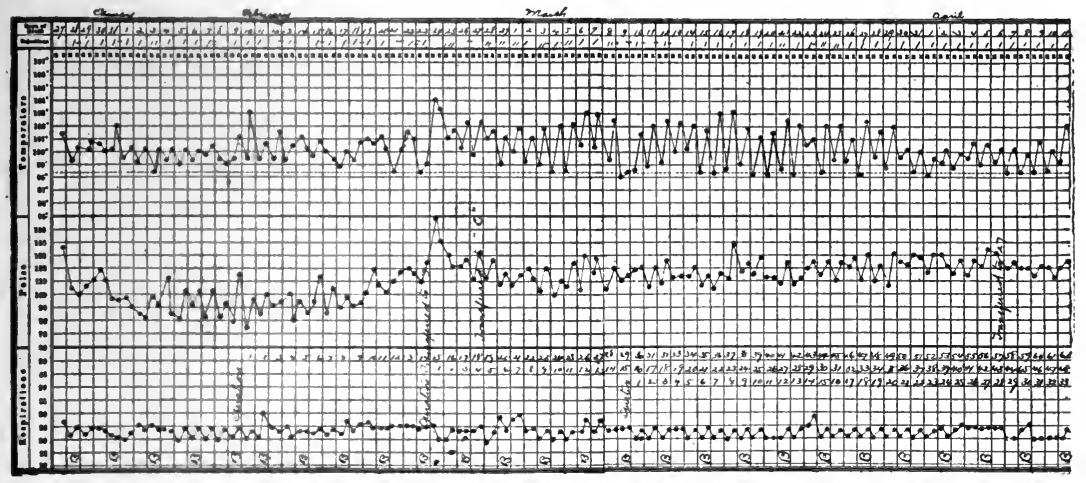

Fig. 91.-Chart of case 226.

reaction was done a number of times, always with negative results. Nevertheless, typhoid could not positively be excluded.

Since the symptoms came on soon after her confinement, there seemed good reason to believe that the case might be one of mild septicemia, pelvic in origin. The dilating and curetage were done with this idea in mind, but no improvement followed, and the diagnosis remained altogether in doubt.

Mesenteric and peritoneal tuberculosis are especially common in recent immigrants of the Italian race, and it is impossible to exclude this diagnosis, though there were no signs of fluid in the peritoneal cavity, no palpable glandular masses, and only a moderate general abdominal spasm, rather more marked in the lower half.

Outcome.-The patient was examined under ether on the twentythird of February, and a mass was felt in the region of the cecum. The abdomen was then opened, and the mass found to consist of caseous 
glands intimately adherent to the cecum. Microscopic examination proved tuberculosis. After a long illness the patient finally made a perfect recovery.

Diagnosis.-Pericecal tuberculosis.

\section{Case 227}

A carpenter of twenty-seven entered the hospital February I7, I907, with an excellent family history and past history. He drinks one or two pints of beer a day, rarely a glass of whisky. His habits are otherwise good.

Two weeks ago he "got a cold," and felt sick enough to go to bed, although free from pain. Since then he has had a slight cough and has

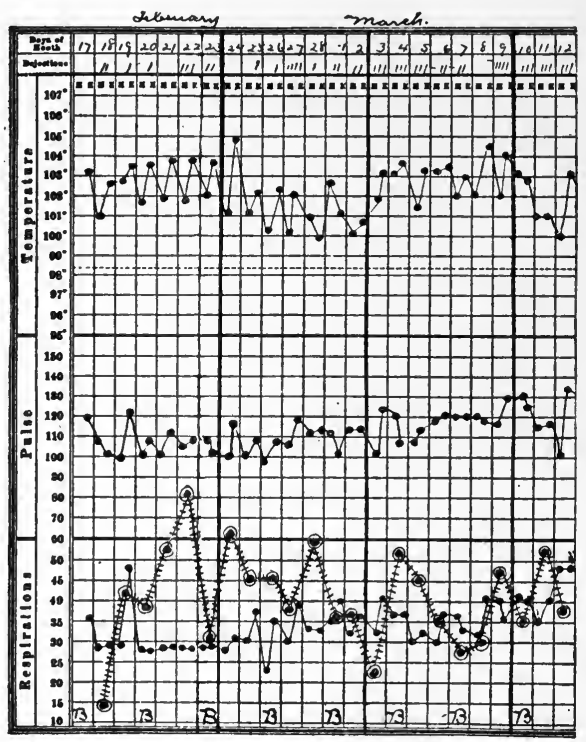

Fig. 92.-Chart of case 227 .

raised a little sputa, which he describes as black and white. He says he feels tired all over, and for the past three days has had some pain in the right axilla and in the region of the right nipple, not increased by deep breathing. To-day his only complaint is of weakness. His appetite is good, his bowels regular, but he thinks he has lost much weight. (For the temperature, see the accompanying chart.)

On physical examination the heart showed nothing abnormal. The left lung was negative, save for a few scattered râles. Throughout the right lung fine crackles were heard, with slightly diminished voice- 
sounds, except at the apex, where they were slightly increased, with a little dulness on percussion. The edge of the liver was felt one finger's breadth below the ribs. Physical examination, including two examinations of sputa, was otherwise negative. The Widal reaction was always negative. The leukocytes numbered I2,400 on February I7th; I3,000 on February 18th; I6,500 on February 22d; II,900 on February 26 th.

Discussion. - It seems natural to associate the fever and the rather indefinite pulmonary signs as cause and effect, but it is hard to see how these signs can be considered sufficient to represent a pneumonia, an acute pulmonary tuberculosis, or an empyema, which are about the only lung diseases one would think of in this connection. Tuberculosis seems perhaps the more probable of the three, but we have no positive evidence of this in the sputa or elsewhere.

Let us attack the problem from a different point of view. As I have elsewhere shown, ${ }^{1}$ there are but three obscure continued fevers in New England which last over two weeks-typhoid, tuberculosis, and pyogenic infections (sepsis). The other fevers, such as those due to meningitis, to acute articular rheumatism, to leukemia, pernicious anemia, syphilis, or malignant disease, are rarely "obscure"-that is, they show, as a rule, some obvious local lesions as their cause. Returning then to our case with this clue, it seems that we may exclude typhoid because of the continued leukocytosis, the continued absence of the Widal reaction, the excellent appetite, the absence of splenic enlargement, and the time of year.

Sepsis is not so easily excluded, but the great majority of cases show either- $(a)$ a definite localized focus or source of infection, or $(b)$, in the absence of such focus, a much more serious clinical picture. This patient does not seem much sick, especially when we compare his condition with that of patients with generalized pyogenic infection without demonstrable source.

Can pulmonary tuberculosis which shows its presence by signs as slight and as few as in the present case be yet responsible for such marked and continued pyrexia? Experience shows that it can. Nothing is more remarkable, as one studies a large series of cases of pulmonary tuberculosis, than the discrepancies between the amount of lung involved and the amount of constitutional disturbances, such as fever, prostration, emaciation, indigestion. Some patients in whose lungs two or three lobes are obviously infiltrated feel scarcely sick at all, and keep about their work for many months. Others, in whom we can scarcely discover enough physical signs to assure the diagnosis, are utterly

1 See Reference on p. 403. 
prostrated, drenched with sweats, constantly febrile, unable to digest, and rapidly emaciate. Presumably these differences are due in part to the variations in individual resistance, in part to the nature of the secondary infection ingrafted upon the original tuberculosis.

Outcome.-After many examinations tubercle bacilli were finally demonstrated February $25^{\text {th }}$ in a small speck of mucus which accompanied about 30 c.c. of fresh blood. No typical signs of solidification appeared until March 6th. March $\mathrm{I}_{3}$ th he was discharged worse.

Diagnosis.-Phthisis.

\section{Case 228}

A teacher of thirty-four, of good family history, entered the hospital December I7, I906. He had been told about eight years ago that he

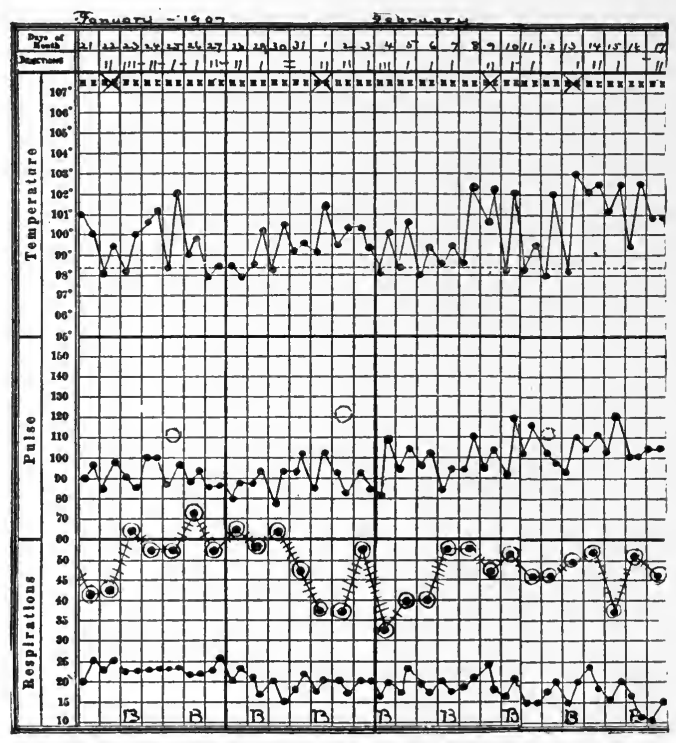

Fig. 93.-Chart of case 228.

had a weak heart. He had gonorrhea five years ago, syphilis eight years ago.

Five weeks ago he "took cold," had a slight cough and fever, occasionally a little pain in the right knee, later in various other parts of the body, never constant or definite. He kept at work until five days ago, when he took to bed on the advice of his physician. He now feels some aching all over his body; he has no appetite, much constipation.

The palpable cardiac impulse extends as low as the sixth space in the nipple line. There is harsh, systolic murmur, best heard at the 
apex, but also audible all over the chest. The pulmonic second sound is slightly accentuated. Dr. H. F. Vickery, who had previously seen him, stated that this murmur has been here for at least fourteen years.

Physical examination is otherwise negative except for a leukocytosis of 19,200 , and a fever ranging between $\mathrm{IOI}^{\circ}$ and $\mathrm{IO}^{\circ} \mathrm{F}$. (See Fig. 93.)

On December 23d, slight dulness and slight increase of voice were made out at the left pulmonary apex. The patient says he has worked very hard for more than a year and is tired out. He now sleeps most of the time, but complains of no discomfort.

There was no change in his condition for the next month. $\mathrm{He}$ remained cheerful, his sleepiness gradually wore off and his appetite returned, but he continued to have fever.

Pneumovaccines were given, beginning March $\mathbf{I} /$ th, but produced no improvement. After the I2th of March the temperature became subnormal, and remained so for most of the following month, though the leukocyte'count was persistently high, varying between ro,000 and 34,000. On the twenty-fourth of March the red cells were 3,012,000, the hemoglobin, 50 per cent. Of the white cells, 92 per cent. were polynuclear and the rest lymphocytes.

Discussion. - Another case exhibiting at the time of entrance a fever and nothing else. The constant leukocytosis makes it possible to exclude typhoid, and the other features of the examination rule out practically everything else except tuberculosis and some form of pyogenic infection. The patient slept so large a portion of his time during the first month of his stay in the hospital that meningitis was at times suspected, but at no time were there any physical signs tending to confirm this suspicion.

The pulmonary signs described under the date of December $23 \mathrm{~d}$ are such as are found in a great number of sick people if the examination is conducted with the utmost care in a quiet room. At the right apex they would have no significance whatever. At the left they call for more consideration, but are not in themselves sufficient to make us seriously fear pneumonia or tuberculosis.

Whenever a continued fever accompanies a cardiac murmur such as that here described, there is reason to fear that a vegetative endocarditis is at work. But in the present case we have reason to believe that the murmur has existed for at least fourteen years, so that its association with this fever may not be significant. On the other hand, the-severe secondary anemia and the constant leukocytosis give us reason to believe that the old process, which was recognized upon the 
mitral valve fourteen years ago, has again become active, like some hitherto quiescent volcano.

Outcome.-Beginning with March 29th, he had a great deal of vomiting, the vomitus containing. considerable blood on one occasion. At this time there was little or no pulse to be felt in the right arm, although in the left it was fairly strong. Vomiting ceased within a few days, but the patient was left exceedingly emaciated and weak. Two purplish areas developed April I4th on the dorsum of the left foot; they disappeared during the day. Another appeared on the heel in the same afternoon. The patient began to be delirious about this time and he died on the twenty-first of April.

Autopsy showed polypous endocarditis of the mitral valve; multiple infarcts of the spleen and kidneys; hypertrophy and dilatation of the heart.

Diagnosis.-Malignant endocarditis.

\section{Case $229+$}

A housewife of sixty-seven entered the hospital February Io, I909. She has seemed to be perfectly well until this morning, although she has noticed that her feet swell from time to time, and has been aware that she passed unusually large quantities of urine. She has had no headache and no vomiting.

This morning she awoke at four o'clock, saying that she did not feel well. Within a short time she had several convulsions and became comatose.

Physical examination showed a red, parched tongue, the heart's apex one inch outside the nipple line, the action regular and slow; there were no murmurs and apparently no increase in pulse tension, but the blood-pressure was I75 mm. Coarse and medium râles were scattered throughout both lungs.

During the examination the patient had a general clonic convulsion, with frothing at the mouth, biting of the tongue, dilatation of the pupils, incontinence of urine and feces. The urine contained sugar, and had a marked reaction for acetone and diacetic acid. Gravity, IO2I; albumin, a slight trace; sediment, negative. The blood showed 25,000 white cells per c.mm.

The course of the temperature is seen in the accompanying chart (Fig. 94).

Cheyne-Stokes' breathing began soon after the patient entered the hospital, and the aortic second sound was noted to be very loud. There 
was no evidence of meningitis, and a blood culture was negative. Convulsions followed each other in rapid succession.

Within an hour of the time of entrance the patient was bled, I4 ounces of blood being taken from the arm, and 2 pints of normal salt solution containing 5 drams of sodium bicarbonate were put into the vein. Convulsions, however, continued until the eleventh, when, under copious sweating by means of hot-air baths, and subpectoral infusions of salt solution, she began to improve steadily.

On the thirteenth she was conscious, though confused. Sugar, acetone, and diacetic acid were gone from the urine, in the sediment of which many hyaline, fine and coarse granular casts were found,

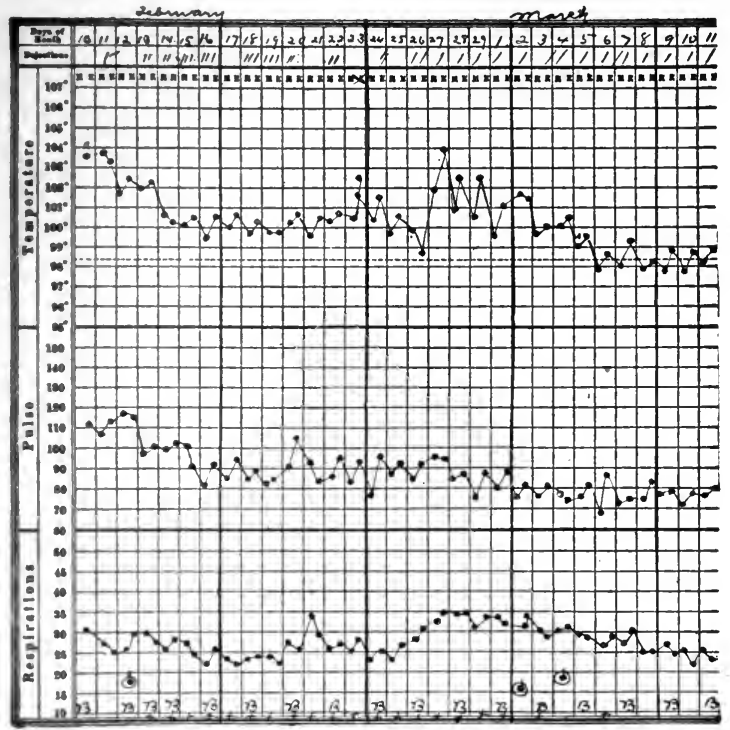

Fig. 94.-Chart of case 229.

some with fat adherent. The left pupil was now larger than the right, though both reacted normally.

On the fourteenth she was at times rational, at times in a muttering delirium. She could swallow and took milk well, but had no control over the sphincters, and was occasionally noisy and profane. The white count had dropped to 8000 .

On the eighteenth she had hallucinations both of sight and hearing, but when spoken to answered rationally.

On the twenty-second she was up in a chair, free from any paralysis or anesthesia, quite rational in the day-time, although a little irrational at night. She had now regained control of the rectal sphincter. 
On the twenty-fifth the urine showed no casts; leukocytosis was still absent. The Widal reaction was entirely negative.

On March 9th she was able to walk about very well, and was to have gone home. Át three o'clock she sank into a chair with a very poor pulse, and had a short convulsion, lasting only fifteen seconds, but followed by hallucinations of sight and hearing. She then suddenly came to, remarked that she was afraid she had made a fool of herself, asked what had struck her, and remained quiet and rational.

On March I2th she was discharged.

Discussion.-The features of this case may be summarized as continued fever with epileptiform attacks and glycosuria.

The last item may be dealt with first. A patient seen for the first time with coma or convulsion should always be catheterized and the urine examined for albumin and sugar, yet I have known a very large number of mistakes arising from an inference made because either albumin, sugar, or both were found to be present. It should always be remembered that convulsions or coma, whatever their cause, frequently give rise to glycosuria, to albuminuria, or to both conditions. One must have other evidence before concluding that diabetes or nephritis is present. Such evidence is to be sought in the condition of the heart, in the previous history, and in the result of subsequent examinations of the urine, which, in the present case, were negative, as indeed they usually are in patients seen for the first time in convulsions or coma. The acetone and diacetic acid are not easily to be accounted for, as we have no evidence that the patient has been starving herself, and her vomiting is very recent.

Subsequent examinations of the urine showed no sufficient evidence of renal disease. A trace of albumin and a few casts were present from time to time, but the amount and gravity of the urine were normal, and in my opinion it has been amply demonstrated that albumin and casts in a woman of this age are not in themselves evidence of renal disease, ${ }^{1}$ although they are perfectly consistent with such a diagnosis, and do not in any way exclude it.

Attacks of convulsions and coma in an elderly person whose heart shows some evidence of enlargement should always lead us to scrutinize the veins of the neck and to listen very carefully over the precordia

1 F. C. Shattuck, Boston Med. and Surg. Jour., I894, vol. cxxx, p. 613: "On the Urine of Persons over Fifty Years of Age." William Osler, New York Medical Jour., I90I, lxxiv, p. 949: "On the Advantages of a Trace of Albumin and a few Tube-casts in the Urine of Certain Men Above Fifty Years of Age." 
for evidences of heart block (Adams-Stokes disease). In the present case no such evidence was forthcoming.

Meningitis may begin as suddenly as this, with fever and convulsions as the chief evidence of its presence. (See Case 266, p. 519.) Although there were no positive evidences of meningitis in this case, lumbar puncture was done, and a sterile fluid almost free from cells spurted out under considerable pressure. No micro-organisms could be demonstrated in the sediment. The very transient character of the leukocytosis is also evidence against any type of meningitis except that due to tuberculosis.

Typhoid fever was difficult absolutely to exclude. The patient's age and the time of year, the initial leukocytosis and the convulsionsall were unusual and atypical, but none positively excluded the disease.

Looking over the case as a whole, and taking account of the high blood-pressure, the absence of any focal symptoms and the intermittence of the cerebral manifestations, it seems to me that this case may best be classed as one of the group denominated by Pal as vascular crises $^{1}$ of the cerebral form. Pal's monograph (which does not seem to me to have received the attention which it deserves) describes in detail a large number of cases in which the diagnosis of cerebral hemorrhage, embolism or thrombosis would ordinarily be made, yet in which the autopsy showed no gross organic lesion in the brain, no hemorrhage, softening or vascular occlusion. He shows that similar crises would reasonably be supposed to occur in cases of lead-poisoning (lead encephalopathy), in nephritis (transient uremic hemiplegia, aphasia, or amaurosis), as well as in arteriosclerotic cases with diminished elasticity of the ressels and high blood-pressure. Presumably, as he argues, the colic of lead-poisoning, the gastric crises of tabes dorsalis, and many of the acute attacks of abdominal pain occurring without any other explanation in arteriosclerotics may be likewise explained as abdominal vascular crises, while the various forms of angina pectoris and of intermittent claudication may reasonably be considered as pectoral or peripheral crises of the same type. Vascular spasm is in all cases assumed as the fundamental change.

Diagnosis.-Vascular crisis.

\section{Case 230 t}

A girl three years old entered the hospital May 5, 1908. The child was perfectly well until the day before, when vomiting, headache, and abdominal pain were complained of. Last night the vomiting

1 Gefässkriesen, J. Pal, Liepsic, I905 (S. Hirzel). 
continued, although she took food well. The bowels were natural and there were no convulsions.

Physical examination showed nothing wrong in the throat or ears, a normal heart, a slight dulness at the right apex extending down to the third rib in front and to the spine of the scapula behind. Over this area there was bronchial breathing and increased fremitus.

The course of the temperature was as seen in the accompanying chart (Fig. 95).

White count, 38,400 on May 5 th; 50,000 on May 7 th; 79,200 on May I 3 th; 69,000 on May I6th; 39,000 on May I8th; 23,000 on May 20th; I7,000 on May 23d.

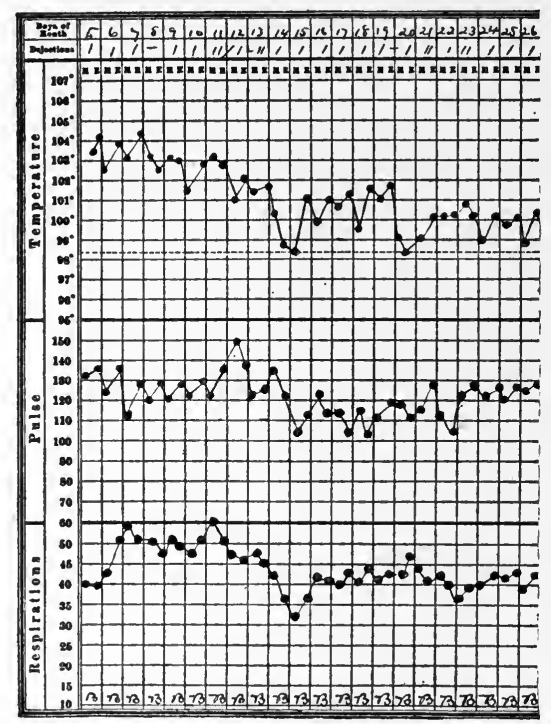

Fig. 95.-Chart of case 230 .

On the ninth of May the lower left lobe became likewise involved. On the eighteenth an aural consultant found double otitis media and opened both drums. On the twenty-second, though both ears were discharging freely, the temperature still remained high. Dulness and diminished breathing were then discovered at the right base.

The child's general appearance was very poor.

On the twenty-fourth the dulness and diminished respiration at the right base had increased, although there were no signs of anything abnormal in the front of the chest. A needle introduced into the back drew pus containing many extracellular pneumococci. A pure culture of pneumococci was obtained from this fluid. 
Discussion.-Obviously, this child's illness began with a pneumonia, continued with a double otitis media, and ended with an empyema. The case is introduced mainly in order to call attention to the very typical chart, which exhibits, between the thirteenth and twenty-fourth, the variations which used to be interpreted as an unresolved pneumonia, but which of late years have been shown to be practically always associated with a development of a postpneumonic empyema.

The diagnosis of unresolved pneumonia was made at the Massachusetts General Hospital: II times from I900 to I905, 5 times from I905 to Oct., I909.

I feel quite convinced that the cases which I used to designate as "unresolved pneumonia" were all, or practically all, mistakes, the actual lesion being postpneumonic empyema.

Outcome.-The chest was opened on the twenty-seventh and a large amount of pus evacuated, after which the temperature promptly fell to normal. The discharge ceased in three weeks. The week after this the wound was healed and the child went home well.

Diagnosis.-Pneumonia and general pneumococcus infection.

\section{Case $231+$}

A laborer of twenty-four entered the hospital April 25, 1908. In June, 1907, he had had rheumatism for a week. Two weeks before the present illness he had had a bad sore throat. Ten days ago he began to have tenderness and pain in both knees and ankles, which compelled him to go to bed. Later, his hands, lips and shoulders became affected, the pain preventing sleep. During the past week he has had four nosebleeds.

Physical examination showed that the tonsils were large and soft, but not red. Cardiac impulse extended to the fifth space, but did not pass the nipple. There was no enlargement to the right. The first sound was replaced by a murmur. The pulmonic second sound was reduplicated. The murmur was also heard in the axilla. Lungs and abdomen showed nothing abnormal.

The joints of both hands, wrists, and the right knee and both ankles were swollen, hot, slightly reddened, and tender.

White cells were 16,600 .

The course of the temperature is seen in the accompanying chart (Fig. 96).

On the second of May, under strontium salicylate, ro grains every hour, the patient seemed almost well, and was about rfady to go home 
when a loud friction-rub, roughly synchronous with the heart's action, was heard along the left edge of the sternum, on the level of the fourth and fifth rib. There was no pain and no fever. The white cells were II,,$\infty 0$. The friction-rub persisted for two weeks, but was never accompanied by any pain.

On the eighth of May he began to have considerable dyspnea, and crackling râles appeared at the right apex, in front, and throughout the whole left lung. He became rather cyanotic. His white cells rose to 29,000 .

On the ninth pain appeared in the right upper quadrant of the abdomen, together with rigidity and slight distention. Nothing could be made out on palpation.

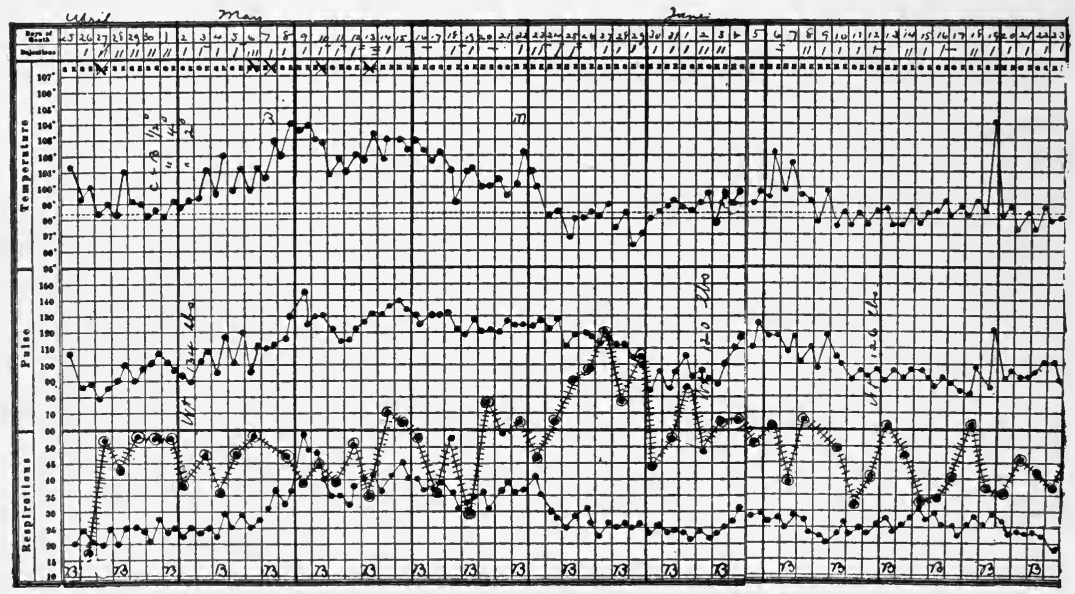

Fig. 96.-Chart of case 23I.

On the eighteenth of May the leukocytes were still 29,000. The patient was up in a chair a good deal of the time, and fairly comfortable, but slept little and did not seem to gain strength. The abdominal distention was very obstinate and difficult to overcome. His hands and feet began about this time to show considerable edema. From the twentieth of May he gained steadily, although his white cells remained high, and on the twenty-ninth of May were still 24,000.

On the eighth of June his lungs were clear, the heart showed the murmur previously described, but no pericarditis.

On the twelfth of June he had a second attack of sore throat. On the nineteenth his tonsils were removed, following which a whitish membrane formed over the stump. Nevertheless, he continued to improve, and on the twenty-fourth was discharged well. 
Discussion.-The sequence of events here may be summarized as follows: After a previous attack of acute arthritis the present illness begins with tonsillitis, which leads immediately to a second attack of arthritis associated with an equivocal cardiac murmur which may or may not be due to endocarditis. In May he develops a friction-rub, due, presumably, to pericardial exudate. Later we have edema of the lungs and cyanosis, due in all probability to an invasion of the myocardium by the same infectious agent which has already attacked the pericardium, and perhaps the endocardium (pancarditis). The abdominal symptoms lead us to conjecture that the gall-bladder may have become infected, or that a mild degree of peritonitis-such as often occurs as part of a general sepsis-may also be present. Finally, the illness winds up with a second attack of sore throat.

We have here an excellent example of a septic infection due to some unknown but presumably attenuated type of pyogenic organism. One structure after another is attacked, yet the patient's resistance is such that he overcomes the invasion again and again, and may be left in the end nearly or quite as strong as he was in the beginning. In case he overcomes altogether this present attack, the chief danger is that the myocardium or the kidney will be permanently scarred, so that in later life a "chronic" myocarditis or nephritis will appear apparently out of a clear sky. In practice we often see this second chapter without the first, as the infection has been passed through without being designated as anything more important than "the grip" or "a common cold."

Diagnosis.-Sepsis.

\section{Case 232}

A stableman sixty-two years old entered the hospital February Io, 1908. He has always been well. He denies venereal disease. His habits are good. For the past four or five days he has noticed fever and severe cough, with yellow sputa. This morning he began to have severe pain in the lower right chest, associated with shortness of breath, but was able to walk to the hospital. The course of his temperature is seen in the accompanying chart.

Physical examination showed slight cyanosis; rapid, labored breathing; the right pupil larger than the left, and reacting sluggishly to light. The tongue came out somewhat to the right. There was well-marked Riggs' disease. The heart's apex extended $\mathrm{I}_{\frac{3}{4}}$ inches outside the nipple line in the fifth space; the right border of dulness not made out; the heart was otherwise negative. The right lung was dull below the nipple line in the front and axilla, and up to a corresponding point in 
the back. Tactile and vocal fremitus were diminished. Breathing was bronchial, especially near the upper border of dulness. Many fine crackles were heard throughout both chests.

The liver and other abdominal viscera were normal, though the bellywall was held rather rigid.

The sputa was mucopurulent. It contained no tubercle bacilli and very few pneumococci.

The patient did not seem very sick, but was slightly delirious at night.

On February r6th the physical signs and temperature were unchanged. The patient was alert, active, and did not seem to feel sick.

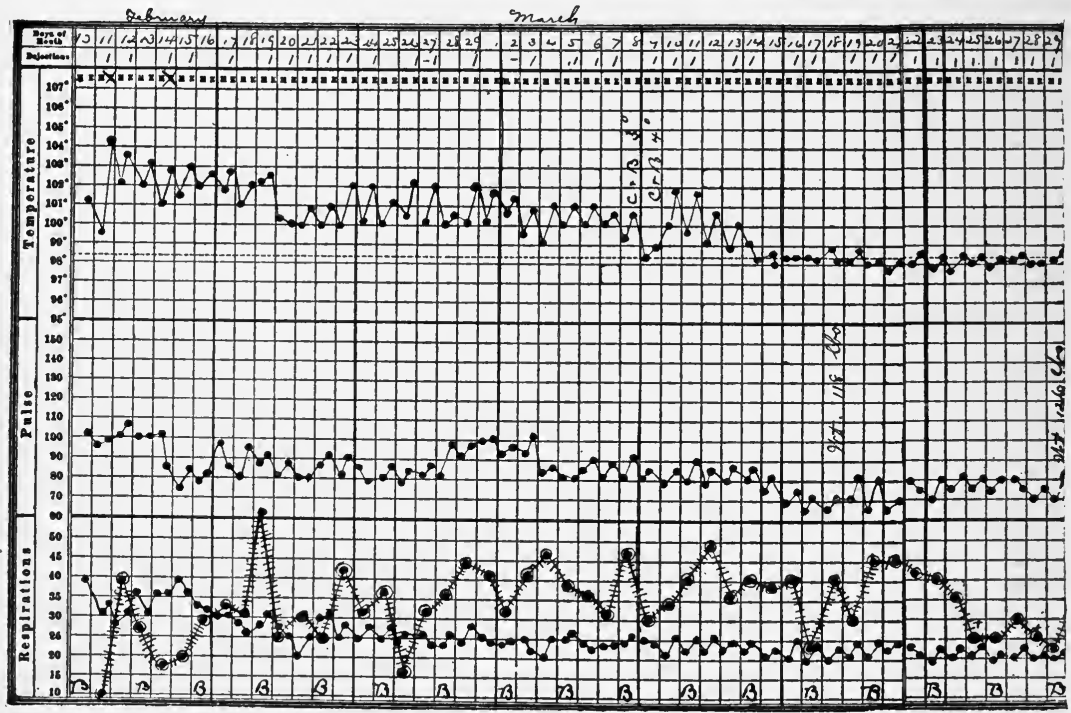

Fig. 97.-Chart of case $23^{2}$.

$X$-ray showed no evidence of pleural effusion. The sputa, repeatedly examined, showed nothing abnormal.

On March 8th he was sitting up, but there was no change in the physical signs.

On March 3 oth the dulness is perhaps a little less. There are no râles. He feels quite well, has no cough, and is discharged.

Leukocytes: February IIth, I7,800; February I4th, I9,400; February I6th, 27,400; February I8th, I5,700; February 20th, I3,900; February 22d, 3I,100; February 25th, I4,700; February 29th, 24,200; March 4th, I0́,300; March 7th, Io,900; March IIth, II,4c0.

Discussion.-This case is introduced chiefly on account of the remarkable temperature chart and the equivocal signs in the chest. The 
rapid, labored respiration, the cyanosis, the bronchial breathing, and the high initial fever are strongly suggestive of pneumonia, but it is very unusual to find the vocal and tactile fremitus diminished over pneumonic solidification.

The long duration of the fever, the absence of any rusty sputa, the moderate constitutional symptoms, and the signs at the base of the lung are very characteristic of a pleural effusion, serous or purulent; yet the $x$-ray, which usually shows a shadow corresponding to such an exudate, was negative at the time when the physical signs were exactly as above described. In view of the outcome of the case I do not see how we can make any other diagnosis than pleurisy, and in view of the negative $x$-ray examination it seems quite possible that we are dealing with a plastic exudate resulting finally in thickening from scar formation.

Diagnosis.-Pleural effusion.

\section{Case 233}

A child of five entered the hospital May 20, 1908. His father had just had typhoid fever and his mother pneumonia. They are both at the Massachusetts Hospital. One sister is now having measles at the City Hospital. The child was perfectly well until last night, when he became fererish, lost his appetite, and at nine o'clock vomited. Since then he has been drowsy, with slight cough, and has vomited several times more. $\mathrm{He}$ complains of no pain anywhere.

The course of the temperature as seen in the accompanying chart (Fig. 98).

Physical examination showed head, chest, and abdomen negative. There was no rigidity of the neck; no Kernig's sign. There were many small red spots scattered over the trunk and limbs,

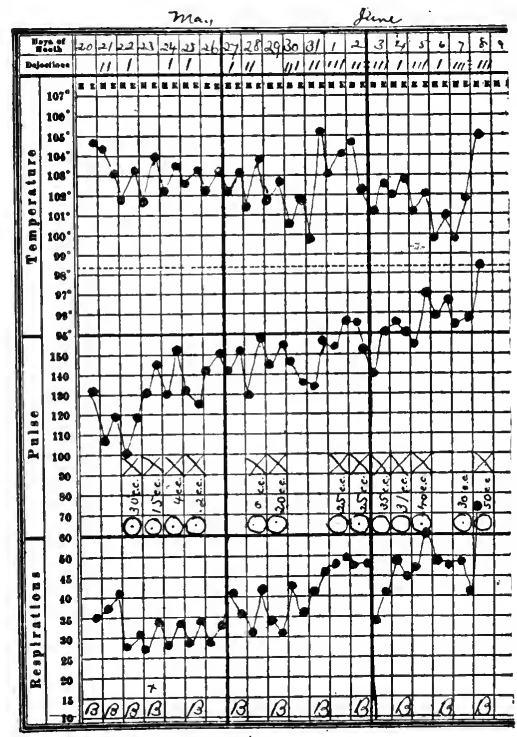

$X=30$ C.C. FLEXNER'S serum introduced.

$\odot=$ LUMBAR PUNCTURE AND AMOUNT OF FWID OBTTINED.

Fig. 98. - Chart of case 233 . not disappearing on pressure.

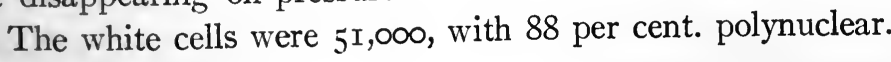

Negative urine. 
After entrance the child was drowsy and continued to vomit frequently and with great suddenness. On the twenty-first he became slightly delirious.

Discussion.-In view of the other cases of fever in the family, one would naturally conjecture that this child has contracted either typhoid, measles, or pneumonia. The eruption is apparently hemorrhagic, not macular or papular, and this, with the absence of coryza, conjunctivitis, and Koplik's spots, is sufficient to exclude measles even at the outset, before the long course of the fever had shown us that some more serious infection must be at work.

Typhoid may be unconditionally excluded by the presence of a high and continued leukocytosis.

Of pneumonia there are no signs, though the herpes; the fever and leukocytosis suggest it. In children pneumonia is aımost never "central," perhaps because it is easier to reach the depths of their lungs by the ordinary methods of physical examination.

The vomiting and sluggishness, as well as the sudden onset, are rather characteristic of meningitis, but against this is the normal flexibility of the neck, the absence of any ham-string contractions and of any complaint of headache-all very constant symptoms. No further certainty can be arrived at without lumbar puncture.

In all doubtful fevers occurring in young children one should investigate the ear-drums, and, especially in girl babies, the urine, with reference to presence of. pus and bacteria. In the present case the latter examination was made, not the former. We were thrown off our guard because the child did not complain of its ears, nor, indeed, of any pain, and because there was no discharge.

Outcome. - Not until May 22d was there any evidence of rigidity in the neck. In the afternoon of the twenty-second lumbar puncture was done and 20 c.c. of turbid fluid obtained. In the sediment of this fluid 92 per cent. of polynuclear cells were found, and many Gramnegative diplococci were seen within and without the cells. Flexner's serum was injected, and the boy seemed brighter next day; but Kernig's sign was present on both sides, and slight internal strabismus had appeared.

Herpes appeared upon the lips on the twenty-fourth. On the twenty-sixth he was taking nourishment freely, and wanted to sit up and go home. The neck was less rigid and strabismus gone; the pulse was of excellent quality, though rapid.

The white count, May 23d, was 42,000; on the twenty-sixth, 2I,000; on the twenty-eighth, 39,000 . 
Lumbar puncture was done seven times more in the course of the next three weeks, and Flexner's serum repeatedly injected. The amount of fluid obtained was usually large -35 to 40 c.c.

The patient seemed to be doing well until the eighth of June, when be became rapidly worse and died. Autopsy showed meningitis, double otitis media, and a very large thymus.

Diagnosis.-Epidemic meningitis.

\section{Case 234x}

A child of six entered the hospital August 2, I907. He has always been well until nine days ago, when he woke near midnight, feverish and vomiting. Five days ago his temperature was found to be $104^{\circ} \mathrm{F}$. Four days ago it was $103^{\circ} \mathrm{F}$. In the middle of the day he had less fever than at night. The last two nights he has slept fairly well. Before that he was rather restless. All the time his appetite has been good, but he has had only liquids. His bowels have been moved by cathartics and since the first he has had no vomiting, no nosebleed, no pain. He has lost considerably in weight.

The course of the temperature as seen in the accompanying chart (Fig. 98).

Physical examination was entirely negative; urine, normal. White cells, $\mathrm{r}_{5}, 000$. No Widal reaction.

By the eighth of August his temperature was normal and the child seemed perfectly well. The treatment consisted of laxatives and alcohol sponges.

Discussion.-All general practitioners see many cases like the above. Ordinarily, they are spoken of as "grip" if they occur in winter, and as "indigestion" or "ptomain poisoning" if they occur in summer. Both these usages seem to me unfortunate, in that they tend to delay the progress of medical knowledge. In the vast majority

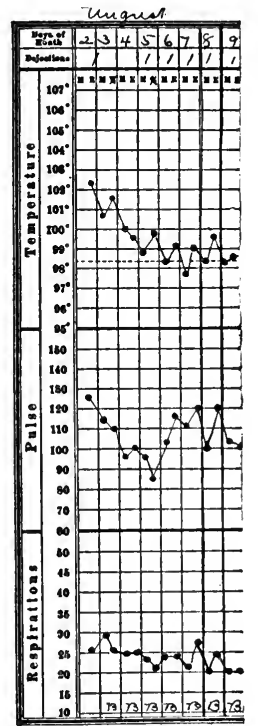

Fig. 99.-Chart of case 234 . of cases there is not the slightest scientific warrant for either diagnosis. The bacteriologic or chemical evidence on which alone such diagnoses could be based is practically never secured, and the terms are used mainly to satisfy the family.

It seems to me much wiser, as well as more truthful, to state that in such a case we are dealing with an unknown infectious disease. (See p. 4rr.) Ptomain poisoning is just now a very fashionable diagnosis, 
and a phrase which the laity loves to brandish about. People are quite proud to have suffered from such an illness. But all this does not advance the progress of medicine, and tends in the long run to discredit our profession.

I have seen similar fevers in which a Widal reaction was obtained, and to which, therefore, the term "abortive typhoid" was quite justifiably applied. If there is a pharyngitis, a tonsillitis, or a bronchitis, an inflammation of the frontal sinus, a jaundice, or a diarrhea, an infection of the urinary passages or a subcutaneous abscess at any point, the fever may properly be considered as a manifestation of one of these local disturbances. In the absence of such it should, I think, be made clear primarily to ourselves and also to our patients that the disease has at present no name, and cannot be identified with any trouble previously known.

Diagnosis.-Unknown infection.

\section{Case 235}

A school-boy of fourteen entered the hospital December I5, I907. He has always previously been well. Four days ago he began to have

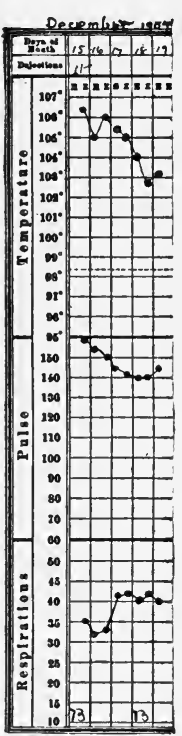

Fig. 100. - Chart of case 235 . pain in the right lower quadrant; it was not very severe, but has persisted to the present time. He vomited once the first day and twice the second day; he has been feverish throughout. He has had no cough, no sore throat and no pain except as above described. The bowels have moved every day. He was sent into the hospital with a diagnosis of appendicitis. At entrance, there was slight tenderness in the right iliac fossa, but without any spasm.

Rectal examination was negative; white count, 20,000; Widal reaction negative.

During the night the patient became slightly delirious and the temperature rose to $106.2^{\circ} \mathrm{F}$.

Physical examination showed at the right base slight dulness, slightly diminished tactile, slightly increased vocal, fremitus, and a few moist râles. Chest and abdomen were otherwise negative. The right kneejerk could not be obtained; the left could be obtained only with difficulty.

The patient.was very delirious, quarreling with imaginary persons, and reaching out for objects in the air. There was no stiffness of the 
neck, no Kernig's sign. The pupils have been markedly dilated throughout, but have been equal and reacted well to light.

Discussion.-What infectious diseases are most common in boys of this age?

(I) Pyogenic sepsis, with or without a focus in bone, joint, or heart valve.

(2) Pneumococcous infections, with or without a demonstrable pneumonia.

(3) Meningitis (otitic, epidemic, or tuberculous).

(4) Typhoid.

(5) Appendicitis.

(6) Unknown infections.

The latter are perhaps the commonest of all.

Though the pain is referred to the right iliac fossa, the presence of a temperature of $106^{\circ} \mathrm{F}$. and of an active delirium is distinctly against appendicitis. We are on our guard also against the mistake, so common in patients of this age, of overlooking a pneumonia or a pleurisy because the abdominal pain often associated with these infections in children occupies so prominent a place in the clinical picture.

Meningitis might begin in this way, and the delirium and the absence of knee-jerks, together with the very high fever and leukocytosis, are quite consistent with that diagnosis. We are surprised, however, whenever we find meningitis without stiffness of the neck or Kernig's sign, especially if the patient is fourteen or younger, for these nervous manifestations are much more apt to be early and well marked in the fevers of children than in those of adults. Even meningeal irritation without actual meningitis often makes a child assume the posture of meningitis. The absence of headache, herpes, and eye changes is also somewhat against meningitis. Nevertheless, this disease can be ruled out only in case lumbar puncture shows no evidence of infection.

A general septicemia, associated either with a pneumococcus or one of the varieties of streptococcus, is the next most reasonable hypothesis. Without blood culture one cannot get any greater certainty in this direction, but the signs in the lung, though in themselves slight, are sufficient to incline us toward a belief that a pneumococcous infection is present. It seems now to be quite clear that the existence or the degree of lung consolidation is quite a secondary and accidental matter in infections due to the pneumococcus. We are dealing in all cases probably with a general infection carried by the blood. In the lung it may arouse no special reaction, may produce a slight bronchitis or bronchopneumonia, or may bring about the solidification of an entire 
lobe. But if all the pneumococcous infections were recognized and classified, we should probably find that those attended by a frank pneumonia are in the majority.

Outcome.-The blood showed a pure culture of pneumococci. By the nineteenth signs of solidification were obvious at the right base. Lumbar puncture showed nothing. The child died on the same day on which solidification became obvious.

Diagnosis.-Pneumonia.

\section{Case 236}

A carpenter of thirty-nine entered the hospital January I8, I90\%. His family history is good. For the last three or four years he has had considerable cough in the morning, with greenish sputa. $\mathrm{He}$ denies venereal disease. He takes a pint of whisky three times a week. Three days ago he began to be chilly, stopped work and went to bed. Two days ago he began to have pain in the region of the heart and in

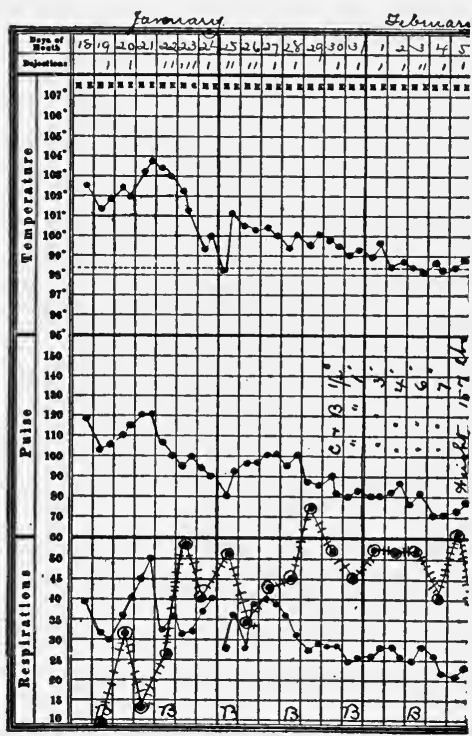

Fig. IOI.-Chart of case 236 . the right axilla. To-day he has been spitting up reddish, frothy material. His cough has not kept him awake at night. The course of his temperature is seen in the accompanying chart.

Physical examination showed cyanosis, a negative heart, thickened arterial walls, many coarse and medium bubbles and squeaks throughout both lungs, diminished resonance in the lower right back and axilla. The upper part of the right front was hyperresonant, the lower part somewhat dull, with much-diminished breath-sounds and voice-sounds over the area between the third and fifth ribs. Abdomen negative.

Over both lower legs there were -many patches of brownish pigment from the size of a nickel to that of the palm of the hand; over the shins there were three white scars, two inches long, $\frac{1}{2}$-inch wide, surrounded by brownish pigmentation. The sputum showed a great variety of bacteria, but nothing of special importance. Leukocytes, 25,300, with 94 per cent. of polynuclear cells. 
On the twenty-third, as noted, the temperature remained high, the pulse irregular; the patient was cyanotic and very noisy. The physical signs remained as before. The sputum was constantly blood-stained.

Discussion.-There is no way in which we can make alcohol responsible for the present fever. Some cases of delirium tremens are febrile, but we have neither tremor nor, at the start, any delirium, and there has been no special accumulation nor increase of alcohol.

The syphilitic infection suggested by the scars on the leg has nothing to support it in the rest of the clinical picture.

Though the signs in the lungs are by no means typical of pneumonia, there seems sufficient reason to take as a working hypothesis the disease which used to be called "central pneumonia." The postmortem evidence seems to be insufficient to justify the belief that in any considerable number of cases solidification actually begins in and remains for a time confined to the central portion of the lung. The conception of "central pneumonia" is derived, I think, mostly from "hind-sight" in cases characterized first by the symptoms, and only later by the physical signs of pneumonia.

It seems to me more reasonable to suppose that in most of the cases usually designated as "central pneumonia" we are dealing, in fact, with a general pneumococcous infection which produces in the lung no lesions whatever or only a moderately severe bronchitis. When a crisis occurs in such a case and the temperature falls suddenly to normal, we are very apt to argue that this proves the case to have been one of lobar pneumonia. I believe, however, that the familiar crisis is characteristic of the pneumococcous infection itself, whether or not it is localized, for it is a very familiar observation that the signs of solidification are often unchanged for many hours after the occurrence of the crisis. It has long been recognized that the dyspnea and cyanosis which cease so suddenly with the crisis, even though the lung signs remain the same, must be due not to the lack of lung space for aëration, but to general toxemia. The same is true, I believe, both of the fever and of the crisis.

Outcome.- On the twenty-seventh the patient was much better. On the third of February he was up and about the ward, and on the sixth he went home well.

Diagnosis.-Pneumococcous infection. $\nmid$

\section{Case 237}

A school-boy six years old was seen December 14, 1906; he had never been ill previously. He has not been well for five days, complaining of pain in his legs and abdomen. The doctor said it was appendi- 
citis. His bowels have not moved for four days. To-day his parents noticed red blotches on his face and body, which they say he does not scratch.

Physical examination is entirely negative, except that the whole body is covered with red, discrete patches from the size of a pea to that of a silver dollar, apparently elevated and surrounded by evidences of scratching.

The urine was negative.

The white cells were 25,200 on the fourteenth; 24,600 on the sixteenth; 27,600 on the twenty-second; I7,000 on the twenty-sixth.

Widal's reaction was slightly suggestive, but not positive. The coagulation time of the blood was three and three-quarters minutes with the Brodie-Russell instrument. Throughout his stay in the hospital the

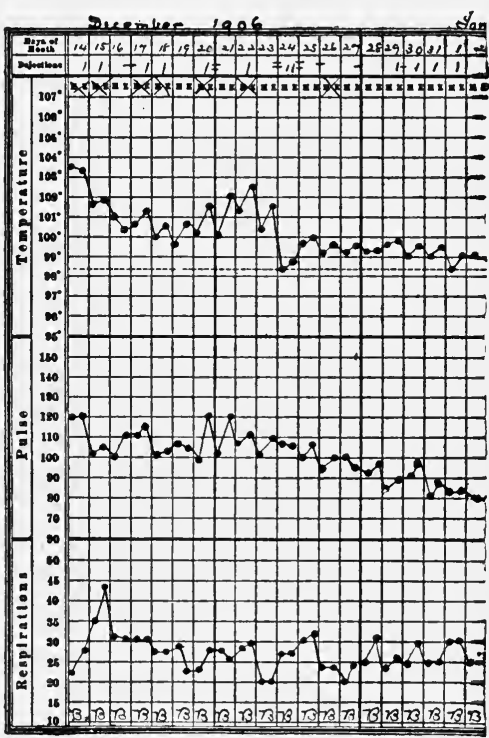

Fig. 102.-Chart of case 237 . patient had no other symptoms or signs.

He had numerous crops of spots up to the twenty-second of December. After that they ceased, and after a few days he seemed so well that he was discharged on the second of January.

Discussion.-In the absence of all physical signs of visceral disease it seems reasonable to associate this fever with the profuse crop of urticarial lesions. The most important lesson from such cases is the recognition that the disease which underlies urticaria can, and often does, produce fever. The other and less obvious results of the urticarial group of lesions discussed so fully by Osler in a series of important papers ${ }^{1}$ must always be borne in mind when the history of the case or the inspection of the skin gives us any knowledge of what we are dealing with.

Symptoms resembling appendicitis, gall-stones, perforating peptic ulcer, pneumonia and many milder affections of the respiratory and gastro-intestinal tract may be produced, when wheals or edematous patches appear in the internal organs as well as in the skin.

Diagnosis.-Urticarial fever.

1 Amer. Jour. Med. Sci., I895, vol. cxxxvii, p. 629 ; Brit. Jour. Dermatol, London, I900, vol. xii; Amer. Jour. Med. Sci., I904, vol. cliv, p. I. 


\section{Case 238}

A butcher of twenty-one entered the hospital May 24, I908. $\mathrm{He}$ has never been sick before. His habits are good. Since early yesterday morning he has had fever, headache, sore throat, slight cough with whitish sputa, and severe, deep-seated pains all over his body. $\mathrm{He}$ has vomited several times. His bowels moved once this morning. The course of his temperature is seen in the accompanying chart. His throat was red, showed no exudate and no swelling. Physical examination was otherwise entirely negative.

Discussion.- Some time in the course of his medical experience every physician, confronted with a case like that here described, has occasion to ask himself the question:

"Can a man be as sick as this and nothing wrong with him but a red throat?" In such cases we rack our brains to see what possible diagnosis we have forgotten. We examine the patient again and again in search for some more extensive organic lesion. But if all these efforts are vain, we are driven in time to the conclusion that a "simple red throat" may be a pretty serious affair. The case here quoted is one of the milder type, but others which begin just as innocently develop into the most virulent type of generalized sepsis. The conclusion is that so frequently

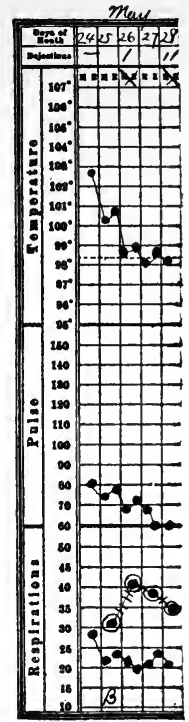

Fig. 103.-Chart of case 238 . emphasized in these pages, viz., that few "local" infections are really local even from the start, that they usually sow their wild oats very widely before settling down, and that this settling may be only temporary.

Outcome.-The patient was given half an ounce of castor oil, followed by three 5 -grain doses of phenacetin at hourly intervals, with a grain of caffein to each dose. In two days he seemed to be entirely well.

Diagnosis.-Pharyngeal (and transpharyngeal) infection.

\section{Case $239-$}

A printer of eighteen entered the hospital June I, I908; his family history and past history were good. He is a heavy smoker of cigarets, and chews a good deal of tobacco besides. Nine days ago he began to have a ticklish throat, then a cough and "cold in his head," which 
soon became severe enough to make him give up work. He has been "up and down" until two days ago, when he took to bed for good. Seven days ago his right foot began to be painful. Yesterday he began to complain of pain in the region of his heart, and his breathing was accompanied by a groan. His cough, at the same time, became much worse, and his fever higher. Last night and to-day he has been somewhat delirious. He has had no chill, no abdominal pain, no vomiting or diarrhea. The course of his temperature is seen in the accompanying chart (Fig. 104). The white cells were 55,700, with 90 per cent. of polynuclear cells. The urine showed a very slight trace of albumin, but no casts. The tonsils were enlarged and the pharynx reddened. The

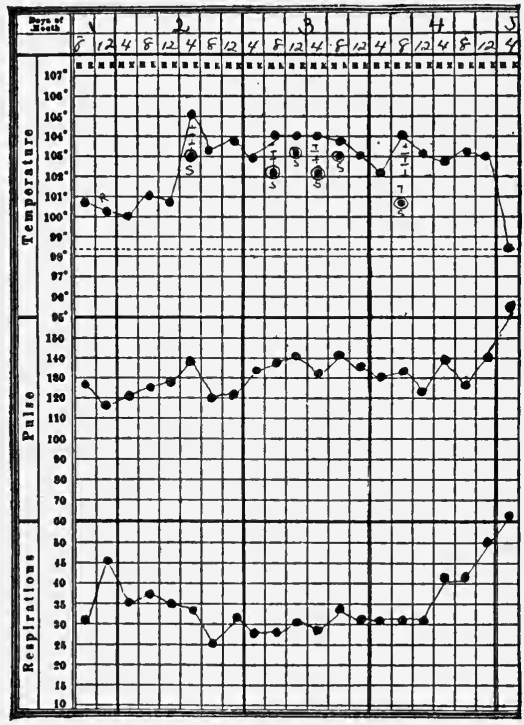

Fig. I04.-Chart of case 239 .

heart-sounds were regular, rapid and distant. There were no murmurs. Respiration was rapid and groaning, the nostrils moving with each breath. There was slight dulness in the left back below the angle of the scapula, a trifling increase of voice-sounds and of tactile fremitus. The respiration was normal. Over the lower axilla and in the precordia a very intense double friction sound was heard synchronous with respiration.

The abdomen and extremities were negative, except that on the top of the right foot was a swollen, reddened, painful area the size of a dollar. Movements of the toes seemed to be painful.

On June $3 \mathrm{~d}$ the pleural rub had disappeared. Respiration seemed 
to be distant in the left back, and there were many coarse râles in the lower right back. Cyanosis was very marked. On the fourth the right border of cardiac dulness had moved to the right at least an inch, and a pericardial friction was heard to the right of the sternum, while pleural friction had returned in the left axilla. There was no definite evidence of fluid or solid in the lungs. Many fine purpuric spots developed on the trunk and limbs during the forenoon of July 4 th.

Discussion. - In cases like this we are prone to ask the muckraker's familiar question: "Where did he get it?" The illness is so sudden, so severe, yet there is so little to account for it.

It appears that an infection showing itself first as a sore throat was scattered thence to the joints, the pericardium, the pleura and the subcutaneous tissues. In any one of these and in many other places a more definite localization might have occurred, as is shown by the course of other similar cases. But here apparently there was a very widespread attack, not wholly successful (i.e., not producing any very obvious or extensive disease) in any one locality. For some unknown reason infections which do not become "localized" often seem to be the worst in case they are not of the very mildest type. Those which "localize" make up the great middle class of moderately severe but not fatal infections.

In all such cases our diagnosis must remain vague unless the results of blood culture are positive.

Outcome.-On the fourth of July a blood culture showed a profuse growth of the streptococcus pyogenes. The patient died next day.

Diagnosis.-Streptococcus sepsis.

\section{Case 240}

A Russian girl baby, twenty-three months old, entered the hospital December 13,1907 . She had never been sick before; three days ago she became sleepy and feverish, with considerable dyspnea, but no cough. She has had no appetite and has vomited twice. The body has been very hot, the feet and hands cold. The bowels have been moved by cathartics, the stools being blackish. Physical examination of the chest, abdomen, and extremities was entirely negative.

White cells, I8,000; urine, not obtained.

Discussion.-Although the history and the physical examination appear to have been conscientiously made in this case, two allessential points are omitted. It is because of these that I introduce the case.

(I) Sick babies do not complain of their ears even when they are 
seriously diseased. The uninitiated are apt to expect that a baby with otitis media will indicate in some way that its ears are painful, even

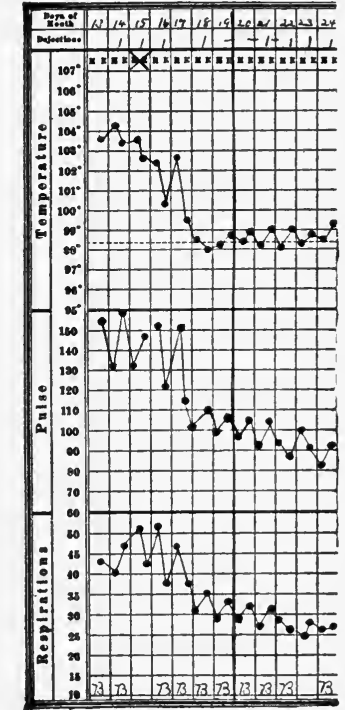

Fig. 105.-Chart of case 240.

when it is too young to talk, but experience shows that the baby rarely puts its hands to its head or gives any other sign that it knows what ails it.

(2) Especially in girl babies suffering from obscure fevers we should always remember the urinary tract and the possibility of infection, hematogenous or ascending. The difficulties of collecting and examining the urine of young babies often lead to its being disregarded, to the great detriment of the child, since most of the milder urinary infections can be cured in their earlier stages by the administration of urotropin and an abundance of water.

Outcome.-On the nineteenth a purulent discharge was seen in each ear. Temperature, pulse, and respiration promptly fell to normal and remained so. The child had been fretful up to this time, but after the discharge became quiet. By the twenty-fourth the ears had ceased discharging and the child went home well.

Diagnosis.-Otitis media.

\section{Case 241}

A school-girl of fourteen with a negative family history entered the hospital March I4, I907. She has had measles, chicken-pox, and whooping-cough. For the last six months she has complained of frontal headache each day after school. The pain goes away by bed-time. On January 24, I906, the child had adenoids removed, without any improvement either in her hearing or in her general health. Her appetite has been poor since her measles seven years previously. Two months ago she had a discharge from one ear. For a week or two there has been tenderness in the sides of her neck and under her jaws.

On February 8th she began to have constant headache, with fever and drowsiness. These symptoms have continued erer since.

On physical examination the child looked sick and "toxic," the sublingual gland large and tender, both submaxillaries also large. The glands in the axillæ and groin were slightly enlarged. There was a soft 
systolic murmur at the apex. The heart was otherwise normal, likewise the lungs and abdomen. There was no tenderness over the mastoids; the white cells were $\mathrm{r} 2,400,65$ per cent. of which were polynuclear; there was no anemia; the urine was normal, Widal reaction negative. Her throat and ears were carefully examined, but nothing abnormal was found there.

Discussion. - No doubt this girl has had eye-strain and presumably she has had adenoids and otitis media, but there seems no reason to believe that the otitis, the adenoids, or any of her previous infections are the cause of the present glandular enlargement.

The main question is whether we are to connect the headache, fever, and other constitutional symptoms with the presence of these glands. Glandular fever is a diagnosis to be made only when no other causes can be found either for the adenitis or the fever.

Such an adenitis is common enough as part of an infection arising from the mouth or throat, or as the residual result of such. But at the present time there seems to be no source of infection in the mouth or throat, no bad teeth, no alveolar abscess, no tonsillitis or otitis.

There is no enlargement of the parotid glands, no history of exposure to mumps, and none of the periglandular infiltration usually seen in that disease.

The glands may possibly be tuberculous, but we have no evidence of tuberculosis elsewhere, no softening or sinus formation, no adherence to the skin or surrounding tissues; the sublingual gland, moreover, is not often involved in tuberculosis unless as a part of a very extensive process.

Leukemia can be ruled out by the blood examination. Hodgkin's disease rarely occurs with just this distribution. It cannot, however, be positively excluded save by the outcome of the case. The absence of any history or lesions of syphilis makes it reasonable to exclude that disease in a girl of this age.

As a result of this reasoning we are left with an unexplained fever and glandular enlargement. To this combination of symptoms, when running a rather short, self-limited, and usually favorable course, the name of glandular fever may be given.

Outcome.-By the twenty-third she was sitting up in her chair and the glands were much smaller. By the twenty-fifth she was free from all symptoms. The glands were still palpable, but now were hard and free from tenderness.

Diagnosis.-Glandular fever (?). 


\section{Case 242}

A factory hand, twenty-six years old, entered the hospital February 22, I908. His family history and past history are negative, his habits good. He has been more constipated than usual for the past four months, the bowels moving once in four or five days. Two weeks ago he began to have headache and poor appetite. Eight days ago he began to have steady, moderate pain at the costal margin, not referred elsewhere. Five days ago he noticed that his eyes were yellow.

Physical examination is negative, except for moderate jaundice of the skin and conjunctivæ, accompanied by rigidity of the right rectus near the ribs. There is tenderness and dulness on percussion, extending $I_{2} \frac{1}{2}$ inches below the rib margin in the nipple line. The edge of the spleen is not felt at entrance, but on the twenty-third of February it was easily felt and considered to be sharp. The urine contains bile, but is not otherwise remarkable. The stools are not clay colored. By February 26th the tenderness and spasm had disappeared, but the jaundice was still present at the time of his discharge, March 17 th, although it had become very much less intense.

The treatment consisted of 20 grains of sodium phosphate three times a day, $\frac{1}{2}$ grain of calomel every fifteen minutes for ten doses, followed by $\frac{1}{2}$ ounce of magnesium sulphate. A diet free from fat was also given.

Discussion.-When we have a case of jaundice of short duration, not following upon or resulting in any other recognizable disease such as gall-stones, obstructive cancer, syphilis or cirrhosis of the liver, we are apt to call it catarrhal jaundice.

Just what this means we do not know. Many cases like the present one have a considerable degree of fever, many more have jaundice for some days preceding the onset of digestive symptoms, so that it seems hardly reasonable to suppose that a gastroduodenitis has extended up the bile-duct and occluded it, according to the classic conception of the disease. It seems more reasonable to believe that the jaundice is one feature of an infectious cholangitis or some other type of hematogenous infection.

Diagnosis.-Catarrhal jaundice.

\section{Case 243}

A housemaid of twenty-one entered the hospital April 30, 1907, complaining of abdominal pain. Physical examination indicated the presence of fluid, and operation showed a diffuse tubercular peritonitis. 
The patient had fever for two weeks, after which she became afebrile and was transferred to the medical wards.

At both bases there was diminished breathing, fine râles and, on the right side, dulness. The abdomen was of board-like rigidity, with shifting dulness in each flank and general tenderness. The patient when received was vomiting everything taken. She was starved for twenty-four hours without relief. Washing her stomach was also of no benefit, and many drugs were tried ineffectually. Soon after the first of June the patient spontaneously ceased vomiting, began to be more comfortable and to take food. The course of her temperature, meantime, is seen in the accompanying chart (Fig. I06). On the ninth of

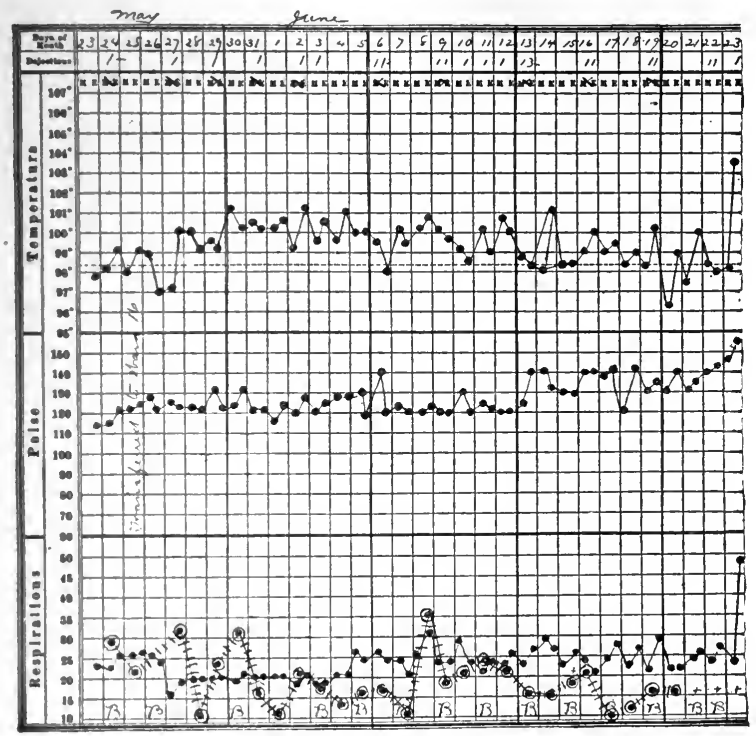

Fig. I06.-Chart of case 243 .

June slight dulness and prolonged expiration were noticed at the left apex in front and behind. By the fifteenth she had developed a leftsided otitis media, which was pronounced to be tubercular by the aural consultant, Dr. H. L. Morse. On June 23d, a purulent vaginal discharge and slight pericardial friction were made out.

Discussion.- In the discussion of previous cases I have repeatedly referred to the special points of election at which a general infection is prone to settle or break out (joints, heart, pleura, gall-bladder, peritoneum, subcutaneous tissues, kidney).

In the present case we have indications of a similarly wide distribution of lesions. The patient has already been operated on for the relief 
of a tubercular peritonitis. It seems reasonable, therefore, to suppose that tuberculosis is also the infection at work at the present time in various other tissues.

Outcome.-The patient died on the twenty-fourth of June. Autopsy showed general miliary tuberculosis and tubercular peritonitis, tuberculosis of the tubes, retroperitoneal and mesenteric lymph-glands, tuberculous ulcers of the rectum. The infection of the middle ear was not tuberculous, but was of streptococcous origin.

Diagnosis.-Miliary tuberculosis.

\section{Case $244 x$}

A longshoreman, twenty-one years old, entered the hospital August 24, I9ro. He has been to sea a good deal, especially to the West Indies,

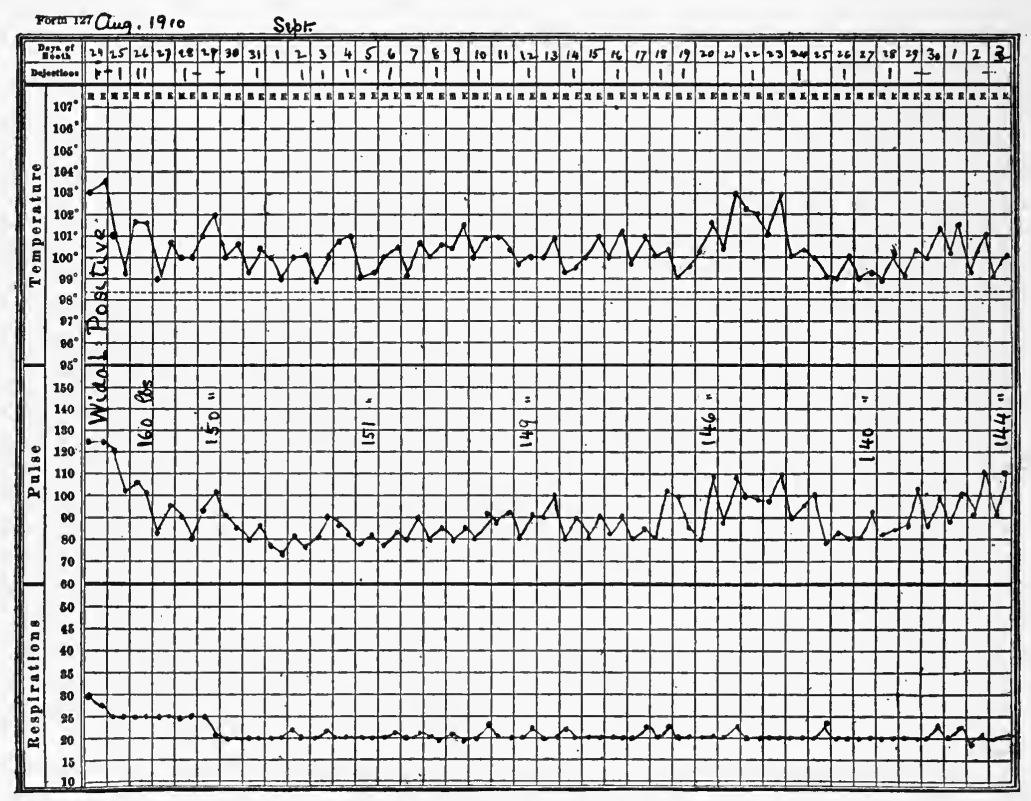

Fig. 107.-Chart of case 244 .

but within the past year has not been further south than Georgia. Left there eighteen days ago and landed at Boston eight days ago, where he at once fell ill, with severe pain in the back and legs, which has lasted ever since. Feverish and sleepless. One nosebleed. No chills. Diarrhea began two days ago, but ceased yesterday.

Three or four weeks ago he had a boil on his left hand, but it healed promptly. A similar boil appeared three days ago on his right hand. 
Family history, past history, and habits good. Denies venereal disease.

Physical examination showed an abundant crop of rose spots on the trunk. The axillary lymph-glands were slightly enlarged. The spleen was not felt, but seemed distinctly enlarged on percussion.

Widal reaction positive $(\mathrm{I}-50)$. White cells, I0,000, among which there were 59 per cent. of polynuclears, 33 per cent. of large lymphocytes, 7 per cent. of small lymphocytes, and I per cent. of eosinophiles. The stained smear was otherwise negative.

The urine averaged 50 ounces in twenty-four hours. At entrance it contained a large trace of albumin, and in the sediment very numerous fine and coarse granular casts with cells, blood and fat adherent. A week later the albumin and casts disappeared and did not return. The stools were negative.

The temperature is seen in the accompanying chart.

By August 3 oth the boils on his hand were healed, but two more appeared on the back. Culture from the pus obtained on opening them showed Staphylococcus aureus.

Discussion.-Typhoid seemed obviously the diagnosis, though the patient (like many other typhoid patients) was never "typhoidal," i. e., stupid, in the least; but September 2 Ist, as the temperature looked uncharacteristic and showed no permanent trend either up or down, we tried another Widal test and found it negative. This test was again negative on the $22 \mathrm{~d}$ and the leukocytes were found to number 10,000. The absence of Widal's test and of leukopenia at this period of the disease seemed very suspicious, also the repeatedly negative Diazo reaction.

Paratyphoid (alpha and beta strains) was tested for twice, with negative results. There was a slightly suggestive agglutination test with a strain of colon bacilli.

Tuberculosis was next searched for, but the lungs, the osseous, lymphoid, and genital tissues were entirely negative.

October $3 \mathrm{~d}$ we tried von Pirquet's cutaneous test, which proved negative. Blood-cultures were also negative.

"Bed fever" (a temperature which disappears when the patient gets up and moves about) was tested for by getting this patient out of bed, but after several days his fever was' as high as ever.

Typhoid remained the diagnosis, though a most unsatisfactory one.

Outcome.-October 7 th the blood was again examined for malaria, and estivo-autumnal crescents were found in abundance. The original blood-smear of August 24th was then hunted up and found to contain 
estivo-autumnal (ring-form) parasites, which had been overlooked on the first examination. The original Widal reaction remains unexplained.

The absence of chills, the presence of Widal's reaction, and the false report on the blood-smear are responsible for my mistake in this case.

Diagnosis.-Estivo-autumnal malaria.

TAble XI.-Long Fevers. Signs and Symptoms.

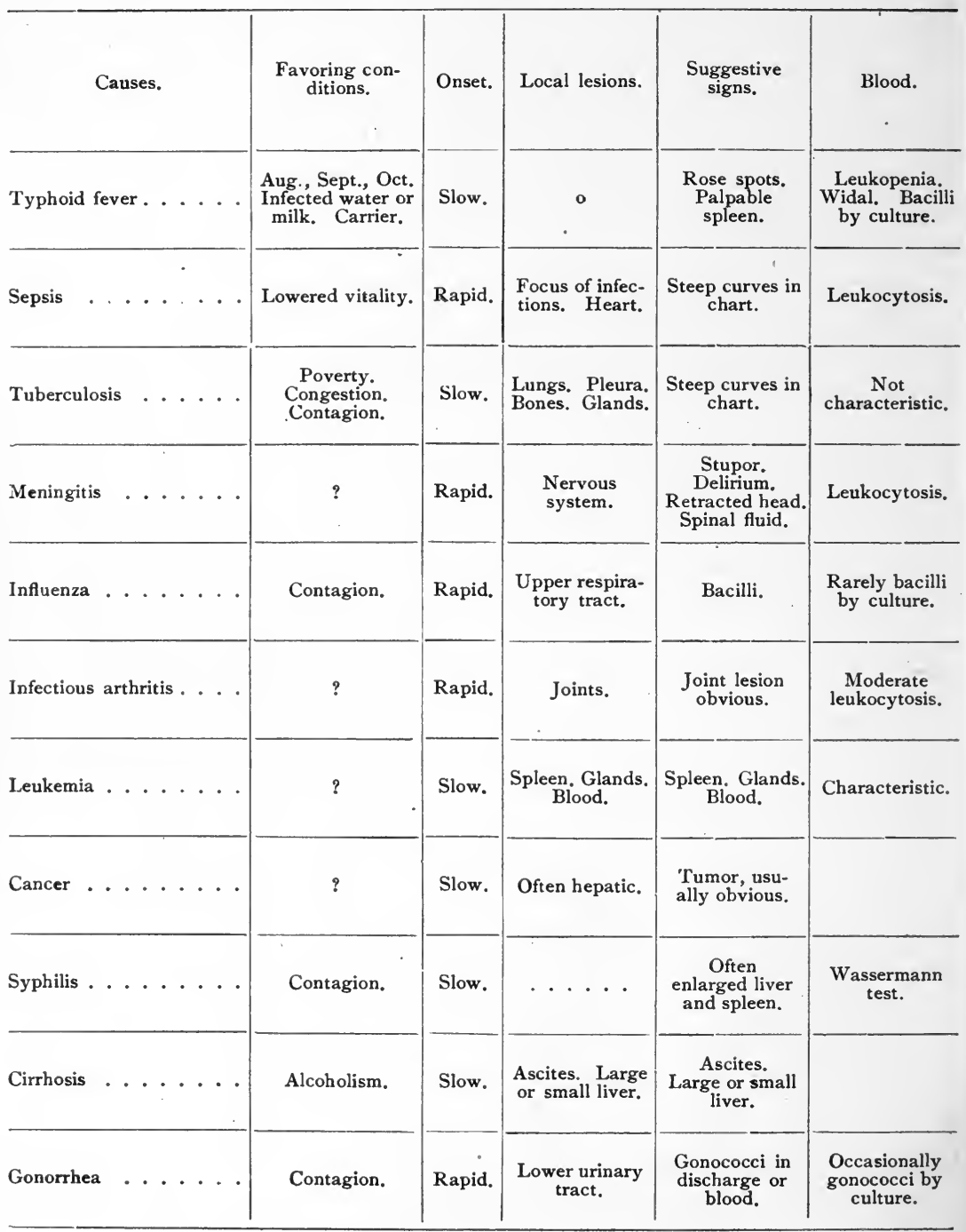





\section{Causes of Chills}

$\left.\begin{array}{l}\text { 1. "NERVOUSNESS" } \\ 2 \text { PYOGENIC SEPSIS }\end{array}\right\} \begin{gathered}\text { CASES TOO MANY AND TOO VAGUELY ENUMERABLE FOR GRAPHIC } \\ \text { REPRESENTATION. }\end{gathered}$

3. PHTHISIS

1171

4. TYPHOID (ONSET)

5. PNEUMONIA

6. BILIARY SEPSIS

7. MALARIA (IN NEW ENGLAND)

Many other infectious diseases at the onset. 


\section{CHAPTER XIV}

\section{CHILLS}

THE rapid clonic spasm of many muscles which may be the only mark of a chill is not to be clearly distinguished from a tremor such as many normal persons are subject to when excited. A subjective sense of cold and an abnormal temperature may or may not accompany the tremor. The chill accompanying gall-stone colic is often seen without pyrexia. Even when the chill marks the onset of an infectious disease the temperature is not always elevated. Hence the distinction between "nervous chills" and those due to infection is sometimes to be made only by the accompanying signs and by the later developments. All chills except those of nervous origin are soon followed by fever.

"Creeping" chills, or chilly sensations without any definite tremor or any chattering of the teeth, are much commoner and less distinctive than the true or "shaking" chill ("Schüttelfrost").

As a rule, when chills are the evidence of infection they accompany an abrupt rather than a gradual rise of temperature. The sudden high fevers of malaria, pneumonia, tonsillitis and pyogenic sepsis are more often attended with a chill than the more gradual rise seen in typhoid or pleurisy.

Among the causes of chills are:

(a) Pyogenic Infections.-These are doubtless the commonest. Under this heading comes, in all probability, most of those occurring in tuberculosis (as a result of secondary infections) as well as those due to appendicitis, septic wounds, renal and hepatic suppurations, tonsillitis vegetative endocarditis, phlebitis, empyema, and erysipelas.

(b) Malaria probably ranks next, especially in the tropical and subtropical regions.

(c) Severe pain (as in renal or biliary colic) may lead to a chill or follow it, even when no infection or fever is demonstrable. In some cases chill (or vomiting) seems to replace the colic as a sort of equivalent.

(d) "Urethral chills," such as often follow the passage of a catheter, sccur in persons who show nothing of a nervous or hysteric taint, yet there may be no fever with or after them. Probably the pain and irritation are enough to set the nervous system "on edge," even if it be ordinarily stolid. 


\section{Case 245}

A baker of thirty-eight entered the hospital February 6, I908. His family history, past history and habits were good.

January 29th he had a sudden chill in the evening. The next morning he vomited his breakfast. Since then he has been having severe chills about twice a day and has vomited a great deal. $\mathrm{He}$ has been lounging about the house, but has not been in bed during the day-time; he has had no cough and no pain.

On examination he is found to be splendidly deveroped. His left pupil is slightly irregular, both pupils reacting somewhat sluggishly to

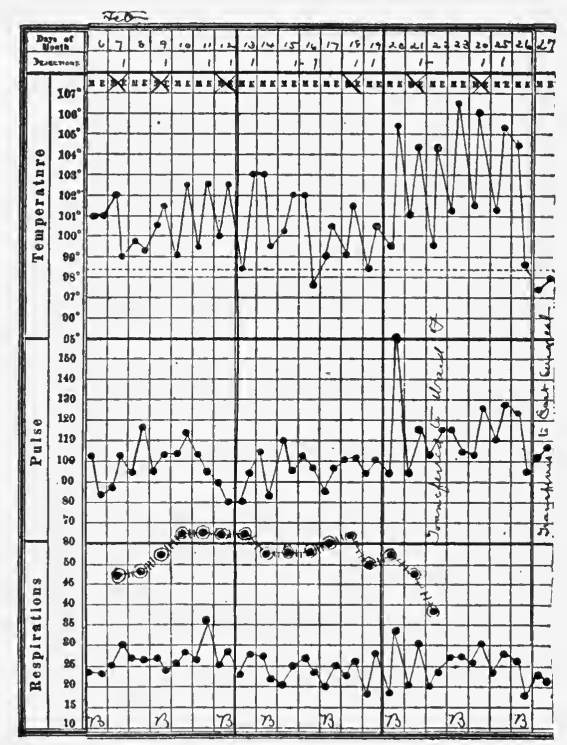

Fig. 108.-Chart of case 245 .

light. The heart is altogether negative. The lungs show slight dulness and many crackling râles at the left base behind, but no other signs. White cells, 17,000 . The rest of the physical examination and the urine are normal.

The sputa shows many pneumococci, some streptococci; no tubercle bacilli.

On the tenth there were no new physical signs. The leukocyte count continued high- 7,600 .

On the twelfth there was distant but distinct bronchial breathing at the left base.

The patient received 190 grains of quinin in twenty-four hours: 
but continued to have chills. On the fourteenth, after a very severe chill lasting half an hour, the rectal temperature was $107^{\circ} \mathrm{F}$. The white cells had now risen to 23,900 , and the patient continued to have daily chills lasting from forty to forty-five minutes, the temperature reaching $106.5^{\circ} \mathrm{F}$. each time and remaining there several hours.

On the sixteenth exploratory puncture of the chest was done twice, the needle entering solid lung each time. From the blood withdrawn by the second tapping a smear was made which showed many pneumococci, both within and outside the leukocytes.

On the twenty-second of February $x$-ray showed a very high diaphragm on the right side, and a shadow behind the scapula between the

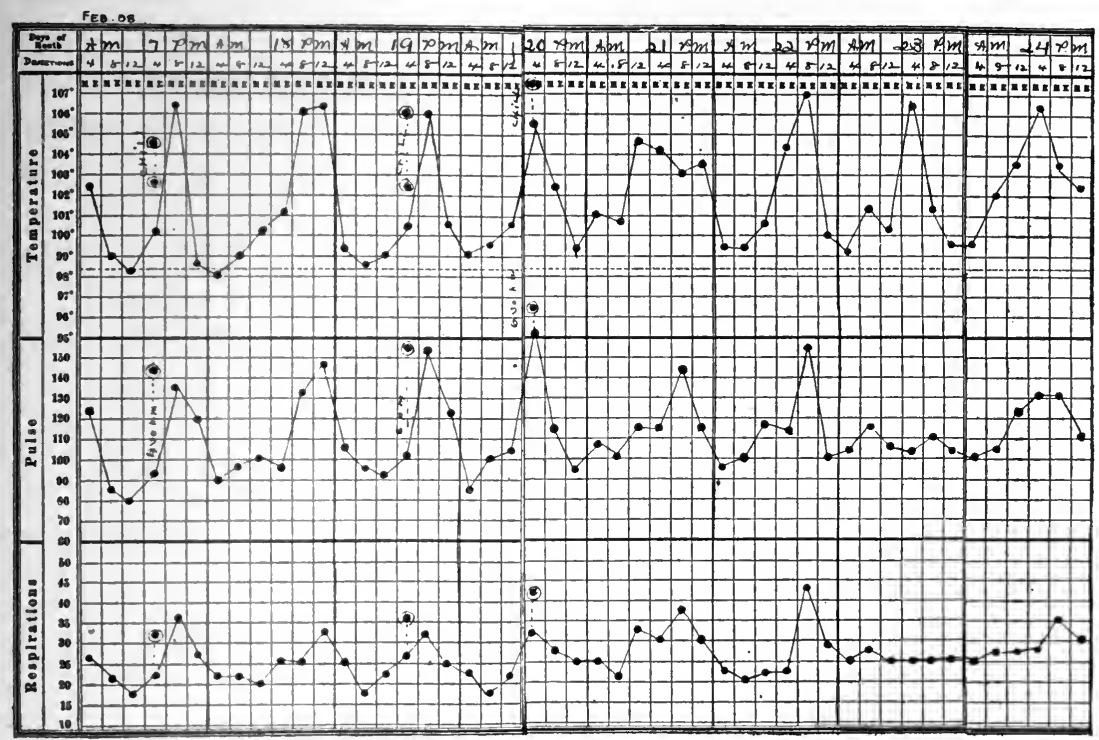

Fig. 109.-Four-hourly chart of case 245 .

fifth and seventh ribs. Erysipelas developed on the twentieth of February and spread all over the face.

Discussion.-When a patient has two chills a day and a leukocytosis ranging from $\mathrm{I} 7,000$ to 23,000 , the administration of large doses of quinin is altogether unjustifiable. There is no reasonable possibility of malaria.

Pulmonary tuberculosis often produces chills, which not infrequently come as close together as in this case, and sometimes recur at exactly the same hour each day, so that the unwary are led to diagnose malaria and to waste time and strength in attempting to stop the chills with quinin. The signs in the lungs of this patient are not at all character- 
istic of phthisis, and the sudden onset of the symptoms would be quite inexplicable if the chills were due to that disease. Chills occurring in pulmonary tuberculosis come after the disease has made its presence evident for many weeks or months previously.

By far the most significant point in this case is the evidence obtained by $x$-ray examination, which appears to prove that the source of the chill lies in or near the liver. That this is usually the source of chills for which no obvious cause can be found is, I think, the general experience. When we have searched the blood, the sputa, the subcutaneous tissues, the ears, and the heart for a septic focus and found none, it usually turns out that the source of infection lies in septic thrombosis of the portal vein, in hepatic abscess, single or multiple, or in a subphrenic abscess.

We cannot be more definite than this in the present case.

Outcome.-On the twenty-seventh a needle introduced through the eighth right space below the angle of the scapula drew pus. A rib was resected March 2d, and a pus-cavity the size of the fist was drained.

The patient continued to have chills, and died on the twenty-sixth. Autopsy showed multiple abscesses of the lung and of the liver.

Diagnosis.- Hepatic and pulmonary abscess.

\section{Case 246}

A Swedish housewife of fifty-six, who had passed the menopause six years previously and had been otherwise well all her life, entered the hospital December 3I, I907. She says that one night last spring while in bed, but not asleep, she "began to feel queer." In a short time she was seen to shake violently all over, complaining at the same time of headache and vertigo. These chills recurred five times during that month. After them she felt weak and had abdominal pain, extending up through the chest to the throat. During the past summer she was much better, but three months ago she went to bed on account of increasing pain, weakness and chills; she has stayed there ever since.

Her most troublesome symptoms are headache, vertigo and constant ringing in her ears. She has a poor appetite and often vomits, though the vomiting seems to have no distinct relation to pain nor to the time of eating. Of late she has a good deal of dyspnea, cough, and expectoration, though the latter is never bloody. She sleeps poorly, and her urine is scanty. She has lost about ten pounds in weight.

Nevertheless, when examined, she was found to be rather obese. Her lips were pale and bluish; the heart's dulness extended one-half inch 
outside the nipple line in the fifth space, five inches to the left of midsternum. A blowing, systolic murmur was heard at the apex, but was not transmitted more than an inch or two in any direction. The pulmonic second was not accented. The pulse tension appeared normal.

In the right back, below midscapula, there was dulness without any other signs. Throughout the left back there were a good many fine, crackling râles. Hemoglobin, 80 per cent. The white cells were 16,200 at entrance; three days later they were I2,300. The urine and the stained blood-smear were normal. Vaginal examination was also negative. The temperature was below $99.5^{\circ} \mathrm{F}$. throughout.

Discussion.- In some respects this case reminds us of the previous one. We have chills, associated with obscure, bilateral, pulmonary signs. Just as we were getting ready to investigate these by means of the $x$-ray they cleared up entirely; otherwise our diagnosis might have remained long in doubt, although with chills lasting nearly nine months we should feel pretty sure that more definite and extensive changes would by this time be demonstrable in the lungs were they the source of the trouble.

Endocardial infection should always be suspected when chills of obscure origin are found to be associated with a cardiac murmur and a slight enlargement of the organ, especially if, as in the present case, there is a leukocytosis. Against this, however, is the long duration of the symptoms and the obesity of the patient. A person who has had ulcerative endocarditis for nine months is not likely to be obese. Further, the course of the temperature is altogether uncharacteristic of a cardiac infection, especially one which would appear to be of long duration. .

In view of these facts and of the nature of the attacks it is fair to suppose that they may have been due to some form of "nerves." But since the patient is well past middle life it is altogether probable that there is some arteriosclerotic change underlying the nervous symptoms.

Outcome.-By the fifth of January the lungs were entirely clear and the patient was able to sit up. After that she complained only of pain in the small of the back, which was greatly relieved by crossstrapping with adhesive plaster. January I6th she went home well.

Diagnosis.-Hysteria (with arteriosclerosis?)

\section{Case 247}

A housewife of thirty-nine, of good family history and past history. entered the hospital November 8 , 1907. She got a cold in the head two weeks ago and was hoarse for a day or two; she then began to cough 
up considerable yellow sputa. During the whole illness (from two weeks ago until yesterday) she had two chills every day at irregular intervals, with shivering, chattering of the teeth and profuse sweating thereafter. At present she feels sore throughout the whole chest, especially beneath the lower half of the breast-bone. The course of the temperature is seen in the accompanying chart.

Physical examination was entirely negative. The sputum showed very large numbers of influenza bacilli both within and outside of the cells. The blood and urine were normal.

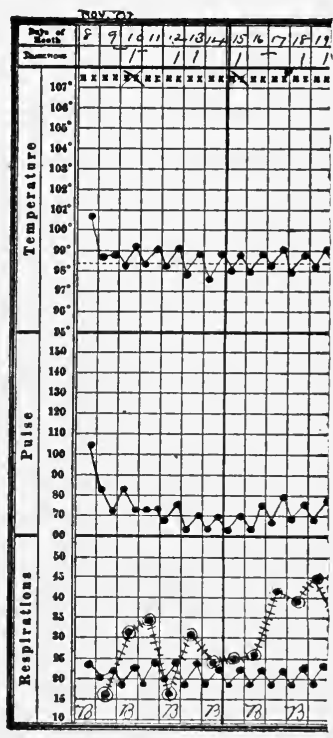

Fig. Iro.-Chart of case 247 .

By the thirteenth the patient was improving slowly under laxatives, heroin for cough and bitter tonics for appetite, but it was not till the nineteenth that she was able to go home practically well.

Discussion.-One investigates in a case like this the ordinary causes for multiple chills: concealed sepsis with or without endocardial localization, malaria, tuberculosis, otitis media, liver disease (including gallstones), renal infection, and nervousness. Since none of these is to be found, we must fall back upon the evidence of an influenza infection, which is the best clue we have to the nature of the chills, although the amount and gravity of the infection seem disproportionately slight when compared with the violence of the constitutional reaction manifested in the chills.

It is to be remembered that the make-up of the individual,-what is often called his "temperament," - and especially his nervous system, influence the degree and character of the reaction against any infection, such as influenza. Any shock or painful experience, such as death, flood or fire, will leave one person quite unmoved, will deprive a second of the power of sleep and make a third irritable and nervous; in a fourth it may provoke that curious motor spasm known as a "nervous chill," and in a fifth may determine an actual convulsion of the type ordinarily called "hysteric." All these reactions represent differences in the degree of sensitiveness of the motor nervous system.

But there are similar differences in the way in which different temperaments react to an attack from within-namely, a bacterial invasion. Those whose motor responses are excessive in the presence of the ordinary 
annoyances and discomforts of life are apt to show a similar exaggeration of the normal response in their constitutional reaction against an infectious disease. In the present case it was learned by subsequent questioning that this woman had been in the habit of having chills whenever any kind of slight ailment affected her. This is perhaps a less common idiosyncrasy than that by reason of which certain persons, whom every physician meets in the course of his practice, show a very high temperature reaction when they catch cold or have a slight digestive upset.

In the discussion of some of the previous cases I have said that most diagnoses of "grip" or influenza seem unjustified, because the bacteriologic evidence is quite insufficient. I do not feel quite sure that we are right in making the diagnosis of influenza even upon such evidence as is presented in the present case. Since the epidemic of I889-90 the influenza bacillus has been a regular inhabitant of the upper airpassages of practically the whole population in New England. The fact that we find it in the sputa in connection with one or another type of disease is, therefore, in itself, of little significance. When the germ occurs in large numbers, both within and outside the leukocytes, and when the other varieties of organisms found are in very small numbers, it is probably justifiable to consider the infection one of influenza.

Diagnosis.-Influenza.

\section{Case 248}

A chambermaid of thirty-eight was first seen November 6, 1907. Two years ago she was in the Massachusetts Eye and Ear Infirmary for nine days with an acute inflammation of the left middle ear, which was lanced several times. Her hearing remained good afterward.

Four days ago she had a severe chill, followed by sweating, vertigo, ringing and buzzing in both ears. The next day there was severe pain in the left ear. Since that time she has felt feverish, and during the last two days has had eight chills and has vomited several times.

During the last two days she has had a dull ache in the left ear, extending down her neck to the left side of the throat. This morning and yesterday morning she went to the Eye and Ear Infirmary, but no trouble was found with the ears. Throughout her illness she has had insomnia, anorexia, and constipation.

Physical examination showed an obese woman with normal pupils. The heart's impulse was felt with difficulty in the fourth space, just outside the midclavicular line. There were no murmurs or accentuations. The lungs were normal. There was some epigastric tenderness, 
but nothing else of importance in the abdomen. The urine ranged between 40 and 60 ounces in twenty-four hours, the specific gravity varying between 1005 and 1023. Albumin was sometimes absent, sometimes present in very slight traces. Casts were sometimes absent, sometimes numerous and of the hyaline and granular type, some of them having cells adherent. In the majority of the examinations it was exceedingly difficult to find any casts at all.

The blood-pressure at entrance was 230. The fundus oculi was normal on the right. On the left there was a large area of opaque nerve-fibers at the lower edge of the disk.

Discussion.-The foreground of this case consists of chills and ear pains, the background of various signs pointing toward a chronic nephritis. The high blood-pressure and the ocular changes are especially important in this latter respect. The urine is equivocal and dubious. The condition of the heart is not characteristic, though suggestive of slight enlargement.

Taking for granted that there is an underlying chronic nephritis, what is the cause of the chill? Otitis media is naturally our first and very decided suspicion, but the high character of the work done at the Massachusetts Eye and Ear Infirmary makes us confident that we may rely upon their negative report regarding the ears.

Nothing is said in the text about an examination of the blood for malaria or for leukocytosis, because these examinations had not been undertaken at the time when I first saw the case. They both turned out, however, to be negative.

Knowing the proneness of all cases of chronic nephritis to an invasion by an infectious disease, it seems natural to assume that some such infection was present in this case, although we do not find definite evidence of its whereabouts. There remains, however, one further possibility: the chills may be a direct result of nephritis without any infection. Chills and convulsions are first cousins. In fact, a chill may be described as a generalized clonic spasm of very short excursion. In view of this it seems more than possible that the excessively high blood-pressure which existed for a short time in this case may have determined the onset of chills, as we well know that a similar rise of pressure often determines the onset of convulsions. In view of the outcome of the case, without further evidence of infection, this hypothesis deserves consideration.

Outcome.-On the thirteenth the blood-pressure was still markedly elevated,-rgo,- - though she had been having daily hot-air baths and was purged every second day with an ounce of magnesium sulphate. 
On the twenty-seventh the blood-pressure had fallen to I55. The $_{55}$ patient had no symptoms except slight tenderness and weakness in her legs. She was then allowed to go home.

Diagnosis.-Chronic glomerulonephritis.

\section{Case 249}

A school-boy of fifteen, whose father died of consumption, was first seen April i5, 1908. He was always well until two weeks ago, when he began to have headache, backache, and soreness all over his body. Since then he has had several chills, with chattering teeth. Yesterday he vomited; throughout he has slept well. There has been no nosebleed. (See Fig. III.)

When examined, the face was flushed, the throat reddened and covered with a mucopurulent secretion, the glands in the axillæ and the right epitrochlear enlarged, the neck not stiffened. The heart was negative except for a soft, systolic murmur in the pulmonary area, the lungs entirely negative, likewise the abdomen.

The white cells were I3,400; Widal reaction negative, blood culture negative.

Discussion.-Among the infections common in boys of fifteen, which are most often obscure?

(a) Endocarditis, with or without arthritis or chorea.

(b) Tuberculosis, especially of the bone, glands, or peritoneum.

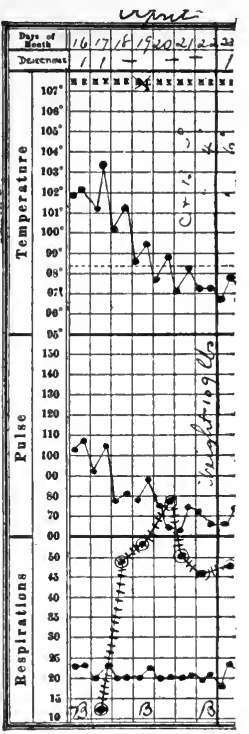

Fig. IIr.-Chart of case 249 .

(c) Otitis media.

(d) Brief febrile maladies to which no name can be given at present, and in which no definite localization in any organ is found (febricula, "gastric fever," "grip").

This latter group is the most numerous of all.

The present case seems to have been investigated sufficiently to exclude with considerable confidence any tuberculous or endocardial infection. No search, however, appears to have been directed toward excluding otitis, a possibility which should never be forgotten in youth and infancy. In the otitis of the adult our attention is usually called at once to a source of the trouble by the occurrence of severe earache.

Outcome.-On the eighteenth the left ear began to discharge and the other followed suit soon after; by the twenty-fourth the ears 
had stopped draining, and the hearing and the boy seemed practically well. At no time was there any considerable amount of pain in or near the ears.

Diagnosis.-Otitis media.

\section{Case 250}

A Russian clothes-presser of twenty-three was first seen September 28, r907. His family history and past history were excellent. He stated that he has never been sick until sixteen days ago, when he was seized while. at work with a severe chill, followed by a profuse sweating. Since then he has had a slight chill twice a week. Occasionally he has sharp

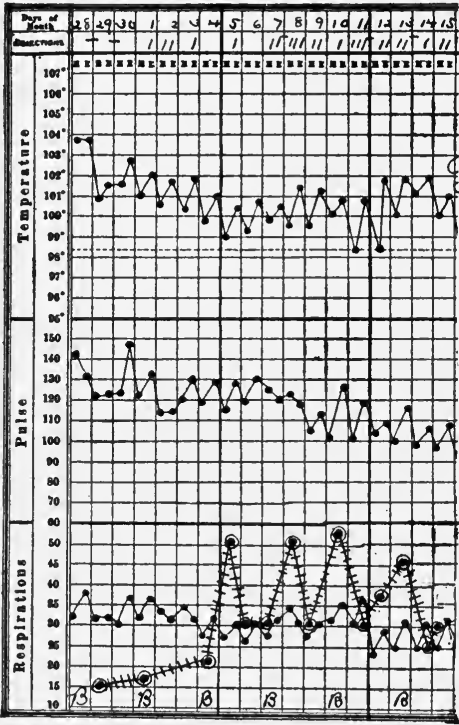

Fig. I12.-Chart of case 250 .

pain in the right side of the chest if he happens to take a specially deep inspiration.

When examined, the patient was well nourished. (See Fig. II3.) The glands in the axillæ were as large as lima-beans. There was marked acne on the back and sides of the chest. The heart showed nothing abnormal. The lungs showed occasional scattered râles throughout, somewhat more numerous in the right front.

White cells I0,200; Widal reaction and stained smear, negative.

Urine, negative. The next morning the lungs were as in the accompanying diagram (Fig. II4). The pulse was rapid and dicrotic, the patient alert and anxious. Each day

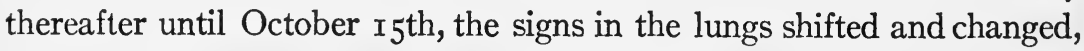
râles coming and going, patches of bronchial breathing appearing and disappearing.

Discussion.-The physical signs suggesting possible causes for this patient's chills are those in the lymphatic glands and in the lungs. These possibilities should first be investigated, though we must bear in mind that some of the other and more obscure causes mentioned in previous cases may be here at work.

Chills may be associated with glandular enlargement in lymphoid leukemia, in Hodgkin's disease (with infection of the glands), and in glandular tuberculosis; occasionally also in syphilis. Leukemia is 


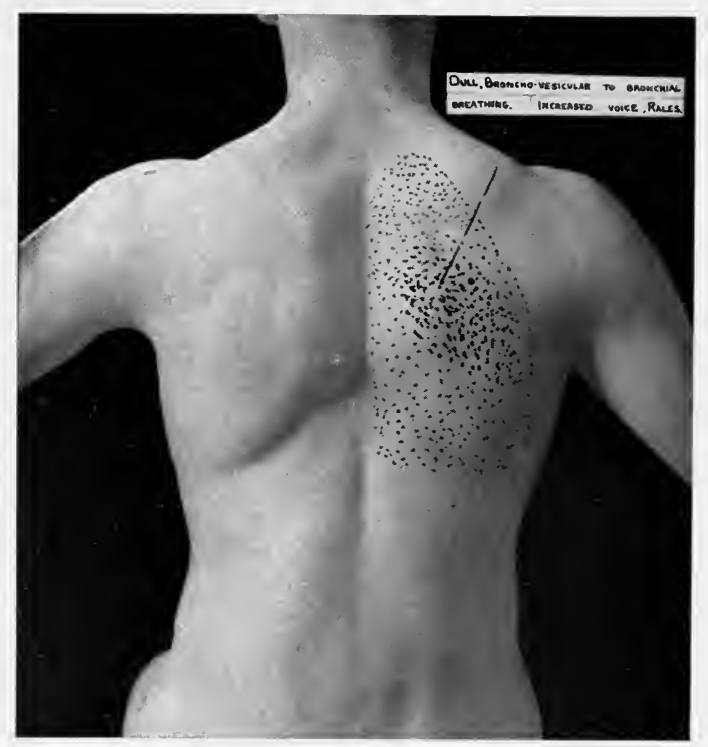

Fig. Ir 3.-Physical signs in Case 250. (See also Fig. 114.)

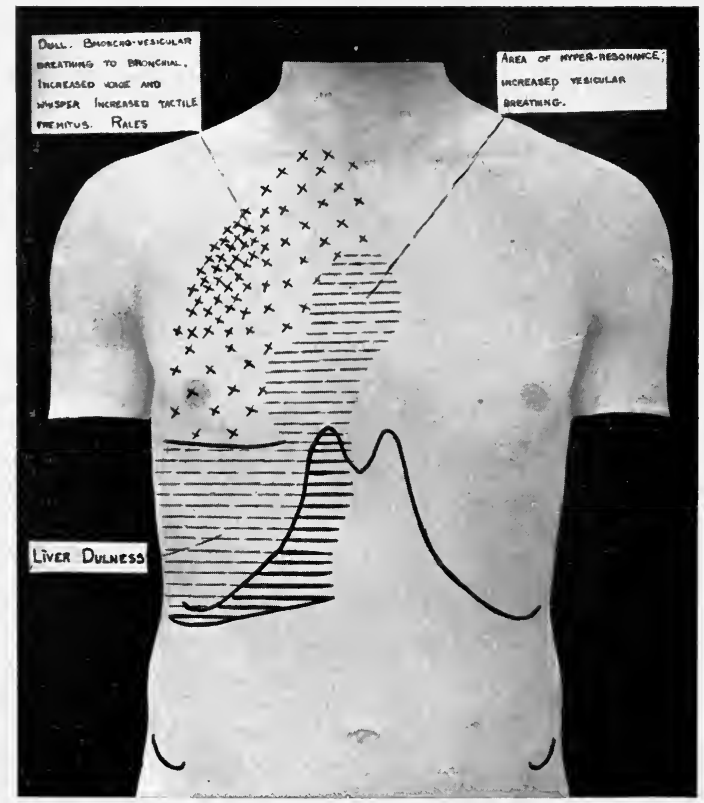

Fig. I I4.- Physical signs in Case 250. Adenitis, chills, and chest pain; no cough. 
here excluded by the blood examination. Hodgkin's disease never manifests itself in the axillary glands alone, and the same is true of syphilis. Glandular tuberculosis cannot here be excluded, though in an adult it rarely causes such marked constitutional disturbance unless other tissues are involved. Of course, the internal glands-bronchial, mesenteric, etc.-would be assumed as affected in addition to those of the axilla.

The pulmonary signs are most like those ordinarily seen in acute bronchitis with bronchopneumonia, but the duration of the disease, which is more than a month at least, is hardly consistent with this idea, and makes us suspect a pulmonary tuberculosis. Further evidence can be obtained only by the sputum examination. 'The cutaneous tuberculin reaction seems to be of little value in patients of this age, and the fever precludes our trying the subcutaneous reaction.

Outcome.-When first questioned, the patient stated that he had no cough whatever, so that when the physical examination was undertaken we had practically no clue from his history regarding the source of the chills. Later he acknowledged that he did cough occasionally, and on the thirteenth of October a little glairy sputum, resembling saliva, was obtained. It seemed hardly worth examination, but to our surprise a few tubercle bacilli were found in it. The patient passed out of observation October I6th, his condition having become steadily worse meantime.

Diagnosis.-Phthisis.

\section{Case 251}

A man of sixty-eight, a dealer in sponges, was seen February I, I908. Thirty years ago he had "Bright's disease" and was sick for a year. He has had "malaria" off anc" on for thirty-five years; otherwise he has been well until seven years ago, when he began to have "stomach trouble," which has become worse in the last three months. This is characterized by pain and discomfort in the epigastrium, coming after each meal and lasting two or three hours. His appetite has been very poor, and for six weeks he has lived mostly on liquids. He never vomits or is jaundiced.

He passes urine eight or ten times at night. He has considerable frontal headache, no cough or dyspnea. A year ago he weighed ${ }_{17} 8$ pounds; now he weighs I34; he thinks he has lost chiefly in the past three months. Four weeks ago he began to have chills coming every day about 4 P. M. For two weeks he has taken 20 grains of quinin every day.

When examined, the patient was found to be emaciated, with a dry, 
rough, pale skin. There was no dulness or bronchial breathing anywhere in the chest. There were crackling râles at the right apex in front, scattered, dry whistling sounds below the right clavicle, and harsh respiration at both bases.

The hemoglobin was 75 per cent. No malarial organisms were found after repeated examinations. The urine was normal; Widal reaction negative. At entrance the patient's temperature was normal. (See Fig. II5.) It soon rose and remained elevated throughout his stay in the hospital. By the stomach-tube examination no fasting contents were recovered. The stomach held 44 ounces, its lower border reaching

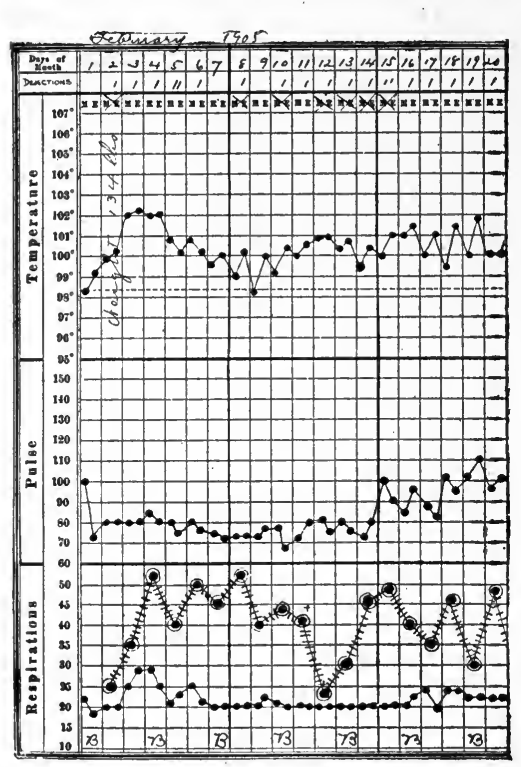

Fig. II5.-Chart of case 251 . just below the navel. After a test-meal, free hydrochloric acid was 0.05 per cent., total acidity, o.I9 per cent. The guaiac test was negative. In the warm bath an edge, distending with respiration, was felt in the right hypochondrium.

The patient was given Io grains of quinin every four hours, but his temperature was not much affected.

On the fourteenth of February there was bronchovesicular breathing in the second right intercostal space near the sternum, with intense whispering bronchophony and coarse consonating râles after cough. Otherwise the sounds were as at entrance.

Discussion.-Since the patient has apparently had malaria for a good many years and is now moderately anemic, it is proper to assume - until proved to the contrary-that the chills of which he now complains are of malarial origin. This idea, however, was decisively disproved by the blood examination, on which we can entirely rely in such cases if it is made by any one who has had the proper training.

Can there be any connection between the stomach, of which he complains so much, and these chills? In answer, we may say, I think, that unless the disease has extended far beyond the stomach (e.g., subphrenic abscess from perforated peptic ulcer) that organ is incapable of producing such a clinical picture. Phlegmonous gastritis-that very rare disease-produces a far more fulminating and virulent type of 
infection. The other stomach diseases may be disregarded in our efforts to explain the chills.

Since the edge of the liver is felt, it is proper to inquire whether any infection in or near that organ may be at the bottom of this patient's troubles. Provided the patient has no leukocytosis (a point on which we are still ignorant), liver infections, whether rising from the gallbladder, from the intestine, or otherwise-are improbable. The upper border of the organ, especially in the axilla and back, is almost always raised, as percussion will demonstrate, if there is any infection in or near the liver. In doubtful cases the $x$-ray examination may help us to determine the outline and position of the organ.

The pulmonary signs do not seem at first examination to be of any special significance. Many patients of sixty-eight present similar abnormalities, off and on, without complaining of anything in particular. In the present case, however, their persistence and the absence of any other important lesions discoverable by physical examination lead us to focus attention upon the lungs. Apparently the conditions, whatever their nature, are steadily getting worse.

In patients of this age we are rather apt to forget the frequency and importance of tuberculosis. Statistics show that, contrary to the impression current, both among the laity and among medical men, tuberculosis is just as prone to occur in the latter decades of life as in the earlier. In this case we are tempted still further away from the track of the truth because the patient says practically nothing about cough-indeed, denies that he has had any previous to the first of February. In all probability this is a mistake, and in view of the steady increase in the lung-signs during the three weeks' period of observation, tuberculosis seems the most reasonable diagnosis.

Outcome.-On the eighteenth a small accumulation of free fluid appeared in the peritoneal cavity. There was no circulatory weakness to account for it. The patient was very fussy and hard to please, declaring that he had no saliva and no secretion from his stomach. On the twenty-first he became much discouraged and insisted upon going home. Tubercle bacilli were never found in his sputa during the three weeks of his stay in the hospital.

Diagnosis.-Phthisis (?).

\section{Case 252}

An Irish laborer. fifty-four years old lost his father of consumption and one sister of the same disease. His wife and one daughter are now sick with "colds." He was first seen February I3, 1907. 
Thirty-eight years ago he was seven weeks in bed with "rheumatism," and had stiff and painful joints for three years thereafter. Thirteen years ago he had a right-sided "pleurisy," but was well in a few days.

$\mathrm{He}$ denies venereal disease, but for ten years has been unable to hold his urine for any considerable length of time in the day, though he passes it only twice at night. As a rule, he does not drink liquor to excess, but a week ago he got drunk and stayed so for two days.

Two weeks ago he began to have chills, several occurring during one night, accompanied by cough and a thick white sputum. He was unable to lie down on account of the distress across the upper abdomen.

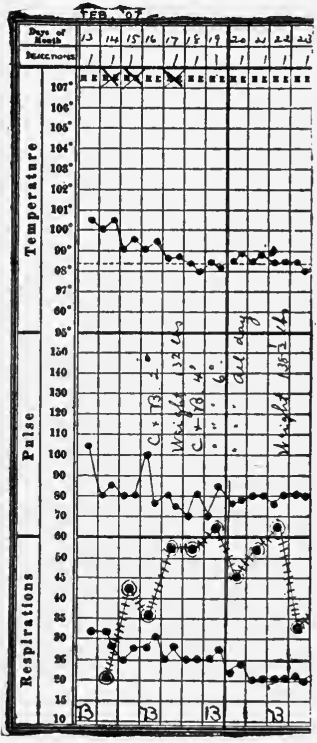

Fig. II6.-Chart of case $25^{2}$.

Two days later he had a sharp pain in the lower part of both chests, increased by cough or breathing. Pain ceased two days ago in the left chest, but persisted in the right. He has been in bed for the past three days, complaining of pain, cough, and weakness.

The course of the temperature is seen in the accompanying chart.

Physical examination showed no enlargement of the heart and no murmurs, though the heartsounds were irregular in force and rhythm. The brachials were very tortuous and showed a lateral exertion, with apparently an increased tension. There was slight dulness in the lower part of both backs and at the right base in front, over which area there are a few crackles, while below the right nipple there was heard an indistinct friction-rub, which on the seventeenth had become rougher and more easily audible.

Discussion.--The common causes of obscure chills, such as malaria, deep-seated suppurations, acute endocarditis, tuberculosis, and nervousness, must all be canvassed in a case of this kind, but there is very little to substantiate our belief in any one of them. A number of points, however, not fully stated in the printed -account must be further investigated.

(a) Nothing is said about the urine. In men of this age an old urethral stricture, with or without prostatic hypertrophy, urinary retention and chronic cystitis, often leads, through an ascending infection, to pyelonephritis, and thus to chills like those here described. Investigation of the urine, however, showed no evidence of any such disease.

(b) Nothing is said about the size of the liver. Pain in the upper 
portion of the abdomen, associated with chills, should always make us look for evidence of liver abscess, gall-stone disease, or subphrenic suppuration. We should expect a leukocytosis in connection with any of these types of infection. Nothing is said about the leukocyte count in the history printed above. As a matter of fact, however, both the blood and the size of the liver appeared to be normal.

(c) It is well known that alcoholism is often associated with nightsweats and sometimes with chills. So far as I know, however, both of these phenomena are of nervous or vasomotor origin, and do not depend upon any variations of temperature, such as are shown in the chart.

On the whole, the chest signs seem the most significant, now that we have excluded some of the other possibilities. Evidently there has been some pleurisy on the right side, possibly on both sides, though double pleurisy is not a common condition. As to the nature of this pleurisy, it is hard to get any definite information; perhaps only the outcome will decide. The pleurisy might be of the type closely associated with lobar pneumonia, although we have no signs of that disease. Many cases of obscure septic infection by pyogenic organisms affect all the serous membranes and joint surfaces to a greater or less extent, passing rapidly from one to another. Some such infection may well have been present here. Tuberculous pleurisy is also a possibility regarding which we can obtain decisive information only by following the case for a long time.

Outcome.-On the fifteenth a fine friction-rub was also heard in the left lower axilla, and this persisted until the twentieth.

The patient was given a tight swathe for four hours and a teaspoonful of a mixture consisting of phosphate of codein, 8 grains, potassium citrate, 3 drams, syrup of hydriodic acid, 4 ounces. This, with a bitter tonic for his appetite, made him able to leave the hospital on the twentyfourth.

Diagnosis.-Double pleurisy (septic?).

\section{Case 253}

A court officer seventy-six years old, of good family history and past history, entered the hospital March I8, 1908. His habits are good. He has had chills occasionally ever since the Civil War.

This morning, about eight o'clock, while on the train, he was seized with a violent chill, not followed by sweating. Since then he has felt very sick and is still chilly, but has no pain anywhere. About noon he vomited four times.

At no time has there been any cough. 
Physical examination showed in the left back, below the angle of the scapula, slight dulness, distant bronchovesicular respiration, increased fremitus, and medium-sized crackling râles. There were a few elevated red patches the size of almonds, covered with crusts, about the left ankle and shin. The white cells were 18,900 ; urine was normal. The temperature was as seen in the accompanying chart (Fig. II7).

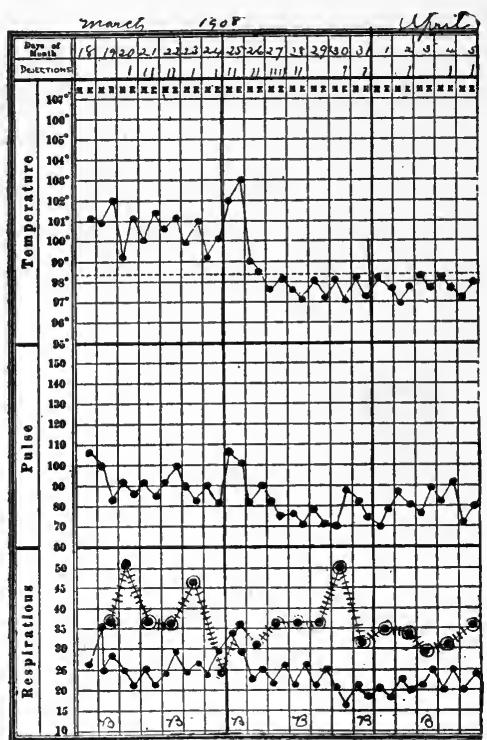

Fig. II 7.-Chart of case 253 .

There was a fine trembling all over whenever the man moved. There was no cough and no sputa.

Discussion.-The point which I wish to insist upon in this case is that the history and the symptoms give no indication of the diagnosis.

With a careful physical examination it becomes tolerably obvious that we are dealing with a lobar pneumonia (although the signs are not very marked), but the diagnosis must rest wholly on signs, as there is no cough, rusty sputa, or pain in the side. The chill was confidently attributed by the patient to the malaria which he acquired in the Civil War.

Although I have referred to the disease here present as "lobar pneumonia," it is more than probable that the infection is not so definitely localized and involves only local congestion with pleurisy and perhaps some bronchopneumonia. The main bulk of the infection works free in the blood-stream.

The old skin lesions about the left ankle have no relation to the symptoms complained of in this case. They probably represent a chronic eczema.

Outcome.-On the twenty-first the white cells were 17,400 , and on the twenty-sixth, 27,000. That night the temperature fell by crisis and he convalesced without incident.

The treatment consisted of strychnin, $\frac{1}{40}$ grain, three times a day, whisky, I ounce, every four hours. The bowels were moved by glycerin enemata.

Diagnosis.-Pneumonia. 


\section{Case 254}

A cook, forty-six years old, was seen May I, 1907. She has one child living and well; one died in infancy. She has had two miscarriages. Her family history is excellent.

Three years ago the patient had a severe sore throat which had to be lanced fifteen or twenty times. Since that time her voice has been thick.

Seven weeks ago she began to have chills and sweating every day or two, accompanied by persistent nausea and vomiting. For the past three weeks she has been troubled chiefly with cough, thoracic and epigastric pain. Throughout her illness she has had moderate irregular fever and epigastric tenderness. Her temperature is seen in the accompanying chart (Fig. II8).

The patient was obese, pale, incoherent, and almost comatose. There was marked photophobia, so that the pupillary reactions could not be obtained. Through the soft palate there was a median perforation the size of a quarter of a dollar. Behind it broad white bands, probably old adhesions, could be seen. The heart-sounds were faint and valvular in quality. No murmurs were heard and no enlargement found; the lungs showed nothing abnormal. Blood-pressure, I Iо.

In the abdomen there was general tenderness, especially marked in the epigastrium, where vague resistance was felt behind the spastic muscles. The reflexes were normal. The white count was I6,000; polynuclears, 70 per cent. There were no malarial parasites. The urine averaged 20 ounces in twenty-four hours, IOI3 in specific gravity;

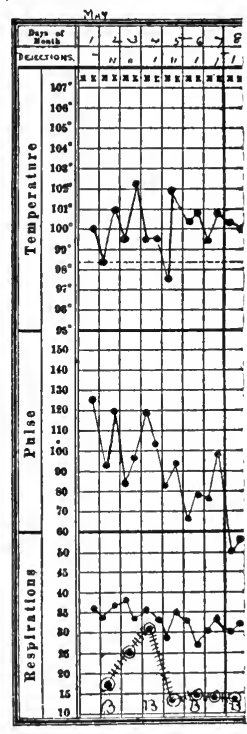

Fig. II 8.-Chart of case 254 . albumin, O.I per cent., a few coarse granular and epithelial casts. The Widal reaction was negative.

Discussion.-This patient had been working very hard for a number of years without vacation, and the diagnosis of her attending physician was general exhaustion. But the condition of the urine made it at once evident that something more serious was going on. Although the patient is febrile, the condition of the urine cannot be explained thereby, as its characteristics are not those ordinarily associated with fever. On the other hand, it does not seem like any of the more familiar types of nephritis. It has not the concentration and bloody sediment seen in 
most cases of acute nephritis, while against chronic nephritis is the absence of any cardiac enlargement or hypertension.

Leaving for the time undecided the problem of the renal condition, we may start from one of the most certain and reliable physical signs present in the case, viz., the perforation of the soft palate and the adhesions between it and the posterior pharyngeal wall. This condition [practically pathognomonic of syphilis unless there is a history of the patient's having swallowed some caustic], the long-standing hoarseness of the voice, the chronic sore throat, and the miscarriages may well be accounted for in the same way.

Experience shows it a fairly safe rule to assume that any acute manifestations occurring in a patient with unmistakable lesions of a past syphilis, are part and parcel of the same infection. There are, of course, exceptions to this rule, but they are not numerous. If now we return to the attempt to explain the condition of the kidneys, we notice that the urine shows the characteristics traditionally associated with a syphilitic type of nephritis involving amyloid change. In this kind of disease cardiac hypertrophy usually does not occur, though the urine has the main features of chronic nephritis.

We get no hint as to the cause of the chills unless it be the epigastric tenderness which might be associated with hepatic syphilis. I have known this disease to produce chills as well as fever, and in the absence of any more obvious cause we may conjecture that something of the kind is going on here. It is quite possible, however, that some acute pyogenic infection may have supervened.

Outcome.-The white count steadily rose during the week of her illness, reaching 25,000 on the fourth of May; 30,800 on the sixth; 37,500 on the eighth. The urine became smoky or bloody, the albumin rising to 0.8 per cent. despite the sweating and purging.

In the last two days the pupils ceased to react and the lungs filled with coarse crackles. The patient died on the ninth; no autopsy.

Diagnosis.-Visceral syphilis.

\section{Case 255}

An Irish housemaid of thirty-six, whose father died of cancer, was seen August 30, 1907. She has had no previous illness. The catamenia are usually painful in the first three days, but not otherwise abnormal.

She has called herself perfectly well until a week ago, when she awoke with a stiff neck, fever, and pain in her neck, back and hips. She worked that day, but the next day had to go to bed, and has been growing worse since. She has been seen four times by her physician, 
who thought at first that she had malaria, as she had frequent chills during the first three days. More recently he thought it might be pneumonia.

The bowels have moved only three times in the past week. Last night she had a chill worse than any of her previous ones. She vomited a great deal and slept very little.

(For the course of the temperature see the accompanying chart, Fig. Irg.)

The patient's hair is nearly all white. The left pupil is larger than the right, both reacting normally. She has marked Riggs' disease. The throat is red, the tonsils somewhat enlarged. The chest shows

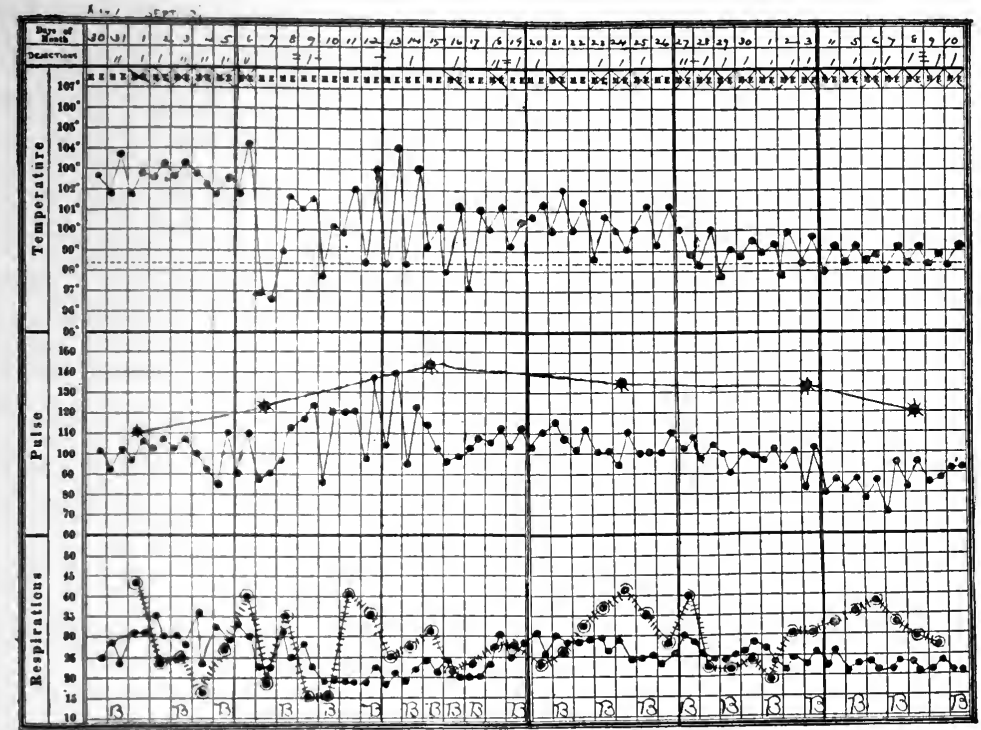

* Blood pressure

Fig. II9.-Chart of case 255 .

nothing abnormal. The abdomen is rather full below the umbilicus and slightly tender throughout. The spleen is not palpable. The most distressing symptom is headache. Her head can be bent only a short distance forward or sidewise, and then with evident pain.

There is also considerable stiffness of the spine and Kernig's reaction is present on both sides.

Lumbar puncture was done, and $32 \mathrm{~cm}$. of clear fluid withdrawn. No cells or organisms were found in the sediment. The white cells at entrance were 16,200. On September $2 d$ they were 9600; on the sixth, I3,500; on the eighth, I2,600.

The fundus oculi was normal. 
On the sixth of September she passed about 30 ounces of blood by rectum. The temperature fell, and she became very thirsty and perspired profusely.

The next day her extremities were cold and her pulse of very poor quality, but she had no further hemorrhage, and did very well after the sixteenth. (See accompanying chart-Fig. II9-of the temperature.)

Discussion.-Meningitis is very strongly suggested, and cannot be positively excluded, but the characteristics of the fluid obtained by lumbar puncture are strongly against every type of meningitis except that due to tuberculosis, and not characteristic even of that.

The intestinal hemorrhage is like that occurring in typhoid fever, and the meningeal symptoms might be explained as meningismus, i. e., irritation of the meninges from congestion, toxemia, or edema, without actual inflammation. Uncomplicated typhoid practically never produces such a leukocytosis as is here present, and the Widal reaction is absent, although this fact is not so significant in a case like this seen early in the course of the disease, as it would be in the later weeks of the fever. The description of the abdomen is quite consistent with the signs usually present in tuberculous peritonitis, yet the clinical picture, seen as a whole, is very different from that of peritoneal tuberculosis. In the latter disease the symptoms and signs are confined almost wholly to the abdomen itself, while in this case there is much to call our attention elsewhere.

A rigid search was, of course, made for any local infection which might cause the chills. All the familiar situations in which deepseated suppuration conceals itself (the ears, the deeper portions of the axilla, the perirectal tissues, the hepatic region, the urinary tract, the pericardium) were examined, with negative results.

Finding no other satisfactory diagnosis, we naturally return to typhoid with some complication producing chills. What can that complication be? In the reports of the Johns Hopkins Hospital (vol. $\mathrm{v}, \mathrm{p} .445$ ) is a study of chills occurring in typhoid fever. The following causes are discussed:

(a) Chills at the onset of the disease.

(b) Chills at the onset of a relapse.

(c) Chills at the onset of complications (phlebitis, cholecystitis, pleurisy, pneumonia, otitis, periostitis).

(d) Chills as a result of treatment (antipyretics, antityphoid vaccination, and intravenous saline infusion).

(e) Chills due to concurrent malaria. 
(f) Chills due to unknown cause (sepsis?) in protracted cases.

In the present case we must classify the chills under the last heading $(f)$.

Outcome.-On the sixteenth of September the Widal reaction became positive. On the twenty-fifth of September it was noticed that for a number of days she had been passing only from one-third to onefifth as much fluid by the urethra as she took in by mouth, although there was no considerable amount of sweating and the bowels were normal. This remarkable retention of fluid was, doubtless, necessary in order to make up for the losses suffered both by hemorrhage and as a result of the fever itself. She continued to improve steadily and went home perfectly well on the third of November.

Diagnosis.-Typhoid.

\section{Case 256}

A glazier of twenty-four, whose father died of consumption, was seen November I3, I906. He has been perfectly well all his life, but takes two or three glasses of whisky and three or four of beer every day.

The temperature curve is seen in the following four-hourly chart:

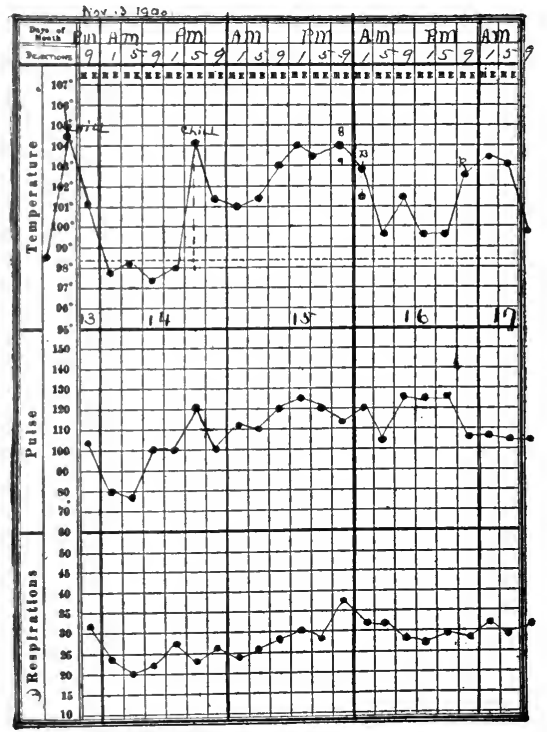

Fig. 120.-Chart of case $25^{6}$.

Ten days ago he had a chill, followed by sweating and weakness. These chills have recurred ever since that time, usually between 7 and 8 P. M. The chills are accompanied by pain in the left side of the 
chest and in the back. They usually last an hour and are accompanied by vomiting. He has been in bed two days.

Physical examination was entirely negative. White cells, 7000. Hemoglobin, 75 per cent.

Malarial parasites were repeatedly sought for, but never found. The polynuclear cells made up 88 per cent. of the leukocyte percentage. Widal's reaction was always negative. The patient looked sick and toxic. No diagnosis was made.

On the seventeenth of November, in the course of a routine examination of all parts of the body, an area of reddening and brawny induration was found in the left buttock, extending along the perineum and up to the groin. The patient had made no complaint of pain in this region. The white cells were now I7,000.

Discussion.-In cases characterized by chills and fever, when malaria and neurasthenia can be excluded, the only proper course for the physician is to keep on looking, day after day, by repeated and searching physical examinations, for some local cause. Typhoid fever is, of course, a possibility in a case of this kind, but the high percentage of polynuclear cells and the continued absence of a Widal reaction, after a period of at least two weeks from the beginning of the illness, makes this unlikely.

The left chest was repeatedly examined for evidence of pleurisy or empyema, but at no time were there any physical signs of disease discoverable there.

Tuberculosis and meningitis were considered, but could not be verified.

The point at which the suppuration was finally found is, I think, a rather frequent one in cases of this kind. Sometimes we fail to find it because the patient's modesty and our own too limited physical examination gives us no hint. In other cases I believe that the suppuration actually causes no pain or recognizable physical sign until it reaches the surface of the body, or, at any rate, the subcutaneous tissues. Another possibility, which the more frequent use of blood cultures of late years has brought to our attention, is that the chills were produced by a non-localized bacteremia which later manifests itself as an abscess.

Outcome.-Operation, November r8th, liberated nearly a pint of pus. The temperature fell at once, and the patient went home well three weeks later.

Diagnosis.-Ischiorectal abscess. 
$$
44^{\circ}
$$

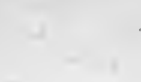

$44^{\circ}$ 


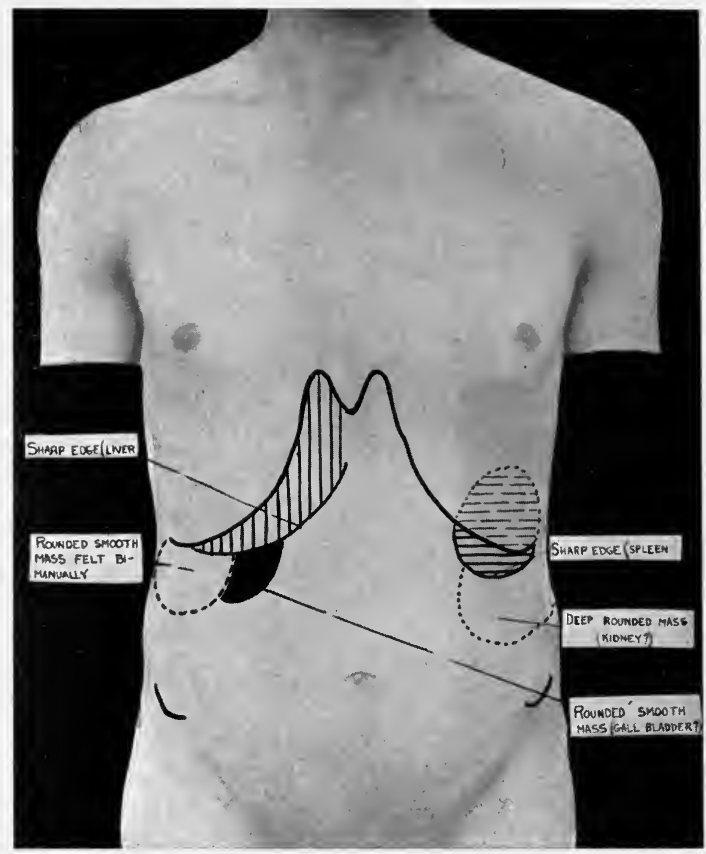

Fig. I21.-Results of physical examination in Case $257 . \quad$ Complaints: chills and "oldfashioned stomach trouble." 


\section{Case 257}

An old lady of seventy-one was first seen September 28, 1909. Twenty years ago, following the menopause, she had two or three chills at intervals of forty-eight hours. No other symptoms. Since that time she has had one or more similar attacks every year without known cause or relation to seasons. Quinin has often been given her, but has noappreciable effect.

No other symptoms occurred until five weeks ago, when she had an attack of what she called "ordinary old-fashioned stomach trouble," $i$. e., an epigastric pain which "cut its way through the right side to the back." There was vomiting with this. These attacks have recurred every second day ever since. The pain is usually controlled by drugs. Her appetite has been failing for five weeks. Her stools have never been light colored, and she has never been jaundiced. Now she feels well and strong. (See Figs. I2I and I22.)

Discussion.-The patient was an exceedingly intelligent and frisky old lady who said what she meant and meant what she said, so that I believe that she really had had chills off and on for twenty years - a remarkably interesting history.

In a woman of a different type one might suspect that these chills were of the nervous variety, but no one who conversed for any length of time with this patient could entertain such a supposition.

There was absolutely nothing in the physical examination to suggest any source or cause for the chills. She had never been in a tropical

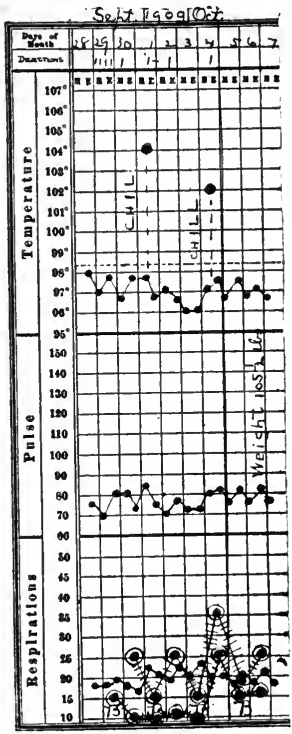

Fig. 1 22.-Chart of case 257 . country where she could have acquired relapsing fever. She had no sign whatever of Hodgkin's disease. There is but one other common cause for a relapsing or recurring type of fever, such as we may assume to have accompanied this patient's chills, viz., gall-stones.

This latter possibility-gall-stone disease-is borne out by the attack of so-called stomach trouble, for many gall-stone pains are referred, . as in this case, to the epigastrium. The most surprising feature, however, of the case and the greatest difficulty with our diagnosis of gall-stones is the entire absence of jaundice. It is true, of course, that manyperhaps most-cases of gall-stone run their course without jaundice, 
but the particular type of trouble which is prone to produce recurrent attacks of fever with chills-common duct stone with infection-almost always causes jaundice.

Outcome.-As the patient refused operation and soon left the hospital with a rather low opinion of the modern medical profession, we have no absolute proof that our diagnosis of gall-stones is correct, but I feel no considerable doubt of it, for we learned later that in a previous attack one of her physicians had found yellowing of the conjunctivæ and bile in the urine.

Diagnosis.-Gall-stones.

\section{Case 258}

A married woman of thirty-one was seen October 7 , I909. She has been working excessively hard for six weeks, caring for four children at home and working to support them during the illness of her husband.

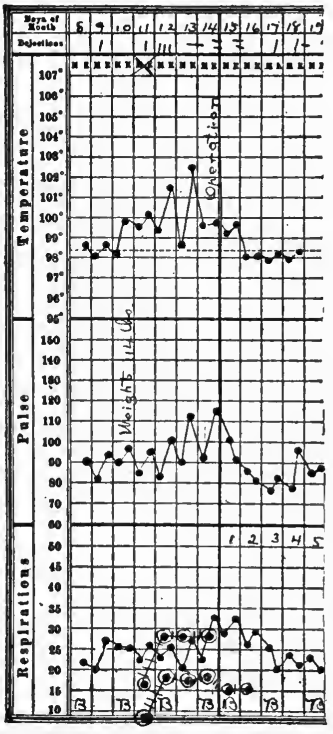

Fig. I23.-Chart of case 258.

Has been very shor: of food and sleep.

One week ago she had a sudden severe chill and vomiting. Three days ago began to cough, raising considerable yellow or brownish sputa. No pain, no headache, no chill, or vomiting, after the first day. Now suffers from great exhaustion, anorexia, constipation, and racking cough which disturbs sleep.

Examination.-(See Fig. I23.)

Marked herpes labialis. Chest and belly negative. Urine and sputa negative. Leukocytes October 8, II, 500 ; October I3th, I9,000. No localizing evidence.

Discussion.-Since we were unable to find any evidence of lobar pneumonia or of bronchopneumonia, we began to think that the case must be one of those uncharted, unnamed infectious diseases ordinarily called "grip." There was not a particle of evidence pointing to any part of the body as the seat of an abscess or inflammation. I rather think this was due, in part, to the fact that we had not in mind any list of the "likely places" where experience has shown that obscure suppurations are prone to occur. Among these we should have remembered a deep axillary abscess.

Nevertheless, in view of the symptoms with which the disease began 
-the cough, sputa, herpes, and vomiting-I do not believe that the inflammation was localized from the start in the axillary region. In other words, when we were looking most fruitlessly for a local source of infection, there was no such local source. That was a later chapter. Perhaps in the earlier stages of the disease a blood culture would have revealed the actual nature of the trouble.

Outcome.-October I4th a swollen, tender mass was found in the left axilla. On the surface of the indurated tissues a few small glands could be felt; underneath, a deep fluctuation (?) was detected. Incision released several ounces of very foul pus and revealed a cavity extending far back under the scapula. The temperature fell after the establishment of the drainage, and in ten days the patient was well.

Diagnosis.-Deep axillary abscess.

TABLE XII.-CHILls. Signs and Symptoms.

\begin{tabular}{|c|c|c|c|c|}
\hline Causes. & Fever. & Blood. & Local signs. & Relief. \\
\hline "Nervousness" ..... & $\circ$ & Negative. & $\circ$ & $\begin{array}{l}\text { Reassurance. } \\
\text { Discipline. }\end{array}$ \\
\hline Pyogenic sepsis . . . . & Remittent. & Leukocytosis. & $\begin{array}{l}\text { At source of infec- } \\
\text { tion, or in heart. }\end{array}$ & $\begin{array}{l}\text { Absorption or } \\
\text { drainage. }\end{array}$ \\
\hline Phthisis . . . . . . & Remittent. & $\begin{array}{l}\text { Usually } \\
\text { leukocytosis. }\end{array}$ & Lungs. Sputa. & Hygienic measures. \\
\hline Pneumonia $\ldots \ldots$ & Continued. & $\begin{array}{l}\text { Usually } \\
\text { leukocytosis. }\end{array}$ & Lungs. Sputa. & $\begin{array}{l}\text { By progress of } \\
\text { disease. }\end{array}$ \\
\hline Gall-stones ..... & Remittent. & Not characteristic. & $\begin{array}{c}\text { Colic. } \\
\text { Jaundice (?). } \\
\text { Enlarged gall- } \\
\text { bladder (?). }\end{array}$ & $\begin{array}{l}\text { Morphin. Opera- } \\
\text { tion. Passage of } \\
\text { stone. }\end{array}$ \\
\hline 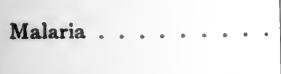 & Intermittent. & $\begin{array}{l}\text { Parasites. } \\
\text { Leukopenia. }\end{array}$ & Enlarged spleen. & Quinin. \\
\hline Typhoid fever (onset) ... & Continued. & $\begin{array}{l}\text { Leukopenia. } \\
\text { Widal test. } \\
\text { Bacilli by culture. }\end{array}$ & $\begin{array}{c}\text { Rose spots. } \\
\text { Enlarged spleen. }\end{array}$ & $\begin{array}{l}\text { By progress of } \\
\text { disease. }\end{array}$ \\
\hline
\end{tabular}




\section{CHAPTER XV}

\section{COMA}

\section{EXAmination of COMATOSE OR CONVULSIVE PATIENTS}

\section{CERTAIN HOARY ERRORS TO BE AVOIDED}

I. MaKe no diagnostic inferences from squints or inequalities of the pupils, and be cautious in all conclusions drawn from pupillary contractures or dilatations. In the majority of comatose cases the state of the pupils gives us no valuable information. Lack of response to light is proportional to the depth of the coma, and in hysteric states the responses are usually normal.

2. Conjugate deviation of the head and eyes has at present no diagnostic value. I have seen it in sunstroke and in uremia when the autopsy showed no local lesions whatever.

3. Stertorous breathing (often mere snoring) means simply deep coma from any cause. It is not characteristic of apoplexy or of any other disease.

4. Albumin or sugar in urine with or without casts have usually no significance. They are far more often seen in non-uremic than in uremic cases, for they may occur in deep coma from any of its numerous causes. In uremic cases we have the history, the condition of the heart and fundus oculi, and usually the evidences of dropsy to guide us.

5. Hemorrhage from the ear often accompanies a coma due to fracture of the base, but it is by no means pathognomonic of this condition, as injuries to the tympanum or external auditory meatus may also cause bleeding.

6. Hemiplegia, aphasia, and Jacksonian epilepsy may occur in coma due to uremia or other non-localized brain irritation. They are not proof of focal disease.

\section{CAUSES OF COMA AND CONVULSIONS}

These two manifestations of cerebrospinal disturbance cannot well be studied separately, since practically all causes of coma are also causes of convulsions and vice versâ. 


\section{Causes of Coma}

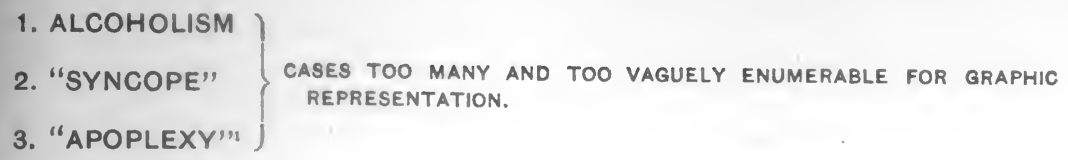

5. UREMIA

6. MENINGITIS

7. OPIUM-POISONING (ACUTE) $\}$ I

8. DEMENTIA PARALYTICA $\}$ I

9. BRAIN TUMOR I

10. SUNSTROKE

${ }^{1}$ Although we have treated at the Massachusetts Hospital only 62 cases for coma due to apoplexy during the six years covered by this report, the statistics of mortality from apoplexy convince me that this disease must be among the commonest causes for coma. The patients are treated at home. 

The following exceptions may be'noted:

(a) Opium and sunstroke cause coma, but very rarely convulsions.

(b) Strychnin and tetanus cause convulsions, but rarely coma.

Otherwise the whole list of diseases shown in the table below are causes of both symptoms.

\section{CAUSES OF COMA AND CONVULSIONS}

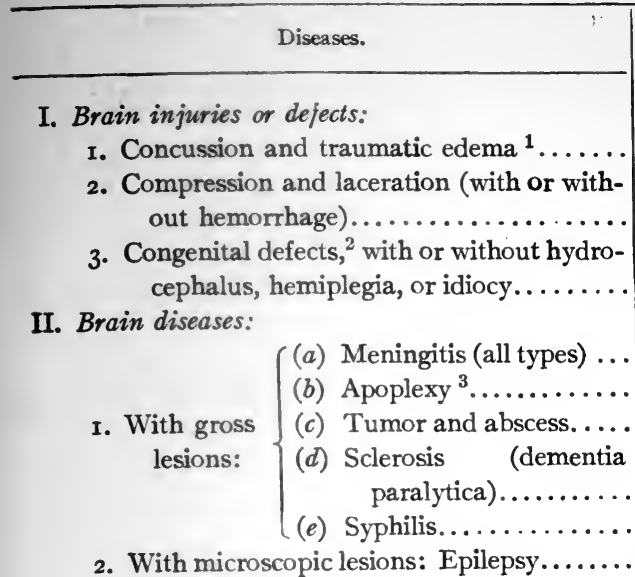

III. Infectious diseases (e. g., tetanus, typhoid, typhus, pyogenic sepsis, pneumonia, trypanosomiasis, malaria, etc.):

IV. Poisons:

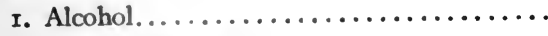

2. Illuminating gas $\ldots \ldots \ldots \ldots \ldots \ldots \ldots \ldots$

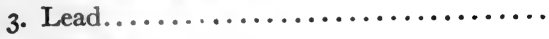

4. Opium

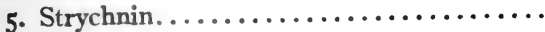

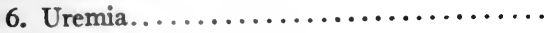

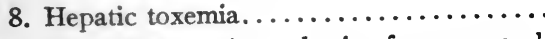

9. Diabetic acidosis, cachexia of cancer, and

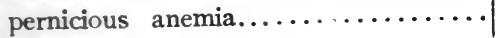

V. Syncope and cardiac weakness:

I. "Simple fainting"...

2. Stokes-Adams' disease..............

3. Valvular or myocardial disease.

4. Pleural irritation (as during irrigation)

VI. Sunstroke.

VII. Digestive and infectious disorders of infancy...

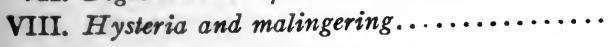

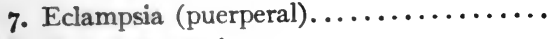

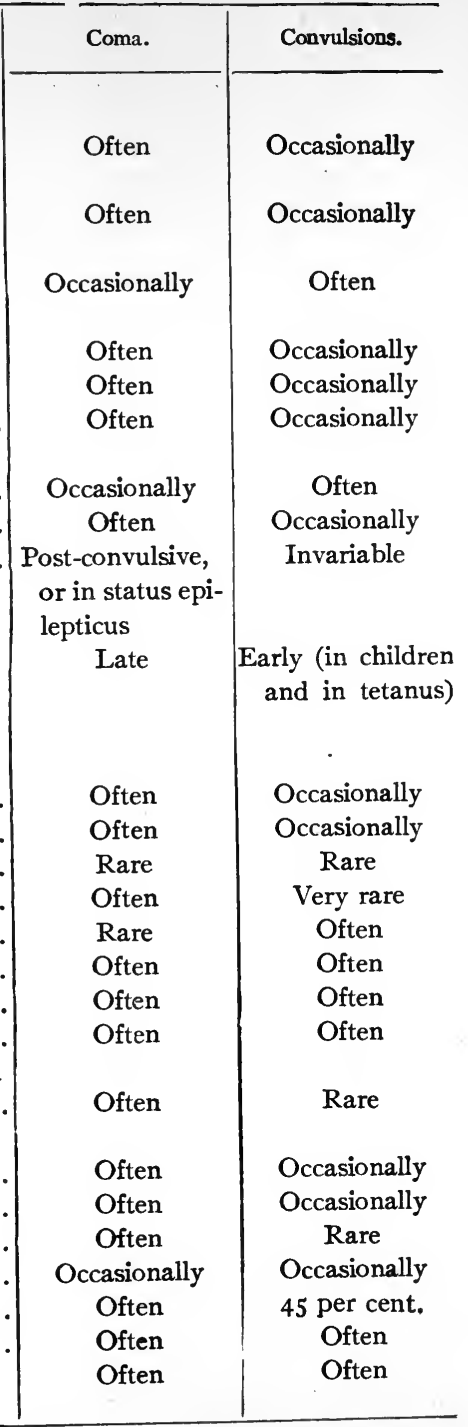

2 Including birth palsies and accidents.

1 Also called "serous meningitis."

Including cerebral hemorrhage, thrombosis, embolism, and softening. 
In the vast majority of adults the cause of any coma or convulsion will be found to be:

(a) A brain disease, injury, or defect.

(b) An infection.

(c) A poison.

(d) A form of cardiac insufficiency.

The details included in these four headings, together with three other and less common types, are shown in the table on page 497 .

\section{ETIOLOGICAL STUDY OF FOUR HUNDRED DEATHS IN COMA}

Skull fracture................................ I44

Cerebral hemorrhage........................... 95

Miscellaneous:

Meningitis.............................. 26

Lobar pneumonia. ........................ 2I

Uremia............................. I9

Cardiovascular disease....................... I4

Cerebral circulatory conditions.................. I4

Delirium tremens........................ I2

Atrophic cirrhosis; fatty liver................... II

Diabetic coma........................... 7

Brain abscess............................ 6

Traumatic intracranial hemorrhage.............. 4

Fractured ribs and sequelæ..................... 4

Fractured vertebræ....................... 3

Senility; bronchopneumonia..................... 3

Suppurative mastoiditis...................... 2

Acute miliary tuberculosis...................... 2

Puerperal sepsis........................... I

Lung abscess............................. I

Tuberculous peritonitis ..................... I

Carcinoma of the prostate................... I

Addison's disease ......................... I

Bronchiectasis.................... I

Otitis media; edema of the brain.................. I

Eclampsia ............................. I

Erysipelas ..................... I

Epilepsy............................ I

Poisoning. ............... I

Pulmonary tuberculosis...................... I

Fractured mandible, phlegmonous inflammation of the face... I I6I Total.............................. 400

The valuable paper by Drs. Bissell and LeCount, published in the Journal of the American Medical Association, Feb. I7, I9I7, contains the tables shown on pages 498 and 499 listing the causes of death in 400 obscure cases of obscure coma. The writers exciuded many comas due to common poisonings, such as the corrosive illuminating gas or morphin. 

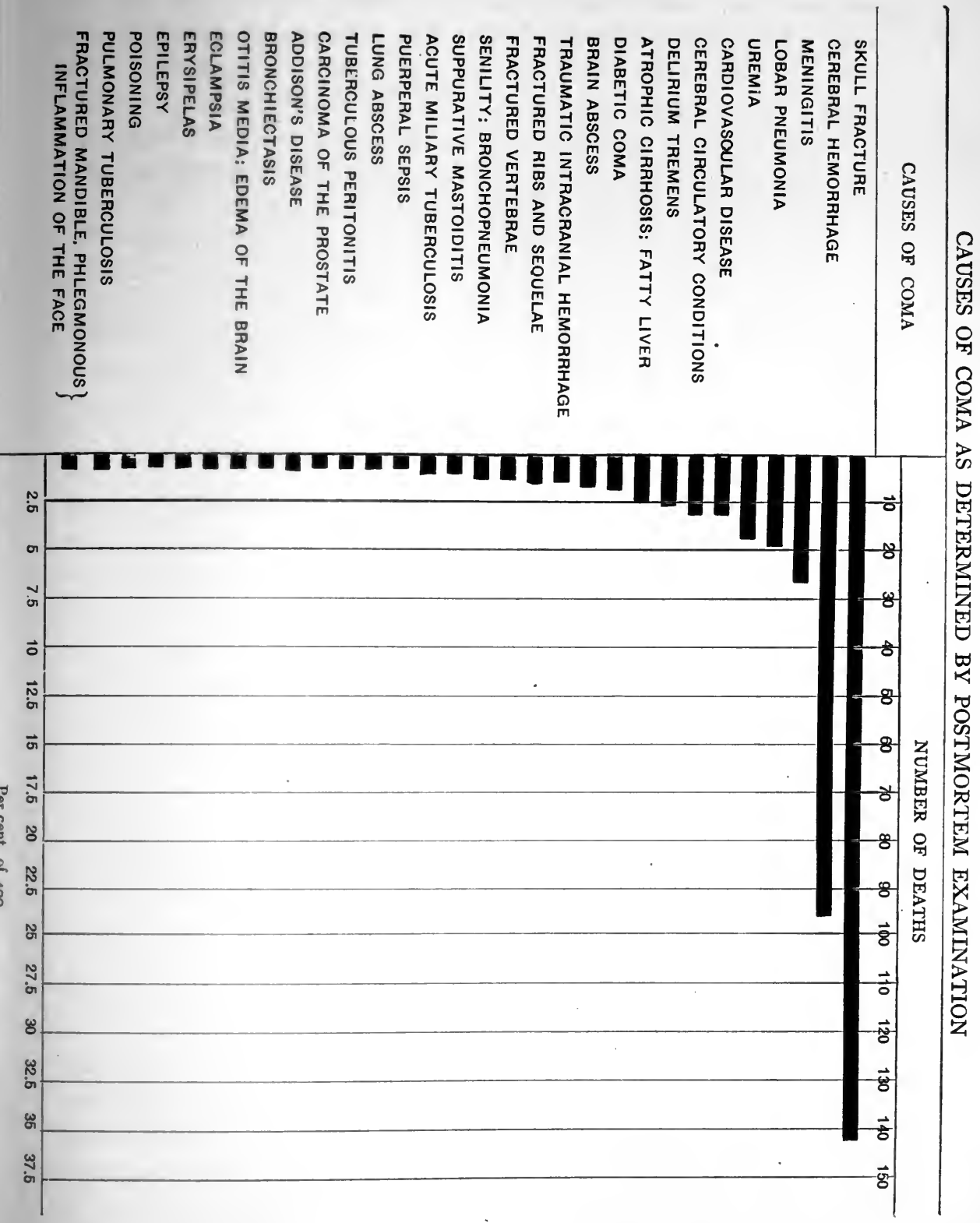
They point out that in less than 5 per cent. of these 400 cases was death due to uremia. Also that in 60 per cent. of these 400 deaths in coma, skull fracture or cerebral hemorrhage was the cause. Sixty-nine per cent. of these were correctly diagnosed during life. They find that the accuracy of the clinical diagnosis is little affected by the length of time under observation. This is in accordance with my own observations and reverses the opinion expressed in a former paper in the Journal of the American Medical Association under date of March 27, I9I5, by the same writers.

\section{VALUABLE CLUES}

I. The History.-It is always of the greatest importance to question carefully any available relatives or friends; indeed, it is usually more valuable than the physical examination. Most of the mistaken diagnoses in comatose or convulsive states are due, in my experience, to the lack of a good history.

(a) In comatose patients with head injuries it is essential, though often impossible. to ascertain whether the injury caused the coma or the coma the injury. A man falls from a scaffolding and strikes his head. Did he fall because he was already unconscious-perhaps from cerebral hemorrhage? His comrades may be able to tell us. Another useful datum in "head cases" is the order of symptoms, and their relation to the time of the injury. In concussion and traumatic edema the coma is immediate and any focal symptoms (paralysis, aphasia, etc.), come later. In traumatic cerebral hemorrhage there is often an interval of hours or days between the injury ( $e . g$., a boxer's blow) and the focal paralysis which next makes its appearance. Coma comes later still.

(b) A clear history of syphilis is obviously an important clue.

(c) The mental and motor changes of dementia paralytica should always be carefully inquired into when an "epilepsy" or a "fainting spell" appears for the first time after the fortieth year. Fainting and epilepsy almost never begin after forty.

(d) Cases of poisoning by alcohol, opium, lead, or gas are usually seen under conditions which make the history (and, therefore, the diagnosis) clear. But in police stations, where the Saturday-night "drunks" are gathered in, the question, "Drunk or dying?" not infrequently arises. Cases of alcoholic pneumonia-more or less comatose and delirious-are treated and die as "common drunks" because the temperature and the lung signs are not investigated. Cerebral hemorrhage may occur during a drinking bout, and the obvious odor of alcohol 
may then prevent our making any distinction between the drunk and the dying.

(e) Uremia, without any previous history of the ordinary symptoms of nephritis, is, I believe, a very rare occurrence, when the physical examination leads to the diagnosis of uremia and the history does not support such a diagnosis. This opinion is strongly supported by the studies of Bissell and LeCount, referred to on page 498. Out of 46 diagnoses of uremia, in cases coming to autopsy, only 8 were substantiated postmortem, and 5 of these 8 correct diagnoses were apparently "chance shots." The true diagnoses in the other cases wrongly diagnosed as uremia were as follows: Cerebral hemorrhage, 16; skull fracture, 4 ; cirrhosis of the liver, 4 ; meningitis, 3 ; pneumonia, 2 ; mastoiditis, 2 ; miliary tuberculosis, 2 ; delirium tremens, I ; cancer of the breast, I; puerperal sepsis, I ; cerebral thrombosis, I; Addison's disease, I.

Thirty per cent. of the cases dying of cerebral hemorrhage were mistakenly diagnosed as uremia. This was by far the commonest mistake of diagnosis in the cases which actually turned out to be cerebral hemorrhage.

\section{INFECTION IN UREMIA AND OTHER CHRONIC DISEASES}

Further study of the 8 cases in which the writers found the diagnosis of uremia substantiated at autopsy shows that 5 of the 8 were apparently thrown into the uremic state by the occurrence of an acute infection on top of a previously existing non-uremic nephritis.

This is an example of a very important pathologic law whereby the balance of forces which keeps us alive in the course of a chronic disease may be upset by an acute infection. Patients with arteriosclerosis whose hearts and kidneys are diseased but yet are quite sufficient for the needs of ordinary life and probably good for many more years of tolerable function, are often brought to the very verge of death by the occurrence of an acute tonsillitis, an acute gall-bladder infection, an acute epididymitis, a dental sepsis, or some other infection. This is the foundation for that peculiar relationship between gall-bladder disease and cardiac failure which Dr. R. H. Babcock of Chicago was the first to point out and which has been abundantly substantiated by the observations of later observers.

One sees the same thing in diabetes wherein the occurrence of an acute infection may upset the otherwise satisfactory metabolism and throw the patient into coma. 
- Probably the sudden demise in cases of otherwise satisfactorily compensated liver cirrhosis is often due similarly to an acute streptococcus infection.

What we see in all these cases is something like this: Despite severe and incurable disease of the heart, kidney, liver or metabolic system, nature has brought about a state of good compensation, renal, hepatic, cardiac, or metabolic. This, if undisturbed, may persist and allow the patient a comfortable and happy existence for many years. But the margin of reserve is slight. It takes but little to upset the balance. A broken bone and the confinement and disability resulting may lead to death in an elderly patient, though it would be easily and promptly recovered from in a younger person, free from arteriosclerosis. A sudden change of diet and environment, a mental shock, an overwhelming grief, or a streptococcus infection-any of these may upset the delicate balance and reduce the margin of reserve to zero.

2. The Physical Examination.-(a) The temperature is most apt to throw light upon the case if a normal reading is found, for thereby we can usually exclude the acute infections as causes of coma or convulsions. Very high temperatures $\left(107^{\circ}, \mathrm{II} 0^{\circ}, \mathrm{II} 5^{\circ} \mathrm{F}\right.$.) are strongly suggestive of sunstroke if the weather gives any countenance to the idea.

(b) A slow pulse occurs especially with tumors, injuries, and infections of the brain; less often in opium-poisoning.

(c) Evidence of cardiac hypertrophy and vascular hypertension are of importance as suggesting that the brain or the kidney is the source of the trouble.

(d) The presence of a lead line and of basophilic stippling in the red corpuscles is occasionally of the greatest importance, and should always be sought for in doubtful cases.

(e) Evidences of hemiplegia (unilateral increase of knee-jerk, Babinski's reaction, increased or diminished muscular tonicity on one side of the body, unilateral analgesia) point toward the brain, but not necessarily toward any gross lesion therein, since hemiplegia may occur without any such lesion in uremia and in epidemic meningitis.

$(f)$ Lesions suggesting syphilis are sometimes discoverable in the bones, glands, skin, or nasopharynx. The presence of such lesions gives us ground for suspecting that similar disease of the brain may be responsible for the coma or convulsions which we are studying.

(g) Spinal puncture may give us information of life-saving value, as, for example, in epidemic meningitis. More often it may help us to identify a syphilitic or metasyphilitic lesion.

3. The Recognition of Hysteric States.-There is only one way 
of being comatose, and, save for the peculiarities of individuals, there are no distinguishing marks or qualities in any of the varieties of coma above referred to. Their causes are distinguished by the accompanying physical signs or by the history, not by the characteristics of the coma itself.

Hysteric states are rarely true coma, and the distinction, which may be of considerable importance, rests mainly upon the following points:

(a) By appropriate stimulus the patient can be roused. This stimulus may be a pail of water or a well-chosen remark. The patient may, however, be quite insensible to pain, and apparently so to noise or light.

(b) The motions or attitudes during the apparently unconscious period are usually semivoluntary or purposive. Grasping movements and efforts at resistance, as when, for example, the nose and mouth are covered, are especially characteristic. The clonic spasms which so often occur in coma are not seen in hysteria. Hypertonicity and opisthotonos are frequently seen.

(c) Tremor or flickering of the eyelids and rolling up of the eyeballs are very common.

(d) In falling, the patients almost never hurt themselves, and during the convulsions there is rarely any biting of the tongue or relaxation of the sphincters. Often there is confused talk or screaming.

I will now exemplify a few of the causes of coma; many others will be found in the chapter on Convulsions, because the spasm was more striking than the coma in these cases.

\section{Case 259}

A Russian housewife of forty-eight, whose father died of a "cold in his foot," 1 had typhoid fever in childhood, but has otherwise been well all her life. She passed the menopause three years ago.

For three or four months she has complained of "rheumatic" pains in her limbs, with headache, constipation, and loss of appetite. For two or three days she has had distress about the precordia. To-day at I P. M. this distress increased until she was forced to lie down, following which she became comatose. After two or three minutes she regained consciousness and screamed violently for several minutes on account of precordial pain, which apparently did not radiate at all.

These attacks recurred every ten to fifteen minutes until seven in the evening. She vomited six or seven times during the afternoon, and when seen at II P. M., complained of palpitation and a sense of weakness about her heart.

${ }^{i}$ Gangrene presumably. 
A physician who saw her in one of her "fainting attacks" previous to her entering the hospital said that she was practically pulseless during the period of unconsciousness.

Subsequently it was learned that she had been subject to fainting attacks for over thirty years, and had had a goiter for the same length of time.

Physical examination revealed the tumor above referred to, which was about the size of a hen's egg, situated in the median line, smooth, rounded, not tender, moving with the larynx on swallowing.

Upon inspection the heart's impulse was very diffuse, apparently extending a half inch outside the nipple in the fifth space. There were no murmurs, and physical examination was otherwise negative; blood and urine normal.

The patient presented chiefly the picture of exhaustion. She complained of various pains in her arms and legs. The pulse during most of her stay in the hospital was very irregular, but there was no repetition of the attacks of syncope.

On February 7 th, when the pulse was IIo, there was noticed a pulsation in the jugular veins, filling from below at exactly twice the rate of the arterial pulse. On the eighth there were three beats in the neck for every one at the wrist.

Discussion.-Fainting attacks often repeated usually turn out to be due to some important underlying disease. One should always look with great suspicion upon any attack so designated if it is known to have occurred frequently. Hysteria is perhaps the disease which turns out most frequently to be the cause of attacks of this nature, but I have known also cases of nephritis, of cerebral tumor, and of epilepsy which have been called "fainting attacks" for months or years before the real cause was recognized.

In the present case, since physical examination is so nearly negative, the most important diagnostic feature is the report by the physician who watched her in one of these fainting attacks and noticed that she was practically pulseless. In the absence of any obvious valvular disease, this observation should lead us to suspect disease of the myocardium and to study very carefully the condition of the neck veins during attack.

Outcome.-February $7_{\text {th }}$ it was noticed that the jugular veins filled from below and pulsated at exactly twice the rate of the arterial pulse. After two weeks' rest in bed and the administration of iodid of potash the patient seemed much benefited. She was seen two years later and stated that she had had no recurrence of the "fainting fits," although from once a month to once in three months she had had severe attacks 
of precordial pain relieved by rest and applications of heat. She now passes water two or three times each night, and has had various attacks of infectious arthritis and one of erysipelas. The goiter remains unchanged.

Diagnosis.-Stokes-Adams' disease.

\section{Case 260}

An unmarried Russian seamstress of twenty, whose mother died of diabetes, lost her father and one sister of tuberculosis. Three brothers and one sister are well.

The patient herself became much run down five years ago and was sent into the country, apparently for suspected tuberculosis, though her cough was not very persistent, and her sputa was never examined. She was first seen April I4, I908.

Four days ago she became excited and fell unconscious. There were no convulsion and no paralysis, but she was somewhat rigid during the attack, and she has since then been in bed and has vomited everything that she has taken by mouth. Constant vertigo and palpitation, with epigastric pain, have been her complaints.

On examination, the heart's apex was found one inch outside the midclavicular line in the fifth space. There was a presystolic thrill and murmur at the apex. The sounds were very irregular, and at times amounted to delirium cordis. The pulmonic second sound was sharply accentuated. At times a systolic murmur was heard, following the very sharp first sound at the apex. As is shown in the accompanying chart (Fig. I24) many heart-beats failed to reach the wrist. ${ }^{1}$ There were fine crackling râles at the base of both lungs, especially on the left side.

The abdomen was tender and rigid in the right hypochondrium, and dulness extended two inches below the costal margin; no edge was felt. Blood and urine normal.

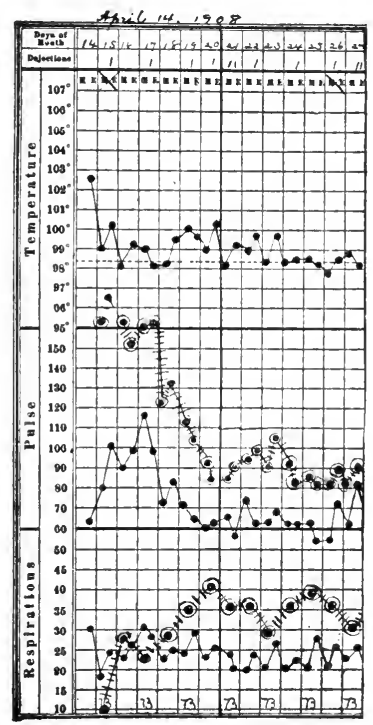

Fig. 124.-Chart of case 260 .

Despite the extreme rapidity and irregularity of the heart, there was no cyanosis or orthopnea.

${ }^{1}$ In this chart the line immediately above that representing the pulse stands for the number of heart-beats counted at the apex. 
Discussion.-The essential features here are the tuberculous family history, the "fainting fit," and the present condition of the heart.

The latter shows all the evidences of mitral stenosis rather poorly compensated. Probably she has to thank this heart trouble for her freedom from tuberculosis.

To what should we attribute the attack of coma? In many respects it resembles an hysteric attack. Patients who faint very seldom do so as the result of valvular heart disease, although there is no symptom except "pain about the heart," which so often alarms a patient about his cardiac condition. Of the numerous patients who have consulted me believing that they had heart trouble because of the occurrence of "fainting fits," I have never found heart disease in a single case. The sufferings of this patient are, therefore, all the more interesting. Careful inquiry into her previous history showed that she had had similar attacks in childhood, and had always had a decided tendency toward hysteria. In view of this it is probably true to say that her heart disease was only a contributing cause of her "fainting fits."

It must not be forgotten, however, that in cases of mitral stenosis a thrombus often forms in one of the left auricular appendages, whence a bit is detached and carried to the brain, producing embolic hemiplegia. Possibly a minute embolus or a group of such emboli might produce a "fainting fit" without paralysis, but this conjecture is so far wholly unsupported.

Outcome.-The patient was given $\frac{1}{6}$ grain of morphin subcutaneously and fed on milk and lime-water in small amounts. The bowels were moved by small doses of calomel, followed by a suds enema.

On the fourteenth of May the heart-beats all reached the wrist. On the twenty-third she was able to walk about, and had no complaints. Diagnosis.-Mitral disease (and hysteria?).

\section{Case 261}

A club waiter forty years old has been at work as usual during the last five days, as is learned from the manager of the club where he was employed. He was first seen August 7, 1907. He has done no heavy work, and nothing is known of his previous history. It has been noticed that his color is poor, and he has expressed a fear that he might have a breakdown. Night before last he had an attack of dyspnea, from which he recovered, however, without medical attendance. The next day he did his work as usual, but seemed irritable and rude, so that he was warned by the manager. This morning at 5 o'clock he was found lying on the grass outside the club. He said that he went out there to 
get the air. He seemed very short of breath, but walked to the porch and sat down. On his way thence to the hospital he became unconscious, and at the time of his entrance was almost moribund.

His nutrition was excellent, his color dusky. The heart's apex was in the anterior axillary line, in the sixth space. The sounds were very irregular in force and rhythm; no murmurs were heard. Tracheal râles were so loud as to make examination of the heart and lungs very difficult. Respiration was very rapid and irregular. The liver seemed to be slightly enlarged.

Outcome.-The patient died within a few hours. Autopsy showed chronic endocarditis of the mitral valve, with mitral stenosis; general arteriosclerosis; hypertrophy and dilatation of the heart; hydrothorax; hydropericardium; cicatrices in the liver.

Discussion.-Why was this patient's death so sudden? The vast majority of cardiac cases die in their beds after prolonged periods of dropsy and dyspnea. Now and then a case either of the valvular type (chronic endocarditis), of the arteriosclerotic, or of the syphilitic types dies suddenly.

In a number of such cases no coronary disease, no pulmonary embolism, and no other sufficient cause for sudden death can be found postmortem. I have seen so many fruitless examinations of this sort that I no longer count on the pathologist to explain by mechanical causes the sudden death in cardiac cases.

Some ultramechanical, perhaps some chemical, explanation must be sought.

Diagnosis.-Chronic valvular disease. Sudden heart failure from unknown cause.

\section{Case 262}

An Irish housemaid of twenty-five was seen November 30, I909. The previous morning she had seemed perfectly well and in good spirits. At 9 P. M., November 29 th, she suddenly became unconscious and fell to the floor, though her brother caught her, so that her head did not strike. After this she vomited several times without regaining consciousness.

This morning she roused enough to moan and complain of severe headache, but soon lapsed into coma again.

Examination.-Temperature, Iог. $6^{\circ} \mathrm{F}$; pulse, IIо; respiration, 22.

Blood-pressure, $245 \mathrm{~mm}$. Hg. Leukocytes, 28,000. Urine clear, acid, ro20; very large trace of albumin; no sugar. Sediment negative. Left pupil slightly larger than right. Both react sluggishly to light. 
Left arm and leg moved but little on sensory stimulation. Babinski's reaction on the left. Knee-jerks and Achilles jerks absent.

Physical examination was otherwise negative.

Lumbar puncture was done, and blood-tinged serous fluid spurted nearly a foot through the needle. The sediment of this fluid showed no excess of leukocytes and no organisms in cover-slip or culture. Venesection gave no relief.

Fundus examination showed double optic neuritis.

Discussion.-Coma, leukocytosis, fever, and hypertension with pupillary inequality and apparently a left hemiplegia are the essentials of this case.

The negative character of the spinal fluid is sufficient to exclude meningitis.

Diagnoses of uremia made under these conditions always turn out wrong. The reasons for this I have discussed more fully on p. $5^{26}$.

The content of the spinal fluid makes syphilis unlikely, especially in a girl with no previous history or lesion suggesting that disease.

Apoplexy, using this old term to include cerebral hemorrhage, thrombosis, or embolism, with or without softening, is practically unknown in a girl of this age so long as the heart is negative.

The double optic neuritis with the very high blood-pressure and the normal heart points strongly toward cerebral tumor.

Outcome.-The patient died suddenly of respiratory failure December $2 \mathrm{~d}$. The temperature, pulse, respiration, and leukocyte count remained practically as at entrance. After lumbar puncture she moved all parts of her body freely, complained of headache, and answered a few questions clearly and with comprehension.

It was learned later that she had had several attacks of vomiting during the past summer, and that her eye-sight had not been good.

Diagnosis.-Cerebral tumor (?). 


\begin{tabular}{|c|c|c|c|c|c|c|c|c|c|c|}
\hline 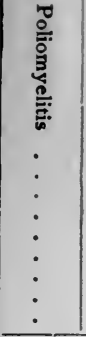 & $\begin{array}{l}\forall \\
\stackrel{0}{0} \\
\frac{6}{0} \\
\vdots \\
\vdots \\
\vdots \\
\vdots \\
\vdots \\
\vdots\end{array}$ & 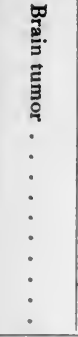 & 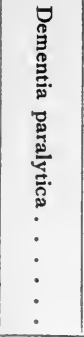 & 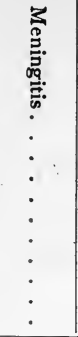 & $\begin{array}{l}C \\
\mathbb{1} \\
\vdots \\
\vdots \\
\vdots \\
\vdots \\
\vdots \\
\vdots \\
\vdots\end{array}$ & 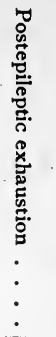 & 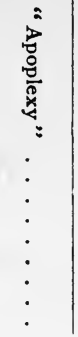 & 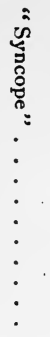 & $\begin{array}{l}\frac{2}{0} \\
\vdots \\
0 \\
0.5 \\
3 \\
\vdots \\
\vdots \\
\vdots \\
\vdots \\
\vdots\end{array}$ & 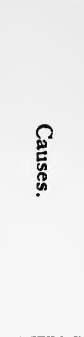 \\
\hline 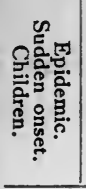 & 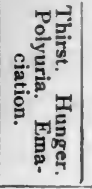 & 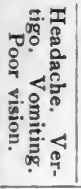 & 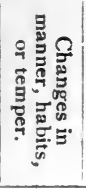 & 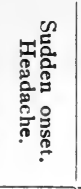 & 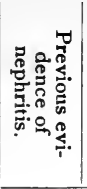 & 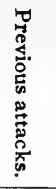 & 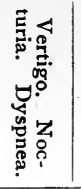 & 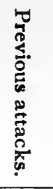 & 苞 & 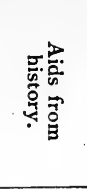 \\
\hline+ & 0 & 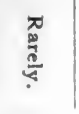 & ० & + & 足 & $\circ$ & 邑 & 0 & 。 & 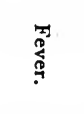 \\
\hline 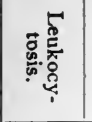 & 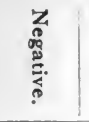 & 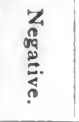 & 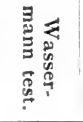 & 它总 & 它 & 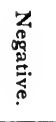 & 它 & 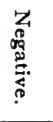 & 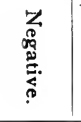 & $\begin{array}{l}\sigma_{0}^{\prime} \\
\vdots \\
\vdots\end{array}$ \\
\hline$:$ & $\bullet$ & 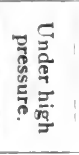 & 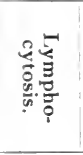 & 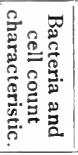 & 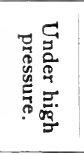 & 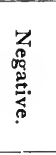 & . & 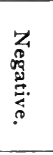 & 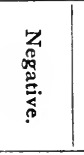 & 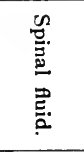 \\
\hline 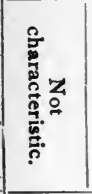 & 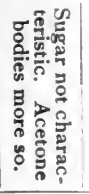 & 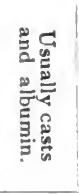 & 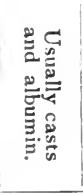 & 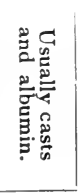 & 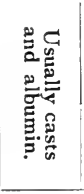 & 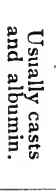 & 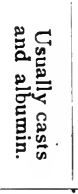 & 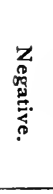 & 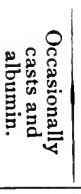 & S. \\
\hline 。 & $\circ$ & 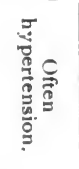 & ० & ० & 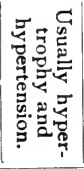 & $\circ$ & 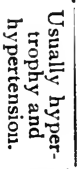 & $\circ$ & ० & 产魚 \\
\hline$\circ$ & 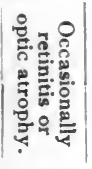 & 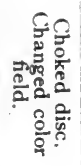 & 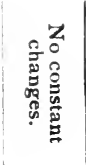 & 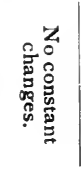 & 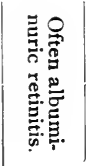 & $\circ$ & 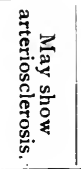 & $\circ$ & $\circ$ & 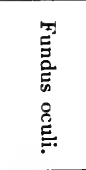 \\
\hline 율 & 。 & 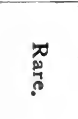 & $\circ$ & + & 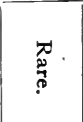 & $\circ$ & $\circ$ & $\circ$ & $\circ$ & 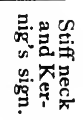 \\
\hline 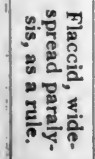 & 0 & 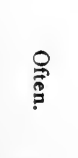 & ० & 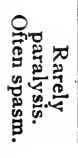 & 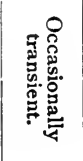 & $\circ$ & 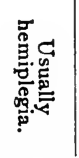 & $\circ$ & $\circ$ & 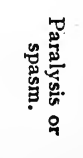 \\
\hline
\end{tabular}




\section{CHAPTER XVI}

\section{CONVULSIONS}

\section{Case 263}

AN Irish longshoreman of fifty entered the hospital November I7, 1907. He has been a steady, hard drinker for many years, but denies venereal disease, and has been otherwise well. Four years ago, he says, he had a fit, which lasted twenty minutes; nothing of the sort has occurred since. All last week he drank hard. He spent Saturday night at the Salvation Army rooms. On Sunday,

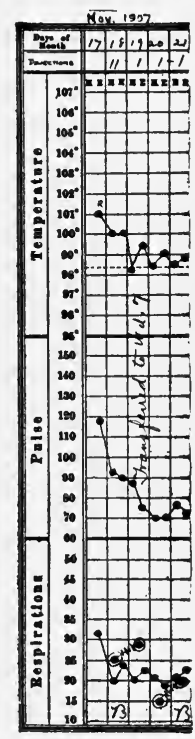

Fig. 125.-Chart of case 263 . November $\mathrm{I} 7 \mathrm{th}$, while attending a Salvation Army meeting, he became unconscious.

When examined at 5:25 P. M., November 17 th, he was still unconscious and in convulsions, at times confined to the right side, later general, and succeeding each other without intermission.

There was a deep, bleeding cut on the chin, and a shifting strabismus of the eyes. Marked hyperresonance of the lungs made it impossible accurately to estimate the size of the heart. Its sounds were also obscured by snoring râles, but the arteries showed no evidence of degeneration, and physical examination was generally negative. For temperature, see the accompanying chart (Fig. I25). The blood showed nothing abnormal.

The urine was pale, IO2I in specific gravity, with the slightest possible trace of albumin. One finely granular cast was found in the sediment.

Blood-pressure was $125 \mathrm{~mm}$. Hg.

Discussion.- In the absence of any obvious localizing brain symptoms or signs, epilepsy is naturally our first thought, especially as we know that an attack of epilepsy is apt to be precipitated by acute alcoholism. But if we are to take the history on its face value and understand that there has been but one attack previously, it seems unlikely that a man of this age would become epileptic so recently. 


\title{
Causes of Convulsions
}

\author{
1. ECLAMPSIA (PUERPERAL)
}

2. INFANTILE SPASM

3. ALCOHOLISM

\section{EPILEPSY}

5. UREMIA

6. HYSTERIA

7. MENINGITIS 

In the urinary examination quoted nothing is said about sugar, but even if it were known to be present, its quantity cannot be large in view of the specific gravity of the urine, and it may be further stated that, although diabetes may produce convulsions, it practically never does so "out of a clear sky" - that is, in patients who have not previously known themselves to be ill or suffered any of the cardinal symptoms of diabetes.

Meningitis might begin in this way, and the chart is consistent therewith, but a knowledge of the results of lumbar puncture would be necessary before any such diagnosis could be made, since we have none of the ordinary manifestations of meningitis (cervical rigidity, Kernig's sign, squints and pupillary changes, headache, vomiting, and delirium).

Of lead-poisoning, of dementia paralytica, brain tumor, or abscess -all of which might cause similar convulsions-we have no evidence either in the history or in the physical examination. Hysteria and trauma need not be considered.

With the exclusion of these alternatives the most reasonable hypothesis remaining is that the alcohol is the source of the trouble. From conversations with physicians who attend the Saturday-night drunks in city prisons my own more limited experience of "rum fits" is fortified in making the following division into three groups:

(a) An alcoholic debauch may make a person hysteric and so precipitate hysteric convulsions.

(b) Alcohol may bring on one of the regular epileptic attacks in a patient already suffering from that disease.

(c) A true "rum fit" may be produced by alcohol in a person not epileptic or hysteric. These fits are presumably due to the cerebral changes ("wet brain," vascular crises) produced by alcohol.

The present case seems to belong to the third group.

Outcome.-The patient was bled about 20 ounces, and an equal amount of saline solution was injected. About ro P. M. he regained consciousness and remained thereafter practically normal. Later he admitted that his present trouble began on the second night of his last spree. The bystanders say that there was no cry at the time he fell and the sphincters were not relaxed. He was discharged well on the twenty-second.

Diagnosis.-Alcoholism.

\section{Case 264}

A housemaid of twenty, of good family history and past history, entered the hospital February 12, 1908. She has been irregular in her 
menstruation for the past six years, and has been subject, during that period, to frequent severe left-sided headaches and to attacks of unconsciousness. These attacks are apt to occur on the first day of menstruation, but they may come in the middle of the intermenstrual period. She has considerable pain in the lower abdomen during the first two days of menstruation, otherwise the function is not abnormal. During her attacks of unconsciousness she falls, but sometimes can get up and steady herself by taking hold of something. She is then apt to become violent, going out of her head, frothing at the mouth, often gripping her throat with her hands. sometimes bleeding from the mouth and nose. She has never injured herself nor passed urine during an attack. The attacks last from a few minutes to a few hours.

Her last menstruation ceased five days ago. Two days ago, while sweeping, she felt dizzy, fell down, and says she remembers no more until she was seen at the hospital. From her friends it was learned that after her fall she was put to bed, where she threw herself about and talked incoherently all day. At 7 in the evening she apparently came to, and was taken home from her place of work at II P. M. She again became unconscious and lay still in bed with limbs rigid.

Yesterday she awoke and said she felt well. She went to work at 8 o'clock in the morning, but an hour later again fell unconscious and rigid and remained so until this morning at 6 , when she was partially aroused by an enema of soapsuds, but became again unconscious after twenty minutes. She has taken no food for four days, according to her own statement. She has been very constipated for years. During these attacks she says her feet are apt to swell, but at other times they are never swollen.

Physical examination showed a well-nourished girl, herpes on her lips, dozing most of the time, apparently rational when aroused, but apathetic and complaining of headache and abdominal pain.

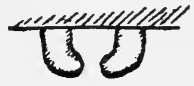

Fig. 126.-Diagrammatic representation of upper median incisors in case 264. They are set far apart and are malformed, curving toward each other at the ends.

The upper and median incisor teeth are malformed. The other teeth are in fair condition. (See Fig. I26.) The pupils are dilated and react sluggishly to light and distance. The vault of the palate is very high and narrow. She is a mouth-breather. The heart's apex is in the fourth interspace, one-half inch outside the midclavicular line. The sounds are of good quality. At the apex there is a loud systolic 
murmur, heard clearly in the axilla, faintly in the pulmonary area. This murmur, or a similar one, is heard in the tricuspid area, and there it seems to be of a higher pitch and different quality. In the tricuspid area the first sound is much louder and sharper than in the mitral area. The pulmonic second sound is slightly accentuated. There is a systolic venous pulse in the neck. The radial pulses are not in any way remarkable. The abdomen and reflexes are not abnormal. Sensation is apparently normal.

During the first night after her arrival the patient complained of headache and severe abdominal pain. Half an hour later she began to grow rigid and clutched the blankets firmly. She then became apparently unconscious. Her pulse was 76 , respiration 44 . When the lighted lamp was held in front of her she closed her eyes more tightly. When they were forcibly opened, the balls were found to be rolled up, and she turned her head as if to avoid the light. The rigidity of the arms could be overcome, though with difficulty. It seemed to be partially voluntary. A pin could be passed through a fold of the skin without causing any change of expression or any motion.

After half an hour of this rigidity the patient became again sensitive to pain and would reply to questions.

On the twenty-first she complained of a severe headache, which immediately disappeared under the ethyl chlorid spray. The attention then became concentrated on the abdominal pain. After that the headache did not return and she said the treatment cured her.

Discussion.- The symptoms in this case and the description of the attack remind us very strongly of hysteria. Two other possibilities, however, must first be considered.

Can the malformation of the incisor teeth be interpreted as a lesion of congenital syphilis and the convulsions be also due to that disease? This is very improbable, for aside from these attacks there is nothing in the patient's condition or history to suggest syphilis, a disease which, in its congenital form, almost always appears earlier than in the twentieth year. The nature of the attack, moreover, is not at all characteristic of cerebral syphilis, of which more anon. The malformed teeth are not of the Hutchinsonian type.

Can the tricuspid regurgitation, evidenced by systolic venous pulsation in the neck, the swollen feet, and the murmur in the tricuspid area, account for the attack? In answering this question it must first be noticed that the tricuspid regurgitation must be slight if, indeed, it exists at all in any pathologic sense. Many observers believe that a slight degree of tricuspid regurgitation is physiologic. As a result of 
various strains or accidents we may conceive that this physiologic reflux is more or less exaggerated, but one can hardly believe that its effects. would be so disproportionately concentrated upon the brain as to produce convulsions without bringing about any more obvious stasis in the other internal organs.

With the exclusion of these two possibilities we may conclude that the attack was due to hysteria. The present case exemplifies many of the characteristic signs by which hysteric convulsions have traditionally been differentiated from those due to the other causes discussed in this chapter. Such characteristics are:

(a) The absence of deep coma.

(b) The semivoluntary and semiconscious nature of the motions (e. g., such as to prevent her hurting herself in falling, grasping motions, talking, resistance of efforts to open the eyelids).

(c) The absence of any biting of the tongue, any relaxation of the sphincters, prespasmic cry, or aura.

These characteristics hold good in probably the large majority of hysteric cases, but it must be realized that these and all the other signs by which we have sought to differentiate hysteric from epileptic convulsions may fail us. Attacks which, on the whole, must be judged hysteric may occur in the night during sleep, may be accompanied by biting of the tongue and all the ordinary evidences of an epileptic fit. I have recently known such a case in which the cause of the spasms was found to reside in a partly subconscious knot of morbid ideas, acquired in childhood, reënforced by the abnormal conditions of a girls' boardingschool, and finally removed as a result of psycho-analysis and Freud's cathartic method. Attacks which can be thus abolished must be recognized, I suppose, as belonging to the hysteric group.

The reasonable conclusion is that, in doubtful cases, we cannot rely upon the precise nature of the movements or on individual features of attacks to differentiate hysteria and epilepsy. Only by a study of the possible causes in the patient's mental life and by the therapeutic test (i. e., the attempt to remove these causes) can the nature of the malady be determined. This is in line with Babinski's conception of hysteria.

Diagnosis.-Hysteria.

\section{Case 265}

A married woman forty-eight years old entered the hospital January I, I908. Her last menstrual period was in the preceding September, and she has apparently reached the menopause. She has had nervous prostration three times, the last time two years ago. Five days ago 
she was taken with influenza and confined to bed. She was first seen by her physician three days ago, when she suddenly collapsed from "acute heart failure" and pain in the back.

The doctor found her much exhausted, pale, very dyspneic, and partially stuporous. At frequent intervals, three times within an hour, the pain in her back became very severe; the muscles of the trunk were rigid and all the muscles of the body twitched convulsively. Nitroglycerin afforded much relief. The next day the paroxysms of pain continued and she lost the use of her limbs. For twenty-four hours she had retention of urine, and 40 ounces were finally drawn by catheter. Within the last twenty-four hours the use of her limbs has in part returned, but the pain in her back continues and is sharp when she tries to move. The attacks of pain are still accompanied at times by muscular twitching.

When first seen, her temperature was $102.2^{\circ} \mathrm{F}$. During the first day in the hospital it was $100^{\circ} \mathrm{F}$.

Visceral examination was negative, as were reflexes, the urine, and the blood. Blood-pressure, I35 to I4O. The pharynx, larynx, and trachea were markedly injected, and there was herpes on the lips.

By the fifth of January she was much better and able to be up, but was very unwilling, even on the thirteenth, to leave the hospital. On the thirtieth she was able to do so.

Discussion.-There are some indications of an acute infection here, especially the red throat and the herpes on the lips. It is a very familiar fact that in children the onset of acute infectious diseases often produces a typical epileptiform convulsion. It seems possible that the present attack may be the equivalent of such a convulsion, modified by the age of the patient, although the precise nature of the infection is unknown ("influenza").

Some facts in the case, however, suggest a different type of convulsion. The intense pain in the back, the rigidity of the trunk muscles, the temporary loss of power in the limbs, the retention of the urine, are all of them symptoms consistent with some type of organic disease of the spinal cord or its membranes. The difficulty with this idea is that closer scrutiny of the symptoms fails to find any arrangement among them corresponding to any known disease, while their outcome seems to show that no permanent lesion has occurred in the central nervous system.

By the results of physical examination it is possible to exclude organic brain disease, such as meningitis, dementia paralytica, abscess or tumor, and apoplexy. There is no evidence of poisoning by lead 
nor of any organic disease of the heart and kidney. There has apparently been no previous attack resembling the present one, and an epilepsy beginning at forty-eight is always an improbable diagnosis.

In view, therefore, of the negative result of physical examination directed to reveal the ordinary organic causes of convulsions we may conclude that this attack is of functional type, ordinarily, though somewhat loosely, designated as "hysteria." This diagnosis, however, does not end our study of the actual nature of this attack.

Two points are of especial interest in relation to this particular example of the vague type of spasms known as "hysteric" or "functional." We have, in the first place, to consider the possible influence of the psychic elements which may have been introduced quite unconsciously by her physician and friends. It will be noted that she is said to have suddenly "collapsed" as a result of "acute heart failure." Now these phrases have a very great effect upon the mind of a patient and thereby upon his symptoms. We often see what a great benefit may be produced in a patient when we persuade him that his headache is not in "the base of his brain," but merely in the nape of his neck, or that the pain in the left side of his chest is not "around the heart," but merely in the stomach or in the ribs.

A corresponding aggravation of symptoms is pretty sure to follow if, by chance, such phrases as "collapse" or "acute heart failure" are let loose in the patient's vicinity, whether they are from the patient's own lips and merely corroborated by the physician, or whether the patient overhears them in the conversation of relatives or bystanders. Particularly in their early stages, functional attacks may be greatly relieved if we call a spade a spade, rather than an agricultural instrument. To make light of symptoms which our physical examination assures us are not of serious importance may shorten by many days the patient's illness, while, on the other hand, suggestions conveyed by a grave and serious expression reflected from the doctor's face to the family, by the terminology used or permitted by the doctor, or by the nature of the remedies employed, may greatly aggravate and prolong the patient's sufferings. For example, I remember a case of postoperative pleurisy in which the patient, who was high strung and pretty well tired out previous to the operation, began to breathe very rapidly, so that the nurse in charge brought in a can of oxygen and administered it at regular intervals. The patient took for granted that this was done by the doctor's orders. But his previous hospital experience, in connection with an appendix operation, had led him to associate the arrival of the oxygen can with the most serious and even terminal stages 
of disease. He had noticed that pretty soon after the oxygen can was carried into a patient's room the patient himself was carried out dead.

As a result of putting two and two more or less unconsciously together in this way, my patient became greatly alarmed about himself, was hardly able to breathe, and totally unable to sleep. Soon after, his physician came in, was greatly surprised at the sight of the oxygen can, promptly ordered it out, and irritably asked the nurse, in the patient's hearing, what on earth she "had brought that thing in for when there was not the slightest need for it." The patient soon afterward went to sleep, and awoke next morning much improved. He afterward confessed to the writer how the sight of the oxygen can had affected him, and how profoundly its removal had relieved him.

The other point of interest in this case is the relief by nitroglycerin. In Pal's book on Vascular Crises, to which I have already referred, he shows that any type of vascular spasm, cerebral, cardiac, pulmonary, abdominal, or peripheral, may be relieved by the administration of nitroglycerin, and uses the fact of such relief as corroborative evidence of the nature of the attack. Now this patient is apparently at the menopause, a period in which disturbances of vasomotor balance are notoriously frequent, manifold, and annoying. Is it not possible that this attack was of the nature of a vascular crisis induced by the onset of an acute infection at an especially sensitive period of life? In the present state of our knowledge no definite answer can be given to this question.

Diagnosis.-Hysteria.

Case $266 x$

An unmarried woman, thirty-eight years old, was seen in consultation at 8 A. M., September 26, I909.

She had suffered all her life from periodic headaches occurring every two to four weeks, more especially at the time of menstruation. Aside from these attacks, she had never been sick, and seemed to be vigorous in every respect. On the nineteenth of May she went to bed with one of her regular headaches, so it seemed. During the day she had seemed as well as usual. About I o'clock in the morning her sister, who slept in the same room with her, was aroused by some curious sound, and found the patient unconscious and in a convulsion. When seen in consultation at 8 o'clock A. M., she was conscious, but very drowsy and heavy. Between I A. M. and 8 A. M. she had had six general tonic-clonic convulsions, five of them accompanied by complete loss of consciousness, each lasting about a minute, and followed by profound relaxation with deep relaxed breathing. 
Physical examination of the chest and abdomen was negative. The reflexes were all somewhat exaggerated, especially on the left side, and at times Babinski's reaction could be elicited on the left. The pupils were moderately dilated, the left larger than the right, and responded rather sluggishly to light stimulus. When conscious, she was aware of no pain, and although she had been more or less nauseated, there had been no vomiting. The urine drawn by catheter showed specific gravity of IOI4, O.I25 per cent. of albumin, a moderate number of hyaline casts, some with granules or cells adherent. There was no edema anywhere. The blood-pressure was $138 \mathrm{~mm}$. $\mathrm{Hg}$.

The convulsions were usually preceded by some shaking of the left hand, extending thence to the foot and leg, and then becoming general. The eye-grounds were not examined. The temperature was IoI. $3^{\circ} \mathrm{F}$; pulse, Ioo between convulsions, becoming rapid and feeble during and after them.

The blood showed I6,000 white cells, 8I per cent. of which were polynuclear.

The bowels were moved by enema, and showed nothing of importance in the intestinal evacuation.

Discussion.-When I saw this patient, a diagnosis of uremia had already been made by the attending physician. Against this I immediately rebelled in my own mind, even in advance of accurate physical examination. For this prejudice I had two reasons: First, I had recently heard one of the wisest and most experienced clinicians in the world say that he had never known a diagnosis of uremia, made when the patient was seen for the first time in coma, to turn out correct. By this he meant that the correct diagnoses of uremia are those made in chronic cases, not those made in patients who, out of a clear sky, without any previous complaints, have suddenly fallen in coma or convulsions.

My second reason was that in the study of 1500 postmortem examinations made at the Massachusetts General Hospital within the last ten years I have been unable to find a single case in which the diagnosis of "acute uremia" had been confirmed at autopsy. I found many in which this diagnosis was shown to be erroneous.

This mistake results, no doubt, because nearly all cases in which coma or convulsions suddenly supervene, show albuminuria and casts, sometimes in great abundance. This is true whatever the cause of the seizure.

Nocturnal epilepsy with status epilepticus seemed very improbable because the patient's sister had been in the habit of sleeping in the same 
room with her for some years, and had never known of any similar attack, nocturnal or diurnal.

The absence of any previous cardiovascular or renal disease, the normal condition of the heart and blood-pressure, the absence of any history or present evidence of syphilis, made apoplexy and vascular crises somewhat improbable.

Cerebral tumor and abscess have been known to begin or rather to show themselves for the first time with symptoms like those here described, and there is nothing by which we can positively exclude these lesions. We should expect, however, a higher blood-pressure. Fundus examination, which was not made in this case, might be of decided assistance, since the vast majority of cases in which a cerebral tumor produces convulsions also show optic neuritis (choked disk).

What is the significance of the leukocytosis here present? Experience has shown me that the leukocyte count is practically valueless as a source of information in cases involving coma or convulsions. Whatever the cause of these symptoms, leukocytosis is practically always present. In cerebral hemorrhage, for example, it is almost constant. In this case, therefore, as in others, I disregarded it.

Utterly in the dark as to the diagnosis, it seemed to me possible that some light might be shed upon it, or possibly some relief given to the patient's symptoms, by lumbar puncture. As will be shown by the outcome, this puncture turned out to be of critical, indeed, I think, of life-saving, importance. Its value in this case was such that I shall in future never be content unless it is done in every doubtful case involving coma and convulsions.

The fever here recorded has as little diagnostic value as the leukocytosis. It is in nowise indicative of an infectious process, but occurs with equal frequency in all types of coma and in all diseases producing convulsions.

Outcome.-A needle introduced into the spinal cord drew 37 c.c. of clear, transparent, colorless fluid. Of the cells contained within the sediment of this fluid seventy-eight per cent. were polynuclear, and both within and without the leukocytes a diplococcus was seen which corresponded with the diplococcus of epidemic meningitis.

Flexner's antimeningitic serum was injected several times. The patient made a complete recovery, although for a few hours on the sixth day she became suddenly and completely blind. The attack, however, left no untoward effects behind it.

Diagnosis.-Epidemic meningitis. 


\section{Case 267}

A plumber of sixty-two, one of whose children died of consumption, had been an intermittent hard drinker for many years, taking rather more of late-sometimes a quart of whisky a day. He entered the hospital January 29, I908. Every night for two or three years he has filled a chamber vessel with pale urine and has been seen to drink much more water than formerly. He has had no headache, no cough, no vomiting, but has belched a good deal of late. His eye-sight has been good.

At 2 o'clock on January $29^{\text {th }}$ he had a convulsion in a street car, and was brought at once to the hospital, where he immediately had a

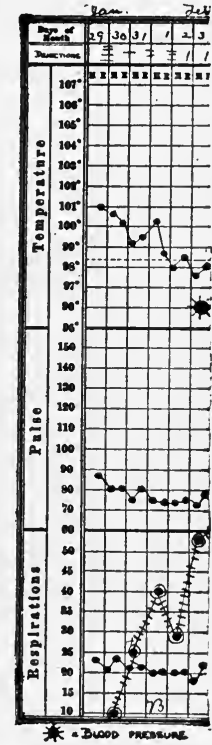

Fig. 127.-Chart of case 267 . second convulsion, after which he was very restless, struggling and throwing himself about on the table. At first he said nothing and did not answer questions, but later he swore profusely. There was no odor of alcohol or of acetone on the breath. The right pupil was larger than the left; both reacted well to light and accommodation. The tongue was very dry and red. No enlargement of the heart was made out, and there seemed to be no accentuation of either second sound. Blood-pressure was I $70 \mathrm{~mm}$. $\mathrm{Hg}$. The artery walls were palpable and tortuous. The lungs were hyperresonant throughout. Breath-sounds were accompanied by many medium, crackling râles over both lungs. The abdomen was held somewhat rigidly, but nothing abnormal was detected.

During this examination he had a third convulsion lasting about three minutes, quite epileptiform in type, with coma. The course of the temperature is seen in the accompanying chart (Fig. I27).

White cells were I6,500; hemoglobin, 80 per cent

The urine was of high gravity, with a very slight trace of albumin, but no casts at all.

Discussion.-In such a hard drinker the question of "rum fits" must be entertained. The patient has, however, no odor of alcohol and no history of a recent increase in the amount of alcohol consumed. This is not an acute debauch of the Saturday-night type, such as makes "rum fits" so common in our police stations. . It is a long-standing habit, which would probably not begin to produce these especial effects at the age of sixty-two. 
The condition of the urine is not characteristic, but the history of excessive nocturia and the high blood-pressure makes us suspect chronic nephritis as the ultimate cause of this attack. When the lungs are universally hyperresonant, it is very difficult to make out the size of the heart. Hence there may well have been a cardiac hypertrophy in this case, although none was discovered.

Of the organic diseases involving the brain and its membranes, we have no definite evidence. This does not by any means exclude them, but makes it impossible for us to move any nearer toward a diagnosis of any one of them until further signs appear. An examination of the fundus oculi might give great assistance, likewise lumbar puncture.

$\mathrm{My}$ reasons for ignoring the diagnosis ordinarily made under these conditions - i. e., acute uremia-have already been given (see p. $5^{20}$ ), and may here be simply summarized by saying that $I$ do not believe that any such condition exists. Uremia is a chronic affair.

As a working diagnosis, therefore, sufficient to guide treatment in the emergency, a vascular crisis favored by the underlying nephritis seems reasonable.

Crackling râles were to be heard throughout both lungs in this case. Their significance deserves some discussion. Any one who has seen many cases of sudden coma, with or without convulsions, must have noticed that we can almost always hear these scattered râles, whatever the nature of the attack. Their number and the extent of their distribution seem to depend upon the severity of the attack and the depth of the coma, rather than upon its cause. I have seen them in sunstroke, alcoholic and narcotic poisoning, apoplexy, brain tumor, plumbism, meningitis, and various other conditions, with or without a fatal issue. I am not now referring merely to the tracheal râles or snoring sounds attributable merely to the coma which prevents the patient from clearing the throat or closing his mouth, but rather to finer sounds audible with a stethoscope over the backs alone in milder cases and over the entire chest in severer ones.

No adequate explanation for these râles, so far as I am aware, has ever been given, but the rapidity with which they appear and disappear seems to point to some vascular condition which affects the lungs directly, rather than through any change in the heart's action. Certainly they are not always associated with cardiac lesions, but may be associated with the most forcible and efficient action of that organ.

Outcome.-The patient was lightly etherized, and a subpectoral infusion of four pints of normal saline solution was given. The bowels were moved by magnesium sulphate, and a hot-air bath was administered. 
He nad to be partially restrained, owing to his desire to get up, but by February $2 \mathrm{~d}$ he was entirely rational, and the restraint was removed.

$X$-ray showed cardiac hypertrophy. Hypertension and an excess of light-weight urine persisted.

On February 4th he was allowed to go home.

Diagnosis.-Chronic interstitial nephritis; vascular crisis.

\section{Case 268}

A shoemaker of twenty-seven entered the hospital June 28, I908. $\mathrm{He}$ had been in the hospital in the previous December for an attack similar to the present. Four weeks ago his knee became swollen and painful, and with this he was in bed for two weeks. Since then he has been walking with crutches. Yesterday the left elbow also became swollen and painful. This afternoon he had a convulsion, for which he was brought to the hospital. He had similar attacks in April and in February. It has been noticed that he passed an increased amount of urine, that he had to get up seven or eight times each night for this purpose, and that he had had edema of the legs for three weeks and almost constant headache. He has vomited four or five times a week since January. He has no dyspnea.

At entrance the patient held the left arm across his body in a condition of moderate spasm. He could move it but slightly. The left elbow was swollen and tender, the whole arm and axilla also slightly tender, the dorsum of the left hand swollen, the grip very weak. There was no other evidence of paralysis or weakness.

The heart's apex extended $\frac{1}{2}$ inch outside the nipple-line in the fifth space, the right border $I_{2}^{\frac{1}{2}}$ inches to the right of midsternal line. A systolic murmur was audible at the base, faint at the apex. There was no accentuation of either second sound. The right pulse was somewhat larger than the left. Blood-pressure was I $40 \mathrm{~mm}$. $\mathrm{Hg}$.

During the nine days of the patient's stay in the hospital the urine varied from IOO6 to IOI7 in specific gravity, amounting to 50 or 60 ounces in twenty-four hours. Albumin, from 0.7 per cent. to 2.4 per cent.; there were no casts. There were depressed scars on the right tibia from the knee to the ankle, also one an inch above the inner condyle of the left tibia. The blood was negative.

Discussion.-The age of the patient and the condition of the heart and urine apparently make it clear that we are dealing with a case of chronic glomerular nephritis. (See Appendix C, on The Classification of the Types of Nephritis.) If this be the case, the arthritic symp- 
toms are probably due to a low-grade infection, favored by the weakening of resistance which chronic nephritis usually entails.

In view of these conclusions it would seem reasonable to interpret the attack as uremic, since the patient has had previous symptoms indicating renal insufficiency, viz., headache, vomiting, nocturia and edema. It seems altogether probable that some chemical retention will account for the sudden appearance of cerebral symptoms in a case like this. (For fuller discussion of this matter see p. 520.)

The scars upon the shins naturally direct our search toward other evidences of syphilis, but as none such are forthcoming the possibility must be left open.

Outcome.-There was no repetition of the convulsions while in the hospital-from June 28 th to July 7 th.

The treatment consisted of hot-air baths, purgation, and diet.

Diagnosis.-Chronic interstitial nephritis; uremia.

\section{Case 269}

A child of fourteen months, who had never been sick previously, entered the hospital January I2, 1907. She fell down two days ago in a convulsion and has since then appeared to be very sick, crying much of the time, and extremely thirsty. Yesterday she had the "shivers," but no convulsion. 'The last twenty-four hours she has not seemed to recognize her mother, and has vomited occasionally. Whenever she is touched anywhere, she cries as if hurt. There is no discharge from either ear.

On examination, numerous white circular scars are scattered over the body.

There is convergent strabismus, and vision is apparently impaired. Considerable mucopurulent secretion can be seen in the pharynx. There is no mastoid tenderness, no retraction or rigidity of the neck;

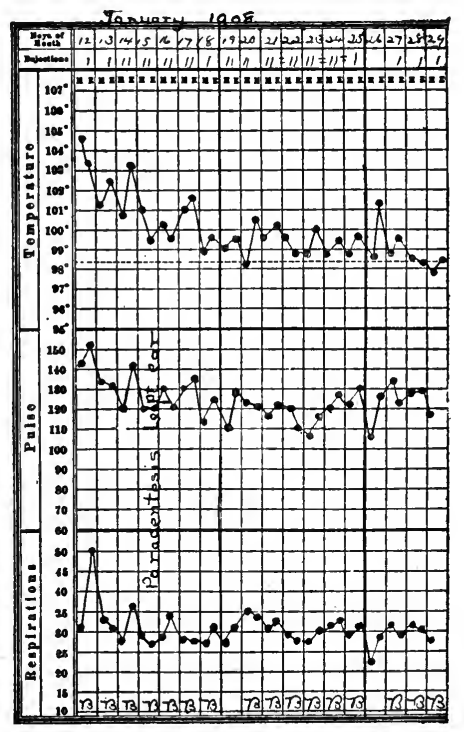

Fig. 128.-Chart of case 269. there is a slight fulness under the angle of the left lower jaw; moderate rickety rosary; coarse squeaks and bubbling sounds are heard through- 
out both lungs. Physical examination, including the reflexes, is otherwise negative.

The white cells are 18,300 ; the temperature range is seen in the accompanying chart (Fig. I28).

Discussion.-It used to be the fashion to attribute most of children's convulsions to teething or colic, and it is still generally believed that digestive upsets may be sufficient to produce convulsions which in older individuals would have a much more serious significance. In the present case there is no evidence that the teeth or any part of the digestive tract are connected with the seizure.

Rickets has been made responsible for almost every symptom and ill to which a baby's flesh is heir. On p. 4I4 I have already referred to a case of fatal urinary infection in which, owing to the presence of a rickety rosary and some slight errors in diet, the clinical diagnosis was rickets. I have known similar mistakes made in various other cases in which a slight epiphyseal enlargement was present. The moral seems to me to be that one should not explain any severe illness as due to rickets unless there is other evidence of that disease beside a rosary.

The child is thirsty, suggesting fever, has a leukocytosis, and a good many râles in its lungs. Râles may sometimes be the only auscultatory evidence of bronchopneumonia. Might not this case be one of infectious bronchopneumonia with convulsions at the onset? I have previously noted, however, that râles of this type generally distributed throughout the lungs are present in practically all cases of coma. This child is apparently semicomatose, and might easily, therefore, have many râles of this type without the existence of any pneumonia. Moreover, the râles of bronchopneumonia are only distinctive when grouped in discrete patches and associated with a good deal more cyanosis and dyspnea than are present in this case.

In an adult, meningitis would naturally be considered, and even in a baby of this age it can be by no means excluded. Other and commoner causes for convulsions should, however, be first investigated.

The most important of these causes is otitis media. I have already referred in a previous case to the fact that children suffering from otitis do not usually indicate in any way what part of their body is affected. It is, therefore, all the more important that we should write upon the tablets of our memory, in such a form that it will never be forgotten when we are dealing with children, the motto: "Remember the ears."

Outcome.-Examination of the ears by a specialist showed otitis media on the left. Paracentesis allowed the escape of a little bloody 
and purulent fluid, after which the child was much more comfortable, though there was still some deafness in the left ear.

On the eighteenth a swelling, apparently a gland, appeared at the angle of the left jaw. It did not extend up in front of the ear and was not tender. Ten days later it was still persistent, although the baby seemed otherwise entirely well.

Diagnosis.-Otitis media.

\section{Case 270}

A freight-handler of forty-seven was first seen on June 2, I905. $\mathrm{He}$ has had a markedly alcoholic history-five or six whiskies a day. He denies venereal disease. He has been well up to six months ago, when he began to complain of shortness of breath and cough, with a "rush of blood" to the head. Two months ago his eye-sight began to fail, and glasses seemed to do him no good. Within the past year he has had to pass water twice each night.

Ten days ago he felt too sick to go to work, vertigo, dyspnea, weakness, and nausea being his chief symptoms. This continued until three days ago, when he felt better and went out for a walk, but on returning he had a convulsion lasting three minutes.

For the past week he has been forgetful and incoherent at times. At noon to-day, while sitting in his yard, he went into a convulsion, with coma and snoring breathing. This convul-

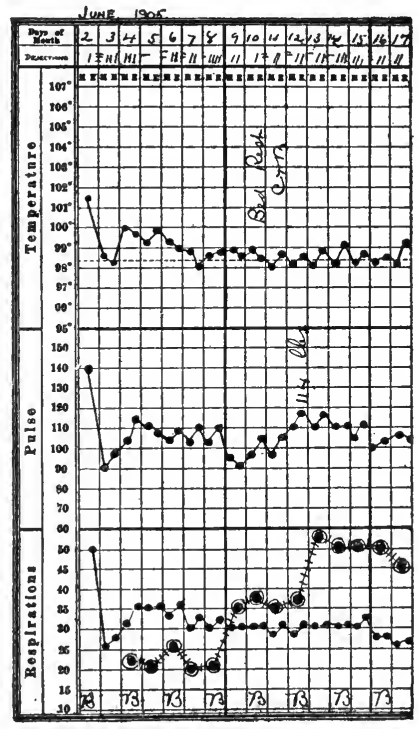

Fig. I29.-Chart of case 270. sion recurred before 4 o'clock, when he was brought to the hospital, still unconscious.

Physical examination showed good nutrition, slight enlargement of the glands of the neck, axillæ, and groins, nothing abnormal in the chest except a few coarse râles at the base of the lungs.

The abdomen and extremities were not remarkable; reflexes normal. (See Fig. 129.)

The white cells were 27,000 ; urine, 20 ounces in twenty-four hours: specific gravity, I022; the slightest possible trace of albumin; a few 
hyaline casts. Examination of the fundus oculi showed nothing remarkable.

The patient was treated with milk diet, hot-air baths, and magnesium sulphate.

By June 7 th he was fairly clear in mind, and by the eleventh seemed perfectly well. There was no special change in the urine, but the white cells had come down to 13,000 .

The patient was seen again on the eighteenth of August, I906. He had remained in good health and had no trouble with his eyes until about two weeks ago, when he "began to feel queer" and had frequent attacks of vomiting. Three days ago he had two convulsions, and has had several of the same since. Between them he has been drowsy and remembers nothing. His pupils were at this time irregular, the left larger than the right, the speech thick. He was unable to repeat his alphabet or to repeat sentences said aloud to him. His bloodpressure was $125 \mathrm{~mm}$. Hg.

Physical examination otherwise was as when last seen, except that the white cells were now 8000 .

Discussion.-In all cases where a convulsion is the presenting symptom we must first determine the question of epilepsy, in case it seems possible to exclude all gross organic changes or chemical poisons as causes for the convulsion. In any man of this age, however, we should always be very skeptical of a diagnosis of epilepsy. Why should it begin at forty-seven, when it is well known that the vast majority of cases of epilepsy begin in youth or young adult life. Only if no other possible explanation can be found is such a diagnosis justifiable in a patient of this age.

Although the patient is markedly alcoholic, there seems no evidence of any unusual indulgence, such as might determine at this time a "rum fit."

As there seems to be no residual paralysis or focal symptom, we have no right to conclude that hemorrhage or tumor is present. The normal condition of the fundus and the absence of any long-standing headache, vomiting, or vertigo strengthen the evidence against cerebral tumor.

The study of the blood and urine reveals no evidence of plumbism, diabetes, or nephritis. The latter disease is still further debarred from consideration (in its chronic form) by the low blood-pressure. Acute inflammatory changes (meningitis) do not deserve consideration, even in view of the leukocytosis present at the time he was first seen, for leukocytosis occurs in all acute cerebral seizures.

Attacks of unexplained convulsions associated with marked forget- 
fulness, incoherence, irregular pupils, and some disturbance of speech should always lead us to investigate, by further tests, the possibility of dementia paralytica. Such tests are, especially, the condition of the hand-writing as compared with previous years, the presence or absence of slight changes in manner or habits, the cellular constituents of the spinal fluid, and the Wassermann reaction.

Outcome.-August 24th he was extremely cordial and polite, even effusive, but some of his words were slurred, as if he were drunk, and his talk was decidedly muddled. He says he feels excellently well-better than for ten years.

On the second of September he escaped from the hospital and went to early mass, clad only in his red wrapper and carpet slippers. $\mathrm{He}$ returned immediately after service and did not seem to realize that he had done anything unusual. The later course of the case confirmed the diagnosis of dementia paralytica:

Diagnosis.-General paralysis.

\section{Case $271+$}

A manufacturer of sixty-two, with a good family history, entered the hospital January 2, I908. He says he has always been "tougher than a boiled owl," though he had diphtheria when a child, followed by a paralysis of both legs. His habits are excellent.

Three.weeks ago he had the "grip," and when nearly over it eight days ago caught a fresh cold, and began to have pain in both wrists, knees and the left shoulder, the pain not severe, but catching him when he moves. He has had no other symptoms.

On the morning of entrance, at 9.15, he had a series of short general epileptiform convulsions, lasting from five to ten seconds. During these the pulse fell to 22 and was very irregular. There were periods of fifteen to twenty seconds when no pulse could be felt and no heartbeat heard; following this came an epileptiform convulsion lasting from three to five seconds, then from seven to ten slow, full beats of the heart; the whole cycle would then be repeated. The convulsions were accompanied by momentary loss of consciousness, with flushing of the face; there was no cyanosis, orthopnea, drooling, or incontinence. The breathing throughout was deep and regular. The convulsions lasted all day and until after midnight, when they became less frequent, occurring at I A. M. and 5 A. M.

Physical examination showed a powerful, obese man, without glandular enlargement, with pupils altogether normal, and dry, brown tongue. The heart-sounds were almost inaudible. The heart's impulse 
was neither visible nor palpable; by percussion there was no evidence of cardiac enlargement. The belly was negative.

The blood-pressure was only $95 \mathrm{~mm}$. $\mathrm{Hg}$. and the arteries were barely palpable. The lungs were hyperresonant everywhere, and contained many scattered râles. The knee-jerks were not obtained, even with reënforcement; the Achilles jerk was likewise absent.

The joints of the left shoulder, knee, ankle, and the right wrist were slightly red, swollen, tender, and very painful on motion.

The course of the temperature is seen in the accompanying chart (Fig. I30).

The white cells were I5,000 at entrance; 18,500 two days later. The joint symptoms rapidly improved under sodium salicylate, to grains

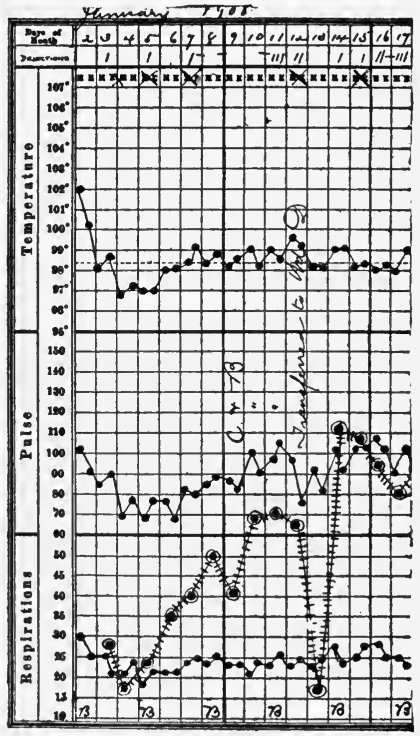

Fig. $130 .-$ Chart of case $27 \mathrm{I}$. every hour, and a dram of potassium citrate every four hours, with mild laxatives.

Discussion.-The striking point about this convulsion is its association with a very slow pulse and periods of pulselessness. Almost any variety of convulsion may be associated or followed by slow pulse, that is, by a reduction in the number of beats to 60 or even 50 a minute, but a pulse of 22 , such as that here recorded, has a very special significance, particularly when the general condition of the patient, both before and after the convulsion, shows no evidence of heart failure. Stokes-Adams' disease is always the first working hypothesis to be considered.

Confirmation of this diagnosis can be obtained only by the study of the venous pulse in the neck, which was not undertaken in this case, so that no certainty can be arrived at. Nevertheless, it is altogether probable that if such a study had been undertaken, evidence that the auricle beat more frequently than the ventricle would have been found.

Two other points in the case are of interest: the joint symptoms and the absence of deep reflexes. The latter is probably to be explained as a result of the diphtheric neuritis of his childhood. The normal condition of his pupils, the good control of the sphincters, the absence of characteristic sensory symptoms are sufficient to exclude tabes. 
The occurrence of multiple arthritis, accompanied by fever and leukocytosis, and promptly disappearing during the administration of salicylate, is of interest to me because I have several times observed such an attack simultaneously with a paroxysm of Stokes-Adams' disease. The fact may be a mere coincidence, though one may also conjecture that the blood changes accompanying infection may interfere with the transmission of impulses through a previously diseased bundle of His.

Outcome.- The patient slept a great deal during the first ten days of his stay in the hospital. After that he gradually regained his appetite and strength until, by the eighteenth, he seemed altogether normal and was allowed to go home.

Diagnosis.-Stokes-Adams' disease (?).

\section{Case 272}

A cook, sixty-eight years old, was first seen September 9, r907. Six brothers and three sisters died of unknown causes. Three sisters and two brothers are well.

For eighteen years he has had fits without known cause. In them he falls suddenly and usually without warning. Occasionally he bites his tongue, sometimes he "shakes."

The attacks last from a few minutes to an hour, and are usually accompanied by coma. They may come from once a week to once in three months; the last attack was two months ago. He denies venereal disease. He drinks two or three glasses of ale a day. Eight weeks ago he began to have swelling of his legs and abdomen, and this has steadily increased ever since. He has passed urine once or twice at night for twenty years. He worked until yesterday.

The patient was ill-nourished, pale, with normal pupils and a heart extending $\frac{3}{4}$ inch outside the left nipple and $5^{\frac{1}{4}}$ inches from the median line; sounds

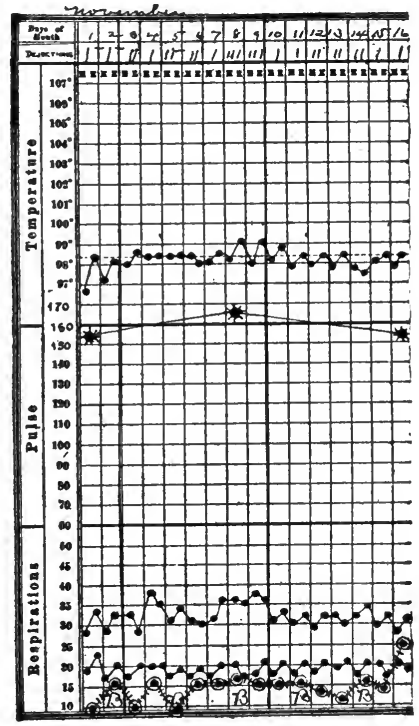

Fig. 131.-Chart of case 272 . regular, slow, and forcible. A moderate systolic murmur was heard all over the precordia, loudest at the apex; blood-pressure $162 \mathrm{~mm}$. Hg. The arteries were palpable and tortuous above the elbow. In the left back there was dulness up to the eighth 
rib in midaxilla, and to the seventh rib on the right. Over the dull area breath sounds and crackling râles were heard; fremitus was feeble. There was dulness in the flanks, shifting with change of position; marked soft edema of the legs and lower eyelids. By tapping, four quarts of straw-colored serum with a specific gravity of roog were withdrawn from the abdomen. In the sediment of this fluid lymphocytes made up 82 per cent.

About 2 A. M. on the $\mathrm{r}_{3}$ th of September the patient became unconscious, and had general epileptiform convulsions, lasting about ten minutes. There was no incontinence or biting of the tongue. Within the next two days he had six more such attacks. Each began with groaning respiration, which in a short time altogether ceased, so that the patient became almost black in the face, the pulses ceasing, the heart-sounds inaudible, the tongue protruded. Soon he breathed again; the heart-beat rose rapidly to 80 in a minute, then again fell to about 25. A short time after the beginning of the attack the muscles of the face moved convulsively, the whole body became rigid and then shook. The urine and feces were passed involuntarily. The whole attack lasted from half a minute to two minutes, and was followed by unconsciousness lasting some hours. In one of these attacks the breathing ceased for over two minutes by actual observation. The heart and pulse began again before the respiration.

Discussion.-This patient had been seen by several physicians on account of the convulsions from which he had suffered so long, and it had been so far assumed that the diagnosis was epilepsy. I have already called attention to the rarity of epilepsy beginning after the attainment of middle age. This patient's fits, it will be noticed, began when he was fifty. Presumably; therefore, some cause for them can be found.

The evidences of arterial degeneration at the peripheral, the moderate elevation of blood-pressure, and the age of the patient make it proper to consider cerebral arteriosclerosis or general arteriosclerosis with vascular crises as possible cause for these attacks. Their long duration, however, is against this supposition, and the fact that there is stasis, evidenced by the signs in the lungs, the abdomen, and the legs, points also against vascular crisis, since such crises usually cease when stasis begins.

Dementia paralytica rarely produces attacks extending through anything like so long a period of years. It is true that the disease may run a long course, but a duration of eighteen years after the appearance of convulsions and without any more marked mental or motor symptoms than are here recorded is contrary to all experience. 



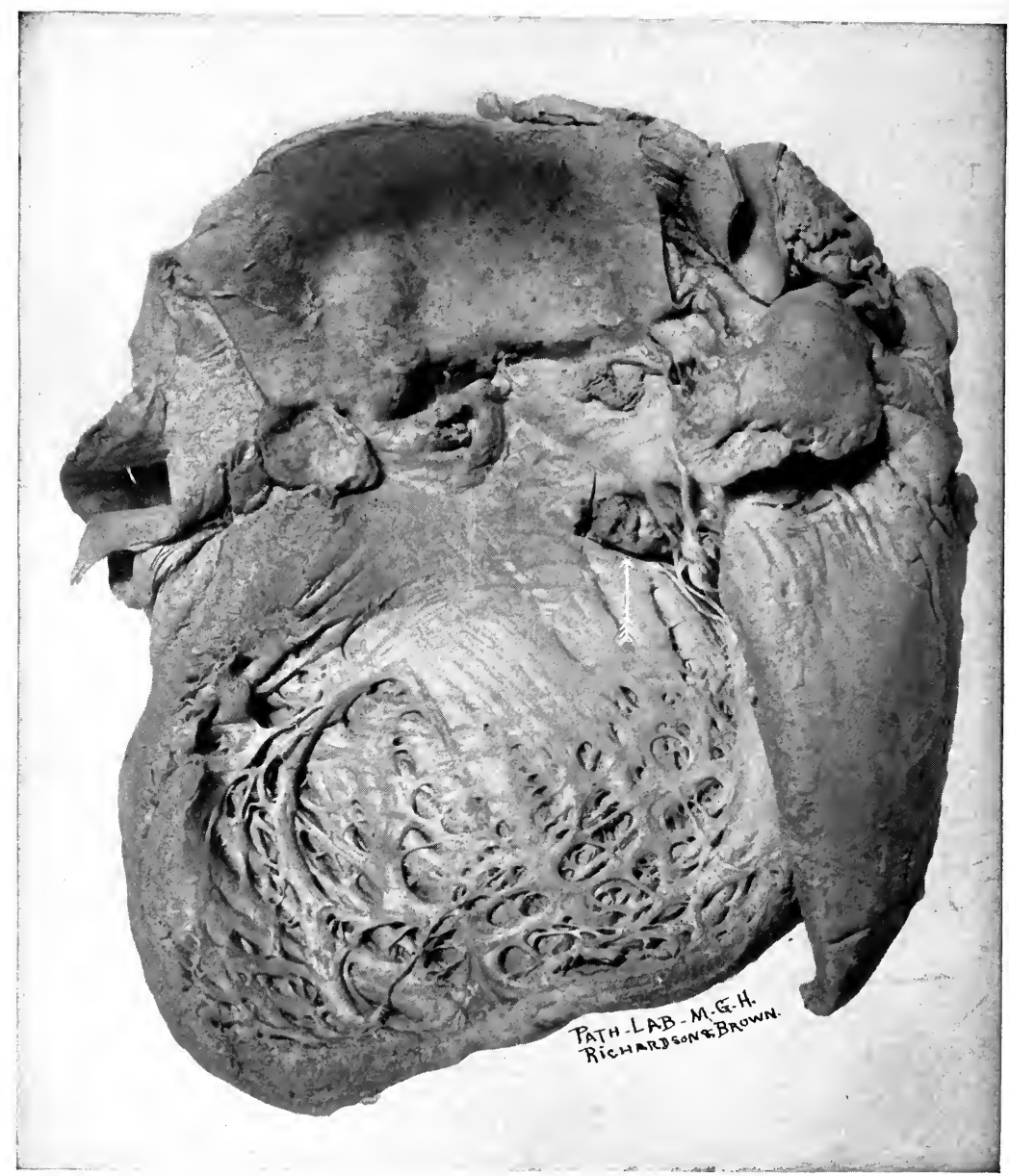

Fig. I32.-Calcareous ridge involving the bundle of His in a case of Stokes-Adams' disease. The arrow points to the ridge. A bit has been cut out for microscopic examination. (Photograph by Lewis A. Brown. Used by kind permission of Dr. H. F. Vickery and the Boston Medical and Surgical Journal.) 
The most important fact for the differential diagnosis is the cessation of the pulse-beat observed during the attacks which occurred in the hospital. This is very suggestive of Stokes-Adams' disease, but needs, of course, the confirmation obtainable by the study of the venous pulse in the neck.

Outcome.-Between attacks it was noticed that the pulse in the veins of the neck was to the radial pulse as 2 is to $\mathrm{r}$, or as 3 is to 2. Synchronous tracings confirmed this.

The urine averaged 20 ounces in twenty-four hours, specific gravity I020, the slightest possible trace of albumin, many hyaline, granular, and epithelial casts; leukocytes, 8000. By fluoroscopic examination the auricular beat was counted at 62 , while the pulse was 25. The fluid in the abdomen rapidly reaccumulated, and had to be tapped several times before the patient's death, December 15. Autopsy showed cirrhosis of the liver and a calcareous ridge in the region of the bundle of His.

Diagnosis.-Stokes-Adams' disease. ${ }^{1}$

\section{Case 273}

A child of three years, of good family history, was first seen May 12, 1908. She has always been subject to colds, but was otherwise well until yesterday afternoon, when she had a convulsion, more or less relieved by a mustard bath. Later she vomited twice and was somewhat feverish. This morning she began to cough and to breathe rapidly. The course of the temperature is seen in the accompanying chart (Fig. I33).

The tonsils are large and covered with a whitish exudate. There are small tender glands on each side of the neck. The neck muscles are not at all rigid. The heart shows no enlargement in any direction. In the pulmonary area a very loud systolic murmur is heard, completely replacing the first sound, and transmitted to all parts of the chest. There is no thrill. The pulmonic second sound appears to be much accentuated and reduplicated.

Physical examination was otherwise negative; the white cells, Io,700.

Discussion.-Besides the convulsion, the essential symptoms seem to be cough and dyspnea, associated with all the evidences of an acute tonsillitis and a cardiac murmur.

Before concluding that the convulsion merely expresses a reaction on the part of the symptom against the onset of the infectious tonsillitis,

1 This case was reported by Dr. H. F. Vickery in the Boston Medical and Surgical Journal, Oct. I, 1908, and is reproduced here by kind permission of the doctor and the journal. 
we must exclude the other and more serious possibilities. Meningitis is one of these, but there seems to be nothing definite except the convulsion itself to support this idea. Children are very prone to show cervical rigidity, with a retraction of the head, strabismus, and Kernig's sign, even when meningitis is not present; but the absence of all these symptoms is strongly against meningitis.

Can we connect the cardiac abnormalities, hinted at by the murmur, with the convulsions? Such a connection might be made out in case there were evidence of embolism of the brain or lung, of

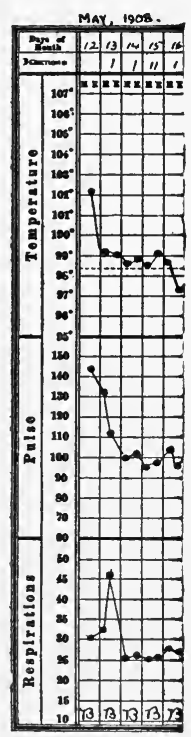

Fig. I33.-Chart of case 273 . marked cerebral anemia, or of a broken compensation involving the accumulation of $\mathrm{CO}_{2}$ in the cerebral circulation. But we have no reason to believe that any of these conditions exist, and I can think of no other way to connect the cerebral and cardiac symptoms.

The ears were examined without showing anything abnormal. The urine showed only the ordinary results of fever. It seemed probable, therefore, that our original supposition was correct, and that the onset of the tonsillitis was in itself sufficient to explain the convulsions.

What is to be said of the heart murmur? Very loud murmurs in the pulmonary area are usually the result of congenital heart disease. This is probably the best diagnosis to make in the present case, although one would feel much surer of it if any cyanosis and thrill were observable. It is usually unwise to attempt any further or finer description of the anatomic conditions in congenital heart disease. Autopsy seldom confirms the details of our diagnosis. Those who have seen most cases are generally least willing to commit themselves regarding a particular lesion or combination of lesions which is producing the trouble.

Outcome.-There was no repetition of the convulsion, and within a week the child seemed to be perfectly well, though the cardiac murmur persisted unchanged.

Diagnosis.-Tonsillitis and congenital heart disease.

\section{Case 274}

A married woman of thirty-four with three healthy children was seen September 8, I909.

She has had no miscarriage. Her husband denies syphilis. Five years ago began to have convulsions-typical "epilepsy." Considerable headache in this time, and occasional diplopia. 
Cureted for dysmenorrhea five years ago with relief to the dysmenorrhea, but none to the fits. Had tried many doctors without relief. For past six months no general convulsions, but attacks of twitching of the right hand and wrist, apparently provoked by excessive use.

Examination.-Choked discs. Urine and blood-pressure normal. Lumbar puncture gives clear, non-cellular fluid under pressure. Viscera normal. Later she developed paralysis of external rectus and ptosis on the left, with contracted pupil. Wassermann test and blood negative.

Died September 9.

Discussion.-This patient is at the age when ordinary epilepsy is common. The most notable feature in the case, however, and the most important from the diagnostic point of view, is the change in the nature of the spasm within the past six months. The attacks from which she now suffers have been diagnosed as "writer's cramp," for she is of a very nervous type, and has done a great deal of writing and sewing of late.

As soon as we had observed one of the attacks, however, it became obvious that it had nothing to do with "writer's cramp," that it was wholly involuntary, and possessed all the characteristics of Jacksonian epilepsy. Localized spasms of this type are often seen immediately preceding an attack of ordinary generalized epilepsy; in fact, pretty much all epileptic

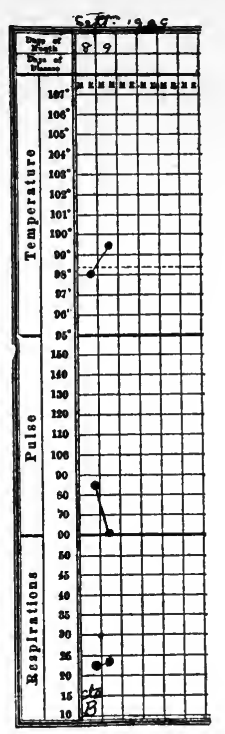

Fig. 134.-Chart of case 274 . attacks begin in some single group of muscles. It is only when the convulsion fails to spread beyond the original group that we attribute localizing significance to it, and begin seriously to consider a circumscribed lesion, such as tumor, cyst, meningeal adhesions, abscess, or softening.

The presence of choked discs, with a normal urine and bloodpressure and normal cerebral spinal fluid obtained by lumbar puncture, points strongly toward brain tumor. Were cerebrospinal syphilis, tabes, or paresis present, the spinal fluid would in all probability show an excess of cells. The negative Wassermann reaction is also of some significance, although in this, as in so many other fields, negative evidence is far less valuable than positive.

Meningitis does not give rise, so far as I am aware, to Jacksonian epilepsy, and the content of the spinal fluid suffices to exclude it.

There seems to be nothing left but cerebral tumor, and no important 
evidence against it. At first sight the long duration of her illnessthe five years of typical "epilepsy"-seems confusing, but there are now on record a considerable number of cases in which cerebral tumor has been demonstrated at autopsy after a number of years of headache and convulsive attacks, like those here described.

Diagnosis.-Cerebral tumor.

\section{Case 275}

A physician of fifty was seen October 28 , I908. He has always been well until eight years ago, when he had a good deal of pain in his lumbar region and down the back of his right leg. For this he consulted Dr. J. E. Goldthwait, who put him into a plaster jacket, with marked relief.

After this he was well until three years ago, when he had "some kind of a spasm," the nature of which cannot be more accurately learned. In July, 1907, he was seized with some sort of an attack during the night while in his bath-room. He found himself on the floor, and was unable to get to his feet, but crawled back to bed. Ever since that time it has been noticed that his gait is somewhat shuffling or shambling, especially when he is much fatigued.

In September, 1907 , he had an attack of catarrhal jaundice. A consultant saw him at that time and considered it a case of "brain-fag." Muscular power, sensation, and the pupils were then examined and found to be normal. During the next six months, however, he had a number of attacks of vomiting without obvious cause and without relation to meals. They did not, however, prevent his carrying on a very active practice, in which he has been engaged up to the present time.

In September, I908, he had a bad nose-bleed, and next day seemed very weak, with profuse sweating and marked pallor. To these nosebleeds he says he has been subject all his life. Many other members of his family have a similar tendency.

October 27 , I908, the day before the one on which I saw him, he was seized about 6 P. M. with a general epileptiform convulsion, and within the next twenty-four hours he had nine similar attacks. The first convulsion followed immediately upon the eating of a very heavy meal. After it he was comatose, and between the subsequent convulsions he did not fully regain consciousness until about three hours before the time at which I saw him.

On examination he was normally conscious, intelligent, and cheerful. There was no paralysis anywhere, and the tendon reflexes were normal. 
The pupils immobile. The heart, lungs, and abdominal viscera showed nothing worthy of note except that the aortic second sound was sharp and ringing, and the pulse of high tension. As he spoke to me in answer to questions I noticed an occasional stumbling, and now and then the elision of a syllable. He said he felt quite well, and wanted to make some medical calls that afternoon.

On subsequent inquiry it was learned that his urine had been examined several times in the past eighteen months and always found to contain a trace of albumin. He had sometimes had a little trouble in urination, and once last summer involuntary defecation took place.

Discussion.-Every physician sees many cases of this type, if one omits the history of the convulsion. In the absence of such convulsions as were here described the diagnosis of neurasthenia is very frequently made. Such a diagnosis, in my opinion, is never justified when the patient's symptoms first appear at or after middle life. Nervous weakness under these conditions means organic disease with nervous manifestations. The underlying trouble is most often cardiovascular disease, with or without a demonstrable arteriosclerosis. The persistent elevation of the blood-pressure, which is almost always to be found, should put us on our guard against the mistake of supposing the patient to be "merely nervous."

In all such cases, however,-and more especially when a convulsion has occurred,-we should make such inquiries as would serve to determine whether any evidence of beginning dementia paralytica is present. In a physician carrying on an active and successful practice it may seem hardly justifiable to consider so serious a disease, but in the present patient inquiry brought out the following points:

(a) His hand-writing, always indistinct, had now become so illegible that apothecaries were frequently unable to decipher his prescriptions. (I fear this happens to many not demonstrably the victims of dementia paralytica.)

(b) His wife had noticed that he had recently become entirely incapable of making up his accounts or doing even simple sums in arithmetic.

(c) Despite the vagueness and inaccuracy of his account books, he was very cheerful, if not optimistic, upon money matters, though his wife declared with tears that he had little ground for such optimism.

(d) He has fallen into the habit of dropping asleep while at work, or even in the midst of a conversation, and his attention at all times is short-lived and wandering. (This is true, also, of many other members of his family, and, indeed, of the human family.) 
(e) His memory and decisiveness of action have been gradually failing for many months.

(f) It has been noticed that he drops things very frequentily, and complains that he has no feeling in his fingers.

In view of these mental and psychomotor changes the diagnosis of dementia paralytica seemed to me clear.

Outcome.-The patient went away for a few weeks on a vacation soon after I saw him. He then returned to Boston and tried to resume practice, aided by a very old friend, a physician, who, for friendship's sake, was willing to go everywhere with him and make good his mistakes.

On February 13, I909, he had another convulsion, and March Ioth a general tonic clonic spasm, without loss of consciousness. After this he gradually improved in strength, gait, and ability to write, but his personal habits-previously most correct-became somewhat untidy. May 25th he went to his old home on a Maine farm, where he passed the summer in reasonable comfort. October 3, r909, he seemed in unusually good spirits, but at midnight he had a series of convulsions and died within a few hours.

Diagnosis.-Dementia paralytica.

\section{Case 276}

July 9, I906, I was called to a small town in the southern part of Massachusetts, on the outskirts of which lived a farmer whose wife I was asked to see for the relief of convulsions of unknown origin. She was a woman of twenty-eight, of excellent family history, and had always up to this time been well and strong. Nine weeks previously she had borne her first child, parturition and the convalescence from it being normal.

Three weeks before I saw her she consulted her physician on account of persistent headache, an entirely new symptom for her. A week later she was noticed to be distinctly pale. Iron was prescribed, and she seemed to be doing well until eight days ago, when she had an attack of vomiting without any known reason. Such attacks have recurred every day or two since that time.

Five days ago she had her first epileptiform convulsion, which was followed within twelve hours by a second convulsion involving only the muscles of the right half of the body. Four days ago a similar unilateral convulsion occurred. Yesterday she had a generalized convulsion without loss of consciousness. The urine has been several times examined. It is always rather scanty, but has never shown any 
albumin. The gravity has averaged I024, the color rather darker than normal. She has voided 27 ounces in the past twenty-four hours.

Between the convulsive attacks she seems pretty well, though rather weak. There has been no pain at any time and no paralysis.

Physical examination of the chest and abdomen was entirely negative. The reflexes and pupils were normal; the hemoglobin, 50 per cent; the urine free from albumin, and otherwise as above described.

Discussion.-Naturally, our first attempt in such a case is to relate the convulsions in some way to the recent childbirth, but this seems, on reflection, rather far fetched, as the woman was in perfect health for the six weeks following parturition.

Uremia seems to be excluded by the absence of any cardiac hypertrophy or urinary changes. Unfortunately, the blood-pressure was not measured. To the palpating finger the arterial tension seemed unusually low.

Without an examination of the fundus oculi one cannot speak with confidence against the possibility of brain tumor; but there is really little to suggest it, local symptoms being entirely absent, the headache being very moderate and unaccompanied by vertigo.

In the physical examination and in the reasoning process above reproduced one essential step has been omitted primarily, because in the first fifteen minutes of my study of this case it was altogether forgotten, also because it never occurred to the mind of the attending physician. Both of us forgot to consider lead-poisoning. After my first unsatisfactory and fruitless review of the case I began again and went over the patient systematically from head to foot. On the gums I found this time a typical lead-line, which I had previously omitted to look for, because lead-poisoning is associated in my mind chiefly with those who work in some trade involving the use of lead. A young woman living in the depths of the country and doing no work outside her own house does not necessarily suggest the possibility of leadpoisoning.

After finding the evidence of lead in her gums I began to wonder where she could have acquired the metal. Could it be from drinking water? If so, other members of the family should be affected. I turned at once to the husband, standing at the foot of his wife's bed, and examined his gums. They also showed a typical lead-line, though he had had no symptoms. There was no one else in the house but the baby, who had taken no water and seemed to be quite healthy.

The family then recollected that the water had tasted queer since the previous winter, but they had been using the same well-water for 
the past three years. About roo feet of lead-pipe intervened between the well and the house.

The patient was ordered to drink no more of this water, to take 5 grains of potassium iodid three times a day, and a purge of magnesium sulphate every morning. The convulsions ceased at once, and the patient made a rapid and lasting recovery.

Diagnosis.-Lead-poisoning.

\section{Case 277}

In July, I893, a gentleman of forty-nine entered the hospital with the diagnosis of astasia abasia, made by a neurologist three weeks previously. He remained in the hospital for three months, during most of which time he had partial paralysis of the leg, relaxed sphincters, and a great number of complaints referred to different parts of his body. 'The reflexes were never markedly abnormal, and visceral examination was always negative. He gradually improved until he was able to walk with a cane, left the hospital, and was not seen again until 1903 .

During most of the intervening decade he lived in India or in Egypt, painted a good many pictures, and enjoyed himself thoroughly. In I900 he had an indolent abscess on his forehead, which did not heal after it had been opened, and showed no considerable improvement for six weeks. After that he was given some medicine "with a salty taste," and the abscess promptly healed.

Since 1897 he has been troubled with attacks diagnosed as "petit mal." These occur every two to five days, and last about half a minute. A typical attack begins with slight nausea and a bad taste in the mouth; next he begins to notice a sudden change in the behavior of the people around him. They seem to be walking so as not to disturb him, or creeping toward him. After this, comes a tremor or thrill down the left arm and an involuntary closing of the left thumb and index-finger, with some shaking of the whole hand, so that he may almost drop his newspaper if he is reading one at the time. All the colors of the objects around him become intensified. He does not think that any one about him notices what is going on. Between these attacks he feels pretty well, but occasionally wets his bed at night, and always passes water five or six times after he gets to bed. Occasionally he has noticed that his linen is stained, owing to relaxation of the rectal sphincter.

In 1904 he went abroad and enjoyed himself very much. Various English doctors told him that his troubles were all due to gout. Occa- 
sionally his left ankle gives way under him, but, as a rule, he walks very well. November 7, 1904, he fell unconscious in a water-closet, and for half an hour afterward was drowsy and drooled saliva. January 2I, I905, after four days of excellent spirits and entire absence of the attacks of "petit mal," he woke up in the night with severe pain across his forehead, a very sore tongue, and much sensitiveness of his muscles, especially across the loins. For the whole day following this he was very sleepy and stupid.

February 2oth he started to dine with a friend in Cambridge. The next thing that he knew he found that the electric car in which he was had come to the end of its route, in surroundings which he did not at all recognize. How he got there he had no idea: About three-quarters of an hour had elapsed since he took the car for Cambridge. Next day he noticed that his left.foot dragged a little in walking.

In 1906 he began to have trouble with his rectum, and a tumor was felt high up upon the right. Operation showed an apparently inoperable tumor mass involving a large portion of the rectum and lower sigmoid. An artificial anus was made, after which he was greatly better. Four years later there had been no increase of symptoms. The artificial anus was working excellently well. Occasional attacks of unconsciousness, with or without generalized convulsions, and very many of the seizures called "petit mal," still troubled him.

Discussion.-Twice this patient was given up to die-the first time in 1893 , the second time in 1897 ; yet he is alive and healthy (I910). The most important diagnostic and therapeutic indication in his case was to my mind the so-called abscess on the forehead, which resisted all ordinary treatment and then healed so promptly after the administration of a medicine which had the taste of potassium iodid. The patient had no knowledge of any syphilitic infection, but had lived the type of life in which such infections are acquired. I see no reason to doubt that all his symptoms were due to syphilis in one form or another. First the spinal cord, later the brain, and finally the perirectal tissues . were involved.

The question of operation for the relief of his attacks of "petit mal" was often and seriously considered, but in view of his previous history it seemed probable that the disease was so widely diffused that little could be expected from operative interference.

Diagnosis.-Syphilis. 


\begin{tabular}{|c|c|c|c|c|c|c|c|}
\hline 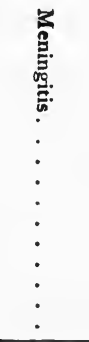 & 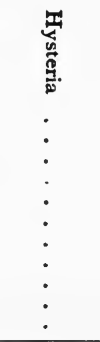 & 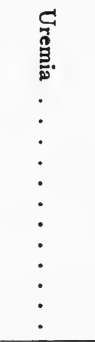 & 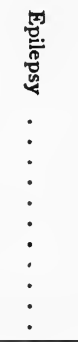 & $\begin{array}{l}\frac{2}{0} \\
\frac{0}{0} \\
\frac{\sigma_{0}^{\circ}}{3} \\
\vdots \\
\vdots \\
\vdots \\
\vdots \\
\vdots\end{array}$ & 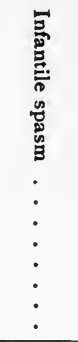 & 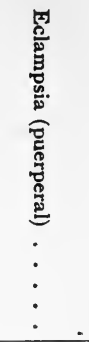 & 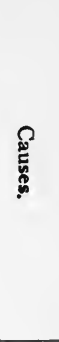 \\
\hline 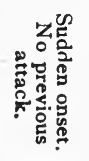 & 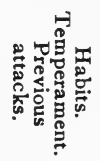 & 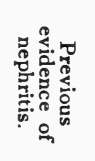 & 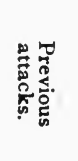 & 范 & 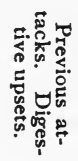 & 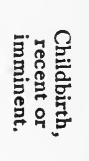 & 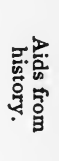 \\
\hline+ & ० & 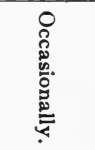 & ० & ० & 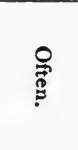 & 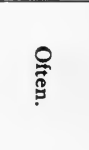 & . \\
\hline 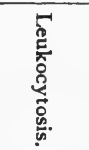 & 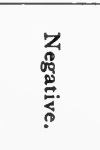 & 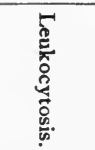 & 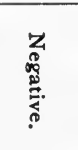 & 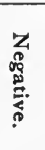 & 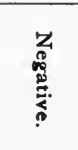 & 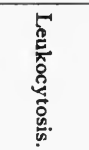 & $\begin{array}{l}\underline{\omega} \\
\vdots \\
\vdots\end{array}$ \\
\hline 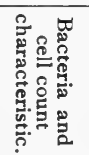 & 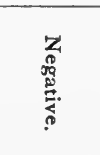 & 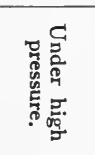 & 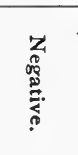 & 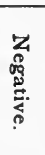 & $\infty$ & 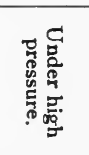 & 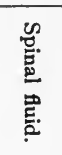 \\
\hline 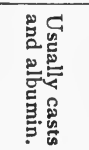 & 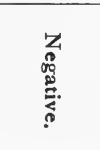 & 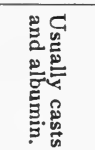 & 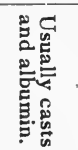 & 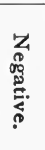 & 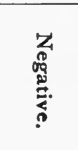 & 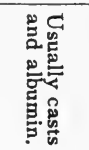 & 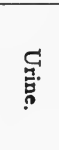 \\
\hline ० & $\circ$ & 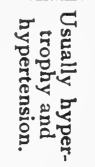 & ० & 0 & 。 & 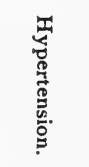 & 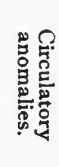 \\
\hline 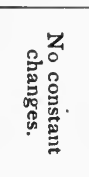 & 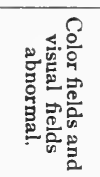 & 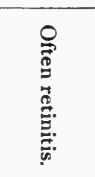 & 。 & 0 & ○ & 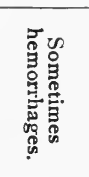 & 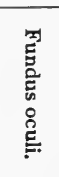 \\
\hline 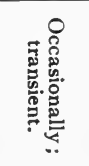 & 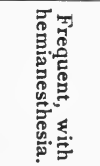 & 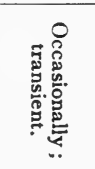 & ० & 0 & 0 & 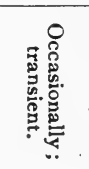 & 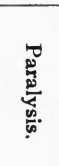 \\
\hline 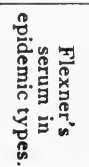 & 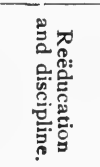 & 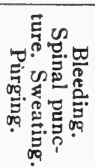 & 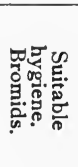 & 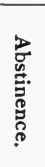 & 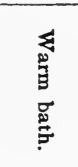 & 可 & $\begin{array}{l}\pi \\
0 \\
\stackrel{0}{\pi} \\
\end{array}$ \\
\hline
\end{tabular}





\section{Causes of Paralysis}

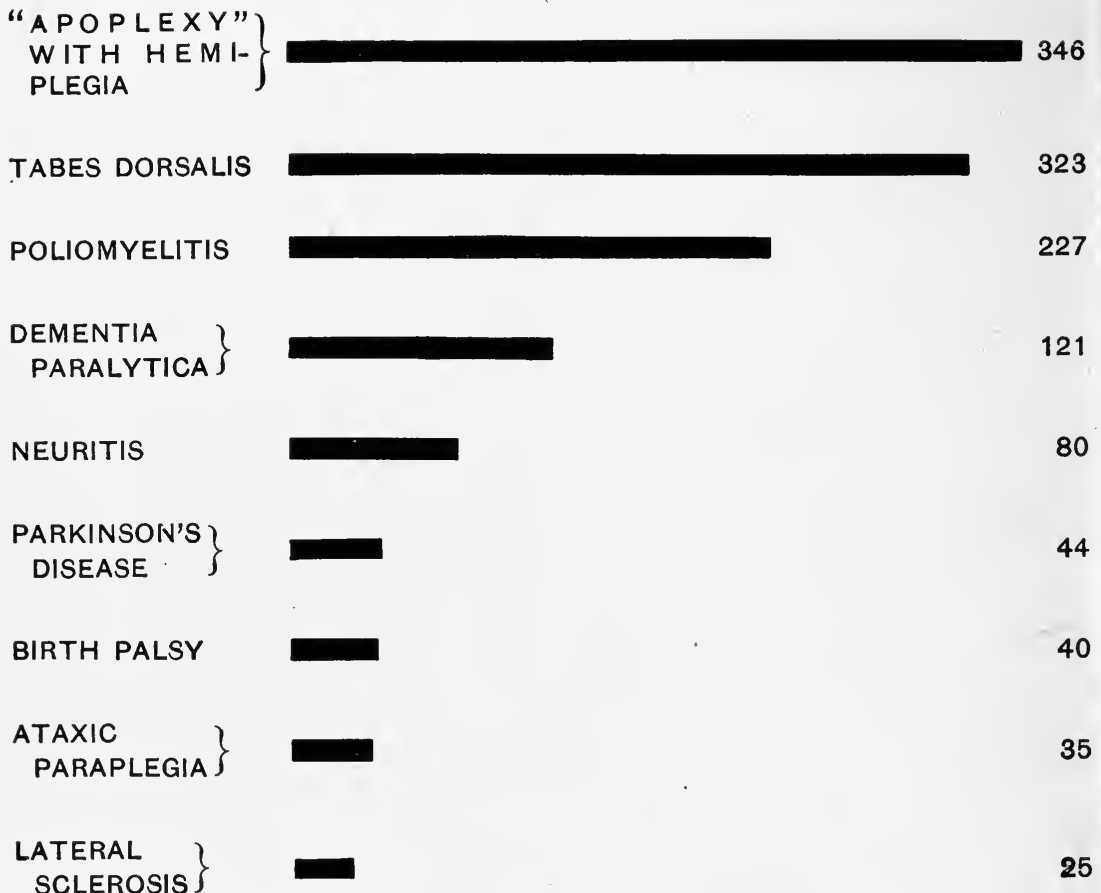

SCLEROSIS $\}$ 


\section{CHAPTER XVII}

\section{WEAKNESS}

So many patients consult a physician complaining primarily of weakness that I have thought it best to discuss it and to illustrate it by cases, although so little is known regarding the manner of its production in the great majority of patients. We must make at the outset a distinction which is often not noticed by patients themselves, the distinctior, namely, between:

(a) Paralysis due to organic lesions of the brain, cord, or peripheral nerve.

(b) Hysteric and psychasthenic "forgetfulness" (Janet), whereby a patient loses control of his motor tract.

(c) Weakness in the narrower sense, excluding $(a)$ and $(b)$, and due to a great variety of defects in nutrition, excretion, or blood-supply.

Of this latter type of functional insufficiency we really have very little knowledge. It is often said that anemia is directly and in itself the cause of many weakened states, yet I had under my care for three years a patient with pernicious anemia who was in the habit of taking a daily swim of about a mile in the Charles River, when his red cells numbered less than $\mathrm{I}, 500,000$ per c.mm. He also walked to and from his work, - a distance of about two miles each way,- -and was very actively engaged as a salesman in the basement of a department store for nearly twelve hours in every twenty-four. In view of this and similar cases it is difficult to believe that anemia is in itself the allimportant cause of weakness such as we should often be led to suppose.

It is also well known that the size of muscles and their firmness have only a rough and general relation to their strength. Some of the most remarkable athletes have small and apparently soft muscles.

In a large group of cases weakness appears as the result of cardiac insufficiency, but even here it is difficult to fix the blame, since dyspnea is so intimately related to the disabilities of which the patient complains.

Fever is likewise associated in our minds, and apparently, in fact, with many cases of weakness, yet, on the other hand, we have all of us dealt with patients who feel much brighter and better when their temperature is elevated than when it is normal. 
Despite all these limitations of our knowledge it is doubtless true that anemia, lack of muscular development, cardiac insufficiency, malnutrition, and fever are in some way connected with the weakness of which our patients complain. Clinically, such complaints are most often associated with the following conditions:

I. Neurasthenia and other psychoneuroses.

2. Tuberculosis.

3. Anemia.

4. Bad hygiene.

5. Nephritis.

6. Valvular heart disease.

7. Convalescence from acute respiratory and other streptococcus infections ("influenza").

8. Diabetes.

9. Hyperthyroidism.

In the latter two diseases we have the striking phenomenon of loss of weight and strength despite good appetite. Besides those above listed, one sees now and then a patient complaining only of weakness, yet proving, on examination, to have typhoid fever. The same is true of myxedema and not infrequently of obesity.

\section{Case 278}

An expressman, thirty years old, of good family history and good nabits, had pneumonia seventeen years ago, and again seven years ago. Six years ago he passed a life-insurance examination and was told that his lungs were sound. He was first seen October 29, I906.

$\mathrm{He}$ has been feeling entirely well until about two years ago, when he began to get weak, lost his appetite, and felt some nausea and faintness. He kept at work, however, until October, 1905, when he went to his father's home in New York State, was out-of-doors hunting, and felt much stronger and better, but still was not cured.

Some time after this he had jaundice. He was treated with calomel, but did not improve. He went back to work in February, 1906, but in May broke down again, and since then has never been able to work more than three weeks at a time on account of weakness.

At no time has he had any pain, but his weakness gradually became so troublesome that six weeks ago he gave up work for good.

His appetite has been poor throughout this illness, but for the past three weeks he has eaten almost nothing because he cannot bear the sight of food. He has had no vomiting, no pain anywhere, and his bowels have moved regularly once a day.

For the past two weeks he has been very sleepy, and found it difficult 
to keep awake in the day-time. For the past two weeks he has taken beef-juice in teaspoonful doses, but no other food. He has had no drugs except sodium phosphate. Of this, he says he has used half a bushel-basket full of bottles.

A year ago he weighed 125 pounds, now he weighs ro3.

On examination the patient is emaciated. His breath shows an odor like acetone. His skin is of a dark yellow hue.

His heart is negative, except that the sounds are very faint. His pulse is of very low tension; his blood-pressure, $50 \mathrm{~mm}$. $\mathrm{Hg}$.

The edge of the liver is just palpable below the ribs on full inspiration. The knee-jerks are absent.

There is no edema. The urine is negative.

The temperature is as seen in the accompanying chart.

Red cells are 5,040,000; white cells, I7,200; hemoglobin, 85 per cent.; 69.5 per cent. of the leukocytes polynuclear, the remainder lymphocytes, the majority of which are very large. The blood is otherwise normal, repeated search for malarial parasites being fruitless.

The stools showed nothing abnormal except a slight reaction with the guaiac test. The vomitus showed no hydrochloric acid; nothing else of interest.

Extreme weakness was practically his only symptom.

Discussion.-The marked gastric symptoms complained of by this patient direct our search first toward some cause in the gastro-intestinal tract.

(a) Anorexia nervosa often produces a condi-

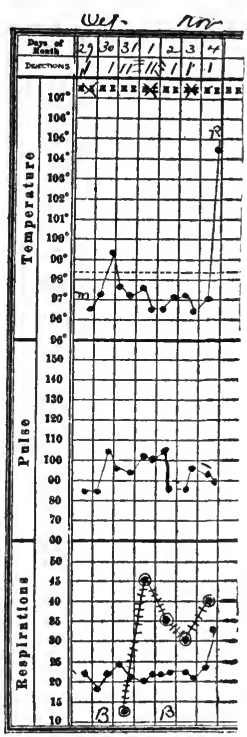

Fig. 135.-Chart of case 278 . tion even more serious than the one now under discussion. Indeed, it is not infrequently fatal. But in a person of this age, sex, and manner of life it is, so far as I know, unknown.

(b) Cancer of the stomach may occur at this age or even earlier, though such an occurrence is very rare. One of its earliest symptoms is often a complete loss of appetite, such as this patient suffered. The absence of hydrochloric acid in the vomitus would seem to support this hypothesis. On the other hand, in a patient so markedly emaciated we should certainly expect to feel a tumor, especially as the symptoms seem to have lasted two years. Other gastric symptoms-such as 
stasis and vomiting-would certainly have appeared by this time in the great majority of cases of gastric cancer.

(c) The enormous amount of sodium phosphate which this patient had taken might cause us to conjecture that he has poisoned himself with the drug were there any evidence that it is capable of producing toxic symptoms; but so far as I know there is no such evidence.

The patient's yellow pallor reminded me strongly of some of the cases of chronic malarial poisoning which I had seen in soldiers returning from the Cuban war, but the results of blood examination absolutely excluded malaria.

As there was no discoloration of the conjunctivæ and no bile in the urine, we did not consider a chronic hemolytic jaundice. The low blood-pressure and the great emaciation were such as one often sees in the latest stages of some form of tuberculosis. There were no lesions, however, discoverable by physical examination, and no fever.

The absence of knee-jerks was not explainable by any of the diagnoses which we considered. There was no sufficient reason to consider tabes, as there were no sensory, pupillary, or sphincteric changes and no pain. Very possibly he may have passed through an attack of peripheral neuritis at some previous time, but there was no reason to connect it with the present symptoms.

Addison's disease produces the lowest blood-pressure that has been observed, so far as I know, in any disease previous to the moribund state. It is often associated with gastric symptoms like those from which this patient has suffered. The discoloration of the skin is usually more marked than that here described, but as it is well known that Addison's disease can occur without any pigmentation at all, it is well always to remember the disease in any differential diagnosis of cases characterized by extreme weakness of obscure origin.

Outcome.-The face and hands were a good deal darker colored than the rest of the body; there was no increase at the body folds.

In the mouth was a small patch of dark-brown color on the inside of the cheek, near the corner of the mouth, also some clusters of minute brownish points on the inside of the cheeks near the junction of the teeth, and a few on the hard palate. The patient looked like one in the last stages of malignant disease or tuberculosis. At times he would suddenly feel much better.

The patient was put on forced feeding by mouth and rectumwhisky, I ounce every four hours, strychnin, $\frac{1}{30}$ grain every four hours and seemed better until the fourth of November, when he developed fever and chill, became delirious, and soon died. 
Autopsy showed tuberculosis of the adrenal glands; obsolete tuberculosis of the apices of the lungs.

Diagnosis.-Addison's disease.

\section{Case 279}

A freight truckman, thirty-eight years old, entered the hospital July I, 1906. He had formerly used alcohol in moderation, but had used none for over a year until it was prescribed by a doctor during the present illness. He denies venereal disease, and has been well until twelve days ago, when he began to feel weak, mean, and seedy. Three days ago he had to give up work on account of weakness, night-sweats accompanied by constant frontal headache, pain all over him (especially in the back), anorexia, nausea, and vomiting.

Physical examination was negative, except that the knee-jerks and abdominal reflexes were not obtained. The nutrition is fair.

Blood, urine, temperature, pulse, and respiration were normal.

Despite his weakness and prostration, there was noticeable during the examination an unusual degree of nervous alacrity. Any direction given him was executed with lightning speed and almost with violence.

Discussion.-The symptoms of the onset seem like those of an acute infectious disease, especially pneumonia or typhoid, and although fever was absent, we made rigorous and repeated search for visceral evidences of some such infection. Nothing came to light, however, and we were obliged to look elsewhere for a cause of the symptoms. In cases of this kind it is always well to consider:

(a) Neurasthenia or some other type of psychoneurosis.

(b) Poisoning by morphin or some other drug.

Though abstractly possible, the psychoneuroses were practically easy to exclude when we were face to face with this burly, lethargic, hard-working wage-earner.

Of morphinism there was no hint, either in the history or in his present condition. He showed none of the vague longings, irritable complaints of widely distributed pain, itching about the face, scars of hypodermic punctures, pallor, emaciation, insomnia, or other evidences of the morphin habit.

On the other hand, another poison-alcohol--was distinctly suggested by the absence of knee-jerks, when considered in connection with his mental state. The peculiar alertness and alacrity, shown by alcoholics immediately previous to an attack of delirium tremens, is difficult to convey by description, but easily recognized by any one who has once or twice seen it. In the present case it was very marked, and 
was associated also with a very noticeable smoothness and satiny texture of the skin, a sign often of great value in patients who deny alcoholism, but present other evidences which make us suspect it.

Outcome.-Although the patient indignantly denied any recent alcoholic excess, he began to show the nervous symptoms of approaching delirium tremens two days after his entrance to the hospital, and in spite of considerable doses of potassium bromid. These symptoms abated, however, within two or three days, when he was able to go home in much better condition.

Diagnosis.-Alcoholism.

\section{Case 280}

A bridge-tender, fifty-eight years old, of good family history, has had "chronic rheumatism," and especially "sciatic rheumatism," in the right leg at irregular intervals for ten years or more. Otherwise he has always been well and strong, and his habits have been good.

Ten months ago he began to notice a weakness so marked that at times he came near fainting. This weakness was most noticeable in the legs, but he has felt tired all over. For the last six weeks he has felt, on exertion, a rather severe pain in his chest, near the lower part of the breast-bone, accompanied by shortness of breath, which compels him to stop whatever he is doing. The pain ceases after a few moments' rest. Hearty food also brings on this pain, which comes on immediately after eating and lasts for an hour or more.

He has no cough, no vomiting; is usually somewhat constipated; the bowels move once in two or three days.

Two years ago he weighed 250 pounds; he still weighs $215 . \mathrm{He}$ gets up three or four times at night to pass water.

The temperature, pulse, respiration, urine, and blood-pressure all were normal. The heart showed no enlargement, and its sounds were of fair quality. There was a faint systolic murmur at the apex, transmitted a few inches to the left; no accentuation of either sound at the base. The edge of the liver was felt two inches below the costal margin; the abdomen is otherwise negative, likewise the lungs, reflexes, and extremities.

Rectal examination showed prominent external hemorrhoids, but no evidence of bleeding.

Examination of the stomach with a gastric tube showed nothing abnormal, either physically or chemically.

The red cells were 2,520,000; white cells, 8000; hemoglobin, 30 per cent.; differential count normal. The stained specimen showed marked 
achromia, very slight variations in the size of the red cells, but nothing else abnormal.

Discussion.-The history of sciatica and the complaint of especial weakness in the legs naturally lead us to consider peripheral neuritis. No such diagnosis can be made, however, when the reflexes are normal and all sensory symptoms are absent, as in this case.

Arteriosclerosis must occur to us whenever a patient of fifty-eight complains of substernal pain and general weakness. Possibly there is some arteriosclerosis in this patient, but I do not see that we can be sure of it or that we can connect it with his present symptoms, since his blood-pressure is low, his heart negative, and symptoms of stasis absent.

But for the blood-examination this patient would present almost precisely the picture of pernicious anemia; even the substernal pain, which he complains of, is sometimes seen in that disease, apart from arteriosclerosis or nephritis. The blood-picture, however, is that of secondary anemia, and compels us to make a most careful search for its cause.

That search should be directed so as to ascertain whether syphilis, malignant disease, hepatic cirrhosis, or any disease involving hemorrhage is present. All these except the last could easily be excluded, but in view of past experience I always look with particular care for evidence of hemorrhoids when the problem is to find the cause for the anemia of a middle-aged patient. I recollect three persons suffering from anemia of unknown cause and totally unaware of any trouble from piles, which, nevertheless, turned out subsequently to be the source of frequent long-standing hemorrhages. In all these cases the anemia was cured by treating the piles and stopping the hemorrhage. The same turned out to be true in the present case, the moral of which is that careful examination of the rectum with a speculum should always be made when we are searching for the cause of an obscure anemia.

Outcome.- It was learned subsequently that the patient had had bleeding piles off and on for at least four years. For some unknown reason he omitted to mention this fact. Operation was advised, but refused.

Diagnosis.-Secondary anemia. Piles.

\section{Case 281}

A post-office clerk of sixty-three entered the hospital November ז4, 1907. He was in the hospital first in I9OI with genito-urinary tuberculosis, and again in 1904 for stone in the bladder. He seems to have recovered entirely from both his previous troubles. For the 
past year he has been losing strength and weight. Twenty years ago he weighed 196 pounds; five years ago, I70; one year ago, 167; and now, I28. From July ist to November Ist of this year he was unable to work. For the past two weeks he has been at work again, but had to give up to-day. He has no other symptoms of any kind, and has noticed no pallor or pigmentation of the skin.

The patient is somewhat pale and much emaciated. A systolic murmur is heard at the apex, but not transmitted. The heart is other-

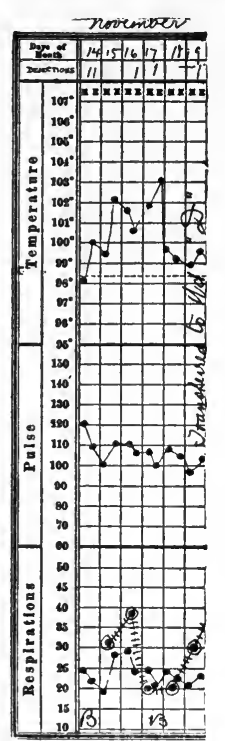

Fig. I36.-Chart of case $28 \mathrm{I}$. wise negative, as are the lungs. The arteries are rough and tortuous. The pulse appeared to be one of high tension. Hemoglobin, 25 per cent.

The abdomen and extremities show nothing abnormal. The course of the temperature is seen in the accompanying chart (Fig. 136). The urine averaged 30 ounces in twenty-four hours, with a specific gravity of ror2; a slight trace of albumin was found, but no casts.

Discussion.-In looking for a cause for the anemia here present we notice that the kidneys do not seem to be doing much work, and might be rash enough to assume that some type of nephritis is responsible for the symptoms. It is true that nephritis may be in itself the cause of very intense anemia, but is there any sufficient evidence that this man has a nephritis at all? The total solids excreted are certainly very deficient, but this may be the result merely of insufficient food. Although we know very little about his diet, it is safe to assume that he does not eat enough to give him the normal output of urinary solids.

Emaciation is at least as important a feature as the anemia in this patient. $\mathrm{He}$ is at the age when very considerable emaciation often occurs merely as the result of the aging process- $i$. e., of arteriosclerosis. Such, at any rate, seems to me the reasonable conclusion as we observe the rapid loss of weight which takes place in a large proportion of elderly persons without any corresponding change in the diet. It must be confessed, however, that the evidence of arteriosclerosis in this patient is not conclusive. Many patients whose arteries are rough and tortuous turn out, postmortem, to have very little arteriosclerosis, and the high pulse tension which would seem to verify, to a certain extent, the hypothesis of arteriosclerosis, was based merely on digital examination-a most unreliable procedure. 
Pernicious anemia is probably the commonest cause of an extreme reduction in the hemoglobin percentage at the age of sixty-three. In most cases of pernicious anemia, emaciation is comparatively slight; sometimes it is absent altogether. But this fact does not by any means suffice to exclude pernicious anemia in this case. The blood must be much more carefully investigated.

Outcome.-The red cells were found to number 863,000 , so that the hemoglobin, though very low; was yet relatively high (color index, r.4). The leukocytes numbered 4200,58 per cent. of which were polynuclear, with $4 \mathrm{I}$ per cent. of lymphocytes and I per cent. of mast cells. During a differential count of 200 cells, four normoblasts and two megaloblasts were found. The red cells were of huge size, deeply stained, and much deformed. Many of them contained basophilic granulations or showed diffuse abnormal staining reaction.

The blood-pressure was only $100 \mathrm{~mm}$. $\mathrm{Hg}$. The patient rapidly failed and died on the twenty-first.

Diagnosis.-Pernicious anemia.

\section{Case 282}

An Irish housewife of forty-two, of good family history, had malaria fifteen years ago; a still-born child last May; no other children or miscarriages.

Her chief complaint at the present time is of weakness, affecting especially her back. She entered the hospital on April 27, 1908. Six months previously she had had a good many dizzy spells, with insomnia and much nervousness. At that time she was five weeks in a hospital, but no diagnosis was made. At the present time she has a good appetite and sleeps well.

Her catamenia are regular, but she believes herself to have some pelvic disease, and vomits occasionally without relation to food.

Physical examination shows an obese woman, with a dry skin and numerous rose-colored papules scattered over the front of the chest and abdomen. The course of the temperature is seen in the accompanying chart. The chest and abdomen showed nothing abnormal. The reflexes, blood, and urine were negative. Vaginal examination showed no pelvic disease.

Discussion.- The papules here described had all the characteristics of rose spots, and would have passed perfectly well for the exanthem of typhoid fever had any pyrexia been present. In the absence of fever no obvious explanation was found for them. It may be worth stating here that, even in febrile conditions, the rose spot, although most valuable 
as confirmatory evidence of typhoid, is by no means pathognomonic of that disease. The typhoid bacillus is not the only germ which is prone to settle beneath the skin and produce the hyperemic area known as a rose spot. I have seen the same thing in pyogenic sepsis many times, and in tuberculosis once.

The patient is stated to be obese. Is this enough to account for her weakness? Occasionally one sees persons for whose exhaustion and incapacity no other cause can be found. But I have never known a patient to enter a general hospital on this account. Further, there has

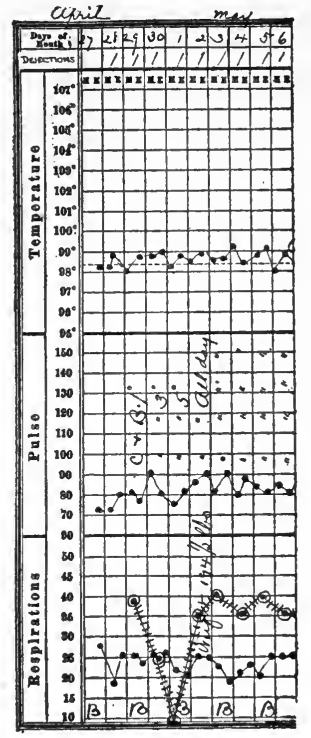

Fig. 137.-Chart of case 282. been no special increase in the amount of fat during the period occupied by her illness.

Myxedema is sometimes mistaken for obesity, and often causes a very troublesome weakness. In the present case, however, we have no good reason to believe that myxedema is present. There are no cutaneous or mental symptoms, no subnormal temperatures, nor special sensitiveness to cold. The facial expression is unchanged.

If physical examination, repeatedly and conscientiously performed, is wholly negative in a case of this kind, it is proper to investigate the mental condition of the patient. Subconscious fears and internal tensions may be enough to account for all the troubles of which this patient complains, though we should never assume anything of the kind until every other possibility has been exhausted. In the search for a psychic cause it is never sufficient to ask a patient such a question as, "Are you worrying about anything?" or "Have you anything on your mind?" The worries which do the most harm physically are those of which the patient is partially or quite unconscious. Of course, the only proof that our diagnosis is right, when we believe we have succeeded in drawing out of the depths of a patient's consciousness some submerged cause of internal strife, is the physical results. If immediate improvement follows, it is reasonable to suppose that we have hit upon the source of the trouble.

Outcome-It developed later that after her child was born and she had left the hospital, she was told that she was "in a bad way"; this idea fermented in her mind and apparently was the basis of her present troubles. 
During two weeks' observation she seemed to be perfectly well, and after being officially reassured, her sensations corresponded to her good digestive condition.

Diagnosis.-Apprehension.

\section{Case 283}

An Irish chambermaid of twenty-two, of good family and past history, was seen December 18, 1906. She came to the United States four months ago. Her menstruation began at the age of fifteen and has always been regular, but her last period occurred on the steamer during her passage to America.

A month ago she began to feel weak and unfit for work. This weakness was accompained by a palpitation on any exertion and sometimes by faintness. She has been very constipated all through her sickness, but has had no vomiting or other gastric symptoms, and no cough or fever, so far as she is aware.

On examination the girl is well nourished, with bright red cheeks, but somewhat pale and slightly bluish lips. The glands are palpable in the neck, axillæ and groins, but not enlarged. The heart seems to be of normal size, its action regular, but there is a rough systolic murmur heard best at the base and transmitted to the left axilla. The pulmonic second sound is distinctly louder than the aortic.

The lungs show scattered coarse râles.

Visceral examination is otherwise negative. The patient weighed $165 \frac{1}{2}$ pounds. Her pulse, temperature, respiration and urine were normal throughout three weeks' observation.

Discussion.-Could this girl be pregnant? The amenorrhea, weakness, palpitation, and fainting are consistent with that diagnosis, which could only be confirmed, however, in case the uterus was found to be demonstrably enlarged. We should expect also some gastric disturbances and changes in the breasts. Since none of these necessary confirmations appear to be present, we must look for some other cause for the amenorrhea.

Vegetative endocarditis produces general weakness without localizing symptoms. It had been considered by the attending physician on account of the rough murmur over the base of the heart. But such a diagnosis needs a great deal more evidence before we can be content with it. The pulmonic second sound, though louder than the aortic, did not appear to be abnormal, and there was no fever, chills or cardiac enlargement. Regarding the leukocytes, which should be increased in number if endocarditis is present, we have as yet no information. 
Cases of early tuberculosis often have a history very much like this, and one should always examine the pulmonary apices with especial care in such a case. But without fever, loss of weight, gastric disturbances or cough we should not be warranted in entertaining any further the hypothesis of tuberculosis, nor in suggesting it to the patient or her family. Diffuse râles in both lungs are not what we expect to find in early tuberculosis, except in the miliary form, and then with much more virulent symptoms.

If the patient were pale, we should naturally suspect chlorosis. Everything else in the case seems consistent with that idea. Can a patient with bright red cheeks have chlorosis or any other form of anemia? Most certainly, and it is for this reason that I have introduced the case. Many like it are overlooked, I believe, because we have not the habit of making routine hemoglobin estimations. The color of the face is no guide. The majority of pale people are not anemic, and many anemics are not pale.

Outcome.-The red cells are 3,364,000; white cells, 3200; hemoglobin, 35 per cent. The stained specimen shows marked achromia, no nucleated cells, no abnormal staining or abnormal shapes. The differential count is also normal.

It was subsequently learned that before coming to this country she had always been used to out-of-door life, though during her work here she had been closely confined.

Under Blaud's pill, Io grains three times a day, the red cells had risen by the fifth of January to $4,400,000$, the hemoglobin to 60 per cent., and the girl felt entirely well.

Cascara was needed at the beginning of the treatment, but not after the first week.

Diagnosis.-Chlorosis.

\section{Case 284}

A Syrian thirty-seven years old entered the hospital June 27, I906. He has had many touches of malaria, and takes three whiskies a day. Otherwise his history was not notable until seven months ago, when he began to lose strength and got run down. For the past three months he has been rapidly growing weaker. At no time has he had any pain or other localizing symptoms except at the very beginning of his illness, when he had a rather indefinite pain in the right shoulder and right axilla. This passed off within a few weeks, but has returned again of late.

He has no cough and no dyspnea, but within the past week he has 
taken $\frac{1}{4}$ grain of morphin every night to make him sleep. For four or five days he has been in bed. Two weeks ago he noticed for the first time that his feet were swollen.

On examination the man was emaciated. The right chest was flat in front below the third rib and below the spine of the scapula above. Breath-sounds and voice-sounds were absent over the same area.

The heart's impulse was in the fifth space, and reached $\mathrm{I}_{4}^{\frac{3}{4}}$ inches outside the nipple-line. The right border could not be determined. The pulmonic second sound was accentuated. There were no murmurs. Blood-pressure, I40.

The abdomen was held rigidly throughout. It was tympanitic, not tender. There was soft edema of the lower legs and feet, also some over the sacrum. The white cells were 15,000 ; hemoglobin, 85 per cent. The course of the temperature is seen in the accompanying chart.

Discussion.-The essential features of the case are: fever, weakness, displacement of the cardiac apex, edema of the feet, and apparently fluid in the right chest.

Pleurisy is naturally our first thought, but we are puzzled by the absence of pain, cough, or dyspnea, and by the presence of swelled feet. Can the latter symptoms be the result of a pleurisy, or must we suppose that both the swollen feet and the thoracic fluid are the results of some common cause, perhaps disease of the heart or kidney?

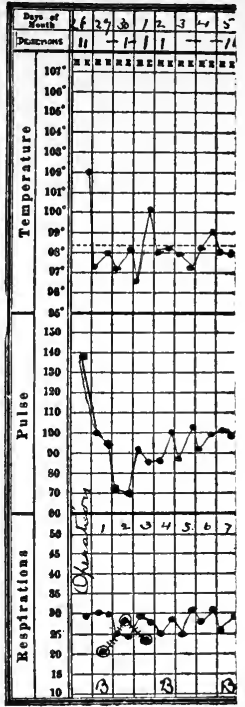

Fig. I38.-Chart of case 284 .

If the heart is diseased, we should expect either a murmur, a change in blood-pressure, an arhythmia, or some other evidence besides dropsy. Further, it is difficult to explain the fever as the result of heart disease unless there is a vegetative endocarditis, in which case there should be a murmur, though this is not invariable.

No more positive evidence can be obtained without tapping the chest. The characteristics of the fluid thus presumably to be obtained should decide the question. Meantime it may be suggested that in children such a group of symptoms would be clearly indicative of empyema. What happens frequently in children may occur now and then in adults.

Outcome.-Exploratory puncture showed foul pus on the twentyeighth. Next day a rib was resected, and several pints of the same fluid removed. The pus showed no growth on ordinary culture-media. 
The patient did well for the week following operation, and went home with a small discharging sinus. His further progress could not be traced.

Diagnosis.-Empyema [tuberculous?].

\section{Case 285}

A boy of four years, with good family history, entered the hospital May 27, 1908. He had always been well until nineteen days ago, when he complained of being tired, and seemed listless and disinclined to play. Soon after this he began to be feverish, especially at night.

For the past fifteen days he has been in bed. At no time has he complained of any pain. His appetite has been good, his bowels regular, and he sleeps well. He has had a little dry cough for a week. (The course of the temperature is seen in the accompanying chart, Fig. 139.)

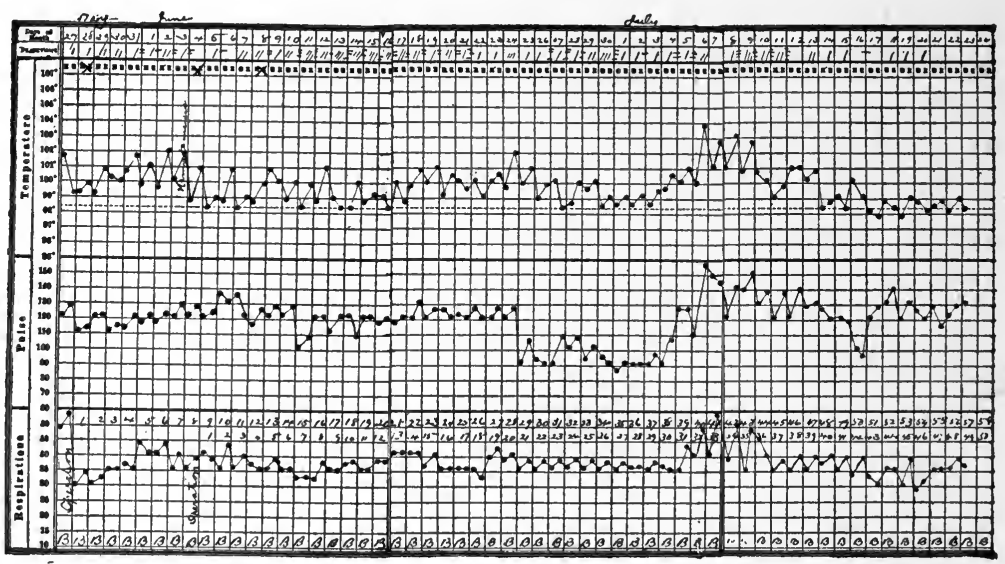

Fig. I39.-Chart of case 285 .

Physical examination shows a well-nourished child, with pink cheeks and a freckled face. His left tonsil is enlarged, his throat somewhat reddened. The heart's impulse is in the fourth interspace, just outside the nipple-line. The organ seems otherwise normal.

The right chest in front is flat below the fourth interspace, the line of flatness rising in the axilla, so that it reaches to the top of the chest behind. Respiration is normal over the left chest, harsh in the front of the right chest, above the line of flatness; below that it is nearly absent.

Just below the midscapular level behind, there is a spot of bronchial breathing and a few crackling râles. Voice-sounds are slightly in- 
creased at this point. Elsewhere they are absent. The edge of the liver is felt one finger's-breadth below the ribs.

Discussion.-One could hardly make a mistake regarding the diagnosis in this case if he placed his reliance upon physical signs. What I desire specially to point out is the misleading character of the history. The child has no pain in the side, almost no cougb, no dyspnea, nothing to call one's attention rather to one part of the body than to another.

Relying on the physical signs, we cannot doubt that there is fluid in the right chest. The small spot of bronchial breathing near midscapula is not in the least in contradiction to this diagnosis. Indeed, we generally hear bronchial breathing in some part of the chest of a young child when fluid is present in large amounts. Pneumonia, the only other disease which we could consider at all, never has so insidious an onset or so prolonged a course in young children.

When we know that a child's chest contains fluid and have no reason to suspect disease of the heart or kidney, empyema is far commoner than serous pleurisy. Insidious serous effusion, so common in adults as a result of tuberculosis, is distinctly rare in infancy, while the pneumococcus infections leading to empyema are common, and present, as a rule, just such a clinical picture as I have here reproduced.

Outcome.-On the twenty-eighth the chest was opened, with the escape of ro ounces of pus teeming with a growth of pneumococci. The child's convalescence was prolonged and often interrupted by the retention of pus in subsidiary cavities, owing to unsatisfactory drainage. Recovery was ultimately complete.

Diagnosis.-Empyema.

\section{Case 286}

A Scottish salesman, seventy years old, of good family history and past history, entered the hospital November 8, I906. He gave up work six months ago on account of progressive weakness. About a month later he noticed that gas gathered in his stomach about twenty minutes after eating, causing considerable noise and some nausea. He has at no time any pain, but has gradually become weaker, paler, and more short of breath. Within the past three weeks his legs have swollen; his skin has turned yellow and itched. Ten months ago he weighed 195 pounds, now he weighs 155 .

Examination showed obvious loss of weight; skin pale, and of a yellowish tinge; no demonstrable jaundice, the color being more like that of pernicious anemia. The heart showed a systolic murmur, 
audible all over the precordia and in the left axilla. The sounds were faint and distant. There were no evidences of enlargement and no irregularity. Visceral examination was otherwise negative, except for a slight puffiness of the face and hands, and a moderate, rather brawny edema of the lower legs.

The red cells were 2,328,000; white cells, 5000; hemoglobin, 25 per cent. There was a very marked serum ring around the bloodstain as I took the hemoglobin test by the Tallqvist scale. The differential count showed polynuclears, 59 per cent.; lymphocytes, 4I per cent. The red cells showed very marked achromia, moderate deformities in shape, no abnormal staining reactions, no blasts. The urine was altogether negative. A small amount of brownish material which gave a positive guaiac reaction was found in the fasting stomach. The organ held $5^{2}$ ounces, and after a test-meal showed no free $\mathrm{HCl}$.

Discussion.-Here we have the symptoms of pernicious anemia but the blood-picture does not correspond. It is a familiar puzzle and an important one. As a result of a fairly extensive experience in dealing with this particular problem I think it may be stated that it is the part of wisdom to follow the indications of the blood-examination in such cases. Primary anemia does not produce a blood-picture like that here described in patients of this age. The most distinctive features are the achromia and the low color index.

Assuming, then, that we are dealing with secondary anemia, what is its cause? In men of this age severe secondary anemia is produced usually by cancer, syphilis, or hemorrhage. The anemia of nephritis or of cirrhosis usually occurs in younger persons. Since we have no evidence whatever either of syphilis or hemorrhage, cancer is the most probable diagnosis. But what is the seat of the tumor? Such slight indications as we possess seem to point to the stomach. The recovery of a material reacting positively to the guaiac test, the absence of hydrochloric acid, the slight enlargement of the stomach, the nausea and flatulence tend to confirm this indication.

The case is of special interest because of the absence of pain and vomiting. It tends to substantiate the old rule, which bids us suspect gastric cancer whenever a patient, previously free from digestive disturbances begins in later life to have any gastric symptoms, however slight.

Outcome.-The patient was given forced feeding and oxygen by rectum in the hope of inhibiting the growth of anaërobic bacteria, but on the fourteenth of November he was so weak that he could not walk alone. The blood examination then showed: Red cells, I,820,000; 



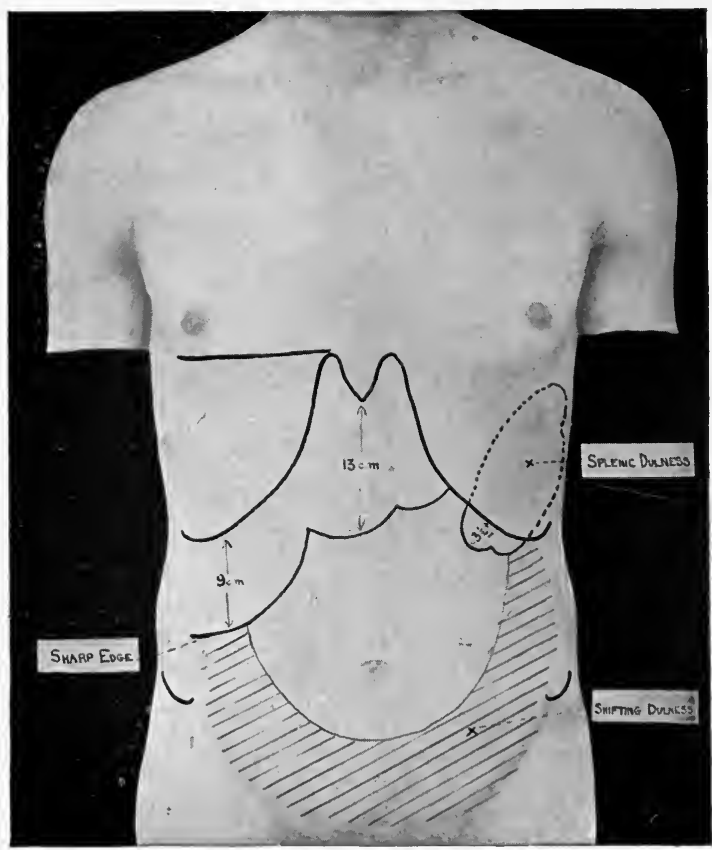

Fig. 141.-Findings in a case characterized chiefly by weakness and swollen belly (four weeks' duration). 
white cells, 3200; hemoglobin, I2 per cent. The stained specimen showed essentially the same as the previous examination.

The patient died on the seventeenth. Autopsy showed a large, cauliflower growth at the pylorus, almost obstructing its lumen; carcinoma histologically.

Diagnosis.-Gastric cancer.

\section{Case 287}

An Italian housewife, fifty years old, entered the hospital June $\mathrm{I} 7$, 1907. She began to complain four weeks ago of weakness and fatigue. It was also noticed that she passed very little urine.

For three weeks she has been in bed, complaining mostly of weakness, accompanied by anorexia, insomnia, and constipation. Her mouth is dry and she is very thirsty. There is a dull, constant epigastric pain. Last week she vomited twice small quantities of green fluid. She has no headache; her eye-sight is good. The urine continues scanty.

On examination the patient is found to be somewhat emaciated. Her chest shows nothing abnormal except a few crackles and squeaks in the lower part of each lung.

The abdomen is protuberant, the umbilicus pushed out, and there is shifting dulness in the flanks. The condition of the epigastrium is shown in the accompanying diagram, and the temperature in the accompanying chart (Fig. I40).

The blood and urine show nothing abnormal. The circumference of the abdomen at the level of the navel is $80 \mathrm{~cm}$.

Discussion.-Cirrhosis of the liver was the "snap diagnosis" in this case, suggested, of course, by the insidious onset of extensive ascites. Although this disease cannot be excluded from consideration, there

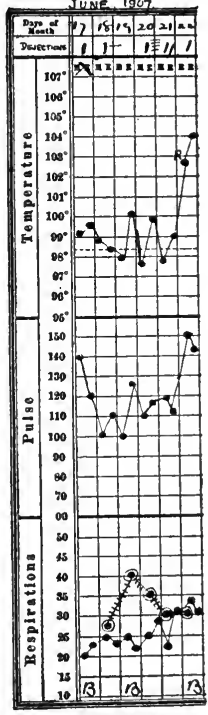

Fig. 140.-Chart of case 287 . are a number of points against it. The most important is the patient's pain, a symptom of which we hear practically nothing in cirrhosis. Further, Italian wine, which is all that this patient has taken, does not often produce cirrhosis. Finally, the surface of the liver, which is stated to be rough on palpation, is not characteristic of cirrhosis from the clinical point of view. The hob-nails of the hob-nail liver are almost never to be felt through the abdominal wall. I have known many. cases where they were felt, but not one of these cases turned out to be 
cirrhosis. The supposed hob-nails proved to be nodules of fat in the abdominal wall or irregularities due to cancer or syphilis.

The course of the disease seems very short and rapid for syphilis. The transition from perfect health to great prostration, with ascites, anorexia, and vomiting is rarely brought about by syphilis within four weeks. Of course, it may well be that the history is inaccurate, especially as it was obtained through an interpreter. If we disregard the history, the chief evidence against syphilis is the absence of any luetic lesions in other parts of the body. Without the therapeutic test, however, it is impossible positively to exclude syphilis in this case.

Tuberculous peritonitis is not common at this age, rarely produces so much prostration, and would not explain the enlargement of the liver and spleen, or what we take to be such. Cancer of the liver would explain most of the symptoms, and would account for the rapid march of the malady. It is surprising, however, that no more marked gastric symptoms are complained of. Hepatic cancer usually shows itself as a later development in an illness characterized by months of severe digestive disturbance. Possibly the cancer may have originated in some "silent," deep-seated organ, whence it was extended by metastasis to the liver.

Banti's disease is always to be suspected when an Italian is found to be suffering from enlargement of the liver and spleen with ascites. It must be admitted, however, that the clinical picture of Banti's disease has seldom been clearly recognized except by Italian writers. It is essential, at all events, that we should be able to demonstrate an enlargement of the spleen preceding by a considerable period the onset of ascites and hepatic hypertrophy. Nothing of the kind can be shown in the present case.

The most reasonable diagnosis, therefore, appears to be cancer of the liver, origin unknown.

Outcome.-June I8th it was learned that she had received large doses of mercury and potassium iodid before entrance, hence this clue was not followed up any further. The patient died on the twentysecond. Autopsy showed primary cancer of the liver with metastases in the mesenteric glands, spleen, pancreas, lungs and thyroid.

Diagnosis.-Cancer of the liver.

\section{Case 288}

A Jewish married woman, thirty-four years old, was first seen February 4, I907. Her father died of kidney disease; her family 
history is otherwise good. She had chlorosis when she was seventeen. She has had three children, the youngest five years old.

For eighteen months she has felt weak, and in that time she has lost about 30 pounds. Her appetite has been poor throughout this period. At times she has been feverish. She often has a bitter taste in her mouth after eating. Four weeks ago she had a severe left-sided headache; since then she has heard roaring noises in her head, and has felt still weaker than before.

Two weeks ago she had two similar attacks within a week, and the headache has been continuous for the last five days. With each of these attacks of headache she has vomited, but there has been no other pain. When her eyes were examined recently at the Eye and Ear Infirmary they were found to be normal. Throughout the eighteen months of her illness she has had diarrhea alternating with constipation.

The patient was poorly nourished, the skin brightly colored. Visceral examination was wholly negative, as was the blood examination. The urine was free from albumin and casts; twenty-four-hour amount normal.

Discussion.-Weakness and loss of weight with headache and digestive symptoms are complained of by innumerable Jewesses of this age without our being able to discover any more definite cause than their self-starvation and a psychoneurotic constitution. If the physical examination is wholly negative and no drug habits can be discovered, the case will have to be treated on this basis.

We must first make sure, however, that nothing of any importance has been omitted from our physical examination. Are we quite certain that no hints of larval hyperthyroidism are to be found? No tremor, tachycardia, profuse sweating without cause or slight thyroid enlargement? All these signs were searched for in the present case, with negative results.

One all-essential point, however, is omitted in the account of the case printed above, because it was absent from the record presented to me when I saw the case in consultation. There is no record of the test for sugar nor of the specific gravity of the urine, which turned out to be ro4o.

Outcome.-Five and a half per cent. of glucose was found in the urine. In the course of a month, however, this disappeared under a diet of increased fats and diminished carbohydrates. The headache, which had been throughout her chief complaint, disappeared as soon as the urine became sugar-free. In the course of the month under my observation she gained six pounds.

Diagnosis.-Diabetes mellitus. 


\section{Case 289}

A school-boy of fifteen was first seen on November 28, 1907. His family history is good, and he has always been well until three weeks ago, when he began to complain of weakness, headache, vertigo, and slight nausea.

Six weeks ago he weighed 99 pounds; now he weighs 8I. His appetite is good, his bowels regular, and there is no vomiting.

Physical examination shows emaciation and mental dulness.

The edge of the liver is felt on inspiration. Physical examination, including the blood, is otherwise negative. The urine is pale, its quantity from 3500 to 5000 c.c. in twenty-four hours, the specific gravity never far from I030; amount of sugar, 5.5 per cent., gradually rising to 7.5 per cent. during the five weeks of his stay in the hospital.

Discussion.-This case is introduced merely as a further exemplification of the fact that diabetes may occur without any of the cardinal symptoms on which we often rely for diagnosis. This boy complained of no thirst, had no increase of appetite, and, so far as he knew, no polyuria. The diagnosis was simple enough as the result of a routine examination, including, as all such examinations should, a test for sugar.

Of some interest, I think, is the outcome of the treatment, which, though it sufficed merely to prolong the boy's life for a month, undoubtedly did accomplish as much as this. Such a respite is sometimes of very great importance when a relative wishes to come from a distance or when a financial matter has to be finished up.

I may call attention also to the convulsions which occurred as a part of the terminal acidosis. Diabetes is not often mentioned among the possible causes of convulsions, because there is so rarely any diffculty in recognizing spasms of this type, occurring as they do at the end of a prostrating illness, the nature of which is not likely to have been in doubt. When a convulsion occurs "out of a clear sky" in a patient not known previously to be ill, it practically never turns out to be due to diabetes.

The purpura noted in the outcome was doubtless of the cachectic type.

Outcome.--As he showed every sign of impending coma at entrance, he was saturated as rapidly as possible with sodium bicarbonate, given both by mouth and intravenously. Sodium bicarbonate, 250 c.c. of a 2.5 per cent. solution, was given, and next day 350 c.c. of a 5 per cent. solution of glucose was administered intravenously. This was followed by a very marked improvement. 
On account of the very marked acidosis the patient was given an unmodified diet, the bowels kept open by enemata, and his appetite stimulated by bitter tonics. He was kept out-of-doors daily, well wrapped up, in bed.

December roth the diet was slightly restricted, omitting starchy soups, though cereals, bread, milk and potatoes were given without restriction. Chewing-gum was allowed on his request, and gave much relief to the dryness of the mouth.

Impending coma was again relieved on the fifteenth by a treatment similar to that previously given, but it seemed wise not to attempt further to restrict the diet.

He steadily lost weight and strength, and any exertion made him drowsy.

On the thirtieth numerous purpuric spots appeared on the trunk, arms and legs, and he began to have drowsiness, which rapidly increased to complete coma, after which he had a series of general clonic convulsions lasting from thirty to sixty seconds each. At midnight he died.

Diagnosis.-Diabetes mellitus.

\section{Case 290}

An Irish painter of thirty entered the hospital November 25, 1907. His mother died of cancer, and he has lost one sister of consumption. $\mathrm{He}$ is a hard drinker every Saturday, rarely drinking during the week. $\mathrm{He}$ had gonorrhea three times, but denies syphilis.

For six weeks he has complained of weakness in his legs and backhe says his legs won't hold him up. His joints are lifeless and he cannot go upstairs. There is no swelling of his joints, and he has no pair except on stretching the muscles. He has lost no weight. His appetite is good, and he feels well except for the above complaint. He quit work two weeks ago, but has not been in bed.

Physical examination shows slight irregularity of both pupils, without any other abnormality there. There is no lead line.

The heart is slow-6o to the minute, with a prolonged diastolic pause. The artery walls are firm, but not nodular; lungs normal, abdomen rather spastic, not otherwise remarkable. There is wellmarked left varicocele.

The knee-jerks are absent even on reënforcement. Kernig's sign present on the right; well-marked Romberg sign; superficial reflexes lively; no Babinski; temperature, pulse, respiration; and urine are normal. 
Discussion.-Most patients who come to us complaining of weakness in the legs have some disease of the nervous system. Occasionally a case of diabetes or of cardiac trouble brings this symptom into the foreground, but, as a rule, all general diseases outside the central nervous system have some chief complaint or complaints other than weakness.

Among diseases of the nervous system some type of neuritis is suggested because the pupils are normal, the reflexes diminished, and the symptoms bilateral. The occupation of the patient naturally prejudices us in favor of lead-poisoning, but as there is no lead line, no colic, no special involvement of the extensor muscles (toe-drop), we are inclined to canvas the other possible causes of neuritis first.

As an alcoholic he has a perfect right to alcoholic neuritis, though we see no special reason why it should come on now rather than sooner. Most, if not all, cases of alcoholic neuritis, however, present some sensory symptoms. This patient has none.

Tabes dorsalis is very unlikely on account of the normal reaction of the pupils and the entire absence of sensory symptoms such as usually occupy the foreground in tabes.

One possibly decisive test has been omitted-blood examination. Alcoholic neuritis rarely if ever produces any marked basophilic stippling of the red cells; saturnine neuritis practically always does. To this question, therefore, it is reasonable next to turn our attention.

Outcome.-Blood examination showed hemoglobin, 70 per cent.; leukocytes, 5500. In the stained smear the red cells exhibited marked achromia and a great deal of stippling, but no other abnormalities.

Under potassium iodid-ro grains thrice daily-the patient began to improve at once, and by the thirteenth of December could walk fairly well, though a slight exertion put him out of breath.

Diagnosis.-Lead-poisoning (?).

\section{Case 291}

A married woman, forty-three years old, with an excellent family history, past history, and good habits, entered the hospital October I3, r906. She had always been well until a year ago, when she noticed that she was gradually growing weak. She had no pain anywhere; her appetite remained good and her bowels regular; but some months later she noticed that the abdomen was increasing in size and that she was short of breath on exertion. At this time she was much annoyed by noises in her left ear and by attacks of vertigo. Throughout the past year her weakness has steadily increased and is her only complaint at the present time. 
$-$

$5^{6}$ 


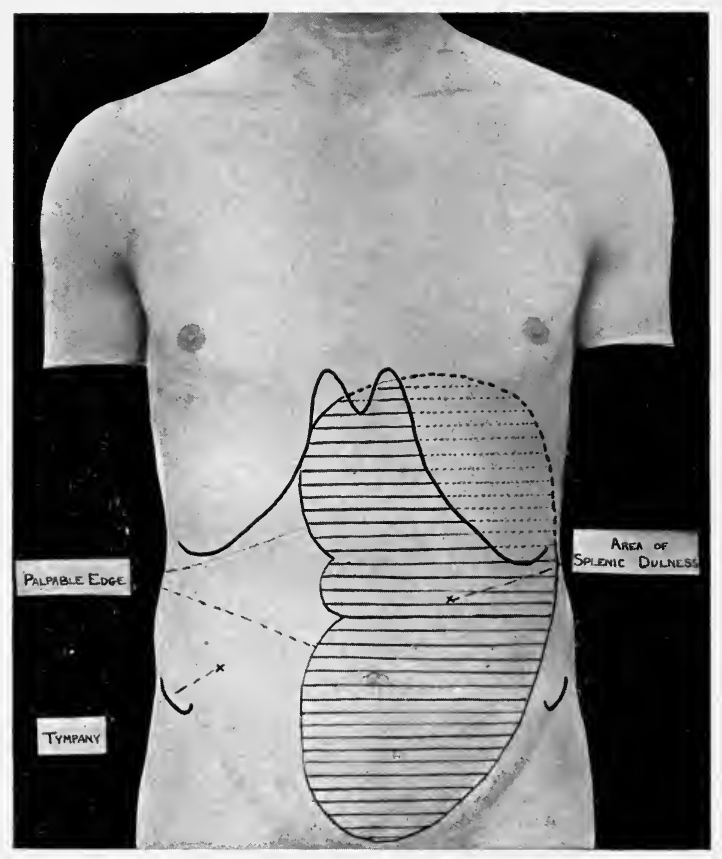

Fig. 142.- Showing results of percussion and palpation in a patient complaining only of weakness and abdominal enlargement. 
Four years ago she weighed I60 pounds, now she weighs II 7 .

On examination the patient is somewhat pale, but the hemoglobin shows 70 per cent. She is well nourished.

The heart is negative, save for a soft systolic murmur, best heard in the third left interspace, and not transmitted. The vessels of the neck pulsate rather strongly. The lungs are entirely negative. The abdomen shows a marked prominence on the left side, and dulness as shown in the accompanying diagram (Fig. I42).

Discussion.-One could hardly make a mistake in the diagnosis of this case unless one were in the habit of relying on symptoms rather than on the results of physical examination. No one could fail to notice the abdominal tumor if he had palpated the abdomen with any care. No one with any knowledge of physical examination could have any doubt that that tumor was due to splenic enlargement.

Splenic enlargement associated with such evidences of anemia as this patient presents is characteristic of three diseases seen in temperate climates, and among those who have never visited the tropics. The huge "ague-cake" of chronic estivo-autumnal malarial or kala-azar need not be considered in any patient who has never been out of New England. Leukemia, splenic anemia, and syphilis are the only diseases which we need to consider. Leukemia can be instantly recognized by the blood examination. Splenic anemia and visceral syphilis may be almost indistinguishable unless other evidence of syphilis can be obtained from the history or in the physical examination.

Outcome.-Examination of the blood showed: red cells, 2,656,000; white cells, 652,000 . Differential count showed polynuclears, 54 per cent.; myelocytes, $3^{8}$ per cent.; eosinophiles, 3.5 per cent.; mast cells, 2.5 per cent.; lymphocytes, 2 per cent.

The red cells were well stained and showed no special abnormalities. This blood-picture did not change appreciably during the month in which the patient was under observation.

The patient was much more comfortable as the result of an abdominal support which held up the enlarged spleen.

Under $x$-ray treatment she seemed to be getting steadily better until the ninth of November, when she had a slight pain in the left back, which later in the evening became severe; morphin, $\frac{1}{8}$ grain by mouth, was vomited.

Respiration was slightly quickened. At 2 o'clock in the morning of November roth the patient had a chill and vomited. The pulse rose to 130 ; respiration was very rapid; temperature, $99.4^{\circ} \mathrm{F}$. Morphin, $\frac{1}{6}$ grain subcutaneously, gave some relief; but at 4.50 the respiration 
suddenly ceased. No cause for this sudden death was found either before or after the autopsy, which showed the lesions of myelogenous leukemia.

Diagnosis.-Myeloid leukemia.

\section{Case 292}

A married woman of forty-four was first seen on July I0, I907. Her family history is entirely uneventful. She was said to have had cerebrospinal meningitis when a child, and has ever since been subject to headaches and nose-bleeds. The menopause occurred five years ago. She has had no children and no miscarriages.

She takes two glasses of beer a day, but never takes whisky. She drinks the Boston city water through a lead pipe.

Two weeks ago she became so weak that she fell to the floor, striking the back of her head. She was unconscious for about an hour, and woke up in bed, where she had been put by her husband. Since then she has been unable to stand unless supported, although she can move her legs readily in bed.

During the past four days her arms and fingers have become numb and lifeless. She can hold a knife and fork, but she cannot lift a glass of water. Her speech has not been affected, and sphincteric control is perfect. She has no headache and sleeps well. The bowels are constipated; her appetite is poor.

On examination the patient shows loss of weight; the arteries are palpable and tortuous above the elbow; the heart, lungs, and abdomen are negative, except for a sharp edge felt underneath the right ribs. The knee-jerks are not obtained. There is general tenderness over the nerve-trunks of the legs. The grip of both hands is weak, and the extensors of the wrist are likewise weak.

There is no lead line. The blood shows no stippling. The urine is normal and contains no arsenic.

Discussion.-Evidently this is a different type of weakness from that of the cases we have been previously studying. It is referred more definitely to legs, and appears suddenly. Especially when we take account of the condition of the nervous system, as revealed by physical examination, we are clear that the case does not belong with those in which weakness is due to cardiac or toxemic conditions.

The possible connection of lead with the trouble is naturally our first thought, since the history mentions a lead pipe. It must be remembered, however, that in the vast majority of cases, water coming through lead pipe between the street main and the facet within the house does 
not become impregnated with lead and does no harm to any one. More over, in this patient the tissues most sensitive to lead and most apt to show its influence as soon as poisoning begins, are here evidently untouched. There are no changes in the gums or in the blood, no colic, no arthritis, no encephalopathy.

Arsenical poisoning has now gone out of fashion, partly, I believe, because the neurologists have grown tired of it and are fonder of the term "neurasthenia" or "psychoneurosis," partly because our wallpapers are now freer from arsenical dyes. The absence of arsenic from the urine in the present case would probably be accepted as conclusive evidence against the presence of arsenical poisoning. On the other hand, it must be remembered that a considerable proportion of the community often passes arsenical urine from time to time while in perfect health, so that the demonstration of the mineral is by no means proof of arsenical poisoning. Negative evidence is here better than. positive.

Epidemic poliomyelitis is apt to occur in the hot summer months, in one of which this patient was attacked. It is, however, very rare at her age, does not often attack both legs or produce such incomplete paralysis of the muscles involved. Tenderness over the nerve-trunks is not common.

The tenderness just referred to enables us to rule out other types of myelitis which would in any case be unlikely to produce so mild a disturbance of motion, without increase of reflexes or involvement of the sphincters. Alcoholic neuritis would produce practically all the symptoms here complained of, but the amount of alcohol which she admits having consumed seems insufficient to produce so severe a trouble. If no other cause can be discovered, however, we may have to disbelieve her story.

Beyond any reasonable doubt she has a multiple neuritis; as she has had no fever, we cannot call it an infectious type of neuritis, and all other varieties, except that referred to in the last paragraph, appear to have been excluded. On the whole, alcoholic neuritis seems the most reasonable diagnosis.

Outcome.-Upon cross-questioning the patient later admitted that she had been taking four bottles of ale daily for a number of months. As a result of continued abstinence, with good hygiene, she recovered entirely in the course of three months.

Diagnosis.-Alcoholic neuritis. 


\section{Case 293}

A freight-handler of twenty-six entered the hospital August 14, I907. He had always been previously well except for an attack of fever five years ago. He began to lose his appetite and his strength two weeks ago. A week ago he was so weak that he gave up work.

In the last ten days he has vomited almost everything he has eaten and has had very little appetite. He has also had a cold in his head, with a little cough and pain in the right side of the chest. His bowels are regular, but he sleeps poorly.

Physical examination shows good nutrition; the heart's apex is in the fifth space in the nipple-line, but the heart-sounds are also distinctly heard to the right of the sternum.

There is dulness at the base of the right lung below the angle of the scapula, accompanied by diminished vocal and tactile fremitus, diminished breath-sounds, and crackling râles.

Physical examination, including the blood and urine, is otherwise negative.

Discussion.-Without the physical examination we have no clue. Even with it there seems to be but little to account for so much prostration, for it will be noted that the breath-sounds are audible, though diminished over the whole of the affected side. We must be dealing either with a very small accumulation of fluid or with a plastic pleurisy resulting in thickening. Is this enough to explain so much weakness and fever? (See chart on page 6r.)

Yes, it certainly is enough, as experience has repeatedly shown us, because it implies with reasonable certainty other tuberculous lesions in the lung itself, in the internal lymphatic glands, or elsewhere. Very probably that attack of fever five years previously was also due to tuberculosis, possibly also pleural in situation, though nothing of the kind was recognized at that time.

Of course, we must run over in our minds and exclude by our questions or physical tests the other familiar causes of weakness, such as anemia, psychoneurotic conditions, diabetes, concealed sepsis, and other infections. But this I think we can do with the aid of the data here presented. Doubtless we are right in being influenced to favor the diagnosis of tuberculosis in this case by our knowledge that many other cases demonstrated by the lapse of time to be tuberculous have begun just in this way.

Outcome.-On the thirty-first a needle was inserted in the back, $\frac{3}{4}$ inch below the angle of the scapula. The needle passed through 
fully $I \frac{1}{2}$ inches of thick, gritty, pleural exudate before any fluid was obtained, and only $\frac{1}{2}$ ounce came out.

The course of the temperature is seen in the accompanying chart (Fig. 143).

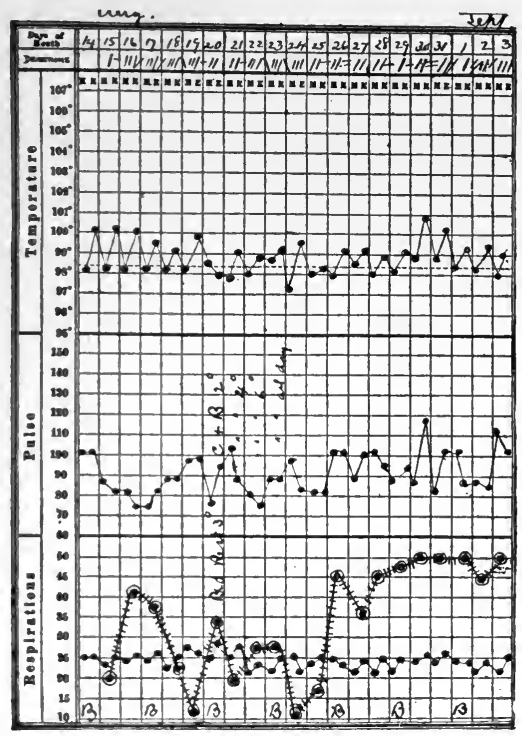

Fig. 143.-Chart of case 293.

The patient rapidly improved, and was discharged on the second of September.

Diagnosis.-Chronic plastic pleurisy.

\section{Case 294}

A shipper of thirty-eight was first seen April 9, I908. He has been in the habit of taking 20 glasses of beer a day, but his past history, as well as his family history, is otherwise negative. He has had pneumonia three times, the last time four years ago. A week ago he had the grip, from which he is now convalescing. He now feels pretty well, but weak.

Five days ago some albumin was found in his urine and he was put upon a milk diet. Lately he has vomited his milk. There has been no swelling of the legs or face. A year ago he weighed rgo pounds, now he weighs I7o. He has had a good deal of cough and sputa during the past week, but he thinks not previously.

On physical examination he was found to be rather stout. The 
heart is negative. The condition of the lungs is shown in the accompanying diagrams (Figs. I44, I45).

The abdomen was held firmly, and showed some dulness in the flanks, which, however, did not shift with change of position.

The leukocytes were 15,600 ; hemoglobin, 85 per cent.; temperature, $97 \cdot 4^{\circ} \mathrm{F}$; pulse, 96; respiration, 24; blood-pressure, I25 mm. $\mathrm{Hg}$. Urine negative.

The liver dulness extended from the sixth rib to the costal margin. The edge of the organ was palpable. The sputa showed large numbers of pneumococci; no tubercle bacilli on repeated examination. The ocular tuberculin reaction was negative.

Discussion.-This man is said to have had the "grip." Can his weakness be accounted for merely as a result of that disease? In the epidemic of influenza occurring in 1889 and 1890 the convalescence was notoriously slow and painful, but within the past few years I do not believe that we have had any cases of that type, so that I should doubt very much, even before scrutinizing the results of physical examination, any explanation of this patient's weakness as the result of such a type of influenza as could have been acquired in 1908 .

Such an albuminuria as is recorded above is quite often seen after mild attacks of tonsillitis or nasopharyngitis- $i$. e., a common cold. The urinary findings, therefore, do not imply that the previous infection has been anything more serious than a cold. But the physical signs in the lungs certainly do imply something more, and can be explained only as the result of some type of pneumonia or as the results of tuberculosis.

Delayed resolution in pneumonia is so rare that one should never make the diagnosis with confidence unless empyema, especially in the interlobar form, pulmonary abscess, and tuberculosis can be excluded. The negative tuberculin reaction is here of very considerable value. The negative results of sputum examination are also of some importance, especially as their number is considerable.

These two facts, together with the absence of fever and the presence of a transient albuminuria, should incline us to decide against tuberculosis, and in favor of some acute infection of the lungs, now probably in the stage of convalescence.

Outcome.-April $\mathrm{I} 7$ th the signs in the lungs were much less marked. April 28th examination showed nothing abnormal, and the patient felt quite well.

Diagnosis.-Convalescence from pneumonia. 


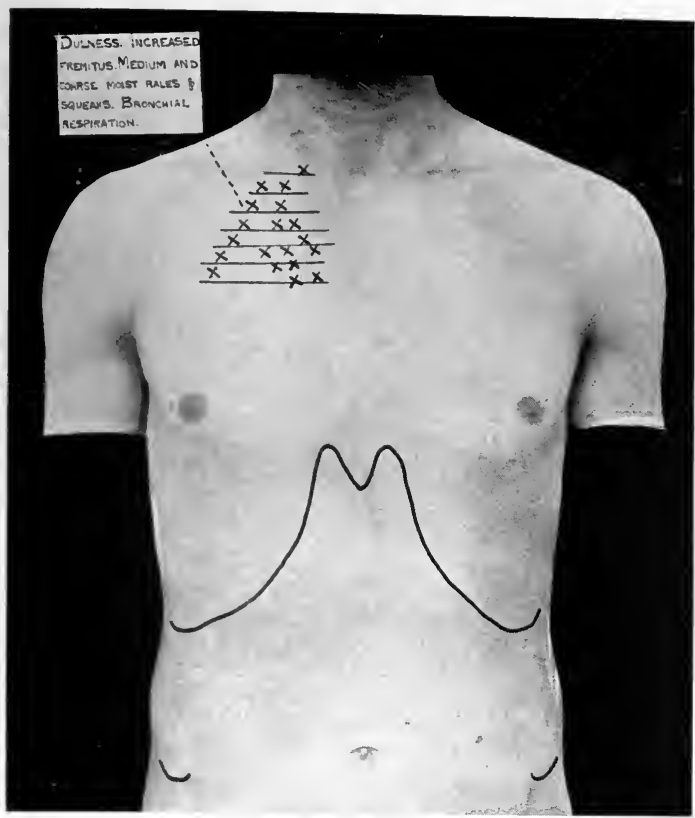

Fig. 144.-Represents what was found to explain weakness following an attack called "grip." (See also Fig. I45.)

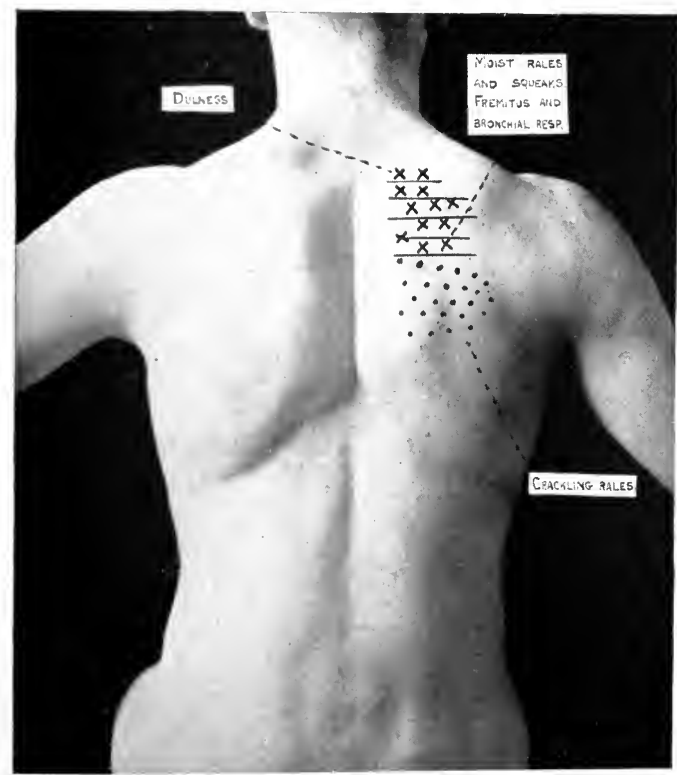

Fig. 145.-Signs found in the back (Case 294) of a patient complaining of weakness after an attack of "grip." (See Fig. I44.) 



\section{Case 295}

A Jewish peddler of seventeen, never previously sick, entered the hospital July 6, 1907 .

He was in bed four months ago for a few days on account of a slight cough and expectoration, which was never bloody. After a week he returned to work, but then he had pain in his legs, relieved by flat-foot plates. He still felt very weak and run down, and through the aid of the Social Service Department was sent to a farm, whence he returned after two weeks unimproved; indeed, since his return has been getting weaker. He is very easily tired and short of breath on exertion.

He says he has pains all over, a very poor appetite, cannot sleep, and is feverish and chilly at times.' (See Fig. 146.)

The patient was found to be poorly nourished. Physical examination was otherwise entirely negative, save for sharp lateral curvature of the spine to the right in the midscapular region. Tuberculin (o.I, I, 5, and Io milligrams) was injected subcutaneously, but was not followed by any rise of temperature or any constitutional symptoms. The blood and urine were entirely normal. Investigation of the gastric functions with a stomach-tube showed no fasting contents, a capacity of 40 ounces, and after a test-meal: free

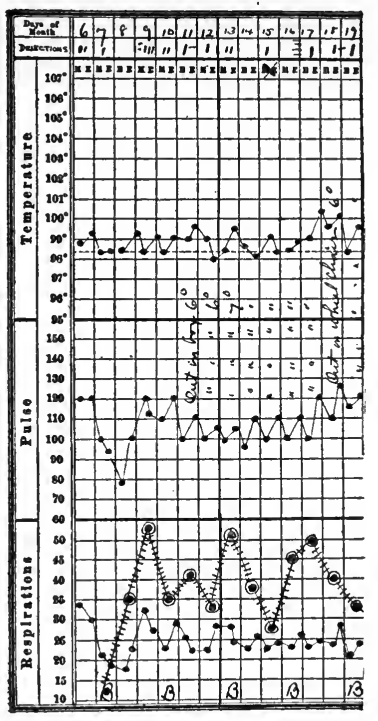

Fig. 146.-Chart of case 295 . $\mathrm{HCl}$, 0.18; total acidity, 0.44 .

Discussion.-The onset of this illness is very characteristic of tuberculosis. Cough, fever, anorexia, weakness, shortness of breath, chilliness, insomnia - all point in that direction. It will need the strongest kind of evidence to convince us that this boy is free from the tuberculous taint.

By continued observation, however, by repeated examinations of the lungs, and especially by the negative results of tuberculin injections, it was possible, in my judgment, to exclude tuberculosis.

The gastric functions were then carefully studied, but nothing of any importance as evidence of disease was discovered. By the study of the blood and urine we were able further to narrow the field of possibilities. No evidence of syphilis or other infectious disease 
could be found. The eyes and ears were examined; with negative results.

After all these tests had turned out negative, it seemed justifiable to return to that most obvious and yet most perilous diagnosis, a functional nervous derangement. The age of the patient and his race doubtless helped to justify such a diagnosis. The more one sees of adolescence, the more one is astonished at the apparent gravity but eventual transiency of the physical and mental symptoms exhibited by some healthy people at that period. Boys and girls who turn out quite healthy, sensible, and reliable in adult life, may be almost inconceivably weak, vacillating, hypochondriacal, and turbulent at that period. All their vital forces seem to be slowing down or hobbling along as if about to stop altogether. This applies to all races, but more especially to the Jews.

Outcome.-By the twelfth of July he was eating better and behaving as if he had some strength. He had received up to that time no drugs except an occasional dose of veronal, to grains, for insomnia. His blood-pressure on the twenty-second was II5 mm. Hg. At this time he looked and felt much better, but did not gain in weight. Potassium iodid was tried in large doses, but produced no improvement.

After eight weeks of observation, with careful study of the case, we were convinced that the patient's mental attitude had a great deal to do with his condition. After some reëducation he was discharged much relieved.

Diagnosis.-Psychoneurosis.

\section{Case 296}

A married woman of forty-three, of good family history, entered the hospital May 22, 1908. She had "inflammation of the bowels" twelve years ago and was sick for two months. Before and since that time she has been well until two weeks ago, when she began to feel tired and weak all over. She has had no pain anywhere, but her appetite has been poor. For a week she has noticed chilly sensations, with a scanty, highcolored urine. Two days ago she had a sore throat and took to her bed. Now the sore throat has disappeared. She has not been exposed to typhoid fever, so far as she knows. She has no cough.

The course of the temperature is seen in the accompanying chart (Fig. 147). A systolic murmur is heard all over the heart's area, loudest in the pulmonary area. The aortic second is louder than the pulmonic second sound. The heart's apex is in the midclavicular line, $4 \frac{1}{2}$ inches to the left of midsternum. 
The arteries and lungs show nothing abnormal. There is some dulness in the flanks, but this does not shift with change of position. The abdomen is otherwise negative. Blood cultures and Widal reaction were persistently negative. The white cells at entrance were 10,700, the urine normal. The urine was 25 to 30 ounces in twenty-four hours, specific gravity roro, no albumin, no casts or cells.

Menstruation came on about the time she was first seen, and was profuse, but not abnormal.

Discussion.-It is safe to assume that the fever and the weakness should be grouped together as the result of a common cause.

Though there is a systolic murmur over the precordia, it is not so situated or so supported by other physical signs as to be in itself satisfactory evidence of endocarditis or of any other cardiac lesion.

Tuberculous peritonitis would account for many of the symptoms, but we have no physical signs sufficient to justify any such hypothesis. In an abdomen which is otherwise negative, dulness in the flanks means nothing of importance unless it shifts with change of position.

We tried our best to make this case fit the diagnosis of typhoid fever, but could never obtain any positive evidence of it.

Urinary infection seemed very improbable, as the sediment of the urine showed nothing pathologic. No culture, however, was made from it, and if another cause for fever and weakness had not been discovered, bacteriologic investigation of the urine would have been in order.

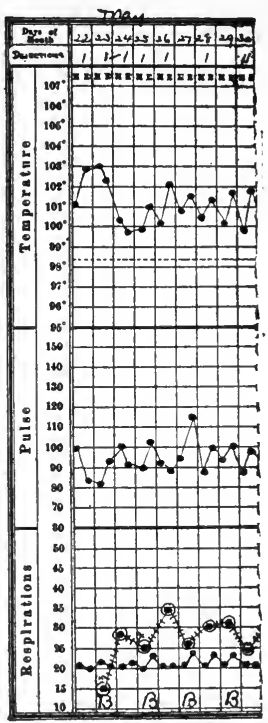

Fig. 147.-Chart of case 296.

The reader will, I hope, have noted that one method of physical examination, essential as part of a thorough study in any obscure case, is here omitted. Doubtless it was this mistake which postponed our making the correct diagnosis. I refer, of course, to the pelvic examination.

Outcome.-The cause of the weakness and fever remained quite unexplained until May 3oth, when the leukocyte count was discovered to have risen to 30,000 . This at last suggested a vaginal examination, which showed that the uterus was considerably enlarged. To its left a mass, the size of an orange, apparently attached to the fundus, extended upward. Another rounded mass seemed to be attached to the anterior uterine wall. 
An uncomplicated fibroid tumor would not have produced so much weakness and fever. Were it strangulated, degenerated, suppurating, or in process of producing a localized peritonitis, there should have been pain. Fibroid must, therefore, be ruled out or recognized as a subordinate part of the diagnosis. Cyst of the ovary or the broad ligament should produce more acute symptoms if its pedicle were twisted, and less fever if it were in a normal condition.

In view of these considerations, pelvic suppuration, probably dependent upon a pus-tube, seems the most probable diagnosis.

Operation June $2 \mathrm{~d}$ revealed a large pelvic abscess to the left of the uterus and a pus-tube on the right. There were also two small fibroid tumors attached to the fundus uteri. These were shelled out, the pus was drained, and the patient made a good recovery.

Diagnosis.-Pus-tube.

\section{Case 297}

An Italian laborer of forty-eight, of good family history and past history, was first seen October II, I907. He had a nose-bleed ten days ago, and has since then suffered from general malaise and weakness,

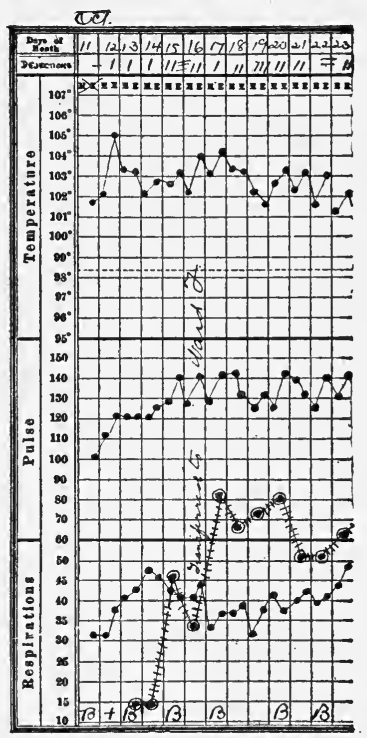

Fig. I48.-Chart of case 297 . with moderate headache, ill-defined abdominal pain, and slight cough.

Physical examination showed a swarthy, well-developed man, breathing rapidly but easily. (See accompanying chart, Fig. I48.) He was almost without complaint when seen, though his face was flushed, his breath very offensive. The cardiovascular system was negative. Breathing throughout the right back seemed more feeble than in the left, otherwise the lungs were entirely negative, as was the abdomen.

The white cells were 7000; hemoglobin, 85 per cent.; Widal reaction, negative; the urine averaged 50 ounces in twenty-four hours; specific gravity, Ior 3 ; there was the slightest possible trace of albumin; very many hyaline and granular casts, some with cells, and a small amount of fat adherent.

On the succeeding day the patient coughed up some stringy yellow sputa containing many cocci and bacilli, but nothing distinctive. 
An expert examination of the upper air-passages showed the foul breath to be due to atrophic rhinitis.

On the fifteenth the patient began to raise mouthfuls of thick, reddishgray, odorless pus, looking like that from gangrene of the lung, but without the characteristic odors. It contained a variety of organisms, but no tubercle bacilli. On the seventecinth the lungs were so full of coarse, bubbling râles that the heart-sounds were inaudible. On the same day friction, synchronous with the heart-sounds, appeared in the precordia. The right axilla was duller than the left.

On the eighteenth the abdomen began to be distended, the neck to be slightly stiff. The face expressed extreme anxiety and dread. On the eighteenth the sputum began to have a foul odor, and the right thigh became generally tender on motion and somewhat swollen.

On the nineteenth the white cells had risen to I4,900. On the twentieth an abscess appeared on the top of the left shoulder, and a similar one, painful, indurated, and red, appeared in the right groin below Poupart's ligament.

The patient lost strength rapidly; cyanosis and a foul, frequent diarrhea developed.

Discussion.- This case begins just like a typhoid, and at first there seemed to be nothing else that we could call it, although there was no Widal reaction, no rose spots or splenic enlargement, no bacilli by blood culture, and nothing characteristic about the temperature-curve. But as we could find no signs of tuberculosis, septicemia, syphilis, or any other type of obscure fever, our best guess was typhoid during the first four days of the illness.

When the pus began to come up and was found to be free from tubercle bacilli, we began to search for further evidence of pulmonary abscess. Nothing localizing could be found, but this, as experience has shown, is often the case in abscess of the lung. Our present methods of physical examination-even when supplemented by radiographyare not sufficiently accurate to reveal the presence of pulmonary abscess in all cases. We may have a little patch of dulness and diminished breathing, or râles may be heard over a circumscribed area; but nothing characteristic is often found, especially when the abscess is multiple and small. It is quite possible that the pus came entirely from the bronchi in this case.

When the peripheral abscesses began to appear, our attention was no longer concentrated on the lungs, and it began to be clear that we were dealing with a general infection. When the pericarditis developed, there was no longer any reasonable doubt of the diagnosis.

Outcome.-Blood cultures made on the twenty-first of October 37 
showed the staphylococcus aureus without any admixture of other organisms. The same coccus was obtained from the external abscesses. On the twenty-fourth the patient died, no benefit having been obtained from an autogenous vaccine.

Diagnosis.-Staphylococcus sepsis.

\section{Case 298}

An unmarried stenographer of twenty-nine had lost her mother of typhoid fever and one sister of acute tuberculosis two and one-half years ago. She was first seen by me March 12, I908.

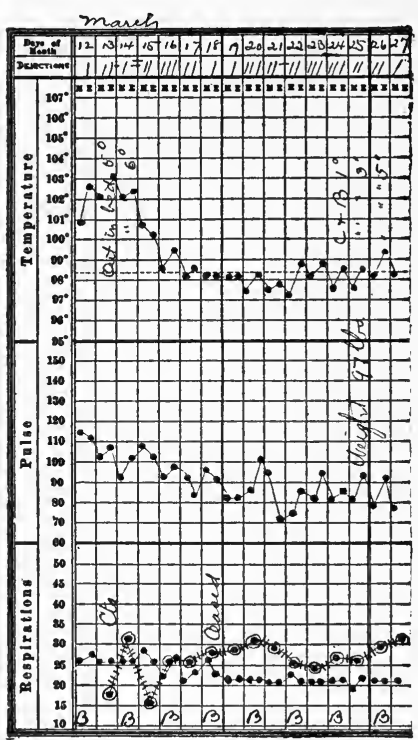

Fig. I49.-Chart of case 298.

The patient had bronchitis for a whole year when twelve years old, but has since been well until the previous fall, when she became run down, lost appetite, and had some pain in the left upper chest. Her chief complaint at this time was of weakness. She went to the country and remained there two months, with some improvement, so that she was able to go to work again on January I3, I908; but as soon as she took up her work again she began to lose appetite, and felt very tired and often chilly at night after her work. She had no cough and no pain, and continued to work until two days ago, when she noticed fever and headache and began to cough and raise yellow. sputa:

Yesterday evening her temperature was said to have been $104^{\circ} \mathrm{F}$. For the last two days she has had no sputa. She has now no pain anywhere. (For the course of the temperature see the accompanying chart, Fig. I49.)

The patient is well-nourished, ruddy; the heart and vessels show nothing abnormal. Over the right clavicle in front, and above the spine of the scapula behind, there is slight dulness, increased whisper, increased vocal and tactile fremitus, bronchovesicular breathing, and a few fine crackling râles. Kernig's isthmus and the excursion of the lung are equal on the two sides.

Physical examination, including the blood and urine, is otherwise negative. 
Discussion.-Many patients of this type have to suffer by reason of a mistaken diagnosis of general debility, neurasthenia, or "grip."

The latter diagnosis is difficult positively to exclude. A few cases are on record which prove that an influenzal bronchopneumonia may be so localized at the apex of a lung as to simulate tuberculosis. Such cases, however, are very rare, and for practical purposes may be disregarded.

The physical signs of this case, though strongly suggestive of tuberculosis, are not in themselves, and in the absence of any longer pyrexia, conclusive. The so-called bronchitis, which lasted a whole year during the patient's childhood, doubtless inclines us to interpret any dubious pulmonary signs as evidence of tuberculosis. But it must be remembered that such signs may be the harmless residual effects of an old burnt-out process which do not necessarily signify anything of importance at the present time. One of the most difficult tasks that I know of in connection with pulmonary diagnosis is to distinguish, by physical signs alone, the scars of an old healed process from the evidences of a new and threatening one. In many cases the differentiation of the two is impossible until the progress of the symptoms supplements our physical examination.

Despite several negative examinations of the sputa, our provisional diagnosis was phthisis, the most decisive point in our minds being the sharp, crackling quality of the râles, although they were elicited only by cough.

Outcome.-After repeated negative examinations of the sputa, tubercle bacilli were finally found March Igth. On the twenty-seventh she went to a sanatorium for the tuberculous.

Diagnosis.-Phthisis.

\section{Case 299}

A housemaid of twenty-four entered the hospital July I5, I908. Her family history and past history are good. She has one child eight months old. Ever since this baby was born she has complained of weakness. Though the labor was normal and not difficult, she has been able to walk since the baby was born but a few steps, owing to muscular weakness and edema of the legs. These troubles have been notable for two months, and have been accompanied by dyspnea on exertion. Pallor has also been noticed ever since the baby was born. For the past month she has also had some pain in the chest on taking a deep breath. She has no other pain. Her appetite is good, her bowels are regular, her sleep is fair. The course of the temperature is seen in the accompanying chart (Fig. I50). 
The patient is emaciated, pale, has one large submental gland, and several small postcervical glands. The heart shows no enlargement and no murmurs. The sounds are regular, clear, rapid. The bloodpressure is $100 \mathrm{~mm}$. $\mathrm{Hg}$. The lungs are negative except for one or two squeaking râles above the right clavicle and at the right base behind, with slight dulness, and diminished breathing.

In the abdomen there is dulness at the sides, which, however, does not shift to any extent with change of position.

There is a right lateral curvature of the spine, projecting somewhat backward, and involving the twelfth dorsal and the first, second, and third lumbar vertebræ. A pelvic examination is negative. Blood

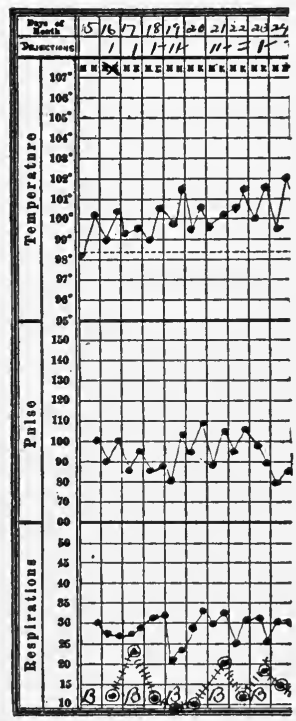

Fig. I50.-Chart of case 299. examination shows marked achromia, and some variation in size and shape. Urine is normal. Reflexes normal.

On the eighteenth there was distinct evidence of fluid in the abdomen, and the signs at the apex of the lung were no less evident.

Discussion.-At first sight the cardiac symptoms appear to be in the foreground. The edema, the dyspnea, the ascites, and the low blood-pressure all point in this direction, but the examination of the heart gives no support to the idea that any type of heart disease is present.

There is a good deal to suggest tuberculosis, especially the rather equivocal pulmonary signs and the association of ascites with fever. On the other hand, if the belly fluid were due to tuberculous peritonitis, we should expect pain, tenderness, or spasm, none of which is present.

From the blood examination it appears that the patient is anemic, and much of her weakness is doubtless due to this cause, but the details of the blood examination are such as to compel us to seek some further cause for the anemia itself.

The spinal deformity might be either the result of some old quiescent trouble or of a more recent disease. Since there are reasons to suspect tuberculosis in other parts of the body, the thought of Pott's disease should cross our minds. This leads straight to an $x$-ray examination as the next step in the study of the case.

Outcome. $-X$-ray of the spine showed telescoping of the vertebræ. It was subsequently learned that this prominence in the back had existed 
for three years and had been accompanied by some pain in the back at the beginning of an attack of "lumbago."

The patient was much relieved by a plaster jacket.

Diagnosis.-Vertebral tuberculosis.

\section{Case 300}

A carriage painter of thirty-seven entered the hospital February 20, I908. His father died of ulcer of the stomach, his mother of pneumonia, one sister and one cousin of pulmonary tuberculosis. The patient has always been perfectly well. He denies venereal disease and has good habits. Two years ago he overworked, and has since had much to worry him, especially his wife's sickness (heart disease) and the death of one of his children. Apparently, as a result of these troubles, he has been gradually running down, and last October had to quit work on account of general weakness and stomach trouble. He has an excellent appetite, but frequently vomits, especially in the morning before breakfast. His bowels are loose, moving usually two or three times a day. For three months he has been short of breath and has a little cough and expectoration. For two months he has been troubled with numbness in his hands and feet. For three weeks he has had night-sweats. His average weight is 135 pounds. At present he weighs II8 pounds.

Physical examination showed a sallow, somewhat emaciated young man with partial right wrist-drop; the chest entirely negative. The abdomen was very rigid throughout, tympanitic, but not tender. The blood was examined 20 or 30 times at weekly intervals. At entrance the red cells were 1,062,000. From that point they rose by March 24th to $1,880,000$, after which they gradually declined, reaching 570,000 on the twenty-second of June. The white cells ranged between 4000 and 7000. The hemoglobin at entrance was 55 per cent., and remained always relatively high.

The differential count showed nothing remarkable. In the stained specimen the red cells showed considerable stippling, some achromia in the smaller cells, and deep staining of the larger ones. Megaloblasts were always present and in excess of the normoblasts.

The urine was normal throughout. The stools were not remarkable. Parasites were repeatedly searched for, but never found.

The skin showed a brownish pigmentation, which gradually increased in tint, though no arsenic was given during his stay in the hospital.

The ocular tuberculin reaction was negative; the urine and stools contained no lead. Throughout his stay in the hospital he complained of nothing but weakness and numbness of the extremities. 
By the thirteenth of May he had a chill, the temperature rising to ro4. $6^{\circ} \mathrm{F}$. Previous to that time it had ranged between $98^{\circ}$ and $100^{\circ}$ F. After that there was a moderate pyrexia $-99^{\circ}$ to $10 I^{\circ} \mathrm{F}$.-for about one-half of the rest of his stay. No cause for the chill was found.

Discussion.-Carriage painters often get lead-poisoning. Because of this patient's occupation, as well as for other reasons presently to be mentioned, plumbism is the first possibility which calls for investigation. Our patient has a wrist-drop, various troubles with his stomach, and stippling of the red blood-corpuscles, all of which signs point toward lead. On the other hand, the blood-picture is distinctly that of primary anemia. His gums show no lead line and his wrist-drop turns out on inquiry to be an affair of very long standing. The degree of anemia, moreover, aside from its type, is greater than that seen in any but the severest cases of lead-poisoning, such as exhibit extensive paralyses and encephalopathy. Finally, the absence of lead in the stools and urine decisively excludes plumbism.

The brownish pigmentation of the skin, steadily increasing at a time when no arsenic was given, suggests Addison's disease of the suprarenal capsules, rather than the anemia first described by him. The weakness and stomach trouble are quite in harmony with this idea. On the other hand, suprarenal disease is never, so far as I am aware, associated with so severe an anemia except in the acutest and most fulminating cases. In the great majority the anemia is very moderate. The negative ocular tuberculin reaction helps to convince us that we are not dealing with the commonest type of Addison's disease-suprarenal tuberculosis.

Pernicious anemia, then, seems to be the most reasonable diagnosis, although the patient is rather younger than most of those who suffer from this type of anemia. The chill and sudden rise of temperature on the thirteenth of May puzzled us somewhat. Fever, it is true, is the rule in pernicious anemia, but not so sudden and sharp a rise. Probably it is to be explained as the result of some secondary infection favored by the great weakening of general resistance. I have seen a good many similar attacks in the last few years, all of them passing off, as in this case, without any indication of their source.

Outcome.-The edema of the feet and the general weakness increased steadily, so that by the first of July he was confined to bed. Death occurred July igth.

Autopsy showed the usual lesions of pernicious anemia. The suprarenal capsules were normal.

Diagnosis.-Pernicious anemia. 


\section{Case 301}

A real-estate agent thirty-six years old consulted me February I5; 1909, complaining of weakness and headache. Although he has an excellent appetite and perfect digestion, he has lost 25 pounds in the last two years, I4 pounds of which were lost within the last three months.

His family history is good except that his father died of tuberculosis.

His past history was uneventful until September, Igor, when he had typhoid fever. In 1904 hẻ had a good deal of pain in his shoulders, which passed off, however, within a few months, though no diagnosis or treatment was given. For the next three years he was quite well, but in December, $190_{7}$, he had pains in his left arm, diagnosed as neuritis. By reason of this he was kept out of work in January and February, 1908, and in August and September of the same year.

November 7, 1908, he had an attack of vomiting, accompanied by severe headache, and was kept in bed a week. Ever since that time he has been troubled by headache, which is worse on waking in the morning, and usually clears off at noon. It affects especially the vertex and the frontal region, but is not changed in any way by the position, by diet, or by the weather. His eyes have been examined by a specialist and pronounced entirely normal except for a horizontal nystagmus of almost minute excursion. His nose has also been carefully examined, but nothing found.

He had no fever at any time, but his physician tells him that his pulse is rarely below roo. From time to time he has had slight jaundice. Since November $7^{\text {th }}$ he has felt unable to work, and since December he has passed urine once or twice every night after bed-time.

Physical examination shows rather poor nutrition; the internal viscera are entirely negative, except that the pulse is r ro-not an unusual rate during an office consultation in patients of any tendency to nervousness. The knee-jerks are unusually lively; the blood-pressure, ${ }_{55} \mathrm{~mm}$. $\mathrm{Hg}$.

The urine is of normal color; 40 ounces in twenty-four hours; specific gravity, I023, no albumin, no sugar. Several subsequent examinations showed essentially the same conditions. Blood normal.

Discussion.-Loss of weight with a good appetite is a rather rare combination of symptoms. Diabetes is its only quite familiar cause, and that disease can be immediately ruled out of consideration in view of the urinary findings.

Aside from diabetes I have met with this combination of symptoms in persons who are losing a great deal of sleep by reason of pain or 
emotional strain, in certain stages of arteriosclerosis, and in hyperthyroidism. This patient's headaches never prevented his sleeping. He had no special causes for anxiety, and seemed to be in good spirits. There was no good reason to suspect arteriosclerosis, and no external evidence of that disease.

Hyperthyroidism (Graves' disease) should always be suspected when a patient loses weight despite a good appetite, especially if there is any tendency, as in this case, toward tachycardia. As I examined this patient with special reference to hyperthyroidism, I found no trace of goiter or exophthalmos, butquite a definite fine tremor of the fingers when extended.

Outcome.-Under a régime of overfeeding and rest the patient's headaches became much less frequent, his pulse slower, and his weight increased. In October, I909, he was back at work.

Diagnosis.-Hyperthyroidism (Graves' disease).

\section{Case 302}

I was consulted, September 4, I906, by a widow aged sixty-four, whose chief and most distressing complaint was weakness. Her weight had shown no change; her appetite was, she said, "too good," and her sleep excellent. She had no pain, cough, or vomiting, but she had been losing strength steadily for years, and for the past twelve months had been decidedly short of breath. In I89I she had been treated by Dr. Arthur T. Cabot for hemorrhoids, which never bled at all, as far as she knew, until two years ago, when there began to be some bleeding each month for a period of three or four days. For the past five months, however, there has been no bleeding whatever.

Her color has been noticeably abnormal for at least six years. Four years ago, she says, it was worse than it is now. Headaches have bothered her some part of every day for many years. They are aggravated by walking, and affect especially the occipital region. She is markedly constipated, and notices a good deal of mucus in the stools. Two years ago she had an illness which she fears was a "shock," and since that time she talks slowly and with difficulty. All her symptoms are aggravated in winter, and she feels the cold very much, though not more, she says, than most ladies of her age.

Examination showed a yellow, waxy pallor of the skin. The patient was somewhat obese, but nothing wrong was detected in the internal viscera or in the urine. Blood examination showed:

Red cells, 3,600,000; leukocytes, 6000; hemoglobin, 45 per cent. In the stained specimen there were 66 per cent. of polynuclear cells, 32 
per cent. of lymphocytes, and 2 per cent. of eosinophiles. The red corpuscles showed marked achromia and slight deformities. There were no nucleated forms or abnormal staining reactions.

Discussion.-The case was sent to me as one of pernicious anemia, and her appearance bore out this diagnosis. The blood examination, however, did not, but was indicative rather of a secondary type of anemia. It did not seem to me that there was enough hemorrhage (assuming the history to be correct) to account for this anemia.

Rectal examination and the study of the stools showed no evidence of a rectal or intestinal cancer, and her good nutrition and freedom from pain or diarrhea made it unnecessary to consider this diagnosis further.

I learned, during a subsequent visit, that she had difficulty in making fine motions with her fingers. Following up this hint I tested the functions of motion, sensation, reflex action, and nutrition without getting any new information except that the skin was very dry and the nutrition of the finger-nails notably poor. The association of this condition of the skin with slowness of speech naturally suggested myxedema.

On questioning her I then learned that her hair had been coming out very fast, though she had thought and said nothing of it, supposing that her age accounted for the loss. It appeared, further, that she never perspired unless the thermometer was above $90^{\circ} \mathrm{F}$., a temperature very grateful to her feelings.

Outcome.-The patient was given thyroid extract, 2 grains three times a day, gradually increased to 5 grains three times a day. January I6th she reported herself as wonderfully better. March $25^{\text {th }}$ she wrote that her hair was growing tremendously, so that it was now thick and dark. Her speech had greatly improved, and her waxy pallor had disappeared. Within a short time she was perfectly well, and has remained so up to the present time (I9IO). She still takes thyroid extract regularly.

Diagnosis.-Myxedema.

In the table which ends the chapter and in the diagram which begins it I have grouped causes of paralytic weakness without any attempt to tabulate the cardiac or hemic types of weakness. 


\begin{tabular}{|c|c|c|c|c|c|c|c|c|c|}
\hline 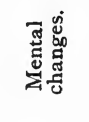 & 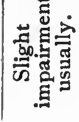 & 0 & ० & + & 0 & 0 & 起 & 0 & 0 \\
\hline 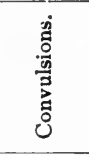 & 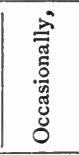 & ० & $\circ$ & + & ० & ० & $\begin{array}{l}\text { 苞 } \\
\text { 苔 }\end{array}$ & 0 & 0 \\
\hline 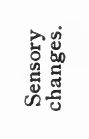 & $\begin{array}{l}\text { 尝。 } \\
\text { 点。 }\end{array}$ & + & $\circ$ & 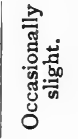 & + & $\circ$ & $\circ$ & ० & 0 \\
\hline 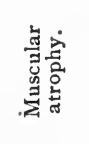 & 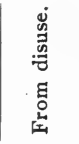 & ० & + & $\circ$ & + & ० & 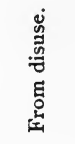 & 0 & 0 \\
\hline 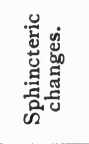 & 离。 & + & ○ & 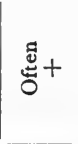 & ○ & $\circ$ & ० & $\begin{array}{l}\dot{\grave{d}} \\
\text { हैँ }\end{array}$ & 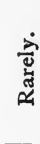 \\
\hline 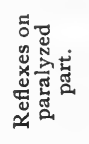 & + & $\circ$ & $\circ$ & + & 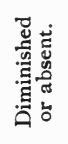 & 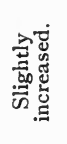 & + & + & + \\
\hline 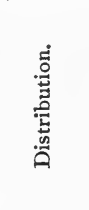 & 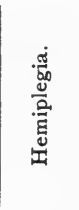 & 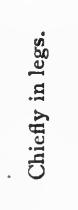 & 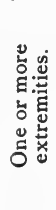 & 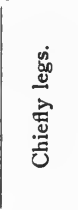 & 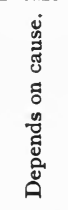 & 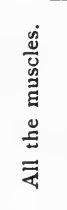 & 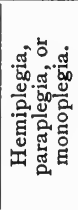 & 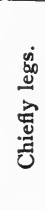 & 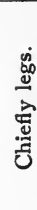 \\
\hline 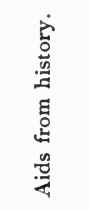 & 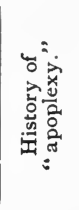 & 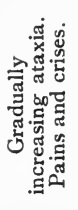 & 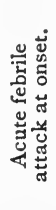 & 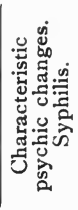 & 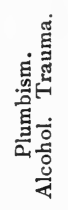 & 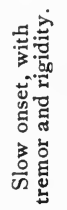 & 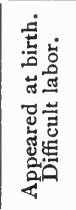 & 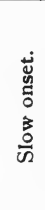 & 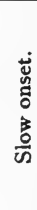 \\
\hline 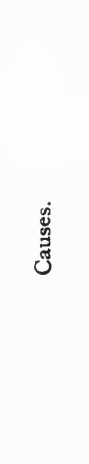 & 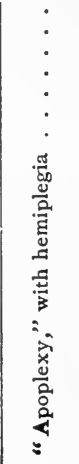 & 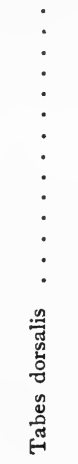 & $\begin{array}{l}\vdots \\
\vdots \\
\vdots \\
\vdots \\
\vdots \\
\vdots \\
: \\
\stackrel{0}{0} \\
\stackrel{0}{0} \\
\stackrel{0}{\circ}\end{array}$ & 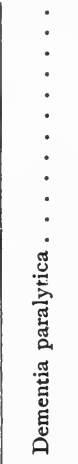 & $\begin{array}{c}\vdots \\
\vdots \\
\vdots \\
\vdots \\
\vdots \\
\vdots \\
\vdots \\
\vdots \\
\vdots \\
\vdots \\
\text { 苛 }\end{array}$ & 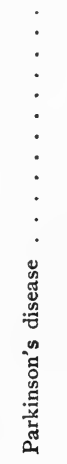 & 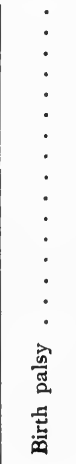 & 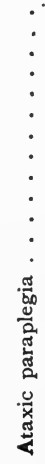 & 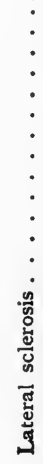 \\
\hline
\end{tabular}





\section{Causes of Cough}

2. "BRONCHITIS"

3. MITRAL DISEASE

4. TONSILLITIS

5. PLEURISY

6. PHARYNGITIS

7. MYOCARDIAL WEAKNESS $\}$

8. PNEUMONIA

9. AORTIC REGURGITATION $\}$

10. "INFLUENZA"

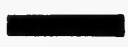

11. ASTHMA

12. EMPHYSEMA 


\section{CHAPTER XVIII}

\section{COUGH}

THERE are, of course, many causes of cough which do not raise diagnostic puzzles, and are, therefore, not suitable for this book. Thus:

(a) "A common cold" or mild infection of the upper air-passages. whether by the streptococci or other organisms, may produce cough by irritating the pharynx, larynx, trachea, and larger bronchi. Diagnosis is suggested by direct inspection of these parts, and by the absence of signs in the lungs and other viscera. It is clinched by the short, mild course of the affection.

(b) In infants and children diffuse bronchitis often stuffs the lungs with squeaking or crackling râles, with or without considerable constitutional signs. To exclude pneumonia is here the chief diagnostic task. Occasionally this cannot be done. Usually the absence of marked constitutional signs (continued fever, marked leukocytosis, cyanosis, drowsiness) and of the physical evidence of solidification in any part of the lungs excludes lobar pneumonia, but bronchopneumonia may exist without any demonstrable physical signs.

(c) The more obvious "text-book" pictures of phthisis and pneumonia have not been included.

(d) The so-called "stomach coughs," "uterine coughs," "liver coughs," and other "reflex" irritations from a distance have not yet demonstrated themselves in my experience.

(e) Nasal coughs and aural coughs still linger on in the pages of textbooks, but I can find no convincing evidence that they exist.

\section{VARIETIES OF COUGH}

(a) The distinction between a loose or productive cough, which is associated with sputa [unless the patient is too weak or too young to raise any], and a $d r y$ or unproductive cough, is very familiar.

(b) The brassy or laryngeal cough is a loud, ringing, usually unproductive effort, associated most often with aneurysms or tumors pressing upon the trachea, and occasionally with mitral stenosis, the engorged left auricle exerting pressure upon the left recurrent laryngeal nerve. It is not by any means distinctive, but in conjunction with other and more precise signs it may help us to recognize a source of pressure in the mediastinum. For this reason it is often called a "pressure" cough. 
(c) Nervous cough is sometimes a life-long habit, showing itself especially when the individual is embarrassed or when he desires to preempt a pause in the conversation. Many patients will undergo a long siege of questioning and physical examination without showing any sign of cough until we ask them whether this symptom is troublesome. With the first words of their answer there comes a cough.

Yet it must be remembered that in some cases of incipient phthisis the cough seems to be of the nervous variety, and is believed to be such by the patient and his family. The matter can be settled only by careful watching and repeated examination.

(d) A barking cough often occurs in children at or before the age of puberty. It has no special significance, though it often gives rise to much alarm. Its explanation is not known, and it may be associated with any of the commoner lesions of the upper respiratory tract.

(e) A prolonged suffering from cough is usually due to phthisis, to emphysema, or chronic bronchitis with bronchiectasis; occasionally to cardiac insufficiency or pulmonary abscess.

$(f)$ Cough on exertion is usually due to heart disease, but may be the result of any of the causes mentioned in the last paragraph.

(g) Cough on change of position, accompanied by a profuse discharge of sputum, usually indicates pulmonary abscess or bronchiectasis.

(h) Winter cough recurring each year is usually characteristic of bronchiectasis or arteriosclerosis with weakened heart. The cavities remain comparatively dry and harmless in the summer-time, but are prone to become infected, usually with the influenza bacillus, in the winter-time. This is the affection usually known as chronic bronchitis, though a considerable percentage of the cases so diagnosed are really due to pulmonary tuberculosis.

\section{Case 303}

A mule spinner of forty-five, of good family history and past history, was seen November 14, 1907. He took gas as an anesthetic twenty weeks ago and had all his teeth pulled out. He had no trouble at the time, but a week later he began to have pain in the right side of the chest, worse on deep breath. Two weeks after the anesthetic he began to cough, and noticed that a bad odor and bad taste came into his mouth; next day he coughed so as almost to choke him. Four days after this he began to raise more foul sputum of a dark, greenish-brown color, with dark-red portions in it. On the third day he coughed up half a large bowlful during the night. Sometimes the sputum came rushing up in large amounts with very little cough. The pain in the right side, mean- 
$5^{9^{\circ}}$ 


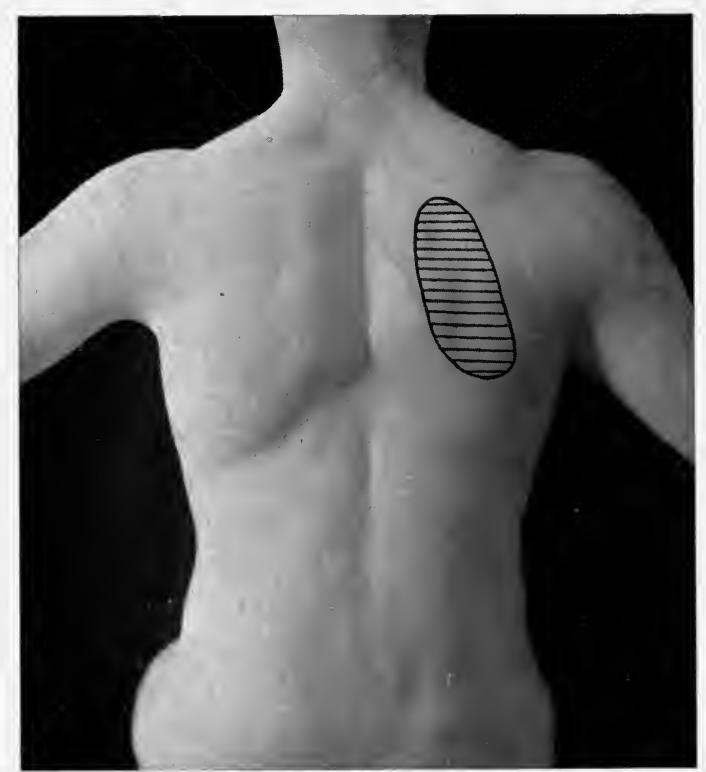

Fig. 152.- Area of rough and prolonged expiration in Case $3 \circ 3$. 
time, had become less, the diminution coinciding with the period of excessive sputa.

Eight weeks ago the cough diminished. His sputa became yellow and less foul, and his appetite improved, as did all his other symptoms, until five weeks ago, when, as he stooped to lace his shoe, blood filled his mouth, and he spit up half a cupful of it. Four hours after he raised about the same amount, and this continued for a couple of days in decreasing quantities. Since then he has not raised any more blood, but his appetite has been very poor and his cough frequent.

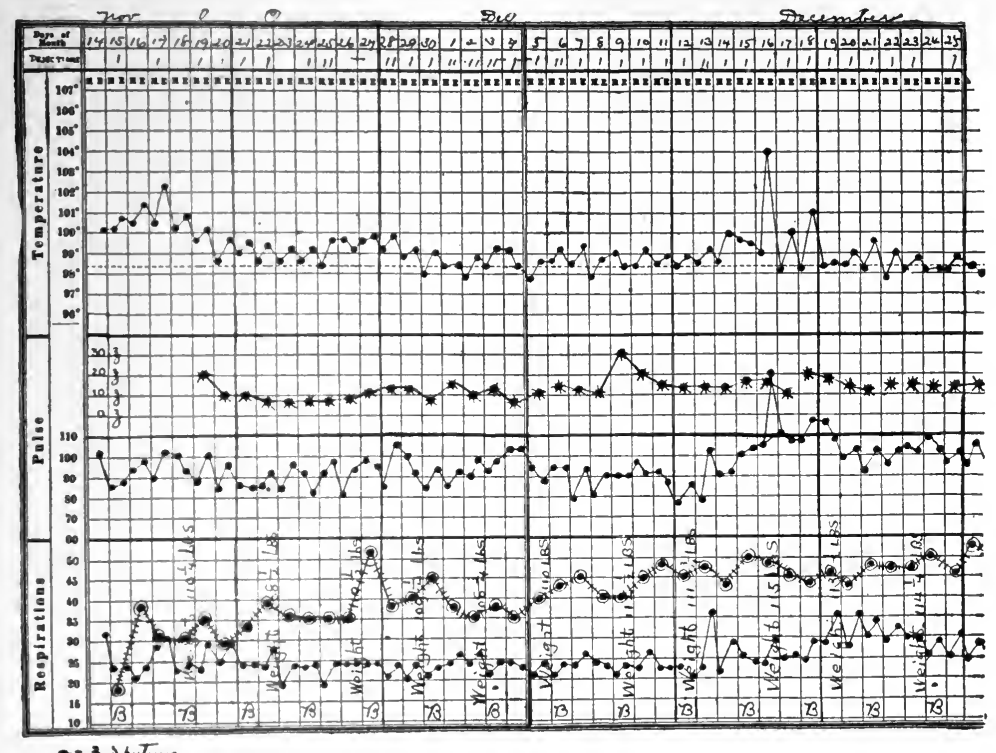

Fig. I51.-Chart of case 303 .

Two and one-half weeks ago the pain in the right side again became severe, and he had to get up in the night in order to get breath. Since then he has been short of breath on any exercise, and his sputum has again been foul and dark, as at first.

$\mathrm{He}$ has had fever for fifteen weeks, off and on, with occasional nightsweats.

He gave up work on July 20 th on account of weakness, but he has lost very little weight, as he was always thin. The course of the temperature is seen in the accompanying chart.

The patient is emaciated, the breath rather foul, the heart negative, the right supraclavicular space deeper and more capacious than the left. A friction-rub is felt in the right axilla, and there is dulness throughout 
the right chest. In the right supraclavicular space and axilla the percussion-note is almost flat. Over the area shown in the diagram (Fig. I52) expiration is rough and prolonged, and there is a coarse friction-rub extending through both inspiration and expiration over the whole right axilla, and heard more faintly in front and behind.

During his stay in the hospital the patient raised daily Io to 30 ounces of mucopurulent, foul sputa, containing no elastic fibers or tubercle bacilli. Its color was as described by the patient.

Later, on the twenty-eighth, the odor was a good deal less marked.

$X$-ray shows a deep shadow in the region of the right scapula, agreeing practically with the area shown in the diagram.

Discussion.-Cough with foul sputa ejected in large quantities and associated with fever and sweating suggests four possibilities:

(a) Pulmonary abscess, with or without gangrene.'

(b) Empyema rupturing through the lung.

(c) Phthisis with large cavity.

(d) Bronchiectasis.

Empyema and phthisis are improbable because of the history and the condition of the sputa. The signs are not situated in the parts generally most affected by phthisis, and when that disease produces a cavity large enough to contain so much sputa, it is practically certain to show bacilli in great numbers. Empyema is almost always postpneumonic, and we have nothing to suggest that this patient has ever had pneumonia.

The sudden onset and the localization of the signs speak against the ordinary type of bronchiectasis, namely, that associated with a chronic bronchitis and affecting a great many bronchi almost equally. Blood is far less likely to be raised in bronchiectasis than in abscess or tuberculosis.

After discussing these alternatives, pulmonary abscess seems by far the most reasonable diagnosis. We have no clear conception of the reason or method of its origin; it is difficult to connect it with the taking of gas as an anesthetic unless we suppose that, under the influence of the gas, he swallowed something "the wrong way." The history gives no hint of this. Only by surgical interference could one make the diagnosis any more certain, and the results of such interference are not brilliant enough to make us willing to urge it unless other forms of treatment are obviously useless. This has not yet been proved here.

Outcome.-The patient improved a good deal in weight and strength

1 Pulmonary abscess and pulmonary gangrene are essentially the same disease. The one may pass into the other at any time if the organisms favoring gangrene supervene. 
under forced feeding and sleep out-of-doors. He left the hospital on December 27,1907 .

May I8, I9I0, he writes: "I have not fully recovered from my sickness. I am able to go around, but the trouble in my lung has not healed yet. I still cough and spit as much as ever. I have had a number of hemorrhages since I left the hospital. My stomach keeps all right, and I can eat most anything that comes along. My•doctor says he thinks my lung will heal up in time."

Diagnosis. - Pulmonary abscess.

In connection with this case (303) I have received the following interesting communication from Dr. J. B. Hodgkin, of Urbanna, Va.

"A lady patient of mine who wished some teeth extracted went to one of the gas specialists for that service. As she came back to me in a few hours after the extraction she seemed well, but as I did not see her for some two weeks and as I heard that she was ill after leaving my office, I was interested to know about her case. She told me that soon after she left my office (I did not give the gas or do the extracting) she began coughing violently, and the attack was so severe that she sent for her physician, who pronounced hers a case of acute bronchitis. The next day, after a violent fit of coughing, she coughed up a tooth that the extractor had dropped into the larynx. Fortunately she thus got rid of what would, had it remained in the bronchus, have produced just such a case as you describe in your Case 303."

\section{Case 304}

A mill foreman thirty-eight years old was seen May 4, rgo8. He had "congestion of the left lung" twelve years ago. His personal history and family history are otherwise excellent.

In December, 1906, he had a "bad cold" with a severe dry cough which has continued in spells ever since. He sometimes coughs so hard that he faints away, and it is very difficult for him to get his breath at these times; yet he may go for a week without any cough whatever. His appetite is good, his bowels regular and he sleeps well, except during the spells of coughing. He has no digestive or urinary symptoms, and has lost no weight.

Physical examination shows a finely developed, strong-looking man, with a hoarse voice and occasional ringing cough. There are numerous dark-red papules scattered over the chest. The pupils are equal, circular, and react normally. Harsh, noisy respiration is heard over the whole of both lungs. There seems to be some slight dulness toward the top of the right axilla. The abdomen is slightly distended and held rather firmly. It is tympanitic throughout. The patient seems entirely 
comfortable, except for the coughing spells, at times excessively severe. The urine is negative. The white cells are from 12,000 to 15,000 . There are no abnormal areas of dulness or pulsation. The heart is negative. The right pulse is distinctly larger than the left; indeed, the left is hardly palpable.

The patient went back to business on March gth.

Discussion.--Intense paroxysmal cough in children usually means pertussis; in adults one would not make such a diagnosis without a very circumstantial history unless we had heard the typical "whoop."

If the patient had had bronchitis for so long a period, he should be either better or worse. He should have more sputa and more signs in the lungs.

If tuberculosis were at work, there would be more emaciation, fever, and other constitutional symptoms. In tuberculosis, moreover, the cough is not often so violent, paroxysmal, and intermittent.

Obscure and violent cough is often due to pleural irritation, such as occurs at the onset of acute pleurisy or when foreign bodies irritate the surface of the lung. But there seems no evidence of any source of irritation in this case.

Malignant disease of the lung, pleura, or mediastinal glands should always be considered in obscure diseases of the respiratory tracts. Diagnosis, however, is impossible unless there is a pleural effusion, some pulmonary signs corresponding to an infiltration of the lung or pleura, or radiating pressure pains. Except for the slight dulness, made out rather doubtfully toward the top of the right axilla, we have nothing corresponding to any circumscribed pulmonary or pleural lesions. This questionable dulness is not a sufficient basis for any diagnostic hypothesis.

Most significant in this case, as in any involving hoarseness and a ringing paroxysmal cough, is the difference between the two pulses. Indeed, in the presence of these three symptoms we should always suspect aneurysm, with malignant tumor as a less probable alternative. It is possible, of course, that the difference of the pulses may represent nothing but a congenital anomaly. Such idiosyncracies are not uncommon, but they are rarely associated with the rest of the symptom group above described. To arrive at any greater certainty regarding the diagnosis we need, first of all, an examination of the vocal cords. If one cord is found to be in the cadaveric position, we may conclude that the left recurrent laryngeal nerve is being pressed upon by an aneurysm or a tumor. Further evidence would be furnished by $x$-ray examination.

Outcome.-Radioscopy showed a pulsating shadow corresponding 
to that ordinarily found in aneurysm of the aortic arch. Examination of the vocal cords showed no paralysis of the recurrent laryngeal nerve and no obstruction of the trachea. March gth the patient went back to business, considerably improved by his rest, possibly also by the potassium iodid which he took in ro-grain doses throughout the period of treatment.

A notable feature of this case is the absence of any pain or any sign of pressure other than the cough, the hoarseness, and the inequality of the pulses.

Diagnosis.-Aneurysm.

\section{Case 305}

A school-girl seven years old has had "asthma" about once a month for the past two years, but has otherwise been well.

Two weeks ago she caught cold and had a bad cough, but stayed at school until a week ago. Yesterday her cold was worse and she went to bed. To-day she has vomited three times. Her bowels are loose as a result of "Father John's Medicine."

At entrance, November 9, I907, the breathing was rapid, but not labored; cheeks flushed, lips dry and fissured. There was a crop of herpetic vesicles about one corner of the mouth. The glands in the neck, axillæ, and groins were somewhat enlarged. The heart's impulse extended to the nipple-line in the fourth space. Its action was regular, and the sounds were of good quality. The pulmonic second sound was louder than the aortic second. In the right back the breathing was rough from the apex to the angle of the scapula, accompanied by squeaks and increase of voice-sounds. The abdomen was flat, spastic, and very tender throughout, especially in the right lower quadrant. Nothing else could be felt. By rectum, there was general tenderness, nothing more distinctive.

The course of the temperature is seen in the accompanying chart (Fig. I53).

White cells, 30,200; urine, normal.

A surgeon promptly saw the case and thought that her symptoms were all due to the lung in-

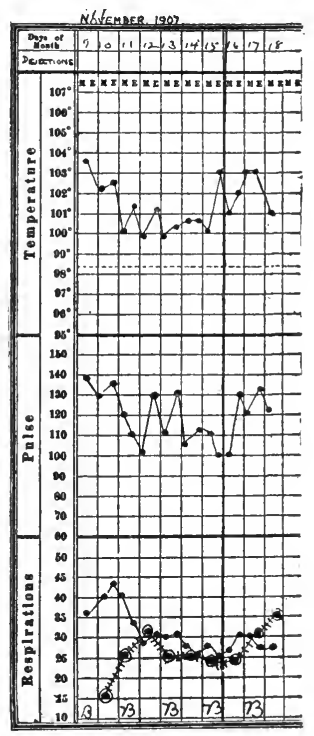

Fig. 153.-Chart of case 305. volvement. Next morning the belly was much less tender, and by the eleventh the lung signs were also very slight.

The child was pale and looked delicate. The sputum was repeatedly examined, without any positive result. 
From the fourteenth to the eighteenth the child got steadily worse; she woke frequently in the night crying with pain, relieved to some extent by flaxseed poultices to the abdomen. There was some dulness, with diminished breathing in the right back and lower axilla. The right thigh was now held flexed upon the abdomen.

Discussion.-We have been warned so often of late that whenever a child seems to have something wrong in his abdomen we should always consider and investigate the chest, that we naturally make the effort to explain in this way all this little girl's symptoms. The thoracic diseases which ordinarily produce abdominal pain in children are pneumonia and pleurisy, but this child shows no evidence of either of these. A moderate degree of bronchitis, such as might be the outcome of an ordinary cold or the beginning of a tuberculosis, is what we find. Neither of these diseases is prone to make the abdomen spastic and tender, but the presence of a herpes makes us wonder whether there may not be some deep-seated pneumonic process which we have overlooked.

As the lungs cleared up, the condition of the abdomen did not. Thereby the focus of attention was shifted, and we began to ask ourselves more seriously what was wrong there. Appendicitis is not common in children of this age, and is not often associated with herpes. Nevertheless, it seems more probable than any other condition. The psoas spasm might be explained in this way, especially as there seems to be no lesion of the hip, spine or urinary tract.

Some discussion arose in this case regarding the significance of the leukocyte count. Since children have naturally a higher leukocyte count and greater diurnal variations than adults, some of those who saw this child were in doubt whether a count of 30,000 was markedly abnormal under the circumstances. It seemed to me clear, however, that such a count should be interpreted as a well-marked leukocytosis, since, in children of this age, the blood has practically always attained to conformity with the adult type.

Outcome.- On the eighteenth the abdomen was opened and about 4 ounces of pus were removed from the region of the appendix.

There is no question that bronchitis was also present here, and after the study of a good many similar cases-some of which developed appendicitis, others endocarditis or multiple arthritis, while still others remained as an unlocalized pyogenic infection of the blood-streamit seems to me at least possible that the appendicitis which results in a case like that here discussed represents the outcome or localization of a general pyogenic infection.

Diagnosis.-Bronchitis and appendicitis. 


\section{Case 306}

A Russian picture-frame maker, thirty-three years old, who has been three years in this country, entered the hospital September 24, 1907. He has never been sick before. His habits and family history are excellent. He was suddenly seized, three weeks ago, with chills and fever and pain through both sides of his chest. The next day he began to cough, and the pain became confined to the left chest. The appetite remained good, but for the last two nights he has slept poorly on account of dyspnea, which makes it almost impossible for him to lie down.

When examined, he was breathing jerkily. His lungs were hyperresonant throughout, expiration prolonged, feeble, and accompanied by squeaks and crackles. In the left axilla, from the fifth rib downward, a friction-rub could be felt and heard. It was most marked during expiration. Visceral examination was otherwise negative.

The white cells were 5000; the urine normal; there was no fever during his week in the hospital. The patient was given an ice-bag over the painful side; $\frac{1}{2}$ grain of codein every two hours when needed; fluid extract of grindelia robusta, 20 minims every twenty minutes for four doses; then 30 minims every three hours. His chest pain was relieved by strapping the chest.

Discussion. - When pain is complained of in both chests by one who is suffering from a cough, it usually represents the result of muscular soreness due to the cough itself. Occasionally it is produced, like headache and backache, by the infectious agent which has caused the cough. At first sight it seems that pleurisy would be a simpler explanation, at any rate for the left-sided pain; but as we scrutinize the report more carefully and note that the friction was most marked during expiration, we begin to doubt whether it really was a friction-i.e., whether it was due to a pleurisy. Pleural frictions are'almost never exaggerated during expiration. The end of inspiration is the favorite time for their appearance and their usual period of maximum intensity. But there is another phenornenon not infrequently mistaken for pleural friction, and especially apt to occur during expiration-I mean the snoring râle which can often be felt as well as heard, and which is apt to occur in chests presenting the group of physical signs here recorded.

The most salient point, however, about this case is the presence of cough without fever. Such a cough, associated with the group of signs just referred to, is especially characteristic of the spasmodic or asthmatic type of bronchitis. Indeed, one would proceed straight 
way to make this diagnosis, provided he had adequately considered two other possibilities which should always haunt us when we make a diagnosis of asthma or asthmatic bronchitis. I refer to:

(a) Syphilis involving aortic aneurysm or stenosis of a bronchus.

(b) Pulmonary tuberculosis.

Any one who remembers, as I do, the disgrace of being confronted at autopsy with aneurysm or syphilitic stenosis of the bronchus in a case which he has treated durirg life for asthma will never be hasty again in making the latter diagnosis. The wheezing and coughing produced by one of the varieties of syphilis above referred to may be clinically identical with those of ordinary bronchial asthma. The treatment often relied upon for asthma (large doses of potassium iodid) may still further mislead us through the improvement it produces in syphilitic infections. Indeed, one sometimes is led to wonder whether this is not the explanation for the reputation of potassium iodid in the treatment of asthma.

In a paper by Dr. Cleaveland Floyd ${ }^{1}$ we are warned how frequently cases of asthma and emphysema with chronic bronchitis mask the development of a pulmonary tuberculosis.

Both these possibilities were considered in the present case, and everything was done to discover evidence of their presence. Nothing of the kind came to light, however, and with reasonable certainty these haunting possibilities may be excluded by the outcome.

Outcome.-By the thirtieth of September the physical signs were gone and the patient was allowed to go home. His health has since then remained good, though he has occasional attacks of wheezing.

Diagnosis.-Bronchitis and asthma.

\section{Case 307}

A hospital nurse, twenty-eight years old, was seen May 4, 1907. She was never sick until three weeks ago, when she had "grip," but kept on duty until the right ear began to ache twelve days ago; the drum was tapped ten days ago, with a copious discharge of pus containing streptococci. At the right base, below the angle of the scapula, were numerous crackling râles. Later these râles gradually extended over the rest of both lungs. The white cells ranged between 18,000 and 21,000 . Physical examination was otherwise negative. Now she complains of anorexia, insomnia, cough, fever, and weakness. There is no longer any tenderness about the ear.

On the tenth the patient was mildly delirious, respiration shallow

${ }^{1}$ Boston Med. and Surg. Jour., I909, vol. clxi, p. 467. 
and almost stertorous, pulse falling steadily, but of poor volume and tension. Acute redness and tenderness now developed over the tendons of both wrists. All this time there had been a continuous discharge from the right ear, but there was apparently good drainage and no mastoid tenderness or edema.

The patient's extreme nervousness suggested cerebral irritation. Accordingly, on the tenth of March the right mastoid was opened and curetted, a good deal of pus being found and removed. The lateral sinus was laid bare, and a needle introduced into it. Pure sterile blood was withdrawn. Infection of the sinus was thus excluded.

Discussion.- In this patient we find the signs of bronchitis only, but she is obviously too sick for mere bronchitis of the ordinary type. This particular combination of the signs of bronchitis with constitutional manifestations too grave to be thus explained is very familiar in young children, and under those conditions is well known to mean bronchopneumonia, provided evidence of disease in other organs is wanting. In adults this particular combination or contradiction is much less common.

It is quite possible that this patient had bronchopneumonia, but we do not need to assume it, for the lesions of the middle ear and of the tendon-sheaths furnish abundant evidence of a generalized pyogenic infection sufficient to explain why this patient is so sick.

It is just within the bounds of possibility for a generalized tuberculosis to begin in this way, but the presence of streptococci in the aural discharges and the absence of tubercle bacilli from the sputa give us no ground for following this idea any further.

After the drainage of the mastoids the pulmonary signs did not clear up, and the sputum was repeatedly reëxamined for evidence of tuberculosis, always, however, with negative results. In an older person with a bigger heart we should have been probably in considerable doubt whether the pulmonary râles were due to edema or to inflammation, to a transudate, or an exudate. Not infrequently these two states are so mixed up in elderly people that the fine gradations between bronchitis, edema, hypostatic pneumonia, and lobar pneumonia cannot be distinguished. In the present case, however, there is no occasion for any such speculation. The heart was of good strength throughout.

Outcome.-In the course of two weeks the patient's recovery was nearly complete, though some râles remained in the lung, even after the mastoids were entirely healed.

Diagnosis.-Streptococcus bronchopneumonia. 


\section{Case 308}

A school-boy of seventeen entered the hospital January 20 , I908. He had had "pneumonia" when he was four years old, and again when he was seven. Four years ago he had "general peritonitis," for which he was operated upon at the Boston City Hospital. He also had measles and mumps in infancy.

For the last three years he has been bothered by a persistent cough with abundant sputum. The cough is severe enough to make him vomit about once a week after breakfast. He loses much sleep on account of the cough.

Five times in the past year his sputum has been blood-streaked, the last time four days ago, when there were small black clots in it. He has no night-sweats, and, so far as he knows, no fever. (See chart for temperature.)

His appetite has been good, his bowels regular, his sleep good except when disturbed by cough.

Physical examination shows that the boy is distinctly undersized. His present weight is 76 pounds. He has a "chicken breast." The

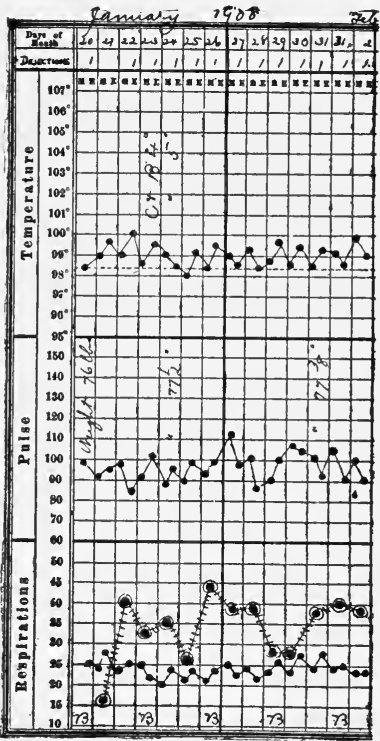

Fig. I54.-Chart of case 308 . heart is negative. The lungs are tympanitic throughout, with scattered râles of various sizes, especially numerous in the right axilla and at the right base behind. There are two operation scars in the right and in the left lower quadrant of the abdomen. The fingers are markedly clubbed, the nails curved horizontally and laterally; slight clubbing of the toes also. The blood and urine are normal. His sputum is of a very offensive odor.

Discussion.-Chronic cough associated with clubbed fingers in a young boy with a sound heart means usually one of three things:

(a) Chronic bronchitis with bronchiectasis.

(b) Chronic pleurisy, serous or purulent.

(c) Phthisis.

A pulmonary abscess could not have lasted so long without producing more definitely circumscribed physical signs in the affected lung. We should be practically sure, also, to find a history of the discharge of large quantities of sputum within a short time when the cavity was emptied out. 
Returning now to the three alternatives mentioned above, it seems certain that the physical signs would be far more marked and extensive if pulmonary tuberculosis had been at work for three years. Of course, the sputa must be carefully and repeatedly examined. A dozen negative examinations in succession would constitute strong evidence against tuberculosis.

All types of pleurisy can be easily ruled out. There would be marked flattening of one chest, displacement of the heart, and much more characteristic physical signs than are here reported.

If tubercle bacilli are proved to be absent, the only reasonable conclusion will be that this boy is suffering from a bronchiectasis, probably with secondary infection of the dilated bronchi by influenza, the usual inhabitant of such diseased tubes. Between the enlarged bronchi, pulmonary tissue becomes condensed and more and more atrophic.

Conclusive evidence may very possibly be obtained by means of $x$-ray examination, which is especially valuable in young subjects with thin chest-walls.

Outcome.--Examination of the abundant purulent sputa was performed many times. Pneumococci, streptococci, and influenza bacilli were present always; tubercle bacilli never. $X$-ray examination showed shadows suggestive of a number of dilated bronchi. The diagnosis of bronchiectasis seemed reasonably certain.

A vaccine made from the influenza bacilli isolated from the boy's sputa, was injected a number of times, but had no obvious effect except to increase the amount of sputum, a change which was noted after each injection.

This patient reëntered the hospital September 3, 1908, and died of pneumonia after an illness of six days.

Diagnosis.-Bronchiectasis.

\section{Case 309}

A rag-sorter of fifty-six entered the hospital June I3, I907. He has always been well except for a slight cough during the last three years. His family history is excellent. He has been much more annoyed than usual during the last four weeks by a cough accompanied by viscid, scanty sputa. He has had pain, first in the right chest, now in the left. There has been no fever, but much weakness. The arteries are palpable and tortuous, his fingers clubbed. Scattered throughout the lungs are many fine and coarse râles; the lungs are generally hyperresonant, the breathing strongly suggestive of emphysema. 
The râles are more numerous at the base of each axilla. Near the vertebral border of the left scapula there is a patch of pure bronchial breathing about the size of a silver dollar. (See Figs. ${ }_{55}$ and ${ }_{556 .)}$

The leukocytes are $17, \infty 00$; urine, normal; sputa abundant, mucopurulent, containing a few pneumococci; nothing else of interest.

Discussion.-The history, the social condition of the patient, the physical signs, and the clubbed fingers suggest a chronic bronchitis with bronchiectasis, but from the nature of the present complaints it would seem that something more acute must be going on, especially as there is a patch of bronchial breathing in the left back. How is this to be explained?

Bronchopneumonia and tuberculosis are the chief possibilities. Of tuberculosis there is as yet no evidence, but we have not yet watched the case long enough to have any right to confidence on this point. Cases beginning with signs like these often continue for months and years without any proof of our suspicions of tuberculosis, until finally a sputum examination is positive. Many such cases deserve to be treated as tuberculous long before we can prove them to be so.

It is quite possible, however, that we are dealing in this case with nothing more dangerous than one of those attacks of bronchopneumonia so apt to occur from time to time in the course of a chronic bronchitis with bronchiectasis. Indeed, it is sometimes convenient to divide this disease into three phases:

(a) The summer phase.

(b) The winter phase.

(c) The bronchopneumonic attacks.

In summer it may be nothing but a little wheezing induced by exertion or by laughing; in winter we get infection of the bronchiectatic cavities with influenza; profuse purulent discharge and paroxysms of coughing, diurnal and nocturnal, are the result.

At any time there may be acute febrile attacks, with or without definitely localized, demonstrable foci of solidification, such as are here described. The vast majority of such attacks run a favorable course within a few weeks. They are associated with a good deal more wheezing and a more abundant nummular sputum than is usual in lobar pneumonia.

Outcome.-By June 2 Ist the signs had practically disappeared from the left chest, and the patient, though not well, was in approximately the same condition as before his acute attack. He was accordingly allowed to go home.

Diagnosis.-Bronchitis; bronchopneumonia, bronchiectasis, and emphysema. 


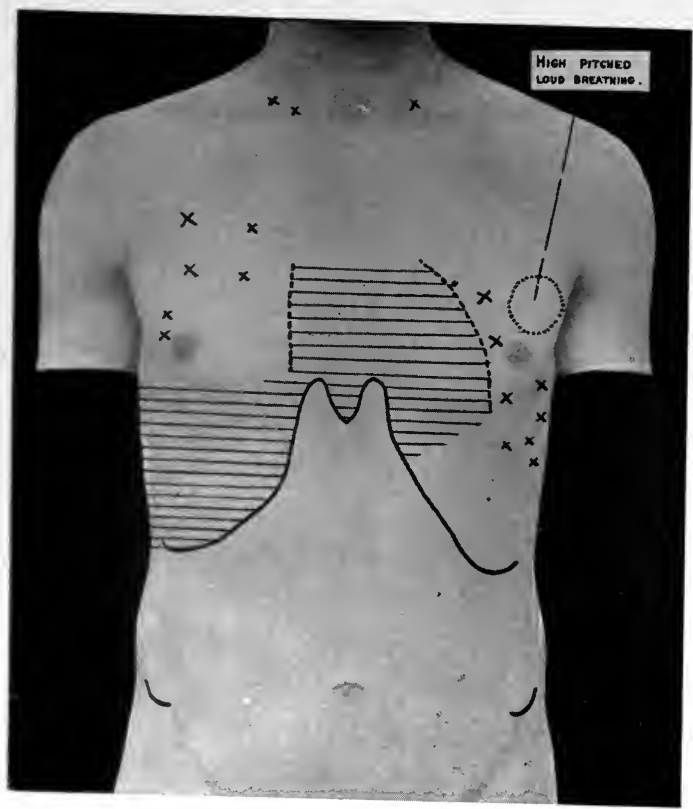

Fig. I55--Areas of cardiac and hepatic dulness in Case 309; also position of râles (crosses).

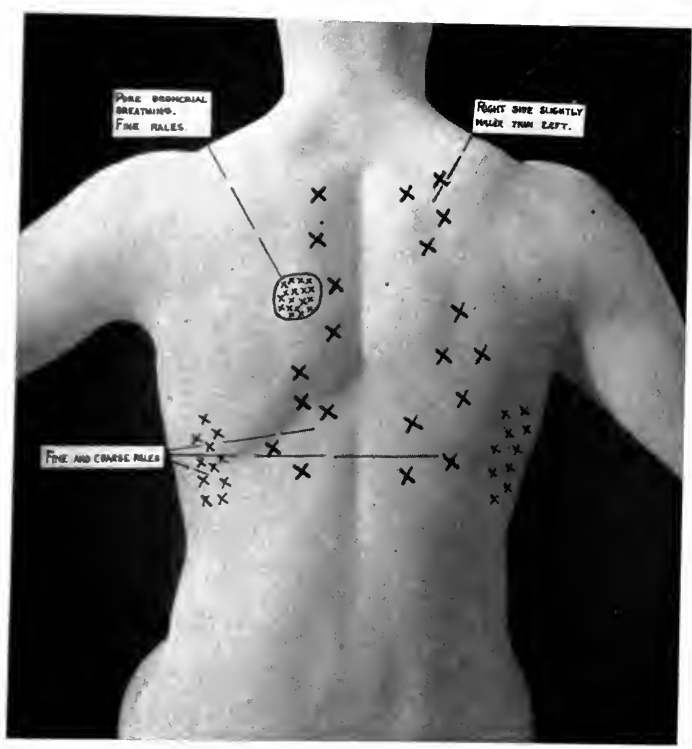

Fig. 156.-Results of auscultation and percussion in Case 309. Three years' cough; clubbed fingers. 



\section{Case 310}

A nurse of twenty-four entered the hospital May 5, 1907. She has always previously been well, and has an excellent family history. For a week she has had a bad cold, with headache, loss of appetite, cough, and frothy white sputum.

The course of the temperature is seen in the accompanying chart. The breathing above the third rib, in the right front, is much diminished, occasionally of cog-wheel type, and accompanied by crackles and squeaks. There is a friction-rub in the right axilla. Visceral examination is otherwise negative, as is the blood, the urine and the sputum. Gradually an area of dulness developed in the right axilla and spread over the whole right chest by the thirteenth of May, with flatness below midscapula and intense bronchial breathing. Many crackles and friction-sounds were heard over this area. The white cells continued low (7000). The sputum was repeatedly examined, with negative results.

On the nineteenth a trocar was introduced in the right posterior axillary line, $\frac{1}{2}$ inch below the angle of the scapula. It appeared to enter solid lung, and no fluid was withdrawn.

By the twenty-second she had regained her appetite, and although pale and emaciated, was in good spirits. The physical signs were as previously described, except that the râles and the pleural rubs were now practically

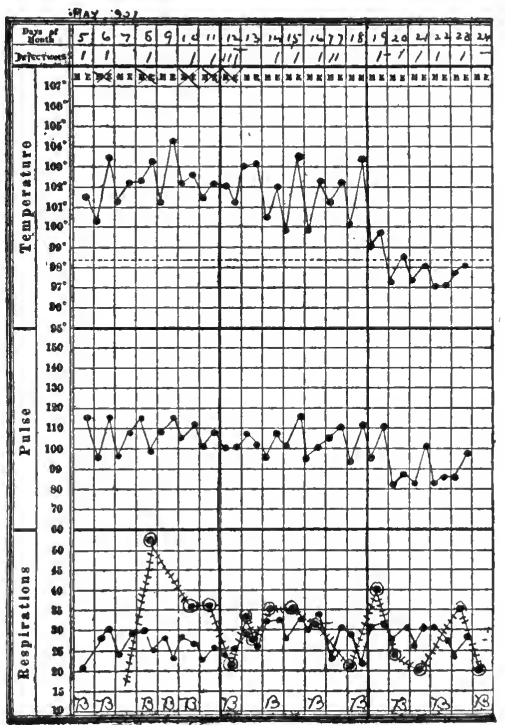

Fig. 157.-Chart of case 310. gone.

By the twenty-fifth dulness was less marked; the breathing bronchovesicular or vesicular.

On the twenty-ninth dulness persisted in the right axilla and a little in front, but there was none in the back, and the breath-sounds were there normal, while in front they were still bronchovesicular, with an occasional crackle.

Discussion.-The case looks alarmingly like one of consumption, in spite of its acute onset. The physical signs are by no means distinc- 
tive, but through the earlier part of the disease are perfectly consistent with tuberculosis. One could only attain greater certainty at this period of the disease by repeated sputum examinations and by the cutaneous tuberculin tests (valuable, if negative).

The onset is very unlike that of ordinary pneumonia. Flatness on percussion, such as was observed about the thirteenth of May, almost never occurs in true pneumonia, and in the great majority of cases indicates free fluid. I am alluding here to flatness used in the strict sense, and in contradistinction from dulness. It was for this reason that the exploratory puncture was done despite the presence of bronchial breathing and râles. It is of great importance to remember that fluid has again and again been obtained through a needle inserted at a point where bronchial breathing, râles, or both were clearly audible.

The result of tapping excluded fluid at that date, though it seems to me quite possible that an effusion had previously been present. The tapping seems to me entirely justified, for there was a good deal in the aspects of the case on the nineteenth, which suggested a postpneumonic empyema and only tapping could rule this out.

Once this result was obtained, the balance of probabilities was again in favor of pneumonia. One further possibility, however, remained, viz., interlobar empyema, a complication always difficult of recognition, though not uncommon.

How is the low white count to be explained? The patient was never in that condition of desperate illness which we associate with most cases of pneumonia without leukocytosis. Indeed, she was never in any condition calling for anxiety. In all probability the disease was due to some organism other than the pneumococcus. Clinically, the course was distinctly atypical. Both the physical signs and the leukocyte count were distinctly "queer," but not enough is known as yet regarding the pneumonias due to organisms other than the pneumococcus to enable us to recognize the definite types, such as streptococcous pneumonia or influenzal pneumonia.

Outcome.-By June $2 \mathrm{~d}$ the breath-sounds were everywhere normal, the râles gone, the dulness very slight. Her convalescence was uneventful thereafter.

Diagnosis.-Pneumonia.

\section{Case 311}

A clerk of eighteen entered the hospital June 24, I907. His family history was excellent. He had never been sick until the present time. A week ago he woke up coughing and raising bloody sputum; in all, 
about two teaspoonfuls. He had then no pain, vomiting, or fever. From this time on he continued to have cough and began to be short of breath. He has been weak and has kept his bed. (See chart for temperature, and diagram for condition of the lungs.).

Physical examination was otherwise negative; white cells, 8000; urine normal. The sputa was examined three times for tubercle bacilli, but nothing found. The patient had a good appetite, and did not seem especially sick. During his stay in the hospital he raised no more blood, but the signs extended until most of the left lung was involved. Later the base cleared very much, but at the left apex, both front and back, there remained bronchial breathing, crackling râles, and increased fremitus. Near the anterior fold of the right axilla amphoric breathing and "cracked-pot sound" were obtained.

Discussion.-The onset is not typical of any of the commoner respiratory diseases, and pneumonia should have fever and leukocytosis from the beginning, even when cough and sputa are absent. On the other hand, the signs remind us more of pneumonia than of anything else,

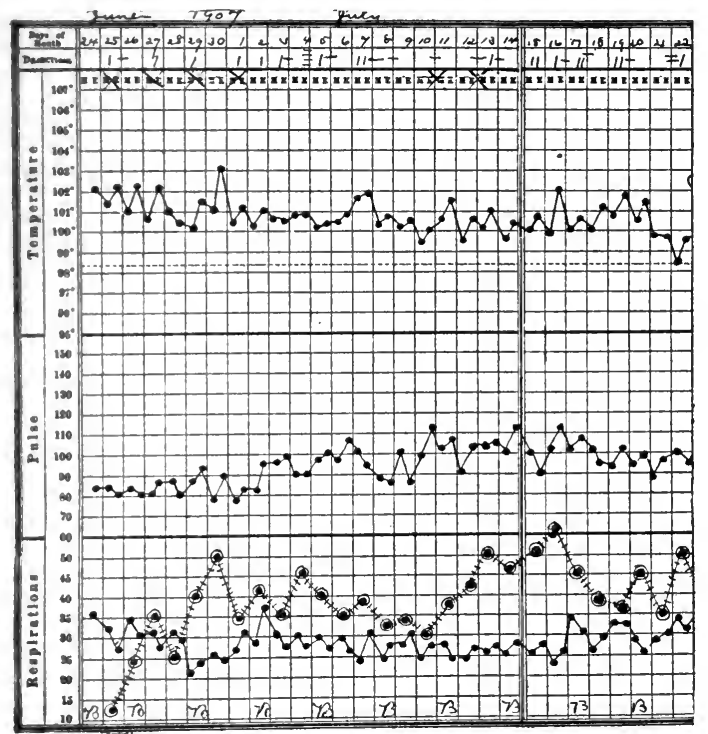

Fig. 158.-Chart of case 3Ir.

and as the condition persists it is natural to ask whether we may not be dealing with a failure of resolution. I have already said, however, in the discussion of previous cases, that unresolved pneumonia usually turns out, in my experience, to be a mistake in diagnosis, the actual condition being postpneumonic empyema. 
One would hardly make a diagnosis of this latter condition, however, unless one had better reason to believe in the original pneumonia. But there is no such difficulty with the diagnosis of pulmonary abscess which may be next considered. This disease may begin acutely and without any hint of a cause. Blood may be raised, as in the present case, and as there are no typical signs of abscess, we cannot quarrel with those which this patient shows. Abscess may exist with almost any combination of signs and without any signs at all.

Nevertheless, abscess of the lungs, arising in this way without any known cause and without lesions in other organs, is distinctly rare and the character of the sputa is not at all typical. One expects a larger amount of pus and a foul odor.

Acute tuberculosis cannot be excluded. We very rarely observe so rapid a progress in tuberculous disease, and the negative examinations of sputa are of considerable though not of decisive importance. Most cases of tuberculosis beginning with hemoptysis present no physical signs at all within the first two or three weeks. Accordingly, the ordinary course of affairs is as follows: The patient is much alarmed by the hemoptysis, and soon calls upon a physician for examination of the lungs. This examination reveals nothing whatever. The temperature is normal, the blood-spitting already beginning to seem ancient history as the patient now feels perfectly well. The doctor allows his wish to be the father of his hope, and, humoring the patient's urgent desire to be told that he is not tuberculous, gives a clean bill of health and surmises that the blood came from the throat.

Thus a golden opportunity is lost, and the patient is not treated for tuberculosis until the more obvious signs make their appearance some months later.

In the present case there is nothing that we can do but persist in the sputum examinations. Either tubercle bacilli will appear or the sputum will become foul and take on the other characteristics of abscess.

Outcome.-Only on the fourth examination-July $2 \mathrm{~d}-$ were tubercle bacilli discovered. The patient went home July $23 \mathrm{~d}$ considerably worse.

I take this opportunity of enumerating and discussing briefly the causes of hemoptysis. Leaving on one side the cases in which only slight streaks or fragments of blood appear, mixed with mucopurulent sputa, and also the cases in which blood, obviously derived from the nasal cavities, is expectorated, we may group practically all the cases of hemoptysis occurring in temperate climates under the following three headings: 


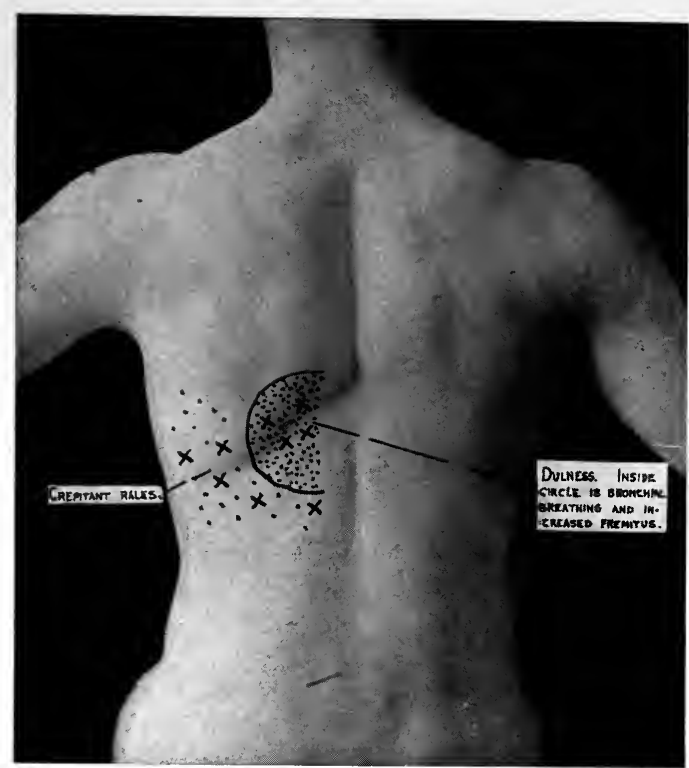

Fig. 159.- Chest signs in Case 311. History: seven days' cough; bloody sputa. 

(a) Hemoptysis due to tuberculosis.

(b) Hemoptysis due to pulmonary infarct, usually from mitra] disease.

(c) Hemoptysis from pulmonary abscess (non-tuberculous).

The last two groups can usually be recognized with ease by the history and the attending physical signs, cardiac or pulmonary. Practically all the cases of hemoptysis which we puzzle over are later ex. plained as tuberculosis or tlse remain wholly unexplained. In the unexplained group should be placed those traditionally charged up to vicarious menstruation, to hysteria, and other mythical causes.

In hemorrhagic diseases, such as purpura, scurvy, hemophilia, leukemia, and in the hemorrhagic forms of the exanthemata, we may have blood-spitting, but diagnostic puzzles rarely arise in these diseases. Occasionally a case of uremia obeys the mandate of nature to lower blood-pressure by any and all methods, so that pulmonary hemorrhage, instead of the ordinary uremic nose-bleed, occurs. There could be no difficulty in recognizing the source of such a hemorrhage unless we omitted to study the heart and kidneys.

In many cases a patient is alarmed by the expectoration of blood which the physician sees, at his first examination, to come from a spongy gum. In various forms of stomatitis the patient may awake in the morning to find a blood-stain on the pillow. This often excites great alarm, but the most casual examination of the mouth should make clear the source of the bleeding. Nocturnal epilepsy, however, should also be remembered in such a case, as the patient may be himself quite unaware of the fit.

Summing up this discussion, I wish to emphasize the point that there is but one important cause of obscure hemoptysis, viz., tuberculosis. If the source of a pulmonary hemorrhage is not made clear by the examination of the heart, lungs, gums, and nasopharynx, and if it is not obviously the expression of some infectious or constitutional malady, it is in all probability the first sign of phthisis. I do not deny that the causes of hemoptysis are numerous, but I assert that the causes of genuinely obscure hemoptysis in temperate climates may be reduced to one-pulmonary tuberculosis. I may refer in this connection to the cureful study of F. T. Lord, ${ }^{1}$ in which it is demonstrated that in the great majority of cases in which a young person has a pulmonary hemorrhage, recovers at once, and remains well for the rest of his life, postmortem examination proves the bleeding to have been due to tuberculosis which healed without ever producing further symptoms.

Diagnosis.-Pneumonic phthisis.

${ }^{1}$ Boston Med. and Surg. Jour., I909, vol. clxi, p. 57 I. 


\section{Case 312}

A Canadian brakeman, twenty-seven years old, was jammed October 31, 1907, between two freight-cars and sustained severe contusions over the sacrum and left thigh. The day after the injury he had a severe chill; another occurred while in the ambulance on his way to the hospital, November 9 th.

For the past ten days, while in the surgical wards, he has had cough and continued fever.

Signs of solidification were found at last near the angle of the right scapula, associated with a good deal of pain in that side. There was no dyspnea at any time.

When seen November 9 th the white cells were 18,600 . The patient's appearance distinctly suggested phthisis, but repeated examinations of the sputa showed no tubercle bacilli, and by the seventeenth of Novem-

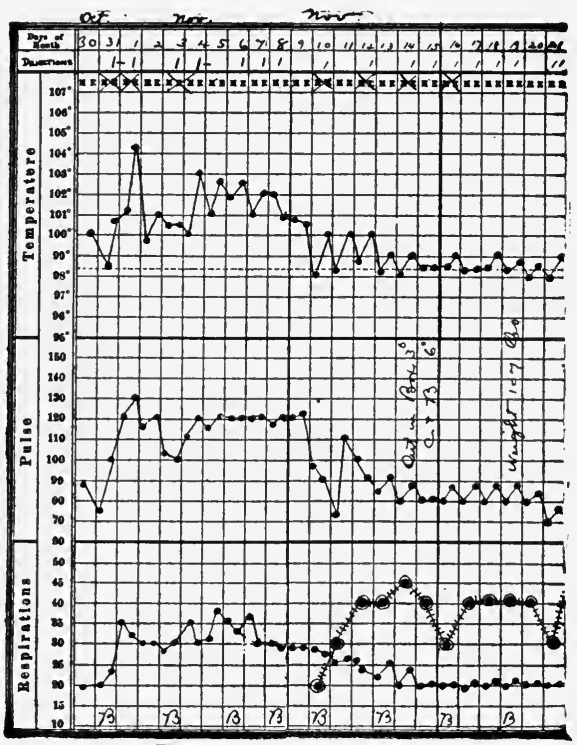

Fig. I60.-Chart of case $3^{\text {I } 2 \text {. }}$

ber the lung signs had nearly cleared up, though rough breathing and a few râles were still heard in the upper left lung.

On the twentieth, there was dulness and diminished respiration over a small area at the right base. A needle was introduced here and penetrated thick, hard pleura into apparently normal lung. Soon after this all signs disappeared. The patient was able to go home on the twenty-second, entirely well. 
Discussion.-It is natural to ask at the outset whether this patient's symptoms may be due to trauma; so severe an accident might easily have wounded the lung. But the facts seem to be against this hypothesis. Apparently, his chest was not injured, and if we take the record on its face value, this is conclusive. At the postmortem table, however. one sometimes sees strange revelations in cases of this kind. Fractured ribs and fractured pelvis, wholly unsuspected during life, I have seen demonstrated postmortem to the great chagrin of all concerned.

It is difficult to suggest in the printed record the strongly tuberculous appearance of this patient. Any one in the habit of judging by facies and the general look of the patient could hardly have doubted that he was phthisical. If one adopted this hypothesis, one would have to say that the tuberculous process was "lighted up" by the accident. We have good reason to believe that a patient's general power of resistance may be notably diminished by such an accident as this, so that he becomes much more subject to infectious disease of any kind.

Doubtless this idea has been overworked in the innumerable suits for damages brought against steam and electric-car roads by persons who have been injured. The lawyer for the plaintiff can always succeed in finding some doctor who will swear that the pulmonary tuberculosis from which the patient now suffers in an advanced form did not exist before the accident and must have been produced by it. But without believing that anything of this kind often happens, one must admit its possibility.

In the present case we must confess that the signs are quite compatible with tuberculosis, though by no means typical of it. Further light can be obtained only by the results of repeated sputum examination and by the cutaneous tuberculin test.

Pleural effusion was seriously considered here, as is indicated by the fact that the chest was punctured. The question, "Have we fluid or solid in this chest?" is much more frequently a difficult one than textbooks would lead us to suppose. A small effusion at the base of the lung may so compress and condense the pulmonary tissue above it that all the signs of solidification are present. This is equally true in dropsical effusions due to heart disease and associated with edema of the lung itself. I have known many such cases mistaken for pneumonia.

In view of the outcome of the case, it seems more than probable that the whole affair represented that type of pneumonia known as "traumatic" or "surgical," and, due to that very lowering of resistance by traumatism which I have hinted above, is often falsely lugged in to explain a long-standing phthisis. Doubtless it is because we are so 
familiar with the fact that trauma can cause pneumonia by favoring infection that we invoke the same theory quite unjustifiably in tuberculosis.

Many cases of "traumatic pneumonia" have much more insidious onsets than this, not only without dyspnea, but without pain or cough, and sometimes without fever. The latter is especially apt to occur in elderly persons and remains wholly undiscovered unless routine physical examination, performed as a sort of daily house-cleaning, brings the disease to light.

Diagnosis.-Traumatic pneumonia.

\section{Case 313}

A granite-cutter of sixty-five entered the hospital February I7, I908. His family history and past history were excellent. He has had a winter cough for twenty-years, to which he has grown so used that he thinks little of it; but for six weeks his accustomed "bronchitis" has been somewhat worse than usual, and for the last three weeks he has done but little work. He has distress after each meal, and abdominal pain when he coughs hard. During the night he has to pass water every hour, and it burns him. For many years he has had cramps in his legs. He says they are most troublesome at the time of the new moon, and last through the first quarter. When young, he weighed I75 pounds; now he weighs I23, but he says he has lost no weight of late years.

The patient's face is pitted with small-pox; eyes show complete arcus senilis on both sides. His pupils are small and irregular, the right larger than the left. Both react to light and distance.

The heart-sounds are somewhat indistinct, but a careful examination of the organ shows nothing else of importance.

The blood-pressure is $135 \mathrm{~mm}$. Hg. The arteries are palpable and tortuous. The condition of his lungs is shown in the accompanying diagram. Temperature, blood, and urine are normal. Underneath the right rib margin there is a dull, resistant, firm mass, which shifts little, if at all, with respiration. When examined in a warm bath, this tumor disappears, but there is still more resistance in the muscles of that region than elsewhere. The sputum is very profuse and purulent. The patient seems weak and sleeps much of the time.

Discussion.-Cough without fever is usually of no great importance, especially in a person who has had it every winter for twenty years. Under these conditions it is natural to assume it an old man's bronchiectasis with a more recent (possibly influenzal) infection of the cavities. The physical signs are by no means typical of this condition here, but 


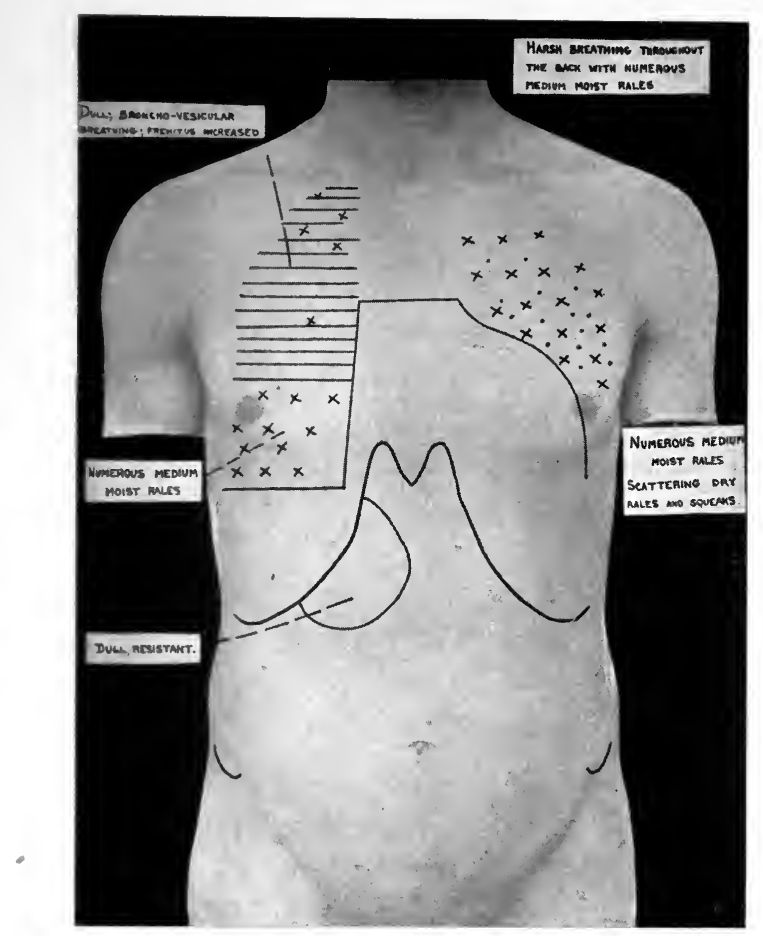

Fig. 161.-Signs demonstrated in Case 3г3. Complaint: twenty years' "winter cough." 

July-AUG. 1909

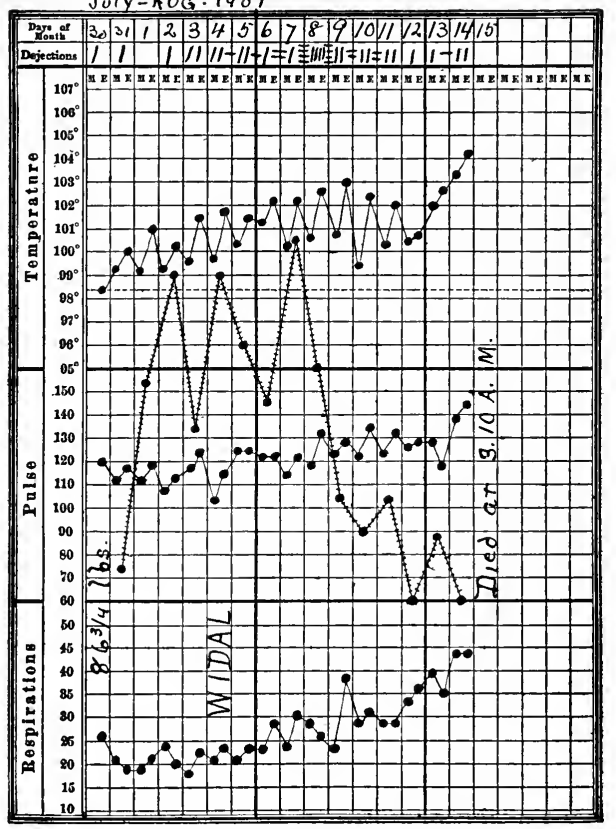

Fig. I6Ia.-Diabetes mellitus, accompanying miliary tuberculosis. 
they will do in case the sputa proves negative and no other good reason for the cough can be adduced.

In men of this age it is often difficult to distinguish " $a$ heart cough" from " a lung cough." Cardiac weakness favors stasis in the lungs, with malnutrition and increased susceptibility to infection. On the other hand, any infection of the bronchial tract leads to increased work for the heart and thus perhaps to cardiac stasis. This patient apparently has arteriosclerosis (palpable and tortuous arteries), and his heartsounds are said to be feeble. But, on the whole, this does not seem to me enough to make us consider the heart seriously as a cause for his cough.

It is of the greatest importance to remember that tuberculosis may at any time become ingrafted upon the lungs of a patient who has suffered for many years from nothing more serious than a winter cough. The only safe plan is to assume each time that one sees such a patient that he may have contracted tuberculosis recently, and to test this possibility by repeated examinations of the sputa as well as by a temperature chart and a study of the pulmonary signs. In the statistics of Dr. Cleaveland Floyd, already referred to, this point is well illustrated.

Outcome.-On the third examination, tubercle bacilli were found in the sputum.

Diagnosis.-Phthisis.

\section{Case 314}

A widow of thirty-five entered the hospital July 30, 1907. Her husband died of hemorrhage from the lungs. Her family history is excellent. She had pneumonia six years ago, in the Portland, Maine, Hospital. Since the birth of her last child, three years ago, she has had no menstruation. For a year she has been coughing and raising much phlegm, but never any blood. Three weeks ago she began to cough less, but has been much "choked up" and has felt very weak. She has a splendid appetite and rarely vomits. Her bowels are usually regular, but she passes water very frequently, both day and night. She says she once weighed 200; at entrance she weighed 86 . She says she has not an ache or a pain, and complains at present only of great general weakness.

On examination the patient is found to be emaciated, the skin dry and rough, the pupils irregular, neither reacting to light, the right larger than the left. The heart and lungs show nothing abnormal. The abdomen is full, resistant in the upper half, soft and tympanitic below. The liver dulness extends from the sixth rib to the umbilicus, 
and the edge of the organ is easily felt there. The white cells are 4200; hemoglobin, 70 per cent. The urine contains no albumin and no casts; specific gravity, IO25; it contains considerable sugar. On the fourth of August there was a positive Widal reaction, absolute loss of motility, and agglutination in one hour in dilutions of $\mathrm{I}: \mathrm{IO}$ and $\mathrm{I}: 50$. On this date there were many fine, moist râles at the base of each lung; a small abscess formed at the top of the right little finger. It was opened and a pure culture of staphylococcus obtained. The sputum was repeatedly examined, with negative results.

On August 8th there was sudden severe pain in the hypogastrium, with a falling temperature, a rising pulse, and increasing abdominal distention. All symptoms disappeared after two hours.

By the thirteenth she was much worse, very toxic, noisy and slightly delirious, with muscular tremor, veins bloated, râles growing more numerous, and abdomen more distended. The diacetic acid which was present in the urine at entrance had now disappeared, and the sugar had fallen to 2 per cent.

On the fourteenth of September a patch of bronchovesicular respiration with crepitant râles was heard in the right axilla, and there was slight external strabismus. She died on the fifteenth, the diagnosis being typhoid fever, diabetes mellitus, bronchopneumonia.

At autopsy there was found miliary tuberculosis of the lungs, spleen, and kidneys, fatty liver-no evidence whatever of typhoid. The patient had stated positively that she had never had typhoid fever.

Discussion.-I did not see this case during life, and I have no reason to believe that my diagnosis would have been any nearer correct than that which was made. Everybody was "bowled over" by the Widal reaction, and assumed that the case was one of typhoid fever. Looking back now from the standpoint of the autopsy, it is worth while to consider by what signs we might have been warned against the mistake which we made.

Obviously, we were dealing with a case of diabetes and not merely with a symptomatic glycosuria. The long-standing weakness and emaciation, despite a splendid appetite, point to this conclusion. But diabetes is very seldom associated with typhoid infection. I have not been able to find any such case in the records of the Massachusetts General Hospital, though Curschmann has observed such.

It is notorious that there is another infectious disease which diabetics are especially prone to catch-viz., tuberculosis. Of this, there is little evidence in the present case, yet it should be noted that the patient has been coughing and expectorating for a year, and that the pulmonary 
signs, although not at present characteristic, are compatible with tuberculosis. When the strabismus appeared in the last days of the patient's life, the suggestion of tuberculosis became inevitable. Before that the repeated negative examinations of the sputa threw us off the track, and the lung signs were interpreted as a typhoid bronchitis.

As I review the results of autopsy experience in diabetes and recall the number of mistakes, more or less similar to that made in the present case, I feel inclined to formulate the rule that any pulmonary signs (obviously not those of pneumonia) occurring in a diabetic should be assumed to be due to tuberculosis, especially if the patient is in an advanced stage of this disease.

The Widal reaction remains a mystery, and furnishes an example of the dangers attendant upon our modern habit of placing almost exclusive reliance on signs of this kind in diagnosis. If this case had occurred prior to $I \$ 96$, it is probable that the mistake would not have been made. We should have turned more attention upon the past history and the present signs, as seen in the light of our general knowledge of the complications usually occurring in diabetes.

Diagnosis.-Miliary tuberculosis and diabetes.

\section{Case 315}

A married woman of thirty-eight was first seen April I, 1907. She was never sick until seven years ago, when she had a sore on the genitals and in her throat. At that time her hair came out.

Two years ago she had an operation upon her breast-bone at the Carney Hospital. For two years she has had severe headaches, with "fits and faint spells." These last sometimes seem to be brought on by anger or excitement.

In December, I906, she gave birth to a child, which died three days later at the Infants' Hospital and was said to have had syphilis.

For the past three months she has had a painful cough, with nightsweats and thick yellow sputum. She is weak, dyspneic, constipated, eats and sleeps poorly, has many headaches, and faints when she gets angry.

On examination the patient is obese and shows enlarged glands in the neck, axillæ, and groins. The inner third of the right clavicle is missing. An old operation scar occupies its site. On cough, the lung projects through the hole thus left. The heart and peripheral blood-vessels show nothing abnormal. Over an area extending from the right apex to the third rib in front and to the scapula behind, expiration and inspiration are very noisy and strident. There are occasional crackling râles in 
this area. Elsewhere the lungs are negative. The sputum shows many intracellular influenza bacilli, a few pneumococci, no tubercle bacilli.

A letter to the Carney Hospital showed that the lump excised from the clavicle, which before operation had been taken to be tuberculosis, showed gumma when examined histologically.

It was also learned that the patient had been eight months in the Worcester Insane Asylum some years ago.

Tuberculin, Io milligrams, was injected, with negative results. (For temperature see the accompanying chart, Fig. I62.)

The patient was given mercury; also iodid of potash in doses increased from to to Ioo grains three times a day. By this treatment, symptoms and signs very markedly improved, so that by the eighteenth of April

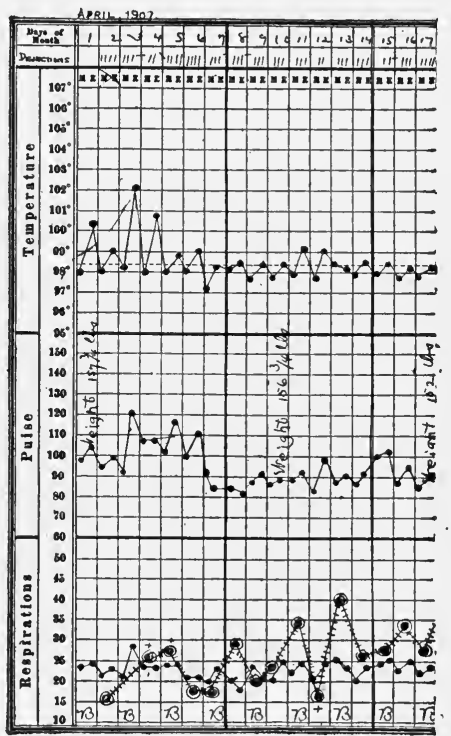

Fig. 162.-Chart of case 315 . she was able to leave the hospital:

The physical signs at this time consisted of bronchovesicular breathing and a few medium râles at the right apex.

$X$-ray report by Dr. Walter Dodd:

"Apices.-Both present hazy appearance. Left more marked than right. At base of the right lung there was a dense shadow observed which started about I inch from median line at level of sixth space. Shadow was deep seated.

Diaphragm. - Excursion on left side normal. Could not see diaphragm on right side. When patient coughed the shadow at its base moved upward about I inch and receded immediately."

Discussion.- It was clear enough that this patient was suffering mainly from syphilis, but what of the pulmonary conditions? Tuberculosis, as is well known, often complicates syphilis, owing to the diminution of resisting power brought about by the syphilis. Ordinary types of bronchitis, due to the influenza bacillus or other organism, are also of frequent occurrence in syphilitics. There are no physical signs or clinical features characteristic of pulmonary syphilis, so that the diagnosis can never be made with any confidence.

Experience seems to me to show that it is safe to assume pulmonary complications of this kind to be due to syphilis, provided, of course, that we are convinced by sputum examination or otherwise that the case is not 
one of tuberculosis. It was shown some years ago by Dr. E. G. Janeway that patients who have fever, night-sweats, and pulmonary signs like those ordinarily seen in phthisis may promptly recover under antisyphilitic treatment, after residence in a sanatorium for tuberculosis has failed to benefit them at all. It seems, therefore, the wiser course to give every syphilitic the benefit of the doubt, and treat him with mercury and potassium iodid while we continue to search his sputum for tubercle bacilli. If marked and rapid improvement takes place under this treatment, we may conjecture that we have been dealing with a case of pulmonary syphilis, but as the pathologic anatomy of that disease is practically unknown, it is difficult to make any positive statement on the matter.

A point of great interest in this case is the patient's habit of fainting when she gets angry. Ordinarily, one would call attention to such a symptom as indicating a hysteric basis for any other complaints which the patient might express. In this case we have reason to believe that organic brain disease of syphilitic origin is present. Yet the patient faints when she gets angry. It may be that a closer psychologic study of the case would show that the fit of anger-like the fits of running which immediately precede some epileptic attacks-is the first symptom, not the cause, of the subsequent loss of consciousness. In anger we are only partly ourselves; in fainting we cease to be ourselves at all.

Diagnosis.-Syphilitic disease of the lung.

\section{Case 316}

A gardener sixty-one years old, with an excellent family history, entered the hospital June 24, I908. He states that he had dropsy three years ago, while at the Boston City Hospital.

Five months ago he caught a bad cold, and has had a troublesome cough, with profuse sputa, dyspnea, and poor appetite ever since. For three months he has had orthopnea. The cough often keeps him awake.

On examination he was found to be emaciated, orthopneic, markedly cyanotic, and breathing with much difficulty; the rate was from 40 to 50 a minute. The lungs showed many coarse bubbling râles on both sides. There was very slight dulness and harsh breathing above and below the right clavicle in front, also slight dulness and prolonged low-pitched respiration at the right apex behind. There was slight general abdominal tenderness, and the edge of the liver could be felt $\mathrm{I} \frac{1}{2}$ inches below the ribs. On percussion, the upper border was at the sixth rib. (For temperature, see the accompanying chart, Fig. I63.) 
The white cells at entrance were 29,000; the urine, sp. gr. I009 to ror 4,30 ounces in twenty-four hours, with a slight trace of albumin, many hyaline and coarse granular casts. The heart's impulse and dulness were felt in the fifth space, inside the nipple-line. A systolic murmur was audible at the apex, and the pulmonic second sound was accentuated. The first apex sound was very loud and sharp. The arteries were palpable and tortuous, with a lateral excursion in the brachials.

Discussion.-At this patient's age, with the evidences of cardiac and vascular disease furnished by the physical examination, and in view of the history of dropsy three years previously, it

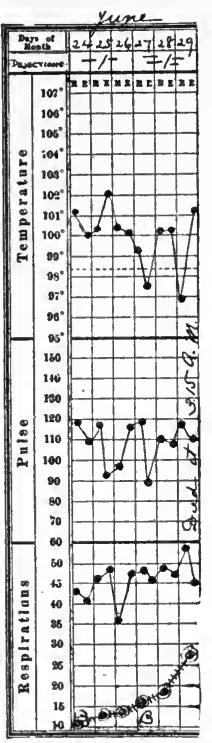

Fig. I63.-Chart of case 316 . would be natural to assume at the outset that the pulmonary signs are due to stasis and insufficient heart action.

Against this idea, however, is the presence of fever and leukocytosis, neither of which should be produced by the degenerative, non-infectious type of heart trouble which we expect in people of this age. The acute vegetative types of endocarditis and the myocardial infections which might produce fever and leukocytosis along with pulmonary stasis in a younger patient, are rarely seen at sixty-one unless as terminal infections. The present illness, however, has been going on for five months, and cannot be called terminal.

We are apt to forget the possibility of tuberculosis in people who have managed to worry through sixty years of life without acquiring it, but recent statistics give us no excuse for this form of absentmindedness, and warn us to search the sputum of every patient who has any cough, whatever his age, especially when the pulmonary signs seem to be most marked at the apex of the lung involved.

Outcome.-The sputa showed a few tubercle bacilli, though it had in other respects the characteristics of pulmonary abscess and was at times excessively foul. The patient lost ground rapidly after entering the hospital, and died on June zoth.

Autopsy showed tuberculosis of the lungs, chronic interstitial nephritis, hypertrophy and dilatation of the heart, tubercular ulcers of the intestine, and hypernephroma.

Diagnosis.-See last paragraph. 


\section{Case 317}

A weaver of twenty-four was seen August 20, 1907. He had "stomach trouble" three or four years ago. He has otherwise been well.

A week ago he began to have cough, headache, and, after two days, vomiting, the vomitus containing some blood. Three days ago he had a chill, pain in his left side, loss of appetite, insomnia, and constipation. He has been in bed three days.

In the left back, close to the spine of the scapula, are heard harsh respiration and a few fine râles. The lungs are otherwise negative. Palpation of the abdomen causes severe paroxysms of cough. The blood and urine are negative. (The temperature is as seen in the accompanying chart.)

The next morning an erythematous eruption appeared in the right half of the trunk, arms, and legs. This disappeared after calomel $\frac{1}{2}$ grain every half-hour for six doses, but soon broke out again, the wheals being, however, less numerous the second time.

On the twenty-fifth of August the upper lip suddenly became much swollen. The patient was given calcium chlorid, I gram three times a day, Carlsbad salts, I dram three times a day. On the twenty-sixth he was practically well.

Discussion.-This case is introduced merely to exemplify one of the unusual manifestations of urticaria. I have previously illustrated the manifestations of urticarial lesions in the intestinal tract. (See p. 74.) In the present case we have good reason to believe that the

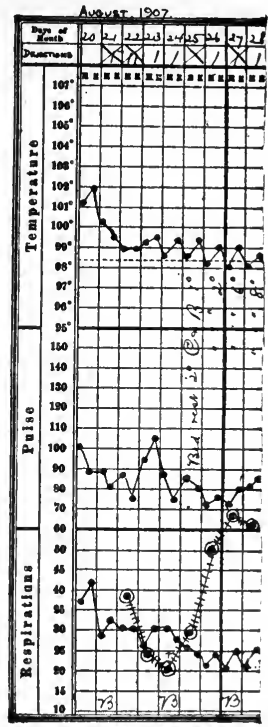

Fig. I64.-Chart of case 3 I 7 . bronchial tree was the seat of similar lesions, producing patches of edema which corresponded to the signs recorded in the text.

It may be of some importance not to forget the possibility of a cough produced in this way, since it would be likely to yield to treatment of the same type, which, in some cases, is found to be effective against the cutantous wheals, viz., catharsis and attention to diet.

Diagnosis.-Internal urticaria.

Dr. Lawrason Brown of Saranac Lake, N. Y., gives me permission to add here certain of his admirable theses on the diagnosis of pulmonary tuberculosis.

"I. An appearance of ruddy health does not exclude tuberculosis. 
"2. In any patient with constitutional symptoms (i. e., loss of weight, strength and color, dyspepsia unexplained, slight fever, rapid pulse), no matter of what he complains, the possibility of tuberculosis must be kept constantly in mind.

"3. Prolonged and intimate exposure to tuberculosis at any time of life, but especially in childhood, and in home or workshop or office, is vastly more important in diagnosis than 'unassociated' or 'noncontact' heredity.

"4. Prolonged contact with tuberculosis may lead to infection, but debilitating conditions are necessary usually to cause this to develop into clinical tuberculosis.

"5. Constitutional or general symptoms lead us to a diagnosis of tuberculosis, while the localizing symptoms point out the organs involved.

"6. The history or presence of certain complications, as fistula in ano, pleurisy, dry, or especially with effusion, adenitis, a discharging ear, coming on painlessly, are all strongly suggestive of tuberculosis.

"7. Loss of color, prolonged exposure to tuberculous infection, especially in childhood, with a history of swollen glands at that time, the more recent subjection to debilitating conditions, the presence of unequivocal constitutional and localizing symptoms, with or without the aforementioned complications, demand a diagnosis of pulmonary tuberculosis even though no abnormal physical signs are present in the lungs.

"8. The importance of physical examination in the diagnosis of pulmonary tuberculosis has been overemphasized.

"9. Symptoms are a better and more accurate guide to activity than physical signs.

"Iо. Symptoms without physical signs demand treatment, while physical signs without symptoms require only careful watching.

"II. Slight but persistent rise in temperature and increase in pulse rate are often present early in the disease.

" 12 . The usual weight of a patient who develops pulmonary tuberculosis is often 10 pounds below the normal weight for his height and age.

"I3. Failure to interpret rightly the significance of symptoms, to detect the presence of abnormal physical signs, can be condoned; but failure to ask for and examine the sputum repeatedly in any patient with chronic cough is inexcusable.

"I4. Absence of tubercle bacilli in the sputum means only that bronchial ulceration has not yet occurred. 
"I5. Auscultation and inspection are the most important procedures in the detection of abnormal physical signs.

"I6. Auscultation is more important than inspection, and the detection of râles by the auscultation of the inspiration following cough is the most important procedure in the detection of physical signs of early pulmonary tuberculosis.

"I7. The disease is always practically more extensive than the physical signs indicate.

"I8. Abnormal physical signs in one apex should be considered as due to pulmonary tuberculosis until proved not to be, while those at the base should be looked on as non-tuberculous until definitely proved to be tuberculous.

"I9. When sputum is lacking, or when tubercle bacilli are absent on repeated examinations, the possibility of the presence of bronchiectasis, hyperthyroidism, syphilis and influenza, and more rarely pulmonary tumor and Hodgkin's disease, should be borne in mind.

"20. It may be impossible to determine definitely the presence or absence of clinical tuberculosis." 


\begin{tabular}{|c|c|c|c|c|c|c|c|c|c|c|c|c|}
\hline 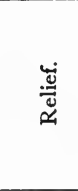 & 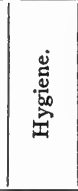 & 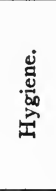 & 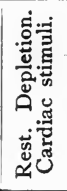 & 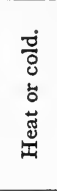 & 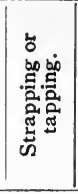 & 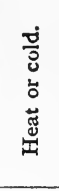 & 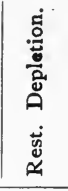 & 思 & 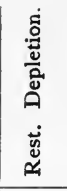 & 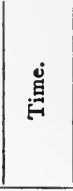 & 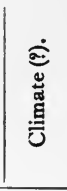 & \\
\hline 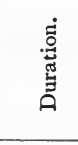 & 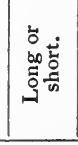 & 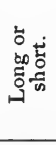 & 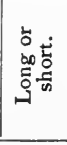 & 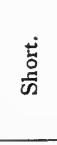 & 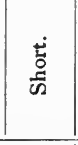 & 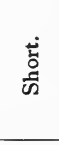 & 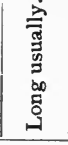 & 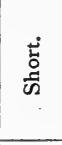 & $:$ & $\begin{array}{l}\text { t. } \\
\text { \&્ }\end{array}$ & 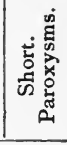 & $\dot{00}$ \\
\hline 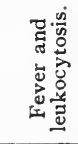 & + & + & $\circ$ & + & 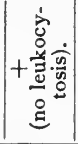 & + & $\circ$ & + & $\circ$ & + & 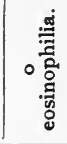 & $\circ$ \\
\hline 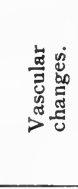 & o & $\circ$ & 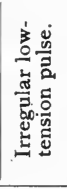 & $\circ$ & $\circ$ & $\circ$ & 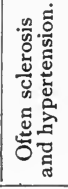 & $\circ$ & 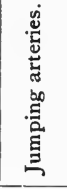 & $\circ$ & $\circ$ & $\circ$ \\
\hline 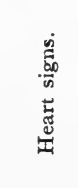 & $\circ$ & $\circ$ & 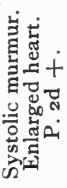 & $\circ$ & $\circ$ & $\circ$ & 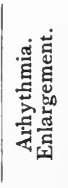 & $\circ$ & 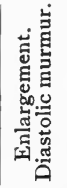 & $\circ$ & $\circ$ & 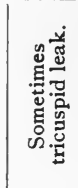 \\
\hline 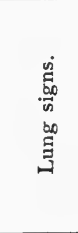 & 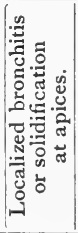 & 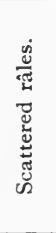 & 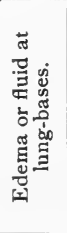 & $\circ$ & 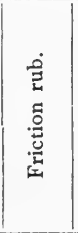 & 。 & 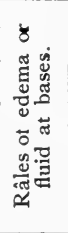 & 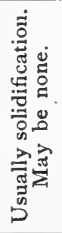 & 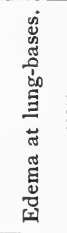 & 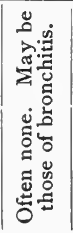 & 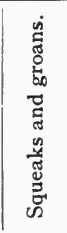 & 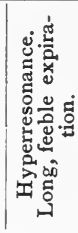 \\
\hline 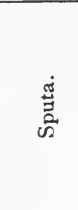 & 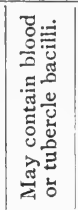 & 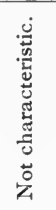 & 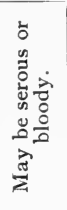 & 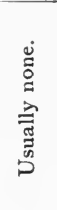 & 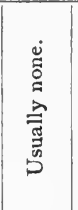 & 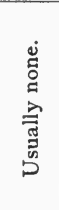 & 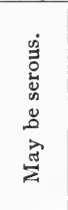 & 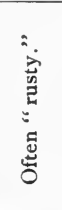 & 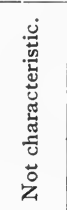 & 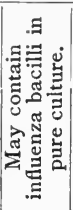 & 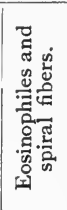 & 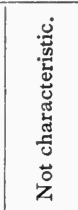 \\
\hline 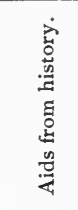 & 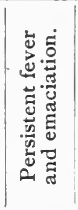 & 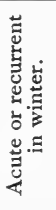 & 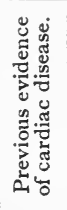 & 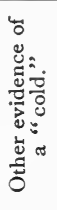 & 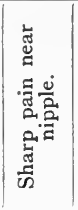 & 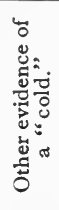 & 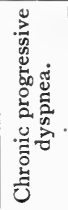 & 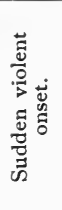 & 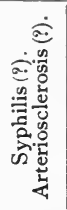 & 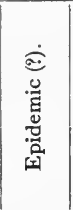 & 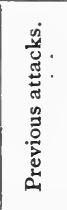 & 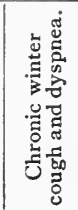 \\
\hline 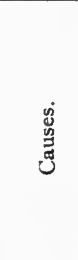 & 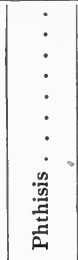 & 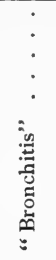 & 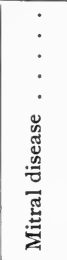 & 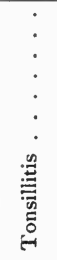 & 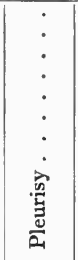 & 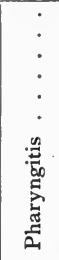 & 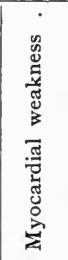 & 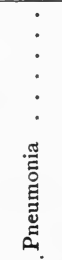 & 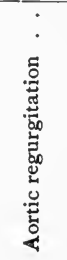 & 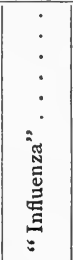 & 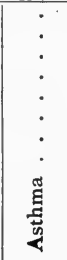 & 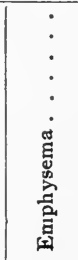 \\
\hline
\end{tabular}





\section{Causes of Vomiting}

1. TOXEMIA OF PREGNANCY

2. "ACUTE DYSPEPSIA"

3. ALCOHOLISM

4. SEA-SICKNESS

5. ONSET OF INFECTIOUS DISEASES

6. POSTOPERATIVE "SHOCK"
CASES TOO MANY AND TOO VAGUELY ENUMERABLE FOR GRAPHIC REPRESENTATION.
7. GASTRIC NEUROSIS $\}$

8. ACUTE APPENDICITIS

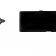

9. CARDIAC DISEASE

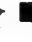

10. PEPTIC ULCER

11. "GASTRITIS"

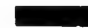
309 209

12. INTESTINAL OBSTRU C-
TION 167

13. GASTRIC CANCER

14. UREMIA

15. TABES

42 


\section{CHAPTER XIX}

\section{VOMITING}

THE act of vomiting must be distinguished, in the first place, from the easy and more or less habitual regurgitation of the normal stomachcontents. In young infants this presents itself as the familiar phenomenon of "spilling over"; in older persons it is often a matter of habit, quite controllable when explained, for, curiously enough, there are those who act as though they believed that the stomach-contents have a divine right to be ejected whenever it reaches the mouth. A little wholesome advice is here of value.

In estimating the significance of vomiting we must take account also of the patient's temperament and of some other habits besides that just mentioned.

(a) There are people who have learned early in their career the trick of emptying their stomachs on slight provocation, with or without the aid of a finger in the throat. To such people the slightest gastric discomfort, the faintest retrosternal or esophageal irritation, is the signal for a voluntary emptying of the stomach.

(b) At the other extreme, temperamentally or physiologically, are those who may have gone through thirty or forty years of life without ever tasting the experience of emesis. In a person of this type we may be led to underestimate the importance of certain symptoms merely because he does not vomit.

The first of these temperamental extremes is especially prominent in the gastric neuroses, and when the existence of such a condition is satisfactorily established, it may be our chief duty to make the patient control the act. This can be accomplished sometimes by a simple explanation, sometimes by scolding, sometimes by a sort of mental counterirritation, the result of getting the patient busy; sometimes by subpectoral infusions of saline solution, which the patient is distinctly warned must continue until the vomiting stops. To break a habit is the main object, whatever method is adopted. I have known an apparently sensible working-man of thirty-five who vomited continuously until four months had elapsed, and 55 pounds of his weight had vanished, all from habit alone-a habit which was broken without much difficulty in the course of a week's hospital treatment with subpectoral infusions as above described. 


\section{IMPORTANT FACTORS IN THE PRODUCTION OF VOMITING}

Three prominent elements may be distinguished:

(a) Cerebral.

(b) Gastro-intestinal.

(c) Pharyngeal.

Most of the so-called "reflex" causes of vomiting may be arranged without much violence under the first of these headings. The vomiting due to intense pain, that induced by fright and other strong emotions or by fatigue, can probably be accounted for in this way. The majority of toxic varieties of vomiting belong in the same group-e.g., the vomiting of pregnancy, cyclic or paroxysmal vomiting, and that accompanying migraine and hyperthyroidism.

The pharyngeal factor is especially important in the morning vomiting which accompanies many cases of alcoholism, but which is 'very often due to the accompanying pharyngitis caused by smoking. The patient has a smoker's throat, which he rasps and scrapes in the clearing-out process when he wakes in the morning. The pharyngeal irritation finally produces emesis.

Together with the ordinary gastric causes of vomiting, we must remember the cases in which chronic or acute intestinal obstruction, with or without peritonitis, causes the stomach to empty itself. With many of the intestinal neoplasms we may have symptoms very closely simulating those of cancer of the stomach, and the examination of the gastric contents and functions may still further confuse us, since gastrectasis, hypomotility, and achlorhydria may be found. The vomiting due to acute appendicitis or to strangulated hernia is probably of the same type.

Finally, we may mention the prolonged attacks of emesis accompanying the gastric crisis of tabes dorsalis, the explanation of which is not as yet clear.

\section{Case 318}

An Irish bartender of forty entered the hospital January I6, I908. He had catarrhal jaundice three times several years ago. His father died of pleurisy. The patient has been a very hard drinker, consuming a quart of whisky and I7 large beers daily. He denies venereal disease.

For many years he has had stomach trouble. He vomits early in the morning, and often after eating any ordinary food, so that at present he practically lives on liquor. He cannot remember to have been intoxi- 
cated so that he could not do his work and take care of himself. His appetite for everything but liquor is very poor.

The bowels move two or three times a day.

$\mathrm{He}$ is very shortwinded, and for two weeks has noticed scantiness of his urine, enlargement of his belly, and some swelling of the feet, face, legs, and hands. A week ago he noticed that his conjunctivæ were getting yellow.

Two years ago he weighed I95 pounds; now he weighs 236 .

Physical examination showed a satin-like skin; no jaundice; feeble heart-sounds; soft and apparently normal arteries; blood-pressure, IIo. There was nothing abnormal in the lungs. He had an enormous dome-shaped abdomen, with slight dulness in the flanks, showing perhaps a little shift with change of position. The edge of the liver not felt, though there is dulness for $2 \frac{1}{2}$ inches below the right costal margin. There was slight edema of both legs.

Discussion.- This seems at first sight an obvious case of alcoholism, but on closer study we notice that the heart-sounds are feeble, that he has dyspnea, that the face and extremities are edematous, and that, despite persistent vomiting, there has been a marked gain in body weight. All these facts - and especially the last one-point toward cardiac disease, which, as we know, is frequently a cause of persistent vomiting.

But what cardiac disease can it be? There is no evidence of a valvular lesion or of a weakened heart due to kidney trouble. Chronic fibrous myocarditis was, in earlier years, a favorite diagnosis in cases of this kind, but the autopsy so seldom confirmed it that many of us are growing more cautious. Personally, I am unwilling to make a diagnosis of chronic myocarditis unless there is convincing evidence of arteriosclerosis, and unless all other causes of cardiac weakness can be satisfactorily excluded. But there is no evidence here of arteriosclerosis, and no good reason for the heart to be weak merely as a result of alcohol.

In any such patient cirrhosis must be considered, especially as it might help to explain the persistent vomiting. Cirrhosis is always a difficult disease to exclude, since we know that it usually exists for years before it produces any symptoms. All we can say in a case like this is that we have no positive evidence of it, such as ascites, enlargement or shrinkage of the liver, hematemesis.

Of course, the gastric functions must be more thoroughly investigated if the patient does not promptly yield to treatment based on some other hypothesis. But after this survey of the case we have obtained, as it seems to me, so little positive evidence of any disease other than alcoholism 
that the first experiment to be tried-all treatment is an experimentis an anti-alcoholic régime. If that fails, the next step should be to pass a stomach-tube and examine the physical and chemical functions of the stomach; next, if that proves negative, to try the effect of cardiac stimulation preceded by depletion.

Outcome.-Alcohol was withdrawn entirely at entrance. The patient was given a diet of liquids and soft solids, with to minims of the tincture of capsicum before each meal, an ounce of magnesium sulphate early each morning, and 2 drams of paraldehyd every afternoon. In four days he was able to eat without vomiting and sleep without medicine. In a week he felt perfectly well, but was much surprised to discover that he could exist without rum.

Diagnosis.-Alcoholism.

\section{Case 319}

A housemaid of twenty-four entered the hospital October II, I906. She had always been well except for habitual constipation, the bowels moving once in from two to six days. She has taken no breakfast for some weeks and has occasionally vomited. A week ago the patient was married. Her last menstruation was August I4th.

At 3 o'clock this afternoon she began to vomit, and soon after had a sudden sharp abdominal pain, with faintness, and in the course of the day six loose movements of the bowels.

Examination was negative save for slight dulness in the right flank, slight tenderness of the epigastrium and along the right side. At McBurney's point tenderness was very marked on deep pressure, and there was spasm over this area.

Vaginal examination shows much tenderness high up on the right side, but no mass.

The white cells were 16,800; urine, negative; temperature, pulse, and respiration normal. The breasts were somewhat large.

Discussion.-The vomiting here might well be due to pregnancy, but no one could make any such stitement without any further evidence than is furnished by the physical signs here reported. If the history is taken as correct, - - that is, if her last menstruation was August I4th,- there is hardly time enough for the development of an ectopic gestation, which should, moreover, show more definite signs on vaginal examination or more characteristic evidence of hemorrhage.

Patients of this type not infrequently take large doses of irritating cathartic medicine in the attempt to produce a miscarriage. The loose movements of the bowels and the vomiting might be thus accounted 
for. No history of this kind, however, could be elicited, and the patient seemed to be telling the truth.

The patient's habitual constipation might account for a good deal of vomiting, but hardly for such an acute attack or for tenderness and spasm in the right iliac fossa.

One always hesitates to make a diagnosis of appendicitis when the temperature and pulse are normal and when there has been no previous attack. Nevertheless, in the presence of very marked tenderness, with spasm at McBurney's point and leukocytosis, appendicitis seems the most reasonable diagnosis. A pyosalpinx might produce very much the same physical signs, but would probably show a mass or induration by vagina, and would not, in all probability, come on so acutely without any other or previous symptoms.

Outcome.-Operation October I2th showed acute appendicitis. There were no old adhesions.

Diagnosis.-Appendicitis.

\section{Case 320}

A married woman of fifty-three with an excellent family history entered the hospital September 28, 1907. She has been strong and healthy all her life, with the exception of a double inguinal hernia, for which she was successfully operated upon in May, 1907. Since childhood she has been in the habit of passing water once at night after bedtime.

About a year ago she began to have attacks of vomiting, in which she was unable to retain any kind of food, the vomitus consisting at first of the food previously eaten, unmixed with mucus or blood. Last fall the vomiting ceased altogether, and she was in the Massachusetts General Hospital for a Colles' fracture. During her stay here nothing abnormal was noted in the digestive functions, but after her return home vomiting began again, and has gradually grown more frequent up to the present time. She has vomited exery day through the past summer-sometimes three times a day. At no time has there been any pain except after eating a large amount, and then only slight distress. There have been no eructations of gas and no swelling of any part of the abdomen, though the epigastrium has been somewhat tender on pressure for two months.

Fifteen months ago she weighed I24 pounds; four months ago, Ir6; now she weighs roo pounds. Her bowels have gradually become constipated. She has noticed no jaundice. She has never considered herself nervous. 
The temperature and pulse were as seen in the accompanying chart. The patient was poorly nourished and pale, though her hemoglobin was 75 per cent. and the white cells were 7300 . The heart and lungs showed nothing abnormal. In the upper abdomen was a hard, tender mass, descending with respiration. (See Figs. I65 and I66.)

Physical examination, including the urine, was otherwise negative. The stools showed no occult blood. Vaginal and rectal examinations were negative. Through the stomach-tube only I $3_{3}$ ounces of water could be introduced without extreme pain, retch

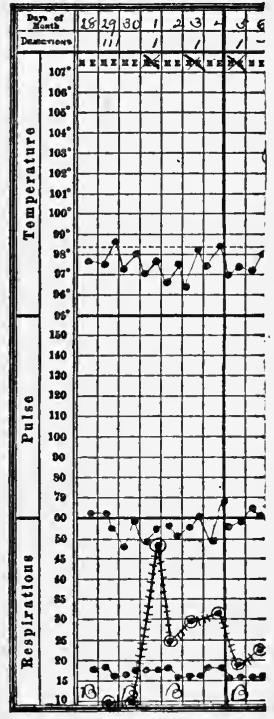

Fig. I67.-Chart of case 320. ing, and struggling. There was no food in the fasting stomach. After an Ewald test-meal the gastric contents showed no free acid of any kind. The benzidin test for blood was positive; the wash-water used for lavage returned slightly blood-stained.

Discussion.-The early part of this history reminds us of a gastric neurosis, because one isolated symptom-vomiting - seems to make up the whole clinical picture. When any single symptom, such as vomiting, gaseous eructation, diarrhea, or constipation persists over a considerable period of time with little or no background of other interconnected symptoms, it usually turns out that we are dealing with a neurosis-that is, with a morbid habit. We can make such a statement, however, only when we have exhausted all the resources of physical diagnosis without finding any evidence of organic disease.

We cannot attribute the vomiting to constipation or to the exhaustion produced by any constitutional or infectious disease, since we have no evidence of these conditions. A consultant suggested the possibility of cerebral tumor, and the fundus oculi was examined with this possibility in view. Neither there nor elsewhere, however, could we find.any support for the assumption of brain disease.

As soon as the epigastric mass was clearly made out and the possibility of its being due to a fecal accumulation was excluded by free catharsis, it began to be pretty evident that the vomiting was due either to ulcer or cancer of the stomach. This became still more certain when it was recognized that the capacity of the stomach was diminished and its secretion of hydrochloric acid abolished. It remained to decide the question: cancer or ulcer? Such a tumor is often produced by 

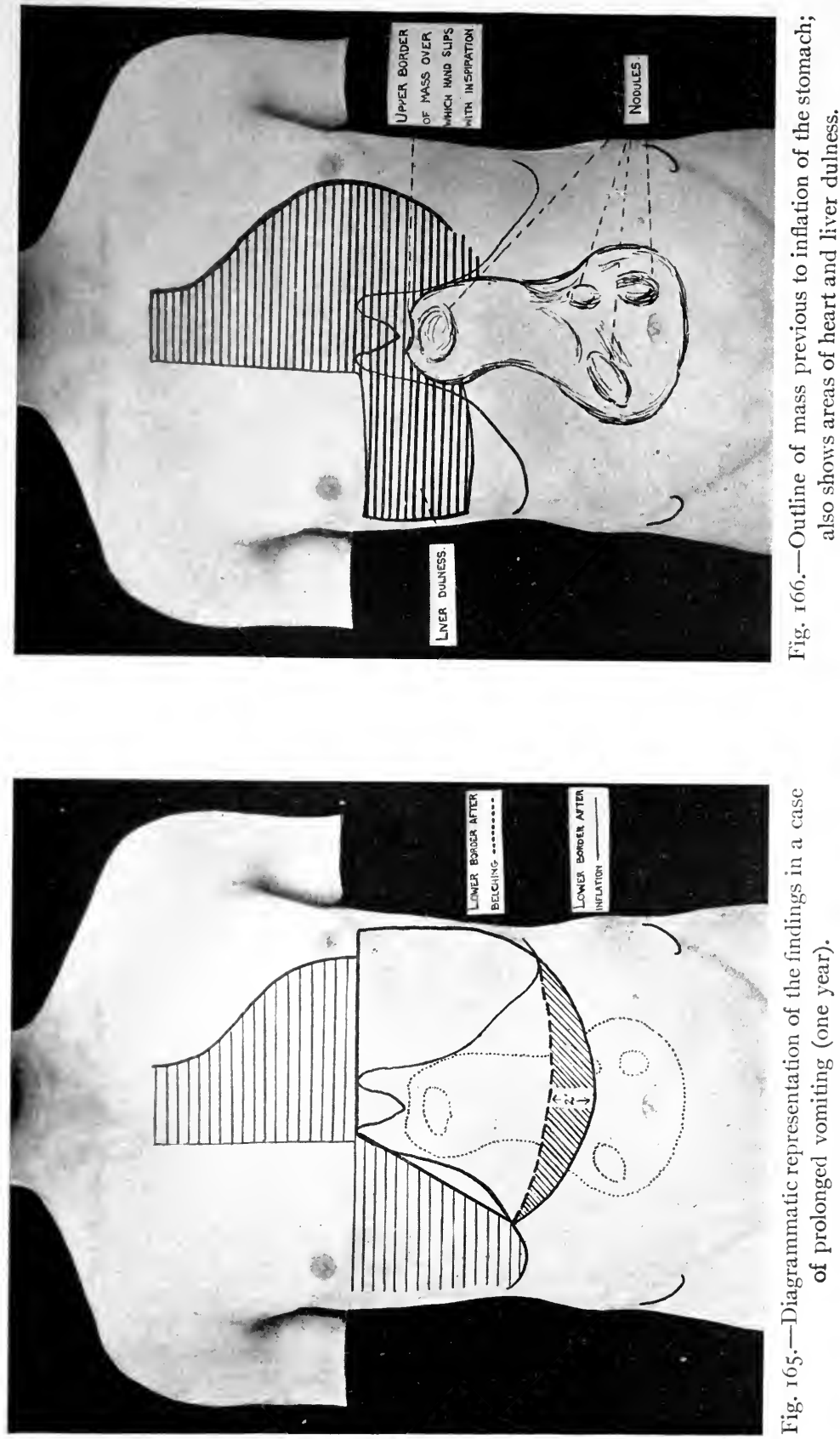

a perigastric exudate around an old ulcer. But the history is distinctly against ulcer and in favor of cancer. Ulcers seldom begin in persons who have lived to fifty-two years without gastric disturbances. They are hardly ever characterized by vomiting without pain, and in the earlier stages of their course there are usually long periods of freedom from symptoms and marked relief (even during the acute stages of the disease) immediately after the taking of food.

One of the things that made it rather difficult to realize that cancer was really the most reasonable diagnosis in this case was the entire absence of pain. This, however, is by no means unprecedented. A number of similar cases have been recorded.

Outcome.-Dr. Maurice Richardson made a diagnosis of cancer of the lesser curvature, without obstruction of the pylorus, and advised against operation of any kind.

Under liquid and soft solid diet, with small doses of calomel and magnesium sulphate, hydrochloric acid, 20 minims after food, the patient ceased vomiting on October $4^{\text {th }}$ and felt a great deal better.

Diagnosis.-Gastric cancer.

\section{Case 321}

A married woman of thirty-five entered the hospital March 24, I908. She lost one brother by phthisis in the previous December. She has had two children and no miscarriages. Her youngest child is seven years of age.

Three years ago she was in St. Elizabeth's Hospital for ten weeks on account of stomach trouble; dilatation and curetting of the uterus were done.

Five years ago she weighed I44 pounds. A week ago she weighed I IO.

During these past five years she has been having attacks of vomiting, at first only before each menstrual period, later at other times. Her vomitus was watery and contained undigested food, but never any food eaten twenty-four hours before. She has not been free from vomiting for more than two weeks since I903. The vomiting relieves sharp epigastric pain, which is usually worse after eating. In 1905 she vomited two cupfuls of dark blood. On a milk diet this ceased, but returned eleven weeks later. In November, 1907, she again vomited blood and had epigastric pain and tenderness. She was confined to bed a week at that time; she has not vomited since, but still has epigastric pain and tenderness, worse after food. Her appetite is poor; the bowels move only with enemata. She has no headache. Her eye-sight is good. 
Physical examination is negative save for a blowing systolic murmur limited to a small area near the apex of the heart. There is slight epigastric tenderness, but no spasm. The right kidney is easily felt, its lower pole being on the level of the navel.

After a test-meal the gastric contents showed free $\mathrm{HCl}$, o.I4 per cent.; total acidity, .25 per cent.; guaiac test, negative. The stools were also free from blood.

The patient did excellently well on a diet of milk and eggs, following roughly the formula of Lenhartz.

On the second of May she seemed well and was allowed to go home.

The patient returned to the hospital August 3, I908, stating that, two weeks after leaving the hospital before, she had an attack of vomiting with the menstrual period, and this vomiting had continued for the two subsequent periods, though she was perfectly well between them. The vomiting seemed to be entirely independent of the taking of food. The vomitus contained no blood. At the time of these attacks she had a good deal of epigastric pain, and has a little pain all the time, slightly relieved by food and accompanied by gaseous and sour eructations. She sleeps poorly, on account of nervousness, she believes.

Physical examination shows marked pallor, the red cells, 3,050,000; hemoglobin, 45 per cent. Two normoblasts, also considerable achromia and deformities, were seen while making the differential count of 200 white cells. The polynuclear cells were 82 per cent. The urine was negative.

The stools showed a slight but constant reaction to guaiac.

Stomach-tube examination showed essentially the same condition as in the previous spring. There were no fasting contents. The patient did excellently well on a diet of crackers and milk.

Discussion.- In marked contrast to the previous case, the stomach trouble is here of long duration-five years or more-and occurs in a young woman. In the early stages of the disease, and to a certain extent throughout, the pain seems to be connected with menstruation, as if it were a "reflex" nervous disturbance associated with the nervous tension of that period. The relief of pain by vomiting and the attack of hematemesis in 1905 still further support the diagnosis of peptic ulcer or hyperchlorhydria, which had been already suggested by the previous history.

In August, 1908, her symptoms still suggest chiefly peptic ulcer, but we have now one symptom not easily accounted for on that hypothesis, viz., the very marked anemia. There has been no evidence of 
hemorrhage for three years, and peptic ulcer does not produce anemia unless there is sharp bleeding.

It is not likely to produce anemia by oozing or discharging blood in small quantities. The marrow readily makes up these losses. Neither is it likely that a large amount of blood might have been poured out at one time and discharged wholly by rectum without the patient being aware of it. Such rectal hemorrhages produce so much weakness and thirst that the patient is usually made aware that something has happened.

Nevertheless, it must be admitted that everything else in the case save this one fact-an unexplained anemia-points to chronic ulcer, and perhaps the anemia alone is not of sufficient diagnostic significance to outbalance the other indications which favor ulcer. But since the trouble has gone on so long and recurs so frequently under dietetic treatment it seems as if the patient should be given the benefit of an exploratory laparotomy, especially as there is at least a possibility that the anemia may be due to something more serious- $i$. $e_{\text {., }}$ to gastric cancer.

Outcome.-August I4th the abdomen was opened. A cancerous mass was found on the posterior wall of the stomach, with metastases in the omentum. Gastro-enterostomy was done. A month later the patient was reported as eating well, sleeping well, and gaining in weight.

Diagnosis.-Gastric cancer.

\section{Case 322}

A widow of forty-five entered the hospital August I4, I906. She lost her husband of consumption twelve years ago. Sixteen years ago she had an attack similar to the present one, which was cured in two weeks. A year ago she had another attack, and was in the hospital for three weeks on rectal feeding, during which her weight fell from I37 to II 7 pounds. Since then she has been dieting carefully, has felt pretty well, and has not been troubled by indigestion.

Nine days ago she was suddenly seized with sharp abdominal pain and vomiting. This pain has recurred frequently since that time. It is relieved by vomiting, but shows no other relation to food. Occasionally it requires morphin. In her attack of sixteen years ago she vomited blood, but there has been none since. For the past three months she has had occasional attacks of diarrhea, the movements being preceded by pain and often containing blood.

For the past two days she has been fed exclusively by the rectum. Her weight is now 120 pounds. 
On examination the patient is very well nourished, but rather neurasthenic. There is a soft systolic murmur at the apex of the heart. The pulmonic second sound is larger than the aortic second. The heart shows no enlargement. The lungs are negative. The right side of the abdomen is held rather rigid, owing to marked tenderness, greatest in the middle quadrant.

Physical examination, including the vagina and rectum, is otherwise negative, as are the blood, urine, temperature, pulse, and respiration.

The patient in the early days of her treatment seemed markedly neurasthenic, but this ceased after the cessation of the menstrual flow, and she was able to take solid food in moderate amounts for three days.

After this the patient began to vomit a great deal. Nutrient enemata caused much distress and seemed to aggravate the vomiting. On the fourth day she was able to take some champagne and some albuminwater flavored with sherry, without any vomiting. The vomitus was found to be strongly acid, and consisted mostly of clear mucus.

Sodium bicarbonate relieved her pain somewhat, but it was vomited after a short time. Only morphin gave relief.

After a test-meal the stomach-contents showed an abundance of free hydrochloric acid and a positive guaiac reaction. The stools were foul smelling, dark, and bloody.

On the twentieth the patient complained a great deal of abdominal cramps. The stool at this time was found to contain much fresh blood and a considerable amount of pus and mucus. The blood showed: Red cells, 3,676,000; white cells, Io,000; hemoglobin, 65 per cent.

The stained specimen showed nothing remarkable except achromia. Further study of the stools showed that they contained almost no fecal matter.

The subsequent examination of the abdomen showed on the right side, low down, something which felt like hard lumps, which, however, disappeared with the rumbling of gas. The capacity of the lower bowel was measured with warm water, and only a pint could be introduced. There seemed to be no distention of the transverse colon, but the region of the ascending colon was persistently distended. The attempt to introduce more than a pint of water by rectum was repeatedly unsuccessful.

Discussion.-We see occasionally in adults those unexplained attacks of summer diarrhea and vomiting which are so common in young children; but we do not expect them to persist for nine days. True, this patient is neurasthenic, so the record states, and that might account for a great deal, especially as the attack has happened to occur at the 
menstrual period. During the earlier days of her treatment we accounted for her symptoms in this way. Among the other possibilities considered was hyperchlorhydria, which will next be discussed.

The previous attack of hematemesis was described by the patient as similar to the present trouble. The illness of 1905 also suggests hyperchlorhydria or ulcer. The stomach-contents now show a positive guaiac reaction and a large amount of free hydrochloric acid. There is also blood in the stools. When all these facts came to light, we were inclined to switch over from the idea of gastric neurosis to that of peptic ulcer, all the more so when it turned out that sodium bicarbonate relieved her pain.

It was not until the second week of treatment that the intestinal symptoms began to seem more important, especially as we could find no good cause for the well-marked secondary anemia. There had been no recent hemorrhage to account for it, and it did not appear that the diarrhea had lasted long enough in any of her previous attacks or in the present one to produce so much anemia. It was, however, the condition of the stools, especially the presence of pus, and the remarkably small amount of fecal matter which led us further to investigate the possibility of intestinal neoplasm. When we found that, on two separate occasions, not more than a pint of water could be introduced into the rectum, even when slowly and carefully given, the suspicion of intestinal neoplasm low down in the colon became such that operation was advised.

Outcome.-The abdomen was opened on the twenty-seventh of August, revealing a cancer of the sigmoid.

This case and several others which I saw about the same time were very instructive to me because I had never been warned of the possibility and the danger of getting our attention so focused on the gastric manifestations of what turns out to be intestinal obstruction that the possibility of the latter does not occur to us. If once we get a false start, we may find a good deal to confirm us in our mistake. For example, in a recent case, which turned out, like the present one, to be due to a cancer of the sigmoid, everything seemed at first to point to the stomach. A stomach-tube was passed, and proved the presence of gastric enlargement and the absence of free hydrochloric acid. The patient was fifty years of age, and had never had any gastric symptoms before the present year. All these facts seemed to point so strongly to gastric cancer that we neglected to make a sufficiently careful examination of the abdomen or to put well-directed questions concerning bowel movements, the exact location of pain, and intestinal noise. 
"Gastric neurosis" is the diagnosis on the record of another case of causeless vomiting and diarrhea in an elderly woman, who was promptly relieved in the hospital by a few days of diet and quiet (1908). Dr. E. A. Codman insisted that intestinal cancer was present, though no tumor was felt. The patient remained perfectly well for several months, but Dr. Codman's diagnosis was ultimately verified.

Diagnosis.-Cancer of the sigmoid.

\section{Case 323}

A waitress of nineteen, born in Maryland, entered the hospital January 14, 1907. She has a good family history and past history up to eighteen months ago, when she began to lose strength and to have dizzy spells, especially after breakfast. Her appetite continued good, and she felt in other respects well until about two months ago, when she began to have nausea, coming on about five minutes after eating, and relieved at the end of about an hour by vomiting the food just taken, together with considerable phlegm. She never vomits except after breakfast, although she has considerable distress after the other meals.

She has worked until a week ago, and still feels well enough when her stomach is empty. She gets up once each night to pass water. She has noticed a somewhat tender spot in the epigastrium. For three months she has been somewhat pale and short of breath on exertion.

On examination the patient was found to be pale. There was a faint systolic murmur in the pulmonary area; otherwise the chest is negative; likewise the abdomen and urine.

The blood showed red cells, 4,012,000; white, 6800; hemoglobin, 55 per cent. Differential count normal; marked achromia; no other changes.

Discussion.-We may rule out without further consideration the long list of organic diseases, such as cancer, tuberculosis, and brain tumor, of which vomiting is a symptom. The first point to be noticed on the positive side is that nausea of this type, though not of this duration, is often seen in the early months of pregnancy - a possibility which should never be forgotten when we are dealing with obstinate digestive disturbances. I once saw a patient who had been for some weeks under the care of a distinguished specialist in gastric troubles, whose treatment made it evident that he had never considered the possibility of pregnancy, though the patient had, during the period of his treatment, an amenorrhea and all the other usual evidences of early pregnancy. When I saw her the condition was quite obvious. 
Nothing is said about menstruation in this case, but inquiry showed that it had been absent for the past three months. This fact, together with the stomach trouble and a certain degree of bad conscience, had considerably alarmed the patient. It was found on examination, however, that there was no uterine enlargement and none of the other evidences of early pregnancy.

Many cases like this turn out on careful study to be the result of the disturbances produced by constipation. There was nothing in the history, however, to indicate that she was constipated. The testimony of a patient is not always reliable on this point. Fecal movements may occur daily, and yet be so insufficient in amount that a considerable accumulation takes place. One has no right to assume this unless physical examination or the inspection of the stools demonstrates it.

Some eager surgeons would consider the evidence here presented as sufficient for a diagnosis of peptic ulcer or chronic appendicitis, but there is not the relief by food so characteristic of the earlier stages of peptic ulcer, while the symptoms are too continuous and include too little suffering from pain to give us any genuine indication of chronic appendicitis.

But for the abnormalities of the blood-picture one would here be forced to say that physical examination is negative, a conclusion which lies very near to the decision: gastric neurosis. Is that slight anomaly in the hemoglobin percentage and in the stained smear sufficient to account for so much gastric disturbance? Yes, it certainly is, if we take it as the outward and visible sign of a more extensive and less comprehended malady, to which we give the superficial and unsatisfactory name of chlorosis. Experience has often shown that chlorosis may produce vomiting as severe as that complained of by this girl, and in the absence of any other obvious cause one should plan treatment upon this hypothesis.

Outcome.-The patient was put to bed and given $\frac{3}{4}$ grain calomel every fifteen minutes for eight doses, followed the next morning by I ounce of magnesium sulphate. Liquid and soft solid diet, with nux and gentian before meals, was perfectly well borne. The patient had no vomiting, no gastric symptoms of any kind, and by the twentythird was up and had an excellent appetite, although her blood showed no gain whatever.

On the tenth of February the patient went home much stronger and better, although her hemoglobin was only 70 per cent.

Diagnosis.-Chlorosis. 


\section{Case 324}

An unoccupied Irish girl of nineteen, of good family and past history, entered the hospital June 7, I908. Five weeks ago she began to have abdominal pain and frequent vomiting. The pain was colicky and not localized. It seemed to have no relation to food, and had only occurred three or four times in the past five weeks, lasting an hour or two. Yet since the pain ceased she has continued to vomit almost daily whether she eats or not. Yesterday she vomited five times, though she took only milk. The vomitus sometimes consists of undigested food, sometimes of a yellowish or blackish sour fluid. It has never been red or brown. The vomiting seems to have no relation to the time or the character of food. The catamenia are regular.

The appetite remains fair, the bowels constipated, moving every day or two by enemata only.

On examination the patient is obese, ruddy. There is slight general tenderness over the lower abdomen, with some voluntary spasm, preventing satisfactory palpation.

Physical examination, including the temperature, pulse, respiration, blood, urine, and stools, was not otherwise remarkable, though the stools were found to contain many bismuth crystals.

Discussion.-A diagnosis seems to me impossible here without a therapeutic test. We should first get the bowels started and watch to see if that does not check all the other symptoms. Suppose, then, that the vomiting still continues, and we are still unable to find any physical cause for it despite our most painstaking examination of the internal viscera and despite the absence of any discoverable cause, such as morphin, malaria, or starvation, what course should be pursued?

It is almost inevitable to assume that the case must represent some type of neurosis and to plan treatment accordingly, yet this is never a satisfactory basis of action unless we can obtain other evidence of neurotic constitution besides the vomiting itself. I was greatly impressed, a few months ago, by the outcome of a case in which, owing to the negative results of repeated and searching examinations, we made the diagnosis of gastric neurosis and used a great deal of moral suasion. Yet the man proceeded to die, and the autopsy (No. 25I4, Massachusetts General Hospital autopsy records) showed absolutely no cause for death. I do not believe for a moment that our diagnosis was right here, yet it would be difficult to avoid making the same mistake again.

In the present case we acted on the principle that it is always wise 
to remove any possible cause for the patient's symptoms before com. mitting ourselves absolutely to any diagnosis. The result is shown by the outcome.

Outcome.-As soon as the bowels were thoroughly evacuated by cascara, olive oil, I ounce twice a day, and enemata, vomiting ceased; and within a week the patient was eating ravenously without any distress or nausea. The bowels continued to be very sluggish. She was advised to eat a great deal of green vegetables and as much fat as she could tolerate.

Diagnosis.-Constipation (neurosis?).

\section{Case 325}

A housewife of thirty-eight, of good family history, was first seen April 2 I, 1908. She had pleurisy six years ago and a still-born child a year ago, since when she has been nervous and troubled with indigestion. For three weeks she has had a great deal of indigestion, accompanied by "smothering feelings around the heart." For ten days she has vomited everything taken into the stomach, averaging ten attacks in twenty-four hours. Even water is rejected. She has sometimes vomited as much as a quart of undigested food and once about a teaspoonful of bright blood which her physician said came from her throat. Her indigestion and smothering feelings are worse after eating and are relieved by vomiting. She is very constipated and belches much gas. During these three weeks there has been palpitation of the heart. At the beginning of this spell, ten days ago, she also had numbness of the right leg and left arm for two days.

When sixteen years old she had swollen glands in the neck, which discharged for about two years.

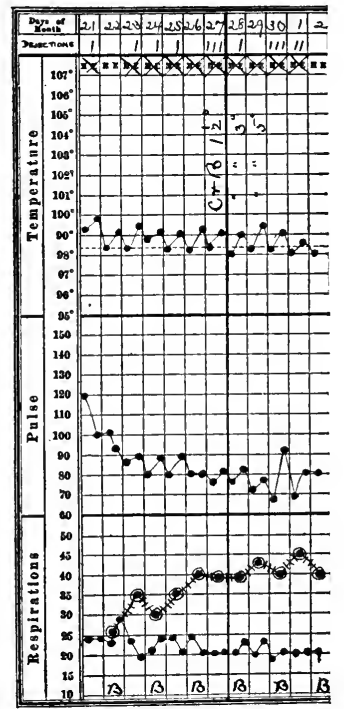

Fig. I68.-Chart of case 325 .

The patient is obese, the right pupil slightly larger than the left, both reacting normally. There are four small irregular scars on the left side of the neck.

The heart's apex is in the fifth space, $\frac{1}{2}$ inch outside the midclavicular line. The sounds are normal. There are no murmurs. The lungs 
and abdomen are normal; reflexes are normal. The stools give no reaction to guaiac.

(For temperature see.Fig. I68.)

Discussion.-An essential element in diagnosis is here omitted: we have no account of the urine or of the blood-pressure, although there is apparently a slight cardiac enlargement which might suggest a chronic nephritis as the cause of the vomiting. This hypothesis, however, was at once upset by the negative result of urinalysis.

Gastro-intestinal troubles of the type here recorded are not infrequently the first and most obscure manifestations of a tuberculous infection. It is very probable that she suffered from a cervical adenitis, tuberculous in origin, when she was sixteen. The history of a discharge from swollen neck glands and especially the duration of the discharge, together with the present evidence of scars in the neck, leaves no considerable reason for doubt upon this point. If she is correct in supposing that she had pleurisy eight years ago, the probability of tuberculosis still lingering somewhere in the system is still further increased.

I do not think it is possible absolutely to exclude tuberculosis as the cause of symptoms in this case, and I am aware that some persons would consider the variations in temperature shown in the accompanying chart as sufficient to constitute additional evidence favoring tuberculosis. On the whole, however, it seems to me that the evidence is insufficient. A very large number of patients, demonstrably not tuberculous, have as much temperature as this chart shows owing to any of a variety of causes. Repeated examinations of the lungs and other viscera revealed absolutely nothing. The patient's nutrition was excellent. I very much doubt whether she had any more tuberculosis than the rest of us-i.e., whether it was present in any active form or was responsible for any of her symptoms.

The still-born child and the irregular pupils compel us to consider for a moment the question of syphilis, but neither on questioning nor by examining the sites at which syphilis most often leaves evidences of itself could we find any reason further to entertain this suspicion.

The patient presented many neurotic characteristics not easily to be described. Whether these were the cause of the constipation or its results I cannot say; the question seems to me usually unanswerable in such cases. The sensible thing to do, however, is to attack the symptom-complex at any and all of its vulnerable points. Let us begin with the constipation.

Outcome.-The patient was given a high oil enema, 6 ounces, 
followed by a suds enema. Her gastric distress was relieved by $\frac{1}{2}$ dram of essence of peppermint. She was given fluid extract of cascara and the enemata continued daily. Under this treatment the patient was able to take liquid and soft solid diet and by the twenty-fifth could eat anything with relish.

By the twenty-ninth all symptoms had disappeared, although the patient still felt somewhat weak.

Diagnosis.-Constipation (neurosis?).

\section{Case 326}

A Canadian bolt-maker twenty-seven years old entered the hospital October I4, 1907. He lost one sister of consumption some years ago. His mother now suffers from "asthma"; otherwise his family history is good, and he himself has always been well, save that for the past five years he has hawked up a good deal of yellow material from his throat. He smokes and chews five cents' worth of tobacco a day. His habits are otherwise good.

Eight days ago, while at work and in his usual health, he became nauseated and vomited, the vomitus consisting of the food last eaten. He kept at work that day but felt weak and has not tried to work since then. The first night he felt feverish, but he has not noticed this since that time. The nausea and vomiting, however, have continued and have been especially troublesome in the morning. He has no severe pain, but a slight soreness in the epigastrium, rather more to the right than to the left, ascribed by him to retching. There has been no chill and no cough. His bowels have been rather loose for the last two days. He has not been jaundiced.

On examination the temperature, pulse, respiration, and blood are all normal. The urine shows a very slight trace of bile, and on careful examination of the eyes a slight yellowing of the conjunctivæ over the peripheral portion of the eyeball is discerned. Near the iris there is no yellowness.

The tonsils are slightly enlarged and reddened. The heart and lungs show nothing abnormal. The abdomen is slightly rigid just below the right costal margin and there is some tenderness at that point. No soreness or spasm is felt elsewhere. The liver dulness extends one or two fingerbreadths below the costal margin, but no liver-edge can be felt.

Discussion.-The presenting symptoms are vomiting and bile in the urine. Whether we shall call the condition jaundice depends upon our definition of this word. In all the more marked cases in 
which the conjunctiva is stained by bile-pigment, the discoloration extends not only over the deeper and less easily visible portions of the sclera, but up to the outer border of the iris. In milder cases there is a ring of white or bluish-white, unstained sclera around the iris. But if the yellow coloration outside this ring is well marked, we do not ordinarily hesitate to call it jaundice. The doubtful cases are those in which it is only by drawing back the eyelids and by getting the patient to turn the eye as far as possible to one side that any yellow coloration can be seen. In most of these cases the tint is, moreover, a very pale one. Our hesitation is further increased because we find so many cases of this type, if once our curiosity is aroused tolook for them. Nevertheless, it seems to me that the only defensible course is to use the word jaundice whenever any degree of yellow discoloration is visible in the sclera.

Proceeding on this basis we may say that the case under consideration is characterized by vomiting and jaundice occurring without other notable symptoms in a workingman of twenty-seven. Gall-stones are unusual at this age, and we have no tenderness or palpable mass in the region of the gall-bladder, no evidence of hepatic enlargement, and no characteristic biliary colic. Nothing, indeed, suggests any local trouble except the slight rigidity below the right costal margin, and we have no fever or other constitutional manifestation of infection in the biliary tract.

Under these conditions- $i$. e., when jaundice occurs without any obvious cause, without any marked toxemia or other evidence of infection, without any change in the shape or the size of the liver, and without any evidence of gall-stones-it has long been customary to make a diagnosis of catarrhal jaundice. That the condition so named often gives rise to very persistent nausea with or without vomiting is a familiar fact. Therefore, although we do not know what we mean by the term "catarrhal jaundice" in the sense of understanding its pathology, it is reasonable to use the term in a case of this kind, at any rate as long as nothing more serious appears in sight. If the jaundice does not go off within six weeks, we begin to fear that something more important is behind it-viz., gall-stones or cancer. During those six weeks, therefore, our diagnosis always rests on shaky foundations; indeed, it is never confirmed until the patient is well.

Since examination has revealed no sufficient reason to fear that the vomiting in this case results from any deeper and more obscure lesion of the gastro-intestinal tract, kidney, heart, or brain, catarrhal jaundice seems to be our best working-and-talking hypothesis. 
Outcome.-The patient was given a diet in which carbohydrates and fat were considerably restricted; calomel, $\frac{1}{2}$ grain every fifteen minutes until ten doses were taken, followed in half an hour by $\frac{1}{2}$ ounce artificial Carlsbad salts, and each morning thereafter by 30 grains of sodium phosphate and an enema of plain water made 5 degrees cooler each day up to the limit of tolerance. On the second day he was given dilute muriatic acid 5 minims, with I dram of fluid extract of taraxacum after each meal.

By the twenty-fourth the jaundice and other symptoms had practically disappeared.

Diagnosis. - Catarrhal jaundice.

\section{Case 327}

A sewing woman of thirty-six entered the hospital January 6, I908. She had lost one sister of consumption seven years ago. Her family history is otherwise good. The patient has always been strong and well and was in comfortable circumstances until the time of the San Francisco earthquake in April, 1907, when she lost everything. In the past year, though working very hard at sewing, she has been unable to earn enough to give herself proper food and lodging. Her menstruation has always been regular until recently, but its last appearance was two months ago.

For the past four months she has been very much run down and so nervous that she has not been able to work, though she has not been constantly in bed for any length of time. She was in a hospital during the whole of July and August, and was somewhat better after her stay there, but not able to work.

Up to two weeks ago she had no symptoms except weakness and an occasional headache. Two weeks ago she began to vomit and has continued to do so very frequently every day since, rejecting all that she eats and considerable yellow and whitish material besides. She has seen no blood in the vomitus at any time. Her abdomen is sore all over, but there is no pain anywhere. Since the vomiting began the bowels have moved once in two or three days. She has no headache. Her eye-sight is good. She has taken only cereals, milk, and water for the past two weeks. There has been no considerable loss of weight.

There is a faint tremor of the lips and hands; well-marked arterial pulsation in the neck. The aortic second sound is accentuated. There is considerable pigmentation of the abdominal wall about the navel. The urine shows a strong reaction for acetone and diacetic acid; the amount averages 30 ounces in twenty-four hours; specific gravity, 
from IOI4 to IOI7, with the slightest possible trace of albumin, a few hyaline casts and a trace of sugar, later estimated to be 0.69 per cent.

Visceral examination, including the pelvis, is otherwise negative. The blood shows nothing abnormal.

Discussion.-As we review the results of physical examination, the first point worthy of note is the tremor of the lips and hands and the violent arterial pulsation in the neck. In women of this age such hints should always lead us to examine the eyes for slight degrees of exophthalmos, to scrutinize the neck for unobserved goiter, and to count the pulse under various conditions-all with reference to a possible Graves' disease (hyperthyroidism) in larval form. Such a search was here undertaken, but was fruitless.

The urine contains sugar and acetone bodies. Is it possible that we are dealing with a diabetes, and that the vomiting is due to that disease? Against it we may range the following evidence:

(a) The cardinal symptoms of diabetes-thirst, polyphagia, polyuria, and emaciation-are absent.

(b) The amount of sugar in the urine is very small, although the diet has not been in any way restricted.

(c) Severe constitutional manifestations-such as vomiting and headache-appear in diabetes late in the course of the disease, after the cardinal symptoms have been manifested for a considerable period.

(d) Acetonuria is very common as a result of severe and prolonged vomiting from any cause.

(e) A slight glycosuria-such as that here present-is not at all uncommon in persons of a nervous temperament and under any unusual psychic strain.

There seems, therefore, no sufficient reason to treat this patient as a diabetic. But if we are to disregard the acetonuria, the glycosuria, and the tremor and find no reason for supposing them to point to any organic disease which might account for the vomiting, there seems to be nothing left but that old and much-overworked hypothesisneurosis. This is made a little more plausible than ordinary in the present case because the patient's circumstances, the nature of her work, and the tragedy through which she passed nine months before are such as to favor the development of a nervous breakdown. There seems, on the whole, to be no better basis for work and talk.

Outcome.-The patient was given a diet of milk, one-third limewater, 4 ounces every two hours. The bowels were moved by enemata. Within twenty-four hours she was so much improved that she could take an ordinary mixed diet. Trembling and nervousness markedly 
lessened; acetone, diacetic acid, and sugar disappeared within three days on full diet. She was somewhat sleepless, but was helped by 15 grains of trional for two nights, after which she slept fairly well without any hypnotic.

On the eleventh the patient was allowed to sit up in bed. On the fifteenth she tried to walk, but was very dizzy and weak. On the twentieth she was able to walk, and thereafter gained rapidly.

Diagnosis.-Exhaustion.

\section{Case 328}

A musician of fifty entered the hospital August 4, I906. The family history and past history are good. He denies alcohol and venereal disease.

A week ago, without known cause, he was suddenly seized with colicky epigastric pain, nausea, and vomiting. Since that time he has vomited everything that he has eaten. There has been soreness, but no marked abdominal pain, and no blood in the vomitus.

He gave up work five days ago. This morning he began to hiccup and has continued for the past two hours. He has never had a similar attack. His general health has been good. During the first four days of this attack he had diarrhea.

On examination the patient is thin and wiry. His pupils are slightly irregular and react sluggishly. There is no lead-line. The glands in the neck, axillæ, and groins are palpable, but not enlarged.

The chest and abdomen show nothing abnormal, although there is some tenderness in the lower portion of the abdomen and the sharp edge of the liver is palpable on deep inspiration.

The knee-jerks are lively, the fundus oculi negative, likewise the blood and urine. Within a couple of days the pain was gone, the patient very hungry, yet he vomited when solid food was given to him.

Discussion.-At this man's age the sudden occurrence of vomiting makes us think first of all of cerebral or cardiorenal disease, but we find no confirmation of this idea in the results of objective investigation.

Gall-stones is a possibility to be reckoned with, but on that hypothesis it is hard to explain why the vomiting should have continued for a week after the pain has ceased. The same difficulty confronts us if we try to reason that chronic appendicitis or nephrolithiasis may have produced the pain. For peptic ulcer in the stage of perforation the local manifestations are not sufficiently acute and definite; for any other stage in the course of this disease the symptoms are too violent and the vomiting too continuous. 
I mention the phrase "ptomain poisoning" because I have so frequently heard it used in cases of this kind, as well as in perforative appendicitis, intestinal obstruction, and other acute abdominal emergencies. The phrase seems to be a favorite "blind" behind which our ignorance or error may be concealed. I have never yet known a single case in which the diagnosis was justified by any sufficient chemical examination either of the food supposed to be responsible for the trouble or of the contents of the gastro-intestinal tract.

The patient has not been constipated, exhausted, or neurotic; he is not at all of the type that vomits for lack of any other occupation. We may be forced to make the unsa tisfactory diagnosis of gastroneurosis, but not until all other possibilities are exhausted.

Tabes dorsalis with gastric crisis was at first seriously considered, but our seriousness was disturbed by the liveliness of the knee-jerks. This symptom being out of agreement with our diagnosis, there appeared to be nothing but the sluggish light reaction of the pupils on which to base the diagnosis of tabes. There were no lightning pains, anomalies of sensation, or sphincteric disturbances. Several confirmatory points had, however, been overlooked, as was shown by the outcome.

Outcome.-It was subsequently discovered that the Achilles jerk was absent. The Wassermann reaction was positive, and the spinal fluid showed an excess of lymphocytes.

The vomiting persisted, though less frequently, until the fifth of September. After that it ceased and convalescence was rapid. Repeated examinations of the urine were negative. No treatment that was given seemed to help him.

Diagnosis.-Tabes with gastric crisis.

\section{Case 329}

An Irish laborer of forty-three, of good family history, entered the hospital November I5, I907. He has had no disease of importance, though he has been in several dynamite explosions and sustained various wounds and burns. Within the last eight years he has taken no alcohol, and before that never drank to excess. $\mathrm{He}$ denies venereal disease.

Since the last explosion in which he was involved ten months ago he has vomited once or twice almost every day, generally in the morning before breakfast. The vomitus consists of greenish mucus; it sometimes contains food eaten many hours before. He has seen no blood. $\mathrm{His}$ bowels are rather loose, moving three to seven times a day. $\mathrm{He}$ has no pain, a fair appetite, and he has kept at work until seventeen 
days ago, when the vomiting became almost incessant and he had to give up.

On examination the temperature, pulse, respiration, blood, and urine are normal.

The patient is obese, shows many powder-marks about his right eye and some scars in the cornea of both eyes, which he says are due to the old explosion.

Examination of the nervous system and internal viscera is through-. out negative. No contents could be obtained by the stomach-tube from the fasting stomach, which held only 26 ounces without distress. After a test-meal the gastric contents showed free $\mathrm{HCl}$, O.I28 per cent.

Discussion.-Is it wise to believe the patient's story on the subject of alcoholic indulgence? Is it not more probable that the patient's vomiting is due to the cause usually discoverable in such cases? However this may be, it should be noted that vomiting continued in the hospital after the alcohol had been withdrawn. Moreover, his family and friends confirmed his account of his habits.

I have never known concealed morphinism in a man of this type. Nevertheless, it is always a possibility to be reckoned with in case of unexplained vomiting, especially if there are wide-spread pains, insomnia, and great restlessness associated with it.

A point of special importance is the patient's obesity, which proves pretty conclusively either that his vomiting has occurred on an empty stomach independent of food, or that he has not ejected the whole of many meals. Many a patient fails to take account of the difference between emptying the stomach and merely spilling over, as a baby does, the excess of what has been eaten. This explains the astonishing discrepancy often confronting us between the patient's account-- "I have vomited every meal I have taken for weeks"-and the excellent strength and nutrition of his tissues, and spares us the necessity of assuming that he is lying or consciously exaggerating.

As we go over the case afresh after a fruitless search for organic lesions, we note that his vomiting followed immediately upon a dynamite explosion. Further inquiry may perhaps show that deep impressions made at that time may be connected with the habit and practice of vomiting - that, in other words, we may be dealing with a traumatic neurosis. This must not be assumed without a careful study of the patient's mental attitude, as it is apt to be revealed on close questioning about the accident and what has happened since. It would seem strange that a man who has been through several explosions should lose his nerve for the first time in the last one. Only further inquiry and experi- 
ment can decide. Such an inquiry, though in rather an abbreviated form, was undertaken.

Outcome.-After some preliminary questioning, the house officer gave the patient a long explanation of the theory and practice of traumatic neuroses, explaining the supposedly nervous origin, structure, and development of the trouble. The patient accepted everything that was told him as absolute truth, and began at once to eat and to smoke without any discomfort or vomiting. After four days of entire freedom from symptoms he was discharged well.

Diagnosis.-Traumatic neurosis.

\section{Case 330}

A married woman of fifty-one entered the hospital July I5, Igo8. Her family history is good, and she has always been well, although she has been subject to belching and nausea for many years. "The

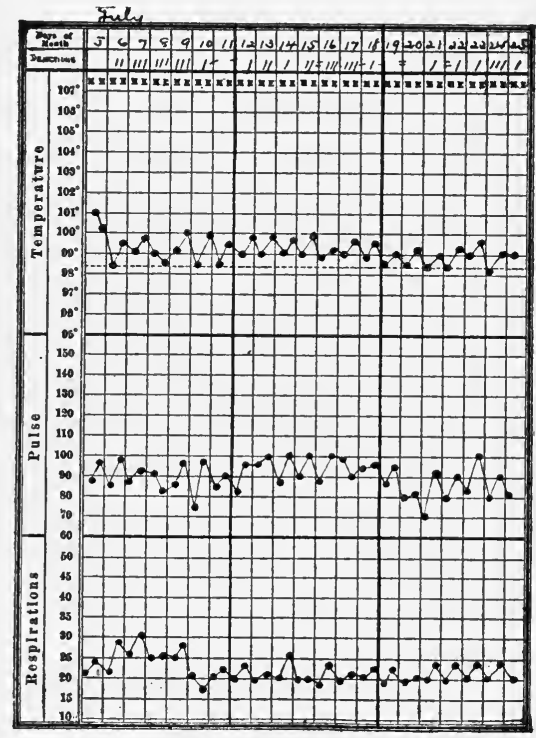

Fig. r69.-Chart of case 330. amount of gas that forms in my stomach is beyond belief," she says. She has taken three cups of coffee and four of tea daily. She passes water two or three times at night. Catamenia ceased fourteen years ago.

During the past winter and spring she was having, as usual, a good deal of trouble with gas and distention of the stomach, especially at night. Toward the end of May she had an attack of "bloating" somewhat severer than the previous ones, accompanied by epigastric pain and by vomiting of a sour fluid. At this time she was put on a milk diet and stayed in bed. Seven weeks ago she was put on rectal feeding, and this was continued in the Hale Hospital at Haverhill for the last four weeks. She has gradually vomited more and frequently, no matter what she eats or drinks, even in the absence of all food by mouth. She has always been allowed to take water by mouth. At the present time her vomitus is green and bitter. About three weeks ago she noticed about a teaspoonful of bright blood in the vomitus. Even morphin, which has been given in considerable quantities for the last week, has 
not sufficed to control vomiting. She continues to have gnawing pain in the epigastrium, running up the sternum to the throat.

On examination, the temperature is $\mathrm{IOI}^{\circ} \mathrm{F}$., the pulse 88 , respiration 24. The patient is obese; the pupils small, but reacting normally. Her lungs and other internal viscera show nothing abnormal. The blood is negative, likewise the urine, except for the presence of a small amount of acetone and diacetic acid. The guaiac test in the stool and vomitus is negative.

Discussion. - In a patient who vomits with a temperature of $\mathrm{I} \mathrm{I}^{\circ} \mathrm{F}$., infectious disease is the first possibility to be investigated. I have repeatedly seen a case which turned out to be pneumonia, but in which constant nausea and vomiting were the only complaints for three days, cough and signs of solidification being wholly absent. Less frequently one sees the same prolonged nausea at the outset of typhoid or malaria, and in children in almost any infection. Even if physical examination is at first wholly negative, we should suspend judgment as long as the temperature remains elevated, and continue to "watch for the development of some distinctive symptom betraying infection. Vomiting itself does not produce fever.

Some type of organic gastric disease or some of the extragastric lesions simulating it should next be looked for, although at present there seems nothing definite enough on which to base a conjecture.

Leaving these possibilities for the present undecided, I wish to call attention to two points of interest:

(a) That she has had morphin enough in the past week to prevent her vomiting from ceasing if, by chance, it showed any tendency to do so.

(b) That she is still obese and has, therefore, in all probability retained and absorbed more food than her account would otherwise lead us to suppose.

Since morphin may check pain or vomiting, many physicians are slow to realize that when continued more than a few days it has a tendency to produce in many persons both pain and vomiting.

The first move, therefore, should be to stop the morphin and study the condition of the patient when free from its influence. The size and functions of the stomach, the temperament and habits of the patient, must be learned. Thus the diagnosis may be more definitely outlined. But as a working hypothesis I think we have already evidence enough to justify us in following the clue given by her account of her own flatulence. A history of this kind usually points to a habit neurosis dependent upon cribbing. 
Outcome.-The patient was found to be swallowing air constantly. She was given at once a liberal diet of liquids and soft solids, with a bitter tonic before meals, fluid extract of cascara for her bowels, and Hoffmann's anodyne, a dram at night, if needed, for gas and distress.

In the four days following her entrance to the hospital the patient vomited only once.

Examination with the tube showed no fasting contents, no enlargement of the stomach, and after a test-meal, free $\mathrm{HCl}$, O.I2. The patient was kept for some time in the hospital on account of the slight fever, which continued for something over two weeks, but in every other way she seemed entirely well, and regained her confidence before the twentyfifth of July, when she was allowed to go home. The cause of fever was not found.

Diagnosis.-Gastric neurosis.

\section{Case 331}

A single woman of thirty, formerly a buyer for a dry-goods house, entered the hospital March I, I907. Four years ago she weighed I25 pounds and was strong and vigorous. She then began to have frequent stomachaches and much doctoring, both of which have continued and got worse each year. She had an osteopath for two years, with considerable relief to her stomach symptoms.

For the last year vomiting has been her chief symptom. It has no relation to the quality, quantity, or time of food. Some days she can eat and retain her meals. Other days even a mouthful makes her vomit.

Three weeks ago she was operated on for a floating kidney. Since then she has vomited everything. She is convinced that the operation was unnecessary and harmed her stomach. She is hungry and sleeps well, but is very weak and weighs only 67 pounds.

On examination the patient is much emaciated and nervous, but not despondent. The glands in the neck, axillæ, and groins are slightly enlarged; the heart-sounds weak and valvular; a systolic whiff is closely confined to the apex region; no enlargement; the pulmonary second sound is slightly louder than the aortic second sound.

The blood-pressure is $95 \mathrm{~mm}$. $\mathrm{Hg}$. Occasional râles are heard over the large bronchi. The lungs are otherwise negative.

The aorta and the iliac arteries are easily palpable, but the abdomen shows nothing abnormal. Hemoglobin, 75 per cent., white cells, 5200. Temperature, pulse, respiration, and urine, normal. 



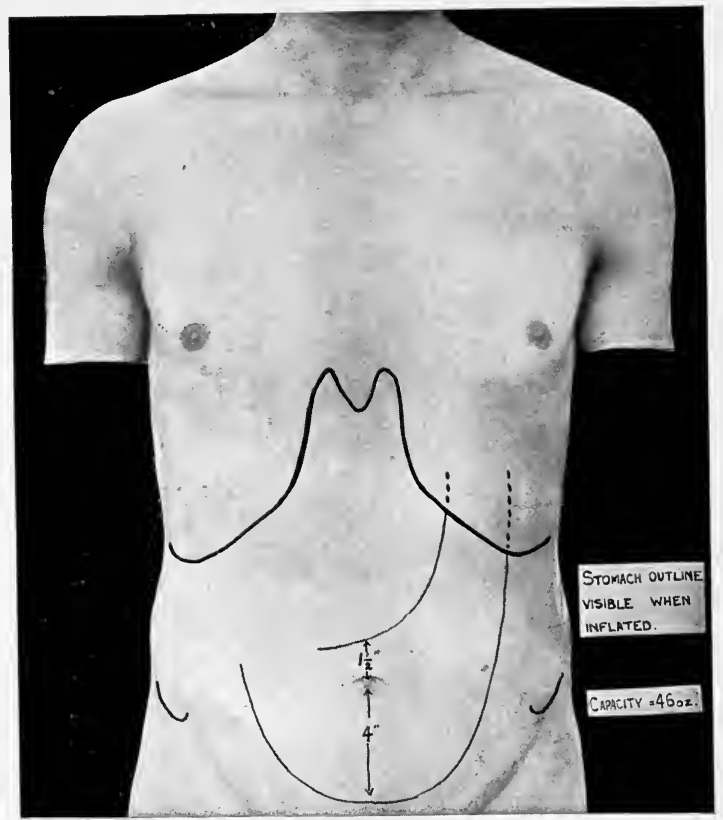

Fig. 171. - Shows percussion outlines (also visible) after inflation of the stomach in Case 33I 
The stomach-tube showed the gastric capacity to be 46 ounces. The outlines of the inflated stomach were as shown in the accompanying diagram (Fig. I7I). There was food in the fasting stomach. $\mathrm{HCl}$ was absent both in the fasting contents and after a test-meal. There was no reaction to guaiac in the stools or gastric contents.

Discussion. - Though there is nothing in the physical signs to suggest tuberculosis, one must always search with extra care for evidences of this disease when a patient is so emaciated and presents such marked gastric symptoms at the age of thirty. The low blood-pressure also points in the same direction, but in the entire absence of fever, and without more definite physical signs in the lungs, abdomen, bones, or glands, we cannot take another step.

Cancer of the stomach is very rare at this age, yet the emaciation, the evidences of gastric stasis, and the absence of hydrochloric acid compel us to give it consideration. As bearing on this question it is of importance to note that the symptoms are of long duration and gradual onset-very unusually so for gastric cancer. The good appetite is another point against cancer, likewise the absence of blood in the stomach-contents and in the stools. The patient is so thin that we should expect to feel a tumor if any were present, especially as the disease has lasted so long. On the whole, it seems well to adopt some other working hypothesis.

The position of the upper gastric border of the stomach proves that we are dealing with gastroptosis as well as gastrectasis, and makes it

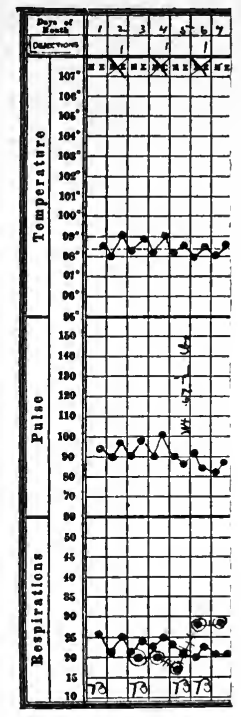

Fig. 1 70.-Chart of case 33 r. quite possible that the enlargement may be due wholly to the dropping. Whether any "benign" form of stenosis is present at the pylorus can be determined only by palpation, and by the results of our efforts at inducing the stomach to empty itself more thoroughly. Even in a warm bath and with the most perfect relaxation of the abdominal walls, no induration could be felt in the region of the pylorus, which was unusually accessible to the hand, owing to the low position of the whole organ (afterward demonstrated by bismuth and $x$-ray picture).

As a result of these investigations and of a good many studies of the patient's mental state it seemed clear that we were dealing with a vicious circle. The patient's fruitless regrets and fulminations about the apparently useless operation doubtless helped to aggravate, and were 
in turn aggravated by, the stasis in her prolapsed stomach. Such a vicious circle usually has some point of least resistance either on the physical or the mental side. We break it by striking at that point, ascertained by experiment or as a result of previous knowledge of similar cases. In the present case it seemed well to attack first the gastric stasis, and, by conquering that, to improve the general nutrition, thereby mitigating the mental fermentation. Other cases may be best attacked from the mental side.

It may be well to say a word in passing of the dangers of undertaking any operation in such a patient except in genuine emergencies. I may here refer to the interesting and suggestive article of Dr. Stuart McGuire on "Latent and Active Neurasthenia in its Relation to Surgery," Jour. Amer. Med. Assoc., March 26, I9io. As the result of a slight orthopedic or cosmetic operation done at an unfavorable time in a neurasthenic patient, I have seen acute and intractable exacerbation of all the patient's previous troubles reinforced by a host of new ones which tortured the patient and his friends for a year thereafter. The present case was a comparatively mild one of this type, but I have no doubt that the operation made her far worse than she was before, whether the kidney remained in place or not.

Outcome.- - Under daily gastric lavage, liquid and soft solid diet for the first two days, and then six meals with dry diet, $\mathrm{r}_{5}$ drops of dilute hydrochloric acid after each meal, and the same amount of tincture of nux vomica before meals, the patient steadily improved.

The food residue in the fasting stomach had diminished by March Igth from ro ounces to 3 ounces. The patient was much less nervous, up and about the ward daily. On the twenty-third she weighed 79 pounds and was very markedly improved.

Diagnosis.-Neurosis; gastroptosis.

\section{Case 332}

An Irish teamster of forty-six, of good family history, entered the hospital February 2I, I908. He has had attacks of stomach trouble like the present nne on and off for ten years; nevertheless he has kept at work practically all the time, has smoked 35 cents' worth of tobacco a week, and averaged one whisky a day. He denies venereal disease.

All through the summer and autumn his stomach was in bad condition, but for the past four weeks he has been having an increasing amount of distress. He vomits almost daily, often four or five times a day, and usually in large amounts-two or three pints at a time. His vomitus consists of food, at times mixed with brownish material. Sometimes he 
has seen in the vomitus food eaten forty-eight hours before. There has been no blood recognized as such.

He has also epigastric pain, which radiates to the back and abdomen, severe, but always relieved by vomiting. Neither pain nor vomiting bears any relation to meals, so far as he knows. His appetite is excellent. He eats everything, as he finds that he vomits as much on a milk diet as when eating solid food. He insists especially that he is all right if he "keeps quiet," but that he finds it hard to get along if he tries to work. Nevertheless, he has worked up to February 2oth. His average weight is 135 pounds. Now he weighs 105, though he does not think that he has lost much weight of late.

He has no symptoms except those above mentioned.

On examination the patient is emaciated, with a dry, somewhat pale skin; the cervical, axillary, and inguinal glands are slightly enlarged. The pupils are normal in all respects. The tongue is clean. The arteries are palpable and tortuous. The brachials show a lateral excursion.

Physical examination is otherwise negative, except for slight rigidity of the right rectus abdominalis.

White cells, 15,400; hemoglobin, 85 per cent.; urine negative; temperature as seen in the accompanying chart.

Examination by means of the stomach-tube showed that the stomach held 60 ounces, though its lower border reached only to the level of the navel after inflation, and no fasting contents were obtained. After an

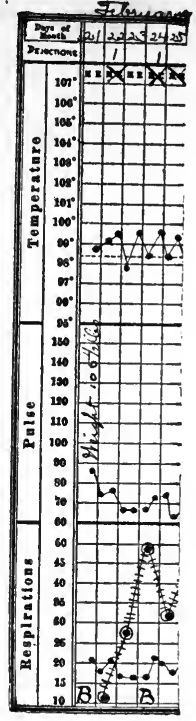

Fig. I72. - Chart of case $33^{2}$. Ewald test-meal the contents showed free $\mathrm{HCl}, 0.266$ per cent.; total acidity, 0.348 ; no reaction to guaiac. On examination in a warm bath a hard, irregular mass the size of a plum was felt in the right upper quadrant of the abdomen. It was very movable, but not tender. It could be grasped in the fingers and moved freely from a point below the umbilicus until it disappeared behind the ribs. It was not obliterated by inflation of the stomach.

Discussion.-We are dealing here with a case of long-standing stomach trouble which leads, in the patient's forty-sixth year, to that type of persistent vomiting which points to gastric stasis and dilatation. It is to be noted that vomiting relieves pain and is associated with a good appetite, a clean tongue, and a high gastric acidity. The presence of a 
very movable intra-abdominal tumor is the chief remaining point of importance.

Tumors of extreme mobility, as to whose nature any doubt is entertained, almost always turn out to be connected with the pylorus. Floating kidney is usually recognized with ease if its mobility is extreme, and there are no other tumors of this type. Those connected with the gallbladder may have considerable mobility, but their other characteristics usually serve to distinguish them.

Tumors at the pylorus result either from cancer or ulcer. Against cancer in this case is the long duration of symptoms, the retention of appetite, the high acidity, and especially the marked relation to exertion; but as we know that cancer may become ingrafted upon ulcer, it does not seem possible to be any surer of our diagnosis without laparotomy. On the whole, though, the evidence points very strongly toward ulcer.

Outcome.-On the twenty-eighth the abdomen was opened. An area of induration, with glandular enlargement in the neighborhood, was found near the pylorus.

Pylorectomy and gastro-enterostomy was done, about $10 \mathrm{~cm}$. of the lower end of the stomach being removed. In this operation there were two ulcers, one about $4 \mathrm{~cm}$. above the pylorus, $1.5 \mathrm{~cm}$. in diameter, with sharply punched-out edges and deep excavations. Its base was firm and gristly, but consisted only of chronic inflammatory tissue. Another ulcer about $2 \mathrm{~cm}$. in diameter, with a similar gristly wall, is just above the pyloric ring.

The patient left the hospital on the twenty-eighth of March, seemingly quite well.

On the fifth of April, I909, the patient reported that he was in good health and working regularly. He has no gastric distress, but has to. eat five times a day in small amounts. He has gained markedly in weight and strength.

Diagnosis.-Gastric ulcer; pyloric stenosis.

\section{Case 333}

A barber, thirty-seven years old, whose father died of Bright's. disease, was first seen June I9, I907, complaining of vomiting spells which began when he was sixteen years old and have continued about. twice a year ever since, though less frequent in the last ten years. $\mathrm{He}$ feels a "lump like lead" in the epigastrium all the time at present, and cannot remember when he did not feel it. All food distresses him about equally. His appetite is good, and he eats slowly and at 
regular intervals. The bowels are always constipated unless he takes laxatives.

Ten days ago he began to vomit without known cause, and has since then rejected everything except malted milk. The vomitus is chiefly phlegm in small amounts. During these ten days he has perspired during the earlier part of the night, and felt very cold the rest of the night. His sleep has been dull and heavy. He thinks he has lost weight. He has been able to do no work for this same period.

The course of the temperature is seen in the accompanying chart.

The patient is well nourished, slightly pale, shows some concretions of blood in the nose. Heart and lungs are negative, likewise the abdomen and the urine. The blood shows 4000 white cells and 60 per cent. of hemoglobin.

Discussion.-Our first impression of this case would be that it is one of chronic indigestion of unknowr cause (gastric neurosis, chronic ulcer, chronic appendicitis), with an acute exacerbation perhaps due to constipation or some temporary nervous disturbance. But for the negative examination of the urine and the absence of headache, one might suspect chronic Bright's disease or cerebral tumor, both of which I have known to show themselves in this way in a patient with a similar history, reaching back indefinitely into boyhood.

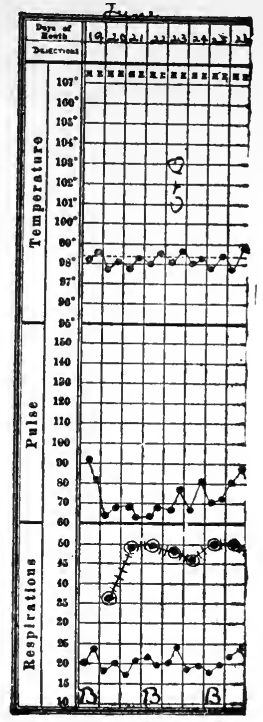

Fig. 173.-Chart of case 333 .

One feature, however, arrests attention: In the present vomiting spell, which seems to be a good deal worse than the rest, he has had night-sweats. Although the daily chart (the temperature being taken morning and evening) shows no fever, it does not cover the period during which he was complaining of night-sweats, and as it was only taken in the day-time, there may well have been a febrile rise at night since his entrance to the hospital, as well as before. This indication should lead us to search for evidences of tuberculosis or other infectious disease, more especially as there is a considerable degree of anemia manifested by the lowered hemoglobin percentage.

Such a lowering of the hemoglobin should always lead us to the study of a stained specimen. To one well trained in routine blood work the stained specimen would have made clear the diagnosis in this case, yet as a matter of fact the blood has already been examined and nothing 
found to suggest what was wrong with the patient. A good example of the importance of competent blood examination is thus furnished in this case by the outcome.

Outcome.-A large number of tertian parasites-especially the young, unpigmented ring forms-were found in the stained blood-smear, together with some large atypical lymphocytes exhibiting phagocytosis of red cells. Had the blood been examined unstained, as is often done in cases of suspected malaria, these parasites would probably not have been recognized.

The patient was given Io grains of quinin and the dose was repeated an hour later. After this he received 5 grains every four hours until his ears began to ring, then enough to keep them ringing for three days. In two days his blood was free from parasites and his vomiting had ceased.

I may add a word here concerning other atypical forms of malaria$i$. e., those which do not begin with the familiar tertian chill. Among the malarial fevers of temperate climates, almost all of which are due to the tertian organism, I have noted the following unusual clinical types:

(a) A case beginning with violent maniacal delirium without other distinguishable symptoms.

(b) Cases beginning with intense headache and stupor, closely simulating the onset of meningitis.

(c) A case beginning with violent pain in both axillary regions and along the lower costal margin.

(d) A group of cases already referred to (see p. I42) which simulated appendicitis, owing to the severe pain in the right iliac region or in the epigastrium.

(e) Cases in which headache, sleepiness, or muscular weakness recurred every day or every other day at the same hour.

In estivo-autumnal malaria persistent diarrhea may be the only striking symptom. A very large number of cases occurring in children are mostly or altogether latent. ${ }^{1}$

In all these atypical cases diagnosis is comparatively simple, provided we are led to make a careful examination of the stained blood-film; without this, diagnosis may be impossible.

Diagnosis.-Tertian malaria.

${ }^{1}$ See Craig, "Latent Infections in Malaria," being Part III, Chapter IV, of his book on Malarial Fevers. 


\section{Case 334}

A sewing woman of fifty-nine, always previously in good health, entered the hospital June 7 , 1906. She felt perfectly well in the morning, four days ago, and went to call upon a friend. While there she was suddenly seized with nausea and vomited several times. She was taken home, went to bed, but felt mean and nauseated all the next day. She has not been able to work, and has been in bed practically all the time since, vomiting a little each day. She feels "all gone" and weak, is very drowsy and sleepy, and has had rather a severe headache since the onset. The bowels are moved with medicine. She gets up once or twice at night to urinate; she has a very slight dry cough.

The patient is obese; the pupils are equal and react normally. The tongue is clean, the heart and arteries normal. There are a few crackling râles in both backs, especially at the bases.

For temperature, see the accompanying chart.

The abdomen and reflexes are normal.

The urine averages 40 ounces in twentyfour hours, IO2I in specific gravity; no albumin; many hyaline and fine granular casts.

Discussion.-The very acute onset and the association with headache and drowsiness suggest some implication of the brain. Meningitis or brain tumor sometimes show themselves for the first time in this way, but when we follow out the tests indicated by these hints, there seems to be nothing to confirm them. Nephritis also might indirectly produce cerebral symptoms like these, and the habit of nocturnal urination, together with the abundance of tube-casts, gives some warrant to this idea. The other characteristics of the urine, however, do not bear it

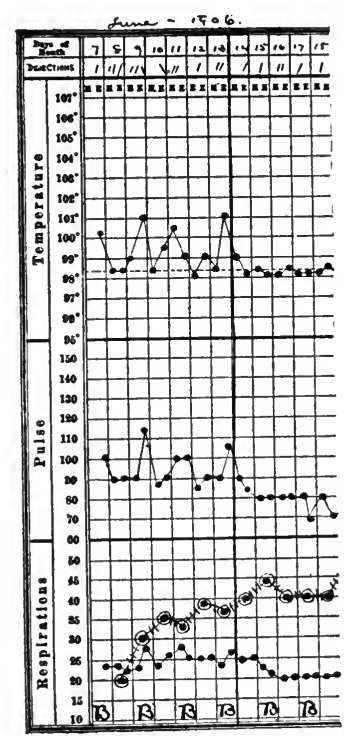

Fig. I74.-Chart of case 334 . out, and as there is fever in the case, the presence of casts can be thus accounted for. The evidence would be more complete upon this point if we had an accurate measurement of the systolic blood-pressure.

Other diseases which often begin in this way are pneumonia and the gastric crises of tabes dorsalis. Occasionally a paroxysm of vomiting is the only manifestation of an attack of nephrolithiasis. None of these clues, however, turned out fruitful when followed up in this case. 
The correct diagnosis was actually suggested for the first time by the temperature chart, which shows, as will be at once obvious, a tertian fever. Such fever is by no means pathognomonic of malaria; I have known it to occur in tuberculosis and in various types of sepsis. Nevertheless, it could hardly fail to remind us that malaria may begin with nausea and vomiting and thus lead us to a careful blood examination.

Outcome.-The day after entrance the patient had a chill, and parasites were looked for, but in vain. It was not until the third day that the malarial parasites were discovered.

On the fourteenth the patient had a rise of temperature and vomited, though she had been taking quinin, 5 grain every six hours, since the eleventh. After that day, however, she had no more fever or vomiting.

Diagnosis.-Malaria (tertian).

\section{Case 335}

A school-boy of sixteen was first seen August 24, 1907. His family history and past history are excellent. Last winter he entered high

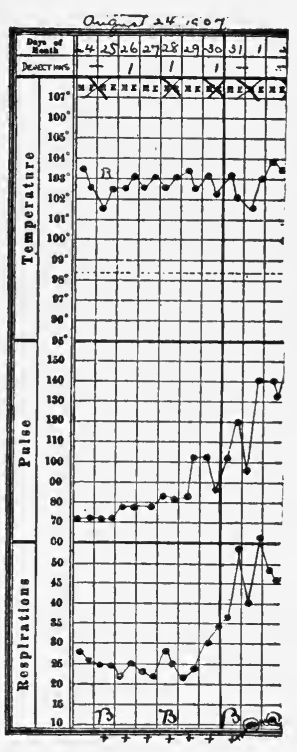

Fig. $175 .-$ Chart of case 335. school and worked very hard. This spring he seemed tired out, and had headaches, supposedly due to eye-strain.

Two weeks ago he began to vomit. For four days he could retain no food, and for a week more vomited each morning. The bowels, meantime, were constipated, but the appetite was good throughout. During these two weeks he has frequently been chilly or feverish, his hands and feet being cold. He has complained of headache and pains in his back and legs, with slight cough. Throughout he has been very weak. For the past three days there has been delirium.

When seen, the patient was in a muttering delirium, with twitching of the face. He was poorly nourished. The course of the temperature is seen in the accompanying chart. The chest showed nothing abnormal. The abdomen was flat, rather tense, tympanitic throughout, and apparently not tender. Reflexes were normal. There were no rose-spots. There was incontinence of urine and feces, but the urine showed nothing abnormal. 
White cells, 7000; hemoglobin, 80 per cent.; Widal reaction, entirely negative.

The case was believed to be one of typhoid fever with meningeal irritation.

Discussion.-When a boy of sixteen is suddenly attacked by fever and vomiting in the middle of summer, no exanthem or other obvious cause being visible, malaria is one of the first diseases to be considered. It was easily excluded, however, in this boy's case by blood examination.

Brain tumor, especially solitary tubercle of the cerebellum, sometimes begins in this way, and without an examination of the fundus one can hardly exclude it with certainty. It would be unusual, however, to find no hint tending to localize the trouble in any particular part of the brain (vertigo, staggering gait, strabismus).

Typhoid fever was the diagnosis actually made in the early days of this illness. The negative Widal reaction seemed to be of no great significance, since this reaction is so often absent in the earlier stages of typhoid. The early onset of delirium and the relaxation of the sphincters were accounted for by meningeal irritation (meningismus). In all such cases, however, experience has taught me that it is wise to do lumbar puncture. Several times I have known typhoid mistaken for tuberculous meningitis and a hopeless prognosis given in consequence. When the child got well and the diagnosis of typhoid became obvious, the family were not pleased with the prognosis previously given by the medical attendant. In the present case the opposite mistake was made, and a falsely hopeful prognosis was given.

Outcome.-On the twienty-seventh his neck was found to be stiff. Lumbar puncture showed Io c.c. of clear, colorless fluid, the sediment of which showed 95 per cent. lymphocytes. A culture of blood-serum remained sterile.

The Widal reaction was tried daily, but continued negative. The ear-drums were examined without result.

On the twenty-ninth the neck became more relaxed and the patient quieter.

A second lumbar puncture, September Ist, gave essentially the same results as before. Some of this fluid was injected into a guinea-pig. The patient became unconscious on the second of December and died on the fourth.

Throughout the first week of his stay in the hospital he was treated for typhoid; later he was fed by stomach-tube, milk, beef-juice, and eggs being introduced in this way.

October $7^{\text {th }}$ the guinea-pig which had received the spinal fluid inter- 
peritoneally was killed, and showed tuberculosis of the spleen and lymphatic gland.

Diagnosis.-Tuberculous meningitis, [presumably with general miliary tuberculosis].

\section{Case 336}

A married woman of thirty-four, of good family history and past history, entered the hospital March 9, I908. She had a miscarriage five weeks ago, in the fourth month of pregnancy, following a hard day's work of washing. There was considerable hemorrhage both before and after the miscarriage. She was curetted at the time, but has been very weak and confined to bed ever since. During the first three or four days after the miscarriage she had two chills, but there has been no fever, so far as she knows.

For the past three weeks she has vomited persistently and has been able to retain no solid food. It is chiefly for this symptom that she seeks advice.

On examination, the pulse, temperature, and respiration are normal. The patient is well nourished, slightly pale, pupils equal and reacting normally, the tongue thickly coated white, the left tonsil slightly enlarged, about half of the teeth missing. The heart's impulse is not seen or felt. The sounds are best heard, and the left border of dulness found, in the fourth space, five inches to the left of the midsternal line, one inch outside the midclavicular line. The sounds are regular and of good quality, the pulse of low tension.

The lungs are normal, the abdomen considerably tender above the symphysis and at McBurney's point. The patient has many varicose veins in the right lower leg, and two white scars said to be due to previous ulcers.

Blood and urine not abnormal.

Discussion.- The salient facts appear to be as follows: Obstinate vomiting following miscarriage and associated with slight enlargement of the heart and abdominal tenderness especially marked in the appendix region.

This group of symptoms does not easily cohere into any of the traditional groups which we call diseases. The cardiac signs are slightobviously too slight to account for the vomiting as a result of passive congestion. There is no kidney change to account for the cardiac. enlargement, or to suggest uremia as a reason for the vomiting. There are no brain symptoms, and though a fundus examination would be a satisfactory completion of our record, it is not urgently called for by 
the other symptoms of the case. Malaria and other infectious diseases are ruled out by the entire absence of fever.

The abdomen deserves, of course, our special attention. Is it possible that the general tenderness may be the result of some type of peritonitis, a low-grade septic infection, or tuberculosis? The evidence is, on the whole, insufficient to justify any such belief. There are no muscular spasm, no free fluid or masses-nothing more, in fact, than one finds after a miscarriage in a great many uncomplicated cases. In the absence of fever, leukocytosis, elevated pulse, or more distinctive local evidences appendicitis seems unlikely.

A more thorough investigation of the pelvis is indicated. Although we know very little about the connections between the vomiting center and the genital tract, connections whereby so-called reflex vomiting of pelvic disease is supposed to arise, it is a very familiar fact that a variety of low-grade inflammatory changes in the puerperal uterus, complicated no doubt to a greater or lesser extent by absorption from incompletely organized thrombi, may lead to well-marked constitutional disturbances, of which vomiting is one. Whether this comes about through the nervous system, by the aid of the vomiting center, and favored by psychic disturbances, or whether it is in some way a more direct result of infection, I know no way of determining at the present time. But, however this may be, it seems clear that, when we have finished our task of excluding the other possibilities mentioned above, the best remaining hypothesis on which we may base treatment is that which assumes that the vomiting is in some way connected with the residual effects of the miscarriage.

Outcome.-The patient was put to bed and given zominims of fluidextract of ergot at once, and I5 minims three times a day after meals. There was some reddish vaginal discharge without odor. The uterus was moderately enlarged, freely movable, and somewhat tender. By the twenty-second the uterus was much smaller and less tender. The vomiting ceased after the third day. The treatment consisted of laxa.. tives and a daily suds enema.

Diagnosis.--Incomplete miscarriage.

\section{Case 337}

A stationary engineer of fifty-seven was first seen January 2I, 1907. $T_{w o}$ of his brothers and one sister died of consumption. His wife is said to have died of tuberculosis of the bowels. He has himself been well except for so-called rheumatic pains referred to the muscles of the back and extremities. These he has had for many years. 
Eight years ago he was poisoned by steaming oxalic acid, and was sick a week, with vomiting, diarrhea, and abdominal pain. Since that time he has had occasional attacks of a similar nature about twice a year, lasting three or four days.

His usual weight is 184 . He has passed urine frequently at night for a number of years.

Seven months ago he began to have frequent attacks of vomiting, coming on quite suddenly during a meal, or soon after it, and without any pain, nausea, or distress. He has vomited every day for some months-often two or three times a day. There has never been any large amount of vomitus, nor any indication of food eaten the previous day. The appetite has been poor, his bowels very loose. He has lost color, weight, and strength very rapidly. Six months ago he was obliged to take to bed, where he remained two months, and got somewhat better. Diarrhea and vomiting improved decidedly, and he gained in weight, but at the end of another month he relapsed and had to take to bed again for most of the succeeding months. His complaints are now the same as they were seven months ago. He has never vomited blood. The character of the food has apparently no effect on the vomiting. Seven months ago he weighed I 84 pounds; four months ago, I42; three months ago, I53; now he weighs I25 pounds.

On examination the patient is pale. The heart's apex is in the fifth space, inside the nipple-line; there are no murmurs, no accentuations of any sound. The pulses are of high tension, the artery wall apparently somewhat thickened. The abdomen shows distinct resistance in the epigastric region and beneath the right costal border. Otherwise it is negative.

The blood showed: Red cells, 2,796,cco; white cells, 9400, of which 84 per cent. are polynuclear, the rest lymphocytes. There is no achromia and no other change except slight deformities in the red cells.

The urine is 30 ounces in twenty-four hours, and contains a large trace of albumin, many pus-cells, no casts. Gravity, тог2-гог6.

The stools are nega tive to guaiac and show no abnormal food residues.

Discussion.-The family history of tuberculosis is so threatening in this case that one would naturally begin diagnostic investigations with a search for evidence of phthisis or some other form of tuberculosis. This was done, but without result.

Since his occupation does not involve any constant exposure to the oxalic acid by which he was poisoned eight years ago, there appears to be no reason to connect his symptoms with this poison. 
The loss of weight, the anemia, the age of the patient, and the method of onset suggest ulcer or cancer of the stomach. The symptoms have lasted so long, however, that stasis would probably have manifested itself before this time. It is remarkable also that the food he takes has apparently no relation to the vomiting.

Gastritis and enteritis, without some obvious cause, such as alcohol, uncompensated cardiac lesions, tropical dysentery, or chronic nephritis, are distinctly rare diseases in a man of this age. There is nothing in the study of the stools or of the vomitus to justify any such belief; neither of these diseases is apt to be accompanied by severe anemia unless a great deal of blood has been discharged.

It is often profitable, in the discussion of such a case, to begin with the well-established fact of secondary anemia and study the rest of the disease from the point of view of the possible cause of such an anemia. I have previously referred to a case (see p. 550) in which severe anemia was produced by long-standing hemorrhoids with bleeding, altogether unknown to the patient. Such a cause was sought for in the present case, but not found.

Obscure anemia in a patient of this age very often turns out to be of cancerous origin. It did not seem to me, at the time that the case came under my observation, that the possibility of gastric cancer had been sufficiently investigated, and, accordingly, I advised further study of the gastric contents and functions. Nothing of importance was elicited, however. The capacity of the stomach was within normal limits, there was no stasis, and although the amount of free hydrochloric acid was very small, this fact could not be interpreted as of any importance in relation to the possibility of cancer, since it could be explained in so many other ways. The guaiac test was negative in the gastric contents.

Chronic nephritis was next considered, since it is a familiar fact that long-standing irritation of the stomach and intestines, with or without a catarrhal inflammation, often complicates, and is the main cause of, distress in this disease. Little could be found to support this idea. There was no demonstrable enlargement of the heart. Unfortunately, the blood-pressure was not measured, so that we could not be certain that our digital impressions really corresponded to hypertension, as they were supposed to do. The urine was not incompatible with nephritis, but not characteristic of it-a very familiar and baffling state of things.

Although the patient's habits were supposedly excellent, the resistarice beneath the right costal border, the unexplained anemia and persistent vomiting, made us speculate concerning the possibility of a cir- 
rhosis, but we found no means of advancing beyond the region of speculation upon this point. In the end the diagnosis was very uncertain. Fewer objections were raised against the diagnosis of chronic nephritis with uremic vomiting than against any other, but none of us felt satisfied.

Outcome.-The patient grew progressively weaker, and died on the twenty-eighth.

Autopsy showed chronic interstitial nephritis, a very firm, rubbery dark liver, enteritis and gastritis, with chronic colitis, and terminal streptococcus septicemia.

Diagnosis.-(See above.)

\section{Case 338}

A barber of thirty-five with an excellent family history and past history, entered the hospital January II, I908. He has been having business reverses for the past four weeks, during which he has slept poorly and become very nervous.

On the night of December 27 he fell down eight or nine stairs and bruised his right hip, which has improved somewhat under poulticing, but is still lame and stiff and has confined him to bed.

The night after this fall he began to vomit, and has continued to do so three or four times a day, and once at night ever since. The vomitus at first consisted of food in considerable amounts; later, of a frothy liquid and mucus. Once or twice there has been a small streak of blood. His bowels meantime have moved from two to six times a day, with considerable griping pain and gas. Throughout there has been a dull, steady pain in the epigastrium, with a sense of dragging when he stands or sits up, but no especial pain. His food has been milk, eggs, oysters, and cool drinks.

On examination, the temperature, pulse, respiration, blood, urine, and internal viscera are all negative, except that the abdomen is held rather rigidly and that there is some flattening of the right lower chest in front, apparently connected with a funnel-breast deformity.

Examination of the stools showed no blood to any test and no abnormal food-remains.

Discussion.-Gastro-enteritis is the usual diagnosis in such a case, but while it is impossible positively to exclude such an affection, I think it is unwarrantable to assume its presence when the stools and the gastric contents furnish no better evidence of inflammation. The presence of mucus in the vomitus and the occasional small streak of blood are in no way distinctive. Almost any case characterized by persistent vomiting shows such products now and then. 
The abdominal rigidity leads us to think, at least for a moment, of some type of peritonitis, but there is not a single other fact to support this idea. Surely there would be some change in the temperature, pulse or blood, were peritonitis present. Moreover, any patient who has recently vomited a good deal is apt to hold his abdomen rather rigidly when the palpating hand explores it.

It is worth noticing that the vomiting came on immediately after the patient had been confined to bed by the injury to his hip. The isolation, the inactivity and the deprivation of all occupation or interest produced by putting a patient to bed will give him a splendid opportunity to dwell upon the worried and depressing events which had previously made him nervous and sleepless. One is justified in laying stress upon these factors in a case of this kind when careful physical examination furnishes no explanation of the symptoms. Treatment should be planned in accordance with the possibility that the symptoms may be psychic in origin, though greatly aggravated, no doubt, by physical exhaustion and starvation.

Outcome.--The patient was given a liquid and soft-solid diet, gentian and nux before meals, and trional, 5 grains, at night for two nights. In the three days following the beginning of this treatment he had no vomiting whatever, and declared that he felt perfectly well. Doubtless the reassurance given him as the result of a negative physical examination contributed to his recovery.

Diagnosis.-Nervous exhaústion.

\section{Case 339}

A motorman of forty-four entered the hospital May 8, I908. His family history and past history are rather uneventful, but he admits that he has lost much weight. Four years ago he weighed 210 pounds; now he weighs $\mathrm{I}_{59}$. He has not worked since October, 1907, because of stomach trouble. For the first two weeks of his illness he vomited everything that he had eaten, the sour vomitus consisting of undigested food, but never containing blood.

Soon after recovering from this he had "the grip," and was in bed for several weeks. Since then he has had wandering pains, "like rheumatism," in the shoulders, hips, and abdomen. He has not been confined to bed, and he was able to walk to the hospital, though he complains of considerable weakness. He has had a cough all winter until last week-since then, none. His appetite is poor, likewise his sleep. The bowels are moved daily. (For temperature, see the accompanying chart.) 
The patient is emaciated, the heart's apex in the fourth interspace, inside the nipple-line, the sounds of good quality. The pulmonic second sound is slightly accentuated.

At the apex of the right lung there are a few fine crackles after cough and a slight prolongation of expiration. In the left back there is a triangular area of dulness, with its apex at the spinal column, $\mathrm{I} \frac{1}{2}$ inches above the lower angle of the scapula; thence it slopes out into the midscapula. Over this area tactile and vocal fremitus are absent, breathing distant and bronchovesicular. Just above this area the voice has a nasal quality. The abdomen, blood, and urine are normal.

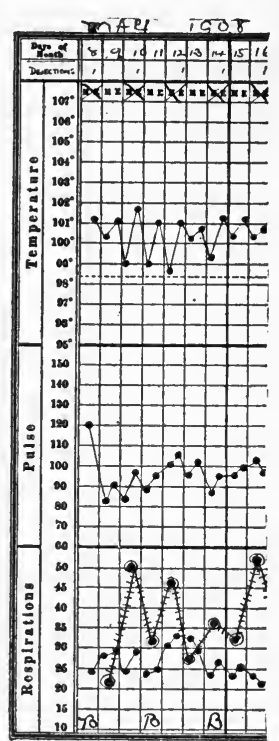

Fig. I76.-Chart of case 339 .

The sputum was twice examined for tubercle bacilli, with negative results.

Discussion.-The onset of well-marked gastric symptoms in a man of forty-four whose stomach has never troubled him previously, should always compel us to consider gastric cancer, but more especially so when the physical examination of the rest of the body reveals nothing which might be a cause for the gastric disturbances. This can hardly be said to be the case here, so that our attention is properly concentrated first upon the extragastric causes which might lead to his present gastric symptoms.

Nothing is said regarding the reflexes in this record. Without a knowledge of their condition it would be impossible for us to exclude tabes dorsalis with gastric crises. Further investigation, however, showed that all the reflexes were normal.

The most important abnormalities discoverable on physical examination are in the lungs, and though these signs are not extensive, they lead us to ask whether so much emaciation and stomach trouble could be produced by any well-known disease affecting the lungs. This question must be answered in the affirmative. Nothing is more familiar than the production of such a clinical picture as a result of pulmonary tuberculosis. But have we evidence of enough pulmonary disease to explain such severe constitutional manifestations? This point I have discussed in previous cases. Experience has made us very familiar with the discrepancy often existing between the extent of the discoverable physical signs and the severity of the constitutional manifestations, 
such as fever, sweats, chills, emaciation, and gastro-intestinal disturbances.

It is quite possible that some degree of secondary gastritis may arise as a result of lowered powers of resistance produced by tuberculosis, but postmortem evidence does not compel us to make any such assumption.

No weight should be laid upon the absence of tubercle bacilli in the sputum examined in this case. Such tests are of importance only when many times repeated.

Outcome. $-X$-ray showed a deep shadow over the lower part of the left chest. The upper margin of this shadow had an irregular outline, and did not suggest the existence of any considerable pleural effusion. There was also a slighter shadow corresponding to the left apex. The patient was discharged to a sanatorium for tuberculosis

Diagnosis.-Phthisis.

\section{Case 340}

A woman of fifty-six, a post-office clerk, of good family history and past history, entered the hospital January 9, I908. She passed the menopause six years ago without trouble. Throughout the past summer she has been bothered by sour stomach and flatulence.

Five days ago she ate canned salmon for supper, and that night, after going to bed, she felt very chilly, headachy, vomited and sweated profusely. The next two days she also vomited a good deal, and during the last two days has been extremely nauseated. Throughout these five days she has had aching all over her body, has slept very little, and has felt feverish. The matter vomited has been either food or a greenishcolored material. The bowels have moved twice in five days. She has eaten practically nothing.

For the last three days she has had a constant, irritating, dry cough.

Physical examination was negative, save that in the left upper chest and in the right axilla, at the extreme base, were a few transient, fine moist rales. Temperature, I02 $^{\circ} \mathrm{F}$.; pulse, Io0; respiration, 20; white cells, 19,500; hemoglobin, 90 per cent.; Widal reaction, negative; urine, negative.

Discussion.-The acute onset of symptoms in this case naturally and properly turns our attention for the time being away from the longstanding causes of vomiting, such as have been discussed in previous cases. It is true that some of these chronic diseases-such as brain tumor, nephritis, gastric cancer, or neurosis-may suddenly be "lighted up" or roused to unusual activity after having remained latent for a long 
time, but few if any of them would then be associated with such marked fever, leukocytosis, and general constitutional signs pointing to infection.

It is reasonable, therefore, to consider whether any well-known infection is prone to begin in this way. Infections of the gastro-intestinal tract are uncommon and still more uncommonly recognizable. Typhoid sometimes starts out with prolonged gastro-intestinal disturbances, but the high leukocyte count is sufficient to exclude any uncomplicated type of this disease. The same is true of acute or incipient tuberculosis, except in the meningeal form. The unknown infections, usually termed "grip" or "ptomain-poisoning," are much less likely to produce such leukocytosis and such continued vomiting than is one possibility-next to be mentioned.

Of all the severe infections which are apt to attack elderly people in or near the month of January, pneumonia is the one most often beginning with gastro-intestinal symptoms alone. The leukocytosis, the cough, and the trifling chest signs are all quite consistent with this idea. Yet no one could make a positive diagnosis of pneumonia from the facts here presented. A blood-culture might enable him to do so, since the pneumococcus is not infrequently to be found in the circulating blood before any evidence of solidification has become manifest in the lungs. Without culture one can only suspect pneumonia, the practical significance of which act consists in what it leads us to say to the family, together with the focusing of our attention on the results of repeated examinations of the lungs. In many cases with exactly such signs as are here recorded, the stethoscope reveals nothing characteristic, but with the free ear against the chest-wall we may be able to detect a distant but quite distinctive tubular breathing.

Outcome.-Twenty-four hours after the above record was taken there appeared in the left upper lobe, below the clavicle in front, dulness, diminished breathing, diminished voice-sounds, and many fine and medium moist râles. Although the breathing was of diminished intensity, the expiration and the whispered voice were high-pitched and relatively intense. The patient had no dyspnea and no pain.

On the eleventh the white cells were 28,000 ; on the thirteenth, 24,600; on the fifteenth, 7600 .

There were no sputa at any time and practically no cough. By the sixteenth the abnormal sounds were less; by the twenty-second they were gone.

The patient complained very much of abdominal distress and numbness. She vomited occasionally on the following days, and it was suggested that she ought to have a special nurse and be fed by the bowel. 
6

66 


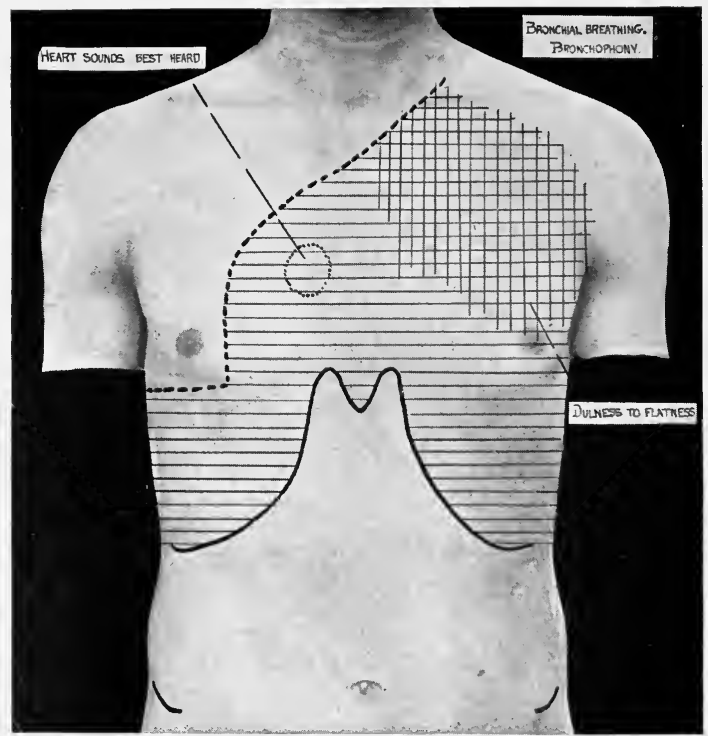

Fig. 178.- Signs recorded in a case characterized apparently by vomiting (eight weeks' duration); only within five days have pain and dyspnea appeared.

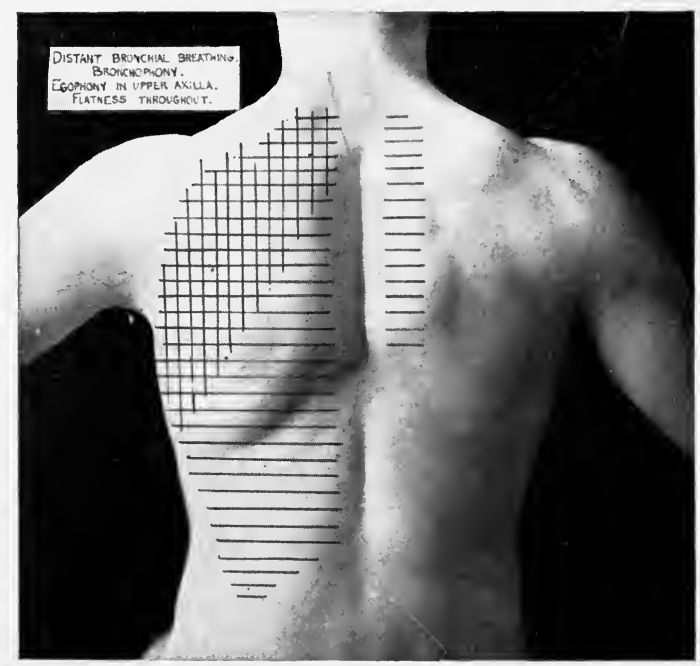

Fig. 179.-Signs as recorded in Case 34I. Note absence of any record of paravertebral dulness (Grocco's sign) on the right. In I906 we had not been taught to look for it. 
February Ist she announced that she would like to sit up, and her appetite began to return; this was not until her temperature, pulse, and respiration had been normal for seventeen days.

On the third of February she complained of being paralyzed all over. On questioning, "paralysis" turned out to mean numbness, and there was not the slightest sign of impaired motion nor of impaired sensation anywhere.

Diagnosis.-Pneumonia.

\section{Case 341}

An unmarried Canadian woman of twenty-seven, of good family history and past history, was first seen November 21, 1906. She has had occasional indigestion for the past two or three years, and of late has been run down and anemic. Catamenia have been absent for the past two and one-half months. For eight weeks she has had nausea and vomiting almost every day, sometimes several times a day. Vomiting may follow food, but at times she retches when the stomach is empty. Loss of weight and strength has been such that ten days ago she was obliged to go to bed, in spite of which vomiting has continued.

Five days ago she began to have dull pain in the lower left chest, increased by deep breathing or cough. At the same time she began to be short of breath. For two weeks she has had cough, occasionally raising thick, yellow, blood-streaked sputum. (See accompanying chart for the temperature.)

On examination the heart's apex is not seen or felt. The right border of cardiac dulness appears to extend $3 \frac{1}{2}$ inches to the right of midsternum. The sounds are clear, but are best heard to the right

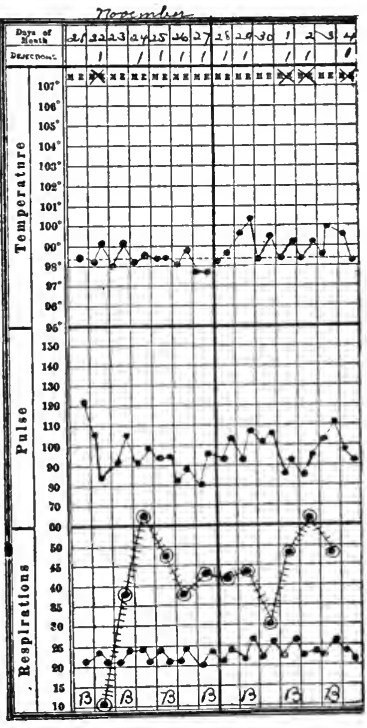

Fig. I77.-Chart of case 34I. of the sternum. The condition of the lungs is shown in the accompanying diagram (Figs. I78 and I79). The abdomen shows nothing abnormal. The reflexes are also normal. The white cells are 5500; the urine negative.

On the twenty-second 74 ounces of yellow, slightly turbid fluid were withdrawn from the chest. Specific gravity was ror8; albumin, 
4 per cent. In the sediment small lymphocytes made up 98.5 per cent. No tubercle bacilli could be demonstrated from the digested clots.

After the tapping the patient ceased vomiting and felt finely. By December Ist there was no vomiting at all; then it returned in occasional attacks, and by the twelfth it was causing a great deal of trouble. Free fluid had reaccumulated in the chest to a slight extent only, but the patient seemed to be losing ground.

Discussion.-The pleurisy which was so easily demonstrable in this patient's chest seemed at first a sufficient reason for her vomiting. We were, therefore, surprised that the vomiting continued after the effusion had been tapped. Neither the temperature chart nor any of the other signs in the case seemed to indicate that the tuberculosis, presumably in the background of this pleural effusion, was the cause of the vomiting.

There appeared to be no reason to suspect organic disease of the stomach, brain, heart, or kidney. There was no constipation or toxemia apparent. We might have been tempted to settle down on the unsatisfactory diagnosis of "gastric neurosis." One fact, however, still remained unexplained-namely, the amenorrhea. This was not to be accounted for by anemia or by any obvious psychic cause. Clearly, it was our duty to investigate the possibility of pregnancy.

Outcome.-Tuberculous salpingitis was suspected. Vaginal examination showed a mass in the pelvis, distinguishable from the uterus, softer and more fluctuant on the right than on the left. A great number of remedies for vomiting were tried, among them sodium bicarbonate, I dram in half a glass of hot water, sipped, Hoffmann's anodyne, I dram in hot water, ginger-ale with sodium bicarbonate in sips, mustard leaf to the epigastrium, bismuth and beta-naphthol, cerium oxalate, 2 grains every two hours, and various diets. Finally, on the fourteenth of December she was put on nutrient enemata and all food by mouth omitted. As the patient continued to vomit and retch at times, though the nutrient enemata were well retained and did not cause discomfort, it seemed best again to explore the pelvis, with a view to freeing the adhesions and perhaps stopping the vomiting in this way. Accordingly, on the twenty-first the abdomen was opened and showed nothing but a large, presumably pregnant uterus, with normal tubes and ovaries. Within five days after this exploratory operation vomiting ceased altogether.

Diagnosis.-Vomiting of pregnancy; pleural effusion. 


\section{Case 342}

A Russian tailor of thirty-seven entered the hospital April 28, 1908 . He has found himself unable to work for several months, because whenever he tries to move about at all he vomits. This vomiting is accompanied by shortness of breath and palpitation. He has also a slight cough, anorexia, constipation, and insomnia. He has noticed no edema.

On examination, the right pupil is slightly larger than the left. The heart's apex is in the fifth space, one inch outside the left nipple. At the apex there is a murmur which lasts through the whole of diastole with a crescendo toward its end. The first apex sound is very sharp, and preceded by a palpable thrill. The pulmonic second sound is not accentuated. There are crackles and squeaks scattered throughout both lungs, especially at the base behind, where there are slight dulness and diminished fremitus.

In the abdomen there is a little dulness laterally when the patient is lying down, this dulness shifting with change of position.

Physical examination, including the blood and urine, is otherwise negative.

Discussion.-Attention should be arrested by an unusual combination of symptoms present in this case-viz., vomiting, excited, apparently, by exertion. This is a very significant grouping of facts, and may be interpreted to mean either that vomiting is dependent upon some circulatory disturbance which exertion increases, or upon the shifting of the position of some viscus when he assumes the upright position.

Since the vomiting is associated with other symptoms suggesting disturbance of the circulation and the physical examination reveals a cause for this disturbance, we must ask whether mitral stenosis, which is apparently the lesion present, is capable of producing such persistent vomiting, even when compensation is not very seriously disturbed. Experience shows that we may answer this question in the affirmative, although, as a rule, troublesome gastro-intestinal symptoms do not occur until rather later in the course of an uncompensated cardiac lesion.

Since physical examination gives us no hint of any other cause for the vomiting, it is proper to treat the patient with reference purely to his circulatory disturbance, and to anticipate that he will stop vomiting when his compensation is improved.

Outcome.-The patient was kept in bed, given an ounce of magne- 
sium sulphate each morning, and a bitter tonic before meals. Occasionally he needed to grains of trional to induce sleep, but no cardiac stimulation seemed called for. After a week's rest he was in good condition and had no symptoms as long as he did not exert himself violently.

Diagnosis.-Mitral stenosis.

\section{Case 343}

A Swedish tailor of fifty, with a good family history and past history. entered the hospital June I2, I907.

About February I, 1907, he began to have abdominal pain and vomiting. At first his pain was relieved by belching sour gas, but it gradually became more severe, especially after eating meat or heavy food, less after milk or crackers.

For the past two months the pain comes three or four hours after eating, and is very severe and grinding, passing from right to left across the epigastrium, and relieved by vomiting, which is apt to occur once or twice every two or three days, usually after a siege of pain. He has often seen in the vomitus food eaten a day or two before. Usually the vomitus contains the unchanged food and bile-stained mucus, never more than a pint at a time, never dark or bloody.

He has constantly lost weight, strength, and color. In February he weighed $\mathrm{I}_{52}$ pounds, now he weighs $\mathrm{I} 3 \mathrm{I}$. He is constipated; sleeps well. He gets up two or three times at night to pass water. His appetite is excellent. He worked until two days ago.

Examination showed emaciation, pallor, negative chest and abdomen.

White cells, 5000; hemoglobin, 75 per cent.; urine, normal. The stomach held 70 ounces of water; after a test-meal, free $\mathrm{HCl}$ was absent on two occasions. Total acidity, O.I2 and 0.2. Stools altogether normal. The fasting stomach contained considerable food remnants.

After a few days in the hospital on a dry diet divided into six meals daily, $\mathrm{HCl}, 20$ minims after meals, and 2 drams of Carlsbad salts each morning, the symptoms disappeared.

There was at no time any visible peristalsis.

Two days after leaving the hospital the pain recurred and a profuse diarrhea began.

On July 24th, I907, he reëntered the hospital, complaining that he had had since his last entry five or six attacks similar to that just described, beginning with severe abdominal pain followed by vomiting and diarrhea. Most of these attacks have followed some indiscretion in diet. As in the previous illness, he has noticed in what he vomits 
food eaten a day or more before. The appetite is good, bowels con. stipated. He sleeps well except during the attacks of pain.

The abdomen now shows marked rigidity and tenderness in the right lower quadrant, but no masses are made out. The hemoglobin has now fallen to 50 per cent.

Discussion.-Gastric cancer is strongly suggested by this history. Here is a man who has lived on peaceful terms with his stomach for forty-nine years and then, without any discoverable cause, begins to have trouble with it. The regular recurrence of pain three or four hours after eating is, however, less characteristic of cancer than of ulcer and some other diseases. Quite obviously we have stasis, emaciation, and anemia, presumably of the secondary type. These facts, together with the absence of hydrochloric acid, still further support the hypothesis of cancer, especially as no hemorrhage or other cause for the anemia is manifest.

Though we were much inclined toward the diagnosis of gastric cancer, the disappearance of symptoms after a few days' stay in the hospital made us more doubtful. Marked improvement does, however, occur in cases of undoubted gastric cancer, and I have often known a false conclusion based upon such improvement. Doubtless the secondary irritation of the gastric mucous membrane resulting from the stasis is itself the cause of many of the symptoms in gastric cancer.. When this stasis is relieved by diet and lavage, the symptoms improve, though the cancer does not.

By the time of his second entrance to the hospital we had begun to notice certain anomalies in the clinical picture, which made it difficult to identify it with that of gastric cancer. First of all, it was notable that the pain passed across the epigastrium from right to left-i.e., against the current of the gastric contents. This is unusual in pyloric obstruction. Next, we were forced to observe that the pain had not the steady, grinding character usually seen in the more painful types of gastric cancer, nor was it confined to that indefinite distress and sense of weight which characterizes the less painful types of the disease. This man's pain came in distinct paroxysms of great severity, separated by intervals of complete freedom. This is more suggestive of intestinal than of gastric cancer. The profuse diarrhea following his last stay in the hospital, and the constipation which has been a feature of his case throughout, fall in very well with this idea.

If we are dealing with chronic intestinal obstruction, as this last interpretation of the symptoms seems to indicate, there is reason to believe that cancer is its cause, both because the great majority of 
such cases occurring in men of this age turn out to be cancerous and because the anemia is not otherwise explicable.

Outcome.-Subsequently a hard mass with indefinite outline was made out on the right side of the abdomen, at the level of the navel.

Rectal examination was negative, but the stools now exhibited a marked reaction to guaiac. After a test-meal the stomach-contents showed free $\mathrm{HCl}$, $0.0 \mathrm{I} 8$.

Leukocytes, 4100, among which 59 per cent. were polynuclear, 30 per cent. lymphocytes, II per cent. eosinophiles.

Operation on the twenty-seventh showed cancer of the ascending colon.

Diagnosis.-Cancer of the ascending colon.

\section{Case 344}

A married woman of fifty years was first seen in September, 1903, for major hysteria. After this she passed out of my ken and I did not see her again until December 8, I908. During this time she had gor into the habit of taking brandy at the rate of about a quart a week at irregular intervals; also a spray of cocain, which she used two or three times a day for most of the time. Besides this, she had a prescription for amylene hydrate, originally given her by Brown-Séquard, and continued by her at intervals ever since.

She has been more or less hors de combat, owing to the influence of hysteria and drugs, for a considerable portion of the time. Two and a half weeks ago she got overtired with shopping and the theater. Within a day or two she began to have persistent vomiting, headache, and great irritability, which at times amounted almost to delirium, and was accompanied by suicidal impulses. This continued until within the present week, when she became very quiet and drowsy most of the time, but continued to vomit. As far as can be estimated, she has consumed about a quart of brandy in the last three days.

For the past four days it has been noticed that the amount of urine has considerably diminished. Yesterday afternoon it was examined and found to contain 0.5 per cent. of albumin. It was smoky in color, and in the sediment were a great many casts, chiefly fine granular and brown granular, with a smaller number of the hyaline and cellular types. It was subsequently learned that she had had albumin in the urine four years previously, but its other characteristics were not known.

For the past twelve hours she had been semiconscious, and as we went upstairs the attending physician said to me that the case seemed to him very grave; he doubted somewhat whether she could be roused. 
We stepped to the bedside. "Mrs. D.," said he, "here is Dr. Cabot. Do you remember seeing him some years ago?" "Yes," said she, "and a bigger fool I never knew." All of which seemed to argue that she was not so badly off after all; yet she soon slipped off again into a semiunconscious condition, in which the pupils reacted very sluggishly.

On physical examination there was decided muscular negativism. The heart and lungs showed nothing abnormal; the blood-pressure was not high; the abdomen was negative. There was no muscular paralysis, but the knee-jerks could not be obtained, and there was an extraordinary degree of flabbiness in the calf muscles-almost no muscle left. Elsewhere nutrition was fair. Blood examination showed nothing wrong in that direction.

Discussion.-As there was no muscular tremor and no suggestion of alacrity in the psychic responses, there seemed no good reason to anticipate delirium tremens.

Uremia was seriously considered, and could not be excluded. The points against it were the low blood-pressure and the muscular relaxation, also the emphatic statement by the husband that she had often "been as bad as this before," but had always come out of it all right when alcohol and drugs were taken away.

On the other hand, it seemed difficult to account for the bad condition of the urine as a result simply of alcoholism and cocain. On the whole, I was inclined to think her uremic and to give a bad prognosis.

Outcome.-Alcohol and drugs were stopped, and she was kept on a diet of milk and water for twenty-four hours, after which she rebelled and nibbled a considerable quantity of various objects. Within a week the urine had cleared up and the patient was decidedly active and bad tempered, but insisted on running her automobile, which she drove herself. So far as could be ascertained, the attack had passed off without leaving her any worse than before, and without producing any permanent damage in her internal viscera.

The case taught me a lesson not unfamiliar to those who deal with acute alcoholism and drug-poisoning, viz., that there is almost no limit to the amount of albumin and casts which may be excreted in the urine of patients during an acute attack of narcotic poisoning, without leaving any evidence of permanent damage to the kidneys after the attack passes off. One should attempt no judgment about the condition of the kidneys until we have been able to eliminate the present effect of alcohol and other narcotics.

Diagnosis.-Hysteria; alcoholism; drug habits. 


\section{Case 345}

Anne K., a laundress of forty-eight, entered the hospital March I4, 1909. She has always been well and has an excellent family history. She passed the menopause a year ago. On the morning of Christmas, I908, after a hard day's work preceding, she vomited "half a washbowl of phlegm" when she first got up. She noticed no food or blood in the vomitus. She breakfasted and worked as usual that day. Through January and February, I909, she considered herself perfectly well, though her bowels had been more than usually constipated, and when once started by catharsis. there had often been a slight diarrhea for several days.

Three weeks ago she took a dose of salts one morning for one of her usual spells of constipation and immediately began to vomit bile-stained phlegm. Her vomiting continued all day, though she kept at work. She spent the next three days in bed and continued to vomit after eating. She then went to a friend's house for a rest. There the vomiting soon ceased, and in five days she was able to resume work.

A week later, March 8, I909, vomiting recurred for the third time. Nevertheless she kept at work until March I2th, the day previous to her entering the hospital. The vomitus has always been small in amount and apparently free from blood or food residue. She says she has had no pain at any time except a "griping" after taking a cathartic.

Appetite poor, sleep excellent, weight (a year ago) I50 pounds. Her mistress says that she has always been reticent and uncomplaining.

On examination her weight was found to be $\mathrm{r}_{5} \mathrm{I}$ pounds, and she seemed distinctly obese, though it was also noted by one of the consultants that "her flesh hung on her body with wrinkles and folds," suggesting that she had previously been heavier.

Physical examination, including the blood and urine, was wholly negative, save for a very slight degree of abdominal distention. After the bowels had been started by a glycerin enema of 4 ounces on March I 3 th. and a high oil enema of 6 ounces March I 5 th, they continued to move normally. The stools were not remarkable either in number or appearance, and showed a negative guaiac reaction March I6th, I 7 th, and 20th. After the I8th she was able to take milk and crackers without any vomiting or distress. March 29th she was taking a full diet without any symptoms, and April ist she was discharged well.

Discussion.-The diagnosis of gastric neurosis and constipation was made in this case. The basis for this was chiefly the negative physical examination, and the prompt recovery of gastric and intestinal function under rest and diet, with enemata. 
We were, therefore, quite astonished and somewhat amused when Dr. E. A. Codman, who saw her March I8th, made a diagnosis of chronic intestinal obstruction, probably due to cancer. Yet the outcome showed that Dr. Codman was in all probability right. The facts which guided him (and should have guided us) to this diagnosis were (a) the presence of slight abdominal distention on March I8th after five days of partial starvation and free catharsis; $(b)$ the occurrence of vomiting in a middleaged woman who had been previously well and had no special cause for a nervous breakdown; $(c)$ the history of periods of constipation alternating with diarrhea; $(d)$ the griping pain which, though attributed by the patient to the action of cathartics, did not seem to Dr. Codman explainable thereby.

Outcome.-After leaving the hospital April Ist, she stayed at a friend's house for three weeks; she then returned to her work as a laundress and seemed perfectly well through May and June. I inquired after her this time and noted, with great satisfaction, her continued good health, confirming, as I thought, my diagnosis of gastric neurosis. In July she had her fourth attack, which came on this time with great suddenness. She was seen at once by a physician who found distention and vomiting, but under his treatment she seemed well again within a few days and resumed work. A few weeks later her mistress happened to go into the laundry and found that Anne had a fifth recurrence of her old trouble-vomiting. Another vacation was advised, but after three weeks' rest at a friend's house her symptoms returned in great force. In this, the sixth attack, the vomiting finally became fecal and there was intense abdominal pain and rapid emaciation. Dr. John T. Bottomley saw her in consultation. In answer to my inquiry he writes as follows:

"She presented a picture of the terminal stage of an intestinal obstruction; distended abdomen, anxious face, fecal vomiting, empty rectum, etc. A mass was indistinctly felt in the upper abdomen and was believed to be malignant."

It seemed too late to operate and the patient died soon after.

Diagnosis.-Chronic intestinal obstruction, probably due to malignant disease.

\section{Case 346}

Called at noon February Io, I9ro, to see a Syrian housewife of twenty-nine (childless) whose groans and cries reached me in the street as I came to the house. I found her writhing in intense pain, referred to the epigastrium, and accompanied by so much retching that I could hardly get her history between the spasms. 
Six days previously she had begun to vomit without obvious causethe act being immediately preceded by epigastric pain and relieved by emesis. The early morning was her worst time in this respect.

Until this morning she had slept well and had suffered no pain between the vomiting spells, but at 5 A. $\mathbf{~ . ~}$. to-day, after a sleepless night, the pain began again and her vomiting no longer relieves it.

Her bowels moved after an enema yesterday, and again this morning.

I examined her as well as I could between her spasms of writhing and vomiting. She was a stout, flushed, powerful woman. No percussion dulness, no tenderness or muscular spasm, no palpable mass could be felt through the belly-walls by rectum or by vagina. The temperature was $97.6^{\circ} \mathrm{F}$., the pulse roo, the respiration 30 . The vomitus consisted of mucus and bile-stained, watery fluid. The urine contained no albumin or sugar. The blood was negative.

The woman's sufferings seemed as intense as those of acute perforative peritonitis, yet I could find no objective evidence of that disease. The pain was such as I have seen in gall-stone disease, yet until this morning it had been merely an accompaniment of the vomiting-and relieved by it.

All the complaints pointed to an abdominal emergency of some kind, yet I could find none on physical examination.

Discussion.-I felt very much puzzled by the case. The loudness and vigor of her cries and her healthy appearance inclined me to believe that she was free from any serious disease. On the other hand, the attending physician had evidently felt much alarmed about her. When he had previously telephoned me and found that it would be an hour before I could get there, he said he was not sure she would live so long. In the hullabaloo going on in the room it was very difficult to think, but as I made the effort, my eye roved about the room and fell upon the center table, which contained a huge Turkish hookah, two boxes of cigarettes, and a hypodermic syringe. The last was the clue that I wanted.

Inquiry showed that it belonged to the doctor, and that he had given morphin once or twice daily for the last five days. As soon as I came to look at the patient with this in mind I saw that her outcries, tossing, and thrashing about the bed, and her vehement, though inchoate desire for something, she knew not what, were very characteristic of what I had so often seen in morphin habitués deprived of their drug. Inquiry showed that she was not a habitué, but had acquired a strong need for the drug in five days.

Outcome.-The subsequent course of the case showed that there was nothing else the matter with her; her original attack of vomiting 
was brought on by overeating and constipation. It had been intensified and prolonged by the unwise use of morphin in a neurasthenic. After the evacuation of a large fecal accumulation, the withdrawal of the morphin, and a sensible diet, she recovered completely, though with a good many relapses.

Diagnosis.-Neurosis; morphin.

TABLE XVIII.-VoMITING. Signs and Symptoms.

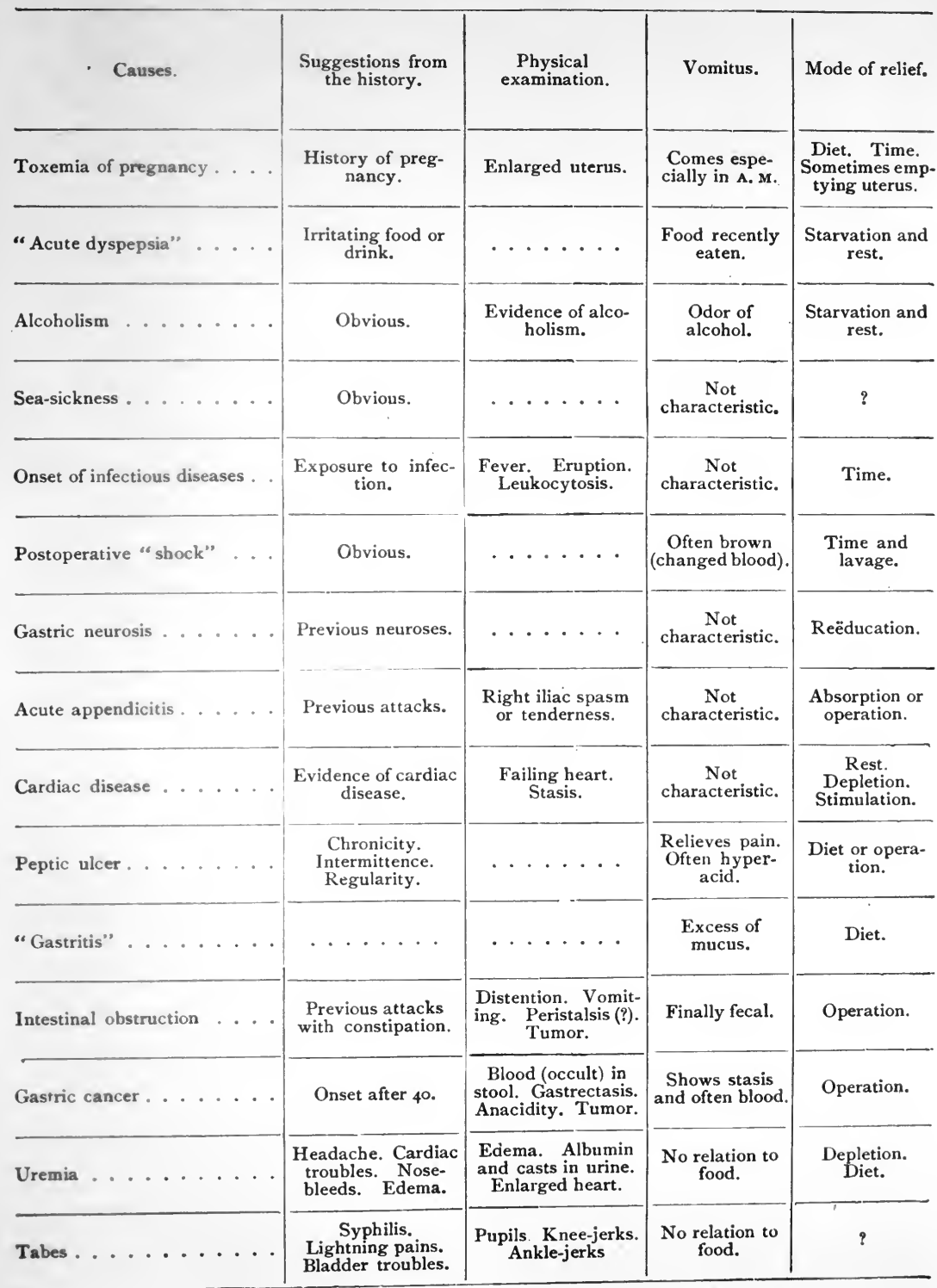




\section{CHAPTER XX}

\section{HEMATURIA}

THE term is applied only when blood is visible in the urine by the unaided eye. It does not apply to cases in which the microscope alone enables us to detect red corpuscles in the urine. It is also distinguished from hemoglobinuria, a rather rare condition, in which the urine contain free hemoglobin, but no corpuscles.

It is important to make sure, before deciding that we are dealing with a case of hematuria, that all admixture of vaginal discharges is avoided.

\section{CAUSES AND TYPES OF HEMATURIA}

Since a very large proportion of the varieties of hematuria are not such as lend themselves to the method of differential diagnosis by case analysis which I have used throughout this book, I shall not be able to exemplify them all by discussable cases presenting diagnostic difficulties. I shall, therefore, mention them in more than usual detail in this introductory section.

It is convenient to divide the cases of hematuria into those due to:

I. Trauma.

2. Nephritis, acute or chronic.

3. Renal disease (other than nephritis).

4. Vesical disease.

5. Hemorrhagic diseases and diseases of the blood.

6. Poisons.

7. General infections.

Traumatic hematuria is most frequently seen as the result of severe crushing accidents, as, for example, when the body has been run over by a heavy wagon. It also occurs as the result of injuries in the perineal region and after instrumentation. It rarely presents any diagnostic difficulties.

In nephritis, hematuria frequently occurs as part of an acute process without leading to any puzzles in its interpretation. In chronic nephritis it may appear out of a clear sky when the underlying disease is quite latent, and under these conditions mistakes of diagnosis often occur. 


\section{Causes of Hematuria}

\section{ACUTE NEPHRITIS'}

2. CHRONIC NEPHRITIS

\section{RENAL CALCU-
LUS}

\section{TUMOR OF THE BLAD-
DER}

5. TUBERCULO-

SIS OF KID-
NEY

\section{TUMOR OF KIDNEY}

9. UNKNOWN

CAUSE

1 The special limitations of my material make it impossible for me to estimate accurately the proportion of cases in which hematuria is a feature of acute nephritis, but I believe it to be the commonest type of bleeding from the urinary tract. 

The best methods of guarding against them will be discussed in connection with some of the cases presented in the latter portion of this chapter.

Excluding nephritis, we have left a group of diseases of the kidney ordinarily termed "surgical," and including-(a) The irritations produced by oxaluria, with or without gravel, or by stone; $(b)$ renal tuberculosis; (c) renal neoplasm; (d) cystic kidney; $(e)$ unknown cause. All these will be exemplified later, and, accordingly, are not further discussed here.

Vesical hematuria may be due to-(a) Cystitis of unknown origin; (b) stone in the bladder; (c) tumors, benign or malignant; (d) acute prostatitis, prostatic cancer, and prostatic hypertrophy; $(e)$ tuberculosis of the bladder; $(f)$ the sudden and complete emptying of an overdistended bladder, as in cases of acute retention; $(g)$ bilharzia disease, which is seen only in tropical climates or in patients recently returned from such a climate. Most of these will be discussed later on in this chapter. Bilharzia disease is recognizable by finding the eggs of the parasites in the urine. When once seen, they are easily remembered.

The vesical forms of hematuria are generally distinguishable from the other types above mentioned because other vesical symptoms, such as urinary frequency, tenesmus, or pain, are associated with the hematuria. This rule, however, is by no means invariable, since renal tuberculosis may produce marked vesical symptoms, while, on the other hand, bladder tumors may remain wholly latent save for the occasional presence of blood in the urine. Prostatic cancer in its early stages produces no symptoms that compel the patient to consult a doctor, only slight frequency or irritation with micturition, such as is discounted and easily borne by elderly men. Hematuria is a late symptom and occurs in only about 14 per cent. of cases. Expert rectal touch will detect the unusual hardness, nodular surface, or fixity of the prostate, but when neuralgic pains in the pelvis, back, or legs make it clear to the patient that he must have medical assistance, the disease is usually too advanced for any comparative help.

In the hemorrhagic diseases, such as hemophilia, scurvy, and the various types of purpura, blood may appear in the urine as well as elsewhere. The diagnosis of the underlying condition is usually obvious, and gives us a clue to the explanation of the hematuria. The same is true of the hematurias occasionally seen in connection with leukemia or pernicious anemia.

A large number of poisons occasionally produce bloody urine, but among the drugs which are often used at the present time there are 
very few that lead to this accident. Cantharides, turpentine, and phosphorus are not often used to-day in such doses as are capable of producing hematuria. In factories where coloring-matters, especially fuchsin, are manufactured, gases are evolved which not infrequently produce a severe hematuria, but obviously not many of us are likely to encounter cases of this origin.

In the hemorrhagic forms of various infectious diseases such as typhoid, typhus, small-pox, yellow fever, and septicemia, blood is occasionally discharged with the urine, but its origin is usually clear enough.

\section{Case 347}

A housemaid of twenty-five, whose mother and one uncle died of consumption, entered the hospital December 23, 1907. She has had bladder trouble for eight weeks. She was in the Boston City Hospital three years ago. Three months after this time she passed a tumbler of clotted blood, but was well in a few days. The same thing occurred again in three months. Nine months ago she again passed liquid and clotted blood for three days, but recovered without treatment. Her last attack was about four weeks ago, and, like the nthers, was over in three days. The attacks have no relation to the time of menstruation. Two days ago she passed a small clot and some bloody urine. After that her urine was normai for twenty-four hours. Yesterday afternoon hemorrhage was again profuse. In these attacks she occasionally has a little pain during or at the end of micturition. There is no frequency of micturition, but she passes urine twice in the course of each night. In all other respects she feels perfectly well.

Her physical examination is negative except for some tenderness in the lower part of the abdomen, especially on the left side. The leukocytes are 8000; hemoglobin, 75 per cent. The urine is not remarkable, except for the presence of a large amount of blood. An $x$-ray of the bladder and kidneys showed no evidence of the presence of stone. Cystoscopy showed that the blood was coming from the right kidney. Catheterization of the ureters gave clear urine from the left, nothing from the right. There was evidence of ulceration here and there in the left upper portion of the bladder. Rectal examination showed tenderness in the region of the right ureter and in the region of the ulceration on the left side of the bladder.

Discussion.-The essential feature of this case is the intermittent hematuria persisting for three years in a woman of twenty-five with long intervals between the attacks and perfect health in the intervals. 
By the study of the urine we obtained no evidence of nephritis, genito-urinary tuberculosis, or lithiasis. There are no bladder symptoms pointing toward tumor, benign or malignant, nor toward any type of cystitis. The $x$-ray evidence supports us in excluding stone.

Under these conditions cystoscopy is obviously indicated. By the combination of cystoscopy and rectal examination we obtained the following additional information: that the blood comes from the right kidney, that the bladder is ulcerated, that the ureter is tender, and probably stenosed. These facts, together with the family history, point distinctly to tuberculosis of the kidney and bladder. There is no other common lesion which produces unilateral renal hematuria with tenderness, partial obstruction of the ureter, and ulceration of the bladder-wall.

In view of this evidence it is not necessary to delay the beginning of treatment until a guinea-pig can be inoculated with the urinary sediment and allowed to "ripen" six weeks. This procedure should, nevertheless, be carried out at once.

Malignant disease of the kidney, especially hypernephroma, has been known to produce hematuria lasting over a period of three years. But with hypernephroma we should not expect ulceration of the bladder or tenderness of the ureter, and after the lapse of three years we should expect local tumor, metastases, and constitutional disturbance.

It should be noted how very good this patient's general condition appears to be, despite the apparently long duration of the disease. In this connection we may remember that in the kidney, as in the lung, tuberculosis may pursue an entirely "silent" and symptomless course. I have recently seen the results of such a tuberculosis at autopsy, although the patient had never had any symptoms remotely suggesting any malady of the genito-urinary tract.

Outcome.-December 28 th the right kidney was cut down upon, found to be enlarged and cystic, the ureter full, enlarged, and in places constricted. It was removed together with the kidney.

The histologic examination of the kidney showed tuberculosis, with complete blocking of the ureter near the kidney.

The patient made an uneventful recovery.

Before the operation a specimen of urine removed by catheter (December r8th) was injected into a guinea-pig. Autopsy, January I 7 th, showed tuberculosis of the animal, and tubercle bacilli were recovered in cover-glass examination.

Diagnosis.- Tuberculosis of the kidney and bladder. 


\section{Case 348}

An Irish shop-clerk of twenty-eight entered the hospital March 28, I908. One brother and one sister died of phthisis. For two years he has noticed frequent micturition. Two months ago, for periods lasting about eight days at a time, he passed bloody urine with clots. There was some pain during micturition. Ten days ago this was repeated twice. There have been no other symptoms. He feels perfectly well.

Physical examination was negative, except for some tenderness on deep pressure in the right flank. On the night when he was first seen he passed some urine entirely free from blood. The next morning the urine was excessively bloody. $X$-ray of the kidneys was entirely negative.

The urine drawn by catheter showed the following characteristics: Amount in twenty-four hours, 26 ounces; color, bloody; specific gravity, I023; albumin, very slight trace; sediment, considerable blood, normal and abnormal. Some pus, no casts. Few acid-fast bacilli.

Cystoscopic examination showed on the anterior wall of the bladder a single small, shallow ulcer surrounded by a reddened area.

Discussion.-The age of the patient and the duration of the case are against malignant disease of the kidney or bladder. The $x$-ray evidence tends to exclude stone. Single circumscribed ulcers of the bladder are not infrequently due to tuberculosis, and the family history supports this idea. Further certainty cannot be obtained without a further study of the urinary sediment for confirmation of the finding of acid-fast bacilli. A guinea-pig inoculated subcutaneously with 35 c.c. of urine on the second of April was killed May IIth, and showed tuberculosis of the lymph-glands, spleen, and liver, from which tubercle bacilli were recovered. There was no evidence of renal disease.

Outcome.-In May; 1908, an ulcer of the bladder-wall, apparently tubercular, was excised. May II, I9Io, the patient's physician writes: "I am more than pleased to let you know that Mr. F. has been feeling very well since his operation. He works every day."

Though positive evidence relates only to the bladder here, it is more than possible that the kidney was also involved.

Diagnosis.-Tuberculosis of the bladder. Renal tuberculosis? 



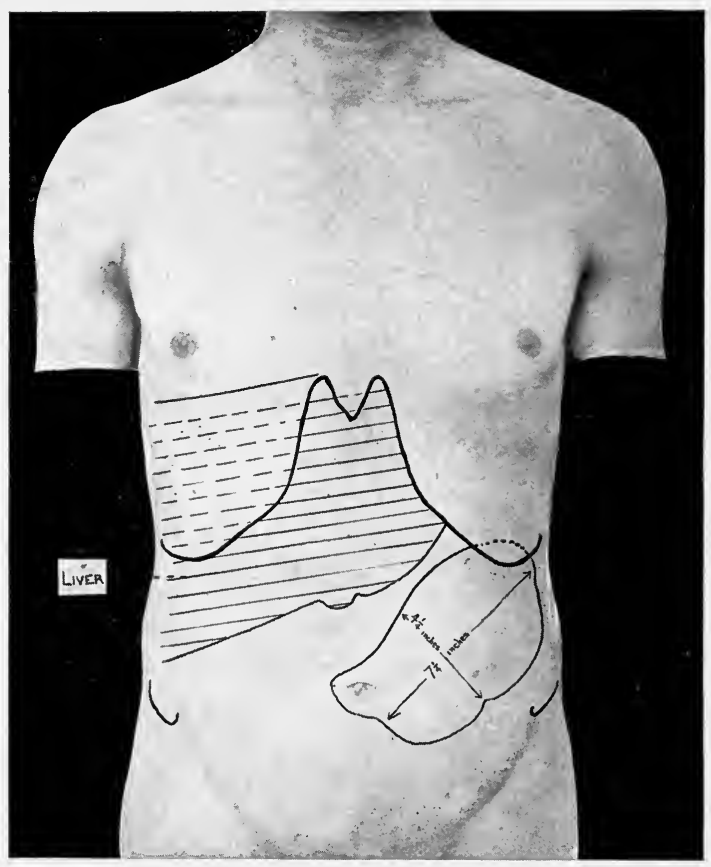

Fig. I80.-Outline plotted by palpation in a case of intermittent hematuria of a week's duration. 


\section{Case 349}

A child of four years was first seen June 29, I908. In the previous July he fell off a ladder, after which he was poorly and was thought to have malaria. Six weeks ago he fell off an express wagon, striking on his left side in the lumbar region. Soon after this he began to pass bloody urine, and within the last six weeks he has had three such attacks, lasting each a couple of days; and soon after his fall, six weeks ago, a lump was noticed on the left side of the abdomen. It has rapidly increased in size since that time. The presence of this lump, together with the fact that he has fever every other day, has led to a diagnosis of malaria. He comes from a very malarious village. He has been rapidly losing weight for the past six weeks.

On physical examination the child shows no anemia and no malarial parasites, but is poorly nourished. Examination of the head, neck, and chest reveals nothing abnormal. The left upper portion of the abdomen is prominent, and contains a large, firm, irregular mass, distending on inspiration. (See Fig. 180.) The air-distended colon passes in front of the tumor. The edge of the liver is felt two inches below the costal margin, in the nipple-line.

Discussion.- The time of year at which this illness occurs, the fever, the age of the child, and the presence of the tumor which may represent enlargement of the spleen, naturally suggest malaria, which was, indeed, the diagnosis at the time when the case was first seen. Malaria of the severer type may be associated with something resembling hematuria,-i. e., with hemoglobinuria,--but there is no blood and no true hematuria, only free hemoglobin discoloring the urine. We must have some other explanation, therefore, for the bloody urine in this case, especially as no malarial parasites were found, despite careful search.

Leukemia might give us a similar clinical picture, and is not infrequently complicated by hematuria. That disease, however, could be excluded in the present case because the stained blood-smear was quite free from any suggestion of leukemia. It is true that leukemia may for a short time exist without a typical blood-picture; under treatment with arsenic or $x$-ray the blood may return altogether to normal, and the same change has been known to occur as the result of a complicating infection (pneumonia, erysipelas, etc.), or even without known cause. All these possibilities, however, are extraordinarily rare, and we have no good reason to consider them here.

Large abdominal tumors occurring in young children are not common. If bilateral, they are usually due to congenital cystic kidneys; if unilateral, 
some form of renal neoplasm is usually the diagnosis. The other possibilities are very few. In this child there is no reasonable doubt that we are dealing with a new-growth of the left kidney. The hematuria, the tumor, the poor nutrition, and the fever are the usual features of such a disease. Renal tuberculosis is almost unknown in a child so young, and would not have produced a tumor of this size without manifesting itself in the urine by the presence of pus and probably by vesical irritation.

Malignant disease of the kidney may be difficult or impossible of diagnosis in its earlier stages, before the tumor is large enough to be palpable. A metastasis in some distant organ, usually in some bone, may be the first hint of the renal neoplasm. In other cases we have long-standing, intermittent hematuria, such as we have already exemplified in genito-urinary tuberculosis. This is unfortunate, because early diagnosis and early operation give the only hope of cure. When the tumor is well marked, as in the present case, the diagnosis is not usually difficult.

Outcome.-The child died July I9, I908. There was no autopsy. Diagnosis.-Renal neoplasm (?).

\section{Case 350}

A married woman of fifty-two, a shoe stitcher by profession, was first in the hospital in 1905 for acute nephritis, recovering at the end of a month. Although there were still a very slight trace of albumin and a few fine granular casts in the urine at the end of her treatment, she felt perfectly well and showed no cardiac enlargement.

She was next seen November 18, 1907, complaining of hematuria. She has worked without a vacation since her last illness, though last spring she fainted several times while working. Since last May she has had much dyspnea on exertion, and has felt very tired most of the time, but has not again fainted. A week ago the sight of her left eye began to blur. She says that she had similar trouble with her right eye twelve years ago. She has noticed a slight swelling of her ankles off and on during the last three months. Her appetite has been poor, and her weight has fallen from 187 pounds last May to Ir9 pounds at the present time.

For the past week she has noticed bloody urine and has had cough and pain in the chest. She has had no headache or vomiting.

On examination the temperature, pulse, and respiration were normal. The apex of the heart was in the fifth interspace, anterior axillary line, the action slightly irregular, with a tendency to gallop rhythm. There 
was a soft systolic murmur at the apex, transmitted to the axilla, and the pulmonic second sound was accentuated.

The blood-pressure was I $75 \mathrm{~mm}$. Hg. $X$-ray of kidneys and bladder negative. The blood was negative.

There were many coarse and medium crackling râles scattered over both chests. Otherwise they were negative. There was no edema. The abdomen and extremities were apparently normal. The urine was smoky, IOII to IOI4 in specific gravity; the twenty-four-hour amount, from 40 to 50 ounces, with albumin from o.I per cent. to 0.9 per cent. In the sediment were enormous numbers of fresh red cells. At first there were no casts. Later on, moderate numbers of hyaline, fine and coarse granular casts appeared, some with cells adherent. The hematuria ceased in ten days. At no time was there any edema, headache, vomiting, or oliguria.

Discussion.-By reason of the symptoms alone a diagnosis of pulmonary tuberculosis had previously been made in this case. The cough, the chest pain, and the emaciation led to this mistake, which was easily rectified when the chart and chest were examined. At no time was there any fever or anything suggesting the usual signs of pulmonary tuberculosis in the lungs.

We had no evidence of the ordinary causes of hematuria, such as stone, tuberculosis, or tumor. There was nothing to call attention to the bladder or to any disease, primary or secondary, in its walls.

The high blood-pressure, the urine, and the condition of the heart are such as we expect to find in chronic nephritis of the glomerulonephritic or interstitial type, especially the former. But we do not ordinarily associate hematuria with chronic nephritis. It is in the acute cases that we expect blood. Nevertheless, it has been repeatedly pointed out, as a result of surgical experience within the past ten years, that the kidneys in chronic nephritis may bleed profusely without any evidence of an exacerbation of the renal disease itself. Why this occurs we have no idea, but a number of surgeons have proved the fact when searching the kidney for evidence of stone or other cause for the hematuria. Doubtless many of the cases of apparently causeless hematuria belong to this group.

Outcome.-The patient was fatigued on the slightest exertion, and was very slow in regaining her strength. Considerable improvement took place, however, in the course of her two months' stay in the hospital. Treatment consisted of diet, with an occasional bitter tonic.

Diagnosis.-Chronic nephritis. 


\section{Case 351}

A lady of seventy, of excellent family history and past history, entered the hospital June 25, 1907. She has had nocturia ( 1 to 2) for ten years. For about a year she has had to pass water frequently-i.e., about every two or three hours in the daytime, and seven or eight times at night. During most of this period she has frequently noticed the presence of fresh blood in her urine, together with small, blackish clots. The urine has never been foul, and there has been no pain or burning on micturition. There have been periods of a month or two during the past year when she was free from her present trouble, but for the last three months it has been constant. She has been gradually losing weight for a number of years, but for the past year her appetite has been very poor and emaciation has been rapid.

On physical examination the heart's apex was not seen or felt. The sounds were best heard in the normal situation. There were no murmurs or other abnormalities. The tension of the pulse was apparently increased, but the blood-pressure was only I 25. There were slight general abdominal tenderness and a small umbilical hernia.

Several examinations of the urins showed essentially the same conditions: Amount in twenty-four hours, 30 ounces; color, very dark brown; specific gravity, Ior6; albumin, 0.3 per cent.; bile and sugar absent; sediment, large amount of fresh blood with numerous round, mononuclear cells, somewhat larger than the erythrocytes. Occasional small macroscopic blood-clots.

Vaginal examination was entirely negative, as likewise was the $x$-ray of the kidneys and bladder. Cystoscopic examination showed ulcerated nodules in the wall of the bladder.

Discussion.-Without a cystoscopic examination no diagnosis is possible in a case of this kind. From her age, the urinary data, and the bladder symptoms, one might suspect bladder stone or malignant disease, but nephritis, genito-urinary tuberculosis, and renal tumor would also be possiblc.

Genito-urinary tuberculosis is very rare at this patient's age. The urine would probably contain more pus and less blood. Renal tumor would probably be palpable after a course as long as that indicated by this patient's history. The condition of the heart and arteries is not such as we expect in chronic nephritis. Acute nephritis is almost unknown at the age of seventy, especially in the absence of any infection or poisoning.

Even without a cystoscope, then, the diagnosis of some bladder 
trouble was rendered probable by the exclusion of renal disease. Bladder stone would probably produce more discomfort than this patient suffered. Cystitis is not likely to produce such long-continued bleeding. Tumor, therefore, seems most probable.

The ocular evidence through the cystoscope left no considerable doubt as to the nature of the case.

Diagnosis.-Cancer of the bladder.

\section{Case 352}

A blameless cook of fifty years entered the hospital January II, I907. She had previously been there in 1896 for "chronic cystitis," but of late years she has been very well. A year ago she fell down stairs, and although she kept about and on her feet after it, she became very much exhausted, had pain in her back, and had to be sent to the Boston City Hospital. Two days after arriving there she passed bloody urine. She went home convalescent six weeks later, and has since then been very well.

Last Sunday (five days ago) she walked two miles to see Mrs. Mary Baker Eddy's home. When she got back her urine was bloody, and she had much pain on micturition, with tenderness over the bladder and in the left lumbar region. She has been passing blood ever since. She is very nervous, and says thar her heart turns somersaults. She has been steadily at work since November Ist the previous year. For the past three years she has persevered in the habit of reading in bed until $20^{\circ}$ 'lock in the morning.

Physical examination shows an obviously cyanotic woman, with slight tenderness in the lower abdomen, but without any other evidences of disease except in the urine, which is bloody and shows in the sediment much fresh blood and triple phosphate crystals. No casts or pus. During her stay in the hospital the urine was approximately 45 ounces in every twenty-four hours, specific gravity from ıого to IоI3, alkaline in reaction, with a very slight trace of albumin. The colon bacillus was recovered in pure culture from the urine. $X$-ray of the kidney and bladder was negative.

The patient had numerous chills, not accompanied by any rise of temperature. The bladder was carefully sounded for stone, but none was found. She was given urotropin ro grains three times a day, abundance of water, and by the twenty-first of January the symptoms were rapidly clearing up.

Discussion.- Such bladder symptoms in a woman of this age are often due to renal tuberculosis, but the alkaline urine, without any 
considerable amount of pus, without fever or renal tumor, incline us to look elsewhere for the cause of the symptoms.

The low gravity urine and the trace of albumin, together with the age of the patient, lead us to consider chronic nephritis with one of the periodic hemorrhages already referred to as an occasional complication of that disease. Against this idea, however, is the absence of any cardiac enlargement and the fact that we can account for the small amount of albumin present as a result of the hematuria itself.

The increase of symptoms following a walk, even though it was a walk to the residence of the founder of Christian Science, suggests bladder stone and led to the careful investigation of the bladder by means of the sound. Since no stone was thus discovered, it did not seem necessary to use a cystoscope.

Bladder cancer or papilloma is possible, and should the symptoms recur a cystoscopic search will be indicated.

It seemed reasonable to try next the therapeutic test, based on the idea that we were dealing with a cystitis of unknown origin, and pending the results of animal inoculation with the urinary sediment.

Outcome.-The sediment of the urine was injected into a guineapig January $23 \mathrm{~d}$. When the animal was killed six weeks later no evidence of the tuberculosis was found. By this time the patient was convalescent, the urine almost normal. On the thirtieth of January she went home apparently well.

Diagnosis.-Cystitis due to the bacillus coli.

\section{Case 353}

A stationary fireman forty-nine years old was first seen on July 22, I909. One sister died of cancer of the stomach; his family history and past history are otherwise good.

Twelve years ago his urine began to be cloudy and occasionally contained blood. At this time there was also pain at the end of micturition on various occasions. He had acute retention nine years ago, and was operated on at the Boston City Hospital by Dr. Watson through a suprapubic incision. He believes that he had spasm of the neck of the bladder. After seven weeks in the hospital he was able to be at work, but his urine has ever since then been passed frequently and in small amounts.

At the present time there is no pain. He passes water about every two hours, and now and then there is blood in it. For the past six months he has had a poor appetite, and has frequently vomited during the day after drinking water. He has also had a great deal of sour 
stomach and belching. Within the past year he has lost 28 pounds, but he still weighs $\mathrm{r} 87$.

There was a systolic murmur at the apex of the heart, transmitted to the axilla, and associated with accentuation of the pulmonic second sound, but without cardiac enlargement. The abdomen showed nothing abnormal. The urine was very turbid, but not at this time bloody. The kidneys were not palpable. The cutaneous and subcutaneous tuberculin reaction was negative.

Cystoscopy showed a normal bladder. Thick pus was seen coming from the left ureter, while normal urine came from the right ureter. $X$-ray of the kidneys and bladder was negative.

Discussion.-The case looks like one of tuberculous kidney, and this disease cannot be positively excluded. In a case which has lasted so long we should expect a palpable kidney, but as we do not know with any accuracy the duration of the disease, this point is not of great importance. More significant is the absence of fever, and especially the absence of tuberculin reaction. The evidence against tuberculosis could be strengthened by repeated search for tubercle bacilli in the urinary sediment and by animal inoculation.

Hematogenous infections of the kidney would explain almost everything in the case except the hematuria, but so far as I am aware hematuria has not yet been reported in connection with this type of disease.

Stones in the kidney may be divided into the "silent" and the "obstreperous"; the largest branching stones are often entirely latent and symptomless, discovered first at autopsy or by the $x$-ray. Their size makes it very unlikely that they will be overlooked in an $x$-ray plate, such as was taken in the present case. On the other hand, the small stones, such as might be missed in $x$-ray examination, are very much more likely to cause pain. In this case we have no pain and no $x$-ray shadows. Stone may be, therefore, in all probability excluded.

What are the other possible causes of a unilateral renal pyuria, such as was here demonstrated by cystoscopy? I can think of but two:

(a) Pyonephrosis of unknown origin.

(b) Renal neoplasm.

Renal suppuration with a normal bladder and without tuberculosis is not common except as a result of hematogenous infection, which I have already discussed and excluded. Pyonephrosis of unknown origin is usually intermittent, like hydronephrosis, the material accumulating for a considerable period while a tumor gradually forms, then emptying with a gush into the bladder, with disappearance of the tumor. There is no history of anything of this kind in the present case. 
Renal neoplasms are not prone to suppurate. Nevertheless, we do find pus in varying amounts accompanying the hematuria or alternating with it. It is impossible, therefore, to exclude neoplasm in this case.

Outcome.-Operation August $3 \mathrm{~d}$ showed that the left kidney was converted into a pus-sac, which at the time was thought to be the result of tuberculosis. Under the microscope, however, the wall of the sac showed the structure of papillary cystadenoma. The kidney and ureter were removed. The recovery from operation was satisfactory, but the patient soon passed out of observation.

Diagnosis.-Papillary cystadenoma of the kidney.

\section{Case 354}

An iron-molder of forty-two entered the hospital April 6, r9o6. His uncle has recently developed bloody urine. His wife has cancer of the uterus. His past history is, nevertheless, uneventful.

One month ago he noticed that his urine was dark in color, and except for one day, it has been of this same tint ever since. There has been no pain at any time, and no other symptoms. No clots or gravel have been noticed. The color of the urine varies a good deal, but is never normal. Cystoscopic examination by Dr. Lincoln Davis showed considerable iniravesical enlargement of the prostate. The bladder was normal. From the right ureter came a jet of blood-tinged fluid, from the left, normal urine.

Except for a mild secondary anemia, physical examination was otherwise entirely negative. The blood, pulse, and temperature were normal. The urine averaged 35 ounces in twenty-four hours, with a specific gravity of I027 to IO3I, a large trace of albumin, but no sugar. The sediment was made up of normal blood and a few leukocytes. Newgrowth of the kidney was thought to be the most likely diagnosis.

$X$-rays of the kidney and bladder were negative. The sediment of the urine was repeatedly investigated for tubercle bacilli, with negative results.

On the eighth of April there was no blood in the urine, but on the tenth it was again present.

During these three days urotropin, Io grains, was given every eight hours, with abundant water.

Discussion.-As a result of the cystoscopic examination we know that the blood comes from the right kidney, not from the bladder. We have no evidence of stone or of tuberculosis in the kidney. The urine and the condition of the heart do not suggest nephritis. We find no calcium oxalate crystals or other source of local irritation. No drug 
or poison capable of inducing hematuria had been ingested. The patient has no form of hemorrhagic or infectious disease, no anemia or leukemia, no cachectic condition, such as might be complicated by renal bleeding.

When all these possibilities are excluded, as is often the case in the differential diagnosis of hematuria, two alternatives remain. We may be dealing with hematuria due to:

(a) Renal neoplasm.

(b) Unknown cause.

It has already been stated, in the discussion of a previous case, that diagnosis of renal new-growths is often impossible until the tumor has reached a considerable size or has produced metastases. There may be months or even years of latency with nothing but an occasional attack of hematuria, perhaps without even this. As no tumor can be felt in this case, we have no definite reason for the diagnosis of newgrowth, but we can by no means be sure of its absence except as the result of exploratory incision.

A very large number of hematurias-perhaps the majority of them all-are due to causes altogether unknown to us at the present time. After we have distinguished and excluded chronic nephritis as a cause of otherwise inexplicable hematuria, wa have left the bleedings due to minute varices or vessels in the renal pelvis. In many cases not even these slight lesions can be found when the kidney is opened at operation or at autopsy. Vague guesses like "vicarious menstruation," smart phrases like "renal epistaxis," do not help us, and for the present we are altogether in the dark regarding the cause of a large group of hematurias.

Outcome.-The kidney was cut down upon April I8th, and found to be entirely healthy both within and without. A month later the patient wrote that he had remained perfectly well since leaving the hospital.

Diagnosis.-Hematuria, cause unknown.

\section{Case 355}

A physician of forty, always previously well, was first seen August 26, 1908. About eleven that morning he noticed that his urine was bloody. The urine passed the night before was normal. He has been having a cold, with some cough and hoarseness for six days, and at the onset of this illness much headache and chilliness. At the present time the cold is practically gone and his urine is exceedingly clear.

Physical examination of the internal viscera was wholly negative.

$X$-ray of the kidneys and bladder showed no stones. The urinary sediment consisted of blood with many large calcium oxalate crystals. 
On the morning of the twenty-seventh the urine was normal in color. Later in the day it was again bloody, the amount of calcium oxalate varying directly with the amount of blood.

Discussion.-In the absence of fever, pyuria, and local bladder symptoms, tuberculosis seems here unlikely. A careful study of the urine and of the kidneys by $x$-ray showed no evidence of stone, nephritis, or vesical parasites (bilharzia disease). Cystoscopy was considered, but since there were no bladder symptoms or other definite indications to guide the search, it was postponed. Malignant disease of the kidney and tuberculosis were considered, but no concrete evidence could be found to support either idea.

We were much impressed with the close parallelism between the degree of hematuria and the amount of calcium oxalate present in the urine from hour to hour and from day to day. So close was this parallelism that it seemed wise to base treatment upon the idea that the calcium oxalate might be causing sufficient irritation to produce the hematuria by some means or other. Nevertheless, we felt by no means convinced that this hypothesis was correct, and looked forward with much interest to its confirmation or refutation through the outcome.

Outcome.-The patient was given a diet from which tomatoes, spinach, berries, cocoa, tea, and pepper were excluded. The carbohydrates of the diet were also moderately limited. Water was given in abundance; also 30 grains of sodium phosphate before each meal. By the twenty-ninth of August the bleeding had wholly ceased. Eighteen months later there had been no return of the symptoms.

Diagnosis.-Renal irritation from oxaluria.

\section{Case 356}

A man of forty-eight, who had had lead-poisoning four years before, entered the hospital January 21, I903.

Eight years ago he had an attack of pain in the region of the right kidney and thiniss he passed a small calculus. Two years ago he began to notice blood in his urine, and this was almost constant for six months. When it stopped for a day or two, he usually had pain over the right lumbar region, relieved when blood reappeared in the urine.

In the next six months hematuria came for about twenty-four hours every week or two. Between these attacks he had dull pain in the right renal region, relieved, as before, by bleeding. When bleeding occurred, he also noted pain at the end of the penis. He was now losing strength and had to give up work. Hematuria continued off and on until two months before he entered the hospital, when it ceased altogether. $\mathrm{He}$ has lost 50 pounds. 
Physical examination showed emaciation, anemia (hemoglobin, 6o per cent.). Temperature, $99^{\circ}$ to IOI.2 ${ }^{\circ} \mathrm{F}$.; chest and extremities normal; in the right hypochondrium a nodular, insensitive mass, extending $9 \mathrm{~cm}$. below the ribs, descending with deep inspiration. Urine bloody, otherwise normal; leukocytes, 6500.

Discussion.-The renal pain makes it altogether probable that the kidney, rather than the bladder, is the source of the hemorrhage. 'The presence of a palpable tumor in the renal region points strongly in the same direction. Tuberculosis, stone, and neoplasm are the chief possibilities.

Stone never produces a tumor having these characteristics; it may lead to a small accumulation of pus and cheesy material in the renal pelvis, but not to anything like the mass here described.

The emaciation, the fever, and the renal tumor are quite consistent with tuberculosis, but we expect pyuria, less profuse bleeding, and more bladder symptoms, such as urinary frequency, burning, and pain. Nevertheless, tuberculosis cannot be excluded without cystoscopy and animal inoculation.

The occurrence of pain relieved by bleeding is uistinctly suggestive of renal tumor, also the long duration of the bleeding without any check. Indeed, the most prolonged attacks of hematuria which we recognize clinically usually turn out to be due to renal neoplasm. In nephrolithiasis the bleeding is usually brief, and accompanies the pain, instead of relieving it.

Outcome.-Operation showed a hypernephroma which weighed I 500 grams, and measured I6 XI4 XI2 cm. This was successfully removed. Nine months later the patient wrote that he was steadily at work and had gained 40 pounds.

Diagnosis.-Hypernephroma.

\section{Case 357}

An Irish stableman of twenty-eight entered the hospital August I8, 1906. His family history and past history are negative.

Two months ago he noticed that his urine was bloody. He experiences a dull pain in his back, more or less constant. A considerable portion of the time since then, he has had pain in the epigastrium, increased by food, and has vomited frequently.

Physical examination of the chest and abdomen is negative. In the right back, just below the angle of the scapula, was a tumor the size of a small English walnut, freely movable beneath the skin, not tender, and was said by the patient to be due to a bullet which entered just below the right nipple in the front and lodged in his back. The patient 
showed evidence of marked anemia, the red cells being $3,300,000$, hemoglobin, 40 per cent., white cells, 9000. The stained specimen showed nothing remarkable except achromia. The urine contained a large amount of normal blood, but no casts. It was sufficient in amount, and not abnormal in any other respect. Tuberculin reaction (cutaneous) negative.

$X$-ray of the kidneys was negative. Through the cystoscope blood was seen spurting from the left ureter. There was no evidence of trouble in the bladder.

Discussion.-Left renal hematuria associated with well-marked secondary anemia occurring in a young man who complains of no bladder symptoms presents a clinical picture distinctly puzzling at the outset. We have done what we could to rule out stone, nephritis, calcium oxalate, and other toxic infectious and constitutional sources of hematuria.

Tuberculosis rarely produces so marked an anemia except in advanced cases with well-marked pyuria, fever, or tumor. Against tuberculosis we have the absence of the above signs and the absence of a tuberculin reaction.

Renal new-growth is always a danger threatening such patients$i$. e., patients with unexplained hematuria. The nodule in the right posterior thorax is not so situated as to correspond with any of the ordinary sites for metastasis from a renal or suprarenal tumor. There seems no good reason to doubt that the patient's idea about the origin of this nodule is correct. If the case be not one of renal tumor, we have no other plausible alternative to suggest. Very possibly it may be one of those cases of "idiopathic" bleeding discussed on p. 693. Further certainty can be obtained only by operation. As the patient does not seem to be improving and has a very considerable degree of anemia, exploratory incision seems justified.

Outcome.-In the pelvis of the left kidney there was found at operation one or two clots of blood. A section of the kidney was removed for examination and showed absolutely normal kidney tissue.

September 2d: The patient, who had made a good recovery from the operation, though he still continued to pass considerable amounts of fresh blood, began to complain of stiffness in his neck and jaw muscles, with pain, and was unable to open his mouth more than an inch. Later in the day he began to have convulsions, and died at 9 P. M.

Autopsy showed ulcer of the stomach, no cause for hematuria, and nothing else of importance.

The results of the autopsy indicate that the anemia was in all probability due, in part at least, to the gastric ulcer. Our attention had been 
completely diverted from this side of the case by the more spectacular symptoms, especially the hematuria. In the retrospect we say to ourselves for the hundredth time that a major operation should never be lightly undertaken.

Diagnosis.-Gastric ulcer. Hematuria; cause unknown.

\section{Case 358}

A Jewish schoolboy ten years old was seen October I5, 1908. He had measles when he was thirteen months old. Six weeks later he began to have incontinence of urine, which he has had ever since. The trouble is mostly nocturnal. This morning his mother saw in his bed some blood, which she thinks was passed during the night. The boy did not know it, but since that time has continued to pass exceedingly bloody urine, with clots.

Physical examination was entirely negative; the urine contained only blood with pus and large mononuclear cells. Catheter specimen remained sterile on culture-media. No tubercle bacilli found. Cystoscopic examination showed general reddening with areas of ulceration. No stone. The stream from each ureter was clear.

Discussion.-Sudden hematuria occurring in a boy of ten without previous evidence of cystitis is distinctly rare. Stone in the bladder was my first thought after going over the case. The renal causes of hematuria, such as have been discussed in the previous pages, are all of them very infrequent in children, with the single exception of renal new-growth, of which we had no evidence in this case.

The results of cystoscopy showed that we had no reason to suspect the kidney as the source of bleeding, and indicated that we were dealing with a cystitis of unknown origin. What may have been the duration of this cystitis we have no means of judging; it gave no signs of its presence until the day on which he was seen, unless, indeed, we reckon the eight years of nocturnal enuresis as such a sign. I see no good reason for considering the enuresis in this light.

Outcome.-The patient was given urotropin, 5 grains three times a day. October $25^{\text {th }}$ the urine was free from blood and the incontinence had almost ceased. By the first of November he seemed perfectly well and entirely able to control the flow of urine. This improvement was subsequently maintained. A guinea-pig inoculated with the urinary sediment was killed seven weeks later and showed no evidence of tuberculosis.

A point of considerable interest in this case is the sudden stoppage of a long-standing and obstinate enuresis. Can we suppose that the cystitis was of a chronic type and represented the cause of the incon- 
tinence? It does not seem probable, for at no time had the boy's symptoms differed from those of any other case of enuresis. He can hardly have had the cystitis since his thirteenth month.

Another possible explanation presents itself: May it not be that the instrumentation itself-the cystoscopy-cured the enuresis? It is a well-known fact that enuresis is prone to cease after an operation of any kind, presumably because the operation makes a strong impression upon the child's gray matter, both cerebral and spinal. In the present case the boy had no reason to suppose that the cystoscopy was done for the relief of enuresis. He knew that he was being treated for the hematuria, and that nothing was said about curing the other and older habit. Nevertheless, he may have drawn his own conclusions in his own way-we cannot tell.

Diagnosis.-Cystitis; enuresis. 


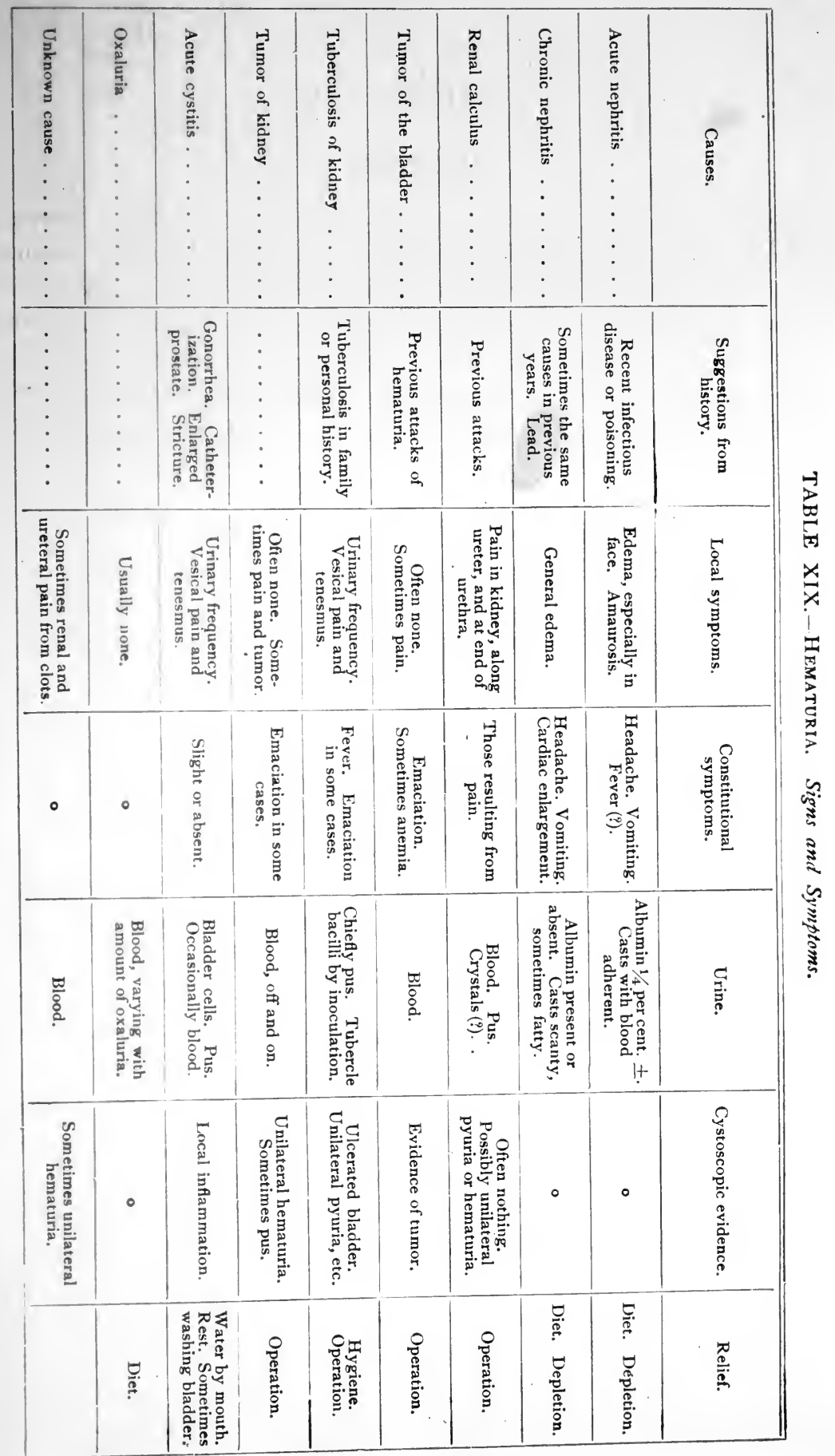




\section{CHAPTER XXI}

\section{DYSPNEA}

Ir is, on the whole, best to maintain the usual distinction between dyspnea and polypnea, and to use polypnea for a quickening of respiration without any evidence of effort or distress, objective or subjective. Such a polypnea is seen most often in infectious fevers, such as typhoid or septicemia, less often in the terminal coma of diabetes and in the other forms of coma due to acidosis, in the infections and gastrointestinal diseases of childhood, occasionally also in nephritis. In its extreme form it is to be witnessed in hysteria, which produces the most rapid breathing to be met with clinically.

Nevertheless, the distinction above explained is neither so sharp nor so logical as we might desire. When a healthy man begins to run, his breathing is first quickened,--polypnea,- -then, at the end of a variable period, it begins to be slightly difficult, until at last true dyspnea is reached. So it is in cases of failing cardiac compensation, and occasionally in general infections such as those above mentioned. In the early stages of the disease the breathing is quickened; later it becomes difficult as well. We might agree to say that dyspnea begins when the accessory muscles of respiration are called into play. But this is rather an arbitrary distinction. The movement of the alæ nasi begins in some patients before the breathing has become even distinctly quickened, yet this movement must, I suppose, be reckoned among those involving accessory muscles of respiration.

For these reasons it seems to me that we can maintain the distinction between dyspnea and polypnea only as one of convenience, and in an approximate sense.

Dyspnea and short breath are not always used as equivalents. Some patients puzzle us very much by complaining of short breath despite our inability to find any sign of disease on physical examination. More careful questioning sometimes brings out the fact that by short breath the patient means a feeling of inability to get as much breath as he thinks is owing him. His breathing may not be quickened or difficult, but he has the sense that he cannot fully fill or distend his lungs. This is complained of most often by those who are overworked, underfed, and short of sleep, or who, for some other reason, have allowed themselves to get run down. Beyond this I have no idea of its explanation. 


\section{Causes of Dyspnea}

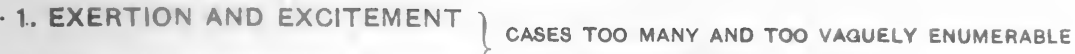

2. INFECTIOUS DISEASES

3. CARDIAC DIS3780 EASE

$$
\}
$$

4. PHTHISIS

5. CHRONIC BRONCHITIS

6. PNEUMONIA 

Among the types of difficult breathing we may distinguish:

(a) Inspiratory dyspnea.

(b) Expiratory dyspnea.

(c) Mixed types.

The last is by far the most common, and is seen in the great majority of uncompensated cardiac cases, in pneumonia, pulmonary tuberculosis, and other cardiac and respiratory affections.

Inspiratory dyspnea is comparatively infrequent, and occurs especially as the result of some obstruction in the upper air-passages, for example, in laryngeal diphtheria, edema of the glottis, "croup," tumors of the larynx, foreign bodies in the larynx, trachea, or primary bronchi, postpharyngeal suppurations, Ludwig's angina, and similar conditions. If the obstruction is below the primary bronchi, we do not see dyspnea of this type.

Expiratory dyspnea, usually accompanied by wheezing noises, occurs chiefly in emphysema and asthma, occasionally in pulmonary edema. It is apt to be more or less paroxysmal, whatever its cause.

\section{CAUSES OF DYSPNEA}

Types of dyspnea may be divided into chemical and mechanical. The former are associated with acioiosis, diabetes, and many children's diseases, also with the auto-intoxication seen in chronic cases of nephritis. Among the mechanical causes of dyspnea the most important is a reduced vital capacity of the lungs, whereby they become stiff and congested. Spirometric tests are sometimes of value by showing us the degree of reduction in the vital capacity of the lungs. Such tests may thus aid our prognosis and our estimation of the degree of cardiac compensation.

Heart disease, phthisis, and pneumonia are doubtless the commonest sources of dyspnea, but one sees the slighter degrees of the condition in a great many anemic or debilitated patients, perhaps as the result of a slight cardiac insufficiency not recognizable by other means.

Very acute and alarming dyspnea is seen at the onset of pneumıthorax, although both the frequency and the distress disappear altogether within the course of a few days or weeks after the thoracic cavities and their contents have adjusted themselves.

Occasionally in miliary tuberculosis one sees a dyspnea so extreme and a rate so rapid that hysteria is sometimes falsely diagnosed. A careful history and a thorough physical examination should set us right.

Increased intrathoracic pressure due to mediastinal tumors, to 
aneurysm, and occasionally to pericardial effusion may produce dyspnea, sometimes of a paroxysmal type, which is difficult to understand, since the cause persists unchanged.

A high diaphragm pushed up by pressure from an accumulation of fluid or gas or by some solid tumor causes a certain amount of polypnea, and occasionally dyspnea, by reducing the amount of space available for respiration.

\section{THE EFFECT OF POSITION AND OF THE TIME OF DAY}

Probably for the reason referred to in the last paragraph (high diaphragm) dyspnea is always increased by the recumbent position and eased by sitting up. In extreme cases the patient leans forward over his knees and much prefers to sit in a chair, owing to the cramped position of his legs in bed. Mountain-climbers at extreme elevations instinctively assume a similar position when endeavoring to rest.

All types of dyspnea are apt to be worse at night. This is not wholly due to the fact that at night the patient is usually trying to assume a recumbent position. Even with bed-ridden patients, whose position varies scarcely at all in the twenty-four hours, dyspnea is much more annoying after dark. This has been explained by Hoover and others as due to the fact that the respiratory center goes to sleep and allows the respiratory act to become almost suspended. The patient then wakes with a horrible gasp. It is in accord with this theory that the most troublesome dyspnea of most cardiac patients is in the earlier hours of the night, when sleep is deepest. Later in the night they can often lie down and get some rest.

\section{CHEYNE-STOKES BREATHING}

Regularly recurrent or periodic variation in the depth and frequency of respiration, with intervals of apnea alternating with dyspnea, receives the name of Cheyne-Stokes breathing. If there is no pause or apneic period, but merely a rhythmic quickening and slowing of respiration, the name of Biot's breathing is applied.

Either of these types of breathing may occur in healthy infants during sleep. In adults they usually complicate severe disease of the heart or kidney, less often of the brain, but also occur in the more critical stages of acute infectious diseases, such as pneumonia, and under these conditions may not be the harbinger of death. In cardiac and renal troubles such breathing is a bad prognostic sign, though I have known it to occur during sleep for many months before the fatal termination. 


\section{Case 359}

A German messenger-boy of twenty-two was first seen February 18, 1908. His family history was negative. Seven years ago he was thrown from a horse and trampled on; his left thigh and many of the ribs on the left side were broken. He was in the hospital for eighteen months, and states that he was unconscious for the first six months of this time In December, 1907, he was in the Boston City Hospital for three days on account of a cough of four days' duration, accompanied by bloodstreaked sputum and pain in the left axilla. Physical examination was negative, and he was discharged in three days. He again entered the same hospital on the ninth of January, Ig08, complaining of chest pain, sore throat, and a slight cough. Careful physical examination and $x$-ray examination showed nothing. Since that time he has felt weak and run down, but has worked steadily up to this morning. For a week he has noticed that his left ear was not as good as is the right, and for the same period he has had sensations of pinching on both sides of the chest near the left nipple, and in the neck a feeling as if it were being scraped.

For the past four days he has had frequent cough with bloody sputum. $\mathrm{He}$ thinks he may have raised as much as half a pint of blood in the whole four days. During this time his breathing has been very rapid. $\mathrm{He}$ has been restless and wakeful at night, and has vomited everything that he has taken. When on his feet, he is dizzy and faint. He has had frequent chilly sensations, but, so far as he knows, no fever. This morning while at work he fainted away, but walked to the hospital without assistance.

On physical examination the patient showed a remarkably rapid respiration, -80 to the minute,though his pulse was only 72 , later slowing down to 50 . Temperature

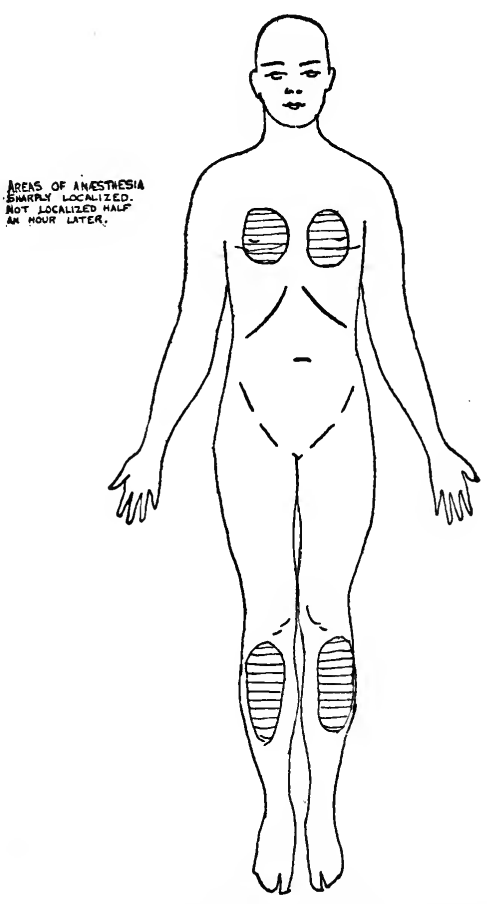

Fig. 18I.-Anesthetic areas demonstrated in a case of polypnea. was $99 \cdot 4^{\circ} \mathrm{F}$. He lay flat upon the accident room table, and seemed to be half asleep, except when spoken to; then he was notably alert, answering all questions in a strong, clear 
voice. There was an occasional slight, dry cough. The throat was somewhat reddened, the pupils equal and reacting normally, the heart absolutely negative, the lungs slightly less resonant in the right front than in the left. In the same region the voice-sounds are very slightly increased. Inconstant bubbling râles are heard in the right front and back. Physical examination, including the nervous system, blood, and urine, was otherwise entirely negative.

During the first week of his stay in the hospital the patient's respiration continued at from 80 to 95 a minute, while his pulse was from 50 to 6o. His hands, arms, and lower legs were cyanotic, congested, mottled, showing marked sluggishness of the capillary circulation. The face was flushed, but not cyanotic. He was as comfortable when lying down as in any other position. The fundus oculi was negative.

Discussion.- Summing up the past history of this boy, we find two sets of events, one or both of which may bear upon his present disturbance of breathing. The first of these, however,-the accident of seven years ago,- - seems rather too much in the past to be of any importance in relation to his present trouble. The pulmonary injuries arising from broken ribs are matters of immediate importance within a few days or weeks after their occurrence: not after the lapse of seven years. Questions of this kind are raised in connection with suits for damages supposedly due to accidental injuries, and it is often claimed that an injury of this kind might be the source of long-standing pulmonary disease. I do not believe that there is any sufficient ground for this idea.

The two attacks of axillary pain with cough are very much more recent, and are probably connected in some way with the present trouble. Tuberculosis is strongly suggested by the hemoptysis, as well as by the nature and position of the pain (pleuritic?). I have known polypnea similar in many respects to that here described, and due, as it afterward turned out, to miliary tuberculosis. This, indeed, was my first thought in the case here under discussion. The absence of dyspnea, the remarkably slow pulse, and the perfect comfort in the reclining position first aroused our skepticism, which was further increased by the nearly negative results of physical examination.

No blood was expectorated during his stay in the hospital, and we had no independent account of the fact of blood-spitting-only the patient's own statement. The other symptoms complained of -the fainting and dizziness, the sensory disturbances in the chest and neck, the curious mental state, and the mottling of the skin-all pointed toward a functional nervous disturbance as at least a part of the cause of these 
symptoms. It will be recalled also that he is stated to have been unconscious for six months after his accident of seven years ago-a condition strongly suggestive of hysteria. This hypothesis accordingly was followed up and led to the additional observations recorded in the outcome.

Outcome.-It was noticed that when actually talking to his friends, his breathing slowed down to the normal, and that any interruption or surprise would slow his respirations for a few seconds. During the night he got but little sleep, but when he dozed off, it was noted that his respiration fell to $2 \mathrm{I}$. The patient's friends say that he has been very peculiar and untruthful for a long time.

The patient's bed was screened off and he amused himself by reading. When thus occupied, his breathing would at times fall to normal. At times his pulse was as slow as 45 and his respiration as rapid as roo. The patient's headache was entirely removed by an ethyl chlorid spray. Areas of absolute anesthesia, as shown in the accompanying diagram, were demonstrated by Dr. Fitz at a clinic. Half an hour later they were not present.

It was later learned that he had worked for a long time in the hospital at Baden, Germany, and was interested in medical subjects; also that seven weeks ago he received news of the death of his father, the last of his immediate relatives. Since then he says he feels that he has no one to live for. On the twenty-fourth of February it was noticed that he had no palatal reflex. On the third of March he said that he had swallowed a safety-pin. He did not say whether it was open or closed. $X$-ray showed a doubtful shadow in his stomach. The patient said he could feel the pin in his throat.

At this time the respiration became more normal and remained so, but the next day he said he could feel the pin in the region of the left sacro-iliac joint. The patient was dry-cupped at this point, and reassured. His respiration still remained normal, and he was allowed to go home.

In view of all these facts we had no hesitation in making the diagnosis of hysteric polypnea. Of the four cases of this disease which have come under my observation, three have occurred in male patients. In all, the rapidity of the breathing was greater than in any but the terminal stages of organic pulmonary or cardiac disease. From these it may be distinguished by the following criteria:

(a) Hysteric polypnea is not associated with any demonstrable lesions of the heart or lungs; the cough is generally very trifling.

(b) The rapid respiration does not continue throughout the twenty- 
four hours; it may often be interrupted, as in the present case, whenever the patient can be led to talk with interest, and frequently ceases during sleep.

(c) Other evidences of hysteria are usually demonstrable-for example, the susceptibility to suggestion, areas of anesthesia, and wide deviations from the truth which seem like ordinary lying, but are more probably due to the peculiar mental state.

(d) The distress, cyanosis, orthopnea, and other evidences that breathing is difficult (true dyspnea) are absent. The breathing is rapid but not labored, and its rapidity is usually much greater than that associated with any organic disease of the heart or lung.

Diagnosis.-Hysteric polypnea.

\section{Case 360}

A collector, sixty-four years old, was first seen March IO, I908. He has had dyspnea for eighteen months. For the past four or five weeks it has become worse, and he has. slept every night in a chair. For two or three weeks he has had a cough with yellowish sputa.

Physical examination shows obvious loss of weight. The left pupil is larger than the right, and slightly irregular. The heart's impulse is in the fifth space, $\frac{3}{4}$ inch outside the nipple. There is no obvious enlargement to the right. The action is irregular and the sounds are indistinct. The second aortic sound cannot be heard. A coarse, discordant, squeaking murmur is heard with systole at the apex, and is transmitted to the axilla. At the base there is a rough systolic murmur, and along the left border of the sternum a low-pitched diastolic murmur. In the third right interspace near the sternum is a systolic thrill. The arteries are palpable, tortuous, and show a lateral excursion. The pulses are of small volume, low tension. There is no capillary pulse. Coarse bubbling râles are scattered throughout both lungs, and there is dulness, diminished respiration, and fremitus at both bases behind. The abdomen shows dulness in the flanks, shifting with change of position.

Discussion.-A long-standing dyspnea in a man of sixty-four associated with a cough which is of very recent origin is almost invariably due to cardiac disease. Since there are well-marked cardiac lesions shown on physical examination, and nothing in the lungs except what is easily explained by passive congestion, it is proper to assume that the heart disease is the cause of the dyspnea, unless evidence is presented suggesting another cause.

A to-and-fro murmur in the upper half of the precordial region, 
associated with absence of the aortic second sound, a systolic thrill, a pulse of small volume, and moderate cardiac enlargement is strong evidence of aortic disease with stenosis and regurgitation. The diagnosis of aortic stenosis is one of those most often made erroneously. - In my opinion it should never be made unless there is also evidence of aortic regurgitation-in other words, so-called pure aortic stenosis probably does not exist. At any rate, I know of no convincing evidence of its xccurrence. Stenosis accompanied by regurgitation is a proper conclusion under two sets of conditions:

(a) In any case exhibiting signs essentially identical with those just given.

(b) In any long-standing rheumatic case showing an aortic regurgitation in a person under twenty-five, whether there are physical signs of stenosis or not. This latter conclusion is the result of postmortem observations. I have never known a case of long-standing heart disease in a young person in which pure aortic regurgitation was discovered at autopsy. Stenosis always accompanies it, because the rheumatic type of endocarditis does not stay long upon the aortic valve without producing stenosis. In older persons aortic regurgitation without stenosis is very common, and the presence of a systolic murmur without the other signs recorded in this case should never be considered as sufficient evidence for the diagnosis of stenosis. In the present case we have all the cardinal signs.

Outcome.- Under rest in bed, with digitalis and purgation, he improved very much within three days. Blood and urine were normal, temperature constantly subnormal; pulse and respiration not remarkable. Under 15 minims of digitalis tincture three times a day the patient was able to be up and about by the twenty-first. On the twenty-fifth digitalis was omitted, and the patient was able to walk about without distress. A half-ounce magnesium sulphate was still given every morning. On the twenty-ninth he was allowed to go home.

Diagnosis.-Aortic stenosis and regurgitation.

\section{Case 361}

A school-girl eight years old was first seen November I9, I907: The mother now has consumption. Two sisters have died of pneumonia. The child had the measles and chicken-pox five years ago. Four years ago she visited the Boston Dispensary and was told that her heart was enlarged. Three years ago she was kept out of school for the whole winter, and seemed about as she is at present, but picked up in the spring. For three weeks she has been short of breath on exertion, and 
has complained that her feet were sore. In the same period she has been growing pale and thin, and has been heard to moan in her sleep. Two or three times in the last four days she has coughed up a teaspoonful of blood.

The course of the temperature, pulse, and respiration are seen in the chart (Fig. I82). The child is very pale, though her hemoglobin is 75 per cent. The apex impulse is difficult to place, but seems to be in the sixth space, $\frac{1}{2}$ inch inside the nipple-line. At the apex there is a palpable presystolic and systolic thrill. When the child is lying down, a systolic impulse can be traced as far out as the seventh space, and nearly to the posterior axillary line. No left border of dulness can be marked out. (See Fig. 184.) Cardiac pulsation can be felt over the dull area to the right of the sternum. A loud to-and-fro friction rub is heard in the exposed space. (See Fig. 183.) In this area the heartsounds cannot be clearly made out. In the anterior axillary line systolic and diastolic murmurs are heard. Posterior to this point the first sound is very sharp. The right lung seems normal. The left lung is hyperresonant at the apex, but below that dull, gradually increasing to flatness

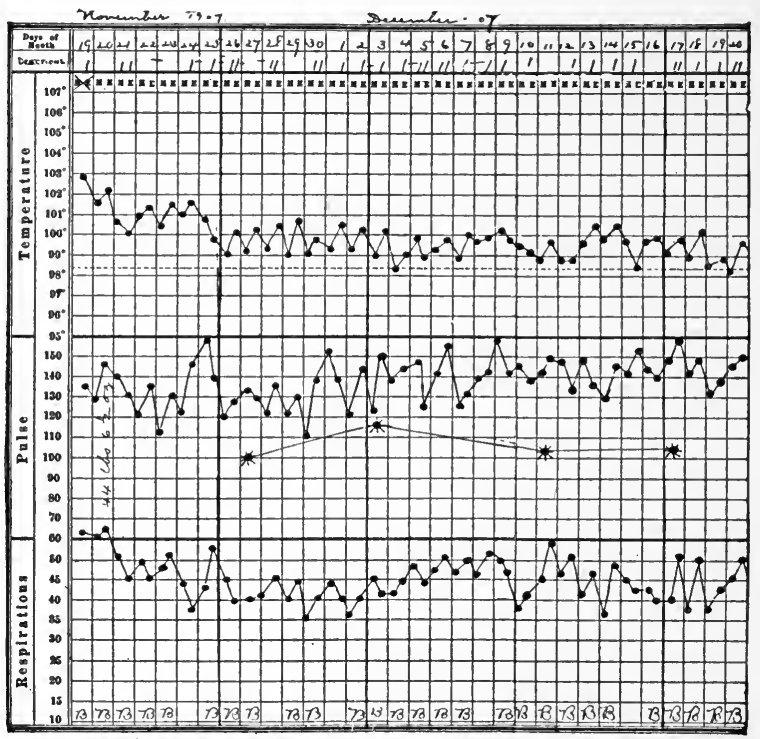

Fig. I82.-Chart of case $36 \mathrm{r}$.

at the base behind, where the breath-sounds are bronchial, though feeble. Above this many fine and medium crackles are heard. The edge of the liver, can_be felt across the upper abdomen, as seen in the diagram. 


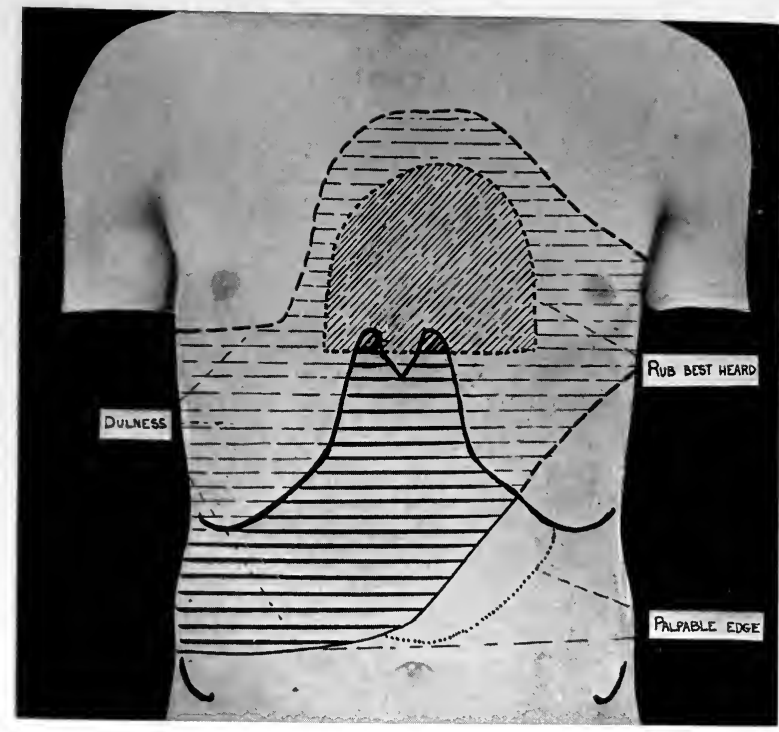

Fig. 183.-Results of palpation, percussion, and auscultation in a patient complaining of three weeks' dyspnea.

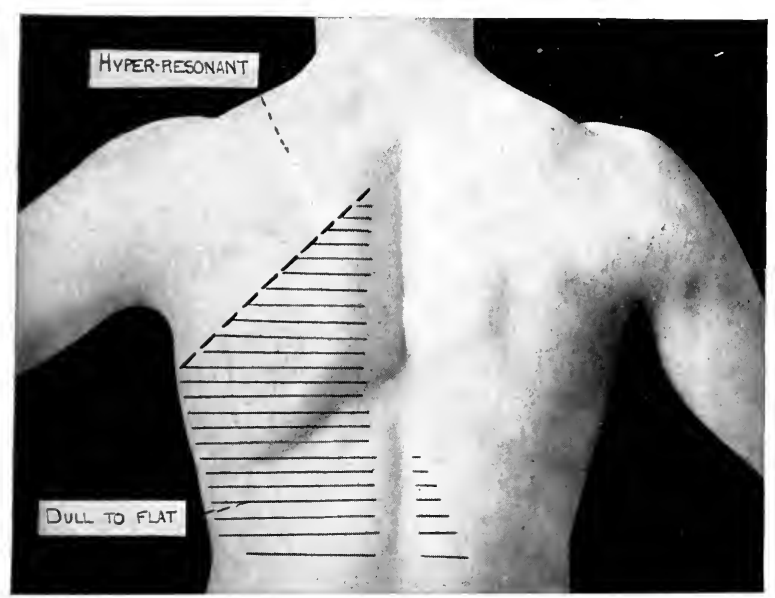

Fig. 184.-Signs in back (Case 361). (See also Fig. 183.) 

Discussion.-As I have definitely stated that a to-and-fro friction rub is audible in the exposed space, there can be no reasonable doubt that.we are dealing here with pericarditis. If the friction were pleuropericardial in origin, one would not describe it by the words here used, and there would be some note regarding its change or disappearance when the breath was held.

But acute seronibrinous pericarditis does not cause dyspnea unless the amount of effusion is large. We see postmortem many a shaggy heart (cor villosum) which has developed as a terminal complication in chronic nephritis without producing any dyspnea whatever. In the present case, therefore, the important question is: What else have we besides such a pericarditis?

It is to be noticed, in the first place, that the child's heart has been known to be enlarged for at least four years; in the second place, that there is apparently decided enlargement now, although it is impossible to say just where the left border is. There is a double apical murmur and thrill which, in itself, aside from the other conditions present, would lead us to suppose that the mitral valve was narrowed and incompetent. But to any one who has had a considerable opportunity to verify his cardiac diagnoses postmortem it will be an old story that, in markedly enlarged hearts, systolic and presystolic murmurs at the apex have no diagnostic value. They may be present with or without valvular lesions.

The observations of the last ten years, especially in England, have made it clear that in the heart troubles of childhood the whole heartendocardium, myocardium, and pericardium-is usually involved. In the inflammatory process attacking the heart, the part borne by the valve is usually far less important than that borne by the myocardium and the pericardium. In other words, pericarditis is much more common than endocarditis in the heart troubles which occur in childhood, with or without joint infection (rheumatism) and chorea. But in all cases the affection of the myocardium is the all-important element, though we have no direct auscultatory evidence of its changes, such as we often have when the endocardium or the pericardium is attacked.

A point of practical importance in these cases is this: No matter what murmurs are present we are not bound to assure ourselves or the family that an incurable cardiac malady is present. If only the myocardium has been severely inflamed, we may see an almost complete restoration of the cardiac functions with the disappearance of the murmurs and a return of the heart almost or quite to its normal size. This result, however, comes only after months of rest and careful watching. In the great majority of cases digitalis does harm. 
Outcome.-Under rest in bed, with tincture of digitalis 5 minims every four hours, the temperature and the friction rub gradually subsided. After the rst of December she was carried out-of-doors each day and gradually improved. By the fifteenth of December the friction rub had disappeared and the sounds in the left lung were reduced to very slight dulness at the extreme left base, with cogwheel respiration, but no râles. On the twenty-first of December the cardiac apex was in the sixth space, $3 \frac{1}{2}$ inches from the median line. There was a palpable thrill and a rough presystolic murmur, followed by a loud systclic murmur at the apex. The child could walk about without dyspnea and seemed nearly well.

In this case presumably the mitral valve was attacked, as well as the myocardium and the pericardium.

Diagnosis.-Infectious endocarditis, myocarditis, and pericarditis. Mitral stenosis and regurgitation.

\section{Case 362}

A married American woman, forty-one years old, was seen March I3, r908. Her family history anc past history are excellent. For six months she has noticed that she has been short of breath. There has been no cough, zo edema, no orthopnea. For four months she has been getting weaker, but has kept at work until four weeks ago, when she fell over at her work. She was not unconscious, but, she says, "I had no pulse and no heart-beat and they worked over me for two hours before I was better." She has been in bed since that time, and while quiet, feels well enough except for a little pain around her heart. There has been some bloody vaginal discharge for a considerable portion of the time in the last five years; there are few days in the month without bleeding, though the amount is small.

Physical examination showed no anemia and nothing demonstrably wrong in the chest or abdomen; normal urine. During the subsequent week she had more or less continuous bloody vaginal discharge, associated with slight anteflexion and retroversion and a little erosion about the cervix.

Under gas and ether on the nineteenth of March a jet of hot steam was introduced into the uterus, the vagina being protected by a continuous stream of cold salt solution passing around the uterine tube during the process. The steam was continued for forty seconds, and after a few minutes' intermission, for thirty-five seconds. Following this the uterine discharge ceased. 
Discussion.-The hyperplastic endometritis which doubtless was present in this case must have produced an undesirable and in some ways debilitating drain upon the system, though it cannot have been. of very great physical importance, since no anemia was produced. But psychically such a drain has a very great effect on most women, especially when the knowledge of its presence is given a solemn and ominous significance by the hints and fears of kindly neighbors who have "seen what such things come to."

Nothing in the physical examination gives us any definite knowledge of an organic disease to which this dyspnea may be made secondary, but it is a fact very familiar to clinicians that "short breath" is complained of by a great many patients in a variety of debilitated conditions. In some of these patients cross-questioning showed that no true dyspnea is present, for by "short breath" they mean not a rapid and difficult respiration, but a certain sensation as if they were unable to draw as full a breath as they desired. It is thus a sensory, not a motor, phenomenon, and as such should be distinguished from true dyspnea. Just what is the significance of this sensation I have no idea. One meets it in a great many neurasthenic persons and sees it pass off under reassurance and work-cure without any change in the condition of the circulatory or respiratory organs.

One also sees a great many cases of true dyspnea which are never fully explained. The symptom is indeed much more common than is often realized, because we often forget to ask for it, and unless questioned, patients often do not mention it. It may be surmised that these unexplained types of dyspnea are due to mild forms of myocardial insufficiency which recover without our being able to be sure that they exist or to recognize their cause. It seems altogether probable $a$ priori that such types of insufficiency occur and that they will assume greater importance in the future.

One often hears from patients the history of an attack like that suffered by this woman four weeks ago-an attack in which a doctor is called "and works over the patient for hours before she is better." From a considerable experience of the outcome of such cases I have come to believe that this very process of "working over people for hours," together with the alarm reflected from the medical attendant to the patient via sympathetic relatives, is itself the cause of most of the symptoms; in other words, I believe that these attacks are largely hysteric in nature, and are much aggravated by the treatment which they receive. If neglected or made light of, such an attack will often 
pass off in a few minutes, but if inhalations of amyl nitrite, alcoholic stimulants by mouth, subcutaneous injections of strychnin, and heat over the precordia are given, the patient takes the hint, faces the worst with courage, and proceeds to suffer accordingly.

Diagnosis.-Hyperplastic endometritis; debility.

\section{Case 363}

A canvasser seventy years old was first seen April II, I908. His family history is excellent. Since his twentieth year he has had epileptic attacks, once in two or three weeks at first, for the past thirty-five years much less frequently. He is unconscious for a few minutes, but never falls, as he knows when his attack is coming. He never bites his tongue, and has no incontinence. Since his twentieth year he has also had involuntary twitching of the muscles of the left hand, for which he wears a glove, with relief. He denies venereal disease. For the past month he has had much dyspnea, increasing within the last few days to orthopnea, and associated with a cough and profuse sputum $-\frac{1}{4}$ of a cupful of thick, greenish sputum in twenty-four hours.

On physical examination the blood-pressure is found to be $160 \mathrm{~mm}$. $\mathrm{Hg}$; the nocturnal urine is more than the diurnal. Respiration is rapid and wheezing; there is a frequent loose cough, with mucopurulent sputum. The heart shows nothing except unusual faintness of the sounds and accentuation of the pulmonic second. The chest is hyperresonant on percussion throughout, obscuring the cardiac dulness. Expiration everywhere is prolonged and accompanied by coarse squeaks and groans. The sputum contained many eosinophiles, many mixed bacteria, no tubercle bacilli.

Discussion.-We have no reason to doubt that this patient has epilepsy, though there are some symptoms which lead us to conjecture that it may be of the secondary type, and that some source of cortical irritation may be present. In all probability, however, this longstanding malady has no special connection with the symptoms from which he now is suffering.

Dyspnea combined with high blood-pressure, nocturia, and innumerable pulmonary râles may be associated with chronic myocardial weakness, with acute pulmonary edema, or with some pulmonary infection (bronchitis and bronchiectasis). Presumably the heart is enlarged in this case since we find blood-pressure high. If the symptoms had appeared with great suddenness in a patient previously in good condition, and if the sputum had been very profuse, watery, and pinkish, acute edema of the lungs would be the most probable diagnosis. But as the onset 
has been a gradual one, we have no reason to consider that mysterious and dangerous disease.

It remains to distinguish between-(a) Dyspnea due to chronic pulmonary stasis with edema, the result of myocardial weakness, and (b) respiratory infection. The pulmonary hyperresonance makes it impossible for us to estimate the size of the heart; accurate auscultation is rarely possible when all the sounds are obscured by noisy râles. In cases of this kind, which are very frequent in general practice, our chief reliance must be upon the pulse. In the present case the pulse was regular, not rapid, or in any other respect remarkable. The cervical veins showed no distention or unusual pulsation. The distribution of the râles in the lungs was not that usually seen in chronic edema due to stasis, and the number of bubbling and crackling sounds was less than that usually heard in edema. The examination of the sputum further inclined us to believe that the dyspnea was due to the condition of the lungs rather than to any form of cardiac insufficiency.

Putting together all these facts, therefore, it appears that the dyspnea is due to emphysema and bronchitis, with very possibly some bronchiectasis as well. One recognizes, however, that the occurrence of such infections is greatly favored in case any weakening of the circulation supervenes, as it is always prone to do in men of this age.

Outcome.-He was given potassium iodid, Io grains three times a day, atropin sulphate $\frac{1}{100}$ grain three times a day, morphin $\frac{1}{6}$ grain occasionally for dyspnea and sleeplessness. By the twentieth his bronchitis was nearly gone and he was having good nights. He was then given a cough mixture in the following recipe for cough:

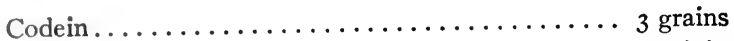

Chloroform ...................... I5 minims

Syrup of wild cherry $\ldots \ldots \ldots \ldots \ldots \ldots \ldots \ldots, 3$ ounces

A teaspoonful of this mixture every two hours, when needed, for cough.

Diagnosis.-Bronchitis and emphysema. Epilepsy.

\section{Case 364}

A housewife, aged twenty-six years, of good family history, was first seen January 16 , r907. She had never been sick in bed until seventeen months ago, when she had "typhoid fever"; at this time she was in bed five weeks. Eleven months ago she had "bronchitis" and was in bed a week. Eight months ago she had pleurisy with effusion, and was tapped, but only about a teaspoonful of clear fluid was drawn for diagnosis. During the past summer she has been somewhat short of 
breath on exertion, with considerable wheezing cough and the raising of thick, greenish phlegm in the morning. For the past month the wheezing and rattling in her chest has been almost constant and not affected by cough. Dyspnea has grown worse, and she gets out of breath very easily. The wheezing comes in paroxysms lasting an hour about twice a day. They are usually brought on by exertion and are relieved by rest or by coughing. She has had no fever, no chills or sweats. Six months ago she weighed II2; now she weighs II5 pounds. She feels well and strong, and has not been confined to bed, but complains that any exertion brings on shortness of breath and wheezing. The course of the temperature is seen in the accompanying chart.

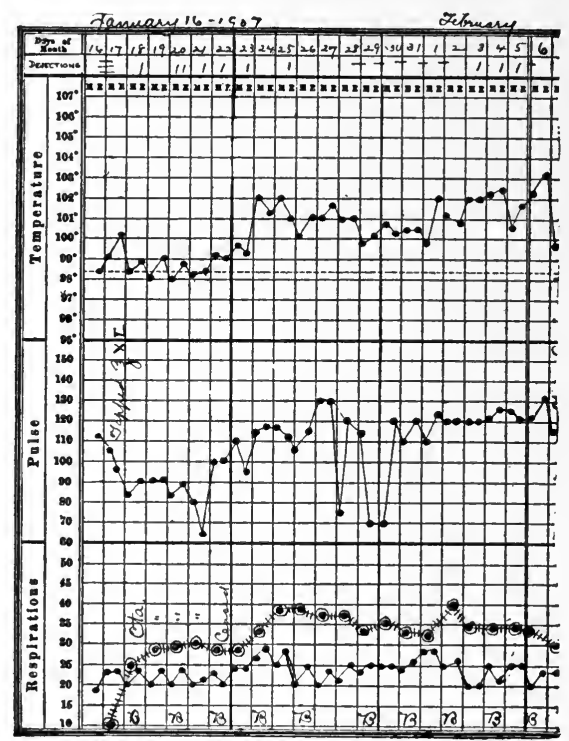

Fig. I85.-Chart of case 364 .

The patient is well nourished, rather nervous, and fidgety. Coarse râles can be heard at some distance from the chest, and the nostrils move with each inspiration. The heart's apex is seen and felt in the fourth space, 5 inches to the left of midsternum, 2 inches outside the nipple. The dulness extends also as low as the sixth rib. The sounds are regular, of good quality, and there are no murmurs. The condition of the lungs is shown in the diagrams (Figs. 186 and I87). The liver dulness extends three fingers' breadths below the costal margin. Its edge is not felt. The blood showed a continuous leukocytosis varying between I 6,000 and 28,000 , with 84 per cent. of polynuclear cells. The urine is not remarkable. The head, abdomen, and extremities are negative. 


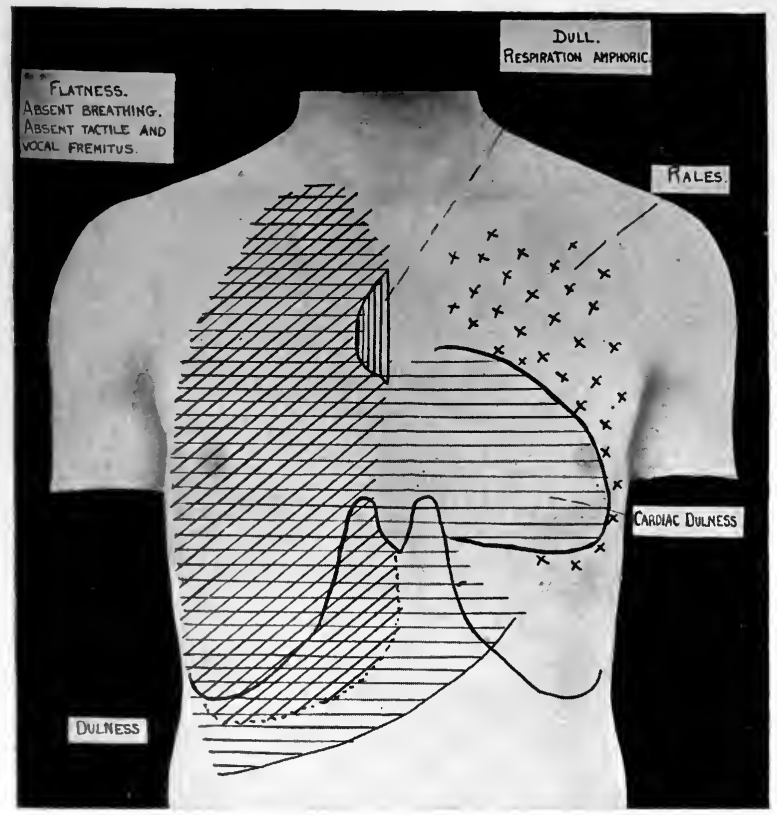

Fig. 186. - Results of physical examination in Case 364. (See also Fig. I87.)

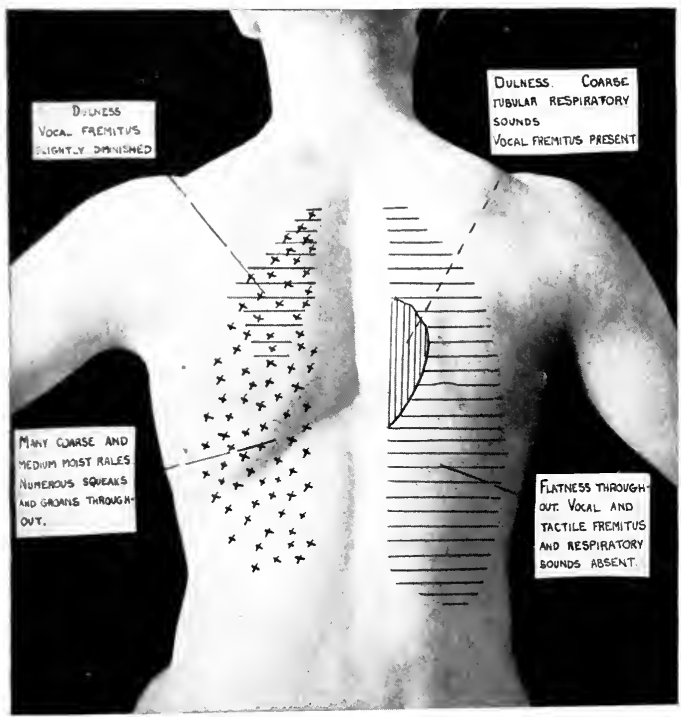

Fig. 187.-Graphic representation of the signs observed in a case characterized by dyspnea (eight months) and wheezing (one month). 

The sputum shows an abundance of various bacteria but no tubercle bacilli.

On the seventeenth the right chest was tapped, and 40 ounces of fluid removed, with great relief to the patient. This fluid was turbid and deposited a considerable whitish sediment. It was odorless, I023 in gravity. The sediment consisted mostly of disintegrated polynuclear cells, very few mononuclears.

Discussion.-This case is characterized by the occurrence of paroxysmal dyspnea and wheezing, brought on by exertion in a patient who otherwise feels well. The physical examination indicates at once that the heart has something to do with it. But when we have such marked signs in the right chest, we must always question whether the displacement of the cardiac impulse is due to hypertrophy and dilatation or to the pressure of an effusion in the right chest. Doubtless the heart's action is embarrassed when it is made to beat in this unusual position, even though no cardiac disease is present. But until we know what is the position of the cardiac apex after we have tapped the pleural effusion, we have no way of being sure of any lesion in the heart itself.

What is the malady in the chest? The leukocytosis indicates that it is not due to hydrothorax or serous pleurisy. The specific gravity of the fluid obtained by tapping and the character of the sediment point to an infection which will soon result in frank pus (empyema).

Aside from traumatic cases, we recognize two types of empyema within which almost all purulent effusions fall:

(a) Postpneumonic.

(b) Tuberculous.

(a) In a considerable portion of the postpneumonic cases the pneumonia is so mild and rapid that it is altogether unrecognized, and the empyema is supposed to be "primary." The study of the fluid, however, almost always reveals pneumococci more or less degenerated, and on careful questioning we can usually elicit a history that strongly suggests the original pneumonia. Latent cases are especially common in children. Practically all the postpneumonic empyemas get well and stay well. Their prognosis is far better than that of serous pleurisy, as I proved some years ago by following up the end-results of a large number of cases of both diseases.

(b) Tuberculous empyema has usually a gradual and insidious onset like that described in this case. The fluid is often at first serous, and the doctor may blame himself (quite unjustly) when it becomes purulent after tapping. In a minority of cases there is obvious tuberculosis of the lung or pneumothorax, preceding the appearance of the 
empyema. Often there is evidence of tuberculosis in other organs. In perhaps the majority of cases, however, it is the failure of the empyema to clear up after drainage which first makes us suspect tuberculosis. I have never known a tuberculous case to get well.

Outcome.-In the sediment of the chest fluid a few small clumps of tubercle bacilli were demonstrated, though none could be found on repeated examination of the sputa.

Though the patient felt very well through the later days of January, and was out-of-doors daily in a chair, her temperature ranged higher and higher, and on the sixth of January a friction-rub was heard all over the precordia. On the seventh the right chest was again tapped. and a thicker and yellower fluid was withdrawn. The patient was then transferred to the surgical ward and several pints of thick, odorless, creamy pus liberated; specific gravity was rozo, the sediment as before, but containing no tubercle bacilli, while pneumococci were easily cultivated from it. For twelve weeks after this the patient continued to run a high, irregular temperature, but finally recovered, and when heard from eighteen months later, was in excellent general health, though with a small discharging sinus. A guinea-pig inoculated with $30 \mathrm{~cm}$. of the chest fluid withdrawn January $\mathrm{I} 7$ th was killed six weeks later, and showed marked glandular tuberculous lesions, from which tubercle bacilli were recovered.

Diagnosis.-Tuberculous empyema and (presumably) phthisis.

\section{Case 365}

A young woman of twenty-two, a typesetter by profession, was seen April 3, I907. Her menstruation has always been irregular, occurring about every six weeks. Two years ago she almost choked to death while eating tea and cake. Ever since that time she has been very short of breath on walking, and suffers a gnawing pain in the left chest and shoulder on any exertion. Cold weather always makes her worse, and some days she can scarcely walk for shortness of breath. This, however, never interferes with her regular occupation. She has no other symptoms. Her appetite, bowels, and sleep are normal.

The heart's impulse and dulness reached to the sixth space, midaxillary line, $8 \mathrm{~cm}$. outside the nipple. The right border of cardiac dulness seemed to be at the right sternal margin. Cardiac action was regular, rapid, II2, the first sound short and valvular, the pulmonic second very much accentuated. There were no murmurs and no venous pulsation in the neck. The pulse was of small volume, moderate tension. Blood-pressure, II5. The lungs were normal, save for an occasional bubbling râle at the left base. There was a trifling edema 
of the hands and feet, together with marked coldness. Blood and urine showed nothing abnormal, and there was no indication of stippling in the red cells.

Discussion.-This patient's right ventricle seems to have given way; at any rate, the heart is enlarged, and the cause for such an enlargement does not appear to lie either in valvular disease, in syphilis, or in any renal affection. Our problem is to find some other etiology.

In a woman of this age we can hardly suppose that we are dealing with a hypertrophy and dilatation due to a chronic fibrous myocarditis. It is true, however, that myocardial weakening, with or without demonstrable fibroid changes, does occur in young people as a result of an acute infectious disease of the same type which we call rheumatism when the joints are involved. When a heart is thus weakened, dyspnea may result either from the gradual and progressive dilatation, or acutely, as the result of some strain, such as mountain climbing.

Chronic adhesive pericarditis, which may occur without the patient's having been aware of its earlier stages, often produces hypertrcphy and dilatation of the heart, with resulting dyspnea. We cannot exclude this disease in the present patient, but there is no definite evidence of it, no retraction of interspaces in any part of the chest during systole, no restriction of the normal cardiac mobility when the patient lies on the left side, no history of acute pericarditis in the past.

We must beware of an incipient Graves' disease (hyperthyroidism) in any case presenting the symptoms here under discussion. The cardinal symptoms (tachycardia, thyroid tumor, exophthalmos, tremor) may be so slight as to be easily overlooked, and the cardiac weakness and enlargement may thus occupy the foreground of the clinical picture. Some evidence of the cardinal symptoms must, however, be detected before we can go beyond a suspicion of Graves' disease. In this case we could find no such evidence.

Acute dilatation of a previously healthy heart I have never known to occur except during acute infectious disfases, such as pneumonia, bronchitis, articular rheumatism, or influenza; yet I have seen a number of cases like that now under discussion in which we had no definite evidence of any disease such as would weaken the myocardium, and were confronted, therefore, with an apparently "primary" dilatation, acute or subacute. So far I have never followed such a case to postmortem examination without finding evidence of a previous myocarditis. When, therefore, we find no causes such as an acute infectious disease, hyperthyroidism, or adherent pericardium, and when valvular disease and nephritis can be excluded, I think we should conclude, as 
I do in the present case, that we are dealing with a chronic myocarditis of unknown origin (syphilitic?), with a complicating acute dilatation. Only by the results of treatment can we ascertain whether the dilatation is temporary or permanent.

Outcome.-Under rest, purgation, magnesium sulphate, and 5 grains of veronal at night, the patient was remarkably improved within four days. On the seventeenth the heart showed no enlargement and no murmur, and the patient was able to walk about without symptoms.

No evidence of syphilitic infection was obtained.

Diagnosis.-Acute cardiac dilatation, cause unknown.

\section{Case 366}

A widow of fifty-two who had lost two sisters of cancer and had previously suffered from typhoid fever, several attacks of pneumonia, and from one severe attack of diphtheria many years ago, was first seen January Io, I908. She had several uterine operations four years ago, the last of which was a partial hysterectomy. Three years ago the left

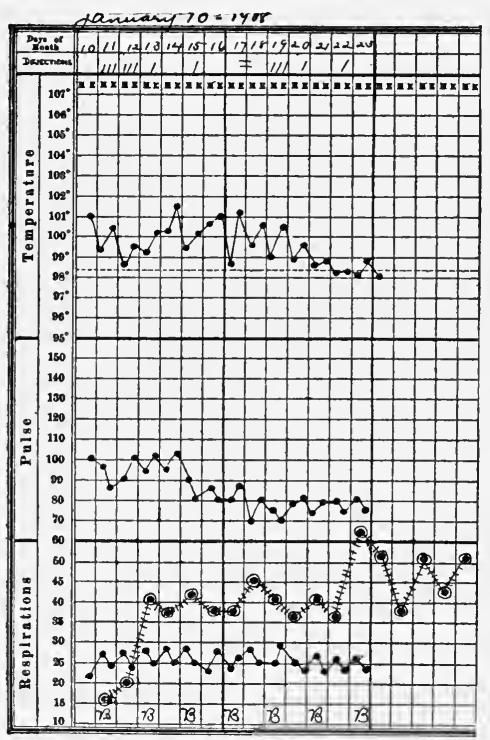

Fig. 188.-Chart of case 366 . kidney was removed on account of an injury to the ureter at previous operation.

For three weeks she has had a cold in the head, with sore throat. A week ago she became dizzy and almost lost consciousness while on the street, but managed to get home, when she had chilliness, sweating, and pains all over her body. Since that time she has had fever, dry cough, nausea, and shortness of breath. The course of the temperature is seen in the accompanying chart. Her throat is reddened and swollen. There is herpes on the nose and upper lip. The glands at the left angle of the jaw are enlarged. The heart-sounds are faint at the apex, but show nothing else abnormal. The heart is not enlarged. The pulse tension appears to be slightly increased. There is slight edema of the lower legs. The abdomen and the urine are negative. Scattered râles in both backs.

Soon after her entrance to the hospital the patient had several 
attacks of inspiratory dyspnea, with a high crowing inspiration and croupy cough.

.Discussion.- - In all the cases discussed so far in this section the dyspnea has been of the ordinary type seen in the vast majority of cases due to pulmonary or cardiac disease. It has been "mixed"-i.e., it has affected both inspiration and expiration alike.

From this, which is by far the commonest type, we distinguish:

(a) Inspiratory dyspnea.

(b) Expiratory dyspnea.

Expiratory dyspnea is seen especially in emphysema, in asthma, and in the cases of bronchitis or bronchiectasis complicated by asthmatic attacks. The breath seems to go in easily enough, but comes out with a prolonged wheeze and so imperfectly that the chest does not return to normal expiratory shape but remains in the position of full inspiration.

Inspiratory dyspnea, such as was present in the case now under discussion, is due always, so far as I am aware, to an obstruction of the upper air-passages-i. e., the pharynx, larynx, trachea, or primary bronchi. The laryngeal types of obstruction are by far the commonest. Among these we may distinguish:

(a) Ordinary catarrhal laryngitis, which in children is called "croup."

(b) Diphtheria, involving the larynx or trachea.

(c) Tumors of the larynx.

(d) Laryngeal syphilis.

(e) Paresis or paralysis of the vocal cords.

(f) Tuberculosis of the larynx.

Next in frequency come the causes which exert pressure upon the trachea or primary bronchi from without. Such causes are found in tumors of the mediastinum and aortic aneurysm. Postpharyngeal abscess, acute or chronic, produces a peculiar type of inspiratory dyspnea, with a "whoop" like that of pertussis, and a curious cough which reminds one of the bark of a small puppy or the cry of some bird ("cri de canard"). I have heard such a sound again and again echoing through the halls of an out-patient department, and seldom found myself wrong in the "snap diagnosis" of postpharyngeal abscess.

Syphilitic stenosis of a bronchus may produce inspiratory dyspnea of a paroxysmal type, which precisely simulates bronchial asthma.

In the present case we have reason to suspect, in advance of an accurate diagnosis by means of the laryngoscope, that acute laryngitis will be found because the patient has evidently been invaded by an acute infection involving the upper air-passages. Such an infection 
very commonly reaches the larynx. Nothing more can be said until the larynx is thoroughly examined, but we have no reason for suspecting any of the other causes listed above.

Outcome.-Tracheotomy instruments were kept at hand, and inhalations of steam, with a laryngeal spray of Dobell's solution, used at frequent intervals. Wine of ipecac, I dram, was given several times with relief when laryngeal dyspnea became extreme. It was later noticed that the palatal reflex was entirely absent. Subsequent examination of the throat and larynx showed a very marked atrophic rhinitis, with acute laryngitis and tracheitis. The patient continued very hoarse until the twenty-eighth of January, though the lungs were nearly clear by the nineteenth. By February 5 th the patient was able to go home.

Diagnosis.-Acute laryngitis.

\section{Case 367}

A school-girl of six was first seen November 29, I907. About an hour before her entrance she was seized with cough, frontal headache, vomiting, and rapid breathing. Previous to that time she had been perfectly well, as far as her mother knows. The child looked healthy, but breathed very rapidly and with a pronounced inspiratory wheeze. The tonsils were large and injected. On account of gagging further examination was impracticable. There was frequent brassy cough. The breathing was everywhere normal. White cells were 15,500; urine, negative. Physical examination was otherwise entirely negative. After a teaspoonful of wine of ipecac the dyspnea promptly ceased. Next day the child was well.

Discussion.-This case is included merely to show what I mean, and what I think most physicians mean, by "croup." Since we have come clearly to distinguish the cases of laryngeal diphtheria which were formerly mistaken for "croup," some clinicians have been inclined to assume that the familiar clinical entity which for generations has passed under that name was abolished. The reason for retaining the name is that in children acute laryngitis is apt to appear at night suddenly, and, as it were, out of a clear sky, and to terminate abruptly before morning, while in adults the clinical picture is quite different because the laryngitis appears and disappears so much more slowly.

This difference is due, doubtless, to the accumulation of adenoid tissues about the throats of children and its subsequent disappearance in adults.

Diagnosis.-Croup. 


\section{Case 368}

A barrel-maker of twenty-three, whose family history, past history, and habits are good, was seized two weeks ago with headache, vertigo, and vomiting. Despite these symptoms he managed to work until a week ago, when he began to be markedly short of breath. In the past two days he has had considerable cough and scanty yellow sputa. The patient mentions no other complaints.

On physical examination the cardiac apex is in the fifth space, $I_{4}^{\frac{1}{4}}$ inches outside the nipple-line. The right border corresponds with the right sternal margin. The aortic second sound is accentuated. Bloodpressure is $175 \mathrm{~mm}$. Hg. The arteries show no sclerosis. The chest is everywhere hyperresonant, expiration prolonged and difficult, accompanied by innumerable squeaks and bubbling sounds. The abdomen is distended and shows shifting dulness in the flanks. The legs were moderately swollen.

The temperature, pulse, and respiration were normal for ten days. The white cells on the twenty-sixth were 16,000 ; after two days of treatment they had fallen to 5000; hemoglobin, 60 per cent. The urine averaged 20 ounces in twenty-four hours, I0r6 in specific gravity. A large trace of albumin was found, and very many hyaline and granular casts, with much fat adherent.

Discussion.-As the dyspnea is here associated with cardiac enlargement, it is proper, first of all, to inquire whether cardiac disease is its cause. We find no evidence of valve trouble. Fibrous myocarditis is not common at this age. Acute dilatation is a diagnosis which we make only as a last resort when no trace of any cause can be found. Adherent pericardium cannot be ruled out, but has no definite facts in its favor. We have no evidence of incipient hyperthyroidism.

The high blood-pressure makes us suspect the kidney, and as soon as we turn our attention to the condition of the urine, we perceive that its characteristics are not those ordinarily associated with heart disease and renal congestion. $I$ have known but two cases of passive renal congestion with a urine of low gravity. The opposite is the almost invariable rule.

Nephritis, then, is in all probability the cause of the dyspnea and the other symptoms, but what type of nephritis is it? Certainly not acute nephritis, since the heart is hypertrophied. Probably not chronic interstitial nephritis, since this disease is rare at twenty-three and is not often associated with any considerable degree of anemia. In all probability we are dealing with the chronic glomerulonephritis of Councilman and Wright, the secondary contracted kidney of the Germans. 
Outcome.-The patient was given magnesium sulphate, 2 ounces, at the time of entrance, and $I_{2}^{\frac{1}{2}}$ ounces every morning thereafter; also hot-air bath every second day. His liquids were limited to two pints daily, and his diet was restricted as for acute nephritis. Under this treatment his tormenting headache, his nervousness, and edema disappeared in four days. His dyspnea persisted three days longer, but was not marked after four days. The dimensions of the heart, however, showed no change. He was allowed to finish his convalescence at home after the fifth of October, the diet no longer restricted.

Diagnosis.-Chronic glomerulonephritis.

\section{Case 369}

An electrician of sixty-two, with an excellent family history, past history and habits, entered the hospital November I2, 1907. He had been perfectly well until he began, nine months ago, to suffer from shortness of breath. Two months ago the dyspnea compelled him to quit work for two weeks, and a month ago he had to give up for good. At times he has been unable to lie down at night. There has been a slight cough, with a little grayish sputum. He has slept very poorly, and has sweated much at night during the past week. Two years ago he suffered for five or six weeks from quite marked edema of the legs at night-time, entirely without any other symptoms. For years he has risen once at night to pass water.

The patient was orthopneic at entrance. The heart's apex was in the fifth interspace, one inch outside the nipple, the right border $I \frac{1}{2}$ inches to the right of the sternal margin. The heart's action was slightly irregular, with a slightly accentuated pulmonic second sound. There were no murmurs. Blood and urine showed nothing abnormal. There was no edema or hydrothorax. The edge of the liver was felt almost on the level of the navel. There was dulness and diminished breathing, voice, and fremitus, with coarse bubbling râles belọw the angle of each scapula. Temperature, pulse, and respiration were normal throughout his stay. The sputa, twice examined, showed a variety of bacteria, but no tubercle bacilli.

Discussion.-This case exemplifies a type extremely common in general practice. Since the urine is normal and the blood-pressure apparently not elevated, we have no good reason to suspect that commonest cause of dyspnea and edema in elderly men-chronic interstitial nephritis. The examination of the heart gives us no reason to believe that the trouble originates in disease of the valves or of the pericardium. We have no chronic pulmonary disease which might weaken the heart, 
especially the right ventricle, in the attempt to force blood through capillaries decimated by emphysema and chronic pneumonitis.

Only one alternative remains, so long as our present conceptions of circulatory disease are adhered to. The myocardium must be insufficient. What the nature of this insufficiency is seems to me wholly problematic. We can no longer assume, as of yore, that a demonstrable fibrous myocarditis underlies the insufficiency of the heart muscle. It has been abundantly proved that we may have fatal myocardial insufficiency without fibrous myocarditis; also that we may have extensive myocarditis without any cardiac weakness. The same thing is true of the microscopic forms of myocardial change: they are very common both with and without the clinical evidences of myocardial weakness, but we have no reason to assume that they are its cause.

The modern studies of defective conduction in cardiac impulses may in time give us the key to our difficulty, but for the present we must state our diagnoses in functional or physiologic terms. When confronted with a case like that above narrated, our diagnosis should be myocardial weakness or myocardial insufficiency, not myocarditis.

Outcome.-Under rest in bed, with $\frac{1}{6}$ grain of morphin at the time of entrance, magnesium sulphate, I ounce, every morning, $\frac{1}{30}$ grain strychnin three times a day, the heart became more regular, stronger, and a well-marked systolic murmur appeared at the apex. By the seventeenth the edema had gone from the lungs, and the patient was well as long as he avoided any exertion.

Diagnosis.-Myocardial insufficiency.

\section{Case 370}

A Russian carpenter of thirty-four, never previously sick, was first seen December 22, I906. While lifting a heavy piece of timber four months ago he felt something "give way in his chest." He was carried home and has not worked since, owing to dyspnea on the slightest exertion, palpitation, and dry cough. He needs three or four pillows at night, and sleeps poorly. His appetite and bowels are normal. $\mathrm{He}$ has no urinary symptoms. Despite treatment his symptoms increased four days ago and he has had complete orthopnea and steady pain under the right costal margin. There has been no edema of the feet at any time. Dyspnea, cyanosis, engorgement of the cervical veins, were the striking features at entrance. The heart's impulse was seen and felt two inches outside the nipple in the sixth interspace. There was delirium cordis. A systolic murmur was heard at the apex and in the axilla. The first sound was very sharp, and occasionally, perhaps one 
beat in every four or five, was preceded by a short presystolic roll. The pulmonic second sound was accentuated and double. There were many more beats audible at the apex than palpable at the wrist. (See chart.) There were many fine bubbling râles at the base of both lungs, and slight dulness at the right base. The tender edge of the liver was felt two inches below the costal margin in the nipple-line. The upper

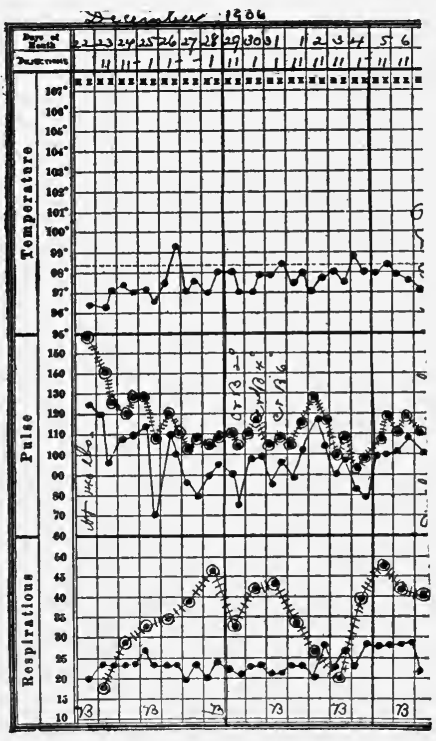

Fig. I 89.-Chart of case 370 . border of the organ was in the fourth interspace. Blood and urine were normal. No ascites.

In the chart (Fig. I89) the line below that representing the temperature stands for the number of heart-beats. Just below this is the radial pulse curve.

Discussion.-The kidney seems to be all right; the heart is obviously diseased. It is there that we should look first for the cause of the dyspnea.

Apparently it is the right ventricle which is laboring hardest. There are no evidences of stasis in the peripheral circulation, but the lungs are evidently congested, while both in the superior and in the inferior vena cava stasis is obvious. The cyanosis and engorgement of the cervical veins betray back pressure in the domain of the superior cava. The pain under the right costal margin, the tenderness, and the enlargement of the liver give evidence that the inferior cava cannot empty properly. All this points to insufficiency of the right ventricle.

When the right ventricle is insufficient, the cause is usually to be found in disease of the mitral valve, much less often in chronic emphysema or other long-standing pulmonary disease. The clinical picture of acute tricuspid regurgitation due to dilatation of the right ventricle without previous mitral disease has been insisted on, especially by Gibson and other writers in Great Britain, but as yet I have not been able to verify their accounts in my own experience.

What form of mitral disease is present in this patient? Besides mitral regurgitation, of which we have all the ordinary classic signs, we have a very sharp first sound, such as rarely accompanies an uncomplicated mitral regurgitation. Even without the occasional occurrence 
of a short presystolic roll we should be right in assuming the presence of mitral stenosis because of the great irregularity of the heart and the sharpness of the first sound at the apex. The doubling of the second sound in the pulmenary area still further justifies this assumption.

Outcome.-The patient was given a dry diet in six meals, tincture of digitalis, ro minims, every six hours, $\frac{1}{6}$ grain morphin subcutaneously, repeated later in the night and on two subsequent nights; magnesium sulphate, I $\frac{1}{2}$ ounces every morning. Under this treatment the heart was much steadier by the twenty-fourth, though still irregular. The area of dulness was smaller, and the left border had retreated almost to the nipple-line. By the twenty-seventh he was able to sleep well without morphin. The presystolic murmur was then much louder, the heart still rapid and irregular. By the third of January the cardiac apex was inside the nipple-line, the patient was able to move about without dyspnea, all the edema had disappeared, and the liver had retreated behind the costal margin. By the seventh he was able to go. home.

Since the heart diminished so markedly in size as the result of treatment, we may assume that we are dealing at the start with a case of acute cardiac dilatation supervening upon a long-standing disease which had narrowed the mitral valve and prevented it from closing tightly.

Diagnosis.-Mitral stenosis and regurgitation. 
TABLE XX.-DyspneA. Signs and Symptoms.

\begin{tabular}{|c|c|c|c|c|}
\hline Causes. & $\begin{array}{l}\text { Suggestions } \\
\text { from history. }\end{array}$ & Local signs. & $\begin{array}{l}\text { Constitutional } \\
\text { disturbances. }\end{array}$ & Relief. \\
\hline Infectious diseases. . . . & $\begin{array}{l}\text { Source of con- } \\
\text { tagion. Mode } \\
\text { of onset. }\end{array}$ & $\begin{array}{l}\text { Depend on what disease } \\
\text { is present. }\end{array}$ & $\begin{array}{l}\text { Fever. Often leu- } \\
\text { kocytosis. Pros- } \\
\text { tration. General } \\
\text { aches. Vomiting. }\end{array}$ & \\
\hline Cardiac disease $\ldots \cdots \cdot$ & Gradual onset. & $\begin{array}{l}\text { Hypertrophy. Arhyth- } \\
\text { mia. Murmurs. Stasis } \\
\text { in lungs, liver, legs. }\end{array}$ & $\begin{array}{l}\text { Weakness. } \\
\text { Insomnia. }\end{array}$ & $\begin{array}{c}\text { Rest. } \\
\text { Depletion. } \\
\text { Stimulation. }\end{array}$ \\
\hline Phthisis ....... & $\begin{array}{l}\text { Family history } \\
\text { of tuberculosis. }\end{array}$ & $\begin{array}{l}\text { Circumscribed apical } \\
\text { bronchitis or solidifica. } \\
\text { tion. }\end{array}$ & $\begin{array}{c}\text { Fever. Emacia. } \\
\text { tion. Dyspepsia. } \\
\text { Weakness. }\end{array}$ & $\begin{array}{l}\text { Hygiene. } \\
\text { Climate. }\end{array}$ \\
\hline Chronic nephritis .... & Gradual onset. & $\begin{array}{c}\text { Cardiac hypertrophy. } \\
\text { Hypertension. Nootu- } \\
\text { ria. Albumin. Edema. } \\
\text { Headache. }\end{array}$ & $\begin{array}{l}\text { Vomiting. } \\
\text { Weakness. }\end{array}$ & $\begin{array}{l}\text { Rest. } \\
\text { Depletion. } \\
\text { Diet. }\end{array}$ \\
\hline $\begin{array}{l}\text { Chronic bronchitis (usually } \\
\text { with bronchiectasis) } \ldots \text {. }\end{array}$ & $\begin{array}{c}\text { Winter } \\
\text { exacerbations }\end{array}$ & $\begin{array}{l}\text { Bilateral râles. } \\
\text { Nummular sputa. }\end{array}$ & $\cdots \cdots \cdot$ & $\begin{array}{l}\text { Climate. } \\
\text { Hygiene. }\end{array}$ \\
\hline Pneumonia $\ldots \ldots$ & Sudden onset. & $\begin{array}{c}\text { Axillary pain. Lobar } \\
\text { or lobular solidification. }\end{array}$ & $\begin{array}{l}\text { Fever. Herpes. } \\
\text { Leukocytosis. } \\
\text { Vomiting. } \\
\text { Weakness. }\end{array}$ & Time. \\
\hline Asthma $\ldots \ldots \cdots$ & $\begin{array}{l}\text { History of } \\
\text { previous } \\
\text { paroxysms. }\end{array}$ & $\begin{array}{l}\text { General hyperreso- } \\
\text { nance. Piping râles. } \\
\text { Eosinophilic sputa. }\end{array}$ & Slight. & $\begin{array}{l}\text { Morphin. } \\
\text { Nitrites. }\end{array}$ \\
\hline Emphysema . . . . & Gradual onset. & $\begin{array}{l}\text { "Barrel chest." Ex- } \\
\text { tension of lung borders. } \\
\text { Hyperresonance. }\end{array}$ & Slight or absent. & \\
\hline
\end{tabular}




\section{CHAPTER XXII}

\section{JAUNDICE}

THERE is no authoritative statement or logical rule which settles the minimum amount of discoloration which shall receive the name "jaundice," but the general consensus of usage applies the term to all cases in which there is distinct yellowing of the conjunctiva, whether the skin and urine show any demonstrable change or not. This conjunctiva yellowing must be distinguished from the yellowish patches of subconjunctival fat to be seen in many eyes.

In the milder cases of jaundice we can see around the iris a ring of bluish-white sclera over which there is no discoloration. In the more intense types the yellow color meets the iris.

Like all judgments depending upon a color test alone, the decision whether or not jaundice is present is by no means an infallible one. Careful inspection of the deeper portions of the conjunctiva in many healthy persons shows a faint shade of yellow from time to time, and it is always more or less arbitrary where we draw the line between this supposedly physiologic condition and true jaundice. If the skin and urine are not discolored, and if none of the symptoms of gastroduodenal catarrh, gall-stones, or cancer are present, it is customary to overlook and disregard many a faint shade of yellow upon the eyeball, but I am not sure that this practice is wise.

\section{TYPES AND CAUSES OF JAUNDICE}

The distinction between a hematogenous and a hepatogenous jaundice has gone, never to return. Its immortal soul survives in the division between-(a) Jaundice which owes its origin in the first instance to an infectious disease, such as puerperal sepsis or malaria, and $(b)$ jaundice due to mechanical obstruction, such as gall-stone or cancer. All jaundice is hepatogenous in its production, but the original cause may be infectious or mechanical.

Doubtless the most common cause of jaundice is the unknown one, which produces it in so large a percentage of all newborn children, and usually occasions no diagnostic difficulties. In clinical work we are apt to be puzzled especially by cases of jaundice resulting from three causes: 
(a) Gall-stones.

(b) Cancer.

(c) So-called catarrhal form.

Less common and less puzzling in diagnosis are the cases due to:

(d) Cirrhosis.

(e) Syphilis of the liver.

All these are of the obstructive type, and are therefore distinguished from the infectious varieties mentioned above. Rare and obscure causes for jaundice are exemplified in:

(f) Acute liver atrophy, with or without poisoning by chloroform or phosphorus.

(g) Weil's and the other types of infectious jaundice of unknown origin.

(h) Family hemolytic jaundice.

The so-called catarrhal jaundice is probably the commonest of all the forms just mentioned. It is also the least understood. The old idea of a catarrh spreading up into the common bile-duct from the duodenum has very little support either in postmortem demonstration or in the clinical course of the disease. Many of the cases bear all the external evidences of a mild general infection and are indistinguishable, when they occur sporadically, from Weil's disease, which is a name given to epidemics of jaundice associated with a fever lasting from four to nine days, a sudden onset with muscular pains and sometimes with a palpable spleen.

Both catarrhal jaundice and Weil's disease are distinguished from acute yellow atrophy of the liver only by their course, and, for aught we know, may be, in fact, mild forms of the same infection. The liver is notoriously strong in its power of regeneration after injury, and it may well be that the cases which we now term acute yellow atrophy represent merely its occasional failures, while catarrhal jaundice and Weil's disease exemplify its much more frequent victories over some of the poisons that lead to necrosis.

Painless jaundice, coming on insidiously and lasting more than six weeks in elderly persons, is strongly suggestive of cancer in the biliary passages.

\section{ASSOCIATED SYMPTOMS}

A slow pulse, a tendency to mental depression and to uncontrollable oozing from any wounded surface, are usually associated with the severer types of jaundice. More troublesome and more interesting is the itching, which is frequently but by no means always a concomitant of jaundice. A patient of mine suffered two attacks of severe jaundice 


\section{Causes of Jaundice}

1. ICTERUS NEONATORUM

2. SEPSIS

3. GALL-STONES

4. "CATARRHAL
JAUNDICE"

5. CANCER OF LIVER

CASES TOO NUMEROUS AND TOO VAGUELY ENUMERABLE FOR GRAPHIC REPRESENTATION.

369

131

57
6. CIRRHOSIS

48

7. CANCER OF

BILE-DUCTS

AND GALL-

BLADDER

8. CANCER OF ?

PANCREAS

9. GASTRIC CAR-
CINOMA

12

$\left.\begin{array}{l}\text { 10. CANCER OF } \\ \text { DUODENUM }\end{array}\right\}$ 

within six months. Both were due to gall-stones and ran approximately the same course, but in one he was tormented with itching, in the other he was wholly free from it. About half the cases itch and half do not. This proves to my satisfaction that the itching is not due merely to the presence of bile in the skin and subcutaneous tissues. Some other and less constant factor must be present when itching occurs.

\section{INTENSITY OF JAUNDICE}

As a rule, the deepest discoloration occurs in complete and permanent occlusion of the bile-ducts by cancer. In gall-stones the depth of the yellow staining is apt to vary from week to week. In the so-called catarrhal forms the color is usually paler, but there are striking exceptions to this rule. The jaundice of infectious disease, of hepatic cirrhosis, and syphilis is generally moderate in degree.

\section{Case 371}

A laboratory worker, forty-seven years of age, entered the ward July 30, I906, with the following history. Two weeks ago, while on his vacation, he felt some discomfort after eating and lost his appetite. A day or two later his skin turned yellow, his urine dark. Five days after this his stools became clay-colored. He has vomited only twice, yesterday and the day before. He has no pain. His bowels move daily. He feels very mean and seedy and is troubled with itching. Two weeks ago he weighed I6I pounds, now he weighs I42. He has never had an attack similar to this.

On examination, the edge of the liver is easily felt below the costal margin. The jaundice is fairly well marked. In other respects the physical examination, including blood and urine, is negative. The patient seemed unaccountably weak and continued so even up to the twelfth of August. At that time his color began to fade.

Discussion.-A jaundice of four weeks' duration, associated with enlargement of the liver, loss of weight, and marked prostration in a man forty-seven years old.

He has had no pain, no chill, fever, or palpable gall-bladder in this attack. He has had no previous seizures. So far as this evidence goes, it is against the diagnosis of stone, though it is perfectly possible that stone may exist.

Malignant disease was much feared by the patient, who could not understand why he was so weak and thin unless there was some very serious disease underlying his symptoms. But of malignant disease, 
as of stone, we have no positive evidence. After four weeks of complete jaundice one expects to find ascites, enlarged gall-bladder, or nodular liver if the jaundice be due to malignant disease. The absence of any history of previous stomach trouble is also a comforting consideration, since malignant disease involving the liver is usually preceded by cancer of the stomach. In particular, however, it should be said that emaciation during an attack of jaundice is no evidence whatever regarding its cause and is just as likely to occur in a gall-stone attack as in malignant disease.

Of the other common causes of jaundice, such as cirrhosis, syphilis, or acute infectious disease (toxemic jaundice), we have no evidence.

Under these conditions, when we have exhausted our efforts in the attempt to find evidence of stone, cancer, and the other less common causes of jaundice, we fall back upon the old term "catarrhal jaundice," one of the most insecurely founded of all the diagnoses that are in good standing at the present time. If the facts were known, it would probably turn out that a considerable number of the cases called "catarrhal jaundice" are really due to stone and that the remainder are, like purpura, the expression of various unnamed infectious processes. Jaundice is almost as general and indistinctive a manifestation as fever.

Outcome.-By the sixteenth of August the patient's appetite had returned, and after a short vacation in the country he came back to work apparently in perfect health. Up to the present time (May, I9IO) he has remained entirely well.

Epicrisis.-This case is fairly illustrative of two points:

(a) The vague opportunism of our diagnoses of catarrhal jaundice, which must be changed at any moment if more distinctive symptoms pointing to gross organic disease make their appearance. At best such a diagnosis is justified only by the outcome of the case, and at any time we may have to eat our words if colic, ascites, or a palpable mass appears.

(b) The frequency of emaciation in jaundice of any type. I have known a physician to be seriously alarmed about his own condition during the course of an attack of jaundice, obviously due to gall-stone, because, as he said, "How can a little stone stuck in a duct make me lose 40 pounds in two months?" Nothing but the removal of the stone and his rapid return to his former weight and health convinced him: Whether the emaciation in cases of this kind is wholly the result of anorexia and insufficient food, or whether there is some more obscure reason connected with the functions of the liver, I do not know.

Diagnosis.-Catarrhal jaundice. 


\section{Case 372}

A stableman of forty-two entered the hospital June 16, 1908. Within three years the patient has had three attacks of rather persistent indigestion, characterized by sharp, colicky pain localized about the umbilicus and sometimes needing morphin. He says he has never been jaundiced. Two years ago he weighed I80 pounds; two months ago, I70, now he weighs 134 . He averages two glasses of beer a day and twenty cents' worth of tobacco a week.

For the past two weeks he has been in bed most of the time, complaining of drowsiness, anorexia, slight fever, and aches all over his body, especially in the lumbar region. He saw a physician for the first time four days ago, who told him that he was jaundiced. At that time his skin began to itch and his stools to be a light clay color. The urine has been dark for a week. The course of the temperature is seen in the accompanying chart.

Examination showed moderate jaundice and nothing else except that the white cells were

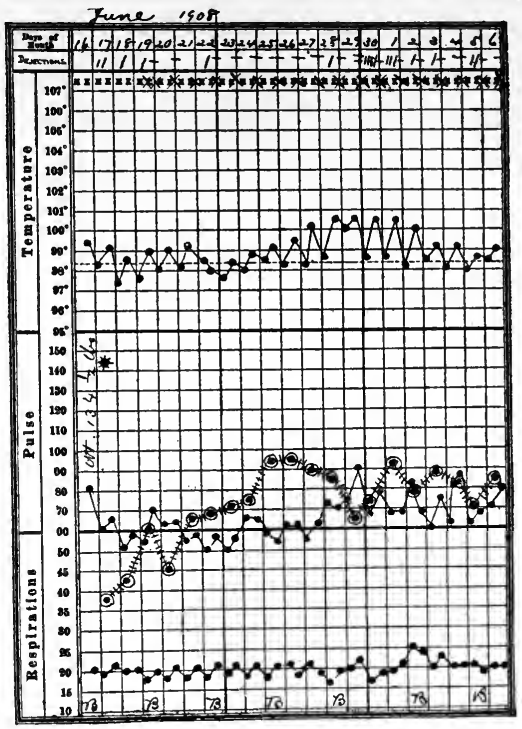

Fig. 190.-Chart of case 372 . I7,000 at entrance, declining three days later to 12,000 . The urine was always normal, except for the presence of bile. Blood-pressure was $145 \mathrm{~mm}$. $\mathrm{Hg}$.

Discussion.-In the discussion of a previous case I referred to the humiliating fact that in many cases of jaundice we have to wait for time to show whether our conjectures are right or not. Experience has shown that most cases of so-called "catarrhal" jaundice clear up within six weeks, and that most of those which run over this limit turn out to be due to gall-stones or malignant disease. The period referred to is, of course, a perfectly arbitrary one, based upon averages, and with very little anatomic evidence to support it. The present case ran its course within this traditional limit without the development of any pain, ascites, nodular mass, or toxemic state.

Nevertheless, we can by no means be sure that the attack was not 
due to gall-stones, especially as he has had three previous seizures which remind us very distinctly of that disease. If, at any time within the next few years, the patient has a typical gall-stone attack, it will seem more than likely that the present attack, as we view it in retrospect, was also due to gall-stones.

Although the relation of alcohol to cirrhotic liver (a possible cause of jaundice) is not clear, we certainly know enough to say that this patient has not taken enough beer to put him in peril of chronic interstitial hepatitis. Of the nature and development of that disease we know so little from a clinical standpoint that we are unable to make positive statements about its earlier stages and their relation to symptoms like those here described. Certainly, however, no one would be justified in giving more than passing consideration to cirrhosis in the present case.

Outcome.-On the twenty-seventh the jaundice was much less marked and the patient was hungry. On the eighteenth of July the jaundice had completely disappeared, the patient had gained six pounds since entrance, and felt entirely well.

On the first of September he reported that he had been perfectly well and had worked ever since leaving the hospital. His weight was I $5^{\mathrm{I}}$ pounds. There was no evidence of jaundice.

Diagnosis.-Catarrhal jaundice.

\section{Case 373}

A schoolboy of thirteen, always previously well, consulted a physician with the statement that for two months he had been jaundiced and had intermittent dull pains across the upper abdomen. At the onset of his illness he had a chill and considerable vomiting for three days, but these symptoms have not recurred. He was first seen September 4, I907.

Examination showed deep jaundice. The spleen and liver easily felt. (See accompanying diagram, Fig. I9r.) The edge of the spleen was hard, the whole organ freely movable. The white cells numbered 3800; hemoglobin, Ioo per cent.; stained specimen normal, as was the urine. The feces were not bleached. Later it was ascertained that a year ago he had had chills and fever every other day for a considerable period.

The boy was given liquid and soft solid diet, sodium phosphate, $\frac{1}{2}$ dram every morning, and under this treatment rapidly improved. $\mathrm{He}$ was able to take full diet by the seventh, was much less jaundiced by the ninth, and by the fourteenth had no visibie discoloration of the skin or conjunctivæ. In view of the history it seemed best to give him $\mathrm{I} \frac{1}{2}$ grains of quinin three times a day for ten days. 


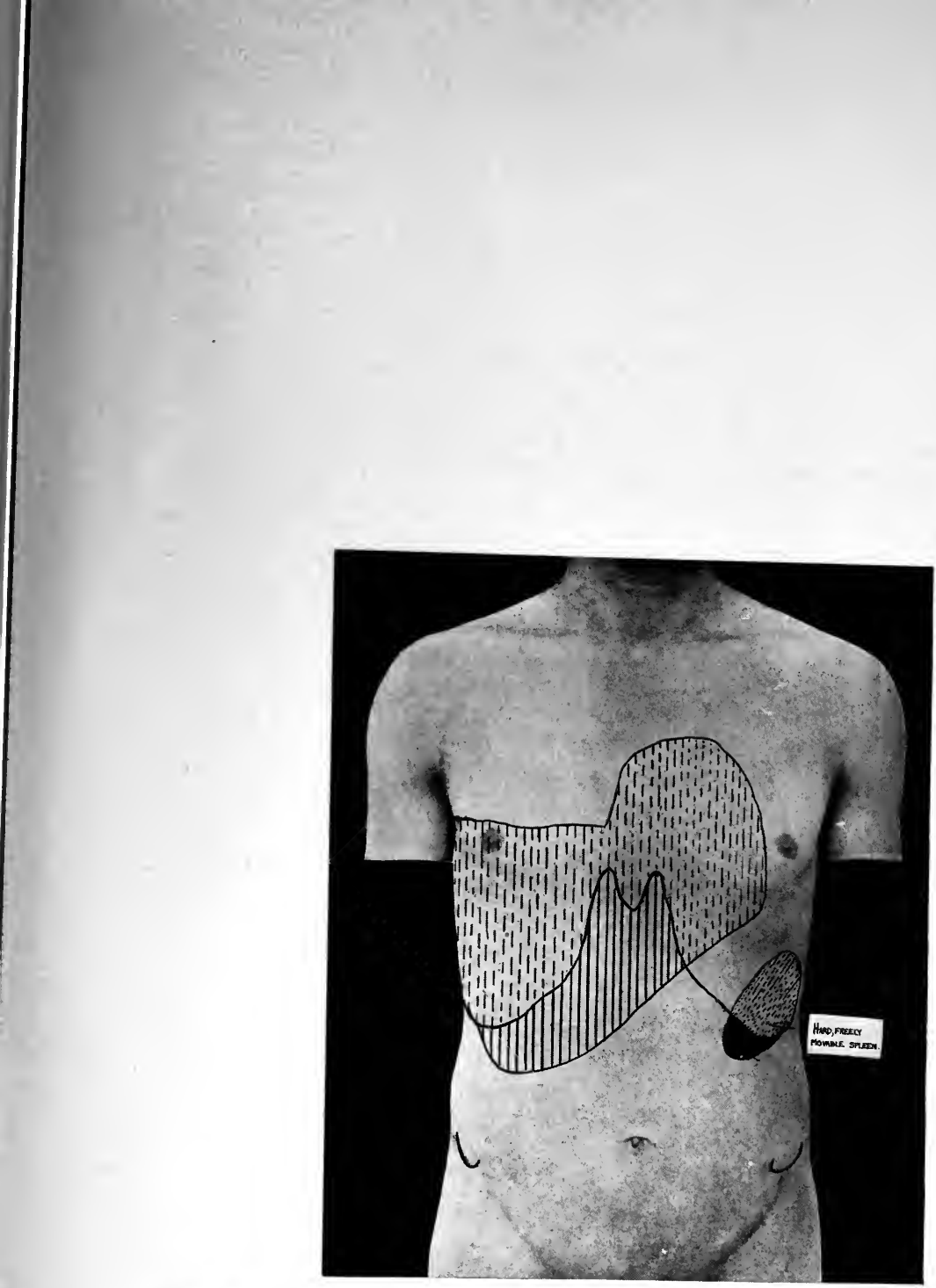

Fig. 191.-Shows results of physical examination in a case of jaundice of two months' duration. 

Discussion.-This patient had absolutely nc complaints at the time when this history was written. His parents wanted to know why his eyes were yellow, but he himself did not feel sick at all.

The presence of an enlarged hard spleen and the history of a chill at the onset remind us that malaria is a not infrequent cause of jaundice. Yet certainly at the present time he has no active malarial infection. Is it possible that the icterus may be a relic of a past malaria? Certainly in the more severe types of the disease jaundice often persists when no parasites are discoverable in the peripheral circulation and when the temperature is steadily normal. In the milder tertian infections of New England one does not often see this, especially when the attack has not destroyed enough corpuscles to lower the hemoglobin, which was Ioo per cent. in this case. On the whole, therefore, this hypothesis seems unlikely.

Gall-stones are rarely found in boys of this age. The pains which previously troubled him were never such as to suggest biliary colic, and the fact that the stools were never decolorized makes it seem improbable that the stone has never blocked the ducts.

Catarrhal jaundice may occur at this age, though it is not common. If we use the term to include any brief icterus of unknown origin and sporadic occurrence, it will doubtless cover such cases as this, even though no digestive symptoms, such as should accompany a gastroduodenal catarrh, were complained of. In all probability the jaundice represents one manifestation of an acute infectious disease. Had it occurred in an epidemic form, it might have passed as Weil's disease.

Outcome.-On October r6th the boy returned to the physician to report that two days previously he had had a chill and fever. The boy had gained considerably in flesh, and looked entirely well. The spleen was still palpable, the liver no longer so. Malarial parasites were now demonstrated in the blood. In view of this fact it seems, on the whole, probable that his jaundice was due after all to malaria.

Diagnosis.-Tertian malaria.

\section{Case 374}

A housewife of thirty-eight, of good family history and past history, entered the hospital September 3, 1907. She began six months ago to have what she calls "a ball" in the stomach, starting at the epigastrium and moving toward the left hypochondrium. Apparently she has steady, dull epigastric pain, not radiating, accompanied by heart-burn. anorexia, a bad taste in the mouth, distress and flatulence after eating, constipation, and occasional vomiting of the food last taken. Through- 
out this six months she has had jaundice, varying in intensity. She has had also occasional chills, followed by profuse sweating, and always by an increase in the jaundice and in the color of the urine. At no time has she had any sudden or sharp pain anywhere. She has lost 3o pounds in the last six months. She sleeps poorly on account of flatulence.

Examination shows an obese, jaundiced woman, with an indefinite resistance under the costal margin and in the median line. Physical examination, including the temperature, pulse, respiration, blood, and urine, is otherwise negative, save for the presence of bile in the urine.

Discussion.-If this jaundice had not already lasted for six months, it would very probably deserve to be called "catarrhal," as no doubt it was called in the earlier weeks of its occurrence. No one maintains, however, that the term "catarrhal" should be extended to cover cases of six months' duration.

The variations in the intensity of the jaundice and the occurrence of chills without malarial parasites in the blood lead us to favor the diagnosis of gall-stones. But can one have gall-stones without any colic; indeed, without any pain except such as might be attributed to flatulence? Thanks to the surgeon we may now answer this question with an unqualified affirmative. Colic is a common but by no means an invariable accompaniment of cholelithiasis.

The loss of weight which occurs in all forms of jaundice has been discussed in the previous cases and shown to have in itself no diagnostic significance. The age, the sex, and the obesity all favor the diagnosis of gali-stones.

Outcome.-Operation September 6th revealed a stone in the lower end of the common duct. The bile-passages were otherwise free, the liver and pancreas not abnormal. The patient's convalescence was uneventful, and after October 6th she seemed and remained entirely well.

Diagnosis.-Gall-stones.

\section{Case 375}

An Irish housekeeper of thirty-eight, whose husband now is consumptive, entered the hospital August 29, 1907. She has always been well, and has a good family history. For nine years she has had frequent sick headaches, accompanied by vomiting. For two years she has had gradual loss of weight and strength, her weight declining from I59 pounds two years ago, to IIg, at the present time. 


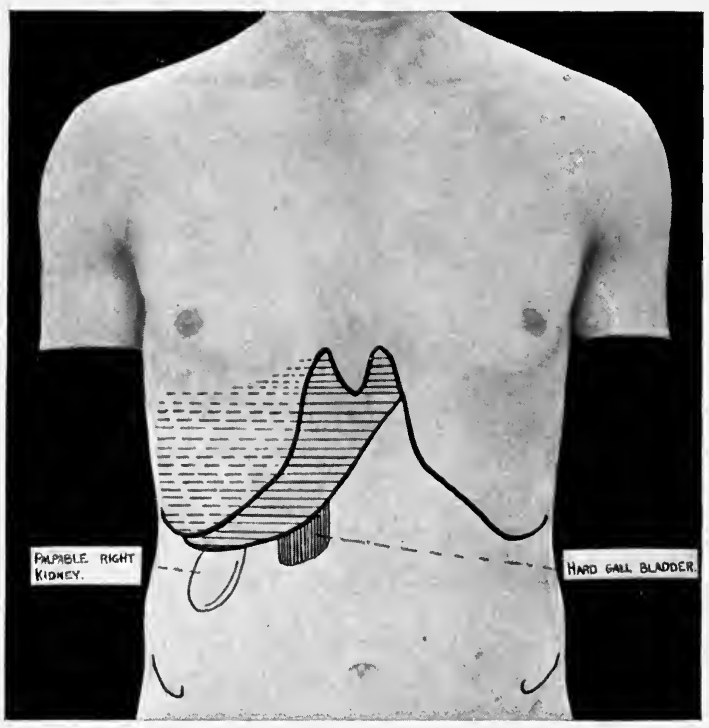

Fig. 192.- Shows conclusions drawn from examination of a case of intermittent painless jaundice lasting three months. 
For three months she has had jaundice, varying in intensity. She has had no pain at any time. Two hours after eating she not infrequently vomits, the vomitus consisting only of food. Her appetite is good. The bowels move with the aid of sodium phosphate. She sleeps well. Her present complaint is of weakness and jaundice.

Physical examination shows the jaundice to be heavy. The chest is negative. The condition of the abdomen is shown in the accompanying diagram, Fig. 192. Blood and urine are normal. The coagulation time of the blood is one minute five seconds with the Brodie-Russell instrument.

Discussion.-A gradual decline in weight and strength during a period of two years, leading up to a jaundice of three months' duration and of variable degree, and accompanied by occasional attacks of vomiting, is rather an unusual clinical picture in a woman of thirtyeight. Why should the loss of weight have preceded the jaundice unless some form of malignant disease is present? Yet if any such disease were present it should, by this time, show more obvious evidence of itself. Without ascites, marked gastric symptoms, or palpable tumor we certainly cannot make a diagnosis of such terrible significance to the patient. Yet from our own minds it is difficult to exclude the thought of cancer.

Cirrhosis or syphilis of the liver would probably show more definite signs of their presence after an illness of this length.

Under such conditions, when a jaundice has lasted rather too long to be calied "catarrhal," yet has not produced any of the more ominous evidences of cancer, cirrhosis, or syphilis, the outcome usually shows that we are dealing with gall-stones. We have to be governed largely by statistical evidence in such cases; direct examination yields very little of value. Indeed, there is no class of diseases in which we depend so largely upon the history and upon general statistical experience as we do in diseases of the liver. Physical examination plays here a smaller part than in the diseases of any other organ with which we deal on terms of any confidence.

Outcome.-At operation, September 6th, several large stones were found in the gall-bladder and one in the cystic duct. No obvious reason for the jaundice was found; there was no swelling extending down from the blocked cystic duct to the hepatic or to the choledochusan explanation which is often invoked to explain jaundice when the stone is in the cystic duct. No other reason could be found.

Recovery was complete and permanent.

Diagnosis.-Gall-stones. 


\section{Case 376}

An Italian widow of sixty, of negative family history and past history, was first seen March 9, I908. She had noticed a year ago that she was jaundiced and had a swelling in the region of her liver. After two weeks she was cured of her jaundice, but the swelling continued and increased. Seven months ago the jaundice returned and has been present most of the time since. She has not lost much weight, though her appetite is poor. She has much nausea, no pain, and no vomiting. For two weeks she has had edema of the feet.

Physical examination showed a deeply jaundiced patient. Both pupils were irregular, the left larger than the right. Both reacted normally. The heart's action was at times irregular in force and rhythm, and a blowing systolic murmur was audible all over the precordia. The pulmonic second sound was louder than the aortic second, the apex beat in the fifth space just outside the nipple-line. The pulse was of low tension. The lungs showed nothing abnormal. The abdomen was enormously distended, dull in the epigastrium and in the flanks, otherwise tympanitic. The circumference was 40 inches. The umbilicus protruded. The edge of the liver was easily felt 5 inches below the costal rnargin. Its surface was smooth, hard, not tender, somewhat irregular. The spleen was not felt. There was considerable soft edema of the legs and of the anterior abdominal wall. The shape of the abdomen suggested encysted rather than free fluid. Blood and urine were normal.

On the eleventh 233 ounces of fluid were withdrawn by tapping the abdomen. It was bile-stained, Ioog in specific gravity. The sediment showed 60 per cent. of lymphocytes to 40 per cent. endothelial cells. After tapping, the surface of the liver was apparently smooth, and extending down from its edge in the region of the gall-bladder was a dense, fluctuant, rounded mass the size of an apple, not tender. (See Fig. I93.) After tapping, the fluid rapidly reaccumulated. The patient seemed entirely comfortable, complaining of nothing at all. She was again tapped on the third of April and I64 ounces removed, the characteristics of the fluid being essentially the same as before. About this time she ran a moderate, irregular fever, reaching as high as $\operatorname{IOI}^{\circ} \mathrm{F}$. at night, and being normal in the morning. This subsided after about ten days.

Discussion.-With jaundice of seven months' duration, a liver markedly enlarged, ascites, and swelled legs, we should have no considerable doubt that malignant disease is present were it not for the 


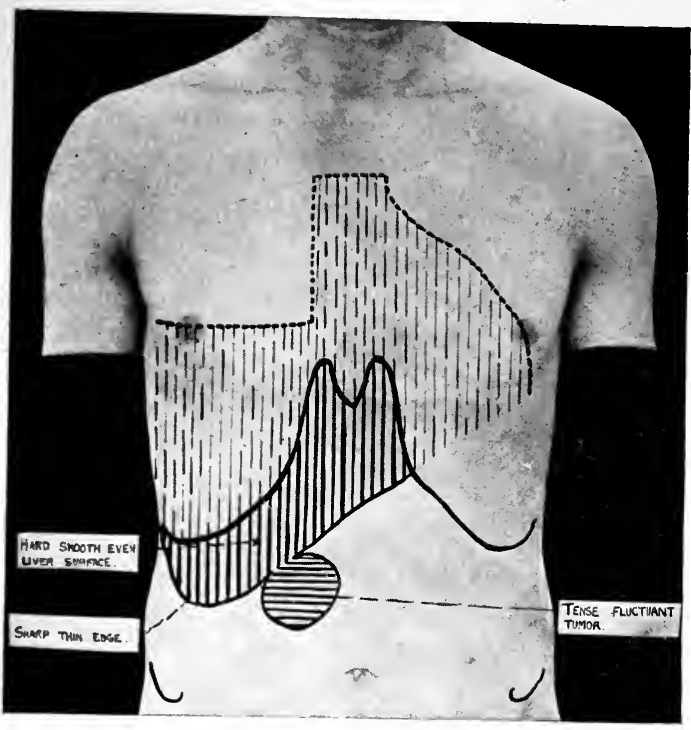

Fig. 193.-Results of physical examination in Case 376 . Jaundice has lasted one year. No pain or emaciation. 

fact that the patient has also an enlarged and irregular heart, whose action is presumably insufficient to maintain satisfactory circulation. The ascites and edema may be due to cardiac weakness rather than to malignant disease.

On the other hand, the irregularity of the liver surface, if it be a fact, is of decisive importance in this connection; for such irregularity, if gross enough to be palpable through the abdominal wall, means one of two things in practically all cases, viz., hepatic cancer or hepatic syphilis. If the first physical examination be correct, then, the cardiac condition is probably not responsible for the dropsy.

As between cancer and syphilis, we should be influenced, in the first place, by statistical considerations; cancer is by far the commoner of the two as the cause of jaundice and ascites. The absence of splenic enlargement is also against syphilis. Less important is the absence of any history of syphilis and of any evidence of its ravages in other parts of the body.

If we are dealing with cancer, what is its site? Probably not the substance of the liver, since hepatic cancer is usually secondary to similar disease at the pylorus. This patient has been free from gastric symptoms. If not in the liver itself, the cancerous obstruction which has produced the jaundice is probably at one of three points:

(a) At the duodenal papilla.

(b) In the head of the pancreas, compressing the common duct.

(c) In the gall-bladder or bile-ducts themselves. Beyond this we cannot go.

Outcome.-April I2th, after more than a month in a hospital, she showed absolutely no loss of weight and we were rather shaken in our confidence that cancer was the correct diagnosis. Nevertheless, operation was performed April I 4 th, as the patient showed no signs of improvement after a thorough course of antisyphilitic treatment. A cancer of the head of the pancreas was found.

Diagnosis.-Pancreatic cancer.

\section{Case 377}

An American timekeeper of twenty began, in I903, to have epigastric pain, after eating, in intermittent spells lasting a month or two. In January, 1907 , this pain became much worse, and he vomited fresh blood. In March, 1907, he entered the hospital and was found to have a marked hyperchlorhydria, which, taken in connection with the above symptoms, led to an operation, which showed adhesions about the pylorus and considerable thickening of the pylorus, without evidence 
of cancer. A posterior gastro-enterostomy was accordingly done. The patient did excellently well, and went home free from symptoms on the seventh of April, I907.

He returned a year later (April 24, I908) with the following history: A month ago became suddenly jaundiced immediately after taking some sulphur and molasses. He has remained so ever since, though his color has been becoming lighter. At the same time he has complained of rather dull pain, felt from time to time in the lower abdomen. For a week this has been absent. 'For two weeks he has not worked, and has been on a milk diet. During this time he has had a slight cough, with yellowish or greenish sputum. He has a great deal of itching, and has noticed that his urine is dark. Yesterday at half-past four he ate a very hearty dinner. Later in the day he vomited and gradually became unconscious, possibly from the effects of morphin which was given in the evening. There is no headache, no fever, no loss of weight.

Examination shows normal temperature, pulse, and respiration. There is marked jaundice. The patient is semicomatose, with dilated pupils which do not react. The chest is negative. The abdomen is level, rather rigid, tympanitic; nothing else is made out. The liver flatness reaches from the fifth rib to the seventh space, measuring $2 \frac{1}{2}$ inches in vertical diameter in the nipple-line. The urine is high in color, specific gravity I020, with a very slight trace of albumin in the sediment, no casts, a large amount of leucin and tyrosin. The white cells are 9200; hemoglobin, 75 per cent.

Discussion.-We will take first the second chapter of this patient's case, after his recovery from the gastro-enterostomy.

In the earlier weeks of his jaundice the brittle and unsatisfactory term "catarrhal" was as usual applied, and one could hardly have done otherwise until the appearance of one very distinctive and ominous symptom, stupor. None of the milder and more curable causes of jaundice produce this symptom. We never meet it in the catarrhal forms, in gall-stones, or in malignant disease previous to the terminal stages. In the infectious forms of jaundice, stupor appears only near a fatal issue.

Only in two forms of liver disease which are accompanied by jaundice does stupor appear-in cirrhosis and in acute yellow atrophy. Either of these diseases might be present here, although we have no history of alcoholism such as usually appears to enter into the causation of cirrhosis, at any rate, as one factor. Another point against cirrhosis and in favor of acute yellow atrophy, is the rapidity of the shrinkage apparently demonstrable by percussion of the liver outlines. Shrinkage of the 
liver can very seldom be demonstrated during life. When the area of liver dulness appears to be less than normal it usually turns out to be obscured by a distention of the colon which pushes the liver backward out of reach of the percussing finger.

In the present case, however, the area of dulness was again and again measured, and showed apparently a progressive shrinkage. This fact, taken in connection with the deep jaundice, the increasing coma and the presence of leucin and tyrosin in the urine, made us tolerably confident that a rapid atrophy of the liver was in progress. No hint of its etiology was obtained; the patient had not inhaled chloroform or ingested phosphorus in any form.

Outcome.-On the morning of the twenty-sixth the liver dulness was only $\mathrm{x} \frac{3}{4}$ inches in vertical diameter; the jaundice had considerably decreased. The patient continued semicomatose. On the afternoon of the twenty-sixth he developed edema of the lungs and died.

Autopsy showed acute yellow atrophy of the liver, obsolete tuberculosis of the right lung and bronchial lymphatic glands, acute degeneration of the kidneys.

Diagnosis.-Acute yellow atrophy of the liver.

TABLE XXI.-JAUNDICE. Signs and Symptoms.

\begin{tabular}{|c|c|c|c|c|}
\hline Causes. & $\begin{array}{l}\text { Favoring } \\
\text { conditions. }\end{array}$ & $\begin{array}{l}\text { Accompanying } \\
\text { signs (local). }\end{array}$ & $\begin{array}{l}\text { Constitutional } \\
\text { manifestations. }\end{array}$ & Relief. \\
\hline Sepsis . . . . . . & $\begin{array}{l}\text { Severe } \\
\text { infection. }\end{array}$ & $\begin{array}{l}\text { Depends on type } \\
\text { of infection. }\end{array}$ & $\begin{array}{c}\text { Fever. I.eukocyto- } \\
\text { sis (usually) } \\
\text { Chills. Anemia. }\end{array}$ & $\begin{array}{l}\text { Drainage. } \\
\text { Time. }\end{array}$ \\
\hline Gall-stones ....... & $\begin{array}{l}\text { Fat old women. } \\
\text { Typhoid. }\end{array}$ & $\begin{array}{l}\text { Biliary colic, often. } \\
\text { Tenderness, often. } \\
\text { Enlarged gall- } \\
\text { bladder, often. }\end{array}$ & $\begin{array}{l}\text { Chills, often. } \\
\text { Fever, often. } \\
\text { Emaciation, often. }\end{array}$ & $\begin{array}{l}\text { Passage of } \\
\text { stone. } \\
\text { Operation. }\end{array}$ \\
\hline "Catarrhal jaundice" . . . & $?$ & $\begin{array}{l}\text { Sometimes } \\
\text { enlarged liver. }\end{array}$ & $\begin{array}{l}\text { Depression. } \\
\text { Slow pulse. }\end{array}$ & $\begin{array}{l}\text { Tonic (under } \\
\text { six weeks). }\end{array}$ \\
\hline Cancer of liver . . . . . & $\begin{array}{c}\text { Previous } \\
\text { gastric cancer. }\end{array}$ & $\begin{array}{l}\text { Enlarged nodular } \\
\text { liver. Gastric } \\
\text { tumor (?). }\end{array}$ & $\begin{array}{l}\text { Emaciation. } \\
\text { Fever. Anemia. }\end{array}$ & 0 \\
\hline Cirrhosis of liver . . . . . & Alcoholism. & $\begin{array}{l}\text { Liver usually en- } \\
\text { larged. Portal } \\
\text { stasis. Splenic } \\
\text { tumor. }\end{array}$ & $\begin{array}{l}\text { Emaciation. } \\
\text { Anemia. }\end{array}$ & $\begin{array}{l}\text { Tapping. } \\
\text { Operation (?). }\end{array}$ \\
\hline $\begin{array}{l}\text { Cancer of bile-ducts and gall- } \\
\text { bladder, pancreas, or duo- } \\
\text { denum }\end{array}$ & $\begin{array}{l}\text { Later middle } \\
\text { life. }\end{array}$ & Big gall-bladder. & $\begin{array}{l}\text { Emaciation. } \\
\text { Anemia. }\end{array}$ & • \\
\hline
\end{tabular}




\section{CHAPTER XXIII}

\section{NERVOUSNESS}

THE uses of this word are so vague and various that one may be seriously misled unless one cross-questions the patient as to just what he means when he calls himself "nervous." Thus, for example, nervousness may be-

(a) Motor, wholly or largely. The patient may have what is called "the fidgets," and be unable to keep still or to control the motions of some part of his body, as in Sydenham's chorea, or in the habit choreas and muscular twitchings so common in neurotic people. The tremors of general paralysis or hyperthyroidism are sometimes referred to by the patient as "nervousness." When we are tired, especially in the evening, it is sometimes almost impossible to keep the feet and legs quiet. Sleep is the cure for this type of motor nervousness. A similar fidgety state is seen in anemia from hemorrhage.

(b) Sensory. When people start at any slight noise or jar, when they are abnormally sensitive to light, to odors and tastes, they often speak of themselves as "nervous."

(c) Psychic. Perhaps the commonest usage of the word "nervousness" is in connection with a variety of predominantly psychic manifestations, such as lack of self-control, emotionalism, fearfulness, causeless and transient depression, irritability, and the sense of unreality in things.

(d) Visceral and secretory neuroses often occur in connection with one or another of the types above mentioned, and may constitute the most prominent part of the clinical picture, but they are not apt to be referred to by the patient as "nervousness." The patient is more apt to believe them due to some more or less serious organic disease.

Interpretation of Nervousness.- The most important point is that identical nervous symptoms may occur with or without organic disease behind them. A patient whose underlying malady is arteriosclerosis or chronic glomerulonephritis may yet present typical symptoms of hysteria or neurasthenia, and the latter are so insistent and so irritating that we are apt to neglect a thorough search for something else in the background.

7 Mistakes are especially apt to occur in persons over forty years of 
age. When nervousness of any type has appeared for the first time after the fortieth year in a patient who has never previously shown anything of the sort, a diagnosis of neurasthenia or hysteria usually turns out wrong or seriously insufficient and leads us to give a faulty prognosis and to misdirect our treatment.

In younger persons nervousness is often falsely treated as the complete diagnosis when, in fact, it is merely an expression of an underlying pulmonary tuberculosis. A great many of the cases of nervous dyspepsia and of pallor miscalled "anemia" turn out to be the earliest manifestations of tuberculosis.

There is a great deal of skepticism among surgeons as to the existence of any such entity as nervousness or psychoneurosis without definite anatomic basis. Surgeons are constantly "discovering" that this or that lesion in the abdomen is the "real cause" of what the rest of us foolishly call neurasthenia. But it is interesting to note that these surgical discoveries come and go with great frequency. They have, I believe, no considerable foundation in fact. Nevertheless the instinct back of them is, in my opinion, correct. They instinctively and rightly seek a cause back of the psychoneurosis. But this cause is more probably chemical, not surgical or mechanical. I have hopes that the development of our knowledge of the ductless glands will increase our understanding and our control in the so-called psychoneuroses. I have very little faith in the psychogenic or purely mental origin of any long-standing complaint.

Other types of mistake will be exemplified in the cases to follow.

\section{Case 378}

A Russian housemaid of twenty-four, with a good family history, was first seen April 27, 1907. She has never been strong. She has suffered from headaches and dysmenorrhea for the past seven years. These pains just now are not so troublesome as formerly. Of late she has been weak, faint, and worn out. Two weeks ago she was operated upon for hemorrhoids at the Boston City Hospital. Since that time she has had a great deal of nervousness with pain scattered throughout various parts of her body ("Schmerzen Uberall"), pressure about the heart, eructations of gas, dryness of the mouth, and the frequent discharge of pale urine. Her appetite is rather poor, the bowels regular. The patient has a cyanotic look. At entrance to the hospital her "cribbing," nervous vomiting, convulsive chills, and moans disturbed the whole ward. Visceral examination was negative. The course of the temperature is seen in the chart on page 746 . 
Discussion.-Obviously, we are dealing with a psychoneurosis, but are we sure that there is nothing behind it? We are told that

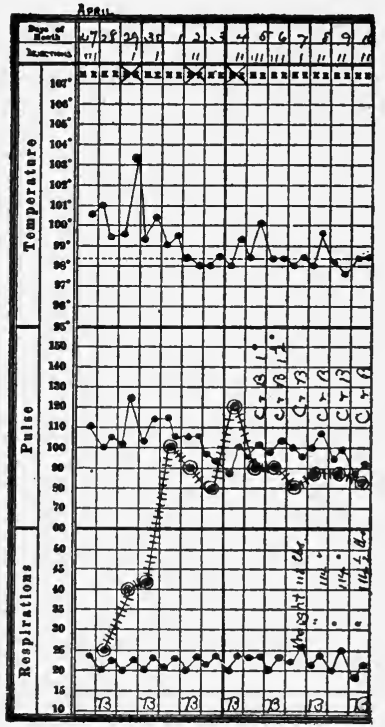

Fig. 194.-Chart of case 378 . visceral examination is negative, but visceral examination is not always taken to include a study of the blood and urine. Among the possibilities which could be eliminated only by such a study are the following:

(a) Chlorosis.

(b) Trichiniasis.

(c) Tuberculosis, perhaps involving the genito-urinary tract.

(d) Nephritis.

(e) Hyperthyroidism (Graves' disease).

(f) Diabetes.

(g) Chronic poisoning by acetanilid or other drugs.

Chlorosis might give very similar symptoms, but it was here easily excluded by the blood examination.

Trichiniasis, suggested by the wide-spread pain, was not positively to be excluded, as no search was made either in muscle or venous blood for the embryo trichinella. The blood showed no eosinophilia and we were diverted from following this hypothesis any further because another and commoner disease soon appeared as a cause for the symptoms.

The urine showed nothing to justify any suspicion of genito-urinary tuberculosis. Pulmonary tuberculosis may certainly exist for a considerable period in the lungs without our having any definite evidence of it. In the present case we could find no such evidence, though we could by no means exclude the earlier "silent" stages of the disease.

Nephritis, drug-poisoning, and hyperthyroidism were easily excluded by a study of the history, blood, and urine.

As soon as the urine was tested for sugar we came upon the object of our search, the cause, to all appearances, of the patient's "nervousness."

Outcome. - The urine was 40 ounces in twenty-four hours at the time of entrance; specific gravity, I028; 4.I per cent. of sugar; 0.2 albumin; in the sediment much pus, no casts. She was given paraldehyd, $\frac{1}{2}$ dram, twice a day, sodium bromid, 20 grains three times a day, and by May 2d was much quieter and said she felt better than for months, though she still cribbed. Under an antidiabetic diet the urine was 
freed from sugar in eight days; the weight increased from II 2 to I $2 \mathrm{I}$. and the reactions for acetone and diacetic acid were present for the first week of treatment and as long as any carbohydrates remained in the diet, disappeared. Her speech and nervous control greatly improved, and by the nineteenth of May she seemed like a different person, sleeping soundly without any hypnotic, and perfectly content with her diet. She was allowed to go home on the twenty-second of May, the urine being free from albumin and sugar, though remaining distinctly increased in amount -80 to $\mathrm{r}$ ro ounces on the average. The gain in weight was 8 pounds in sixteen days.

The question might be asked: "Are we dealing here with diabetes or merely with glycosuria?" Since the distinction is wholly one of time, only the outcome can furnish us with the answer to this question. Acetone and diacetic acid appear much more frequently in the longstanding glycosurias, which we call diabetes, than in the transient form. There is no doubt that neurotic, high-strung people are more often the subject of temporary glycosurias than are the more phlegmatic members of the community. On the other hand, the nervousness may, well be symptomatic, the result rather than the cause.

It will be noted that much pus was found in the urinary sediment. Such a datum should always be fullowed up, and the first thing to do is to make sure that the pus comes from the urine and not from the vaginal secretions. In the present case a specimen of urine drawn by catheter was found to be free from pus on the same day when the specimen spontaneously passed had contained it. Accordingly, no further investigation was made, and as the glycosuria improved, the pus disappeared.

Diagnosis.-Diabetes mellitus.

\section{Case 379}

A married Irish woman, forty years old, with an excellent family history, entered the hospital September I7, 1907. She has been nervous all her life, especially since her seventeenth year. She is very easily worried or frightened, and has many weak spells. Last winter she felt underneath her right breast and in the right back a pain, which was sharp on deep breath. This lasted a month. When nervous, she cries very easily. She has been married twenty-three years and has had nine children. Her last period was nine months ago. She takes about six cups of tea a day, but no alcohol. Since her husband was admitted to the Massachusetts Hospital last January she has been much overworked, taking boarders and caring for her children. For the past two months she has been especially nervous, and felt more tired in the mcrning than at night. She has slept very little, and her food has seemed to stop 
at the bottom of her breastbone and to lie there as a heavy load. For the past week she has vomited everything that she has taken, though her diet has been reduced to milk and weak tea. Of late, she has had no pain anywhere except a tired ache between her shoulderblades. Her appetite is fair; the bowels move once or twice a day. When nervous, she passes urine very frequently. For the past two months she has had a slight cough with a little white sputum.

On physical examination the pupils are somewhat irregular, but react normally. The tongue comes out straight, but has a marked coarse tremor. The throat is reddened and atrophic; the heart is negative, except for a slight accentuation of the aortic second sound, The iungs are negative, save for slightly higher-pitched respiration at the left apex and occasional scattered râles throughout. The abdomen is held rather stiffly, but is otherwise negative, save for a swelling extending from the left anterior-superior spine to the neighborhood of the symphysis, and giving a marked impulse on cough. It is easily reducible by pressure.

Discussion.-As this woman has had no menstruation for nine months and is obviously not pregnant, our first thought is that the symptoms may be due to the menopause, that very convenient but dangerous explanation for such a-multitude of heterogenous symptoms. Such a diagnosis should never be made until every other reasonable alternative has been excluded. Moreover, the vasomotor symptoms usually present as a part of any disturbance dependent upon the menopause are not at all marked in this case. Only the nervous frequency of urine suggests them.

It was the fashion, a few years ago, to explain a great number of debilitated conditions as the result of the abuse of tea, especiaily when the physician was able triumphantly to point out that the patient kept the tea.pot on the stove continuously and took a "nip" every now and then throughout the day, thus getting the full benefit of a strong decoction of tannin. In the eleven years of out-patient service involving four years of female medical clinic I have seen less than half a dozen cases in which the symptoms appeared to me due to the abuse of tea. Doubtless it often does harm by taking the place of food, and in the present case this is distinctly suggested. Overwork may likewise have been a factor in her breakdown.

The hypotheses suggested in the last paragraph cannot be definitely refuted, but against them the following consideration may be urged. The overwork and the tea-drinking have lasted for many years, the symptoms for less than one year. Why should the breakdown have 
come just at this time, after the patient had borne her overwork and faulty habits for so many years without apparent detriment? Some new and determining factor must have come into the case-the same factor, I believe, which accounts for most of the seemingly causeless dyspepsias and run-down conditions which we are apt to attribute to this or that food, overstrain, or a surgical lesion. In a large number of these cases tuberculosis later makes itself obvious; in many others, I believe, it is conquered by the patient's own vitality, while we think we are curing his dyspepsia or his "debility" with one or another remedy.

The pulmonary signs in this case are, indeed, very slight. If precisely the same signs were present at the right apex, one could not, with confidence, attribute any meaning to them; but at the left apex even the slightest abnormalities are of importance if unaccounted for by any pathologic condition of the heart or abdominal organs. Even signs so slight as this should make us follow the patient very carefully and examine the lungs, the sputa, and the temperature chart for confirmatory evidence. If, after we have dune nur best by such an examination, the condition of the lungs seems still doubtful, and no other diagnosis has in the mean time suggested itself, we should always treat the case as tuberculosis.

Outcome.-For some days no sputum could be obtained, but in the first satisfactory specimen tubercle bacilli were demonstrated. The inguinal hernia, present on the left side, was fairly well held up by a truss. Diagnosis.-Phthisis.

\section{Case 380}

A telephone girl of eighteen entered the hospital September 2I, I907. One brother of fifteen is said to have consumption. Two grandfathers and one grandmother died of consumption, the last one ten years ago. The patient has always been strong and healthy. She had typhoid fever eight years ago, measles four years ago, followed by a mastoid operation. Her hearing is excellent. Her menstruation has been irregular for the past two or three years, and painful, so that she has to be in bed two or three days each month. She has recently been in the surgical wards, and has been curetted.

Since early childhood she has had a poor appetite, constipation, distress, and burning stomach without regard to the character of food or the time of taking it. She has sick headaches every two to five weeks.

Eight weeks ago she fainted when she got up in the morning, and did not work that day. After working the next day she took to bed, where she has remained since, vomiting almost every fifteen minutes, 
day and night (?). Rectal feeding has been carried out. She has no definite pain, but her vomiting is preceded by a burning sensation at the epigastrium. For the last three days there has been partial loss of vision. She cannot recognize persons or see more than their outlines. There has also been gradual loss of ambition and slowness of speech.

On examination the patient is fairly nourished, the skin dry and harsh, the pupils widely dilated, but reacting normally. The chest and abdomen show nothing abnormal. An attempt was made to pass a stomach-tube, but the patient struggled violently and pulled it out. It was finally replaced, and fasting contents, consisting of mucus and white froth, obtained; no food. After a test-meal there was no free $\mathrm{HCl}$ by any test, no lactic acid, and no blood. Inflation showed that the stomach was not in any way enlarged.

Examination of the fundus showed an optic neuritis in the right eye, numerous small hemorrhages about the disc, and one large one near the nerve in the left eye. The urine was entirely negative, likewise the blood.

During the first two days after admission the patient vomited four or five times, after that very rarely, the vomitus consisting of colorless mucus. Salt solution, 8 ounces every six hours, was well retained by rectum, and the skin soon began to be less dry. After this the patient took milk and lime-water in small amounts for the first few days, after that cereals and gruels. By October Ist eggs were added, and by the tenth she was taking plenty of all sorts of food and the enemata were omitted.

On October Ist the patient complained of numbness below the waist, later of numbness over the whole body, but there was no diminution of the pain sense. She had one hysteric attack, with tremor of the muscles, following rigidity and slight opisthotonos. The patient seemed irrational and fretful, at times spoke very slowly. Her vision was restored by the sixth, and her appetite was then excellent. She seemed in a very pleasant state of mind, constantly expressing her gratitude to the nurses.

On the ninth of October a tumor was noticed, rising above the pubes almost to the umbilicus. A catheter was introduced, and 85 ounces of high-colored urine with a heavy sediment was withdrawn. Eleven hours later 59 ounces of urine were withdrawn. At this time she said that she was unable to move her legs, and had to be turned in bed. Soon after she had involuntary defecation. On the thirteenth she was somewhat improved, but said she could not speak above a whisper. About that time a rectovaginal fistula developed. Vomiting began 
again on the seventeenth of October, and was accompanied by cyanosis and difficult respiration. The same day tracheal râles were audible. She seemed too weak to clear them.

Under strychnin, $\frac{1}{30}$ grain, and atropin, $\frac{1}{100}$ grain, this attack passed off and she breathed normally, though she continued to vomit in small amounts and the pulse was not of good quality.

Seen by a neurologic consultant on the seventeenth of October, the diagnosis was hysteria plus some toxic process.

Discussion.-The advent of marked slowness of speech is an unusual symptom, which should always attract our attention. It occurs in myxedema, in many depressed and melancholic states, in multiple sclerosis, and occasionally in hysteric states. In multiple sclerosis it is apt to be associated with nystagmus, increased reflexes, and intention tremor, none of which is present here. This girl was rarely depressed or hysteric, and showed none of the cutaneous or mental symptoms of myxedema.

The stomach symptoms were very marked and had led to a diagnosis of gastric ulcer before she entered the hospital. The quick clearing up of the gastric symptoms under treatment, however, and the absence of any gastric or rectal hemorrhage and of any evidence of stasis, makes it obvious that, at all events at the present time, her chief sufferings are not due to that cause.

Hysteria naturally occurs to our minds in a patient who has muscular tremor and opisthotonos, is fretful and irritable, and has a great deal of unaccountable vomiting. But the condition of the fundus oculi cannot be thus explained, despite the dictum of the neurologic consultant. What, then, is the cause of the optic neuritis and retinal hemorrhages?

Brain tumor might account for her vomiting and for the psychic disturbances. We should expect, however, to find headache, vertigo, and focal disturbances of some kind (localized paralysis, spasm, anesthesia, aphasia of some type, astereognosis).

Of meningitis we have no important evidence, and there is nothing in the case definitely to suggest syphilis.

Nephritis is the only other common cause of retinal hemorrhage, with or without optic neuritis, but of this neither the heart nor the urine gave us at the outset any hint. Later the urine was so constantly involuntary that none was collected for examination. In the light of the outcome it would appear that such an examination might have been of the greatest importance.

Outcome.-A few days after this she began to have fever ranging from $99^{\circ}$ to $10 \mathrm{I}^{\circ} \mathrm{F}$., and continuing until the day of her death, October 
27 th. Autopsy showed a chronic nephritis with suppuration, an extensive bronchopneumonia, and an obsolete tuberculosis of the mesenteric lymph-glands. The stomach, brain, and cord were normal.

This case is one of those which have most strongly impressed upon me the dangers lurking in the diagnosis of "hysteria." It is the fourth case that I have known to die with this diagnosis. In two of these absolutely nothing was found postmortem, and in these we might, if we were so inclined, consider the diagnosis verified. But when we say "hysteria," we ordinarily mean a disease which cannot in itself, and apart from starvation (as in anorexia nervosa), prove fatal. To my mind these cases simply indicate some of the blind spots in our diagnostic retina.

Diagnosis.-Suppurative nephritis.

\section{Case 381}

A widow of sixty, first seen October 9, 1907, has been treated in the out-patient department of the hospital for some time under the diagnosis of neurasthenia. She has always been a healthy woman, except that she has had eight miscarriages, attributed by her to horseback riding during pregnancy. She has five living children, all bealthy. Last autumn she had an attack of diarrhea and vomiting, and was in the Chelsea Hospital for a week. Since that time she has been more or less run down. In March, I907, she fell into a hole in the floor, bruising her foot and right side, and since that time has had occasional pains in the right side of the chest, sometimes severe enough to make her leave off her corsets. For the past seven weeks she has had a great deal of nausea and has been extremely nervous. Five weeks ago she was examined in the out-patient department and told that she was "simply nervous." A week later her right chest was tapped, and $2 \frac{1}{2}$ quarts of bloody fluid withdrawn. An equal amount of the same character was removed six days later.

On examination the patient was excellently nourished, slightly obese, the face flushed, the mucous membranes of good color; the lips and finger-tips somewhat cyanotic. The heart's impulse was in the eighth interspace, behind the anterior axillary line, $6 \frac{1}{4}$ inches to the left of midsternum. The right border could not be determined. The sounds were normal. The whole right chest was dull, with flatness in the lower half, and rapid and shallow respiration. Breath-sounds were very indistinct, vocal and tactile fremitus absent, except at the apex. The left lung seemed to be normal. Physical examination was otherwise negative, including blood and urine. The chest was at once aspirated, and IIC 
ounces of bloody fluid, with a specific gravity of rorg, was removed. Differential count of the sediment showed lymphocytes, 97 per cent., endothelial cells, 3 per cent. No tubercle bacilli could be found in the sediment of the digested clot. Under ordinary culture-media the fluid remained sterile, and in a guinea-pig to minims of the sediment produced no disease in six weeks. In four days the fluid had reaccumulated, and tapping had to be repeated about every four days until November 9th.

Discussion.-But for this patient's age, it would be natural to assume, after reading the history and previous to the physical examination, that we are dealing with a traumatic neurosis which originated in the accident of March, I907. In my judgment, however, it is always unwise to make a diagnosis of any type of neurosis when the symptoms arise first after the fiftieth year. I have never known such a diagnosis confirmed. The mental characteristics of this patient were, indeed, very much those which we associate with the neuroses, but diagnoses based upon mental characteristics alone are always most vulnerable, even within the field of the alienist, still more markedly so outside it.

After the chest was tapped we assumed that the patient was suffering from a pleural effusion of the ordinary (i.e., tuberculous) type. Even in advance of our own physical examination, however, we ought to have suspected that something more serious was in the background. Ordinary tuberculous effusions (i.e., 99 per cent. of all the serous effusions which we meet with) are rarely bloody, and very rarely reaccumulate within six days. One tapping suffices, in the vast majority of cases, and bloody fluid does not suggest tuberculosis, despite the oft-copied statement of many text-books.

The age of the patient and the rapid reaccumulation of the bloody fluid should have suggested to us at once the diagnosis of malignant disease involving the pleura, lungs, or mediastinal glands.

Doubtless there was a period (before any fluid had accumulated) when diagnosis was difficult or impossible, and when the psychic peculiarities were sufficient to explain, though not to excuse, the diagnosis of neurosis. At this period our proper attitude would have been expressed by saying, "We do not know."

Outcome.-The $x$-ray showed a diffuse shadow over the whole right side, and an unexplained mass near the hilum of the left lung. The patient had frequent dyspnea, more or less relieved by morphin, amyl nitrite, and oxygen.

Autopsy showed endothelioma of the pleura, with extension into the lungs, pericardium, diaphragm, right thoracic wall, bronchi, and 
retroperitoneal lymphatics, liver, stomach, and left adrenal; acute serofibrinous pericarditis and general arteriosclerosis.

Diagnosis.-(See last paragraph.)

\section{Case 382}

A housewife of thirty-three was first seen November 4, 1907. She has lost one sister of consumption. Her family history was otherwise excellent, and she had never been ill except that four years ago she had blood-poisoning after childbirth, but recovered entirely in three months. Fifteen months ago she gave birth to a child after a normal labor. She felt unusually well during the pregnancy. Inımediately after she became very nervous, with spells of trembling and restlessness lasting an hour, once or twice a day. These symptoms persisted until four months later; the child, who had been doing excellently well at the breast, had to be weaned. During this period she also had severe burning micturition, but since the weaning of the child this has not troubled her. Four months ago she ate a considerable quantity of green corn, and was at once attacked with diarrhea, four or five green watery movements a. day and five or six at night. This diarrhea persisted until two weeks. ago, when it was diminished by medicine, and for the past two days her bowels have not moved. She has been in bed for the past five weeks,

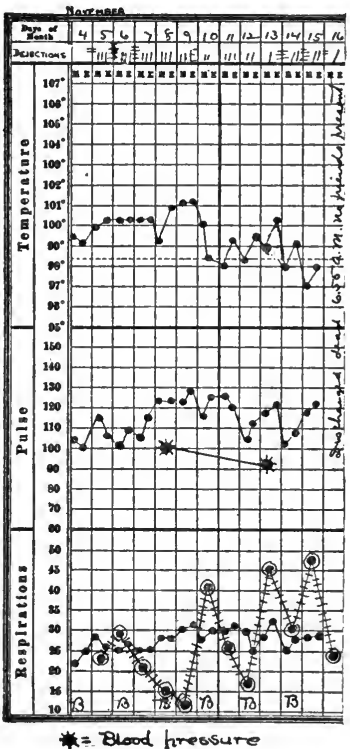

Fig. I95.-Chart of case 382 . complaining chiefly of dizziness, weakness, rumbling in her head, and dryness of the mouth. For two weeks she has had a cough and raised considerable greenish sputa.

The course of the temperature is seen in the accompanying chart (Fig. I95). The patient is pale and emaciated, the tongue moist and slightly excoriated along the anterior edge, the mouth and throat otherwise normal. A systolic murmur is heard over all the precordia, loudest in the pulmonary area, otherwise the heart shows nothing abnormal. The lungs, abdomen, and reflexes are normal. There is slight spinal curvature in the dorsal region, with a concavity toward the left. Examination of the sputa shows nothing abnormal. The same is true of the urine.

Discussion.-The family history, the spinal curvature, the fever, greenish sputa, and painful micturition might be taken as hints of a 
tuberculous infection, though its localization is not clear, and nothing in the further study of the case gives support to any such hypothesis.

Acute endocarditis might produce a murmur with the characteristics here described, although it is much more common to find it in the mitral or aortic area. Especially when fever, without known cause, is present, any cardiac murmur must be thought of in the light of a possible endocarditis. Yet in this case we cannot advance beyond the stage of conjecture with such a diagnosis, as we have nothing but the facts just mentioned by which to support it. Leukocytosis, evidences of peripheral embolism, tender finger-pads (Osler), marked urinary abnormalities, chills, and sweats are all absent.

Doubtless if this patient had been of the male sex, the diagnosis would have been much earlier suspected. Her recent labor, and the rather indefinite ill health which we are accustomed to tolerate in many women at such a time without feeling obliged to make a diagnosis, probably prevented her physician from thinking earlier of the importance of a blood examination. the tell-tale indications of which are revealed in the outcome.

Outcome.-The blood showed red cells, 640,000; white cells, 6500; hemoglobin, I3 per cent. A differential count of 200 white cells showed polynuclears, 57 per cent.; lymphocytes, 43 per cent.; two megaloblasts and one normeblast were seen during this count. The red cells were markedly oversized, deformed, abnormally stained, and stippled. The fundus oculi showed numerous retinal hemorrhages. The patient steadily failed, and died on the sixteenth of November, without any marked change in the symptoms.

Diagnosis.-The diagnosis was not in doubt after the first blood examination, as all the characteristics of pernicious anemia were present.

\section{Case 383}

An optician of fifty-six was first seen May 28, I907. He has lost one brother of consumption, and his wife died of the same disease. The patient had pleurisy seven years ago, and was sick with it for three or four days. Seven years ago he began to have nervous depression, and he has never been quite free from it since. He has had periods of depression and despondency, and has been confined to bed many times for from one to eight weeks. His habits are good. He denies venereal disease.

Ten days ago he began to feel "all smashed up"-considerable headache, nausea but no vomiting, stiffness of the legs and neck, shortness of breath, insomnia (apparently due to nervousness), and 
moderate constipation. His appetite has been good, and he has been very anxious to make a business trip to Ohio, but has been prevented by this present illness. Of late his hands and arms have begun to tremble, and his left foot drags a little when he walks.

On examination, the patient is well developed and nourished. His right pupil is slightly irregular and larger than the left; both react normally. The heart's apex is in the fifth space; just outside the nippleline. There is no enlargement to the right. The heart-sounds are irregular in force and rhythm; no murmur or accentuation. Blood normal. Blood-pressure is $\mathrm{I} 20 \mathrm{~mm}$. Hg. The lungs and abdomen are negative. In the left axilla there is a gland the size of a walnut. The left leg is moved with great difficulty. Sensation is everywhere good. There is fibrillary twitching over the arms and body, and a coarse twitching of the hands and face. The patient is very sleepy, but when aroused, speaks without difficulty. A neurologic consultant said, "probably psychoneurosis," but advised us to continue observation before concluding that there is nothing further.

Discussion.-From the family history, from the previous attack of pleurisy, and from the presence of an enlarged gland in the left axilla, tuberculosis is naturally the first cause for this man's nervousness which we are led to consider. Such a consideration, however, proves fruitless, as nothing in the physical examination bears it out.

Some cerebral lesion was the next thing that occurred to me in studying the case, especially in view of the headache, the nausea, the irregular and unequal pupils, and the paresis of the left leg. The mental state, moreover, was very abnormal, cspecially considering the age at which it first appeared, and the muscular tremor seems likewise significant of a lesion of the central nervous system. Had the blood-pressure been high, we should doubtless have thought of chronic nephritis as soon as the slight cardiac enlargement was discovered, but the normal pulse tension threw us at first off the track.

We remained in the dark regarding the diagnosis, trying to figure out some type of thrombosis, softening, or slight hemorrhage in the brain which could account for the condition of the left leg. Dementia paralytica was considered, but the mental state, the pupils, and reflexes were not at all characteristic of this condition; nor, on the other hand, were they wholly inconsistent with it. At this point most of our difficul. ties were cleared up by the receipt of a full report upon the condition of the urine, details of which follow.

Outcome.-The urine averaged 60 ounces in twenty-four hours; specific gravity, I0II; the slightest possible trace of albumin was found, 
and a rare, finely granular cast. There was marked soft edema of the feet and lower legs. By rest in bed, ro minims of digitalis every four hours, I ounce of magnesium sulphate every morning, diuretin, I 5 grains four times a day, and limitation of liquids, the patient was greatly improved by the second of June. By the third the edema was gone. By the ninth he was sitting up, feeling well, passing a large amount of urine, his heart's action much stronger and steadier, his leg motions nearly normal. On the eighteenth he was allowed to go home in excellent spirits.

Diagnosis.-Chronic interstitial nephritis. 



\section{APPENDIX A}

As mentioned in the Introduction, certain statistical articles and monographs were used in the preparation of the "gridiron" diagrams. The most important were as follows:

I. Rolleston: "Diseases of the Liver" (W. B. Saunders Co.).

2. Bramwell: "Clinical Studies," Jan. I, rgro, vol. viii, part ii, p. 97.

3. Garceau: "Renal Tumors" (Appleton, I9ro).

4. Howard A. Kelly: "The Vermiform Appendix" (W. B. Saunders Co.).

5. Tauquerel des Planches: "Monograph on Plumbism," Paris, I839.

6. Starr: "Text-book of Diseases of the Nervous System."

7. F. C. Shattuck: "Tuberculous Peritonitis," Trans. Assoc. Amer. Physicians, 1902, p. I37.

8. Thomas McCrea: Article on "Typhoid Fever" in vol. ii of Osler's "Modern Medicine."

9. Naunyn: "Klinik der Cholelithiasis," Leipsig, I892.

I0. Greenough and Joslin: Article on "Gastric Ulcer," Boston Med. and Surg. Jour., Oct. 19, I899.

II. E. A. Codman: "Subdeltoid Bursitis," Boston Med. and Surg. Jour., May 3I, I906.

I 2. Berger: "Cccupation Neuroses," Osler's "Modern Medicine," vol. vii, p. 793.

13. Osler: Article on "Aneurysm," Medical Chronicle, I906, vol. xi, p. 69.

14. Osler: Lumleian Lecture on "Angina Pectoris," Lancet, March I2, I9IO.

15. Henry Phipps' Institute: "Annual Reports" (Igo6-1909).

r6. Musser and Norris: Article on "Pneumonia," Osler's "Modern Medicine," vol ii, p. $5^{6} 3$.

17. Dickinson: “Uremia," in “Allbutt's System," r897, vol. v, p. $3^{67}$.

I8. Savill: Lectures on "Hysteria, Etc." (Wm. Wood, I9o9).

r. F. T. Lord: "Diseases of the Pleura," Osler's "Modern Medicine," vol..iii, p, 780.

20. Keyes: "Diseases of the Genito-urinary Organs" (Appleton, r910).

2r. Bevan: "Renal Tuberculosis," Jour. Amer. Med. Assoc., Oct. 9, Igo6.

22. Albarran: "Les Tumeurs du Rein," Paris, I903.

23. Robson and Cammidge: "Diseases of the Pancreas."

24. Benj. Tenney: "Renal and Ureteral Calculi," Boston Med. and Surg. Jour., June 8 , rgo5.

25. F. B. Greenough: "Herpes Zoster," Boston Med. and Surg. Jour., Dec. 5, I889.

\section{APPENDIX B}

\section{Typhoid Régime at the Massachusetts General Hospital (West Medical Service)}

I. Bed until temperature is normal.

2. Four-hourly chart.

3. Four-hourly mouth-wash and spray. 
(a) Swab tongue, cheeks, and gums with equal parts ofBoric acid (saturated aqueous solution), )

Lemon-juice, Glycerin,

(b) Dobell's solution, Water, \}on a cotton stick. 3 parts $\}$ Sprayed with an atomizer,

4. Four-hourly bath whenever temperature reaches or exceeds $102.5^{\circ} \mathrm{F}$. (by mouth).

Baths are given with water and 40 per cent. alcohol, equal parts. Duration, twenty minutes (if reaction is good).

Temperature of bath, $90^{\circ} \mathrm{F}$, if mouth temperature is $102.5^{\circ} \mathrm{F}$.

Temperature of bath, $85^{\circ} \mathrm{F}$. " " $\quad$ " $103.0^{\circ} \mathrm{F}$.

Temperature of bath, $80^{\circ} \mathrm{F}$. " " " $\quad 103.5^{\circ} \mathrm{F}$.

Temperature of bath, $75^{\circ} \mathrm{F}$. " " $\quad$ I04. $0^{\circ} \mathrm{F}$. or more.

5. Suds enema every second day, if needed.

6. Cocoa butter to lips, p. r. n.

Typhom Diet (as Introduced by Dr. F. C. Shattuck in I897 ${ }^{1}$ ).

\section{Breakfast:}

I. One egg on a slice of toast with butter.

2. One of the following drinks-

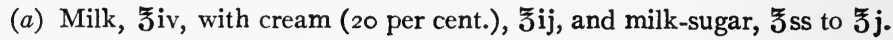

(b) Malted milk, כ̃v.

(c) Cocoa.

(d) Coffee.

(e) Mellin's food.

II. IO A. M.:

Lemonade, orangeade, grape-juice, or albumen-water with $气$ jij, milk-sugar.

\section{Dinner:}

I. Milk, cream, and sugar mixture as above.

2. One of the following solids-

(a) Egg with toast and butter, as above.

(b) Minced chicken with toast and butter, as above.

(c) Ice-cream.

(d) Blanc mange or wine jelly with sugar and at least $5 \mathrm{j}$ of cream.

IV. 3 P. M.:

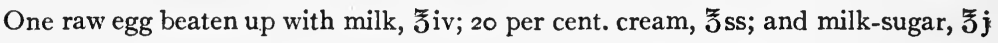

\section{Supper:}

I. Milk, cream, and sugar mixture, as at breakfast.

2. Baked apple or banana-whip, with $\tilde{z} \mathrm{j}$ cream, or one-half slice of toast with butter.

iI. During the Night:

Two drinks consisting of--

$\left.\begin{array}{l}\text { Albumen-water, } \\ \text { Grape-juice, } \\ \text { Lemonade, } \\ \text { Orangeade, }\end{array}\right\}$ with milk and sugar, $\mathbf{3}$ j.

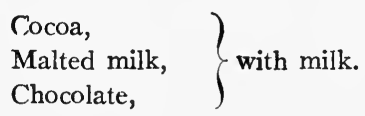

This diet supplies about 2900 calories.

${ }^{1}$ F. C. Shattuck, Journal of the Amer. Med. Assoc., July Io, I897. 


\section{Régime for Cases of Peptic Ulcer-Gastric or Duodenal-as Used in the West Medical Wards, Massachusetts General Hospital}

$A$. Rest in bed.

$B$. Diet as follows:

I. For first three days give every two hours (when awake)-

Milk, 2 ounces, with two powdered sida-crackers ( $2 \frac{1}{2}$ inches square) and

Cane-sugar, I teaspoonful (if relished).

2. For next two or three weeks every two hours-

Milk, 6 to 8 ounces, with four powdered soda-crackers and

Cane-sugar, 1 to 2 teaspoonfuls (if desired).

3. For next two months (more or less) a diet consisting of average portions of the following articles--

Milk and crackers, as above.

Cornmeal mush with cream and sugar or salt.

Potato purée.

Milk with whites of 2 eggs.

Soft custard.

Chocolate.

Pea purée.

Water is given according to the patient's desire.

c. For pain or sour burning eructation a saucer of sodic bicarbonate and a spoon are put at the bedside and the patient is told to take the soda in amounts sufficient to relieve him.

\section{APPENDIX C}

\section{THE CLASSIFICATION OF NEPHRITIS}

Throughout this book I have adopted the classification used in the autopsy records of the Massachusetts General Hospital, and identical in its main outlines with that used by Senator and by Mallory. The gist of it appears to be as follows:

Leaving out of account the acute destructive lesions, such as may be produced by mercurial poisoning, suppurative nephritis, etc., the renal lesions distinguishable by a group of clinical and anatomic characteristics are:

I. Glomerulo-nephritis, early or late.

2. Arteriosclerotic nephritis.

The former results from an injury produced in the glomeruli by some irritantusually the poison of an acute infectious disease, such as scarlet fever, pneumonia, or acute endocarditis. Edema, anemia, and often uremic manifestations occur. In cases lasting over six weeks there is usually cardiac hypertrcphy. If the injury to the glomeruli is reiatively slight and limited to a few, recovery takes place after an acute or, rarely, a subacute course of the disease. If the injury is more serious, the disease may go on in a latent and well-compensated form for months or years, finally ending with a burst of "acute" symptoms (edema, anemia, uremia, cardiac failure). In the more chronic cases the histology of the kidney and the condition of the heart and urine may closely resemble those of the type next to be described

In arteriosclerotic nephritis the lesions are not diffusely distributed. Some glomeruli remain relatively intact. These are in various stages of sclerosis and disintegration. The vascular lesions are relatively marked. In the end stage the contracted kidney, resulting from this process, may be indistinguishable from the end stage of a glomerulonephritis. To either of these conditions, in their terminal stage of contraction, the term "chronic interstitial nephritis" is often applied, but it would seem to me better to give up that term altogether and to refer simply to a "contracted kidney of unknown origin," when the histology does not enable us to state whether in its earlier stages the process was glomerular or vascular. 



\section{INDEX}

[Words and page numbers printed in heavy type correspond to illustrative cases; other words and numbers, to minor discussions.]

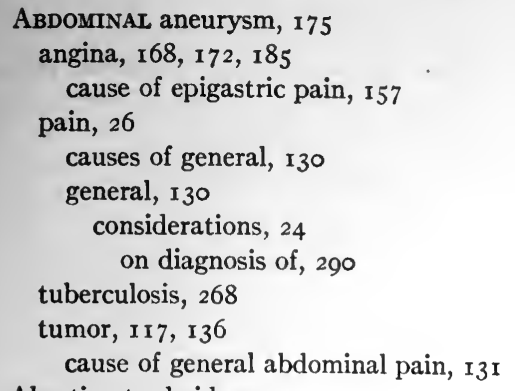

Abortive typhoid, 452

Abscess, brain, cause of coma, 499 cerebral, $52 \mathrm{I}$

deep axillary, 340,492

"fixation," 2II

hepatic, 90, 234, 235, 322, 470, 483

ischiorectal, $374,406,489$

liver. See Abscess, hepatic.

lung. See Abscess, pulmonary.

multiple renal, i 13

perinephric, $87,94,322,419$

cause of lumbar pain, $8 \mathbf{I}$

perirectal, 4 I 9

pulmonary, 222, 470, 570, 590, 600, 606 cause of coma, 499

renal, I I 3

subdiaphragmatic, $I 37,233,322,483$

cause of right hypochondriac pain, 208

tubal, 267

tuberculosis with, 344

Acetanilid, 4I

Acid (oxalic) poisoning, 660

Actinomycosis, 324

Acute yellow atrophy of liver, 74 I

Adams-Stokes' disease, 443, 503, 529, 53 I

Addison's disease, 546, $5^{82}$

cause of coma, 499

Adenitis, 316

mesenteric, 363

syphilitic, 285

Adenoma, simple, of thyroid, 50
Adherent pericardium, 220, 723

Adhesions, pelvic, cause of left iliac pain, 280 of right iliac pain, $26_{3}$

pleural, I88, 305

pleuropericardial, I 88

pyloric, I77

cause of epigastric pain, 157

Adhesive pericarditis, 52

chronic, 719

Adolescence cause of headache, 35

Air, bad, cause of headache, 33

Alcohol cause of headache, 33

Alcoholic gastritis, 2 I 8

neuritis, 379, 566, 568

cause of pain in legs and feet, 357

Alcoholism, 78, I6r, r70, I 73, I80, 197, I99, $274,365,396,455,483,510,528,549$, 645,672

cause of coma, 495

of convulsions, 494

of vomiting, 622

Amyloid metamorphosis of liver, 322

Anal fistula, 32I

Anemia, I42

cause of headache, 32

pernicious, I48, I $52,551,560,585,754$

secondary, I 53,550

Aneurysm, I68, I93, 296, 301, 303, 332, 343, $346,349,35$ I, 593

abdominal, 175

aortic, 87, I I I, I44, 300

called rheumatism, 333

cause of brachial pain, 329

of lumbar pain, Iro

of spine, 120

thoracic, 319,347

Angina abdominalis, I68, I72, I85

cause of epigastric pain, $\mathrm{I}_{57}$

pectoris, 26, I80, I85, 299, 30I, 3I2, 3I7,

323,349

cause of brachial pain, 329, 33 I

functional, 247 
Angina pectoris, low, I7 I

Anginal pain, four occasions for, 350

Anginoid pain, $26^{\circ}$

Ankle, sprained, cause of pain in legs and feet, 357

Anorexia nervosa, 444

Aorta, dynamic,. I43, I45 syphilitic heart and, 300

Aortic aneurysm, 87, I I I, I44, 300

insufficiency, $30 \mathrm{I}$

regurgitation cause of cough, 588

stenosis and regurgitation, 708

valves, fibrous endocarditis of, 219

Apoplexy, 395, 509, 52I

cause of coma, 495

with hemiplegia cause of paralysis, 544

Appendicitis, 90, 94, I०3, I33, I35, I38, I40, I82, 263, 266, 267, 27I, 272, 362, 427, $453,626,659$

acute, 65,268

cause of short fever, 4 Io of vomiting, 622

healed, I95

\section{and bronchitis, 595}

of general abdominal pain, I3I

of right iliac pain, 263

chronic, I56, I94, I95, I96, 635, 643, 653

fear of, cause of right iliac pain, $26_{3}$

Appendicular colic, 274

Appendix, "high," 2 I I , 2 I3, 240

cause of right hypochondriac pain, 208

Apprehension, 553

Arm and shoulder, neoplasm of, cause of brachial pain, 329

Arms, pain in, 328

Arsenical poisoning, 569

Arterial spasm, 30

Arteriosclerosis, 31, 32, 52, 184, 305, 359, $55 \mathrm{I}, 552,6$ I I, 625

cerebral, 532

general, 532

Arthritis, $67, \mathrm{I} 83,337,349,385$

acute, cause of short fever, 4 Io of hip, 375

atrophic, 340, 356, 390

cause of pain in legs and feet, 357

gonorrheal, $67,356,398$

hypertrophic, $340,356,390$

cause of lumbar pain, $8 \mathrm{I}$

of pain in legs and feet, 357

spinal, 9 I

radiations from, cause of axillary pain, 293
Arthritis, infectious, 387

cause of long fever, 409

of pain in legs and feet, 357

of hip, 374

of left sacro-iliac joint, rog

of spine, rog

cause of lumbar pain, 8I

osteo-. See Osteo-arthritis.

pneumococcus, 38I

rheumatic, $67,371,390$

sacro-iliac, 182

of shoulder-joint, 344

various types of, cause of brachial pain, 329

\section{Artificial menopause, 306}

Ascending colon, cancer of, 670

Ascites, 319

Astasia abasia, 540

Asthma, bronchial, 320

and bronchitis, 597

cause of cough, 588

of dyspnea, 70I

Ataxic paraplegia cause of paralysis, 544

Atrophic arthritis, 340, 356, 390 cause of pain in legs and feet, 357

cirrhosis cause of coma, 499

Atrophy of liver, acute yellow, 74I

Attempted miscarriage, 626

Attention, expectant, 210

Atypical forms of malaria, 654

"Auto-intoxication," 305

Axillary abscess, deep, 340, 492

pain, 292

BAвсоCK, Dr. R. H., 50I

Bacillus coli cause of cystitis, 689

Back, acute sprain of, cause of lumbar pain, 8I

strain, 97

Backache, "functional," 80, 87, 105

"kidney group," 83,87

"orthopedic group," $83,87,92$, 103, I 20, I 22

"postural," 80

"pressure group," 83,87 , I I0, I II, I 20, I 2 I

sacro-iliac, 80

"uterine," 80

Bad air cause of headache, 33

hygiene, 134

Balance, defective, cause of lumbar pain, 8I

Banti's disease, 562

Base of skull, fracture of, 55 
Basedow's disease, 52

"Bed fever," 4I 2, 465

Bile-ducts, cancer of, cause of jaundice, $73 \mathrm{I}$

"Biliousness," 35 cause of headache, 33

Birth palsy cause of paralysis, 544

Bissell, Dr., 498, 5 이

Bladder cancer, $282,688,690$ gall-. See Gall-bladder.

stone in, $283,689,690,697$

tuberculosis of, $284,682,684,694$

tumor of, cause of hematuria, 679

Blood. See Hematuria, Hemoptysis, etc. cultures, 75

Bones, tuberculosis of, $35 \mathrm{I}$

Bowel, cancer of, 87, 173. See also Colon. ulcer of, 87

Boys of fourteen and fifteen, infections common in, 453, 477

Brachial neuralgia, 342, 343 neuritis, 344

Brain abscess cause of coma, 499 concussion of, 56 edema cause of coma, 499 softening, 78

tumor, 56, 62, 71, 76, 78, 431, 507, 521, $528,534,539,628,653,655,657,75^{1}$ cause of coma, 495 of headache, $33,38,47$

"wet," 78

"Brain-fag," 536

Breuer, 378

Brewer, G. E., IoI

Bright's disease, chronic, $258,359,638,653$, 686,690

Broken rib, 296

Bronchial asthma, 320

Bronchiectasis, 592, 599, 610, 6ro

cause of coma, 499

influenzal infection of, 308

Bronchitis, 308, 594, 6I4

acute, 479

cause of short fever, 4 Io

and appendicitis, 595

and asthma, 597

and emphysema, 714

bronchopneumonia, bronchiectasis, and emphysema, 60I

cause of cough, 588

chronic, 222

cause of dyspnea, 70I

Bronchopneumonia, 526
Bronchopneumonia, bronchiectasis, emphysema, and bronchitis, 601

cause of coma, 499

streptococcus, 598

Brown, Dr. Lawrason, 6r7

Bubo, 286

"Burning pain," 227

Bursitis, subacromial, 335, 339, 341, 342, 344 cause of brachial pain, 328,329

Calculus. See Stone.

Cancer, 99, 262, 273, 739

of bile ducts cause of jaundice, $73 \mathrm{I}$

of bladder, 282, 688, 690

of bowel, 87,173

cause of long fever, 409

of colon, 20I, 256, 259, 26r, 285, 2i.7, 6.3 I, 670

of duodenum cause of jaundice, 73I

of gall-bladder cause of jaundice, 731

gastric, $87,146,159,162,164,166,160$, I 78,187, I92, I99, $227,256,547,559$,

$627,629,649,661,664,671$

cause of epigastric pain, 157

of jaundice, $73 \mathrm{I}$

of vomiting, 622

hepatic, I6o, 20I, 215, 233, 376, 4I6, 56r, $734,74 \mathrm{I}$

cause of jaundice, 73 I

of right hypochondriac pain, 208

intestinal, $149,162,281,585$

of liver. See Cancer, hepatic.

of pancreas, $189,228,234,740$

cause of jaundice, 731

of prostate cause of coma, 499

prostatic, 68I

rectal or intestinal, $5^{85}$

of rectum, 152

recurrent intestinal, 148

of sigmoid, $285,287,63 \mathbf{x}$

cause of left iliac pain, 280

of spine cause of lumbar pain, 8I

of splenic flexure, 256, 259

of stomach. See Cancer, gastric.

of uterus, 387

Cardiac cases, sudden death in, 507

dilatation, acute, 52, 30I, 318, 718, 723

disease, 4I, 230, 557, 580, 616, 625, 723,

724

cause of dyspnea, 701

of vomiting, 622

hypertrophy and dilatation, 52, 30I

insufficiency, 7 I 5 
Cardiorenal disease, 643

Cardiovascular disease cause of coma, 499

Caries sicca, 339, 344

Catarrhal jaundice, 214, 462, 639, 73I, 733, 735, 737, 738, 742

"Cathartic method," 378

Cecal region, tuberculosis of, 265

Cellulitis, 34I, 346

infectious, 337

Central pneumonia, 455

Cerebral abscess, $52 \mathrm{I}$

arteriosclerosis, 532

circulatory conditions cause of coma, 499 concussion, 56

cause of headache, 35

hemorrhage, $7 \mathrm{I}, 77$

cause of coma, 499

softening, 78

syphilis, 58

tumor. See Tumor, brain.

cause of headache, 33, 38, 47

Cerebrospinal syphilis, 397

Cervical rib, 332, 342, 343, 344, 346 cause of brachial pain, 329, 33I

Charcot joint, 389

Charts and diagrams, explanation of, 22

Chest, malignant disease of, 316,320

wall, malignant disease of, 324

Childbirth cause of lumbar pain, 8I

Children's fevers, 425

Chills, 469

causes of, 469

"creeping," 469

"nervous," 469

occurring in typhoid fever, 488

Chlorosis, $163,403,555,634,746$

Cholangitis, 156,214

Cholecystitis, I03, I56, I94

acute, 223

cause of right hypochondriac pain, 208

typhoid, 2I4, 242

Choledochus, stone in ductus, 228, 236

Cholelithiasis, $87,115,167,168,172,173$, I77, I78, I79, I85, I90, 201, 203, 210, 2I4, 239, 256, 269, 3І I, 49 I, 643, 733, $736,737,738$

cause of chills, 468 of epigastric pain, 157

of jaundice, 73I

of right hypochondriac pain, 208

with perforations, I8I

Circumflex paralysis, 339, 344

Cirrhosis, atrophic, cause of coma, 499
Cirrhosis of liver, 147, 190, 200, 217, 229, 236, 250, 322, 56I, 625, 66I, 739, 742 cause of jaundice, $73 \mathrm{I}$

of long fever, 409

sudden death in, 502

Claudication, intermittent, cause of pain in legs and feet, 357

Clubbed fingers, 396

Cobb, Farrar, Ior

Cocain habit, I34

"Cclds, common," 49 cause of short fevers, 4IO

Colic, 26, 526

appendicular, 274

intestinal, 156

lead-, 133

renal, 146

Colica mucosa cause of right iliac pain, 263

Colitis, chronic, $\mathrm{I}^{2} 2$

mucous, 130, I33, 273

perforative, I5I

tuberculous, 148

Colon bacillus infection, 105

cancer of, 20I, 256, 259, 26I 285,287 , 631,670

Column, spinal. See Spinal column.

Coma, 494

causes of, 495, 499

deaths in, etiologic study, 498

diabetic, 499

Comatose or convulsive patients, examination of, 494

"Common colds," 49 cause of short fevers, 4IO

Common duct, stone in, 228,236

infections cause of headache, 35

Concomitant nephritis, 60

Concussion of brain, 56

Congestion, gastrohepatic, due to cirrhosis or cardiac disease cause of epigastric pain, 157

hepatic, 200, 322

cause of right hypochondriac pain, 208

Constipation, I35, I54, I62, I64, I65, I69, I 73, I76, I78, I99, 202, 284, 627,636, 637,674

cause of epigastric pain, I 57 of general abdominal pain, I3I of headache, 32

(neurosis?), 636, 637

Constitutional headache, 49

Constriction, pain with a sense of, 26

Contusion, 34I 
Convalescence from pneumonia, 571

Convulsions, 5 io

causes of, 494

Convulsive patients, examination of coma-

tose or, 494

Costal tuberculosis, 324

Cough, 589

causes of, 588

"heart," 6II

mixture, 715

varieties of, 587

Courvoisier's law, Igo

Craig, Charles F., 654

"Cramp, writer's," 535

Crisis, gastric, I3I, I69, 643, 655, 664

vascular, 3I, I84, 393, 440, 52 I, 522, 532

Croup, 722

Cultures, blood, 75

Curschmann, 6 2

Curvature, spinal, 386

Cyst, hydatid, of liver, 225, 234

ovarian, 27I, 273, 286

cause of left iliac pain, 280

of right iliac pain, 263

ruptured, 270

strangulated, 287

with twisted pedicle, 267, 269

renal. See Cystic kidneys.

Cystadenoma, papillary, of kidney, 690

Cystic kidneys, congenital, 87, I I 7, 225, 250, 260

Cystitis, 682, 689, 697

cause of hematuria, 679

chronic, 284

due to bacillus coli, 689

gonorrheal, 284

Death in coma, etiologic study, 498

sudden, in cardiac cases, 507

in cirrhosis of liver, 502

Debility, 103, 122, I6I, 229, 231, 573, 712

Defective balance cause of lumbar pain, 8I

Deforming osteitis, 49

Deformity, round-shoulder, 348

Degree of pain, 25

Delirium, salicylate, 370

tremens, 673

cause of coma, 499

Dementia paralytica, $68,70,532,536,756$

cause of coma, 495

of paralysis, 544

Dercum, Dr. C. T., 8I
Diabetes mellitus, 52, I42, 368, 442, 51 3 , $5^{28}, 562,564,583,611,642,745$

Diagnoses, causes of incorrect, 17

Diagnosis, vulnerability of all differential, 19

Diagrams and charts, explanation of, 22

Diaphragm, dome of, pain from, 30

Diarrhea cause of epigastric pain, $I_{57}$ of general abdominal pain, $\mathrm{I}_{3} \mathrm{I}$

Diet, Lenhartz's, Dr. H. F. Hewes's modification of, 257

Dilatation, acute cardiac, 50, 52, 30I, 318, 718, 723

Diseases frequently diagnosed as rheumatism, 334

not considered in this book, $2 \mathrm{I}$

Dislocation of humerus, 34I

Displacement of liver, 225

Distomiasis, $32 \mathrm{I}$

Disturbances of sensation, 29

Diverticulitis, 288

Dome pain; 30

Dorsal tabes, I34, I64, 393, 566

with gastric crises, 655,664

Dropsy, 50, 305, 36I

pleural, 3 i 8

Drug habits, 672

poisoning, 746

Ductus choledochus, stone in, 228, 236

Dunn, Dr. Charles Hunter, 22I

Duodenal ulcer, I33, I42, I62, I66, I69, I70, I 72, I 79, 216, 238

Duodenum, cancer of, cause of jaundice, $73 \mathrm{I}$

Dynamic aorta, 143, 145

Dysentery, 405

Dysmenorrhea, 26

cause of left iliac pain, 280

of right iliac pain, 263

Dyspepsia, I56, I80, 323

Dyspnea, 700

causes of, 701,703

inspiratory and expiratory, $72 \mathrm{I}$

Dysuria, 431

EClampsia (puerperal) cause of coma, 499 of convulsions, $5^{\text {II }}$

Ectopic gestation cause of left iliac pain, 280

Edema, acute pulmonary, 309

of brain cause of coma, 499

Edinger, 36

Effusion, dropsical, 305

pericardial, 209, 318

pleural, 303, 304, 310, 359, 447, 609, 667, 753 
Effusion, pleural, purulent, 322

Embolism, 7I

Emotional excitement, its relation to pain, 27

Emphysema, 90, 304, 601, 714

cause of cough, 588

of dyspnea, 7 or

Empyema, 233, 322, 437, 490, 558, 592

interlobar, 423, 604

necessitatis, 324

postpneumonic, 64, 423, 604, 605,

717

tuberculous, 556, 715

Endocardial fever, 428

infection, 473

Endocarditis, 97, I83, 381, 477, 555, 575, 709

acute, 755

fibrous, of mitral and aortic valves, 219

gonorrheal, 306

mitral, $37 \mathrm{I}$

ulcerative (or malignant), $94, \mathbf{4 3 8}$

Endometritis, hyperplastic, 712

Endothelioma, pleural, 752

Enteritis, 66I

cause of epigastric pain, 157

of general abdominal pain, I3I

gastro-, 145, 662

tuberculous, 260

Enuresis, 697

Epidemic meningitis, 58, 449, 519

poliomyelitis, 569

Epididymitis, tubercular, II 7

Epigastric pain, I60 causes of, 157

Epilepsy, 510, 516, 528, 532, 535, 714

cause of coma, 499 of convulsions, 5 I I

Jacksonian, 398, 535

nocturnal, 520

Erysipelas cause of coma, 499 of short fever, 4 ro

Erythematous lesions, 74

"Essential" headache, 49

Estivo-autumnal malaria, 428, 464

Evidences of pain, 24

Evils of obesity, resulting, 379

Examination of comatose and convulsive patients, 494

Excitement cause of dyspnea, 701 emotional, and its relation to pain, 27

Exertion cause of dyspnea, 701

Exhaustion, 485, 64I
Exhaustion, nervous, 662

postepileptic, cause of coma, 495

"Expectant attention," 2 IO

Expiratory and inspiratory dyspnea, 721

Extra-uterine pregnancy, 266

cause cf general abdominal pain, I3I of right iliac pain, 263

Eye-strain cause of headache, 33, 43, 68

FACE, phlegmonous inflammation of, cause of coma, 499

Fatigue cause of brachial pain, 329

of headache, 32, 33, 536

of lumbar pain, $8 \mathrm{I}, 83$

poisons, 32

Fatty liver cause of cirrhosis, 499

metamorphosis of liver, 322

"Febricula," I40

Fecal impaction, 432

Feet, pain in legs and, 356

Femur, sarcoma of, 389

Fevers, 407

"bed-," 4I 2, 465

of children, 425

endocardial, 428

gall-stone, $4 \mathrm{I} 6$

glandular, 460

long, 407, 409

non-infectious, $4 \mathrm{I} 3$

relapsing, $4 \mathrm{I} 2$

short, 407, 410, 4II

slow, 305

streptococcus, $4 \mathrm{I} 3$

terminal, $4 \mathrm{I} 3$

thrombus, 413

toxic, $4 \mathrm{I} 3$

urticarial, 465

Fibroid tumor of uterus, $176,267,287$

uterine, 176,267

Fibrous endocarditis of mitral and aortic valves, 219

myocarditis, chronic, 625, 719, 723

Fingers, clubbed, 396

Fistula in ano, $32 \mathrm{I}$

Fixation abscess, 2 II

Flat-foot, 372, 377, 385, 39I, 394, 40 I cause of pain in legs and feet, 357

Flatulence, 204, 248, 31 7, 323

cause of axillary pain, 293

Flint, Austin, 220

Floating kidney, 229, 277, 652

Floyd, Dr. Cleaveland, 598, 6I I

Food, relation of pain to taking of, 28 
Food-strain, acute, 378

Fracture of base of skull, 55

of humerus, $340,34 \mathrm{I}$

of mandible cause of coma, 499

of skull cause of coma, 499

Fractured pelvis, 372

rib cause of axillary pain, 293

of coma, 499

Freud, S., 378

Frontal sinus, disease of, cause of headache, 43

Functional affection of spine, r ro angina pectoris, 247

backache, 80,8 I , 87, I05, I ro, I 20

neurosis, 269

GALL-BLADDER, cancer of, cause of jaundice, $73 \mathrm{I}$

disease, $x$ 59, r6o

enlargement of, 225

gangrenous, I 79

infection, 133,403

Gall-stone disease, 483,676

fever, 4 I 6

Gall-stones. See Cholelithiasis.

"Gastralgia," r67

Gastrectasis, 68

Gastric cancer. See Cancer, gastric.

crisis, tabes dorsalis with, I3I, I69, 643, 655,664

flatulence, 204

hepatic congestion due to cirrhosis or cardiac disease cause of epigastric pain, I57

neurosis, $165,192, \mathbf{9} 93,261,628,635,644$, $646,653,668,674$

cause of epigastric pain, I 57

of general abdominal pain, I3 I

of vomiting, 622

tumor, II 7

ulcer, 87, r6o, r9o, 194, 629, 650, 66r, 695, $75 \mathrm{I}$

perforated, 90, $28 \mathrm{I}$

Gastritis, 66I

aicoholic, 218

cause of vomiting, 622

phlegmonous, 480

Gastro-enteritis, I45, 662

Gastro-intestinal disease, psychic causes in, r66

tract, infection of, 666

Gastroptosis, 648

"Gefässkrisen," 30
Genito-urinary tuberculosis, 683, 688, 746

Gestation, ectopic, cause of left iliac pain, 280

Glandular fever, 460

tuberculosis, 343, 479

Glomerulonephritis, chronic, 50, 6r, 475, 723

Goiter, 52

Gonorrhea, I4 I, I83, 262, 375, 382, 390, 393 cause of long fever, 409

Gonorrheal arthritis, $67,356,398$

cystitis, 284

endocarditis, 306

Gout, 371, 379, 382, 393, 540

cause of pain in legs and feet, 357

Graves' disease (hyperthyroidism), 50, 563, 583, 619, 642, 719, 723, 746

Gregg, Dr. Donald, 338

"Grip," 55, 93, 97, I 40, 298, 409, 420, 45 I, 473, 492, 5I7, 572, 666

Gumma, hepatic, I6o

Gummatous tumor, 35 I

HABIT, cocain, I 34

drug, 672

"Habit" pain, 29, 86, 32I

Hagenbauch, 226

Head, Henry, 29, $25^{2}$

Headache, 32

causes of, 33

constitutional, 49

due to trauma, 45

"essential," 49

general considerations on, 32

indurative, 33,35

its position and nature, 38

"neuralgic," 72

"neurasthenic," 49, 55, 68

of psychic origin, 48,74

"rheumatic," 36, 43

"sick," 5 I

syphilitic, 44

of unknown origin, 68

uremic, 50

vasomotor, 26, 37

"Heart cough," 6i I

Heart, dilated, difference between pericardial effusion and, 3 I 8

disease, 4I, 230,' 557, 580, 616, 625, 723,

724

congenital, 533

hypertrophy and dilatation, 50, 52, 30I

syphilitic, and aorta, 300

weak, 309 
Heberden's nodes, 396

Hematogenous infection of kidney, 87,93 , I00, 69I

Hematoma, 34I

Hematuria, 678

cause unknown, 692, 695

causes and types, 678

Hemolysis, 373

Hemolytic jaundice, chronic, 548

Hemoptysis, causes of, 606

Hemorrhage, 560

cerebral, 7I, 77

cause of coma, 499

intracranial traumatic, cause of coma, 499

Hemorrhagic conditions, 32 I

Hemorrhagica, purpura, 32 I

Hemothorax, 305

Hepatic abscess, 9o, 234, 235, 322, 470, 483 atrophy (acute), 74I

cancer, I60, 20I, 215, 233, 376, 4I6, 56r, 734, $74 \mathrm{I}$

cause of jaundice, 731

of right hypochondriac pain, 208

congestion, 200, 322

cause of right hypochondriac pain, 208

diseases, 429

displacement, 225

gumma, syphilitic, 159

infection, 48I

and pulmonary abscess, 470

syphilis. See Syphilis, hepatic.

Hepatitis, chronic interstitial, 736

Herpes zoster, 87, 94, 366

cause of lumbar pain, 8I

"High" appendix, 2II, 2I3, 240

cause of right hypochondriac pain, 208

Hip, acute arthritis of, 375

infection of, 374

disease (tuberculous), 98, 375

infectious arthritis of, 374

History of injury sometimes misleading, 345

Hodgkin's disease, 322, 46I, 479, 49I, 6I9

Humerus, dislocation of, 34I

fracture of, 339, 34I

osteomyelitis of, cause of brachial pain, 329

sarcoma of, 344, 345

septic osteomyelitis of, 340

tuberculosis of, 339, 342

Hunger cause of headache, 32, 33

Hydatid cyst of liver, 225, 234

infection, 217
Hydronephrosis, 87, I 7 7, 225

cause of right hypochondriac pain, 208

Hydrothorax, 222, 7I 7

Hygiene, bad, 134

Hyperchlorhydria, I62, I68, I70, I73, 255, 26I

cause of epigastric pain, I 57

Hyperemia, vascular, 26

Hypernephroma, 259, 683, 694

metastatic, 350

Hyperperistalsis, 26

intestinal, 156

Hyperplastic endometritis, 712

Hypertension, nephritic, 3I

Hyperthyroidism. See Graves' disease.

Hypertrophic arthritis, $340,357,390$

cause of lumbar pain, 8I

of pain in legs and feet, 357

spinal arthritis, $9 \mathrm{I}$

radiation from, cause of axillary pain, 293

spondylitis, 83

Hypertrophy and dilatation of heart, 50 , 52,301

Hypochlorhydria, 170, 255, 630, 633

Hypochondriac pain, left, 245

right, 209

Hysteria, 58, 391, 472, 504, 506, 513, 516, $61_{5}, 672,75 \mathrm{I}$

cause of convulsions, $5 \mathrm{It}$

and epilepsy, ${ }_{516} 6$

minor, 240

Hysteric affection of spine, I ro

polypnea, 705

suggestibility, $5 \mathrm{I} 8$

ICTERUS neonatorum cause of jaundice, 73I

Iliac pain, left, $28 \mathrm{I}$

right, 263

Impaction, fecal, $43 \mathrm{I}$

Incarcerated uterus, I 24

Incomplete miscarriage, 658

Indigestion, 45I

cause of epigastric pain, 157

of headache, 32,33

Indurative headache, 33,35

Infantile paralysis, 397

spasm cause of convulsions, 5 II

Infarct, renal, 87

Infection, acute, of hip, 374

cause of headache, 35

colon bacillus, ro5

endocardial, 473 
Infection, gall-bladder, 403

of gastro-intestinal tract, 666

general, III, I2O, 2 II

hydatid, 217

in uremia, 501

influenzal, of small bronchiectasis, 308

of liver, $48 \mathrm{I}$

localized, 459

perirenal, cause of right hypochondriac

pain, 208

pharyngeal, 457

pneumococcus, 454

general, 443

of puberty, 453,477

pyogenic, 370

general, 369

renal, 87,100, 101, 254, 414, 424, 69 I

cause of right hypochondriac pain, 208

"scattering," cause of long fever, 409

staphylococcus, 66

streptococcus, 37

terminal, 359

unknow $\mathrm{n}, 55,96,297,45 \mathrm{I}$

urinary, 106, 575

Infectious arthritis, 387

cause of long fever, 409

of pain in legs and feet, 357

of hip, 374

of sacro-iliac joint, Io9

of spine, 109

cause of lumbar pain, $8 \mathrm{I}$

cellulitis, 338

disease cause of dyspnea, $70 \mathrm{r}$

of lumbar pain, $8 \mathrm{r}, 86$

of pain in legs and feet, 357

onset, cause of headache, 33,35

of vomiting, 622

\section{endocarditis, 709}

osteo-arthritis, acute, I 20

polyarthritis, acute, 334

spondylitis, 109, I12

thrombosis, 338

Inflammation, pain due to, 334

Influenza, 55, 93, 96, 140, 298, 407, 420, 45I,

473, 492, 517, 572, 579, 619, 666

cause of cough, 588

of long fever, 409

of short fever, 410

Influenzal infection of small bronchiectases, 308

Injury, history of, sometimes misleading, 345

Insolation cause of headache, 35
Inspiratory and expiratory dyspnea, 73I

Insufficiency, aortic, 3 or

myocardial, 62, 715, 724

Intensity of jaundice, 733

Intercostal neuralgia, 323

cause of axillary pain, 293

Interlobar empyema, 423, 604

Intermittent claudication cause of pain in legs and feet, 357

Interpretation of nervousness, 744 symptoms and their, 29

Interstitial hepatitis, chronic, 736

nephritis, chronic, 522, 524, 659, 723, 724, 755

Intestinal cancer, $149,162,281$

recurrent, 148

colic, 156

hyperperistalsis, 156

obstruction, 52, 153, I54, I64, 202, 204

cause of epigastric pain, $\mathrm{I} 57$

of general abdominal pain, I 3 I

of vomiting, 622

chronic, I37, I69, I9I, 67 I

stricture, $\mathrm{I} 77$

Intracranial hemorrhage, traumatic, cause of coma, 499

Intrathoracic tumor, 342

Irritation, pleural, 594

Ischiorectal abscess, $374,406,489$

JACKSONIAN epilepsy, 398, 535

Janeway, Dr. E. G., 6r 5

Jaundice, 729

catarrhal, 2 I 4, 462, 639, 73I, 733, 735, 737,

738,742

chronic hemolytic, 548

intensity of, 733

painless, 730

symptoms associated with, 730

types and causes of, 729

Jaw, fractured, cause of coma, 499

Johns Hopkins Hospital report, 488

Jeint, Charcot, 389

infection of left sacro-iliac, ro9

of shoulder, 344

Joints, syphilitic disease of, 37 I

KIDNEY abscess, II3

congenital cystic, $87,117,225,250,260$

floating, 229, 277, 652

group of backaches, 83,87

hematogenous infections of, $87,69 \mathrm{I}$ 
Kidney neoplasm, 87, 213, 225, 258, 260, Liver, infection of, 48I $283,685,692,693,696,697$

papillary cystadenoma of, 690

pus in, 314

tuberculous, 314

stone in. See Nephrolithiasis.

"surgical," I I4

tuberculous, $60,87,114,213,225,247$, $258,260,276,283,682,684,686,689$, 691,696

cause of hematuria, 679

tumor. See Renal tumor.

Knee, septic, 389

sprained, cause of pain in legs and feet, 357

\section{Laryngitis, acute, 720}

Lead colic, I33

Lead-poisoning, 60, 87, III, I34, I42, I46, I53, I60, I63, I69, I72, I77, I78, I80, 246, 26I, 299, 397, 434, 528, 538, 565, $568,5^{82}$

cause of epigastric pain, 157

of general abdominal pain, I3I

Le Count, Dr., 498, 5 or

Left hypochondriac pain, 245 iliac pain, 28I

Leg bones, sarcoma of, cause of pain in legs and feet, 357

Legs and feet, pain in, 357

Lenhartz's diet, Dr. H. F. Hewes' modification, of, 257

Leukemia, 74, 25I, 256, 259, 286, 322, 416, $46 \mathrm{I}, 478,685$

cause of long fever, 409

lymphoid, I 46

myeloid, 25I, 566

Leukocytosis, 476

Lipoma, 393

"Lithemia," 35

Lithiasis. See Stone.

Liver abscess, 90, 234, 235, 322, 470, 483

acute yellow atrophy of, $74 \mathrm{I}$

amyloid metamorphosis, 322

cancer of. See Liver, malignant disease of. cirrhosis of, $147,190,200,217,229,236$, 250, 322, 56I, 625, 66I, 739, 742

sudden death in, 502

congestion of, 200

displacement of, 225

enlargement of, 322

fatty metamorphosis, 322

hydatid cyst of, 225, 234

malignant disease of, 160, 201, 215, 233, 376, 4I6, 56!, 734, 74I

passive congestion of, 200, 322

syphilis of. See Syphilis, hepatic.

"torpidity of," 35

tumors of, 233

Lobar pneumonia, 318, 319

cause of coma, 499

"Localized" infections, 459

"Long fevers," 407, 409

Lord, F. T., 607

Lovett and Reynolds, $8 \mathrm{I}$

Lumbago, 37, 81, 83, 87, 92, 98, го4, І1о, II 2 , II 5 , II 6 , I 21,300

cause of lumbar pain, 8I

and sciatica, 402

Lumbar neuralgia, 87

neuritis, 87

pain, 80

causes of, $8 \mathrm{I}$

due to aneurysm, I Io

to fatigue, $8 \mathrm{r}$

to functional causes, I Io

to infectious disease, 86

to osteo-arthritis, I Io, 368

to parturition, 86

infectious group, 93

orthopedic group. See Backache, orthopedic group.

postoperative, 86,88

pressure group, 87 , I10, I I I I 20, I 2 I

psychoneurotic, 85

renal group, 92

Lung, abscess of, 222, 470, 577, 590, 600, . 606

cause of coma, 499

malignant disease of, 434,594

syphilitic disease of, 613

Lymphangitis, 338

cause of short fever, 410

Lymphoid leukemia, 146

Lymphoma, malignant, $35^{2}$

Malaria, 4I, 49, 55-57, 6I, 67, 75, 76, 78, I93, 4I $5,420,435,47 \mathrm{I}, 476,480,487$, $647,657,659,685$

atypical forms, 654

cause of general abdominal pain, I3I

estivo-autumnal, 428,464

in New England cause of chills, 468

tertian, I 23, I50, 298, 652, 655, 736

Malarial poisoning, chronic, 548 
Malignant disease, $43,60,346,348$ of chest-wall, 316, 320, 324 of kidney, 213, 258, 283, 684 of liver, 160, 20r, 215, 233, 376, 416, $56 \mathrm{r}, 734,74 \mathrm{I}$

of lung, 434, 594

of mediastinal glands, 434, 594

of pleura, 434, 594

growth in or near spinal column, 87,89 , 92

lymphoma, 352

Malingering, $4 \mathrm{II}$

Mastoiditis, suppurative, cause of coma, 499

McGuire, Dr. Stewart, $65^{\circ}$

McKenzie, James, 29

Measles, $45^{\circ}$

Mediastinal glands, malignant disease of, $335,434,594$

tumor, 333,353

cause of brachial pain, 329

Melancholia, 198

Meningismus, 314, 488 complicating typhoid, I 24

Meningitis, $56,70,73,78,123,365,397,439$, $443,453,488,490,5^{1} 3,5^{26}, 528,534$, $535,655,75 \mathrm{I}$

acute, 295

cause of coma, 495, 499

of convulsions, 5 I I

of headache, 33

of long fever, 409

epidemic, 58, 449, 5 19

tuberculous, $54,57,58,76$, I6r, 656

Menopause, 748

artificial, 306

Menstruation cause of headache, 33, 35

vicarious, $32 \mathrm{I}$

Mesenteric adenitis, 363

gland, tuberculosis of, 363,435

tabes, I3I, 268, 364, 375

Metamorphosis, amyloid, of liver, 322

fatty, of liver, 322

Metastatic hypernephroma, $35^{\circ}$

Metatarsalgia, Morton's, cause of pain in legs and feet, 357

Methemoglobinemia, 40

Migraine, 38, 39, 40, 47, 62

Miliary tuberculosis, 57, 75, 243, 308, 462, 6ri, 706

acute, cause of coma, 499

Miscarriage, attempted, 626

incomplete, 658
Mitral and aortic valves, fibrous endocarditis of, 219

disease, 505

cause of cough, 588

endocarditis, $37 \mathrm{I}$

stenosis, 52, 669, 709, 725

Morphin, 675

Morphinism, 99, 549, 645

Morton's metatarsalgia cause of pain in legs and feet, 357

Motion, its relation to pain, 27

Mucous colitis, 130, r33, 263, 273

Multilocular ovarian cyst, 286

Muscular lesions, 349

pains, 334

strain, I 2 I

Myelitis, 569

acute, 397

Myeloid leukemia, 251, 566

Myocardial insufficiency, 62, 724

weakness cause of cough, 588

Myocarditis, 310, 709

chronic fibrous, 625,719

fibrous, 723

Myositis, chronic, 37

septic, $33^{8}$

Myxedema, 4r, 554, 584

NEOPLASM, II I, 34I

of arm and shoulder cause of brachial pain, 329

of kidney, $87,685,69$ I, 692

mediastinal, 335

pelvic, 386

retroperitoneal, cause of right hypochondriac pain, 208

spinal, 87 , r 20

Nephritis, 52, 62, r6I, 204, 442, 485, 528, $55^{2}, 655,683,688,746$

acute, 723

cause of headache, 33,47

of hematuria, 679

chronic, $258,353,638,653,686,69 \circ$

cause of dyspnea, 7 or

of hematuria, 679

glomerulo-, 50, 6I, 475, 723

interstitial, $\mathbf{5 2 2}, \mathbf{5 2 4}, \mathbf{6 5 9}, 723,724,755$

suppurative, 749

Nephrolithiasis, 59, 60, 87, I13, I21, 212 ,

257, 276, 283, 36r $, 643,655,69 \mathrm{I}, 695$

cause of hematuria, 679

of lumbar pain, 8I

of right hypochondriac pain, 208 
Nephrolithiasis, psoas spasm due to, 36 I

"Nervous chills," 469

Nervous exhaustion, 662

Nervousness, 420,744

cause of chills, $4 \leqslant 8$

interpretation of, 744

Neuralgia, 70, 325, 336, 337

brachial, 342,343

cause of brachial pain, 329

intercostal, 323

cause of axillary pain, 293

lumbar, 87

trigeminal, cause of headache, 33, 35

"Neuralgic" headache, 72

pain, 26

Neurasthenia, I $16,537,632$

Neurasthenic affection of spine, I Io headache, $49,55,68$

Neuritis, 95, 99, 107, 202, 388

alcoholic, 379, 566,568

cause of pain in legs and feet, 357

brachial, 343

cause of paralysis, 544

lumbar, 87

peripheral, 548, 55 I

saturnine, 566

with herpes zoster, 366

Neurosis, I30, I36, I4I, I54, I62, I65, I80, $213,252,275,316,648,675$

cause of epigastric pain, 157

and constipation, 636,637

functional, 269

of spine, 120

gastric, $165, \mathbf{1 9 2}, \mathbf{1 9 3}, 26 \mathrm{I}, 628,635,644$, $646,653,668,674$

cause of epigastric pain, ${ }_{5} 5$

of general abdominal pain, I3I

of vomiting, 622

occupation, cause of brachial pain, 328 , 329

postoperative, 138

traumatic, 33I, 34I, 644, 753

New-growth of kidney, 225, 260, 692, 696, 697

pelvic, 368

renal. See New-growth of kidney.

New-growths cause of lumbar pain, xro

Nocturnal epilepsy, 520

OBesity, 554

resulting evils of, 379

Obstruction, chronic intestinal, I37, I69, I9I, 67I, 674
Obstruction, intestinal, 52, I53, I54, I64, 202, 204

cause of epigastric pain, 157

of general abdominal pain, I3I

Occupation, effect of, its relation to pain, 27

neurosis cause of brachial pain, 328, 329

Opium-poisoning, 232

cause of coma, 495

Orchitis, syphilitic, I 75

Orthopedic group of backaches, 83, 87, 92, 93, I03, I 20, I 22

Osler on "Urticarial Lesions," 456

Osteitis deformans, 49

tuberculous, 390

Osteo-arthritis, I 22, 387

acute, i ro

infectious, I 20

cause of lumbar pain, iro

spinal, 87,98, п 6,368

Osteomyelitis, 66, 67

cause of pain in legs and feet, 357

of humerus cause of brachial pain, 329

septic, 340, 344-346, 389

of humerus, 340

of rib, 324

tuberculous, 339, 34I, 346, 389

of rib, 324

Otitis media, $295,459,476,477,525$

cause of coma, 499

of headache, 35

Ovarian cyst, $27 \mathrm{I}, 273,286$

ruptured, 270

strangulated, 287

with twisted pedicle, 267, 269, 286

cause of left iliac pain, 280

of right iliac pain, 263

tumor, 262

Overwork, 748

Oxalic acid poisoning, 660

Oxaluria, renal irritation from, $\mathbf{6 9 3}$

cause of hematuria, 679

Paget's disease, 49

Pain, 24

abdominal, 26

anginal, four occasions for, $35^{\circ}$

anginoid, 26

in arms, 328

axillary, 293

"burning," 227

causes of general abdominal, I3I

darting, 26

degree of, 25 
Pain, diaphragm dome, 30

dome, 30

due to inflammation, 334

epigastric, 157

evidences of, 24

functional lumbar, I Io

general abdominal, $\mathbf{x}_{3} \mathbf{r}$

considerations on, 24

on diagnosis of, 290

habit, 29, 86, 32 I

in left hypochondrium, 245

in legs and feet, 357

left iliac, $28 \mathrm{r}$

lumbar, 8o, 8r, 86

muscular, 334

nerve, 334

neuralgic, 26

radiations of, 30

its relation to effect of emotional excitement, 27

of motion, 27

of occupation, 27

of season and weather, 28

to poison of body, 27

to taking of food, 28

to time of day, 27

relief of, 28

rhythmically recurring, 26 .

right hypochondriac, 209

iliac, 262

shooting, 26

theories regarding its production, 29

thoracic, 26

throbbing, 26

types of, 26

with a sense of constriction, 26

Painless jaundice, 730

Pal, J., 29, 519

Pancreas, cancer of, $189,228,234,74^{\circ}$ cause of jaundice, $73 \mathrm{I}$

Pancreatitis, acute, I 80

cause of epigastric pain, 157

chronic, 189

Papillary cystadenoma of kidney, 690

Papilloma, 690

Paralysis, causes of, 544

circumflex, 339

general, $68,70,527,532,536,75^{6}$

infantile, 397

Paranephric abscess, 87

Paraplegia, ataxic, cause of paralysis, 544

Paratyphoid, 465

Parkinson's disease cause of paralysis, 544
Paroxysmal tachycardia, 62, 63

Parturition, 125, 539

cause of lumbar pain, 86

Passive congestion of liver, 200, 322

Paul, W. E., and G. L. Walton, 32

Pelvic adhesions cause of left iliac pain, 263

of right iliac pain, 208

new-growth, 368,386

peritonitis, 176

thrombosis, 373

Pelvis, fractured, $\mathbf{3 7 2}$

Peptic ulcer, I73, I77, I78, I85, I88, I93, I99, 202, 204, 227, 232, 250, 256, 630, $633,635,643$

cause of epigastric pain, 156,157

of vomiting, 622

perforated, $90, \mathrm{I} 80,28 \mathrm{I}$

Perforative colitis, I5 I

peritonitis, $137,146,153,197,676$

Pericardial effusion, 209, 318

and dilated heart, difference between, 3 I 8

Pericarditis, I80, I86, 317, 709

acute, $\mathrm{I} 82$

adhesive, 52, 220, 719, 723

cause of epigastric pain, 157

Pericecal tuberculosis, 148, 237, 262, 265,

434

Perinephric abscess, 87, 93, 322, 419 cause of lumbar pain, 8I .

Periosteal lesions, 349

Periostitis, 344

syphilitic, 42, 403, 404

cause of headache, 33

of pain in legs and feet, 357

Peripheral neuritis, $548,55^{\mathrm{I}}$

thrombosis, 373

Perirectal abscess, 4 I9

Perirenal infection cause of right hypochondriac pain, 208

Peristalsis (visible), 3 I 2

Peritonitis, I03, I77, I80, 659, 663

acute perforative, 676

general, I5 I, I 53

cause of general abdominal pain, $\mathrm{I}_{3} \mathrm{I}$

pelvic, 176

perforative, $137,146,153,197$

tuberculous, $136,144,154,161,175,177$, I9I, 202, 25I, 3I9, 427, 435, 464, 488,

$562,575,580$

cause of coma, 499

of general abdominal pain, I3 I 
Pernicious anemia, $148,152,551,560,581$, Pneumonia cause of cough, 588

585,754

Pertussis, 549

"Petit mal," 540

Pharyngeal infection, 457

Pharyngitis, acute, cause of short fever, 410 cause of cough, $5^{88}$

Phlebitis, 346

cause of pain in legs and feet, 357

Phlegmonous gastritis, 480

inflammation of face cause of coma, 499

Phthisis, I35, 221, 233, 302, 306, 308, 320, $32 \mathrm{I}, 347,4 \mathrm{I} 7,42 \mathrm{I}, 436,47 \mathrm{r}, 478,479$, 578, 592, 598, 600, 604, 608, 6ro, 6r5, 6г7-6r9, 663, 687, 7×5, 746, 747

cause of coma, 499

of chills, 468

of cough, 588

of dyspnea, 7 or

pneumonic, 604

Piles, 550

Plastic pleurisy, chronic, 570

Pleura, endothelioma of, $75^{2}$

malignant disease of, 434, 594

Pleural adhesions, I88, 307

dropsy, 3 I 8

effusion, 303, 304, 305, 310, 359, 447, 609, $667,717,753$

purulent, 322

irritation, 594

thickening, $6_{5}$

chronic, 248

Pleurisy, 90, III, I 25, I 83,2 ro, 255, 256, 259, 297, 298, 315, 317, 321, 376, 453, 490, 557, 596, 597, 600

cause of axillary pain, 293

of cough, 588

chronic plastic, 321,570

double, $48 \mathrm{I}$

tuberculous, 432,483

Pleurodynia, 297, 323, 327

Pleuropericardial adhesions, I88

Plumbism. See Lead-poisoning.

Pneumococcus arthritis, 381 infection, 454

general, 443

Pneumonia, 86, 90, 102, I23, 183, 210, 255, 292, 296, 298, 303, 308, 310, 381, 405, $415,437,439,443,449,450,452,455$, 483, 487, 492, 549, 559, 596, 603, 605, $647,655,665$

cause of axillary pain, 293

of chills, 468 of dyspnea, 7 or

of short fever, 4 ro

central, $4 \dot{5} 5$

convalescence from, $\mathbf{5 7 1}$

lobar, 3I8, 423

cause of coma, 499

traumatic, 608

unresolved, 65

diagnosis made at the Massachusetts

General Hospital, 445

Pneumothorax, tubercular, 89, 302

Poisoning, arsenical, 569

cause of coma, 499

chronic malarial, 548

drug, 746

lead-. See Lead-poisoning.

opium-, 232

oxalic acid, 660

"ptomain," 305, 45 I, 644, 666

sodium phosphate, 548

tea-, 748

Poisons of fatigue, 32

Poliomyelitis, 424

cause of paralysis, 544

of short fever, 410

epidemic, 569

Polyarthritis, acute infectious, 334

Polycythemia, 4I

Polypnea, hysteric, 705

Position of body, relation of pain to, 27 and nature of headache, 38

Postepileptic exhaustion cause of coma, 495

Postoperative lumbar pain, 8r, 86

neurosis, 138

shock cause of vomiting, 622

Postpneumonic empyema, 64, 423, 604, 605, 7 I 7

Postural group of backaches, 80

Pott's disease, 87, 99, I00, I08, I I०, I I I, 353, 364

Pregnancy, I 24, 262, 266, 273, 555, 626, 634 extra-uterine, 266 cause of general abdominal pain, I3I of right iliac pain, 263

toxemia of, cause of vomiting, 622

vomiting of, 667

Presenting symptom, I 7

Pressure group of backaches, 83,87 , Iro, I I I, I 20, I 2 I

Prolapsed uterus, I 24

Prostate, cancer of, 68I

cause of coma, 499 
Prostatitis, chronic, 87

Pseudoleukemia, 286

Psoas spasm due to nephrolithiasis, 36r, tear, 363

Psychic cause of headache, 48, 74 causes in gastro-intestinal disease, 166 origin, headache of, 48,74 trauma, 328

Psycho-analysis, 378

Psychoneurosis, I 20, 134, 138, 182, 230, 371, $387,549, \mathbf{5 7 3}, 745$

cause of headache, 33 of right iliac pain, 263

Psychoneurotic lumbar pain, 85

"Ptomain poisoning," 305, 45 I, 644, 666

Puberty, infection of, 453,477

Puerperal sepsis cause of coma, 499

Pulmonary abscess, 222, 470, 577, 590, 600, 606

cause of coma, 499

disease, 714

edema, acute, 310

tuberculosis. See Phthisis.

tumor, 6r9

Pupillary changes, 68

Purpura hemorrhagica, 321

\section{Pus-kidney, 314}

Pus-tube, 574

cause of left iliac pain, 280

of right iliac pain, 263

Pyelitis, IOI

Pyelonephritis, 482

Pyloric adhesions, 177

cause of epigastric pain, 157

spasm, 156

stenosis, 650

Pyogenic infection, 370

$$
\text { general, } 369
$$

sepsis cause of chills, 468

Pyonephrosis, 87, 225, 69 r

cause of right hypochondriac pain, 208

with stone, 246

Pyosalpinx, 262, 272

Radiations of pain, 30

Rectal cancer, 152,585

Recurrent intestinal cancer, 148

Recurring pain, rhythmic, 26

Regurgitation, aortic, cause of cough, 588

stenosis and, 708

mitral stenosis and, 709, 725

tricuspid, 5 I 5
Relapsing fever, 4I 2

Relief of pain, 28

Renal abscesses, II 3

colic, $87, \mathbf{1 4 6}$

cyst, 87, II 7 225, 246, 260

disease, 120, 122, 20I, 220, 227, 310, 442

group of lesions, III

lumbar pain, 92

infarct, 87

infection, IOO, IOI, IO2, 254, 4I 4, 424

cause of right hypochondriac pain, 208

hematogenous, 87,93 , 100, $69 \mathrm{I}$

irritation from oxaluria, 693

lesions, I IO, I 22, 363

neoplasm. See Neoplasm of kidney.

new-growth. See Nerw-growth of kidney.

stasis, $5^{2}$

stone. See Nephrolithiasis.

cause of lumbar pain, $8 \mathrm{I}$

suppuration cause of lumbar pain, 8I

tuberculosis. See Kidney, tuberculosis of.

tumor, 276,688

cause of hematuria, 679

of lumbar pain, 82

Retroperitoneal glands, tumors of, $20 \mathrm{r}$

neoplasms cause of right hypochondriac pain, 208

sarcoma, Ir 6

tumor, 225

cause of lumbar pain, $8 \mathrm{r}$

Retroverted uterus, 124

Reynolds, E., 8I

Rheumatic arthritis, 67, 37I, 390

"Rheumatic headache," 36,43

Rheumatism, 37, 67, 210, 334, 350, 370, 380

aneurysm called, 333

diseases frequently diagnosed as, 334

sciatic, 368

Rhythmic recurring pain, 26

Rib, broken, 293, 296

cause of coma, 499

cervical. $332,342,343,346$

cause of brachial pain, 329

septic osteomyelitis of, 324

tuberculous osteomyelitis of, 324

Rickets, 49, 4I4, 526

Right hypochondriac pain, 209

iliac pain, 262

Rose spots, 553

Round-shoulder deformity, 348

"Rum-fits," 513, 522

Ruptured ovarian cyst, 270 
SACRO-ILIAC arthritis, I 82

backache, 80

disease, $87,92,98$, I04, I 10, I22, 387

cause of lumbar pain, 8I

joint, infectious arthritis of left, Io9 lesion, 368

lesions cause of right hypochondriac pain, 208

strain, $97,98,40$ r

Salicylate, delirium from, 370

Salpingitis cause of short fever, 4 ro

tuberculous, 668

Sarcoma, III

of femur, 389

of humerus, 344,345

of leg bones cause of pain in legs and feet, 357

retroperitoneal, II 6

of testis with metastases, 174

Saturnine neuritis, 566

"Scattering" infections cause of long fevers, 409

Schmidt, Rudolf, 31,87

"Sciatic rheumatism," 366

Sciatica, 365, 366, 402

cause of pain in legs and feet, 357

primary, 387

Sclerosis, lateral, cause of paralysis, 544

Sea-sickness cause of vomiting, 622

Season and weather, their relation to pain, 28

Secondary anemia, $153,55^{\circ}$

Senility cause of coma, 499

Sensation, disturbances of, 29

Sepsis, 49, 93, 372, 400, 408, 435, 437, 445, $447,453,659$

cause of jaundice, $73 \mathrm{I}$

of long fever, 409

puerperal, cause of coma, 499

pyogenic, cause of chills, 468

staphylococcus, $\mathbf{5 7 6}$

streptococcus, 457

with thrombi, 312

Septic infection. See Sepsis.

knee, 389

myositis, 338

osteomyelitis, $340,344,345,346,389$

of humerus, 340

of rib, 324

thrombosis, 418

Septicemia. See Sepsis.

Serous pleurisy, 305, 7I7

"Shingles," 87
"Shingles" cause of lumbar pain, 8I

"Shock," postoperative, cause of vomiting, 622

Shooting pain, 26

Short fevers, 409, 4II

Shoulder deformity, round-, 348

Shoulder-joint, arthritis of, 344

"Sick-headache," 5 I

Sigmoid, cancer of, $285,287,63 \mathrm{x}$

cause of left iliac pain, 280

Sinusitis, 43, 56, 70, 72

cause of headache, 33

of short fever, 410

Skull, fracture of base of, 55

cause of coma, 499

"Slow fever," 305

Softening, cerebral, 78

Spasm, arterial, 30

infantile, cause of convulsions, 5 I I

psoas, due to nephrolithiasis, $36 \mathrm{I}$

Spinal aneurysm, I 20

arthritis, hypertrophic, 9 I radiations from, cause of axillary pain, 293

column, disease of, 324

malignant growth in or near, $87,89,92$, I 20

curvature, 386

disease, 104

osteo-arthritis, 87,98 , I 6

tuberculosis, 87, 93, I08-II0, II7, I19, I22, I38, I 87, 348, 579

cause of general abdominal pain, I3I of lumbar pain, 8I

Spine, functional affection of, I Io neurosis of, I 20

infectious arthritis of, 109

cause of lumbar pain, $8 \mathrm{I}$

osteo-arthritis of lumbar, 368

Spleen, obsolete tuberculosis of, 50

tumor of, 252

Splenic enlargement with anemia, 567

flexure, cancer of, 256,259

Spondylitis, 297

acute, 120

hypertrophic, 83

infectious, I09, II 2

typhoidal, II 7

Sprain, acute, of back, cause of lumbar pain, 8I

Sprained ankle cause of pain in legs and. feet, 357

knee cause of pain in legs and feet, 357 
Spurious fevers, $4 \mathrm{II}$

Staphylococcus infection, 66 sepsis, 576

Starvation, 324

Stasis, 319, 6r6 renal, 52

Stenosis, aortic, 708 mitral, 52, 669, 709, 725 pyloric, 650

Stiff neck, 37

Stokes-Adams' disease, $443,503,529,53 \mathrm{I}$ Stomach, cancer of. See Cancer, gastric. tumor. See Gastric tumor. ulcer of. See Gastric ulcer.

Stone in bladder, $283,688,689,697$ in common duct, 228, 236 gall-. See Cholelithiasis. in kidney. See Nephrolithiasis. pyonephrosis with, 246 renal. See Nephrolithiasis. in ureter, 268, 272 cause of left iliac pain, 280 of right hypochondriac pain, 208

Strabismus, 69 iliac pain, 263

Strain, acute foot, 378 back, 97 eye-, cause of headache, 33, 43, 68 muscular, $12 \mathrm{I}$

sacro-iliac, 97, 98, 40I

Strangulated ovarian cyst, 287

Streptococcus bronchopneumonia, 598 infection, 37

fever in, $4 \mathrm{I} 3$

meningitis, 123

sepsis, 3 I 2,457

Stricture, intestinal, 177

Subacromial bursitis, 335, 339, 34I, 342, 344

cause of brachial pain, 328,329

Subdiaphragmatic abscess, I37, 233, 322, 483

cause of right hypochondriac pain, 208

Sudden death in cardiac cases, 507

Suggestibility, hysteric, 5 I 8

Sunstroke cause of coma, 495

Suppurative nephritis, 749

Surgical kidney, II4

Symptom, presenting, $\mathbf{I}$

Symptoms associated with jaundice, 730

and their interpretation, 29

Syncope cause of coma, 495
Syphilis, $44,46,55,74,76,78,120, \mathrm{I} 36, \mathrm{I} 4 \mathrm{I}$, 159, 182, I91, 21 7, 218, 220, 238, 250, $344,361,376,388,391,392,395,415$, 421, 427, 430, 455, 461, 479, 508, 515, $540,560,562,638,619,75 \mathrm{I}$ cause of long fever, 409 cerebral, 58

\section{cerebrospinal, 397}

hepatic, 189, 201, 224, 739, 74I of liver. See Syphilis, hepatic.

visceral, 485

with stenosis of a bronchus, 598

Syphilitic adenitis, 285

disease of joints, $37 \mathrm{I}$

of lung, $6 \mathbf{r}_{3}$

gumma, hepatic, I59

headache, 44

heart and aorta, 300

orchitis, 175

periostitis, 42, 403, 404

cause of headache, 33

of pain in legs and feet, 357

Tabes dorsalis, $134,164,180,185,202,247$, $261,274,370,393^{\circ}, 530,548,566$

cause of pain in legs and feet, 357

of paralysis, 544

of vomiting, 622

with gastric crises, I3r, I69, 643, 655, 664

mesenterica, 268, 364, 375

cause of epigastric pain, ${ }_{57} 7$

of general abdominal pain, $\mathrm{I}_{3} \mathrm{I}$

peritonitis cause of epigastric pain, 157

Tachycardia, paroxysmal, 62, 63

Tapeworm, I67

Tea-poisoning, 748

Teething, $5^{26}$

Temperament, its influence on the reaction against infections, 474

Tenosynovitis cause of pain in legs and feet, 357

Terminal infection, 359

fevers, 4I3

Tertian malaria. See Malaria, tertian.

Testis, sarcoma of, with metastases, 174

Tests to make in puzzling cases of headache, 40

Tetanus, 126

Thickening, chronic pleural, 248

pleural, 65

Thomas, H. M., 32

Thoracic aneurysm, 319, 347 
Thoracic pain, 26

Throat, disease of, $32 \mathrm{I}$

Throbbing pain, 26

Thrombophlebitis, 342

Thrombosis, infectious, 338

pelvic, 373

peripheral, 373

septic, of lateral sinus and jugular vein, 418

Thrombus fevers, $4 \mathrm{I} 3$

Thyroid, simple adenoma of, 50

Time of day, relation of pain to, 27

Tinea versicolor, 230

Tonsillitis, 49, 93, 295, 313, 399, 533

cause of cough, 588

of short fever, 4 IO

"Torpid liver," 35

Toxemia and pregnancy cause of vomiting, 622

Toxic fevers, $4 \mathrm{I} 3$

Trauma, 258

headache due to, 47

psychic, 328

Traumatic neurosis, 331, 34I, 644, 753 pneumonia, 608

Trichiniasis, $67,338,746$

Tricuspid regurgitation, 5I5

Trigeminal neuralgia cause of headache, 33,35

Tubal abscess, 267

Tube, purulent infection of, 574

tuberculosis of right, 272

Tuberculosis, 43, 49, 75, 77, 273, 340, 361, $382,388,389,391,396,420,427,429$, 439, 461, 465, 477, 490, 548, 556, 572, 573, 594, 599, 6о2, 604, 614, 617-619, $638,649,653,659,660,666,706,755$, 756

abdominal, 268

acute, 606

of bladder, 284, 682, 684, 694

of bones, $35 \mathrm{I}$

cause of coma, 499

of long fever, 409

of pain in legs and feet, 357

of cecal region, 265

costal, 324

general, 364, 375

genito-urinary, $683,689,746$

glandular, 343,479

of hip, 375

of humerus, 339, 342

of kidney. See Kidney tuberculosis.
Tuberculosis of mesenteric gland, 363,435 miliary, 57; 75, 243, 308, 462, 611, 706 acute, cause of coma, 499

obsolete, of spleen, 50

pericecal, I48, 237, 262, 265, 434

peritoneal. See Tuberculous peritonitis. pulmonary. See Phthisis.

renal. See Kidney, tuberculous.

spinal, 87, 93, I08-I 10, I I 7, I 19, I22, I38, I $87,348,579$

cause of general abdominal pain, I3I of lumbar pain, 8I

of tube, $\mathbf{2 7 2}$

vertebral. See Tuberculosis, spinal.

with abscess, 344

Tuberculous colitis, 148

empyema, 556, 715

enteritis, 260

epididymitis, II 7

kidney. See Kidney, tuberculous.

meningitis, 54, 57, 58, 76, I6I, 656

osteitis, 390

osteomyelitis, 339, 34I, 346, 389

of rib, 324

peritonitis, 136, I44, I59, I6I, 175, I77, I9I, 202, 25 I, 319, 425, 435, 464, 488, $562,575,580$

cause of coma, 499

of general abdominal pain, $\mathrm{I} 3 \mathrm{I}$

pleurisy, 432, 483

pneumothorax, 89, 302

pus kidney, 3I4

salpingitis, 668

Tumor, $332,343,683$

abdominal, I 17,136

cause of general abdominal pain, I3I

of bladder cause of hematuria, 679

brain, $56,62,71,76,78,431,507,521,528$,

$534,539,628,653,655,657,75$ I

cause of coma, 495

of headache, 33, 47

cerebral. See Tumor, brain.

fibroid, of uterus, I 76, 267

gastric, II 7

gummatous, $35^{\circ}$

intrathoracic, 342

of kidney. See Tumor, renal.

of liver, 233

mediastinal, 333,353

cause of brachial pain, 329

ovarian, 262

pulmonary, 6ro

renal, 276,688 
Tumor, renal, cause of hematuria, 679 of lumbar pain, 8I

retroperitoneal, 225

cause of lumbar pain, 8I

of retroperitoneal glands, $20 \mathrm{I}$

of spleen, $25^{2}$

stomach. See Gastric tumor.

Types and cause of hematuria, 678

of jaundice, 73I

of pain, 26

Typhoid, 49, 53, 57, 58, 66, 73, 76, 78, 86, 92, 97, 109, 124, 137, 139, 298, 305, 307, $365,408,417,419,421,431,435,437$, $439,443,450,475,486,490,549,553$, $575,577,612,647,657,666$

abortive, $45^{2}$

\section{afebrile, $43 \mathrm{I}$}

\section{brief, 428}

cause of long fever, 409

chills occurring in, 488

cholecystitis, 214, 242

diarrhea and tenderness due to fecal impaction in, 432

meningismus complicating, I 24

onset cause of chills, 468

with relapse, 426

Typhoidal spondylitis, I I 7

\section{UICER of bowel, 87}

chronic, 653

peptic, 173, I77, I78, I85, I88, I93, I99, $202,204,227,232,250,256,6.30,633$. 635,643

cause of epigastric pain, 156, I 57 of vomiting, 622

of duodenum, I33, 142, I62, 166, I69, I 70, I $72,179,216,238$

of stomach, $87,160,190,194,629,650$, $66 \mathrm{I}, 695,75 \mathrm{I}$

perforated gastric, $90,180,281$

Ulcerative endocarditis, 94,428

Unknown cause of hematuria, 692, 695

infection, 55, 96, 297, 45 I

headache of, 69

Unlocalized and wide-spread infections, 2 II

Unresolved pneumonia, 65

diagnoses made at the Massachusetts

General Hospital, 445

Uremia, 3I, 56, 62, 7I, 204, 520, 524, 539, 658,673

cause of coma, 495, 499

of convulsions, 5 II

of vomiting, 622
Uremia, infection in, 5or

Uremic headache, 50

Ureter, stone in, 268, 272

cause of right hypochondriac pain, 208

Urethritis, 349, 394, 397

Urinary infection, 106, 575

Urticaria, internal, 617

Urticarial fever, 455

lesions, 74

discussed by Osler, $45^{6}$

Uterine fibroid, 176,267

group of backaches, 80

Uterus, carcinoma of, $\mathbf{3 8 7}$

fibroid tumor of, I 76,267

fibromyoma of, 287

prolapsed, retroverted, incarcerated, pregnant, I 24

Valves, fibrous endocarditis of mitral and aortic, 219

Valvular disease, chronic, 506

Varicose veins, 286

cause of pain in legs and feet, 357

Vascular crisis, 3I, 184, 393, 440, 52I, 522, 532

hyperemia, 26

Vasomotor headaches, 26, 37

Vertebræ, fracture of, cause of coma, 499

Vertebral tuberculosis. See Tuberculosis, spinal.

Vicarious menstruation, 32I

Visceral syphilis, 485

Volvulus, 153

Vomiting, 623 important factors in production of, 624 of pregnancy, 667

Vulnerability of all differential diagnosis, ig

Walton, G. L., 32

Weak heart, 309

Weakness, 545

causes of, 544

Weather and season, relation to pain, 28

"Wet brain," 78

Wide-spread and unlocalized infections, 2 I I

"Writer's cramp," 535

Yellow atrophy of liver, acute, 74I

Zoster, herpes, $87,95,366$ cause of lumbar pain, $8 \mathrm{I}$ 





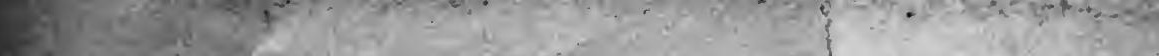

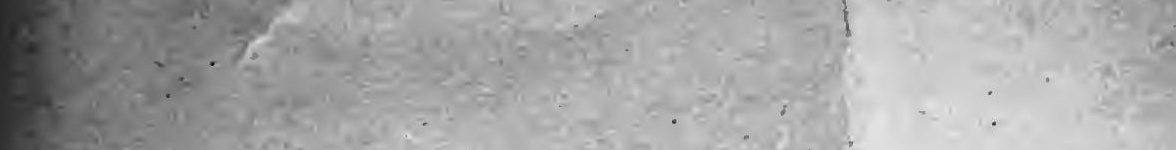

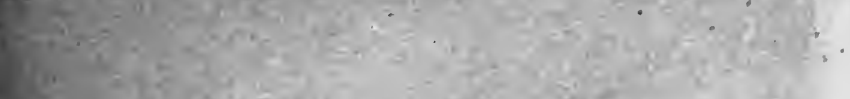

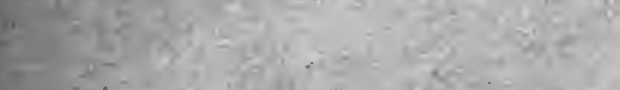

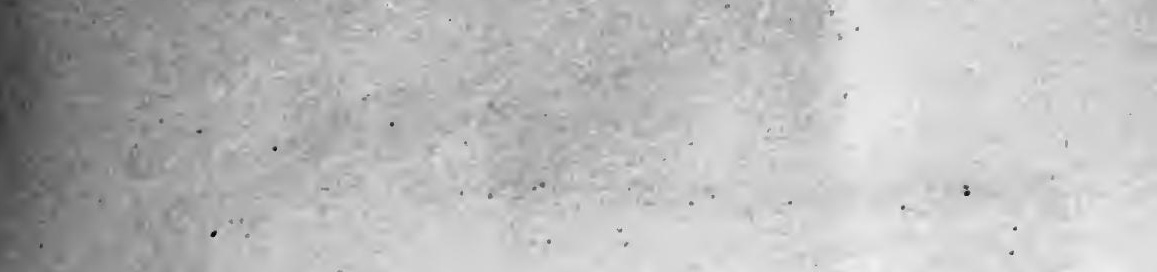

$x^{2}+2=$

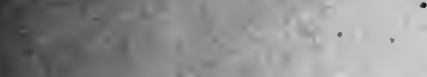

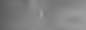

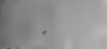

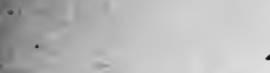





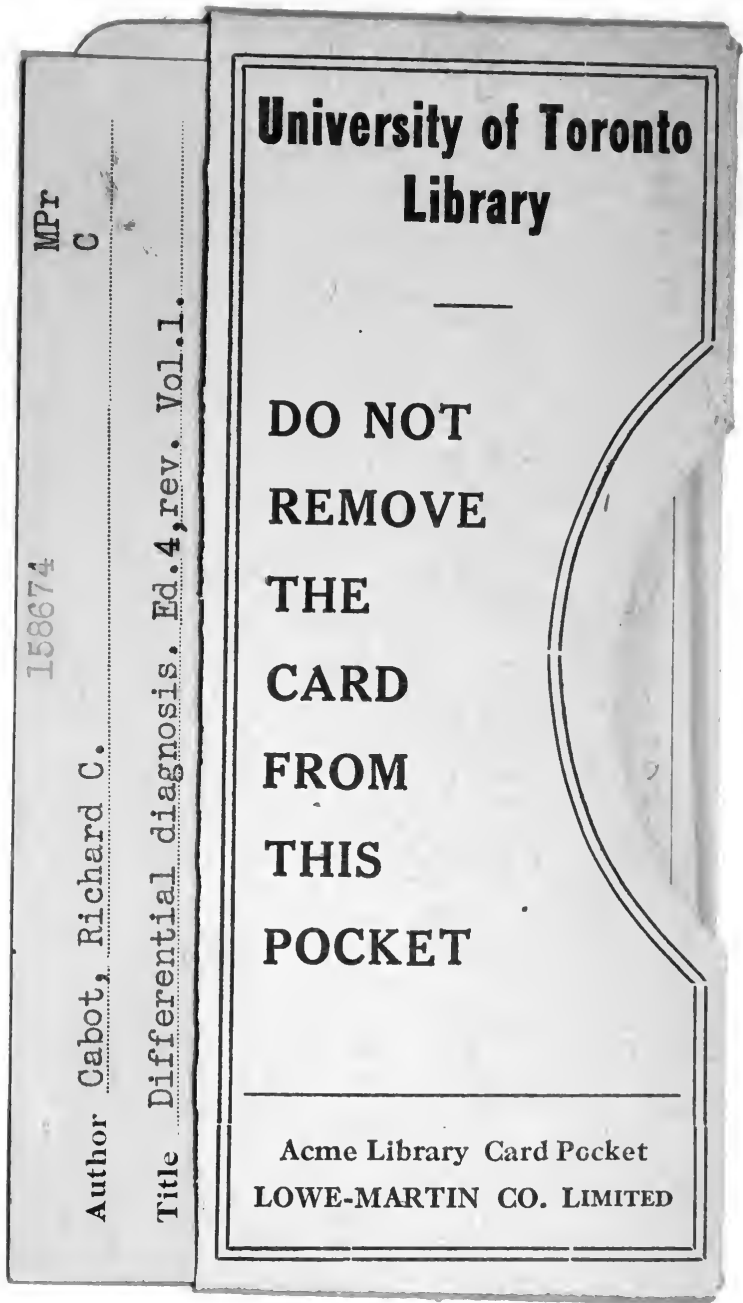


